\title{
Stereoselective Functionalization of Racemic Cyclopropylzinc Reagents via Enantiodivergent Relay Coupling
}

\author{
Lun An, ${ }^{1, \uparrow}$ Fei-Fei Tong, ${ }^{1, \uparrow}$ Shu Zhang $^{2}$ and Xingang Zhang ${ }^{1, *}$ \\ ${ }^{1}$ Key Laboratory of Organofluorine Chemistry, Center for Excellence in Molecular Synthesis, Shanghai Institute of \\ Organic Chemistry, University of Chinese Academy of Sciences, Chinese Academy of Sciences, 345 Lingling Road, \\ Shanghai 200032, China. \\ ${ }^{2}$ School of Materials and Energy, University of Electronic Science and Technology of China, 2006 Xiyuan Avenue, \\ West High-Tech Zone, Chengdu, Sichuan 611731, China. \\ ‘'Lun An and Fei-Fei Tong contributed equally.
}

Correspondence to: xgzhang@mail.sioc.ac.cn 


\section{Table of Contents}

1. General information and materials 4

2. Optimizations of nickel-catalyzed enantioselective cross-coupling of racemic cis-cyclopropylzinc reagent $( \pm$ ) cis-1a with gem-difluoropropargyl bromide 2a (Tables S1-S4) ...... .5

3. Synthesis of racemic cis-cyclopropylzinc reagents $( \pm)$ cis-1 and alkyl electrophiles 2 .................7

3.1. Preparation of racemic $c i s$-cyclopropylzinc reagents $( \pm)$ cis-1 ......................................................... 7

3.1.1 General procedure for the synthesis of 2,2-dibromocyclopropanes (General Procedure A)................ 7

3.1.2 General procedure for the synthesis of $c i s$-bromocyclopropanes (General Procedure B)................... 11

3.1.3 General procedure for the preparation of cyclopropylzinc reagents $( \pm$ ) cis-1 (General Procedure C) 16

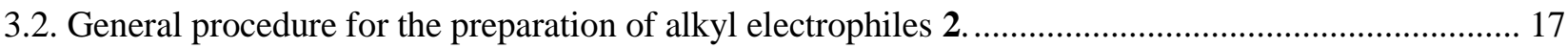

3.2.1 General procedure for the preparation of gem-difluoropropargyl bromides (General Procedure D).. 17

3.2.2 General procedure for the preparation of secondary propargyl bromides (General Procedure E) ...... 17

4. Control experiments to determine the origin of the stereoselectivity in the nickel-catalyzed coupling. .22

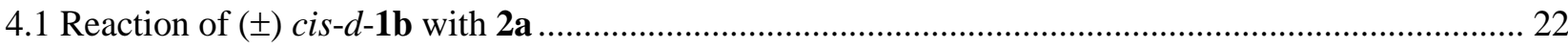

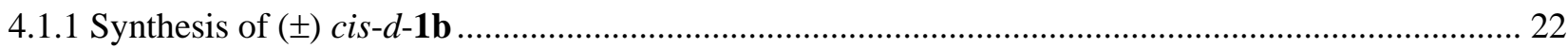

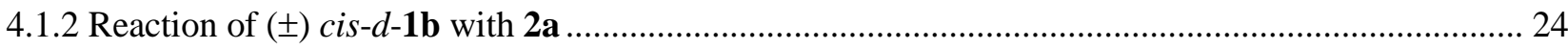

4.2 Reactions of $\mathbf{1 l}$ with 2a using either enantiomer of $i \operatorname{Pr}-\mathrm{PyB}$ ox as the chiral ligand............................. 25

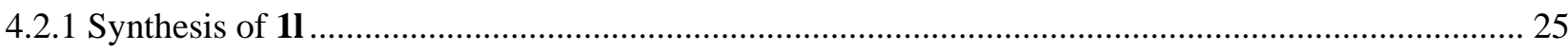

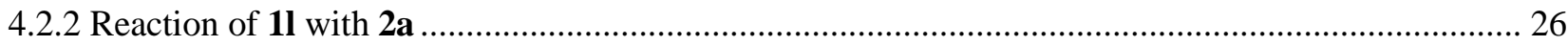

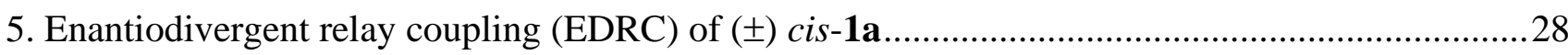

6. Ni-catalyzed enantioselective cross-coupling of racemic cyclopropylzinc reagents with alkyl bromides 31

6.1 General procedure for $\mathrm{Ni}$-catalyzed enantioselective cross-coupling of racemic cyclopropylzinc reagents $( \pm)$ cis-1 with primary propargyl and allyl bromides (General Procedure F)......................... 31

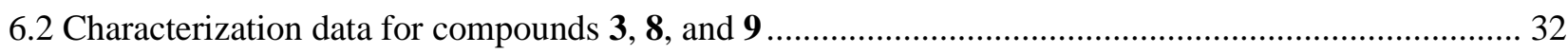

6.3 General procedure for $\mathrm{Ni}$-catalyzed enantioselective cross-coupling of racemic cyclopropylzincs $( \pm)$ cis-1 with secondary propargyl bromides (General Procedure G).................................................... 46

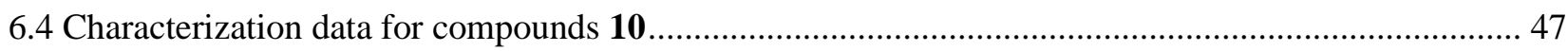

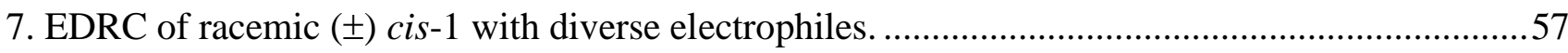


8. Diverse transformations of chiral cyclopropanes ............................................................... 70

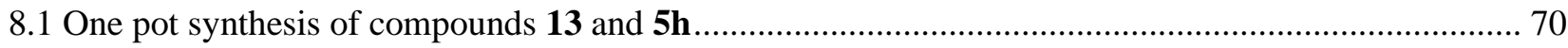

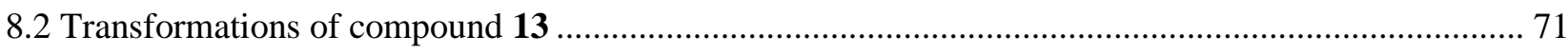

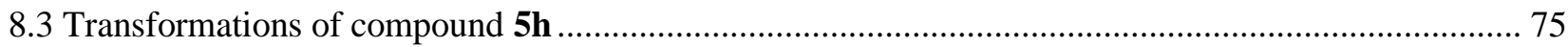

9. Crystal data and structure refinement for compounds $\mathbf{5 a}, \mathbf{5 g}, \mathbf{6}, \mathbf{1 1}, \mathbf{1 2}$, and $\mathbf{1 5 f}$.....................83

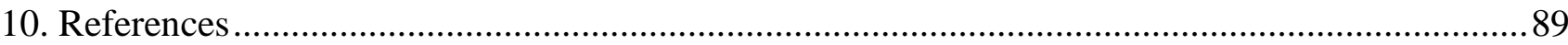

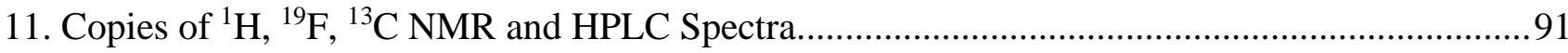




\section{General information and materials}

General Information: ${ }^{1} \mathrm{H}$ NMR and ${ }^{13} \mathrm{C}$ NMR spectra were recorded on a Bruker AM 400 and Agilent MR 400 spectrometer. ${ }^{19} \mathrm{~F}$ NMR was recorded on an Agilent MR 400 spectrometer $\left(\mathrm{CFCl}_{3}\right.$ as an external standard and low field is positive). The chemical shifts $(\delta)$ are given in parts per million (ppm) relative to $\mathrm{CDCl}_{3}\left(7.26 \mathrm{ppm}\right.$ for $\left.{ }^{1} \mathrm{H}\right)$ or $\mathrm{TMS}\left(0 \mathrm{ppm}\right.$ for $\left.{ }^{1} \mathrm{H}\right)$ and $\mathrm{CDCl}_{3}\left(77.0 \mathrm{ppm}\right.$ for $\left.{ }^{13} \mathrm{C}\right)$, and coupling constants $(J)$ are reported in Hertz $(\mathrm{Hz})$. The following abbreviations were used to explain the multiplicities: $\mathrm{s}=$ singlet, $\mathrm{d}=$ doublet, $\mathrm{t}=$ triplet, $\mathrm{dd}=$ doublet of doublets, $\mathrm{m}=$ multiplet, br $=$ broad, app. $\mathrm{t}=$ apparent triplet. NMR yield was determined by ${ }^{19} \mathrm{~F}$ NMR using fluorobenzene as an internal standard before working up the reaction mixture. Mass spectra was recorded by National Center for Mass Spectrometry in Shanghai Institute of Organic Chemistry. High performance liquid chromatography was performed on Waters 2487-600E, Waters ACQUITY UPC2, and Agilent Series HPLC, using PC-1, PC-3, PA-2, ID-3, IB, IG, ADH, OJH chiral column eluted with a mixture of acetonitrile/water or hexane/isopropyl alcohol. Optical rotation was measured on Rudolph-Autopol I and Autopol VI. Flash column chromatography was performed on silica gel (particle size 200-300 mesh) and eluted with petroleum ether/ethyl acetate (EtOAc) or petroleum ether/dichloromethane (DCM).

Materials: All reagents were used as received from commercial sources unless specified otherwise, or prepared according to literatures as described below. $\mathrm{ZnCl}_{2}(1.0 \mathrm{M}$ solution in $\mathrm{THF}$, MkSeal) was purchased from Shanghai Macklin Biochemical Co., Ltd. Anhydrous THF was purchased from J\&K Scientific Ltd (Tetrahydrofuran, 99.9\%, Super-Dry, stabilizer free, J\&K sealed bottle). 
2. Optimizations of nickel-catalyzed enantioselective cross-coupling of racemic cis-cyclopropylzinc reagent $( \pm)$ cis-1a with gem-difluoropropargyl bromide 2a (Tables S1-S4)

Table S1. Ligand effect on the reaction ${ }^{a}$

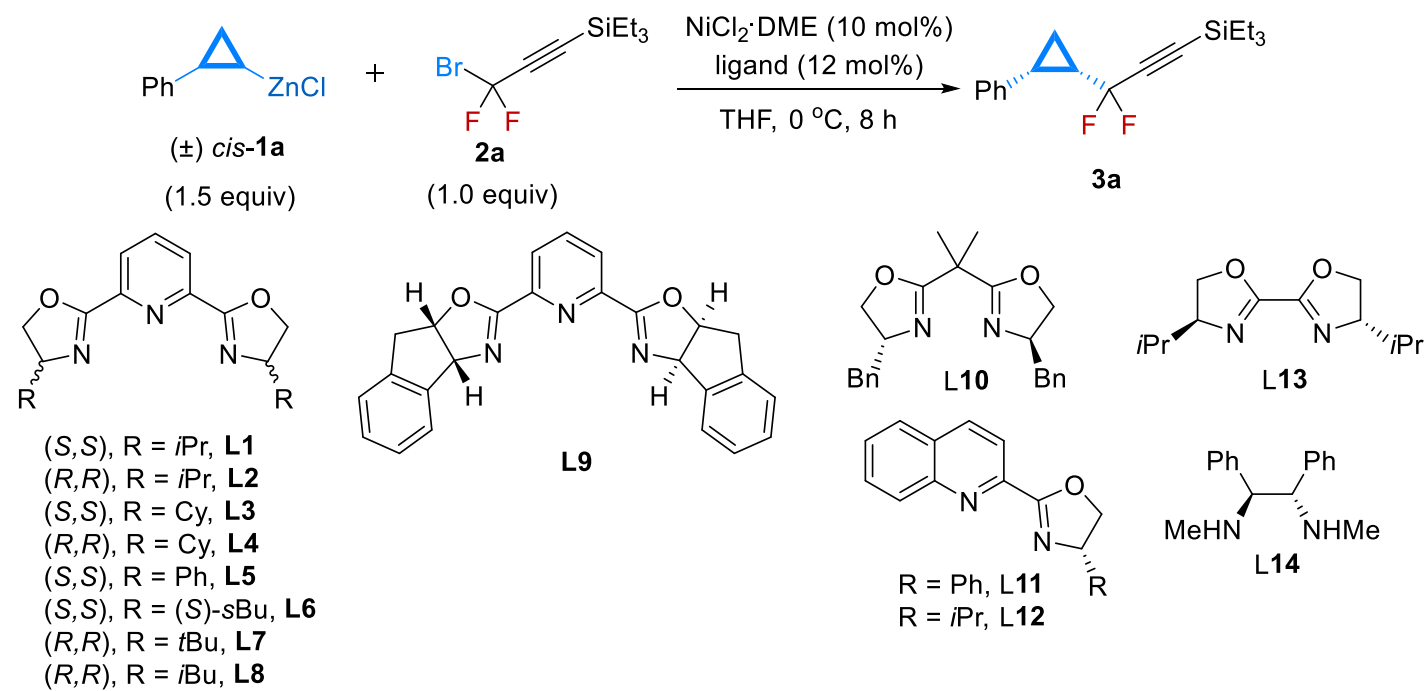

\begin{tabular}{cccc}
\hline Entry & Ligand & 3a, Yield $(\%)^{b}$ & 3a, $\mathrm{er}^{c}$ \\
\hline 1 & L1 & 84 & $91: 9$ \\
2 & L3 & 82 & $90: 10$ \\
3 & L5 & 0 & - \\
4 & L6 & 84 & $92: 8$ \\
5 & L7 & 0 & - \\
6 & L8 & 86 & $68: 32$ \\
7 & L9 & 93 & $72: 28$ \\
8 & L10 & 0 & - \\
9 & L11 & 4 & - \\
10 & L12 & 6 & - \\
11 & L13 & 16 & $75: 25$ \\
12 & L14 & 0 & - \\
\hline
\end{tabular}

${ }^{a}$ Reaction conditions (unless otherwise specified): 1a $(0.33 \mathrm{M}$ in THF; $0.45 \mathrm{mmol}, 1.5$ equiv), $2 \mathbf{a}(0.3 \mathrm{mmol}, 1.0$ equiv), THF $(2 \mathrm{~mL}) .{ }^{b}$ Determined by ${ }^{19} \mathrm{~F}$ NMR using fluorobenzene as an internal standard. ${ }^{c}$ Determined by chiral HPLC. 
Table S2. Screening of the nickel sources ${ }^{a}$

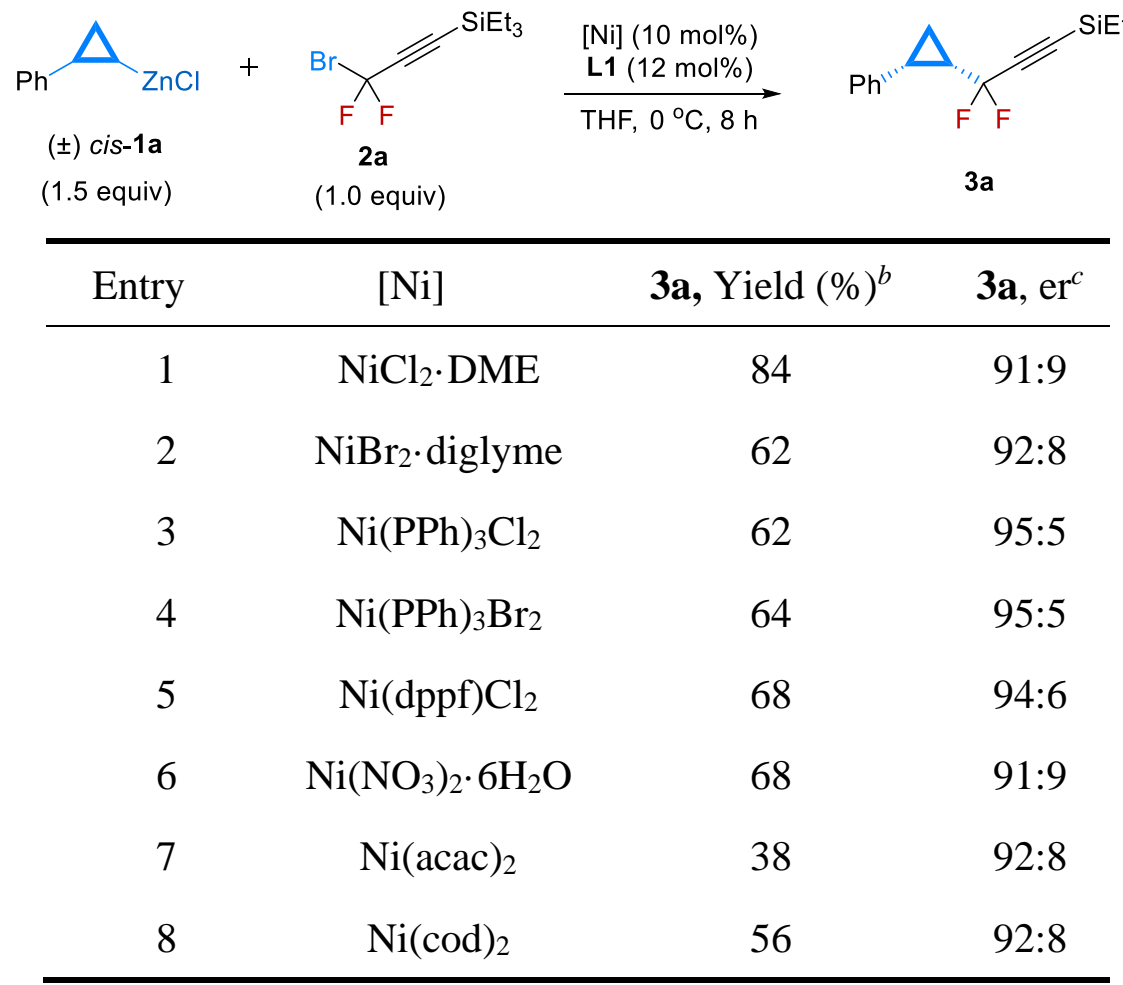

${ }^{a}$ Reaction conditions (unless otherwise specified): $1 \mathbf{a}(0.33 \mathrm{M}$ in THF; $0.45 \mathrm{mmol}, 1.5$ equiv), $2 \mathbf{a}$ (0.3 mmol, 1.0 equiv), THF ( $2 \mathrm{~mL}) .{ }^{b}$ Determined by ${ }^{19} \mathrm{~F}$ NMR using fluorobenzene as an internal standard. ${ }^{c}$ Determined by chiral HPLC.

Table S3. Optimization of reaction temperature ${ }^{a}$

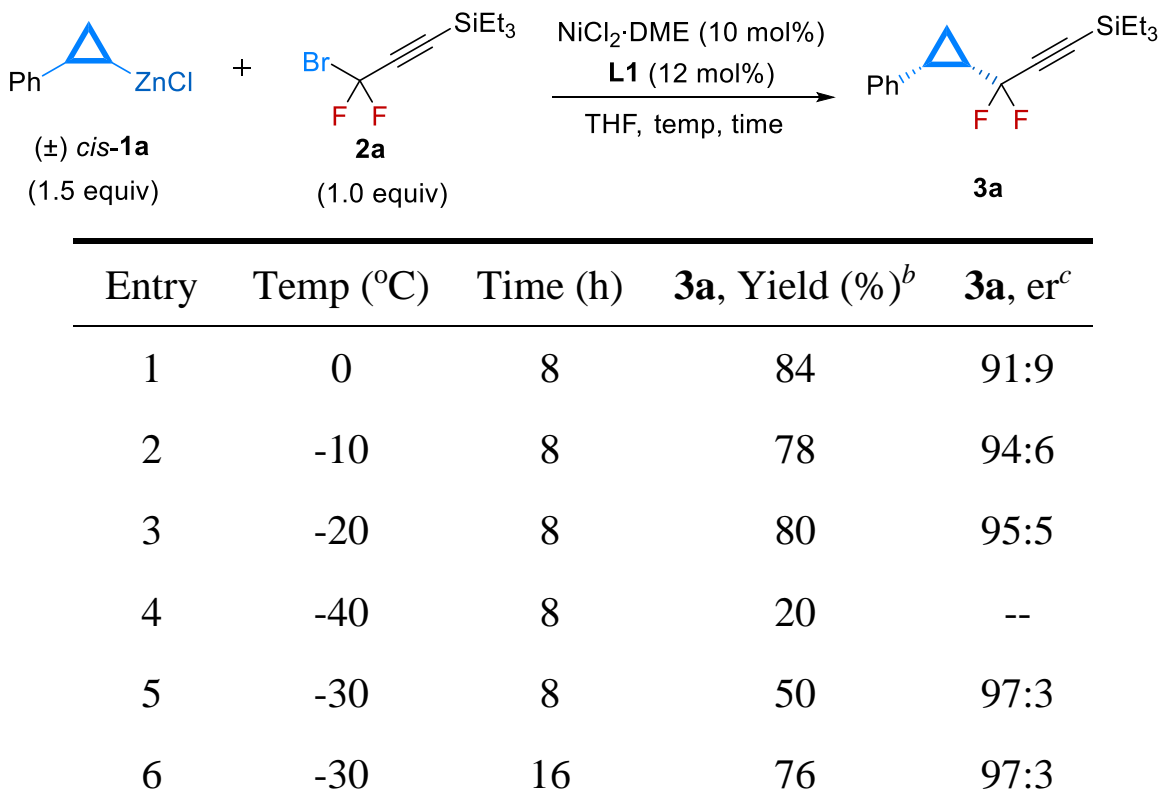

${ }^{a}$ Reaction conditions (unless otherwise specified): $1 \mathbf{a}(0.33 \mathrm{M}$ in THF; $0.45 \mathrm{mmol}, 1.5$ equiv), $2 \mathbf{a}(0.3 \mathrm{mmol}, 1.0$ equiv), THF ( $2 \mathrm{~mL}) .{ }^{b}$ Determined by ${ }^{19} \mathrm{~F}$ NMR using fluorobenzene as an internal standard. ${ }^{c}$ Determined by chiral HPLC. 
Table S4. Optimization of the loading amount of $( \pm)$ cis-1a ${ }^{a}$

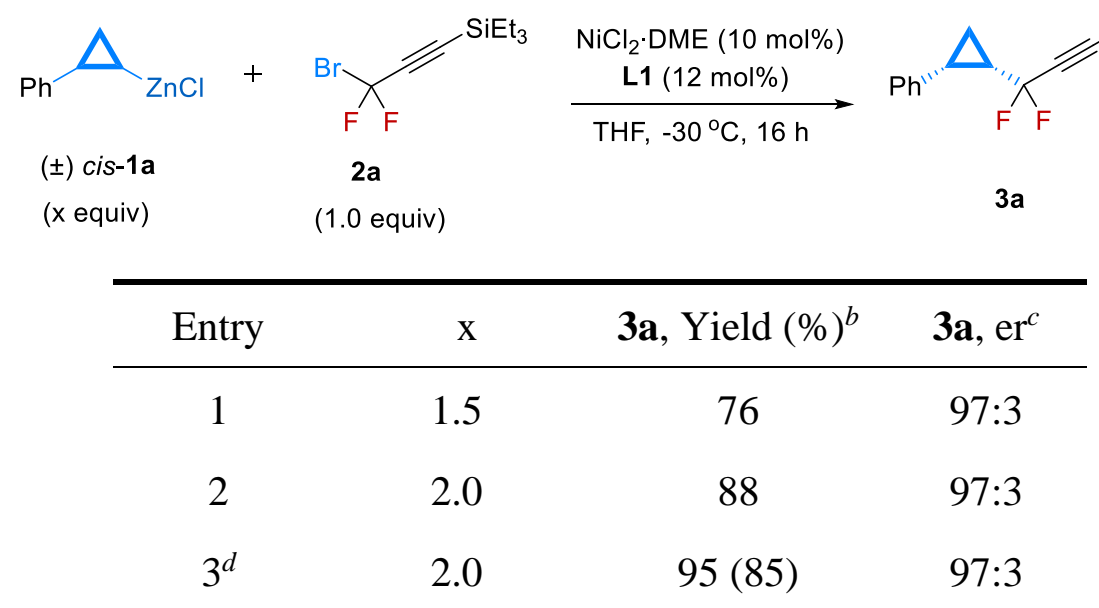

${ }^{a}$ Reaction conditions (unless otherwise specified): 1a ( $0.33 \mathrm{M}$ in THF; $0.45-0.60 \mathrm{mmol}, 1.5-2.0$ equiv), $\mathbf{2 a}(0.3 \mathrm{mmol}, 1.0$ equiv), THF ( $2 \mathrm{~mL}$ ). ${ }^{b}$ Determined by ${ }^{19} \mathrm{~F}$ NMR using fluorobenzene as an internal standard. ${ }^{c}$ Determined by chiral HPLC. ${ }^{d}( \pm)$ cis-1a was added dropwise over 20 minutes.

3. Synthesis of racemic cis-cyclopropylzinc reagents $( \pm)$ cis-1 and alkyl electrophiles 2.

3.1. Preparation of racemic cis-cyclopropylzinc reagents $( \pm)$ cis-1.

\subsubsection{General procedure for the synthesis of 2,2-dibromocyclopropanes (General Procedure A)}

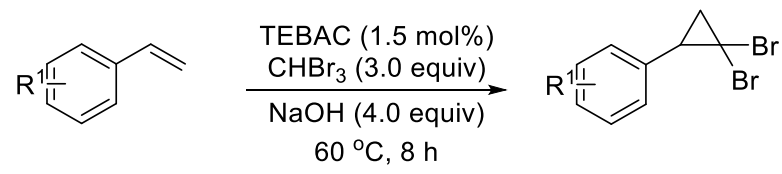

In a $500 \mathrm{~mL}$ round bottom, aqueous $\mathrm{NaOH}(4.0$ equiv, $20.0 \mathrm{M}$ ) was added dropwise to a stirred mixture of alkene (1.0 equiv), bromoform (3.0 equiv) and triethylbenzylammonium chloride (TEBAC) (0.015 equiv) at $0{ }^{\circ} \mathrm{C}$. The reaction mixture was stirred at room temperature for $30 \mathrm{~min}$, and then heated to $60{ }^{\circ} \mathrm{C}$. After stirring at $60{ }^{\circ} \mathrm{C}$ overnight, the reaction mixture was quenched with $\mathrm{H}_{2} \mathrm{O}$ and extracted with $\mathrm{CH}_{2} \mathrm{Cl}_{2}$ (3 times). The combined organic layers were washed with brine, dried over anhydrous $\mathrm{Na}_{2} \mathrm{SO}_{4}$, filtered and concentrated. The residue was purified by distillation or silica gel chromatography to give the pure product. 
(2,2-Dibromocyclopropyl)benzene. [known compound ${ }^{1 \mathrm{a}}$ ]: The reaction was carried out according to the General Procedure A on $200 \mathrm{mmol}$ scale. The product $(43.0 \mathrm{~g}$, $78 \%$ yield) was purified by distillation as a colorless oil. ${ }^{1} \mathrm{H} \mathrm{NMR}\left(400 \mathrm{MHz}, \mathrm{CDCl}_{3}\right) \delta 7.40-7.29$ (m, 3 H), $7.28-7.23$ (m, 2 H), $2.96(\mathrm{dd}, J=10.4,8.4 \mathrm{~Hz}, 1 \mathrm{H}), 2.13(\mathrm{dd}, J=10.5,7.8 \mathrm{~Hz}, 1 \mathrm{H}), 2.01$ (app. t, $J=8.0 \mathrm{~Hz}, 1 \mathrm{H}) .{ }^{13} \mathrm{C} \mathrm{NMR}\left(101 \mathrm{MHz}, \mathrm{CDCl}_{3}\right) \delta 135.9,128.9,128.3,127.6,35.9,28.4,27.2$.

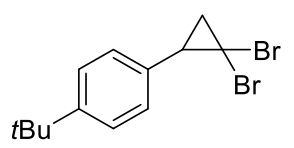

1-(tert-Butyl)-4-(2,2-dibromocyclopropyl)benzene. [known compound $\left.{ }^{2}\right]$ : The reaction was carried out according to the General Procedure A on $100 \mathrm{mmol}$ scale. The product ( $26.5 \mathrm{~g}, 80 \%$ yield) was purified by silica gel chromatography (Petroleum ether) as a colorless oil. ${ }^{1} \mathrm{H}$ NMR (400 MHz, $\left.\mathrm{CDCl}_{3}\right) \delta 7.39(\mathrm{~d}, J=8.2 \mathrm{~Hz}, 2 \mathrm{H}), 7.20(\mathrm{~d}, J=8.1 \mathrm{~Hz}, 2 \mathrm{H}), 2.97$ $-2.87(\mathrm{~m}, 1 \mathrm{H}), 2.13(\mathrm{dd}, J=10.5,7.8 \mathrm{~Hz}, 1 \mathrm{H}), 2.00$ (app. t, $J=8.0 \mathrm{~Hz}, 1 \mathrm{H}), 1.34(\mathrm{~s}, 9 \mathrm{H}) .{ }^{13} \mathrm{C}$ NMR (101 MHz, $\left.\mathrm{CDCl}_{3}\right) \delta 150.5,132.9,128.5,125.2,35.6,34.5,31.3,28.9,27.3$.

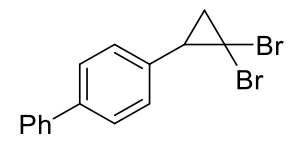

4-(2,2-Dibromocyclopropyl)-1,1'-biphenyl. The reaction was carried out according to the General Procedure A on $65 \mathrm{mmol}$ scale. The product $(18.1 \mathrm{~g}$, $80 \%$ yield) was purified by silica gel chromatography (Petroleum ether) as a white solid (m.p. 70-72 $\left.{ }^{\circ} \mathrm{C}\right) .{ }^{1} \mathrm{H}$ NMR $\left(400 \mathrm{MHz}, \mathrm{CDCl}_{3}\right) \delta 7.67-7.59$ (m, $\left.4 \mathrm{H}\right), 7.50-7.44(\mathrm{~m}, 2 \mathrm{H}), 7.39(\mathrm{~d}, J=7.8 \mathrm{~Hz}, 1$ $\mathrm{H}), 7.35(\mathrm{~d}, J=7.8 \mathrm{~Hz}, 2 \mathrm{H}), 3.05-2.98(\mathrm{~m}, 1 \mathrm{H}), 2.23-2.15(\mathrm{~m}, 1 \mathrm{H}), 2.10-2.04(\mathrm{~m}, 1 \mathrm{H}) .{ }^{13} \mathrm{C}$ NMR $\left(101 \mathrm{MHz}, \mathrm{CDCl}_{3}\right) \delta 140.5,140.4,134.9,129.3,128.8,127.4,127.0,126.9,35.6,28.5,27.4$. MS (DART): m/z (\%) 352, $350\left(\mathrm{M}^{+}\right), 271,273,191$ (100), 192, 165, 115. HRMS: Calculated for $\mathrm{C}_{15} \mathrm{H}_{12} \mathrm{Br}_{2}: 349.9306$; Found: 349.9299.

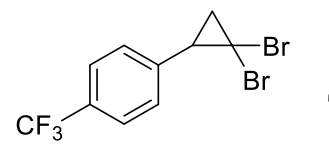

1-(2,2-Dibromocyclopropyl)-4-(trifluoromethyl)benzene. [known compound $\left.{ }^{3}\right]$.

The reaction was carried out according to the General Procedure A on $60 \mathrm{mmol}$ scale. The product (7.6 g, 37\% yield) was purified by silica gel chromatography (Petroleum ether) as a pale yellow oil. ${ }^{1} \mathrm{H}$ NMR $\left(300 \mathrm{MHz}, \mathrm{CDCl}_{3}\right) \delta 7.62(\mathrm{~d}, J=8.0 \mathrm{~Hz}, 2 \mathrm{H}), 7.38(\mathrm{~d}, J=7.9 \mathrm{~Hz}, 2 \mathrm{H})$, 3.00 (appt, $J=9.3 \mathrm{~Hz}, 1 \mathrm{H}), 2.25-2.15$ (m, $1 \mathrm{H}$ ), 2.05 (app. t, $J=8.1 \mathrm{~Hz}, 1 \mathrm{H}) .{ }^{13} \mathrm{C} \mathrm{NMR}(101 \mathrm{MHz}$, $\left.\mathrm{CDCl}_{3}\right) \delta 139.9(\mathrm{q}, J=1.3 \mathrm{~Hz}), 129.8(\mathrm{q}, J=32.6 \mathrm{~Hz}), 129.3,125.3(\mathrm{q}, J=3.8 \mathrm{~Hz}), 124.1(\mathrm{q}, J=$ 272.0 Hz), 35.5, 27.5, 27.0. ${ }^{19} \mathrm{~F} \mathrm{NMR}\left(376 \mathrm{MHz}, \mathrm{CDCl}_{3}\right) \delta-62.6(\mathrm{~s}, 3 \mathrm{~F})$. 


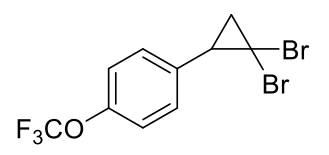

1-(2,2-Dibromocyclopropyl)-4-(trifluoromethoxy)benzene. The reaction was carried out according to the General Procedure A on $50 \mathrm{mmol}$ scale. The product (7.0 g, 39\% yield) was purified by silica gel chromatography (Petroleum ether) as a colorless oil. ${ }^{1} \mathrm{H}$ NMR (400 MHz, $\left.\mathrm{CDCl}_{3}\right) \delta 7.28(\mathrm{~d}, J=8.8 \mathrm{~Hz}, 2 \mathrm{H}), 7.21(\mathrm{~d}, J=8.5 \mathrm{~Hz}, 2 \mathrm{H}), 2.99-2.90(\mathrm{~m}, 1 \mathrm{H})$, $2.17(\mathrm{dd}, J=10.5,7.8 \mathrm{~Hz}, 1 \mathrm{H}), 1.99$ (app. t, $J=8.0 \mathrm{~Hz}, 1 \mathrm{H}) .{ }^{13} \mathrm{C} \mathrm{NMR}\left(101 \mathrm{MHz}, \mathrm{CDCl}_{3}\right) \delta 148.6$ $(\mathrm{q}, J=1.6 \mathrm{~Hz}), 134.6,130.3,120.71,120.70,120.4(\mathrm{q}, J=257.3 \mathrm{~Hz}), 35.2,27.56,27.55 .{ }^{19} \mathrm{~F}$ NMR $\left(376 \mathrm{MHz}, \mathrm{CDCl}_{3}\right) \delta-57.9$ (s, $\left.3 \mathrm{~F}\right) . \mathrm{MS}(\mathrm{EI}): \mathrm{m} / \mathrm{z}(\%)$ 362, $360\left(\mathrm{M}^{+}\right), 358\left(\mathrm{M}^{+}\right), 281,279,200(100)$, 199, 133, 131. HRMS: Calculated for $\mathrm{C}_{10} \mathrm{H}_{7} \mathrm{OF}_{3} \mathrm{Br}_{2}$ : 357.8816; Found: 357.8820.

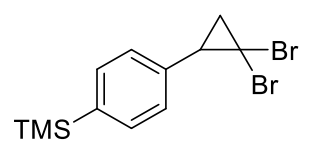

(4-(2,2-Dibromocyclopropyl)phenyl)trimethylsilane. The reaction was carried out according to the General Procedure A on $43.7 \mathrm{mmol}$ scale. The product $(6.8 \mathrm{~g}$, $43 \%$ yield) was purified by silica gel chromatography (Petroleum ether) as a colorless oil. ${ }^{1} \mathrm{H}$ NMR $\left(300 \mathrm{MHz}, \mathrm{CDCl}_{3}\right) \delta 7.51(\mathrm{~d}, J=7.8 \mathrm{~Hz}, 2 \mathrm{H}), 7.23(\mathrm{~d}, J=7.6 \mathrm{~Hz}, 2 \mathrm{H}), \delta 2.67$ (app. t, $J=9.4 \mathrm{~Hz}, 1$ H), 1.86 (dd, $J=10.4,7.8 \mathrm{~Hz}, 1 \mathrm{H}), 1.75$ (app. t, $J=8.0 \mathrm{~Hz}, 1 \mathrm{H}), 0.27$ (s, $9 \mathrm{H}) .{ }^{13} \mathrm{C}$ NMR $(101 \mathrm{MHz}$, $\left.\mathrm{CDCl}_{3}\right) \delta 139.8,136.4,133.2,128.2,35.9,28.5,27.3,-1.1 . \mathrm{MS}$ (DART): m/z (\%) 366, 364 $\left(\left[\mathrm{M}+\mathrm{NH}_{4}\right]^{+}\right), 301,299$ (100). HRMS: Calculated for $\mathrm{C}_{12} \mathrm{H}_{20} \mathrm{NBr}_{2} \mathrm{Si}$ : 363.9726; Found: 363.9724 .

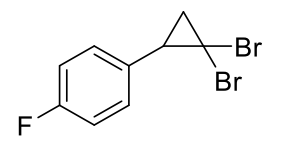

1-(2,2-Dibromocyclopropyl)-4-fluorobenzene. [known compound $\left.{ }^{1 \mathrm{a}}\right]$ : The reaction was carried out according to the General Procedure A on 200 mmol scale. The product (45.0 g, 77\% yield) was purified by distillation as a colorless oil. ${ }^{1} \mathrm{H}$ NMR (400 MHz, $\left.\mathrm{CDCl}_{3}\right) \delta 7.25-7.19(\mathrm{~m}, 2 \mathrm{H}), 7.09-7.01(\mathrm{~m}, 2 \mathrm{H}), 2.96-2.88(\mathrm{~m}, 1 \mathrm{H}), 2.14(\mathrm{dd}, J=10.5,7.8 \mathrm{~Hz}$, $1 \mathrm{H}), 1.96$ (app. t, $J=8.0 \mathrm{~Hz}, 1 \mathrm{H}) .{ }^{13} \mathrm{C} \mathrm{NMR}\left(101 \mathrm{MHz}, \mathrm{CDCl}_{3}\right) \delta 162.1$ (d, $\left.J=246.7 \mathrm{~Hz}\right), 131.8$ (d, $J=3.2 \mathrm{~Hz}), 130.5(\mathrm{~d}, J=8.3 \mathrm{~Hz}), 115.2(\mathrm{~d}, J=21.7 \mathrm{~Hz}), 35.2,28.1(\mathrm{~d}, J=1.4 \mathrm{~Hz}), 27.4 .{ }^{19} \mathrm{~F} \mathrm{NMR}$ $\left(376 \mathrm{MHz}, \mathrm{CDCl}_{3}\right) \delta-114.3--114.5(\mathrm{~m}, 1 \mathrm{~F})$.

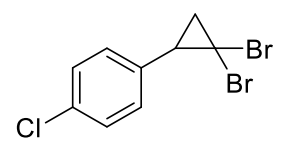

1-Chloro-4-(2,2-dibromocyclopropyl)benzene. The reaction was carried out according to the general procedure A on $180 \mathrm{mmol}$ scale. The product $(34.0 \mathrm{~g}$, $61 \%$ yield) was purified by distillation as a colorless oil. ${ }^{1} \mathrm{H} \mathrm{NMR}\left(400 \mathrm{MHz}, \mathrm{CDCl}_{3}\right) \delta 7.33(\mathrm{~d}, J=$ $8.4 \mathrm{~Hz}, 2 \mathrm{H}), 7.19(\mathrm{~d}, J=8.3 \mathrm{~Hz}, 2 \mathrm{H}), 2.96-2.86(\mathrm{~m}, 1 \mathrm{H}), 2.15(\mathrm{dd}, J=10.5,7.8 \mathrm{~Hz}, 1 \mathrm{H}), 1.97$ (app. t, $J=8.0 \mathrm{~Hz}, 1 \mathrm{H}) .{ }^{13} \mathrm{C} \mathrm{NMR}\left(101 \mathrm{MHz}, \mathrm{CDCl}_{3}\right) \delta 134.5,133.5,130.2,128.5,35.2,27.8,27.4$. 
MS (EI): m/z (\%) 310, $308\left(\mathrm{M}^{+}\right), 275,231,149,115$ (100). HRMS: Calculated for $\mathrm{C}_{9} \mathrm{H}_{7} \mathrm{ClBr}_{2}$ : 307.8603; Found: 307.8606.

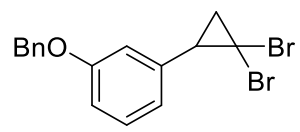

1-(Benzyloxy)-3-(2,2-dibromocyclopropyl)benzene. The reaction was carried out according to the General Procedure A on $71.3 \mathrm{mmol}$ scale. The product (16.3 $\mathrm{g}, 60 \%$ yield) was purified by silica gel chromatography (Petroleum ether/EtOAc $=50: 1$ ) as a colorless oil. ${ }^{1} \mathrm{H}$ NMR (400 MHz, $\left.\mathrm{CDCl}_{3}\right) \delta 7.43(\mathrm{~d}, J=7.2 \mathrm{~Hz}, 2 \mathrm{H}), 7.38(\mathrm{t}, J=7.3 \mathrm{~Hz}, 2 \mathrm{H}), 7.32$ $(\mathrm{t}, J=7.1 \mathrm{~Hz}, 1 \mathrm{H}), 7.29-7.23(\mathrm{~m}, 1 \mathrm{H}), 6.96-6.91(\mathrm{~m}, 1 \mathrm{H}), 6.89-6.83(\mathrm{~m}, 1 \mathrm{H}), 2.92(\mathrm{dd}, J=$ 10.3, 8.5 Hz, $1 \mathrm{H}), 2.10$ (dd, $J=10.5,7.8 \mathrm{~Hz}, 1 \mathrm{H}), 1.97$ (app. t, $J=8.0 \mathrm{~Hz}, 1 \mathrm{H}) .{ }^{13} \mathrm{C}$ NMR $(101$ $\left.\mathrm{MHz}, \mathrm{CDCl}_{3}\right) \delta 158.7,137.5,136.8,129.3,128.6,128.0,127.5,121.5,115.7,113.8,70.0,35.9,28.2$, 27.3. MS (EI): m/z (\%) $382\left(\mathrm{M}^{+}\right), 91$ (100), 77, 65. HRMS: Calculated for $\mathrm{C}_{16} \mathrm{H}_{14} \mathrm{OBr}_{2}: 379.9411$; Found: 379.9403.

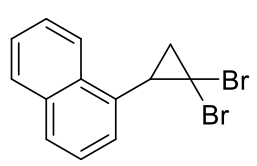

1-(2,2-Dibromocyclopropyl)naphthalene. The reaction was carried out according to the General Procedure A on $75 \mathrm{mmol}$ scale. The product (10.5 g, 43\% yield) was purified by silica gel chromatography (Petroleum ether) as a white solid (m.p. 78-80 $\left.{ }^{\circ} \mathrm{C}\right) .{ }^{1} \mathrm{H}$ NMR $\left(400 \mathrm{MHz}, \mathrm{CDCl}_{3}\right) \delta 8.23(\mathrm{~d}, J=8.4 \mathrm{~Hz}, 1 \mathrm{H}), 7.84(\mathrm{~d}, J=8.2 \mathrm{~Hz}, 1 \mathrm{H}), 7.77(\mathrm{~d}, J=$ $8.2 \mathrm{~Hz}, 1 \mathrm{H}), 7.66-7.58(\mathrm{~m}, 1 \mathrm{H}), 7.55-7.46(\mathrm{~m}, 1 \mathrm{H}), 7.38-7.30(\mathrm{~m}, 1 \mathrm{H}), 7.11(\mathrm{~d}, J=7.1 \mathrm{~Hz}, 1$ $\mathrm{H}), 3.23-3.12(\mathrm{~m}, 1 \mathrm{H}), 2.19(\mathrm{dd}, J=10.3,7.6 \mathrm{~Hz}, 1 \mathrm{H}), 2.10$ (app. t, $J=7.9 \mathrm{~Hz}, 1 \mathrm{H}) .{ }^{13} \mathrm{C}$ NMR $\left(101 \mathrm{MHz}, \mathrm{CDCl}_{3}\right) \delta 133.6,133.4,133.1,128.5,128.3,126.7,126.1,125.6,125.1,124.6,34.6,28.5$, 26.8. MS (EI): m/z (\%) $326\left(\mathrm{M}^{+}\right), 245,165$ (100). HRMS: Calculated for $\mathrm{C}_{13} \mathrm{H}_{10} \mathrm{Br}_{2}$ : 323.9149; Found: 323.9151.

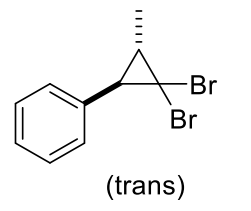

trans-2-Methyl-3-phenyl-1,1-dibromocyclopropane. The reaction was carried out according to the General Procedure A on $67 \mathrm{mmol}$ scale. The product $(11.7 \mathrm{~g}, 60 \%$ yield) was purified by silica gel chromatography (Petroleum ether) as a colorless oil. ${ }^{1} \mathrm{H}$ NMR (400 MHz, $\left.\mathrm{CDCl}_{3}\right) \delta 7.37-7.27(\mathrm{~m}, 3 \mathrm{H}), 7.27-7.19(\mathrm{~m}, 2 \mathrm{H}), 2.45(\mathrm{~d}, J=8.4 \mathrm{~Hz}, 1 \mathrm{H})$, $1.95(\mathrm{dq}, J=8.4,6.2 \mathrm{~Hz}, 1 \mathrm{H}), 1.45(\mathrm{~d}, J=6.2 \mathrm{~Hz}, 3 \mathrm{H}) .{ }^{13} \mathrm{C} \mathrm{NMR}\left(101 \mathrm{MHz}, \mathrm{CDCl}_{3}\right) \delta$ 136.4, 128.6, 128.2, 127.4, 42.2, 38.9, 30.3, 17.4. MS (EI): m/z (\%) 292, 290, $288\left(\left[\mathrm{M}+\mathrm{NH}_{4}\right]^{+}\right), 211,209,131,130$ (100), 129, 115. HRMS: Calculated for $\mathrm{C}_{10} \mathrm{H}_{10} \mathrm{Br}_{2}$ : 287.9149; Found: 287.9147. 


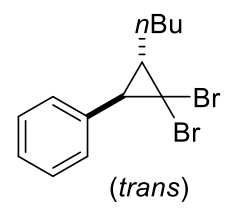

trans-2-Butyl-3-phenyl-1,1-dibromocyclopropane. The reaction was carried out according to the General Procedure A on $68.6 \mathrm{mmol}$ scale. The product $(14.8 \mathrm{~g}, 65 \%$ yield) was purified by silica gel chromatography (Petroleum ether) as a colorless oil. $1.90-1.80(\mathrm{~m}, 2 \mathrm{H}), 1.67-1.50(\mathrm{~m}, 3 \mathrm{H}), 1.43(\mathrm{~h}, J=7.1 \mathrm{~Hz}, 2 \mathrm{H}), 0.95(\mathrm{t}, J=7.2 \mathrm{~Hz}, 3 \mathrm{H}) .{ }^{13} \mathrm{C}$ NMR $\left(101 \mathrm{MHz}, \mathrm{CDCl}_{3}\right) \delta 136.5,128.7,128.2,127.4,41.5,38.0,35.7,32.4,30.4,22.4,14.1 . \mathrm{MS}$ (EI): m/z (\%) $332\left(\mathrm{M}^{+}\right), 277,275,273,129,115$ (100), 91. HRMS: Calculated for $\mathrm{C}_{13} \mathrm{H}_{16} \mathrm{Br}_{2}$ : 329.9619; Found: 329.9618.

\subsubsection{General procedure for the synthesis of cis-bromocyclopropanes (General Procedure B)}

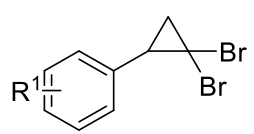

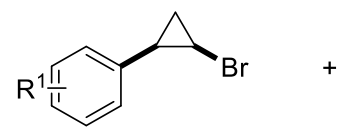

$( \pm)$-cis

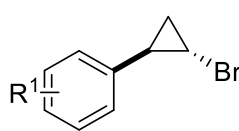

$( \pm)$-trans

Ethylmagnesium bromide (2.0 M in THF; 1.3 equiv) was added dropwise with a dropping funnel to a stirring solution of the 2,2-dibromocyclopropane (1.0 equiv) and $\mathrm{Ti}(\mathrm{O} i \mathrm{Pr})_{4}(10 \mathrm{~mol} \%)$ in anhydrous THF (1.0 M) under argon at $0{ }^{\circ} \mathrm{C}$. The mixture turned dark brown gradually (Note: Slow addition of the ethylmagnesium bromide to the reaction is essential). The reaction mixture was stirred at room temperature for $6 \mathrm{~h}$, and was quenched with $10 \%$ aqueous $\mathrm{HCl}$ solution. THF was removed under reduced pressure and the residue was extracted twice with $\mathrm{CH}_{2} \mathrm{Cl}_{2}$. The combined organic layers were washed with sat. $\mathrm{NaHCO}_{3}$ and brine, then dried over $\mathrm{Na}_{2} \mathrm{SO}_{4}$, filtered and concentrated. The residue was purified by column chromatography on silica gel to afford the cis isomers (Note: Generally, the trans-isomers are less polar than their corresponding cis-isomers. The cis/trans isomers can be readily separated by silica gel column).

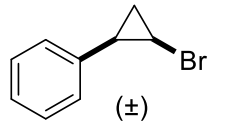

(( \pm )-(cis)-2-Bromocyclopropyl)benzene. [known compound $\left.{ }^{1 \mathrm{a}}\right]$ : The reaction was carried out according to the General Procedure B on 225 mmol scale. The product (15.5 g, 35\% yield) was purified by silica gel chromatography (Petroleum ether) as a colorless oil. ${ }^{1} \mathrm{H}$ NMR (400 MHz, $\left.\mathrm{CDCl}_{3}\right) \delta 7.37-7.30(\mathrm{~m}, 2 \mathrm{H}), 7.29-7.22(\mathrm{~m}, 3 \mathrm{H}), 3.31(\mathrm{td}, J=7.5,4.6 \mathrm{~Hz}, 1 \mathrm{H})$, $2.37-2.26(\mathrm{~m}, 1 \mathrm{H}), 1.63-1.54(\mathrm{~m}, 1 \mathrm{H}), 1.33(\mathrm{td}, J=7.2,4.6 \mathrm{~Hz}, 1 \mathrm{H}) .{ }^{13} \mathrm{C} \mathrm{NMR}(101 \mathrm{MHz}$, $\left.\mathrm{CDCl}_{3}\right) \delta 137.1,129.2,127.9,126.8,24.0,22.1,14.1$ 


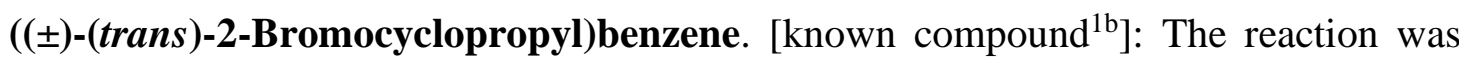

$( \pm) \quad$ carried out according to the General Procedure B on $116 \mathrm{mmol}$ scale. The product (4.7 g, $23 \%$ yield) was purified by silica gel chromatography (Petroleum ether) as a colorless oil. ${ }^{1} \mathrm{H}$ NMR (400 MHz, $\left.\mathrm{CDCl}_{3}\right) \delta 7.35$ - 7.27 (m, $\left.2 \mathrm{H}\right), 7.25$ - 7.18 (m, $\left.1 \mathrm{H}\right), 7.11-7.04$ (m, $\left.2 \mathrm{H}\right), 3.09$ $3.00(\mathrm{~m}, 1 \mathrm{H}), 2.46-2.36(\mathrm{~m}, 1 \mathrm{H}), 1.58-1.42(\mathrm{~m}, 2 \mathrm{H}) .{ }^{13} \mathrm{C} \mathrm{NMR}\left(101 \mathrm{MHz}, \mathrm{CDCl}_{3}\right) \delta 139.8$, $128.5,126.5,126.8,26.8,21.7,18.9$.

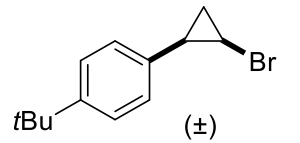

$( \pm)$

1-(( \pm -(cis)-2-Bromocyclopropyl)-4-(tert-butyl)benzene. The reaction was carried out according to the General Procedure B on $30 \mathrm{mmol}$ scale. The product ( $3.1 \mathrm{~g}, 41 \%$ yield) was purified by silica gel chromatography (Petroleum ether) as a colorless oil. ${ }^{1} \mathrm{H}$ NMR (400 MHz, $\left.\mathrm{CDCl}_{3}\right) \delta 7.36(\mathrm{~d}, J=8.2 \mathrm{~Hz}, 2 \mathrm{H}), 7.18(\mathrm{~d}, J=8.1 \mathrm{~Hz}, 2 \mathrm{H}), 3.35-3.27(\mathrm{~m}, 1 \mathrm{H})$, $2.32-2.24(\mathrm{~m}, 1 \mathrm{H}), 1.61-1.54(\mathrm{~m}, 1 \mathrm{H}), 1.36-1.28(\mathrm{~m}, 10 \mathrm{H}) .{ }^{13} \mathrm{C} \mathrm{NMR}\left(101 \mathrm{MHz}, \mathrm{CDCl}_{3}\right) \delta$ 149.5, 134.0, 128.8, 124.8, 34.4, 31.3, 24.2, 21.6, 14.3. MS (EI): m/z (\%) $252\left(\mathrm{M}^{+}\right), 237,173(100)$. HRMS: Calculated for $\mathrm{C}_{13} \mathrm{H}_{17} \mathrm{Br}$ : 252.0514; Found: 252.0507.

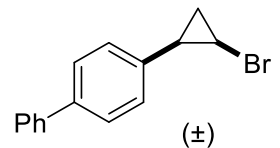

4-((士)-(cis)-2-Bromocyclopropyl)-1,1'-biphenyl. The reaction was carried out according to the General Procedure B on $51 \mathrm{mmol}$ scale. The product $(2.2 \mathrm{~g}, 16 \%$ yield) was purified by silica gel chromatography (Petroleum ether) as a white solid (m.p. 64-65 ${ }^{\circ} \mathrm{C}$ ). ${ }^{1} \mathrm{H}$ NMR (400 MHz, $\left.\mathrm{CDCl}_{3}\right) \delta 7.63(\mathrm{~d}, J=7.7 \mathrm{~Hz}, 2 \mathrm{H}), 7.59(\mathrm{~d}, J=8.2 \mathrm{~Hz}, 2 \mathrm{H}), 7.46(\mathrm{t}, J=7.6 \mathrm{~Hz}$, $1 \mathrm{H}), 7.39-7.31(\mathrm{~m}, 3 \mathrm{H}), 3.36(\mathrm{td}, J=7.6,4.6 \mathrm{~Hz}, 1 \mathrm{H}), 2.41-2.33(\mathrm{~m}, 1 \mathrm{H}), 1.68-1.60(\mathrm{~m}, 1 \mathrm{H})$, $1.38(\mathrm{td}, J=7.1,4.7 \mathrm{~Hz}, 1 \mathrm{H}) .{ }^{13} \mathrm{C} \mathrm{NMR}\left(101 \mathrm{MHz}, \mathrm{CDCl}_{3}\right) \delta 140.8,139.5,136.2,129.5,128.7$, 127.1, 127.0, 126.6, 24.1, 21.8, 14.4. MS (EI): m/z (\%) $272\left(\mathrm{M}^{+}\right), 193$ (100), 178, 165, 115. HRMS: Calculated for $\mathrm{C}_{15} \mathrm{H}_{13} \mathrm{Br}: 272.0201$; Found: 272.0209 .

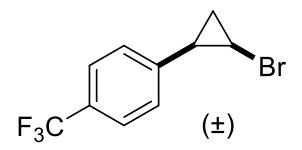

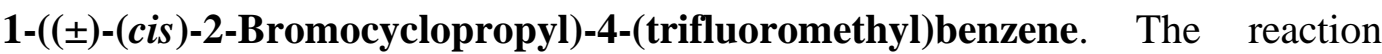
was carried out according to the General Procedure B on $19.1 \mathrm{mmol}$ scale. The product (1.01 g, 20\% yield) was purified by silica gel chromatography (Petroleum ether) as a colorless oil. ${ }^{1} \mathrm{H}$ NMR $\left(400 \mathrm{MHz}, \mathrm{CDCl}_{3}\right) \delta 7.58(\mathrm{~d}, J=8.0 \mathrm{~Hz}, 2 \mathrm{H}), 7.35(\mathrm{~d}, J=8.0 \mathrm{~Hz}, 2 \mathrm{H}), 3.35$ $(\mathrm{td}, J=7.6,4.7 \mathrm{~Hz}, 1 \mathrm{H}), 2.41-2.32(\mathrm{~m}, 1 \mathrm{H}), 1.71-1.61(\mathrm{~m}, 1 \mathrm{H}), 1.37(\mathrm{td}, J=7.2,5.2 \mathrm{~Hz}, 1 \mathrm{H})$. ${ }^{13} \mathrm{C} \mathrm{NMR}\left(101 \mathrm{MHz}, \mathrm{CDCl}_{3}\right) \delta 141.3(\mathrm{q}, J=1.3 \mathrm{~Hz}), 129.5,128.9(\mathrm{q}, J=32.4 \mathrm{~Hz}), 124.8(\mathrm{q}, J=3.8$ 
$\mathrm{Hz}), 124.2(\mathrm{q}, J=271.9 \mathrm{~Hz}), 23.4,21.9,14.7 .{ }^{19} \mathrm{~F} \mathrm{NMR}\left(376 \mathrm{MHz}, \mathrm{CDCl}_{3}\right) \delta-62.4(\mathrm{~s}) . \mathrm{MS}(\mathrm{EI}): \mathrm{m} / \mathrm{z}$ (\%) $264\left(\mathrm{M}^{+}\right)$, 245, 185 (100), 165, 115. HRMS: Calculated for $\mathrm{C}_{10} \mathrm{H}_{8} \mathrm{~F}_{3} \mathrm{Br}$ : 263.9761; Found: 263.9771.

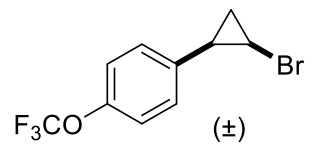

1-((土)-(cis)-2-Bromocyclopropyl)-4-(trifluoromethoxy)benzene. The reaction was carried out according to the General Procedure B on $18.6 \mathrm{mmol}$ scale. The product (1.5 g, 29\% yield) was purified by silica gel chromatography (Petroleum ether) as a colorless oil. ${ }^{1} \mathrm{H}$ NMR (400 MHz, $\left.\mathrm{CDCl}_{3}\right) \delta 7.25(\mathrm{~d}, J=8.5 \mathrm{~Hz}, 2 \mathrm{H}), 7.16(\mathrm{~d}, J=8.6 \mathrm{~Hz}, 2 \mathrm{H}), 3.30(\mathrm{td}, J=$ 7.6, $4.6 \mathrm{~Hz}, 1 \mathrm{H}), 2.33-2.25(\mathrm{~m}, 1 \mathrm{H}), 1.64-1.57(\mathrm{~m}, 1 \mathrm{H}), 1.28(\mathrm{td}, J=7.2,4.6 \mathrm{~Hz}, 1 \mathrm{H}) .{ }^{13} \mathrm{C}$ NMR $\left(101 \mathrm{MHz}, \mathrm{CDCl}_{3}\right) \delta 148.1,135.9,130.5,120.5(\mathrm{q}, J=258.1 \mathrm{~Hz}), 120.394,120.388,23.5$, 21.4, 14.6. ${ }^{19} \mathrm{~F}$ NMR (376 MHz, $\left.\mathrm{CDCl}_{3}\right) \delta$-57.9 (s). MS (EI): m/z (\%) $280\left(\mathrm{M}^{+}\right), 201(100), 115$. HRMS: Calculated for $\mathrm{C}_{10} \mathrm{H}_{8} \mathrm{OF}_{3} \mathrm{Br}: 279.9711$; Found: 279.9705.

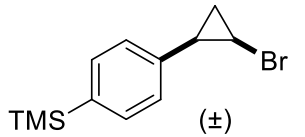

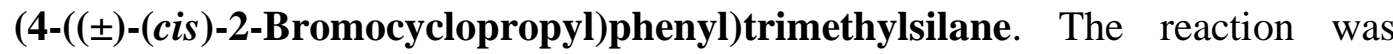
carried out according to the General Procedure B on $16.7 \mathrm{mmol}$ scale. The product ( $1.30 \mathrm{~g}, 30 \%$ yield) was purified by silica gel chromatography (Petroleum ether) as a colorless oil. ${ }^{1} \mathrm{H}$ NMR (400 MHz, $\left.\mathrm{CDCl}_{3}\right) \delta 7.49(\mathrm{~d}, J=8.0 \mathrm{~Hz}, 2 \mathrm{H}), 7.23(\mathrm{~d}, J=7.7 \mathrm{~Hz}, 2 \mathrm{H}), 3.32(\mathrm{td}, J=7.6,4.6$ $\mathrm{Hz}, 1 \mathrm{H}), 2.34-2.25(\mathrm{~m}, 1 \mathrm{H}), 1.62-1.55(\mathrm{~m}, 1 \mathrm{H}), 1.33(\mathrm{td}, J=7.2,4.7 \mathrm{~Hz}, 1 \mathrm{H}), 0.30-0.24(\mathrm{~m}, 9$ H). ${ }^{13} \mathrm{C}$ NMR $\left(101 \mathrm{MHz}, \mathrm{CDCl}_{3}\right) \delta 138.6,137.7,132.9,128.5,24.1,22.1,14.3,-1.1 . \mathrm{MS}(\mathrm{EI}): \mathrm{m} / \mathrm{z}$ (\%) $268\left(\mathrm{M}^{+}\right), 255,253,189,115,73$ (100). HRMS: Calculated for $\mathrm{C}_{12} \mathrm{H}_{17} \mathrm{BrSi}$ : 268.0283; Found: 268.0280 .

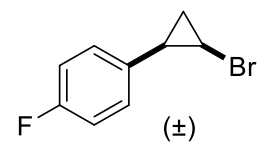

1-(( \pm -(cis)-2-Bromocyclopropyl)-4-fluorobenzene. [known compound $\left.{ }^{1 \mathrm{a}}\right]:$ The reaction was carried out according to the General Procedure B on $50 \mathrm{mmol}$ scale. The product (3.2 g, 30\% yield) was purified by silica gel chromatography (Petroleum ether) as a colorless oil. ${ }^{1} \mathrm{H}$ NMR (400 MHz, $\left.\mathrm{CDCl}_{3}\right) \delta 7.24-7.18(\mathrm{~m}, 2 \mathrm{H}), 7.07-6.99$ (m, $\left.2 \mathrm{H}\right), 3.30$ (td, $J=$ 7.5, $4.6 \mathrm{~Hz}, 1 \mathrm{H}), 2.33-2.24(\mathrm{~m}, 1 \mathrm{H}), 1.64-1.55(\mathrm{~m}, 1 \mathrm{H}), 1.28(\mathrm{td}, J=7.1,4.6 \mathrm{~Hz}, 1 \mathrm{H}) .{ }^{13} \mathrm{C}$ NMR $\left(101 \mathrm{MHz}, \mathrm{CDCl}_{3}\right) \delta 161.7(\mathrm{~d}, J=244.9 \mathrm{~Hz}), 132.8(\mathrm{~d}, J=3.1 \mathrm{~Hz}), 130.7(\mathrm{~d}, J=8.0 \mathrm{~Hz}), 114.8(\mathrm{~d}, J$ $=21.4 \mathrm{~Hz}), 23.8,21.3,14.4 .{ }^{19} \mathrm{~F}$ NMR $\left(376 \mathrm{MHz}, \mathrm{CDCl}_{3}\right) \delta-115.96--116.07$ (m, $\left.1 \mathrm{~F}\right)$. 
$\overbrace{( \pm)}$

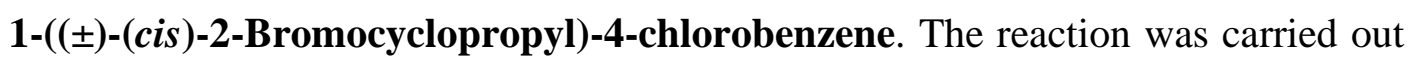
according to the General Procedure B on $100 \mathrm{mmol}$ scale. The product $(6.95 \mathrm{~g}$, $30 \%$ yield) was purified by silica gel chromatography (Petroleum ether) as a colorless oil. ${ }^{1} \mathrm{H}$ NMR $\left(400 \mathrm{MHz}, \mathrm{CDCl}_{3}\right) \delta 7.30(\mathrm{~d}, J=8.3 \mathrm{~Hz}, 2 \mathrm{H}), 7.17(\mathrm{~d}, J=8.2 \mathrm{~Hz}, 2 \mathrm{H}), 3.30(\mathrm{td}, J=7.4,4.9 \mathrm{~Hz}, 1$ H), $2.32-2.24(\mathrm{~m}, 1 \mathrm{H}), 1.64-1.56(\mathrm{~m}, 1 \mathrm{H}), 1.32-1.25(\mathrm{~m}, 1 \mathrm{H}) .{ }^{13} \mathrm{C} \mathrm{NMR}\left(101 \mathrm{MHz}, \mathrm{CDCl}_{3}\right) \delta$ 135.7, 132.6, 130.5, 128.1, 23.7, 21.5, 14.4. MS (EI): m/z (\%) 232, $230\left(\mathrm{M}^{+}\right), 151,115$ (100). HRMS: Calculated for $\mathrm{C}_{9} \mathrm{H}_{8} \mathrm{ClBr}$ : 229.9498; Found: 229.9501.

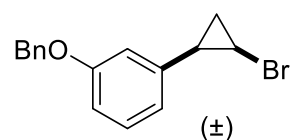

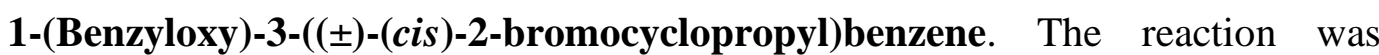
carried out according to the General Procedure B on 40.0 mmol scale. The product $(1.82 \mathrm{~g}, 15 \%$ yield $)$ was purified by silica gel chromatography (Petroleum ether/EtOAc $=$ 50:1) as a colorless oil. ${ }^{1} \mathrm{H}$ NMR (400 MHz, $\left.\mathrm{CDCl}_{3}\right) \delta 7.44(\mathrm{~d}, J=6.9 \mathrm{~Hz}, 2 \mathrm{H}), 7.41-7.36(\mathrm{~m}, 2 \mathrm{H})$, $7.32(\mathrm{t}, J=7.1 \mathrm{~Hz}, 1 \mathrm{H}), 7.27-7.21(\mathrm{~m}, 1 \mathrm{H}), 6.92-6.80(\mathrm{~m}, 3 \mathrm{H}), 5.06(\mathrm{~s}, 2 \mathrm{H}), 3.29(\mathrm{td}, J=7.6$, $4.6 \mathrm{~Hz}, 1 \mathrm{H}), 2.32-2.23(\mathrm{~m}, 1 \mathrm{H}), 1.57-1.52(\mathrm{~m}, 1 \mathrm{H}), 1.29(\mathrm{td}, J=7.1,4.6 \mathrm{~Hz}, 1 \mathrm{H}) .{ }^{13} \mathrm{C} \mathrm{NMR}$ $\left(101 \mathrm{MHz}, \mathrm{CDCl}_{3}\right) \delta 158.5,138.7,137.0,128.9,128.5,127.9,127.5,121.9,115.9,113.0,69.9,23.9$, 22.1, 14.3. MS (EI): m/z (\%) 304, $302\left(\mathrm{M}^{+}\right)$, 225, 223, 91 (100). HRMS: Calculated for $\mathrm{C}_{16} \mathrm{H}_{15} \mathrm{OBr}$ : 302.0306; Found: 302.0311.

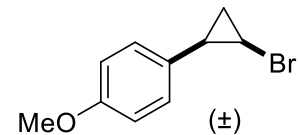

(士)-cis-1-(2-Bromocyclopropyl)-4-methoxybenzene. The reaction was carried $( \pm) \quad$ out according to the General Procedure B on $40.0 \mathrm{mmol}$ scale. The product $(2.25$ $\mathrm{g}, 25 \%$ yield) was purified with silica gel chromatography (Petroleum ether) as a as a colorless oil. ${ }^{1} \mathrm{H}$ NMR $\left(400 \mathrm{MHz}, \mathrm{CDCl}_{3}\right) \delta 7.18(\mathrm{~d}, J=8.7 \mathrm{~Hz}, 2 \mathrm{H}), 6.89(\mathrm{~d}, J=8.7 \mathrm{~Hz}, 2 \mathrm{H}), 3.82(\mathrm{~s}, 3 \mathrm{H}), 3.29$ $(\mathrm{td}, J=7.5,4.5 \mathrm{~Hz}, 1 \mathrm{H}), 2.30-2.20(\mathrm{~m}, 1 \mathrm{H}), 1.67-1.51(\mathrm{~m}, 1 \mathrm{H}), 1.27(\mathrm{td}, J=7.1,4.5 \mathrm{~Hz}, 1 \mathrm{H})$. ${ }^{13} \mathrm{C} \mathrm{NMR}\left(101 \mathrm{MHz}, \mathrm{CDCl}_{3}\right) \delta 158.2,130.0,128.9,113.1,54.9,24.3,21.1,14.0 . \mathrm{MS}(\mathrm{EI}): \mathrm{m} / \mathrm{z}(\%)$ $226\left(\mathrm{M}^{+}\right), 147$ (100), 91, 77. HRMS: Calculated for $\mathrm{C}_{10} \mathrm{H}_{11} \mathrm{OBr}$ : 225.9993; Found: 225.9988 .

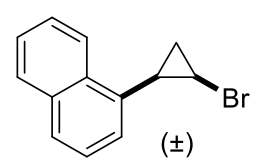

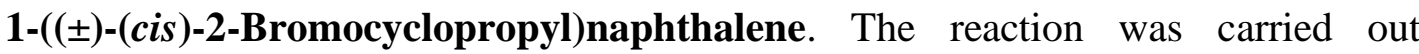
according to the General Procedure B on $32.5 \mathrm{mmol}$ scale. The product (1.7 g, 21\% yield) was purified by silica gel chromatography (Petroleum ether) as a white solid (m.p. $\left.45-46{ }^{\circ} \mathrm{C}\right) .{ }^{1} \mathrm{H}$ NMR $(400 \mathrm{MHz}$, Chloroform- $d$ ) $\delta 8.26(\mathrm{t}, J=6.8 \mathrm{~Hz}, 1 \mathrm{H}), 7.93(\mathrm{t}, J=7.5 \mathrm{~Hz}, 1$ 
H), $7.85(\mathrm{t}, J=7.6 \mathrm{~Hz}, 1 \mathrm{H}), 7.66-7.52(\mathrm{~m}, 2 \mathrm{H}), 7.52-7.44(\mathrm{~m}, 1 \mathrm{H}), 7.43-7.35(\mathrm{~m}, 1 \mathrm{H}), 3.58$ $(\mathrm{td}, J=7.3,4.5 \mathrm{~Hz}, 1 \mathrm{H}), 2.75-2.66(\mathrm{~m}, 1 \mathrm{H}), 1.79-1.71(\mathrm{~m}, 1 \mathrm{H}) .1 .61-1.52(\mathrm{~m}, 1 \mathrm{H}) .{ }^{13} \mathrm{C} \mathrm{NMR}$ $\left(101 \mathrm{MHz}, \mathrm{CDCl}_{3}\right) \delta 133.9,133.4,133.3,128.5,127.6,126.6,126.0,125.8,125.2,124.2,23.1,20.4$, 13.8. MS (EI): m/z (\%) $247\left(\mathrm{M}^{+}\right), 167$ (100), 152, 82. HRMS: Calculated for $\mathrm{C}_{13} \mathrm{H}_{11} \mathrm{Br}: 246.0044$; Found: 246.0054.

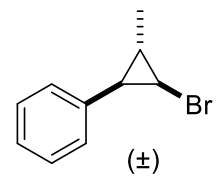

(( \pm )-(cis)-2-bromo-anti-3-methylcyclopropyl)benzene. The reaction was carried out according to the General Procedure B on $38 \mathrm{mmol}$ scale. The product $(1.27 \mathrm{~g}, 16 \%$ yield) was purified by silica gel chromatography (Petroleum ether) as a colorless oil. ${ }^{1} \mathrm{H}$ NMR (400 MHz, $\mathrm{CDCl}_{3}$ ) $\delta 7.35$ - 7.28 (m, $\left.2 \mathrm{H}\right), 7.27$ - 7.23 (m, $\left.1 \mathrm{H}\right), 7.24-7.17$ (m, $\left.2 \mathrm{H}\right), 3.06$ - $3.01(\mathrm{~m}, 1 \mathrm{H}), 2.01$ (app. $\mathrm{t}, J=7.2,1 \mathrm{H}), 1.68-1.58(\mathrm{~m}, 1 \mathrm{H}), 1.27(\mathrm{~d}, J=6.2 \mathrm{~Hz}, 3 \mathrm{H}) .{ }^{13} \mathrm{C} \mathrm{NMR}$ $\left(101 \mathrm{MHz} \mathrm{CDCl}_{3}\right) \delta 137.3,128.9,127.8,126.6,30.8,30.1,22.5,17.8 . \mathrm{MS}(\mathrm{EI}): \mathrm{m} / \mathrm{z}(\%) 212,210$ $\left(\mathrm{M}^{+}\right)$, 184. 171, 131 (100), 91. HRMS: Calculated for $\mathrm{C}_{10} \mathrm{H}_{11} \mathrm{Br}: 210.0044$; Found: 210.0046 .

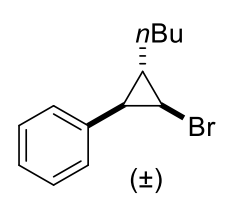

(( \pm )-(cis)-2-bromo-anti-3-butylcyclopropyl)benzene. The reaction was carried out according to the General Procedure B on $38.5 \mathrm{mmol}$ scale. The product (2.24 g, $23 \%$ yield) was purified by silica gel chromatography (Petroleum ether) as a colorless oil. ${ }^{1} \mathrm{H}$ NMR (400 MHz, $\left.\mathrm{CDCl}_{3}\right) \delta 7.32(\mathrm{t}, J=7.2 \mathrm{~Hz}, 2 \mathrm{H}), 7.27-7.19$ (m, $\left.3 \mathrm{H}\right), 3.06$ (dd, $J=7.7,3.8$ Hz, $1 \mathrm{H}$ ), 2.03 (app. t, $J=7.0,1 \mathrm{H}), 1.65-1.53$ (m, $2 \mathrm{H}), 1.52-1.34(\mathrm{~m}, 5 \mathrm{H}), 0.92$ (t, $J=7.2 \mathrm{~Hz}, 3$ H). ${ }^{13} \mathrm{C}$ NMR $\left(101 \mathrm{MHz} \mathrm{CDCl}_{3}\right) \delta 137.5,129.0,127.9,126.6,32.7,30.7,29.8,29.1,28.1,22.4,14.0$. MS (EI): m/z (\%) $252\left(\mathrm{M}^{+}\right), 175,173,119,117$ (100). HRMS: Calculated for $\mathrm{C}_{13} \mathrm{H}_{17} \mathrm{Br}$ : 252.0514; Found: 252.0505.

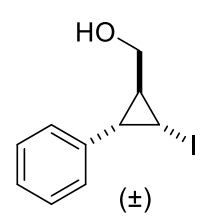

(( \pm )-(1S,2R,3S)-2-Iodo-3-phenylcyclopropyl)methanol. [known compound ${ }^{4}$ ]: To a stirred suspension of iodoform (17.3 g, $44 \mathrm{mmol}, 4.4$ equiv) in anhydrous $\mathrm{CH}_{2} \mathrm{Cl}_{2}$ (48 $\mathrm{mL}$ ) was added diethylzinc ( $22 \mathrm{mmol}, 2.2$ equiv) at room temperature with a water bath. The yellow suspension was stirred at room temperature for $10 \mathrm{~min}$, after which a solution of cinnamyl alcohol (1.34 g, $10 \mathrm{mmol}, 1.0$ equiv) in anhydrous $\mathrm{CH}_{2} \mathrm{Cl}_{2}(5 \mathrm{~mL})$ was added at room temperature. The reaction flask was removed from the water bath and wrapped with aluminum foil. The yellow suspension was allowed to stir at room temperature for $24 \mathrm{~h}$. The reaction was quenched 
with saturated aqueous $\mathrm{NH}_{4} \mathrm{Cl}(10 \mathrm{~mL})$. The organic layer was separated and the aqueous layer was extracted with $\mathrm{Et}_{2} \mathrm{O}(3 \times 50 \mathrm{~mL})$. The combined organic layers were dried over $\mathrm{MgSO}_{4}$, filtered and concentrated. The residue was purified by flash chromatography (Petroleum ether/EtOAc $=4: 1)$ to give the title compound (700 mg, 25\%) as a red oil. This compound is known ${ }^{4}:{ }^{1} \mathrm{H}$ NMR (400 MHz, $\left.\mathrm{CDCl}_{3}\right) \delta 7.37-7.28(\mathrm{~m}, 3 \mathrm{H}), 7.19(\mathrm{~d}, J=7.0 \mathrm{~Hz}, 2 \mathrm{H}), 3.81(\mathrm{dd}, J=11.4,6.0 \mathrm{~Hz}, 1 \mathrm{H}), 3.75(\mathrm{dd}, J$ $=11.4,6.1 \mathrm{~Hz}, 1 \mathrm{H}), 2.92(\mathrm{dd}, J=8.2,4.7 \mathrm{~Hz}, 1 \mathrm{H}), 2.08-2.00(\mathrm{~m}, 1 \mathrm{H}), 1.96-1.88(\mathrm{~m}, 1 \mathrm{H}), 1.61$ (br, $1 \mathrm{H})$.

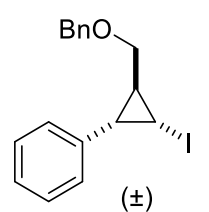

(( ()$-(1 S, 2 S, 3 R)-2-((B b e n z y l o x y)$ methyl)-3-iodocyclopropyl)benzene.

[known compound $\left.^{4}\right]$ : To a solution of $(( \pm)-(1 S, 2 R, 3 S)$-2-iodo-3-phenylcyclopropyl)methanol (700 mg, $2.5 \mathrm{mmol}, 1.0$ equiv) in anhydrous THF (15 mL) was added $\mathrm{NaH}(60 \%$ dispersion in mineral oil, $120 \mathrm{mg}, 3.0 \mathrm{mmol}, 1.2$ equiv) in portions at $0{ }^{\circ} \mathrm{C}$ with stirring. The resulting suspension was stirred at $0{ }^{\circ} \mathrm{C}$ for $1 \mathrm{~h}$. Benzyl bromide (600 mg, $3.5 \mathrm{mmol}, 1.4$ equiv) was added slowly at $0{ }^{\circ} \mathrm{C}$. The reaction mixture was stirred at room temperature for $12 \mathrm{~h}$ in darkness. The reaction was quenched with water and the aqueous layer was extracted with $\mathrm{Et}_{2} \mathrm{O}(3 \mathrm{x} 20 \mathrm{~mL})$. The combined organic layer was washed with brine $(10 \mathrm{~mL})$, dried over $\mathrm{Na}_{2} \mathrm{SO}_{4}$, filtered and concentrated. The residue was purified by flash chromatography (Petroleum ether/EtOAc $=40: 1)$, affording the title compound (728 mg, $80 \%$ yield) as a pale yellow oil. Analytical data in accordance with literature data ${ }^{4}{ }^{1} \mathrm{H}$ NMR $\left(400 \mathrm{MHz}, \mathrm{CDCl}_{3}\right) \delta 7.37-7.28(\mathrm{~m}, 8 \mathrm{H}), 7.19(\mathrm{~d}, J=7.0 \mathrm{~Hz}, 2 \mathrm{H})$, 4.58 (s, $2 \mathrm{H}), 3.68(\mathrm{dd}, J=10.5,5.6 \mathrm{~Hz}, 1 \mathrm{H}), 3.59(\mathrm{dd}, J=10.5,6.0 \mathrm{~Hz}, 1 \mathrm{H}), 2.92(\mathrm{dd}, J=8.1,4.7$ $\mathrm{Hz}, 1 \mathrm{H}), 2.07-1.99(\mathrm{~m}, 1 \mathrm{H}), 1.94-1.88(\mathrm{~m}, 1 \mathrm{H}) .{ }^{13} \mathrm{C} \mathrm{NMR}\left(101 \mathrm{MHz}, \mathrm{CDCl}_{3}\right) \delta$ 138.5, 138.0, $128.8,128.4,127.9,127.7,127.6,126.9,72.6,71.1,28.0,26.0,-1.7$.

\subsubsection{General procedure for the preparation of cyclopropylzinc reagents $( \pm)$ cis-1 and $( \pm)$} trans-1 (General Procedure C)

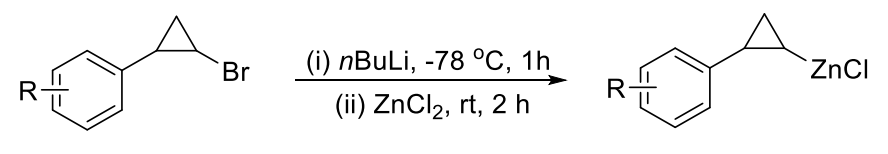

$( \pm)$-cis or $( \pm)$-trans

$( \pm)$-cis-1 or $( \pm)$-trans-1

To a solution of cyclopropylbromide (10.0 mmol, 1.0 equiv) in anhydrous THF ( $4 \mathrm{~mL})$ was added $n \operatorname{BuLi}\left(2.5 \mathrm{M}\right.$ in hexane; $4.4 \mathrm{~mL}, 11.0 \mathrm{mmol}, 1.1$ equiv) under $\mathrm{Ar}$ at $-78{ }^{\circ} \mathrm{C}$. The reaction mixture 
was stirred at $-78{ }^{\circ} \mathrm{C}$ for $1 \mathrm{~h}$, then a solution of $\mathrm{ZnCl}_{2}$ (1.0 M in THF; $12 \mathrm{~mL}, 12.0 \mathrm{mmol}, 1.2$ equiv) was added to the reaction mixture at $-78{ }^{\circ} \mathrm{C}$. The reaction mixture was allowed to warm to room temperature and stirred for another $2 \mathrm{~h}$. The concentration $(0.30 \sim 0.34 \mathrm{M})$ of these cyclopropylzinc reagents were titrated with $\mathrm{I}_{2}$ according to Knochel's method. ${ }^{5}$ The cyclopropylzinc solution can be stored at $0{ }^{\circ} \mathrm{C}$ under argon for several weeks without deterioration.

\subsection{General procedure for the preparation of alkyl electrophiles 2.}

\subsubsection{General procedure for the preparation of gem-difluoropropargyl bromides (General} Procedure D)

$$
\mathrm{R}=+\mathrm{CF}_{2} \mathrm{Br}_{2} \underset{-78^{\circ} \mathrm{C}}{\stackrel{n \mathrm{BuLi}, \mathrm{THF}}{\longrightarrow}} \mathrm{R}=\mathrm{CF}_{2} \mathrm{Br}
$$

The preparation of gem-difluoropropargyl bromide is according to the literature. ${ }^{6}$ To a solution of terminal alkyne (1.0 equiv) in anhydrous THF (0.8 M) was added dropwise $n \operatorname{BuLi}(2.5 \mathrm{M}$ in hexane; 1.1 equiv) under $\mathrm{Ar}$ at $-78{ }^{\circ} \mathrm{C}$. After stirring for $60 \mathrm{~min}$ at $-78{ }^{\circ} \mathrm{C}$, the reaction mixture was cooled to $-196{ }^{\circ} \mathrm{C}$ (liquid $\mathrm{N}_{2}$ bath) and $\mathrm{CF}_{2} \mathrm{Br}_{2}$ (2.1 equiv) was added to the mixture. The resulting reaction mixture was warmed to room temperature gradually and stirred overnight. The reaction was then quenched with saturated aqueous $\mathrm{NH}_{4} \mathrm{Cl}$ and the solvent $\mathrm{THF}$ was evaporated. The residue was extracted with $\mathrm{CH}_{2} \mathrm{Cl}_{2}$ and the combined organic layers were washed with brine, dried over $\mathrm{Na}_{2} \mathrm{SO}_{4}$, filtered and concentrated. The residue was purified with silica gel chromatography to give pure product.

\subsubsection{General procedure for the preparation of secondary propargyl bromides (General}

\section{Procedure E)}

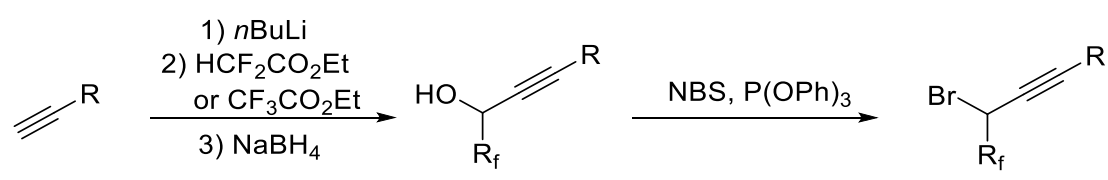

\section{Procedure $^{7}$}

Step 1: To a solution of terminal alkyne (1.0 equiv) in anhydrous THF (0.8 M) was added dropwise $n \operatorname{BuLi}\left(2.5 \mathrm{M}\right.$ in hexane; 1.05 equiv) under an Ar atmosphere at $-78{ }^{\circ} \mathrm{C}$. After the reaction mixture was stirred for $30 \mathrm{~min}$ at $-78{ }^{\circ} \mathrm{C}$, ethyldifluoroacetate or ethyltrifluoroacetate (1.2 equiv) was added 
and the resulting mixture was stirred for $60 \mathrm{~min}$ at the same temperature. Then, $\mathrm{NaBH}_{4}$ was added at $-78{ }^{\circ} \mathrm{C}$ and the reaction mixture was slowly warmed to room temperature and stirred overnight. The reaction was quenched by aqueous $\mathrm{NH}_{4} \mathrm{Cl}$ solution, extracted with EtOAc and concentrated. The residue was purified by silica gel chromatography to give the corresponding alcohol.

Step 2: To a solution of NBS (1.6 equiv) in DCM was added dropwise $\mathrm{P}(\mathrm{OPh})_{3}\left(1.6\right.$ equiv) at $0{ }^{\circ} \mathrm{C}$. After stirring for $20 \mathrm{~min}$, the corresponding alcohol was added dropwise to the reaction mixture. Then, the reaction mixture was heated to $55^{\circ} \mathrm{C}$ and refluxed for $18 \mathrm{~h}$. Silica gel was added to quench the reaction and the product was purified by silica gel chromatography.

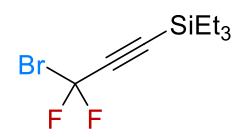

$2 \mathbf{a}$

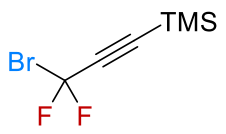

$\mathbf{2 b}$

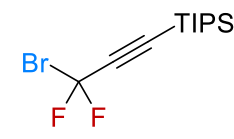

2c

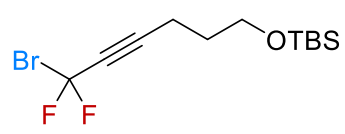

2d

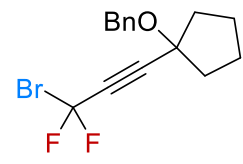

$2 e$

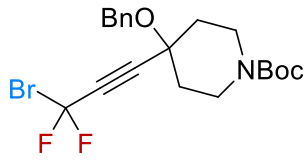

$2 f$
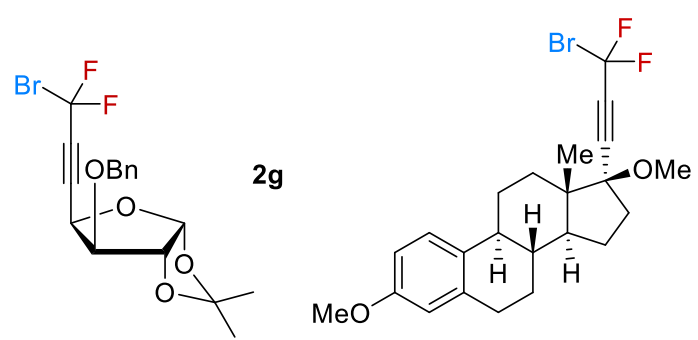

2h

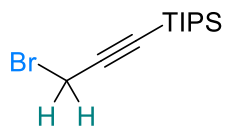

2i

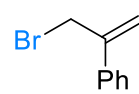

2j

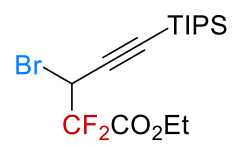

$2 p$

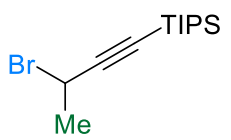

2k

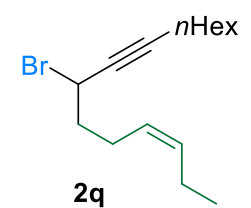
$2 q$

2I

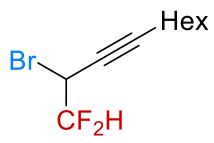

$2 m$

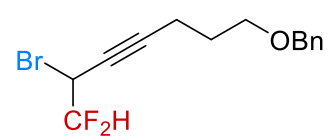

$2 n$

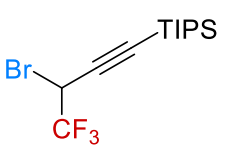

20

Note: Compounds $\mathbf{2} \mathbf{a}-\mathbf{2} \mathbf{d}^{6}, \mathbf{2} \mathbf{h}^{6}, \mathbf{2} \mathbf{i}^{9}, \mathbf{2} \mathbf{j}^{10}, \mathbf{2} \mathbf{k}^{11}$ were prepared according to literatures.

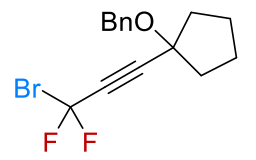

(((1-(3-Bromo-3,3-difluoroprop-1-yn-1-yl)cyclopentyl)oxy)methyl)benzene (2e).

The reaction was carried out according to General Procedure D on $10.0 \mathrm{mmol}$ scale. The product $(2.0 \mathrm{~g}, 61 \%$ yield) was purified by silica gel chromatography (Petroleum ether/EA = 20:1) as a colorless oil. ${ }^{1} \mathrm{H}$ NMR $\left(400 \mathrm{MHz}, \mathrm{CDCl}_{3}\right) \delta 7.42-7.27(\mathrm{~m}, 5 \mathrm{H}), 4.61-4.58(\mathrm{~m}, 2 \mathrm{H})$, $2.24-2.14(\mathrm{~m}, 2 \mathrm{H}), 2.08-1.96(\mathrm{~m}, 2 \mathrm{H}), 1.90-1.74(\mathrm{~m}, 4 \mathrm{H}) .{ }^{13} \mathrm{C} \mathrm{NMR}\left(101 \mathrm{MHz}, \mathrm{CDCl}_{3}\right) \delta$ 138.0, 128.4, 127.75, 127.69, $101.4(\mathrm{t}, J=289.6 \mathrm{~Hz}), 92.7(\mathrm{t}, J=5.7 \mathrm{~Hz}), 80.0(\mathrm{t}, J=1.6 \mathrm{~Hz}), 77.7(\mathrm{t}$, $J=38.2 \mathrm{~Hz}), 67.6,39.0,23.4 .{ }^{19} \mathrm{~F} \mathrm{NMR}\left(376 \mathrm{MHz}, \mathrm{CDCl}_{3}\right) \delta-31.84--31.87$ (m, $\left.2 \mathrm{~F}\right) . \mathrm{MS}(\mathrm{EI}): \mathrm{m} / \mathrm{z}$ 
(\%) 330, $328\left(\mathrm{M}^{+}\right), 300,298,221,219,199,91$ (100). HRMS: Calculated for $\mathrm{C}_{15} \mathrm{H}_{15} \mathrm{OF}_{2} \mathrm{Br}$ : 328.0274; Found: 328.0279.

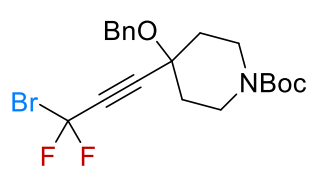

tert-Butyl-4-(benzyloxy)-4-(3-bromo-3,3-difluoroprop-1-yn-1-yl)piperidine-1

-carboxylate (2f). The reaction was carried out according to General Procedure

D on $41.2 \mathrm{mmol}$ scale. The product (9.34 g, 51\% yield) was purified by silica gel chromatography (Petroleum ether/EtOAc $=10: 1)$ as a yellow semisolid. ${ }^{1} \mathrm{H}$ NMR $\left(400 \mathrm{MHz}, \mathrm{CDCl}_{3}\right)$ $\delta 7.39-7.34$ (m, 4 H), 7.33 - 7.29 (m, 1 H), 4.65 - 4.61 (m, 2 H), 3.76 - 3.66 (m, 2 H), $3.42-3.33$ $(\mathrm{m}, 2 \mathrm{H}), 2.08-1.98(\mathrm{~m}, 2 \mathrm{H}), 1.96-1.86(\mathrm{~m}, 2 \mathrm{H}), 1.47(\mathrm{~s}, 9 \mathrm{H}) .{ }^{13} \mathrm{C} \mathrm{NMR}\left(101 \mathrm{MHz}, \mathrm{CDCl}_{3}\right) \delta$ 154.5, 137.5, 128.5, 127.9, 127.8, $101.1(\mathrm{t}, J=290.3 \mathrm{~Hz}), 90.7(\mathrm{t}, J=5.7 \mathrm{~Hz}), 79.9$, $79.4(\mathrm{t}, J=38.7$ $\mathrm{Hz}), 72.0(\mathrm{t}, J=1.4 \mathrm{~Hz}), 66.5,40.4$ (br), 39.5 (br), 35.6 (br), 28.4. ${ }^{19} \mathrm{~F}$ NMR $\left(376 \mathrm{MHz}, \mathrm{CDCl}_{3}\right) \delta$ -32.8 (s, 2 F). MS (EI): m/z (\%) 463, 461, 446, $444\left([\mathrm{M}+\mathrm{H}]^{+}\right), 407$ (100), 405. HRMS: Calculated for $\mathrm{C}_{20} \mathrm{H}_{25} \mathrm{O}_{3} \mathrm{NBrF}_{2}$ : 444.0980; Found: 444.0978.

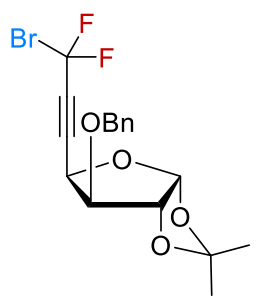

\section{(3aR,5R,6aR)-6-(Benzyloxy)-5-(3-bromo-3,3-difluoroprop-1-yn-1-yl)-2,2-dimet} hyltetrahydrofuro[2,3-d][1,3]dioxole $(\mathbf{2 g})$. The reaction was carried out according to General Procedure D on 19.7 mmol scale. The product (4.13 g, 52\% yield) was purified by silica gel chromatography (Petroleum ether/EtOAc $=10: 1)$ as a white solid (m.p. 48-49 $\left.{ }^{\circ} \mathrm{C}\right) .{ }^{1} \mathrm{H}$ NMR (400 MHz, $\left.\mathrm{CDCl}_{3}\right) \delta 7.44-7.29(\mathrm{~m}, 5 \mathrm{H}), 6.00(\mathrm{~d}, J=3.6 \mathrm{~Hz}, 1 \mathrm{H})$, $4.98-4.92(\mathrm{~m}, 1 \mathrm{H}), 4.77(\mathrm{~d}, J=12.2 \mathrm{~Hz}, 1 \mathrm{H}), 4.71(\mathrm{~d}, J=12.0 \mathrm{~Hz}, 1 \mathrm{H}), 4.66-4.61(\mathrm{~m}, 1 \mathrm{H})$, $4.11-4.06(\mathrm{~m}, 1 \mathrm{H}), 1.48(\mathrm{~s}, 3 \mathrm{H}), 1.32$ (s, $3 \mathrm{H}) .{ }^{13} \mathrm{C} \mathrm{NMR}\left(101 \mathrm{MHz}, \mathrm{CDCl}_{3}\right) \delta 136.7,128.5,128.1$, 127.9, 112.4, 105.1, $101.1(\mathrm{t}, J=290.2 \mathrm{~Hz}), 84.8(\mathrm{t}, J=5.7 \mathrm{~Hz}), 82.4,82.3,79.4(\mathrm{t}, J=38.9 \mathrm{~Hz})$, 72.6, $70.4(\mathrm{t}, J=1.5 \mathrm{~Hz}), 26.8,26.1 .{ }^{19} \mathrm{~F} \mathrm{NMR}\left(376 \mathrm{MHz}, \mathrm{CDCl}_{3}\right) \delta-33.34--33.47$ (m, $\left.2 \mathrm{~F}\right) . \mathrm{MS}$ (DART): m/z (\%) $422(100), 420\left(\left[\mathrm{M}+\mathrm{NH}_{4}\right]^{+}\right), 405,403\left([\mathrm{M}+\mathrm{H}]^{+}\right)$. HRMS: Calculated for $\mathrm{C}_{17} \mathrm{H}_{18} \mathrm{O}_{4} \mathrm{BrF}_{2}$ : 403.0351; Found: 403.0353.

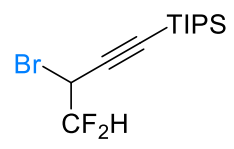

(3-Bromo-4,4-difluorobut-1-yn-1-yl)triisopropylsilane (2l). The reaction was carried out according to General Procedure E on 40.0 mmol scale. The product ( $4.8 \mathrm{~g}$, $37 \%$ yield, two steps) was purified by silica gel chromatography (Petroleum ether) as a colorless oil. ${ }^{1} \mathrm{H}$ NMR $\left(400 \mathrm{MHz}, \mathrm{CDCl}_{3}\right) \delta 5.82(\mathrm{td}, J=55.8,4.2 \mathrm{~Hz}, 1 \mathrm{H}), 4.61(\mathrm{td}, J=9.8,4.2 \mathrm{~Hz}, 1 \mathrm{H}), 1.10-$ 
$1.06(\mathrm{~m}, 21 \mathrm{H}) .{ }^{13} \mathrm{C} \mathrm{NMR}\left(126 \mathrm{MHz}, \mathrm{CDCl}_{3}\right) \delta 112.5(\mathrm{t}, J=248.8 \mathrm{~Hz}), 97.6(\mathrm{t}, J=4.7 \mathrm{~Hz}), 93.5$, 60.4, $34.0(\mathrm{t}, J=28.6 \mathrm{~Hz}), 18.4,11.0 .{ }^{19} \mathrm{~F} \mathrm{NMR}\left(376 \mathrm{MHz}, \mathrm{CDCl}_{3}\right) \delta-117.81(\mathrm{dd}, J=55.8,9.8 \mathrm{~Hz}, 2$ F). MS (EI): m/z (\%) $324\left(\mathrm{M}^{+}\right), 281,143,77(100)$. HRMS: Calculated for $\mathrm{C}_{13} \mathrm{H}_{23} \mathrm{BrF}_{2} \mathrm{Si}: 324.0720$; Found: 324.0709 .

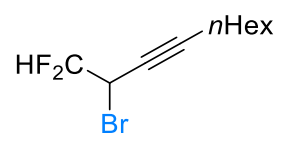

2-Bromo-1,1-difluorodec-3-yne (2m). The reaction was carried out according to

General Procedure E on $5.0 \mathrm{mmol}$ scale. The product $(387 \mathrm{mg}$, 30\% yield, two steps) was purified by silica gel chromatography (Petroleum ether) as a colorless oil. ${ }^{1} \mathrm{H}$ NMR (400 $\left.\mathrm{MHz} \mathrm{CDCl}_{3}\right) \delta 5.79(\mathrm{td}, J=55.9,4.2 \mathrm{~Hz}, 1 \mathrm{H}), 4.61(\mathrm{tdt}, J=9.9,4.3,2.3 \mathrm{~Hz}, 1 \mathrm{H}), 2.27(\mathrm{td}, J=7.1$, $2.3 \mathrm{~Hz}, 2 \mathrm{H}), 1.63-1.46(\mathrm{~m}, 2 \mathrm{H}), 1.43-1.33$ (m, $2 \mathrm{H}), 1.33-1.24(\mathrm{~m}, 4 \mathrm{H}), 0.89$ (t, J = 6.9 Hz, 3 H). ${ }^{13} \mathrm{C}$ NMR $\left(126 \mathrm{MHz}, \mathrm{CDCl}_{3}\right) \delta 112.6(\mathrm{t}, J=248.2 \mathrm{~Hz}), 92.0,71.8(\mathrm{t}, J=5.1 \mathrm{~Hz}), 34.7(\mathrm{t}, J=$ $28.6 \mathrm{~Hz}$ ), 31.2, 28.4, 28.0, 22.5, 18.9, 13.9. ${ }^{19} \mathrm{~F} \mathrm{NMR} \mathrm{(376} \mathrm{MHz,} \mathrm{CDCl}_{3}$ ) $\delta-117.6$ (ddd, $J=287.6$, 55.8, 9.8 Hz, 1 F), -118.3 (ddd, $J=287.6,56.0,9.8 \mathrm{~Hz}, 1 \mathrm{~F}) . \mathrm{MS}(\mathrm{EI}): \mathrm{m} / \mathrm{z}(\%)$ 254, 237, 225, 131 (100). HRMS: Calculated for $\mathrm{C}_{10} \mathrm{H}_{13} \mathrm{BrF}_{2}$ :252.0325; Found: 252.0318.

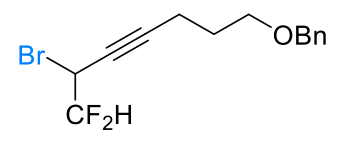

(((6-Bromo-7,7-difluorohept-4-yn-1-yl)oxy)methyl)benzene (2n). The reaction was carried out according to General Procedure E on $19.0 \mathrm{mmol}$ scale. The product (1.2 g, 20\% yield, two steps) was purified by silica gel chromatography (Petroleum ether/EtOAc $=20: 1)$ as a colorless oil. ${ }^{1} \mathrm{H} \mathrm{NMR}\left(400 \mathrm{MHz}, \mathrm{CDCl}_{3}\right) \delta 7.40-7.27(\mathrm{~m}, 5 \mathrm{H}), 5.76(\mathrm{td}$, $J=55.9,4.0 \mathrm{~Hz}, 1 \mathrm{H}), 4.58(\mathrm{tdt}, J=10.0,4.0,2.4 \mathrm{~Hz}, 1 \mathrm{H}), 4.52(\mathrm{~s}, 2 \mathrm{H}), 3.56(\mathrm{t}, J=6.0 \mathrm{~Hz}, 2 \mathrm{H})$, $2.43(\mathrm{td}, J=7.0,2.4 \mathrm{~Hz}, 2 \mathrm{H}), 1.84(\mathrm{p}, J=6.4 \mathrm{~Hz}, 2 \mathrm{H}) .{ }^{13} \mathrm{C} \mathrm{NMR}\left(126 \mathrm{MHz}, \mathrm{CDCl}_{3}\right) \delta 138.4,128.4$, 127.64, 127.61, $112.6(\mathrm{t}, J=248.4 \mathrm{~Hz}), 91.2,73.0,72.1,68.3,34.5(\mathrm{t}, J=28.7 \mathrm{~Hz}), 28.2,15.8 .{ }^{19} \mathrm{~F}$ NMR (376 MHz, $\left.\mathrm{CDCl}_{3}\right) \delta-117.6(\mathrm{ddd}, J=275.7,55.8,9.8 \mathrm{~Hz}, 1 \mathrm{~F}), \delta-118.4(\mathrm{ddd}, J=271.5,55.8$, $10.1 \mathrm{~Hz}, 1 \mathrm{~F}) . \mathrm{MS}(\mathrm{EI}): \mathrm{m} / \mathrm{z}(\%) 317\left([\mathrm{M}+\mathrm{H}]^{+}\right), 237,91$ (100). HRMS: Calculated for $\mathrm{C}_{14} \mathrm{H}_{14} \mathrm{BrF}_{2} \mathrm{O}$ : 315.0196; Found: 315.0205.

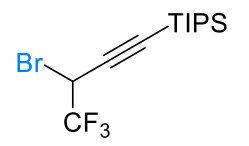

3-Bromo-4,4,4-trifluorobut-1-yn-1-yl)triisopropylsilane (2o). The reaction was carried out according to General Procedure E on $40.0 \mathrm{mmol}$ scale. The product $(6.65$ g, $48 \%$ yield, two steps) was purified by silica gel chromatography (Petroleum ether) as a colorless oil. ${ }^{1} \mathrm{H}$ NMR $\left(400 \mathrm{MHz}, \mathrm{CDCl}_{3}\right) \delta 4.80$ (q, $\left.J=6.1 \mathrm{~Hz}, 1 \mathrm{H}\right), 1.10-1.05(\mathrm{~m}, 21 \mathrm{H}) .{ }^{13} \mathrm{C}$ NMR $(126$ 
$\left.\mathrm{MHz}, \mathrm{CDCl}_{3}\right) \delta 121.7(\mathrm{q}, J=278.0 \mathrm{~Hz}), 95.9(\mathrm{q}, J=1.9 \mathrm{~Hz}), 94.2,31.4(\mathrm{q}, J=39.0 \mathrm{~Hz}), 18.4,11.0$. ${ }^{19} \mathrm{~F}$ NMR (376 MHz, $\left.\mathrm{CDCl}_{3}\right) \delta-72.5$ (d, $\left.J=6.1 \mathrm{~Hz}\right) . \mathrm{MS}(\mathrm{EI}): \mathrm{m} / \mathrm{z}(\%) 342\left(\mathrm{M}^{+}\right), 301$ (100), 272, 115, 77. HRMS: Calculated for $\mathrm{C}_{13} \mathrm{H}_{22} \mathrm{BrF}_{3} \mathrm{Si}$ : 342.0626; Found: 342.0634 .

\section{Synthesis of $2 p$}
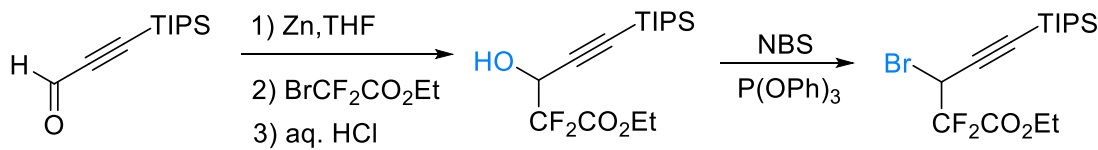

$2 p$

\section{Procedure $^{7}$}

Step 1: To a mixture of 3-(triisopropylsilyl)propiolaldehyde (1.05 g, $5.0 \mathrm{mmol}, 1.0$ equiv) and zinc powder (975 mg, $15.0 \mathrm{mmol}, 3.0$ equiv) in anhydrous THF (5 mL) was added ethyl 2-bromo-2,2-difluoroacetate (2.03 g, $10.0 \mathrm{mmol}, 2.0$ equiv) dropwise under Ar at room temperature. The reaction mixture was stirred at room temperature for 1 hour and was quenched with saturated aqueous $\mathrm{NH}_{4} \mathrm{Cl}$. The mixture was concentrated, extracted with EtOAc and purified with silica gel chromatography to afford alcohol.

Step 2: To a solution of NBS (715 mg, $4.0 \mathrm{mmol}, 1.6$ equiv) in $\mathrm{CH}_{2} \mathrm{Cl}_{2}$ was added dropwise $\mathrm{P}(\mathrm{OPh})_{3}$ (1.24 g, $4.0 \mathrm{mmol}, 1.6$ equiv) at $0{ }^{\circ} \mathrm{C}$. After the reaction was stirred for $20 \mathrm{~min}$ at the same temperature, the alcohol was added dropwise. Then, the reaction mixture was heated to $55{ }^{\circ} \mathrm{C}$ and refluxed for $18 \mathrm{~h}$. Silica gel was added to quench the reaction and the product $(0.535 \mathrm{~g}, 27 \%$ yield for two steps) was purified by silica gel chromatography (Petroleum ether/DCM = 5:1) as a colorless oil. ${ }^{1} \mathrm{H}$ NMR $\left(400 \mathrm{MHz}, \mathrm{CDCl}_{3}\right) \delta 5.00(\mathrm{t}, J=11.2 \mathrm{~Hz}, 1 \mathrm{H}), 4.43-4.31(\mathrm{~m}, 2 \mathrm{H}), 1.37(\mathrm{t}, J=7.1 \mathrm{~Hz}, 3$ $\mathrm{H}), 1.10-1.05(\mathrm{~m}, 21 \mathrm{H}) .{ }^{13} \mathrm{C} \mathrm{NMR}\left(126 \mathrm{MHz}, \mathrm{CDCl}_{3}\right) \delta 161.2(\mathrm{t}, J=32.1 \mathrm{~Hz}), 111.2(\mathrm{dd}, J=$ 259.1, 258.5 Hz), 96.6 - 96.5 (m), 94.5, 63.7, 34.2 (dd, $J=29.9,29.9 \mathrm{~Hz}), 18.4,13.9,11.0 .{ }^{19} \mathrm{~F}$ NMR $\left(376 \mathrm{MHz}, \mathrm{CDCl}_{3}\right) \delta-107.63(\mathrm{dd}, J=251.8,12.1 \mathrm{~Hz}, 1 \mathrm{~F}),-108.84(\mathrm{dd}, J=251.7,10.1 \mathrm{~Hz}, 1 \mathrm{~F})$. MS (EI): m/z (\%) 353 ([M- $\left.\left.{ }^{i} \mathrm{Pr}\right]^{+}, 100\right), 255,131$. HRMS: Calculated for $\mathrm{C}_{13} \mathrm{H}_{20} \mathrm{BrF}_{2} \mathrm{SiBr}: 353.0384$; Found: 353.0388. 


\section{Synthesis of $2 q$}

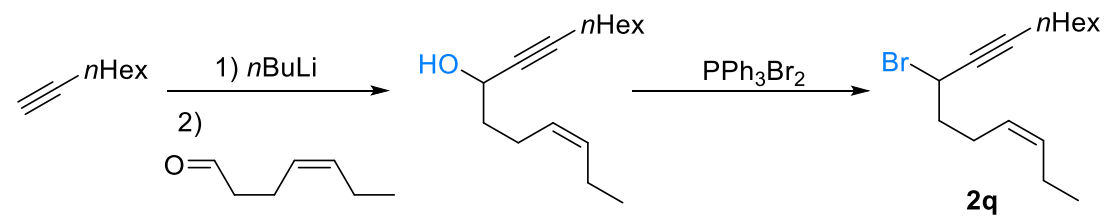

\section{Procedure $^{8}$ :}

Step 1: To a solution of oct-1-yne $(1.1 \mathrm{~g}, 1.0$ equiv, $10 \mathrm{mmol})$ in anhydrous THF $(0.8 \mathrm{M})$ was added dropwise $n \operatorname{BuLi}\left(4.2 \mathrm{ml}, 2.5 \mathrm{M}\right.$ in hexane; 1.05 equiv) under $\mathrm{Ar}$ at $-78{ }^{\circ} \mathrm{C}$. The mixture was stirred at same reaction temperature for $30 \mathrm{~min},(Z)$-hept-4-enal (1.34 g, 1.2 equiv, $12 \mathrm{mmol})$ was then added. After stirring for $20 \mathrm{~min}$, the reaction was quenched by aqueous $\mathrm{NH}_{4} \mathrm{Cl}$ solution. The reaction mixture was extracted with EtOAc. The combined organic layers were dried over $\mathrm{Na}_{2} \mathrm{SO}_{4}$, filtered and concentrated. The residue was purified by silica gel chromatography to give the corresponding alcohol.

Step 2: To the alcohol solution in $\mathrm{CH}_{2} \mathrm{Cl}_{2}(50 \mathrm{ml})$ was added $\mathrm{PPh}_{3} \mathrm{Br}_{2}(4.2 \mathrm{~g}, 10 \mathrm{mmol})$ at room temperature. The reaction mixture was stirred until the alcohol was consumed up monitored by TLC. Silica gel was then added to quench the reaction and the product (1.1g, $38 \%$ two steps) was purified by silica gel chromatography (Petroleum ether) as a colorless oil. ${ }^{1} \mathrm{H}$ NMR $\left(400 \mathrm{MHz}, \mathrm{CDCl}_{3}\right) \delta 5.51$ $-5.35(\mathrm{~m}, 1 \mathrm{H}), 5.32-5.21(\mathrm{~m}, 1 \mathrm{H}), 4.51(\mathrm{tt}, J=6.8,2.0 \mathrm{~Hz}, 1 \mathrm{H}), 2.35-2.16(\mathrm{~m}, 4 \mathrm{H}), 2.13-$ $1.90(\mathrm{~m}, 4 \mathrm{H}), 1.54-1.42(\mathrm{~m}, 2 \mathrm{H}), 1.43-1.32(\mathrm{~m}, 2 \mathrm{H}), 1.31-1.20(\mathrm{~m}, 4 \mathrm{H}), 0.95(\mathrm{t}, J=7.6 \mathrm{~Hz}, 3$ H), $0.87(\mathrm{t}, J=6.8 \mathrm{~Hz}, 3 \mathrm{H}) .{ }^{13} \mathrm{C}$ NMR $\left(126 \mathrm{MHz}, \mathrm{CDCl}_{3}\right) \delta 133.4,126.4,88.3,79.2,40.1,37.8$, 31.2, 28.4, 28.3, 25.0, 22.5, 20.5, 18.9, 14.2, 13.9. MS (EI): m/z (\%) $284\left(\mathrm{M}^{+}\right), 205\left([\mathrm{M}-\mathrm{Br}]^{+}\right), 105$ (100). HRMS: Calculated for $\mathrm{C}_{15} \mathrm{H}_{25} \mathrm{Br}$ : 284.1140; Found: 284.1144.

4. Control experiments to determine the origin of the stereoselectivity in the nickel-catalyzed coupling

\subsection{Reaction of $( \pm)$ cis- $d$-1b with $2 \mathrm{a}$}

\subsubsection{Synthesis of $( \pm)$ cis- $d-1 b$}

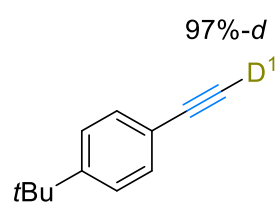

d-1-(tert-Butyl)-4-ethynylbenzene.

[known

compound $^{12}$ ]:

1-(tert-Butyl)-4-ethynylbenzene (6.61 g, $50 \mathrm{mmol}, 1.0$ equiv) was added to ${ }^{n} \mathrm{BuLi}$ (2.5 $\mathrm{M}$ in hexanes; $24.0 \mathrm{~mL}, 60 \mathrm{mmol}, 1.2$ equiv) over 15 minutes at $0{ }^{\circ} \mathrm{C}$. The 
resulting suspension was stirred at $0{ }^{\circ} \mathrm{C}$ for 1 hour, then $\mathrm{D}_{2} \mathrm{O}(5 \mathrm{~mL})$ was added slowly. After stirring at room temperature overnight, the reaction mixture was filtered through a pad of celite. The filtrate was concentrated and the resulting residue was purified by flash silica gel chromatography (Petroleum ether) to give the title compound (5.5 g, 83\% yield; 97\%- $d$ determined by ${ }^{1} \mathrm{H} \mathrm{NMR}$ ) as a colorless oil. ${ }^{1} \mathrm{H}$ NMR (400 MHz, $\left.\mathrm{CDCl}_{3}\right) \delta 7.43(\mathrm{~d}, J=8.1 \mathrm{~Hz}, 2 \mathrm{H}), 7.34(\mathrm{~d}, J=8.3 \mathrm{~Hz}, 2 \mathrm{H}), 3.03$ $(\mathrm{s}, 0.03 \mathrm{H}), 1.31(\mathrm{~s}, 9 \mathrm{H}) .{ }^{13} \mathrm{C} \mathrm{NMR}\left(101 \mathrm{MHz}, \mathrm{CDCl}_{3}\right) \delta 152.0,131.8,125.3,119.0,83.3(\mathrm{t}, J=7.5$ $\mathrm{Hz}), 76.2(\mathrm{t}, J=38.8 \mathrm{~Hz}), 34.8,31.1$.

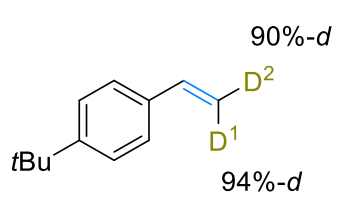

4-tert-Butylstyrene- $\boldsymbol{\beta}, \boldsymbol{\beta}$ - $\boldsymbol{d}_{2}$. To a solution of $9-\mathrm{BBN}$ in THF $(0.5 \mathrm{M}, 100 \mathrm{~mL}$, $50 \mathrm{mmol})$ was added $d$-1-(tert-butyl)-4-ethynylbenzene $(7.24 \mathrm{~g}, 45.5 \mathrm{mmol})$ at $0{ }^{\circ} \mathrm{C}$ under Ar. After the reaction mixture was stirred at $0{ }^{\circ} \mathrm{C}$ for $16 \mathrm{~h}$, AcOD $(2.4 \mathrm{~mL})$ was added. The resulting reaction mixture was stirred at $0{ }^{\circ} \mathrm{C}$ for 2 hours. The reaction was then warmed to room temperature and stirred for $2 \mathrm{~h}$. The mixture was concentrated and the crude residue was purified by silica gel column chromatography to afford the title compound (5.0 g, 68\% yield; 94\% $\mathrm{D}_{\text {cis }}$-incorporation and 90\% $\mathrm{D}_{\text {trans-incorporation }}\left({ }^{1} \mathrm{H} \mathrm{NMR}\right)$ ) as a colorless oil. (Note: $6 \%$ unlabelled $\mathrm{H}_{\text {cis }}$ and $10 \%$ unlabeled $\mathrm{H}_{\text {trans }}$ are indicated in curved parentheses \{\} for $\left.{ }^{1} \mathrm{H} \mathrm{NMR}\right) .{ }^{1} \mathrm{H}$ NMR (400 MHz, $\left.\mathrm{CDCl}_{3}\right) \delta 7.40-7.34(\mathrm{~m}, 4 \mathrm{H}), 6.71(\mathrm{~s}, 1 \mathrm{H}),\{5.85-5.55(\mathrm{~m}, 0.06 \mathrm{H})\},\{5.31-$ $5.01(\mathrm{~m}, 0.10 \mathrm{H})\}, 1.90-0.96(\mathrm{~m}, 9 \mathrm{H}) .{ }^{13} \mathrm{C} \mathrm{NMR}\left(101 \mathrm{MHz}, \mathrm{CDCl}_{3}\right) \delta 150.8,136.3,134.8,125.9$, 125.4, 113.53 - $111.44\left(\mathrm{~m}, \mathrm{CD}_{2}\right), 34.6,31.3 . \mathrm{MS}(\mathrm{EI}): \mathrm{m} / \mathrm{z}(\%) 162\left(\mathrm{M}^{+}\right), 147$ (100), 119, 91, 77. HRMS: Calculated for $\mathrm{C}_{12}{ }^{1} \mathrm{H}_{14}{ }^{2} \mathrm{H}_{2}$ : 162.1378; Found: 162.1380 .

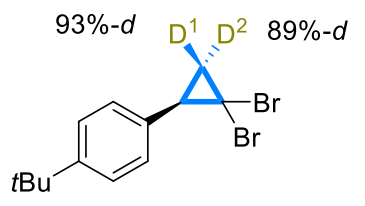

1-(tert-Butyl)-4-(2,2-dibromocyclopropyl-3,3-D 2 )benzene. The title compound (8.05 g, 78\% yield) as a colorless oil is prepared according to the General Procedure A and was purified by silica gel chromatography (Petroleum ether). (Note: $7 \%$ unlabelled $\mathrm{H}_{\text {cis }}$ and $11 \%$ unlabeled $\mathrm{H}_{\text {trans }}$ are indicated in curved parentheses \{\} for ${ }^{1} \mathrm{H}$ NMR ${ }^{1} \mathrm{H}$ NMR). ${ }^{1} \mathrm{H}$ NMR (400 MHz, $\left.\mathrm{CDCl}_{3}\right) \delta 7.38(\mathrm{~d}, J=8.4 \mathrm{~Hz}, 2 \mathrm{H}), 7.19$ $(\mathrm{d}, J=8.6 \mathrm{~Hz}, 2 \mathrm{H}), 2.92(\mathrm{~s}, 1 \mathrm{H}),\{2.11(\mathrm{~d}, J=10.6 \mathrm{~Hz}, 0.11 \mathrm{H})\},\{1.98(\mathrm{~d}, J=8.4 \mathrm{~Hz}, 0.07 \mathrm{H})\}$, $1.36-1.31(\mathrm{~m}, 9 \mathrm{H}) .{ }^{13} \mathrm{C}$ NMR $\left(101 \mathrm{MHz}, \mathrm{CDCl}_{3}\right) \delta 150.5,132.9,128.5,125.2,35.4,34.5,31.3$, 28.7 (Note: the $\underline{C D}_{2}$ was not observed). MS (EI): m/z (\%) 334, 331, 319, 159, 117, 57 (100). HRMS: Calculated for $\mathrm{C}_{13} \mathrm{Br}_{2}{ }^{1} \mathrm{H}_{14}{ }^{2} \mathrm{H}_{2}: 331.9744$; Found: 331.9738 . 
$\overbrace{( \pm)-c i s}^{B r}$

((cis)-2-Bromocyclopropyl-3,3-D $\left.\mathbf{D}_{2}\right)$-4-(tert-butyl)benzene. The title compound

( $2.32 \mathrm{~g}, 38 \%$ yield) as a colorless oil is prepared according to General Procedure B, and was purified by silica gel chromatography (Petroleum ether). ${ }^{1} \mathrm{H}$ NMR (400 $\left.\mathrm{MHz}, \mathrm{CDCl}_{3}\right) \delta 7.37(\mathrm{~d}, J=8.4 \mathrm{~Hz}, 2 \mathrm{H}), 7.20(\mathrm{~d}, J=8.1 \mathrm{~Hz}, 2 \mathrm{H}), 3.30(\mathrm{~d}, J=7.5 \mathrm{~Hz}, 1 \mathrm{H}), 2.28(\mathrm{~d}$, $J=7.5 \mathrm{~Hz}, 1 \mathrm{H}), 1.37-1.33(\mathrm{~m}, 9 \mathrm{H}) .{ }^{13} \mathrm{C} \mathrm{NMR}\left(101 \mathrm{MHz}, \mathrm{CDCl}_{3}\right) \delta 149.5,134.0,128.7,124.8$, 34.4, 31.3, 24.0, 21.4 (Note: the $\underline{\mathrm{CD}}_{2}$ was not observed). MS (EI): m/z (\%) 256, 254, 175, 145, 119, 57 (100). HRMS: Calculated for $\mathrm{C}_{13} \mathrm{Br}^{1} \mathrm{H}_{15}{ }^{2} \mathrm{H}_{2}$ : 254.0639; Found: 254.0636.

\subsubsection{Reaction of $( \pm)$ cis-d-1b with $2 \mathrm{a}$}

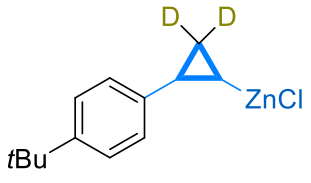

$( \pm)$ cis-d-1b (2.0 equiv)

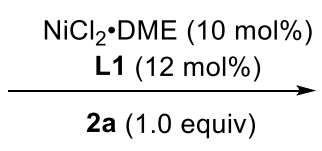

THF, $-30^{\circ} \mathrm{C}$

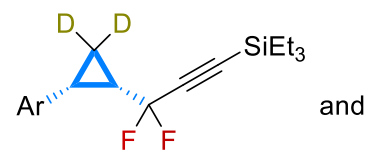
$\mathrm{Ar}=p-\mathrm{BBuC}_{6} \mathrm{H}_{4}$

$\mathbf{d - 3 b}, 80 \%, 95: 5$ er

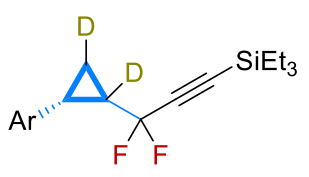

7, not observed

Note: $( \pm)$ cis-d-1b was prepared according to General Procedure C.

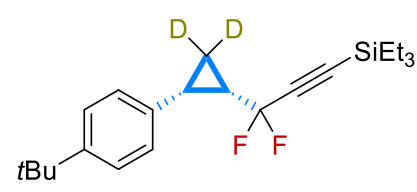

(3-((1S,2R)-2-(4-(tert-Butyl)phenyl)cyclopropyl-3,3-D $)$-3,3-difluoropr op-1-yn-1-yl)triethylsilane $(\boldsymbol{d}-3 \mathbf{b})$. The reaction was carried out on 0.5 mmol scale. The product (145 mg, $80 \%$ yield) was purified with silica gel

chromatography (Petroleum ether) as a colorless oil. $[\alpha]_{\mathrm{D}}{ }^{20}=-11.93\left(c=0.83, \mathrm{CHCl}_{3}\right)$ for a sample with 96:4 er. ${ }^{1} \mathrm{H}$ NMR (400 MHz, $\left.\mathrm{CDCl}_{3}\right) \delta 7.32$ - 7.19 (m, $\left.4 \mathrm{H}\right), 2.35$ (d, J = 9.0 Hz, $\left.1 \mathrm{H}\right), 1.87$ $1.77(\mathrm{~m}, 1 \mathrm{H}), 1.30(\mathrm{~s}, 9 \mathrm{H}), 0.92$ (t, $J=7.9 \mathrm{~Hz}, 9 \mathrm{H}), 0.53$ (q, $J=7.9 \mathrm{~Hz}, 6 \mathrm{H}) .{ }^{2} \mathrm{H} \mathrm{NMR}(77 \mathrm{MHz}$, $\left.\mathrm{CDCl}_{3}\right) \delta 7.26(\mathrm{~s}), 1.54-1.32(\mathrm{~m}), 1.32-1.08(\mathrm{~m}) .{ }^{13} \mathrm{C} \mathrm{NMR}\left(101 \mathrm{MHz}, \mathrm{CDCl}_{3}\right) \delta 149.2,132.7$, $129.6(\mathrm{t}, J=1.5 \mathrm{~Hz}), 124.7,113.5(\mathrm{t}, J=230.4 \mathrm{~Hz}), 97.6(\mathrm{dd}, J=40.2,39.0 \mathrm{~Hz}), 91.4(\mathrm{t}, J=5.2 \mathrm{~Hz})$, 34.4, 31.4, $24.7(\mathrm{dd}, J=33.6,32.0 \mathrm{~Hz}), 20.4(\mathrm{t}, J=2.7 \mathrm{~Hz}), 7.2,3.7$ (Note: the $\underline{C D}_{2}$ was not observed). ${ }^{19} \mathrm{~F}$ NMR $\left(376 \mathrm{MHz}, \mathrm{CDCl}_{3}\right) \delta-73.6(\mathrm{dd}, J=275.9,6.9 \mathrm{~Hz}, 1 \mathrm{~F}),-76.6(\mathrm{dd}, J=275.9$, $14.3 \mathrm{~Hz}, 1 \mathrm{~F})$. MS (DART): m/z (\%) $382\left(\left[\mathrm{M}+\mathrm{NH}_{4}\right]^{+}, 100\right), 345$. HRMS: Calculated for $\mathrm{C}_{22} \mathrm{H}_{34}{ }^{2} \mathrm{H}_{2} \mathrm{NF}_{2} \mathrm{Si}$ : 382.2705; Found: 382.2705. Enantiomeric ratio (96:4 er) was measured by chiral HPLC on PC-1 column $\left(\mathrm{CH}_{3} \mathrm{CN} / \mathrm{H}_{2} \mathrm{O}=70: 30,0.7 \mathrm{~mL} / \mathrm{min}\right.$, $\mathrm{UV}$ detection at $\left.214 \mathrm{~nm}\right)$; retention time $=30.12 \mathrm{~min}$ (major), retention time $=28.46 \mathrm{~min}$ (minor). 


\subsection{Reactions of 11 with 2 a using either enantiomer of $i \operatorname{Pr}-\operatorname{PyB}$ ox as the chiral ligand}

\subsubsection{Synthesis of 11}
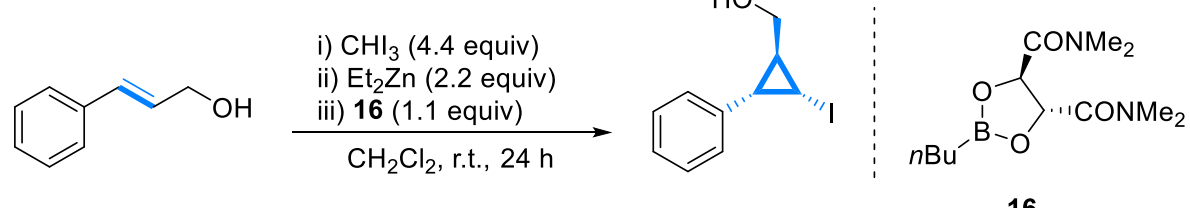

((1S,2R,3S)-2-Iodo-3-phenylcyclopropyl)methanol. [known compound ${ }^{4}$ ]: To a stirred suspension of iodoform (17.32 g, 44 mmol, 4.4 equiv) in anhydrous $\mathrm{CH}_{2} \mathrm{Cl}_{2}$ (48 mL) was added diethylzinc (22 mmol, 2.2 equiv) at room temperature. The yellow suspension was stirred at room temperature for 10 min, then a solution of cinnamyl alcohol (1.34 g, $10 \mathrm{mmol}, 1.0$ equiv) and dioxaborolane 16 (2.97 g, $11 \mathrm{mmol}, 1.1$ equiv) in anhydrous $\mathrm{CH}_{2} \mathrm{Cl}_{2}(5 \mathrm{~mL})$ was added at room temperature. The yellow suspension was allowed to stir at room temperature for $24 \mathrm{~h}$ in darkness. The reaction was quenched with saturated aqueous $\mathrm{NH}_{4} \mathrm{Cl}(30 \mathrm{~mL})$. The resulting two layers were separated and the aqueous layer was extracted with $\mathrm{Et}_{2} \mathrm{O}(3 \times 50 \mathrm{~mL})$. The combined organic layers were transferred into a round-bottomed flask, and a solution containing $60 \mathrm{~mL}$ of $2.0 \mathrm{M}$ aqueous $\mathrm{NaOH}$ and $10 \mathrm{~mL}$ of $30 \%$ aqueous $\mathrm{H}_{2} \mathrm{O}_{2}$ was added in one portion. The resulting biphasic solution was vigorously stirred for 10 min. The two layers were separated and the aqueous layer was extracted with $\mathrm{Et}_{2} \mathrm{O}(3 \times 50 \mathrm{~mL})$. The combined organic layers were washed with $10 \%$ aqueous $\mathrm{HCl}$, saturated aqueous $\mathrm{Na}_{2} \mathrm{~S}_{2} \mathrm{O}_{4}$, saturated aqueous $\mathrm{NaHCO}_{3}$, and brine, dried over $\mathrm{MgSO}_{4}$, filtered and concentrated. The residue was purified by flash chromatography (Petroleum ether/EtOAc $=4: 1)$ to afford the title compound $(1.93 \mathrm{~g}, 70 \%$ yield) as a red oil. ${ }^{1} \mathrm{H}$ NMR $\left(400 \mathrm{MHz}, \mathrm{CDCl}_{3}\right) \delta 7.37-7.27(\mathrm{~m}, 3 \mathrm{H}), 7.19(\mathrm{~d}, J=7.1 \mathrm{~Hz}, 2 \mathrm{H})$, $3.81(\mathrm{dd}, J=11.4,6.0 \mathrm{~Hz}, 1 \mathrm{H}), 3.75(\mathrm{dd}, J=11.4,6.1 \mathrm{~Hz}, 1 \mathrm{H}), 2.92(\mathrm{dd}, J=8.3,4.7 \mathrm{~Hz}, 1 \mathrm{H}), 2.07$ - 1.99 (m, $1 \mathrm{H}), 1.95-1.88$ (m, $1 \mathrm{H}), 1.67$ (br, $1 \mathrm{H})$.
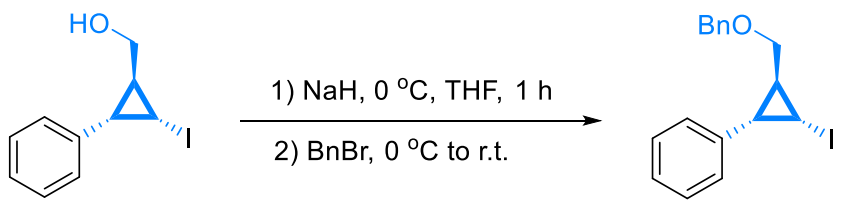

$98: 2$ er

((1S,2S,3R)-2-((Benzyloxy)methyl)-3-iodocyclopropyl)benzene. $\quad\left[\right.$ known $\quad$ compound $\left.^{4}\right]:$ To a solution of alcohol (1.93 g, $7.0 \mathrm{mmol}, 1.0$ equiv) in anhydrous THF (40 mL) was added $\mathrm{NaH}(60 \%$ dispersion in mineral oil, $336 \mathrm{mg}, 8.4 \mathrm{mmol}, 1.2$ equiv) in portions at $0{ }^{\circ} \mathrm{C}$ with stirring. The 
resulting suspension was stirred at $0{ }^{\circ} \mathrm{C}$ for $1 \mathrm{~h}$, then benzyl bromide (1.70 g, $9.8 \mathrm{mmol}, 1.4$ equiv) was added slowly at the same temperature. The reaction mixture was warmed to room temperature and stirred for $12 \mathrm{~h}$ in the darkness. The reaction was quenched with water and the aqueous layer was extracted with $\mathrm{Et}_{2} \mathrm{O}(3 \times 40 \mathrm{~mL})$. The combined organic layers were washed with brine $(50 \mathrm{~mL})$, dried over $\mathrm{Na}_{2} \mathrm{SO}_{4}$, filtered and concentrated. The residue was purified by flash chromatography (Petroleum ether/EtOAc $=40: 1)$ to afford the title compound $\left(2.24 \mathrm{~g}, 88 \%\right.$ ) as a pale yellow oil. ${ }^{1} \mathrm{H}$ NMR (400 MHz, $\left.\mathrm{CDCl}_{3}\right) \delta 7.41-7.27(\mathrm{~m}, 8 \mathrm{H}), 7.20(\mathrm{~d}, J=7.2 \mathrm{~Hz}, 2 \mathrm{H}), 4.59$ (s, $\left.2 \mathrm{H}\right), 3.69$ (dd, $J$ $=10.4,5.6 \mathrm{~Hz}, 1 \mathrm{H}), 3.60(\mathrm{dd}, J=10.4,6.0 \mathrm{~Hz}, 1 \mathrm{H}), 2.93(\mathrm{dd}, J=8.2,4.7 \mathrm{~Hz}, 1 \mathrm{H}), 2.05($ app. t, $J=$ $7.3 \mathrm{~Hz}, 1 \mathrm{H}), 1.97-1.88$ (m, $1 \mathrm{H}) .{ }^{13} \mathrm{C} \mathrm{NMR}\left(101 \mathrm{MHz}, \mathrm{CDCl}_{3}\right) \delta 138.5,138.0,128.8,128.4,127.9$, 127.7 $127.6,126.9,72.6,71.1,28.0,26.0,-1.7$. Enantiomeric ratio (98:2 er) was measured by chiral HPLC on PC-3 column $\left(\mathrm{CH}_{3} \mathrm{CN} / \mathrm{H}_{2} \mathrm{O}=70: 30,0.7 \mathrm{~mL} / \mathrm{min}\right.$, $\mathrm{UV}$ detection at $\left.214 \mathrm{~nm}\right)$; retention time $=19.34 \min$ (major), retention time $=21.15 \min ($ minor $)$.

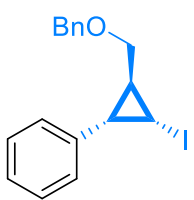

$98: 2 \mathrm{er}$

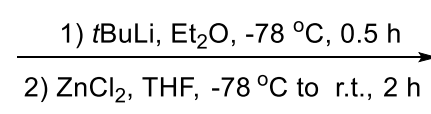

(n) 2 , $\mathrm{THF},-78^{\circ} \mathrm{C}$ to r.t., $2 \mathrm{~h}$

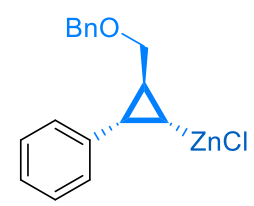

11, $98: 2$ er

Procedure $^{4}$ : To a stirred solution of tert-butyllithium (1.3 $\mathrm{M}$ in pentane, $5.4 \mathrm{~mL}, 7.0 \mathrm{mmol}, 2.2$ equiv) in anhydrous $\mathrm{Et}_{2} \mathrm{O}(4 \mathrm{~mL})$ was added a solution of cyclopropyl iodide (1.16 g, $3.2 \mathrm{mmol}, 1.0$ equiv) in anhydrous $\mathrm{Et}_{2} \mathrm{O}(3 \mathrm{~mL})$ at $-78{ }^{\circ} \mathrm{C}$. The pale yellow solution was stirred at the same temperature for $0.5 \mathrm{~h}$, then a solution of $\mathrm{ZnCl}_{2}(1.0 \mathrm{M}$ in $\mathrm{THF}, 3.4 \mathrm{~mL}, 3.4 \mathrm{mmol}, 1.1$ equiv) was then added at $-78^{\circ} \mathrm{C}$. The reaction mixture was warmed to room temperature and stirred for $2 \mathrm{~h}$. The concentration $(0.3 \mathrm{M})$ was titrated with $\mathrm{I}_{2}$ according to Knochel's method. ${ }^{5}$

\subsubsection{Reaction of 11 with $2 a$}

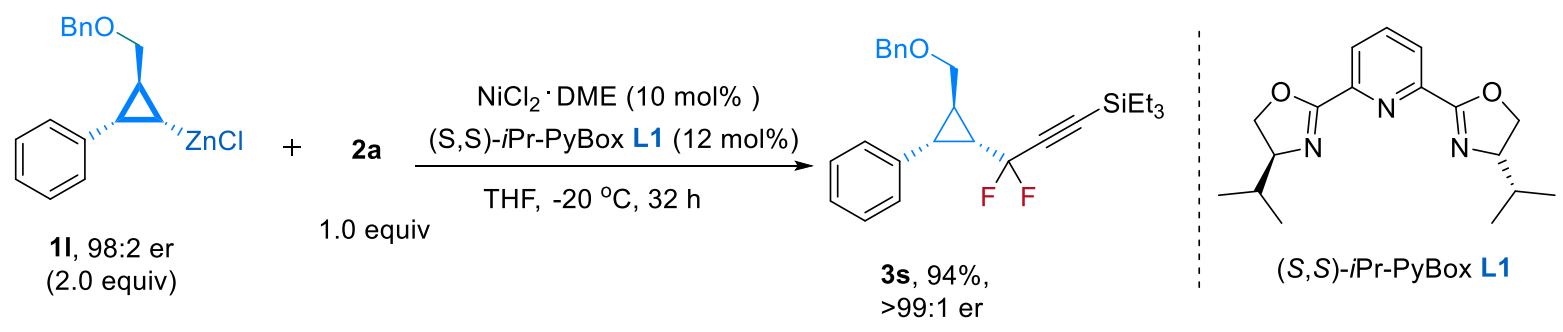


Procedure: To a $25 \mathrm{~mL}$ of Schlenk tube were added $\mathrm{NiCl}_{2} \cdot \mathrm{DME}(6.6 \mathrm{mg}, 10 \mathrm{~mol} \%$ ) and $(S, S)-i$ PrPybox L1 (10.8 mg, $12 \mathrm{~mol} \%)$ in a glovebox. The tube was then taken out from the glovebox. Compound 2a (81 mg, $0.3 \mathrm{mmol}, 1.0$ equiv) and anhydrous THF (2 mL) were added. The mixture was stirred at room temperature for $30 \mathrm{~min}$. The reaction was cooled to $-20{ }^{\circ} \mathrm{C}$ and a solution of 11 (0.30 M in THF, 98:2 er; $2.0 \mathrm{~mL}, 0.6 \mathrm{mmol}, 2.0$ equiv) was added dropwise. After stirring for $32 \mathrm{~h}$, the reaction mixture was diluted with EtOAc $(60 \mathrm{~mL})$ and filtered with a pad of cellite. The filtrate was washed with water $(10 \mathrm{~mL})$, followed by brine $(10 \mathrm{~mL})$. The organic layer was dried over anhydrous $\mathrm{Na}_{2} \mathrm{SO}_{4}$, filtered, and concentrated. The residue was purified by silica gel chromatography to give product $3 \mathrm{~s}$ as a pale yellow oil. The yield $(94 \%)$ was determined by ${ }^{19} \mathrm{~F}$ NMR before working up.

Data for compound 3s: $[\alpha]_{\mathrm{D}}{ }^{20}=11.66\left(\mathrm{c}=0.48, \mathrm{CHCl}_{3}\right)$ for a sample $>99: 1$ er. ${ }^{1} \mathrm{H} \mathrm{NMR}(400 \mathrm{MHz}$, $\left.\mathrm{CDCl}_{3}\right) \delta 7.38-7.15(\mathrm{~m}, 10 \mathrm{H}), 4.60(\mathrm{~s}, 2 \mathrm{H}), 3.68(\mathrm{dd}, J=10.3,5.6 \mathrm{~Hz}, 1 \mathrm{H}), 3.56(\mathrm{dd}, J=10.3,6.3$ $\mathrm{Hz}, 1 \mathrm{H}), 2.42(\mathrm{dd}, J=8.2,7.2 \mathrm{~Hz}, 1 \mathrm{H}), 2.18-2.11(\mathrm{~m}, 1 \mathrm{H}), 1.88-1.78(\mathrm{~m}, 1 \mathrm{H}), 0.92(\mathrm{t}, J=7.9$ $\mathrm{Hz}, 9 \mathrm{H}), 0.53$ (q, $J=7.9 \mathrm{~Hz}, 6 \mathrm{H}) .{ }^{13} \mathrm{C} \mathrm{NMR}\left(101 \mathrm{MHz}, \mathrm{CDCl}_{3}\right) \delta 138.2,135.3,129.8,128.4,127.9$, 127.63, 127.58, 126.6, $112.8(\mathrm{dd}, J=232.3,232.3 \mathrm{~Hz}), 97.6(\mathrm{t}, J=39.7 \mathrm{~Hz}), 91.6(\mathrm{t}, J=5.2 \mathrm{~Hz})$, 72.5, 70.9, $29.6(\mathrm{dd}, J=33.4,31.6 \mathrm{~Hz}), 26.2(\mathrm{t}, J=2.5 \mathrm{~Hz}), 20.5(\mathrm{t}, J=2.1 \mathrm{~Hz}), 7.2,3.7 .{ }^{19} \mathrm{~F} \mathrm{NMR}$ $\left(376 \mathrm{MHz}, \mathrm{CDCl}_{3}\right) \delta-73.5(\mathrm{dd}, J=276.5,7.5 \mathrm{~Hz}, 1 \mathrm{~F}),-77.0(\mathrm{dd}, J=276.5,14.2 \mathrm{~Hz}, 1 \mathrm{~F}) . \mathrm{MS}$ (DART): m/z (\%) $444\left(\left[\mathrm{M}+\mathrm{NH}_{4}\right]^{+}\right)$. HRMS: Calculated for $\mathrm{C}_{26} \mathrm{H}_{36} \mathrm{ONF}_{2} \mathrm{Si}$ : 444.2529; Found: 444.2528. HPLC separation ( $\mathrm{PC}-1, \mathrm{CH}_{3} \mathrm{CN} / \mathrm{H}_{2} \mathrm{O}=70: 30,0.7 \mathrm{~mL} / \mathrm{min}$, UV detection at $214 \mathrm{~nm}$ ): retention time $=34.66 \mathrm{~min}$ (major), retention time $=31.82 \mathrm{~min}$ (minor), 99.7:0.3 e.r.

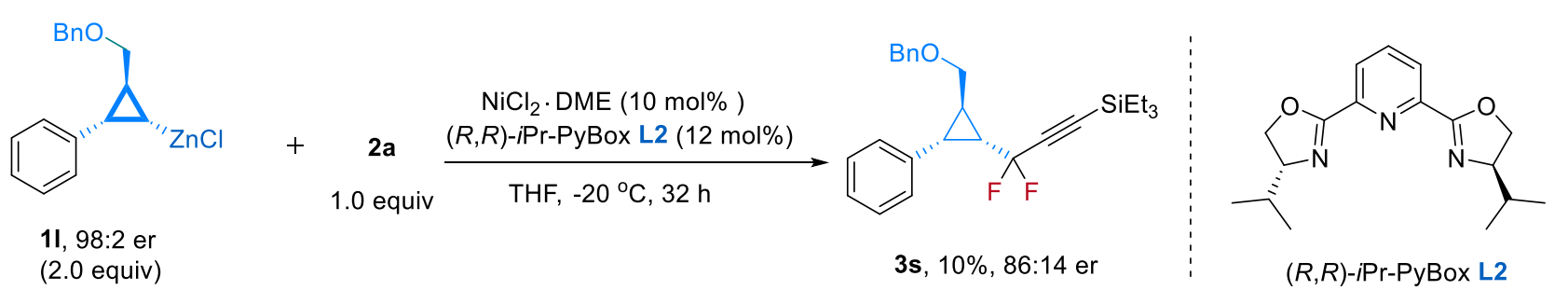

Procedure: To a $25 \mathrm{~mL}$ of Schlenk tube were added $\mathrm{NiCl}_{2} \cdot \mathrm{DME}(6.6 \mathrm{mg}, 10 \mathrm{~mol} \%$ ) and $(R, R)-i$ PrPybox $\mathbf{L} 2(10.8 \mathrm{mg}, 12 \mathrm{~mol} \%)$ in a glovebox. The tube was then taken out from the glovebox. Compound 2a (81 mg, $0.3 \mathrm{mmol}, 1.0$ equiv) and anhydrous THF (2 mL) were added. The mixture was stirred at room temperature for $30 \mathrm{~min}$. The reaction was cooled to $-20{ }^{\circ} \mathrm{C}$ and a solution 
of 11 (0.30 M in THF, 98:2 er; $2.0 \mathrm{~mL}, 0.6 \mathrm{mmol}, 2.0$ equiv) was added dropwise. After stirring for $32 \mathrm{~h}$, the reaction mixture was diluted with EtOAc $(60 \mathrm{~mL})$ and filtered with a pad of cellite. The filtrate was washed with water $(10 \mathrm{~mL})$, followed by brine $(10 \mathrm{~mL})$. The organic layer was then dried over anhydrous $\mathrm{Na}_{2} \mathrm{SO}_{4}$, filtered, and concentrated. The residue was purified by silica gel chromatography to give product $\mathbf{3 s}$ as a pale yellow oil. The yield $(10 \%)$ was determined by ${ }^{19} \mathrm{~F}$ NMR before working up. HPLC separation $\left(\mathrm{PC}-1, \mathrm{CH}_{3} \mathrm{CN} / \mathrm{H}_{2} \mathrm{O}=70: 30,0.7 \mathrm{~mL} / \mathrm{min}\right.$, UV detection at $214 \mathrm{~nm}$ ): retention time $=34.67 \min$ (major), retention time $=30.68 \min$ (minor), $86: 14$ e.r.

\section{Enantiodivergent relay coupling (EDRC) of $( \pm)$ cis-1a}
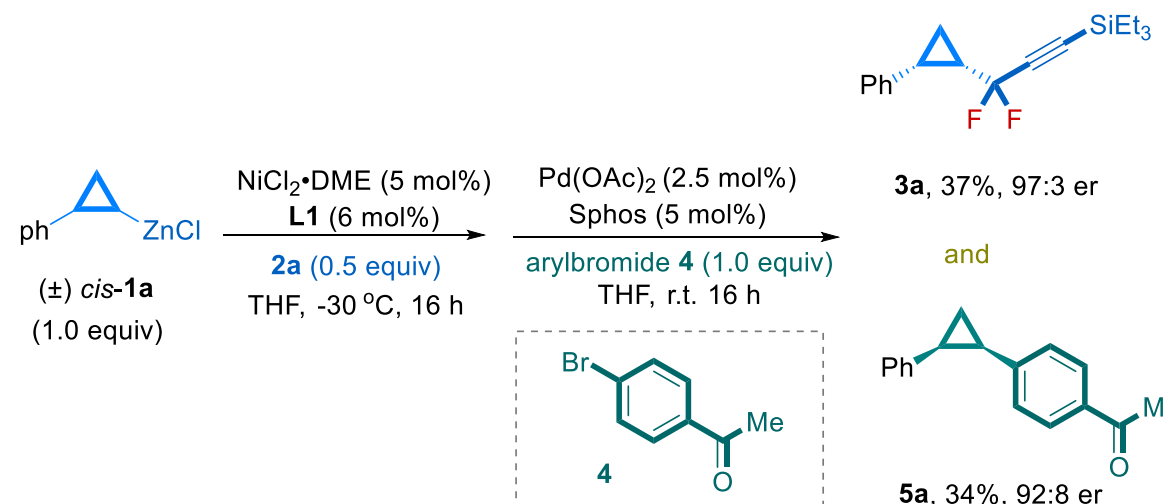

3a, 37\%, 97:3 er

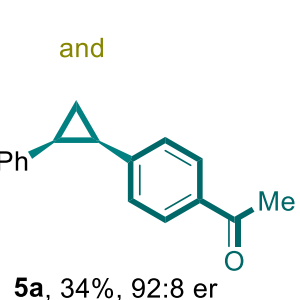

Procedure: To a $25 \mathrm{~mL}$ of Schlenk tube were added $\mathrm{NiCl}_{2} \cdot \mathrm{DME}(6.6 \mathrm{mg}, 5 \mathrm{~mol} \%)$ and $\mathbf{L 1}(10.8 \mathrm{mg}$, $6 \mathrm{~mol} \%$ ) in a glovebox. The tube was then taken out from the glovebox. gem-Difluoropropargyl bromide $2 \mathbf{a}(81 \mathrm{mg}, 0.3 \mathrm{mmol}, 0.5$ equiv) and THF $(2 \mathrm{~mL})$ were added. The resulting mixture was stirred at room temperature for $25 \mathrm{~min}$. The reaction was cooled to $-30{ }^{\circ} \mathrm{C}$ and cyclopropylzinc reagent 1a $(0.6 \mathrm{mmol}, 1.0$ equiv in $1.82 \mathrm{~mL}$ of THF) was added dropwise over a period of $10 \mathrm{~min}$. After stirring at $-30{ }^{\circ} \mathrm{C}$ for $20 \mathrm{~h}$, the above reaction mixture was added to a solution of $\mathrm{Pd}(\mathrm{OAc})_{2}(3.4$ mg, $2.5 \mathrm{~mol} \%)$, Sphos (12.3 mg, $5 \mathrm{~mol} \%$ ) and 1-(4-bromophenyl)ethan-1-one 4 (119 mg, $0.6 \mathrm{mmol}$, 1.0 equiv) in anhydrous THF $(1 \mathrm{~mL})$. The reaction mixture was stirred at room temperature for $16 \mathrm{~h}$ before working up. The reaction was quenched with saturated aqueous $\mathrm{NH}_{4} \mathrm{Cl}$ solution and diluted with $\mathrm{CH}_{2} \mathrm{Cl}_{2}(50 \mathrm{~mL})$. The organic layer was separated and the aqueous layer extracted three times with $\mathrm{CH}_{2} \mathrm{Cl}_{2}$. The combined organic extracts were washed with brine and dried with $\mathrm{Na}_{2} \mathrm{SO}_{4}$, and the solvent was removed. The residue was purified with silica gel chromatography to give compound 3a (68 mg, 37\% yield, 97:3 er; eluent: petroleum ether) and compound 5a (48 mg, 34\% yield, 92:8 er; eluent: petroleum ether/EtOAc $=20: 1$ ). 
Data for compound 3a: pale yellow oil; $[\alpha]_{\mathrm{D}}{ }^{20}=-22.8\left(c=0.62, \mathrm{CHCl}_{3}\right)$ for a sample with 97:3 er. ${ }^{1} \mathrm{H}$ NMR (400 MHz, $\left.\mathrm{CDCl}_{3}\right) \delta 7.32(\mathrm{~d}, J=7.3 \mathrm{~Hz}, 2 \mathrm{H}), 7.27-7.21(\mathrm{~m}, 2 \mathrm{H}), 7.21-7.15(\mathrm{~m}, 1 \mathrm{H})$, $2.46-2.37$ (m, $1 \mathrm{H}), 1.92-1.78(\mathrm{~m}, 1 \mathrm{H}), 1.48-1.40(\mathrm{~m}, 1 \mathrm{H}), 1.28-1.17(\mathrm{~m}, 1 \mathrm{H}), 0.92(\mathrm{t}, J=7.9$ $\mathrm{Hz}, 9 \mathrm{H}), 0.53(\mathrm{q}, J=7.9 \mathrm{~Hz}, 6 \mathrm{H}) .{ }^{13} \mathrm{C} \mathrm{NMR}\left(101 \mathrm{MHz}, \mathrm{CDCl}_{3}\right) \delta 135.9,130.0(\mathrm{t}, J=1.0 \mathrm{~Hz})$, 127.8, 126.5, $113.31(\mathrm{dd}, J=232.0,231.5 \mathrm{~Hz}), 97.6(\mathrm{dd}, J=40.2,39.2 \mathrm{~Hz}), 91.4(\mathrm{t}, J=5.3 \mathrm{~Hz}), 24.8$ $(\mathrm{dd}, J=33.6,31.7 \mathrm{~Hz}), 21.0(\mathrm{t}, J=2.8 \mathrm{~Hz}), 7.2,7.0(\mathrm{t}, J=2.6 \mathrm{~Hz}), 3.7 .{ }^{19} \mathrm{~F} \mathrm{NMR}\left(376 \mathrm{MHz}, \mathrm{CDCl}_{3}\right)$ $\delta-73.92(\mathrm{dd}, J=276.0,7.1 \mathrm{~Hz}, 1 \mathrm{~F}),-77.68(\mathrm{dd}, J=276.3,14.3 \mathrm{~Hz}, 1 \mathrm{~F})$. MS (DART): m/z (\%) 324 $\left(\left[\mathrm{M}+\mathrm{NH}_{4}\right]^{+}\right), 305,287$ (100), 203. HRMS: Calculated for $\mathrm{C}_{18} \mathrm{H}_{28} \mathrm{NF}_{2} \mathrm{Si}: 324.1954$; Found: 324.1952. Enantiomeric ratio (97:3 er) was measured by chiral HPLC on PC-1 column $\left(\mathrm{CH}_{3} \mathrm{CN} / \mathrm{H}_{2} \mathrm{O}=70: 30\right.$, $0.7 \mathrm{~mL} / \mathrm{min}$, UV detection at $214 \mathrm{~nm}$ ); retention time $=22.79$ min (major), retention time $=20.77$ min (minor). (Note: The absolute configuration of compound 3a was determined by single-crystal X-ray diffraction study of compound $\mathbf{6}$, which was derived from 3a by a [3+2] cycloaddition reaction with azide.)

Data for compound 5a: white solid (m.p. $\left.66-68{ }^{\circ} \mathrm{C}\right) ;[\alpha]_{\mathrm{D}}{ }^{20}=35.42\left(c=0.59, \mathrm{CHCl}_{3}\right)$ for a sample with 92:8 er. ${ }^{1} \mathrm{H}$ NMR $\left(400 \mathrm{MHz}, \mathrm{CDCl}_{3}\right) \delta 7.67(\mathrm{~d}, J=8.4 \mathrm{~Hz}, 2 \mathrm{H}), 7.14-7.03(\mathrm{~m}, 3 \mathrm{H}), 6.98(\mathrm{~d}, J$ $=8.4 \mathrm{~Hz}, 4 \mathrm{H}), 2.65-2.57(\mathrm{~m}, 1 \mathrm{H}), 2.54-2.46(\mathrm{~m}, 4 \mathrm{H}), 1.58-1.51(\mathrm{~m}, 1 \mathrm{H}), 1.49-1.43(\mathrm{~m}, 1 \mathrm{H})$. ${ }^{13} \mathrm{C}$ NMR $\left(101 \mathrm{MHz}, \mathrm{CDCl}_{3}\right) \delta 197.7,144.8,137.3,134.5,129.1,128.5,127.8,127.7,125.9,26.4$, 25.4, 24.1, 11.8. MS (EI): m/z (\%) $236\left(\mathrm{M}^{+}, 100\right), 221,193,178,115$. HRMS: Calculated for $\mathrm{C}_{17} \mathrm{H}_{16} \mathrm{O}: 236.1201$; Found: 236.1207. Enantiomeric ratio (92:8 er) was measured by chiral HPLC on ADH column $\left(\right.$ Hexane $/{ }^{i} \mathrm{PrOH}=99: 1,0.7 \mathrm{~mL} / \mathrm{min}, \mathrm{UV}$ detection at $\left.214 \mathrm{~nm}\right)$; retention time $=26.08$ $\min$ (major), retention time $=24.48 \min$ (minor) .

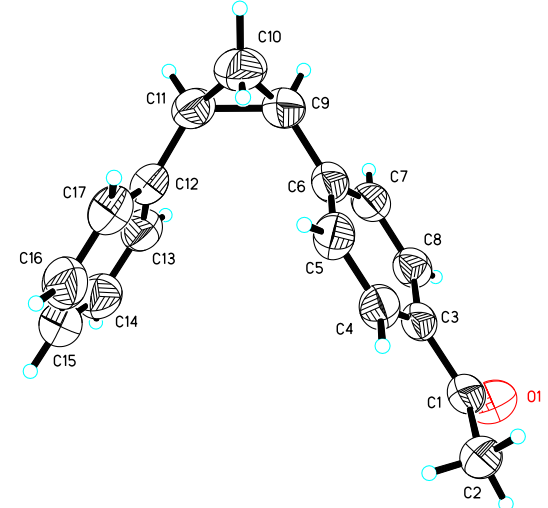




\section{Synthesis of compound 6}

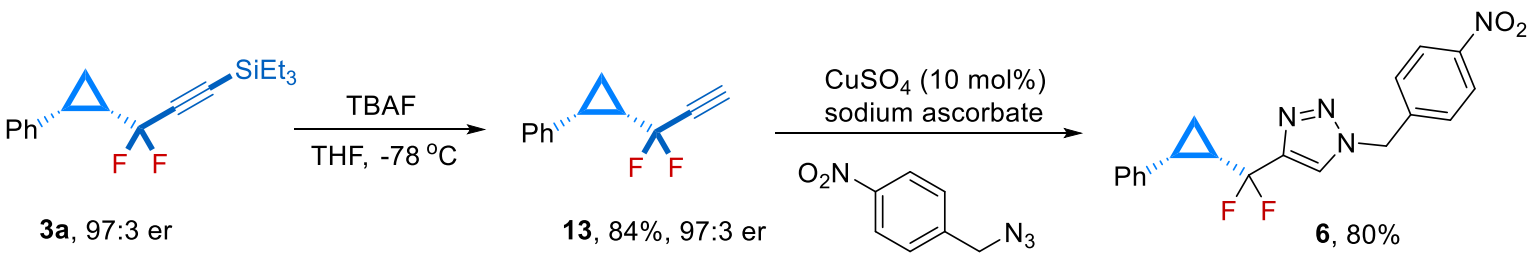

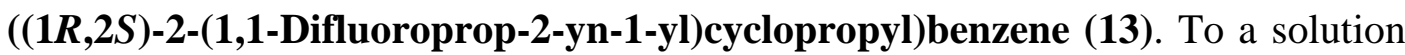
of 3a (960 mg, $3.1 \mathrm{mmol}, 1.0$ equiv) in anhydrous THF (30 mL) was added TBAF (1.0 $\mathrm{M}$ in THF; $3.7 \mathrm{~mL}, 3.7 \mathrm{mmol}, 1.2$ equiv) under Ar at $-78{ }^{\circ} \mathrm{C}$. The reaction mixture was stirred at same reaction temperature for $1 \mathrm{~h}$ and warmed to $-20^{\circ} \mathrm{C}$. The reaction was then quenched with 10 $\mathrm{mL}$ of saturated aqueous $\mathrm{NH}_{4} \mathrm{Cl}$ solution. After stirring for $5 \mathrm{~min}$ at $0{ }^{\circ} \mathrm{C}$, the resulting mixture was extracted with diethyl ether $(3 \times 30 \mathrm{~mL})$. The combined organic layers were washed with brine, dried over $\mathrm{Na}_{2} \mathrm{SO}_{4}$, filtered, and concentrated. The residue was purified by flash silica gel chromatography (Petroleum ether) to give alkyne $13(506 \mathrm{mg}, 84 \%$ yield $)$ as a colorless oil. $[\alpha]_{\mathrm{D}}{ }^{20}=2.78(c=0.79$, $\left.\mathrm{CHCl}_{3}\right)$ for a sample with 97:3 er. ${ }^{1} \mathrm{H} \mathrm{NMR}\left(400 \mathrm{MHz}, \mathrm{CDCl}_{3}\right) \delta 7.35(\mathrm{~d}, J=7.4 \mathrm{~Hz}, 2 \mathrm{H}), 7.31-$ $7.25(\mathrm{~m}, 2 \mathrm{H}), 7.25-7.20(\mathrm{~m}, 1 \mathrm{H}), 2.54(\mathrm{t}, J=5.0 \mathrm{~Hz}, 1 \mathrm{H}), 2.51-2.44(\mathrm{~m}, 1 \mathrm{H}), 1.94-1.82(\mathrm{~m}, 1$ $\mathrm{H}), 1.51-1.45(\mathrm{~m}, 1 \mathrm{H}), 1.30-1.23(\mathrm{~m}, 1 \mathrm{H}) .{ }^{13} \mathrm{C} \mathrm{NMR}\left(101 \mathrm{MHz}, \mathrm{CDCl}_{3}\right) \delta 135.6,130.0,127.8$, 126.6, $113.4(\mathrm{t}, J=231.4 \mathrm{~Hz}), 76.1(\mathrm{t}, J=41.4 \mathrm{~Hz}), 75.6(\mathrm{t}, J=6.6 \mathrm{~Hz}), 24.7(\mathrm{t}, J=31.7 \mathrm{~Hz}), 21.1(\mathrm{t}$, $J=2.7 \mathrm{~Hz}), 6.8(\mathrm{t}, J=3.0 \mathrm{~Hz}) .{ }^{19} \mathrm{~F}$ NMR $\left(376 \mathrm{MHz}, \mathrm{CDCl}_{3}\right) \delta-76.62(\mathrm{ddd}, J=277.9,8.8,4.9 \mathrm{~Hz}, 1$ F), -79.55 (ddd, $J=277.9,13.0,5.2 \mathrm{~Hz}, 1 \mathrm{~F}) . \mathrm{MS}(\mathrm{EI}): \mathrm{m} / \mathrm{z}(\%) 192\left(\mathrm{M}^{+}\right), 117$ (100). HRMS: Calculated for $\mathrm{C}_{12} \mathrm{H}_{10} \mathrm{~F}_{2}$ : 192.0751; Found: 192.0747. Enantiomeric ratio (97:3 er) was measured by chiral HPLC on PC-3 column $\left(\mathrm{CH}_{3} \mathrm{CN} / \mathrm{H}_{2} \mathrm{O}=70: 30,0.7 \mathrm{~mL} / \mathrm{min}\right.$, $\mathrm{UV}$ detection at $\left.214 \mathrm{~nm}\right)$; retention time $=7.25 \mathrm{~min}$ (major), retention time $=8.87 \mathrm{~min}$ (minor) .

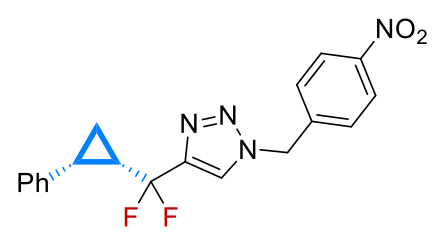

4-(Difluoro-((1S,2R)-2-phenylcyclopropyl)methyl)-1-(4-nitrobenzyl)-1

H-1,2,3-triazole (6). 1-(Azidomethyl)-4-nitrobenzene (122 mg, 0.68 mmol, 1.2 equiv) and 13 (110 mg, $0.57 \mathrm{mmol}, 1.0$ equiv) were dissolved in $t \mathrm{BuOH}(2 \mathrm{~mL})$ and $\mathrm{H}_{2} \mathrm{O}(2 \mathrm{~mL})$, followed by addition a solution of $\mathrm{CuSO}_{4}(9.1 \mathrm{mg}, 10 \mathrm{~mol} \%)$ and sodium ascorbate $(57 \mathrm{mg}, 0.285 \mathrm{mmol}, 0.5$ equiv) in water $(0.3 \mathrm{~mL})$. The reaction was stirred at room temperature for $8 \mathrm{~h}$. After the reaction was quenched with water, the mixture was extracted with EtOAc $(3 \times 30 \mathrm{~mL})$. The combined organic layers were washed with brine, dried over $\mathrm{Na}_{2} \mathrm{SO}_{4}$, 
filtered and concentrated. The crude product was purified by silica gel silica gel chromatography (Petroleum ether/EtOAc $=1: 1)$ to give compound $6(171 \mathrm{mg}, 80 \%$ yield) as a white solid (m.p. 102-103 $\left.{ }^{\circ} \mathrm{C}\right) .{ }^{1} \mathrm{H}$ NMR (400 MHz, $\left.\mathrm{CDCl}_{3}\right) \delta 8.22(\mathrm{~d}, J=8.4 \mathrm{~Hz}, 2 \mathrm{H}), 7.33(\mathrm{~d}, J=8.4 \mathrm{~Hz}, 2 \mathrm{H}), 7.12$ - 6.99 (m, 5 H), 6.94 (s, 1 H), 5.53 (d, J=15.5 Hz, 1 H), 5.41 (d, J=15.5 Hz, 1 H), 2.48 - 2.39 (m, $1 \mathrm{H}), 2.31-2.17(\mathrm{~m}, 1 \mathrm{H}), 1.61-1.68(\mathrm{~m}, 1 \mathrm{H}), 1.39-1.19(\mathrm{~m}, 1 \mathrm{H}) .{ }^{13} \mathrm{C} \mathrm{NMR}\left(101 \mathrm{MHz}, \mathrm{CDCl}_{3}\right)$ $\delta 148.0,145.7(\mathrm{t}, J=34.7 \mathrm{~Hz}), 140.9,136.0,129.4,128.7,127.4,126.0,124.2,121.9(\mathrm{t}, J=2.8 \mathrm{~Hz})$, $118.2(\mathrm{dd}, J=237.6,235.7 \mathrm{~Hz}), 52.9,23.6(\mathrm{dd}, J=32.4,28.5 \mathrm{~Hz}), 20.6(\mathrm{dd}, J=3.8,1.5 \mathrm{~Hz}), 5.5(\mathrm{dd}$, $J=4.7,2.8 \mathrm{~Hz}) .{ }^{19} \mathrm{~F}$ NMR $\left(376 \mathrm{MHz}, \mathrm{CDCl}_{3}\right) \delta-81.9(\mathrm{dd}, J=267.5,10.4 \mathrm{~Hz}),-90.4(\mathrm{dd}, J=267.5$, $14.8 \mathrm{~Hz})$. MS (DART): m/z (\%) $371\left([\mathrm{M}+\mathrm{H}]^{+}, 100\right), 351\left([\mathrm{M}-\mathrm{F}]^{+}\right)$. HRMS: Calculated for $\mathrm{C}_{19} \mathrm{H}_{17} \mathrm{O}_{2} \mathrm{~N}_{4} \mathrm{~F}_{2}$ : 371.1314; Found: 371.1315 .

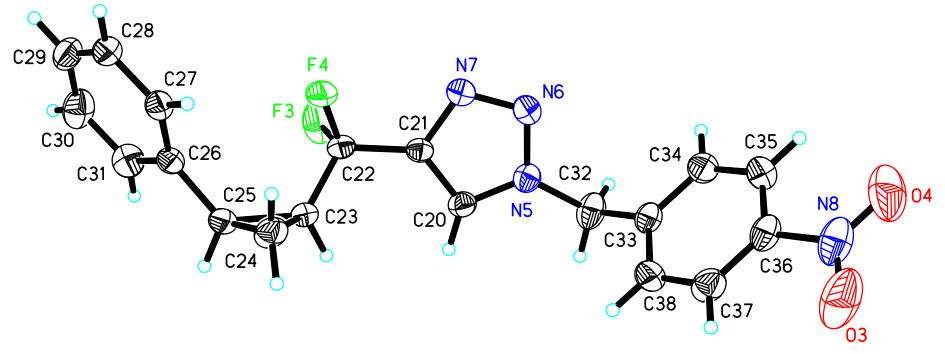

X-Ray Crystal Structure of Compound 6

6. Ni-catalyzed enantioselective cross-coupling of racemic cyclopropylzinc reagents with alkyl bromides

6.1 General procedure for Ni-catalyzed enantioselective cross-coupling of racemic cyclopropylzinc reagents $( \pm$ ) cis-1 with primary propargyl and allyl bromides (General Procedure F).

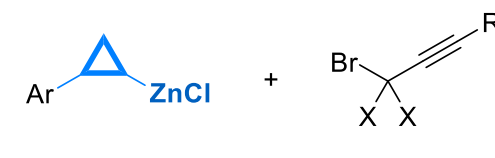

( \pm ) cis- $1,2.0$ equiv

2, 1.0 equiv

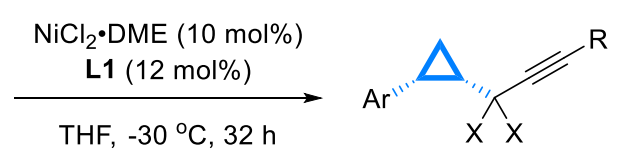

3, $X=F ; 8, X=H$

General Procedure F: To a $25 \mathrm{~mL}$ of Schlenk were added $\mathrm{NiCl}_{2} \cdot \mathrm{DME}(11.0 \mathrm{mg}, 10 \mathrm{~mol} \%)$ and $\mathbf{L 1}$ (18.0 $\mathrm{mg}, 12 \mathrm{~mol} \%$ ) in a glovebox. The tube was then taken out from the glovebox. The corresponding propargyl or allyl bromide $(0.5 \mathrm{mmol}, 1.0$ equiv) and anhydrous THF ( $3 \mathrm{~mL})$ were added. After stirring at room temperature for $25 \mathrm{~min}$, the reaction mixture was cooled to $-30{ }^{\circ} \mathrm{C}$ and 
cyclopropylzinc reagent $( \pm)$ cis-1 $(0.33 \mathrm{M}$ in THF; $1.0 \mathrm{mmol}, 2.0$ equiv) was added dropwise over a period of $15 \mathrm{~min}$. The reaction was stirred at $-30^{\circ} \mathrm{C}$ for $32 \mathrm{~h}$, and was quenched with saturated aqueous $\mathrm{NH}_{4} \mathrm{Cl}$. The resulting mixture was diluted with $\mathrm{CH}_{2} \mathrm{Cl}_{2}(50 \mathrm{~mL})$ and was filtered with a pad of cellite. The filtrate was washed with water $(15 \mathrm{~mL})$, and the organic layer was dried over $\mathrm{Na}_{2} \mathrm{SO}_{4}$, filtered, and concentrated. The residue was purified with flash column silica gel chromatography to give the pure product.

\subsection{Characterization data for compounds 3,8 , and 9}

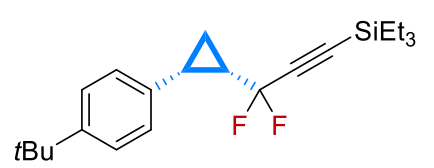

(3-((1S,2R)-2-(4-(tert-Butyl)phenyl)cyclopropyl)-3,3-difluoroprop-1-yn -1-yl)triethylsilane (3b). The product (152 mg, 84\% yield) was purified with silica gel chromatography (Petroleum ether) as a colorless oil. $[\alpha]_{\mathrm{D}}{ }^{20}$ $=-12.6\left(c=0.47, \mathrm{CHCl}_{3}\right)$ for a sample with 97:3 er. ${ }^{1} \mathrm{H} \mathrm{NMR}\left(400 \mathrm{MHz}, \mathrm{CDCl}_{3}\right) \delta 7.30-7.23(\mathrm{~m}, 4$ H), $2.41-2.33(\mathrm{~m}, 1 \mathrm{H}), 1.91-1.74(\mathrm{~m}, 1 \mathrm{H}), 1.49-1.37(\mathrm{~m}, 1 \mathrm{H}), 1.30(\mathrm{~s}, 9 \mathrm{H}), 1.28-1.17(\mathrm{~m}, 1$ $\mathrm{H}), 0.93(\mathrm{t}, J=7.9 \mathrm{~Hz}, 9 \mathrm{H}), 0.53(\mathrm{q}, J=7.9 \mathrm{~Hz}, 6 \mathrm{H}) .{ }^{13} \mathrm{C} \mathrm{NMR}\left(101 \mathrm{MHz}, \mathrm{CDCl}_{3}\right) \delta 149.2,132.7$, $129.6(\mathrm{t}, J=1.0 \mathrm{~Hz}), 124.7,113.5(\mathrm{t}, J=230.3 \mathrm{~Hz}), 97.5(\mathrm{dd}, J=40.3,39.0 \mathrm{~Hz}), 91.5(\mathrm{t}, J=5.2 \mathrm{~Hz})$, 34.4, 31.3, $24.8(\mathrm{dd}, J=33.7,32.0 \mathrm{~Hz}), 20.5(\mathrm{t}, J=2.7 \mathrm{~Hz}), 7.24,7.18(\mathrm{t}, J=2.6 \mathrm{~Hz}), 3.7 .{ }^{19} \mathrm{~F}$ NMR $\left(376 \mathrm{MHz}, \mathrm{CDCl}_{3}\right) \delta-73.7(\mathrm{dd}, J=276.0,6.8 \mathrm{~Hz}, 1 \mathrm{~F}),-76.6(\mathrm{dd}, J=276.0,14.4 \mathrm{~Hz}, 1 \mathrm{~F}) . \mathrm{MS}$ (DART): m/z (\%) $380\left(\left[\mathrm{M}+\mathrm{NH}_{4}\right]^{+}\right)$, 343. HRMS: Calculated for $\mathrm{C}_{22} \mathrm{H}_{36} \mathrm{NF}_{2} \mathrm{Si}$ : 380.2580; Found: 380.2578. Enantiomeric ratio (97:3 er) was measured by chiral HPLC on PC-1 column $\left(\mathrm{CH}_{3} \mathrm{CN} / \mathrm{H}_{2} \mathrm{O}\right.$ $=70: 30,0.7 \mathrm{~mL} / \mathrm{min}, \mathrm{UV}$ detection at $214 \mathrm{~nm})$; retention time $=28.12 \mathrm{~min}$ (major), retention time $=$ $26.56 \min$ (minor).

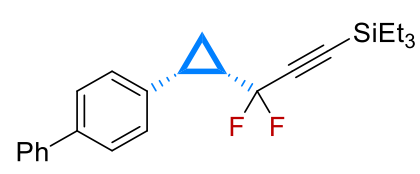

\section{(3-((1S,2R)-2-([1,1'-Biphenyl]-4-yl)cyclopropyl)-3,3-difluoroprop-1-yn} -1-yl)triethylsilane (3c). The product (172 mg, 90\% yield) was purified with silica gel chromatography (Petroleum ether) as a colorless oil. $[\alpha]_{\mathrm{D}}{ }^{20}$ $=-8.9\left(c=0.60, \mathrm{CHCl}_{3}\right)$ for a sample with 97:3 er. ${ }^{1} \mathrm{H} \mathrm{NMR}\left(400 \mathrm{MHz}, \mathrm{CDCl}_{3}\right) \delta 7.61-7.55(\mathrm{~m}, 2$ H), $7.50(\mathrm{dd}, J=8.1,1.6 \mathrm{~Hz}, 2 \mathrm{H}), 7.47-7.38(\mathrm{~m}, 4 \mathrm{H}), 7.33(\mathrm{t}, J=7.3 \mathrm{~Hz}, 1 \mathrm{H}), 2.50-2.42(\mathrm{~m}, 1$ H), $1.97-1.84(\mathrm{~m}, 1 \mathrm{H}), 1.53-1.46(\mathrm{~m}, 1 \mathrm{H}), 1.31-1.25(\mathrm{~m}, 1 \mathrm{H}), 0.91(\mathrm{t}, J=7.9,9 \mathrm{H}), 0.53(\mathrm{q}, J$ = 7.9, $6 \mathrm{H}) .{ }^{13} \mathrm{C} \mathrm{NMR}\left(101 \mathrm{MHz} \mathrm{CDCl}_{3}\right) \delta 140.9,139.3,135.1,130.4,128.6,127.0,126.9,126.5$, $113.5(\mathrm{t}, J=230.4 \mathrm{~Hz}), 97.4(\mathrm{dd}, J=39.9,39.1 \mathrm{~Hz}), 91.8(\mathrm{t}, J=5.1 \mathrm{~Hz}), 24.9(\mathrm{dd}, J=33.8,32.2 \mathrm{~Hz})$, 
$20.7(\mathrm{t}, J=2.7 \mathrm{~Hz}), 7.3(\mathrm{t}, J=2.6 \mathrm{~Hz}), 7.2,3.7 .{ }^{19} \mathrm{~F} \mathrm{NMR}\left(376 \mathrm{MHz}, \mathrm{CDCl}_{3}\right) \delta-73.0--73.9(\mathrm{~m}, 1 \mathrm{~F})$, $-76.1--77.0(\mathrm{~m}, 1 \mathrm{~F})$. MS (EI): m/z (\%) $382\left(\mathrm{M}^{+}\right)$, 362, 193 (100), 91, 77. HRMS: Calculated for $\mathrm{C}_{24} \mathrm{H}_{28} \mathrm{~F}_{2} \mathrm{Si}$ : 382.1928; Found: 382.1931. Enantiomeric ratio (97:3 er) was measured by chiral HPLC on PC-1 column $\left(\mathrm{CH}_{3} \mathrm{CN} / \mathrm{H}_{2} \mathrm{O}=80: 20,0.7 \mathrm{~mL} / \mathrm{min}\right.$, $\mathrm{UV}$ detection at $\left.214 \mathrm{~nm}\right)$; retention time = $26.89 \mathrm{~min}$ (major), retention time $=16.57 \mathrm{~min}$ (minor).

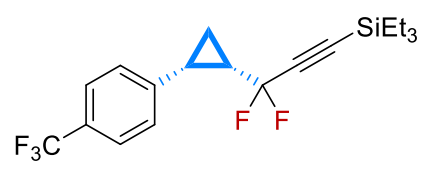

\section{(3,3-Difluoro-3-((1S,2R)-2-(4-(trifluoromethyl)phenyl)cyclopropyl)pro}

p-1-yn-1-yl)triethylsilane (3d). The product (135 mg, 72\% yield) was purified with silica gel chromatography (Petroleum ether) as a colorless oil. $[\alpha]_{\mathrm{D}}{ }^{20}=-3.1\left(c=0.60, \mathrm{CHCl}_{3}\right)$ for a sample with 97:3 er. ${ }^{1} \mathrm{H} \mathrm{NMR}\left(400 \mathrm{MHz}, \mathrm{CDCl}_{3}\right) \delta 7.52(\mathrm{~d}$, $J=8.2 \mathrm{~Hz}, 2 \mathrm{H}), 7.44(\mathrm{~d}, J=8.1 \mathrm{~Hz}, 2 \mathrm{H}), 2.49-2.40(\mathrm{~m}, 1 \mathrm{H}), 2.00-1.87(\mathrm{~m}, 1 \mathrm{H}), 1.52-1.45(\mathrm{~m}$, $1 \mathrm{H}), 1.35-1.27(\mathrm{~m}, 1 \mathrm{H}), 0.91(\mathrm{t}, J=7.9 \mathrm{~Hz}, 9 \mathrm{H}), 0.53(\mathrm{q}, J=7.9 \mathrm{~Hz}, 6 \mathrm{H}) .{ }^{13} \mathrm{C} \mathrm{NMR}(101 \mathrm{MHz}$, $\left.\mathrm{CDCl}_{3}\right) \delta 140.2(\mathrm{q}, J=1.2 \mathrm{~Hz}), 130.3(\mathrm{t}, J=1.3 \mathrm{~Hz}), 128.8(\mathrm{q}, J=32.3 \mathrm{~Hz}), 124.3(\mathrm{q}, J=272.7 \mathrm{~Hz})$, $124.7(\mathrm{q}, J=3.8 \mathrm{~Hz}), 113.1(\mathrm{t}, J=230.7 \mathrm{~Hz}), 97.2(\mathrm{dd}, J=40.1,39.3 \mathrm{~Hz}), 92.1(\mathrm{t}, J=5.2 \mathrm{~Hz}), 25.1$ $(\mathrm{dd}, J=33.8,32.6 \mathrm{~Hz}), 20.8(\mathrm{t}, J=2.6 \mathrm{~Hz}), 7.3(\mathrm{t}, J=2.7 \mathrm{~Hz}), 7.1,3.6 .{ }^{19} \mathrm{~F} \mathrm{NMR}\left(376 \mathrm{MHz}, \mathrm{CDCl}_{3}\right)$ $\delta-62.5(\mathrm{~s}, 3 \mathrm{~F}),-74.0(\mathrm{dd}, J=277.2,7.1 \mathrm{~Hz}, 1 \mathrm{~F}),-76.8(\mathrm{dd}, J=277.2,13.7 \mathrm{~Hz}, 1 \mathrm{~F})$. MS (DART): m/z (\%) $392\left(\left[\mathrm{M}+\mathrm{NH}_{4}\right]^{+}\right), 355$ (100). HRMS: Calculated for $\mathrm{C}_{19} \mathrm{H}_{27} \mathrm{NF}_{5} \mathrm{Si}$ : 392.1827; Found: 392.1824. Enantiomeric ratio (97:3 er) was measured by chiral HPLC on PC-3 column $\left(\mathrm{CH}_{3} \mathrm{CN} / \mathrm{H}_{2} \mathrm{O}\right.$ $=45: 55,0.6 \mathrm{~mL} / \mathrm{min}, \mathrm{UV}$ detection at $214 \mathrm{~nm}$ ); retention time $=128.20 \mathrm{~min}$ (major), retention time $=121.78 \mathrm{~min}$ (minor).

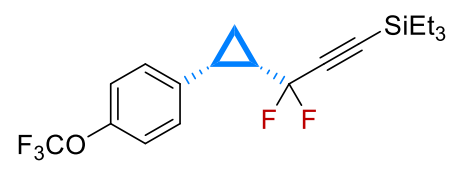

\section{(3,3-Difluoro-3-((1S,2R)-2-(4-(trifluoromethoxy)phenyl)cyclopropyl)} prop-1-yn-1-yl)triethylsilane (3e). The product (137 $\mathrm{mg}, 70 \%$ yield) was purified with silica gel chromatography (Petroleum ether) as a colorless oil. $[\alpha]_{\mathrm{D}}{ }^{20}=-14.0\left(c=0.68, \mathrm{CHCl}_{3}\right)$ for a sample with $96: 4$ er. ${ }^{1} \mathrm{H} \mathrm{NMR}\left(400 \mathrm{MHz}, \mathrm{CDCl}_{3}\right)$ $\delta 7.35(\mathrm{~d}, J=8.4 \mathrm{~Hz}, 2 \mathrm{H}), 7.10(\mathrm{~d}, J=8.3 \mathrm{~Hz}, 2 \mathrm{H}), 2.43-2.35(\mathrm{~m}, 1 \mathrm{H}), 1.95-1.82(\mathrm{~m}, 1 \mathrm{H}), 1.47$ - $1.40(\mathrm{~m}, 1 \mathrm{H}), 1.31-1.23(\mathrm{~m}, 1 \mathrm{H}), 0.93(\mathrm{t}, J=7.9 \mathrm{~Hz}, 9 \mathrm{H}), 0.55(\mathrm{q}, J=7.9 \mathrm{~Hz}, 6 \mathrm{H}) .{ }^{13} \mathrm{C} \mathrm{NMR}$ $\left(101 \mathrm{MHz}, \mathrm{CDCl}_{3}\right) \delta 147.9(\mathrm{q}, J=1.6 \mathrm{~Hz}), 134.7,131.3,120.5(\mathrm{q}, J=256.8 \mathrm{~Hz}), 120.20,120.19$, $113.1(\mathrm{t}, J=231.8 \mathrm{~Hz}), 97.4(\mathrm{dd}, J=40.2,39.5 \mathrm{~Hz}), 91.9(\mathrm{t}, J=5.2 \mathrm{~Hz}), 24.9(\mathrm{dd}, J=33.7,32.1 \mathrm{~Hz})$, $20.3(\mathrm{t}, J=2.7 \mathrm{~Hz}), 7.3(\mathrm{t}, J=2.7 \mathrm{~Hz}), 7.1,3.7 .{ }^{19} \mathrm{~F} \mathrm{NMR}\left(376 \mathrm{MHz}, \mathrm{CDCl}_{3}\right) \delta-57.9(\mathrm{~s}, 3 \mathrm{~F}),-74.0$ 
$(\mathrm{dd}, J=276.9,7.0 \mathrm{~Hz}, 1 \mathrm{~F}),-77.3(\mathrm{dd}, J=276.9,14.1 \mathrm{~Hz}, 1 \mathrm{~F})$. MS (DART): $\mathrm{m} / \mathrm{z}(\%) 408$ $\left(\left[\mathrm{M}+\mathrm{NH}_{4}\right]^{+}\right), 390,371$ (100). HRMS: Calculated for $\mathrm{C}_{19} \mathrm{H}_{27} \mathrm{ONF}_{5} \mathrm{Si}$ : 408.1777; Found: 408.1774 . Enantiomeric ratio (96:4 er) was measured by chiral HPLC on PC-3 column $\left(\mathrm{CH}_{3} \mathrm{CN} / \mathrm{H}_{2} \mathrm{O}=45: 55\right.$, $0.7 \mathrm{~mL} / \mathrm{min}, \mathrm{UV}$ detection at $214 \mathrm{~nm}$ ); retention time $=124.86 \mathrm{~min}$ (major), retention time $=119.11$ $\min$ (minor).

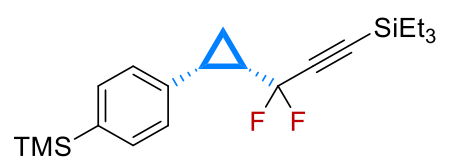

\section{(3,3-Difluoro-3-((1S,2R)-2-(4-(trimethylsilyl)phenyl)cyclopropyl)pro}

p-1-yn-1-yl)triethylsilane (3f). The product (133 mg, 70\% yield) was purified with silica gel chromatography (Petroleum ether) as a colorless oil. $[\alpha]_{\mathrm{D}}{ }^{20}=5.8\left(c=0.58, \mathrm{CHCl}_{3}\right)$ for a sample with 96:4 er. ${ }^{1} \mathrm{H} \mathrm{NMR}\left(400 \mathrm{MHz}, \mathrm{CDCl}_{3}\right) \delta 7.41(\mathrm{~d}, J$ $=7.8 \mathrm{~Hz}, 2 \mathrm{H}), 7.32(\mathrm{~d}, J=7.7 \mathrm{~Hz}, 2 \mathrm{H}), 2.45-2.36(\mathrm{~m}, 1 \mathrm{H}), 1.93-1.80(\mathrm{~m}, 1 \mathrm{H}), 1.49-1.43(\mathrm{~m}$, $1 \mathrm{H}), 1.29-1.16(\mathrm{~m}, 1 \mathrm{H}), 0.91(\mathrm{t}, J=7.9 \mathrm{~Hz}, 9 \mathrm{H}), 0.52(\mathrm{q}, J=7.9 \mathrm{~Hz}, 6 \mathrm{H}), 0.24(\mathrm{~s}, 9 \mathrm{H}) .{ }^{13} \mathrm{C}$ NMR $\left(101 \mathrm{MHz}, \mathrm{CDCl}_{3}\right) \delta 138.2,136.4,132.9,129.4,113.5(\mathrm{t}, J=230.3 \mathrm{~Hz}), 97.3(\mathrm{dd}, J=40.2$, $39.1 \mathrm{~Hz}), 91.7(\mathrm{t}, J=5.2 \mathrm{~Hz}), 24.8(\mathrm{dd}, J=33.9,32.5 \mathrm{~Hz}), 20.9(\mathrm{t}, J=2.7 \mathrm{~Hz}), 7.23,7.17(\mathrm{t}, J=2.5$ $\mathrm{Hz}), 3.6,-1.1 .{ }^{19} \mathrm{~F}$ NMR $\left(376 \mathrm{MHz}, \mathrm{CDCl}_{3}\right) \delta-73.5(\mathrm{dd}, J=276.4,6.7 \mathrm{~Hz}, 1 \mathrm{~F}),-76.1(\mathrm{dd}, J=276.3$, $13.8 \mathrm{~Hz}, 1 \mathrm{~F})$. MS (DART): m/z (\%) $396\left(\left[\mathrm{M}+\mathrm{NH}_{4}^{+}\right]\right.$, 100), 359. HRMS: Calculated for $\mathrm{C}_{21} \mathrm{H}_{36} \mathrm{NF}_{2} \mathrm{Si}_{2}$ : 396.2349; Found: 396.2345. Enantiomeric ratio (96:4 er) was measured by chiral HPLC on PC-1 column $\left(\mathrm{CH}_{3} \mathrm{CN} / \mathrm{H}_{2} \mathrm{O}=80: 20,0.7 \mathrm{~mL} / \mathrm{min}\right.$, UV detection at $\left.214 \mathrm{~nm}\right)$; retention time $=12.68 \mathrm{~min}$ (major), retention time $=13.29 \mathrm{~min}$ (minor).

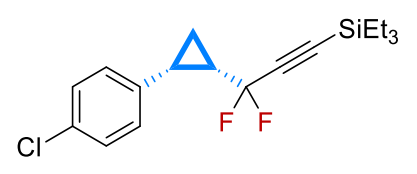

(3-((1S,2R)-2-(4-Chlorophenyl)cyclopropyl)-3,3-difluoroprop-1-yn-1-yl )triethylsilane $\mathbf{( 3 g})$. The product $(131 \mathrm{mg}, 77 \%$ yield) was purified with silica gel chromatography (Petroleum ether) as a colorless oil. ${ }^{1} \mathrm{H}$ NMR $\left(400 \mathrm{MHz}, \mathrm{CDCl}_{3}\right) \delta 7.30-7.19(\mathrm{~m}, 4 \mathrm{H}), 2.41-2.34(\mathrm{~m}, 1 \mathrm{H}), 1.95-1.80(\mathrm{~m}, 1 \mathrm{H}), 1.44-1.38(\mathrm{~m}$, $1 \mathrm{H}), 1.30-1.21(\mathrm{~m}, 1 \mathrm{H}), 0.93(\mathrm{t}, J=7.9 \mathrm{~Hz}, 9 \mathrm{H}), 0.55$ (q, $J=7.9 \mathrm{~Hz}, 6 \mathrm{H}) .{ }^{13} \mathrm{C} \mathrm{NMR}(101 \mathrm{MHz}$, $\left.\mathrm{CDCl}_{3}\right) \delta 134.6,132.4,131.4,127.9,113.3(\mathrm{t}, J=230.6 \mathrm{~Hz}), 97.3(\mathrm{dd}, J=39.3,39.2 \mathrm{~Hz}), 92.0(\mathrm{t}, J=$ $5.1 \mathrm{~Hz}), 24.8(\mathrm{dd}, J=33.0,33.0 \mathrm{~Hz}), 20.3(\mathrm{t}, J=2.7 \mathrm{~Hz}), 7.3(\mathrm{t}, J=2.5 \mathrm{~Hz}), 7.2,3.7 .{ }^{19} \mathrm{~F} \mathrm{NMR}(376$ $\left.\mathrm{MHz}, \mathrm{CDCl}_{3}\right) \delta-73.66(\mathrm{dd}, J=276.8,7.0 \mathrm{~Hz}),-76.54(\mathrm{dd}, J=277.0,13.6 \mathrm{~Hz}) . \mathrm{MS}(\mathrm{EI}): \mathrm{m} / \mathrm{z}(\%)$ $340\left(\mathrm{M}^{+}\right)$, 311, 205, 151, 115 (100). HRMS: Calculated for $\mathrm{C}_{18} \mathrm{H}_{23} \mathrm{~F}_{2} \mathrm{SiCl}$ : 340.1226; Found: 
340.1227. The enantiomeric ratio of $\mathbf{3 g}$ (97:3 er) was determined from $\mathbf{3} \mathbf{g}^{\mathbf{\prime}}$, which was derived from $3 g$ through a [3+2] cyclization with benzyl azide.

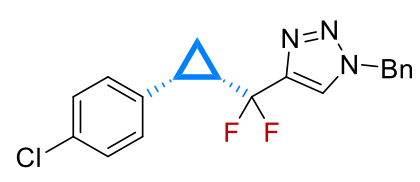

\section{1-Benzyl-4-(((1S,2R)-2-(4-chlorophenyl)cyclopropyl)difluoromethyl)-1}

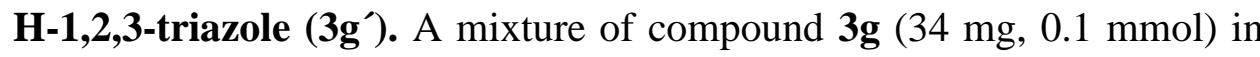
THF (2 mL) was cooled to $-78{ }^{\circ} \mathrm{C}$. A solution of TBAF (1.0 M in THF; $0.12 \mathrm{mmol}, 1.2$ equiv) in THF was added dropwise. The reaction mixture was stirred at $-78{ }^{\circ} \mathrm{C}$ for 1 h. The reaction mixture was then warmed to $-20{ }^{\circ} \mathrm{C}$, and was quenched with saturated aqueous $\mathrm{NH}_{4} \mathrm{Cl}$ solution $(10 \mathrm{~mL})$. After stirring for $5 \mathrm{~min}$ at $0{ }^{\circ} \mathrm{C}$, the resulting mixture was extracted with diethyl ether $(3 \times 30 \mathrm{~mL})$. The reaction mixture was concentrated and filtered a short pad of silica gel using hexane as eluent. The organic solvents were removed, the residue was redissolved in ${ }^{t} \mathrm{BuOH}(2$ $\mathrm{mL})$ and $\mathrm{H}_{2} \mathrm{O}(2 \mathrm{~mL})$. (Azidomethyl)benzene (20 mg, 1.5 equiv), $\mathrm{CuSO}_{4}(3.2 \mathrm{mg}, 20 \mathrm{~mol} \%$ ) and sodium ascorbate $(12 \mathrm{mg}, 0.06 \mathrm{mmol}, 0.6$ equiv) in water $(0.5 \mathrm{~mL})$ were then added. The reaction mixture was stirred at room temperature for $8 \mathrm{~h}$. Water was added. The reaction mixture was extracted with EtOAc (3 times). The combined organic layers were washed with brine, dried over $\mathrm{Na}_{2} \mathrm{SO}_{4}$, filtered and concentrated. The crude product was purified by silica gel chromatography (Petroleum ether/EtOAc $=4: 1)$ to give $3 g^{\prime}$ ( $20 \mathrm{mg}$, 57\% yield for two steps) as a white solid (m.p. 95 $\left.{ }^{\circ} \mathrm{C}\right) .[\alpha]_{\mathrm{D}}{ }^{25}=-2.41\left(c=0.34, \mathrm{CHCl}_{3}\right)$ for a sample with 97:3 er. ${ }^{1} \mathrm{H} \mathrm{NMR}\left(400 \mathrm{MHz}, \mathrm{CDCl}_{3}\right) \delta 7.41-$ $7.39(\mathrm{~m}, 2 \mathrm{H}), 7.26-7.22(\mathrm{~m}, 3 \mathrm{H}), 7.01-6.91(\mathrm{~m}, 5 \mathrm{H}), 5.50(\mathrm{~d}, J=14.7 \mathrm{~Hz}, 1 \mathrm{H}), 5.33(\mathrm{~d}, J=$ $14.7 \mathrm{~Hz}, 1 \mathrm{H}), 2.41-2.32(\mathrm{~m}, 1 \mathrm{H}), 2.31-2.20$ (m, $1 \mathrm{H}), 1.64-1.57$ (m, $1 \mathrm{H}), 1.30-1.24(\mathrm{~m}, 1 \mathrm{H})$. ${ }^{13} \mathrm{C} \mathrm{NMR}\left(151 \mathrm{MHz}, \mathrm{CDCl}_{3}\right) \delta 145.3(\mathrm{t}, J=34.6 \mathrm{~Hz}), 134.7,133.8,131.8,130.8,129.2,129.0,128.2$, 127.6, 121.5, $113.3(\mathrm{t}, J=230.6 \mathrm{~Hz}), 54.2,23.6(\mathrm{dd}, J=32.5,27.7 \mathrm{~Hz}), 20.0(\mathrm{~d}, J=4.1 \mathrm{~Hz}), 5.7(\mathrm{dd}$, $J=5.1,2.5 \mathrm{~Hz}) .{ }^{19} \mathrm{~F} \mathrm{NMR}\left(376 \mathrm{MHz}, \mathrm{CDCl}_{3}\right) \delta-81.23(\mathrm{dd}, J=267.0,10.0 \mathrm{~Hz}, 1 \mathrm{~F}),-92.29(\mathrm{dd}, J=$ 267.0, 15.5 Hz, 1 F). MS (DART): m/z (\%) $(384,382)\left([\mathrm{M}+\mathrm{Na}]^{+}\right), 360$ (100). HRMS: Calculated for $\mathrm{C}_{19} \mathrm{H}_{17} \mathrm{~N}_{3} \mathrm{ClF}_{2}$ : 360.1074; Found: 360.1069. Enantiomeric ratio (97:3 er) was measured by chiral HPLC on IG column $\left(\mathrm{Hexane} /{ }^{i} \mathrm{Pr}_{2} \mathrm{OH}=80: 20,0.7 \mathrm{~mL} / \mathrm{min}\right.$, UV detection at $\left.214 \mathrm{~nm}\right)$; retention time $=14.42 \mathrm{~min}$ (major), retention time $=15.99 \mathrm{~min}$ (minor).

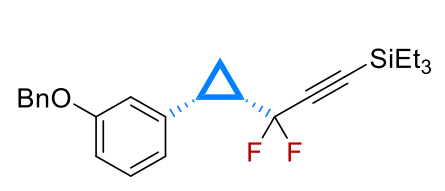

(3-((1S,2R)-2-(3-(benzyloxy)phenyl)cyclopropyl)-3,3-difluoroprop-1-y n-1-yl)triethylsilane (3h). The product (161 mg, 78\% yield) was purified with silica gel chromatography (Petroleum ether/EtOAc $=100: 1)$ as a colorless oil. $[\alpha]_{\mathrm{D}}{ }^{20}=-10.3\left(c=1.01, \mathrm{CHCl}_{3}\right)$ for a sample with 98:2 er. ${ }^{1} \mathrm{H} \mathrm{NMR}\left(400 \mathrm{MHz}, \mathrm{CDCl}_{3}\right)$ 
$\delta 7.46-7.42(\mathrm{~m}, 2 \mathrm{H}), 7.42-7.36(\mathrm{~m}, 2 \mathrm{H}), 7.36-7.29(\mathrm{~m}, 1 \mathrm{H}), 7.17(\mathrm{t}, J=7.9 \mathrm{~Hz}, 1 \mathrm{H}), 7.00-$ $6.93(\mathrm{~m}, 2 \mathrm{H}), 6.83(\mathrm{dd}, J=8.1,2.3 \mathrm{~Hz}, 1 \mathrm{H}), 5.03$ (s, $2 \mathrm{H}), 2.46-2.37$ (m, $1 \mathrm{H}), 1.96-1.79$ (m, 1 H), $1.47-1.38(\mathrm{~m}, 1 \mathrm{H}), 1.28-1.18(\mathrm{~m}, 1 \mathrm{H}), 0.92(\mathrm{t}, J=7.9 \mathrm{~Hz}, 9 \mathrm{H}), 0.54(\mathrm{q}, J=8.0 \mathrm{~Hz}, 6 \mathrm{H})$. ${ }^{13} \mathrm{C} \mathrm{NMR}\left(101 \mathrm{MHz}, \mathrm{CDCl}_{3}\right) \delta 158.4,137.6,137.1,128.7,128.5,127.9,127.5,122.8,116.8,113.4(\mathrm{t}$, $J=230.4 \mathrm{~Hz}), 112.7,97.4(\mathrm{dd}, J=40.5,39.5 \mathrm{~Hz}), 91.6(\mathrm{t}, J=5.3 \mathrm{~Hz}), 69.8,24.8(\mathrm{dd}, J=33.6,32.5$ $\mathrm{Hz}), 21.1(\mathrm{t}, J=2.7 \mathrm{~Hz}), 7.3(\mathrm{t}, J=2.5 \mathrm{~Hz}), 7.2,3.7 .{ }^{19} \mathrm{~F} \mathrm{NMR}\left(376 \mathrm{MHz}, \mathrm{CDCl}_{3}\right) \delta-73.9(\mathrm{dd}, J=$ 276.4, 7.4 Hz, $1 \mathrm{~F}),-76.8$ (dd, $J=276.3,13.7 \mathrm{~Hz}, 1 \mathrm{~F}) . \mathrm{MS}(\mathrm{EI}): \mathrm{m} / \mathrm{z}(\%) 412\left(\mathrm{M}^{+}\right), 91$ (100). HRMS: Calculated for $\mathrm{C}_{25} \mathrm{H}_{30} \mathrm{OF}_{2} \mathrm{Si}$ : 412.2034; Found: 412.2038. Enantiomeric ratio (98:2 er) was measured by chiral HPLC on PC-1 column $\left(\mathrm{CH}_{3} \mathrm{CN} / \mathrm{H}_{2} \mathrm{O}=70: 30,0.7 \mathrm{~mL} / \mathrm{min}\right.$, UV detection at $\left.214 \mathrm{~nm}\right)$; retention time $=42.07 \mathrm{~min}$ (major), retention time $=37.38 \mathrm{~min}$ (minor).

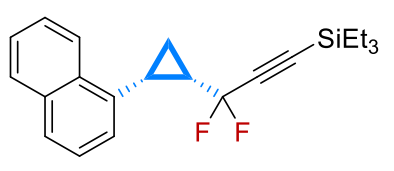

(3,3-Difluoro-3-((1S,2R)-2-(naphthalen-1-yl)cyclopropyl)prop-1-yn-1-yl) triethylsilane (3i). The product (118 $\mathrm{mg}, 66 \%$ yield) was purified with silica gel chromatography (Petroleum ether) as a colorless oil. $[\alpha]_{\mathrm{D}}{ }^{20}=124.6(c=$ 0.63, $\left.\mathrm{CHCl}_{3}\right)$ for a sample with 98:2 er. ${ }^{1} \mathrm{H} \mathrm{NMR}\left(400 \mathrm{MHz}, \mathrm{CDCl}_{3}\right) \delta 8.32(\mathrm{~d}, J=8.3 \mathrm{~Hz}, 1 \mathrm{H}), 7.84$ $(\mathrm{d}, J=7.6 \mathrm{~Hz}, 1 \mathrm{H}), 7.75(\mathrm{~d}, J=8.0 \mathrm{~Hz}, 1 \mathrm{H}), 7.56-7.51(\mathrm{~m}, 1 \mathrm{H}), 7.48(\mathrm{t}, J=7.4 \mathrm{~Hz}, 1 \mathrm{H}), 7.46-$ $7.42(\mathrm{~m}, 1 \mathrm{H}), 7.41-7.35(\mathrm{~m}, 1 \mathrm{H}), 2.78-2.68(\mathrm{~m}, 1 \mathrm{H}), 2.22-2.07$ (m, $1 \mathrm{H}), 1.70-1.63(\mathrm{~m}, 1 \mathrm{H})$, $1.51-1.41(\mathrm{~m}, 1 \mathrm{H}), 0.81(\mathrm{t}, J=7.9 \mathrm{~Hz}, 9 \mathrm{H}), 0.41(\mathrm{q}, J=7.7 \mathrm{~Hz}, 6 \mathrm{H}) .{ }^{13} \mathrm{C} \mathrm{NMR}\left(101 \mathrm{MHz}, \mathrm{CDCl}_{3}\right)$ $\delta 133.5,133.4,132.1,128.3,127.4,126.6(\mathrm{t}, J=1.7 \mathrm{~Hz}), 125.6,125.5,125.0,124.8,113.5(\mathrm{t}, J=$ $230.5 \mathrm{~Hz}), 97.2(\mathrm{t}, J=39.9 \mathrm{~Hz}), 91.7(\mathrm{t}, J=5.1 \mathrm{~Hz}), 25.3(\mathrm{t}, J=33.1 \mathrm{~Hz}), 19.8(\mathrm{t}, J=2.6 \mathrm{~Hz}), 7.3(\mathrm{t}$, $J=2.7 \mathrm{~Hz}), 7.1,3.6 .{ }^{19} \mathrm{~F}$ NMR $\left(376 \mathrm{MHz}, \mathrm{CDCl}_{3}\right) \delta-73.8(\mathrm{dd}, J=276.7,7.5 \mathrm{~Hz}, 1 \mathrm{~F}),-74.8(\mathrm{dd}, J=$ 276.7, $11.3 \mathrm{~Hz}, 1 \mathrm{~F})$. MS (EI): m/z (\%) $356\left(\mathrm{M}^{+}\right)$, 202, 167 (100). HRMS: Calculated for $\mathrm{C}_{22} \mathrm{H}_{26} \mathrm{~F}_{2} \mathrm{Si}$ : 356.1772; Found: 356.1776. Enantiomeric ratio (98:2 er) was measured by chiral HPLC on PC-1 column $\left(\mathrm{CH}_{3} \mathrm{CN} / \mathrm{H}_{2} \mathrm{O}=70: 30,0.7 \mathrm{~mL} / \mathrm{min}\right.$, UV detection at $\left.214 \mathrm{~nm}\right)$; retention time $=60.97 \mathrm{~min}$ (major), retention time $=50.16 \min ($ minor).

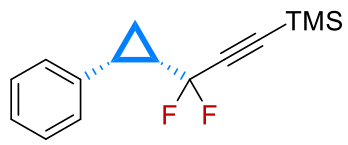

\section{(3,3-Difluoro-3-((1S,2R)-2-phenylcyclopropyl)prop-1-yn-1-yl)trimethylsila}

ne (3j). The product (96 mg, 73\% yield) was purified with silica gel chromatography (Petroleum ether) as a colorless oil. $[\alpha]_{\mathrm{D}}{ }^{20}=-11.5\left(c=0.70, \mathrm{CHCl}_{3}\right)$ for a sample with 97:3 er. ${ }^{1} \mathrm{H}$ NMR $\left(400 \mathrm{MHz}, \mathrm{CDCl}_{3}\right) \delta 7.35(\mathrm{~d}, J=7.4 \mathrm{~Hz}, 2 \mathrm{H}), 7.31-7.25(\mathrm{~m}, 2 \mathrm{H}), 7.25-$ 
$7.18(\mathrm{~m}, 1 \mathrm{H}), 2.49-2.40(\mathrm{~m}, 1 \mathrm{H}), 1.94-1.77(\mathrm{~m}, 1 \mathrm{H}), 1.50-1.42(\mathrm{~m}, 1 \mathrm{H}), 1.30-1.20(\mathrm{~m}, 1 \mathrm{H})$, $0.12(\mathrm{~s}, 9 \mathrm{H}) .{ }^{13} \mathrm{C} \mathrm{NMR}\left(101 \mathrm{MHz}, \mathrm{CDCl}_{3}\right) \delta 135.8,130.1(\mathrm{t}, J=1.0 \mathrm{~Hz}), 127.8,126.5,113.5(\mathrm{t}, J=$ $230.3 \mathrm{~Hz}), 96.0(\mathrm{dd}, J=40.1,38.9 \mathrm{~Hz}), 93.8(\mathrm{t}, J=5.2 \mathrm{~Hz}), 24.6(\mathrm{dd}, J=33.9,32.2 \mathrm{~Hz}), 20.9(\mathrm{t}, J=$ $2.8 \mathrm{~Hz}), 7.1(\mathrm{t}, J=2.6 \mathrm{~Hz}),-0.8 .{ }^{19} \mathrm{~F}$ NMR $\left(376 \mathrm{MHz}, \mathrm{CDCl}_{3}\right) \delta-74.1(\mathrm{dd}, J=276.9,6.8 \mathrm{~Hz}),-76.7$ $(\mathrm{dd}, J=276.9,13.7 \mathrm{~Hz}) . \mathrm{MS}(\mathrm{DART}): \mathrm{m} / \mathrm{z}(\%) 282\left(\left[\mathrm{M}+\mathrm{NH}_{4}\right]^{+}\right), 345,215,187$ (100). HRMS: Calculated for $\mathrm{C}_{15} \mathrm{H}_{22} \mathrm{NF}_{2} \mathrm{Si}$ : 282.1484; Found: 282.1484. Enantiomeric ratio (97:3 er) was measured by chiral HPLC on PC-1 column $\left(\mathrm{CH}_{3} \mathrm{CN} / \mathrm{H}_{2} \mathrm{O}=70: 30,0.7 \mathrm{~mL} / \mathrm{min}\right.$, UV detection at $\left.214 \mathrm{~nm}\right)$; retention time $=13.31 \mathrm{~min}$ (major), retention time $=12.30 \mathrm{~min}$ (minor).

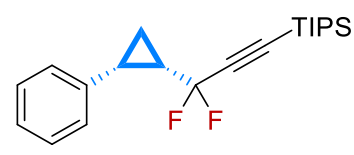

\section{(3,3-Difluoro-3-((1S,2R)-2-phenylcyclopropyl)prop-1-yn-1-yl)triisopropyl}

silane (3k). The product (136 mg, 78\% yield) was purified with silica gel chromatography (Petroleum ether) as a colorless oil. $[\alpha]_{\mathrm{D}}{ }^{20}=-26.2\left(c=0.64, \mathrm{CHCl}_{3}\right)$ for a sample with 96:4 er. ${ }^{1} \mathrm{H}$ NMR (400 MHz, $\left.\mathrm{CDCl}_{3}\right) \delta 7.34(\mathrm{~d}, J=7.6 \mathrm{~Hz}, 2 \mathrm{H}), 7.26-7.21(\mathrm{~m}, 2 \mathrm{H}), 7.20-$ $7.15(\mathrm{~m}, 1 \mathrm{H}), 2.46-2.38(\mathrm{~m}, 1 \mathrm{H}), 1.91-1.80(\mathrm{~m}, 1 \mathrm{H}), 1.48-1.43(\mathrm{~m}, 1 \mathrm{H}), 1.25-1.16(\mathrm{~m}, 1 \mathrm{H})$, $1.07-0.97(\mathrm{~m}, 21 \mathrm{H}) .{ }^{13} \mathrm{C} \mathrm{NMR}\left(101 \mathrm{MHz}, \mathrm{CDCl}_{3}\right) \delta 135.9,129.9(\mathrm{t}, J=1.0 \mathrm{~Hz}), 127.8,126.5$, $113.0(\mathrm{dd}, J=232.0,230.4 \mathrm{~Hz}), 99.0(\mathrm{dd}, J=40.4,39.5 \mathrm{~Hz}), 90.2(\mathrm{t}, J=5.3 \mathrm{~Hz}), 25.1(\mathrm{dd}, J=33.0$, $31.0 \mathrm{~Hz}), 21.2(\mathrm{dd}, J=3.2,2.0 \mathrm{~Hz}), 18.4,10.9,6.8(\mathrm{t}, J=2.6 \mathrm{~Hz}) .{ }^{19} \mathrm{~F} \mathrm{NMR}\left(376 \mathrm{MHz}, \mathrm{CDCl}_{3}\right) \delta$ $-73.9(\mathrm{dd}, J=275.4,7.5 \mathrm{~Hz}),-79.5(\mathrm{dd}, J=275.3,15.0 \mathrm{~Hz}) . \mathrm{MS}(\mathrm{EI}): \mathrm{m} / \mathrm{z}(\%) 348\left(\mathrm{M}^{+}\right), 305,152$, 91, 77 (100). HRMS: Calculated for $\mathrm{C}_{21} \mathrm{H}_{30} \mathrm{~F}_{2} \mathrm{Si}$ : 348.2085; Found: 348.2082. Enantiomeric ratio (96:4 er) was measured by chiral HPLC on PC-1 column $\left(\mathrm{CH}_{3} \mathrm{CN} / \mathrm{H}_{2} \mathrm{O}=70: 30,0.7 \mathrm{~mL} / \mathrm{min}\right.$, UV detection at $214 \mathrm{~nm}$ ); retention time $=33.84 \mathrm{~min}$ (major), retention time $=31.23 \mathrm{~min}$ (minor).

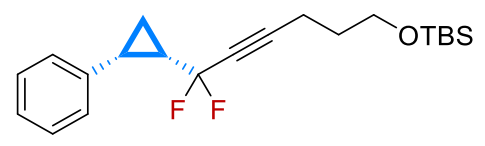

\section{tert-Butyl-((6,6-difluoro-6-((1S,2R)-2-phenylcyclopropyl)hex-4-yn-}

1-yl)oxy)dimethylsilane (31). The product (145 mg, 80\% yield) was purified with silica gel chromatography (Petroleum ether/EtOAc $=50: 1)$ as a pale yellow oil. $[\alpha]_{\mathrm{D}}^{20}$ $=9.7\left(c=0.54, \mathrm{CHCl}_{3}\right)$ for a sample with 93:7 er. ${ }^{1} \mathrm{H} \mathrm{NMR}\left(400 \mathrm{MHz}, \mathrm{CDCl}_{3}\right) \delta 7.33(\mathrm{~d}, J=7.5 \mathrm{~Hz}$, $2 \mathrm{H}), 7.26(\mathrm{t}, J=7.3 \mathrm{~Hz}, 2 \mathrm{H}), 7.18(\mathrm{t}, J=7.2 \mathrm{~Hz}, 1 \mathrm{H}), 3.55(\mathrm{t}, J=5.9 \mathrm{~Hz}, 2 \mathrm{H}), 2.46-2.37(\mathrm{~m}, 1$ H), $2.13-2.01(\mathrm{~m}, 2 \mathrm{H}), 1.89-1.77(\mathrm{~m}, 1 \mathrm{H}), 1.58-1.48(\mathrm{~m}, 2 \mathrm{H}), 1.45-1.38(\mathrm{~m}, 1 \mathrm{H}), 1.28-$ $1.18(\mathrm{~m}, 1 \mathrm{H}), 0.91-0.87(\mathrm{~m}, 9 \mathrm{H}), 0.06-0.02(\mathrm{~m}, 6 \mathrm{H}) .{ }^{13} \mathrm{C} \mathrm{NMR}\left(126 \mathrm{MHz}, \mathrm{CDCl}_{3}\right) \delta 136.2$, 130.0, 127.7, 126.4, $114.4(\mathrm{t}, J=228.9 \mathrm{~Hz}), 89.5(\mathrm{t}, J=6.3 \mathrm{~Hz}), 73.4(\mathrm{t}, J=40.3 \mathrm{~Hz}), 61.2,30.6(\mathrm{t}, J$ 
$=1.8 \mathrm{~Hz}), 25.9,24.9(\mathrm{t}, J=33.9 \mathrm{~Hz}), 21.0(\mathrm{t}, J=2.0 \mathrm{~Hz}), 18.3,14.7(\mathrm{t}, J=2.3 \mathrm{~Hz}), 7.1(\mathrm{t}, J=2.0$ $\mathrm{Hz}),-5.4 .{ }^{19} \mathrm{~F}$ NMR $\left(376 \mathrm{MHz}, \mathrm{DMSO}-d_{6}\right) \delta-77.6(\mathrm{~d}, J=272.7 \mathrm{~Hz}),-78.7(\mathrm{~d}, J=272.7 \mathrm{~Hz}) . \mathrm{MS}$ (EI): m/z (\%) $364\left(\mathrm{M}^{+}\right), 167$ (100), 91, 77. HRMS: Calculated for $\mathrm{C}_{21} \mathrm{H}_{30} \mathrm{OF}_{2} \mathrm{Si}$ : 364.2034; Found: 364.2028. Enantiomeric ratio (93:7 er) was measured by chiral HPLC on PC-3 column $\left(\mathrm{CH}_{3} \mathrm{CN} / \mathrm{H}_{2} \mathrm{O}\right.$ $=70: 30,0.7 \mathrm{~mL} / \mathrm{min}, \mathrm{UV}$ detection at $214 \mathrm{~nm})$; retention time $=10.05 \mathrm{~min}$ (major), retention time $=$ $11.57 \min$ (minor).

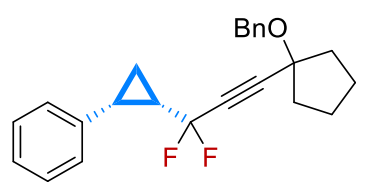

((1R,2S)-2-(3-(1-(Benzyloxy)cyclopentyl)-1,1-difluoroprop-2-yn-1-yl)cyclo propyl)benzene $(\mathbf{3 m})$. The product (156 $\mathrm{mg}, 85 \%$ yield) was purified with silica gel chromatography (Petroleum ether $/$ EtOAc $=100: 1)$ as a colorless oil. $[\alpha]_{\mathrm{D}}^{20}=4.3\left(c=0.48, \mathrm{CHCl}_{3}\right)$ for a sample with $96: 4$ er. ${ }^{1} \mathrm{H} \mathrm{NMR}\left(400 \mathrm{MHz}, \mathrm{CDCl}_{3}\right) \delta 7.37-7.21$ (m, 9 H), 7.18 (t, J = 7.2 Hz, 1 H), $4.35(\mathrm{~s}, 2 \mathrm{H}), 2.47-2.39(\mathrm{~m}, 1 \mathrm{H}), 2.02-1.92(\mathrm{~m}, 2 \mathrm{H}), 1.92-$ $1.81(\mathrm{~m}, 1 \mathrm{H}), 1.77-1.59(\mathrm{~m}, 6 \mathrm{H}), 1.45-1.39(\mathrm{~m}, 1 \mathrm{H}), 1.28-1.20(\mathrm{~m}, 1 \mathrm{H}) .{ }^{13} \mathrm{C} \mathrm{NMR}(101 \mathrm{MHz}$, $\left.\mathrm{CDCl}_{3}\right) \delta 138.5,136.0,130.0(\mathrm{t}, J=1.4 \mathrm{~Hz}), 128.3,127.9,127.6,127.4,126.6,114.3(\mathrm{t}, J=230.2$ Hz), $89.7(\mathrm{t}, J=6.3 \mathrm{~Hz}), 79.9(\mathrm{t}, J=1.8 \mathrm{~Hz}), 77.6(\mathrm{dd}, J=41.5,40.2 \mathrm{~Hz}), 67.1,39.0(\mathrm{t}, J=1.5 \mathrm{~Hz})$, $38.9(\mathrm{t}, J=1.5 \mathrm{~Hz}), 24.9(\mathrm{dd}, J=34.2,32.4 \mathrm{~Hz}), 23.3,21.0(\mathrm{t}, J=2.8 \mathrm{~Hz}), 7.2(\mathrm{t}, J=2.7 \mathrm{~Hz}) .{ }^{19} \mathrm{~F}$ $\operatorname{NMR}\left(376 \mathrm{MHz}, \mathrm{CDCl}_{3}\right) \delta-72.9(\mathrm{dd}, J=274.7,6.8 \mathrm{~Hz}),-75.6(\mathrm{dd}, J=274.8,13.6 \mathrm{~Hz}) . \mathrm{MS}(\mathrm{DART})$ : m/z (\%) $384\left(\left[\mathrm{M}+\mathrm{NH}_{4}\right]^{+}\right.$, 100). HRMS: Calculated for $\mathrm{C}_{24} \mathrm{H}_{28} \mathrm{ONF}_{2}$ : 384.2133; Found: 384.2131 . Enantiomeric ratio (96:4 er) was measured by chiral HPLC on PC-1 column $\left(\mathrm{CH}_{3} \mathrm{CN} / \mathrm{H}_{2} \mathrm{O}=70: 30\right.$, $0.7 \mathrm{~mL} / \mathrm{min}$, UV detection at $214 \mathrm{~nm}$ ); retention time $=25.90 \mathrm{~min}$ (major), retention time $=23.75$ $\min (\operatorname{minor})$.

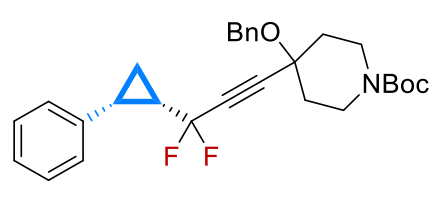

tert-Butyl-4-(benzyloxy)-4-(3,3-difluoro-3-((1S,2R)-2-phenylcyclopro

pyl)prop-1-yn-1-yl)piperidine-1-carboxylate (3n). The product (188 $\mathrm{mg}, 78 \%$ yield) was purified with silica gel chromatography (Petroleum ether $/ \mathrm{EtOAc}=10: 1)$ as a colorless oil. $[\alpha]_{\mathrm{D}}{ }^{20}=-1.6\left(c=0.84, \mathrm{CHCl}_{3}\right)$ for a sample with $96: 4$ er. ${ }^{1} \mathrm{H}$ NMR (400 MHz, $\left.\mathrm{CDCl}_{3}\right) \delta 7.47$ - $6.99(\mathrm{~m}, 10 \mathrm{H}), 4.38(\mathrm{~s}, 2 \mathrm{H}), 3.63$ - 3.49 (m, $\left.2 \mathrm{H}\right), 3.21$ - 3.09 (m, $2 \mathrm{H}), 2.48-2.39(\mathrm{~m}, 1 \mathrm{H}), 1.93-1.81(\mathrm{~m}, 1 \mathrm{H}), 1.76-1.62(\mathrm{~m}, 4 \mathrm{H}), 1.45(\mathrm{~s}, 9 \mathrm{H}), 1.41-1.37$ (m, $1 \mathrm{H}), 1.31-1.21(\mathrm{~m}, 1 \mathrm{H}) .{ }^{13} \mathrm{C} \mathrm{NMR}\left(101 \mathrm{MHz}, \mathrm{CDCl}_{3}\right) \delta 154.5,138.0,135.9,129.9(\mathrm{t}, J=1.5 \mathrm{~Hz})$, $128.4,128.0,127.65,127.63,126.7,114.1(\mathrm{dd}, J=232.1,232.1 \mathrm{~Hz}), 87.8(\mathrm{t}, J=6.1 \mathrm{~Hz}), 79.7,79.6$ 
(dd, $J=41.9,40.6 \mathrm{~Hz}), 71.7$ (t, $J=1.9 \mathrm{~Hz}), 66.1,40.4$ (br), 39.5 (br), 35.7 (br), 28.4, 24.7 (dd, $J=$ 34.0, 32.4 Hz), $21.0(\mathrm{t}, J=2.7 \mathrm{~Hz}), 7.3(\mathrm{t}, J=2.8 \mathrm{~Hz}) .{ }^{19} \mathrm{~F}$ NMR $\left(376 \mathrm{MHz}, \mathrm{CDCl}_{3}\right) \delta-72.7(\mathrm{dd}, J=$ 276.6, $6.8 \mathrm{~Hz}, 1 \mathrm{~F}),-75.2(\mathrm{~d}(\mathrm{br}), J=277.0 \mathrm{~Hz}, 1 \mathrm{~F})$. MS (DART): m/z (\%) $482\left([\mathrm{M}+\mathrm{H}]^{+}\right), 406(100)$. HRMS: Calculated for $\mathrm{C}_{29} \mathrm{H}_{34} \mathrm{O}_{3} \mathrm{NF}_{2}$ : 482.2501; Found: 482.2498. Enantiomeric ratio (96:4 er) was measured by chiral HPLC on PC-1 column $\left(\mathrm{CH}_{3} \mathrm{CN} / \mathrm{H}_{2} \mathrm{O}=70: 30,0.7 \mathrm{~mL} / \mathrm{min}\right.$, UV detection at 214 $\mathrm{nm})$; retention time $=29.83 \mathrm{~min}$ (major), retention time $=27.56 \mathrm{~min}$ (minor).

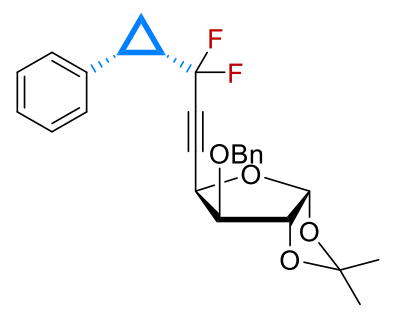

(3aR,5R,6aR)-6-(benzyloxy)-5-(3,3-difluoro-3-((1S,2R)-2-phenylcyclopro pyl)prop-1-yn-1-yl)-2,2-dimethyltetrahydrofuro[2,3-d][1,3]dioxole (3o). The product $(179 \mathrm{mg}, 81 \%$ yield $)$ was purified with silica gel chromatography (Petroleum ether $/$ EtOAc $=10: 1)$ as a colorless oil. $[\alpha]_{\mathrm{D}}{ }^{20}=$ $23.4\left(c=0.66, \mathrm{CHCl}_{3}\right)$ for a sample with 95:5 er. ${ }^{1} \mathrm{H} \mathrm{NMR}\left(400 \mathrm{MHz}, \mathrm{CDCl}_{3}\right) \delta 7.38-7.13(\mathrm{~m}, 10$ H), $5.91(\mathrm{~d}, J=3.7 \mathrm{~Hz}, 1 \mathrm{H}), 4.59(\mathrm{~s}, 2 \mathrm{H}), 4.54-4.49(\mathrm{~m}, 2 \mathrm{H}), 3.79$ (d, J = 3.0 Hz, $1 \mathrm{H}), 2.49-$ $2.40(\mathrm{~m}, 1 \mathrm{H}), 1.94-1.82(\mathrm{~m}, 1 \mathrm{H}), 1.51-1.47$ (m, $1 \mathrm{H}), 1.46(\mathrm{~s}, 3 \mathrm{H}), 1.29$ (s, $3 \mathrm{H}), 1.27$ - 1.16 (m, $1 \mathrm{H}) .{ }^{13} \mathrm{C}$ NMR $\left(101 \mathrm{MHz}, \mathrm{CDCl}_{3}\right) \delta 137.0,136.0,130.2,128.4,127.9,127.8,127.8,126.5,114.3(\mathrm{t}$, $J=230.8 \mathrm{~Hz}), 112.1,104.8,82.6,82.3(\mathrm{t}, J=6.5 \mathrm{~Hz}), 82.2,80.0(\mathrm{t}, J=41.2 \mathrm{~Hz}), 72.6,70.2(\mathrm{t}, J=$ $1.9 \mathrm{~Hz}), 26.8,26.1,24.5(\mathrm{t}, J=32.9 \mathrm{~Hz}), 21.2(\mathrm{dd}, J=2.9,1.7 \mathrm{~Hz}), 7.2(\mathrm{t}, J=3.0 \mathrm{~Hz}) .{ }^{19} \mathrm{~F} \mathrm{NMR}$ $\left(376 \mathrm{MHz}, \mathrm{CDCl}_{3}\right) \delta-76.1(\mathrm{ddd}, J=278.1,10.5,3.9 \mathrm{~Hz}),-77.2(\mathrm{ddd}, J=278.1,9.2,3.8 \mathrm{~Hz}) . \mathrm{MS}$ (EI): m/z (\%) $440\left(\mathrm{M}^{+}\right), 129,91$ (100). HRMS: Calculated for $\mathrm{C}_{26} \mathrm{H}_{26} \mathrm{O}_{4} \mathrm{~F}_{2}$ : 440.1799; Found: 440.1793. Enantiomeric ratio (95:5 er) was measured by chiral HPLC on IG column (Hexane $/{ }^{i} \mathrm{PrOH}$ $=90: 10,0.7 \mathrm{~mL} / \mathrm{min}$, UV detection at $214 \mathrm{~nm}$ ); retention time $=10.28 \mathrm{~min}$ (major), retention time $=$ $11.64 \mathrm{~min}$ (minor).

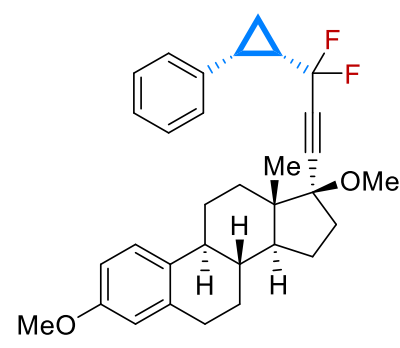

$(8 R, 9 S, 13 S, 14 S, 17 S)-17-(3,3-d i f l u o r o-3-((1 S, 2 R)-2-p h e n y l c y c l o p r o p y l) p$ rop-1-yn-1-yl)-3,17-dimethoxy-13-methyl-7,8,9,11,12,13,14,15,16,17-de

cahydro-6H-cyclopenta $[a]$ phenanthrene $(3 p)$. The reaction was carried out at $-20{ }^{\circ} \mathrm{C}$ on $0.3 \mathrm{mmol}$ scale. The product $(89 \mathrm{mg}, 60 \%$ yield) was purified with silica gel chromatography (Petroleum ether/EtOAc $=100: 1$ ) as a colorless oil. $[\alpha]_{\mathrm{D}}{ }^{20}=-35.4\left(c=0.44, \mathrm{CHCl}_{3}\right)$ for a sample with 97:3 er. ${ }^{1} \mathrm{H} \mathrm{NMR}(400 \mathrm{MHz}$, $\left.\mathrm{CDCl}_{3}\right) \delta 7.36(\mathrm{~d}, J=7.6 \mathrm{~Hz}, 2 \mathrm{H}), 7.31-7.25(\mathrm{~m}, 2 \mathrm{H}), 7.23-7.17(\mathrm{~m}, 2 \mathrm{H}), 6.72(\mathrm{dd}, J=8.6,2.7$ 
$\mathrm{Hz}, 1 \mathrm{H}), 6.63(\mathrm{~d}, J=2.6 \mathrm{~Hz}, 1 \mathrm{H}), 3.78(\mathrm{~s}, 3 \mathrm{H}), 3.28(\mathrm{~s}, 3 \mathrm{H}), 2.88-2.80(\mathrm{~m}, 2 \mathrm{H}), 2.49-2.41$ (m, $1 \mathrm{H}), 2.35-2.27(\mathrm{~m}, 1 \mathrm{H}), 2.21-2.10(\mathrm{~m}, 1 \mathrm{H}), 2.04-1.94(\mathrm{~m}, 1 \mathrm{H}), 1.94-1.82(\mathrm{~m}, 3 \mathrm{H}), 1.79-$ $1.71(\mathrm{~m}, 2 \mathrm{H}), 1.72-1.64(\mathrm{~m}, 1 \mathrm{H}), 1.55-1.28(\mathrm{~m}, 7 \mathrm{H}), 0.84(\mathrm{~s}, 3 \mathrm{H}) .{ }^{13} \mathrm{C} \mathrm{NMR}\left(101 \mathrm{MHz}, \mathrm{CDCl}_{3}\right)$ $\delta 157.4,137.8,136.0,132.3,129.8,127.9,126.6,126.3,114.0(\mathrm{dd}, J=231.5,230.4 \mathrm{~Hz}), 113.7$, $111.5,88.9(\mathrm{t}, J=6.2 \mathrm{~Hz}), 85.4(\mathrm{t}, J=1.7 \mathrm{~Hz}), 81.0(\mathrm{dd}, J=41.4,40.6 \mathrm{~Hz}), 55.2,53.5,49.6,47.8$, 43.4, 39.1, 35.9, 34.2, 29.8, 27.2, 26.4, $25.2(\mathrm{dd}, J=33.5,31.4 \mathrm{~Hz}), 22.7,21.1(\mathrm{t}, J=28.2 \mathrm{~Hz}), 12.6$, $7.0(\mathrm{t}, J=2.5 \mathrm{~Hz}) .{ }^{19} \mathrm{~F}$ NMR $(376 \mathrm{MHz}, \mathrm{CDCl} 3) \delta-72.7(\mathrm{dd}, J=273.6,7.3 \mathrm{~Hz}),-77.6(\mathrm{dd}, J=273.6$, 14.6 Hz). MS (DART): m/z (\%) $508\left(\left[\mathrm{M}+\mathrm{NH}_{4}\right]^{+}, 100\right)$, 471. HRMS: Calculated for $\mathrm{C}_{32} \mathrm{H}_{40} \mathrm{O}_{2} \mathrm{NF}_{2}$ : 508.3022; Found: 508.3018. Enantiomeric ratio (97:3 er) was measured by chiral HPLC on PC-1 column $\left(\mathrm{CH}_{3} \mathrm{CN} / \mathrm{H}_{2} \mathrm{O}=80: 20,0.7 \mathrm{~mL} / \mathrm{min}\right.$, UV detection at $\left.214 \mathrm{~nm}\right)$; retention time = $23.56 \mathrm{~min}$ (major), retention time $=21.29 \min$ (minor).

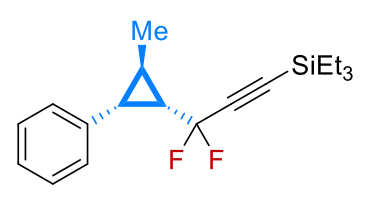

(3,3-Difluoro-3-((1S,2R,3R)-2-methyl-3-phenylcyclopropyl)prop-1-yn-1-yl )triethylsilane (3q). The reaction was carried out at $-20{ }^{\circ} \mathrm{C}$. The product $(128$ $\mathrm{mg}, 80 \%$ yield) was purified with silica gel chromatography (Petroleum ether) as a colorless oil. $[\alpha]_{\mathrm{D}}{ }^{20}=5.0\left(c=0.66, \mathrm{CHCl}_{3}\right)$ for a sample with 98:2 er. ${ }^{1} \mathrm{H}$ NMR (400 MHz, $\left.\mathrm{CDCl}_{3}\right) \delta 7.31(\mathrm{~d}, J=7.5 \mathrm{~Hz}, 2 \mathrm{H}), 7.25(\mathrm{t}, J=7.3 \mathrm{~Hz}, 2 \mathrm{H}), 7.21-7.16(\mathrm{~m}, 1 \mathrm{H}), 2.18(\mathrm{dd}, J=8.8$, $7.2 \mathrm{~Hz}, 1 \mathrm{H}), 1.87-1.79(\mathrm{~m}, 1 \mathrm{H}), 1.65-1.54(\mathrm{~m}, 1 \mathrm{H}), 1.31(\mathrm{~d}, J=6.0 \mathrm{~Hz}, 3 \mathrm{H}), 0.94$ (t, $J=7.9 \mathrm{~Hz}$, $9 \mathrm{H}), 0.55(\mathrm{q}, J=7.8 \mathrm{~Hz}, 6 \mathrm{H}) .{ }^{13} \mathrm{C} \mathrm{NMR}\left(101 \mathrm{MHz}, \mathrm{CDCl}_{3}\right) \delta 136.2,129.7(\mathrm{t}, J=1.3 \mathrm{~Hz}), 127.8$, 126.4, $113.2(\mathrm{t}, J=230.6 \mathrm{~Hz}), 97.9(\mathrm{t}, J=40.4,39.9 \mathrm{~Hz}), 91.2(\mathrm{t}, J=5.2 \mathrm{~Hz}), 32.8(\mathrm{dd}, J=32.7$, $31.6 \mathrm{~Hz}), 29.4(\mathrm{t}, J=2.6 \mathrm{~Hz}), 18.0,15.4(\mathrm{t}, J=2.5 \mathrm{~Hz}), 7.2,3.7 .{ }^{19} \mathrm{~F}$ NMR $\left(376 \mathrm{MHz}, \mathrm{CDCl}_{3}\right) \delta$ $-73.4(\mathrm{dd}, J=275.8,7.8 \mathrm{~Hz}, 1 \mathrm{~F}),-76.6(\mathrm{dd}, J=275.8,14.3 \mathrm{~Hz}, 1 \mathrm{~F}) . \mathrm{MS}(\mathrm{EI}): \mathrm{m} / \mathrm{z}(\%) 320\left(\mathrm{M}^{+}\right)$, 131 (100), 91, 77. HRMS: Calculated for $\mathrm{C}_{19} \mathrm{H}_{26} \mathrm{~F}_{2} \mathrm{Si}$ : 320.1772; Found: 320.1765. Enantiomeric ratio (98:2 er) was measured by chiral HPLC on PC-1 column $\left(\mathrm{CH}_{3} \mathrm{CN}_{2} \mathrm{H}_{2} \mathrm{O}=60: 40,0.7 \mathrm{~mL} / \mathrm{min}\right.$, $\mathrm{UV}$ detection at $214 \mathrm{~nm}$ ); retention time $=63.65 \mathrm{~min}$ (major), retention time $=60.34 \mathrm{~min}$ (minor).

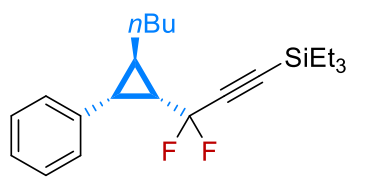

(3-((1S,2R,3R)-2-Butyl-3-phenylcyclopropyl)-3,3-difluoroprop-1-yn-1-yl)t

riethylsilane $(3 \mathbf{r})$. The reaction was carried out at $-20{ }^{\circ} \mathrm{C}$. The product $(116$ mg, 64\% yield) was purified with silica gel chromatography (Petroleum ether) as a colorless oil. $[\alpha]_{\mathrm{D}}{ }^{20}=12.9\left(c=0.51, \mathrm{CHCl}_{3}\right)$ for a sample with 96:4 er. ${ }^{1} \mathrm{H} \mathrm{NMR}(400 \mathrm{MHz}$, 
$\left.\mathrm{CDCl}_{3}\right) \delta 7.30(\mathrm{~d}, J=7.4 \mathrm{~Hz}, 2 \mathrm{H}), 7.24(\mathrm{t}, J=7.1 \mathrm{~Hz}, 2 \mathrm{H}), 7.18(\mathrm{t}, J=7.1 \mathrm{~Hz}, 1 \mathrm{H}), 2.23-2.15(\mathrm{~m}$, $1 \mathrm{H}), 1.81-1.73(\mathrm{~m}, 1 \mathrm{H}), 1.65-1.55(\mathrm{~m}, 1 \mathrm{H}), 1.53-1.46(\mathrm{~m}, 4 \mathrm{H}), 1.45-1.36(\mathrm{~m}, 2 \mathrm{H}), 0.93(\mathrm{t}, J$ $=8.1 \mathrm{~Hz}, 9 \mathrm{H}), 0.55(\mathrm{q}, J=7.9 \mathrm{~Hz}, 6 \mathrm{H}) .{ }^{13} \mathrm{C} \mathrm{NMR}\left(101 \mathrm{MHz}, \mathrm{CDCl}_{3}\right) \delta 136.4,129.8(\mathrm{t}, J=1.4 \mathrm{~Hz})$, 127.8, 126.4, $113.2(\mathrm{t}, J=230.8 \mathrm{~Hz}), 97.9(\mathrm{t}, J=40.1 \mathrm{~Hz}), 91.1(\mathrm{t}, J=5.2 \mathrm{~Hz}), 32.8,31.9(\mathrm{t}, J=32.0$ $\mathrm{Hz}), 31.3,28.5(\mathrm{t}, J=2.5 \mathrm{~Hz}), 22.4,21.2(\mathrm{t}, J=2.4 \mathrm{~Hz}), 14.1,7.2,3.7 .{ }^{19} \mathrm{~F} \mathrm{NMR}\left(376 \mathrm{MHz}, \mathrm{CDCl}_{3}\right)$ $\delta-74.4(\mathrm{dd}, J=275.5,8.7 \mathrm{~Hz}, 1 \mathrm{~F}),-76.7(\mathrm{dd}, J=275.5,13.5 \mathrm{~Hz}, 1 \mathrm{~F})$. MS (DART): $\mathrm{m} / \mathrm{z}(\%) 380$ $\left(\left[\mathrm{M}+\mathrm{NH}_{4}\right]^{+}\right), 362,361,343$ (100). HRMS: Calculated for $\mathrm{C}_{22} \mathrm{H}_{36} \mathrm{NF}_{2} \mathrm{Si}$ : 380.2580; Found: 380.2578 . Enantiomeric ratio (96:4 er) was measured by chiral HPLC on PA-2 column $\left(\mathrm{CH}_{3} \mathrm{CN} / \mathrm{H}_{2} \mathrm{O}=70: 30\right.$, $0.7 \mathrm{~mL} / \mathrm{min}$, UV detection at $214 \mathrm{~nm}$ ); retention time $=14.39 \mathrm{~min}$ (major), retention time $=12.91$ $\min$ (minor).

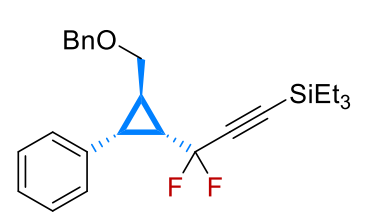

\section{(3-((1R,2R,3R)-2-((benzyloxy)methyl)-3-phenylcyclopropyl)-3,3-difluorop}

rop-1-yn-1-yl)triethylsilane (3s). The reaction was carried out on $0.2 \mathrm{mmol}$ scale at $-20{ }^{\circ} \mathrm{C}$. The product (62 $\mathrm{mg}, 73 \%$ yield) was purified with silica gel chromatography (Petroleum ether/DCM $=3: 1)$ as a colorless oil. $[\alpha]_{\mathrm{D}}^{20}=9.93\left(c=0.59, \mathrm{CHCl}_{3}\right)$ for a sample with 97:3 er. ${ }^{1} \mathrm{H}$ NMR (400 MHz, $\left.\mathrm{CDCl}_{3}\right) \delta 7.38-7.15$ (m, $\left.10 \mathrm{H}\right), 4.60$ (s, $\left.2 \mathrm{H}\right), 3.68$ (dd, J $=10.3,5.6 \mathrm{~Hz}, 1 \mathrm{H}), 3.56(\mathrm{dd}, J=10.3,6.3 \mathrm{~Hz}, 1 \mathrm{H}), 2.42(\mathrm{dd}, J=8.2,7.2 \mathrm{~Hz}, 1 \mathrm{H}), 2.18-2.11(\mathrm{~m}$, $1 \mathrm{H}), 1.88-1.78(\mathrm{~m}, 1 \mathrm{H}), 0.92(\mathrm{t}, J=7.9 \mathrm{~Hz}, 9 \mathrm{H}), 0.53(\mathrm{q}, J=7.9 \mathrm{~Hz}, 6 \mathrm{H}) .{ }^{13} \mathrm{C} \mathrm{NMR}(101 \mathrm{MHz}$, $\left.\mathrm{CDCl}_{3}\right) \delta 138.2,135.3,129.8,128.4,127.9,127.63,127.58,126.6,112.8(\mathrm{t}, J=232.3 \mathrm{~Hz}), 97.6(\mathrm{t}, J$ = 39.7 Hz), $91.6(\mathrm{t}, J=5.2 \mathrm{~Hz}), 72.5,70.9,29.6(\mathrm{dd}, J=33.4,31.6 \mathrm{~Hz}), 26.2(\mathrm{t}, J=2.5 \mathrm{~Hz}), 20.5(\mathrm{t}$, $J=2.1 \mathrm{~Hz}), 7.2,3.7 .{ }^{19} \mathrm{~F} \mathrm{NMR}\left(376 \mathrm{MHz}, \mathrm{CDCl}_{3}\right) \delta-73.5(\mathrm{dd}, J=276.5,7.5 \mathrm{~Hz}, 1 \mathrm{~F}),-77.0(\mathrm{dd}, J=$ 276.5, $14.2 \mathrm{~Hz}, 1 \mathrm{~F})$. MS (DART): m/z (\%) $444\left(\left[\mathrm{M}+\mathrm{NH}_{4}\right]^{+}\right)$. HRMS: Calculated for $\mathrm{C}_{26} \mathrm{H}_{36} \mathrm{ONF}_{2} \mathrm{Si}$ : 444.2529; Found: 444.2528. Enantiomeric ratio (97:3 er) was measured by chiral HPLC on ODH column $\left(\mathrm{Hexane} /{ }^{i} \mathrm{PrOH}=98: 2,0.7 \mathrm{~mL} / \mathrm{min}, \mathrm{UV}\right.$ detection at $\left.214 \mathrm{~nm}\right)$; retention time $=7.57 \mathrm{~min}$ (major), retention time $=8.06 \mathrm{~min}$ (minor).

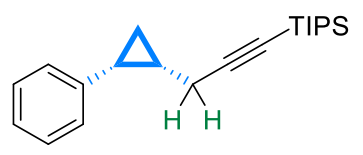

\section{Triisopropyl-(3-((1R,2R)-2-phenylcyclopropyl)prop-1-yn-1-yl)silane (8a).}

The product (125 mg, 80\% yield) was purified with silica gel chromatography (Petroleum ether) as a colorless oil. $[\alpha]_{\mathrm{D}}{ }^{20}=-10.2\left(c=0.51, \mathrm{CHCl}_{3}\right)$ for a sample with $96: 4$ er. ${ }^{1} \mathrm{H}$ NMR (400 MHz, $\left.\mathrm{CDCl}_{3}\right) \delta 7.28$ - $7.23(\mathrm{~m}, 4 \mathrm{H}), 7.21$ - 7.15 (m, $\left.1 \mathrm{H}\right), 2.21$ (ddd, J = 8.6, 8.6, 6.3 Hz, 
1H), $1.99(\mathrm{dd}, J=17.5,6.6 \mathrm{~Hz}, 1 \mathrm{H}), 1.88(\mathrm{dd}, J=17.5,7.5 \mathrm{~Hz}, 1 \mathrm{H}), 1.44-1.34(\mathrm{~m}, 1 \mathrm{H}), 1.11-$ $1.01(\mathrm{~m}, 21 \mathrm{H}), 1.01-0.94(\mathrm{~m}, 1 \mathrm{H}), 0.76-0.69(\mathrm{~m}, 1 \mathrm{H}) .{ }^{13} \mathrm{C} \mathrm{NMR}\left(101 \mathrm{MHz}, \mathrm{CDCl}_{3}\right) \delta 138.3$, $129.5,127.9,126.0,108.3,80.0,21.3,19.8,18.6,17.9,11.3,9.3$. MS (DART): m/z (\%) 331 $\left(\left[\mathrm{M}+\mathrm{NH}_{4}\right]^{+}\right), 314,313\left(\mathrm{M}^{+}, 100\right)$. HRMS: Calculated for $\mathrm{C}_{21} \mathrm{H}_{33} \mathrm{Si}$ : 313.2346; Found: 313.2344. Enantiomeric ratio (96:4 er) was measured by chiral HPLC on PC-3 column $\left(\mathrm{CH}_{3} \mathrm{CN} / \mathrm{H}_{2} \mathrm{O}=70: 30\right.$, $0.7 \mathrm{~mL} / \mathrm{min}$, UV detection at $214 \mathrm{~nm}$ ); retention time $=13.71 \mathrm{~min}$ (major), retention time $=14.97$ $\min$ (minor).

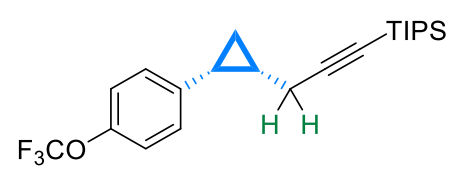

\section{Triisopropyl-(3-((1R,2R)-2-(4-(trifluoromethoxy)phenyl)cyclopropyl} )prop-1-yn-1-yl)silane (8b). The product (168.5 mg, 85\% yield) was purified with silica gel chromatography (Petroleum ether) as a colorless oil. $[\alpha]_{\mathrm{D}}{ }^{20}=-18.2\left(c=1.35, \mathrm{CHCl}_{3}\right)$ for a sample with 97:3 er. ${ }^{1} \mathrm{H}$ NMR $\left(400 \mathrm{MHz}, \mathrm{CDCl}_{3}\right) \delta 7.28(\mathrm{~d}$, $J=8.8 \mathrm{~Hz}, 2 \mathrm{H}), 7.10(\mathrm{~d}, J=8.3 \mathrm{~Hz}, 2 \mathrm{H}), 2.24-2.16(\mathrm{~m}, 1 \mathrm{H}), 2.09(\mathrm{dd}, J=17.5,6.0 \mathrm{~Hz}, 1 \mathrm{H})$, $1.81(\mathrm{dd}, J=17.5,8.2 \mathrm{~Hz}, 1 \mathrm{H}), 1.47-1.35(\mathrm{~m}, 1 \mathrm{H}), 1.12-0.95(\mathrm{~m}, 22 \mathrm{H}), 0.73-0.67(\mathrm{~m}, 1 \mathrm{H})$. ${ }^{13} \mathrm{C}$ NMR $\left(101 \mathrm{MHz}, \mathrm{CDCl}_{3}\right) \delta 147.5,137.1,130.7,120.50,120.48(\mathrm{q}, J=258.0 \mathrm{~Hz}), 80.3,20.6$, 19.7, 18.6, 18.0, 11.3, 9.7. ${ }^{19} \mathrm{~F} \mathrm{NMR}\left(376 \mathrm{MHz}, \mathrm{CDCl}_{3}\right) \delta-58.0$ (s, $\left.3 \mathrm{~F}\right) . \mathrm{MS}(\mathrm{EI}): \mathrm{m} / \mathrm{z}(\%) 396\left(\mathrm{M}^{+}\right)$, 355, 287, 189 (100). HRMS: Calculated for $\mathrm{C}_{22} \mathrm{H}_{31} \mathrm{OF}_{3} \mathrm{Si}$ : 396.2096; Found: 396.2099. Enantiomeric ratio (97:3 er) was measured by chiral HPLC on PA-2 column $\left(\mathrm{CH}_{3} \mathrm{CN} / \mathrm{H}_{2} \mathrm{O}=70: 30,0.7 \mathrm{~mL} / \mathrm{min}\right.$, $\mathrm{UV}$ detection at $214 \mathrm{~nm}$ ); retention time $=14.08 \mathrm{~min}$ (major), retention time $=13.23 \mathrm{~min}$ (minor).

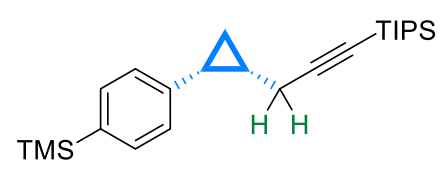

Triisopropyl(3-((1R,2R)-2-(4-(trimethylsilyl)phenyl)cyclopropyl)prop -1-yn-1-yl)silane (8c). The product (138.5 mg, 72\% yield) was purified with silica gel chromatography (Petroleum ether) as a colorless oil. $[\alpha]_{\mathrm{D}}{ }^{20}$ $=-9.4\left(c=0.65, \mathrm{CHCl}_{3}\right)$ for a sample with 97:3 er. ${ }^{1} \mathrm{H} \mathrm{NMR}\left(400 \mathrm{MHz}, \mathrm{CDCl}_{3}\right) \delta 7.4(\mathrm{~d}, J=7.4 \mathrm{~Hz}$, $2 \mathrm{H}), 7.23(\mathrm{~d}, J=8.0 \mathrm{~Hz}, 2 \mathrm{H}), 2.24-2.16(\mathrm{~m}, 1 \mathrm{H}), 2.01(\mathrm{dd}, J=17.5,6.7 \mathrm{~Hz}, 1 \mathrm{H}), 1.90(\mathrm{dd}, J=$ $17.4,7.5 \mathrm{~Hz}, 1 \mathrm{H}), 1.47-1.32(\mathrm{~m}, 1 \mathrm{H}), 1.15-0.95(\mathrm{~m}, 22 \mathrm{H}), 0.78-0.71(\mathrm{~m} 1 \mathrm{H}), 0.32-0.15(\mathrm{~m}$, $9 \mathrm{H}) .{ }^{13} \mathrm{C} \mathrm{NMR}\left(101 \mathrm{MHz}, \mathrm{CDCl}_{3}\right) \delta 139.0,137.5,133.0,128.8,108.3,79.9,21.2,19.8,18.6,18.0$, 11.3, 9.4, -1.1. MS (EI): m/z (\%) $384\left(\mathrm{M}^{+}\right), 299$ (100). HRMS: Calculated for $\mathrm{C}_{24} \mathrm{H}_{40} \mathrm{Si}_{2}$ : 384.2669; Found: 384.2679. Enantiomeric ratio (97:3 er) was measured by chiral HPLC on PA-2 column 
$\left(\mathrm{CH}_{3} \mathrm{CN} / \mathrm{H}_{2} \mathrm{O}=70 / 30,0.7 \mathrm{~mL} / \mathrm{min}, \mathrm{UV}\right.$ detection at $\left.214 \mathrm{~nm}\right)$; retention time $=25.62$ min (major), retention time $=23.32 \mathrm{~min}$ (minor).

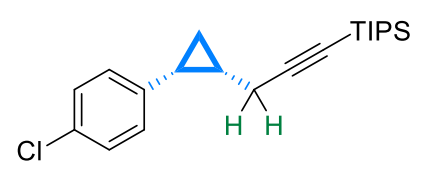

\section{(3-((1R,2R)-2-(4-Chlorophenyl)cyclopropyl)prop-1-yn-1-yl)triisopropy}

Isilane (8d). The product (140 mg, 80\% yield) was purified with silica gel chromatography (Petroleum ether) as a colorless oil. $[\alpha]_{\mathrm{D}}{ }^{20}=-46.19(c=$

0.51, $\mathrm{CHCl}_{3}$ ) for a sample with 97:3 er. ${ }^{1} \mathrm{H} \mathrm{NMR}\left(400 \mathrm{MHz}, \mathrm{CDCl}_{3}\right) \delta 7.26-7.15(\mathrm{~m}, 4 \mathrm{H}), 2.17$ (td, $J=8.6,6.2 \mathrm{~Hz}, 1 \mathrm{H}), 2.07(\mathrm{dd}, J=17.5,6.1 \mathrm{~Hz}, 1 \mathrm{H}), 1.81(\mathrm{dd}, J=17.5,8.1 \mathrm{~Hz}, 1 \mathrm{H}), 1.44-1.36$ $(\mathrm{m}, 1 \mathrm{H}), 1.10-0.96(\mathrm{~m}, 22 \mathrm{H}), 0.71-0.66(\mathrm{~m}, 1 \mathrm{H}) .{ }^{13} \mathrm{C} \mathrm{NMR}\left(101 \mathrm{MHz}, \mathrm{CDCl}_{3}\right) \delta 136.9,131.8$, 130.8, 128.1, 107.9, 80.3, 20.7, 19.7, 18.6, 17.9, 11.3, 9.6. MS (EI): m/z (\%) $346\left(\mathrm{M}^{+}\right), 303(100)$, 151, 115. HRMS: Calculated for $\mathrm{C}_{21} \mathrm{H}_{31} \mathrm{ClSi}$ : 346.1882; Found: 346.1884. Enantiomeric ratio (97:3 er) was measured by chiral SFC on PC-1 column $\left(\mathrm{CO}_{2} / \mathrm{MeOH}=97: 3\right.$, BP Pressure: 2000 psi, 1.0 $\mathrm{mL} / \mathrm{min}$, column temperature: $40{ }^{\circ} \mathrm{C}$, UV detection at $214 \mathrm{~nm}$ ); retention time $=14.92$ min (major), retention time $=14.14 \min ($ minor $)$.

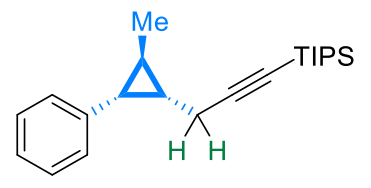

\section{Triisopropyl-(3-((1S,2S,3R)-2-methyl-3-phenylcyclopropyl)prop-1-yn-1-yl} )silane (8e). The product (120 mg, 73\% yield) was purified with silica gel chromatography (Petroleum ether) as a colorless oil. $[\alpha]_{\mathrm{D}}{ }^{20}=45.59(c=0.52$, $\left.\mathrm{CHCl}_{3}\right)$ for a sample with 98:2 er. ${ }^{1} \mathrm{H}$ NMR $\left(400 \mathrm{MHz}, \mathrm{CDCl}_{3}\right) \delta 7.28-7.14(\mathrm{~m}, 5 \mathrm{H}), 1.98-1.89$ $(\mathrm{m}, 3 \mathrm{H}), 1.23(\mathrm{~d}, J=5.3 \mathrm{~Hz}, 3 \mathrm{H}), 1.17-1.10(\mathrm{~m}, 2 \mathrm{H}), 1.10-0.97(\mathrm{~m}, 21 \mathrm{H}) .{ }^{13} \mathrm{C} \mathrm{NMR}(101 \mathrm{MHz}$, $\left.\mathrm{CDCl}_{3}\right) \delta 138.7,129.2,128.0,125.8,108.5,79.9,29.8,26.6,19.2,18.6,18.5,17.7,11.3 . \mathrm{MS}(\mathrm{EI}):$ m/z (\%) $326\left(\mathrm{M}^{+}\right), 283$ (100), 241, 181, 91. HRMS: Calculated for $\mathrm{C}_{22} \mathrm{H}_{34} \mathrm{Si}$ : 326.2430; Found: 326.2440. Enantiomeric ratio (98:2 er) was measured by chiral HPLC on PC-3 column $\left(\mathrm{CH}_{3} \mathrm{CN}_{2} / \mathrm{H}_{2} \mathrm{O}\right.$ $=70: 30,0.7 \mathrm{~mL} / \mathrm{min}$, UV detection at $214 \mathrm{~nm})$; retention time $=15.11 \mathrm{~min}$ (major), retention time $=$ $17.65 \min$ (minor).

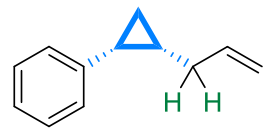

((1R,2S)-2-Allylcyclopropyl)benzene (9a). The product (69 $\mathrm{mg}, 87 \%$ yield) was $=50.33\left(c=0.49, \mathrm{CHCl}_{3}\right)$ for a sample with 97:3 er. ${ }^{1} \mathrm{H}$ NMR $\left(400 \mathrm{MHz}, \mathrm{CDCl}_{3}\right) \delta 7.28-7.23(\mathrm{~m}, 2$ H), $7.21-7.14(\mathrm{~m}, 3 \mathrm{H}), 5.76(\mathrm{ddt}, J=16.6,10.2,6.3 \mathrm{~Hz}, 1 \mathrm{H}), 4.99-4.91(\mathrm{~m}, 1 \mathrm{H}), 4.91-4.85$ (m, 
$1 \mathrm{H}), 2.18(\mathrm{td}, J=8.6,6.2 \mathrm{~Hz}, 1 \mathrm{H}), 1.91-\mathrm{r} 1.81(\mathrm{~m}, 1 \mathrm{H}), 1.71-1.59(\mathrm{~m}, 1 \mathrm{H}), 1.21-1.11(\mathrm{~m}, 1 \mathrm{H})$, $1.01(\mathrm{td}, J=8.4,5.1 \mathrm{~Hz}, 1 \mathrm{H}), 0.73-0.67(\mathrm{~m}, 1 \mathrm{H}) .{ }^{13} \mathrm{C} \mathrm{NMR}\left(101 \mathrm{MHz}, \mathrm{CDCl}_{3}\right) \delta 139.2,137.9$, 129.1, 127.8, 125.7, 114.2, 32.7, 20.9, 17.8, 9.3. MS (EI): m/z (\%) $158\left(\mathrm{M}^{+}\right), 129,117$ (100). HRMS: Calculated for $\mathrm{C}_{12} \mathrm{H}_{14}$ : 158.1096; Found: 158.1099. Enantiomeric ratio (97:3 er) was measured by chiral HPLC on PC-3 column $\left(\mathrm{CH}_{3} \mathrm{CN} / \mathrm{H}_{2} \mathrm{O}=60: 40,0.7 \mathrm{~mL} / \mathrm{min}\right.$, UV detection at $\left.214 \mathrm{~nm}\right)$; retention time $=14.93 \mathrm{~min}$ (major), retention time $=16.97 \mathrm{~min}$ (minor).

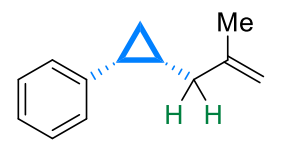

((1R,2R)-2-(2-Methylallyl)cyclopropyl)benzene (9b). The product (72 $\mathrm{mg}, 84 \%$ yield) was purified with silica gel chromatography (Petroleum ether) as a colorless oil. $[\alpha]_{\mathrm{D}}{ }^{25}=97.53\left(c=0.54, \mathrm{CHCl}_{3}\right)$ for a sample with 96:4 er. ${ }^{1} \mathrm{H}$ NMR $\left(400 \mathrm{MHz}, \mathrm{CDCl}_{3}\right) \delta 7.31-$ 7.25 (m, 2 H), $7.24-7.16(\mathrm{~m}, 3 \mathrm{H}), 4.74-4.70$ (m, $1 \mathrm{H}), 4.70-4.67$ (m, $1 \mathrm{H}), 2.23-2.15(\mathrm{~m}, 1 \mathrm{H})$, $1.91(\mathrm{dd}, J=15.8,5.8 \mathrm{~Hz}, 1 \mathrm{H}), 1.67(\mathrm{~s}, 3 \mathrm{H}), 1.53(\mathrm{dd}, J=15.8,8.2 \mathrm{~Hz}, 1 \mathrm{H}), 1.33-1.20(\mathrm{~m}, 1 \mathrm{H})$, $1.07(\mathrm{td}, J=8.4,5.5 \mathrm{~Hz}, 1 \mathrm{H}), 0.76-0.70(\mathrm{~m}, 1 \mathrm{H}) .{ }^{13} \mathrm{C} \mathrm{NMR}\left(101 \mathrm{MHz}, \mathrm{CDCl}_{3}\right) \delta 145.8,139.3$, 129.1, 127.8, 125.6, 109.6, 36.4, 22.8, 20.8, 17.4, 9.7. MS (EI): m/z (\%) $172\left(\mathrm{M}^{+}\right), 157,117$ (100), 91. HRMS: Calculated for $\mathrm{C}_{13} \mathrm{H}_{16}$ : 172.1252; Found: 172.1257. Enantiomeric ratio (96:4 er) was measured by chiral HPLC on OJH column $\left(\mathrm{Hexane} /{ }^{i} \mathrm{PrOH}=100: 0,0.7 \mathrm{~mL} / \mathrm{min}\right.$, UV detection at 214 $\mathrm{nm})$; retention time $=8.27 \mathrm{~min}$ (major), retention time $=8.88 \mathrm{~min}$ (minor)

Ethyl-2-(((1R,2R)-2-phenylcyclopropyl)methyl)acrylate $(9 \mathrm{c})$. The product (71
$\mathrm{mg}, 62 \%$ yield) was purified with silica gel chromatography (Petroleum ether/ $\left.\mathrm{CH}_{2} \mathrm{Cl}_{2}=2: 1\right)$ as a colorless oil. $[\alpha]_{\mathrm{D}}{ }^{20}=58.3\left(c=0.81, \mathrm{CHCl}_{3}\right)$ for a sample with 90:10 er. ${ }^{1} \mathrm{H}$ NMR (400 MHz, $\left.\mathrm{CDCl}_{3}\right) \delta 7.30-7.24(\mathrm{~m}, 2 \mathrm{H}), 7.22-7.15(\mathrm{~m}, 3 \mathrm{H}), 6.10-6.07(\mathrm{~m}, 1 \mathrm{H}), 5.47-$ $5.44(\mathrm{~m}, 1 \mathrm{H}), 4.16(\mathrm{q}, J=7.1 \mathrm{~Hz}, 2 \mathrm{H}), 2.26-2.18(\mathrm{~m}, 1 \mathrm{H}), 2.14(\mathrm{dd}, J=16.3,6.6 \mathrm{~Hz}, 1 \mathrm{H}), 1.91$ $(\mathrm{dd}, J=16.2,7.8 \mathrm{~Hz}, 1 \mathrm{H}), 1.34-1.30(\mathrm{~m}, 1 \mathrm{H}), 1.27(\mathrm{t}, J=7.1 \mathrm{~Hz}, 3 \mathrm{H}), 1.03(\mathrm{td}, J=8.4,5.2 \mathrm{~Hz}, 1$ $\mathrm{H}), 0.79-0.74(\mathrm{~m}, 1 \mathrm{H}) .{ }^{13} \mathrm{C} \mathrm{NMR}\left(101 \mathrm{MHz}, \mathrm{CDCl}_{3}\right) \delta 167.3,140.3,138.7,129.0,127.9,125.8$, $124.4,60.5,30.5,21.1,17.4,14.2$, 9.3. MS (EI): m/z (\%) $230\left(\mathrm{M}^{+}\right), 117$ (100), 91. HRMS: Calculated for $\mathrm{C}_{15} \mathrm{H}_{18} \mathrm{O}_{2}$ : 230.1307; Found: 230.1312. Enantiomeric ratio (90:10 er) was measured by chiral SFC on OJH column $\left(\mathrm{CO}_{2} / \mathrm{MeOH}=90: 10\right.$, BP Pressure: 2000 psi, $1.0 \mathrm{~mL} / \mathrm{min}$, column temperature: $40{ }^{\circ} \mathrm{C}$, UV detection at $214 \mathrm{~nm}$ ); retention time $=7.77$ min (major), retention time $=$ $7.30 \mathrm{~min}$ (minor). 


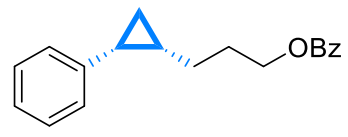

3-((1S,2R)-2-Phenylcyclopropyl)propyl benzoate (9d). The reaction was carried out on $0.5 \mathrm{mmol}$ scale. $\mathrm{CH}_{2} \mathrm{Cl}_{2}(3 \mathrm{~mL})$ and alkyl iodide were used. The crude product was treated with $\mathrm{Et}_{3} \mathrm{~N}$ in PE to remove the excessive alkyl iodide. The solution was filtered and concentrated. The residue was then purified with silica gel chromatography (Petroleum ether/EA $=50: 1)$ to give $9 \mathbf{d}(84 \mathrm{mg}, 60 \%$ yield $)$ as a colorless oil. $[\alpha]_{\mathrm{D}}{ }^{20}=12.03\left(c=0.62, \mathrm{CHCl}_{3}\right)$ for a sample with 81:19 er. 1H NMR (400 MHz, Chloroform-d) $\delta 7.93$ (d, $J=7.6 \mathrm{~Hz}, 2 \mathrm{H}), 7.53$ (t, $J$ $=7.4 \mathrm{~Hz}, 1 \mathrm{H}), 7.41(\mathrm{t}, J=7.7 \mathrm{~Hz}, 2 \mathrm{H}), 7.28-7.10(\mathrm{~m}, 5 \mathrm{H}), 4.20(\mathrm{t}, J=6.5 \mathrm{~Hz}, 2 \mathrm{H}), 2.21-2.10$ (m, $1 \mathrm{H}), 1.82-1.65(\mathrm{~m}, 2 \mathrm{H}), 1.35-1.22(\mathrm{~m}, 1 \mathrm{H}), 1.20-1.08(\mathrm{~m}, 2 \mathrm{H}), 1.44-0.95(\mathrm{~m}, 1 \mathrm{H}), 0.70$ $(\mathrm{q}, J=5.3 \mathrm{~Hz}, 1 \mathrm{H}) .{ }^{13} \mathrm{C}$ NMR $(126 \mathrm{MHz}$, Chloroform-d) $\delta 166.5,139.1,132.7,130.4,129.5,128.9$, 128.2, 127.9, 125.6, 64.7, 28.4, 24.9, 21.0, 18.49, 9.5. MS (EI): m/z (\%) $280\left(\mathrm{M}^{+}\right), 203,159$ (100). HRMS: Calculated for $\mathrm{C}_{19} \mathrm{H}_{20} \mathrm{O}_{2}: 280.1463$; Found: 280.1467. Enantiomeric ratio (81:19 er) was measured by chiral HPLC on PC-1 column $\left(\mathrm{CH}_{3} \mathrm{CN} / \mathrm{H}_{2} \mathrm{O}=55: 45,0.7 \mathrm{~mL} / \mathrm{min}\right.$, UV detection at 214 at $214 \mathrm{~nm}$ ); retention time $=84.03 \mathrm{~min}$ (major), retention time $=78.18 \mathrm{~min}$ (minor).

\section{Reaction of $( \pm)$ trans-1a with 2a}

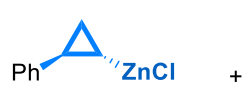

$( \pm)$ trans-1a (2.0 equiv)

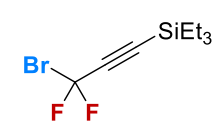

2a (1.0 equiv)

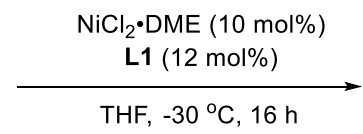

THF, $-30^{\circ} \mathrm{C}, 16 \mathrm{~h}$

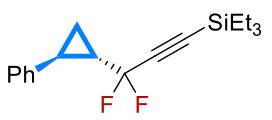

3aa, $82 \%, 65: 35$ er

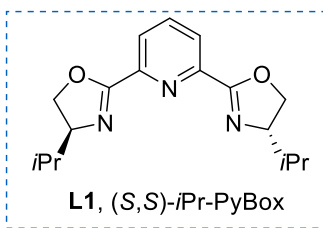

(3,3-Difluoro-3-(2-phenylcyclopropyl)prop-1-yn-1-yl)triethylsilane (3aa). The reaction was carried out on $0.3 \mathrm{mmol}$ scale. The product $(75 \mathrm{mg}, 82 \%$ yield) was purified with silica gel chromatography (Petroleum ether) as a colorless oil (Note: the configuration of 3aa is a relative configuration). $[\alpha]_{\mathrm{D}}{ }^{20}=5.50\left(c=1.00, \mathrm{CHCl}_{3}\right)$ for a sample with $65: 35$ er. ${ }^{1} \mathrm{H}$ NMR $(400 \mathrm{MHz}$, $\left.\mathrm{CDC1}_{3}\right) \delta 7.32-7.27(\mathrm{~m}, 2 \mathrm{H}), 7.24-7.18(\mathrm{~m}, 1 \mathrm{H}), 7.14(\mathrm{~d}, J=7.4 \mathrm{~Hz}, 2 \mathrm{H}), 2.37-2.29(\mathrm{~m}, 1 \mathrm{H})$, $1.92-1.72(\mathrm{~m}, 1 \mathrm{H}), 1.39-1.31(\mathrm{~m}, 1 \mathrm{H}), 1.19-1.11(\mathrm{~m}, 1 \mathrm{H}), 1.02(\mathrm{t}, J=7.9 \mathrm{~Hz}, 9 \mathrm{H}), 0.67(\mathrm{q}, J=$ $7.9 \mathrm{~Hz}, 6 \mathrm{H}) .{ }^{13} \mathrm{C} \mathrm{NMR}\left(126 \mathrm{MHz}, \mathrm{CDC1}_{3}\right) \delta 139.9,128.4,126.6,126.4,114.2(\mathrm{t}, J=230.1 \mathrm{~Hz}), 96.5$ (t, $J=40.1 \mathrm{~Hz}), 91.6(\mathrm{t}, J=5.2 \mathrm{~Hz}), 27.7(\mathrm{t}, J=34.0 \mathrm{~Hz}), 20.4(\mathrm{t}, J=2.6 \mathrm{~Hz}), 11.5,7.3,3.8 .{ }^{19} \mathrm{~F}$ NMR (376 MHz, CDC1 $\left.1_{3}\right) \delta-79.83$ - -79.85 (m, 2 F).MS (EI): m/z (\%) $306\left(\mathrm{M}^{+}\right), 277$ (100), 91. HRMS: Calculated for $\mathrm{C}_{18} \mathrm{H}_{24} \mathrm{~F}_{2} \mathrm{Si}$ : 306.1615; Found: 306.1617. Enantiomeric ratio (65:35 er) was 
measured by chiral HPLC on PC-1 column $\left(\mathrm{CH}_{3} \mathrm{CN} / \mathrm{H}_{2} \mathrm{O}=80: 20,0.7 \mathrm{~mL} / \mathrm{min}\right.$, UV detection at 214 at $214 \mathrm{~nm}$ ); retention time $=13.46 \mathrm{~min}$ (major), retention time $=14.26 \mathrm{~min}$ (minor).

\section{Reaction of alkyl substituted cyclopropylzinc reagent 1l' with 2a}

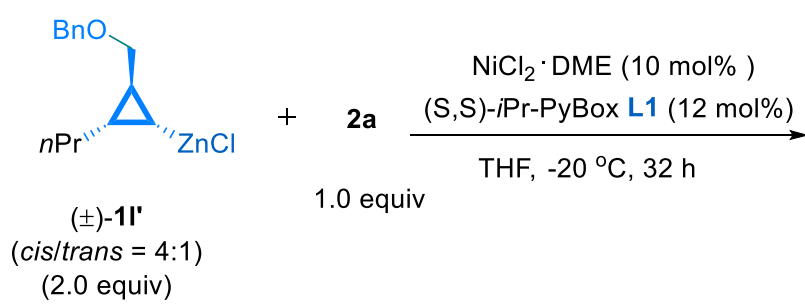

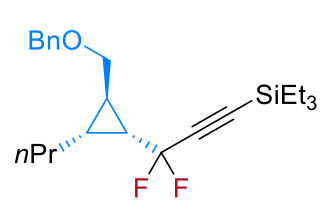

3t, $10 \%, 60: 40$ er

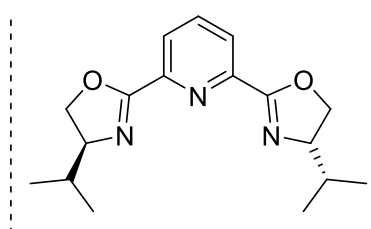

$(S, S)-i P r-P y B o x ~ L 1$

The low yield of $\mathbf{3 t}$ is because of the decomposition of $\mathbf{2 a}$. The preparation of ( \pm )-11' is similar with the procedure for the synthesis of $( \pm)$ cis-11.

6.3 General procedure for Ni-catalyzed enantioselective cross-coupling of racemic cyclopropylzincs $( \pm$ ) cis-1 with secondary propargyl bromides (General Procedure G)

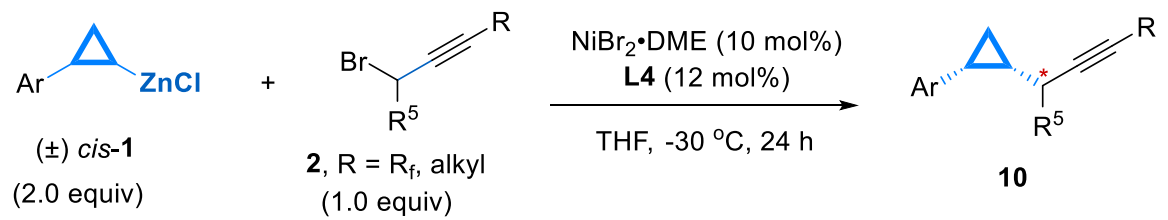

General Procedure G: To a $25 \mathrm{~mL}$ of Schlenk tube were added $\mathrm{NiBr}_{2} \cdot \mathrm{DME}(9.1 \mathrm{mg}, 10 \mathrm{~mol} \%)$ and ligand $[(R, R)$-CyPybox L4 (14.9 mg, $13 \mathrm{~mol} \%)$ for difluoromethyl and difluoroacetyl propargyl bromides; (S,S)-iPrPybox L1 (11.2 mg, $13 \mathrm{~mol} \%$ ) for trifluoromethyl propargyl bromides] in a glovebox. The tube was then taken out from the glovebox. Propargyl bromide $(0.3 \mathrm{mmol}, 1.0$ equiv) and anhydrous 1,4-dioxane $(1 \mathrm{~mL})$ were added. After stirring at room temperature for $25 \mathrm{~min}$, the reaction mixture was cooled to $-20{ }^{\circ} \mathrm{C}$ or $-30{ }^{\circ} \mathrm{C}\left(-20{ }^{\circ} \mathrm{C}\right.$ for difluoromethyl and difluoroacetyl propargyl bromides; $-30{ }^{\circ} \mathrm{C}$ for trifluoromethyl propargyl bromides). cis-Cyclopropylzinc reagent 1 (0.33 $\mathrm{M}$ in THF; $1.0 \mathrm{mmol}, 2.0$ equiv) was added dropwise over a period of $15 \mathrm{~min}$. The reaction was stirred at same reaction temperature for $24 \mathrm{~h}$, and was quenched with saturated aqueous $\mathrm{NH}_{4} \mathrm{Cl}$. The resulting mixture was diluted with $\mathrm{CH}_{2} \mathrm{Cl}_{2}(50 \mathrm{~mL})$ and was filtered with a pad of cellite. The filtrate was washed with water $(15 \mathrm{~mL})$. The organic layer was dried over $\mathrm{Na}_{2} \mathrm{SO}_{4}$, filtered, and concentrated. The residue was purified with silica gel chromatography to give the pure product. 


\section{Synthesis of Triazoles}

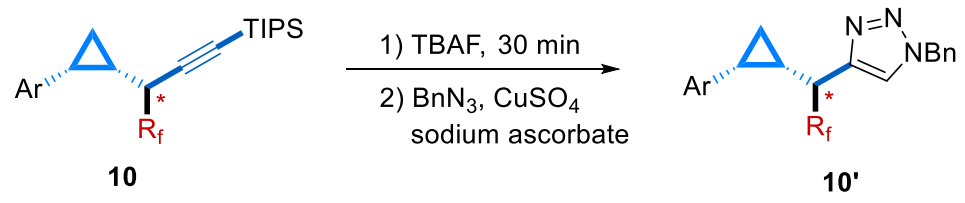

To a solution of $\mathbf{1 0}$ (1.0 equiv) in THF $(0.15 \mathrm{M})$ was added TBAF (1.2 equiv, $1 \mathrm{M}$ in THF) at $0{ }^{\circ} \mathrm{C}$ or $-20{ }^{\circ} \mathrm{C}\left(0{ }^{\circ} \mathrm{C}\right.$ for difluoromethylated compounds; $-20{ }^{\circ} \mathrm{C}$ for trifluoromethylated compounds $)$. After the mixture was stirred at same reaction temperature for $30 \mathrm{~min}, \mathrm{H}_{2} \mathrm{O}(1 \mathrm{~mL})$ was added. The reaction mixture was warmed to room temperature, (azidomethyl)benzene (1.2 equiv), $\mathrm{CuSO}_{4}(0.2$ equiv) and sodium ascorbate ( 0.6 equiv) were added. The resulting mixture was then stirred for $12 \mathrm{~h}$ at room temperature. The reaction mixture was concentrated. The residue was extracted with ethyl acetate. The combined organic layers were washed with brine, dried over $\mathrm{Na}_{2} \mathrm{SO}_{4}$ and filtered. The filtrate was concentrated. The product was purified with silica gel chromatography.

\subsection{Characterization data for compounds $\mathbf{1 0}$}

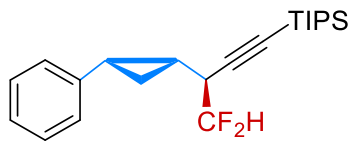

((S)-4,4-difluoro-3-((1R,2S)-2-phenylcyclopropyl)but-1-yn-1-yl)triisoprop

ylsilane (10a). The product (96 mg, 88\% yield, dr >20:1) was purified with silica gel chromatography (Petroleum ether $\left./ \mathrm{CH}_{2} \mathrm{Cl}_{2}=10: 1\right)$ as a colorless oil. $[\alpha]_{\mathrm{D}}{ }^{25}=198.02(c=$ 0.41, $\mathrm{CHCl}_{3}$ ) for a sample with 99.9:0.1 er; ${ }^{1} \mathrm{H} \mathrm{NMR}\left(400 \mathrm{MHz}, \mathrm{CDCl}_{3}\right) \delta 7.35-7.31(\mathrm{~m}, 2 \mathrm{H}), 7.29$ $-7.26(\mathrm{~m}, 2 \mathrm{H}), 7.24-7.21(\mathrm{~m}, 1 \mathrm{H}), 5.78(\mathrm{td}, J=56.3,3.5 \mathrm{~Hz}, 1 \mathrm{H}), 2.38-2.30(\mathrm{~m}, 1 \mathrm{H}), 1.89$ (tdd, $J=14.1,10.9,3.5 \mathrm{~Hz}, 1 \mathrm{H}), \delta 1.47-1.38(\mathrm{~m}, 1 \mathrm{H}), 1.15-1.10(\mathrm{~m}, 1 \mathrm{H}), 1.09-1.04(\mathrm{~m}, 21 \mathrm{H}), \delta$ $0.96-0.89(\mathrm{~m}, 1 \mathrm{H}) .{ }^{13} \mathrm{C} \mathrm{NMR}\left(101 \mathrm{MHz}, \mathrm{CDCl}_{3}\right) \delta 137.2,129.7,128.1,126.5,116.2(\mathrm{t}, J=246.4$ $\mathrm{Hz}), 102.6(\mathrm{t}, J=5.4 \mathrm{~Hz}), 84.9,38.1(\mathrm{t}, J=22.1 \mathrm{~Hz}), 19.4,18.5,18.0(\mathrm{t}, J=3.9 \mathrm{~Hz}), 11.2,8.5 .{ }^{19} \mathrm{~F}$ NMR $\left(376 \mathrm{MHz}, \mathrm{CDCl}_{3}\right) \delta-119.5$ (ddd, $\left.J=274.8,56.2,14.2 \mathrm{~Hz}, 1 \mathrm{~F}\right),-120.3$ (ddd, $J=274.8,56.4$, 13.1Hz, 1 F). MS (EI): m/z (\%) $362\left(\mathrm{M}^{+}\right), 319,247,165,91$ (100). HRMS: Calculated for $\mathrm{C}_{22} \mathrm{H}_{32} \mathrm{~F}_{2} \mathrm{Si}$ : 362.2241; Found: 362.2238. The enantiomeric ratio of 10a (99.9:0.1 er) was determined by 10a', which was derived from 10a through a [3+2] cyclization with benzyl azide.

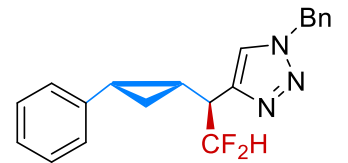

1-benzyl-4-((S)-2,2-difluoro-1-((1R,2S)-2-phenylcyclopropyl)ethyl)-1H-1,2, 3-triazole (10a'). The product ( $46.7 \mathrm{mg}$, 53\% yield) was purified with silica gel chromatography (Petroleum ether/EtOAc= 5:1) as a white solid (m.p. 102-103 
$\left.{ }^{\circ} \mathrm{C}\right) .[\alpha]_{\mathrm{D}}{ }^{25}=117.29\left(c=0.30, \mathrm{CHCl}_{3}\right)$ for a sample with 99.9:0.1 er. ${ }^{1} \mathrm{H} \mathrm{NMR}\left(400 \mathrm{MHz}, \mathrm{CDCl}_{3}\right) \delta$ $7.44-7.35(\mathrm{~m}, 3 \mathrm{H}), 7.23-7.13(\mathrm{~m}, 2 \mathrm{H}), 7.09-7.02(\mathrm{~m}, 1 \mathrm{H}), 7.01-6.98(\mathrm{~m}, 2 \mathrm{H}), 6.88(\mathrm{~d}, \mathrm{~J}=$ $7.3 \mathrm{~Hz}, 2 \mathrm{H}), 6.65(\mathrm{~s}, 1 \mathrm{H}), 6.13(\mathrm{td}, J=56.4,4.6 \mathrm{~Hz}, 1 \mathrm{H}), 5.48(\mathrm{~d}, J=14.9 \mathrm{~Hz}, 1 \mathrm{H}), 5.35(\mathrm{~d}, J=$ $14.9 \mathrm{~Hz}, 1 \mathrm{H}), 2.36$ (tdd, $J=13.9,11.4,4.6 \mathrm{~Hz}, 1 \mathrm{H}) ., 2.26-2.20$ (m, $1 \mathrm{H}), 1.80-1.68$ (m, $1 \mathrm{H})$, $1.25-1.17(\mathrm{~m}, 1 \mathrm{H}), 1.17-1.11(\mathrm{~m}, 1 \mathrm{H}) .{ }^{13} \mathrm{C} \mathrm{NMR}\left(126 \mathrm{MHz}, \mathrm{CDCl}_{3}\right) \delta 143.4(\mathrm{t}, J=4.0 \mathrm{~Hz})$, 137.1, 134.6, 128.9, 128.7, 128.6, 127.8, 127.6, 126.0, 122.3, 117.3 (t, $J=244.4 \mathrm{~Hz}), 53.8,41.1(\mathrm{t}, J$ $=21.0 \mathrm{~Hz}), 19.2,17.2(\mathrm{t}, J=4.5 \mathrm{~Hz}), 8.7 .{ }^{19} \mathrm{~F} \mathrm{NMR}\left(376 \mathrm{MHz}, \mathrm{CDCl}_{3}\right) \delta-119.3(\mathrm{ddd}, J=277.0$, 56.2, 14.4 Hz, 1 F), -120.5 (ddd, $J=256.7,13.2 \mathrm{~Hz}, 1 \mathrm{~F}) . \mathrm{MS}(\mathrm{ESI}): \mathrm{m} / \mathrm{z}(\%) 340.0\left([\mathrm{M}+\mathrm{H}]^{+}\right)$. HRMS: Calculated for $\mathrm{C}_{20} \mathrm{H}_{21} \mathrm{~F}_{2} \mathrm{~N}_{3}$ : 340.1620; Found: 340.1618. Enantiomeric purity (99.9:0.1 er) was measured by chiral HPLC on IF-3 column (Hexanes $/{ }^{i} \mathrm{PrOH}=90: 10,0.7 \mathrm{~mL} / \mathrm{min}$, UV detection at $214 \mathrm{~nm}$ ); retention time $=23.70 \mathrm{~min}$ (major), retention time $=22.50 \mathrm{~min}$ (minor).

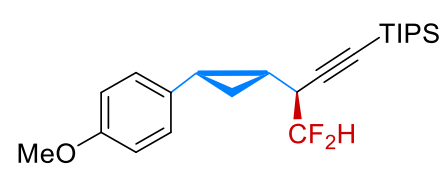

((S)-4,4-Difluoro-3-((1R,2S)-2-(4-methoxyphenyl)cyclopropyl)but-1-y n-1-yl)triisopropylsilane (10b). The product (106 mg, 90\% yield, dr >20:1) was purified with silica gel chromatography (Petroleum ether/DCM= 5:1 $)$ as a colorless oil. $[\alpha]_{\mathrm{D}}{ }^{25}=173.20\left(c=0.50, \mathrm{CHCl}_{3}\right)$ for a sample with 97.5:2.5 er. ${ }^{1} \mathrm{H}$ NMR (400 MHz, $\left.\mathrm{CDCl}_{3}\right) \delta 7.26(\mathrm{~d}, J=8.1 \mathrm{~Hz}, 2 \mathrm{H}), 6.81(\mathrm{~d}, J=8.5 \mathrm{~Hz}, 2 \mathrm{H}), 5.77(\mathrm{td}, J=56.3$, $3.3 \mathrm{~Hz}, 1 \mathrm{H}), 3.79$ (s, $3 \mathrm{H}), 2.35-2.19$ (m, $1 \mathrm{H}), 1.95$ - 1.78 (m, $1 \mathrm{H}), 1.44-1.33$ (m, $1 \mathrm{H}), 1.17$ $1.01(\mathrm{~m}, 21 \mathrm{H}), 0.87-0.83(\mathrm{~m}, 1 \mathrm{H}) .{ }^{13} \mathrm{C} \mathrm{NMR}\left(101 \mathrm{MHz}, \mathrm{CDCl}_{3}\right) \delta 158.3,130.7,129.1,116.2(\mathrm{t}, J$ $=245.5 \mathrm{~Hz}), 113.5,102.7(\mathrm{t}, J=5.4 \mathrm{~Hz}), 84.8,55.2,38.1(\mathrm{t}, J=22.1 \mathrm{~Hz}), 18.5,17.7(\mathrm{t}, J=3.7 \mathrm{~Hz})$, 11.1, 8.6. ${ }^{19} \mathrm{~F}$ NMR $\left(376 \mathrm{MHz}, \mathrm{CDCl}_{3}\right) \delta-119.7$ (d, $\left.J=14.3 \mathrm{~Hz}\right),-119.9$ (d, $\left.J=14.3 \mathrm{~Hz}\right)$. MS (EI): m/z (\%) $392\left(\mathrm{M}^{+}\right), 349\left(\left[\mathrm{M}-{ }^{i} \mathrm{Pr}\right]^{+}\right), 165,147$ (100). HRMS (EI): Calculated for $\mathrm{C}_{23} \mathrm{H}_{34} \mathrm{~F}_{2} \mathrm{OSi}$ : 392.2347; Found: 392.2356. Enantiomeric purity (97.5:2.5 er) was measured by chiral HPLC on ODH column $\left(\right.$ Hexanes $/{ }^{i} \mathrm{PrOH}=100,0.7 \mathrm{~mL} / \mathrm{min}$, UV detection at $214 \mathrm{~nm}$ ); retention time $=21.63$ $\min ($ major), retention time $=20.58 \min ($ minor $)$.

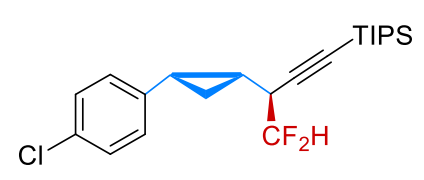

((S)-3-((1R,2S)-2-(4-Chlorophenyl)cyclopropyl)-4,4-difluorobut-1-yn-1 -yl)triisopropylsilane (10c). The product (102 mg, 86\% yield, $\mathrm{dr}>20: 1)$ was purified with silica gel chromatography (Petroleum ether/DCM=10:1) as a colorless oil. $[\alpha]_{\mathrm{D}}{ }^{25}=223.12\left(c=0.52, \mathrm{CHCl}_{3}\right)$ for a sample with 99.9:0.1 er. ${ }^{1} \mathrm{H}$ NMR $(400$ 
$\left.\mathrm{MHz}, \mathrm{CDCl}_{3}\right) \delta 7.28-7.22(\mathrm{~m}, 4 \mathrm{H}), 5.77(\mathrm{td}, J=56.2,3.5 \mathrm{~Hz}, 1 \mathrm{H}), 2.34-2.19(\mathrm{~m}, 1 \mathrm{H}), 1.85(\mathrm{tdd}$, $J=14.1,11.0,3.6 \mathrm{~Hz}, 1 \mathrm{H}), 1.48-1.36(\mathrm{~m}, 1 \mathrm{H}), 1.14(\mathrm{td}, J=8.3,5.9 \mathrm{~Hz}, 1 \mathrm{H}), 1.06-1.05(\mathrm{~m}, 21$ $\mathrm{H}), 0.84-0.85(\mathrm{~m}, 1 \mathrm{H}) .{ }^{13} \mathrm{C} \mathrm{NMR}\left(101 \mathrm{MHz}, \mathrm{CDCl}_{3}\right) \delta 135.8,132.4,131.0,128.2,116.0(\mathrm{t}, J=$ $246.5 \mathrm{~Hz}), 102.2(\mathrm{t}, J=5.6 \mathrm{~Hz}), 85.3,38.1(\mathrm{t}, J=22.3 \mathrm{~Hz}), 18.8,18.5,18.0(\mathrm{t}, J=4.0 \mathrm{~Hz}), 11.1,8.9$. ${ }^{19} \mathrm{~F}$ NMR (376 MHz, $\mathrm{CDCl}_{3}$ ) $\delta-119.4(\mathrm{ddd}, J=275.2,56.1,14.0 \mathrm{~Hz}, 1 \mathrm{~F}),-120.4$ (ddd, $J=275.2$, 56.3, $12.8 \mathrm{~Hz}, 1 \mathrm{~F})$. MS (EI): m/z (\%) $396\left(\mathrm{M}^{+}\right), 353\left(\left[\mathrm{M}-{ }^{i} \mathrm{Pr}\right]^{+}\right), 165$ (100), 105, 91, 77. HRMS: Calculated for $\mathrm{C}_{22} \mathrm{H}_{31} \mathrm{ClF}_{2} \mathrm{Si}$ : 396.1852; Found: 396.1859. The enantiomeric ratio of 10c (99.9:0.1 er) was determined by $\mathbf{1 0} \mathbf{c}^{\prime}$, which was derived from 10c through a [3+2] cyclization with benzyl azide.

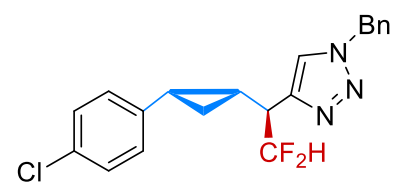

1-Benzyl-4-((S)-1-((1R,2S)-2-(4-chlorophenyl)cyclopropyl)-2,2-difluoroe thyl)-1H-1,2,3-triazole (10c'). The product (56 $\mathrm{mg}$, 58\% yield) was purified with silica gel chromatography (Petroleum ether/EtOAc $=5: 1$ ) as a white solid (m.p. $\left.124-125{ }^{\circ} \mathrm{C}\right) .[\alpha]_{\mathrm{D}}^{25}=142.52\left(c=0.88, \mathrm{CHCl}_{3}\right)$ for a sample with 99.9:0.1 er. ${ }^{1} \mathrm{H}$ NMR (400 MHz, $\left.\mathrm{CDCl}_{3}\right) \delta 7.49-7.37(\mathrm{~m}, 3 \mathrm{H}), 7.25-7.18(\mathrm{~m}, 2 \mathrm{H}), 6.93(\mathrm{~d}, J=8.4 \mathrm{~Hz}, 2 \mathrm{H}), 6.79$ $(\mathrm{d}, J=8.3 \mathrm{~Hz}, 2 \mathrm{H}), 6.75(\mathrm{~s}, 1 \mathrm{H}), 6.08(\mathrm{td}, J=56.4,4.5 \mathrm{~Hz}, 1 \mathrm{H}), 5.51(\mathrm{~d}, J=14.8 \mathrm{~Hz}, 1 \mathrm{H}), 5.35$ (d, $J=14.8 \mathrm{~Hz}, 1 \mathrm{H}), 2.35-2.24(\mathrm{~m}, 1 \mathrm{H}), 2.24-2.15(\mathrm{~m}, 1 \mathrm{H}), 1.83-1.73(\mathrm{~m}, 1 \mathrm{H}), 1.23(\mathrm{td}, J=8.3$, $6.0 \mathrm{~Hz}, 1 \mathrm{H}), 1.12-1.04(\mathrm{~m}, 1 \mathrm{H}) .{ }^{13} \mathrm{C} \mathrm{NMR}\left(126 \mathrm{MHz}, \mathrm{CDCl}_{3}\right) \delta 143.2(\mathrm{t}, J=4.1 \mathrm{~Hz}), 135.8,134.6$, $131.9,130.2,129.2,128.8,128.0,127.8,122.2,117.2(\mathrm{dd}, J=244.9,245.3 \mathrm{~Hz}), 54.0,41.3(\mathrm{t}, J=$ 21.1 Hz), 18.8, $17.2(\mathrm{t}, J=4.6 \mathrm{~Hz}), 9.1 .{ }^{19} \mathrm{~F}$ NMR $\left(376 \mathrm{MHz}, \mathrm{CDCl}_{3}\right) \delta-119.4$ (ddd, $J=277.3,56.2$, $14.4 \mathrm{~Hz}, 1 \mathrm{~F}),-120.4$ (ddd, $J=277.3,56.5,13.0 \mathrm{~Hz}, 1 \mathrm{~F}) . \mathrm{MS}(\mathrm{ESI}): \mathrm{m} / \mathrm{z}(\%) 373.9\left(\mathrm{M}^{+}\right) . \mathrm{HRMS}$ : Calculated for $\mathrm{C}_{20} \mathrm{H}_{19} \mathrm{ClF}_{2} \mathrm{~N}_{3}$ : 374.1230; Found: 374.1228. Enantiomeric purity (99.9:0.1 er) was measured by chiral HPLC on IF-3 column (Hexanes $/{ }^{i} \mathrm{PrOH}=90: 10,0.7 \mathrm{~mL} / \mathrm{min}$, UV detection at $214 \mathrm{~nm}$ ); retention time $=21.24 \min ($ major $)$, retention time $=19.94 \min ($ minor $)$.

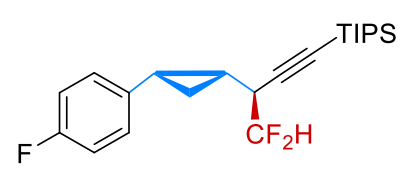

\section{((S)-4,4-Difluoro-3-((1R,2S)-2-(4-fluorophenyl)cyclopropyl)but-1-yn-1-}

yl)triisopropylsilane (10d). The product (76 mg, 67\% yield, dr >20:1) was purified with silica gel chromatography (Petroleum ether/DCM=10:1) as a colorless oil. $[\alpha]_{\mathrm{D}}{ }^{25}=186.10\left(c=0.98, \mathrm{CHCl}_{3}\right)$ for a sample with 99.9:0.1 er. ${ }^{1} \mathrm{H}$ NMR $(400 \mathrm{MHz}$, $\left.\mathrm{CDCl}_{3}\right) \delta 7.30(\mathrm{dd}, J=8.3,5.5 \mathrm{~Hz}, 2 \mathrm{H}), 6.96(\mathrm{t}, J=8.7 \mathrm{~Hz}, 2 \mathrm{H}), 5.77(\mathrm{td}, J=56.3,3.5 \mathrm{~Hz}, 1 \mathrm{H})$, $2.32-2.36(\mathrm{~m}, 1 \mathrm{H}), 1.83(\mathrm{tdd}, J=14.2,10.9,3.5 \mathrm{~Hz}, 1 \mathrm{H}), 1.46-1.35(\mathrm{~m}, 1 \mathrm{H}), 1.17-1.09$ (m, 1 
$\mathrm{H}), 1.07-1.06(\mathrm{~m}, 21 \mathrm{H}), 0.90-0.83(\mathrm{~m}, 1 \mathrm{H}) .{ }^{13} \mathrm{C} \mathrm{NMR}\left(126 \mathrm{MHz}, \mathrm{CDCl}_{3}\right) \delta 161.7(\mathrm{~d}, J=244.5$ Hz), $132.8(\mathrm{~d}, J=2.6 \mathrm{~Hz}), 131.2(\mathrm{~d}, J=8.1 \mathrm{~Hz}), 116.0(\mathrm{t}, J=246.8 \mathrm{~Hz}), 115.0,114.8,102.4(\mathrm{t}, J=$ $5.5 \mathrm{~Hz}), 85.2,38.2(\mathrm{t}, J=22.2 \mathrm{~Hz}), 18.6,18.5,17.80(\mathrm{t}, J=3.3 \mathrm{~Hz}), 11.1,8.8 .{ }^{19} \mathrm{~F}$ NMR $(376 \mathrm{MHz}$, $\left.\mathrm{CDCl}_{3}\right) \delta-116.5(\mathrm{~m}, 1 \mathrm{~F}),-119.4(\mathrm{ddd}, J=275.0,56.0,14.3 \mathrm{~Hz}, 1 \mathrm{~F}),-120.3(\mathrm{ddd}, J=275.4,56.2$, $12.8 \mathrm{~Hz}, 1 \mathrm{~F}) . \mathrm{MS}(\mathrm{EI}): \mathrm{m} / \mathrm{z}(\%) 380\left(\mathrm{M}^{+}\right), 337\left(\left[\mathrm{M}-{ }^{i} \mathrm{Pr}\right]^{+}\right), 165$ (100), 109, 91, 77. HRMS: Calculated for $\mathrm{C}_{23} \mathrm{H}_{31} \mathrm{~F}_{3} \mathrm{Si}$ : 380.2147; Found: 380.2155. The enantiomeric ratio of 10d (99.9:0.1 er) was determined by 10d', which was derived from 10d through a [3+2] cyclization with benzyl azide.

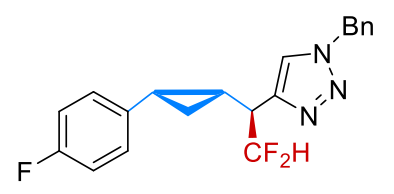

\section{1-Benzyl-4-((S)-2,2-difluoro-1-((1R,2S)-2-(4-fluorophenyl)cyclopropyl)et}

hyl)-1H-1,2,3-triazole (11). The product (35 mg, 49\% yield, $\mathrm{dr}>20: 1$ ) was purified with silica gel chromatography (Petroleum ether/EtOAc $=5: 1)$ as a white solid (m.p. $\left.109-110{ }^{\circ} \mathrm{C}\right) .[\alpha]_{\mathrm{D}}^{25}=103.90\left(c=0.20, \mathrm{CHCl}_{3}\right)$ for a sample with 99.9:0.1 er. ${ }^{1} \mathrm{H}$ NMR (400 MHz, $\left.\mathrm{CDCl}_{3}\right) \delta 7.48-7.34(\mathrm{~m}, 3 \mathrm{H}), 7.24-7.17(\mathrm{~m}, 2 \mathrm{H}), 6.82(\mathrm{dd}, J=8.3,5.5 \mathrm{~Hz}, 2 \mathrm{H})$, $6.78(\mathrm{~s}, 1 \mathrm{H}), 6.65(\mathrm{t}, J=8.6 \mathrm{~Hz}, 2 \mathrm{H}), 6.07(\mathrm{td}, J=56.4,4.5 \mathrm{~Hz}, 1 \mathrm{H}), 5.51(\mathrm{~d}, J=14.8 \mathrm{~Hz}, 1 \mathrm{H})$, $5.36(\mathrm{~d}, J=14.8 \mathrm{~Hz}, 1 \mathrm{H}), 2.35-2.15(\mathrm{~m}, 2 \mathrm{H}), 1.82-1.66(\mathrm{~m}, 1 \mathrm{H}), 1.28-1.17(\mathrm{~m}, 1 \mathrm{H}), 1.09-$ $1.02(\mathrm{~m}, 1 \mathrm{H}) .{ }^{13} \mathrm{C}$ NMR $\left(101 \mathrm{MHz}, \mathrm{CDCl}_{3}\right) \delta 161.2(\mathrm{~d}, J=244.6 \mathrm{~Hz}), 143.3(\mathrm{t}, J=4.1 \mathrm{~Hz}), 134.6$, $132.8(\mathrm{~d}, J=3.1 \mathrm{~Hz}), 130.2(\mathrm{~d}, J=7.9 \mathrm{~Hz}), 129.0,128.7,127.9,122.2,117.2(\mathrm{t}, J=244.6 \mathrm{~Hz}), 114.4$ $(\mathrm{d}, J=21.2 \mathrm{~Hz}), 53.9,41.3(\mathrm{t}, J=21.1 \mathrm{~Hz}), 18.6,16.9(\mathrm{t}, J=4.7 \mathrm{~Hz}), 9.0 .{ }^{19} \mathrm{~F}$ NMR $(376 \mathrm{MHz}$, $\left.\mathrm{CDCl}_{3}\right) \delta-116.6(\mathrm{~m}, 1 \mathrm{~F}),-119.5(\mathrm{ddd}, J=276.9,56.2,14.7 \mathrm{~Hz}, 1 \mathrm{~F}),-120.4(\mathrm{ddd}, J=276.9,55.6$, $13.5 \mathrm{~Hz}, 1 \mathrm{~F})$. MS (ESI): m/z (\%) $357.9\left([\mathrm{M}+\mathrm{H}]^{+}\right)$. HRMS: Calculated for $\mathrm{C}_{20} \mathrm{H}_{19} \mathrm{~F}_{3} \mathrm{~N}_{3}: 358.1526$; Found: 358.1528. Enantiomeric purity (99.9:0.1 er) was measured by chiral HPLC on IF-3 column $\left(\right.$ Hexanes $/{ }^{i} \mathrm{PrOH}=90: 10,0.7 \mathrm{~mL} / \mathrm{min}, \mathrm{UV}$ detection at $\left.214 \mathrm{~nm}\right)$; retention time $=21.31$ min (major), retention time $=20.40 \min ($ minor $)$. 


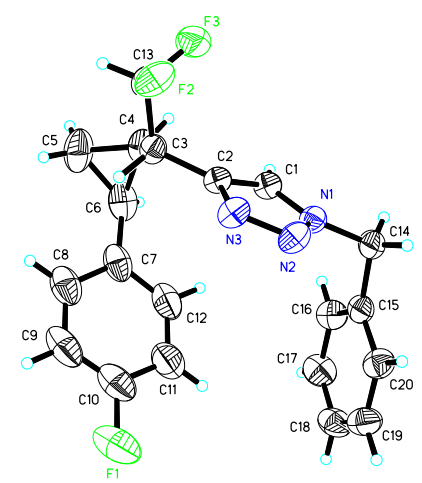

X-ray Crystal Structure of Compound 11

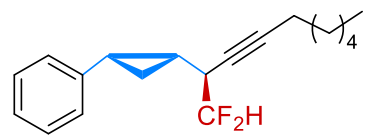

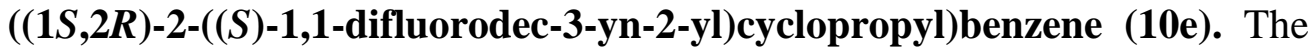
product $(70 \mathrm{mg}, 80 \%$ yield, $\mathrm{dr}=8: 1)$ was purified with silica gel chromatography (Petroleum ether/DCM=10:1) as a colorless oil. $[\alpha]_{\mathrm{D}}^{25}=183.75\left(c=0.24, \mathrm{CHCl}_{3}\right)$ for a sample with 99.7:0.3 er. ${ }^{1} \mathrm{H}$ NMR $\left(400 \mathrm{MHz}, \mathrm{CDCl}_{3}\right) \delta 7.35-7.14(\mathrm{~m}, 5 \mathrm{H}), 5.73(\mathrm{td}, J=56.5$, $3.5 \mathrm{~Hz}, 1 \mathrm{H}), 2.33-2.75(\mathrm{~m}, 1 \mathrm{H}), 2.15-2.11(\mathrm{~m}, 2 \mathrm{H}), 1.89-1.80(\mathrm{~m}, 1 \mathrm{H}), 1.50-1.26(\mathrm{~m}, 9 \mathrm{H})$, $1.14-1.06$ (m, $1 \mathrm{H}), 0.92-0.88(\mathrm{~m}, 4 \mathrm{H}) .{ }^{13} \mathrm{C} \mathrm{NMR}\left(101 \mathrm{MHz}, \mathrm{CDCl}_{3}\right) \delta 137.2,129.6,127.9,126.4$, $116.4(\mathrm{t}, J=245.6 \mathrm{~Hz}), 84.4,74.5(\mathrm{t}, J=5.9 \mathrm{~Hz}), 37.1(\mathrm{t}, J=21.9 \mathrm{~Hz}), 31.3,28.5,28.4,22.6,19.4$, 18.6, $18.1(\mathrm{t}, J=4.2 \mathrm{~Hz}), 14.1,8.5 .{ }^{19} \mathrm{~F}$ NMR $(376 \mathrm{MHz}, \mathrm{CDCl} 3) \delta-120.0(\mathrm{dd}, J=56.5,13.8 \mathrm{~Hz})$. MS (EI): m/z (\%) $290\left(\mathrm{M}^{+}\right), 117$ (100), 91. HRMS: Calculated for $\mathrm{C}_{19} \mathrm{H}_{24} \mathrm{~F}_{2}$ : 290.1846; Found: 290.1849. Enantiomeric purity (99.7:0.3 er) was measured by chiral HPLC on PC-3 column $\left(\mathrm{CH}_{3} \mathrm{CN} / \mathrm{H}_{2} \mathrm{O}=50: 50,0.7 \mathrm{~mL} / \mathrm{min}, \mathrm{UV}\right.$ detection at $\left.214 \mathrm{~nm}\right)$; retention time $=43.29$ min (major), retention time $=49.02 \min ($ minor $)$.

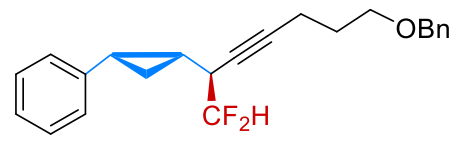

((1S,2R)-2-((S)-7-(Benzyloxy)-1,1-difluorohept-3-yn-2-yl)cyclopropy

l)benzene (10f). The product ( $78 \mathrm{mg}, 73 \%$ yield, $\mathrm{dr}=6: 1$ ) was purified with silica gel chromatography (Petroleum ether/EtOAc $=10: 1)$ as a colorless oil. $[\alpha]_{\mathrm{D}}^{25}=87.27(c=$ 0.33, $\left.\mathrm{CHCl}_{3}\right)$ for a sample with 98.5:1.5 er for the major diastereomer. ${ }^{1} \mathrm{H} \mathrm{NMR}\left(400 \mathrm{MHz}, \mathrm{CDCl}_{3}\right) \delta$ $7.44-7.06(\mathrm{~m}, 10 \mathrm{H}), 5.72(\mathrm{td}, J=56.4,3.4 \mathrm{~Hz}, 1 \mathrm{H}), 4.51(\mathrm{~s}, 2 \mathrm{H}), 3.52(\mathrm{td}, J=6.2,1.9 \mathrm{~Hz}, 2 \mathrm{H})$, $2.37-2.19(\mathrm{~m}, 3 \mathrm{H}), 1.93-1.69(\mathrm{~m}, 3 \mathrm{H}), 1.39-1.27(\mathrm{~m}, 1 \mathrm{H}), 1.14-1.05(\mathrm{~m}, 1 \mathrm{H}), 0.95-0.89(\mathrm{~m}$, $1 \mathrm{H}) .{ }^{13} \mathrm{C} \mathrm{NMR}\left(101 \mathrm{MHz}, \mathrm{CDCl}_{3}\right) \delta 138.5,137.2,129.5,128.4,128.0,127.6,127.5,126.5,116.4(\mathrm{t}$, 
$J=245.7 \mathrm{~Hz}), 83.6,75.0(\mathrm{t}, J=6.0 \mathrm{~Hz}), 73.0,68.8,37.0(\mathrm{t}, J=21.8 \mathrm{~Hz}), 28.7,19.4,18.1(\mathrm{t}, J=4.1$ $\mathrm{Hz}), 15.5,8.5 .{ }^{19} \mathrm{~F}$ NMR $\left(376 \mathrm{MHz}, \mathrm{CDCl}_{3}\right) \delta-119.9$ (dd, $\left.J=14.6,8.8 \mathrm{~Hz}, 1 \mathrm{~F}\right),-120.1$ (dd, $J=14.6$, $8.7 \mathrm{~Hz}, 1 \mathrm{~F}) . \mathrm{MS}(\mathrm{EI}): \mathrm{m} / \mathrm{z}(\%) 354\left(\mathrm{M}^{+}\right), 263\left([\mathrm{M}-\mathrm{Bn}]^{+}\right), 117,91$ (100). HRMS: Calculated for $\mathrm{C}_{16} \mathrm{H}_{17} \mathrm{OF}_{2}$ : 263.1247; Found: 263.1245. Enantiomeric purity (98.5:1.5 er) was measured by chiral HPLC on OJH column (Hexanes $/{ }^{i} \mathrm{PrOH}=97: 3,0.7 \mathrm{~mL} / \mathrm{min}$, UV detection at $214 \mathrm{~nm}$ ); retention time $=22.37 \mathrm{~min}$ (major), retention time $=20.06 \min$ (minor) .

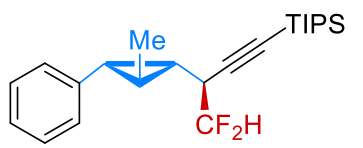

((S)-4,4-Difluoro-3-((1S,2R,3R)-2-methyl-3-phenylcyclopropyl)but-1-yn-1yl)triisopropylsilane $(\mathbf{1 0 g})$. The product $(86 \mathrm{mg}, 76 \%$ yield, $\mathrm{dr}=8: 1)$ was purified with silica gel chromatography (Petroleum ether/DCM=10:1) as a colorless oil. $[\alpha]_{\mathrm{D}}^{25}=$ $165.23\left(c=0.57, \mathrm{CHCl}_{3}\right)$ for a sample with 99.7:0.3 er. ${ }^{1} \mathrm{H} \mathrm{NMR}\left(400 \mathrm{MHz}, \mathrm{CDCl}_{3}\right) \delta 7.47-7.02$ $(\mathrm{m}, 5 \mathrm{H}), 5.78(\mathrm{td}, J=56.4,3.8 \mathrm{~Hz}, 1 \mathrm{H}), 2.05(\mathrm{dd}, J=9.0,5.3 \mathrm{~Hz}, 1 \mathrm{H}), 2.02-1.92(\mathrm{~m}, 1 \mathrm{H}), 1.33-$ $1.26(\mathrm{~m}, 1 \mathrm{H}), 1.24(\mathrm{~d}, J=5.5 \mathrm{~Hz}, 3 \mathrm{H}), 1.19-1.11(\mathrm{~m}, 1 \mathrm{H}), 1.08-1.05(\mathrm{~m}, 21 \mathrm{H}) .{ }^{13} \mathrm{C}$ NMR $(101$ $\left.\mathrm{MHz}, \mathrm{CDCl}_{3}\right) \delta 137.5,129.4,128.0,126.3,116.2(\mathrm{t}, J=246.3 \mathrm{~Hz}), 102.6(\mathrm{t}, J=5.5 \mathrm{~Hz}), 84.6,37.8$ $(\mathrm{t}, J=22.3 \mathrm{~Hz}), 27.8,26.2(\mathrm{t}, J=3.6 \mathrm{~Hz}), 18.5,18.0,16.9,11.1 .{ }^{19} \mathrm{~F} \mathrm{NMR}\left(376 \mathrm{MHz}, \mathrm{CDCl}_{3}\right) \delta$ -119.2 (ddd, $J=274.5,56.2,13.6 \mathrm{~Hz}, 1 \mathrm{~F}),-120.5$ (ddd, $J=274.5,56.5,13.0 \mathrm{~Hz}, 1 \mathrm{~F}) . \mathrm{MS}(\mathrm{EI}): \mathrm{m} / \mathrm{z}$ (\%) 376, 333 ([M- $\left.\left.{ }^{i} \mathrm{Pr}\right]^{+}\right)$, 181, 117 (100). HRMS: Calculated for $\mathrm{C}_{23} \mathrm{H}_{34} \mathrm{~F}_{2} \mathrm{Si}$ : 376.2398; Found: 376.2398. The enantiomeric ratio of $\mathbf{1 0 g}$ (99.7:0.3 er) was determined by $\mathbf{1 0 g}$ ', which was derived from $10 \mathrm{~g}$ through a [3+2] cyclization with benzyl azide.

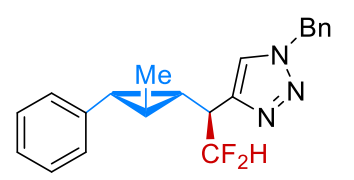

1-Benzyl-4-((S)-2,2-difluoro-1-((1R,2R,3S)-2-methyl-3-phenylcyclopropyl)e thyl)-1H-1,2,3-triazole (10g'). The product (30 mg, 38\% yield) was purified with silica gel chromatography (Petroleum ether/EtOAc $=5: 1$ ) as a white solid (m.p. $\left.121-122{ }^{\circ} \mathrm{C}\right) .[\alpha]_{\mathrm{D}}^{25}=75.85\left(c=0.20, \mathrm{CHCl}_{3}\right)$ for a sample with 99.7:0.3 er. ${ }^{1} \mathrm{H}$ NMR $(400$ $\left.\mathrm{MHz}, \mathrm{CDCl}_{3}\right) \delta 7.47-7.31(\mathrm{~m}, 3 \mathrm{H}), 7.22-7.12(\mathrm{~m}, 2 \mathrm{H}), 7.05-6.97(\mathrm{~m}, 3 \mathrm{H}), 6.86(\mathrm{~d}, J=7.1 \mathrm{~Hz}$, $2 \mathrm{H}), 6.60(\mathrm{~s}, 1 \mathrm{H}), 6.15(\mathrm{td}, J=56.5,4.8 \mathrm{~Hz}, 1 \mathrm{H}), 5.45(\mathrm{~d}, J=14.9 \mathrm{~Hz}, 1 \mathrm{H}), 5.33(\mathrm{~d}, J=14.9 \mathrm{~Hz}, 1$ H), $2.53-2.40(\mathrm{~m}, 1 \mathrm{H}), 1.93(\mathrm{dd}, J=8.4,6.0 \mathrm{~Hz}, 1 \mathrm{H}), 1.53-1.42(\mathrm{~m}, 2 \mathrm{H}), 1.27(\mathrm{~d}, J=5.8 \mathrm{~Hz}, 3$ H). ${ }^{13} \mathrm{C}$ NMR $\left(101 \mathrm{MHz}, \mathrm{CDCl}_{3}\right) \delta 143.7,137.6,134.7,129.0,128.6,128.5,127.9,127.7,125.9$, 122.2, $117.4(\mathrm{t}, J=244.4 \mathrm{~Hz}), 53.8,40.9(\mathrm{t}, J=21.3 \mathrm{~Hz}), 27.8,25.8(\mathrm{t}, J=4.7 \mathrm{~Hz}), 18.1,17.1 .{ }^{19} \mathrm{~F}$ NMR $\left(376 \mathrm{MHz}, \mathrm{CDCl}_{3}\right) \delta-119.1(\mathrm{ddd}, J=276.7,56.2,13.4 \mathrm{~Hz}, 1 \mathrm{~F}),-121.1(\mathrm{ddd}, J=276.7,56.8$, 
13.4 Hz, 1F). MS (ESI): m/z (\%) $354.2\left([\mathrm{M}+\mathrm{H}]^{+}\right)$. HRMS: Calculated for $\mathrm{C}_{21} \mathrm{H}_{22} \mathrm{~F}_{2} \mathrm{~N}_{3}$ : 354.1776; Found: 354.1776. Enantiomeric purity (99.7:0.3 er) was measured by chiral HPLC on IF-3 column $\left(\right.$ Hexanes $/{ }^{i} \mathrm{PrOH}=95: 5,0.7 \mathrm{~mL} / \mathrm{min}, \mathrm{UV}$ detection at $214 \mathrm{~nm}$ ); retention time $=37.46$ min $($ major), retention time $=34.38 \mathrm{~min}$ (minor).

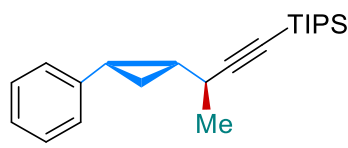

\section{Triisopropyl((S)-3-((1S,2S)-2-phenylcyclopropyl)but-1-yn-1-yl)silane}

(10h). The product ( $85 \mathrm{mg}, 87 \%$ yield, $\mathrm{dr}=9: 1$ ) was purified with silica gel chromatography (Petroleum ether) as a colorless oil. $[\alpha]_{\mathrm{D}}{ }^{25}=-147.12\left(c=0.25, \mathrm{CHCl}_{3}\right)$ for a sample with 99.5:0.5 er. ${ }^{1} \mathrm{H}$ NMR (400 MHz, $\left.\mathrm{CDCl}_{3}\right) \delta 7.36(\mathrm{~d}, J=7.4 \mathrm{~Hz}, 2 \mathrm{H}), 7.29-7.20$ (m, $\left.2 \mathrm{H}\right), 7.18$ $(\mathrm{t}, J=6.8 \mathrm{~Hz}, 1 \mathrm{H}), 2.32-2.22(\mathrm{~m}, 1 \mathrm{H}), 1.53-1.41(\mathrm{~m}, 1 \mathrm{H}), 1.35-1.27(\mathrm{~m}, 1 \mathrm{H}), 1.24(\mathrm{~d}, J=6.9$ $\mathrm{Hz}, 3 \mathrm{H}), 1.14-0.89$ (m, $22 \mathrm{H}), 0.65-0.56$ (m, $1 \mathrm{H}) .{ }^{13} \mathrm{C} \mathrm{NMR}\left(101 \mathrm{MHz}, \mathrm{CDCl}_{3}\right) \delta$ 138.6, 129.8, 127.8, 126.0, 113.1, 79.5, 26.9, 25.6, 21.6, 21.4, 18.6, 11.3, 8.7. MS (EI): m/z (\%) $326\left(\mathrm{M}^{+}\right), 283$ ([M- $\left.\left.{ }^{i} \mathrm{Pr}\right]^{+}\right), 115$ (100). HRMS: Calculated for $\mathrm{C}_{22} \mathrm{H}_{34} \mathrm{Si}$ : 326.2430; Found: 326.2426. Enantiomeric purity (99.5:0.5 er) was measured by chiral HPLC on PC-3 $\left(\mathrm{CH}_{3} \mathrm{CN} / \mathrm{H}_{2} \mathrm{O}=60: 40,0.7 \mathrm{~mL} / \mathrm{min}\right.$, UV detection at $214 \mathrm{~nm}$ ); retention time $=30.41 \mathrm{~min}$ (major), retention time $=25.86 \mathrm{~min}$ (minor).

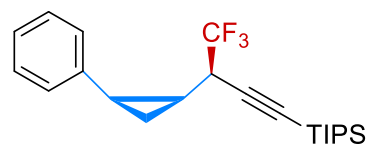

\section{Triisopropyl $((R)-4,4,4-t r i f l u o r o-3-((1 S, 2 R)-2-p h e n y l c y c l o p r o p y l) b u t-1-y n$}

-1-yl)silane (10i). 13 mol\% L1 was used. The product (89 mg, 78\% yield, dr $=40: 1)$ was purified with silica gel chromatography (Petroleum ether) as a colorless oil. $[\alpha]_{\mathrm{D}}^{25}=$ $-189.30\left(c=0.57, \mathrm{CHCl}_{3}\right)$ for a sample with 99.9:0.1 er. ${ }^{1} \mathrm{H} \mathrm{NMR}\left(400 \mathrm{MHz}, \mathrm{CDCl}_{3}\right) \delta 7.39-7.14$ (m, 5 H), $2.39-2.30(\mathrm{~m}, 1 \mathrm{H}), 2.09(\mathrm{dq}, J=10.8,7.7 \mathrm{~Hz}, 1 \mathrm{H}), 1.54-1.45(\mathrm{~m}, 1 \mathrm{H}), 1.17-1.10(\mathrm{~m}$, $1 \mathrm{H}), 1.07-0.99(\mathrm{~m}, 22 \mathrm{H}) .{ }^{13} \mathrm{C} \mathrm{NMR}\left(101 \mathrm{MHz}, \mathrm{CDCl}_{3}\right) \delta 136.7,129.6,128.2,126.7,125.7$ (q, $J=$ $275.9 \mathrm{~Hz}), 100.3(\mathrm{q}, J=2.9 \mathrm{~Hz}), 85.7,38.7(\mathrm{q}, J=30.0 \mathrm{~Hz}), 19.3,18.5,18.3(\mathrm{q}, J=7.2 \mathrm{~Hz}), 11.1$, 8.8. ${ }^{19} \mathrm{~F} \mathrm{NMR}\left(376 \mathrm{MHz}, \mathrm{CDCl}_{3}\right) \delta-70.2(\mathrm{~d}, J=7.7 \mathrm{~Hz})$. MS (EI): m/z (\%) $380\left(\mathrm{M}^{+}\right), 337,165(100)$, 91. HRMS: Calculated for $\mathrm{C}_{22} \mathrm{H}_{31} \mathrm{~F}_{3} \mathrm{Si}$ : 380.2147; Found: 380.2149. The enantiomeric ratio of 10i (99.9:0.1 er) was determined by $\mathbf{1 0 i}$, which was derived from 10i through a [3+2] cyclization with benzyl azide. 


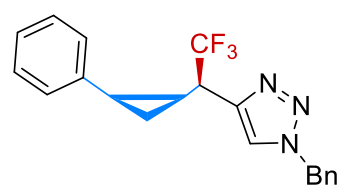

\section{1-benzyl-4-((R)-2,2,2-trifluoro-1-((1S,2R)-2-phenylcyclopropyl)ethyl)-1H-1}

,2,3-triazole (10i'). The product (68 $\mathrm{mg}, 39 \%$ yield) was purified with silica gel chromatography (Petroleum ether/EtOAc= 10:1) as a white solid (m.p.

$\left.105-106{ }^{\circ} \mathrm{C}\right) .[\alpha]_{\mathrm{D}}{ }^{25}=-80.42\left(c=1.40, \mathrm{CHCl}_{3}\right)$ for a sample with 99.9:0.1 er. ${ }^{1} \mathrm{H}$ NMR $(400 \mathrm{MHz}$, $\left.\mathrm{CDCl}_{3}\right) \delta 7.44-7.36(\mathrm{~m}, 3 \mathrm{H}), 7.23-7.13(\mathrm{~m}, 2 \mathrm{H}), 7.05(\mathrm{t}, J=7.2 \mathrm{~Hz}, 1 \mathrm{H}), 6.99$ (t, $J=7.3 \mathrm{~Hz}, 2$ H), $6.85(\mathrm{~d}, J=7.3 \mathrm{~Hz}, 2 \mathrm{H}), 6.78(\mathrm{~s}, 1 \mathrm{H}), 5.50(\mathrm{~d}, J=14.9 \mathrm{~Hz}, 1 \mathrm{H}), 5.37(\mathrm{~d}, J=14.9 \mathrm{~Hz}, 1 \mathrm{H}), \delta$ $2.58(\mathrm{dq}, J=11.3,8.7 \mathrm{~Hz}, 1 \mathrm{H}), 2.33-2.23(\mathrm{~m}, 1 \mathrm{H}), 2.00-1.88(\mathrm{~m}, 1 \mathrm{H}), 1.28-1.20(\mathrm{~m}, 2 \mathrm{H}) .{ }^{13} \mathrm{C}$ NMR $\left(101 \mathrm{MHz}, \mathrm{CDCl}_{3}\right) \delta 141.7,136.6,134.6,129.0,128.8,127.9,127.7,126.4(\mathrm{q}, J=281.9 \mathrm{~Hz})$ 126.2, 122.5, 53.9, $41.8(\mathrm{q}, J=28.2 \mathrm{~Hz}), 19.2,16.8,9.0 .{ }^{19} \mathrm{~F}$ NMR $\left(376 \mathrm{MHz}, \mathrm{CDCl}_{3}\right) \delta-69.2(\mathrm{~d}, J=$ 8.7 Hz). MS (ESI): m/z (\%) 358.1([M+H] $\left.]^{+}\right)$. HRMS: Calculated for $\mathrm{C}_{20} \mathrm{H}_{19} \mathrm{~F}_{3} \mathrm{~N}_{3}$ : 358.1512; Found: 358.1522. Enantiomeric purity (99.9:0.1 er) was measured by chiral HPLC on AY-H column (Hexane/Ethanol/Trifluoroacetic acid $=90: 10: 0.1,1.0 \mathrm{~mL} / \mathrm{min}, \mathrm{UV}$ detection at $220 \mathrm{~nm})$; retention time $=12.21 \mathrm{~min}$ (major), retention time $=8.35 \mathrm{~min}$ (minor).

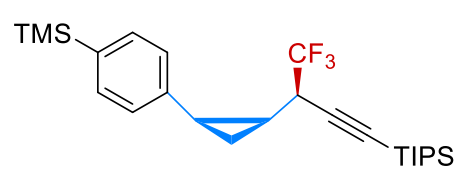

Triisopropyl((R)-4,4,4-trifluoro-3-((1S,2R)-2-(4-(trimethylsilyl)phen yl)cyclopropyl)but-1-yn-1-yl)silane (10j). 13 mol\% L1 was used. The product $(100 \mathrm{mg}, 74 \%$ yield, $\mathrm{dr}=41: 1)$ was purified with silica gel chromatography (Petroleum ether) as a colorless oil. $[\alpha]_{\mathrm{D}}{ }^{25}=-109.34\left(c=0.58, \mathrm{CHCl}_{3}\right)$ for a sample with 99.9:0.1 er. ${ }^{1} \mathrm{H} \mathrm{NMR}\left(400 \mathrm{MHz}, \mathrm{CDCl}_{3}\right) \delta 7.42(\mathrm{~d}, J=7.9 \mathrm{~Hz}, 2 \mathrm{H}), 7.28(\mathrm{~d}, J=7.9 \mathrm{~Hz}, 2 \mathrm{H})$, $2.34(\mathrm{q}, J=8.5 \mathrm{~Hz}, 1 \mathrm{H}), 2.16(\mathrm{dq}, J=10.7,7.7 \mathrm{~Hz}, 1 \mathrm{H}), 1.62-1.46(\mathrm{~m}, 1 \mathrm{H}), 1.19-1.12(\mathrm{~m}, 1 \mathrm{H})$, $1.10-1.02(\mathrm{~m}, 22 \mathrm{H}), 0.25(\mathrm{~s}, 9 \mathrm{H}) .{ }^{13} \mathrm{C}$ NMR $\left(101 \mathrm{MHz}, \mathrm{CDCl}_{3}\right) \delta 138.5,137.2,133.2,128.9$, $125.7(\mathrm{q}, J=280.5 \mathrm{~Hz}), 100.2,85.6,38.7(\mathrm{q}, J=30.0 \mathrm{~Hz}), 29.7,19.3,18.5,18.3,-1.1 .{ }^{19} \mathrm{~F}$ NMR $(376$ $\mathrm{MHz}, \mathrm{CDCl} 3) \delta-70.2(\mathrm{~d}, J=7.7 \mathrm{~Hz}) . \mathrm{MS}(\mathrm{EI}): \mathrm{m} / \mathrm{z}(\%) 452\left(\mathrm{M}^{+}\right), 409\left(\left[\mathrm{M}-{ }^{i} \mathrm{Pr}\right]^{+}\right), 190,175(100)$. HRMS: Calculated for $\mathrm{C}_{25} \mathrm{H}_{39} \mathrm{~F}_{3} \mathrm{Si}_{2}$ : 452.2542; Found: 452.2546. The enantiomeric ratio of $\mathbf{1 0 j}$ was determined by 10j', which was derived from 10j through a [3+2] cyclization with benzyl azide.

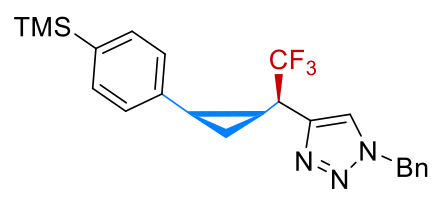

1-Benzyl-4-((R)-2,2,2-trifluoro-1-((1S,2R)-2-(4-(trimethylsilyl)phenyl) cyclopropyl)ethyl)-1H-1,2,3-triazole (10j'). The product (23 mg, 33\% yield) was purified with silica gel chromatography (Petroleum ether/EtOAc $=10: 1)$ as a white solid (m.p. $\left.83-84{ }^{\circ} \mathrm{C}\right) .[\alpha]_{\mathrm{D}}{ }^{25}=128.42\left(c=0.43, \mathrm{CHCl}_{3}\right)$ for a sample 
with 99.9:0.1 er. ${ }^{1} \mathrm{H}$ NMR (500 MHz, $\left.\mathrm{CDCl}_{3}\right) \delta 7.44-7.38(\mathrm{~m}, 3 \mathrm{H}), 7.23-7.20(\mathrm{~m}, 2 \mathrm{H}), 7.16(\mathrm{~d}, J$ $=7.9 \mathrm{~Hz}, 2 \mathrm{H}), 6.84(\mathrm{~d}, J=7.8 \mathrm{~Hz}, 2 \mathrm{H}), 6.78(\mathrm{~s}, 1 \mathrm{H}), 5.52(\mathrm{~d}, J=14.9 \mathrm{~Hz}, 1 \mathrm{H}), 5.39(\mathrm{~d}, J=14.9$ $\mathrm{Hz}, 1 \mathrm{H}), 2.64-2.52$ (m, $1 \mathrm{H}), 2.29-2.22$ (m, $1 \mathrm{H}), 1.99-1.87$ (m, $1 \mathrm{H}), 1.31-1.17$ (m, $2 \mathrm{H}), 0.20$ (s, $9 \mathrm{H}) .{ }^{13} \mathrm{C}$ NMR $\left(101 \mathrm{MHz}, \mathrm{CDCl}_{3}\right) \delta 141.8,138.2,137.3,134.7,132.7,129.2,128.7,127.8,126.4$ $(\mathrm{q}, J=287.7 \mathrm{~Hz}), 122.6,53.9,41.7(\mathrm{~d}, \mathrm{~J}=28.1 \mathrm{~Hz}), 19.2,16.9,8.9,-1.1 .{ }^{19} \mathrm{~F}$ NMR $(471 \mathrm{MHz}$, $\left.\mathrm{CDCl}_{3}\right) \delta-68.9(\mathrm{~d}, J=8.7 \mathrm{~Hz}, 3 \mathrm{~F}) . \mathrm{MS}(\mathrm{ESI}): \mathrm{m} / \mathrm{z}(\%) 430.2\left([\mathrm{M}+\mathrm{H}]^{+}\right)$. HRMS: Calculated for $\mathrm{C}_{23} \mathrm{H}_{27} \mathrm{~F}_{3} \mathrm{~N}_{3} \mathrm{Si}$ : 430.1921; Found: 430.1918. Enantiomeric purity (99.9:0.1 er) was measured by chiral HPLC on IF-3 column (Hexane $/{ }^{i} \mathrm{PrOH}=95: 5,0.7 \mathrm{~mL} / \mathrm{min}, \mathrm{UV}$ detection at $\left.214 \mathrm{~nm}\right)$; retention time $=14.32 \mathrm{~min}$ (major), retention time $=16.58 \mathrm{~min}$ (minor).

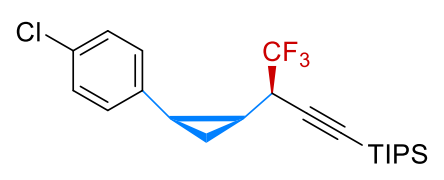

((R)-3-((1S,2R)-2-(4-Chlorophenyl)cyclopropyl)-4,4,4-trifluorobut-1-y n-1-yl)triisopropylsilane (10k). 13 mol\% L1 was used. The product (43 $\mathrm{mg}, 35 \%$ yield, $\mathrm{dr}=11: 1)$ was purified with silica gel chromatography (Petroleum) as a colorless oil. $[\alpha]_{\mathrm{D}}{ }^{25}=-118.71\left(c=1.10, \mathrm{CHCl}_{3}\right)$ for a sample with 98.6:1.4 er. ${ }^{1} \mathrm{H}$ NMR (400 MHz, $\left.\mathrm{CDCl}_{3}\right) \delta 7.26-7.23(\mathrm{~m}, 4 \mathrm{H}), 2.36-2.57(\mathrm{~m}, 1 \mathrm{H}), 2.08(\mathrm{dq}, J=10.8,7.6 \mathrm{~Hz}, 1$ $\mathrm{H}), 1.56-1.48(\mathrm{~m}, 1 \mathrm{H}), 1.22-1.14(\mathrm{~m}, 1 \mathrm{H}), 1.10-1.02(\mathrm{~m}, 21 \mathrm{H}), 1.02-0.94(\mathrm{~m}, 1 \mathrm{H}) .{ }^{13} \mathrm{C}$ NMR $\left(101 \mathrm{MHz}, \mathrm{CDCl}_{3}\right) \delta 135.2,132.6,130.9,128.3,125.5(\mathrm{~d}, J=280.1 \mathrm{~Hz}), 100.0,86.0,38.7(\mathrm{q}, J=$ $30.0 \mathrm{~Hz}), 18.7,18.5,18.3,11.1,9.2 .{ }^{19} \mathrm{~F}$ NMR $\left(376 \mathrm{MHz} \mathrm{CDCl}_{3}\right) \delta-70.2(\mathrm{~d}, J=7.6 \mathrm{~Hz}, 3 \mathrm{~F}) . \mathrm{MS}$ (EI): m/z (\%) $414\left(\mathrm{M}^{+}\right), 371\left(\left[\mathrm{M}-{ }^{i} \mathrm{Pr}\right]^{+}\right), 293,183$ (100). HRMS: Calculated for $\mathrm{C}_{22} \mathrm{H}_{30} \mathrm{ClF}_{3} \mathrm{Si}$ : 414.1757; Found: 414.1765. The enantiomeric ratio of $\mathbf{1 0 k}$ was determined by $\mathbf{1 0 k}$ ', which was derived from 10k through a [3+2] cyclization with benzyl azide.

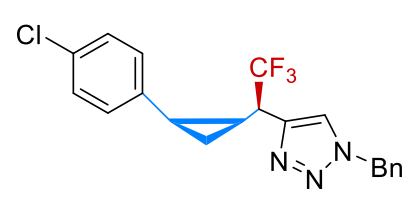

1-Benzyl-4-((R)-1-((1S,2R)-2-(4-chlorophenyl)cyclopropyl)-2,2,2-trifluo roethyl)-1H-1,2,3-triazole (12). The product (38 $\mathrm{mg}, 41 \%$ yield) was purified with silica gel chromatography (Petroleum/EtOAc= 10:1) as a white solid (m.p. $\left.115-116{ }^{\circ} \mathrm{C}\right) .[\alpha]_{\mathrm{D}}{ }^{25}=-235.30\left(c=0.40, \mathrm{CHCl}_{3}\right)$ for a sample with $98.6: 1.4$ er. ${ }^{1} \mathrm{H}$ NMR (400 MHz, $\left.\mathrm{CDCl}_{3}\right) \delta 7.46$ - $7.35(\mathrm{~m}, 3 \mathrm{H}), 7.25-7.16(\mathrm{~m}, 2 \mathrm{H}), 6.92(\mathrm{~d}, J=8.4 \mathrm{~Hz}, 2 \mathrm{H}), 6.87$ $(\mathrm{s}, 1 \mathrm{H}), 6.76(\mathrm{~d}, J=8.3 \mathrm{~Hz}, 2 \mathrm{H}), 5.53(\mathrm{~d}, J=14.8 \mathrm{~Hz}, 1 \mathrm{H}), 5.36(\mathrm{~d}, J=14.8 \mathrm{~Hz}, 1 \mathrm{H}), 2.61-2.41$ $(\mathrm{m}, 1 \mathrm{H}), 2.30-2.20(\mathrm{~m}, 1 \mathrm{H}), 2.02-1.90(\mathrm{~m}, 1 \mathrm{H}), 1.33-1.24(\mathrm{~m}, 1 \mathrm{H}), 1.19-1.11(\mathrm{~m}, 1 \mathrm{H}) .{ }^{13} \mathrm{C}$ NMR $\left(126 \mathrm{MHz}, \mathrm{CDCl}_{3}\right) \delta 141.4,135.3,134.5,132.1,130.2,129.2,128.9,128.0,127.8,126.3$ (q, $J$ 
$=280.4 \mathrm{~Hz}), 122.5,54.0,41.9(\mathrm{t}, J=28.1 \mathrm{~Hz}), 18.7,16.7,9.3 .{ }^{19} \mathrm{~F} \mathrm{NMR}\left(376 \mathrm{MHz}, \mathrm{CDCl}_{3}\right) \delta-69.3$ (d, $J=8.6 \mathrm{~Hz}, 3 \mathrm{~F})$. MS (ESI): m/z (\%) $392.1\left([\mathrm{M}+\mathrm{H}]^{+}\right)$. HRMS: Calculated for $\mathrm{C}_{20} \mathrm{H}_{18} \mathrm{ClF}_{3} \mathrm{~N}_{3}$ : 392.1136; Found: 392.1136. Enantiomeric purity (98.6:1.4 er) was measured by chiral HPLC on IF-3 column $\left(\mathrm{Hexane} /{ }^{i} \mathrm{PrOH}=95: 5,0.7 \mathrm{~mL} / \mathrm{min}, \mathrm{UV}\right.$ detection at $\left.214 \mathrm{~nm}\right)$; retention time $=13.08 \mathrm{~min}$ (major), retention time $=16.31 \mathrm{~min}$ (minor).

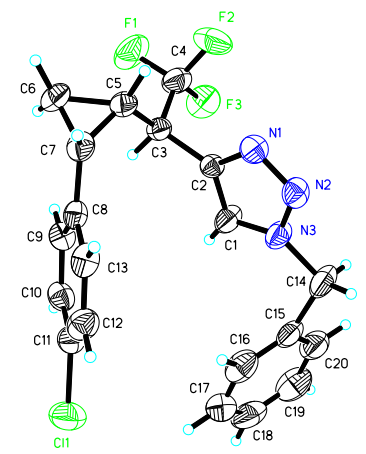

X-ray Crystal Structure of Compound 12

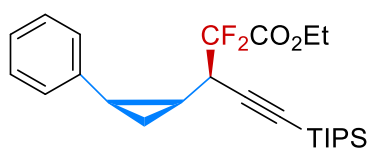

Ethyl(R)-2,2-difluoro-3-((1S,2R)-2-phenylcyclopropyl)-5-(triisopropylsil

yl)pent-4-ynoate (101). The product (104 mg, 80\% yield, dr > 20:1) was purified with silica gel chromatography (Petroleum ether/DCM=5:1) as a colorless oil. $[\alpha]_{\mathrm{D}}^{25}=$ $-164.62\left(c=0.26, \mathrm{CHCl}_{3}\right)$ for a sample with 97:3 er. ${ }^{1} \mathrm{H}$ NMR $\left(400 \mathrm{MHz}, \mathrm{CDCl}_{3}\right) \delta 7.33-7.17(\mathrm{~m}, 5$ H), $4.29(\mathrm{q}, J=7.1 \mathrm{~Hz}, 2 \mathrm{H}), 2.40-2.15(\mathrm{~m}, 2 \mathrm{H}), 1.56-1.46(\mathrm{~m}, 1 \mathrm{H}), 1.32(\mathrm{t}, J=7.1 \mathrm{~Hz}, 3 \mathrm{H})$, $1.13-0.91(\mathrm{~m}, 23 \mathrm{H}) .{ }^{13} \mathrm{C} \mathrm{NMR}\left(101 \mathrm{MHz}, \mathrm{CDCl}_{3}\right) \delta 163.5(\mathrm{t}, J=32.1 \mathrm{~Hz}), 136.7,129.5,128.1$, $126.5,116.5(\mathrm{t}, J=256.6 \mathrm{~Hz}), 101.1,86.0,62.9,38.8(\mathrm{t}, J=24.5 \mathrm{~Hz}), 19.2,18.5,17.8,14.0-13.8$ (m), 11.1, 8.9. ${ }^{19} \mathrm{~F}$ NMR (376 MHz, $\left.\mathrm{CDCl}_{3}\right) \delta-108.94--109.21(\mathrm{~m}) . \mathrm{MS}(\mathrm{EI}): \mathrm{m} / \mathrm{z}(\%) 434\left(\mathrm{M}^{+}\right)$, 391, 129 (100), 115. HRMS: Calculated for $\mathrm{C}_{25} \mathrm{H}_{36} \mathrm{~F}_{2} \mathrm{O}_{2} \mathrm{Si}$ : 434.2453; Found: 434.2461. Enantiomeric purity (97:3 er) was measured by chiral HPLC on PC-3 column $\left(\mathrm{CH}_{3} \mathrm{CN} / \mathrm{H}_{2} \mathrm{O}=70: 30\right.$, $0.7 \mathrm{~mL} / \mathrm{min}$, UV detection at $214 \mathrm{~nm}$ ); retention time $=9.42 \mathrm{~min}$ (major), retention time $=8.60 \mathrm{~min}$ (minor).

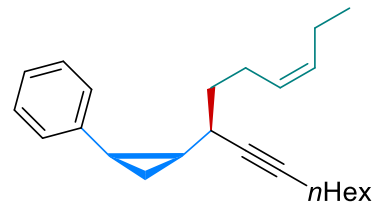

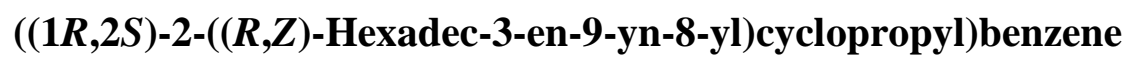

(10m).

The product $(73 \mathrm{mg}, 72 \%$ yield, $\mathrm{dr}=4: 1)$ was purified with silica gel chromatography (Petroleum ether) as a colorless oil. The diastereomers 
could be separated and the data of major diastereomer is presented. $[\alpha]_{\mathrm{D}}^{25}=-74.55\left(c=0.33, \mathrm{CHCl}_{3}\right)$ for a sample with 98.7:1.3 er for the major diastereomer. ${ }^{1} \mathrm{H}$ NMR $\left(400 \mathrm{MHz}, \mathrm{CDCl}_{3}\right) \delta 7.33(\mathrm{~d}, J=$ $7.2 \mathrm{~Hz}, 2 \mathrm{H}), 7.27$ (t, $J=7.4 \mathrm{~Hz}, 2 \mathrm{H}), 7.19$ (t, $J=7.1 \mathrm{~Hz}, 1 \mathrm{H}), 5.43-4.93$ (m, $2 \mathrm{H}), 2.30-2.16(\mathrm{~m}$, $2 \mathrm{H}), 2.13(\mathrm{dt}, J=7.1,3.5 \mathrm{~Hz}, 2 \mathrm{H}), 2.06-1.97(\mathrm{~m}, 3 \mathrm{H}), 1.63-1.51(\mathrm{~m}, 2 \mathrm{H}), 1.50-1.42(\mathrm{~m}, 2 \mathrm{H})$, $1.41-1.15(\mathrm{~m}, 8 \mathrm{H}), 1.03-0.96(\mathrm{~m} 1 \mathrm{H}), 0.95-0.86(\mathrm{~m}, 6 \mathrm{H}), 0.69-0.62(\mathrm{~m}, 1 \mathrm{H}) .{ }^{13} \mathrm{C}$ NMR $(101$ $\left.\mathrm{MHz}, \mathrm{CDCl}_{3}\right) \delta 138.6,132.0,129.7,128.7,127.6,125.8,82.6,81.2,35.7,31.4,31.2,29.0,28.5,24.9$, 24.5, 22.6, 21.2, 20.5, 18.8, 14.3, 14.1, 9.0. MS (EI): m/z (\%) $322\left(\mathrm{M}^{+}\right)$. HRMS: Calculated for $\mathrm{C}_{24} \mathrm{H}_{34}$ : 322.2661; Found: 322.2670. Enantiomeric purity (98.7:1.3 er) was measured by chiral HPLC on PA2 column $\left(\mathrm{CH}_{3} \mathrm{CN} / \mathrm{H}_{2} \mathrm{O}=65: 35,0.7 \mathrm{~mL} / \mathrm{min}\right.$, $\mathrm{UV}$ detection at $\left.214 \mathrm{~nm}\right)$; retention time $=26.40$ $\min$ (major), retention time $=24.98 \min$ (minor) .

\section{EDRC of racemic $( \pm)$ cis-1 with diverse electrophiles.}

\section{EDRC of ( \pm ) cis-1a with gem-difluoropropargyl bromide 2a and allylbromide}

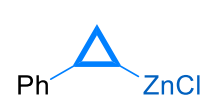

$( \pm)$ cis-1a

1.0 equiv)

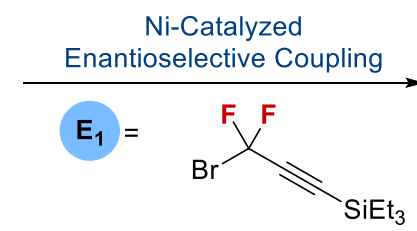

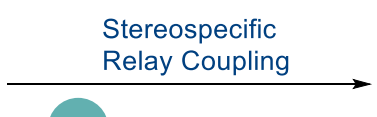

$E_{2}=$ allyl bromide
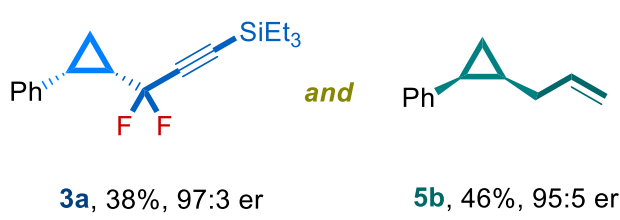

To a $25 \mathrm{~mL}$ of Schlenk tube were added $\mathrm{NiCl}_{2}$. DME (6.6 mg, $\left.5 \mathrm{~mol} \%\right)$ and $\mathbf{L 1}(10.8 \mathrm{mg}, 6 \mathrm{~mol} \%)$ in a glovebox. The tube was then taken out from the glovebox. gem-Difluoropropargyl bromide 2a (81 $\mathrm{mg}, 0.3 \mathrm{mmol}, 0.5$ equiv) and THF ( $2 \mathrm{~mL})$ were added. After stirring at room temperature for $25 \mathrm{~min}$, the reaction mixture was cooled to $-30{ }^{\circ} \mathrm{C}$ and cyclopropylzinc reagent $( \pm)$ cis-1a $(0.6 \mathrm{mmol}, 1.0$ equiv in $1.82 \mathrm{~mL}$ of THF) was added dropwise over a period of $10 \mathrm{~min}$. The reaction mixture was stirred at $-30{ }^{\circ} \mathrm{C}$ for $20 \mathrm{~h}$. Then, the reaction mixture was cooled to $-40{ }^{\circ} \mathrm{C}, \mathrm{CuCN} \cdot 2 \mathrm{LiCl}(0.5 \mathrm{M}$ in THF, $0.3 \mathrm{~mL}, 0.15 \mathrm{mmol}, 0.25$ equiv) was added dropwise. The resulting mixture was stirred for 5 min, allyl bromide (102 mg, $0.84 \mathrm{mmol}, 1.4$ equiv) was added. The reaction mixture was stirred at $-40{ }^{\circ} \mathrm{C}$ for another $1 \mathrm{~h}$ and was slowly warmed to room temperature. After stirring for $8 \mathrm{~h}$, the reaction was quenched with saturated aqueous $\mathrm{NH}_{4} \mathrm{Cl}$ solution and diluted with $\mathrm{CH}_{2} \mathrm{Cl}_{2}(50 \mathrm{~mL})$. The organic layer was separated and the aqueous layer extracted with $\mathrm{CH}_{2} \mathrm{Cl}_{2}$ (three times). The combined organic layers were washed with brine and dried over $\mathrm{Na}_{2} \mathrm{SO}_{4}$, filtered and concentrated. The residue was purified with silica gel chromatography to give $\mathbf{3 a}$ (70 $\mathrm{mg}, 38 \%$ yield, 97:3 er; 
eluent: petroleum ether) as a colorless oil and $\mathbf{5 b}$ (44 $\mathrm{mg}$, 46\% yield, 95:5 er; eluent: petroleum ether) as a colorless oil.

Data for 5b: $[\alpha]_{\mathrm{D}}{ }^{20}=-48.86\left(c=0.35, \mathrm{CHCl}_{3}\right)$ for a sample with 95:5 er. ${ }^{1} \mathrm{H} \mathrm{NMR}\left(400 \mathrm{MHz}, \mathrm{CDCl}_{3}\right)$ $\delta 7.28-7.23$ (m, 2 H), $7.21-7.14(\mathrm{~m}, 3 \mathrm{H}), 5.76(\mathrm{ddt}, J=16.6,10.2,6.3 \mathrm{~Hz}, 1 \mathrm{H}), 4.99-4.91(\mathrm{~m}, 1$ H), $4.91-4.85(\mathrm{~m}, 1 \mathrm{H}), 2.18(\mathrm{td}, J=8.6,6.2 \mathrm{~Hz}, 1 \mathrm{H}), 1.91-1.81(\mathrm{~m}, 1 \mathrm{H}), 1.71-1.59(\mathrm{~m}, 1 \mathrm{H})$, $1.21-1.11(\mathrm{~m}, 1 \mathrm{H}), 1.01(\mathrm{td}, J=8.4,5.1 \mathrm{~Hz}, 1 \mathrm{H}), 0.73-0.67(\mathrm{~m}, 1 \mathrm{H}) .{ }^{13} \mathrm{C}$ NMR $(101 \mathrm{MHz}$, $\left.\mathrm{CDCl}_{3}\right) \delta 139.2,137.9,129.1,127.8,125.7,114.2,32.7,20.9,17.8,9.3$. Enantiomeric ratio (95:5 er) was measured by chiral HPLC on PC-3 column $\left(\mathrm{CH}_{3} \mathrm{CN} / \mathrm{H}_{2} \mathrm{O}=60: 40,0.7 \mathrm{~mL} / \mathrm{min}\right.$, UV detection at $214 \mathrm{~nm}$ ); retention time $=16.85 \min$ (major), retention time $=14.92 \mathrm{~min}$ (minor).

EDRC of $( \pm)$ cis-1a with propargylbromide $2 \mathrm{i}$ and allylbromide

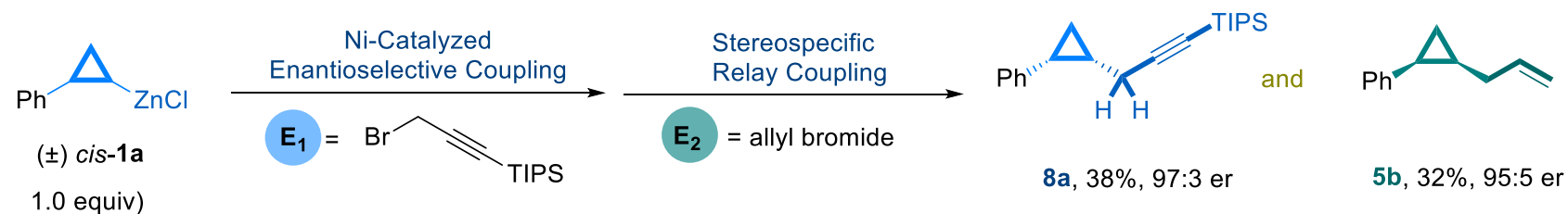

The synthesis of compounds $\mathbf{8 a}$ and $\mathbf{5 b}$ is according to the procedure of EDRC of $( \pm)$ cis $\mathbf{- 1 a}$ with gem-difluoropropargyl bromide 2a and allylbromide. The products $\mathbf{8 a}$ (colorless oil; $71 \mathrm{mg}, 38 \%$ yield, 97:3 er; eluent: petroleum ether) and $\mathbf{5 b}$ (colorless oil; $31 \mathrm{mg}$, 32\% yield, 95:5 er; eluent: petroleum ether) were purified with silica gel chromatography.

\section{EDRC of $( \pm)$ cis-1c with gem-difluoropropargyl bromide 2a and ethyl 2-(bromomethyl)acrylate}

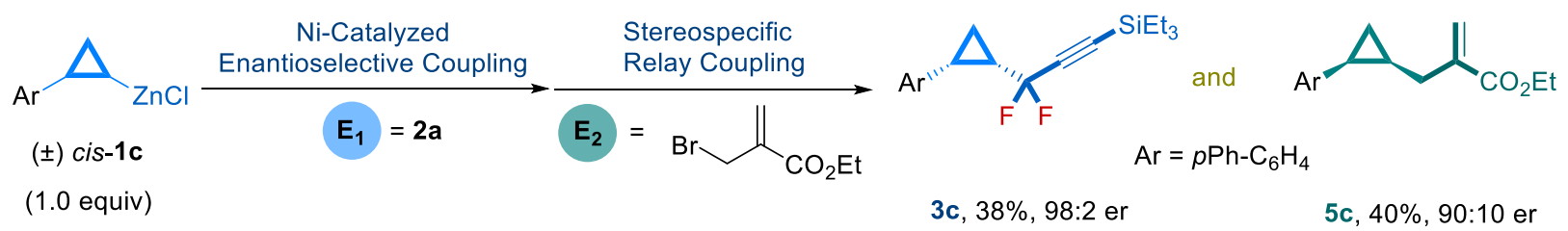

To a $25 \mathrm{~mL}$ of Schlenk tube were added $\mathrm{NiCl}_{2}$. DME (6.6 mg, $\left.5 \mathrm{~mol} \%\right)$ and $\mathbf{L 1}(10.8 \mathrm{mg}, 6 \mathrm{~mol} \%)$ in a glovebox. The tube was then taken out from the glovebox. gem-Difluoropropargyl bromide 2a (81 $\mathrm{mg}, 0.3 \mathrm{mmol}, 0.5$ equiv) and THF ( $2 \mathrm{~mL})$ were added. After stirring at room temperature for $25 \mathrm{~min}$, the reaction mixture was cooled to $-30{ }^{\circ} \mathrm{C}$ and cyclopropylzinc reagent $( \pm)$ cis-1c $(0.6 \mathrm{mmol}, 1.0$ equiv in $1.82 \mathrm{~mL}$ of THF) was added dropwise over a period of $10 \mathrm{~min}$. The reaction was stirred at $-30{ }^{\circ} \mathrm{C}$ for $20 \mathrm{~h}$. Then, the reaction mixture was cooled to $-40{ }^{\circ} \mathrm{C}$ and $\mathrm{CuCN} \cdot 2 \mathrm{LiCl}(0.5 \mathrm{M}$ in $\mathrm{THF}$, 
$0.3 \mathrm{~mL}, 0.15 \mathrm{mmol}, 0.25$ equiv) was added dropwise. The resulting mixture was stirred for $5 \mathrm{~min}$, ethyl 2-(bromomethyl)acrylate (162 mg, $0.84 \mathrm{mmol}, 1.4$ equiv) was added. The reaction mixture was stirred at $-40{ }^{\circ} \mathrm{C}$ for another $1 \mathrm{~h}$ and was slowly warmed to room temperature. After stirring for $8 \mathrm{~h}$, the reaction was quenched with saturated aqueous $\mathrm{NH}_{4} \mathrm{Cl}$ solution and diluted with $\mathrm{CH}_{2} \mathrm{Cl}_{2}(50 \mathrm{~mL})$. The organic layer was separated and the aqueous layer extracted with $\mathrm{CH}_{2} \mathrm{Cl}_{2}$ (three times). The combined organic layers were washed with brine and dried over $\mathrm{Na}_{2} \mathrm{SO}_{4}$, filtered and concentrated. The residue was purified with silica gel chromatography to give 3c (79 mg, 38\% yield, 98:2 er; eluent: petroleum ether) as a colorless oil and 5c (74 mg, 40\% yield, 90:10 er; eluent: petroleum ether/ $\left.\mathrm{CH}_{2} \mathrm{Cl}_{2}=1: 1\right)$ as a colorless oil.

Data for 5c: $[\alpha]_{\mathrm{D}}{ }^{20}=189.35\left(c=0.67, \mathrm{CHCl}_{3}\right)$ for a sample with 90:10 er. ${ }^{1} \mathrm{H}$ NMR $(400 \mathrm{MHz}$, $\left.\mathrm{CDCl}_{3}\right) \delta 7.60(\mathrm{~d}, J=7.3 \mathrm{~Hz}, 2 \mathrm{H}), 7.52(\mathrm{~d}, J=8.1 \mathrm{~Hz}, 2 \mathrm{H}), 7.44(\mathrm{t}, J=7.6 \mathrm{~Hz}, 2 \mathrm{H}), 7.33(\mathrm{t}, J=7.3$ $\mathrm{Hz}, 1 \mathrm{H}), 7.28(\mathrm{~d}, J=8.3 \mathrm{~Hz}, 2 \mathrm{H}), 6.19-5.91(\mathrm{~m}, 1 \mathrm{H}), 5.61-5.39(\mathrm{~m}, 1 \mathrm{H}), 4.18(\mathrm{q}, J=7.1 \mathrm{~Hz}, 2$ H), $2.32-2.15(\mathrm{~m}, 2 \mathrm{H}), 1.98(\mathrm{dd}, J=16.3,7.8 \mathrm{~Hz}, 1 \mathrm{H}), 1.38-1.32(\mathrm{~m}, 1 \mathrm{H}), 1.28(\mathrm{t}, J=7.1 \mathrm{~Hz}, 3$ H), $1.09(\mathrm{td}, J=8.4,5.2 \mathrm{~Hz}, 1 \mathrm{H}), 0.85-0.79(\mathrm{~m}, 1 \mathrm{H}) .{ }^{13} \mathrm{C} \mathrm{NMR}\left(101 \mathrm{MHz}, \mathrm{CDCl}_{3}\right) \delta 167.2,140.9$, $140.3,138.6,138.0,129.4,128.7,127.0,126.9,126.5,124.5,60.5,30.5,20.8,17.6,14.2$, 9.6. MS (EI): m/z (\%) $306\left(\mathrm{M}^{+}\right), 277,232,193$ (100), 115. HRMS: Calculated for $\mathrm{C}_{21} \mathrm{H}_{22} \mathrm{O}_{2}$ : 306.1620; Found: 306.1625. Enantiomeric ratio (90:10 er) was measured by chiral HPLC on PC-3 column $\left(\mathrm{CH}_{3} \mathrm{CN} / \mathrm{H}_{2} \mathrm{O}=70: 30,0.7 \mathrm{~mL} / \mathrm{min}, \mathrm{UV}\right.$ detection at $\left.214 \mathrm{~nm}\right)$; retention time $=20.15$ min (major), retention time $=23.55 \mathrm{~min}$ (minor).

\section{EDRC of ( \pm ) cis-1c with gem-difluoropropargyl bromide 2 a and benzoyl chloride}

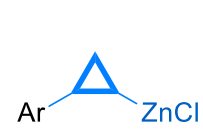

(士) cis-1c

1.0 equiv)

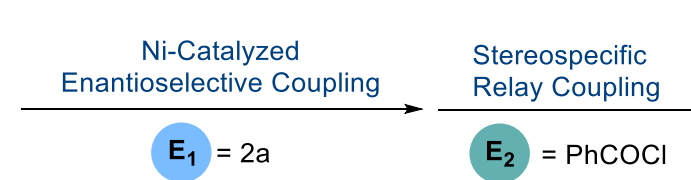

$E_{2}=\mathrm{PhCOCl}$

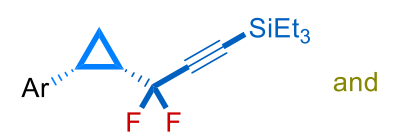

$\mathrm{Ar}=p \mathrm{Ph}-\mathrm{C}_{6} \mathrm{H}_{4}$

$3 c, 39 \%, 97: 3$ er

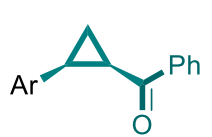

5d, 34\%, 93:7 er

To a $25 \mathrm{~mL}$ of Schlenk tube were added $\mathrm{NiCl}_{2}$. DME (6.6 mg, $\left.5 \mathrm{~mol} \%\right)$ and $\mathbf{L 1}(10.8 \mathrm{mg}, 10 \mathrm{~mol} \%)$ in a glovebox. The tube was then taken out from the glovebox. gem-difluoropropargyl bromide 2a ( $81 \mathrm{mg}, 0.3 \mathrm{mmol}, 0.5$ equiv) and THF ( $2 \mathrm{~mL}$ ) were added. After stirring at room temperature for 25 min, the reaction mixture was cooled to $-30{ }^{\circ} \mathrm{C}$ and cyclopropylzinc reagent $( \pm)$ cis-1c $(0.6 \mathrm{mmol}, 1.0$ equiv in $1.82 \mathrm{~mL}$ of THF) was added dropwise over a period of $10 \mathrm{~min}$. The reaction was stirred at 
$-30{ }^{\circ} \mathrm{C}$ for $20 \mathrm{~h}$. Then, the reaction mixture was cooled to $-78{ }^{\circ} \mathrm{C}$ and $\mathrm{CuCN} \cdot 2 \mathrm{LiCl}(0.5 \mathrm{M}$ in $\mathrm{THF}$, $1.8 \mathrm{~mL}, 0.9 \mathrm{mmol}, 1.5$ equiv) was added dropwise. The resulting mixture was stirred for $30 \mathrm{~min}$, a solution of benzoyl chloride (167 mg, $1.2 \mathrm{mmol}, 2.0$ equiv) in anhydrous THF (1 mL) was added. The reaction mixture was stirred at $-78{ }^{\circ} \mathrm{C}$ for another $1 \mathrm{~h}$ and was slowly warmed to room temperature. After stirring for $8 \mathrm{~h}$, the reaction was quenched with saturated aqueous $\mathrm{NH}_{4} \mathrm{Cl}$ solution and diluted with $\mathrm{CH}_{2} \mathrm{Cl}_{2}(50 \mathrm{~mL})$. The organic layer was separated and the aqueous layer extracted with $\mathrm{CH}_{2} \mathrm{Cl}_{2}$ (three times). The combined organic layers were washed with brine and dried over $\mathrm{Na}_{2} \mathrm{SO}_{4}$, filtered and concentrated. The residue was purified with silica gel chromatography to give 3c (81 mg, 39\% yield, 97:3 er; eluent: petroleum ether) as a colorless oil and 5d (61 mg, 34\% yield, 93:7 er; eluent, petroleum ether/ $\mathrm{CH}_{2} \mathrm{Cl}_{2}=1: 1$ ) as a white solid (m.p. $117-118^{\circ} \mathrm{C}$ ).

Data for 5d: $[\alpha]_{\mathrm{D}}{ }^{20}=-51.11\left(c=0.68, \mathrm{CHCl}_{3}\right)$ for a sample with 93:7 er. ${ }^{1} \mathrm{H} \mathrm{NMR}\left(400 \mathrm{MHz}, \mathrm{CDCl}_{3}\right)$ $\delta 7.97(\mathrm{~d}, J=7.5 \mathrm{~Hz}, 2 \mathrm{H}), 7.60-7.50(\mathrm{~m}, 3 \mathrm{H}), 7.50-7.37$ (m, $6 \mathrm{H}), 7.37-7.28(\mathrm{~m}, 3 \mathrm{H}), 3.20-$ 3.10 (m, $1 \mathrm{H}), 2.99-2.90$ (m, $1 \mathrm{H}), 2.24-2.16$ (m, $1 \mathrm{H}), 1.56-1.49$ (m, $1 \mathrm{H}) .{ }^{13} \mathrm{C} \mathrm{NMR}(101 \mathrm{MHz}$, $\left.\mathrm{CDCl}_{3}\right) \delta 196.0,140.7,139.2,138.5,135.0,132.5,129.4,128.5,128.4,127.9,126.9,126.8,126.5$, 29.2, 27.1, 11.8. MS (EI): m/z (\%) $298\left(\mathrm{M}^{+}\right)$, 105 (100), 77. HRMS: Calculated for $\mathrm{C}_{22} \mathrm{H}_{18} \mathrm{O}$ : 298.1358; Found: 298.1359. Enantiomeric ratio (93:7 er) was measured by chiral HPLC on PC-3 column $\left(\mathrm{CH}_{3} \mathrm{CN} / \mathrm{H}_{2} \mathrm{O}=70: 30,0.7 \mathrm{~mL} / \mathrm{min}\right.$, UV detection at $\left.214 \mathrm{~nm}\right)$; retention time = $12.03 \mathrm{~min}$ (major), retention time $=16.08 \mathrm{~min}$ (minor).

\section{EDRC of $( \pm$ ) cis-1c with gem-difluoropropargyl bromide 2a and (bromoethynyl)benzene}

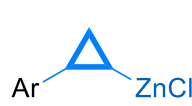

( \pm ) cis-1c

(1.0 equiv)

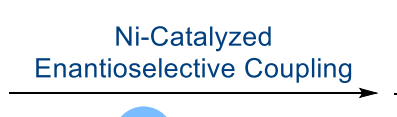

$E_{1}=2 a$

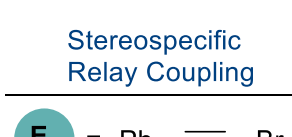

$E_{2}=\mathrm{Ph}=\mathrm{Br}$

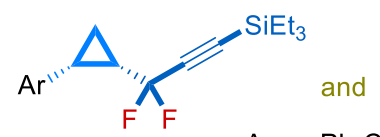

$\mathrm{Ar}=p \mathrm{Ph}-\mathrm{C}_{6} \mathrm{H}_{4}$

$3 c, 36 \%, 97: 3$ er

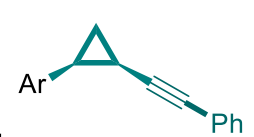

5e, $28 \%, 92: 8$ er

To a $25 \mathrm{~mL}$ of Schlenk tube were added $\mathrm{NiCl}_{2}$. DME (6.6 mg, $\left.5 \mathrm{~mol} \%\right)$ and $\mathbf{L 1}(10.8 \mathrm{mg}, 6 \mathrm{~mol} \%)$ in a glovebox. The tube was then taken out from the glovebox. gem-Difluoropropargyl bromide $2 \mathbf{a}$ (81 $\mathrm{mg}, 0.3 \mathrm{mmol}, 0.5$ equiv) and THF ( $2 \mathrm{~mL})$ were added. After stirring at room temperature for $25 \mathrm{~min}$, the reaction mixture was cooled to $-30{ }^{\circ} \mathrm{C}$ and cyclopropylzinc reagent $( \pm)$ cis-1c $(0.6 \mathrm{mmol}, 1.0$ equiv in $1.82 \mathrm{~mL}$ of THF) was added dropwise over a period of $10 \mathrm{~min}$. The reaction was stirred at $-30{ }^{\circ} \mathrm{C}$ for $20 \mathrm{~h}$. Then, the reaction mixture was cooled to $-78^{\circ} \mathrm{C}$ and $\mathrm{CuCN} \cdot 2 \mathrm{LiCl}(0.5 \mathrm{M}$ in $\mathrm{THF}$, 
$1.8 \mathrm{~mL}, 0.9 \mathrm{mmol}, 1.5$ equiv) was added dropwise. The resulting mixture was stirred for $30 \mathrm{~min}$, (bromoethynyl)benzene ( $217 \mathrm{mg}, 1.2 \mathrm{mmol}, 2.0$ equiv) in anhydrous THF ( $1 \mathrm{~mL})$ was added. The reaction mixture was stirred at $-78^{\circ} \mathrm{C}$ for another $1 \mathrm{~h}$ and was slowly warmed to room temperature. After stirring for $8 \mathrm{~h}$, the reaction was quenched with saturated aqueous $\mathrm{NH}_{4} \mathrm{Cl}$ solution and diluted with $\mathrm{CH}_{2} \mathrm{Cl}_{2}(50 \mathrm{~mL})$. The organic layer was separated and the aqueous layer extracted with $\mathrm{CH}_{2} \mathrm{Cl}_{2}$ (three times). The combined organic layers were washed with brine and dried over $\mathrm{Na}_{2} \mathrm{SO}_{4}$, filtered and concentrated. The residue was purified with silica gel chromatography to give $\mathbf{3 c}$ (75 $\mathrm{mg}, 36 \%$ yield, 97:3 er; eluent: petroleum ether) as a colorless oil and $\mathbf{5 e}$ (48 $\mathrm{mg}, 28 \%$ yield, 92:8 er; eluent: petroleum ether/ $\mathrm{CH}_{2} \mathrm{Cl}_{2}=50: 1$ ) as a pale yellow solid (m.p. $55-57^{\circ} \mathrm{C}$ ).

Data for 5e: $[\alpha]_{\mathrm{D}}{ }^{20}=-68.90\left(c=0.58, \mathrm{CHCl}_{3}\right)$ for a sample with 92:8 er. ${ }^{1} \mathrm{H} \mathrm{NMR}\left(400 \mathrm{MHz}, \mathrm{CDCl}_{3}\right)$ $\delta 7.63(\mathrm{~d}, J=7.5 \mathrm{~Hz}, 2 \mathrm{H}), 7.59(\mathrm{~d}, J=8.1 \mathrm{~Hz}, 2 \mathrm{H}), 7.49-7.40(\mathrm{~m}, 4 \mathrm{H}), 7.36(\mathrm{t}, J=7.3 \mathrm{~Hz}, 1 \mathrm{H})$, $7.23-7.16(\mathrm{~m}, 5 \mathrm{H}), 2.51-2.42(\mathrm{~m}, 1 \mathrm{H}), 2.09-2.01(\mathrm{~m}, 1 \mathrm{H}), 1.51(\mathrm{td}, J=8.4,5.0 \mathrm{~Hz}, 1 \mathrm{H}), 1.38-$ $1.32(\mathrm{~m}, 1 \mathrm{H}) .{ }^{13} \mathrm{C} \mathrm{NMR}\left(101 \mathrm{MHz}, \mathrm{CDCl}_{3}\right) \delta 141.1,139.1,137.3,131.4,128.7,128.7,128.0,127.4$, 127.0, 127.0, 126.5, 123.7, 89.7, 80.4, 23.7, 15.3, 10.4. MS (EI): m/z (\%) $294\left(\mathrm{M}^{+}\right), 215,193(100)$, 178, 165, 115. HRMS: Calculated for $\mathrm{C}_{23} \mathrm{H}_{18}$ : 294.1409; Found: 294.1411. Enantiomeric ratio (92:8 er) was measured by chiral HPLC on PC-3 column $\left(\mathrm{CH}_{3} \mathrm{CN} / \mathrm{H}_{2} \mathrm{O}=80: 20,0.7 \mathrm{~mL} / \mathrm{min}\right.$, UV detection at $214 \mathrm{~nm}$ ); retention time $=15.98$ min $($ major $)$, retention time $=12.30 \mathrm{~min}($ minor $)$.

\section{EDRC of $( \pm)$ cis-1a with gem-difluoropropargylbromide 2a and 1-bromopent-2-yne}

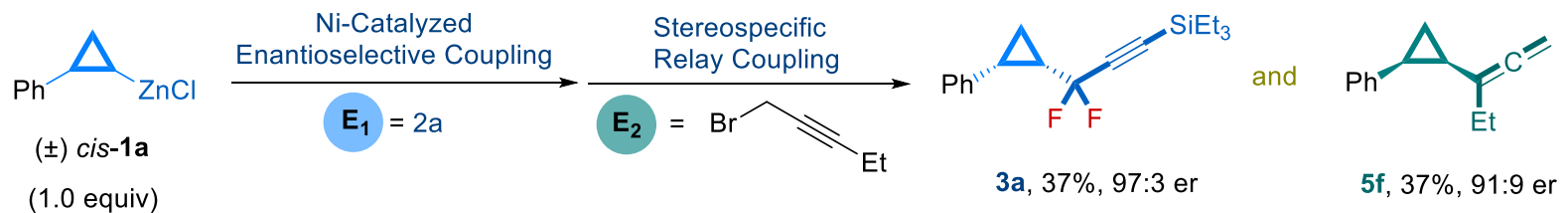

To a $25 \mathrm{~mL}$ of Schlenk tube were added $\mathrm{NiCl}_{2}$. DME (6.6 mg, $\left.5 \mathrm{~mol} \%\right)$ and $\mathbf{L 1}(10.8 \mathrm{mg}, 6 \mathrm{~mol} \%)$ in a glovebox. The tube was then taken out from the glovebox. gem-Difluoropropargyl bromide $\mathbf{2 a}$ (81 mg, $0.3 \mathrm{mmol}, 0.5$ equiv) and THF ( $2 \mathrm{~mL})$ were added. After stirring at room temperature for $25 \mathrm{~min}$, the reaction mixture was cooled to $-30{ }^{\circ} \mathrm{C}$ and cyclopropylzinc reagent $( \pm)$ cis-1a $(0.6 \mathrm{mmol}, 1.0$ equiv in $1.82 \mathrm{~mL}$ of THF) was added dropwise over a period of $10 \mathrm{~min}$. The reaction was stirred at $-30{ }^{\circ} \mathrm{C}$ for $20 \mathrm{~h}$. Then, the reaction mixture was cooled to $-78{ }^{\circ} \mathrm{C}$ and $\mathrm{CuCN} \cdot 2 \mathrm{LiCl}(0.5 \mathrm{M}$ in $\mathrm{THF}$, $1.8 \mathrm{~mL}, 0.9 \mathrm{mmol}, 1.5$ equiv) was added dropwise. The resulting mixture was stirred for $30 \mathrm{~min}$, a 
solution of 1-bromopent-2-yne (177 mg, $1.2 \mathrm{mmol}, 2.0$ equiv) in anhydrous THF (1 mL) was added. The reaction mixture was stirred at $-78{ }^{\circ} \mathrm{C}$ for another $1 \mathrm{~h}$ and was slowly warmed to room temperature. After stirring for $8 \mathrm{~h}$, the reaction was quenched with saturated aqueous $\mathrm{NH}_{4} \mathrm{Cl}$ solution and diluted with $\mathrm{CH}_{2} \mathrm{Cl}_{2}(50 \mathrm{~mL})$. The organic layer was separated and the aqueous layer extracted with $\mathrm{CH}_{2} \mathrm{Cl}_{2}$ (three times). The combined organic layers were washed with brine and dried over $\mathrm{Na}_{2} \mathrm{SO}_{4}$, filtered and concentrated. The residue was purified with silica gel chromatography to give 3a (68 mg, 37\% yield, 97:3 er; eluent: petroleum ether) as a colorless oil and $\mathbf{5 f}$ (41 mg, 37\% yield, 91:9 er; eluent: petroleum ether) as a colorless oil.

Data for 5f: $[\alpha]_{\mathrm{D}}{ }^{20}=-111.06\left(c=1.34, \mathrm{CHCl}_{3}\right)$ for a sample with 91:9 er. ${ }^{1} \mathrm{H}$ NMR $(400 \mathrm{MHz}$, $\left.\mathrm{CDCl}_{3}\right) \delta 7.23(\mathrm{t}, J=7.3 \mathrm{~Hz}, 2 \mathrm{H}), 7.15(\mathrm{t}, J=7.2 \mathrm{~Hz}, 1 \mathrm{H}), 7.08(\mathrm{~d}, J=7.3 \mathrm{~Hz}, 2 \mathrm{H}), 4.75-4.66(\mathrm{~m}$, $1 \mathrm{H}), 4.66-4.57(\mathrm{~m}, 1 \mathrm{H}), 2.39-2.30(\mathrm{~m}, 1 \mathrm{H}), 1.85-1.73(\mathrm{~m}, 1 \mathrm{H}), 1.72-1.56(\mathrm{~m}, 2 \mathrm{H}), 1.23-$ $1.11(\mathrm{~m}, 1 \mathrm{H}), 0.78(\mathrm{t}, J=7.4 \mathrm{~Hz}, 3 \mathrm{H}) .{ }^{13} \mathrm{C} \mathrm{NMR}\left(101 \mathrm{MHz}, \mathrm{CDCl}_{3}\right) \delta 207.1,138.7,128.3,127.4$, 125.5, 103.2, 76.7, 25.5, 24.2, 22.4, 11.8, 9.9. MS (EI): m/z (\%) $184\left(\mathrm{M}^{+}\right), 169,155$ (100), 141, 115, 91. HRMS: Calculated for $\mathrm{C}_{14} \mathrm{H}_{16}$ : 184.1252; Found: 184.1258. Enantiomeric ratio (91:9 er) was measured by chiral HPLC on PC-3 column $\left(\mathrm{CH}_{3} \mathrm{CN} / \mathrm{H}_{2} \mathrm{O}=60: 40,0.7 \mathrm{~mL} / \mathrm{min}\right.$, UV detection at 214 $\mathrm{nm}$ ); retention time $=16.38 \mathrm{~min}$ (major), retention time $=17.75 \mathrm{~min}$ (minor).

\section{EDRC of $( \pm)$ cis-1c with gem-difluoropropargylbromide 2a and NBS}

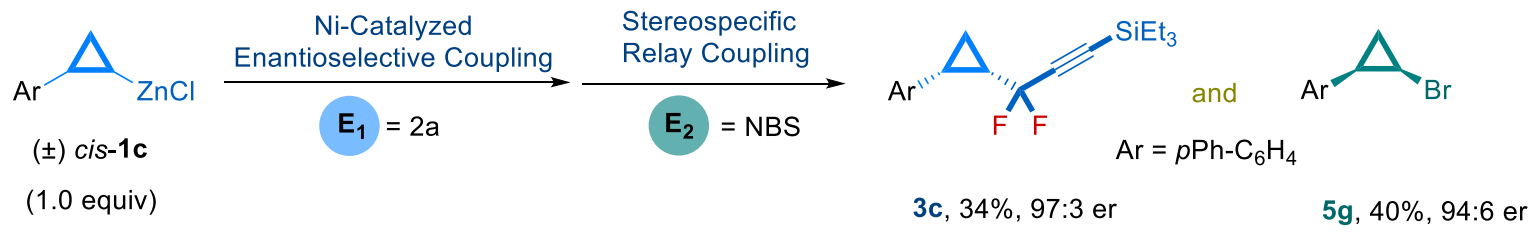

4-((1R,2R)-2-Bromocyclopropyl)-1,1'-biphenyl $(\mathbf{5 g})$. To a $25 \mathrm{~mL}$ of Schlenk tube were added $\mathrm{NiCl}_{2} \cdot \mathrm{DME}(6.6 \mathrm{mg}, 5 \mathrm{~mol} \%)$ and $\mathbf{L 1}(10.8 \mathrm{mg}, 6 \mathrm{~mol} \%)$ in a glovebox. The tube was then taken out from the glovebox. gem-Difluoropropargyl bromide $\mathbf{2 a}(81 \mathrm{mg}, 0.3 \mathrm{mmol}, 0.5$ equiv) and THF ( $2 \mathrm{~mL})$ were added. After stirring at room temperature for $25 \mathrm{~min}$, the reaction mixture was cooled to $-30{ }^{\circ} \mathrm{C}$ and cyclopropylzinc reagent $( \pm)$ cis-1c $(0.6 \mathrm{mmol}, 1.0$ equiv in $1.82 \mathrm{~mL}$ of THF) was added dropwise over a period of $10 \mathrm{~min}$. The reaction was stirred at $-30{ }^{\circ} \mathrm{C}$ for $20 \mathrm{~h}$. A solution of $N$-bromosuccinimide (NBS) (107 mg, $0.6 \mathrm{mmol}, 1.0$ equiv) in anhydrous THF (1 mL) was added at $-30{ }^{\circ} \mathrm{C}$. The reaction mixture was stirred at $-30{ }^{\circ} \mathrm{C}$ for another $1 \mathrm{~h}$. The reaction was then warmed to 
room temperature gradually and stirred overnight. The reaction was quenched with saturated aqueous $\mathrm{NH}_{4} \mathrm{Cl}$ solution and diluted with $\mathrm{CH}_{2} \mathrm{Cl}_{2}(50 \mathrm{~mL})$. The organic layer was separated and the aqueous layer extracted with $\mathrm{CH}_{2} \mathrm{Cl}_{2}$ (three times). The combined organic layers were washed with brine and dried over $\mathrm{Na}_{2} \mathrm{SO}_{4}$, filtered and concentrated. The residue was purified with silica gel chromatography to give $\mathbf{3 c}$ (78 mg, 34 yield\%, 97:3 er; eluent: petroleum ether) and $\mathbf{5 g}$ (65 mg, $40 \%$ yield, 94:6 er; eluent: petroleum ether) as a white solid (m.p. $65-66^{\circ} \mathrm{C}$ ).

Data for 5g: $[\alpha]_{\mathrm{D}}{ }^{20}=-43.64\left(c=0.55, \mathrm{CHCl}_{3}\right)$ for a sample with 94:6 er. ${ }^{1} \mathrm{H} \mathrm{NMR}\left(400 \mathrm{MHz}, \mathrm{CDCl}_{3}\right)$ $\delta 7.62(\mathrm{~d}, J=7.4 \mathrm{~Hz}, 2 \mathrm{H}), 7.58(\mathrm{~d}, J=8.2 \mathrm{~Hz}, 2 \mathrm{H}), 7.45(\mathrm{t}, J=7.5 \mathrm{~Hz}, 2 \mathrm{H}), 7.38-7.29(\mathrm{~m}, 3 \mathrm{H})$, $3.36(\mathrm{td}, J=7.5,4.7 \mathrm{~Hz}, 1 \mathrm{H}), 2.39-2.30(\mathrm{~m}, 1 \mathrm{H}), 1.63(\mathrm{dt}, J=9.3,7.3 \mathrm{~Hz}, 1 \mathrm{H}), 1.38(\mathrm{td}, J=7.1$, $4.8 \mathrm{~Hz}, 1 \mathrm{H}) .{ }^{13} \mathrm{C} \mathrm{NMR}\left(101 \mathrm{MHz}, \mathrm{CDCl}_{3}\right) \delta 140.8,139.6,136.2,129.5,128.7,127.1,127.0,126.6$, 24.1, 21.8, 14.4. Enantiomeric ratio (94:6 er) was measured by chiral HPLC on PC-3 column $\left(\mathrm{CH}_{3} \mathrm{CN} / \mathrm{H}_{2} \mathrm{O}=70: 30,0.7 \mathrm{~mL} / \mathrm{min}, \mathrm{UV}\right.$ detection at $\left.214 \mathrm{~nm}\right) ;$ retention time $=15.41 \mathrm{~min}$ (major), retention time $=16.49 \min ($ minor $)$.

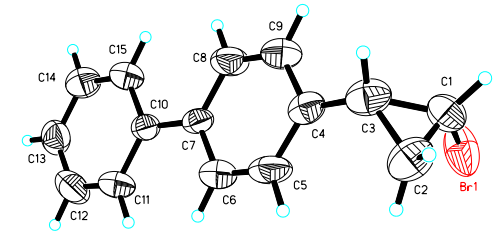

X-Ray Crystal Structure of Compound 5g

\section{EDRC of $( \pm)$ cis-1a with propargylbromide and NBS}

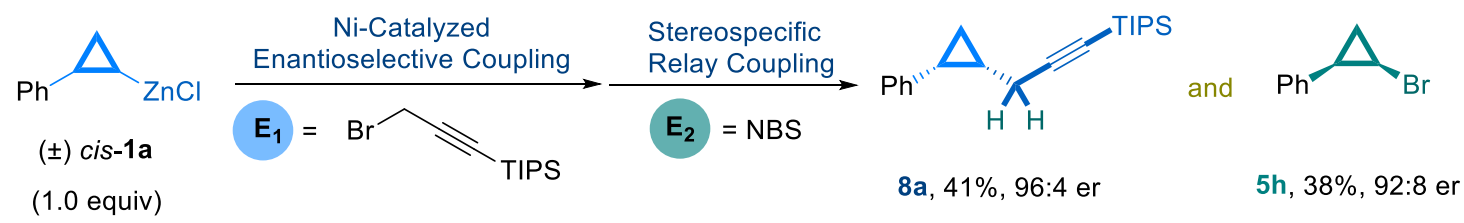

The synthesis of compounds $\mathbf{8 a}$ and $\mathbf{5 h}$ is according to the procedure of EDRC of $( \pm)$ cis-1a with $\mathbf{2 a}$ and NBS. The products $\mathbf{8 a}$ (77 mg, 41 yield\%, 96:4 er; eluent: petroleum ether) and $\mathbf{5 h}$ (45 mg, 38\% yield, 92:8 er; eluent, petroleum ether) were purified with silica gel chromatography.

Data for 5h: $[\alpha]_{\mathrm{D}}{ }^{20}=-49.08\left(c=0.56, \mathrm{CHCl}_{3}\right)$ for a sample with 92:8 er. ${ }^{1} \mathrm{H}$ NMR $\left(400 \mathrm{MHz}, \mathrm{CDCl}_{3}\right)$ $\delta 7.40-7.33$ (m, 2 H), $7.32-7.26$ (m, 3 H), 3.38 - 3.27 (m, 1 H), 2.38 - 2.29 (m, 1 H), $1.64-1.55$ (m, $1 \mathrm{H}), 1.39-1.31(\mathrm{~m}, 1 \mathrm{H}) .{ }^{13} \mathrm{C} \mathrm{NMR}\left(101 \mathrm{MHz}, \mathrm{CDCl}_{3}\right) \delta 137.1,129.2,127.9,126.7,24.0,22.1$, 14.1. Enantiomeric ratio (92:8 er) was measured by chiral HPLC on PC-3 column $\left(\mathrm{CH}_{3} \mathrm{CN} / \mathrm{H}_{2} \mathrm{O}=\right.$ 
60:40, $0.7 \mathrm{~mL} / \mathrm{min}, \mathrm{UV}$ detection at $214 \mathrm{~nm}$ ); retention time $=15.31 \mathrm{~min}$ (major), retention time $=$ $13.95 \min$ (minor).

\section{EDRC of ( \pm ) cis-1c with gem-difluoropropargylbromide 2a and NCS}

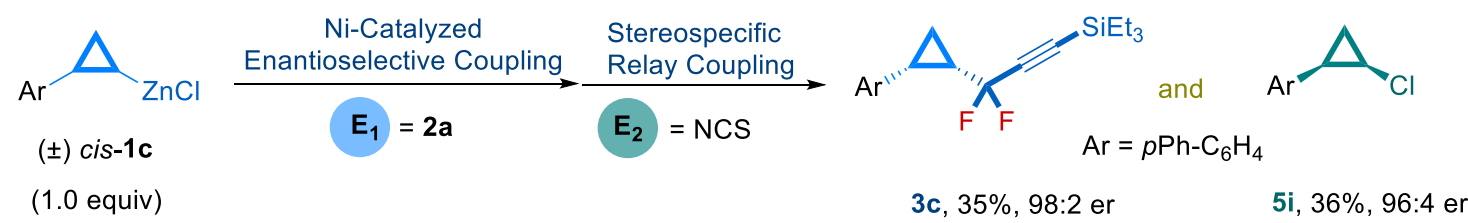

To a $25 \mathrm{~mL}$ of Schlenk tube were added $\mathrm{NiCl}_{2} \cdot \mathrm{DME}(6.6 \mathrm{mg}, 5 \mathrm{~mol} \%)$ and $\mathbf{L 1}(10.8 \mathrm{mg}, 6 \mathrm{~mol} \%)$ in a glovebox. The tube was then taken out from the glovebox. gem-Difluoropropargyl bromide $2 \mathbf{a}$ (81 $\mathrm{mg}, 0.3 \mathrm{mmol}, 0.5$ equiv) and THF ( $2 \mathrm{~mL}$ ) were added. After stirring at room temperature for $25 \mathrm{~min}$, the reaction mixture was cooled to $-30{ }^{\circ} \mathrm{C}$ and cyclopropylzinc reagent $( \pm)$ cis-1c $(0.6 \mathrm{mmol}, 1.0$ equiv in $1.82 \mathrm{~mL}$ of THF) was added dropwise over a period of $10 \mathrm{~min}$. The reaction was stirred at $-30{ }^{\circ} \mathrm{C}$ for $20 \mathrm{~h}$. A solution of $N$-chlorosuccinimide (NCS) $(81 \mathrm{mg}, 0.6 \mathrm{mmol}, 1.0$ equiv) in anhydrous THF $(1 \mathrm{~mL})$ was added at $-30{ }^{\circ} \mathrm{C}$. The reaction mixture was stirred at $-30{ }^{\circ} \mathrm{C}$ for another 1 h. The reaction was then warmed to room temperature gradually and stirred overnight. The reaction was quenched with saturated aqueous $\mathrm{NH}_{4} \mathrm{Cl}$ solution and diluted with $\mathrm{CH}_{2} \mathrm{Cl}_{2}(50 \mathrm{~mL})$. The organic layer was separated and the aqueous layer extracted with $\mathrm{CH}_{2} \mathrm{Cl}_{2}$ (three times). The combined organic layers were washed with brine and dried over $\mathrm{Na}_{2} \mathrm{SO}_{4}$, filtered and concentrated. The residue was purified with silica gel chromatography to give $\mathbf{3 c}$ (73 mg, 35\% yield, 98:2 er; eluent: petroleum ether) and 5i (49.4 mg, 36\% yield, 96:4 er; eluant: petroleum ether/DCM = 20:1) as a white solid (m.p. $\left.70-71^{\circ} \mathrm{C}\right)$.

Data for 5i: $[\alpha]_{\mathrm{D}}{ }^{20}=-27.75\left(c=0.65, \mathrm{CHCl}_{3}\right)$ for a sample with $96: 4$ er. ${ }^{1} \mathrm{H}$ NMR $\left(400 \mathrm{MHz}, \mathrm{CDCl}_{3}\right)$ $\delta 7.67-7.54(\mathrm{~m}, 4 \mathrm{H}), 7.45(\mathrm{t}, J=7.4 \mathrm{~Hz}, 2 \mathrm{H}), 7.39-7.31(\mathrm{~m}, 3 \mathrm{H}), 3.48-3.39(\mathrm{~m}, 1 \mathrm{H}), 2.46-$ $2.36(\mathrm{~m}, 1 \mathrm{H}), 1.57-1.49(\mathrm{~m}, 1 \mathrm{H}), 1.36-1.23(\mathrm{~m}, 1 \mathrm{H}) .{ }^{13} \mathrm{C} \mathrm{NMR}\left(101 \mathrm{MHz}, \mathrm{CDCl}_{3}\right) \delta 140.8$, 139.6, 135.2, 129.6, 128.7, 127.1, 127.0, 126.7, 34.5, 22.6, 14.4. MS (EI): m/z (\%) $228\left(\mathrm{M}^{+}\right), 193$, 178, 152. HRMS: Calculated for $\mathrm{C}_{15} \mathrm{H}_{13} \mathrm{Cl}$ : 228.0706; Found: 228.0712. Enantiomeric ratio (96:4 er) was measured by chiral HPLC on PC-3 column $\left(\mathrm{CH}_{3} \mathrm{CN} / \mathrm{H}_{2} \mathrm{O}=60: 40,0.7 \mathrm{~mL} / \mathrm{min}\right.$, UV detection at $214 \mathrm{~nm}$ ); retention time $=29.34 \mathrm{~min}$ (major), retention time $=26.55 \mathrm{~min}$ (minor) . 


\section{EDRC of $( \pm)$ cis-1a with gem-difluoropropargylbromide $2 \mathrm{a}$ and $\mathrm{I}_{2}$}

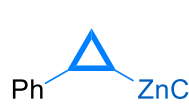

(士) cis-1a

(1.0 equiv)

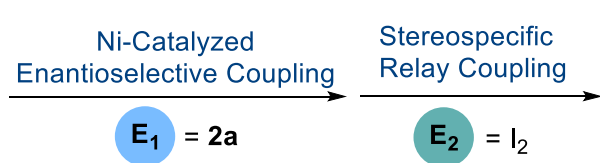

$E_{2}=I_{2}$

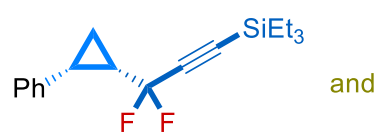

$3 a, 37 \%, 97: 3$ er

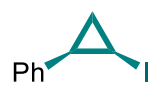

$5 j, 42 \%, 94: 6$ er

To a $25 \mathrm{~mL}$ of Schlenk tube were added $\mathrm{NiCl}_{2}$. DME (6.6 mg, $\left.5 \mathrm{~mol} \%\right)$ and $\mathbf{L 1}(10.8 \mathrm{mg}, 6 \mathrm{~mol} \%)$ in a glovebox. The tube was then taken out from the glovebox. gem-Difluoropropargyl bromide 2a (81 $\mathrm{mg}, 0.3 \mathrm{mmol}, 0.5$ equiv) and THF ( $2 \mathrm{~mL})$ were added. After stirring at room temperature for $25 \mathrm{~min}$, the reaction mixture was cooled to $-30{ }^{\circ} \mathrm{C}$ and cyclopropylzinc reagent $( \pm)$ cis-1a $(0.6$ mmol, 1.0 equiv in $1.82 \mathrm{~mL}$ of THF) was added dropwise over a period of $10 \mathrm{~min}$. The reaction was stirred at $-30{ }^{\circ} \mathrm{C}$ for $20 \mathrm{~h}$ and warmed to $0{ }^{\circ} \mathrm{C} . \mathrm{I}_{2}(228 \mathrm{mg}, 0.9 \mathrm{mmol}, 1.5$ equiv) was added. The reaction mixture was stirred at $0{ }^{\circ} \mathrm{C}$ for another $1 \mathrm{~h}$ and stirred overnight at room temperature. The reaction was quenched with saturated aqueous $\mathrm{Na}_{2} \mathrm{~S}_{2} \mathrm{O}_{3}$ and diluted with $\mathrm{CH}_{2} \mathrm{Cl}_{2}(50 \mathrm{~mL})$. The organic layer was separated and the aqueous layer extracted with $\mathrm{CH}_{2} \mathrm{Cl}_{2}$ (three times). The combined organic layers were washed with brine and dried over $\mathrm{Na}_{2} \mathrm{SO}_{4}$, filtered and concentrated. The residue was purified with silica gel chromatography to give 3a (68 mg, 37 yield\%, 97:3 er; eluent: petroleum) and 5j (61 mg, 42\% yield, 94:6 er; eluant: petroleum ether) as a colorless oil.

Data for 5j: $[\alpha]_{\mathrm{D}}{ }^{25}=-33.28\left(c=0.89, \mathrm{CHCl}_{3}\right)$ for sample with 94:6 er. ${ }^{1} \mathrm{H}$ NMR $\left(400 \mathrm{MHz}, \mathrm{CDCl}_{3}\right) \delta$ $7.39-7.27$ (m, 3 H), $7.24-7.14$ (m, 2 H), 3.01 - 2.92 (m, 1 H), 2.13 - 2.04 (m, $1 \mathrm{H}), 1.76$ - 1.65 (m, $1 \mathrm{H}), 1.36-1.23(\mathrm{~m}, 1 \mathrm{H}) .{ }^{13} \mathrm{C} \mathrm{NMR}\left(101 \mathrm{MHz}, \mathrm{CDCl}_{3}\right) \delta 139.2,129.0,127.9,126.8,21.2,14.8$, -5.0. MS (EI): m/z (\%) $244\left(\mathrm{M}^{+}\right), 117$ (100). HRMS: Calculated for $\mathrm{C}_{9} \mathrm{H}_{9} \mathrm{I}$ : 243.9749; Found: 243.9753. Enantiomeric ratio (94:6 er) was measured by chiral HPLC on PC-3 column $\left(\mathrm{CH}_{3} \mathrm{CN}_{2} \mathrm{H}_{2} \mathrm{O}\right.$ $=70: 30,0.7 \mathrm{~mL} / \mathrm{min}, \mathrm{UV}$ detection at $214 \mathrm{~nm}$ ); retention time $=11.57 \mathrm{~min}$ (major), retention time $=$ $10.82 \min (\operatorname{minor})$. 


\section{EDRC of $( \pm)$ cis-1a with gem-difluoropropargylbromide $2 \mathrm{a}$ and $\mathrm{PhSe}-\mathrm{SePh}$}

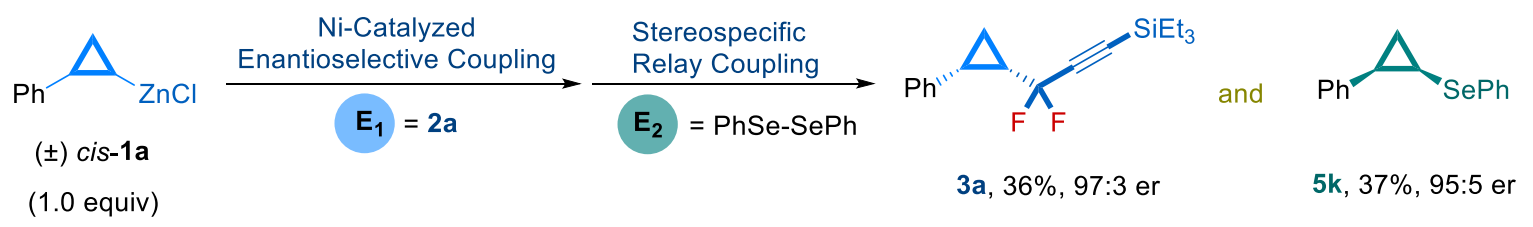

To a $25 \mathrm{~mL}$ of Schlenk tube were added $\mathrm{NiCl}_{2}$. DME (6.6 mg, $\left.5 \mathrm{~mol} \%\right)$ and $\mathbf{L 1}(10.8 \mathrm{mg}, 6 \mathrm{~mol} \%)$ in a glovebox. The tube was then taken out from the glovebox. gem-Difluoropropargyl bromide $\mathbf{2 a}$ (81 $\mathrm{mg}, 0.3 \mathrm{mmol}, 0.5$ equiv) and THF ( $2 \mathrm{~mL})$ were added. After stirring at room temperature for $25 \mathrm{~min}$, the reaction mixture was cooled to $-30{ }^{\circ} \mathrm{C}$ and cyclopropylzinc reagent $( \pm)$ cis-1a $(0.6 \mathrm{mmol}, 1.0$ equiv in $1.82 \mathrm{~mL}$ of THF) was added dropwise over a period of $10 \mathrm{~min}$. The reaction was stirred at $-30{ }^{\circ} \mathrm{C}$ for $20 \mathrm{~h}$. A solution of 1,2-diphenyldiselane (141 $\mathrm{mg}, 0.45 \mathrm{mmol}, 0.75$ equiv) in anhydrous THF ( $1 \mathrm{~mL})$ was added. The reaction mixture was slowly warmed to room temperature and stirred for $8 \mathrm{~h}$. The reaction was quenched with saturated aqueous $\mathrm{NH}_{4} \mathrm{Cl}$ solution and diluted with $\mathrm{CH}_{2} \mathrm{Cl}_{2}$ (50 mL). The organic layer was separated and the aqueous layer extracted with $\mathrm{CH}_{2} \mathrm{Cl}_{2}$ (three times). The combined organic layers were washed with brine and dried over $\mathrm{Na}_{2} \mathrm{SO}_{4}$, filtered and concentrated. The residue was purified with silica gel chromatography to give 3a $(66 \mathrm{mg}$, 36 yield\%, 97:3 er; eluent: petroleum ether) and 5k (61 mg, 37\% yield, 95:5 er; eluant: petroleum ether) as a pale yellow oil.

Data for 5k: $[\alpha]_{\mathrm{D}}{ }^{20}=-17.66\left(c=0.65, \mathrm{CHCl}_{3}\right)$ for a sample with 95:5 er. ${ }^{1} \mathrm{H} \mathrm{NMR}\left(400 \mathrm{MHz}, \mathrm{CDCl}_{3}\right)$ $\delta 7.38-7.32(\mathrm{~m}, 2 \mathrm{H}), 7.29-7.13(\mathrm{~m}, 8 \mathrm{H}), 2.76(\mathrm{td}, J=8.1,5.6 \mathrm{~Hz}, 1 \mathrm{H}), 2.59-2.52(\mathrm{~m}, 1 \mathrm{H})$, $1.65(\mathrm{td}, J=8.4,5.8 \mathrm{~Hz}, 1 \mathrm{H}), 1.32-1.26(\mathrm{~m}, 1 \mathrm{H}) .{ }^{13} \mathrm{C} \mathrm{NMR}\left(101 \mathrm{MHz}, \mathrm{CDCl}_{3}\right) \delta 138.1,131.7$, 130.8, 128.9, 128.7, 127.7, 126.4, 126.1, 22.6, 17.6, 13.3. MS (EI): m/z (\%) $274\left(\mathrm{M}^{+}\right), 117,91$. HRMS: Calculated for $\mathrm{C}_{15} \mathrm{H}_{14}{ }^{74} \mathrm{Se}$ : 268.0320; Found: 268.0326. Enantiomeric ratio (95:5 er) was measured by chiral HPLC on PC-3 column $\left(\mathrm{CH}_{3} \mathrm{CN} / \mathrm{H}_{2} \mathrm{O}=60: 40,0.7 \mathrm{~mL} / \mathrm{min}\right.$, UV detection at 214 $\mathrm{nm}$ ); retention time $=40.97 \min$ (major), retention time $=45.80 \min$ (minor). 


\section{EDRC of ( \pm ) cis-1c with gem-difluoropropargylbromide 2a and $n \mathrm{Bu3SnCl}$}

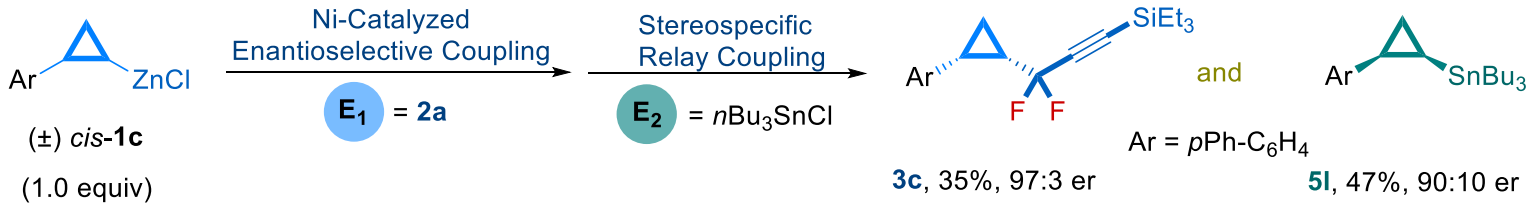

To a $25 \mathrm{~mL}$ of Schlenk tube were added $\mathrm{NiCl}_{2}$. DME (6.6 mg, $\left.5 \mathrm{~mol} \%\right)$ and $\mathbf{L 1}(10.8 \mathrm{mg}, 6 \mathrm{~mol} \%)$ in a glovebox. The tube was then taken out from the glovebox. gem-Difluoropropargyl bromide 2a (81 $\mathrm{mg}, 0.3 \mathrm{mmol}, 0.5$ equiv) and THF ( $2 \mathrm{~mL}$ ) were added. After stirring at room temperature for $25 \mathrm{~min}$, the reaction mixture was cooled to $-30{ }^{\circ} \mathrm{C}$ and cyclopropylzinc reagent $( \pm)$ cis-1c $(0.6$ mmol, 1.0 equiv in $1.82 \mathrm{~mL}$ of THF) was added dropwise over a period of $10 \mathrm{~min}$. The reaction was stirred at $-30{ }^{\circ} \mathrm{C}$ for $20 \mathrm{~h}$. Then, the reaction was cooled to $-40{ }^{\circ} \mathrm{C}$ and $\mathrm{CuCN} \cdot 2 \mathrm{LiCl}(0.5 \mathrm{M}$ in THF, $0.66 \mathrm{~mL}$, $0.33 \mathrm{mmol}, 0.55$ equiv) was added dropwise. The reaction was stirred at $-40{ }^{\circ} \mathrm{C}$ for $20 \mathrm{~min}$, a solution of tributylchlorostannane (147 mg, $0.45 \mathrm{mmol}, 0.75$ equiv) in anhydrous THF (1 mL) was added. The reaction mixture was slowly warmed to room temperature and stirred for $8 \mathrm{~h}$. The reaction was quenched with saturated aqueous $\mathrm{NH}_{4} \mathrm{Cl}$ solution and diluted with $\mathrm{CH}_{2} \mathrm{Cl}_{2}(50 \mathrm{~mL})$. The organic layer was separated and the aqueous layer extracted with $\mathrm{CH}_{2} \mathrm{Cl}_{2}$ (three times). The combined organic layers were washed with brine and dried over $\mathrm{Na}_{2} \mathrm{SO}_{4}$, filtered and concentrated. The residue was purified with silica gel chromatography to give 3c $(64.4 \mathrm{mg}, 35$ yield\%, 97:3 er; eluent: petroleum ether) and $\mathbf{5 l}$ (113 $\mathrm{mg}, 47 \%$ yield, 90:10 er; eluant: petroleum ether) as a colorless oil.

Data for 5l: $[\alpha]_{\mathrm{D}}^{20}=-21.09\left(c=0.65, \mathrm{CHCl}_{3}\right)$ for a sample with 90:10 er. ${ }^{1} \mathrm{H}$ NMR $\left(400 \mathrm{MHz}, \mathrm{CDCl}_{3}\right)$ $\delta 7.59(\mathrm{~d}, J=7.2 \mathrm{~Hz}, 2 \mathrm{H}), 7.49(\mathrm{~d}, J=8.1 \mathrm{~Hz}, 2 \mathrm{H}), 7.44(\mathrm{t}, J=7.7 \mathrm{~Hz}, 2 \mathrm{H}), 7.33(\mathrm{t}, J=7.3 \mathrm{~Hz}, 1$ H), $7.23(\mathrm{~d}, J=8.1 \mathrm{~Hz}, 2 \mathrm{H}), 2.35(\mathrm{td}, J=8.5,5.1 \mathrm{~Hz}, 1 \mathrm{H}), 1.41-1.17(\mathrm{~m}, 14 \mathrm{H}), 1.05-1.00(\mathrm{~m}, 1$ $\mathrm{H}), 0.85(\mathrm{t}, J=7.2 \mathrm{~Hz}, 9 \mathrm{H}), 0.65-0.45(\mathrm{~m}, 7 \mathrm{H}) .{ }^{13} \mathrm{C}$ NMR $\left(101 \mathrm{MHz}, \mathrm{CDCl}_{3}\right) \delta 143.4,141.2$, 138.5, 128.7, 128.1, 126.92, 126.88, 126.8, 29.0, 27.3, 19.1, 13.7, 9.4, 8.7, 7.3. MS (EI): m/z (\%) 427 $\left(\left[\mathrm{M}-{ }^{n} \mathrm{Bu}\right]^{+}\right), 419,311,193,178$ (100). HRMS: Calculated for $\mathrm{C}_{23} \mathrm{H}_{31}{ }^{112} \mathrm{Sn}$ : 419.1474; Found: 419.1476. Enantiomeric ratio (90:10 er) was measured by chiral HPLC on PC-3 column $\left(\mathrm{CH}_{3} \mathrm{CN} / \mathrm{H}_{2} \mathrm{O}=70: 30,0.7 \mathrm{~mL} / \mathrm{min}, \mathrm{UV}\right.$ detection at $\left.214 \mathrm{~nm}\right)$; retention time $=45.05$ min (major), retention time $=52.80 \mathrm{~min}($ minor $)$. 


\section{EDRC of $( \pm)$ cis-1a with secondary propargylbromide 21 and allylbromide}

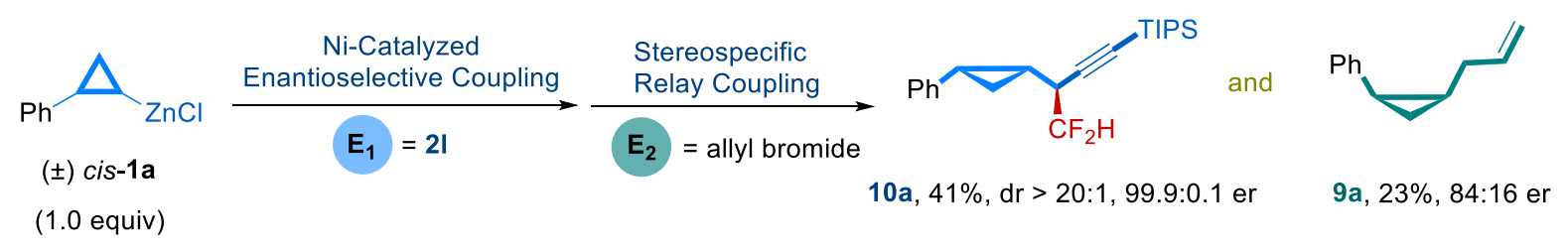

To a $25 \mathrm{~mL}$ of Schlenk tube were added $\mathrm{NiBr}_{2} \cdot \mathrm{DME}(9.1 \mathrm{mg}, 5 \mathrm{~mol} \%)$ and $(R, R)$-CyPybox $(14.9 \mathrm{mg}$, $6.5 \mathrm{~mol} \%$ ) in a glovebox. The tube was then taken out from the glovebox. Secondary propargyl bromide 21 (98 mg, $0.3 \mathrm{mmol}, 0.5$ equiv) and anhydrous 1,4-dioxane (1 mL) were added. After stirring at room temperature for $25 \mathrm{~min}$, the reaction mixture was cooled to $-20{ }^{\circ} \mathrm{C}$. Cyclopropylzinc reagent $( \pm)$ cis-1a $(0.6 \mathrm{mmol}, 1.0$ equiv in $1.82 \mathrm{~mL}$ of THF) was added dropwise over a period of 10 min. The reaction was stirred at same reaction temperature for $24 \mathrm{~h}$. Then, the reaction was cooled to $-40{ }^{\circ} \mathrm{C}$ and $\mathrm{CuCN} \cdot 2 \mathrm{LiCl}(0.5 \mathrm{M}$ in THF, $0.3 \mathrm{~mL}, 0.15 \mathrm{mmol}, 0.25$ equiv) was added dropwise. The reaction was stirred at $-40{ }^{\circ} \mathrm{C}$ for $5 \mathrm{~min}$, allyl bromide (102 $\mathrm{mg}, 0.84 \mathrm{mmol}, 1.4$ equiv) was added. The reaction mixture was stirred at $-40{ }^{\circ} \mathrm{C}$ for another $1 \mathrm{~h}$ and was slowly warmed to room temperature and stirred overnight. The reaction was quenched with saturated aqueous $\mathrm{NH}_{4} \mathrm{Cl}$ solution and diluted with $\mathrm{CH}_{2} \mathrm{Cl}_{2}(50 \mathrm{~mL})$. The organic layer was separated and the aqueous layer extracted with $\mathrm{CH}_{2} \mathrm{Cl}_{2}$ (three times). The combined organic layers were washed with brine and dried over $\mathrm{Na}_{2} \mathrm{SO}_{4}$, filtered and concentrated. The residue was purified with silica gel chromatography to give 10a (90 mg, 41 yield\%, dr > 20:1, 99.9:0.1 er; eluent: Petroleum ether) and 9a (22 mg, 23\% yield, 84:16 er; eluant: Petroleum ether) as a colorless oil. The enantiomeric ratio (84:16 er) of 9a was measured by chiral HPLC on PC-3 column $\left(\mathrm{CH}_{3} \mathrm{CN} / \mathrm{H}_{2} \mathrm{O}=60: 40,0.7 \mathrm{~mL} / \mathrm{min}\right.$, UV detection at $214 \mathrm{~nm}) ;$ retention time $=14.55 \min ($ major $)$, retention time $=16.51 \mathrm{~min}($ minor $)$. Optical rotation value of 9a: $[\alpha]_{\mathrm{D}}{ }^{20}=31.29\left(c=0.75, \mathrm{CHCl}_{3}\right)$ for a sample with $84: 16 \mathrm{er}$.

\section{EDRC of $( \pm)$ cis-1a with secondary propargylbromide 21 and NBS}

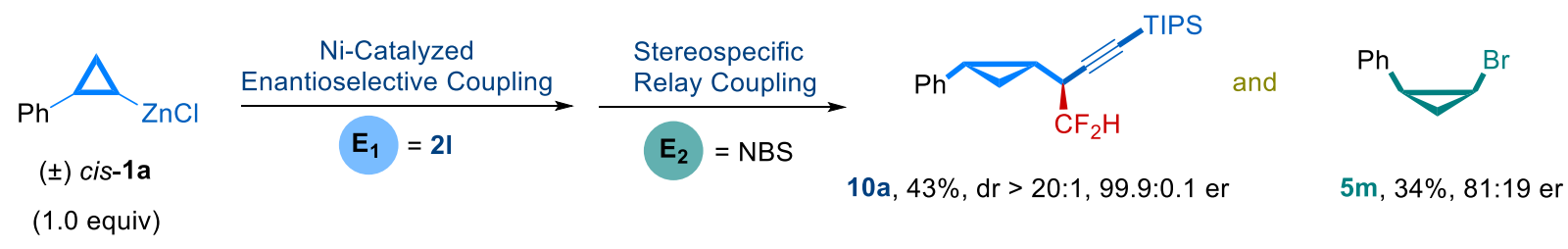

To a $25 \mathrm{~mL}$ of Schlenk tube were added $\mathrm{NiBr}_{2} \cdot \mathrm{DME}(9.1 \mathrm{mg}, 5 \mathrm{~mol} \%)$ and $(R, R)$-CyPybox $(14.9 \mathrm{mg}$, $6.5 \mathrm{~mol} \%$ ) in a glovebox. The tube was then taken out from the glovebox. Secondary propargyl 
bromide 21 (98 mg, $0.3 \mathrm{mmol}, 0.5$ equiv) and anhydrous 1,4-dioxane (1 mL) were added. After stirring at room temperature for $25 \mathrm{~min}$, the reaction mixture was cooled to $-20^{\circ} \mathrm{C}$. Cyclopropylzinc reagent $( \pm)$ cis-1a $(0.6 \mathrm{mmol}, 1.0$ equiv in $1.82 \mathrm{~mL}$ of THF) was added dropwise over a period of 10 $\min$. The reaction was stirred at same reaction temperature for $24 \mathrm{~h}$. Then, the reaction was cooled to $-30{ }^{\circ} \mathrm{C}$ and a solution of NBS (107 $\mathrm{mg}, 0.6 \mathrm{mmol}, 1.0$ equiv) was added. The reaction was stirred at $-30{ }^{\circ} \mathrm{C}$ for another $1 \mathrm{~h}$. The reaction was warmed to room temperature and stirred overnight. The reaction was quenched with saturated aqueous $\mathrm{NH}_{4} \mathrm{Cl}$ solution and diluted with $\mathrm{CH}_{2} \mathrm{Cl}_{2}(50 \mathrm{~mL})$. The organic layer was separated and the aqueous layer extracted with $\mathrm{CH}_{2} \mathrm{Cl}_{2}$ (three times). The combined organic layers were washed with brine and dried over $\mathrm{Na}_{2} \mathrm{SO}_{4}$, filtered and concentrated. The residue was purified with silica gel chromatography to give 10a (93 mg, 43 yield\%, dr > 20:1, 99.9:0.1 er; eluent: Petroleum ether) and 5m (40 mg, 34\% yield, 81:19 er; eluant: Petroleum ether) as a colorless oil.

Data for 5m: $[\alpha]_{\mathrm{D}}{ }^{20}=32.54\left(c=0.66, \mathrm{CHCl}_{3}\right)$ for a sample with 81:19 er. ${ }^{1} \mathrm{H}$ NMR (400 MHz, $\left.\mathrm{CDCl}_{3}\right) \delta 7.39-7.32(\mathrm{~m}, 2 \mathrm{H}), 7.31-7.24(\mathrm{~m}, 3 \mathrm{H}), 3.33(\mathrm{td}, J=7.6,4.6 \mathrm{~Hz}, 1 \mathrm{H}), 2.37-2.29(\mathrm{~m}, 1$ $\mathrm{H}), 1.63-1.55(\mathrm{~m}, 1 \mathrm{H}), 1.34(\mathrm{td}, J=7.2,4.6 \mathrm{~Hz}, 1 \mathrm{H}) .{ }^{13} \mathrm{C} \mathrm{NMR}\left(126 \mathrm{MHz}, \mathrm{CDCl}_{3}\right) \delta 137.1,129.2$, 127.9, 126.8, 24.0, 22.0, 14.1 MS (EI): m/z (\%) $196\left(\mathrm{M}^{+}\right), 117$ (100), 91. HRMS: Calculated for $\mathrm{C}_{9} \mathrm{H}_{9} \mathrm{Br}$ : 195.9888; Found: 195.9894. The enantiomeric ratio $(81: 19$ er) of $\mathbf{5 m}$ was measured by chiral HPLC on PC-3 column $\left(\mathrm{CH}_{3} \mathrm{CN} / \mathrm{H}_{2} \mathrm{O}=60: 40,0.7 \mathrm{~mL} / \mathrm{min}\right.$, UV detection at $\left.214 \mathrm{~nm}\right)$; retention time $=13.87 \mathrm{~min}$ (major), retention time $=15.37 \mathrm{~min}$ (minor).

\section{EDRC of $( \pm)$ cis-1a with secondary propargylbromide $2 \mathrm{k}$ and (bromoethynyl)benzene}

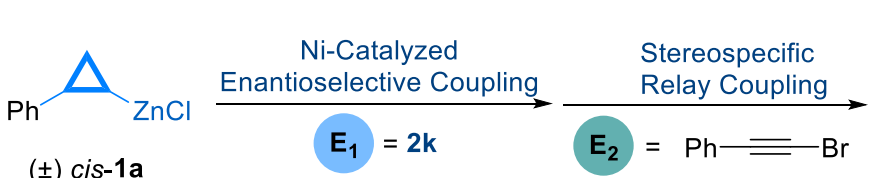

( \pm ) cis-1a

(1.0 equiv)

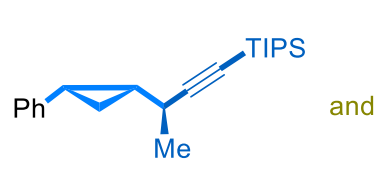

$10 \mathrm{~h}, 42 \%, \mathrm{dr}=9: 1,99.5: 0.5 \mathrm{er}$

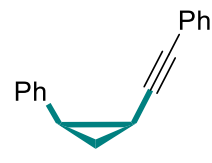

5 n, $23 \%, 80: 20$ er

(((1S,2R)-2-Phenylcyclopropyl)ethynyl)benzene $(\mathbf{5 n})$. To a $25 \mathrm{~mL}$ of Schlenk tube were added $\mathrm{NiBr}_{2} \cdot \mathrm{DME}(9.1 \mathrm{mg}, 5 \mathrm{~mol} \%)$ and $(R, R)$-CyPybox $(14.9 \mathrm{mg}, 6.5 \mathrm{~mol} \%)$ in a glovebox. The tube was then taken out from the glovebox. Secondary propargyl bromide $2 \mathbf{k}$ ( $87 \mathrm{mg}, 0.3 \mathrm{mmol}, 0.5$ equiv) and anhydrous 1,4-dioxane $(1 \mathrm{~mL})$ were added. After stirring at room temperature for $25 \mathrm{~min}$, the reaction mixture was cooled to $-20{ }^{\circ} \mathrm{C}$. Cyclopropylzinc reagent $( \pm)$ cis-1a $(0.6 \mathrm{mmol}, 1.0$ equiv in 
$1.82 \mathrm{~mL}$ of THF) was added dropwise over a period of $10 \mathrm{~min}$. The reaction was stirred at same reaction temperature for $24 \mathrm{~h}$. Then, the reaction was cooled to $-78{ }^{\circ} \mathrm{C}$ and $\mathrm{CuCN} \cdot 2 \mathrm{LiCl}(0.5 \mathrm{M}$ in THF, $1.8 \mathrm{~mL}, 0.9 \mathrm{mmol}, 1.5$ equiv) was added dropwise. The reaction was stirred at $-78^{\circ} \mathrm{C}$ for 30 min, a solution of (bromoethynyl)benzene (217 mg, $1.2 \mathrm{mmol}, 2.0$ equiv) in anhydrous THF (1 mL) was added. The reaction mixture was stirred at $-78{ }^{\circ} \mathrm{C}$ for another $1 \mathrm{~h}$. Then, the reaction was slowly warmed to room temperature and stirred overnight. The reaction was quenched with saturated aqueous $\mathrm{NH}_{4} \mathrm{Cl}$ solution and diluted with $\mathrm{CH}_{2} \mathrm{Cl}_{2}(50 \mathrm{~mL})$. The organic layer was separated and the aqueous layer extracted with $\mathrm{CH}_{2} \mathrm{Cl}_{2}$ (three times). The combined organic layers were washed with brine and dried over $\mathrm{Na}_{2} \mathrm{SO}_{4}$, filtered and concentrated. The residue was purified with silica gel chromatography to give $\mathbf{1 0 h}(83 \mathrm{mg}, 42 \%$ yield, $\mathrm{dr}=9: 1,99.5: 0.5 \mathrm{er}$; eluent: petroleum ether) and 5n (30 mg, 23\% yield, 80:20 er; eluant: petroleum ether) as a colorless oil.

Data for 5n: $[\alpha]_{\mathrm{D}}{ }^{20}=-19.08\left(c=0.26, \mathrm{CHCl}_{3}\right)$ for a sample with 80:20 er. ${ }^{1} \mathrm{H}$ NMR $(400 \mathrm{MHz}$, $\left.\mathrm{CDCl}_{3}\right) \delta 7.37-7.31(\mathrm{~m}, 4 \mathrm{H}), 7.29-7.24(\mathrm{~m}, 1 \mathrm{H}), 7.22-7.18(\mathrm{~m}, 3 \mathrm{H}), 7.18-7.13(\mathrm{~m}, 2 \mathrm{H}), 2.52$ - $2.32(\mathrm{~m}, 1 \mathrm{H}), 2.00(\mathrm{td}, J=8.6,5.8 \mathrm{~Hz}, 1 \mathrm{H}), 1.47(\mathrm{td}, J=8.6,5.1 \mathrm{~Hz}, 1 \mathrm{H}), 1.38-1.25(\mathrm{~m}, 1 \mathrm{H})$.

${ }^{13} \mathrm{C}$ NMR $\left(101 \mathrm{MHz}, \mathrm{CDCl}_{3}\right) \delta 138.0,131.4,128.3,128.0,127.7,127.4,126.3,123.7,89.7,80.2$, 24.0, 15.0, 10.2. MS (EI): m/z (\%) $218\left(\mathrm{M}^{+}, 100\right), 91,77$. HRMS: Calculated for $\mathrm{C}_{17} \mathrm{H}_{14}:$ 218.1096; Found: 218.1088. Enantiomeric ratio (80:20 er) was measured by chiral HPLC on PC-1 column $\left(\mathrm{CH}_{3} \mathrm{CN} / \mathrm{H}_{2} \mathrm{O}=70: 30,0.7 \mathrm{~mL} / \mathrm{min}, \mathrm{UV}\right.$ detection at $\left.214 \mathrm{~nm}\right)$; retention time $=18.53$ min (major), retention time $=21.49 \min ($ minor $)$

\section{Diverse transformations of chiral cyclopropanes}

8.1 One pot synthesis of compounds 13 and $5 \mathrm{~h}$

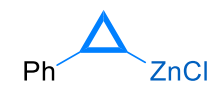

( \pm ) cis-1a (1.0 equiv)

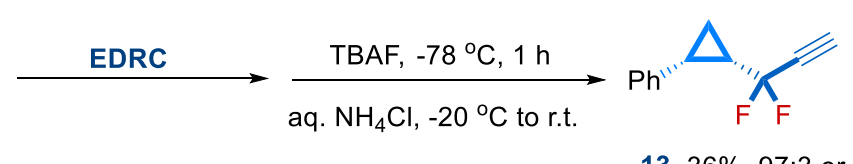

$13,36 \%, 97: 3$ er

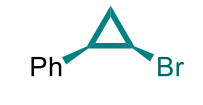

$5 \mathrm{~h}, 38 \%, 92: 8$ er

To a $25 \mathrm{~mL}$ of Schlenk tube were added $\mathrm{NiCl}_{2}$. DME (6.6 mg, $\left.5 \mathrm{~mol} \%\right)$ and $\mathbf{L 1}(10.8 \mathrm{mg}, 6 \mathrm{~mol} \%)$ in a glovebox. The tube was then taken out from the glovebox. gem-Difluoropropargyl bromide $2 \mathbf{a}$ (81 mg, $0.3 \mathrm{mmol}, 0.5$ equiv) and THF ( $2 \mathrm{~mL})$ were added. After stirring at room temperature for $25 \mathrm{~min}$, the reaction mixture was cooled to $-30^{\circ} \mathrm{C}$ and cyclopropylzinc reagent $( \pm)$ cis-1a $(0.6 \mathrm{mmol}, 1.0$ equiv in $1.82 \mathrm{~mL}$ of THF) was added dropwise over a period of $10 \mathrm{~min}$. The reaction was stirred at 
$-30{ }^{\circ} \mathrm{C}$ for $20 \mathrm{~h}$, a solution of NBS (107 mg, $0.6 \mathrm{mmol}, 1.0$ equiv) in anhydrous THF $(1 \mathrm{~mL})$ was then added dropwise at $-30{ }^{\circ} \mathrm{C}$. The reaction mixture was stirred at $-30{ }^{\circ} \mathrm{C}$ for $1 \mathrm{~h}$. The reaction mixture was filtered through a short pad of celite using hexane as the eluent. After removal of the solvents, the residue was redissolved in THF $(3 \mathrm{~mL})$. The resulting mixture was cooled to $-78{ }^{\circ} \mathrm{C}$, a solution of TBAF (1.0 M in THF; 0.36 mmol, 0.6 equiv) in THF was added dropwise. The reaction mixture was stirred at $-78{ }^{\circ} \mathrm{C}$ for $1 \mathrm{~h}$ and then warmed to $-20{ }^{\circ} \mathrm{C}$. The reaction was quenched with $10 \mathrm{~mL}$ of saturated aqueous $\mathrm{NH}_{4} \mathrm{Cl}$ solution. After stirring for $5 \mathrm{~min}$ at $0{ }^{\circ} \mathrm{C}$, the resulting mixture was extracted with diethyl ether $(3 \times 30 \mathrm{~mL})$. The combined organic layers were washed with brine, dried over $\mathrm{Na}_{2} \mathrm{SO}_{4}$, filtered, and concentrated. The residue was purified by flash silica gel chromatography (Petroleum ether) to give $\mathbf{1 3}$ (31 mg, 36 yield\%, 97:3 er) as a colorless oil and $\mathbf{5 h}$ (45 mg, 38\% yield, 92:8 er) as a pale yellow oil.

\subsection{Transformations of compound 13}

\section{Synthesis of compound 14a}

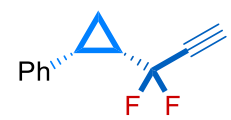

$13,97: 3$ er cat. [Pd], 2-iodopyridine

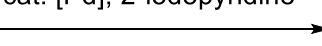

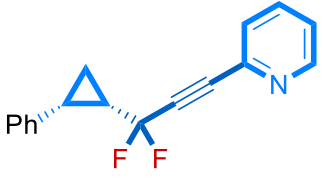

$14 a, 73 \%, 97: 3$ er

2-(3,3-Difluoro-3-((1S,2R)-2-phenylcyclopropyl)prop-1-yn-1-yl)pyridine (14a). To a $25 \mathrm{~mL}$ of Schlenck tube were added $\mathrm{PdCl}_{2}\left(\mathrm{PPh}_{3}\right)_{2}(16.1 \mathrm{mg}, 5 \mathrm{~mol} \%)$ and $\mathrm{CuI}(4.4 \mathrm{mg}, 5 \mathrm{~mol} \%)$. The tube was evacuated and backfilled with Ar for 3 times. 2-Iodopyridine (114 mg, $0.55 \mathrm{mmol}, 1.2$ equiv) and anhydrous $\mathrm{Et}_{3} \mathrm{~N}(2 \mathrm{~mL})$ were added. The resulting mixture was stirred at room temperature for $20 \mathrm{~min}$, a solution of alkyne 13 (90 $\mathrm{mg}, 0.46 \mathrm{mmol}, 1.0$ equiv) in $1 \mathrm{~mL}$ of anhydrous $\mathrm{Et}_{3} \mathrm{~N}$ was then added dropwise. The reaction mixture was stirred at room temperature for $16 \mathrm{~h}$. The reaction mixture was then concentrated. The residue was purified by silica gel chromatography (Petroleum ether/EtOAc $=4: 1)$ to give product $14 \mathbf{a}(91 \mathrm{mg}, 73 \%$ yield $)$ as a brown oil. $[\alpha]_{\mathrm{D}}{ }^{20}=-16.91(c=0.62$, $\left.\mathrm{CHCl}_{3}\right)$ for a sample with 97:3 er. ${ }^{1} \mathrm{H} \mathrm{NMR}\left(400 \mathrm{MHz}, \mathrm{CDCl}_{3}\right) \delta 8.59(\mathrm{~d}, J=4.7 \mathrm{~Hz}, 1 \mathrm{H}), 7.62(\mathrm{td}, J$ = 7.8, 1.6 Hz, $1 \mathrm{H}), 7.38(\mathrm{~d}, J=7.5 \mathrm{~Hz}, 2 \mathrm{H}), 7.29-7.21(\mathrm{~m}, 3 \mathrm{H}), 7.20-7.13(\mathrm{~m}, 2 \mathrm{H}), 2.54-2.45$ (m, $1 \mathrm{H}), 2.03-1.93(\mathrm{~m}, 1 \mathrm{H}), 1.59-1.51(\mathrm{~m}, 1 \mathrm{H}), 1.34-1.24(\mathrm{~m}, 1 \mathrm{H}) .{ }^{13} \mathrm{C}$ NMR $(101 \mathrm{MHz}$, $\left.\mathrm{CDCl}_{3}\right) \delta 150.1,140.8(\mathrm{t}, J=2.4 \mathrm{~Hz}), 136.0,135.8,130.0,127.8,127.7,126.5,124.0,114.2(\mathrm{t}, J=$ 
$232.7 \mathrm{~Hz}), 85.7(\mathrm{t}, J=6.6 \mathrm{~Hz}), 80.6(\mathrm{t}, J=42.0 \mathrm{~Hz}), 24.9(\mathrm{dd}, J=32.6,32.3 \mathrm{~Hz}), 21.2(\mathrm{t}, J=2.2 \mathrm{~Hz})$, $7.0(\mathrm{t}, J=2.5 \mathrm{~Hz}) .{ }^{19} \mathrm{~F}$ NMR $\left(376 \mathrm{MHz}, \mathrm{CDCl}_{3}\right) \delta-76.22(\mathrm{dd}, J=277.2,8.8 \mathrm{~Hz}, 1 \mathrm{~F}),-78.40(\mathrm{dd}, J=$ 276.9, $12.4 \mathrm{~Hz}, 1 \mathrm{~F})$. MS (EI): m/z (\%) $269\left(\mathrm{M}^{+}\right)$, 250, 229 (100), 192. HRMS: Calculated for $\mathrm{C}_{17} \mathrm{H}_{13} \mathrm{NF}_{2}$ : 269.1016; Found: 269.1017. Enantiomeric ratio (97:3 er) was measured by chiral HPLC on PC-3 column $\left(\mathrm{CH}_{3} \mathrm{CN} / \mathrm{H}_{2} \mathrm{O}=70: 30,0.7 \mathrm{~mL} / \mathrm{min}\right.$, UV detection at $\left.214 \mathrm{~nm}\right)$; retention time $=6.38$ $\min$ (major), retention time $=6.70 \mathrm{~min}$ (minor).

\section{Synthesis of compound 14b}

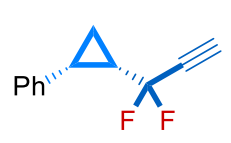

$13,97: 3$ er

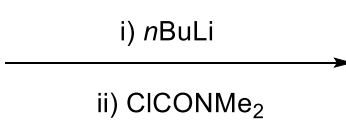

ii) $\mathrm{CICONMe}$

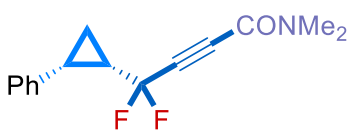

14b, $86 \%, 96: 4$ er

4,4-Difluoro-N,N-dimethyl-4-((1S,2R)-2-phenylcyclopropyl)but-2-ynamide (14b). To a solution of alkyne 13 (96 mg, $0.5 \mathrm{mmol}, 1.0$ equiv) in anhydrous THF (1.5 mL) was added $n \mathrm{BuLi}(2.4 \mathrm{M}$ in hexane; $0.25 \mathrm{~mL}, 0.6 \mathrm{mmol}, 1.2$ equiv) under Ar atmosphere at $-78{ }^{\circ} \mathrm{C}$. The reaction mixture was stirred at $-78{ }^{\circ} \mathrm{C}$ for $60 \mathrm{~min}$. Dimethylcarbamoyl chloride (215 mg, $1.2 \mathrm{mmol}, 4.0$ equiv) was then added at the same temperature. The resulting mixture was warmed to room temperature gradually and stirred overnight. The reaction mixture was quenched with saturated aqueous $\mathrm{NH}_{4} \mathrm{Cl}$. The organic layer was separated and the aqueous layer extracted with $\mathrm{CH}_{2} \mathrm{Cl}_{2}$ (three times). The combined organic layers were washed with brine, dried over $\mathrm{Na}_{2} \mathrm{SO}_{4}$, filtered, and concentrated. The product 14b (113 mg, 86\% yield) was purified with silica gel chromatography (Petroleum ether/EtOAc $=5: 1)$ as a pale brown oil. $[\alpha]_{\mathrm{D}}^{20}=-17.08\left(c=0.76, \mathrm{CHCl}_{3}\right)$ for a sample with 96:4 er. ${ }^{1} \mathrm{H}$ NMR (400 MHz, $\left.\mathrm{CDCl}_{3}\right) \delta 7.34(\mathrm{~d}, J=7.3 \mathrm{~Hz}, 2 \mathrm{H}), 7.25(\mathrm{~d}, J=8.1 \mathrm{~Hz}, 2 \mathrm{H}), 7.22-7.16(\mathrm{~m}, 1$ H), 2.91 (s, $3 \mathrm{H}), 2.88(\mathrm{~s}, 3 \mathrm{H}), 2.54-2.44(\mathrm{~m}, 1 \mathrm{H}), 1.99-1.84(\mathrm{~m}, 1 \mathrm{H}), 1.56-1.48(\mathrm{~m}, 1 \mathrm{H}), 1.33$ - $1.23(\mathrm{~m}, 1 \mathrm{H}) .{ }^{13} \mathrm{C} \mathrm{NMR}\left(101 \mathrm{MHz}, \mathrm{CDCl}_{3}\right) \delta 152.0(\mathrm{t}, J=2.6 \mathrm{~Hz}), 135.4,129.9,127.8,126.6$, $113.7(\mathrm{t}, J=233.0 \mathrm{~Hz}), 81.2(\mathrm{t}, J=42.5 \mathrm{~Hz}), 78.3,37.9,34.0,24.6(\mathrm{dd}, J=32.2,30.8 \mathrm{~Hz}), 21.1(\mathrm{t}, J$ $=2.6 \mathrm{~Hz}), 6.8(\mathrm{t}, J=2.7 \mathrm{~Hz}) .{ }^{19} \mathrm{~F}$ NMR $\left(376 \mathrm{MHz} \mathrm{CDCl}_{3}\right) \delta-77.1(\mathrm{dd}, J=279.7,8.6 \mathrm{~Hz}, 1 \mathrm{~F}),-80.4$ (dd, $J=279.8,13.4 \mathrm{~Hz}, 1 \mathrm{~F}) . \mathrm{MS}(\mathrm{EI}): \mathrm{m} / \mathrm{z}(\%) 263\left(\mathrm{M}^{+}\right), 200,186,171,117$. HRMS: Calculated for $\mathrm{C}_{15} \mathrm{H}_{15} \mathrm{NOF}_{2}$ : 263.1122; Found: 263.1116. Enantiomeric ratio (96:4 er) was measured by chiral HPLC on ID-3 column $\left(\right.$ Hexane $/{ }^{i} \mathrm{PrOH}=90: 10,0.7 \mathrm{~mL} / \mathrm{min}$, UV detection at $\left.214 \mathrm{~nm}\right)$; retention time $=10.99 \min$ (major), retention time $=10.17 \mathrm{~min}($ minor $)$. 


\section{Synthesis of compound $14 \mathrm{c}$}

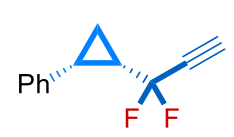

$13,97: 3$ er

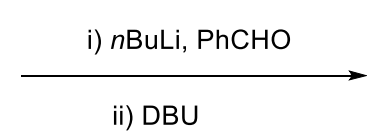

ii) DBU

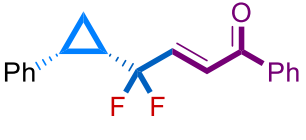

14c, $44 \%, 97: 3$ er

(E)-4,4-Difluoro-1-phenyl-4-((1S,2R)-2-phenylcyclopropyl)but-2-en-1-one $(14 c)^{13}$. To a solution of alkyne 13 (96 mg, $0.5 \mathrm{mmol}, 1.0$ equiv) in anhydrous THF (3 mL) was added $n \mathrm{BuLi}(2.4 \mathrm{M}$ in hexanes, $0.25 \mathrm{~mL}, 0.6 \mathrm{mmol}, 1.2$ equiv) dropwise at $-78{ }^{\circ} \mathrm{C}$ under Ar. After the mixture was stirred at $-78{ }^{\circ} \mathrm{C}$ for $1 \mathrm{~h}$, benzaldehyde $(79.6 \mathrm{mg}, 0.75 \mathrm{mmol}, 1.5$ equiv) in anhydrous THF (1 mL) was added. The reaction mixture was slowly warmed to room temperature and stirred for another $4 \mathrm{~h}$. The mixture was concentrated and the residue was redissolved in THF (2 mL), then DBU (152.2 $\mathrm{mg}, 1.0$ mmol, 2.0 equiv) was added. The resulting mixture was stirred at room temperature for $6 \mathrm{~h}$. The reaction was quenched with saturated aqueous $\mathrm{NH}_{4} \mathrm{Cl}$ and extracted with $\mathrm{CH}_{2} \mathrm{Cl}_{2}$. The combined organic layers were washed with water, dried over $\mathrm{Na}_{2} \mathrm{SO}_{4}$, filtered and concentrated. The residue was purified with silica gel chromatography (Petroleum ether/EtOAc $=30: 1)$ to give $14 \mathbf{c}(65 \mathrm{mg}$, $44 \%$ yield for two steps $)$ as a yellow oil. $[\alpha]_{\mathrm{D}}{ }^{25}=6.72\left(c=0.64, \mathrm{CHCl}_{3}\right)$ for a sample with 97:3 er. ${ }^{1} \mathrm{H}$ NMR $\left(400 \mathrm{MHz}, \mathrm{CDCl}_{3}\right) \delta 7.80(\mathrm{~d}, J=7.6 \mathrm{~Hz}, 2 \mathrm{H}), 7.58(\mathrm{t}, J=7.4 \mathrm{~Hz}, 1 \mathrm{H}), 7.45(\mathrm{t}, J=7.7 \mathrm{~Hz}$, $2 \mathrm{H}), 7.30-7.19(\mathrm{~m}, 4 \mathrm{H}), 7.15(\mathrm{t}, J=6.9 \mathrm{~Hz}, 1 \mathrm{H}), 6.80(\mathrm{dt}, J=15.5,1.9 \mathrm{~Hz}, 1 \mathrm{H}), 6.56(\mathrm{ddd}, J=$ 15.5, 12.3, $10.6 \mathrm{~Hz}, 1 \mathrm{H}), 2.49-2.40$ (m, $1 \mathrm{H}), 1.82-1.68$ (m, $1 \mathrm{H}), 1.52-1.46$ (m, $1 \mathrm{H}), 1.27-$ $1.19(\mathrm{~m}, 1 \mathrm{H}) .{ }^{13} \mathrm{C}$ NMR $\left(101 \mathrm{MHz}, \mathrm{CDCl}_{3}\right) \delta 189.2,138.1(\mathrm{t}, J=28.4 \mathrm{~Hz}), 136.8,135.7,133.4$, 129.6, 128.6, 127.9, 126.6, $126.5(\mathrm{t}, J=7.2 \mathrm{~Hz}), 120.1(\mathrm{dd}, J=241.1,238.3 \mathrm{~Hz}), 23.5(\mathrm{dd}, J=32.5$, $29.1 \mathrm{~Hz}), 20.9(\mathrm{~d}, J=4.2 \mathrm{~Hz}), 5.6(\mathrm{dd}, J=4.6,2.3 \mathrm{~Hz}) .{ }^{19} \mathrm{~F} \mathrm{NMR}\left(376 \mathrm{MHz}, \mathrm{CDCl}_{3}\right) \delta-89.57(\mathrm{dt}, J$ $=249.7,10.3 \mathrm{~Hz}, 1 \mathrm{~F}),-97.38(\mathrm{dt}, J=249.5,13.9 \mathrm{~Hz}, 1 \mathrm{~F}) . \mathrm{MS}(\mathrm{EI}): \mathrm{m} / \mathrm{z}(\%) 298\left(\mathrm{M}^{+}\right), 278,117$ (100). HRMS: Calculated for $\mathrm{C}_{19} \mathrm{H}_{16} \mathrm{OF}_{2}$ : 298.1169; Found: 298.1173. Enantiomeric ratio (97:3 er) was measured by chiral HPLC on PC-3 column $\left(\mathrm{CH}_{3} \mathrm{CN} / \mathrm{H}_{2} \mathrm{O}=70: 30,0.7 \mathrm{~mL} / \mathrm{min}\right.$, UV detection at $214 \mathrm{~nm}$ ); retention time $=8.74 \min ($ major $)$, retention time $=12.86 \min ($ minor $)$. 


\section{Synthesis of compound 14d}

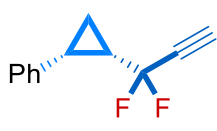

13, $97: 3$ er

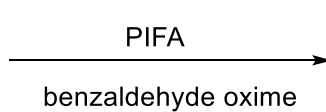

benzaldehyde oxime

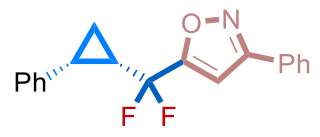

14d, $70 \%, 98: 2$ er

5-(Difluoro((1S,2R)-2-phenylcyclopropyl)methyl)-3-phenylisoxazole $(14 d)^{14}$. To a solution of alkyne 13 (78 mg, $0.5 \mathrm{mmol}, 1.0$ equiv) and benzaldehyde oxime (91 mg, $0.75 \mathrm{mmol}, 1.5 \mathrm{equiv}$ ) in $3.0 \mathrm{~mL}$ of $\mathrm{MeOH} / \mathrm{H}_{2} \mathrm{O}(\mathrm{v} / \mathrm{v}=5: 1)$ was added PIFA (323 mg, $0.75 \mathrm{mmol}, 1.5$ equiv) in three portions ( $3 \times 0.5$ equiv) every two hours at room temperature. After stirring for $8 \mathrm{~h}$, the reaction mixture was diluted with $\mathrm{CH}_{2} \mathrm{Cl}_{2}(20 \mathrm{~mL})$. The resulting mixture was filtered through a pad of cellite and washed with $\mathrm{CH}_{2} \mathrm{Cl}_{2}(10 \mathrm{~mL})$. The combined organic layers were concentrated. The residue was purified with silica gel chromatography (Petroleum ether/DCM = 4:1) to afford compound 14d (109 mg, 70\% yield) as a white solid (m.p. $\left.92-93{ }^{\circ} \mathrm{C}\right) \cdot[\alpha]_{\mathrm{D}}{ }^{20}=-11.52\left(c=0.50, \mathrm{CHCl}_{3}\right)$ for a sample with 98:2 er. ${ }^{1} \mathrm{H}$ NMR (400 MHz, $\left.\mathrm{CDCl}_{3}\right) \delta 7.74-7.66(\mathrm{~m}, 2 \mathrm{H}), 7.50-7.43(\mathrm{~m}, 3 \mathrm{H}), 7.22-7.13(\mathrm{~m}, 5 \mathrm{H}), 6.20$ (s, $1 \mathrm{H}), 2.58-2.50(\mathrm{~m}, 1 \mathrm{H}), 2.10$ (dddt, $J=21.4,14.4,8.8,4.4 \mathrm{~Hz}, 1 \mathrm{H}), 1.71-1.65$ (m, $1 \mathrm{H}), 1.38$ - $1.30(\mathrm{~m}, 1 \mathrm{H}) .{ }^{13} \mathrm{C} \mathrm{NMR}\left(101 \mathrm{MHz}, \mathrm{CDCl}_{3}\right) \delta 166.0(\mathrm{dd}, J=40.0,37.1 \mathrm{~Hz}), 162.0,135.3,130.3$, 129.3, 128.9, 128.1, 127.8, 126.8, 126.6, $116.3(\mathrm{dd}, J=242.3,240.7 \mathrm{~Hz}), 100.9,22.8(\mathrm{dd}, J=30.7$, $27.6 \mathrm{~Hz}), 21.0(\mathrm{~d}, J=3.5 \mathrm{~Hz}), 5.7(\mathrm{dd}, J=5.1,2.0 \mathrm{~Hz}) .{ }^{19} \mathrm{~F} \mathrm{NMR}\left(376 \mathrm{MHz}, \mathrm{CDCl}_{3}\right) \delta-87.44(\mathrm{dd}, J$ $=273.1,12.4 \mathrm{~Hz}, 1 \mathrm{~F}),-95.45(\mathrm{dd}, J=273.1,14.1 \mathrm{~Hz}, 1 \mathrm{~F}) . \mathrm{MS}(\mathrm{EI}): \mathrm{m} / \mathrm{z}(\%) 311\left(\mathrm{M}^{+}\right), 222,206$, 144, 117, 77 (100). HRMS: Calculated for $\mathrm{C}_{19} \mathrm{H}_{15} \mathrm{NOF}_{2}$ : 311.1122; Found: 311.1129. Enantiomeric ratio (98:2 er) was measured by chiral HPLC on PC-3 column $\left(\mathrm{CH}_{3} \mathrm{CN} / \mathrm{H}_{2} \mathrm{O}=70: 30,0.7 \mathrm{~mL} / \mathrm{min}\right.$, $\mathrm{UV}$ detection at $214 \mathrm{~nm}$ ); retention time $=11.40 \mathrm{~min}$ (major), retention time $=15.22 \mathrm{~min}($ minor $)$.

\section{Synthesis of compounds $14 \mathrm{e}$}

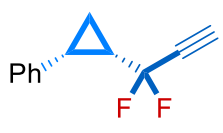

$13,97: 3$ er

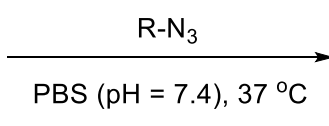

$\operatorname{PBS}(\mathrm{pH}=7.4), 37^{\circ} \mathrm{C}$

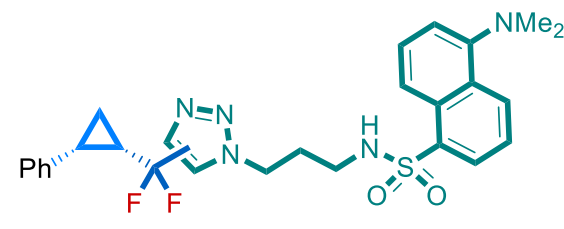

14 e $88 \%$ (regioisomers $6.3: 1$ )

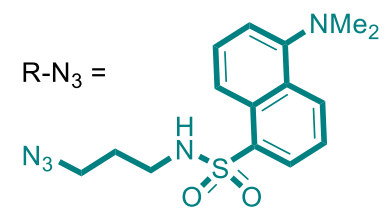

$N$-(3-(4/5-(Difluoro((1S,2R)-(2-phenylcyclopropyl)methyl)-1H-1,2,3-triazol-1-yl)propyl)-5-(dim

ethylamino)naphthalene-1-sulfonamide (14e). To a $25 \mathrm{~mL}$ of Schlenk tube were added alkyne 13 (97 mg, $0.5 \mathrm{mmol}, 1.0$ equiv), Dans $\left(\mathrm{CH}_{2}\right)_{3}-\mathrm{N}_{3}(251 \mathrm{mg}, 0.75 \mathrm{mmol}, 1.5$ equiv), and phosphate 
buffered saline (PBS, $\mathrm{pH}=7.4)(1.5 \mathrm{~mL})$. The tube was screw capped and then vigorously stirred at $37^{\circ} \mathrm{C}$ (oil bath) for $48 \mathrm{~h}$. The reaction mixture was then diluted with water and extracted with EtOAc. The organic layers were dried over $\mathrm{Na}_{2} \mathrm{SO}_{4}$, filtered and concentrated. The resulting crude product was purified by flash chromatography (Pentane/EtOAc $=5: 1$ to $1: 1$ ) to afford the desired products 14e-1 and 14e-2 (236 mg, 88\% combined yield) as a mixture of regioisomers (6:1). ${ }^{1} \mathrm{H}$ NMR (400 $\left.\mathrm{MHz}, \mathrm{CDCl}_{3}\right) \delta 8.53(\mathrm{~d}, J=8.4 \mathrm{~Hz}, 1 \mathrm{H}), 8.28(\mathrm{t}, J=8.5 \mathrm{~Hz}, 1 \mathrm{H}), 8.19(\mathrm{~d}, J=7.1 \mathrm{~Hz}, 1 \mathrm{H}), 7.68(\mathrm{~s}$, $0.3 \mathrm{H}), 7.57-7.45(\mathrm{~m}, 2 \mathrm{H}), 7.21-7.00(\mathrm{~m}, 6 \mathrm{H}), 6.86(\mathrm{~s}, 0.7 \mathrm{H}), 5.71(\mathrm{t}, J=5.8 \mathrm{~Hz}, 0.7 \mathrm{H}), 5.41(\mathrm{t}$, $J=6.0 \mathrm{~Hz}, 0.3 \mathrm{H}), 4.28-4.10(\mathrm{~m}, 2 \mathrm{H}), 2.92-2.77(\mathrm{~m}, 7 \mathrm{H}), 2.54-2.47$ (m, 0.3 H), 2.43- 2.35 (m, $0.7 \mathrm{H}), 2.24-2.13(\mathrm{~m}, 0.7 \mathrm{H}), 2.04-1.79(\mathrm{~m}, 2.3 \mathrm{H}), 1.64-1.57$ (m, $0.7 \mathrm{H}), 1.57-1.50(\mathrm{~m}, 0.3 \mathrm{H})$, $1.34-1.28(\mathrm{~m}, 0.3 \mathrm{H}), 1.27-1.20(\mathrm{~m}, 0.7 \mathrm{H}) .{ }^{13} \mathrm{C} \mathrm{NMR}\left(101 \mathrm{MHz}, \mathrm{CDCl}_{3}\right)($ Note: signals for minor regioisomer are in curved parentheses \{\}$) \delta 151.99,\{151.89\}, 144.75(\mathrm{t}, J=34.5 \mathrm{~Hz}), 136.07,134.87$, 134.51, 134.23, 133.89 - 133.58 (m), 130.61, \{130.43\}, 129.75, 129.51, 129.34, 129.29, 129.16, $128.49,\{128.43\}, 127.86,127.43,126.59,126.02,123.09,\{123.05\}, 122.12,\{118.5\}, 118.4,118.25$ $(\mathrm{t}, J=236.4 \mathrm{~Hz}), 115.20,\{115.17\}, 46.9,\{46.6\},\{45.26\}, 45.23,\{39.9\}, 39.7,29.9,\{29.7\}, 23.6(\mathrm{dd}$, $J=31.5,29.5 \mathrm{~Hz}),\{23.2(\mathrm{dd}, J=28.3,27.0 \mathrm{~Hz})\},\{21.31(\mathrm{~d}, J=3.9 \mathrm{~Hz})\}, 20.6(\mathrm{~d}, J=1.5 \mathrm{~Hz}), 5.92(\mathrm{~d}$, $J=4.5 \mathrm{~Hz}),\{5.7(\mathrm{dd}, J=3.7,2.5 \mathrm{~Hz})\} .{ }^{19} \mathrm{~F} \mathrm{NMR}\left(376 \mathrm{MHz}, \mathrm{CDCl}_{3}\right) \delta-82.43(\mathrm{dd}, J=273.8,13.8 \mathrm{~Hz})$, $-83.09(\mathrm{dd}, J=267.5,12.3 \mathrm{~Hz}),-89.40(\mathrm{dd}, J=267.0,16.1 \mathrm{~Hz}),-94.33(\mathrm{dd}, J=272.6,16.9 \mathrm{~Hz}) . \mathrm{MS}$ (ESI): m/z (\%) $526\left([\mathrm{M}+\mathrm{H}]^{+}\right)$; HRMS: Calculated for $\mathrm{C}_{27} \mathrm{H}_{30} \mathrm{O}_{2} \mathrm{~N}_{2} \mathrm{~F}_{2} \mathrm{~S}$ : 526.2083; Found: 526.2086.

\subsection{Transformations of compound $5 \mathrm{~h}$}

\section{Synthesis of compound 15a}

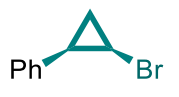

$5 \mathrm{~h}, 92: 8$ er

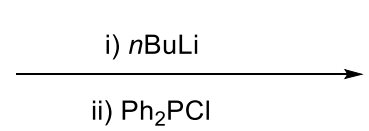

ii) $\mathrm{Ph}_{2} \mathrm{PCl}$

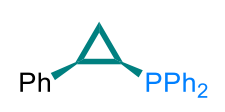

$15 a, 61 \%, 94: 6$ er

Diphenyl((1R,2R)-2-phenylcyclopropyl)phosphine (15a). To a solution of $\mathbf{5 h}(60 \mathrm{mg}, 0.3 \mathrm{mmol}$, 1.0 equiv) in anhydrous THF (1.5 mL) was added $n \mathrm{BuLi}$ (2.4 M in hexane, $0.15 \mathrm{~mL}, 0.36 \mathrm{mmol}, 1.2$ equiv) under $\mathrm{Ar}$ at $-78{ }^{\circ} \mathrm{C}$. After the reaction mixture was stirred at $-78{ }^{\circ} \mathrm{C}$ for $60 \mathrm{~min}$, chlorodiphenylphosphine ( $265 \mathrm{mg}, 1.2 \mathrm{mmol}, 4.0$ equiv) was added to the reaction mixture at the same temperature. The reaction mixture was then warmed to room temperature slowly and stirred overnight. The reaction mixture was quenched with saturated aqueous $\mathrm{NH}_{4} \mathrm{Cl}$. The organic layer was separated and the aqueous layer was extracted with $\mathrm{CH}_{2} \mathrm{Cl}_{2}$ (three times). The combined organic 
layers were washed with brine, dried over $\mathrm{Na}_{2} \mathrm{SO}_{4}$, filtered, and concentrated. The product 15a (55 $\mathrm{mg}, 61 \%$ yield) was purified with silica gel chromatography (Petroleum ether/DCM $=20: 1$ ) as a colorless oil. $[\alpha]_{\mathrm{D}}{ }^{20}=72.00\left(c=0.42, \mathrm{CHCl}_{3}\right)$ for a sample with 94:6 er. ${ }^{1} \mathrm{H} \mathrm{NMR}\left(400 \mathrm{MHz}, \mathrm{CDCl}_{3}\right)$ $\delta 7.50-7.42(\mathrm{~m}, 2 \mathrm{H}), 7.35-7.27$ (m, $3 \mathrm{H}), 7.27$ - $7.14(\mathrm{~m}, 10 \mathrm{H}), 2.63-2.55(\mathrm{~m}, 1 \mathrm{H}), 1.76-1.65$ $(\mathrm{m}, 1 \mathrm{H}), 1.50-1.36(\mathrm{~m}, 2 \mathrm{H}) .{ }^{13} \mathrm{C} \mathrm{NMR}\left(101 \mathrm{MHz}, \mathrm{CDCl}_{3}\right) \delta 139.8(\mathrm{~d}, J=10.7 \mathrm{~Hz}), 139.2(\mathrm{~d}, J=$ $10.7 \mathrm{~Hz}), 138.5(\mathrm{~d}, J=6.7 \mathrm{~Hz}), 132.6(\mathrm{~d}, J=16.5 \mathrm{~Hz}), 132.4(\mathrm{~d}, J=16.3 \mathrm{~Hz}), 129.0(\mathrm{~d}, J=3.4 \mathrm{~Hz})$, $128.3-127.9$ (m), 127.6, 126.1, 22.4 (d, $J=7.1 \mathrm{~Hz}), 18.6$ (d, $J=4.6 \mathrm{~Hz}), 10.7$ (d, $J=12.8 \mathrm{~Hz}) .{ }^{31} \mathrm{P}$ NMR (162 MHz, $\left.\mathrm{CDCl}_{3}\right) \delta$-15.0 (s). MS (EI): m/z (\%) $302\left(\mathrm{M}^{+}\right), 198,117$. HRMS: Calculated for $\mathrm{C}_{21} \mathrm{H}_{19} \mathrm{P}: 302.1224$; Found: 302.1221. Enantiomeric ratio (94:6 er) was measured by chiral HPLC on PC-3 column $\left(\mathrm{CH}_{3} \mathrm{CN} / \mathrm{H}_{2} \mathrm{O}=70: 30,0.7 \mathrm{~mL} / \mathrm{min}, \mathrm{UV}\right.$ detection at $\left.214 \mathrm{~nm}\right)$; retention time $=28.44$ $\min$ (major), retention time $=16.53 \mathrm{~min}$ (minor).

\section{Synthesis of compound $15 \mathrm{~b}$}

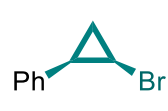

5h, 92:8 er

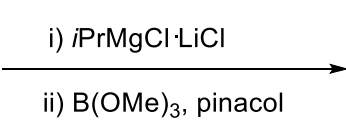

ii) $\mathrm{B}(\mathrm{OMe})_{3}$, pinacol

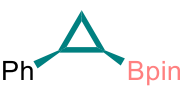

15b, 58\%, 93:7 er

4,4,5,5-Tetramethyl-2-((1R,2S)-2-phenylcyclopropyl)-1,3,2-dioxaborolane $(15 b)^{13}$. A dry and argon-flushed $25-\mathrm{mL}$ Schlenk tube was charged with $i \mathrm{PrMgCl} \cdot \mathrm{LiCl}(1.1 \mathrm{M}$ in THF, $0.5 \mathrm{~mL}, 0.55$ mmol, 1.1 equiv) and anhydrous 1,4-dioxane (50 uL). Compound $\mathbf{5 h}$ (99 mg, 0.5 mmol, 1.0 equiv) was then added at room temperature. The resulting mixture was stirred at room temperature for $8 \mathrm{~h}$. The reaction was then cooled to $-78{ }^{\circ} \mathrm{C}$ and a solution of trimethyl borate $(63 \mathrm{mg}, 0.6 \mathrm{mmol}, 1.2$ equiv) in anhydrous THF (1 mL) was added. The reaction mixture was warmed to room temperature and stirred overnight. Then, a solution of pinacol (71 mg, $0.6 \mathrm{mmol}, 1.2$ equiv) in anhydrous THF (1 $\mathrm{mL})$ was added. After the reaction was stirred for $15 \mathrm{~min}$, glacial acetic acid (1.5 mL) was added and the reaction mixture was stirred at room temperature for another $4 \mathrm{~h}$. The reaction was then quenched with saturated aqueous $\mathrm{NH}_{4} \mathrm{Cl}$. The organic layer was separated and the aqueous layer was extracted with $\mathrm{CH}_{2} \mathrm{Cl}_{2}$ (three times). The combined organic layers were washed with brine, dried over $\mathrm{Na}_{2} \mathrm{SO}_{4}$, filtered, and concentrated. The residue was purified with silica gel chromatography (Petroleum ether/EtOAc $=50: 1)$ to give $\mathbf{1 5 b}(71 \mathrm{mg}, 58 \%$ yield $)$ as a colorless oil. $[\alpha]_{\mathrm{D}}{ }^{20}=-55.82(c=0.57$, $\mathrm{CHCl}_{3}$ ) for a sample with 93:7 er. ${ }^{1} \mathrm{H} \mathrm{NMR}\left(400 \mathrm{MHz}, \mathrm{CDCl}_{3}\right) \delta 7.27(\mathrm{~d}, J=7.3 \mathrm{~Hz}, 2 \mathrm{H}), 7.21(\mathrm{t}, J$ $=7.5 \mathrm{~Hz}, 2 \mathrm{H}), 7.12(\mathrm{t}, J=7.1 \mathrm{~Hz}, 1 \mathrm{H}), 2.35(\mathrm{dt}, J=10.0,7.6 \mathrm{~Hz}, 1 \mathrm{H}), 1.30-1.26(\mathrm{~m}, 1 \mathrm{H}), 1.10$ 
$(\mathrm{td}, J=8.7,4.2 \mathrm{~Hz}, 1 \mathrm{H}), 1.05-0.96(\mathrm{~m}, 6 \mathrm{H}), 0.88(\mathrm{~s}, 6 \mathrm{H}), 0.45(\mathrm{td}, J=9.6,7.3 \mathrm{~Hz}, 1 \mathrm{H}) .{ }^{13} \mathrm{C}$ NMR $\left(101 \mathrm{MHz}, \mathrm{CDCl}_{3}\right) \delta 140.7,128.8,127.6,125.7,82.9,24.7,24.4,21.7,8.9 .{ }^{11} \mathrm{~B}$ NMR $(193$ $\left.\mathrm{MHz}, \mathrm{CDCl}_{3}\right) \delta 32.2$ (br). MS (EI): m/z (\%) $244\left(\mathrm{M}^{+}\right), 229,186,145,117$. HRMS: Calculated for $\mathrm{C}_{15} \mathrm{H}_{21}{ }^{10} \mathrm{BO}_{2}$ : 243.1671; Found: 243.1678. Enantiomeric ratio (93:7 er) was measured by chiral SFC on $\mathrm{OJH}$ column $\left(\mathrm{CO}_{2} / \mathrm{MeOH}=94: 6\right.$, BP Pressure: 2000 psi, $1.0 \mathrm{~mL} / \mathrm{min}$, column temperature: $40{ }^{\circ} \mathrm{C}$, $\mathrm{UV}$ detection at $214 \mathrm{~nm}$ ); retention time $=5.54 \mathrm{~min}$ (major), retention time $=5.81 \mathrm{~min}$ (minor).

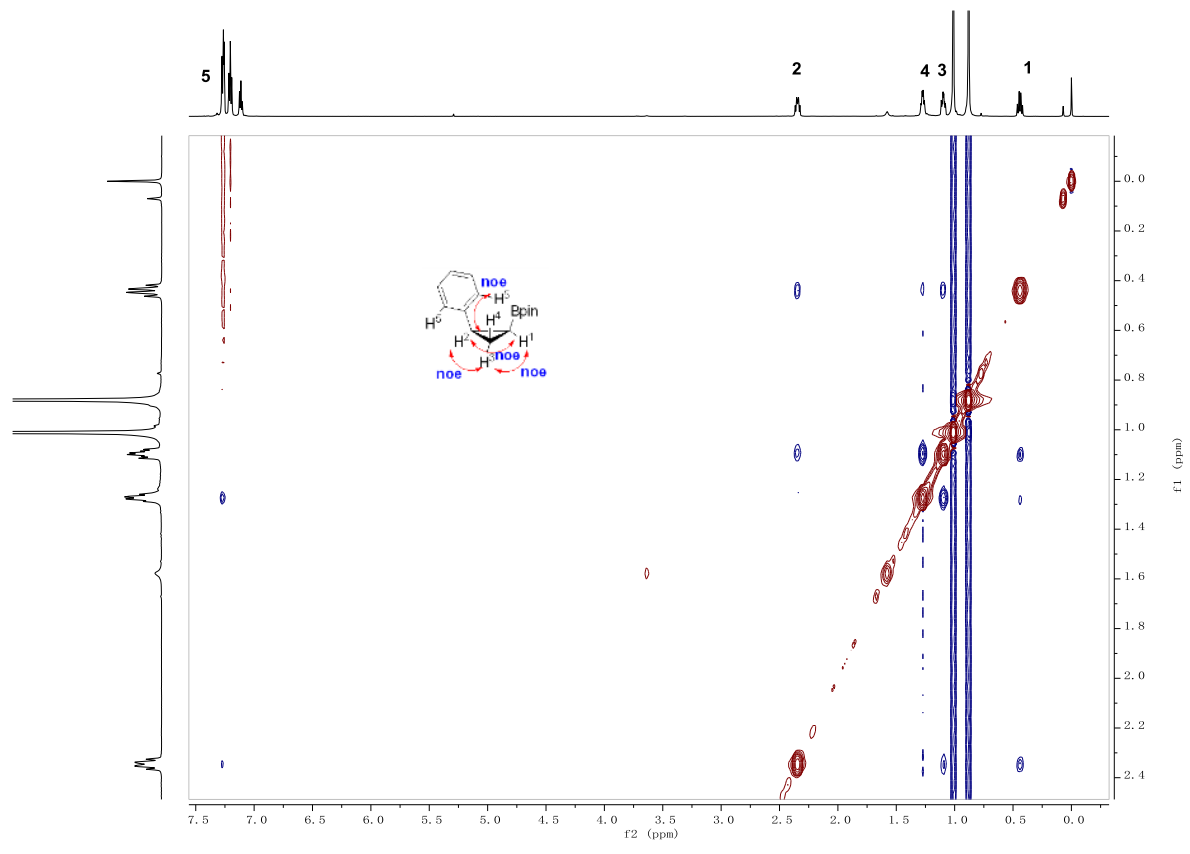

Figure S1. NOE spectrum of compound 15b

\section{Synthesis of compound 15c}

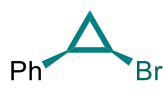

5 h, $92: 8$ er

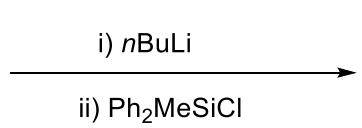

ii) $\mathrm{Ph}_{2} \mathrm{MeSiCl}$

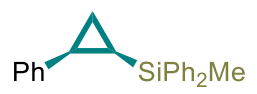

15c, $74 \%, 93: 7$ er

Methyldiphenyl((1R,2R)-2-phenylcyclopropyl)silane (15c). To a solution of $5 \mathbf{h}(60 \mathrm{mg}, 0.3 \mathrm{mmol}$, 1.0 equiv) in anhydrous THF (1.5 mL) was added $n \mathrm{BuLi}(2.4 \mathrm{M}$ in hexane, $0.15 \mathrm{~mL}, 0.36 \mathrm{mmol}, 1.2$ equiv) under $\mathrm{Ar}$ at $-78{ }^{\circ} \mathrm{C}$. After the reaction mixture was stirred at $-78{ }^{\circ} \mathrm{C}$ for $60 \mathrm{~min}$, a solution of chlorodiphenylmethylsilane (280 mg, $1.2 \mathrm{mmol}, 4.0$ equiv) in anhydrous THF (1.5 mL) was added at the same temperature. The reaction mixture was warmed to room temperature slowly and stirred overnight. The reaction mixture was quenched with saturated aqueous $\mathrm{NH}_{4} \mathrm{Cl}$. The organic layer was separated and the aqueous layer was extracted with $\mathrm{CH}_{2} \mathrm{Cl}_{2}$ (three times). The combined organic layers were washed with brine, dried over $\mathrm{Na}_{2} \mathrm{SO}_{4}$, filtered, and concentrated. The product $15 \mathrm{c}$ (70 
mg, 74\% yield) was purified with silica gel chromatography (Petroleum ether) as a colorless oil. $[\alpha]_{\mathrm{D}}^{20}=-49.04\left(c=0.67, \mathrm{CHCl}_{3}\right)$ for a sample with 93:7 er. ${ }^{1} \mathrm{H} \mathrm{NMR}\left(400 \mathrm{MHz}, \mathrm{CDCl}_{3}\right) \delta 7.44(\mathrm{~d}, J$ $=6.8 \mathrm{~Hz}, 2 \mathrm{H}), 7.39-7.24(\mathrm{~m}, 8 \mathrm{H}), 7.10-7.03(\mathrm{~m}, 3 \mathrm{H}), 7.01-6.95(\mathrm{~m}, 2 \mathrm{H}), 2.53-2.45(\mathrm{~m}, 1 \mathrm{H})$, $1.35-1.28(\mathrm{~m}, 1 \mathrm{H}), 1.14-1.09(\mathrm{~m}, 1 \mathrm{H}), 0.69-0.60(\mathrm{~m}, 1 \mathrm{H}),-0.07$ (s, $3 \mathrm{H}) .{ }^{13} \mathrm{C}$ NMR $(101 \mathrm{MHz}$, $\left.\mathrm{CDCl}_{3}\right) \delta 140.2,137.9,137.7,134.60,134.58,129.5,128.9,128.8,127.7,127.6,127.5,125.9,21.0$, 7.6, 4.6, -4.8. MS (DART): m/z (\%) $315\left([\mathrm{M}+\mathrm{H}]^{+}, 100\right), 237$. HRMS: Calculated for $\mathrm{C}_{22} \mathrm{H}_{23} \mathrm{Si}$ : 315.1564; Found: 315.1565. Enantiomeric ratio (93:7 er) was measured by chiral HPLC on PC-3 column $\left(\mathrm{CH}_{3} \mathrm{CN} / \mathrm{H}_{2} \mathrm{O}=70: 30,0.7 \mathrm{~mL} / \mathrm{min}\right.$, UV detection at $\left.214 \mathrm{~nm}\right)$; retention time = $17.30 \mathrm{~min}$ (major), retention time $=15.92 \mathrm{~min}$ (minor).

\section{Synthesis of compound $15 d$}

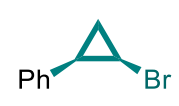

$5 \mathrm{~h}, 92: 8 \mathrm{er}$

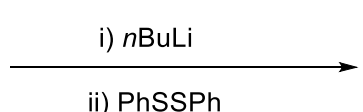

ii) PhSSPh

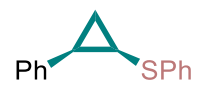

15d, $90 \%, 90: 10$ er

Phenyl((1R,2R)-2-phenylcyclopropyl)sulfane (15d). To a solution of 5 h $(60 \mathrm{mg}, 0.3 \mathrm{mmol}, 1.0$ equiv) in anhydrous THF (1.5 mL) was added $n \mathrm{BuLi}(2.4 \mathrm{M}$ in hexane; $0.15 \mathrm{~mL}, 0.36 \mathrm{mmol}, 1.2$ equiv) under Ar at $-78{ }^{\circ} \mathrm{C}$. After the reaction mixture was stirred at $-78{ }^{\circ} \mathrm{C}$ for $60 \mathrm{~min}$, a solution of diphenyl disulfide (131 mg, $0.6 \mathrm{mmol}, 2.0$ equiv) in anhydrous THF (1.5 mL) was then added at the same temperature. The reaction mixture was warmed to room temperature slowly and stirred overnight. The reaction mixture was quenched with saturated aqueous $\mathrm{NH}_{4} \mathrm{Cl}$. The organic layer was separated and the aqueous layer was extracted with $\mathrm{CH}_{2} \mathrm{Cl}_{2}$ (three times). The combined organic layers were washed with brine, dried over $\mathrm{Na}_{2} \mathrm{SO}_{4}$, filtered, and concentrated. The product $\mathbf{1 5 d}$ (61 mg, $90 \%$ yield) was purified with silica gel chromatography (Petroleum ether) as a yellow oil. $[\alpha]_{\mathrm{D}}^{20}$ $=60.89\left(c=0.59, \mathrm{CHCl}_{3}\right)$ for a sample with 90:10 er. ${ }^{1} \mathrm{H} \mathrm{NMR}\left(400 \mathrm{MHz}, \mathrm{CDCl}_{3}\right) \delta 7.25-7.15(\mathrm{~m}$, $9 \mathrm{H}), 7.09-7.04(\mathrm{~m}, 1 \mathrm{H}), 2.64(\mathrm{td}, J=7.9,5.4 \mathrm{~Hz}, 1 \mathrm{H}), 2.62-2.55(\mathrm{~m}, 1 \mathrm{H}), 1.63-1.54(\mathrm{~m}, 1 \mathrm{H})$, $1.23(\mathrm{dt}, J=6.7,5.5 \mathrm{~Hz}, 1 \mathrm{H}) .{ }^{13} \mathrm{C} \mathrm{NMR}\left(101 \mathrm{MHz}, \mathrm{CDCl}_{3}\right) \delta 137.9,136.8,129.0,128.5,127.6$, 127.1, 126.4, 125.0, 23.7, 21.7, 13.3. MS (EI): m/z (\%) $226\left(\mathrm{M}^{+}\right), 117,91,77$. HRMS: Calculated for $\mathrm{C}_{15} \mathrm{H}_{14} \mathrm{~S}$ : 226.0816; Found: 226.0818. Enantiomeric ratio (90:10 er) was measured by chiral HPLC on $\mathrm{OJH}$ column $(\mathrm{Hexane} / \mathrm{PrOH}=90: 10,0.7 \mathrm{~mL} / \mathrm{min}$, UV detection at $214 \mathrm{~nm})$; retention time = $20.06 \mathrm{~min}$ (major), retention time $=24.73 \mathrm{~min}$ (minor). 


\section{Synthesis of compound $15 \mathrm{e}$}

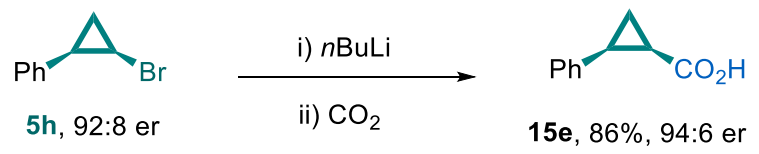

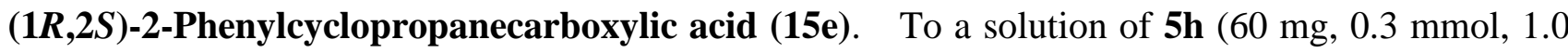
equiv) in anhydrous THF (1.5 mL) was added $n \mathrm{BuLi}(2.4 \mathrm{M}$ in hexane; $0.15 \mathrm{~mL}, 0.36 \mathrm{mmol}, 1.2$ equiv) under Ar at $-78{ }^{\circ} \mathrm{C}$. After the reaction mixture was stirred at $-78{ }^{\circ} \mathrm{C}$ for $60 \mathrm{~min}, \mathrm{CO}_{2}$ was bubbled into the reaction mixture at the same temperature for $30 \mathrm{~min}$. The reaction was then stirred under $1 \mathrm{~atm}$ of $\mathrm{CO}_{2}$ at room temperature for $16 \mathrm{~h}$. The reaction was quenched with saturated aqueous $\mathrm{HCl}(10 \% \mathrm{v} / \mathrm{v}, 10 \mathrm{~mL})$ and diluted with $\mathrm{CH}_{2} \mathrm{Cl}_{2}$. The organic layer was separated and the aqueous layer was extracted with $\mathrm{CH}_{2} \mathrm{Cl}_{2}$ (three times). The combined organic layers were washed with brine, dried over $\mathrm{Na}_{2} \mathrm{SO}_{4}$, filtered, and concentrated. The product $15 \mathrm{e}$ (70 $\mathrm{mg}, 86 \%$ yield) was purified with silica gel chromatography (Petroleum ether/EA $=5: 1$ ) as a white solid (m.p. $102-104{ }^{\circ} \mathrm{C}$ ). $[\alpha]_{\mathrm{D}}{ }^{20}=$ -23.56 $\left(c=0.95, \mathrm{CHCl}_{3}\right)$ for a sample with 94:6 er. ${ }^{1} \mathrm{H} \mathrm{NMR}\left(400 \mathrm{MHz}, \mathrm{CDCl}_{3}\right) \delta 11.11(\mathrm{br}, 1 \mathrm{H})$, $7.27-7.13(\mathrm{~m}, 5 \mathrm{H}), 2.64-2.54(\mathrm{~m}, 1 \mathrm{H}), 2.04-1.95(\mathrm{~m}, 1 \mathrm{H}), 1.67-1.59(\mathrm{~m}, 1 \mathrm{H}), 1.37-1.29(\mathrm{~m}$, $1 \mathrm{H}) .{ }^{13} \mathrm{C} \mathrm{NMR}\left(101 \mathrm{MHz}, \mathrm{CDCl}_{3}\right) \delta 177.3,135.8,129.2,127.9,126.7,26.5,21.4,12.0 . \mathrm{MS}(\mathrm{EI})$ : m/z (\%) $162\left(\mathrm{M}^{+}\right)$, 144, 117 (100). HRMS: Calculated for $\mathrm{C}_{10} \mathrm{H}_{10} \mathrm{O}_{2}$ : 162.0681; Found: 162.0687. Enantiomeric ratio (94:6 er) was measured by chiral HPLC on IG column (Hexane/ ${ }^{i} \mathrm{PrOH}=94: 6,0.7$ $\mathrm{mL} / \mathrm{min}$, UV detection at $214 \mathrm{~nm}$ ); retention time $=13.87$ min (major), retention time $=12.55 \min$ (minor).

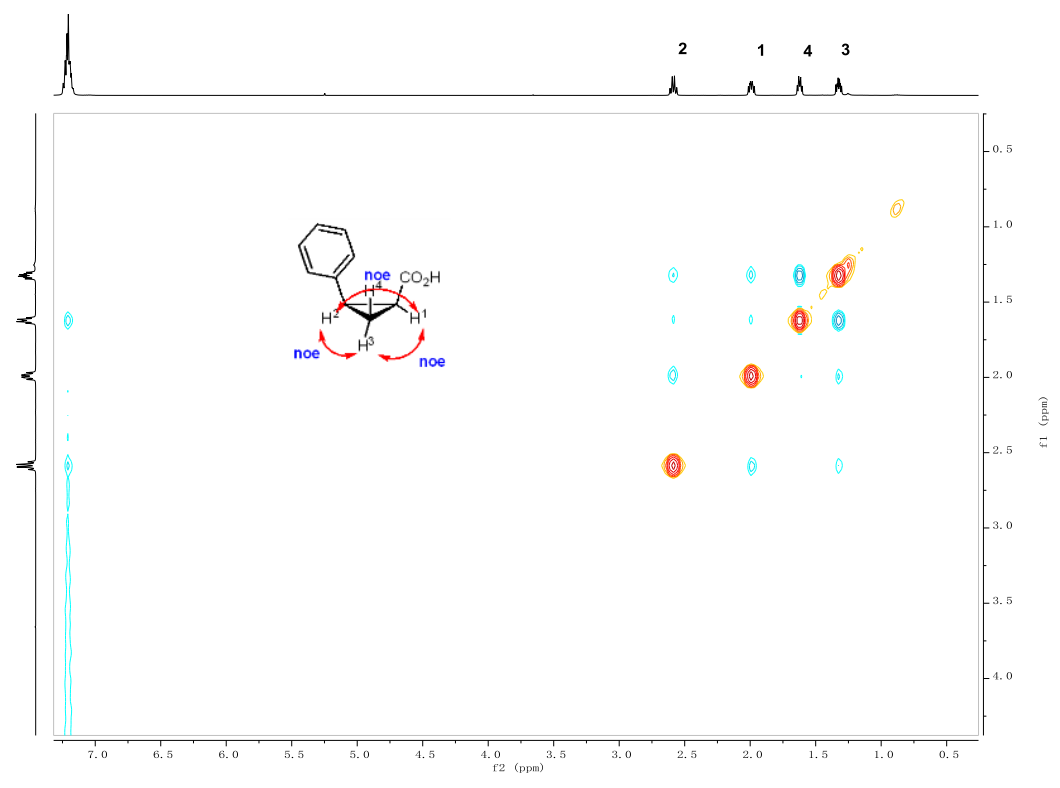

Figure S2. NOE spectrum of compound 15e 


\section{Synthesis of compound $15 f$}

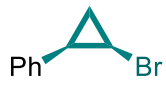

5 h, $92: 8$ er

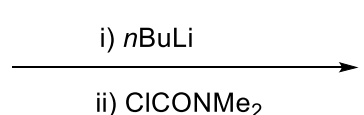

ii) $\mathrm{CICONMe} 2$

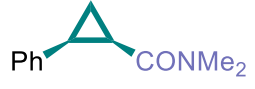

$15 f, 81 \%, 95: 5$ er

$(\mathbf{1 R}, 2 S)-N, \boldsymbol{N}$-Dimethyl-2-phenylcyclopropanecarboxamide (15f). To a solution of $\mathbf{5 h}$ (60 $\mathrm{mg}, 0.3$ mmol, 1.0 equiv) in anhydrous THF $(1.5 \mathrm{~mL})$ was added $n \mathrm{BuLi}(2.4 \mathrm{M}$ in hexane; $0.15 \mathrm{~mL}, 0.36$ mmol, 1.2 equiv) under $\mathrm{Ar}$ at $-78{ }^{\circ} \mathrm{C}$. The reaction mixture was stirred at $-78{ }^{\circ} \mathrm{C}$ for $60 \mathrm{~min}$, after which a solution of dimethylcarbamoyl chloride (129 mg, $1.2 \mathrm{mmol}, 4.0$ equiv) in anhydrous THF $(1.5 \mathrm{~mL})$ was then added at $-78{ }^{\circ} \mathrm{C}$. The reaction mixture was warmed to room temperature slowly and stirred overnight. The reaction mixture was quenched with saturated aqueous $\mathrm{NH}_{4} \mathrm{Cl}$ and diluted with $\mathrm{CH}_{2} \mathrm{Cl}_{2}$. The organic layer was separated and the aqueous layer was extracted with $\mathrm{CH}_{2} \mathrm{Cl}_{2}$ (three times). The combined organic layers were washed with brine, dried over $\mathrm{Na}_{2} \mathrm{SO}_{4}$, filtered, and concentrated. The product $\mathbf{1 5 f}$ (46 $\mathrm{mg}, 81 \%$ yield) was purified with silica gel chromatography (Petroleum ether/EA $=2: 1)$ as a white solid (m.p. $\left.77-78{ }^{\circ} \mathrm{C}\right) .[\alpha]_{\mathrm{D}}{ }^{20}=78.15\left(c=0.41, \mathrm{CHCl}_{3}\right)$ for a sample with 95:5 er. ${ }^{1} \mathrm{H}$ NMR (400 MHz, $\left.\mathrm{CDCl}_{3}\right) \delta 7.25-7.19(\mathrm{~m}, 2 \mathrm{H}), 7.18-7.10(\mathrm{~m}, 3 \mathrm{H}), 2.97$ (s, $3 \mathrm{H}), 2.72(\mathrm{~s}, 3 \mathrm{H}), 2.49-2.40(\mathrm{~m}, 1 \mathrm{H}), 2.19-2.11(\mathrm{~m}, 1 \mathrm{H}), 1.84-1.77(\mathrm{~m}, 1 \mathrm{H}), 1.31-1.24$ $(\mathrm{m}, 1 \mathrm{H}) .{ }^{13} \mathrm{C} \mathrm{NMR}\left(101 \mathrm{MHz}, \mathrm{CDCl}_{3}\right) \delta 168.8,137.4,127.9,127.6,126.1,36.8,35.2,24.0,23.9$, 10.5. MS (EI): m/z (\%) $189\left(\mathrm{M}^{+}\right), 174,144,127,115,91,72$. HRMS: Calculated for $\mathrm{C}_{12} \mathrm{H}_{15} \mathrm{NO}$ : 189.1154; Found: 189.1160. Enantiomeric ratio (95:5 er) was measured by chiral HPLC on IB column $\left(\mathrm{Hexane} /{ }^{i} \mathrm{PrOH}=96: 4,0.7 \mathrm{~mL} / \mathrm{min}\right.$, UV detection at $\left.214 \mathrm{~nm}\right) ;$ retention time $=25.18 \mathrm{~min}$ (major), retention time $=30.78 \min ($ minor $)$. 


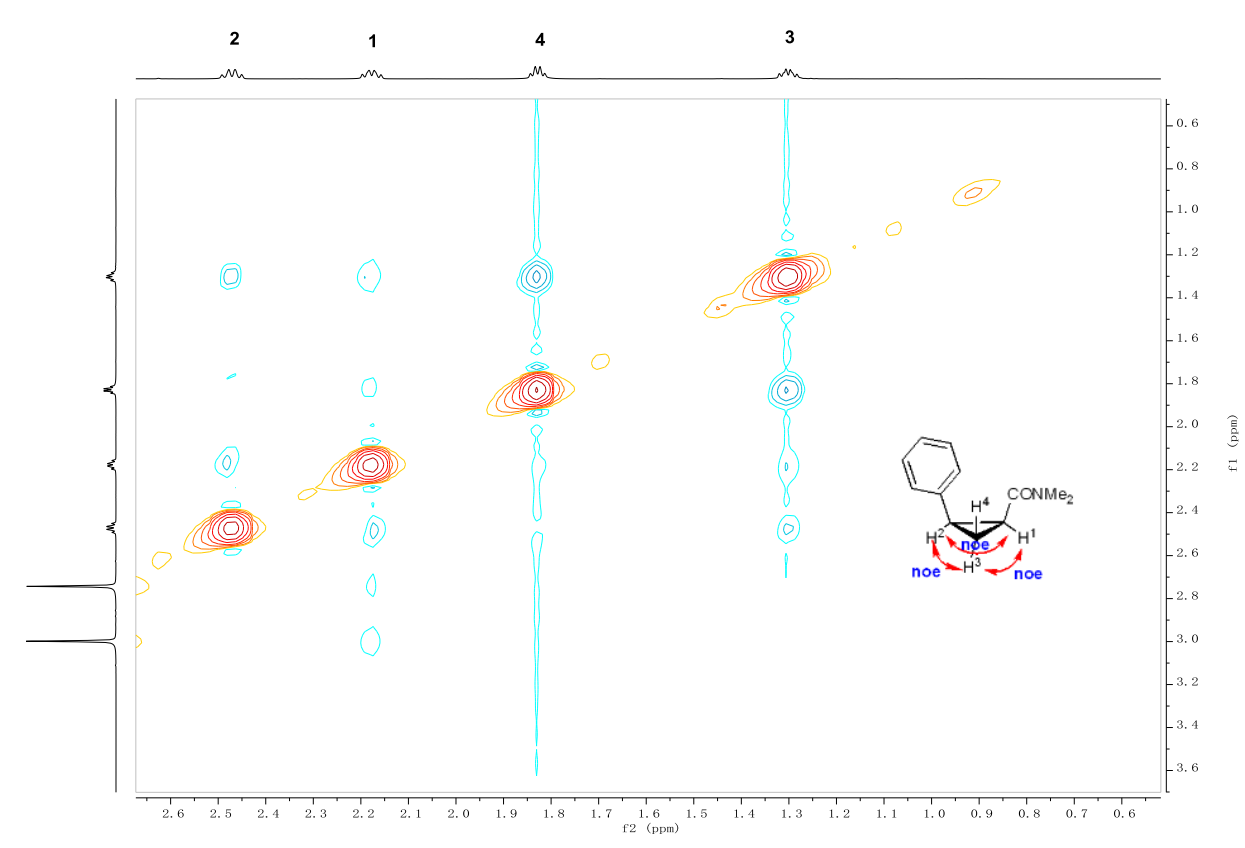

Figure S3. NOE spectrum of compound $15 \mathrm{e}$

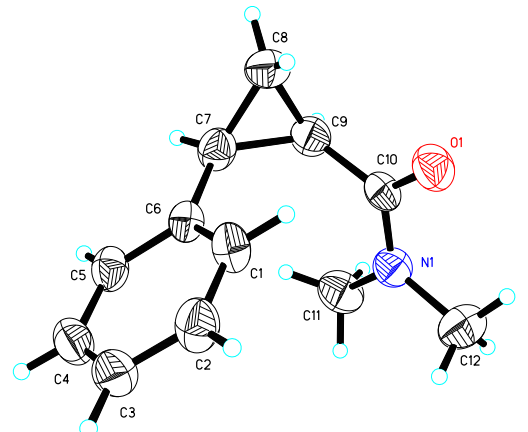

Figure S4. X-Ray Crystal Structure of Compound $15 f$

\section{Synthesis of compound 15g}

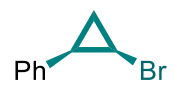

5h, $92: 8$ er

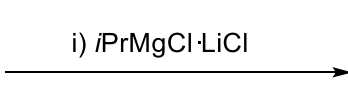

ii) Tosyl cyanide

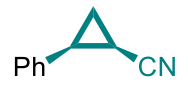

15g, $42 \%, 94: 6$ er

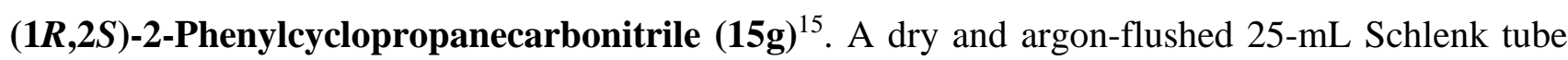
was charged with $i \mathrm{PrMgCl} \cdot \mathrm{LiCl}(1.1 \mathrm{M}$ in THF, $0.5 \mathrm{~mL}, 0.55 \mathrm{mmol}, 1.1$ equiv) and anhydrous 1,4-dioxane (50 uL). Compound $\mathbf{5 h}(99 \mathrm{mg}, 0.5 \mathrm{mmol}, 1.0$ equiv) was then added at room temperature. The resulting mixture was stirred at room temperature for $8 \mathrm{~h}$. The reaction was then cooled to $-40{ }^{\circ} \mathrm{C}$ and a solution of $p$-toluenesulfonyl cyanide $(181 \mathrm{mg}, 1.0 \mathrm{mmol}, 2.0$ equiv) in 
anhydrous THF (1 mL) was added. The resulting mixture was warmed to room temperature slowly and stirred overnight. The reaction mixture was filtered through a pad of celite, the filtrate was concentrated. The product $\mathbf{1 5 g}$ (30 $\mathrm{mg}, 42 \%$ yield) was purified with silica gel chromatography (Petroleum ether/EA $=10: 1)$ as a colorless crystal (m.p. $\left.40-41{ }^{\circ} \mathrm{C}\right) .[\alpha]_{\mathrm{D}}{ }^{20}=-20.00\left(c=0.47, \mathrm{CHCl}_{3}\right)$ for a sample with 94:6 er. ${ }^{1} \mathrm{H}$ NMR $\left(400 \mathrm{MHz}, \mathrm{CDCl}_{3}\right) \delta 7.40-7.34(\mathrm{~m}, 2 \mathrm{H}), 7.34-7.27(\mathrm{~m}, 3 \mathrm{H})$, $2.59-2.49(\mathrm{~m}, 1 \mathrm{H}), 1.88-1.80(\mathrm{~m}, 1 \mathrm{H}), 1.60-1.47(\mathrm{~m}, 2 \mathrm{H}) .{ }^{13} \mathrm{C} \mathrm{NMR}\left(101 \mathrm{MHz}, \mathrm{CDCl}_{3}\right) \delta$ 135.1, 128.5, 127.9, 127.5, 119.4, 23.1, 12.8, 6.3. MS (EI): m/z (\%) $143\left(\mathrm{M}^{+}\right), 115$ (100). HRMS: Calculated for $\mathrm{C}_{10} \mathrm{H}_{9} \mathrm{~N}$ : 143.0735; Found: 143.0734. Enantiomeric ratio (94:6 er) was measured by chiral HPLC on IB column $\left(\mathrm{Hexane} /{ }^{i} \mathrm{PrOH}=94: 6,0.7 \mathrm{~mL} / \mathrm{min}\right.$, UV detection at $214 \mathrm{~nm}$ ); retention time $=16.33 \mathrm{~min}$ (major), retention time $=15.67 \mathrm{~min}$ (minor) . 


\section{Crystal data and structure refinement for compounds 5a, 5g, 6, 11, 12, and $15 f$}

Compound 5a: (The crystal structure of compound 5a has been deposited at the Cambridge Crystallographic Data Centre (CCDC 1974493). The data is available free of charge at www.ccdc.cam.ac.uk/conts/retrieving.html.)
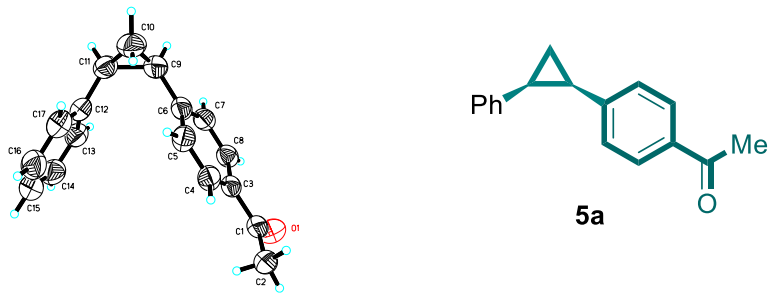

\section{Table S5. Crystal data and structure refinement for compound 5a}

Identification code

Empirical formula

Formula weight

Temperature

Wavelength

Crystal system

Space group

Unit cell dimensions

Volume

Z

Density (calculated)

Absorption coefficient

$\mathrm{F}(000)$

Crystal size

Theta range for data collection

Index ranges

Reflections collected

Independent reflections

Completeness to theta $=67.679^{\circ}$

Absorption correction

Max. and min. transmission

Refinement method

Data / restraints / parameters

Goodness-of-fit on $\mathrm{F}^{2}$

Final $\mathrm{R}$ indices [I $>2 \operatorname{sigma}(\mathrm{I})]$

$\mathrm{R}$ indices (all data)

Absolute structure parameter

Extinction coefficient

Largest diff. peak and hole cu_d8v18913_0m

C17 H16 O

236.30

296(2) K

$1.54178 \AA$

Monoclinic

$\mathrm{C} 2$

$\mathrm{a}=18.7374(6) \AA$

$\alpha=90^{\circ}$.

$\mathrm{b}=9.7813(3) \AA$

$\beta=92.345(2)^{\circ}$.

$\mathrm{c}=14.8207(5) \AA$

2714.01(15) $\AA^{3}$

8

$1.157 \mathrm{Mg} / \mathrm{m}^{3}$

$0.543 \mathrm{~mm}^{-1}$

1008

$0.200 \times 0.180 \times 0.150 \mathrm{~mm}^{3}$

2.984 to $66.990^{\circ}$.

$-22<=\mathrm{h}<=22,-11<=\mathrm{k}<=11,-17<=1<=17$

22471

$4779[\mathrm{R}(\mathrm{int})=0.0529]$

$97.2 \%$

Semi-empirical from equivalents

0.7533 and 0.4694

Full-matrix least-squares on $\mathrm{F}^{2}$

4779 / 1 / 328

1.028

$\mathrm{R} 1=0.0414, \mathrm{wR} 2=0.1038$

$\mathrm{R} 1=0.0503, \mathrm{wR} 2=0.1122$

$0.03(18)$

$0.0077(14)$

0.070 and -0.065 e. $\AA^{-3}$ 
Compound 5g: (The crystal structure of compound 5g has been deposited at the Cambridge Crystallographic Data

Centre (CCDC 1975646). The data is available free of charge at www.ccdc.cam.ac.uk/conts/retrieving.html.)

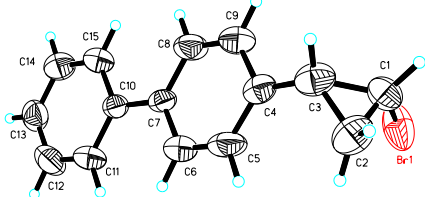<smiles>BrC1CC1c1ccc(-c2ccccc2)cc1</smiles>

Table S6. Crystal data and structure refinement for compound $5 \mathrm{~g}$

Identification code

Empirical formula

Formula weight

Temperature

Wavelength

Crystal system

Space group

Unit cell dimensions

Volume

$\mathrm{Z}$

Density (calculated)

Absorption coefficient

$\mathrm{F}(000)$

Crystal size

Theta range for data collection

Index ranges

Reflections collected

Independent reflections

Completeness to theta $=25.242^{\circ}$

Absorption correction

Max. and min. transmission

Refinement method

Data / restraints / parameters

Goodness-of-fit on $\mathrm{F}^{2}$

Final $\mathrm{R}$ indices [I $>2 \operatorname{sigma}(\mathrm{I})]$

$\mathrm{R}$ indices (all data)

Absolute structure parameter

Largest diff. peak and hole mo_d8v181022_0m

C15 H13 Br

273.16

293(2) K

$0.71073 \AA$

Orthorhombic

P 212121

$\mathrm{a}=5.6464(6) \AA \quad \alpha=90^{\circ}$.

$\mathrm{b}=8.7426(9) \AA \quad \beta=90^{\circ}$.

$\mathrm{c}=24.933(3) \AA \quad \gamma=90^{\circ}$.

$1230.8(2) \AA^{3}$

4

$1.474 \mathrm{Mg} / \mathrm{m}^{3}$

$3.309 \mathrm{~mm}^{-1}$

552

$0.180 \times 0.150 \times 0.050 \mathrm{~mm}^{3}$

2.469 to $25.987^{\circ}$.

$-6<=\mathrm{h}<=6,-10<=\mathrm{k}<=10,-30<=\mathrm{l}<=23$

5975

$2396[\mathrm{R}(\mathrm{int})=0.0629]$

$99.7 \%$

Semi-empirical from equivalents

0.7456 and 0.4435

Full-matrix least-squares on $\mathrm{F}^{2}$

2396 / 0 / 146

1.004

$\mathrm{R} 1=0.0470, \mathrm{wR} 2=0.1174$

$\mathrm{R} 1=0.0884, \mathrm{wR} 2=0.1409$

$0.114(18)$

0.348 and -0.297 e. $\AA^{-3}$ 
Compound 6: (The crystal structure of compound 6 has been deposited at the Cambridge Crystallographic Data Centre

(CCDC 1974494). The data is available free of charge at www.ccdc.cam.ac.uk/conts/retrieving.html.)

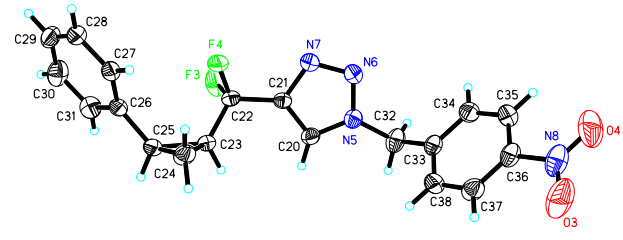<smiles>O=[N+]([O-])c1ccc(Cn2cc(C(F)(F)C3C[C@@H]3c3ccccc3)nn2)cc1</smiles>

Table S7. Crystal data and structure refinement for compound 6

Identification code

Empirical formula

Formula weight

Temperature

Wavelength

Crystal system

Space group

Unit cell dimensions

Volume

$\mathrm{Z}$

Density (calculated)

Absorption coefficient

$\mathrm{F}(000)$

Crystal size

Theta range for data collection

Index ranges

Reflections collected

Independent reflections

Completeness to theta $=67.679^{\circ}$

Absorption correction

Max. and min. transmission

Refinement method

Data / restraints / parameters

Goodness-of-fit on $\mathrm{F}^{2}$

Final $\mathrm{R}$ indices [I $>2 \operatorname{sigma}(\mathrm{I})]$

$\mathrm{R}$ indices (all data)

Absolute structure parameter

Extinction coefficient

Largest diff. peak and hole cu_d8v18156_0m

C19 H16 F2 N4 O2

370.36

296(2) K

$1.54178 \AA$

Triclinic

P 1

$\mathrm{a}=5.4853(3) \AA$ $\alpha=77.847(2)^{\circ}$.

$\mathrm{b}=9.2380(5) \AA$ $\beta=85.000(2)^{\circ}$.

$\mathrm{c}=17.4925(9) \AA$ $\gamma=86.063(2)^{\circ}$.

862.09(8) $\AA^{3}$

2

$1.427 \mathrm{Mg} / \mathrm{m}^{3}$

$0.931 \mathrm{~mm}^{-1}$

384

$0.200 \times 0.130 \times 0.070 \mathrm{~mm}^{3}$

4.903 to $66.982^{\circ}$.

$-6<=\mathrm{h}<=5,-11<=\mathrm{k}<=11,-20<=\mathrm{l}<=20$

24482

$5986[\mathrm{R}(\mathrm{int})=0.0458]$

$97.7 \%$

Semi-empirical from equivalents

0.7533 and 0.5317

Full-matrix least-squares on $\mathrm{F}^{2}$

5986 / 3 / 488

1.043

$\mathrm{R} 1=0.0366, \mathrm{wR} 2=0.0929$

$\mathrm{R} 1=0.0395, \mathrm{wR} 2=0.0960$

$-0.03(6)$

$0.021(3)$

0.192 and -0.171 e. $\AA^{-3}$ 
Compound 11: (The crystal structure of compound 11 has been deposited at the Cambridge Crystallographic Data Centre (CCDC 1974495). The data is available free of charge at www.ccdc.cam.ac.uk/conts/retrieving.html.)

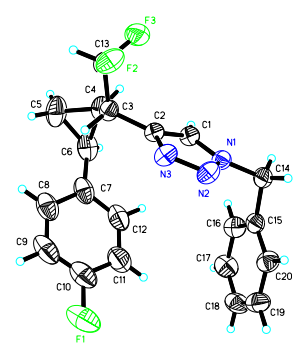<smiles>Fc1ccc(C2CC2C(c2cn(Cc3ccccc3)nn2)C(F)(F)F)cc1</smiles>

11

Table S8. Crystal data and structure refinement for compound 11

Identification code

Empirical formula

Formula weight

Temperature

Wavelength

Crystal system

Space group

Unit cell dimensions

\section{Volume}

Z

Density (calculated)

Absorption coefficient

$\mathrm{F}(000)$

Crystal size

Theta range for data collection

Index ranges

Reflections collected

Independent reflections

Completeness to theta $=67.679^{\circ}$

Absorption correction

Max. and min. transmission

Refinement method

Data / restraints / parameters

Goodness-of-fit on $\mathrm{F}^{2}$

Final $\mathrm{R}$ indices [I $>2 \operatorname{sigma}(\mathrm{I})]$

$\mathrm{R}$ indices (all data)

Absolute structure parameter

Extinction coefficient

Largest diff. peak and hole cu_d8v19326_0m

C20 H18 F3 N3

357.37

293(2) K

$1.54178 \AA$

Orthorhombic

P 212121

$\mathrm{a}=5.57310(10) \AA \quad \alpha=90^{\circ}$.

$\mathrm{b}=10.9291(2) \AA \quad \beta=90^{\circ}$.

$\mathrm{c}=29.6367(4) \AA \quad \gamma=90^{\circ}$.

1805.14(5) $\AA^{3}$

4

$1.315 \mathrm{Mg} / \mathrm{m}^{3}$

$0.847 \mathrm{~mm}^{-1}$

744

$0.180 \times 0.150 \times 0.120 \mathrm{~mm}^{3}$

4.311 to $66.450^{\circ}$.

$-6<=\mathrm{h}<=5,-12<=\mathrm{k}<=11,-32<=\mathrm{l}<=35$

9050

$3080[\mathrm{R}(\mathrm{int})=0.0288]$

$93.8 \%$

Semi-empirical from equivalents

0.7456 and 0.5844

Full-matrix least-squares on $\mathrm{F}^{2}$

3080 / 0 / 236

1.059

$\mathrm{R} 1=0.0383, \mathrm{wR} 2=0.0989$

$\mathrm{R} 1=0.0410, \mathrm{wR} 2=0.1018$

0.01(6)

$0.020(4)$

0.086 and -0.109 e. $\AA^{-3}$ 
Compound 12: (The crystal structure of compound 12 has been deposited at the Cambridge Crystallographic Data Centre (CCDC 1974496). The data is available free of charge at www.ccdc.cam.ac.uk/conts/retrieving.html.)
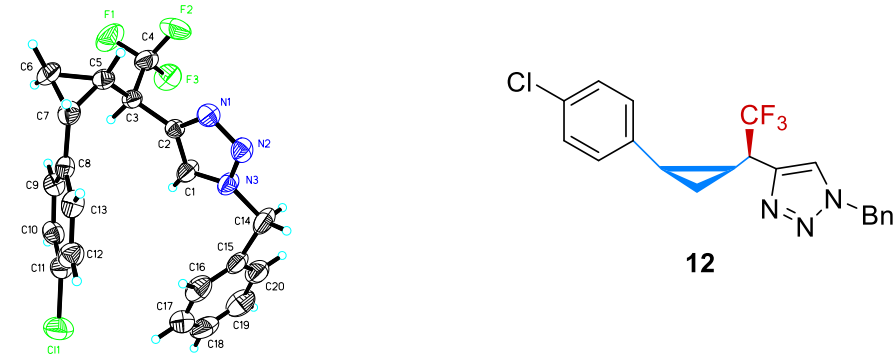

\section{Table S9. Crystal data and structure refinement for compound 12}

Identification code

Empirical formula

Formula weight

Temperature

Wavelength

Crystal system

Space group

Unit cell dimensions

\section{Volume}

Z

Density (calculated)

Absorption coefficient

$\mathrm{F}(000)$

Crystal size

Theta range for data collection

Index ranges

Reflections collected

Independent reflections

Completeness to theta $=25.242^{\circ}$

Absorption correction

Max. and min. transmission

Refinement method

Data / restraints / parameters

Goodness-of-fit on $\mathrm{F}^{2}$

Final $\mathrm{R}$ indices [I $>2 \operatorname{sigma}(\mathrm{I})]$

$\mathrm{R}$ indices (all data)

Absolute structure parameter

Extinction coefficient

Largest diff. peak and hole mo_d8v19958_0m

C20 H17 Cl F3 N3

391.82

293(2) K

$0.71073 \AA$

Monoclinic

P 21

$\mathrm{a}=11.2274(4) \AA \quad \alpha=90^{\circ}$.

$\mathrm{b}=5.6306(2) \AA \quad \beta=104.4610(10)^{\circ}$.

$\mathrm{c}=15.3435(6) \AA \quad \gamma=90^{\circ}$.

939.24(6) $\AA^{3}$

2

$1.385 \mathrm{Mg} / \mathrm{m}^{3}$

$0.241 \mathrm{~mm}^{-1}$

404

$0.200 \times 0.160 \times 0.120 \mathrm{~mm}^{3}$

2.583 to $25.998^{\circ}$.

$-13<=\mathrm{h}<=13,-6<=\mathrm{k}<=6,-18<=\mathrm{l}<=18$

11387

$3644[\mathrm{R}(\mathrm{int})=0.0261]$

$98.8 \%$

Semi-empirical from equivalents

0.7456 and 0.6556

Full-matrix least-squares on $\mathrm{F}^{2}$

3644 / 7 / 254

1.035

$\mathrm{R} 1=0.0384, \mathrm{wR} 2=0.0934$

$\mathrm{R} 1=0.0478, \mathrm{wR} 2=0.1013$

$-0.03(3)$

$0.058(16)$

0.169 and -0.201 e. $\AA^{-3}$ 
Compound 15f: (The crystal structure of compound $15 f$ has been deposited at the Cambridge Crystallographic Data Centre (CCDC 1975279). The data is available free of charge at www.ccdc.cam.ac.uk/conts/retrieving.html.)
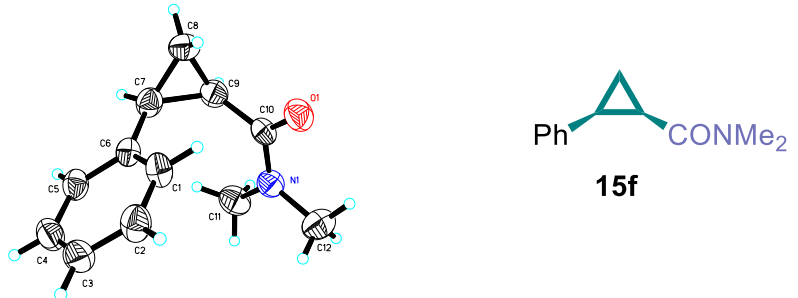

\section{Table S10. Crystal data and structure refinement for compound $15 f$}

Identification code

Empirical formula

Formula weight

Temperature

Wavelength

Crystal system

Space group

Unit cell dimensions

Volume

Z

Density (calculated)

Absorption coefficient

$\mathrm{F}(000)$

Crystal size

Theta range for data collection

Index ranges

Reflections collected

Independent reflections

Completeness to theta $=67.679^{\circ}$

Absorption correction

Max. and min. transmission

Refinement method

Data / restraints / parameters

Goodness-of-fit on $\mathrm{F}^{2}$

Final $\mathrm{R}$ indices [I $>2 \operatorname{sigma}(\mathrm{I})]$

$\mathrm{R}$ indices (all data)

Absolute structure parameter

Extinction coefficient

Largest diff. peak and hole cu_d8v191195_0m

C12 H15 N O

189.25

293(2) K

$1.54178 \AA$

Orthorhombic

P 212121

$\mathrm{a}=7.8115(3) \AA$

$\alpha=90^{\circ}$.

$\mathrm{b}=9.4031(4) \AA$

$\beta=90^{\circ}$.

$\mathrm{c}=14.7571(6) \AA$

$\gamma=90^{\circ}$.

1083.94(8) $\AA^{3}$

4

$1.160 \mathrm{Mg} / \mathrm{m}^{3}$

$0.578 \mathrm{~mm}^{-1}$

408

$0.180 \times 0.160 \times 0.130 \mathrm{~mm}^{3}$

5.579 to $67.464^{\circ}$.

$-9<=\mathrm{h}<=9,-11<=\mathrm{k}<=11,-17<=\mathrm{k}<=16$

9219

$1906[\mathrm{R}(\mathrm{int})=0.0350]$

$97.3 \%$

Semi-empirical from equivalents

0.7456 and 0.5889

Full-matrix least-squares on $\mathrm{F}^{2}$

$1906 / 0 / 129$

1.079

$\mathrm{R} 1=0.0357, \mathrm{wR} 2=0.0999$

$\mathrm{R} 1=0.0368, \mathrm{wR} 2=0.1010$

$0.06(11)$

$\mathrm{n} / \mathrm{a}$

0.092 and -0.092 e. $\AA^{-3}$ 


\section{References}

1. a) Grupe, S.; von Wangelin, A. J. Iron-Catalyzed Synthesis of Cyclopropyl Halides. ChemCatChem 2013, 5, 706-710. b) J. Clayden, D. W. Watson, M. Chambers, Tetrahedron 2005, 61, 3195-3203.

2. Hu, J.; Xie, Y.; Huang, H. Palladium-Catalyzed Insertion of an Allene into an Aminal: Aminomethylamination of Allenes by C-N Bond Activation. Angew. Chem., Int. Ed. 2014, 53, $7272-7276$.

3. Zhao, Z.; Racicot, L.; Murphy, G. K. Fluorinative Rearrangements of Substituted Phenylallenes Mediated by (Difluoroiodo)toluene: Synthesis of $a$-(Difluoromethyl)styrene. Angew. Chem., Int. Ed. 2017, 56, 11620-11623.

4. Beaulieu, L.-P. B.; Zimmer, L. E.; Gagnon, A.; Charette, A. B. Highly Enantioselective Synthesis of 1,2,3-Substituted Cyclopropanes by Using $a$-Iodo- and $a$-Chloromethylzinc Carbenoids. Chem. Eur. J. 2012, 18, 14784-14791.

5. Krasovskiy, A.; Knochel, P. Convenient Titration Method for Organometallic Zinc, Magnesium, and Lanthanide Reagents. Synthesis 2006, 890-891.

6. An, L.; Xu, C.; Zhang, X. Highly Selective Nickel-Catalyzed gem-Difluoropropargylation of Unactivated Alkylzinc Reagents. Nat. Commun. 2017, 8, 1460.

7. Liang, Y.; Fu, G. C. Stereoconvergent Negishi Arylations of Racemic Secondary Alkyl Electrophiles: Differentiating between a $\mathrm{CF}_{3}$ and an Alkyl Group. J. Am. Chem. Soc. 2015, 137, 9523-9526.

8. Smith, S. W.; Fu, G. C. Nickel-Catalyzed Asymmetric Cross-Couplings of Racemic Propargylic Halides with Arylzinc Reagents. J. Am. Chem. Soc. 2008, 130, 12645-12647.

9. Jones, G. B.; Wright, J. M.; Plourde, G. W.; Hynd, G.; Huber, R. S.; Mathews, J. E. A Direct and Stereocontrolled Route to Conjugated Enediynes. J. Am. Chem. Soc. 2000, 122, 1937-1944.

10. Tripathi, C. B.; Mukherjee, S. Catalytic Enantioselective Iodoetherification of Oximes. Angew. Chem. Int., Ed. 2013, 52, 8450-8453.

11. Smith, S. M.; Fu, G. C. Nickel-Catalyzed Negishi Cross-Couplings of Secondary Nucleophiles with Secondary Propargylic Electrophiles at Room Temperature. Angew. Chem., Int. Ed. 2008, 47, 9334-9336. 
12. Teo, W. J.; Ge, S. Cobalt-Catalyzed Diborylation of 1,1-Disubstituted Vinylarenes: A Practical Route to Branched gem-Bis(boryl)alkanes. Angew. Chem., Int. Ed. 2018, 57, 1654-1658.

13. Hariss, L.; Hadir, K. B.; El-Masri, M.; Roisnel, T.; Grée, R.; Hachem, A. Preparation of Imidazo[1,2-a]- $N$-heterocyclic Derivatives with gem-Difluorinated Side Chains. Beilstein J. Org. Chem. 2017, 13, 2115-2121.

14. Guo, W.-H.; Luo, Z.-J.; Zeng, W.; Zhang, X. Access to Difluoromethylene-Skipped 1,4-Diynes with gem-Difluoropropargyl Bromide. ACS Catal. 2017, 7, 896-901.

15. Rauhut, C. B.; Cervino, C.; Krasovskiy, A.; Knochel, P. Stereoselective Preparation of Cyclopropylmagnesium Reagents via a $\mathrm{Br}-\mathrm{Mg}$ Exchange Using $i$-PrMgCl$\cdot \mathrm{LiCl}$ in the Presence of Dioxane. Synlett. 2009, 67-70. 
11. Copies of ${ }^{1} \mathrm{H},{ }^{19} \mathrm{~F},{ }^{13} \mathrm{C}$ NMR and HPLC Spectra

(2,2-Dibromocyclopropyl)benzene

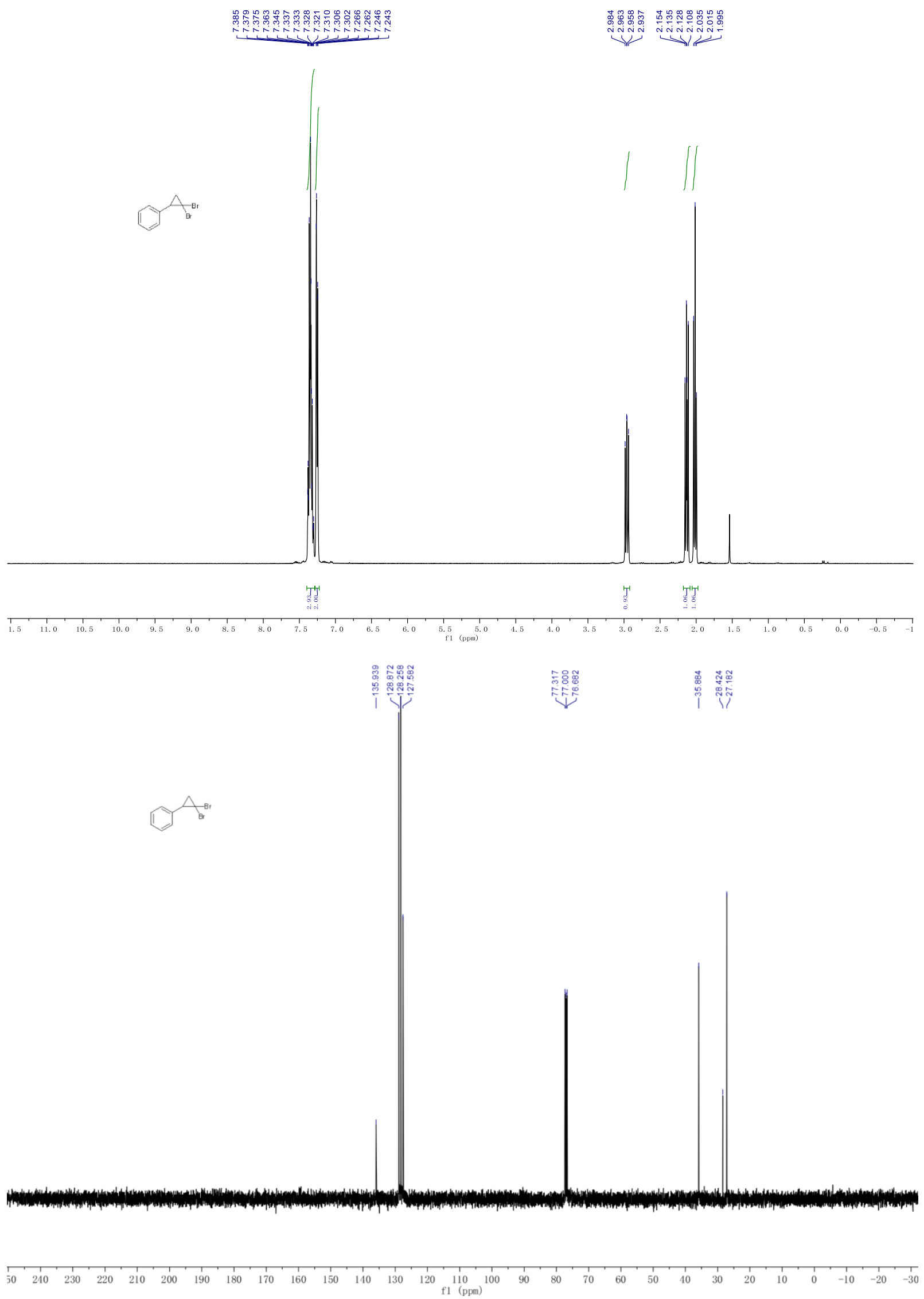


1-(tert-Butyl)-4-(2,2-dibromocyclopropyl)benzene.
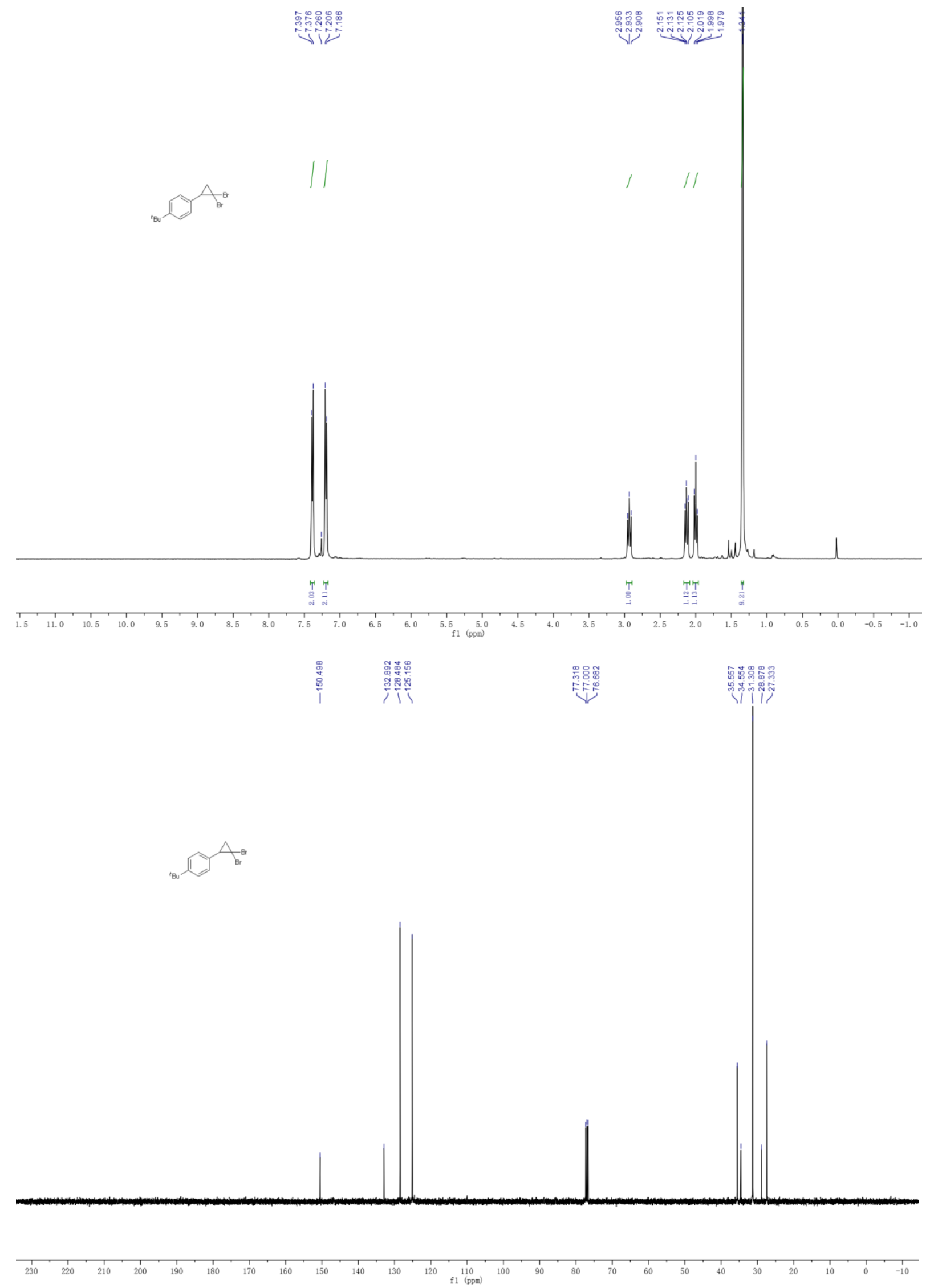
4-(2,2-Dibromocyclopropyl)-1,1'-biphenyl.
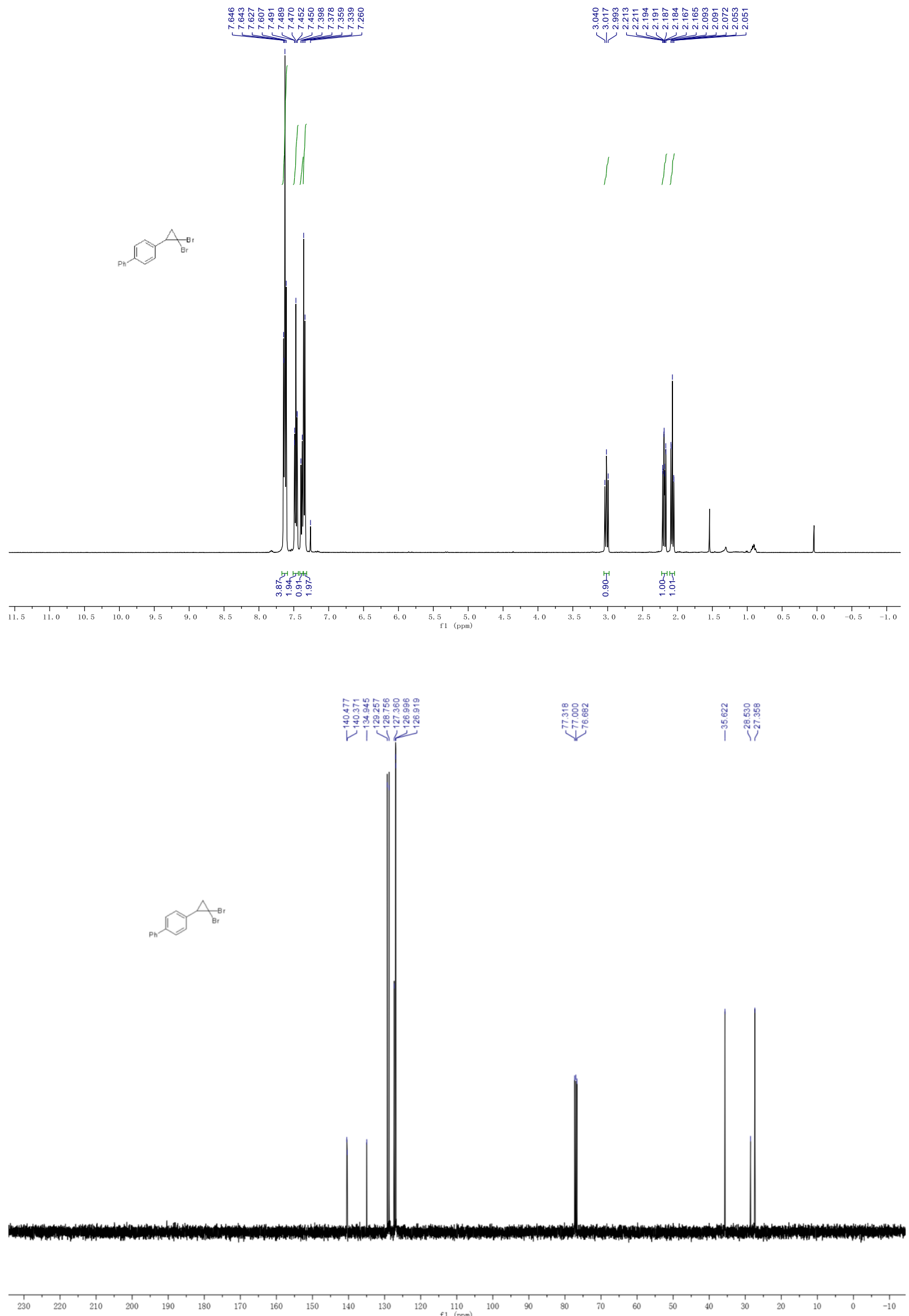
1-(2,2-Dibromocyclopropyl)-4-(trifluoromethyl)benzene.
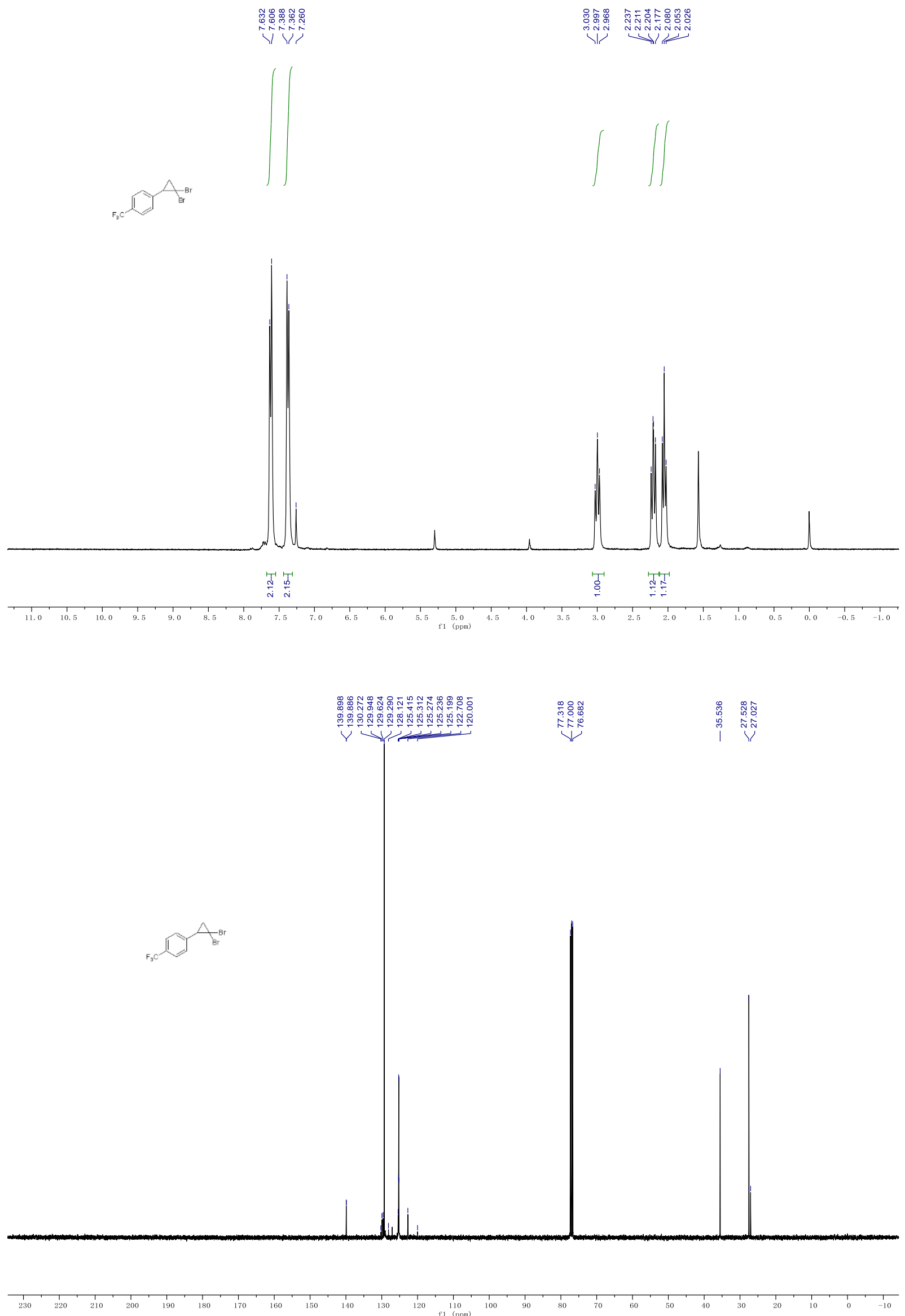

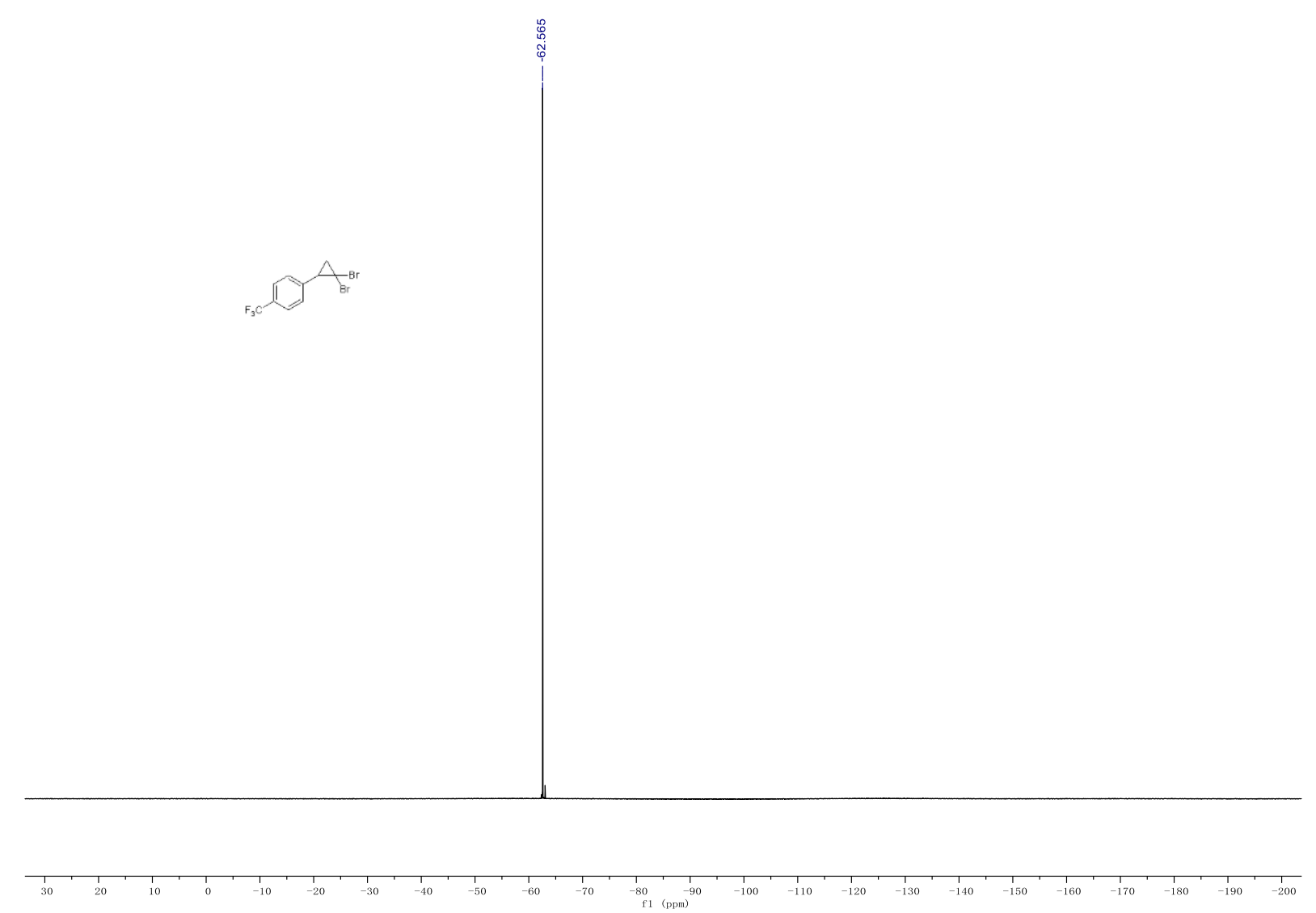

1-(2,2-Dibromocyclopropyl)-4-(trifluoromethoxy)benzene.

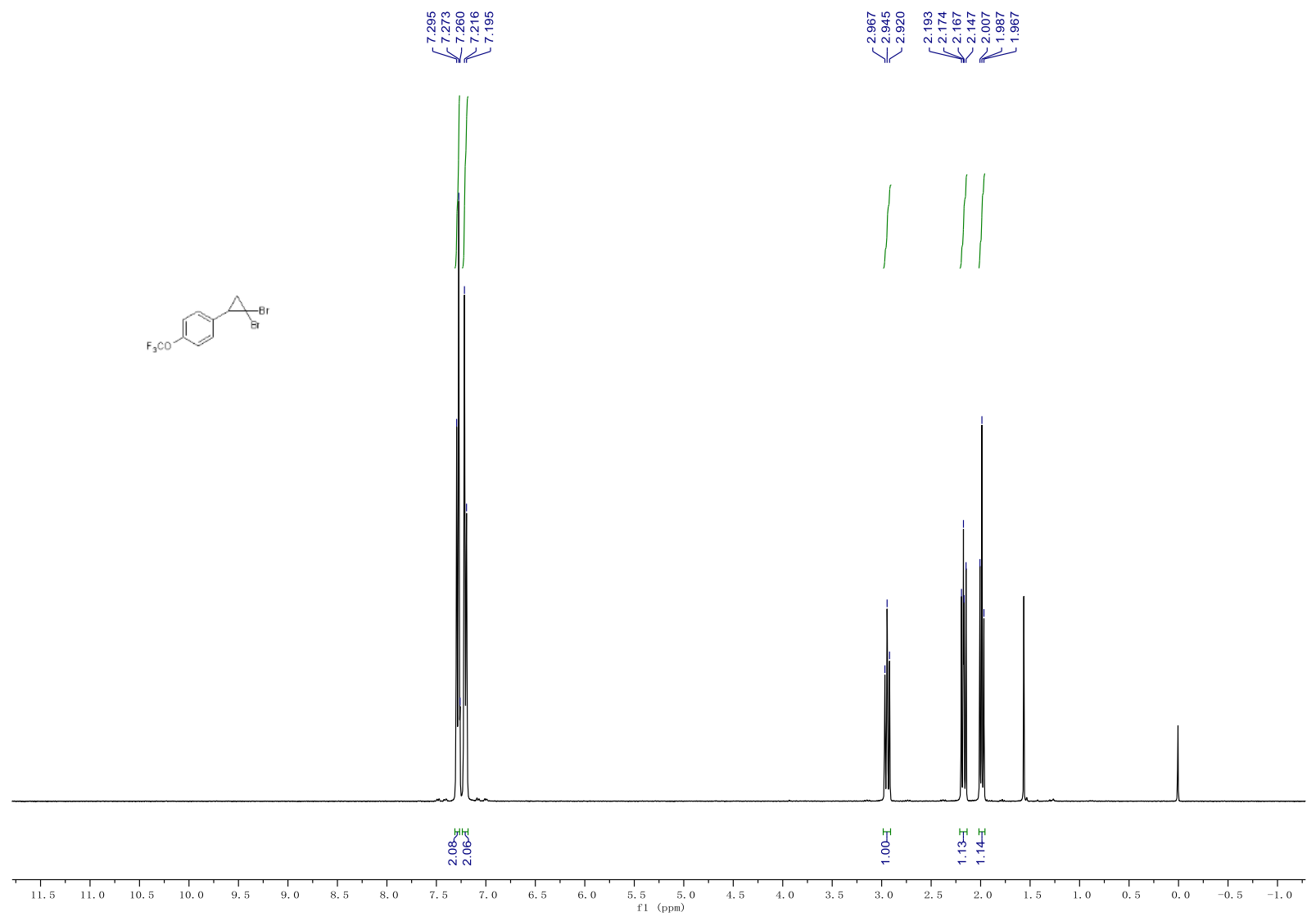



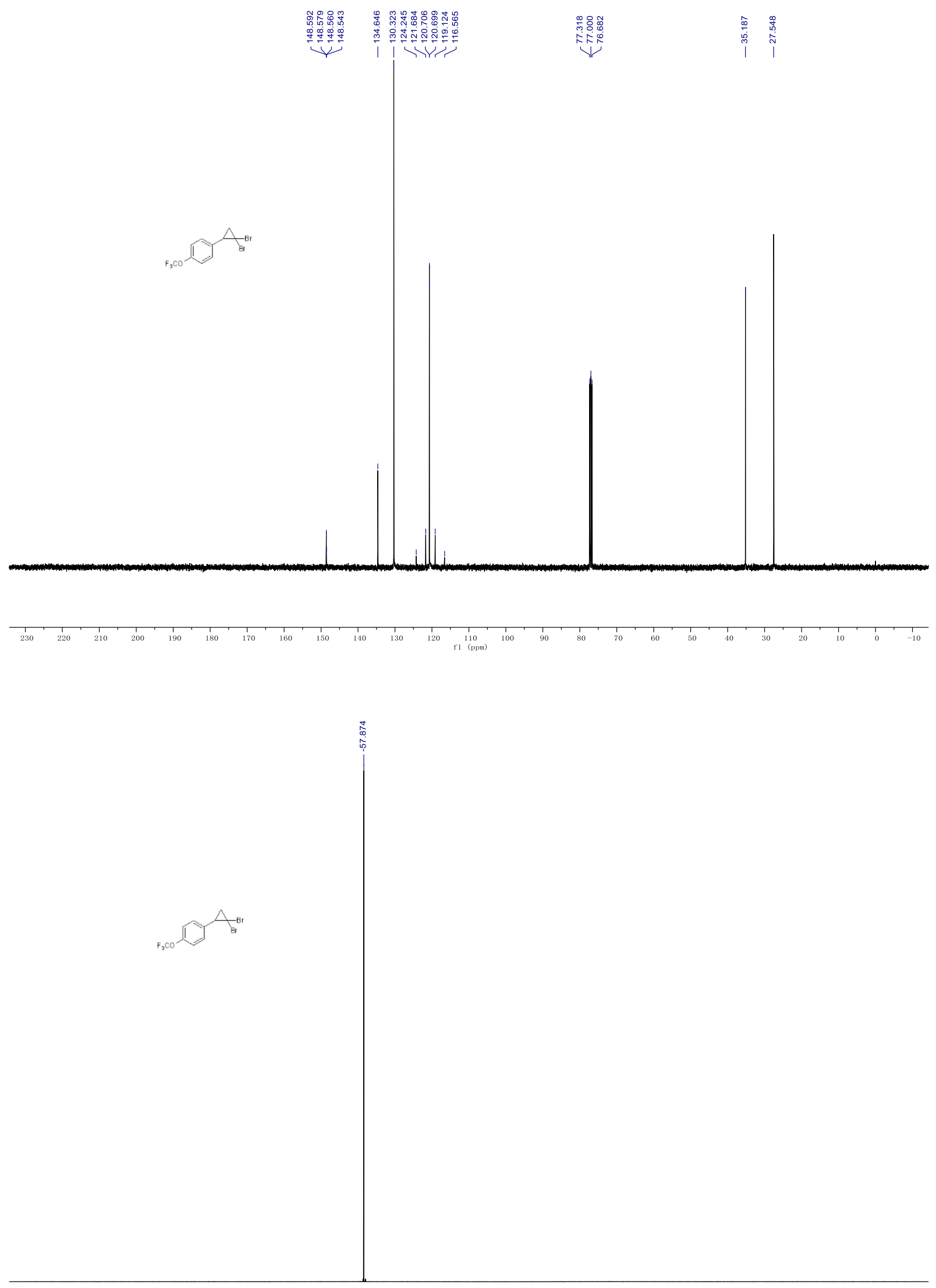

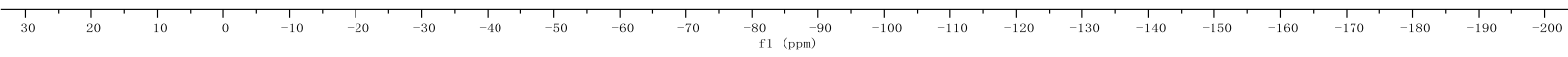


(4-(2,2-Dibromocyclopropyl)phenyl)trimethylsilane.

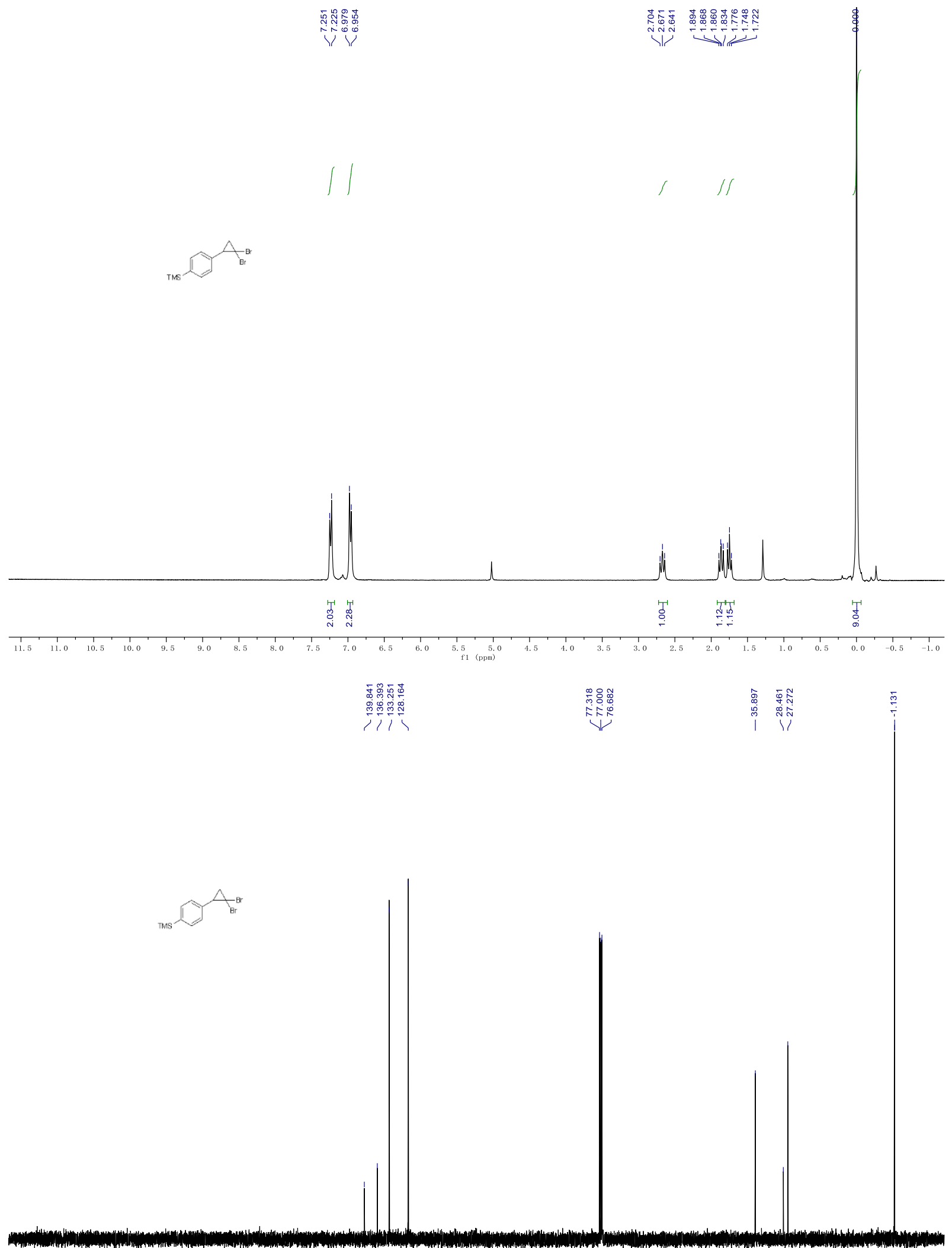

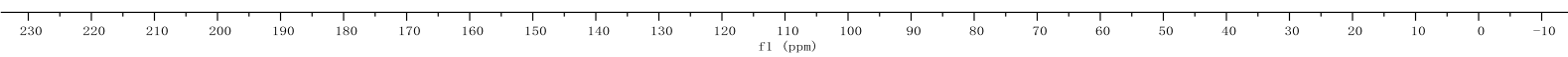


1-(2,2-Dibromocyclopropyl)-4-fluorobenzene.
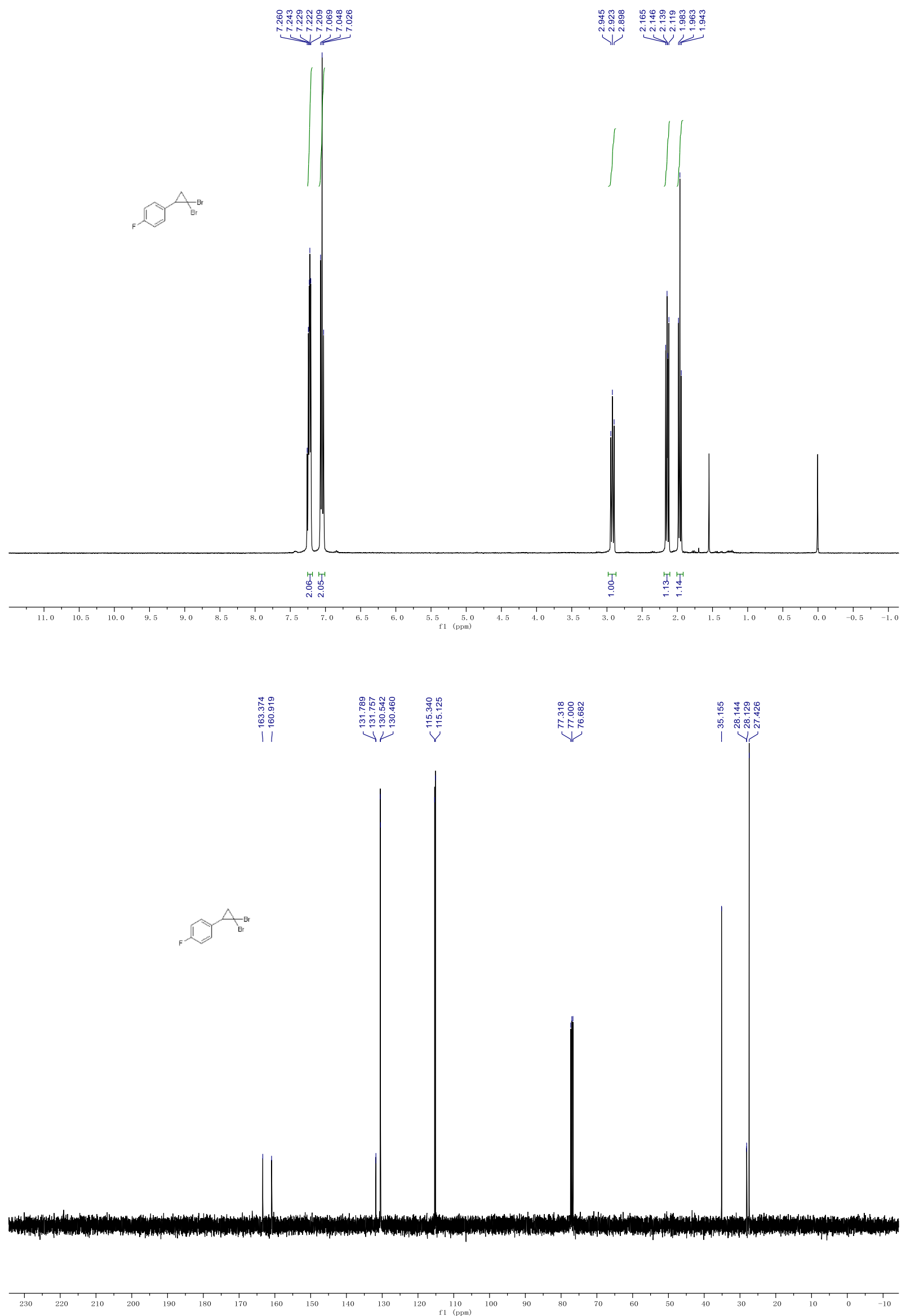

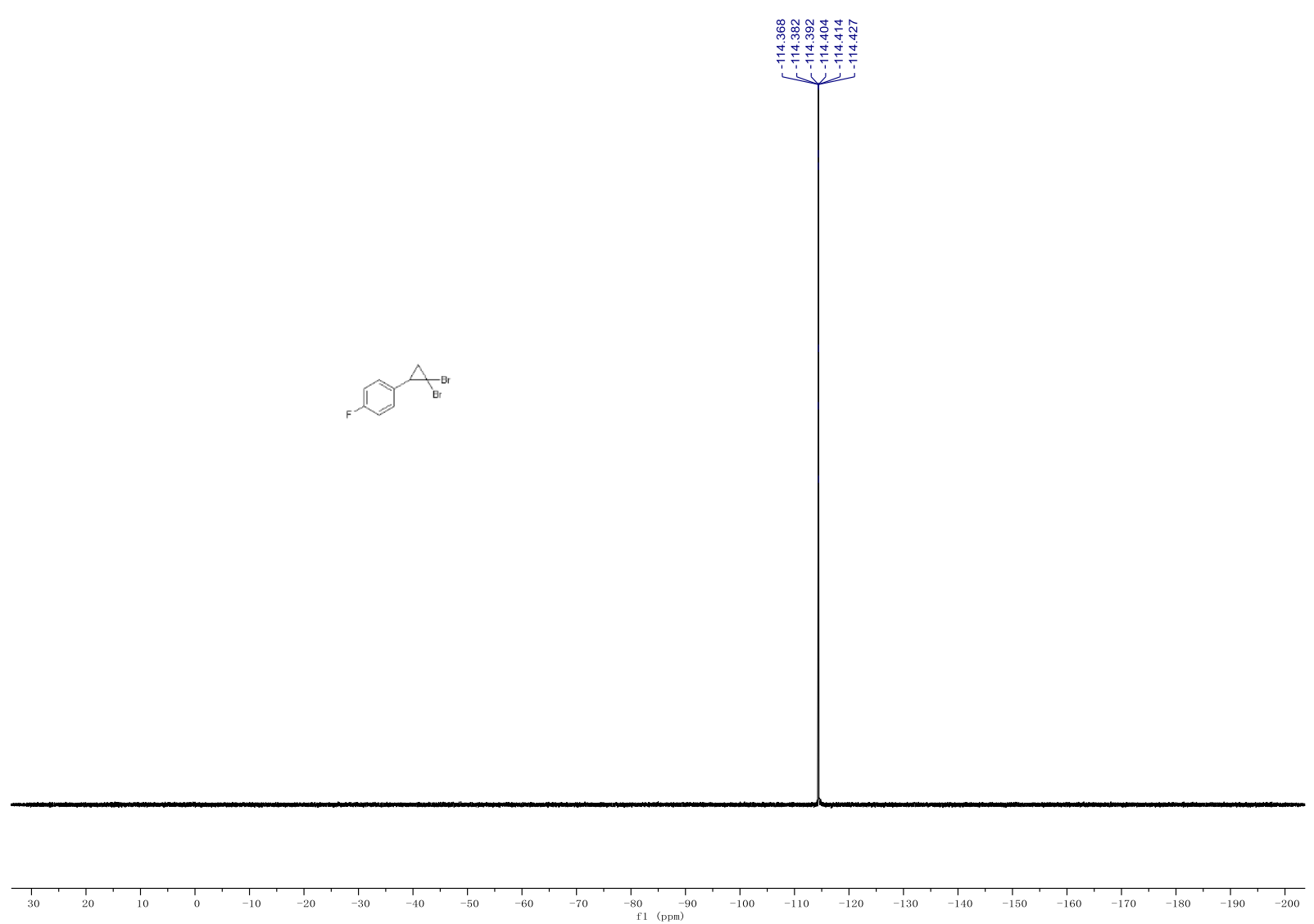

1-Chloro-4-(2,2-dibromocyclopropyl)benzene.

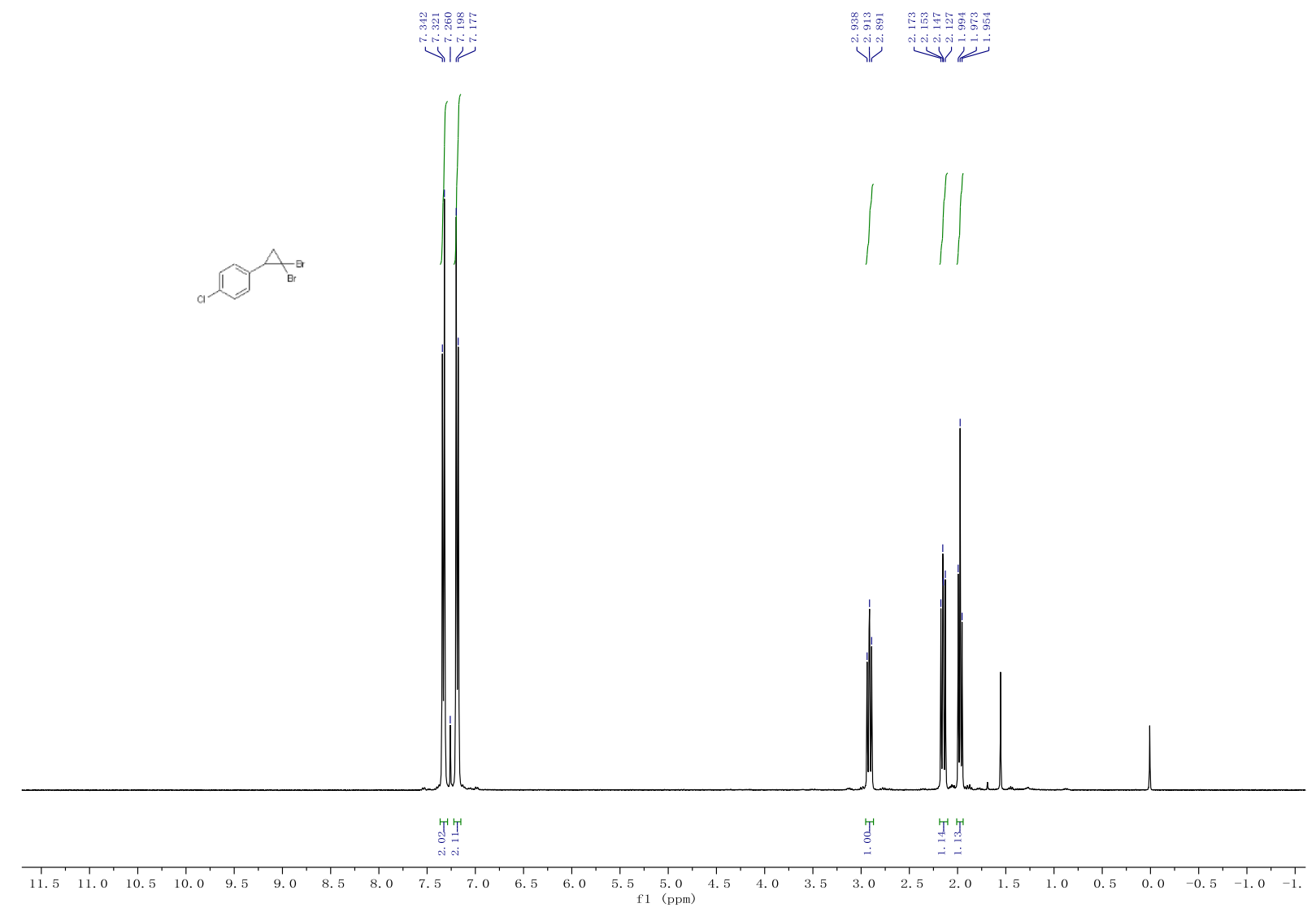




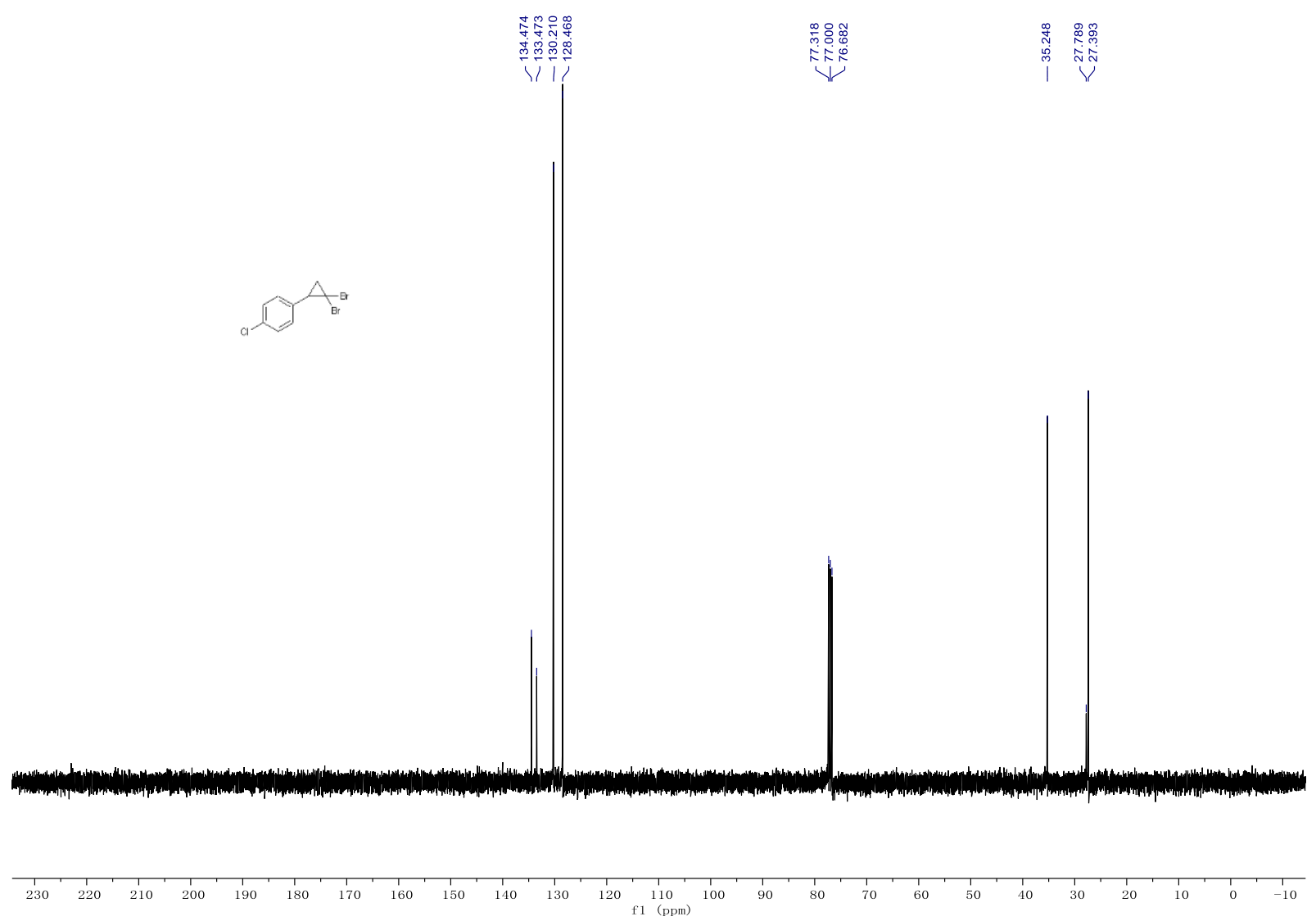

1-(Benzyloxy)-3-(2,2-dibromocyclopropyl)benzene.

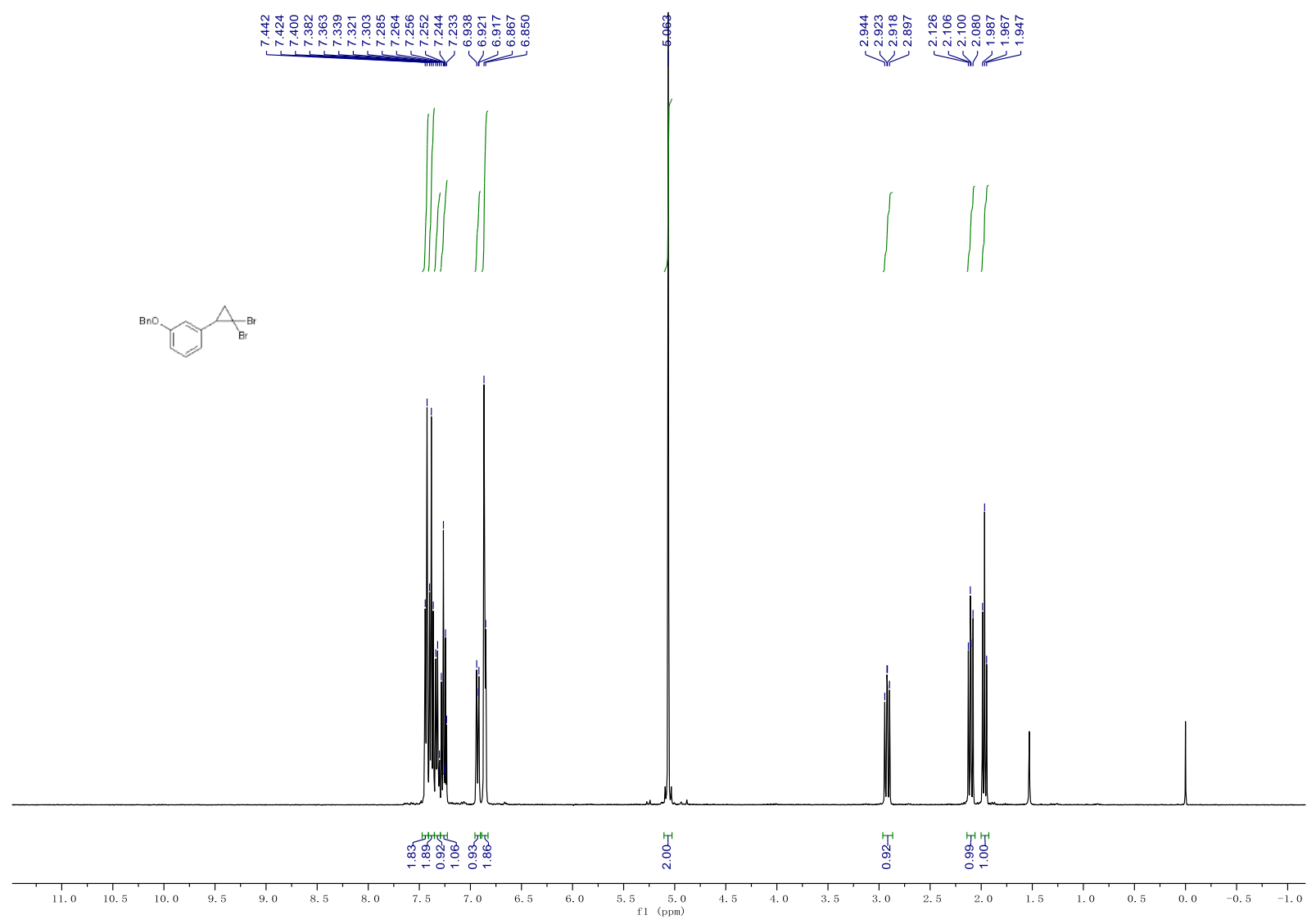



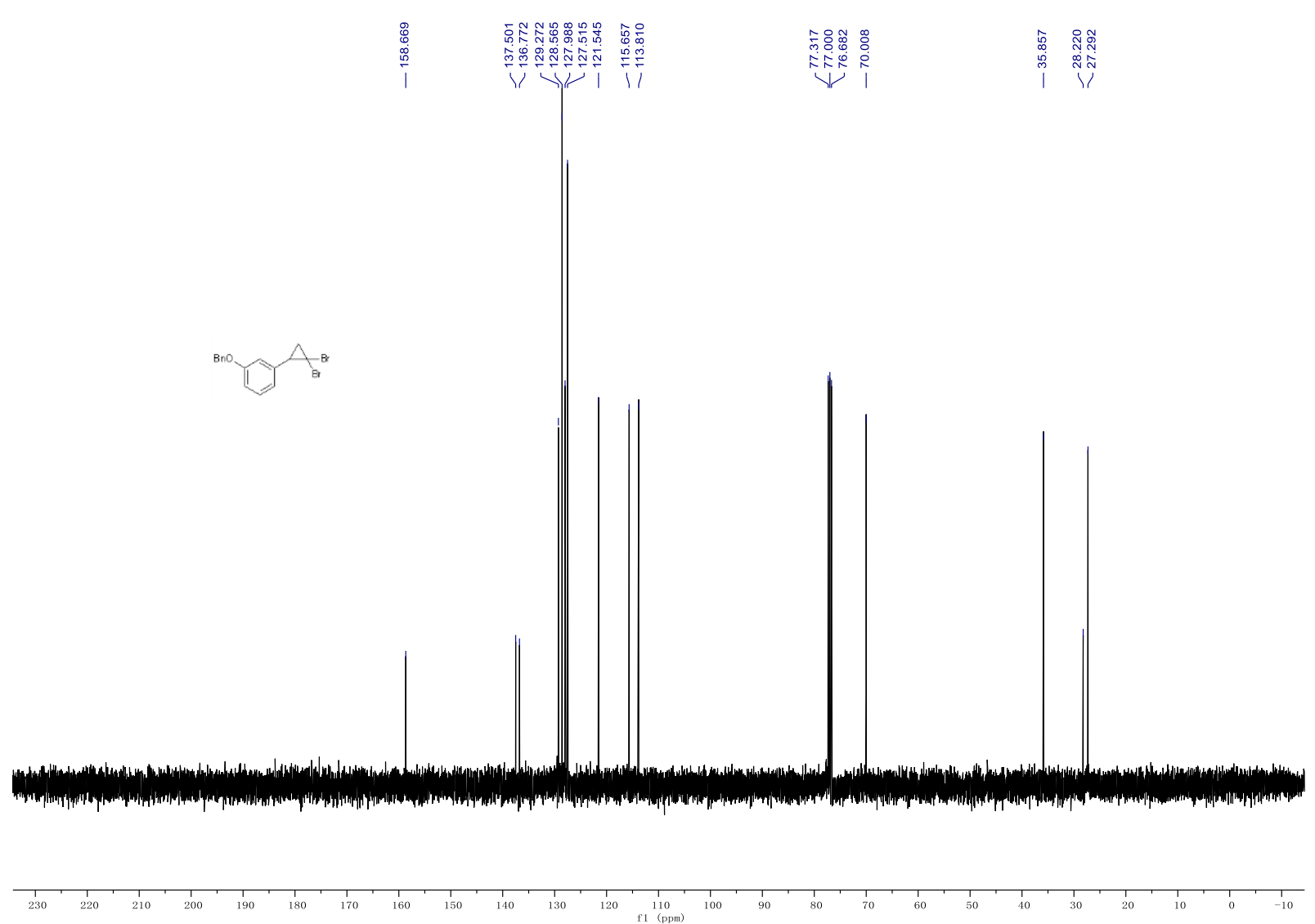

1-(2,2-Dibromocyclopropyl)naphthalene.

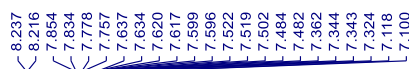

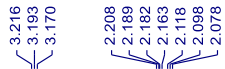

(1) $\triangle_{\mathrm{Br}}^{\mathrm{Br}}$
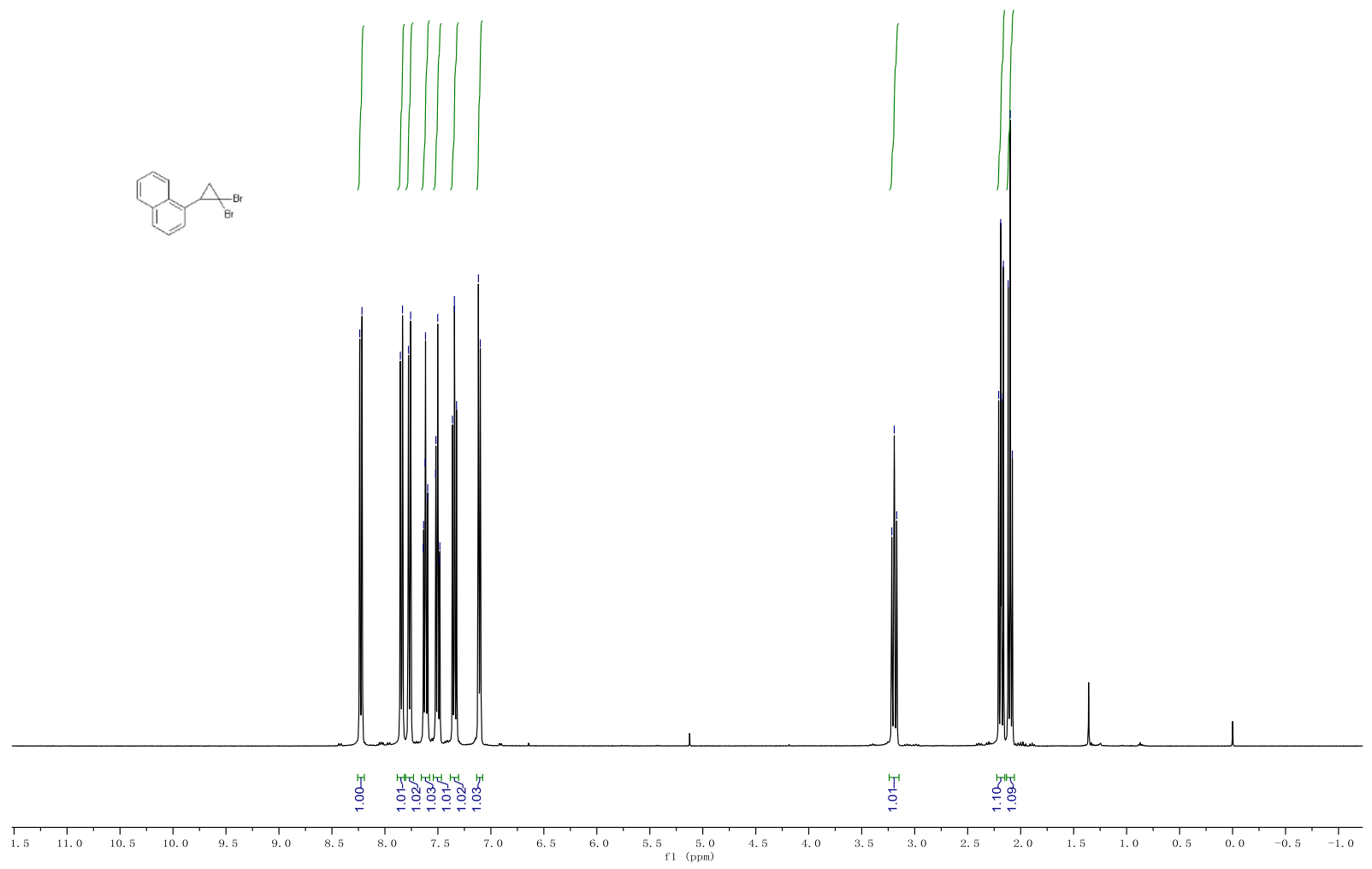

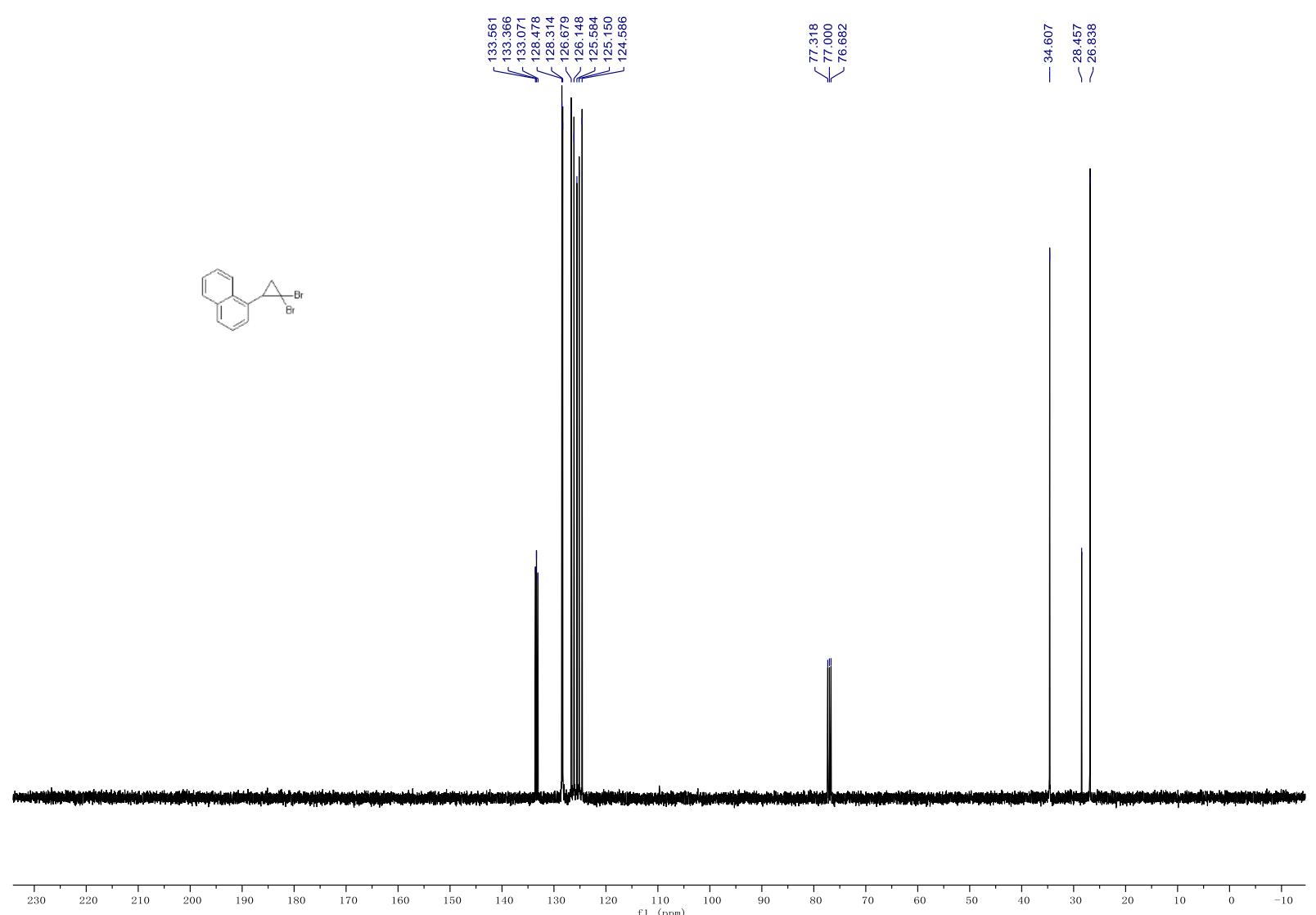

trans-2-Methyl-3-phenyl-1,1-dibromocyclopropane.

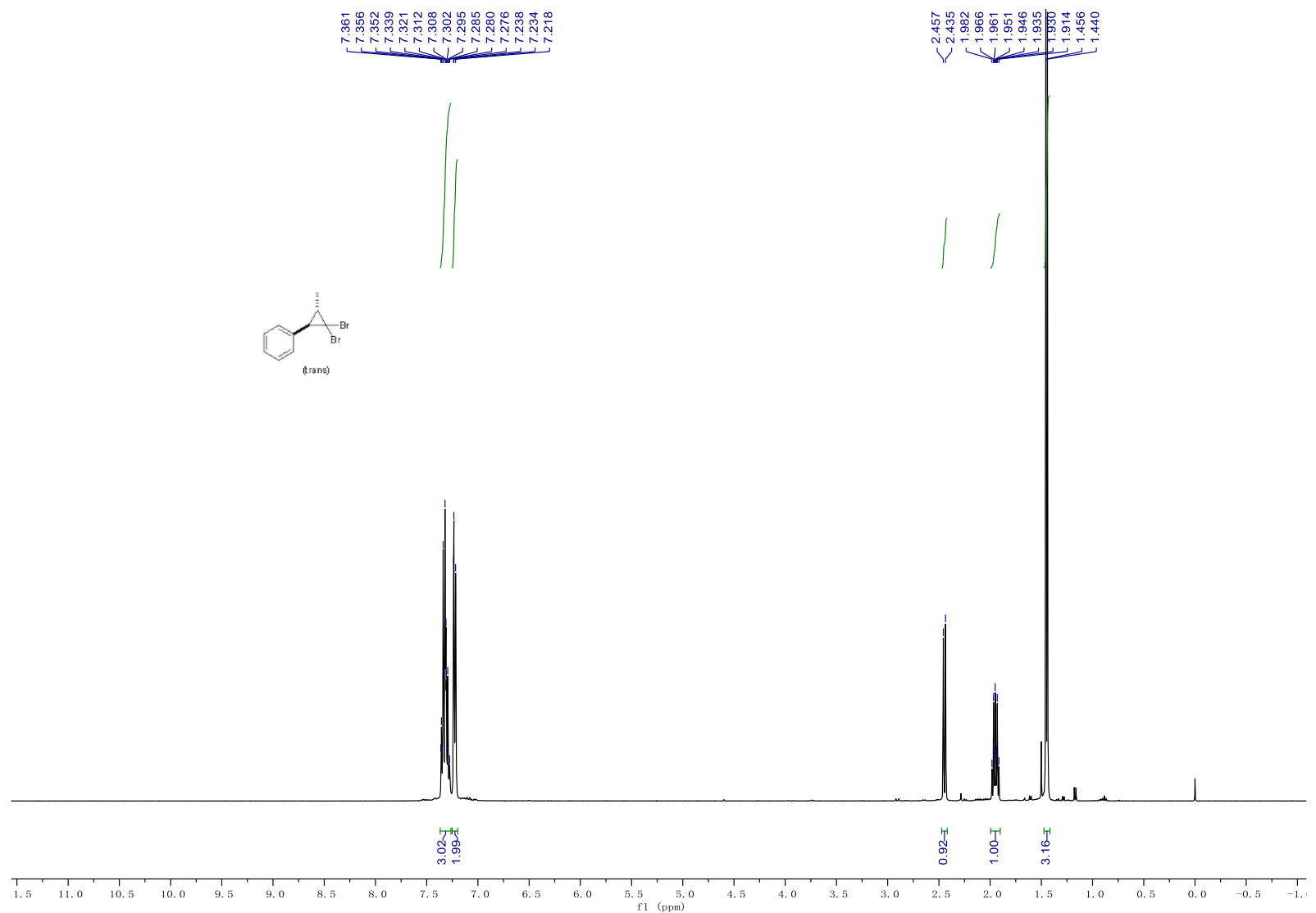



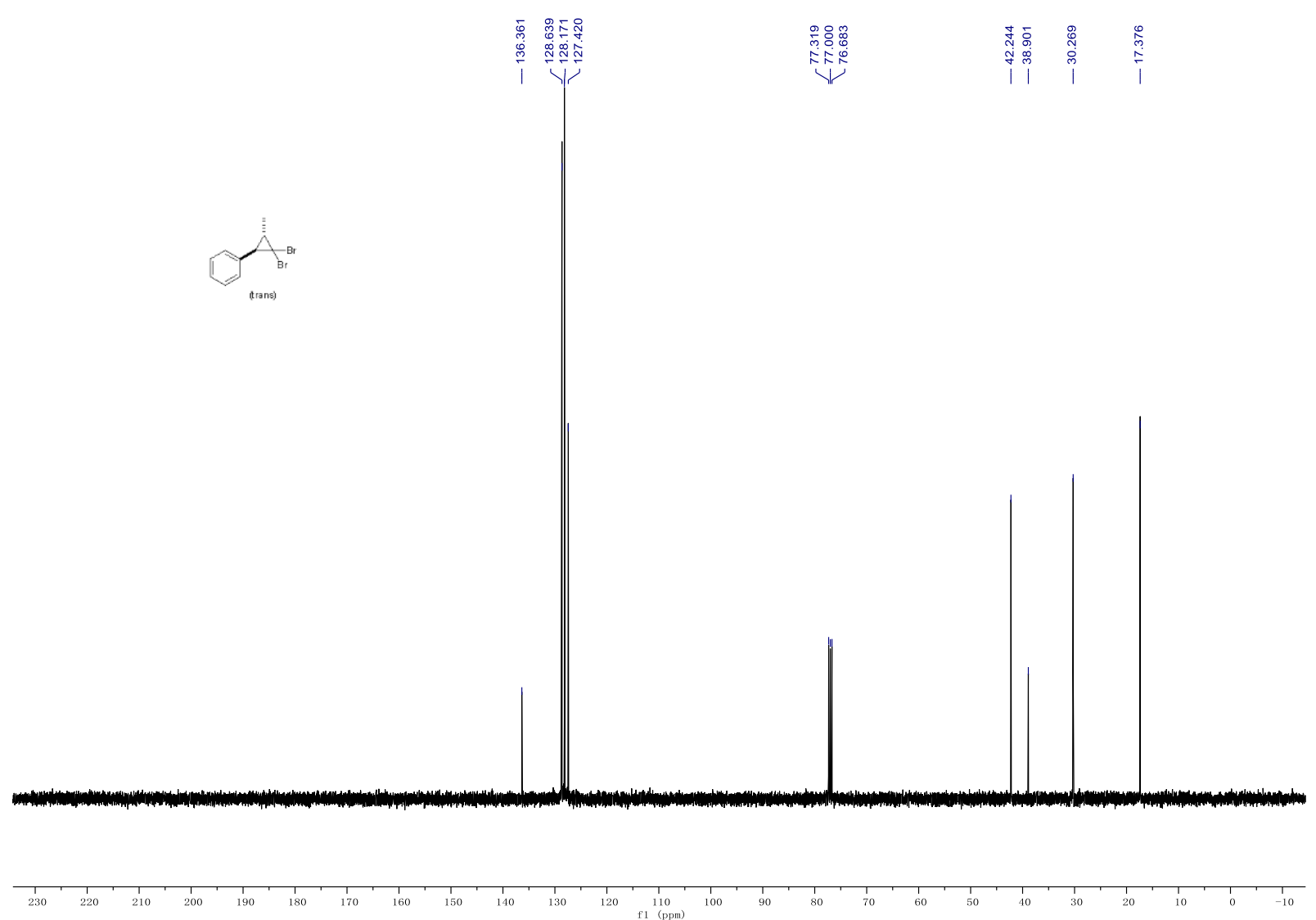

trans-2-Butyl-3-phenyl-1,1-dibromocyclopropane.

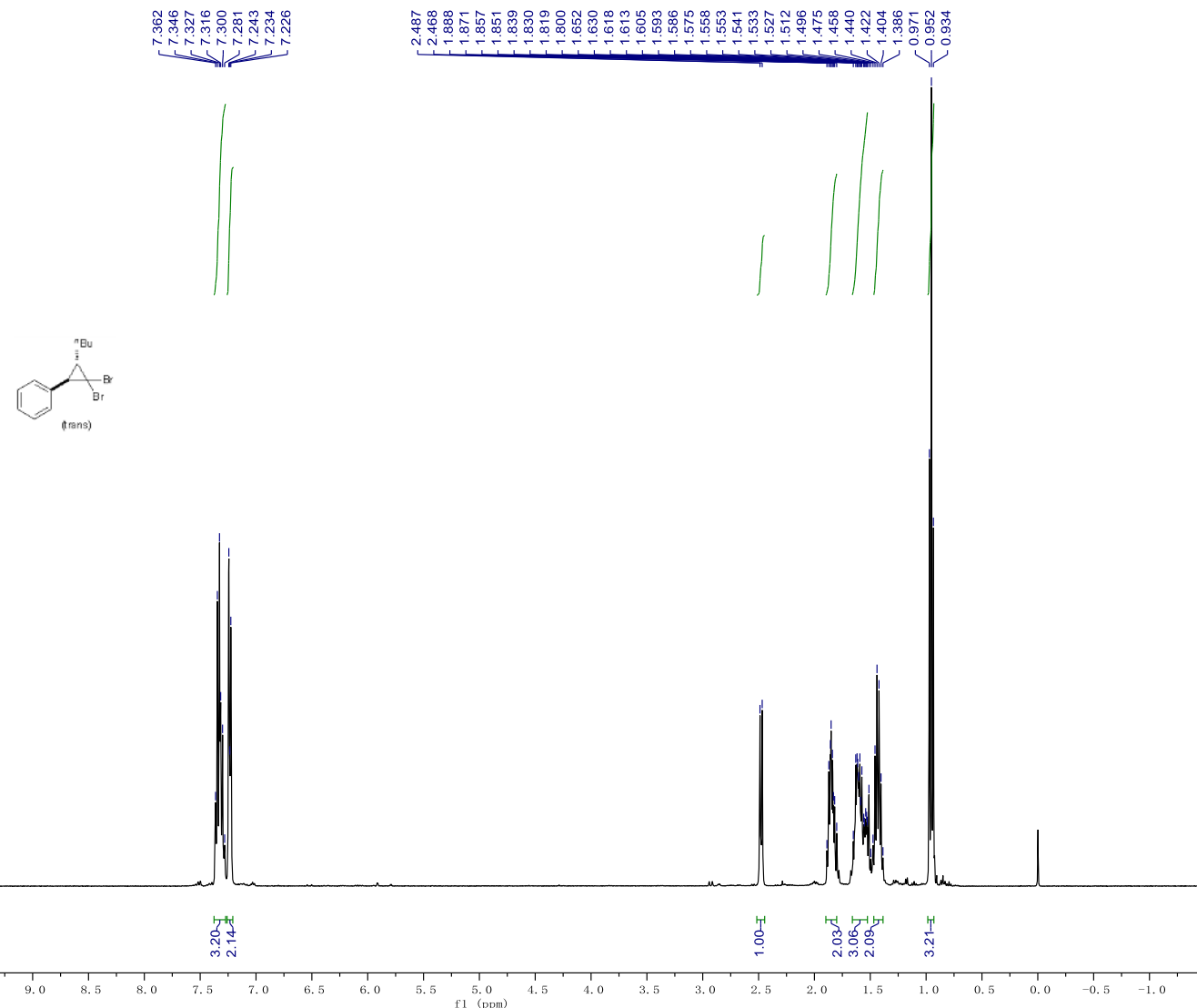

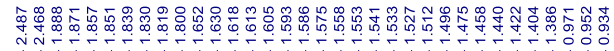



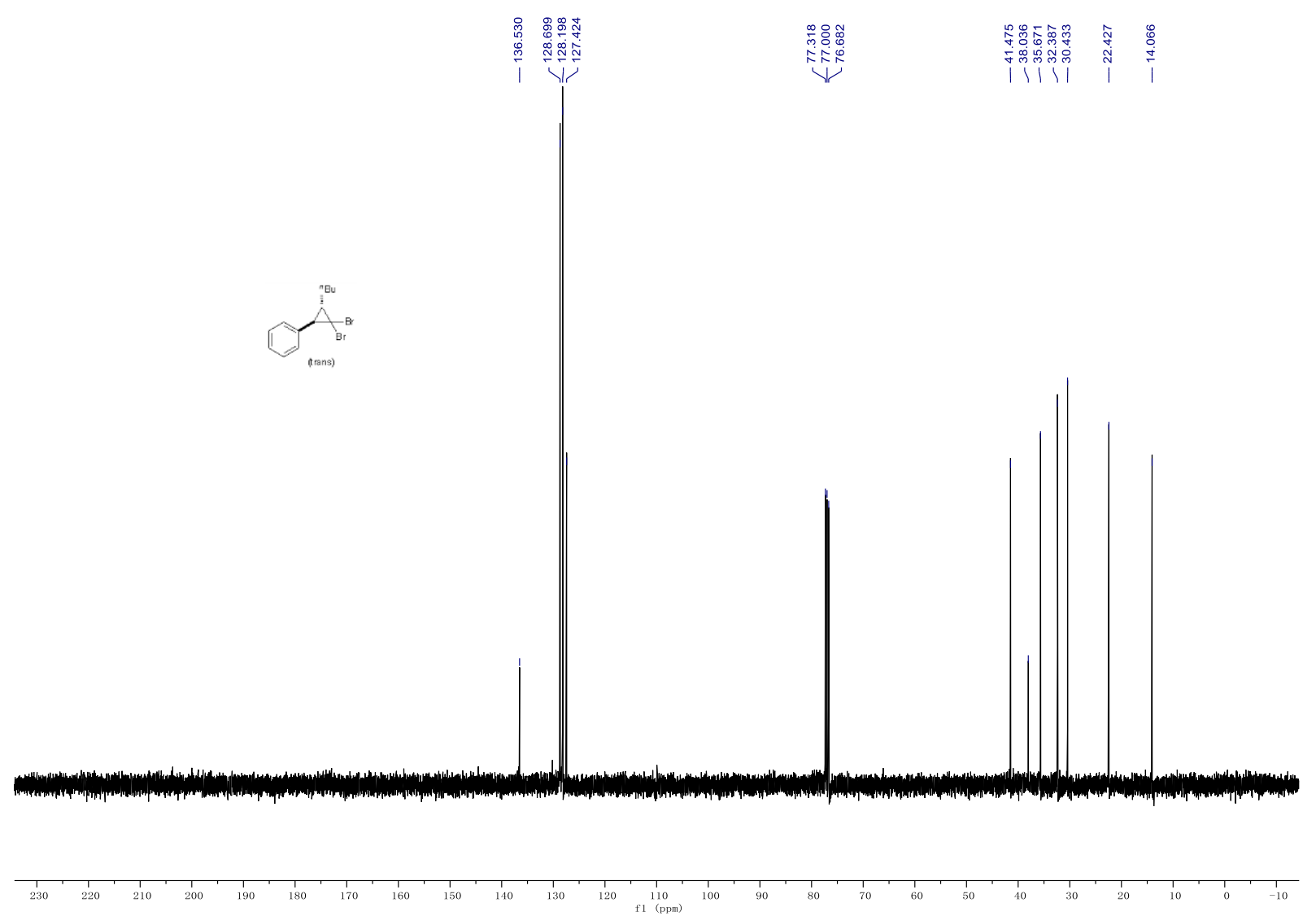

(( \pm$)$-(cis)-2-Bromocyclopropyl)benzene.

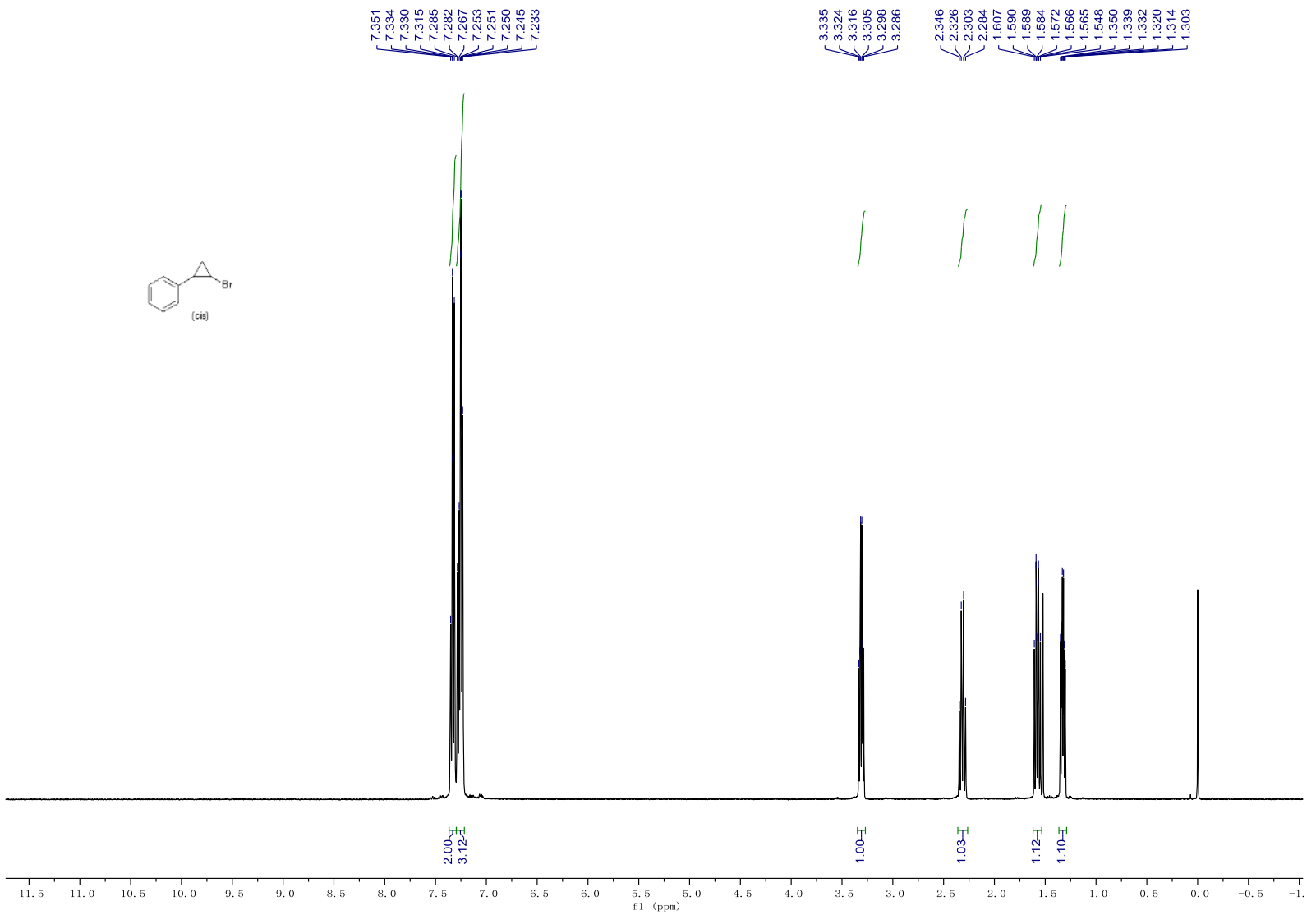



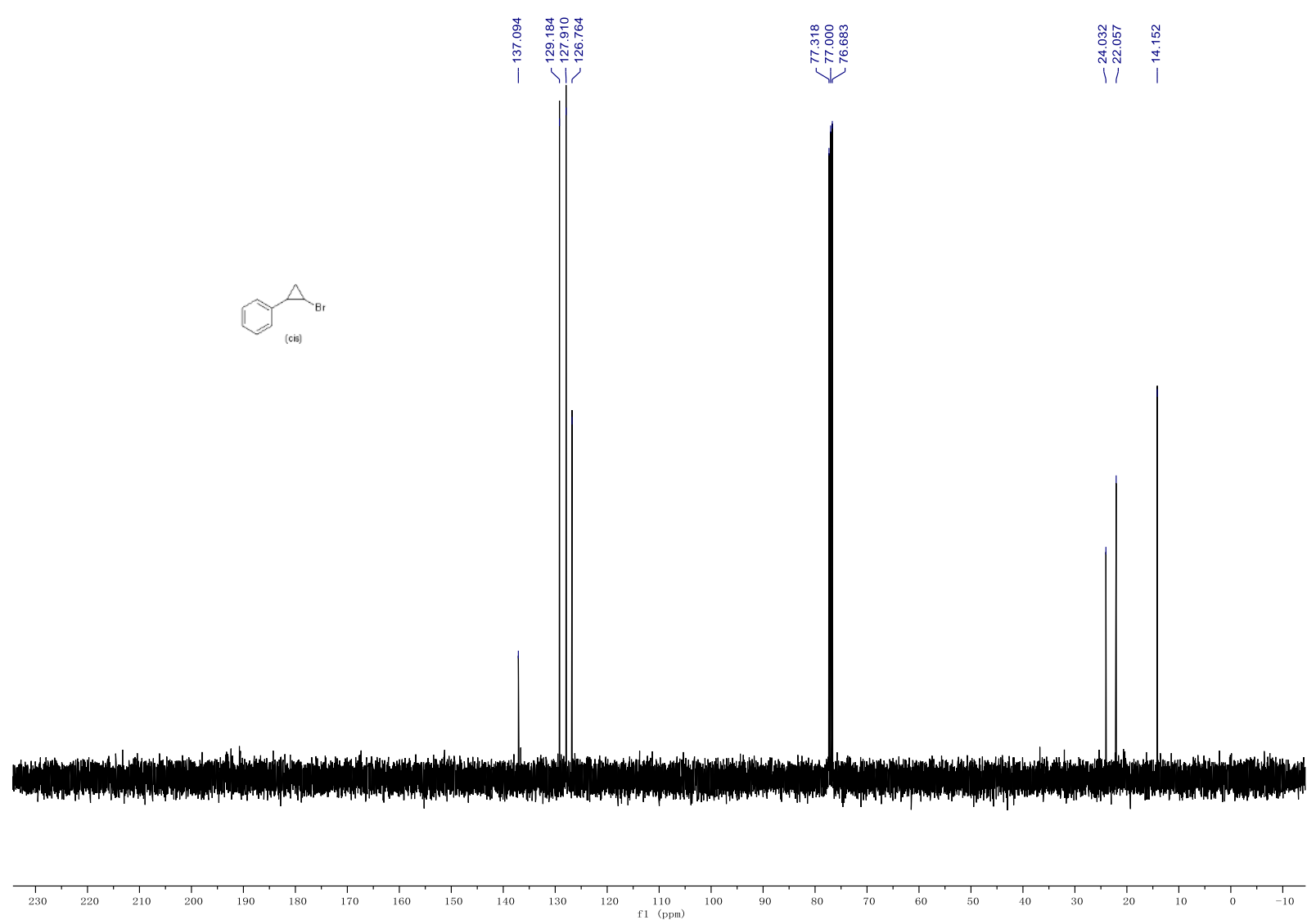

((士)-(trans)-2-Bromocyclopropyl)benzene.

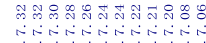

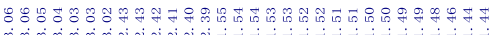
年<smiles>BrC1CC1c1ccccc1</smiles>
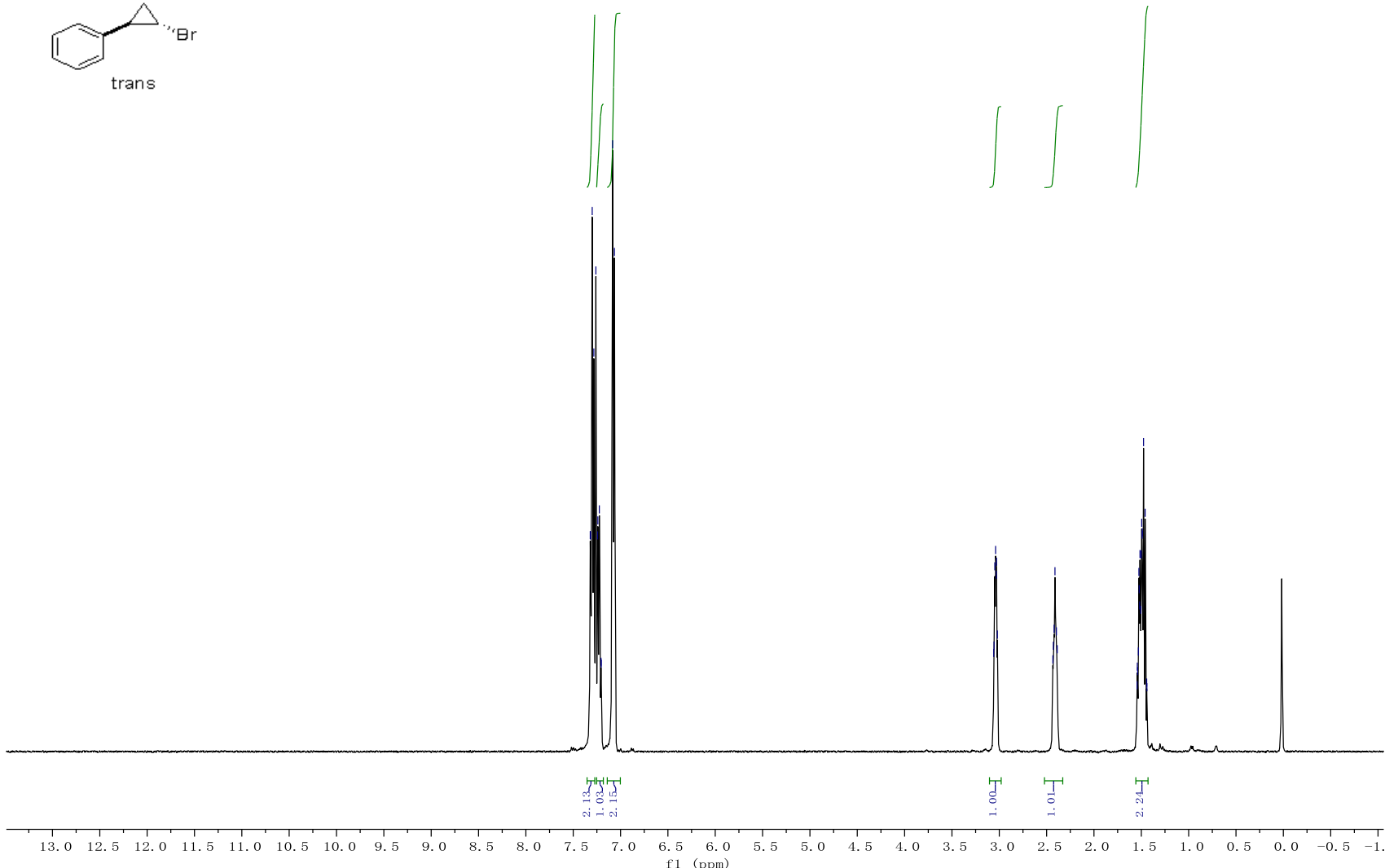


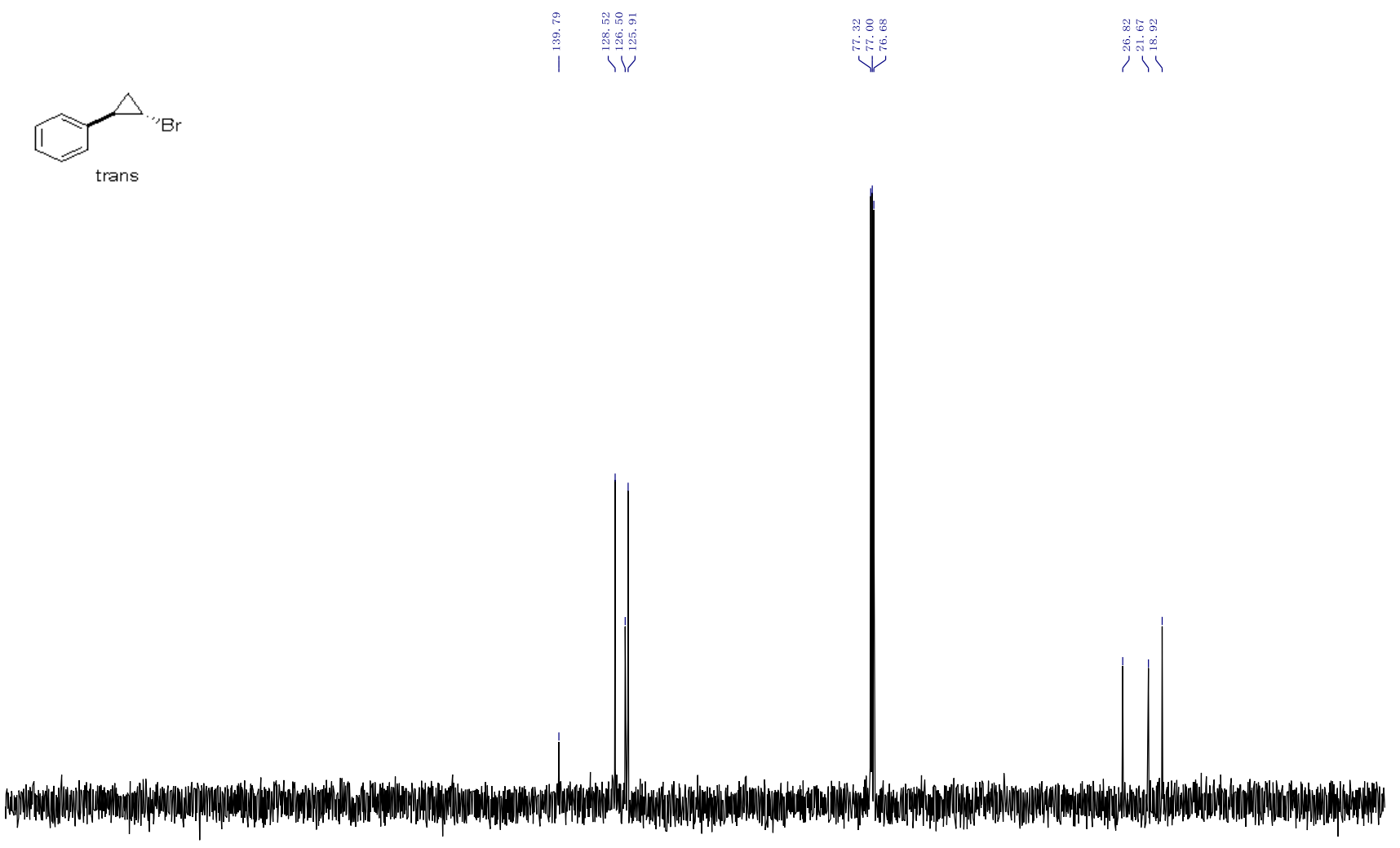

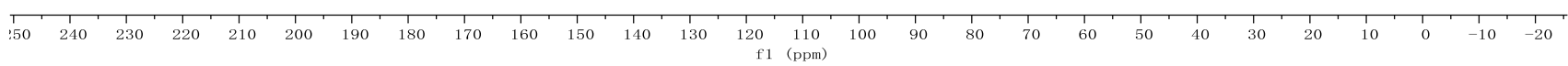

1-(( \pm )-(cis)-2-Bromocyclopropyl)-4-(tert-butyl)benzene.

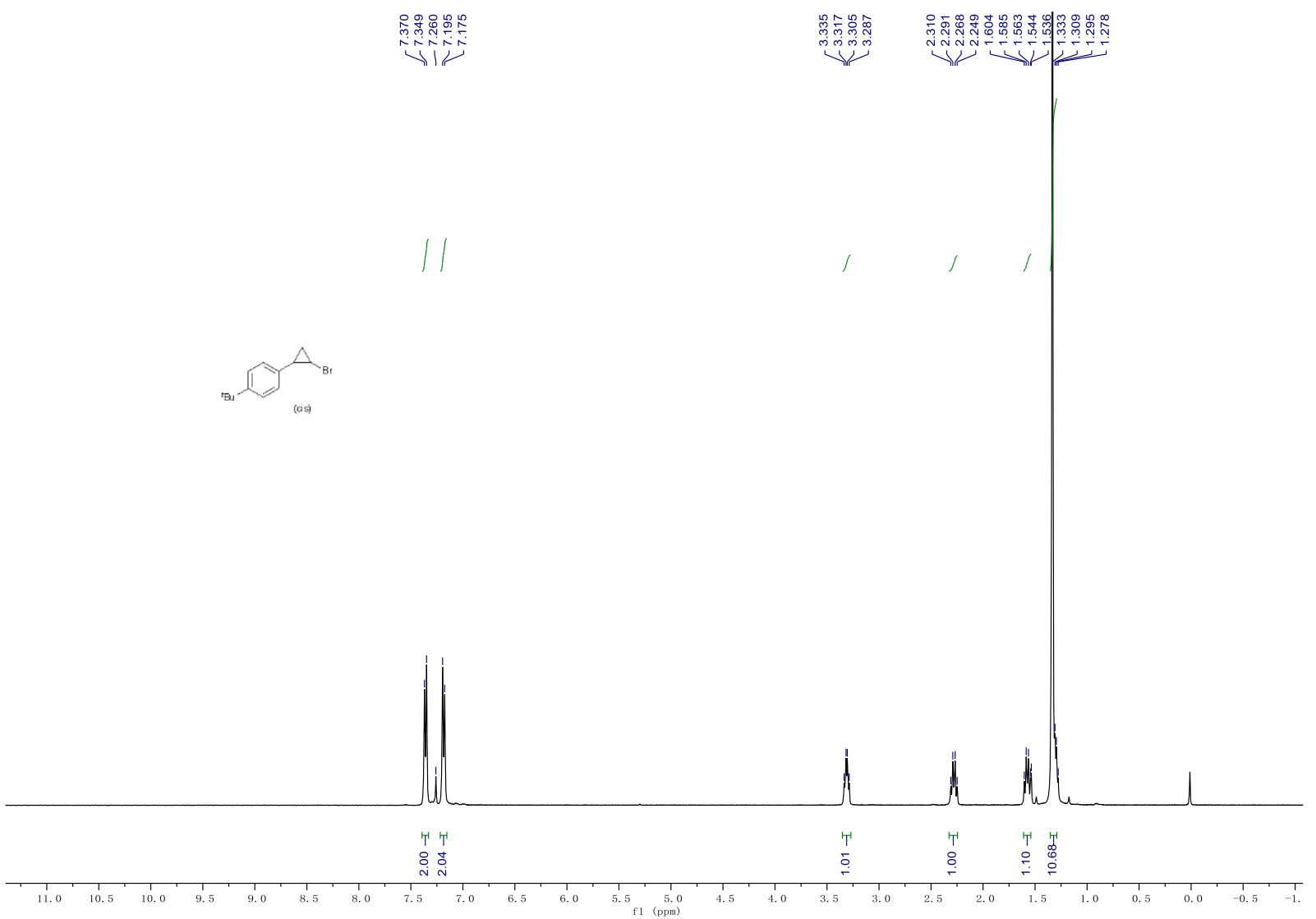



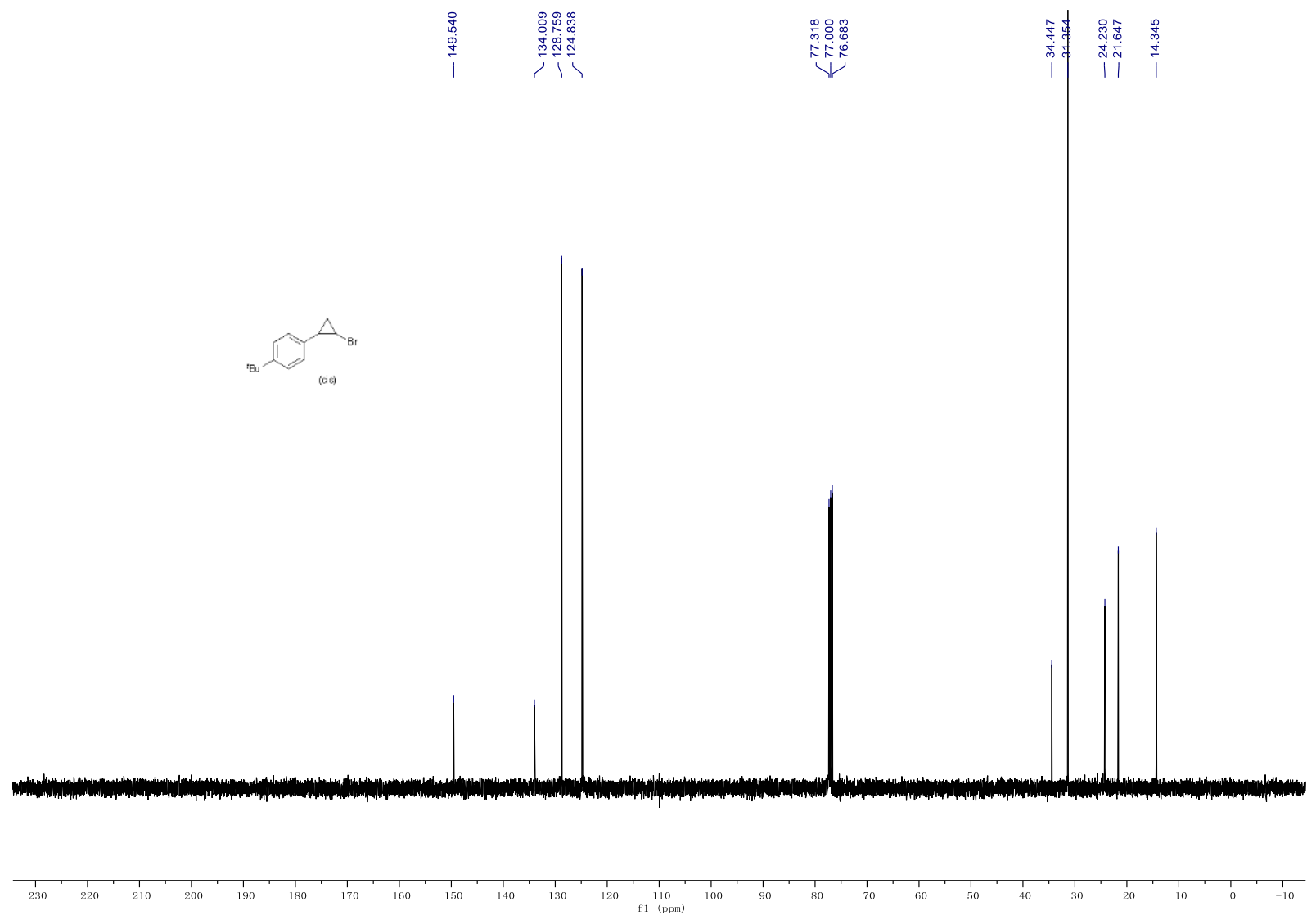

4-((土)-(cis)-2-Bromocyclopropyl)-1,1'-biphenyl.

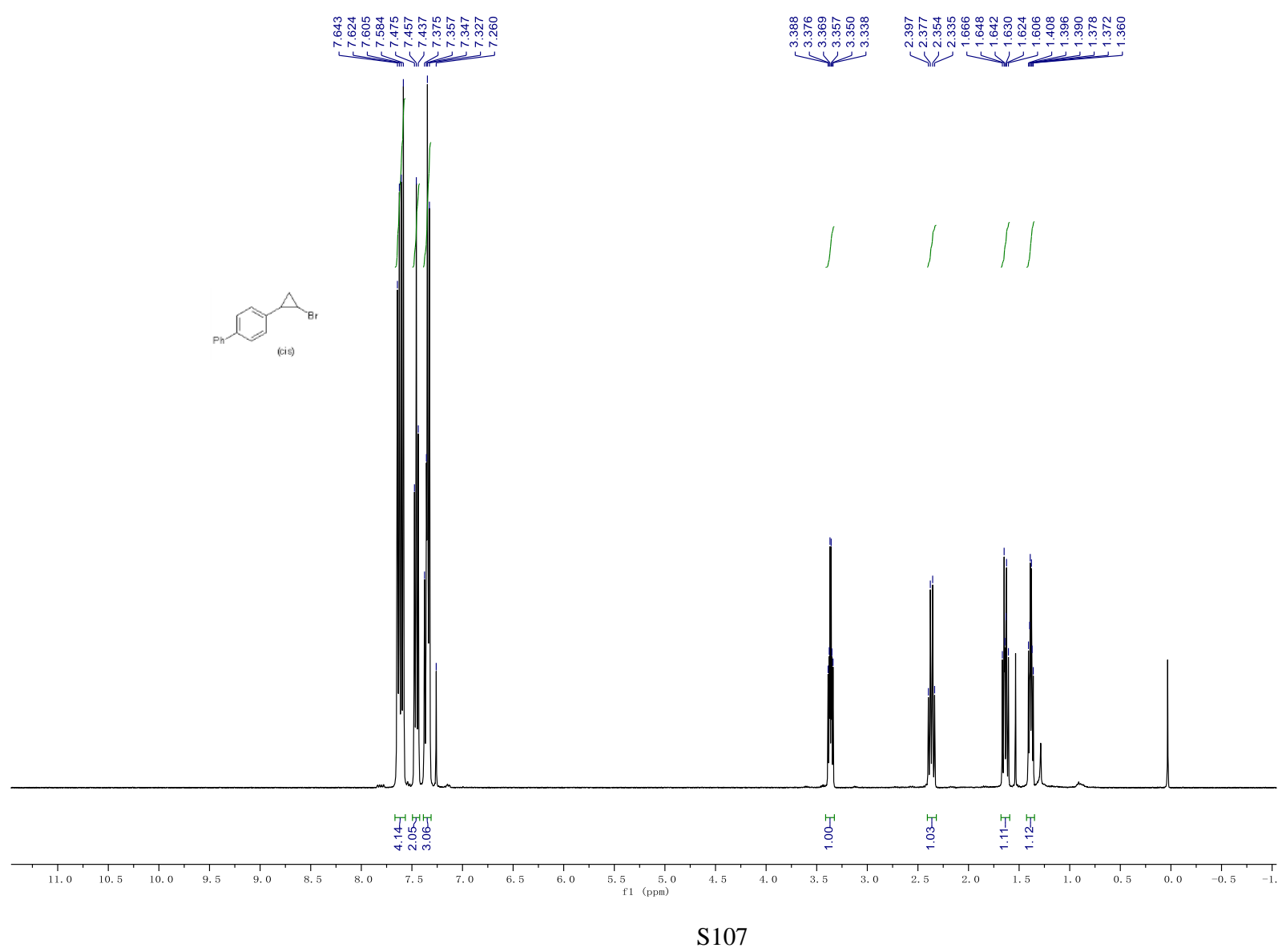



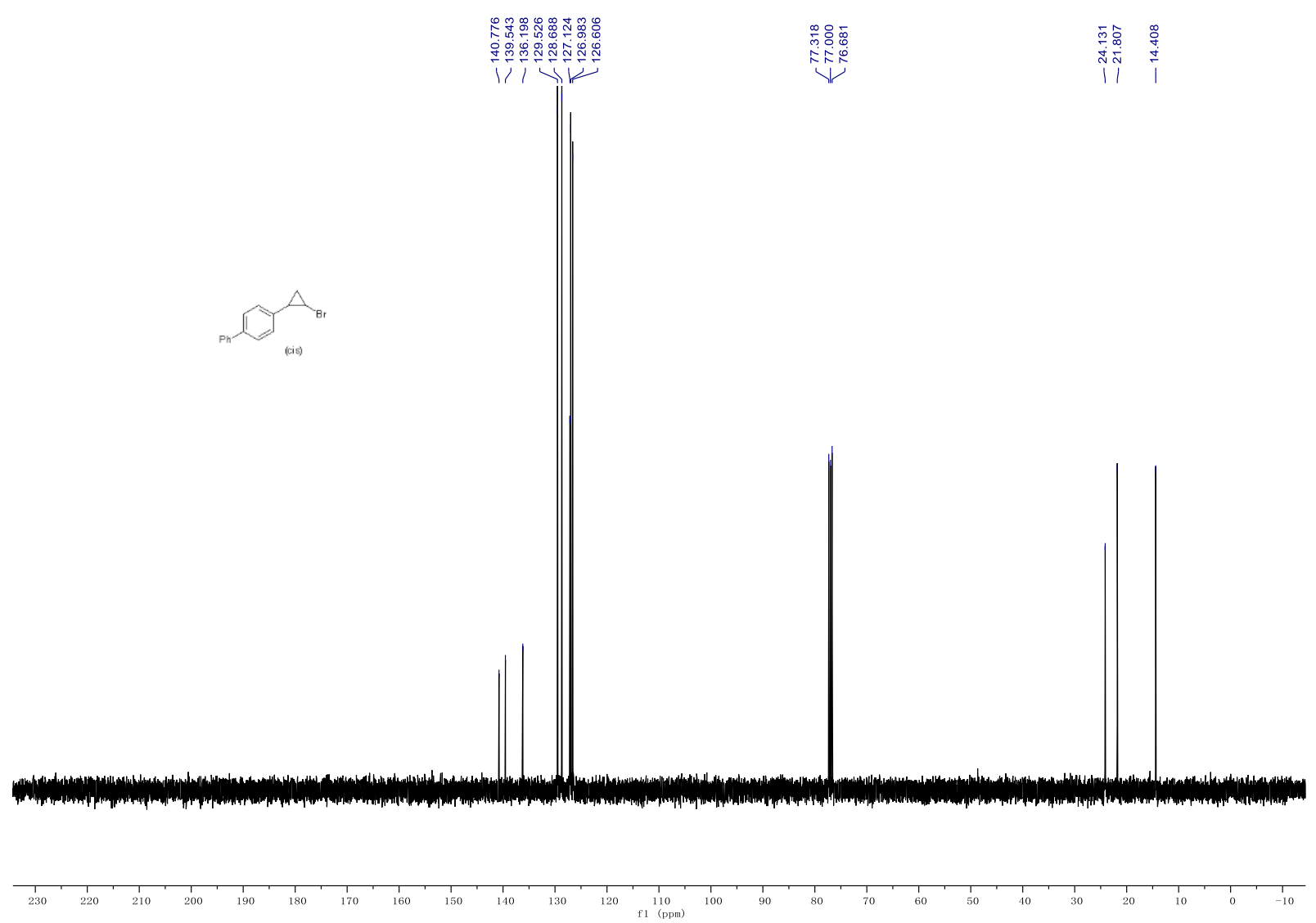

1-(( $)$-(cis)-2-Bromocyclopropyl)-4-(trifluoromethyl)benzene.

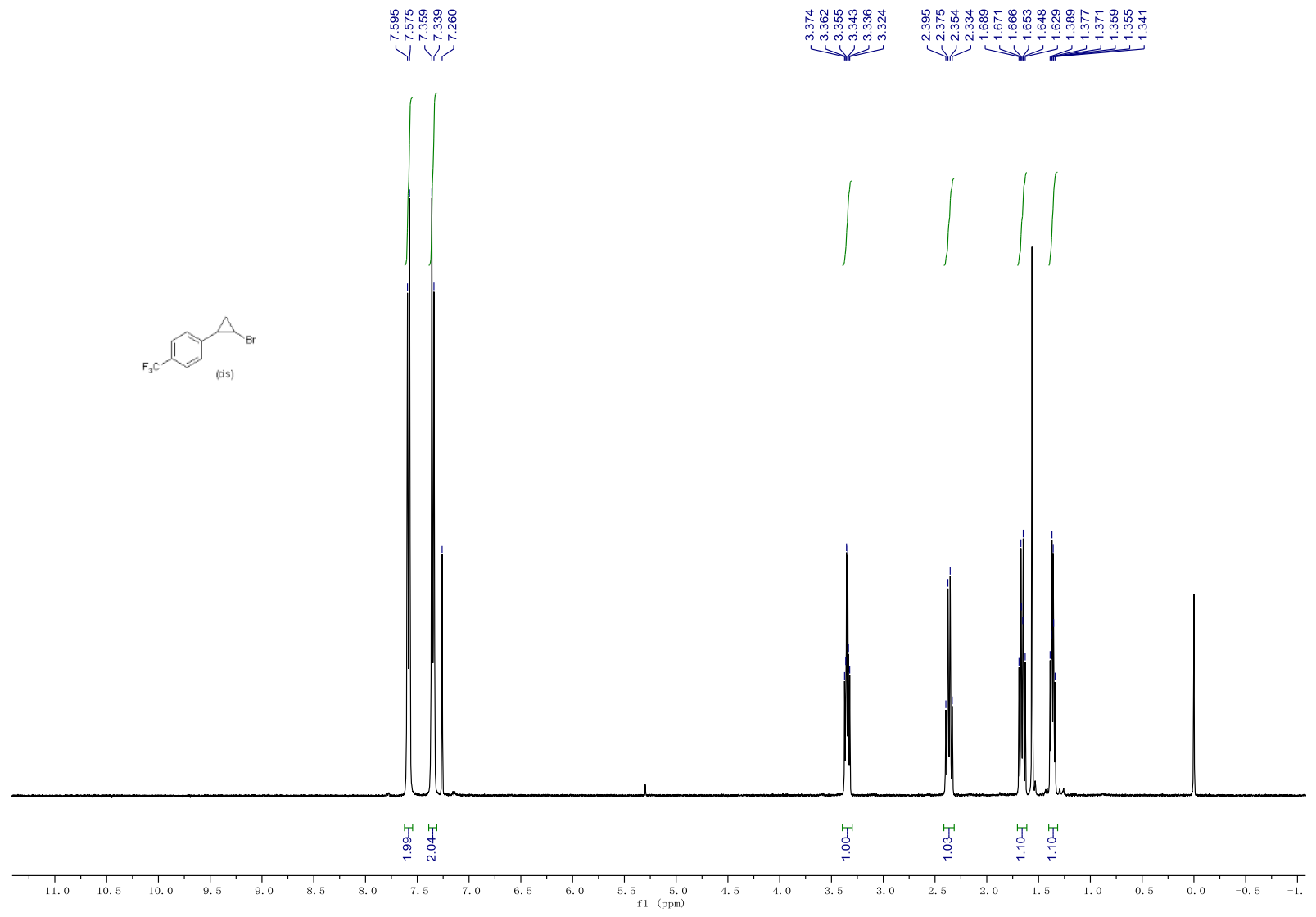



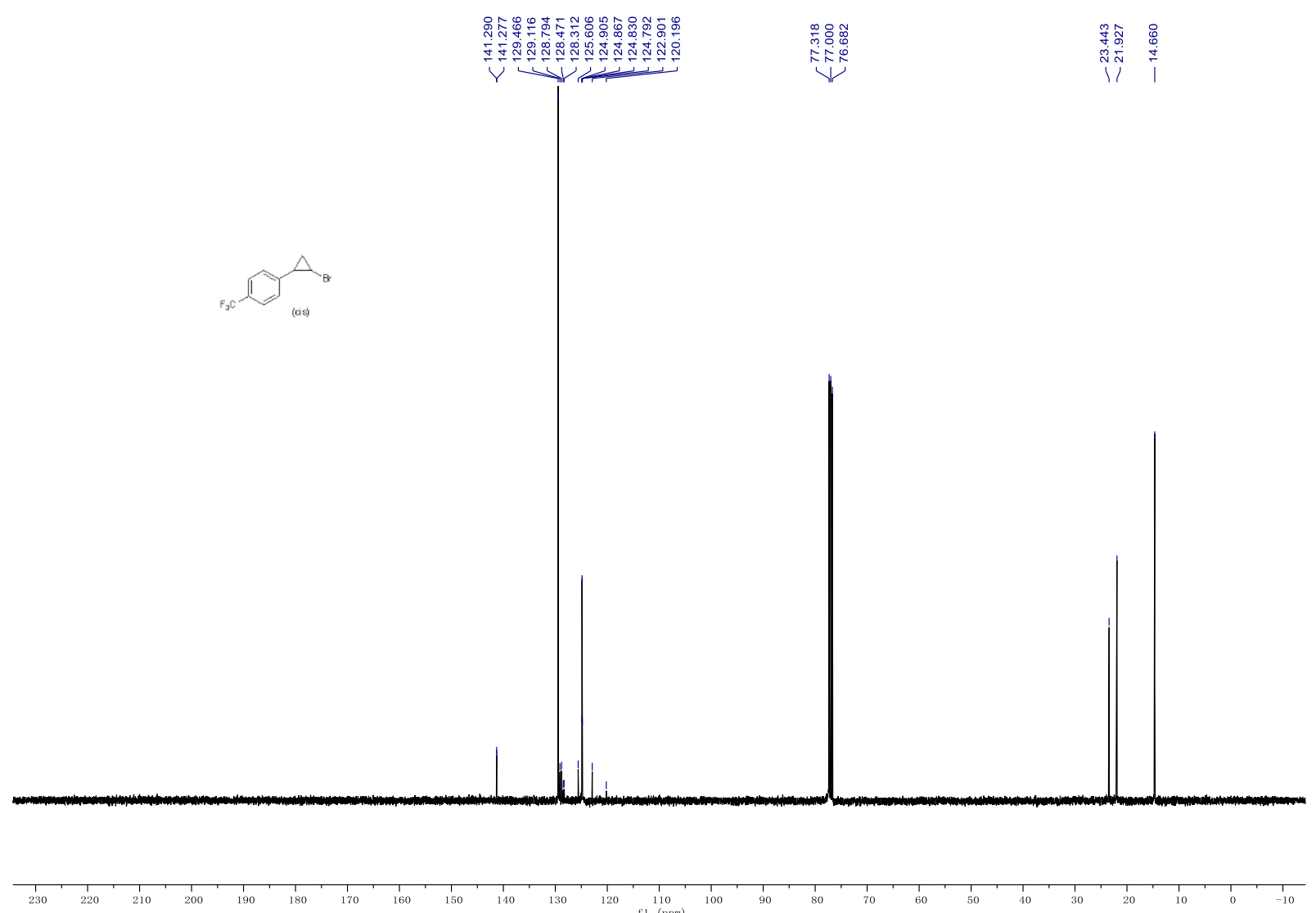

$$
\triangle_{\mathrm{Br}}
$$

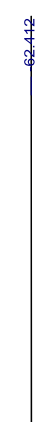


1-((土)-(cis)-2-Bromocyclopropyl)-4-(trifluoromethoxy)benzene.
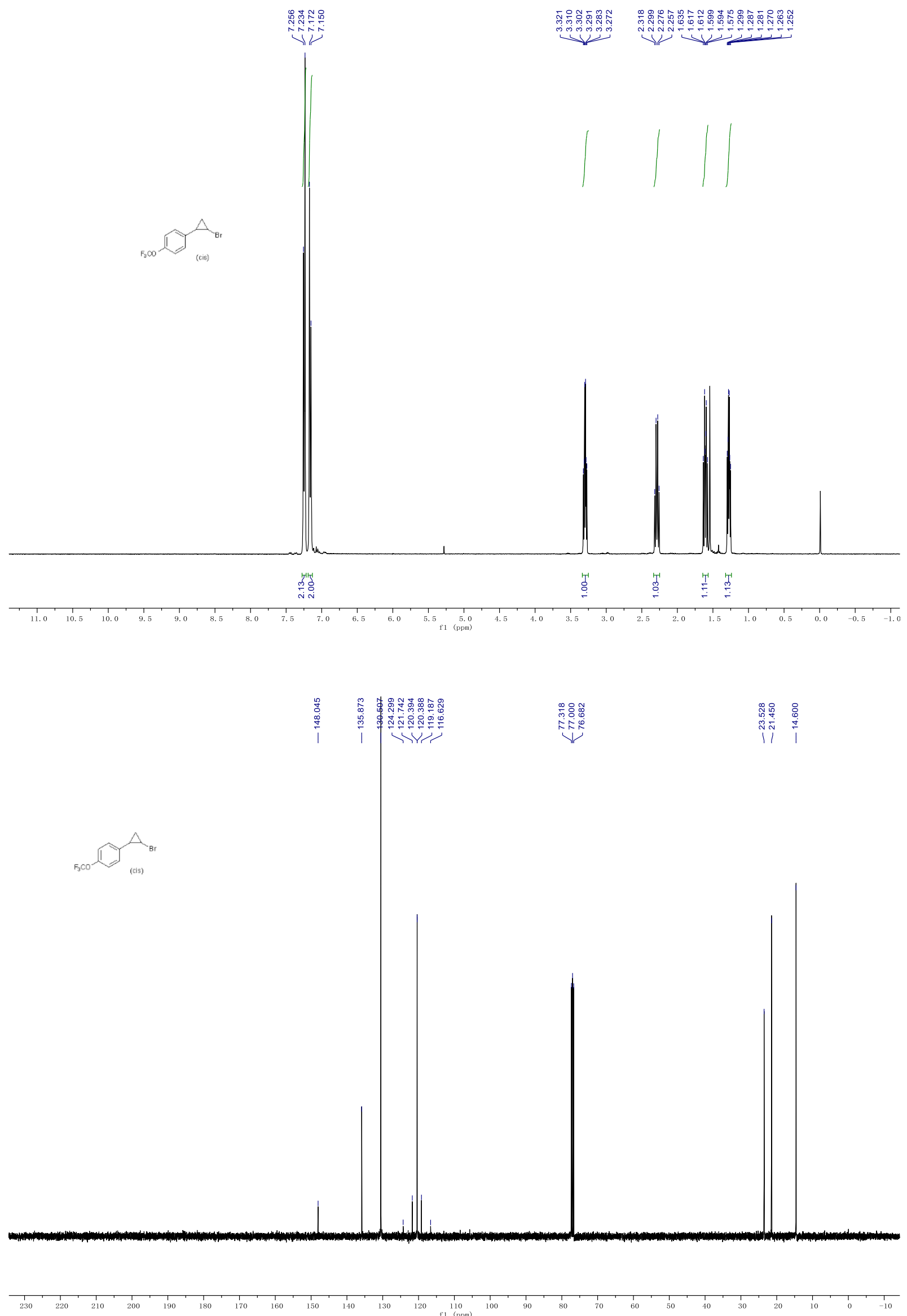

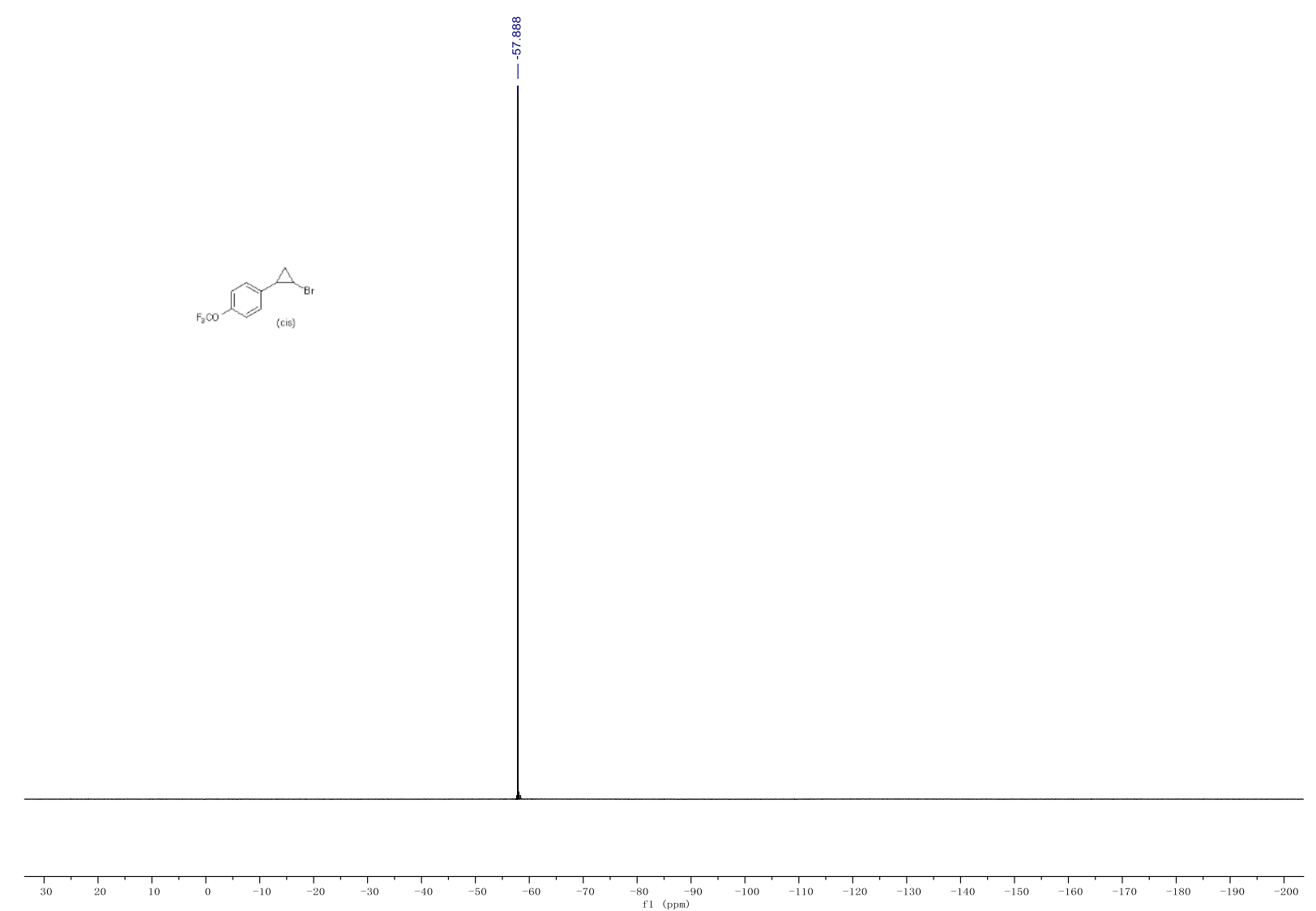

(4-(( $)$-(cis)-2-Bromocyclopropyl)phenyl)trimethylsilane.

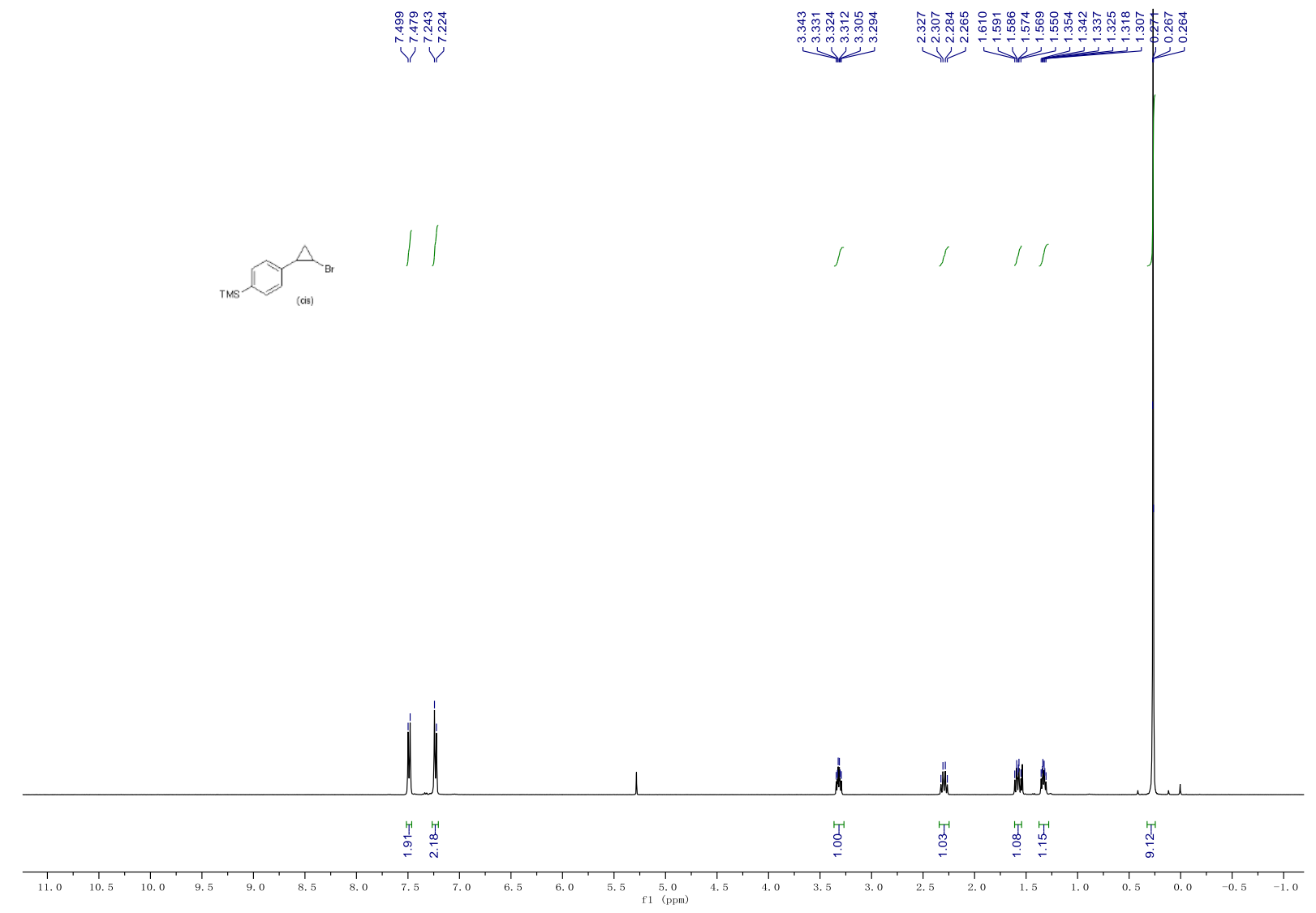



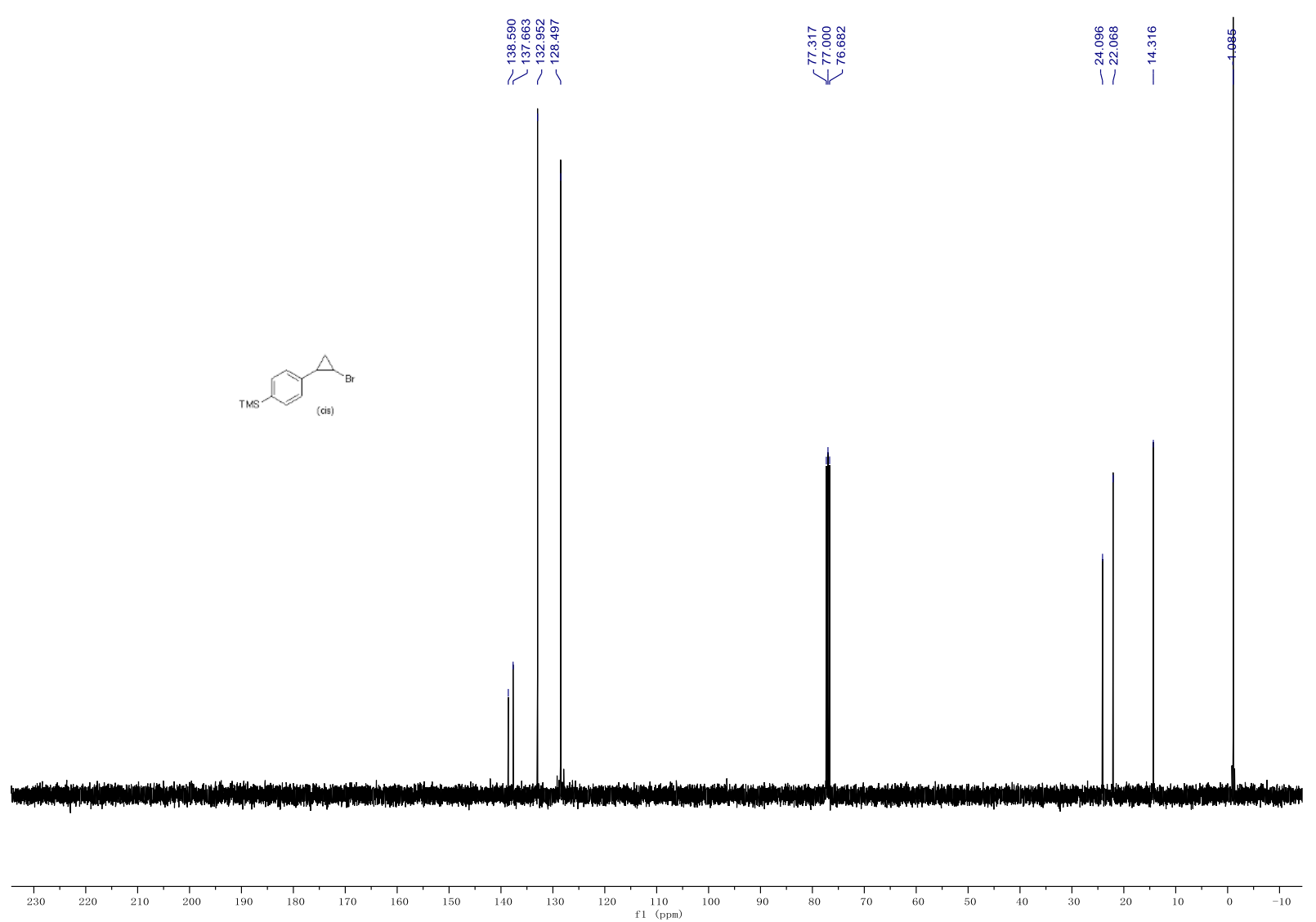

1-(( \pm )-(cis)-2-Bromocyclopropyl)-4-fluorobenzene.

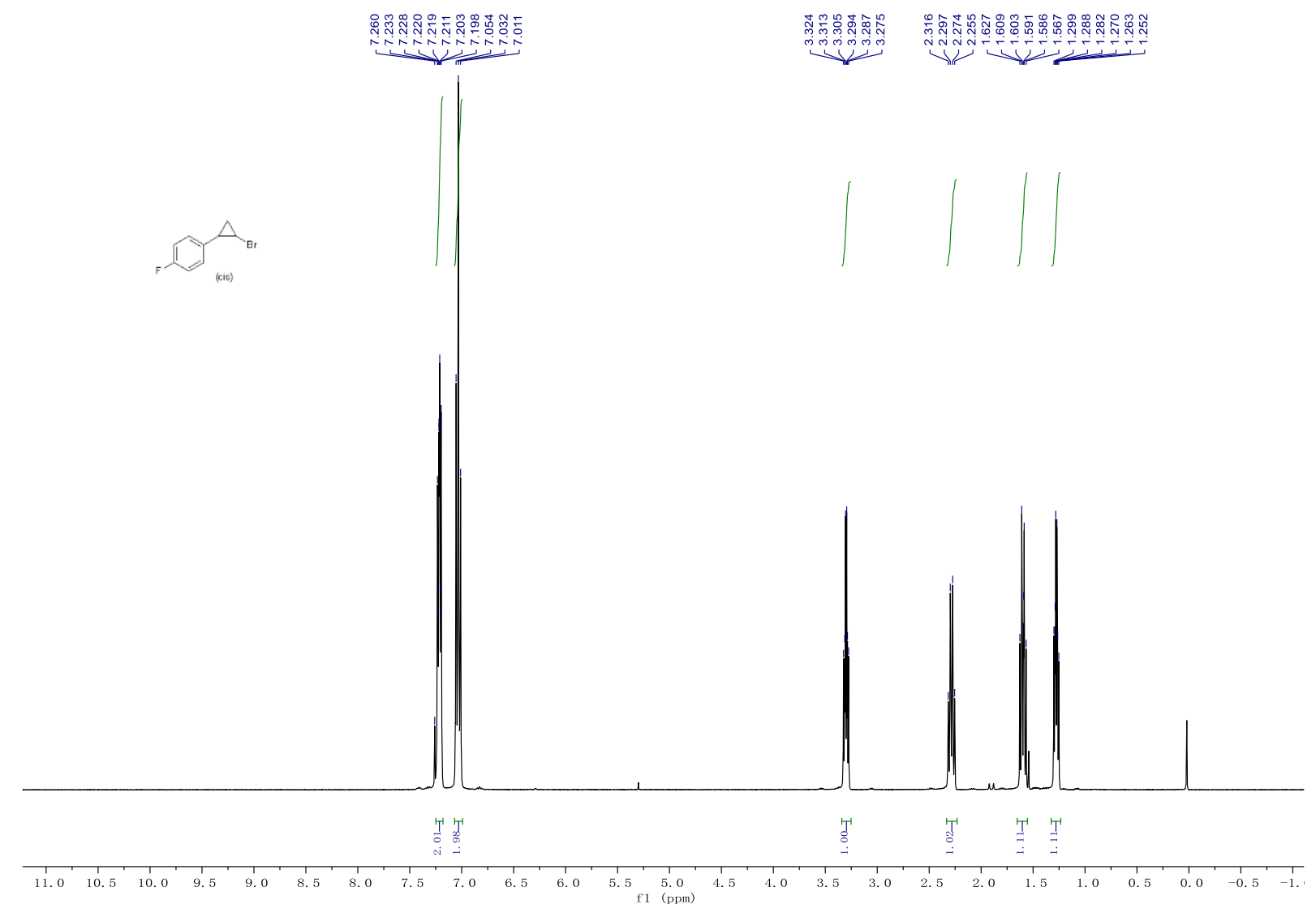



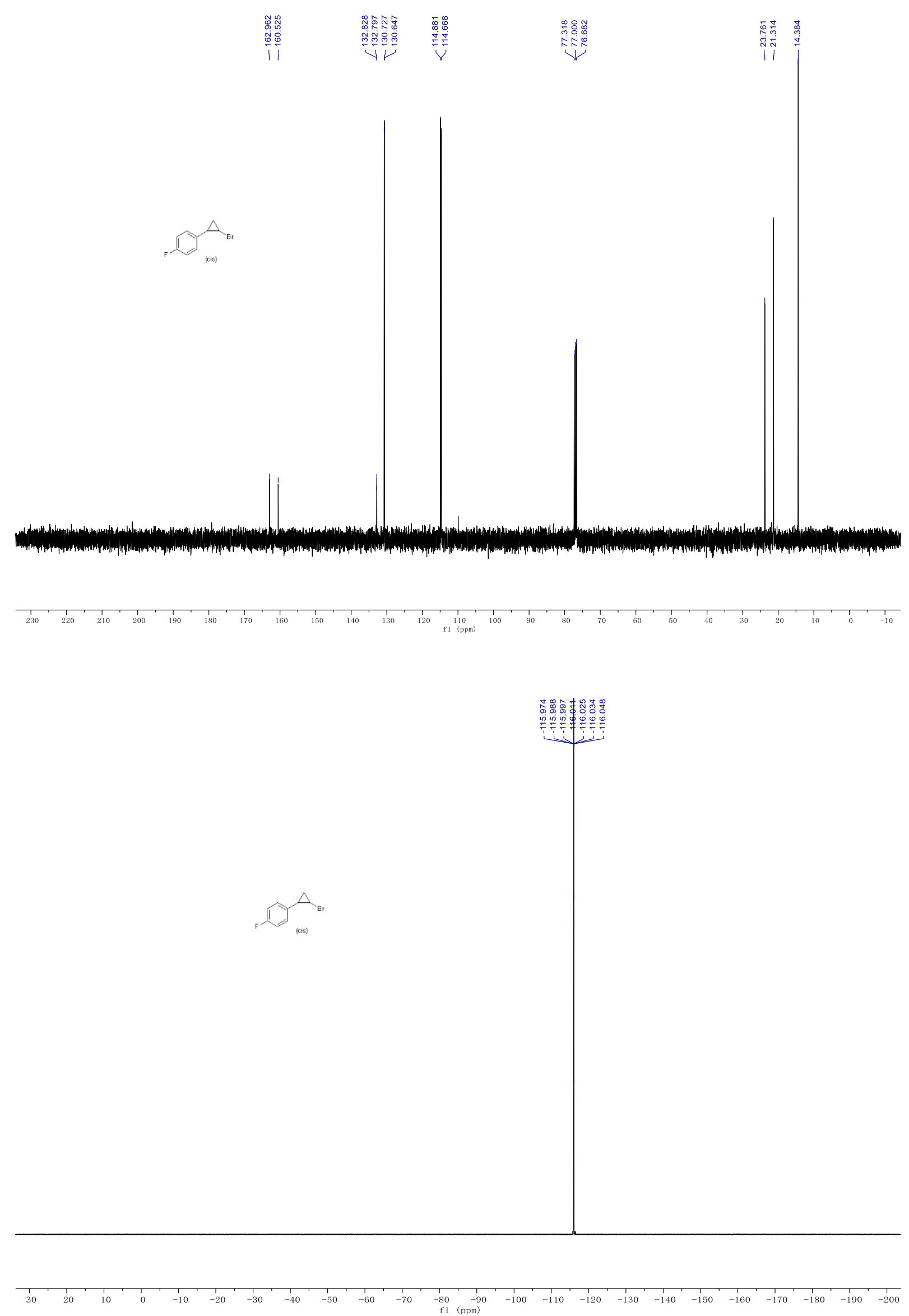
1-((土)-(cis)-2-bromocyclopropyl)-4-chlorobenzene.
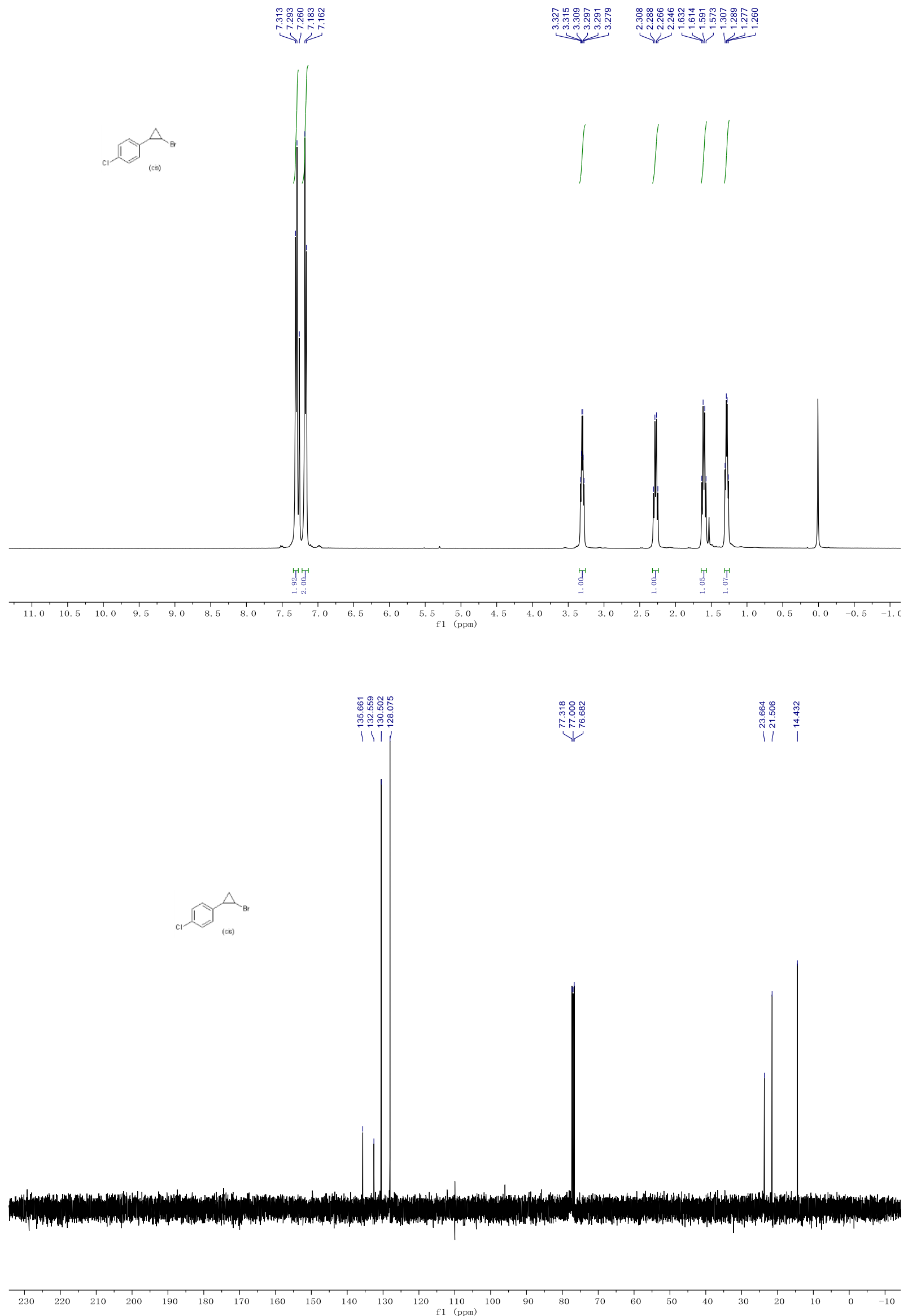
1-(Benzyloxy)-3-(( \pm )-(cis)-2-bromocyclopropyl)benzene.
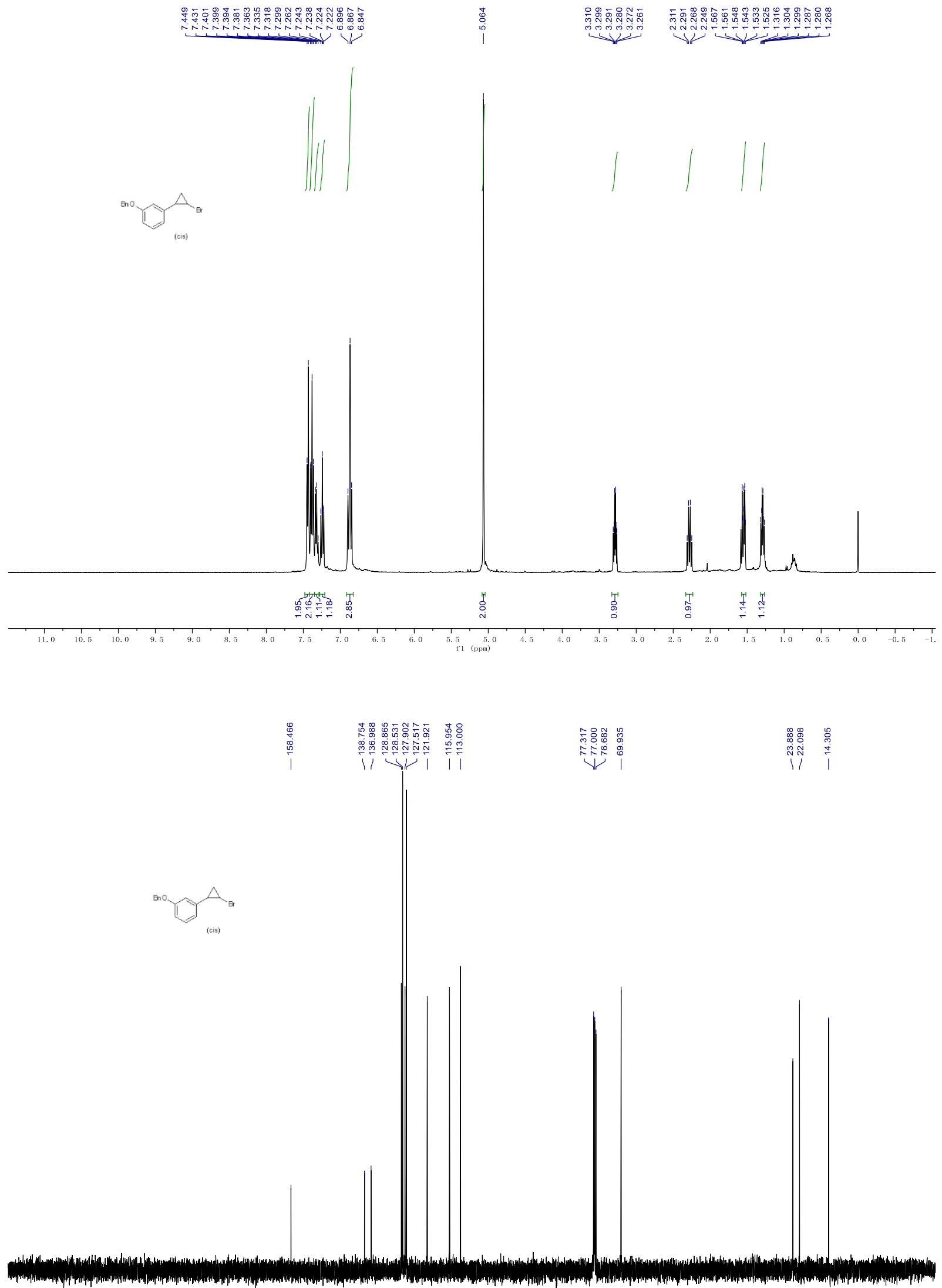

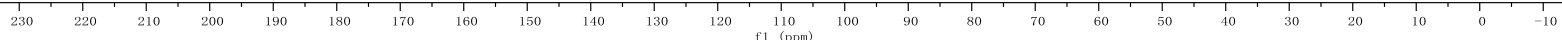


( \pm )-cis-1-(2-Bromocyclopropyl)-4-methoxybenzene.

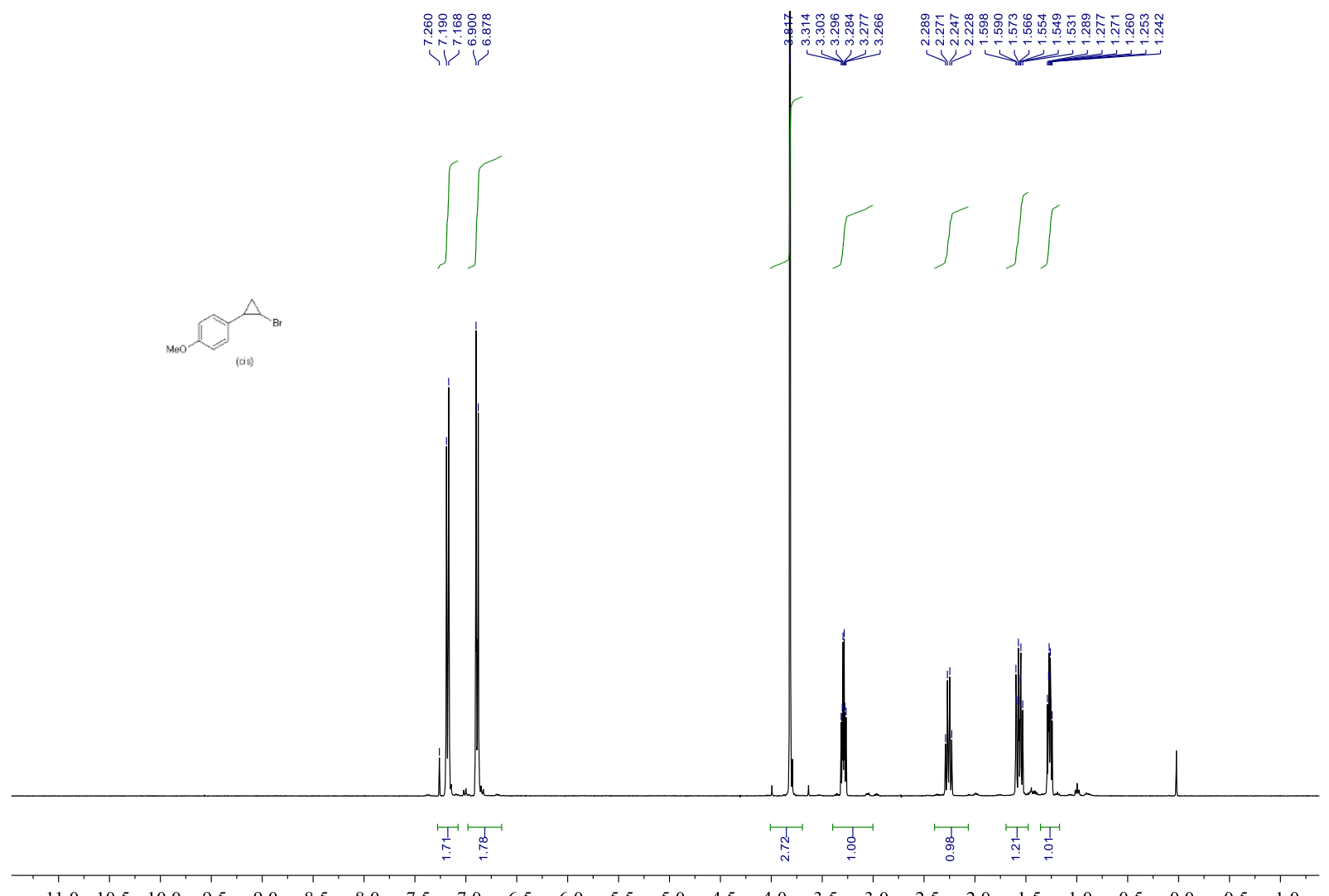

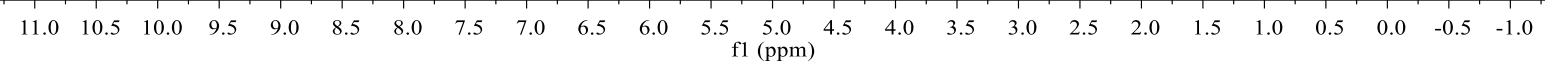

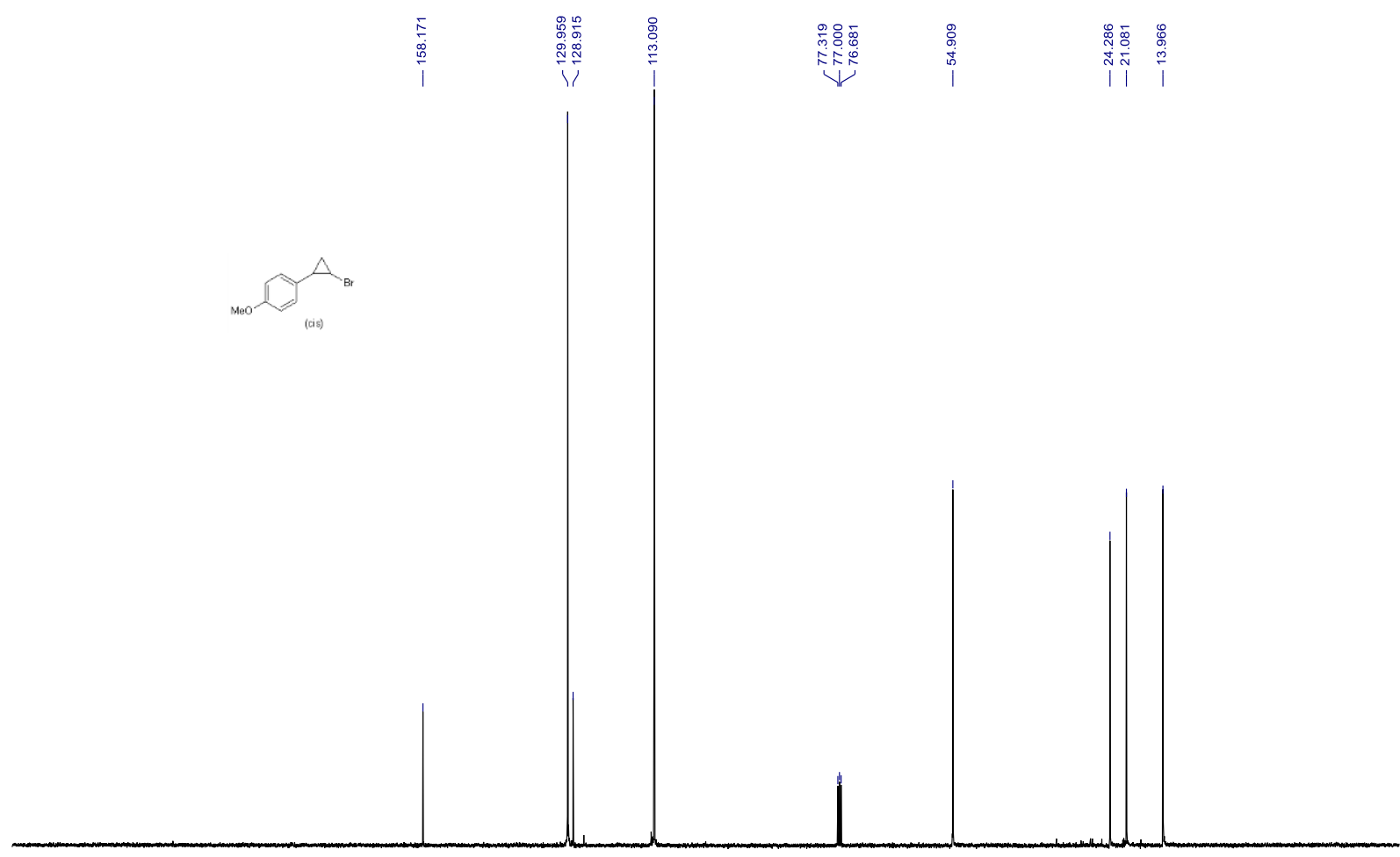

$\begin{array}{lllllllllllllllllllllllllllllllllll}230 & 220 & 210 & 200 & 190 & 180 & 170 & 160 & 150 & 140 & 130 & 120 & 110 & 100 & 90 & 80 & 70 & 60 & 50 & 40 & 30 & 20 & 10 & 0 & -10 & -20 & -31\end{array}$ 
1-((土)-(cis)-2-Bromocyclopropyl)naphthalene.
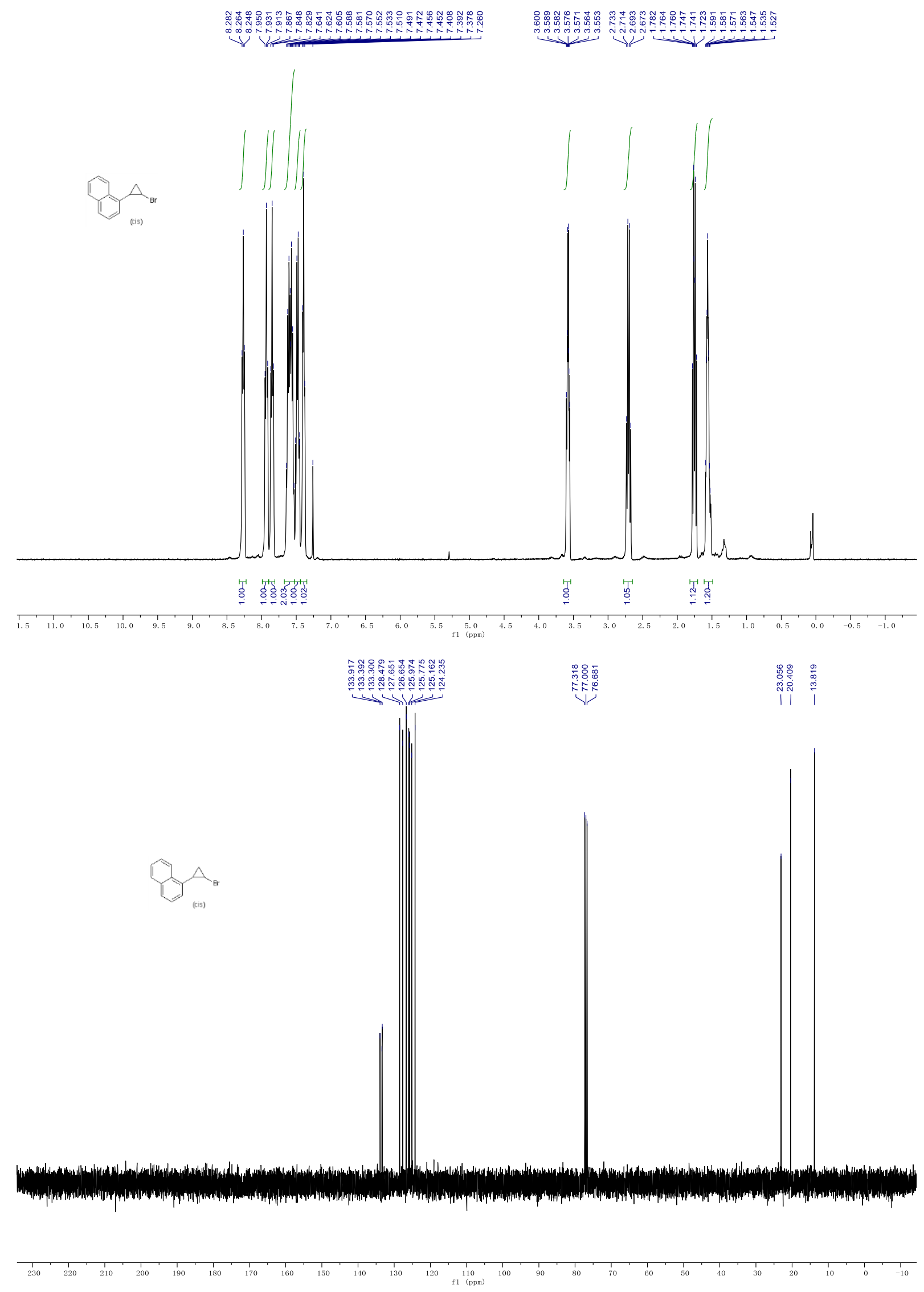
((土)-(cis)-2-bromo-anti-3-methylcyclopropyl)benzene.

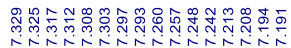

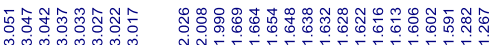

(1)

$\underbrace{2000}$
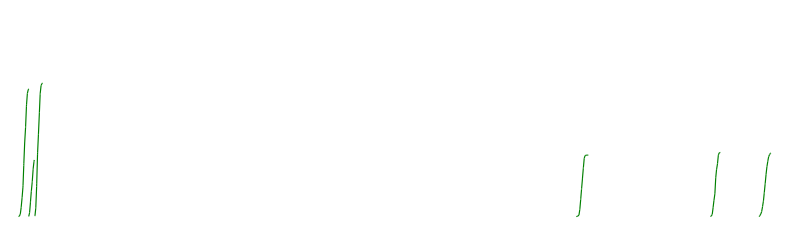

(1)

(cis)
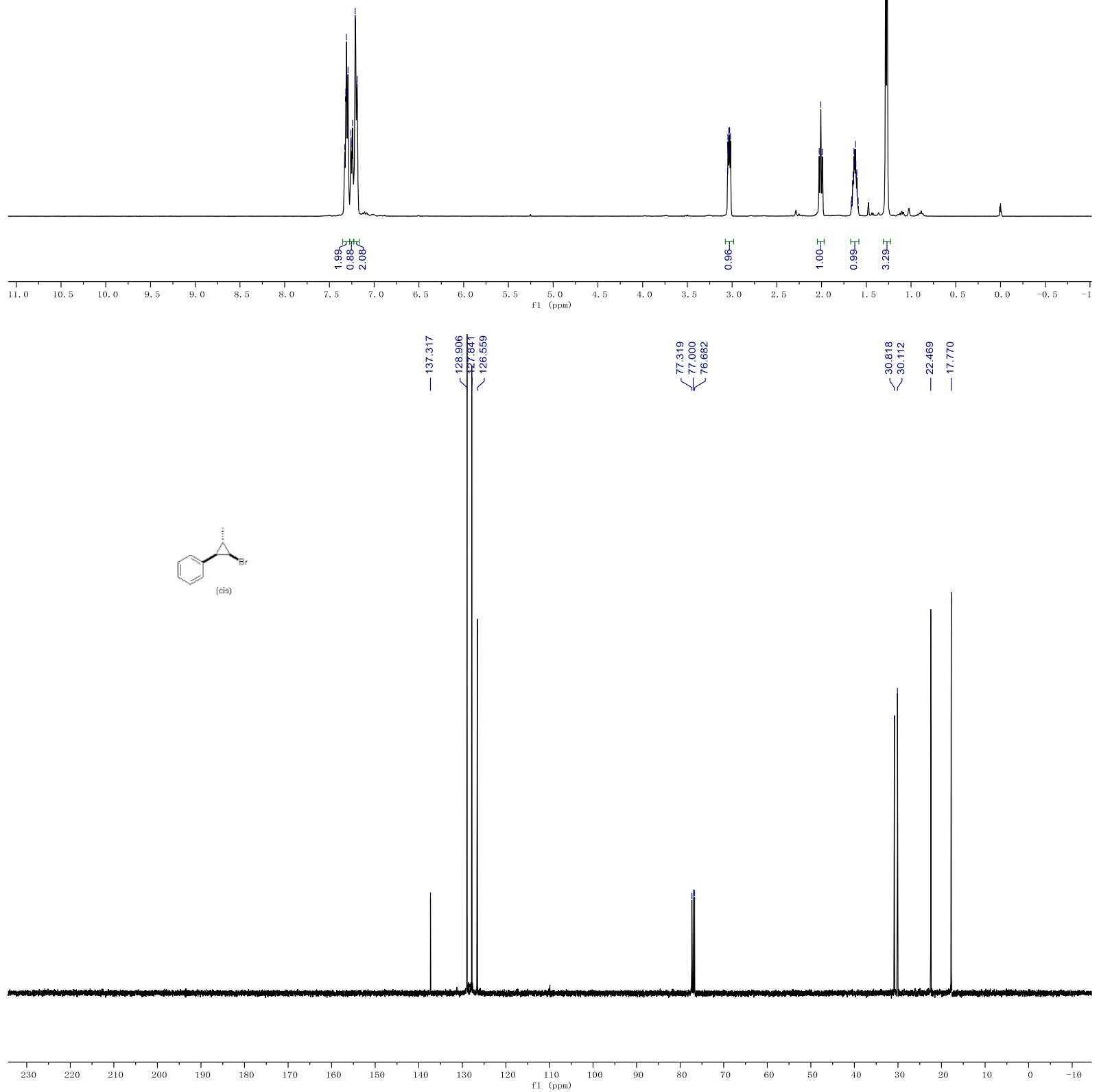
(( \pm -(cis)-2-bromo-anti-3-butylcyclopropyl)benzene.
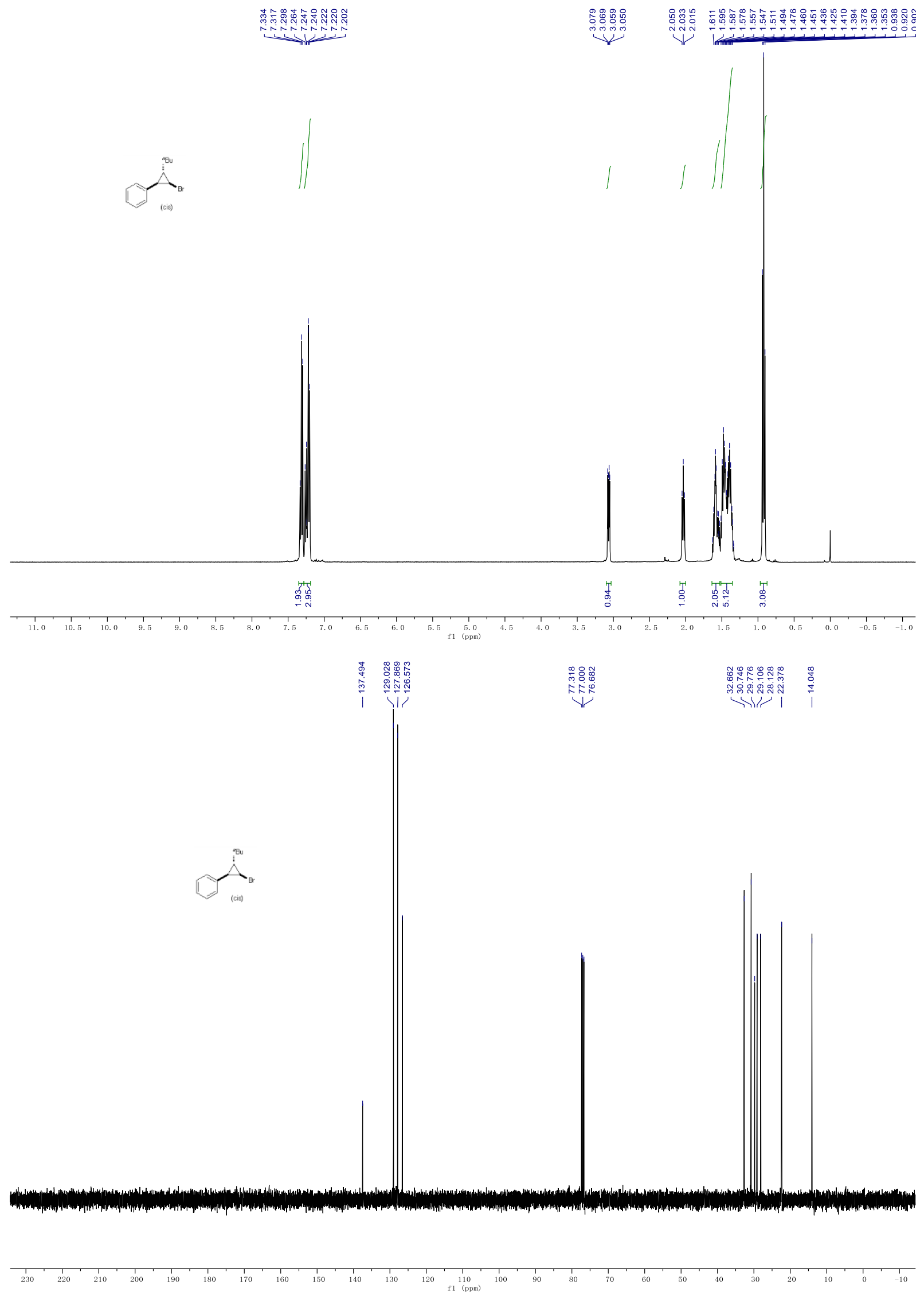
(( \pm$)-(1 S, 2 S, 3 R)-2-(($ Bbenzyloxy $)$ methyl)-3-iodocyclopropyl)benzene.

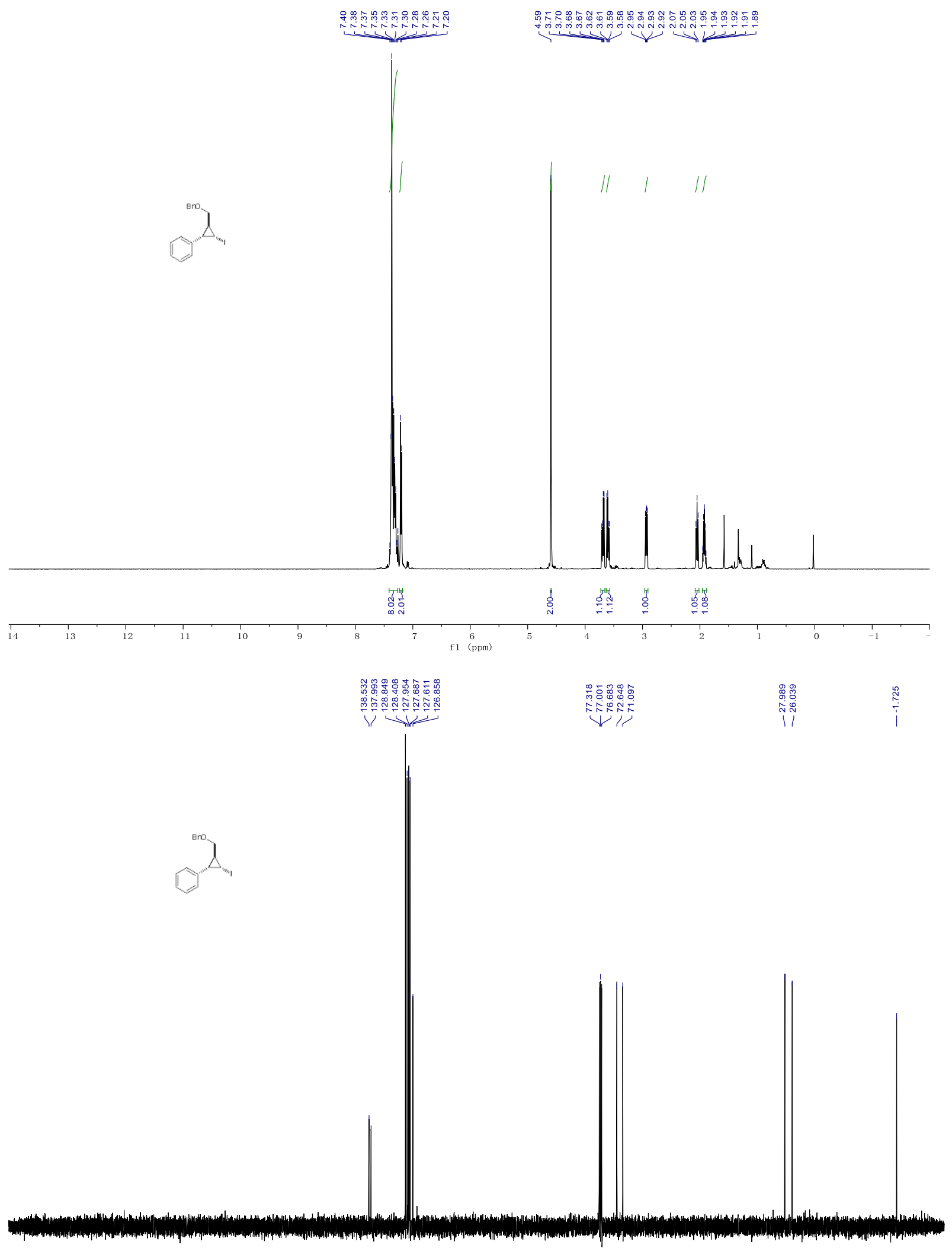

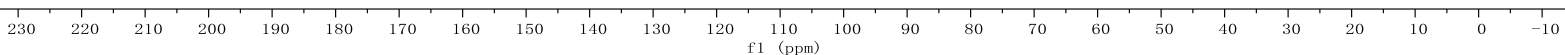


(((1-(3-Bromo-3,3-difluoroprop-1-yn-1-yl)cyclopentyl)oxy)methyl)benzene (2e).
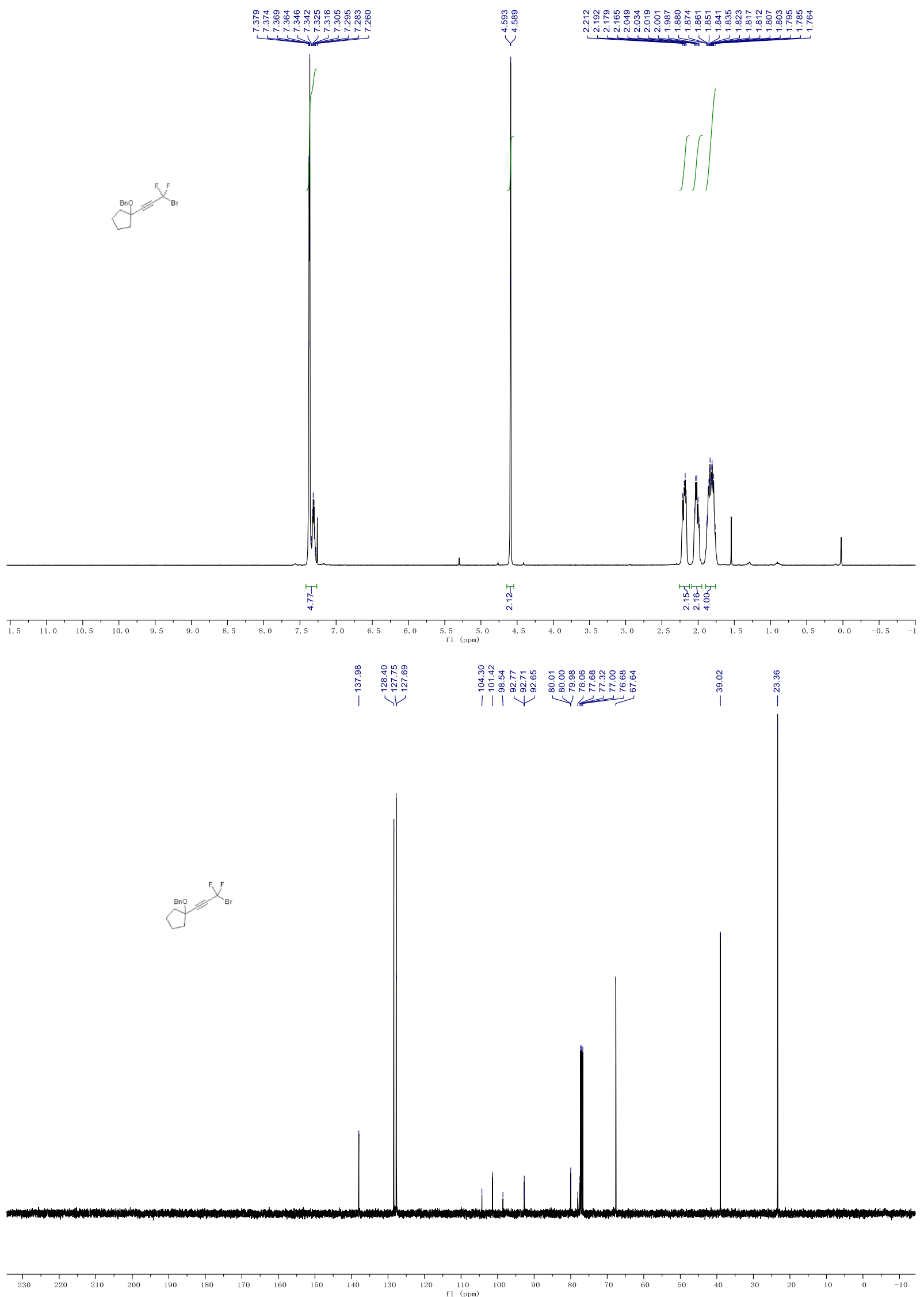


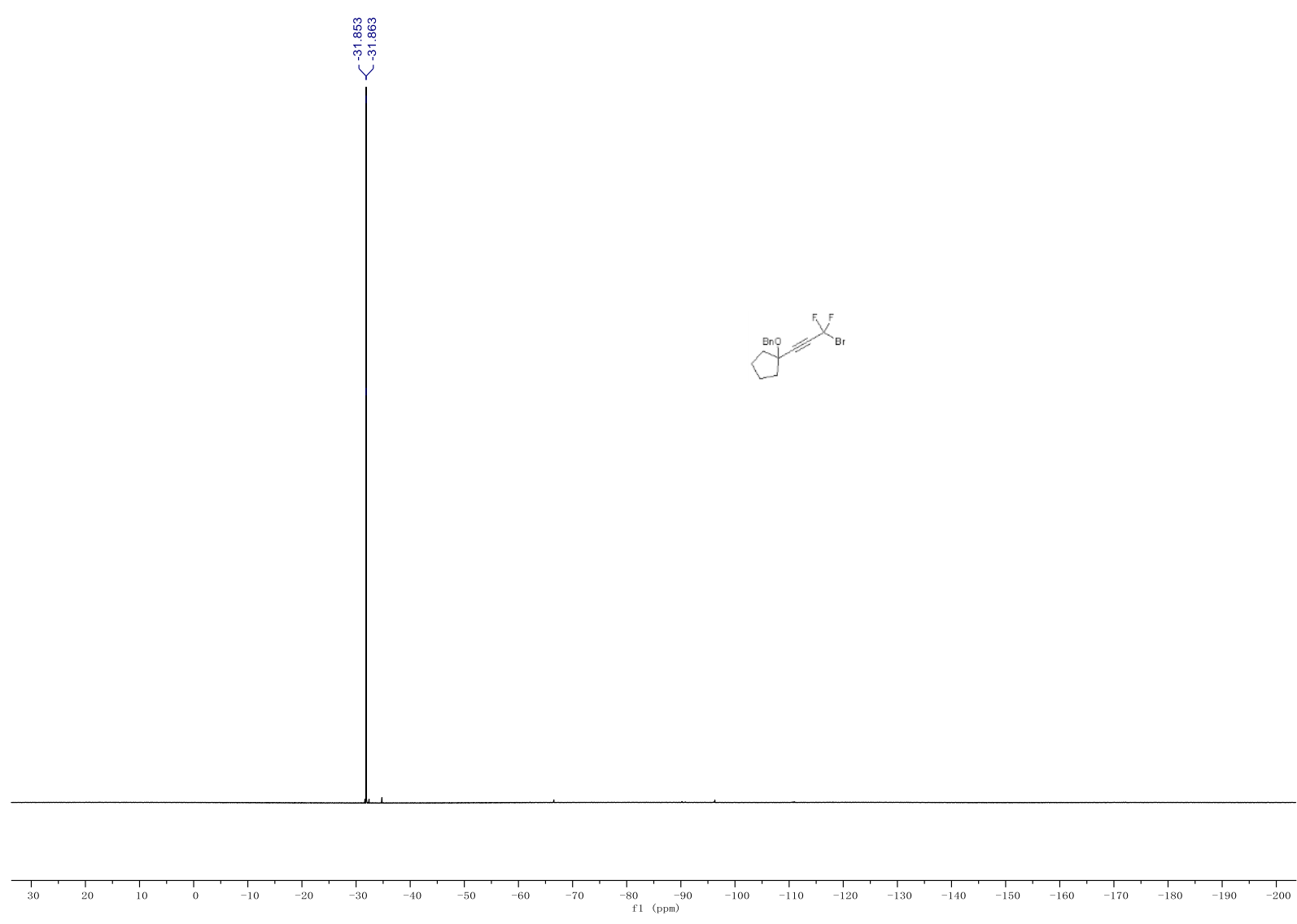

tert-Butyl-4-(benzyloxy)-4-(3-bromo-3,3-difluoroprop-1-yn-1-yl)piperidine-1-carboxylate (2f).

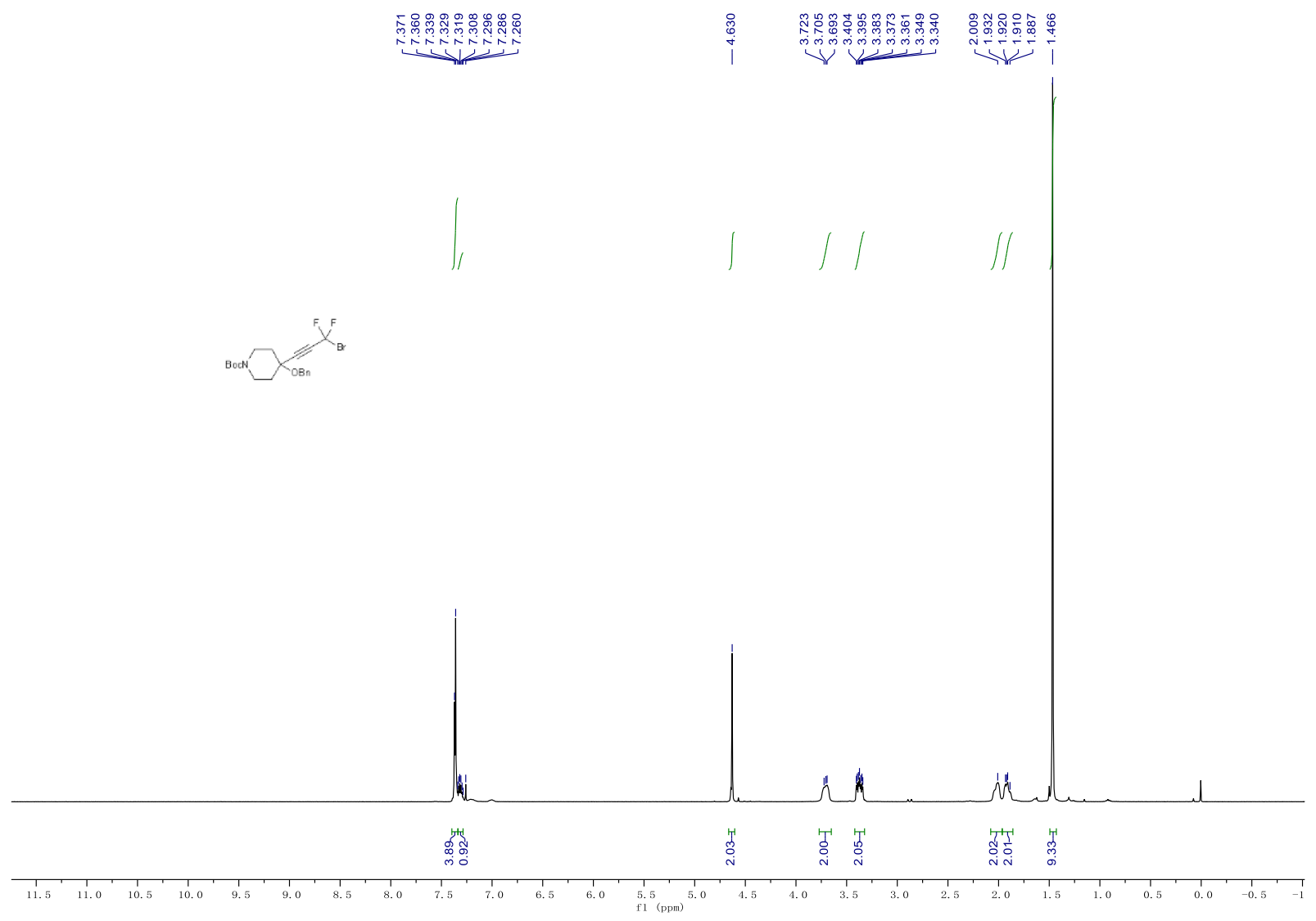



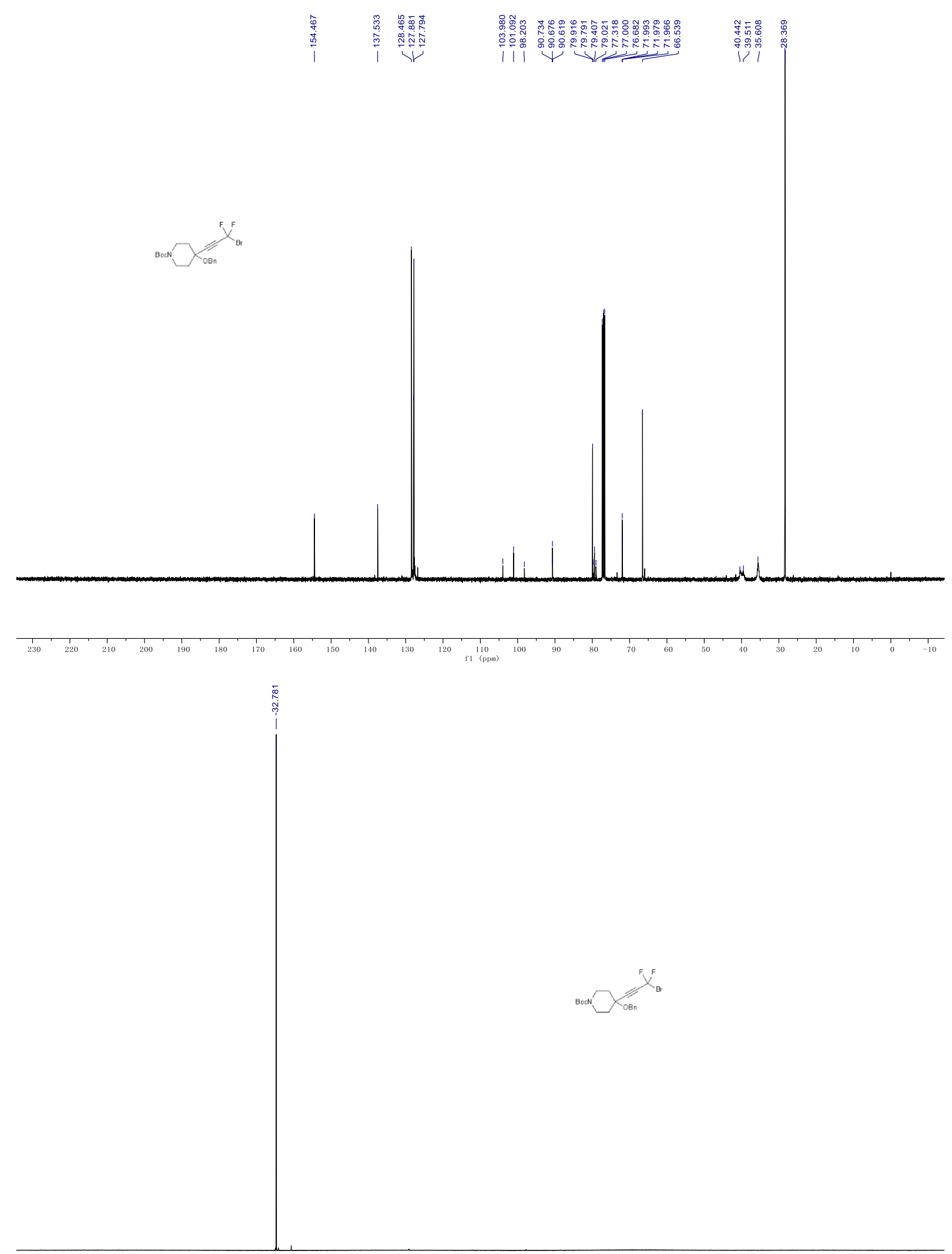

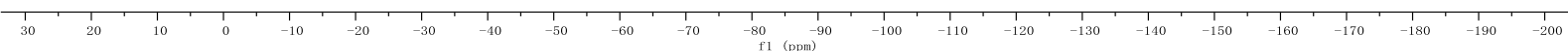


(3aR,5R,6aR)-6-(Benzyloxy)-5-(3-bromo-3,3-difluoroprop-1-yn-1-yl)-2,2-dimethyltetrahydrofur o[2,3-d][1,3]dioxole $(2 \mathrm{~g})$.

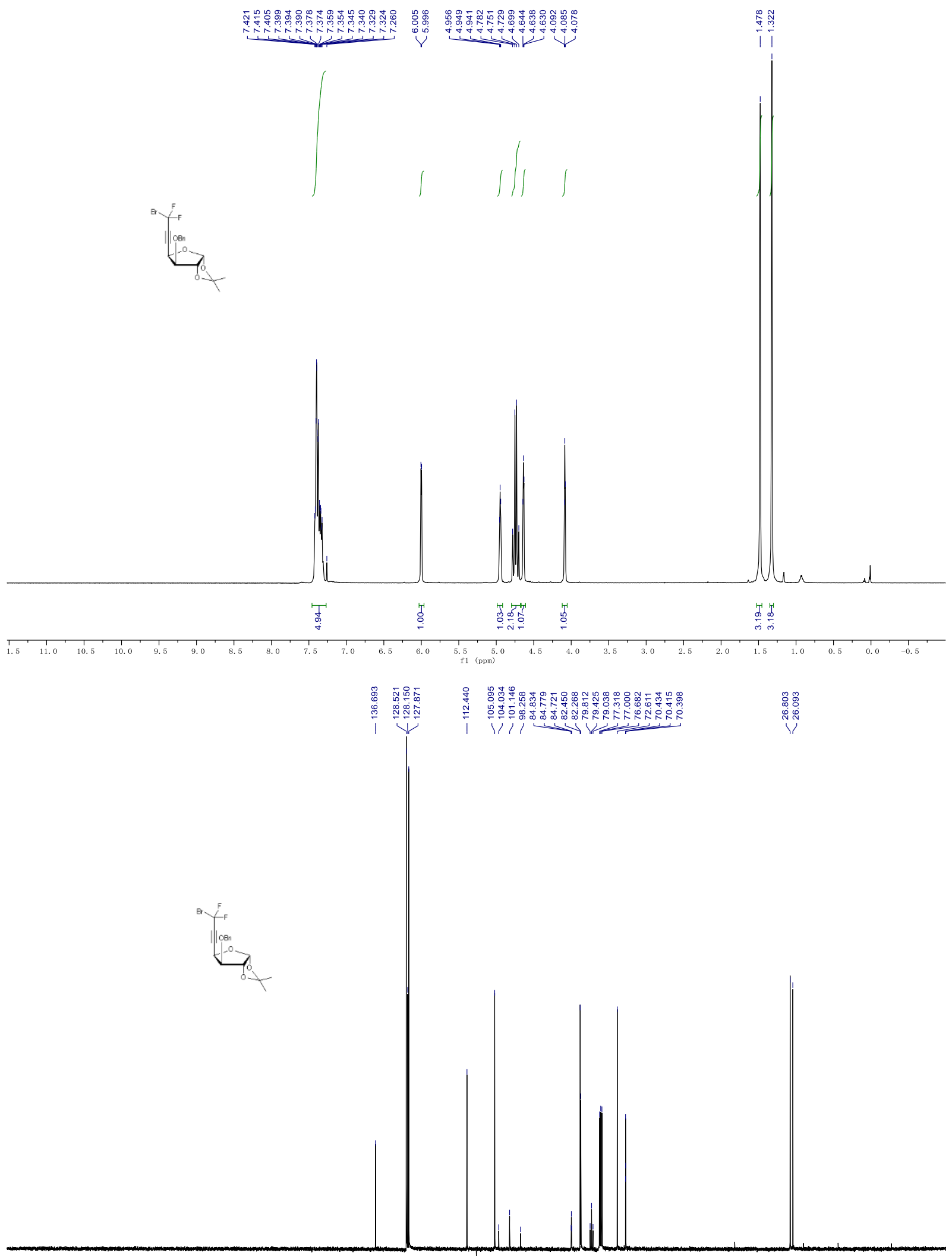

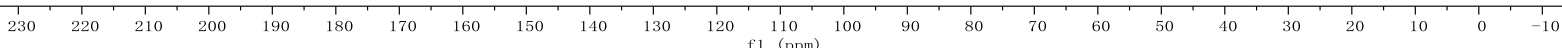



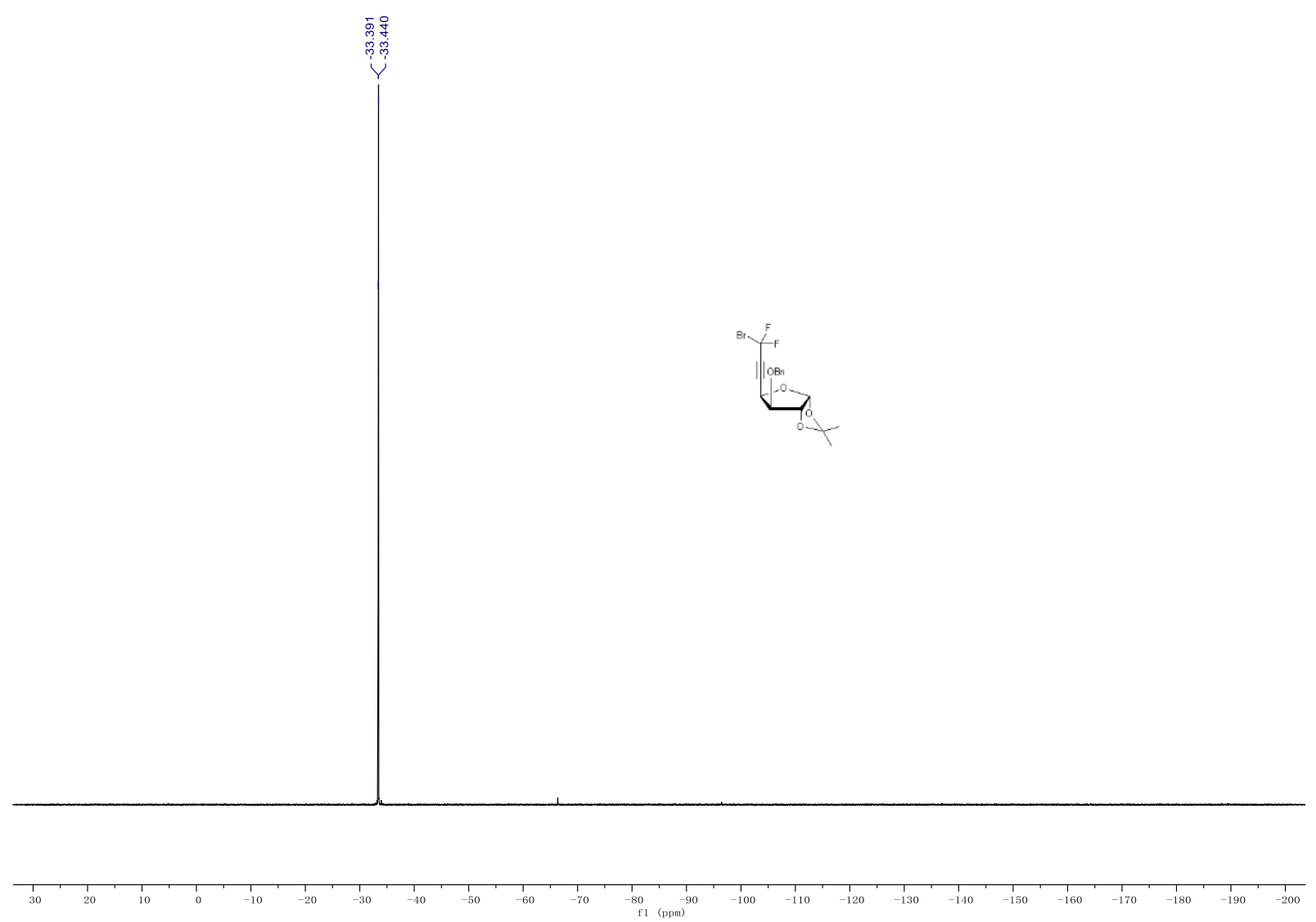

(3-Bromo-4,4-difluorobut-1-yn-1-yl)triisopropylsilane (21).

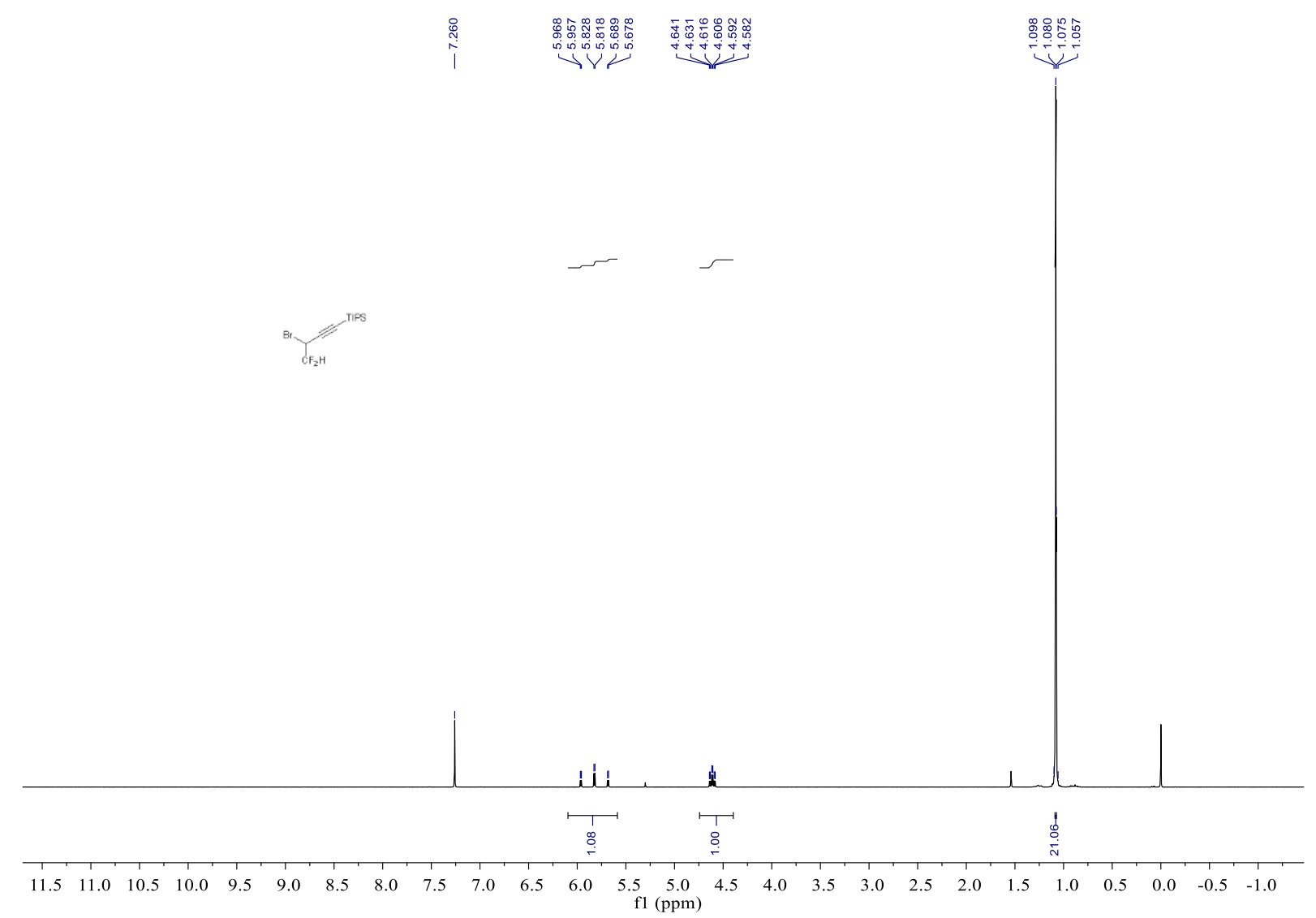




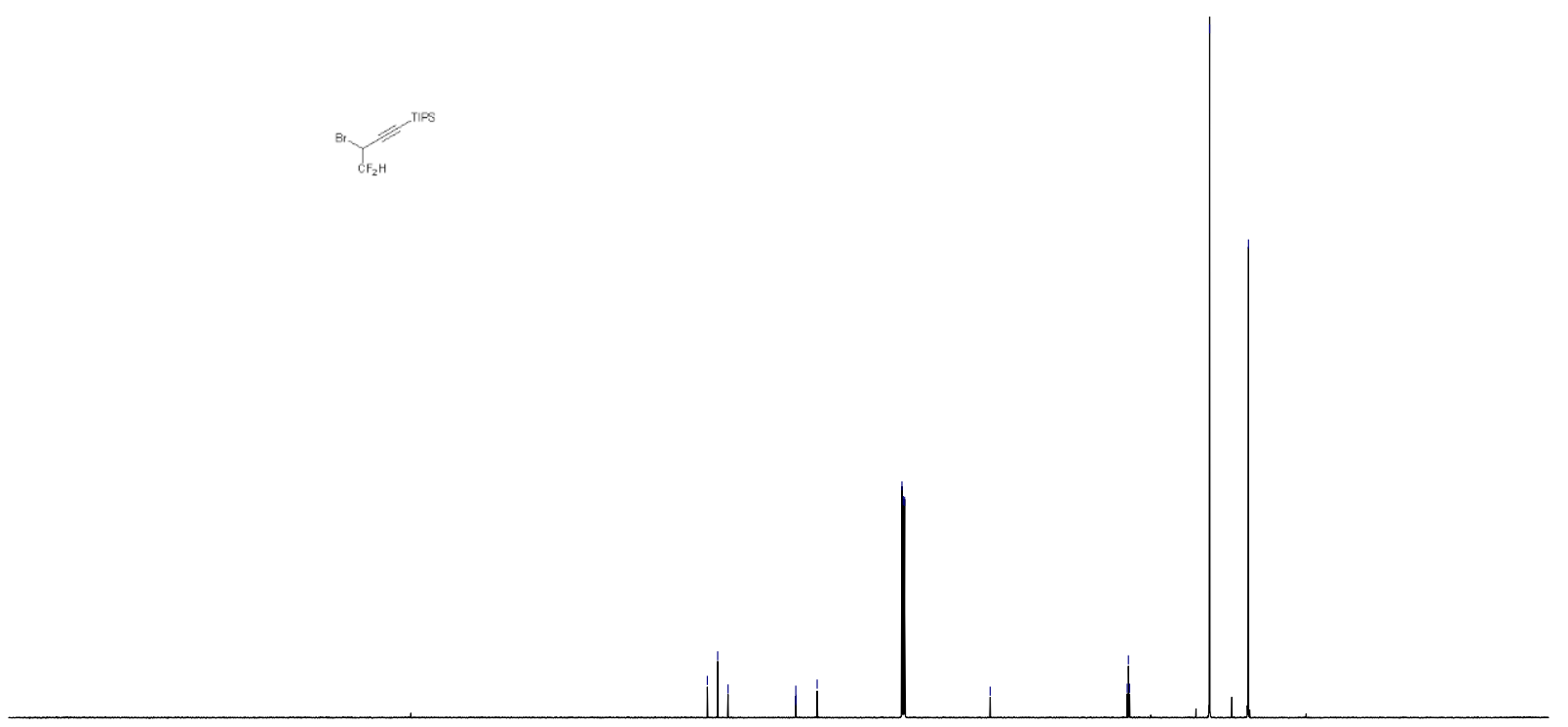

$\begin{array}{lllllllllllllllllllllllllllllllllllllllllll}240 & 230 & 220 & 210 & 200 & 190 & 180 & 170 & 160 & 150 & 140 & 130 & 120 & 110 & 100 & 90 & 80 & 70 & 60 & 50 & 40 & 30 & 20 & 10 & 0 & -10 & -20 & -30 & -40\end{array}$ fl (ppm)

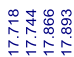

政
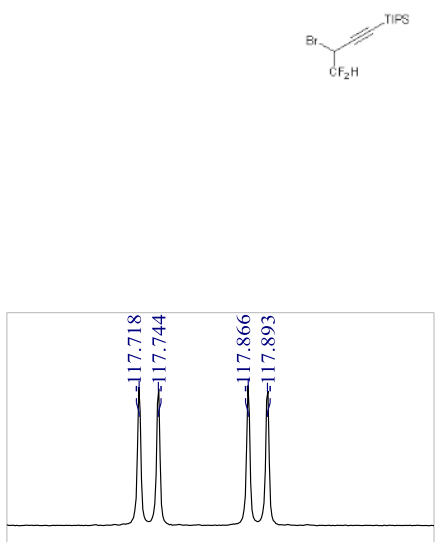

$-117.6-117.7-117.8-117.9-118.0-118.1$

f1 (ppm)

\begin{tabular}{llllllllllllllllllllllllllllllllll}
\hline 30 & 20 & 10 & 0 & -10 & -20 & -30 & -40 & -50 & -60 & -70 & -80 & -90 & -100 & -110 & -120 & -130 & -140 & -150 & -160 & -170 & -180 & -190 & -200
\end{tabular} 
2-Bromo-1,1-difluorodec-3-yne (2m).

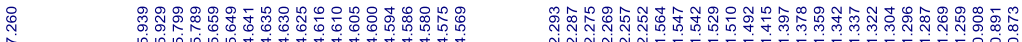

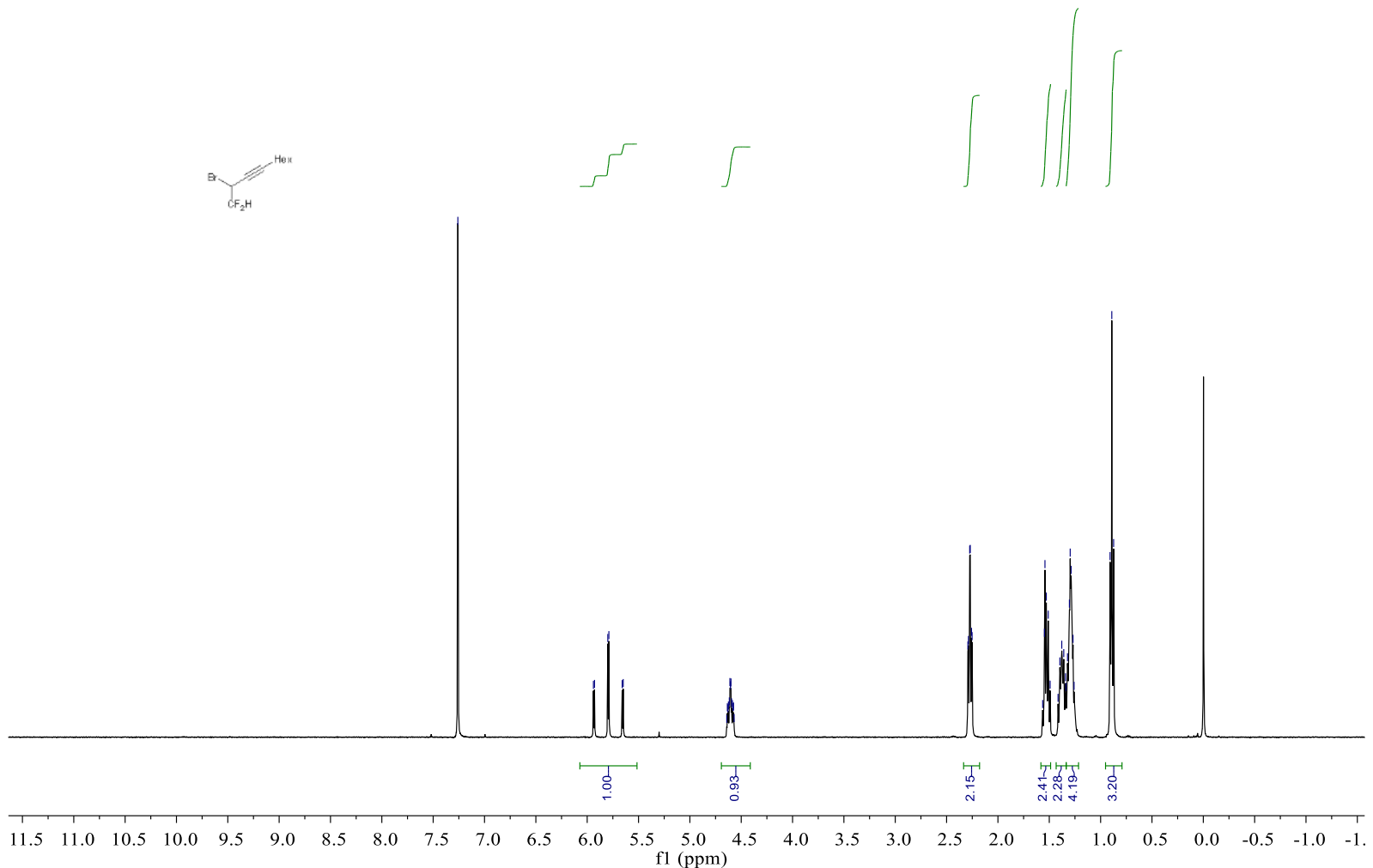

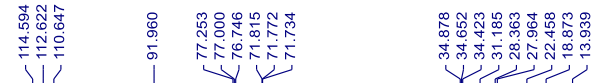

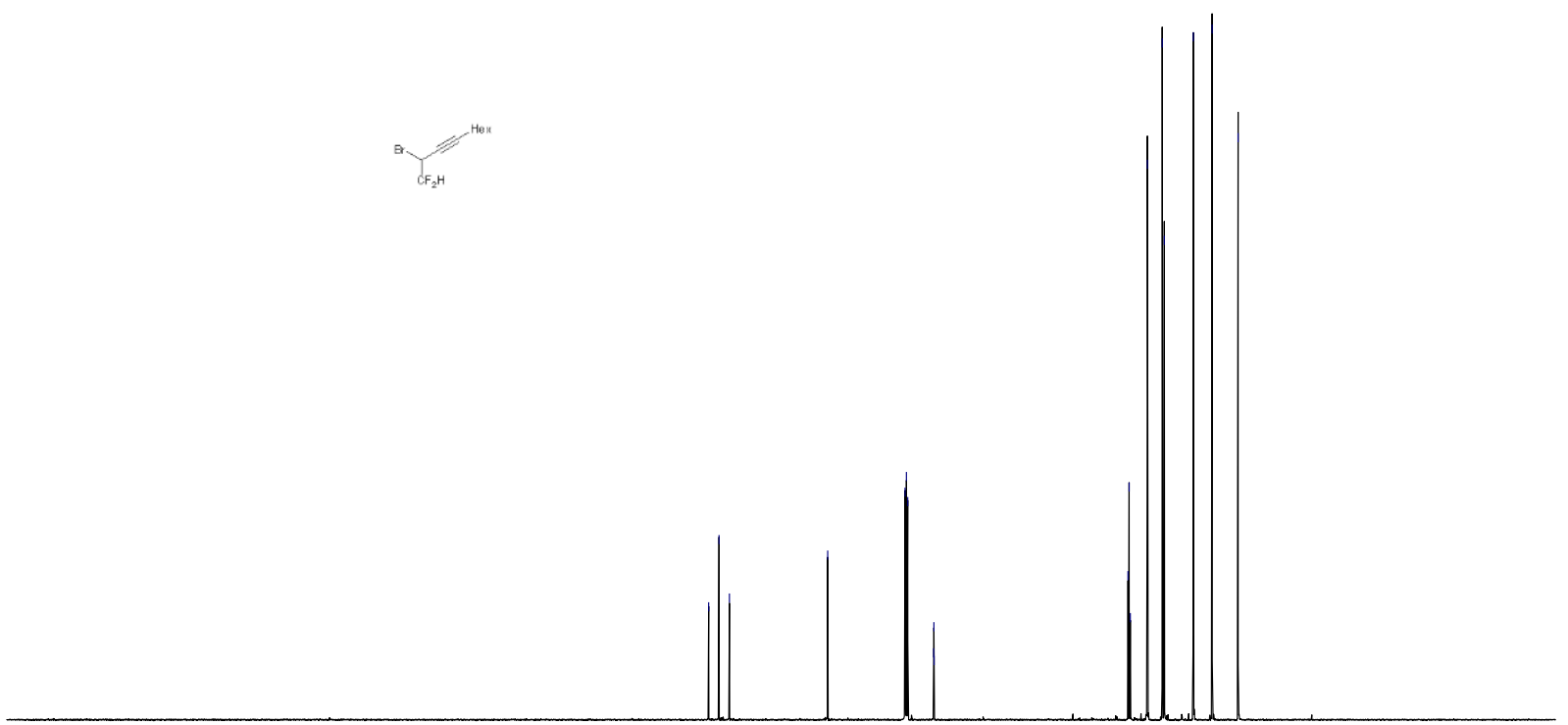

$\begin{array}{llllllllllllllllllllllllllllllll}240 & 230 & 220 & 210 & 200 & 190 & 180 & 170 & 160 & 150 & 140 & 130 & 120 & 110 & 100 & 90 & 80 & 70 & 60 & 50 & 40 & 30 & 20 & 10 & 0 & -10 & -20 & -30 & -40\end{array}$ f1 (ppm) 

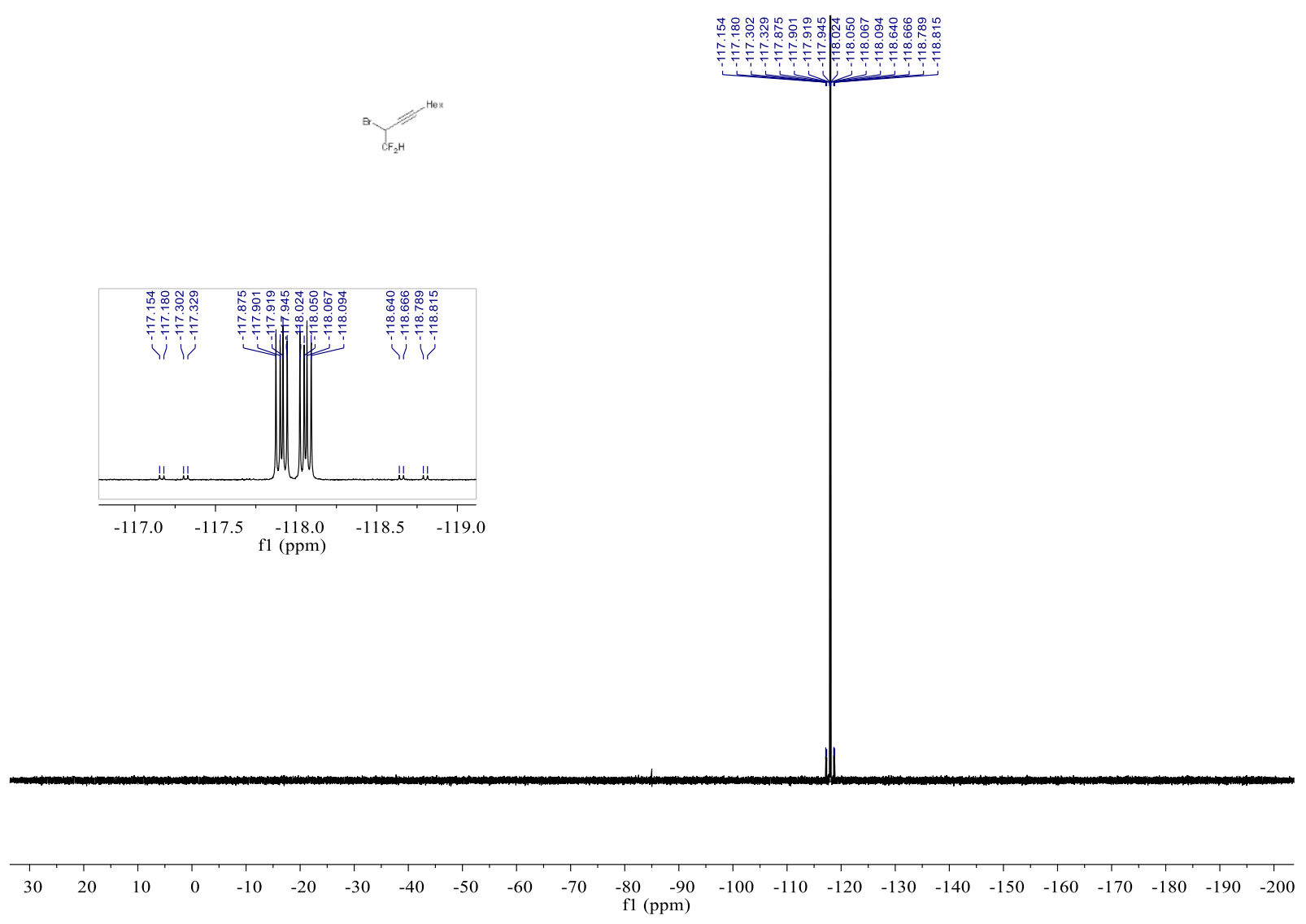

(((6-Bromo-7,7-difluorohept-4-yn-1-yl)oxy)methyl)benzene (2n).

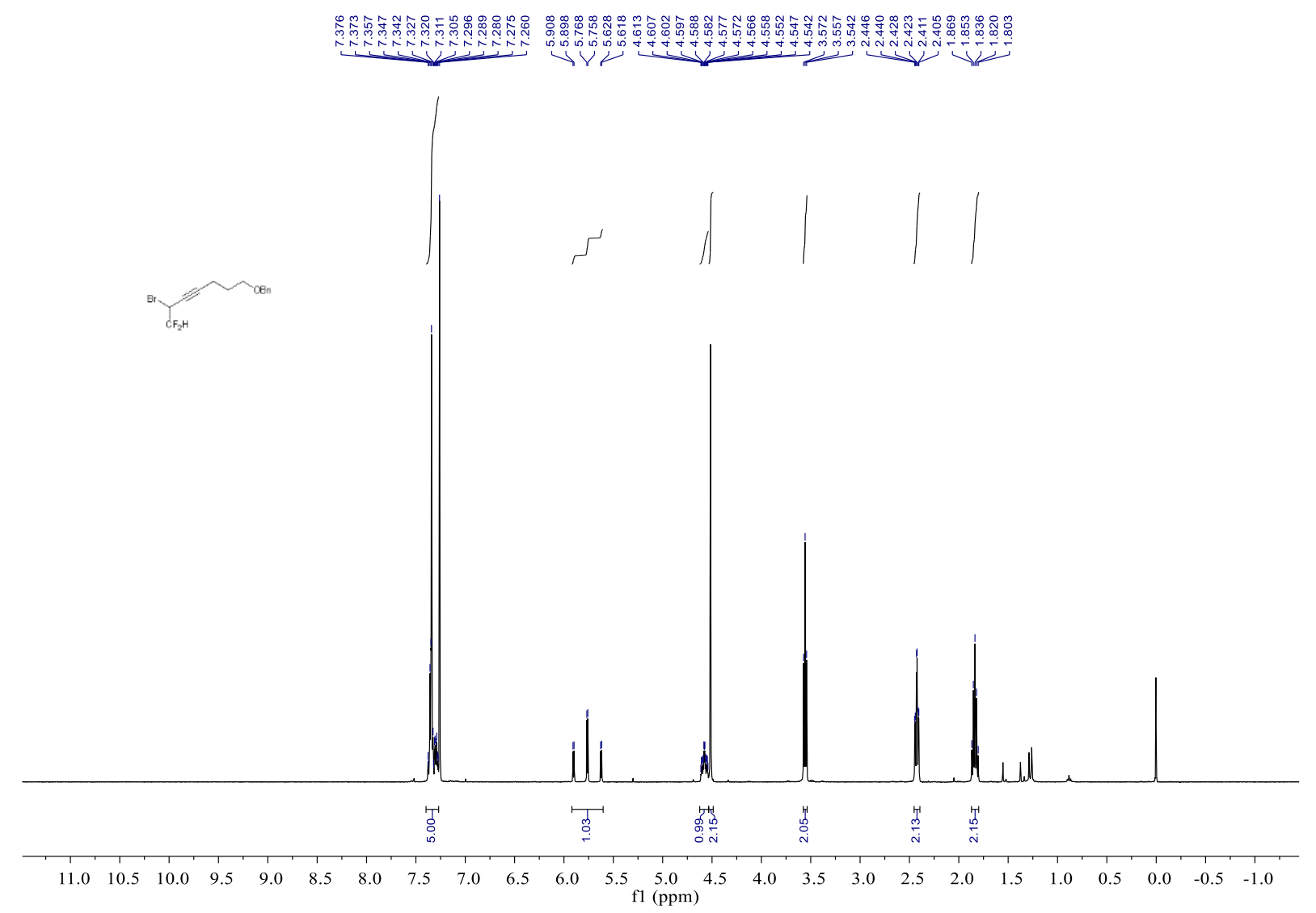




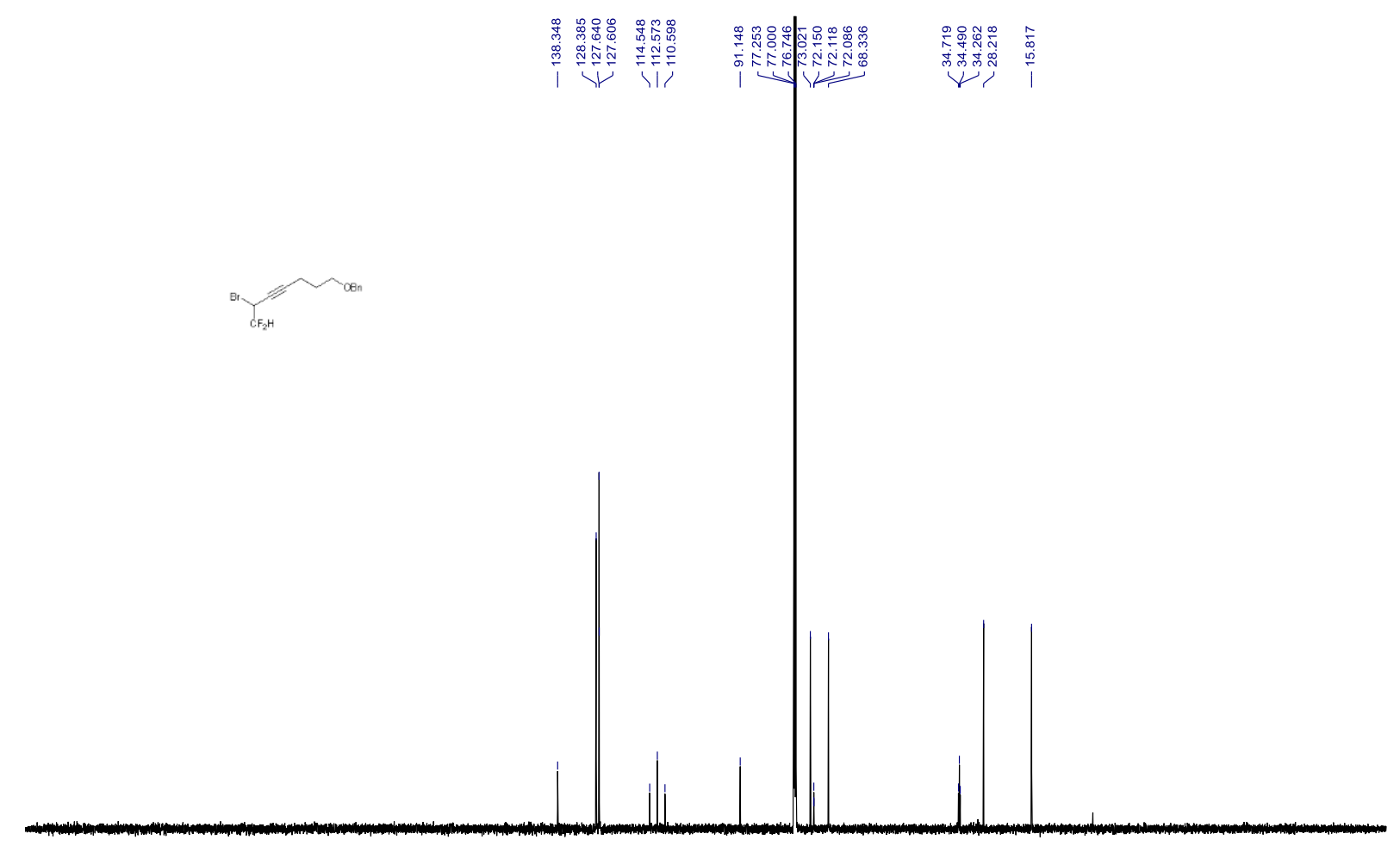

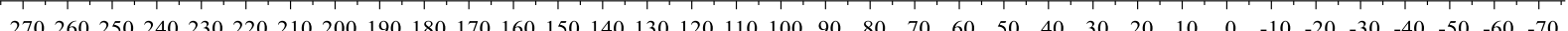
fl $(\mathrm{ppm})$

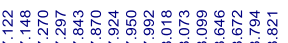

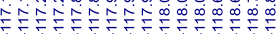
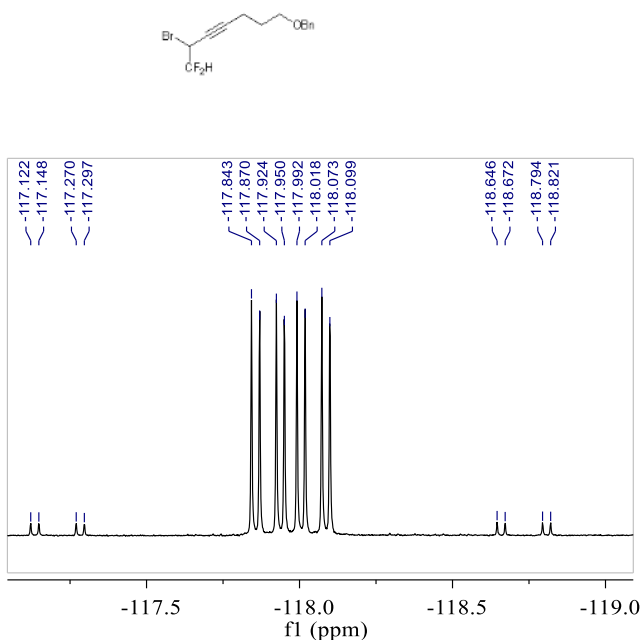

落

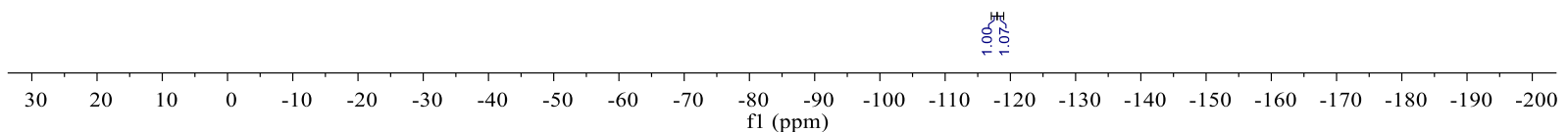


3-Bromo-4,4,4-trifluorobut-1-yn-1-yl)triisopropylsilane (2o).

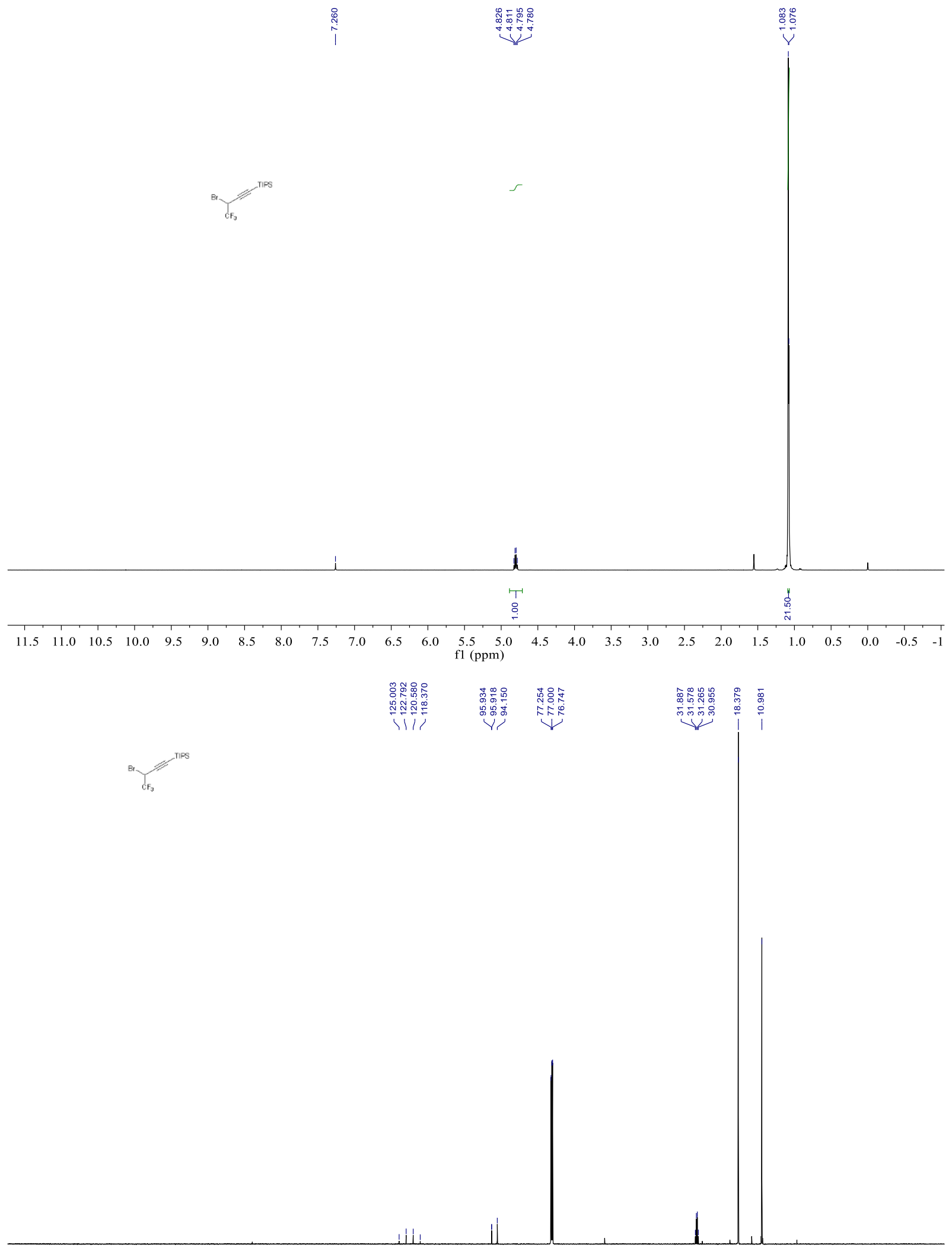

$\begin{array}{lllllllllllllllllllllllllllllllllllll}240 & 230 & 220 & 210 & 200 & 190 & 180 & 170 & 160 & 150 & 140 & 130 & 120 & 110 & 100 & 90 & 80 & 70 & 60 & 50 & 40 & 30 & 20 & 10 & 0 & -10 & -20 & -30 & -40\end{array}$ f1 (ppm) 


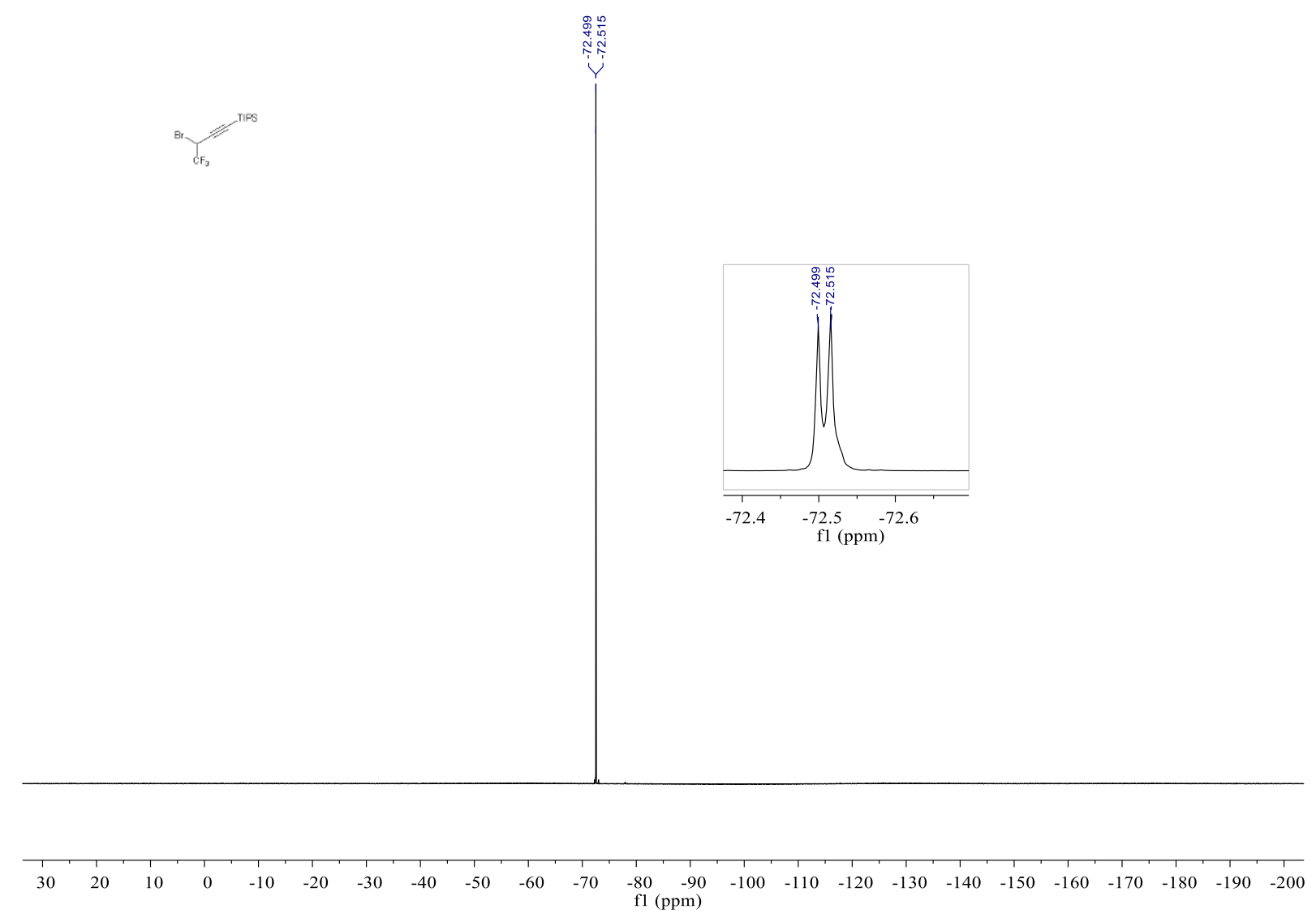

Ethyl 3-bromo-2,2-difluoro-5-(triisopropylsilyl)pent-4-ynoate (2p).

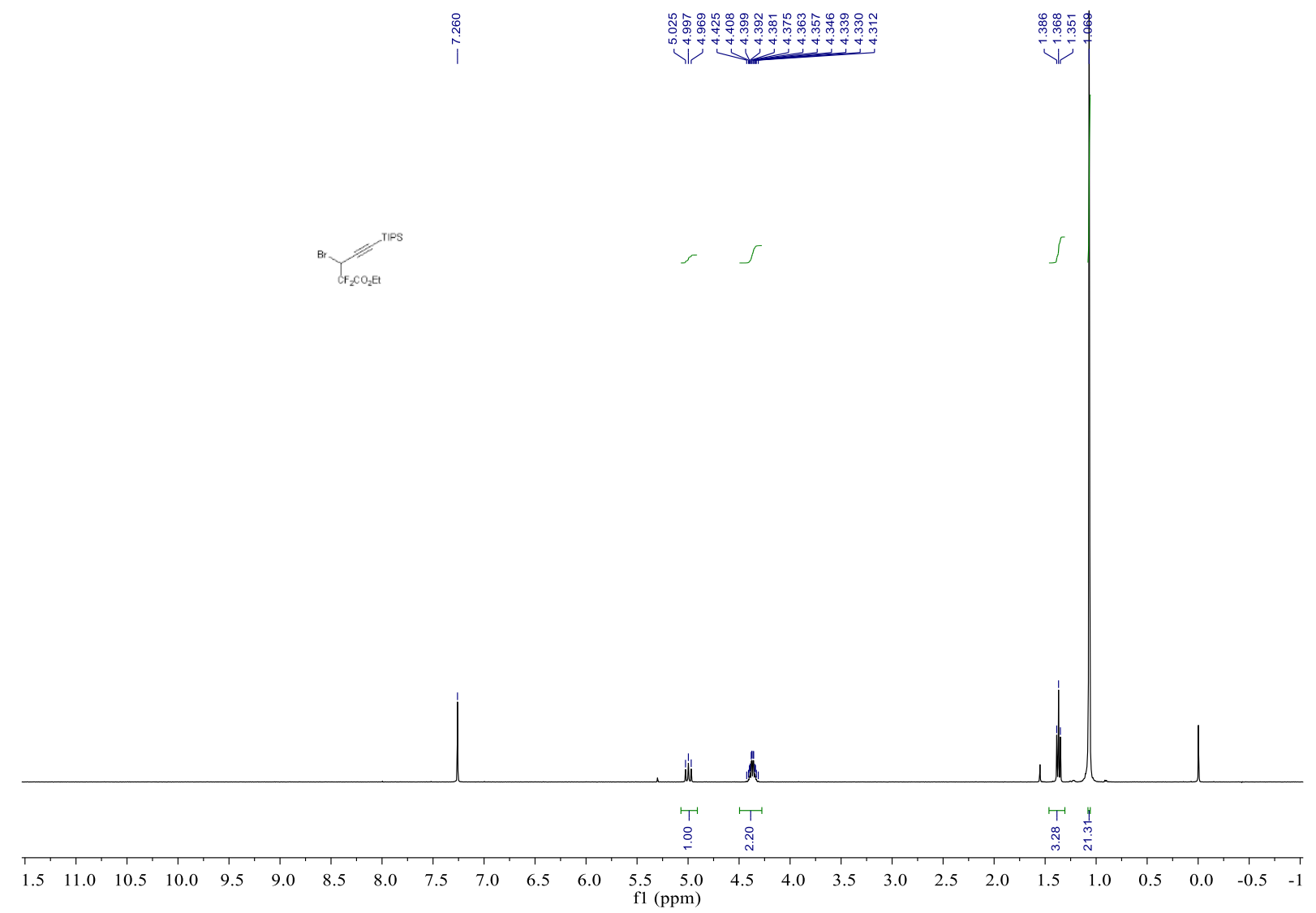



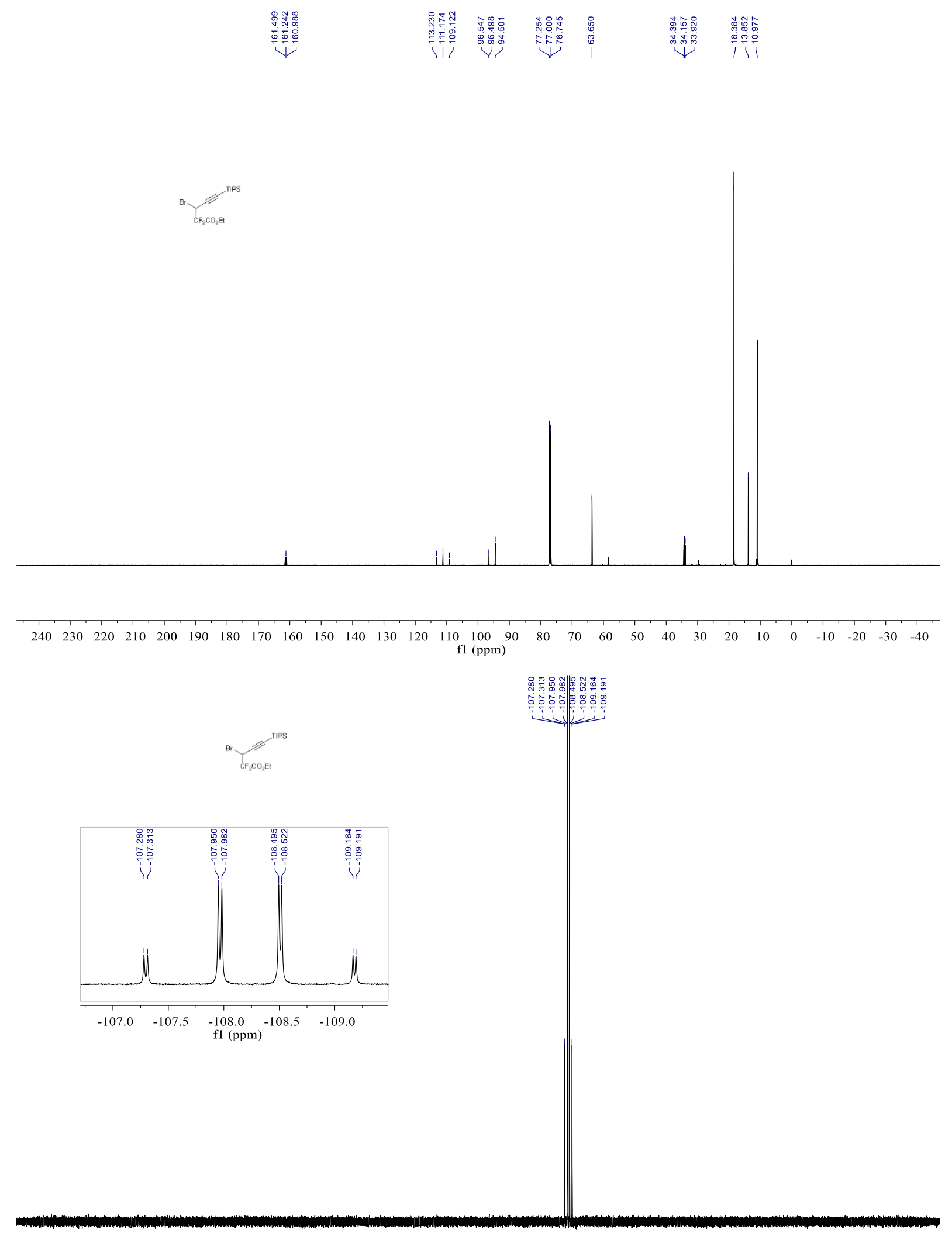

\begin{tabular}{rlllllllllllllllllllllll}
\hline 30 & 20 & 10 & 0 & -10 & -20 & -30 & -40 & -50 & -60 & -70 & -80 & -90 & -100 & -110 & -120 & -130 & -140 & -150 & -160 & -170 & -180 & -190 & -200
\end{tabular} 
(Z)-7-bromopentadec-3-en-8-yne (2q).
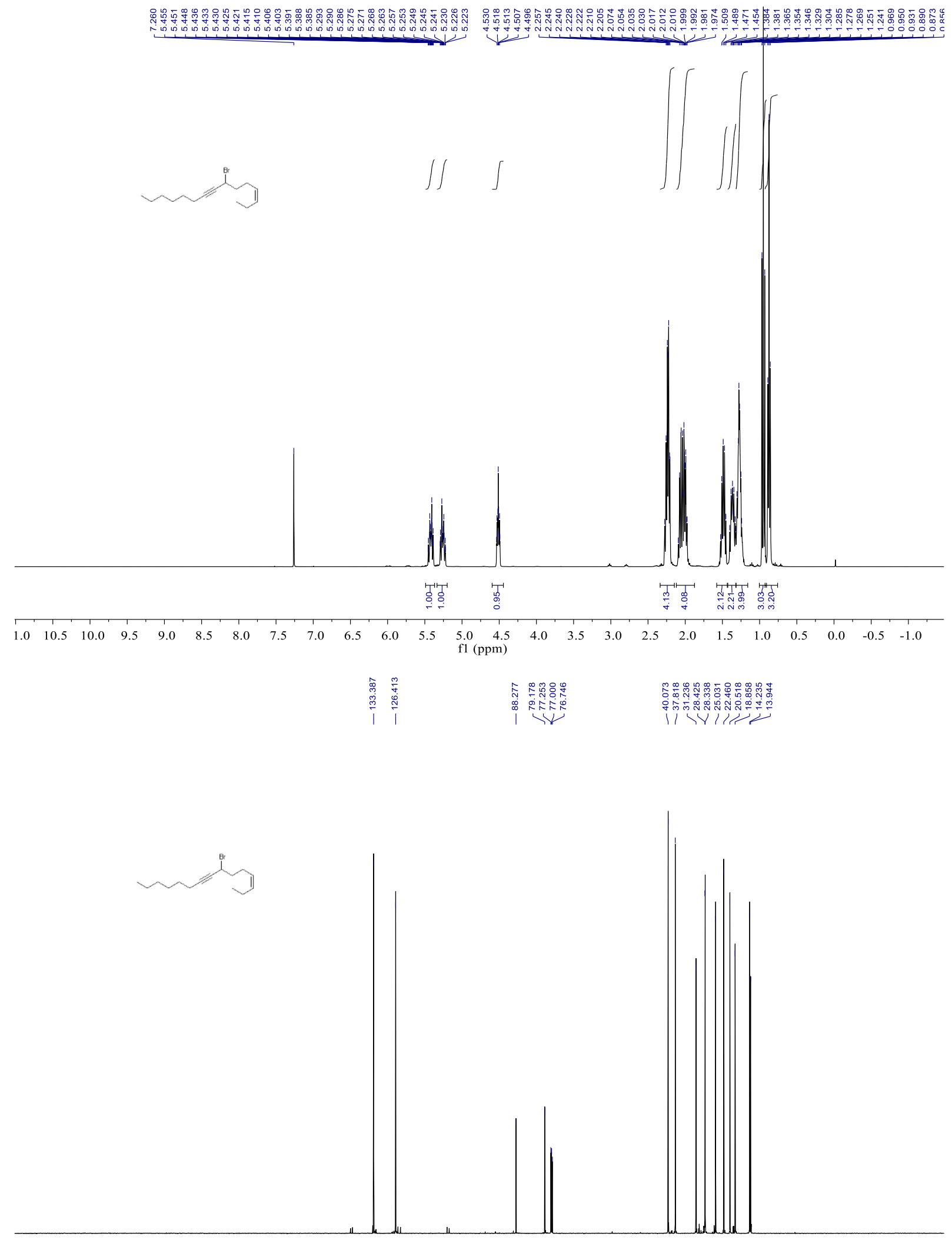

$\begin{array}{llllllllllllllllllllllllllllllllllllllll}240 & 230 & 220 & 210 & 200 & 190 & 180 & 170 & 160 & 150 & 140 & 130 & 120 & 110 & 100 & 90 & 80 & 70 & 60 & 50 & 40 & 30 & 20 & 10 & 0 & -10 & -20 & -30 & -40\end{array}$ 
d-1-(tert-Butyl)-4-ethynylbenzene.
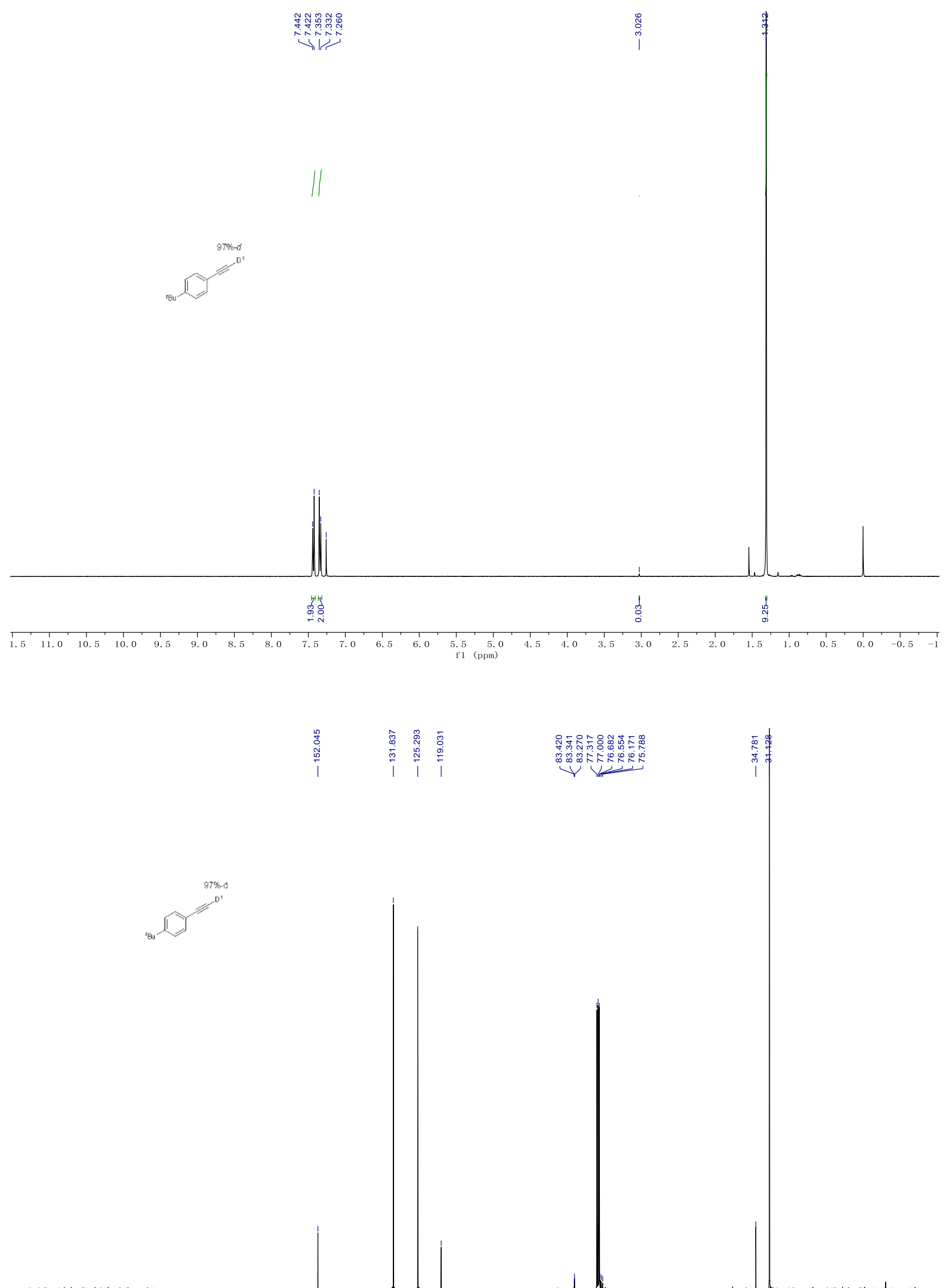

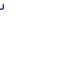


4-tert-Butylstyrene- $\beta, \beta$-D2.

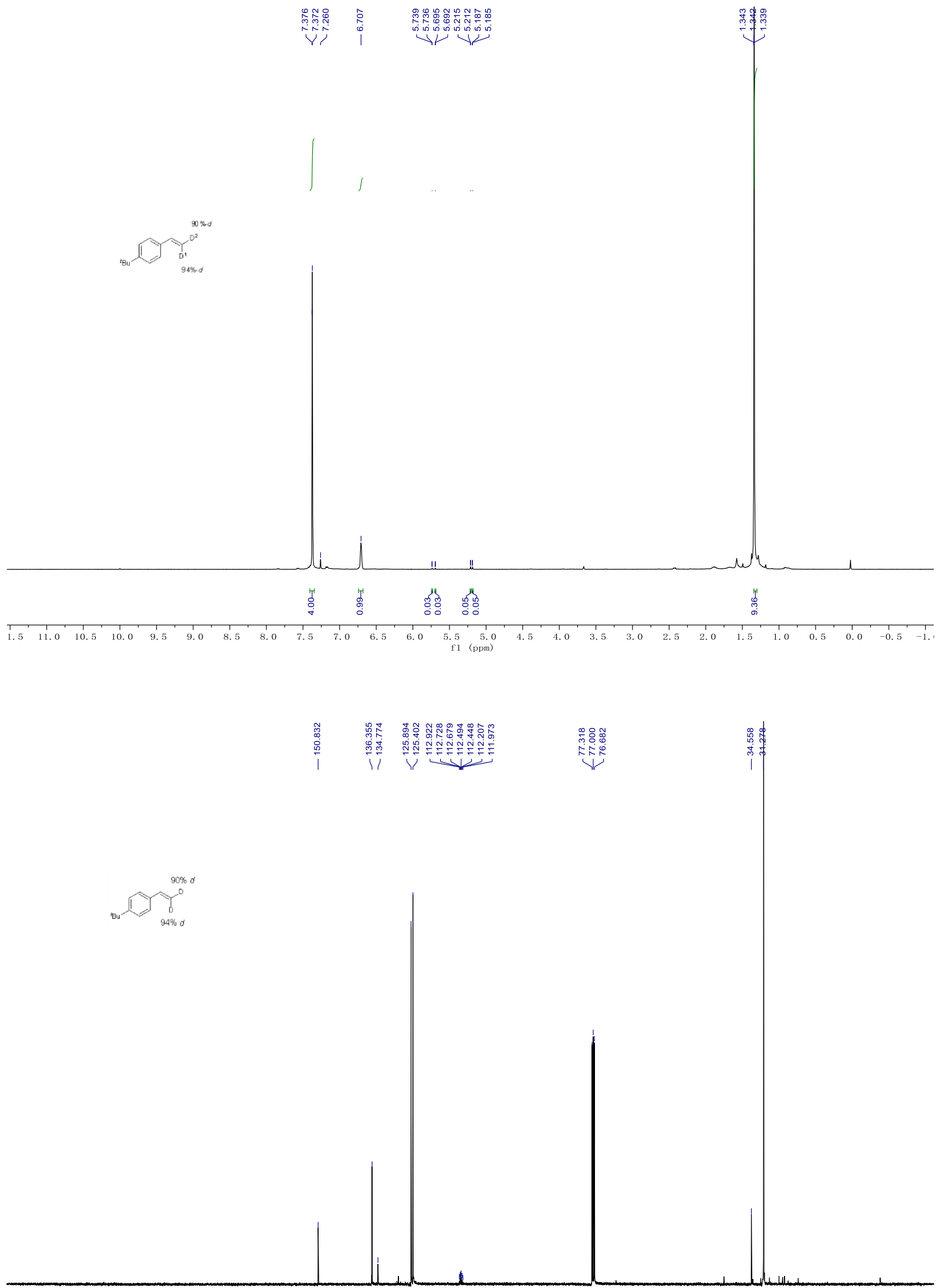

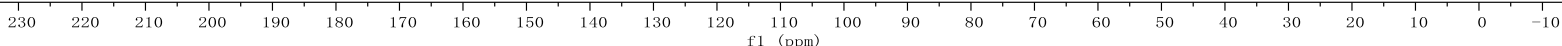


1-(tert-Butyl)-4-(2,2-dibromocyclopropyl-3,3-D 2$)$ benzene.
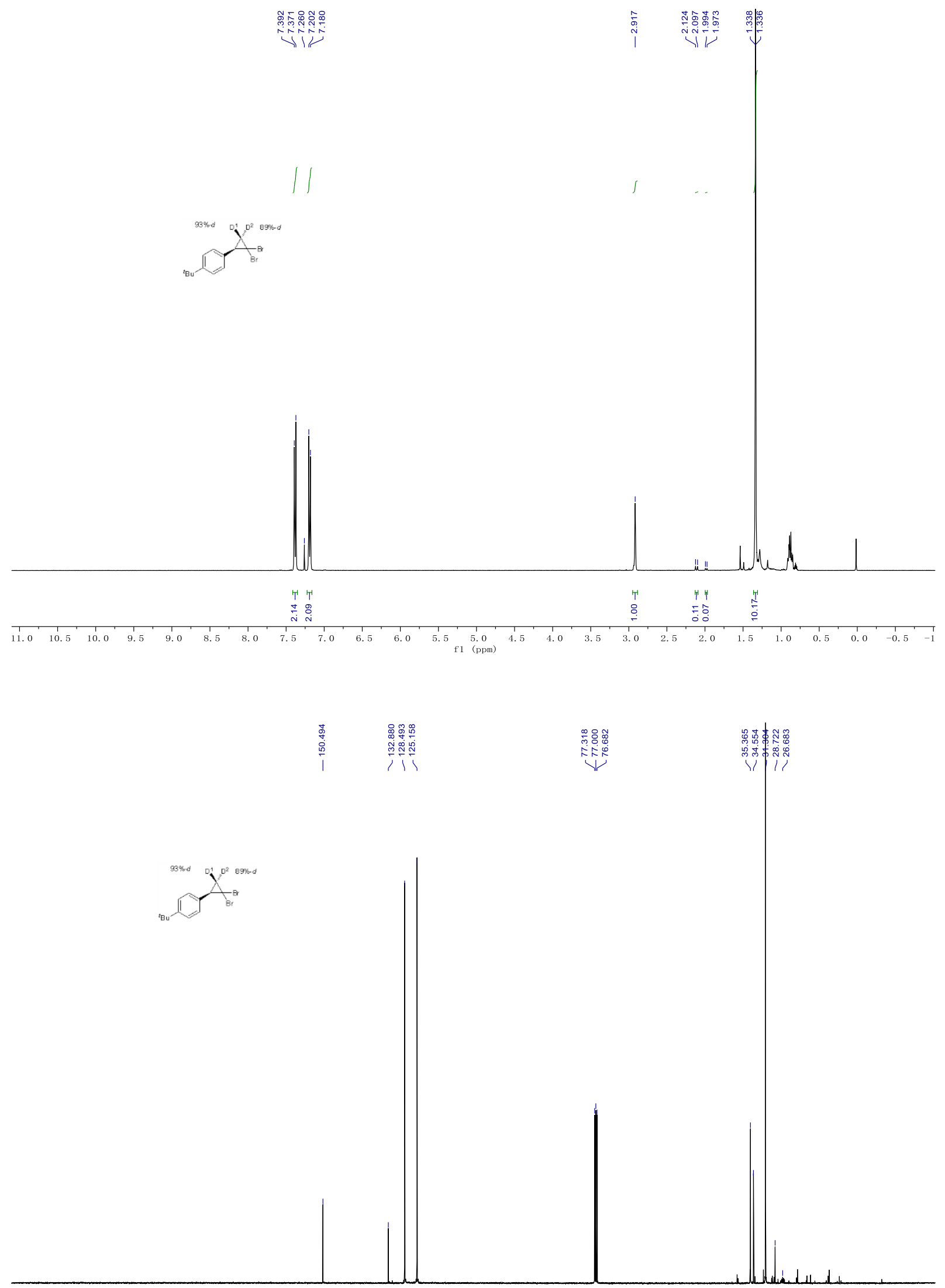

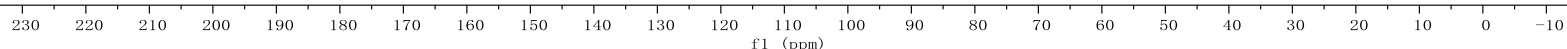


(( \pm )-(cis)-2-Bromocyclopropyl-3,3-D2)-4-(tert-butyl)benzene.
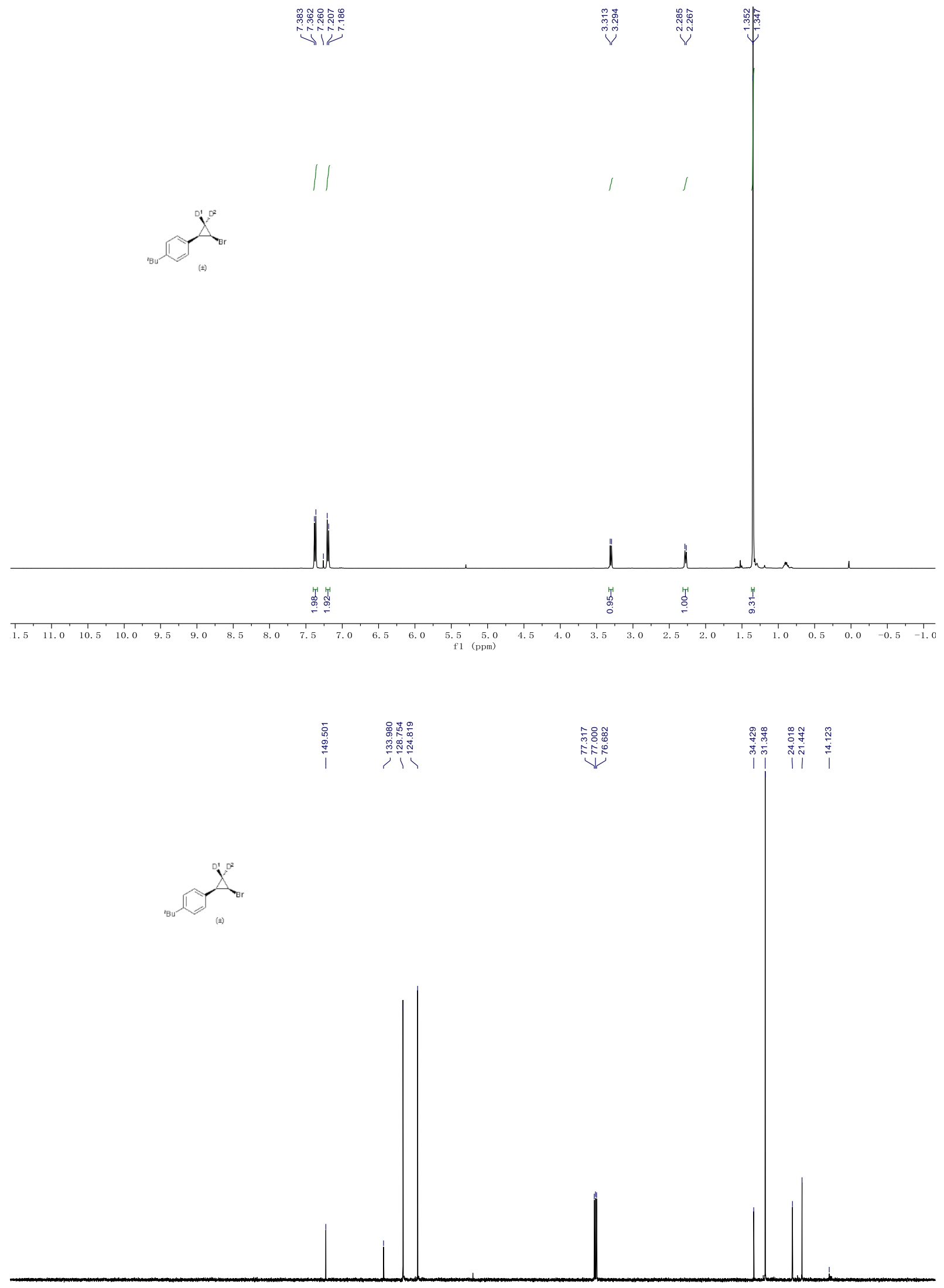

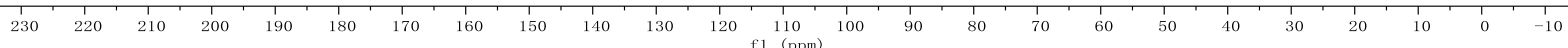




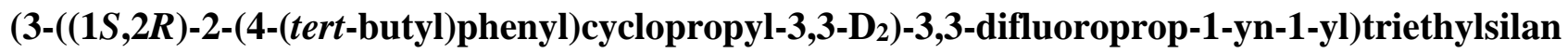
e (d-3b).
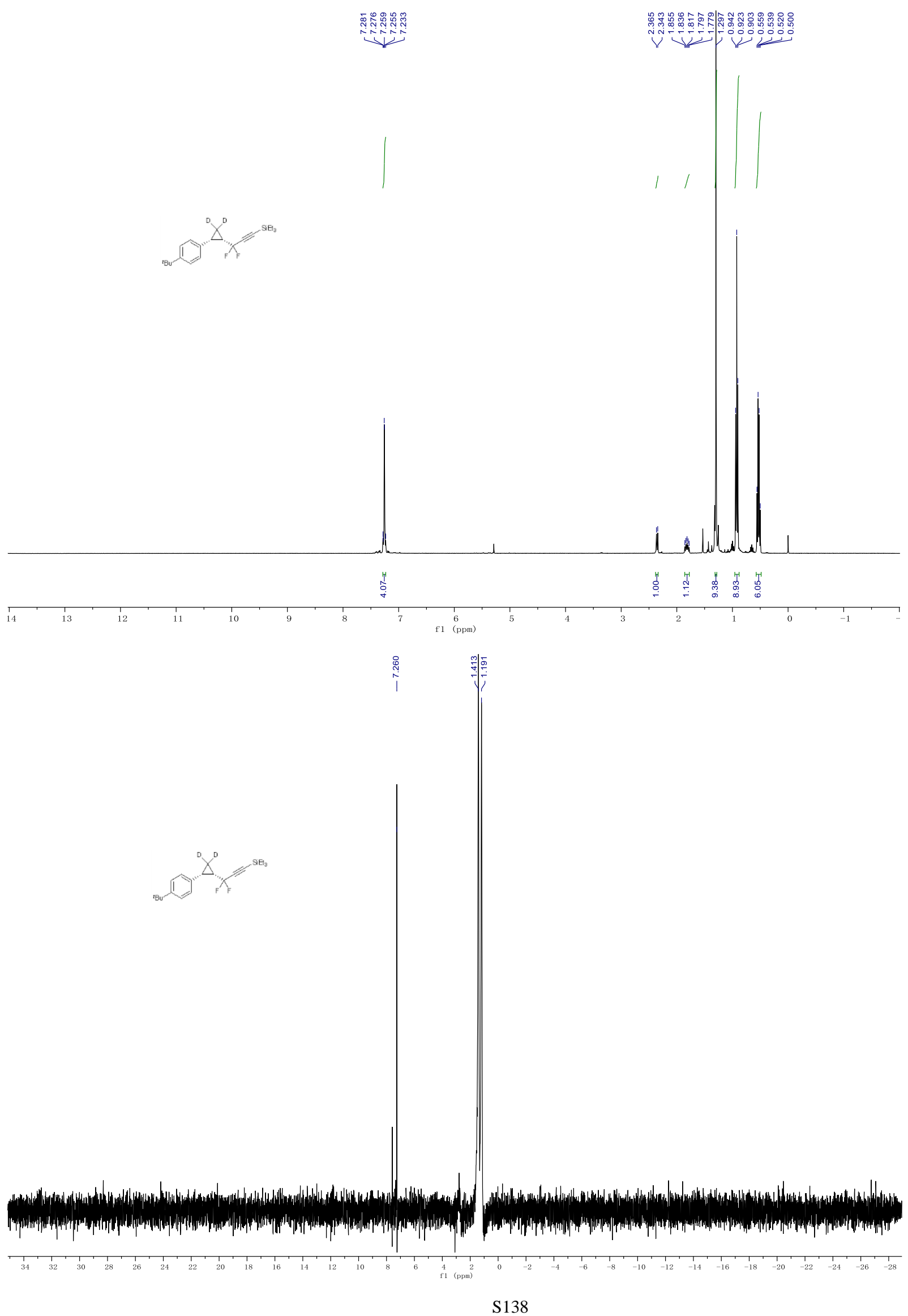

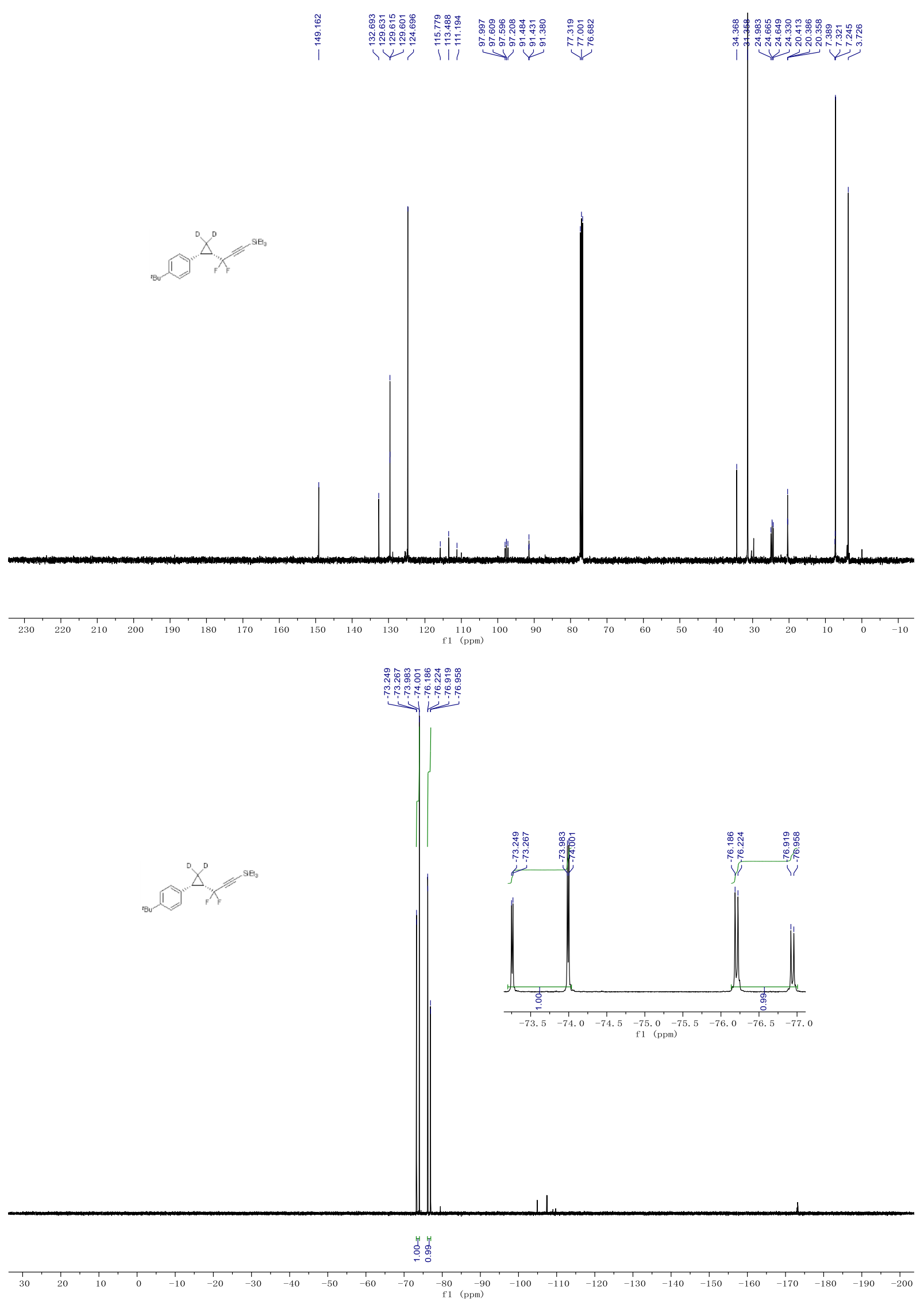


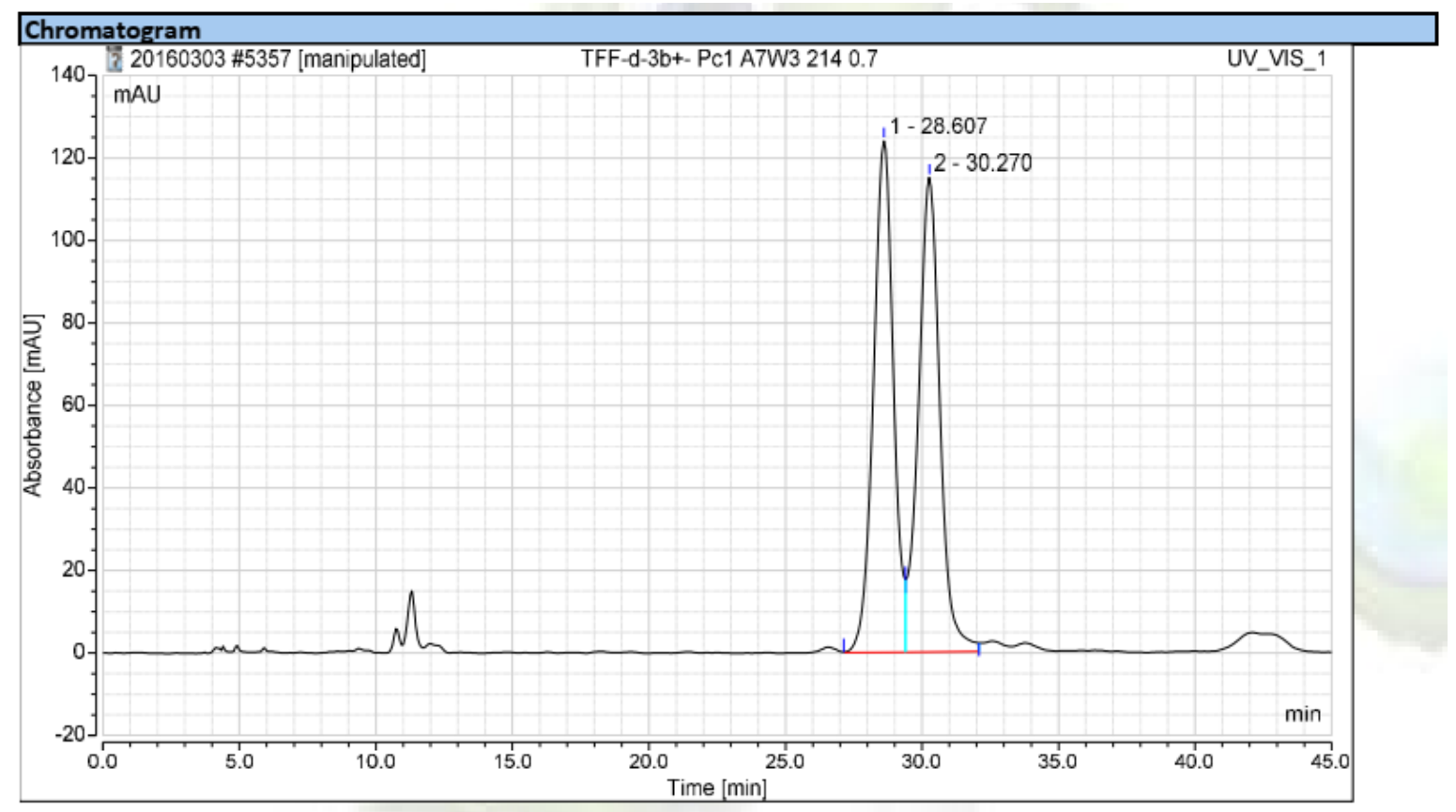

\begin{tabular}{|c|c|c|c|c|}
\hline \multicolumn{5}{|c|}{ Integration Results } \\
\hline No. & $\begin{array}{c}\text { Retention Time } \\
\text { min }\end{array}$ & $\begin{array}{c}\text { Area } \\
\mathrm{mAU}^{*} \mathrm{~min}\end{array}$ & $\begin{array}{l}\text { Height } \\
\text { mAU }\end{array}$ & $\begin{array}{c}\text { Relative Area } \\
\%\end{array}$ \\
\hline 1 & 28.607 & 104.4108 & 124.0063 & 49.909 \\
\hline 2 & 30.270 & 104.7931 & 115.0026 & 50.091 \\
\hline \multicolumn{2}{|c|}{ Total: } & 209.204 & 1401.998 & 100.000 \\
\hline
\end{tabular}

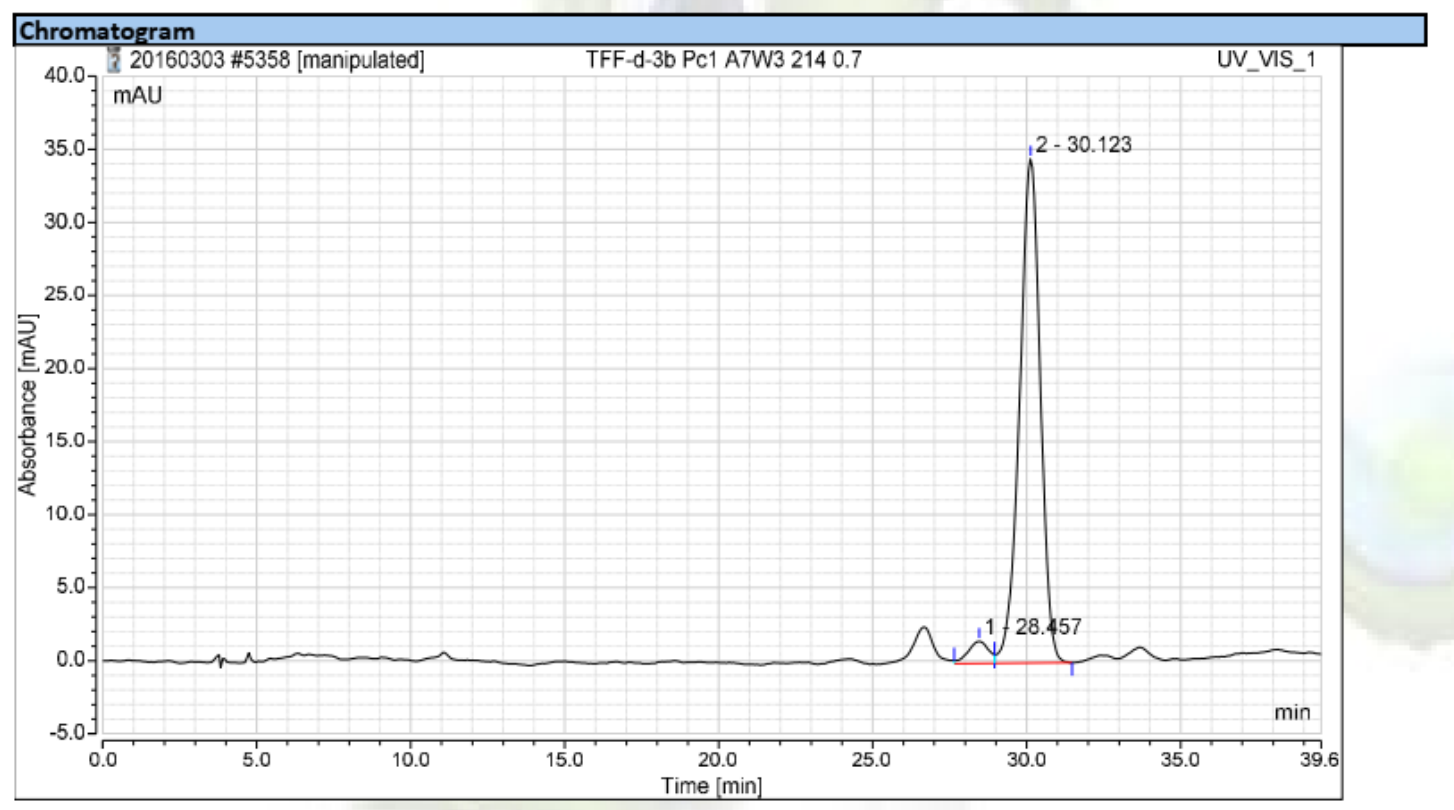

\begin{tabular}{|c|c|c|c|c|}
\hline \multicolumn{5}{|c|}{ Integration Results } \\
\hline No. & $\begin{array}{c}\text { Retention Time } \\
\text { min }\end{array}$ & $\begin{array}{c}\text { Area } \\
\mathrm{mAU}^{*} \min \end{array}$ & $\begin{array}{c}\text { Height } \\
\text { mAU }\end{array}$ & $\begin{array}{c}\text { Relative Area } \\
\%\end{array}$ \\
\hline 1 & 28.457 & 1.1687 & 1.5213 & 4.157 \\
\hline 2 & 30.123 & 26.9441 & 34.4913 & 95.843 \\
\hline Total: & & 28.113 & 1401.998 & 100.000 \\
\hline
\end{tabular}


((1S,2S,3R)-2-((Benzyloxy)methyl)-3-iodocyclopropyl)benzene.
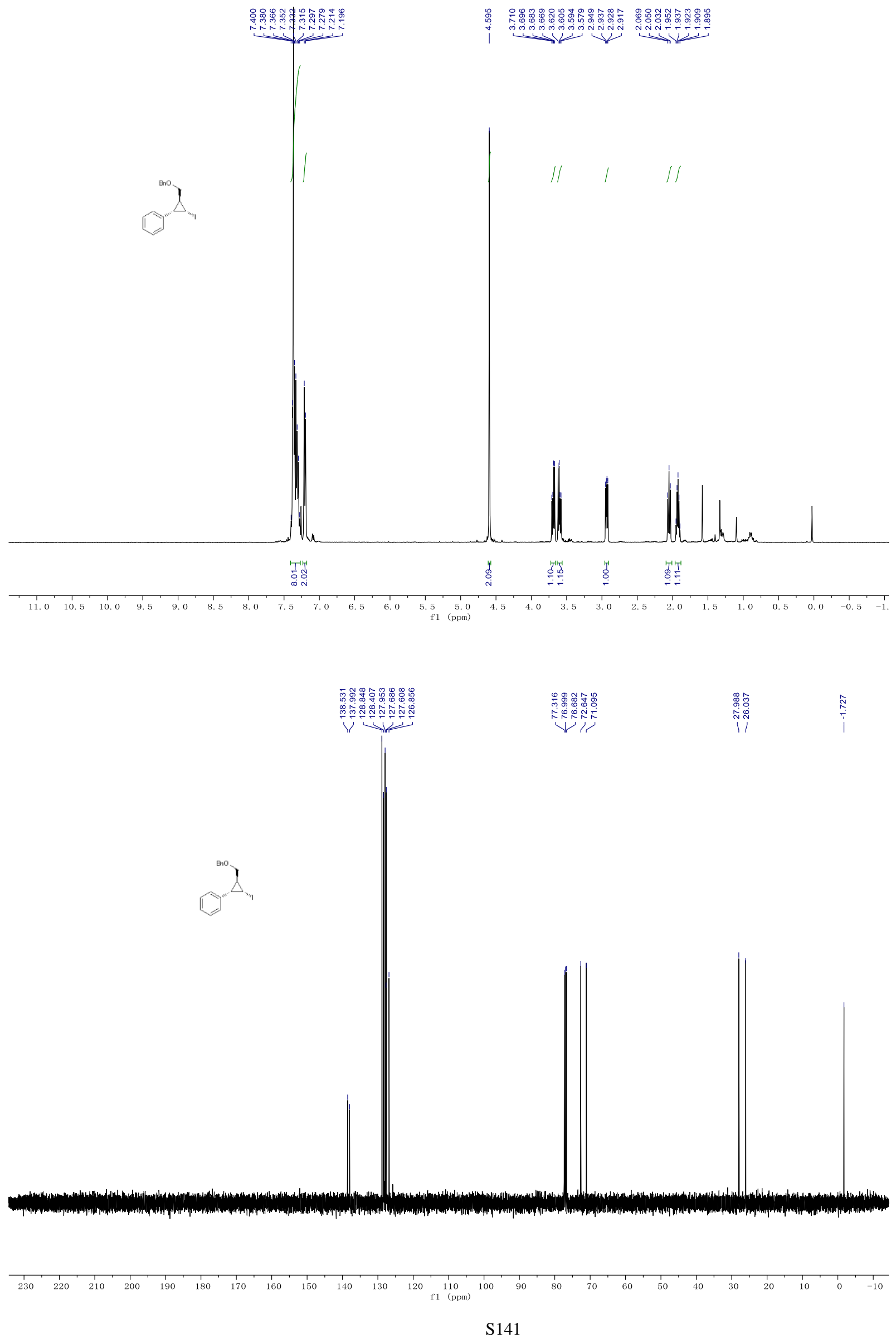


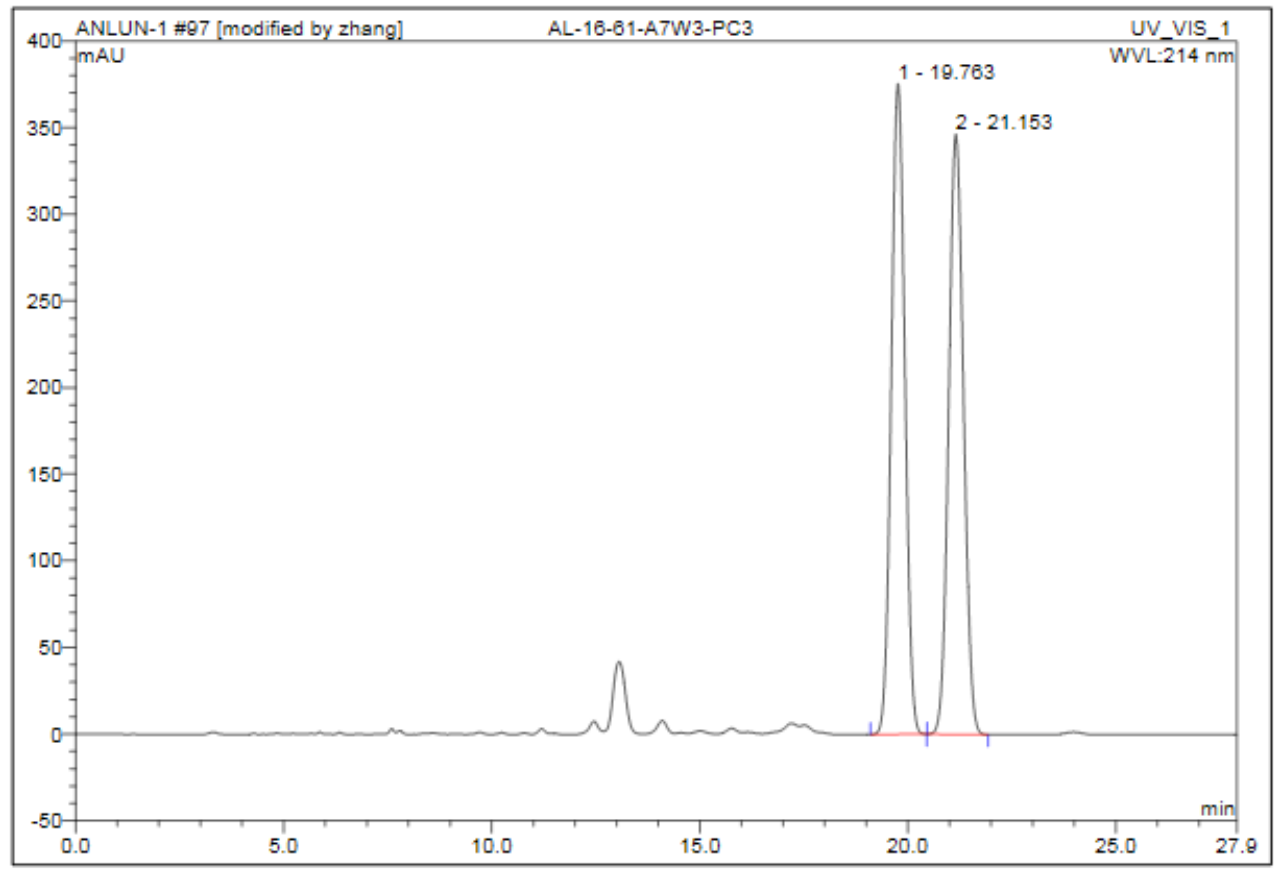

\begin{tabular}{|r|cccrrrr|}
\hline No. & $\begin{array}{c}\text { Ret.Time } \\
\text { min }\end{array}$ & Peak Name & $\begin{array}{c}\text { Height } \\
\text { mAU }\end{array}$ & $\begin{array}{c}\text { Area } \\
\text { mAU*min }\end{array}$ & $\begin{array}{r}\text { Rel.Area } \\
\%\end{array}$ & Amount & Type \\
\hline 1 & 19.76 & n.a. & 375.605 & 142.698 & 49.86 & n.a. & BMB \\
2 & 21.15 & n.a. & 346.440 & 143.473 & 50.14 & n.a. & BMB \\
\hline Total: & & & 722.045 & 286.172 & 100.00 & 0.000 & \\
\hline
\end{tabular}

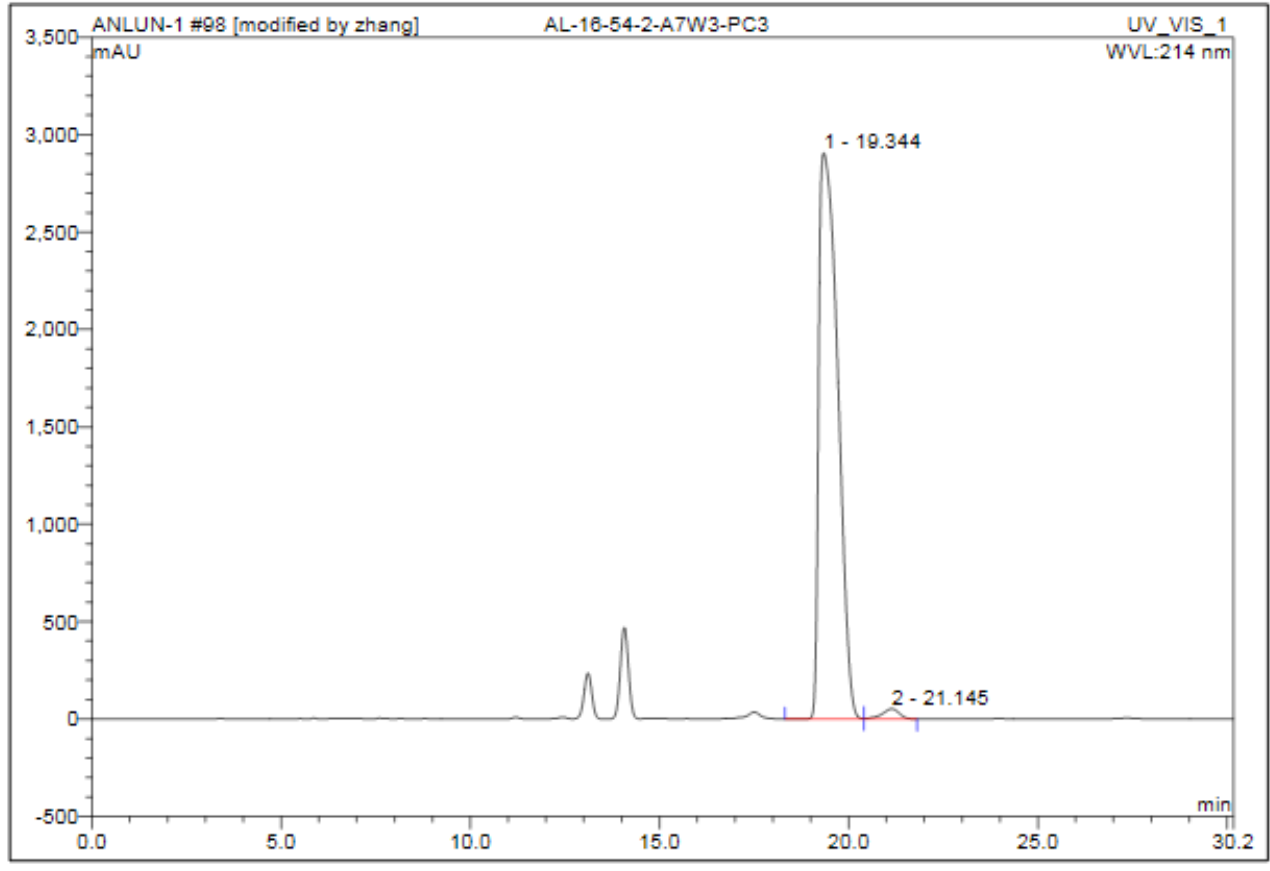

\begin{tabular}{|r|ccrrrrr|}
\hline No. & $\begin{array}{c}\text { Ret.Time } \\
\text { min }\end{array}$ & Peak Name & $\begin{array}{c}\text { Height } \\
\text { mAU }\end{array}$ & $\begin{array}{c}\text { Area } \\
\text { mAU*min }\end{array}$ & $\begin{array}{r}\text { Rel.Area } \\
\%\end{array}$ & Amount & Type \\
\hline 1 & 19.34 & n.a. & 2905.807 & 1687.671 & 98.40 & n.a. & BM $^{*}$ \\
2 & 21.15 & n.a. & 52.524 & 27.500 & 1.60 & n.a. & MB $^{*}$ \\
\hline Total: & & & 2958.331 & 1715.170 & 100.00 & 0.000 & \\
\hline
\end{tabular}


(3-((1R,2R,3R)-2-((Benzyloxy)methyl)-3-phenylcyclopropyl)-3,3-difluoroprop-1-yn-1-yl)triethyl silane (3s).
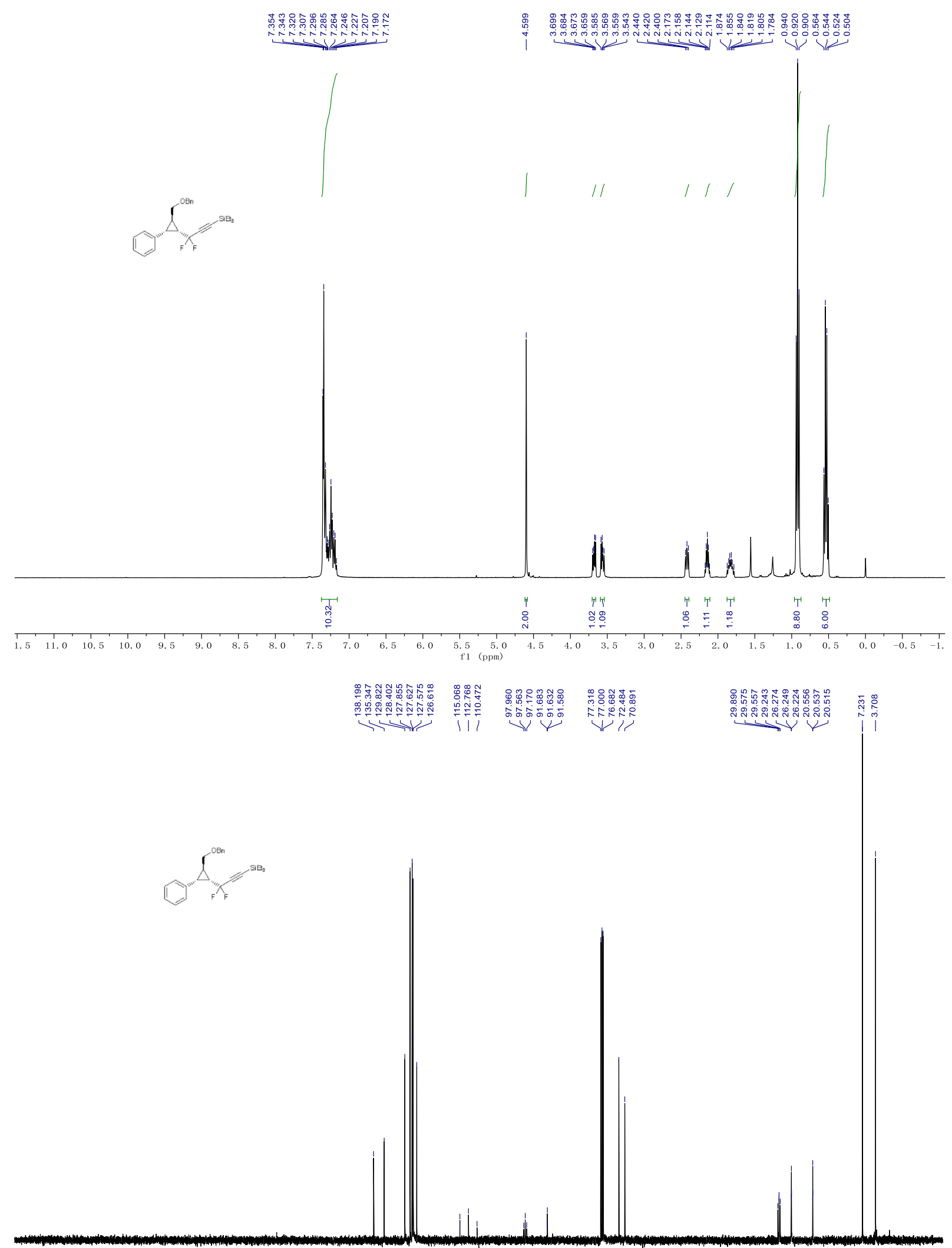

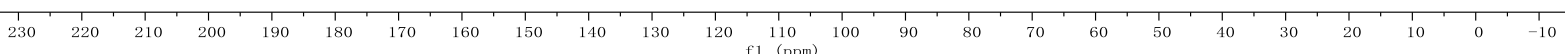



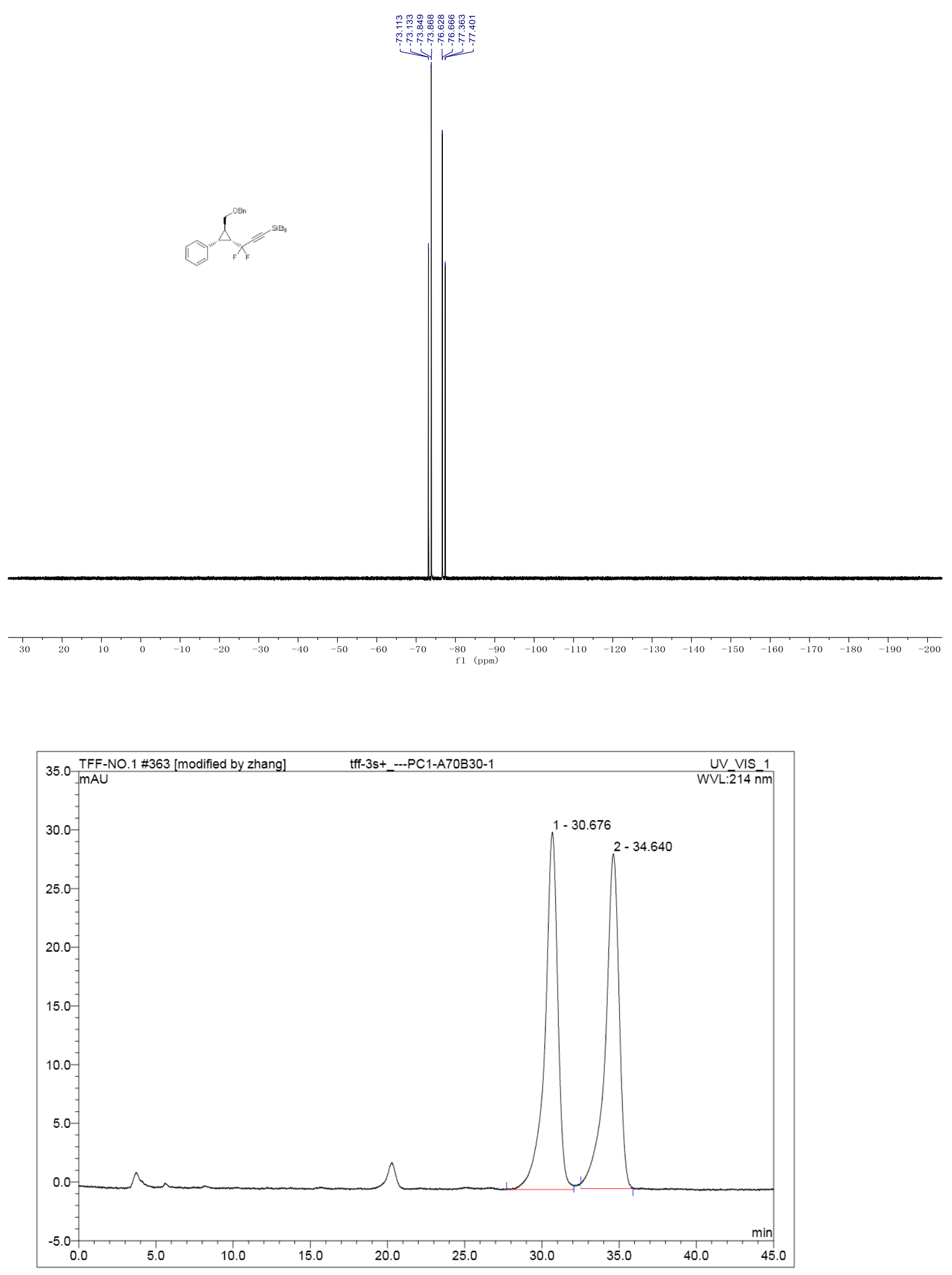

\begin{tabular}{|r|ccrrrrr|}
\hline No. & $\begin{array}{c}\text { Ret.Time } \\
\text { min }\end{array}$ & Peak Name & $\begin{array}{c}\text { Height } \\
\text { mAU }\end{array}$ & $\begin{array}{c}\text { Area } \\
\text { mAU*min }\end{array}$ & $\begin{array}{c}\text { Rel.Area } \\
\%\end{array}$ & $\begin{array}{c}\text { Amount } \\
\%\end{array}$ & Type \\
\hline 1 & 30.68 & n.a. & 30.468 & 29.365 & 49.90 & n.a. & BM $^{*}$ \\
2 & 34.64 & n.a. & 28.548 & 29.486 & 50.10 & n.a. & MB $^{*}$ \\
\hline Total: & & & 59.016 & 58.851 & 100.00 & 0.000 & \\
\hline
\end{tabular}




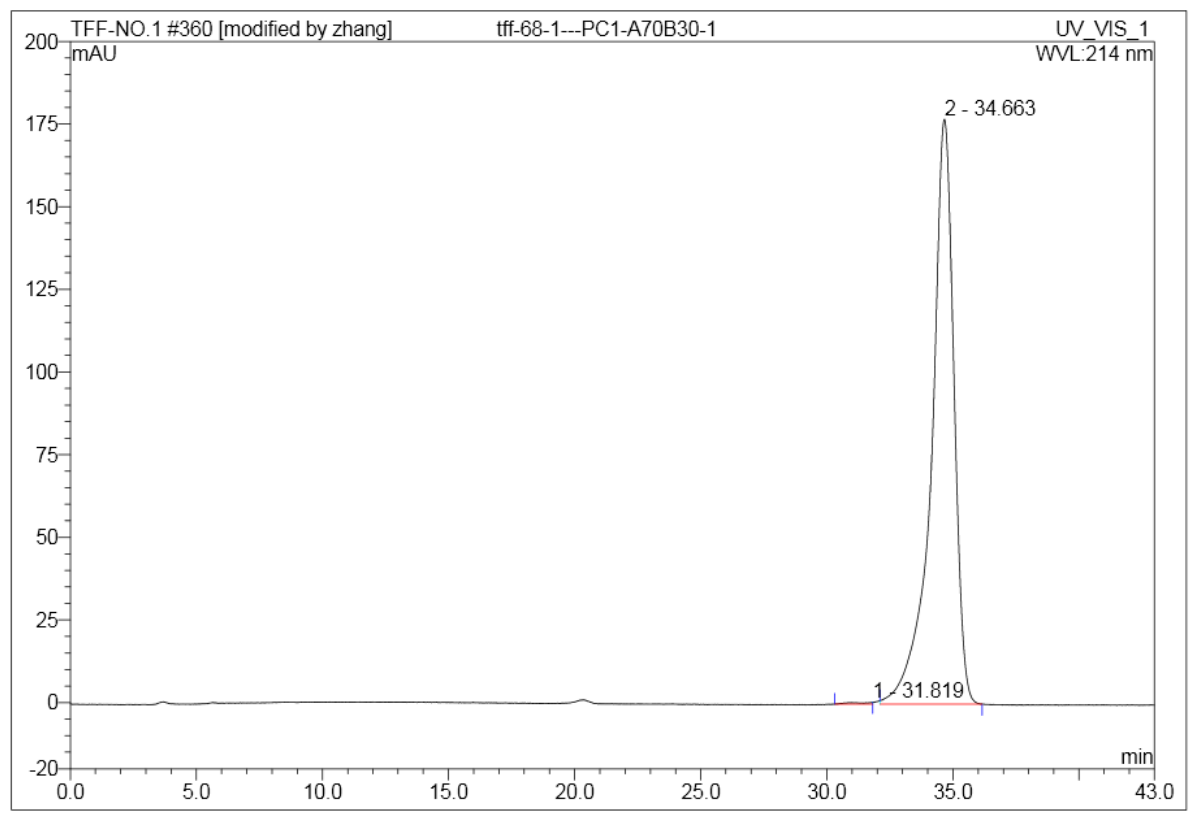

\begin{tabular}{|r|ccrrrrr|}
\hline No. & $\begin{array}{c}\text { Ret.Time } \\
\text { min }\end{array}$ & Peak Name & $\begin{array}{c}\text { Height } \\
\text { mAU }\end{array}$ & $\begin{array}{r}\text { Area } \\
\text { mAU*min }\end{array}$ & $\begin{array}{r}\text { Rel.Area } \\
\%\end{array}$ & $\begin{array}{c}\text { Amount } \\
\%\end{array}$ & Type \\
\hline 1 & 31.82 & n.a. & 0.553 & 0.523 & 0.29 & n.a. & BM $^{*}$ \\
2 & 34.66 & n.a. & 176.935 & 182.610 & 99.71 & n.a. & MB $^{\star}$ \\
\hline Total: & & & 177.488 & 183.133 & 100.00 & 0.000 & \\
\hline
\end{tabular}

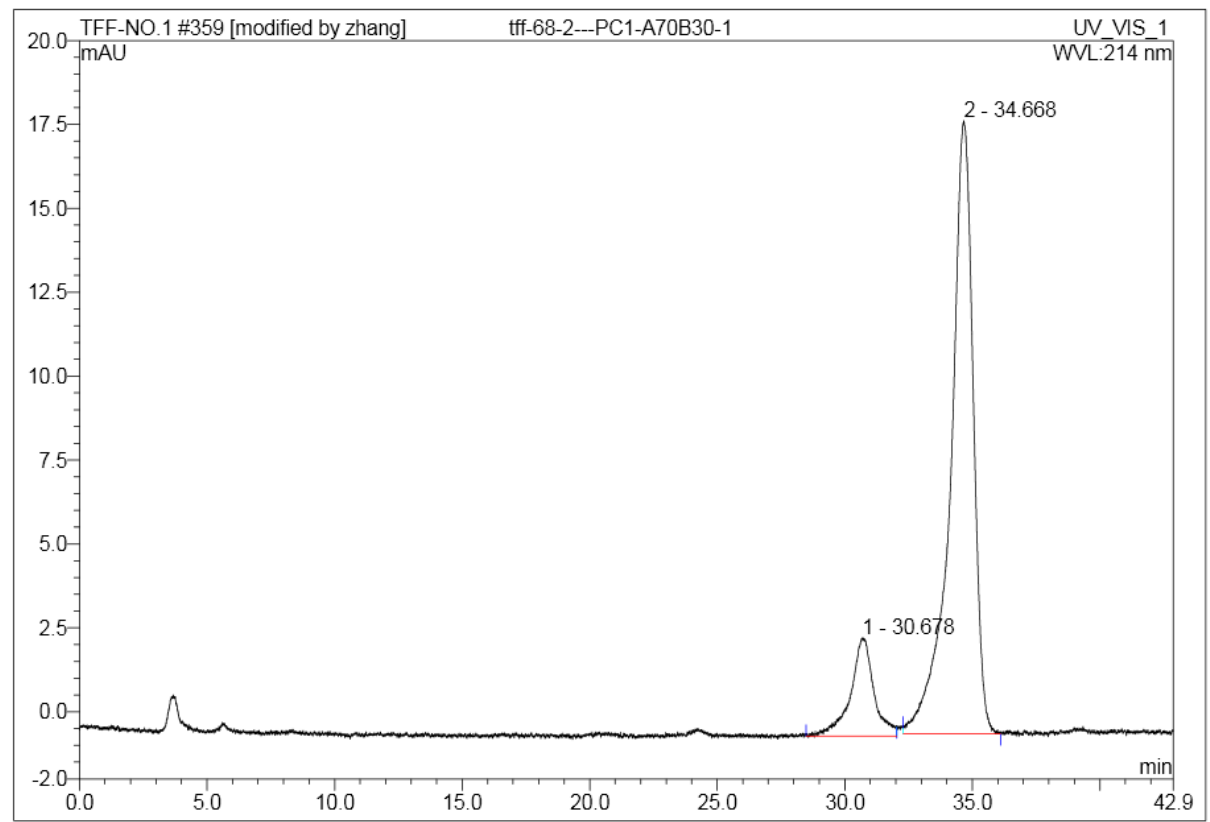

\begin{tabular}{|r|ccrrrrr|}
\hline No. & $\begin{array}{c}\text { Ret. Time } \\
\text { min }\end{array}$ & Peak Name & $\begin{array}{c}\text { Height } \\
\text { mAU }\end{array}$ & $\begin{array}{r}\text { Area } \\
\text { mAU*min }\end{array}$ & $\begin{array}{c}\text { Rel.Area } \\
\%\end{array}$ & $\begin{array}{c}\text { Amount } \\
\%\end{array}$ & Type \\
\hline 1 & 30.68 & n.a. & 2.930 & 3.097 & 14.15 & n.a. & BM $^{*}$ \\
2 & 34.67 & n.a. & 18.273 & 18.796 & 85.85 & n.a. & MB $^{*}$ \\
\hline Total: & & & 21.203 & 21.893 & 100.00 & 0.000 & \\
\hline
\end{tabular}


(3,3-Difluoro-3-((1S,2R)-2-phenylcyclopropyl)prop-1-yn-1-yl)triethylsilane (3a).
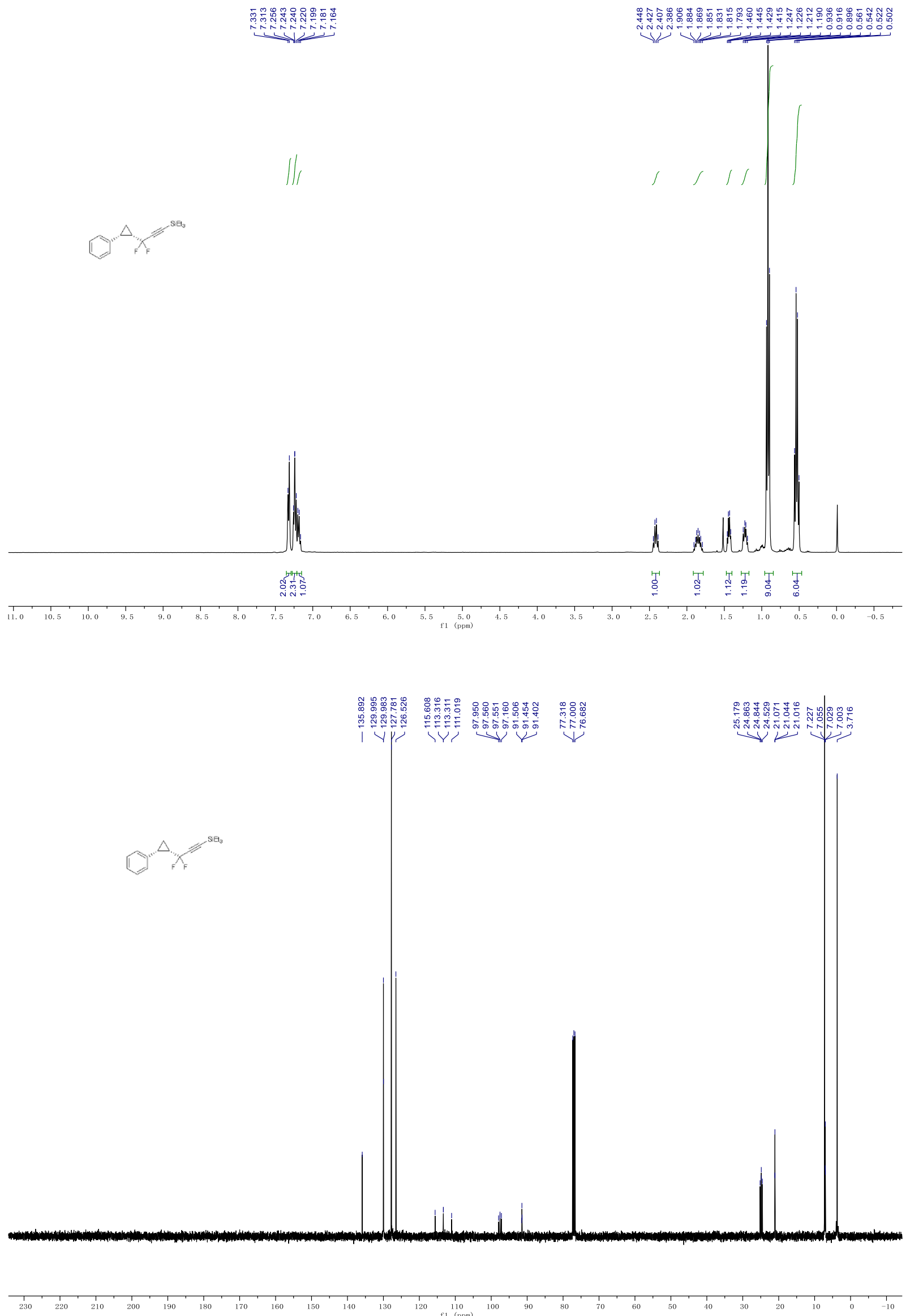

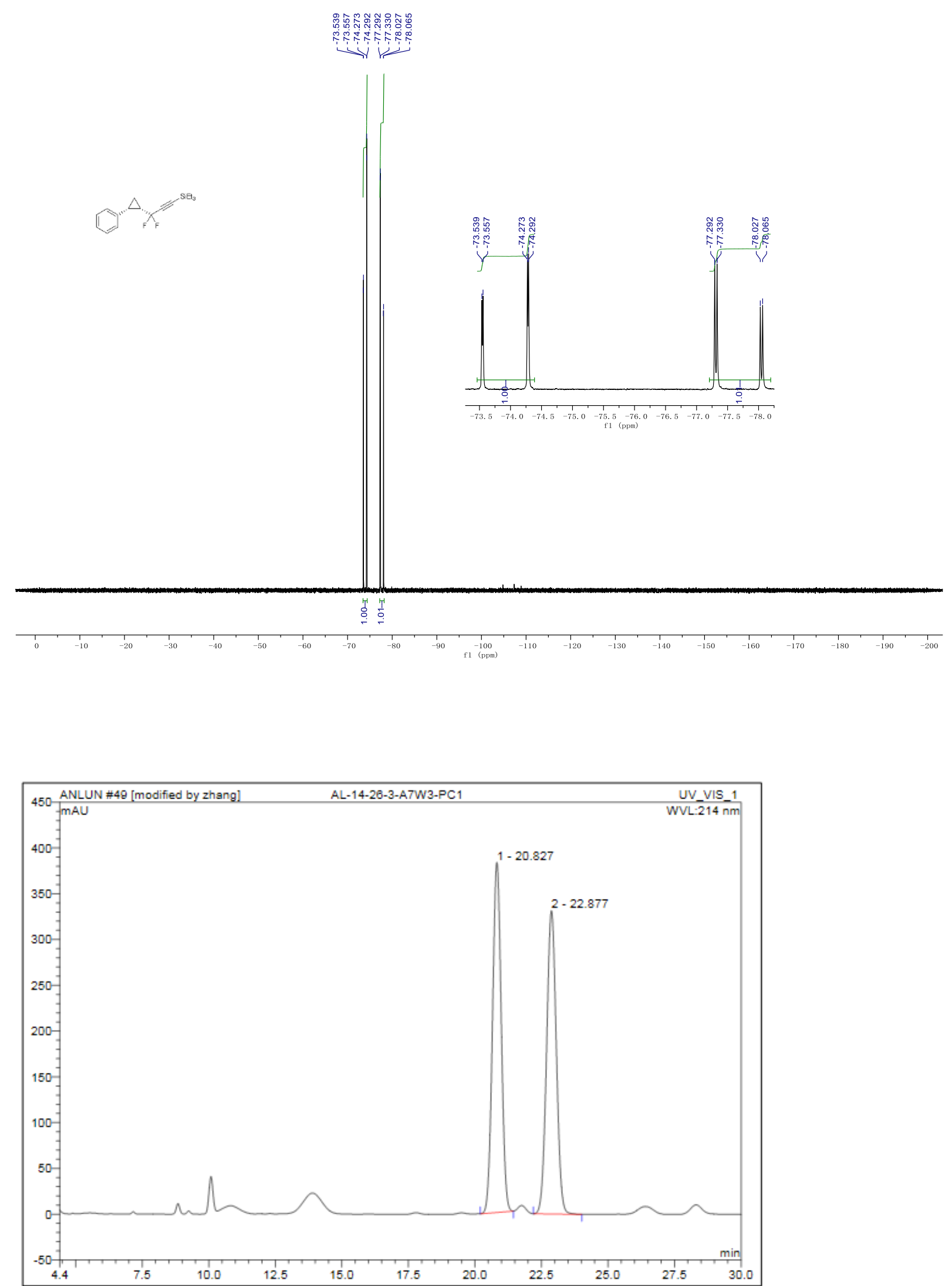

\begin{tabular}{|r|cccrrrr|}
\hline No. & $\begin{array}{c}\text { Ret.Time } \\
\text { min }\end{array}$ & Peak Name & $\begin{array}{c}\text { Height } \\
\text { mAU }\end{array}$ & $\begin{array}{c}\text { Area } \\
\text { mAU*min }\end{array}$ & $\begin{array}{c}\text { Rel.Area } \\
\%\end{array}$ & Amount & Type \\
\hline 1 & 20.83 & n.a. & 382.389 & 144.754 & 50.83 & n.a. & BMB \\
2 & 22.88 & n.a. & 331.165 & 140.040 & 49.17 & n.a. & BMB \\
\hline Total: & & & 713.554 & 284.795 & 100.00 & 0.000 & \\
\hline
\end{tabular}




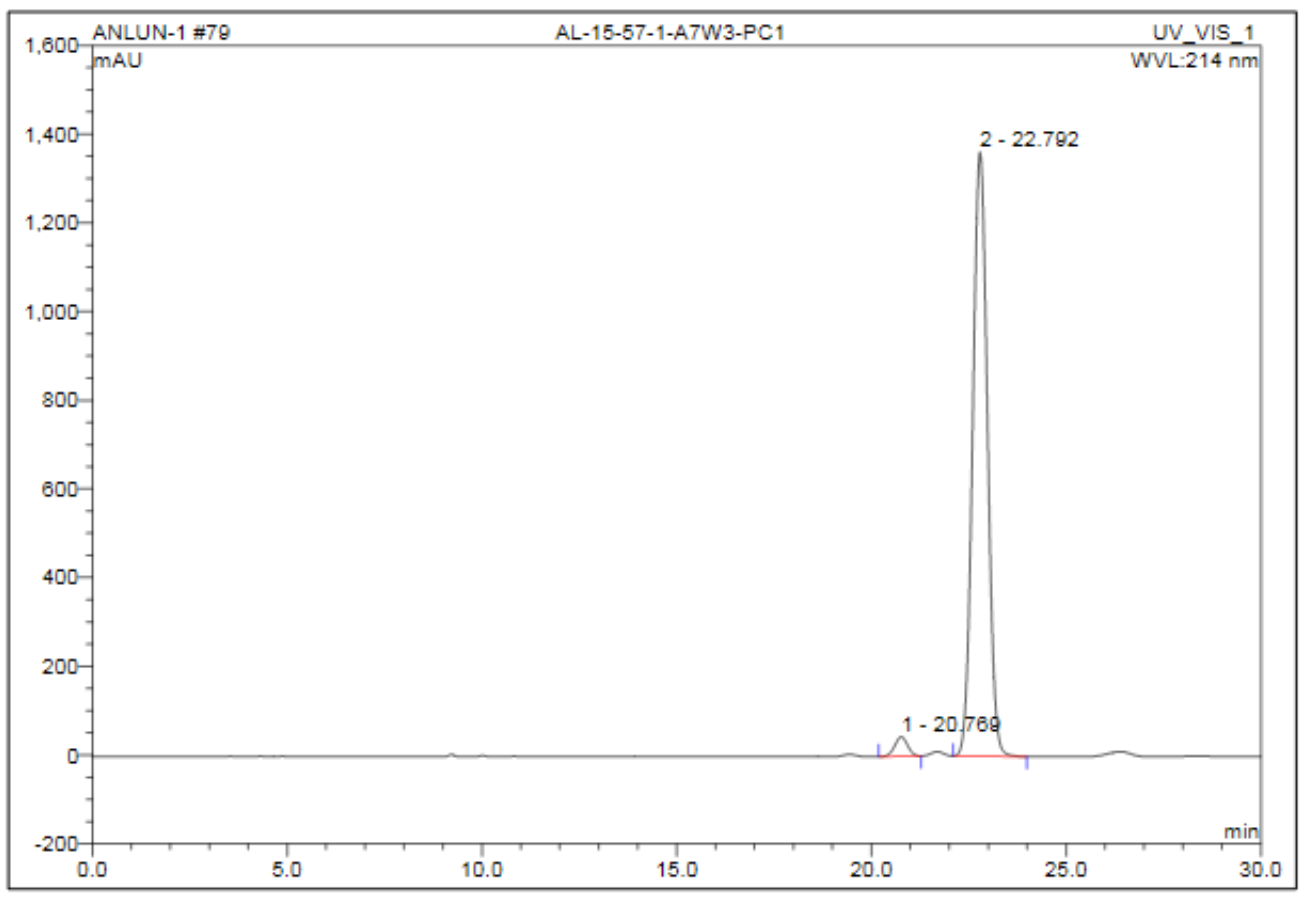

\begin{tabular}{|r|ccrrrrr|}
\hline No. & $\begin{array}{c}\text { Ret.Time } \\
\text { min }\end{array}$ & Peak Name & $\begin{array}{c}\text { Height } \\
\text { mAU }\end{array}$ & $\begin{array}{r}\text { Area } \\
\text { mAU*min }\end{array}$ & $\begin{array}{r}\text { Rel.Area } \\
\%\end{array}$ & Amount & Type \\
\hline 1 & 20.77 & n.a. & 43.701 & 16.691 & 2.72 & n.a. & BMB \\
2 & 22.79 & n.a. & 1362.438 & 597.351 & 97.28 & n.a. & BMB \\
\hline Total: & & & 1406.138 & 614.042 & 100.00 & 0.000 & \\
\hline
\end{tabular}

1-(4-((1R,2S)-2-Phenylcyclopropyl)phenyl)ethenone (5a).

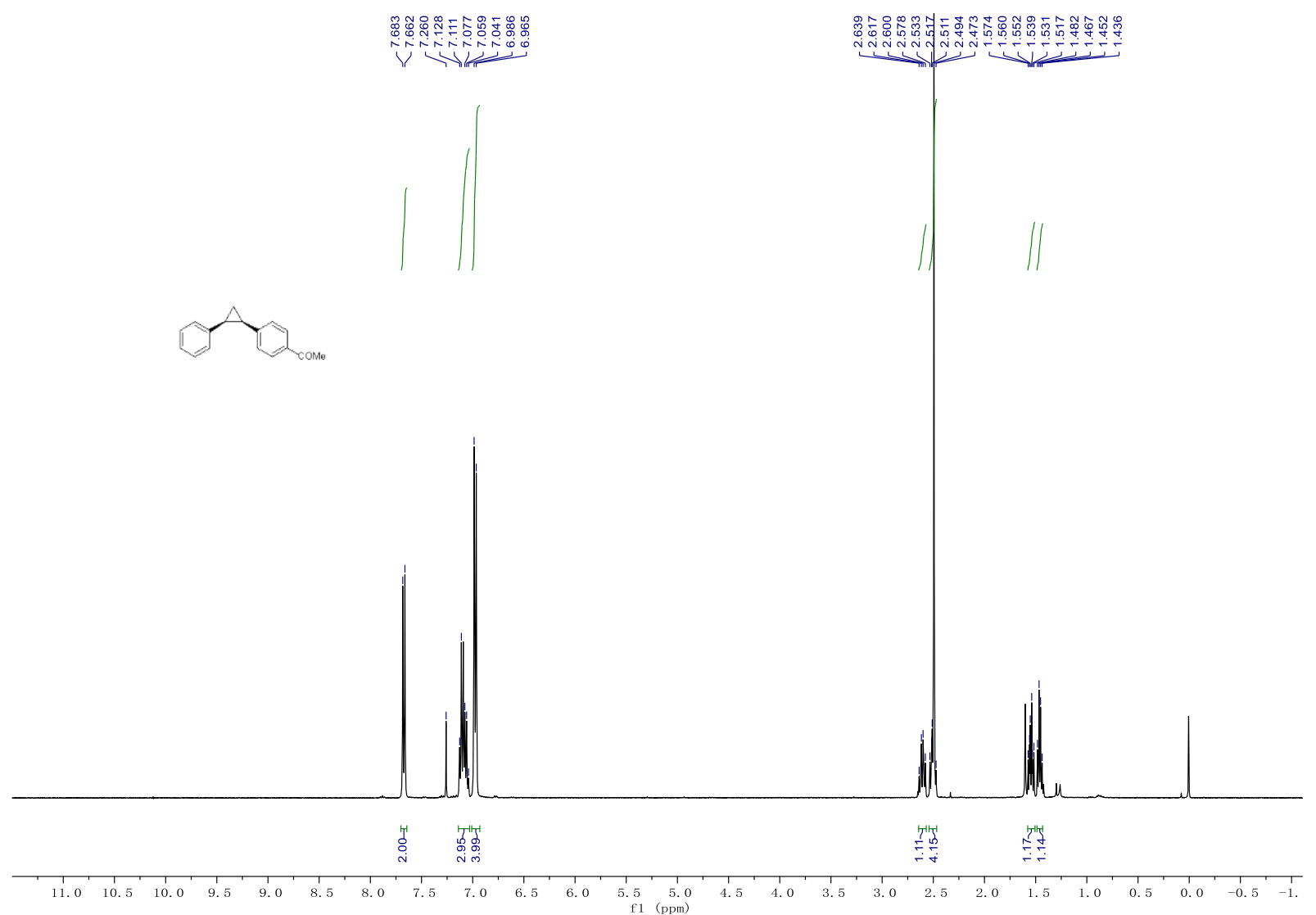




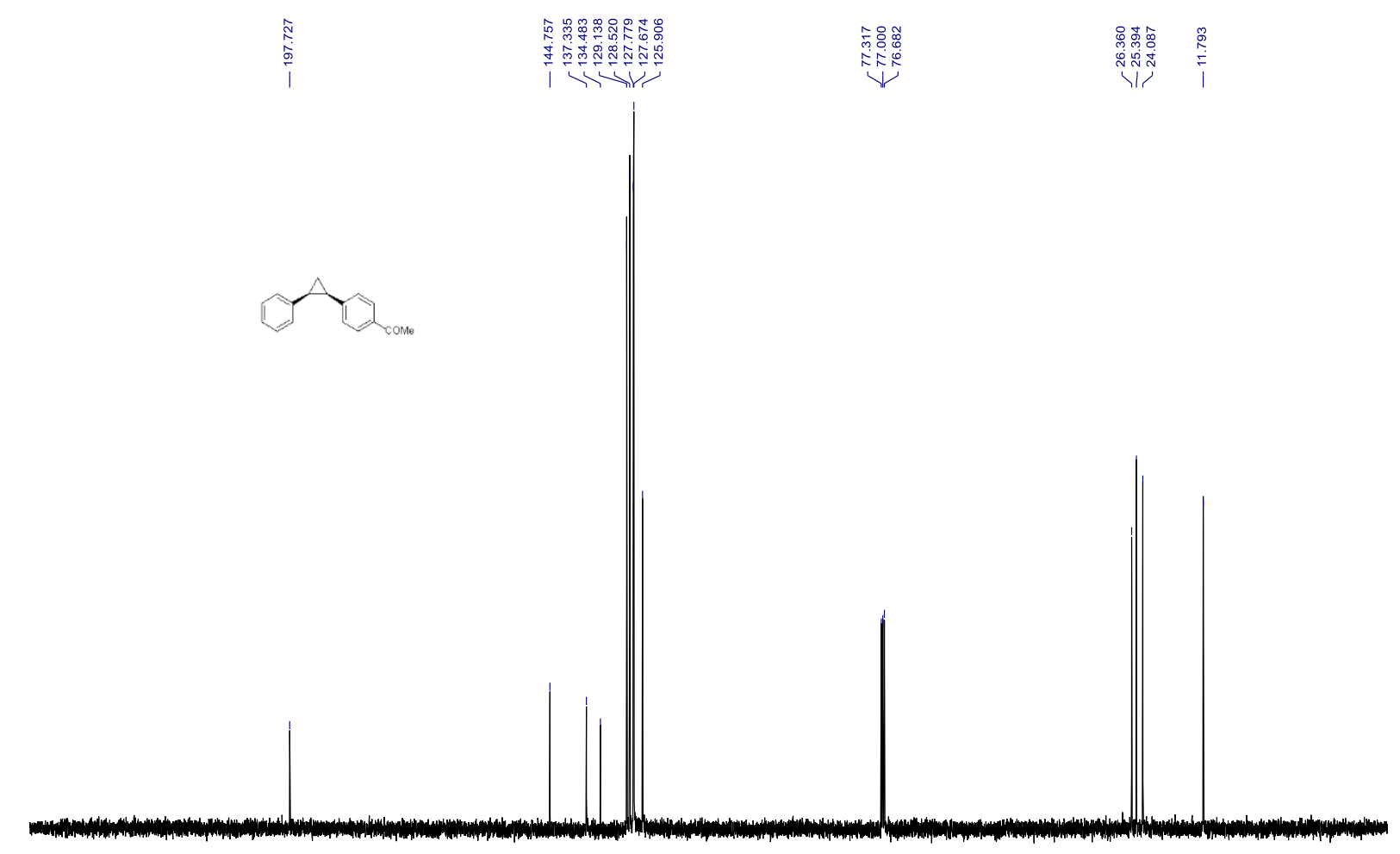

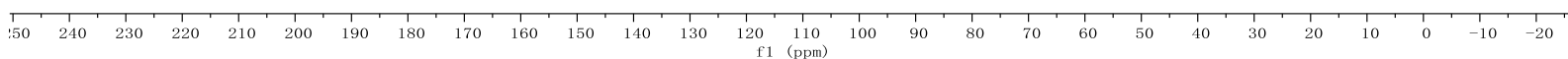

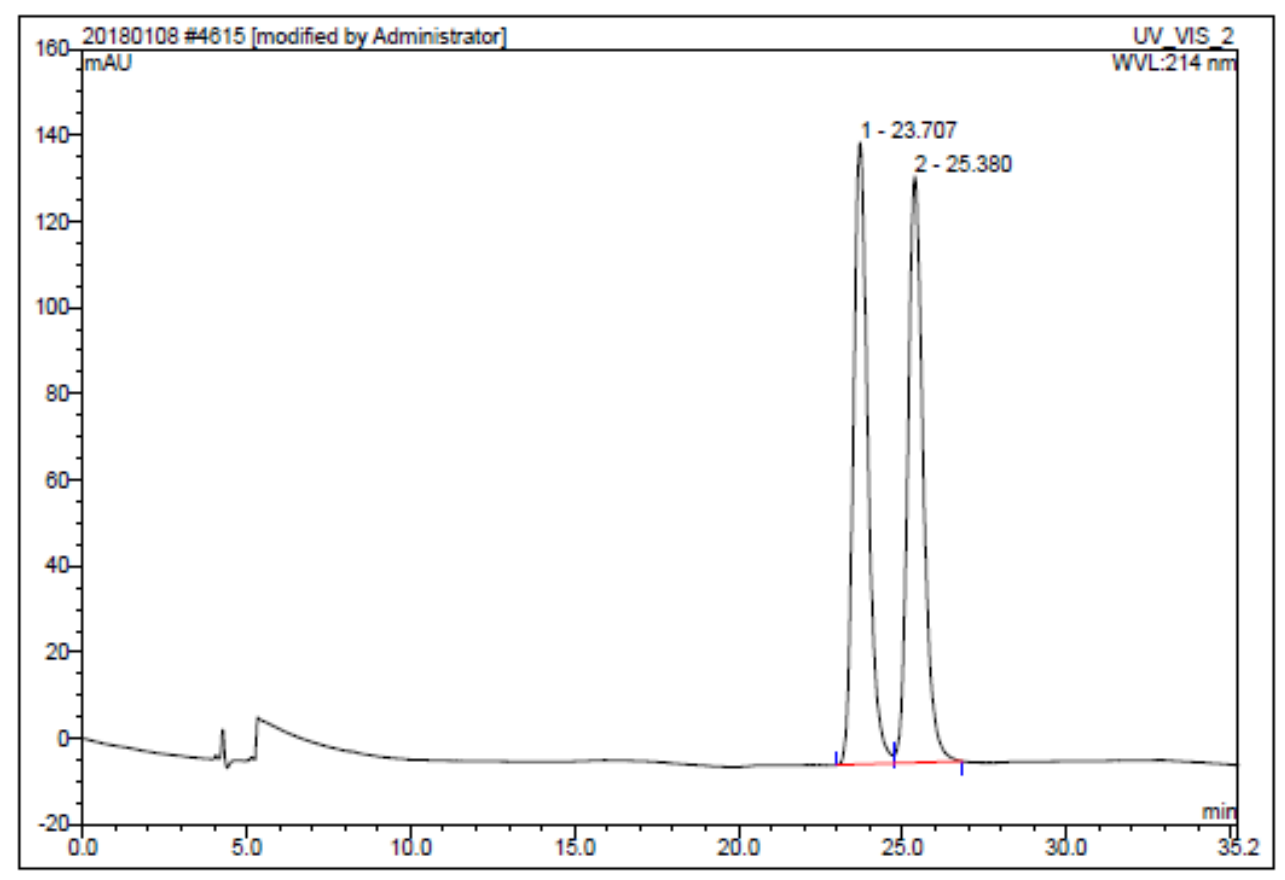

\begin{tabular}{|r|ccrrrrr|r|}
\hline No. & $\begin{array}{c}\text { Ret.Time } \\
\text { min }\end{array}$ & Peak Name & $\begin{array}{c}\text { Height } \\
\text { mAU }\end{array}$ & $\begin{array}{c}\text { Area } \\
\text { mAU*min }\end{array}$ & $\begin{array}{c}\text { Rel.Area } \\
\%\end{array}$ & Amount & Type \\
\hline 1 & 23.71 & n.a. & 144.305 & 74.537 & 50.05 & n.a. & BM $^{\star}$ \\
2 & 25.38 & n.a. & 136.053 & 74.376 & 49.95 & n.a. & MB $^{\star}$ \\
\hline Total: & & & 280.358 & 148.913 & 100.00 & 0.000 \\
\hline
\end{tabular}




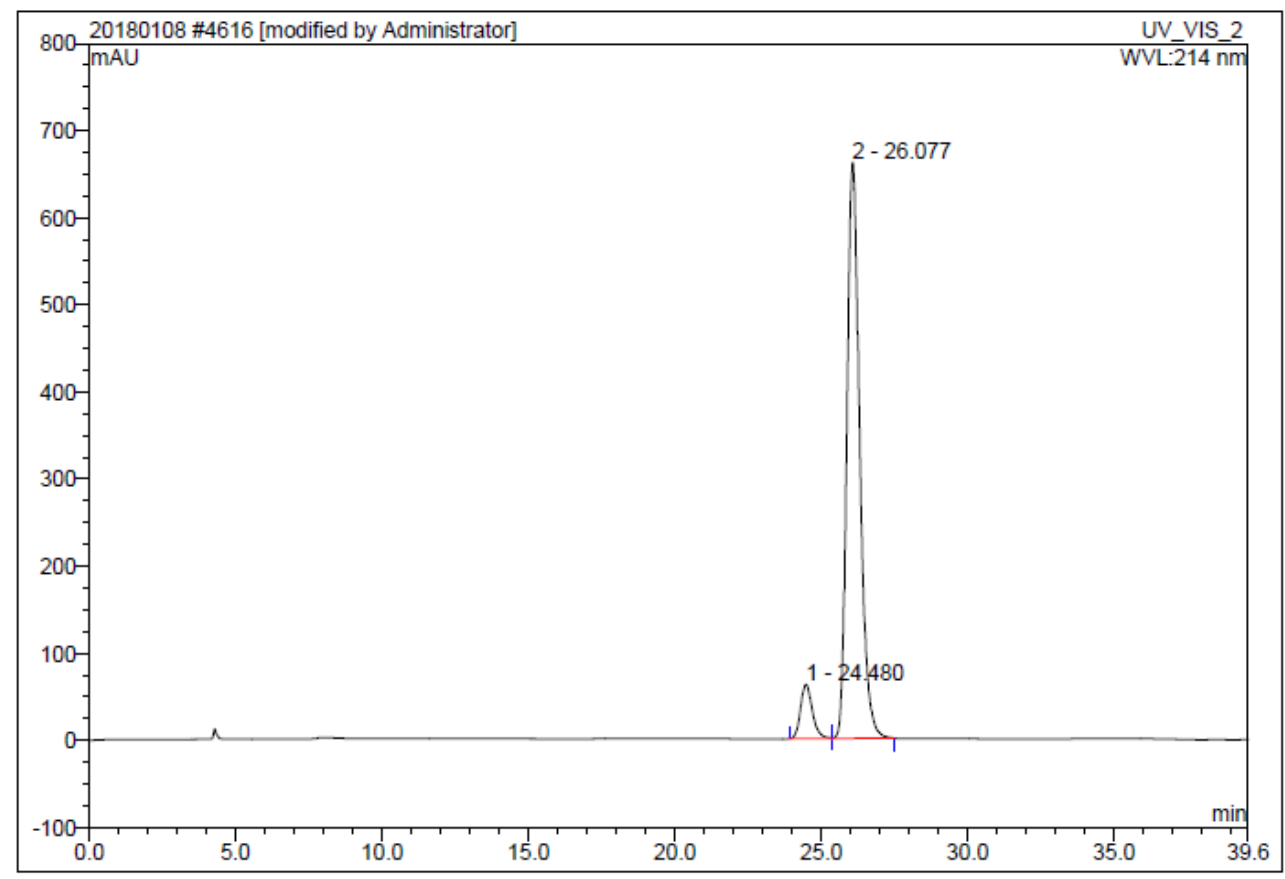

\begin{tabular}{|c|c|c|c|c|c|c|c|}
\hline No. & $\begin{array}{c}\text { Ret.Time } \\
\text { min }\end{array}$ & Peak Name & $\begin{array}{c}\text { Height } \\
\text { mAU }\end{array}$ & $\begin{array}{c}\text { Area } \\
\mathrm{mAU}^{*} \min \end{array}$ & $\begin{array}{c}\text { Rel.Area } \\
\% \\
\end{array}$ & Amount & Type \\
\hline 1 & 24.48 & n.a. & 62.340 & 29.265 & 8.04 & n.a. & BM \\
\hline 2 & 26.08 & n.a. & 661.447 & 334.926 & 91.96 & n.a. & MB \\
\hline Total: & & & 723.787 & 364.191 & 100.00 & 0.000 & \\
\hline
\end{tabular}

((1R,2S)-2-(1,1-Difluoroprop-2-yn-1-yl)cyclopropyl)benzene (13).

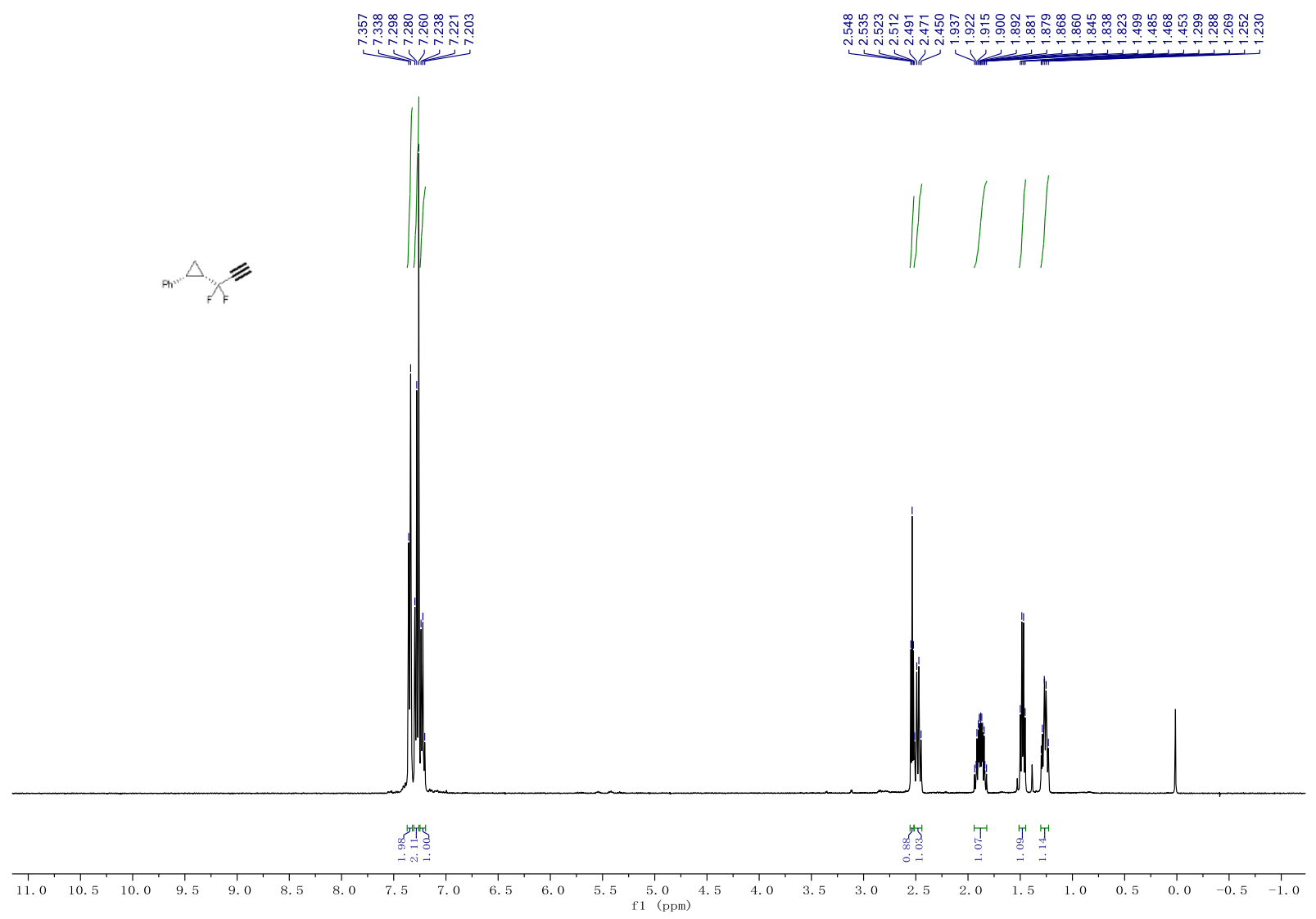



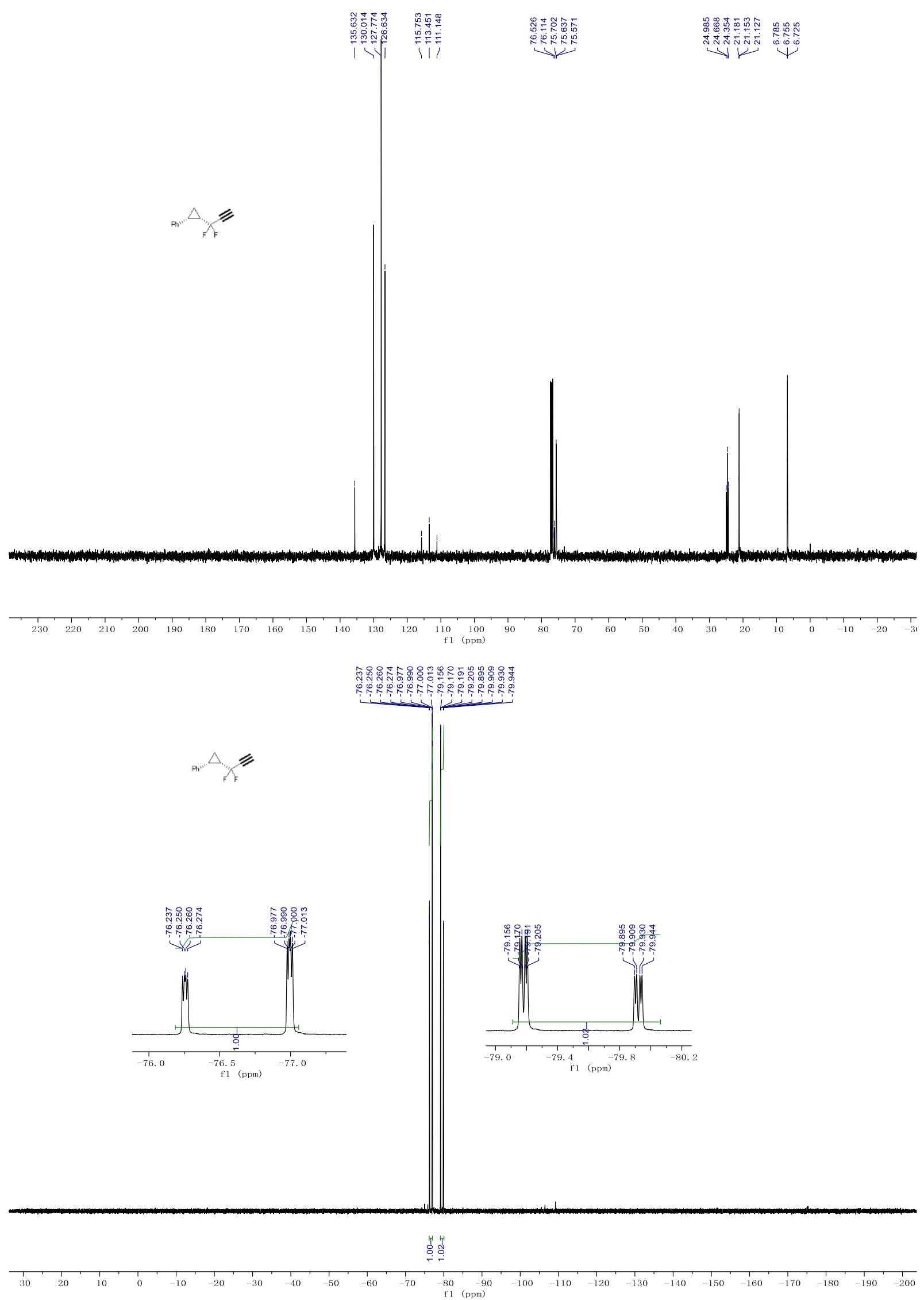


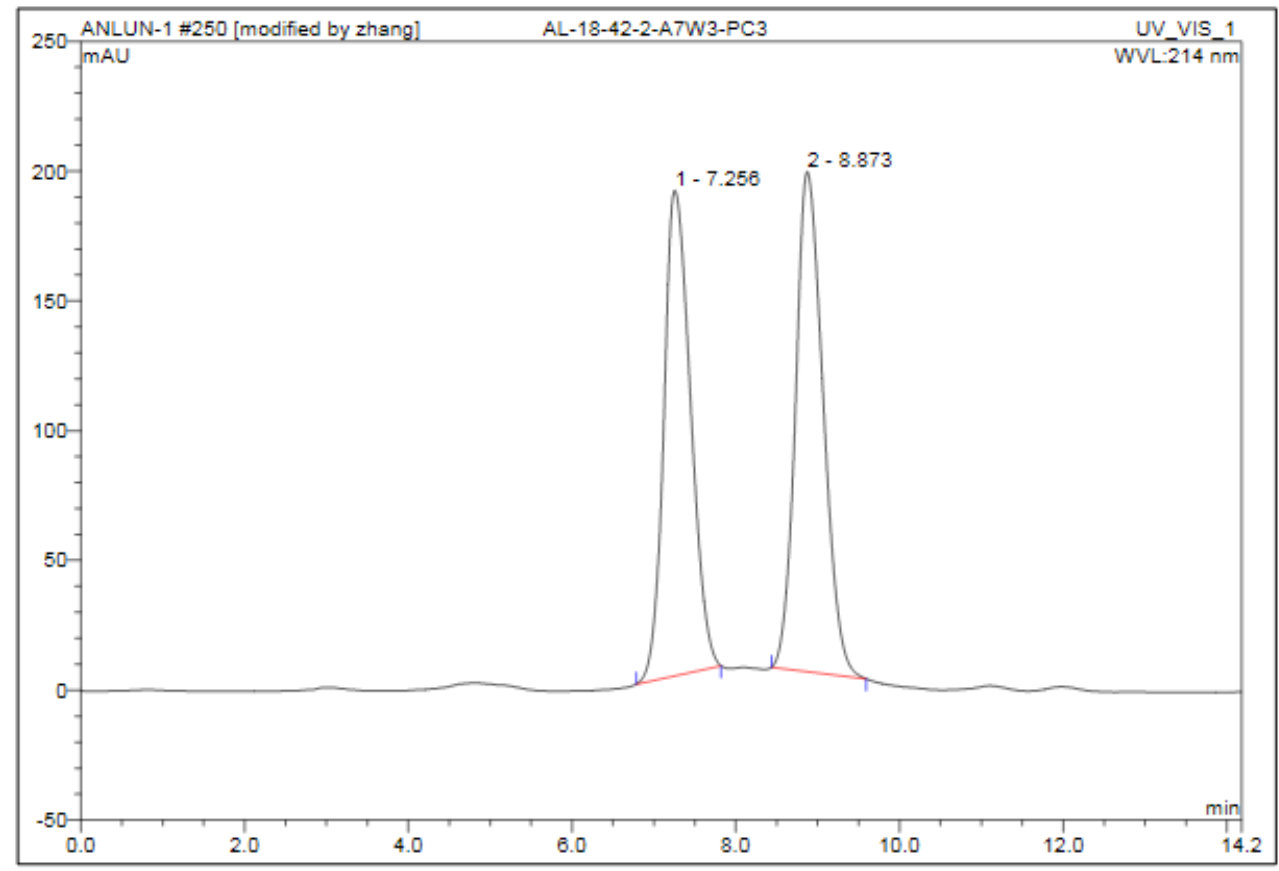

\begin{tabular}{|r|ccrrrrr|}
\hline No. & $\begin{array}{c}\text { Ret.Time } \\
\text { min }\end{array}$ & Peak Name & $\begin{array}{c}\text { Height } \\
\text { mAU }\end{array}$ & $\begin{array}{c}\text { Area } \\
\text { mAU*min }\end{array}$ & $\begin{array}{r}\text { Rel.Area } \\
\%\end{array}$ & Amount & Type \\
\hline 1 & 7.26 & n.a. & 187.014 & 70.523 & 48.35 & n.a. & BMB $^{\star}$ \\
2 & 8.87 & n.a. & 192.644 & 75.331 & 51.65 & n.a. & BMB $^{\star}$ \\
\hline Total: & & & 379.658 & 145.854 & 100.00 & 0.000 & \\
\hline
\end{tabular}

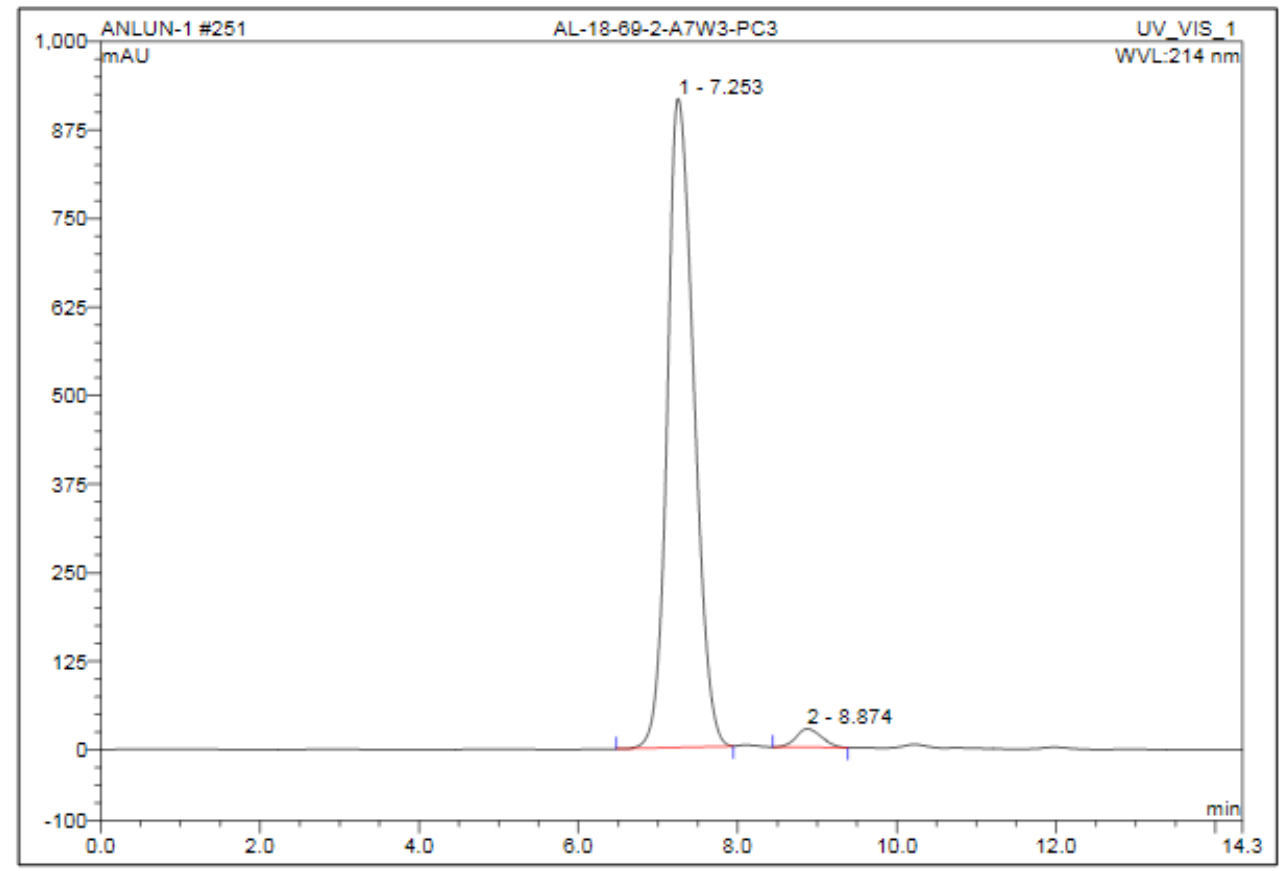

\begin{tabular}{|r|ccrrrrr|}
\hline No. & $\begin{array}{c}\text { Ret.Time } \\
\text { min }\end{array}$ & Peak Name & $\begin{array}{c}\text { Height } \\
\text { mAU }\end{array}$ & $\begin{array}{c}\text { Area } \\
\text { mAU*min }\end{array}$ & $\begin{array}{r}\text { Rel.Area } \\
\%\end{array}$ & Amount & Type \\
\hline 1 & 7.25 & n.a. & 915.750 & 355.067 & 97.33 & n.a. & BMB \\
2 & 8.87 & n.a. & 26.226 & 9.734 & 2.67 & n.a. & BMB \\
\hline Total: & & & 941.976 & 364.801 & 100.00 & 0.000 & \\
\hline
\end{tabular}


4-(Difluoro-((1S,2R)-2-phenylcyclopropyl)methyl)-1-(4-nitrobenzyl)-1H-1,2,3-triazole (6).
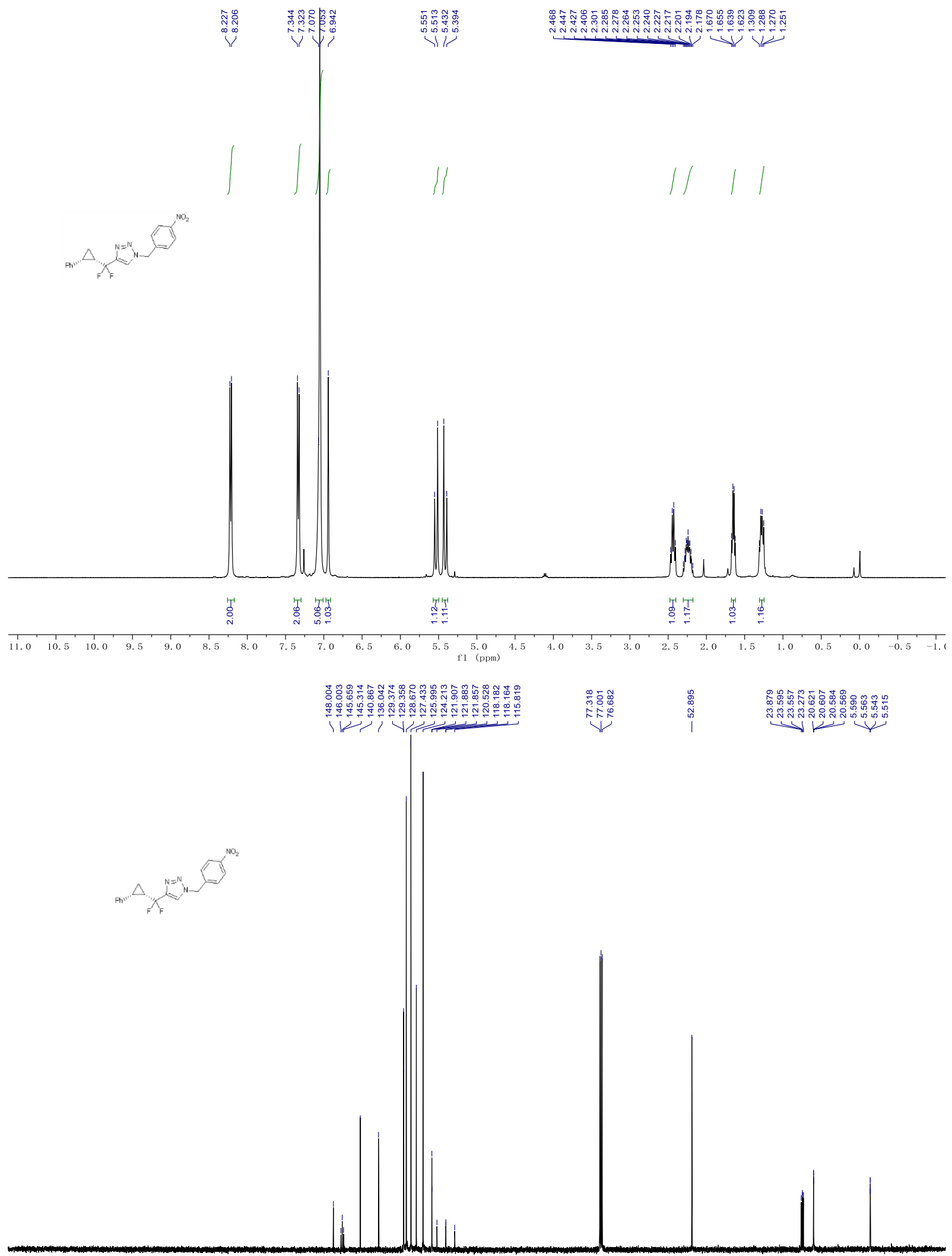

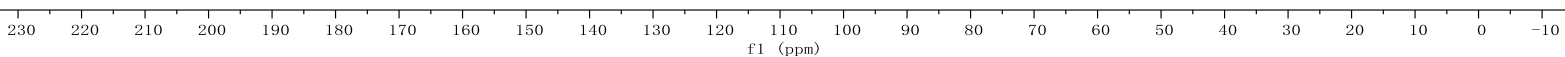




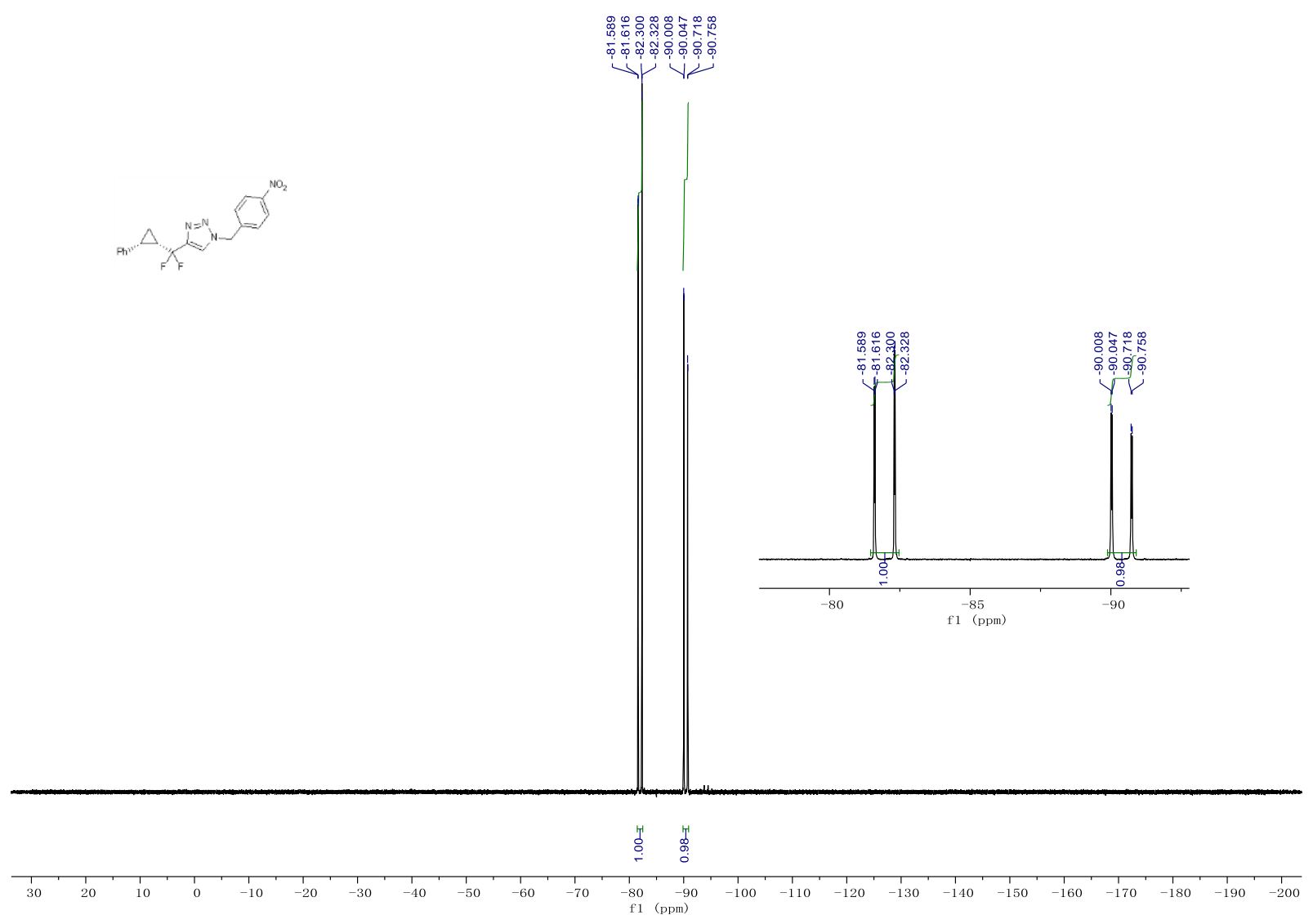

(3-((1S,2R)-2-(4-(tert-Butyl)phenyl)cyclopropyl)-3,3-difluoroprop-1-yn-1-yl)triethylsilane (3b).

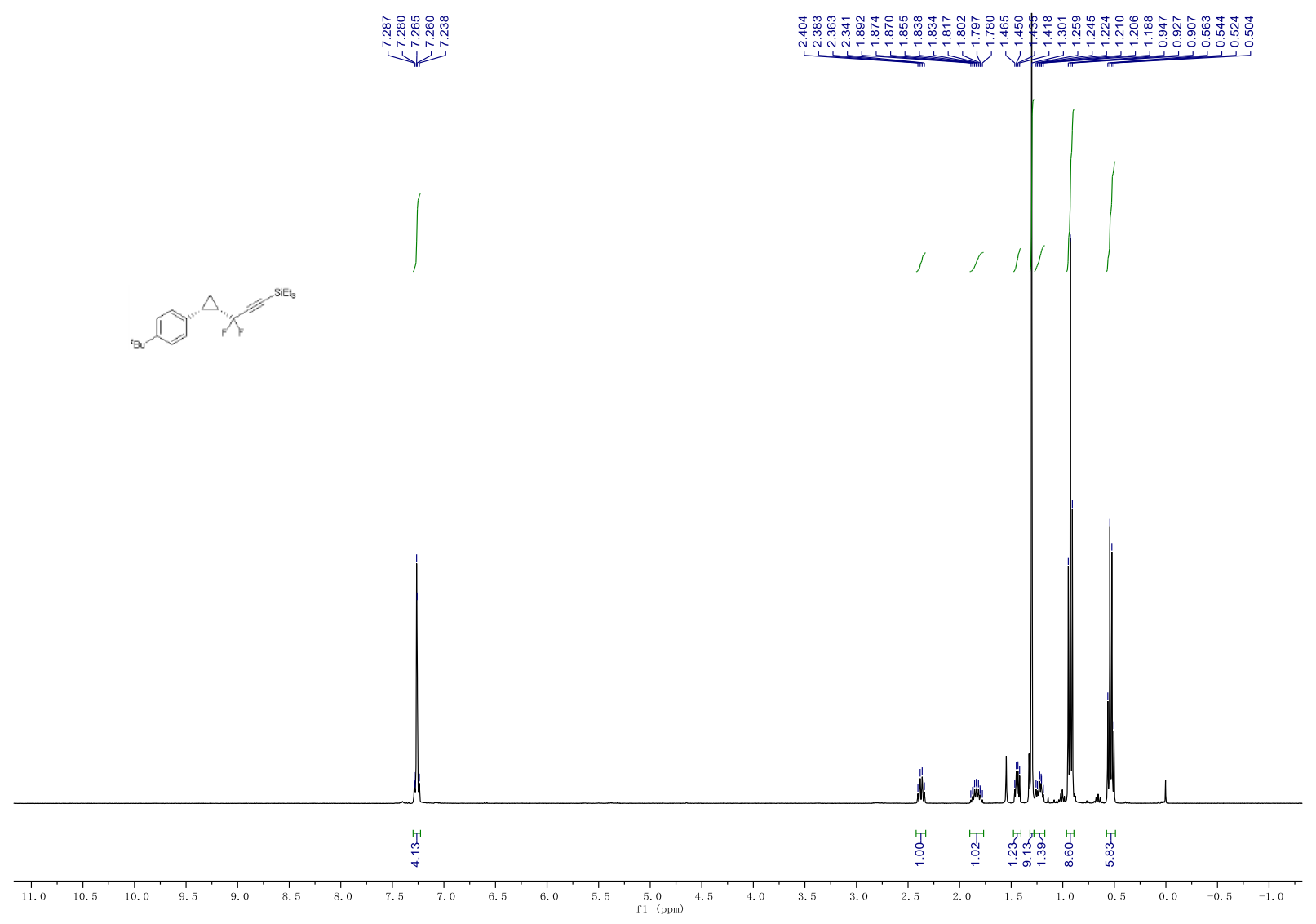



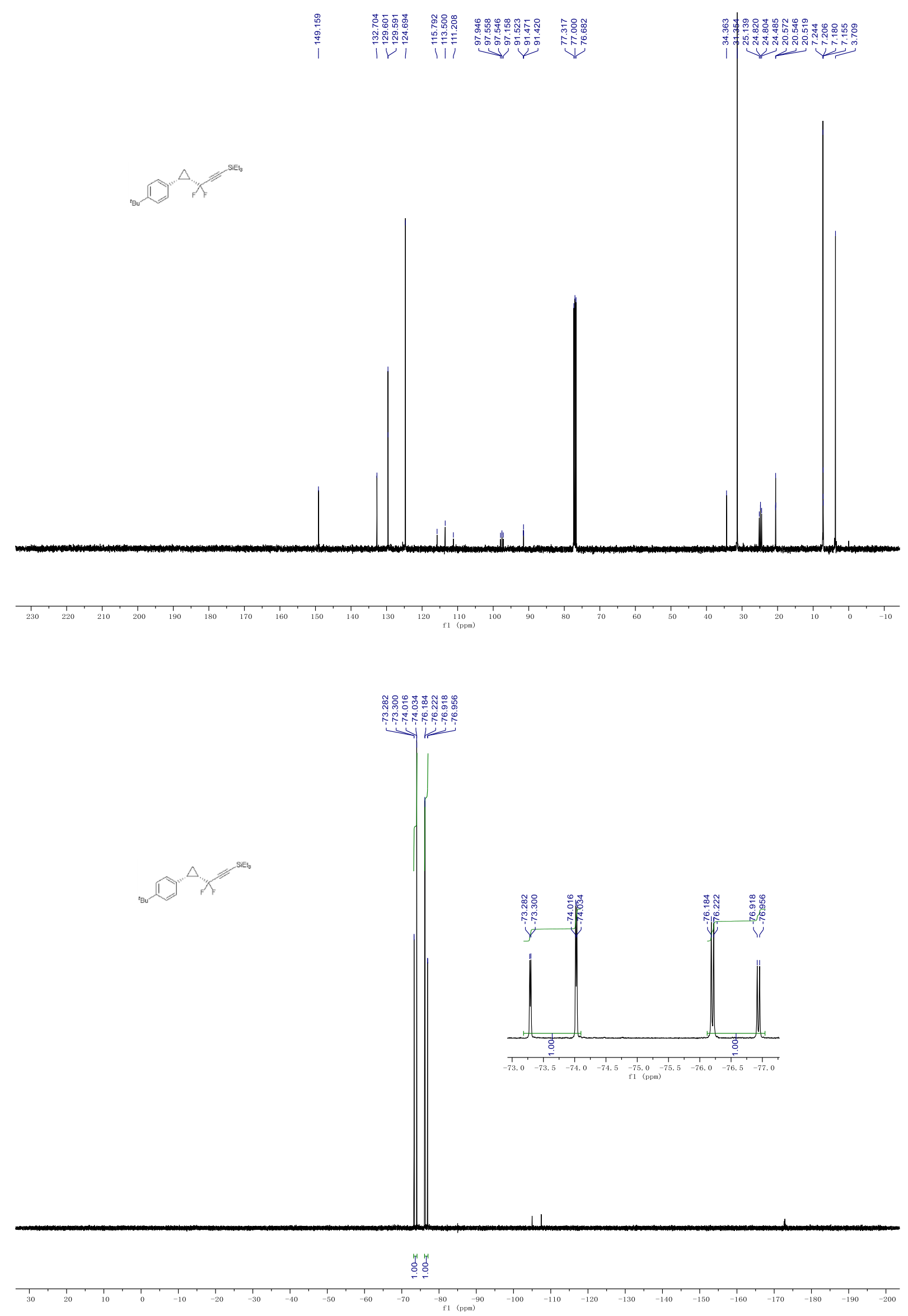


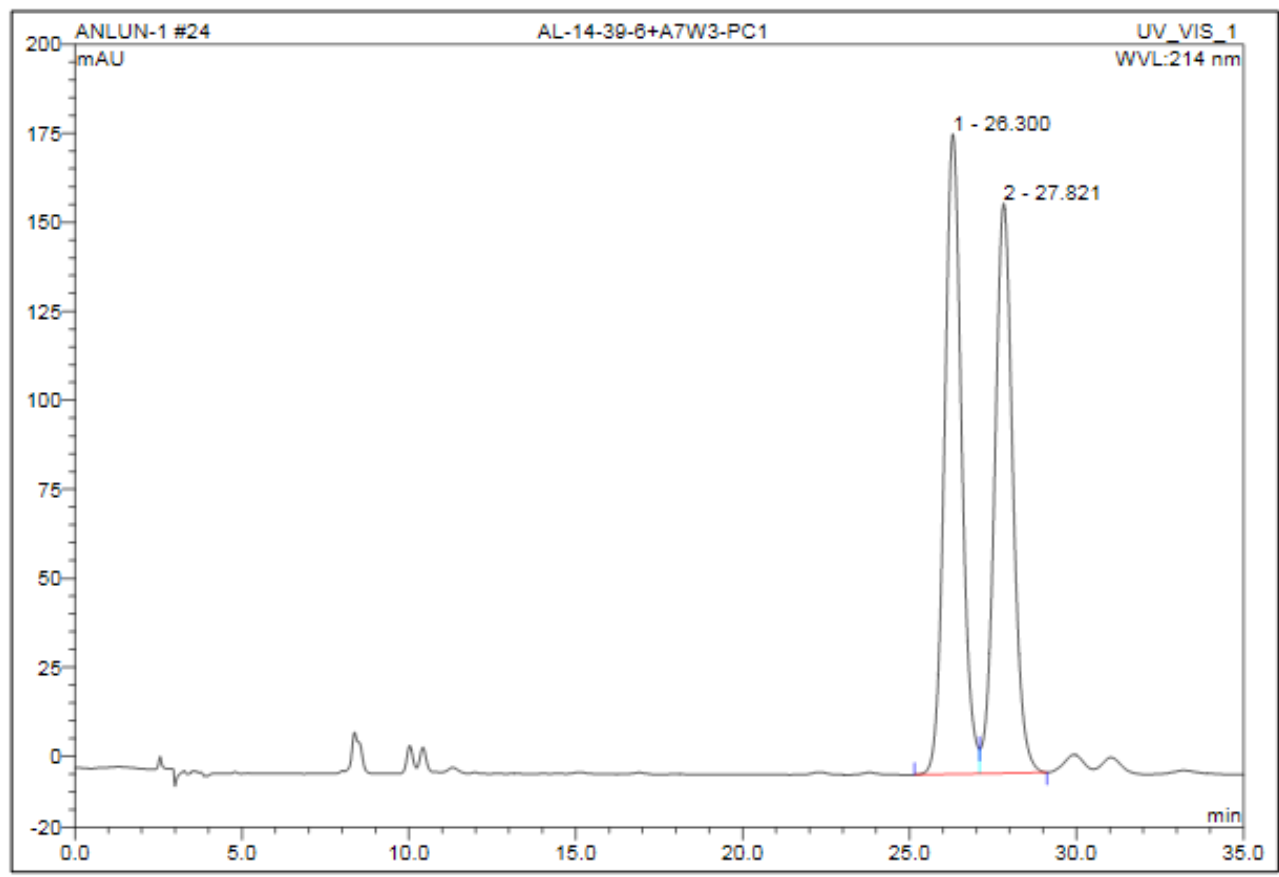

\begin{tabular}{|r|ccccccc|}
\hline No. & $\begin{array}{c}\text { Ret.Time } \\
\text { min }\end{array}$ & Peak Name & $\begin{array}{c}\text { Height } \\
\text { mAU }\end{array}$ & $\begin{array}{c}\text { Area } \\
\text { mAU*min }\end{array}$ & $\begin{array}{c}\text { Rel.Area } \\
\%\end{array}$ & Amount & Type \\
\hline 1 & 26.30 & n.a. & 179.638 & 107.077 & 51.47 & n.a. & BM \\
2 & 27.82 & n.a. & 160.011 & 100.945 & 48.53 & n.a. & MB \\
\hline Total: & & & 339.649 & 208.022 & 100.00 & 0.000 & \\
\hline
\end{tabular}

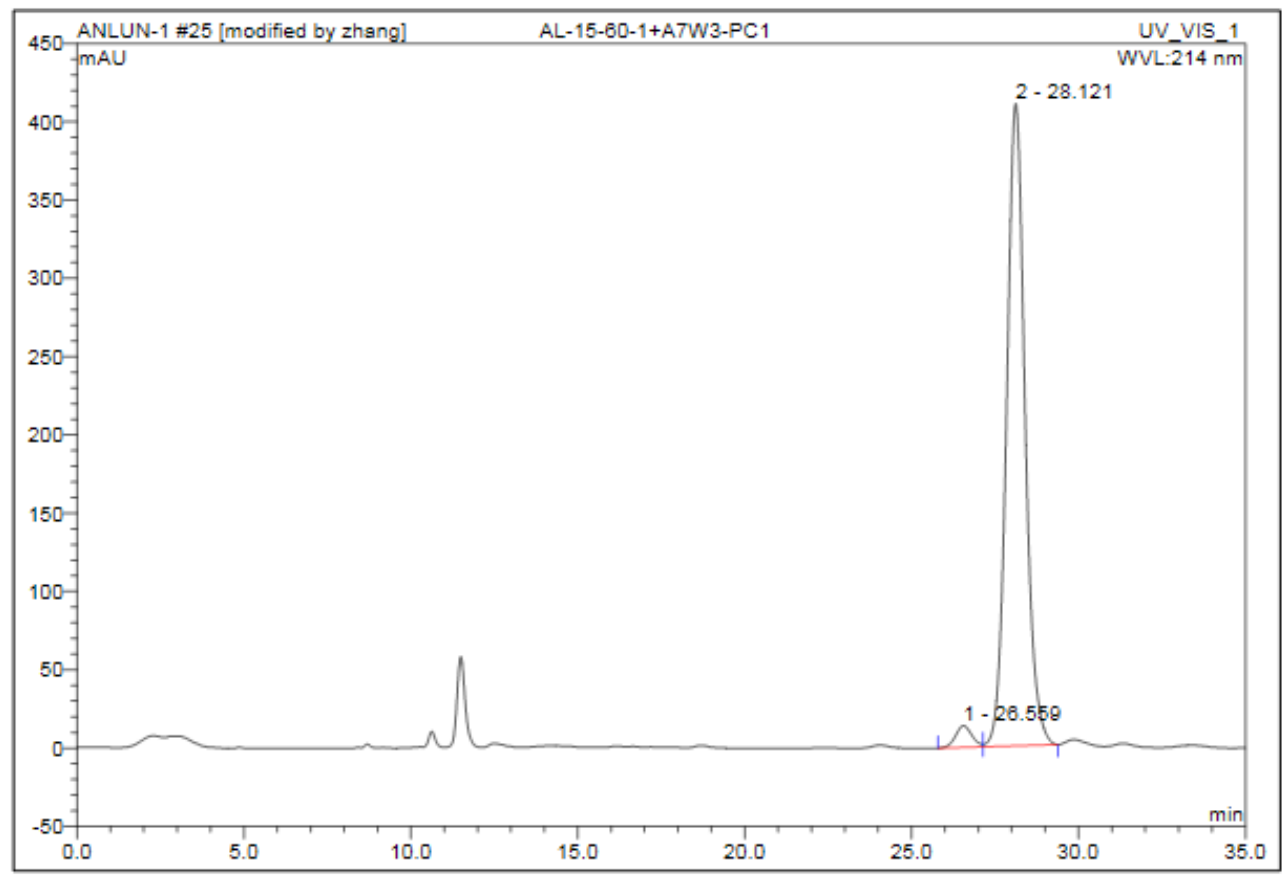

\begin{tabular}{|r|ccrrrrr|}
\hline No. & $\begin{array}{c}\text { Ret.Time } \\
\text { min }\end{array}$ & Peak Name & $\begin{array}{c}\text { Height } \\
\text { mAU }\end{array}$ & $\begin{array}{c}\text { Area } \\
\text { mAU*min }\end{array}$ & $\begin{array}{c}\text { Rel.Area } \\
\%\end{array}$ & Amount & Type \\
\hline 1 & 26.56 & n.a. & 13.727 & 7.797 & 2.89 & n.a. & BM \\
2 & 28.12 & n.a. & 410.170 & 262.094 & 97.11 & n.a. & MB \\
\hline Total: & & & 423.896 & 269.891 & 100.00 & 0.000 & \\
\hline
\end{tabular}


(3-((1S,2R)-2-([1,1'-Biphenyl]-4-yl)cyclopropyl)-3,3-difluoroprop-1-yn-1-yl)triethylsilane (3c).
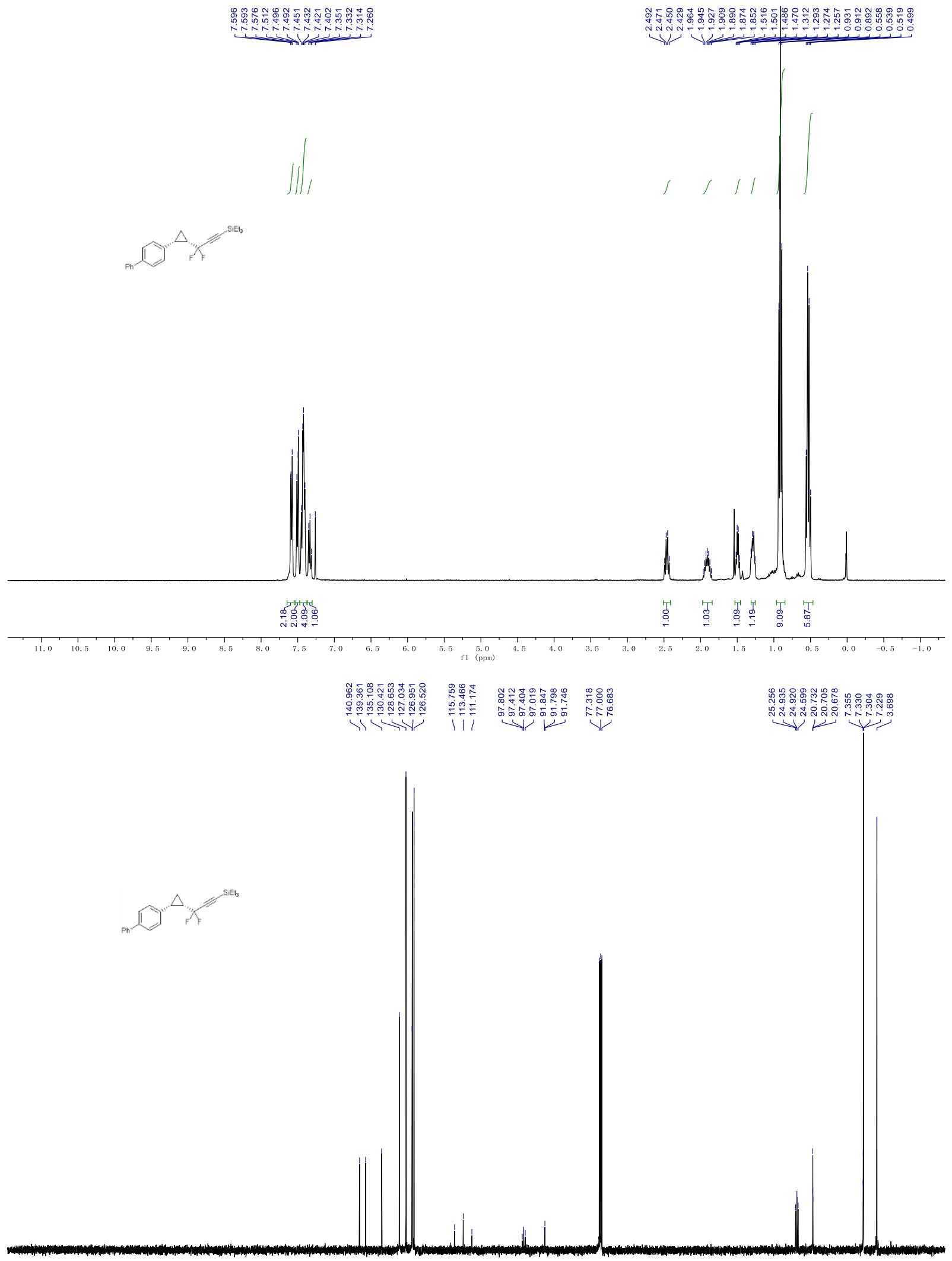

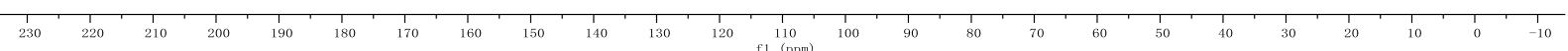



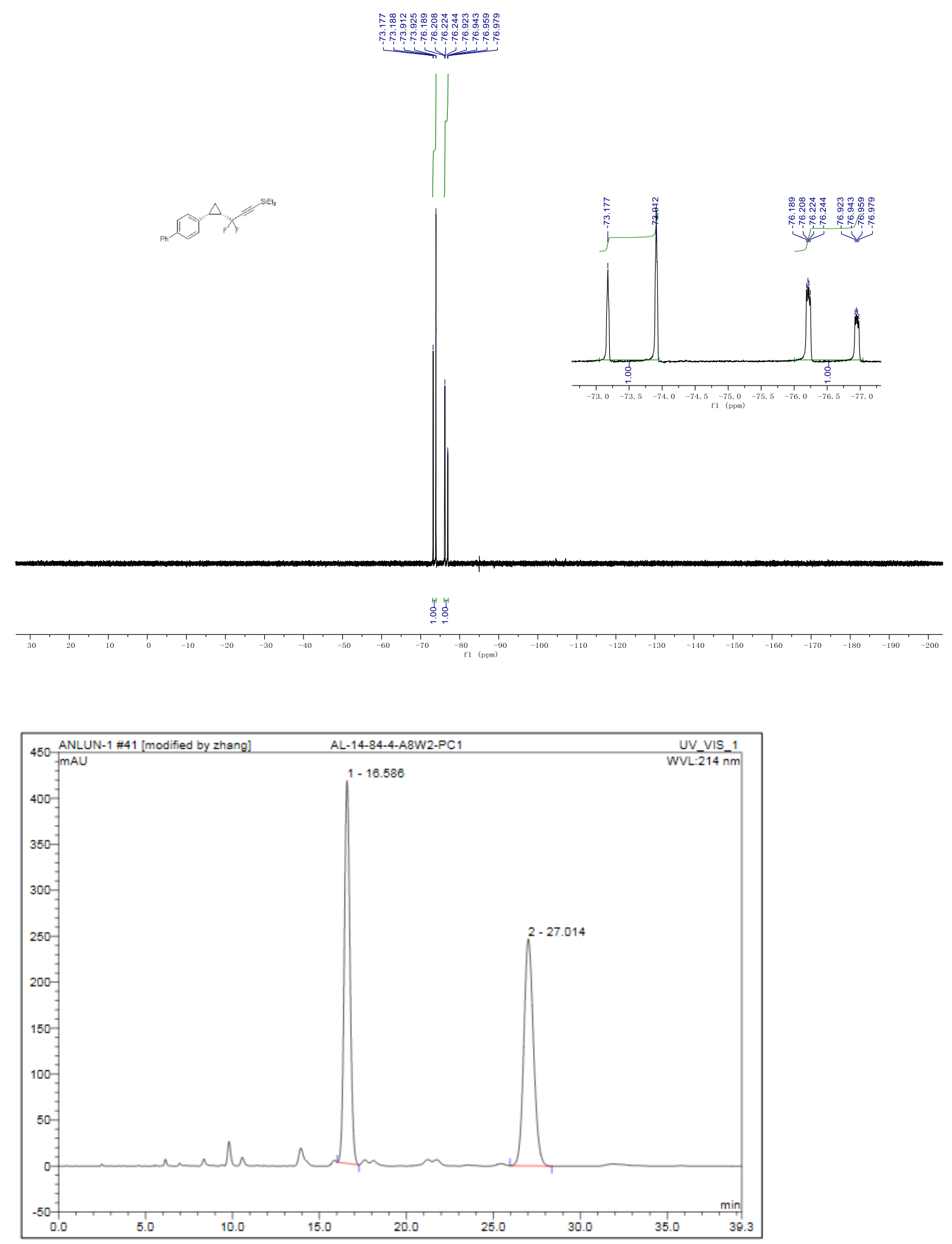

\begin{tabular}{|r|cccrrrr|}
\hline No. & $\begin{array}{c}\text { Ret.Time } \\
\text { min }\end{array}$ & Peak Name & $\begin{array}{c}\text { Height } \\
\text { mAU }\end{array}$ & $\begin{array}{c}\text { Area } \\
\mathbf{m A U}^{*} \text { min }\end{array}$ & $\begin{array}{c}\text { Rel.Area } \\
\%\end{array}$ & Amount & Type \\
\hline 1 & 16.59 & n.a. & 416.533 & 159.616 & 50.06 & n.a. & BMB \\
2 & 27.01 & n.a. & 247.051 & 159.244 & 49.94 & n.a. & BMB \\
\hline Total: & & & 663.584 & 318.860 & 100.00 & 0.000 & \\
\hline
\end{tabular}




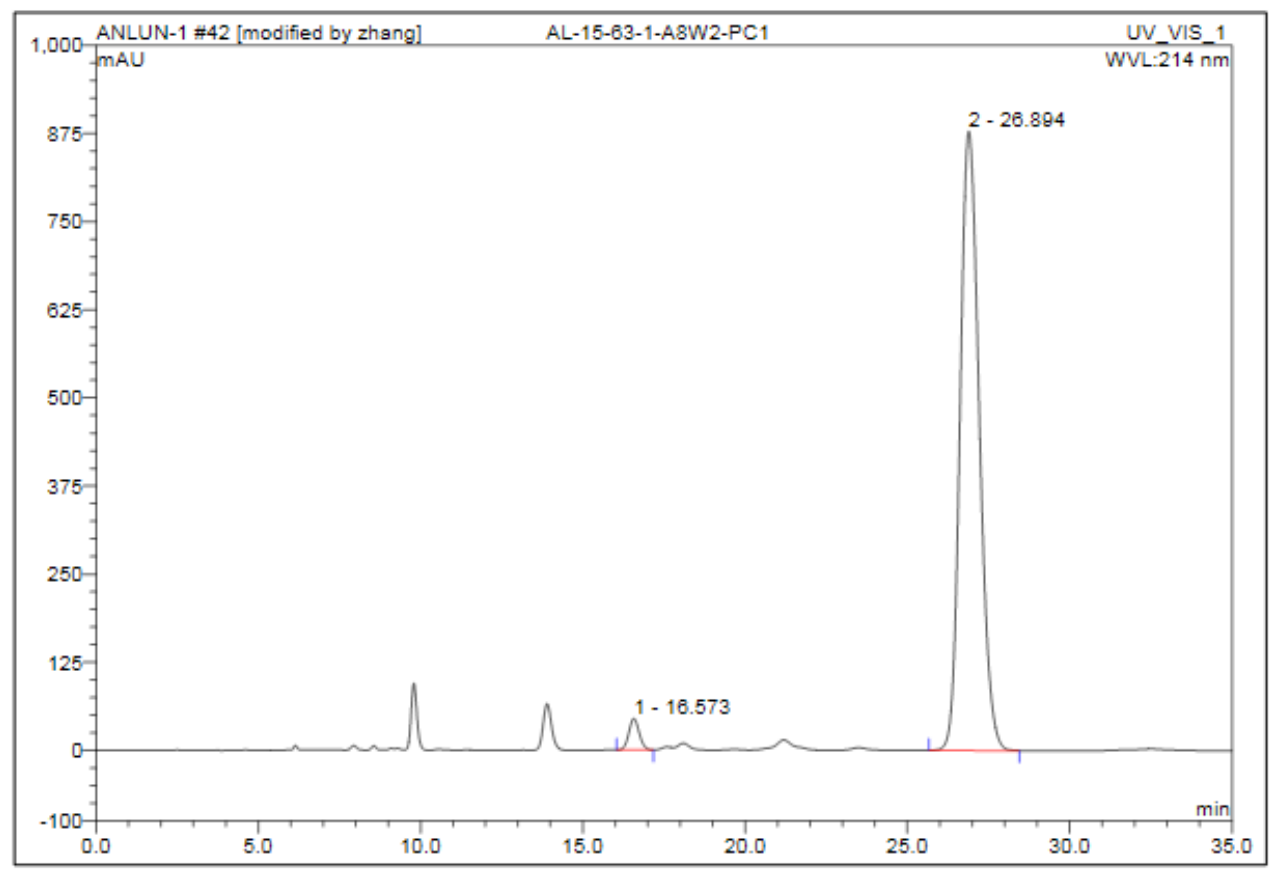

\begin{tabular}{|c|c|c|c|c|c|c|c|}
\hline No. & $\begin{array}{c}\text { Ret.Time } \\
\text { min }\end{array}$ & Peak Name & $\begin{array}{c}\text { Height } \\
\text { mAU }\end{array}$ & $\begin{array}{c}\text { Area } \\
\mathrm{mAU^{* } \operatorname { m i n }}\end{array}$ & $\begin{array}{c}\text { Rel.Area } \\
\%\end{array}$ & Amount & Type \\
\hline 1 & 16.57 & n.a. & 44.515 & 16.699 & 2.71 & n.a. & BMB \\
\hline 2 & 26.89 & n.a. & 878.107 & 599.545 & 97.29 & n.a. & $\mathrm{BMB}$ \\
\hline Total: & & & 922.622 & 616.244 & 100.00 & 0.000 & \\
\hline
\end{tabular}

(3,3-Difluoro-3-((1S,2R)-2-(4-(trifluoromethyl)phenyl)cyclopropyl)prop-1-yn-1-yl)triethylsilane (3d).

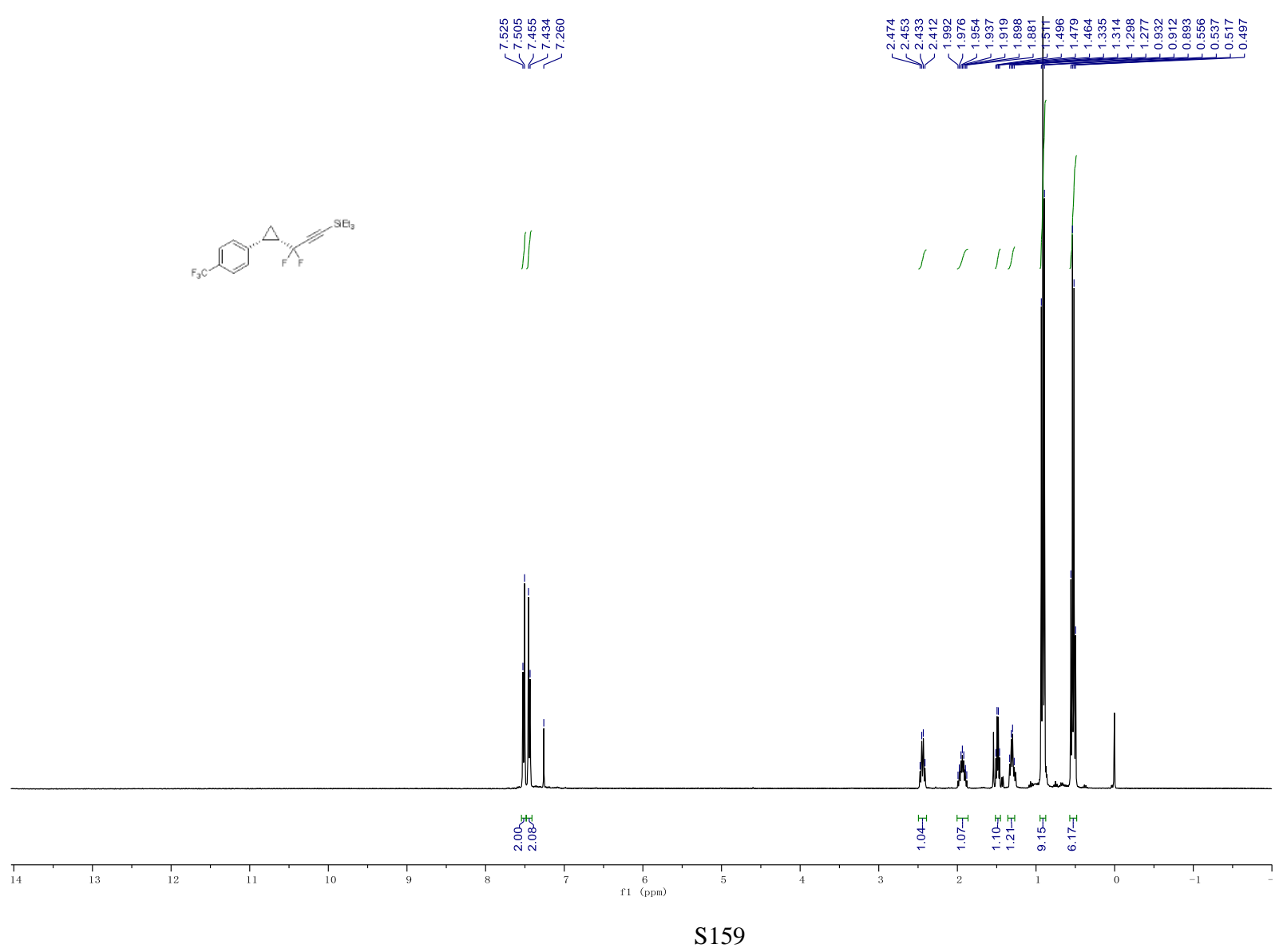


Fon 0
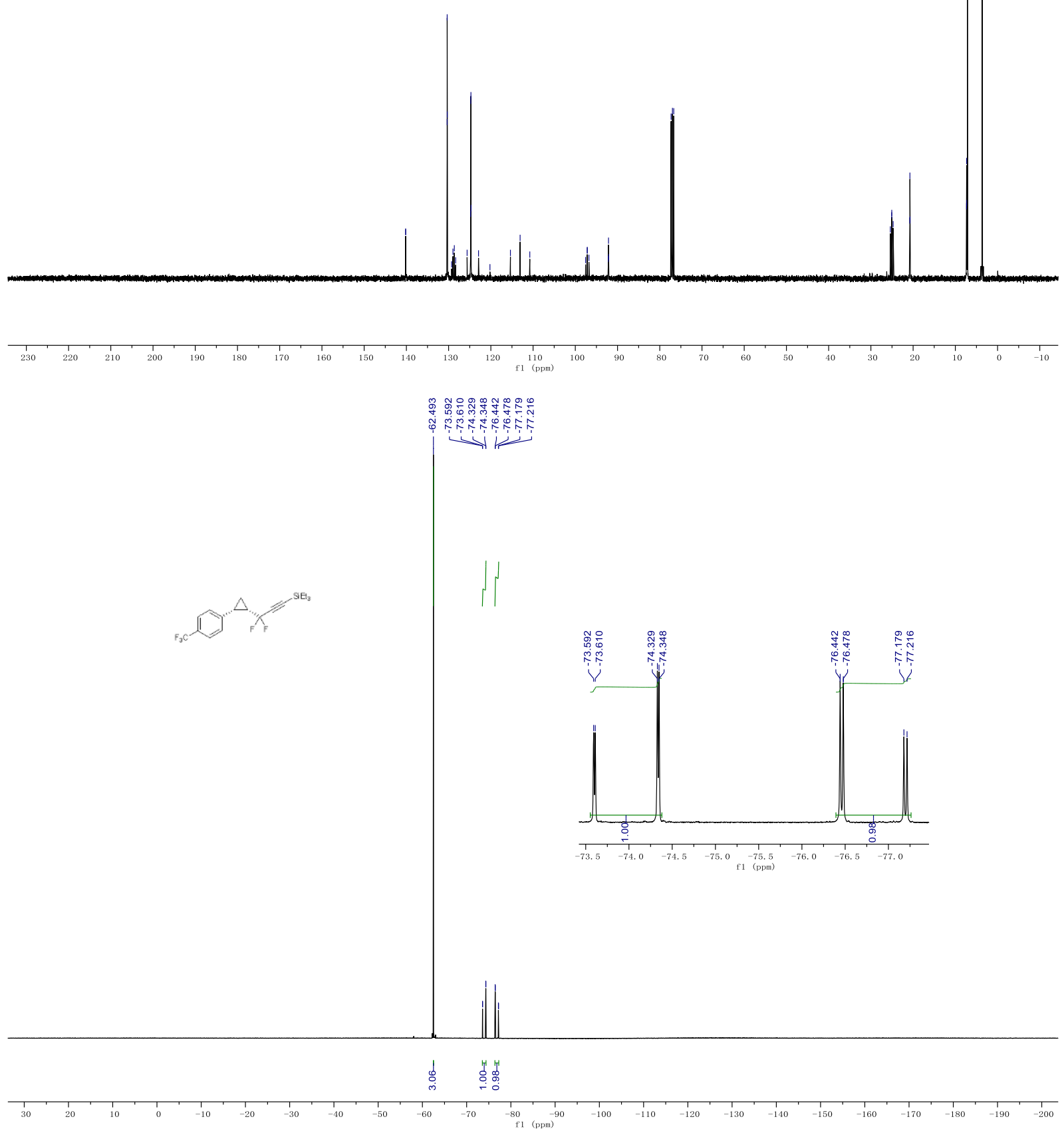


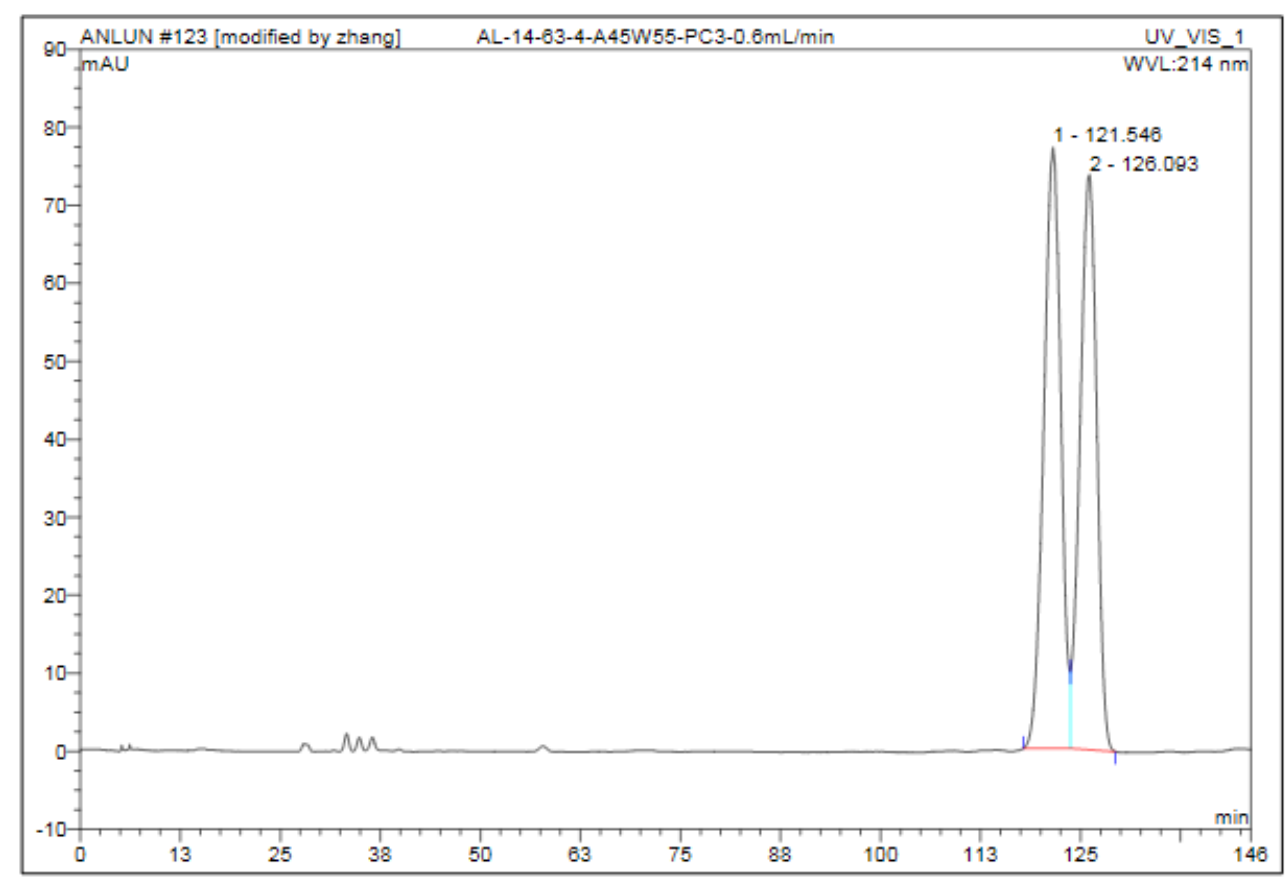

\begin{tabular}{|c|c|c|c|c|c|c|c|}
\hline No. & $\begin{array}{l}\text { Ret.Time } \\
\text { min }\end{array}$ & Peak Name & $\begin{array}{c}\text { Height } \\
\text { mAU }\end{array}$ & $\begin{array}{c}\text { Area } \\
\mathrm{mAU}^{*} \min \end{array}$ & $\begin{array}{c}\text { Rel.Area } \\
\%\end{array}$ & Amount & Type \\
\hline 1 & 121.55 & n.a. & 77.032 & 190.823 & 51.58 & n.a. & $\mathrm{BM}^{*}$ \\
\hline 2 & 126.09 & n.a. & 73.736 & 179.147 & 48.42 & n.a. & $\mathrm{MB}^{*}$ \\
\hline Total: & & & 150.768 & 369.970 & 100.00 & 0.000 & \\
\hline
\end{tabular}

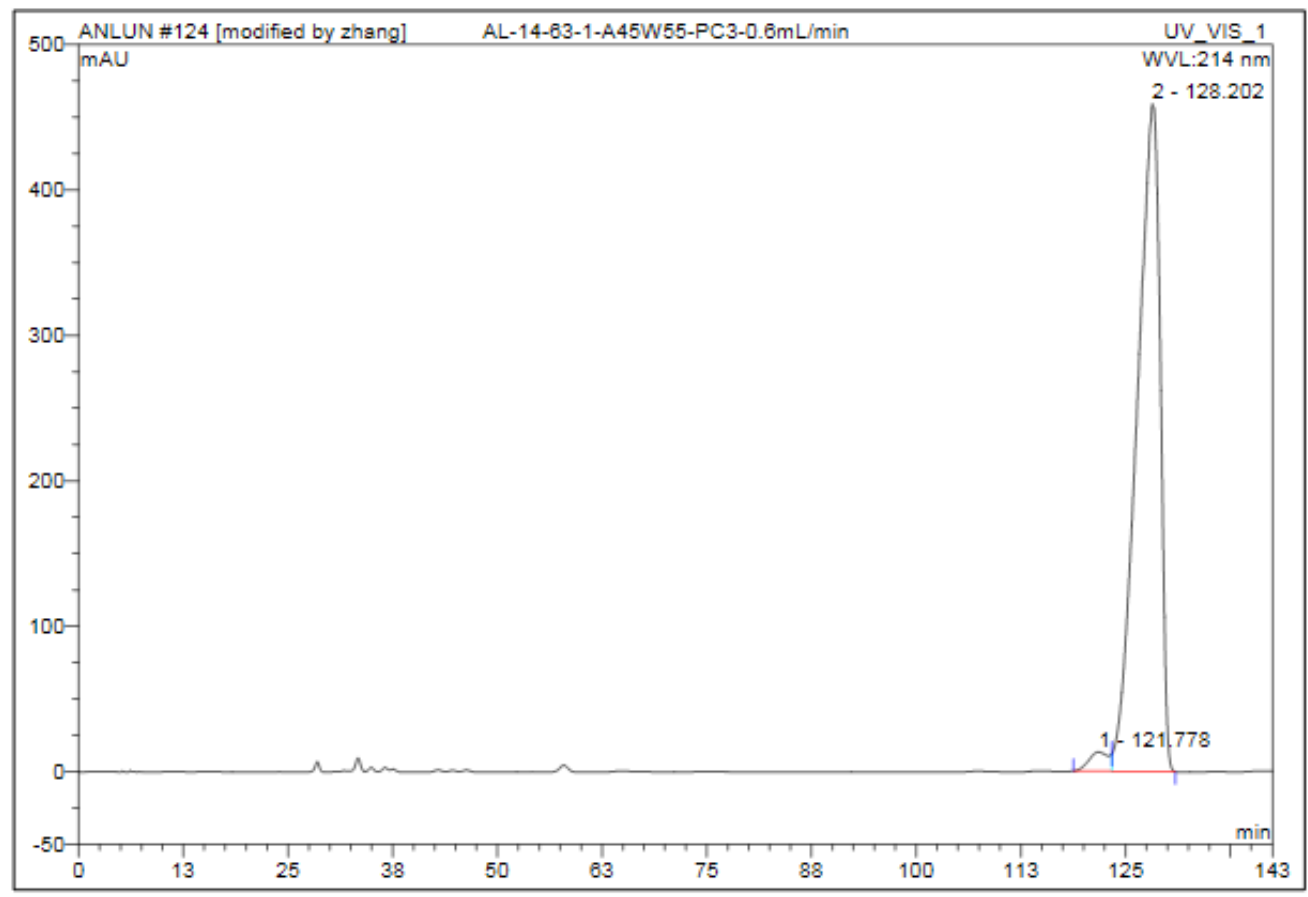

\begin{tabular}{|c|c|c|c|c|c|c|c|}
\hline No. & $\begin{array}{c}\text { Ret.Time } \\
\text { min }\end{array}$ & Peak Name & $\begin{array}{c}\text { Height } \\
\text { mAU }\end{array}$ & $\begin{array}{c}\text { Area } \\
\mathrm{mAU}^{*} \min \end{array}$ & $\begin{array}{c}\text { Rel.Area } \\
\% \\
\end{array}$ & Amount & Type \\
\hline 1 & 121.78 & n.a. & 13.241 & 36.455 & 2.42 & n.a. & $B M$ * \\
\hline 2 & 128.20 & n.a. & 459.008 & 1467.028 & 97.58 & n.a. & $\mathrm{MB}^{*}$ \\
\hline Total: & & & 472.248 & 1503.482 & 100.00 & 0.000 & \\
\hline
\end{tabular}


(3,3-Difluoro-3-((1S,2R)-2-(4-(trifluoromethoxy)phenyl)cyclopropyl)prop-1-yn-1-yl)triethylsila ne (3e).
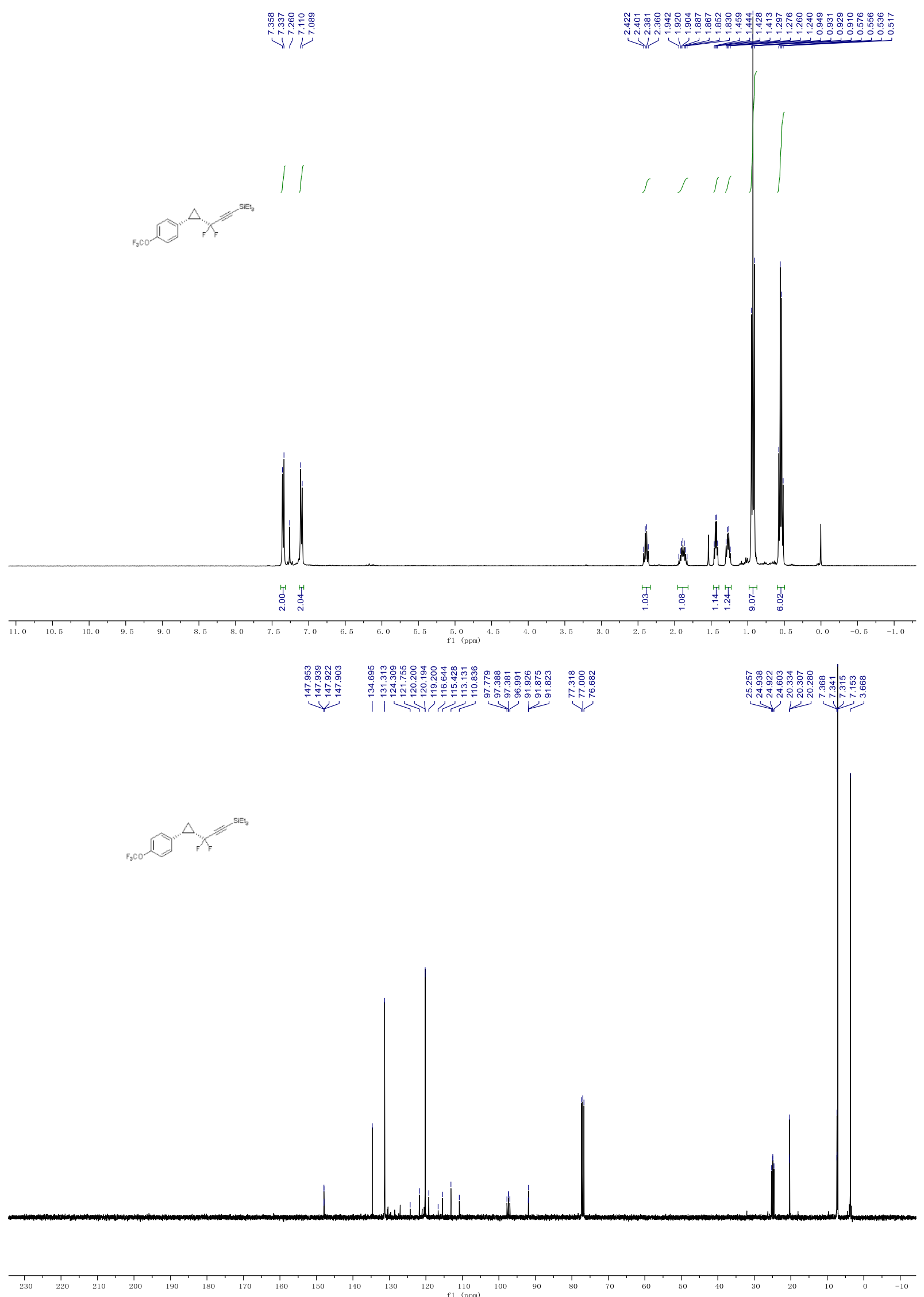

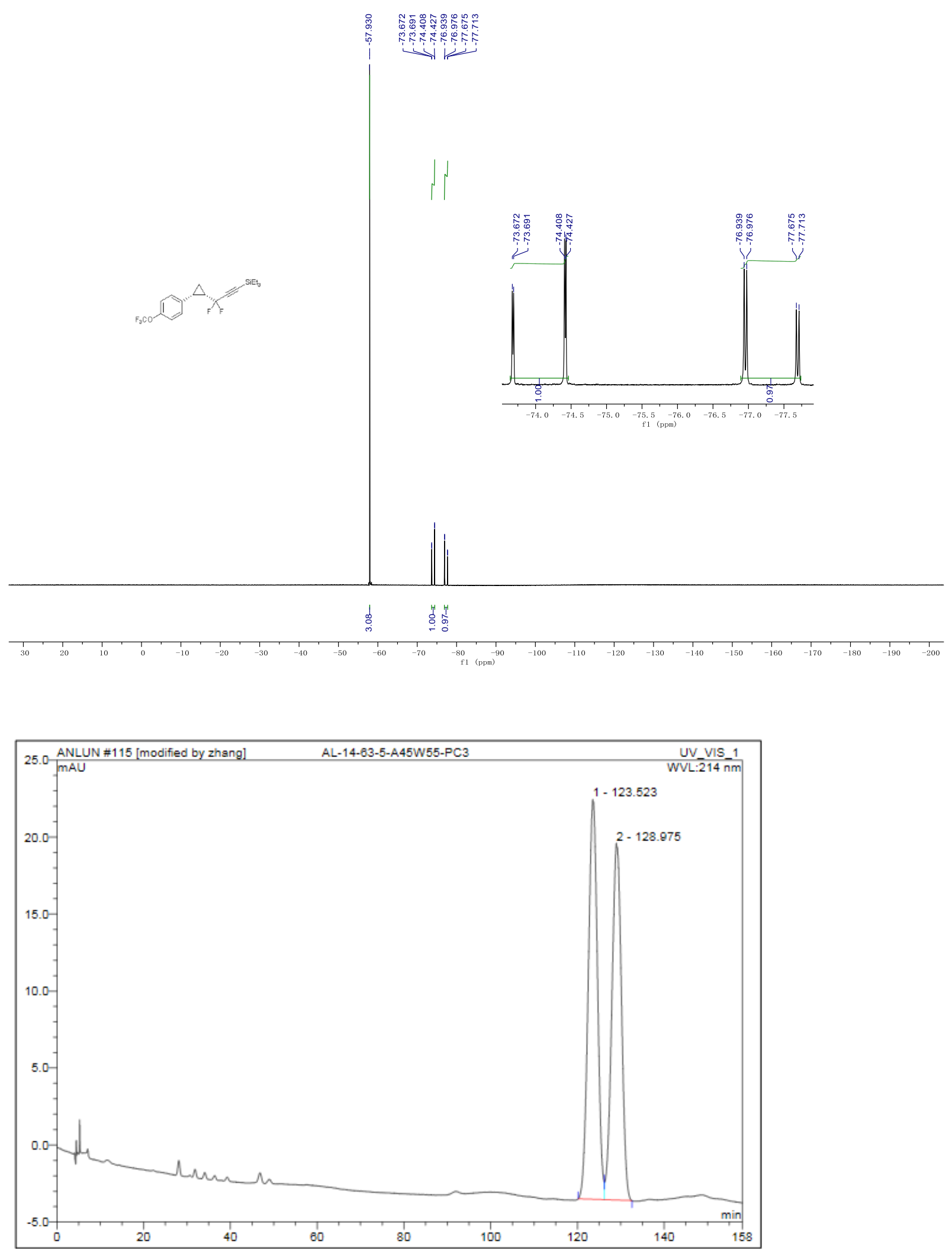

\begin{tabular}{|r|ccrrrrr|}
\hline No. & $\begin{array}{c}\text { Ret.Time } \\
\text { min }\end{array}$ & Peak Name & $\begin{array}{c}\text { Height } \\
\text { mAU }\end{array}$ & $\begin{array}{c}\text { Area } \\
\text { mAU*min }\end{array}$ & $\begin{array}{r}\text { Rel.Area } \\
\%\end{array}$ & Amount & Type \\
\hline 1 & 123.52 & n.a. & 25.968 & 63.899 & 51.70 & n.a. & BM $^{*}$ \\
2 & 128.98 & n.a. & 23.191 & 59.694 & 48.30 & n.a. & MB $^{*}$ \\
\hline Total: & & & 49.159 & 123.594 & 100.00 & 0.000 & \\
\hline
\end{tabular}




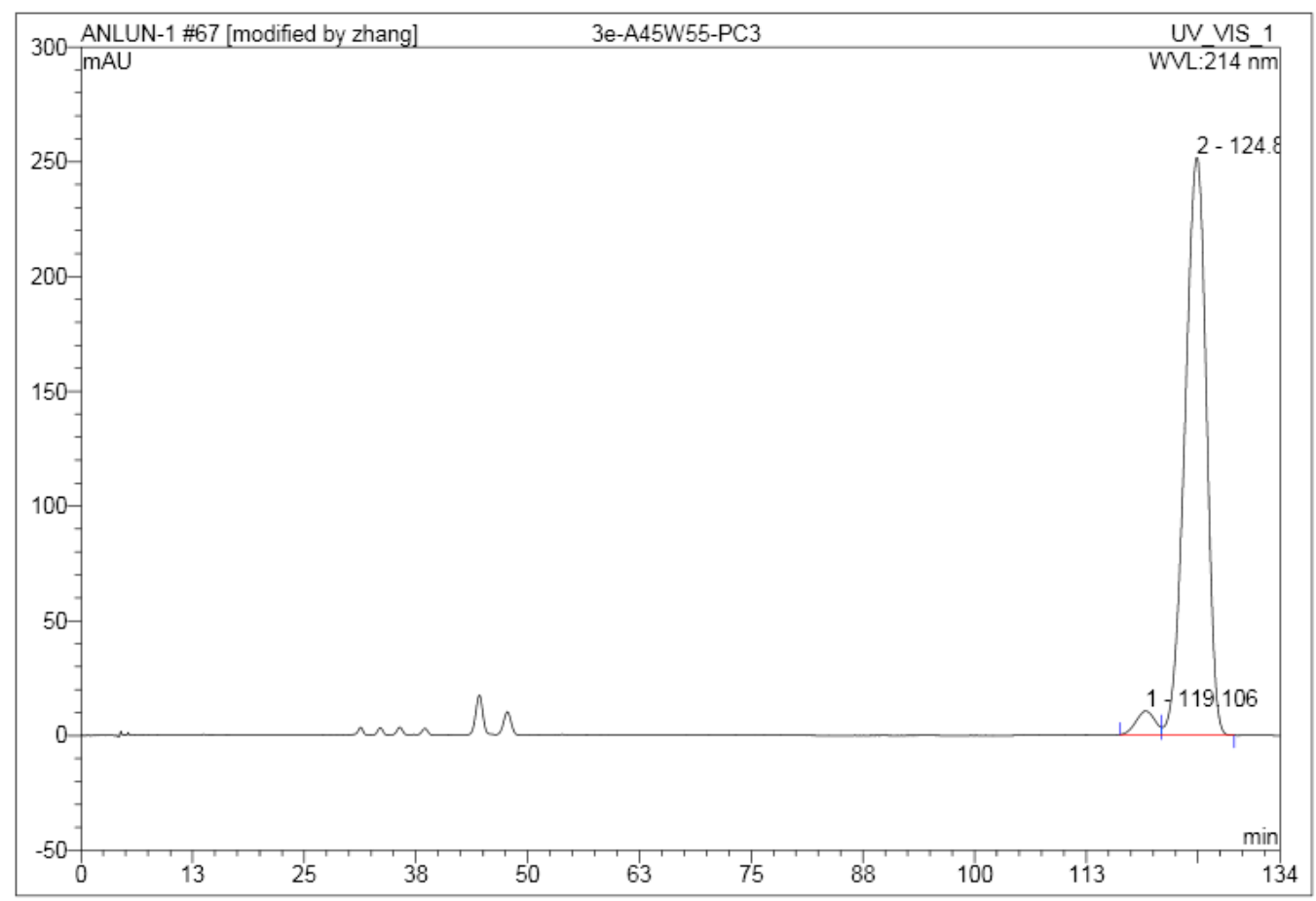

\begin{tabular}{|r|ccrrrrr|}
\hline No. & $\begin{array}{c}\text { Ret. Time } \\
\text { min }\end{array}$ & Peak Name & $\begin{array}{c}\text { Height } \\
\text { mAU }\end{array}$ & $\begin{array}{c}\text { Area } \\
\text { mAU*min }\end{array}$ & $\begin{array}{r}\text { Rel.Area } \\
\%\end{array}$ & Amount & Type \\
\hline 1 & 119.11 & n.a. & 10.457 & 26.081 & 3.60 & n.a. & BM $^{*}$ \\
2 & 124.86 & n.a. & 251.749 & 697.720 & 96.40 & n.a. & MB $^{*}$ \\
\hline Total: & & & 262.206 & 723.801 & 100.00 & 0.000 & \\
\hline
\end{tabular}

(3,3-Difluoro-3-((1S,2R)-2-(4-(trimethylsilyl)phenyl)cyclopropyl)prop-1-yn-1-yl)triethylsilane (3f).

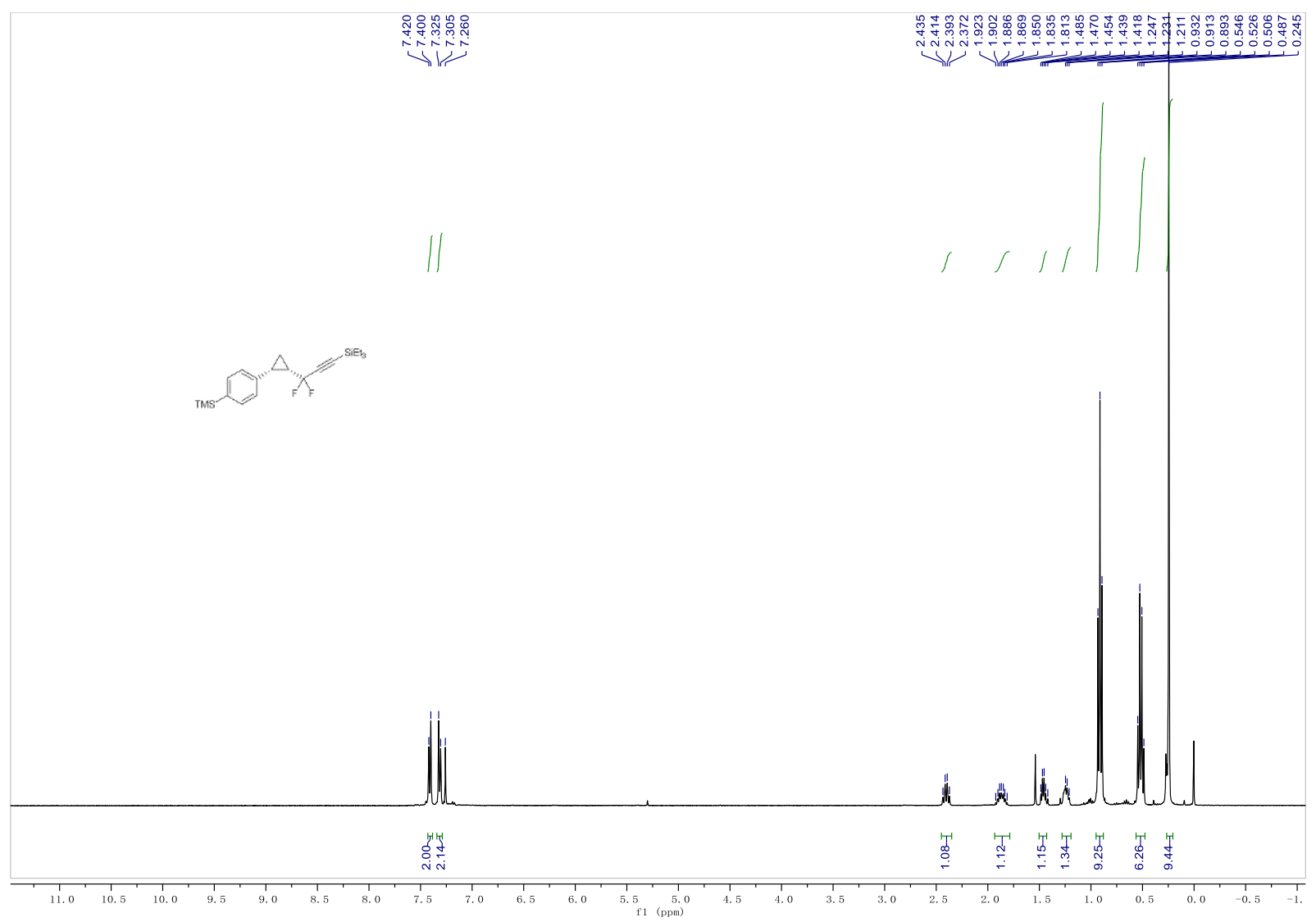



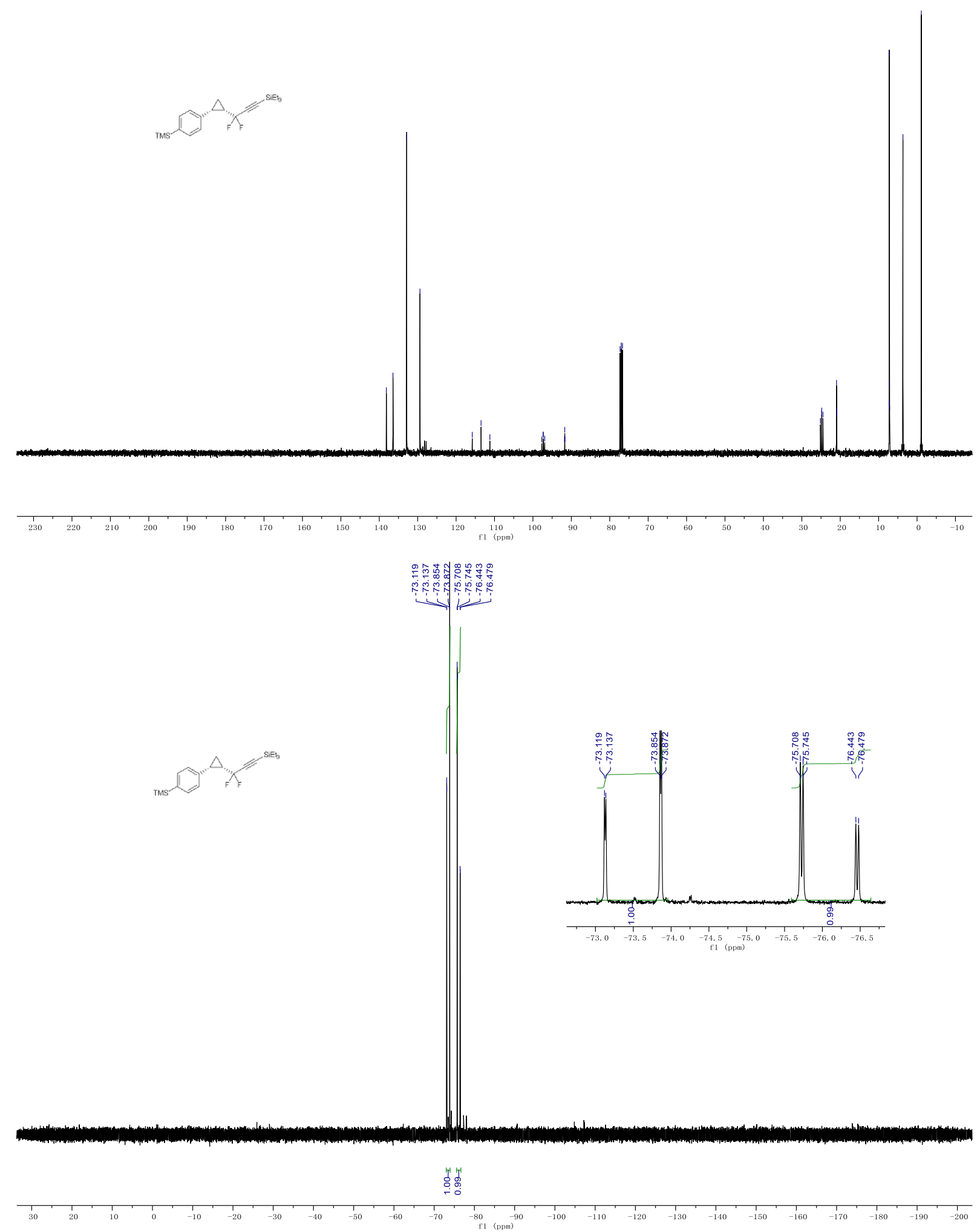


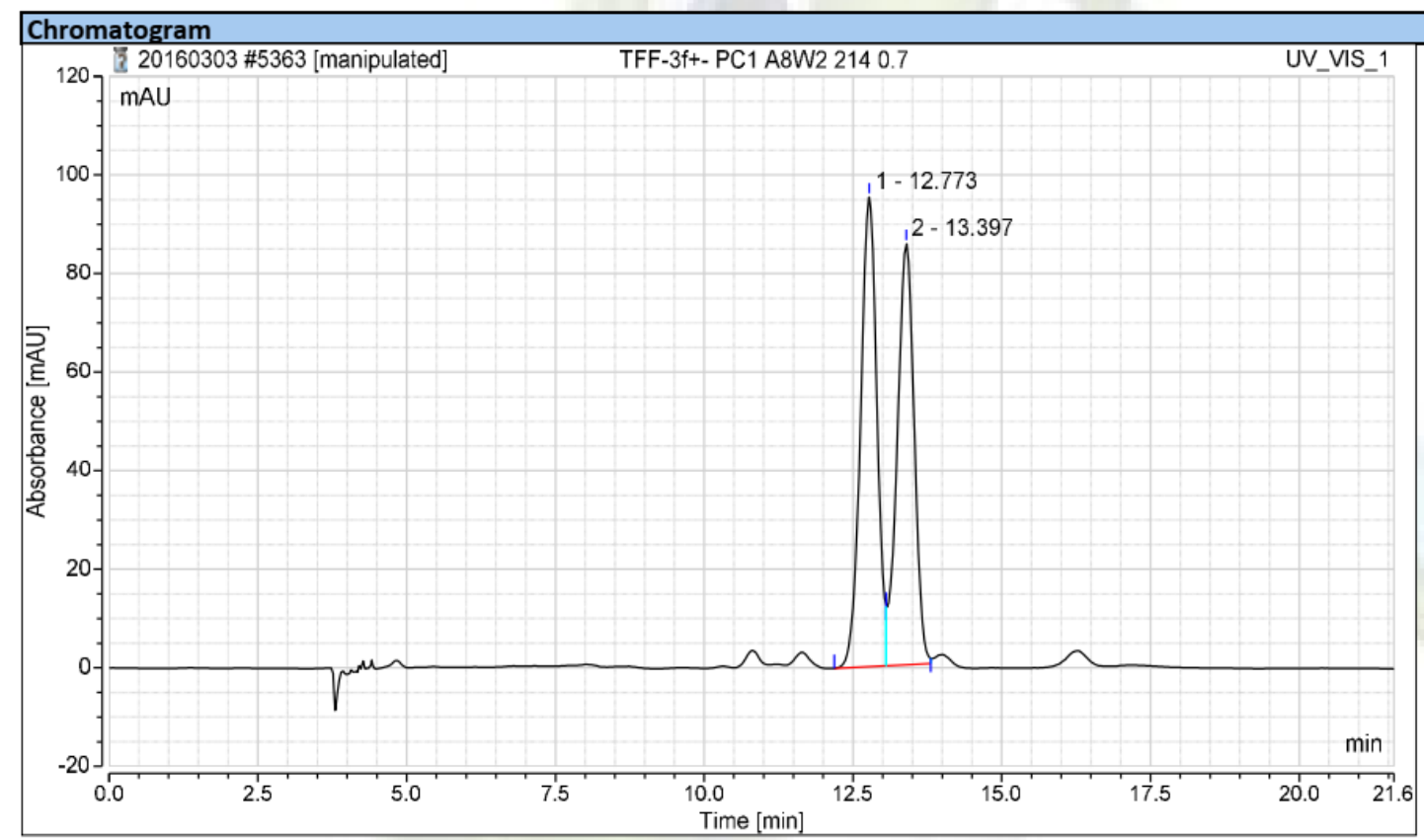

\begin{tabular}{|l|c|c|c|c|}
\hline \multicolumn{5}{|l|}{ Integration Results } \\
\hline No. & $\begin{array}{c}\text { Retention Time } \\
\mathrm{min}\end{array}$ & $\begin{array}{c}\text { Area } \\
\mathrm{mAU}^{*} \min \end{array}$ & $\begin{array}{c}\text { Height } \\
\mathrm{mAU}\end{array}$ & $\begin{array}{c}\text { Relative Area } \\
\%\end{array}$ \\
\hline 1 & 12.773 & 30.2340 & 95.3341 & 51.644 \\
2 & 13.397 & 28.3093 & 85.4777 & 48.356 \\
\hline Total: & $\mathbf{5 8 . 5 4 3}$ & $\mathbf{1 4 0 1 . 9 9 8}$ & $\mathbf{1 0 0 . 0 0 0}$ \\
\hline
\end{tabular}

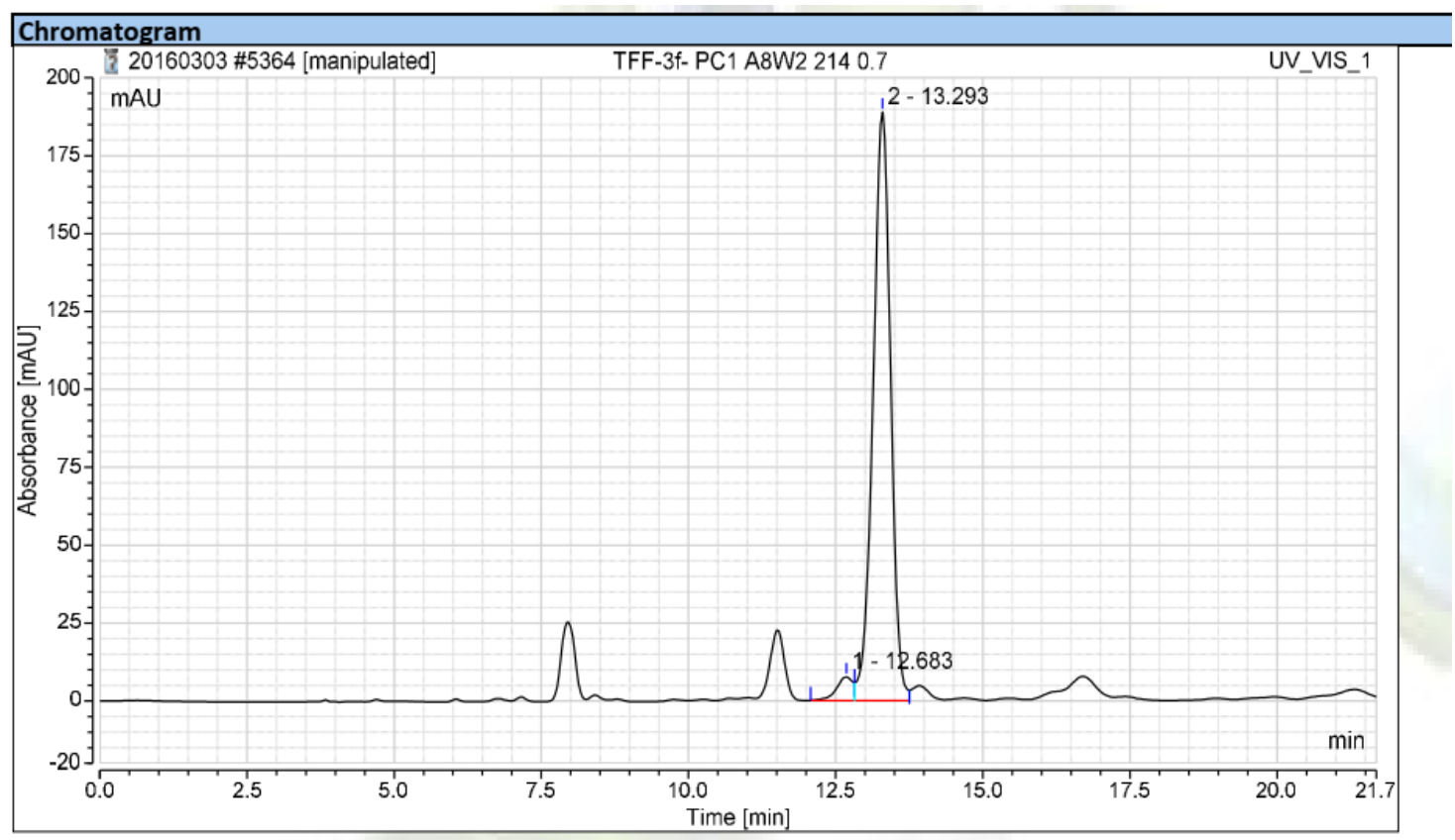

\begin{tabular}{|c|c|c|c|c|}
\hline \multicolumn{5}{|c|}{ Integration Results } \\
\hline No. & $\begin{array}{c}\text { Retention Time } \\
\text { min }\end{array}$ & $\begin{array}{c}\text { Area } \\
\mathrm{mAU* \operatorname {min }}\end{array}$ & $\begin{array}{c}\text { Height } \\
\text { mAU }\end{array}$ & $\begin{array}{c}\text { Relative Area } \\
\%\end{array}$ \\
\hline 1 & 12.683 & 2.4003 & 7.5619 & 3.552 \\
\hline 2 & 13.293 & 65.1683 & 188.8384 & 96.448 \\
\hline \multicolumn{2}{|c|}{ Total: } & 67.569 & 1401.998 & 100.000 \\
\hline
\end{tabular}


(3-((1S,2R)-2-(4-Chlorophenyl)cyclopropyl)-3,3-difluoroprop-1-yn-1-yl)triethylsilane (3g).

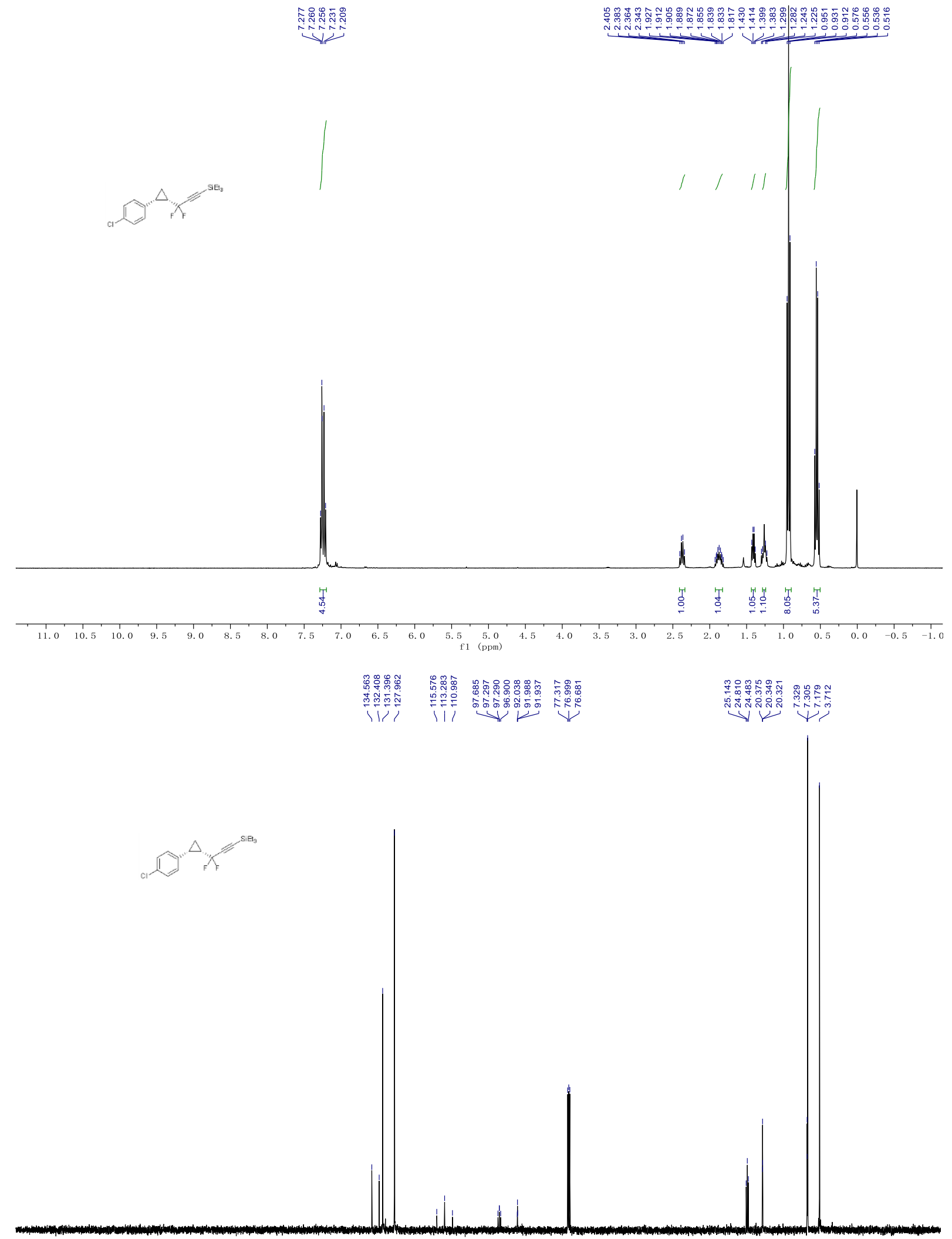

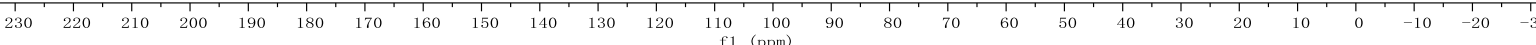




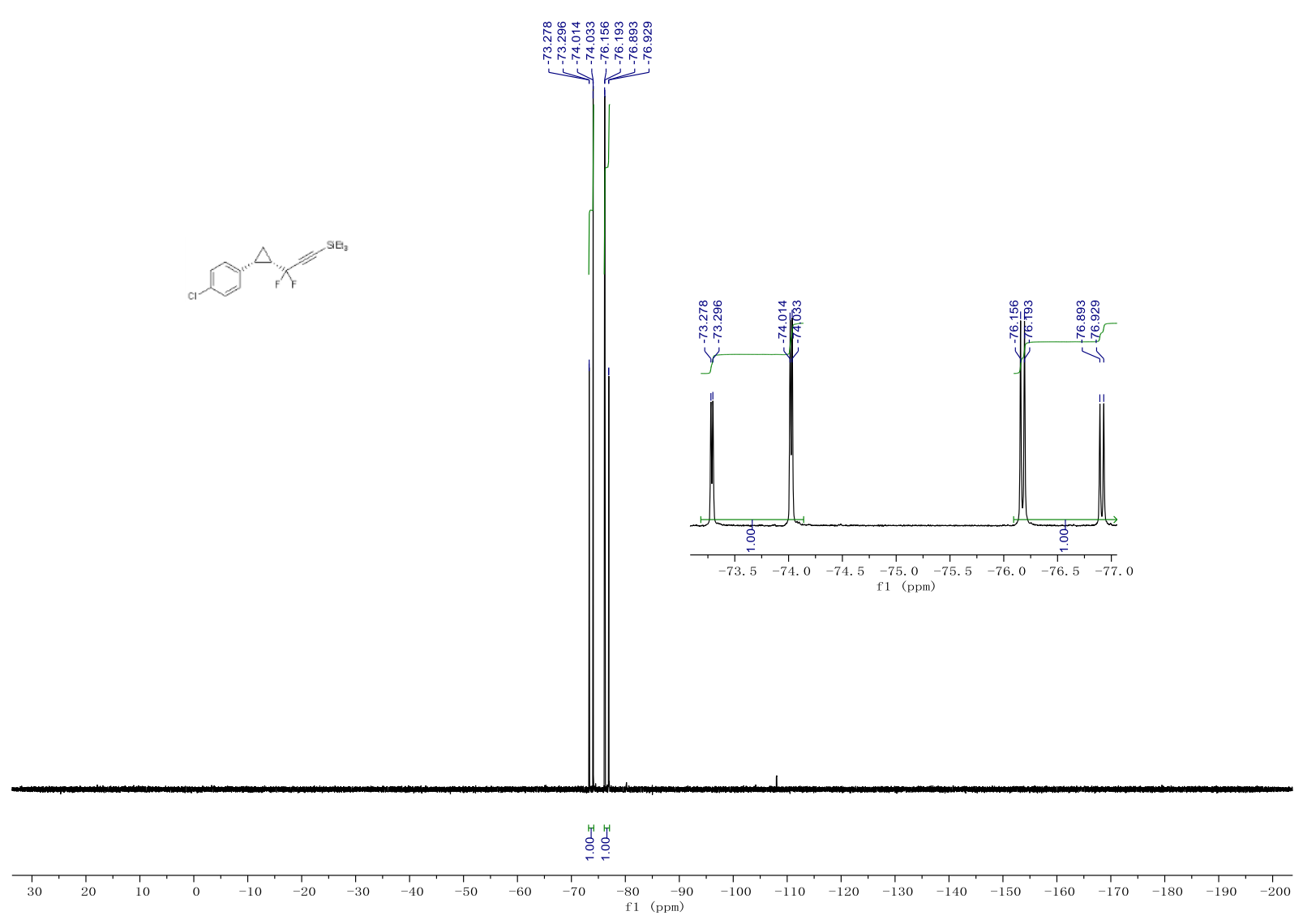

1-Benzyl-4-(((1S,2R)-2-(4-chlorophenyl)cyclopropyl)difluoromethyl)-1H-1,2,3-triazole (3g').

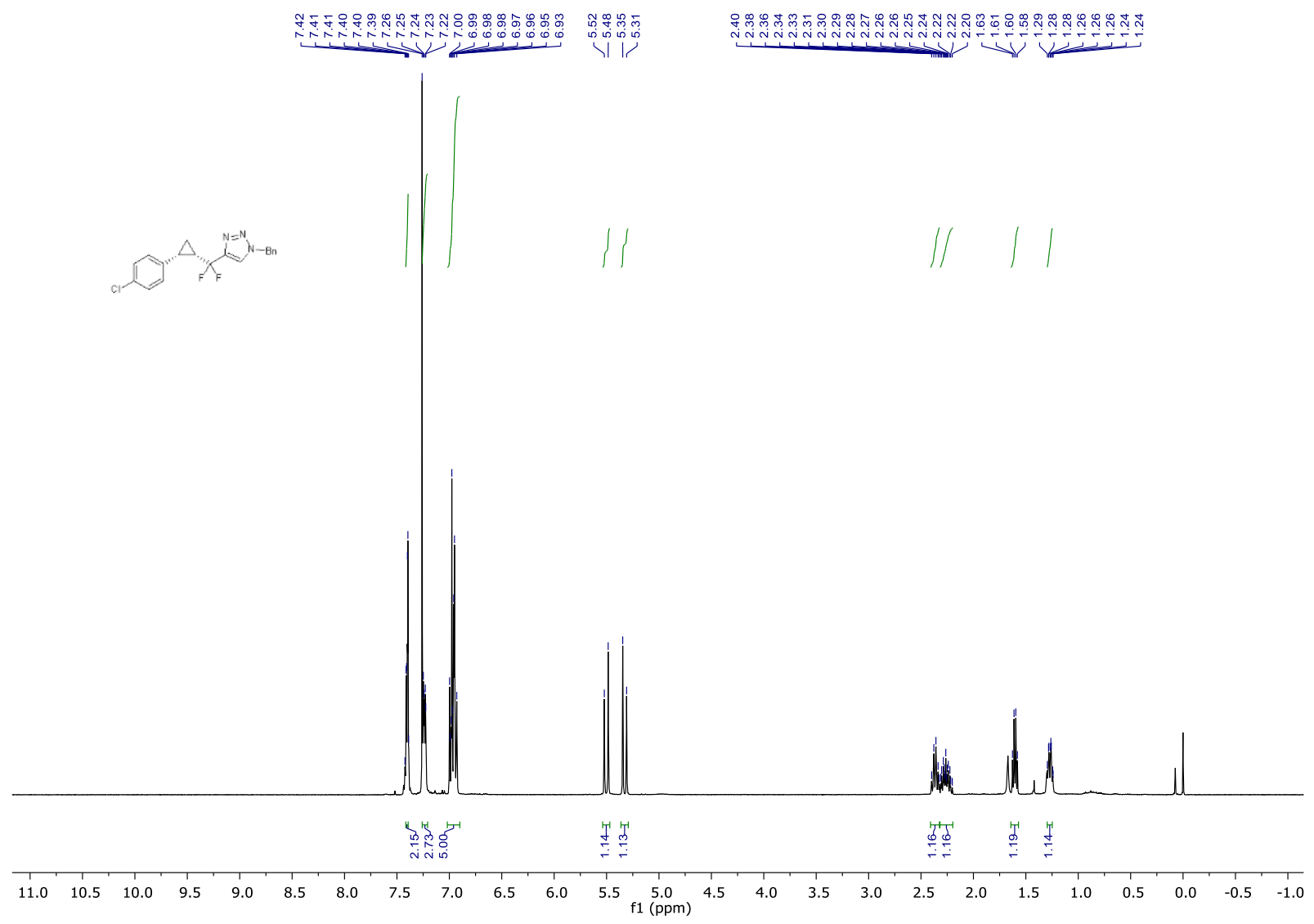




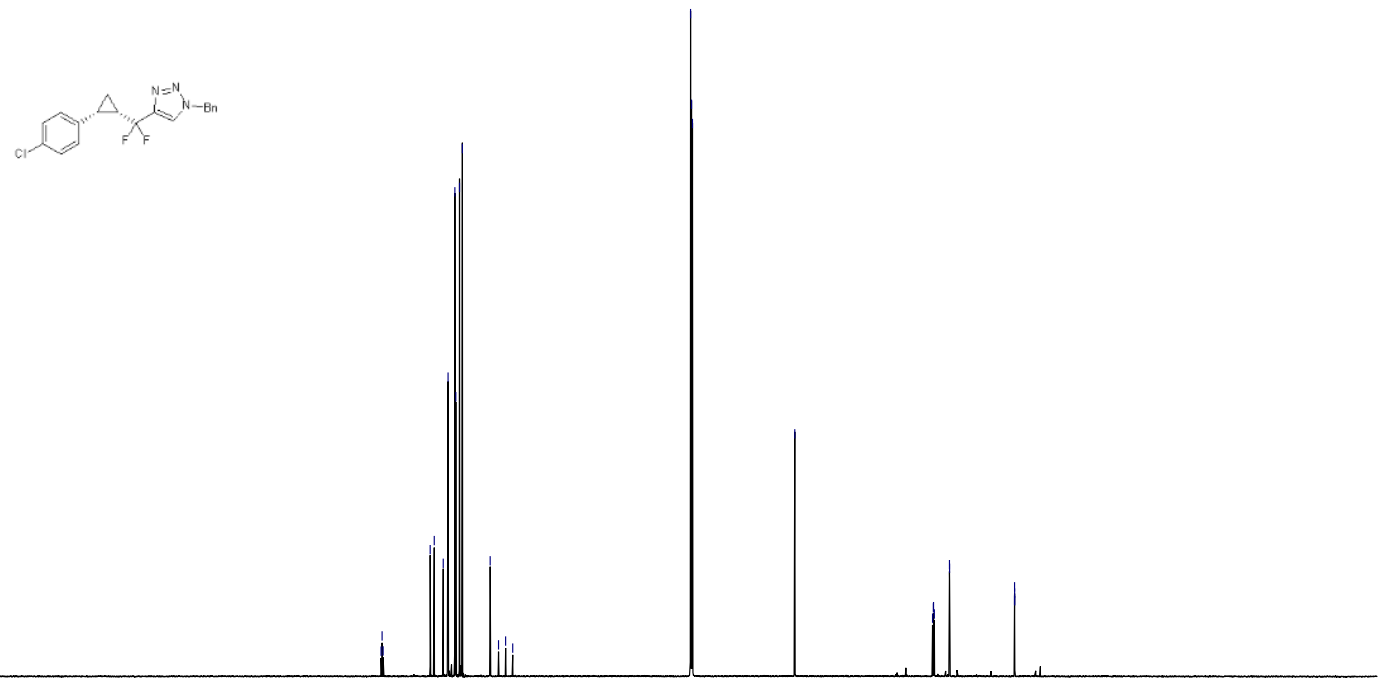

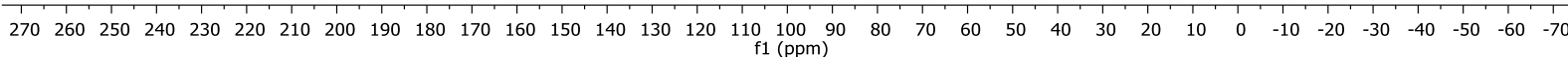

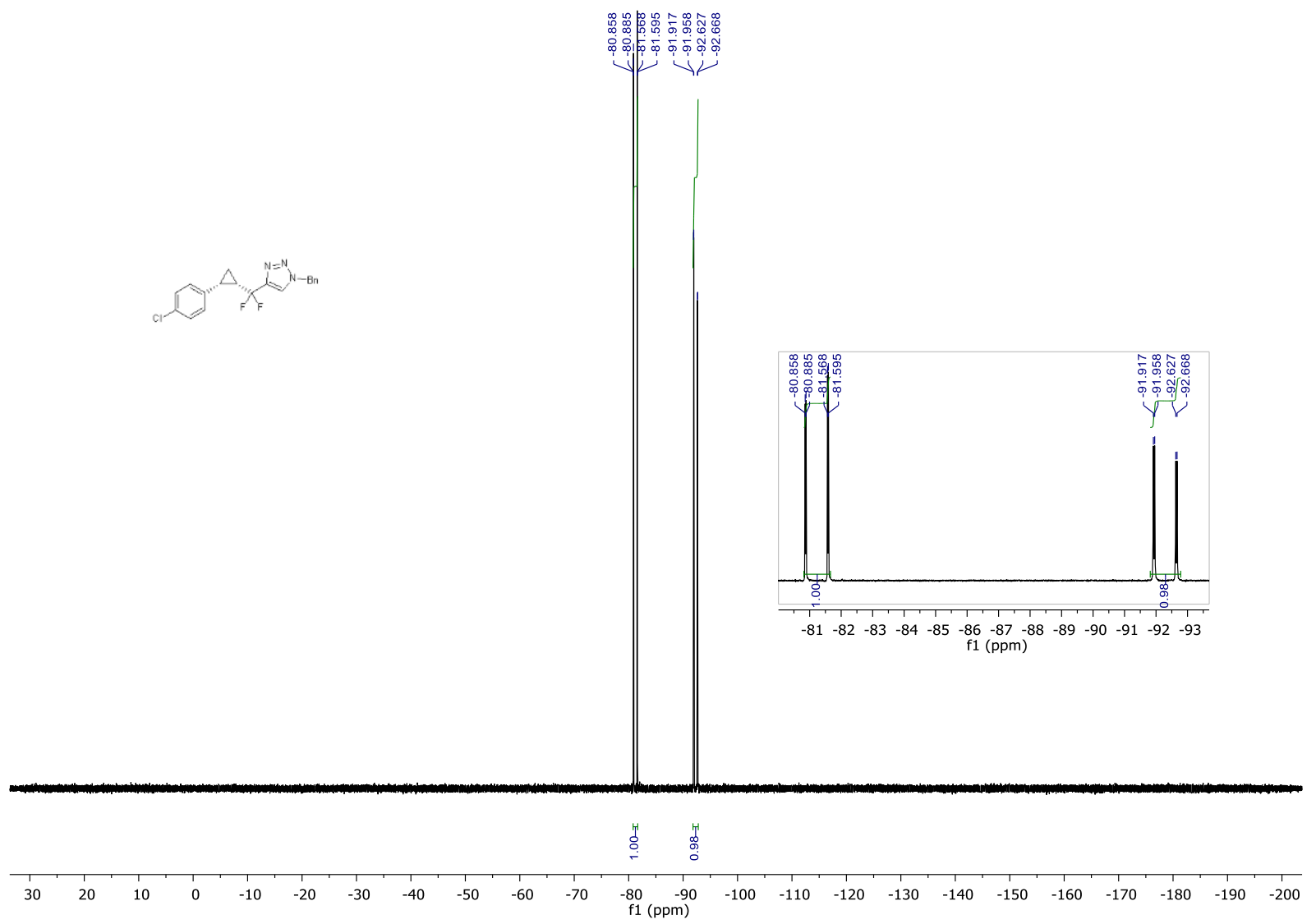




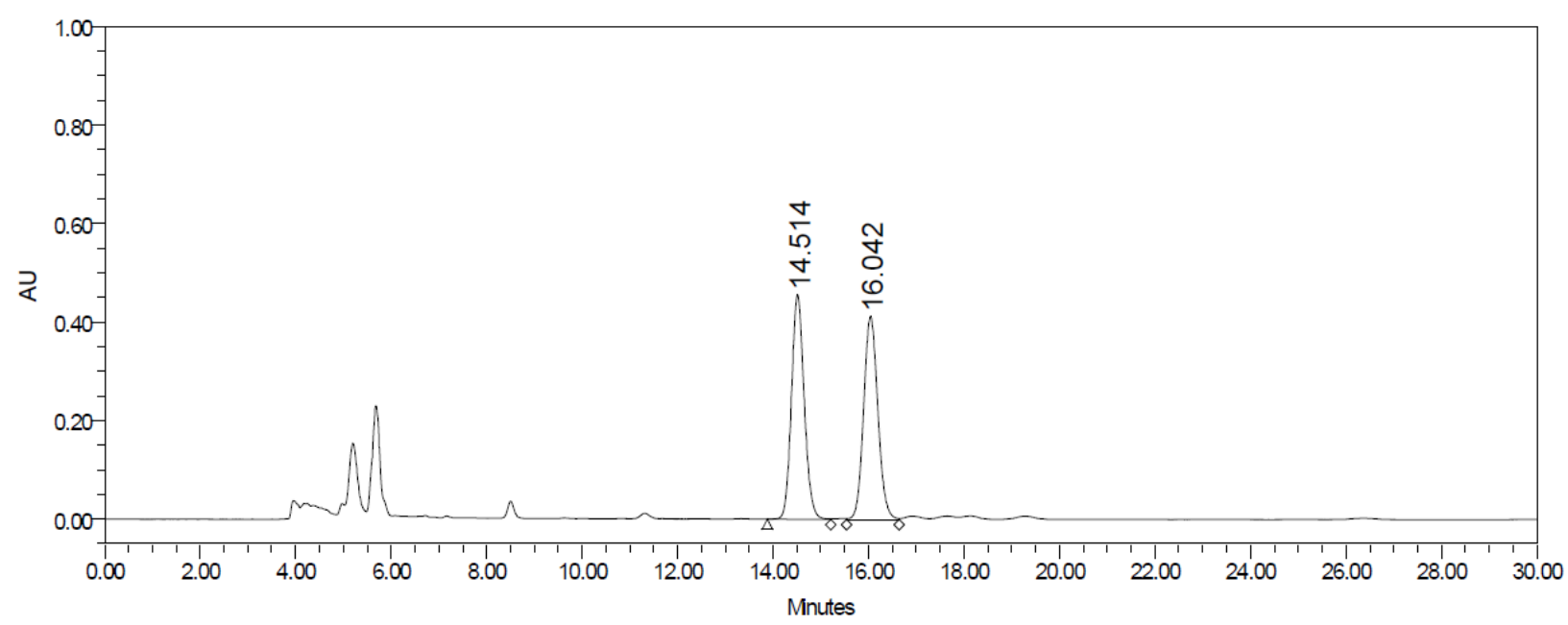

\begin{tabular}{|c|c|c|c|c|}
\hline & RT & Area & \% Area & Height \\
\hline 1 & 14.514 & 8472299 & 49.99 & 455747 \\
\hline 2 & 16.042 & 8476007 & 50.01 & 411768 \\
\hline
\end{tabular}

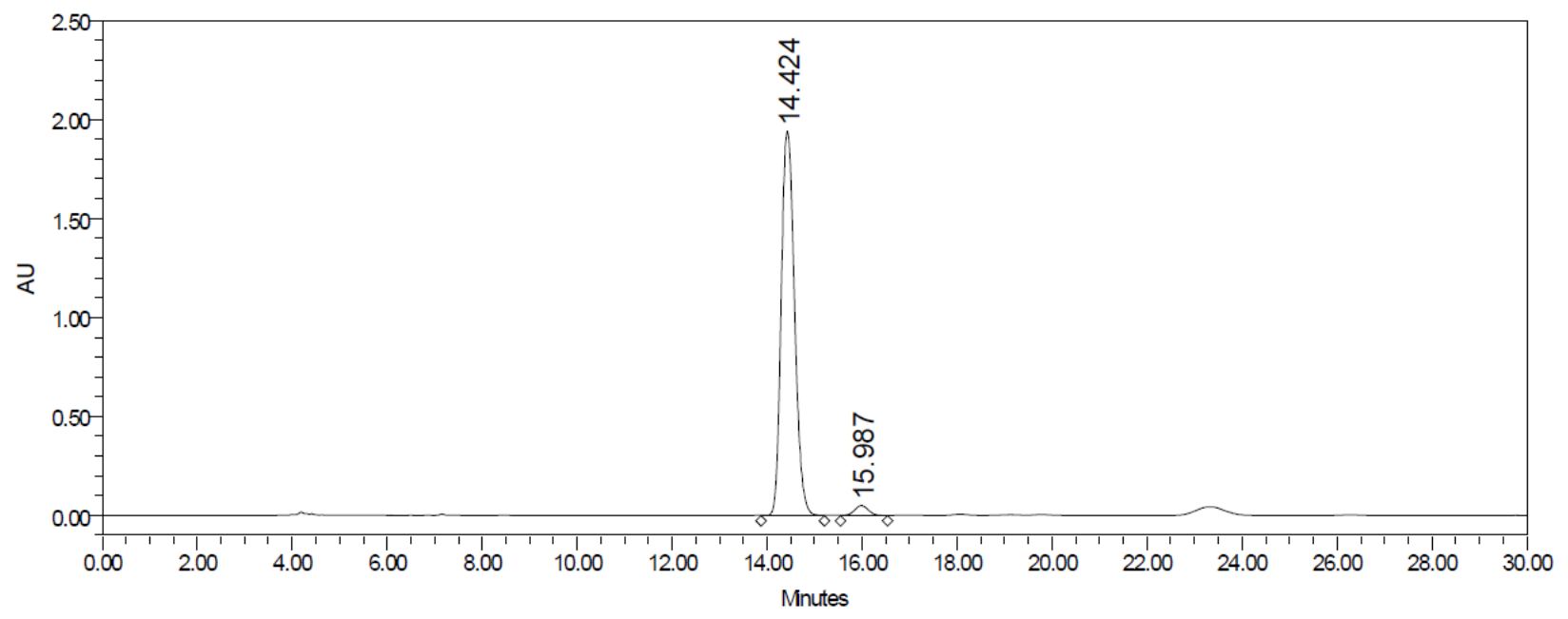

\begin{tabular}{|c|c|c|r|r|}
\hline & RT & Area & \% Area & Height \\
\hline 1 & 14.424 & 37791124 & 97.36 & 1941660 \\
\hline 2 & 15.987 & 1023917 & 264 & 50829 \\
\hline
\end{tabular}


(3-((1S,2R)-2-(3-(Benzyloxy)phenyl)cyclopropyl)-3,3-difluoroprop-1-yn-1-yl)triethylsilane (3h).

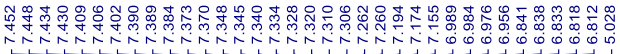

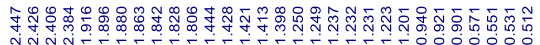

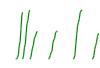

${ }_{F}^{B N O}{ }_{F}^{\text {SEE }}$
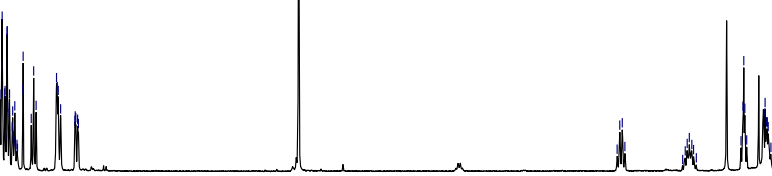

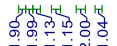

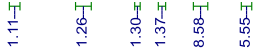

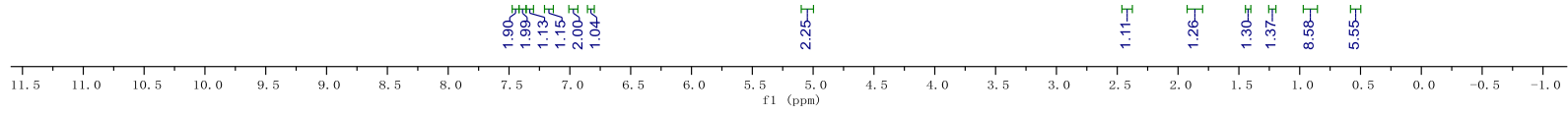

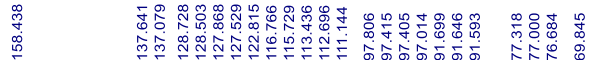

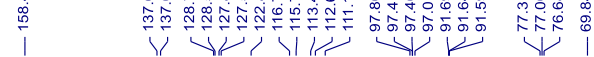
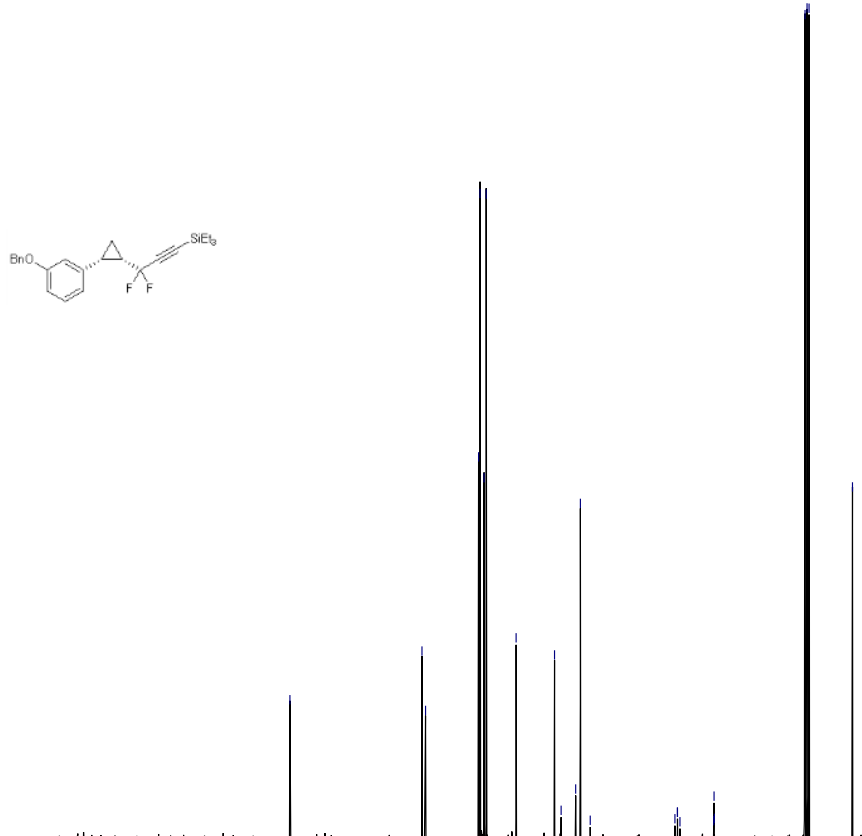

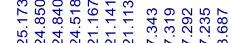

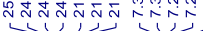
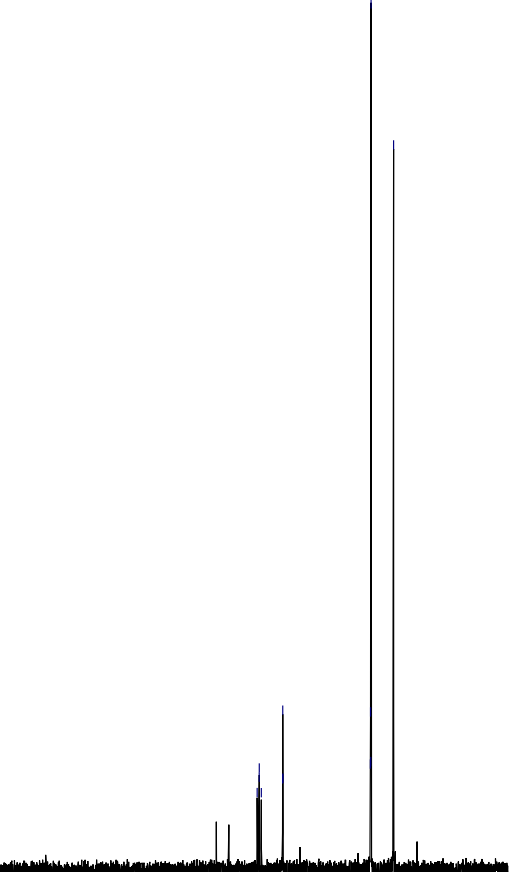

${ }_{230}^{1} \quad{ }_{220}^{1} \quad{ }_{210}^{1} \quad 200$ 

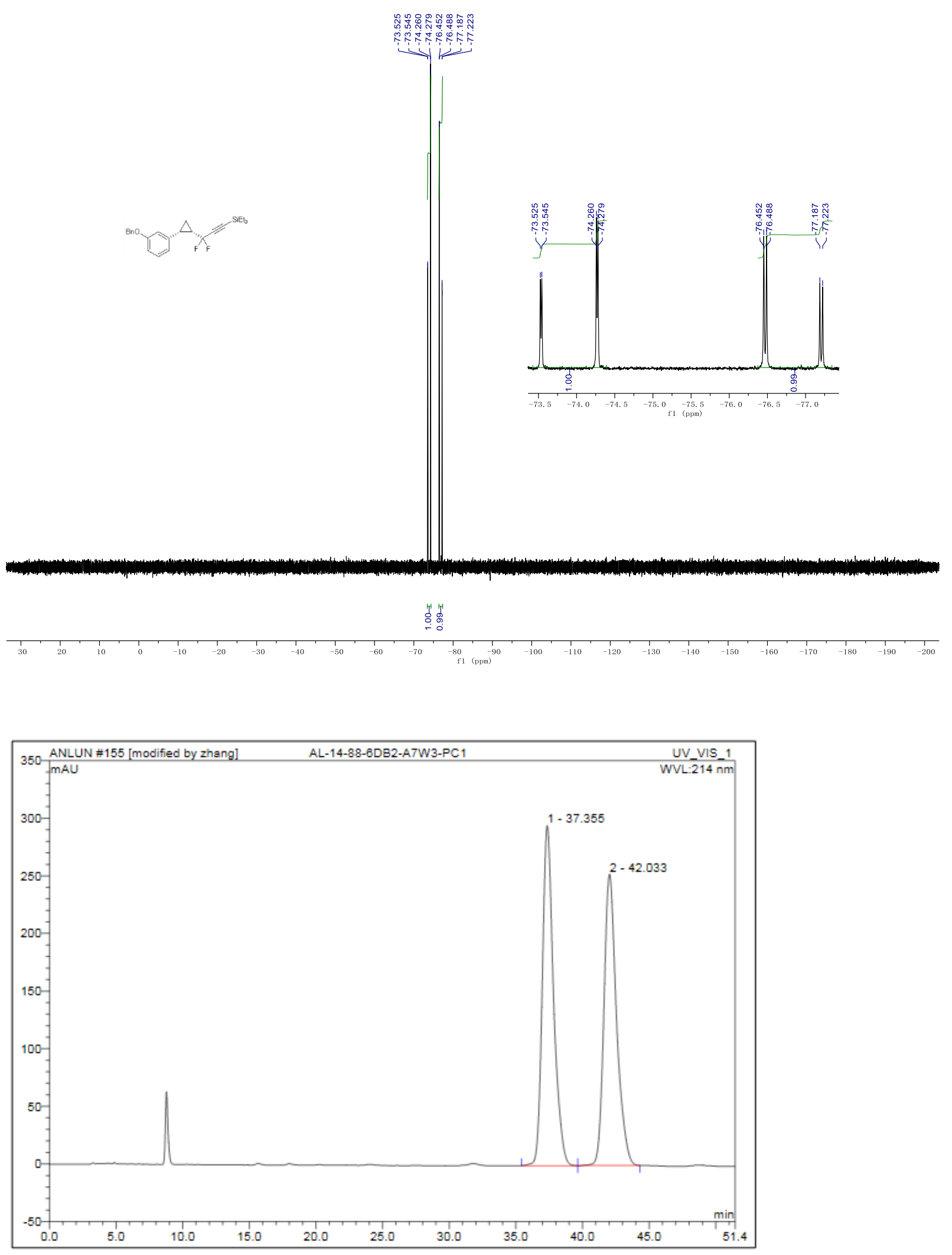

\begin{tabular}{|r|cccrccc|}
\hline No. & $\begin{array}{c}\text { Ret.Time } \\
\text { min }\end{array}$ & Peak Name & $\begin{array}{c}\text { Height } \\
\text { mAU }\end{array}$ & $\begin{array}{c}\text { Area } \\
\text { mAU*min }^{*}\end{array}$ & $\begin{array}{c}\text { Rel.Area } \\
\%\end{array}$ & Amount & Type \\
\hline 1 & 37.36 & n.a. & 294.896 & 287.437 & 50.96 & n.a. & BM \\
2 & 42.03 & n.a. & 252.814 & 276.609 & 49.04 & n.a. & MB \\
\hline Total: & & & 547.711 & 564.046 & 100.00 & 0.000 & \\
\hline
\end{tabular}




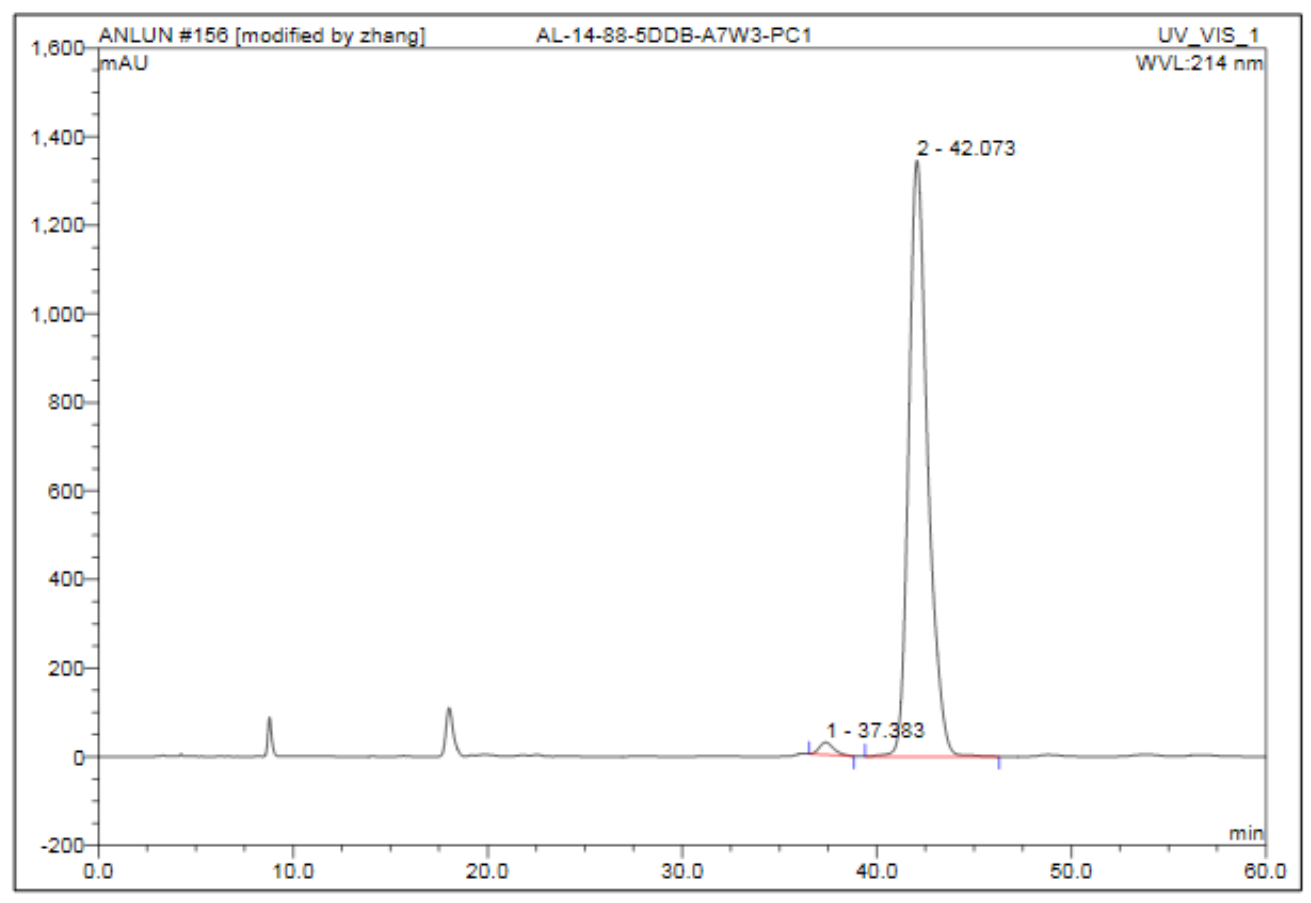

\begin{tabular}{|r|rrrrrrr|}
\hline No. & $\begin{array}{c}\text { Ret.Time } \\
\text { min }\end{array}$ & Peak Name & $\begin{array}{c}\text { Height } \\
\text { mAU }\end{array}$ & $\begin{array}{c}\text { Area } \\
\text { mAU*min }\end{array}$ & $\begin{array}{c}\text { Rel.Area } \\
\%\end{array}$ & Amount & Type \\
\hline 1 & 37.38 & n.a. & 27.881 & 24.390 & 1.57 & n.a. & BMB \\
2 & 42.07 & n.a. & 1346.294 & 1525.128 & 98.43 & n.a. & BMB \\
\hline Total: & & & 1374.175 & 1549.518 & 100.00 & 0.000 & \\
\hline
\end{tabular}

(3,3-Difluoro-3-((1S,2R)-2-(naphthalen-1-yl)cyclopropyl)prop-1-yn-1-yl)triethylsilane (3i).

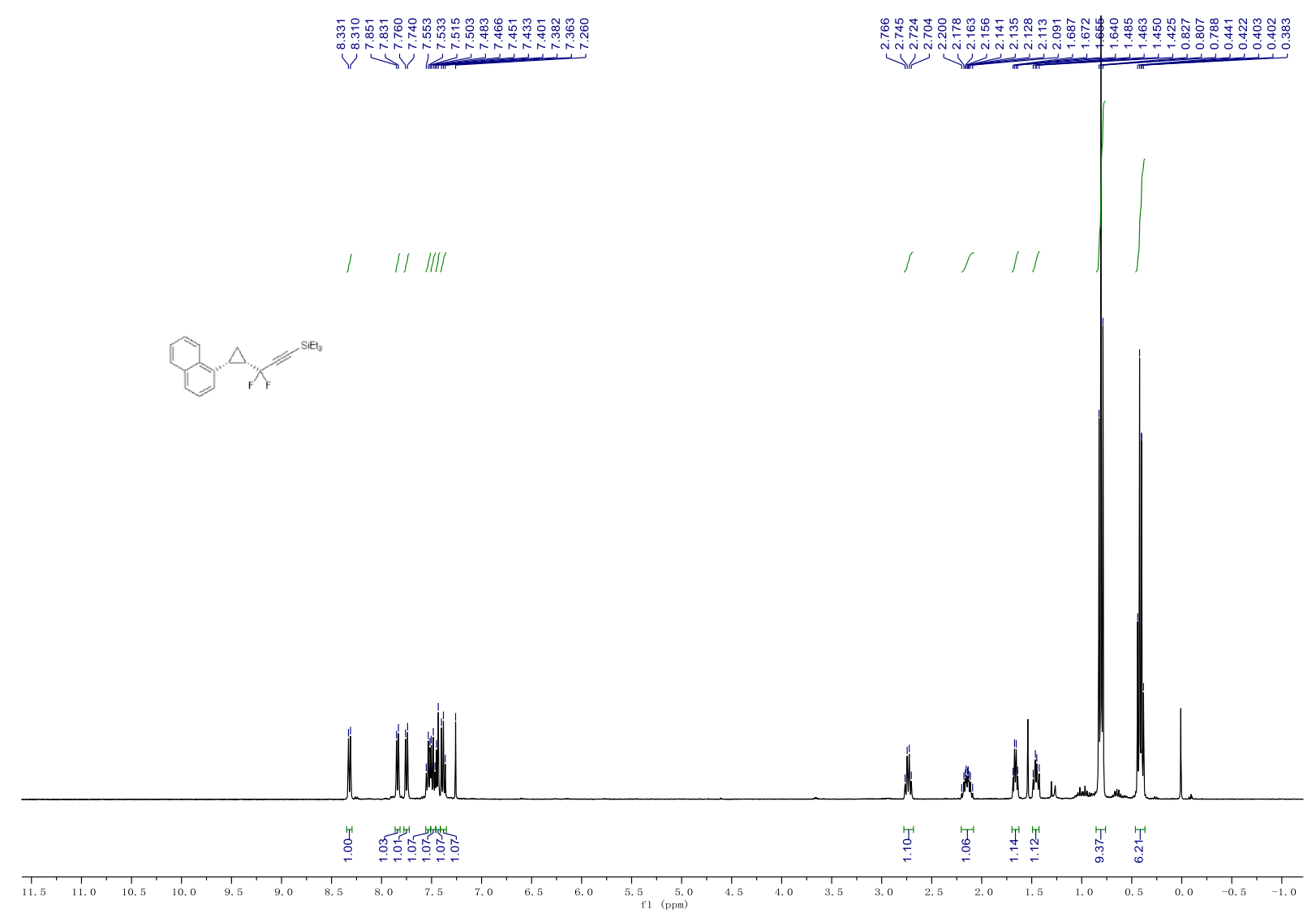



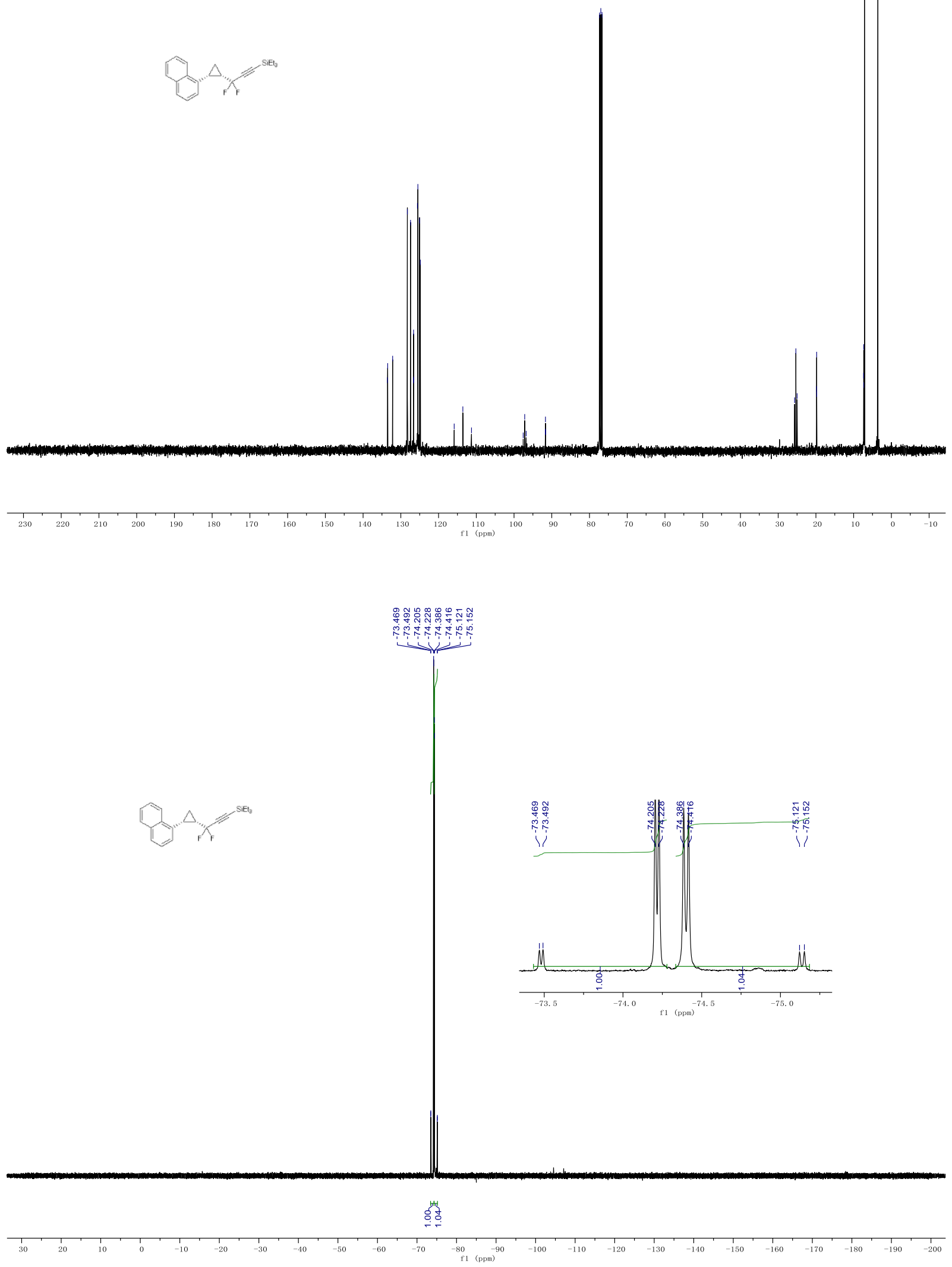


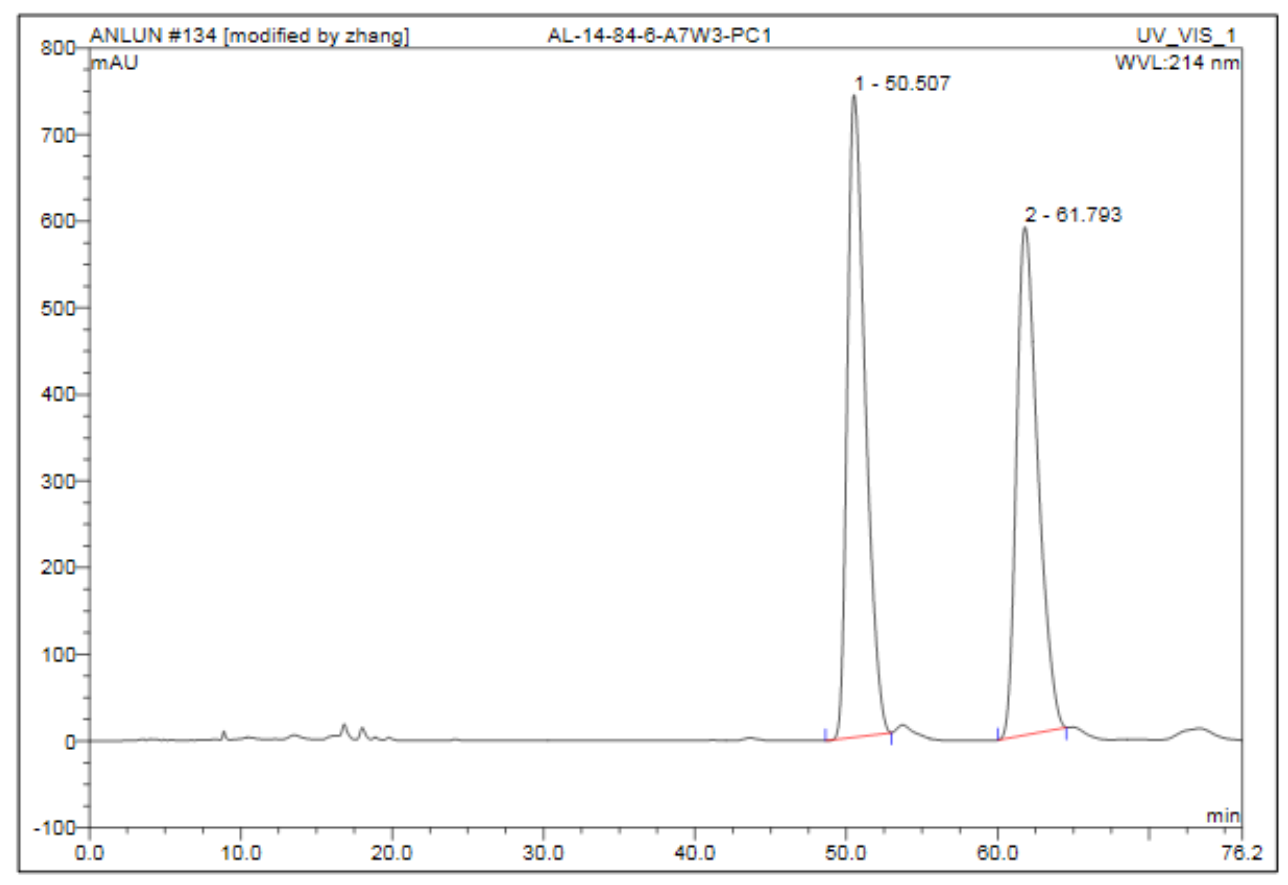

\begin{tabular}{|r|ccrrrrr|}
\hline No. & $\begin{array}{c}\text { Ret.Time } \\
\text { min }\end{array}$ & Peak Name & $\begin{array}{c}\text { Height } \\
\text { mAU }\end{array}$ & $\begin{array}{r}\text { Area } \\
\text { mAU*min }\end{array}$ & $\begin{array}{r}\text { Rel.Area } \\
\%\end{array}$ & Amount & Type \\
\hline 1 & 50.51 & n.a. & 741.606 & 1020.112 & 51.51 & n.a. & BMB $^{\star}$ \\
2 & 61.79 & n.a. & 586.498 & 960.353 & 48.49 & n.a. & BMB $^{*}$ \\
\hline Total: & & & 1328.104 & 1980.466 & 100.00 & 0.000 & \\
\hline
\end{tabular}

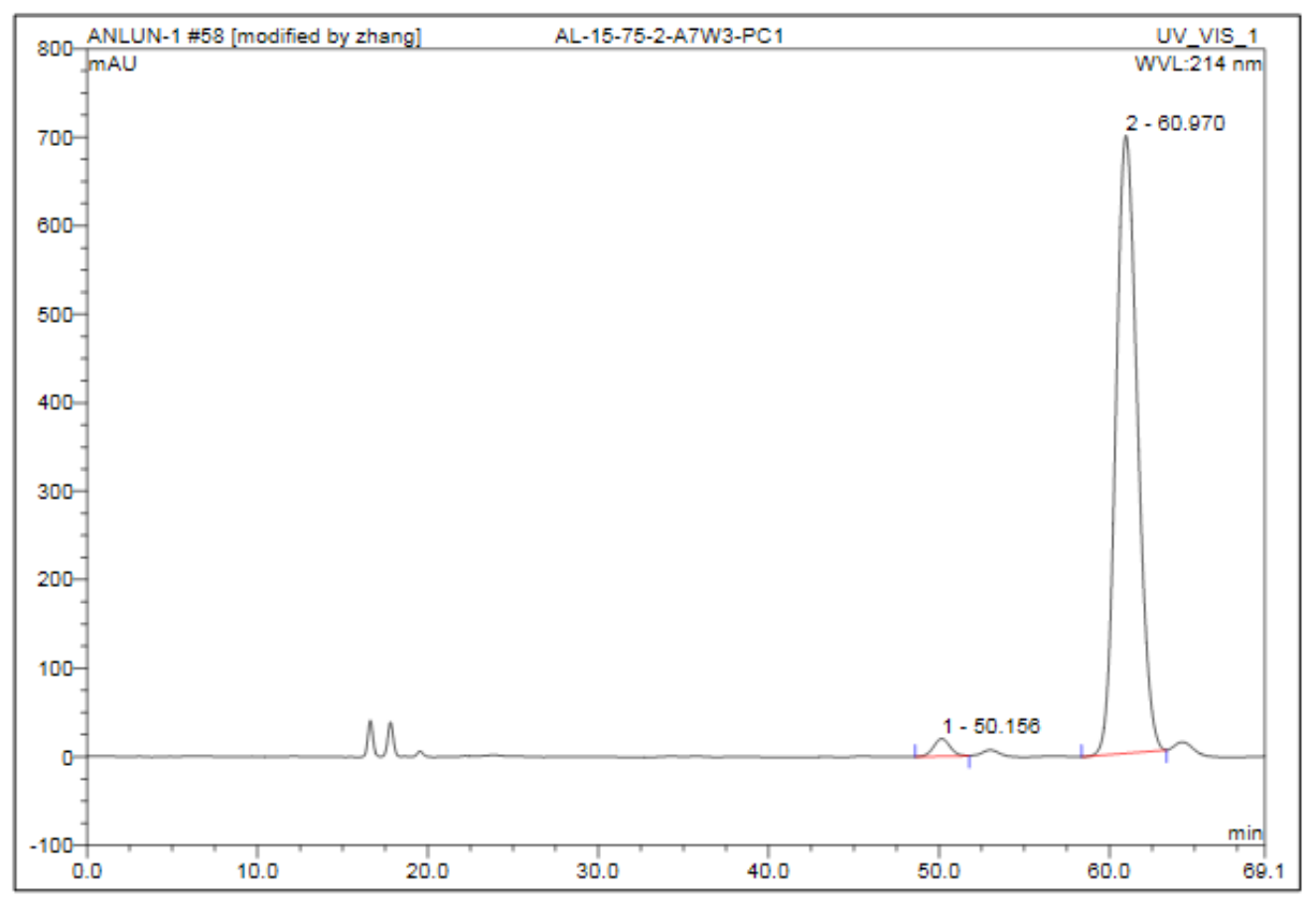

\begin{tabular}{|c|c|c|c|c|c|c|c|}
\hline No. & $\begin{array}{c}\text { Ret.Time } \\
\text { min }\end{array}$ & Peak Name & $\begin{array}{c}\text { Height } \\
\text { mAU }\end{array}$ & $\begin{array}{c}\text { Area } \\
m A U^{\star} \min \end{array}$ & $\begin{array}{c}\text { Rel.Area } \\
\%\end{array}$ & Amount & Type \\
\hline 1 & 50.16 & n.a. & 19.901 & 22.172 & 2.12 & n.a. & BMB \\
\hline 2 & 60.97 & n.a. & 698.330 & 1023.138 & 97.88 & n.a. & $\mathrm{BMB}^{\star}$ \\
\hline Total: & & & 718.230 & 1045.310 & 100.00 & 0.000 & \\
\hline
\end{tabular}


(3,3-Difluoro-3-((1S,2R)-2-phenylcyclopropyl)prop-1-yn-1-yl)trimethylsilane (3j).
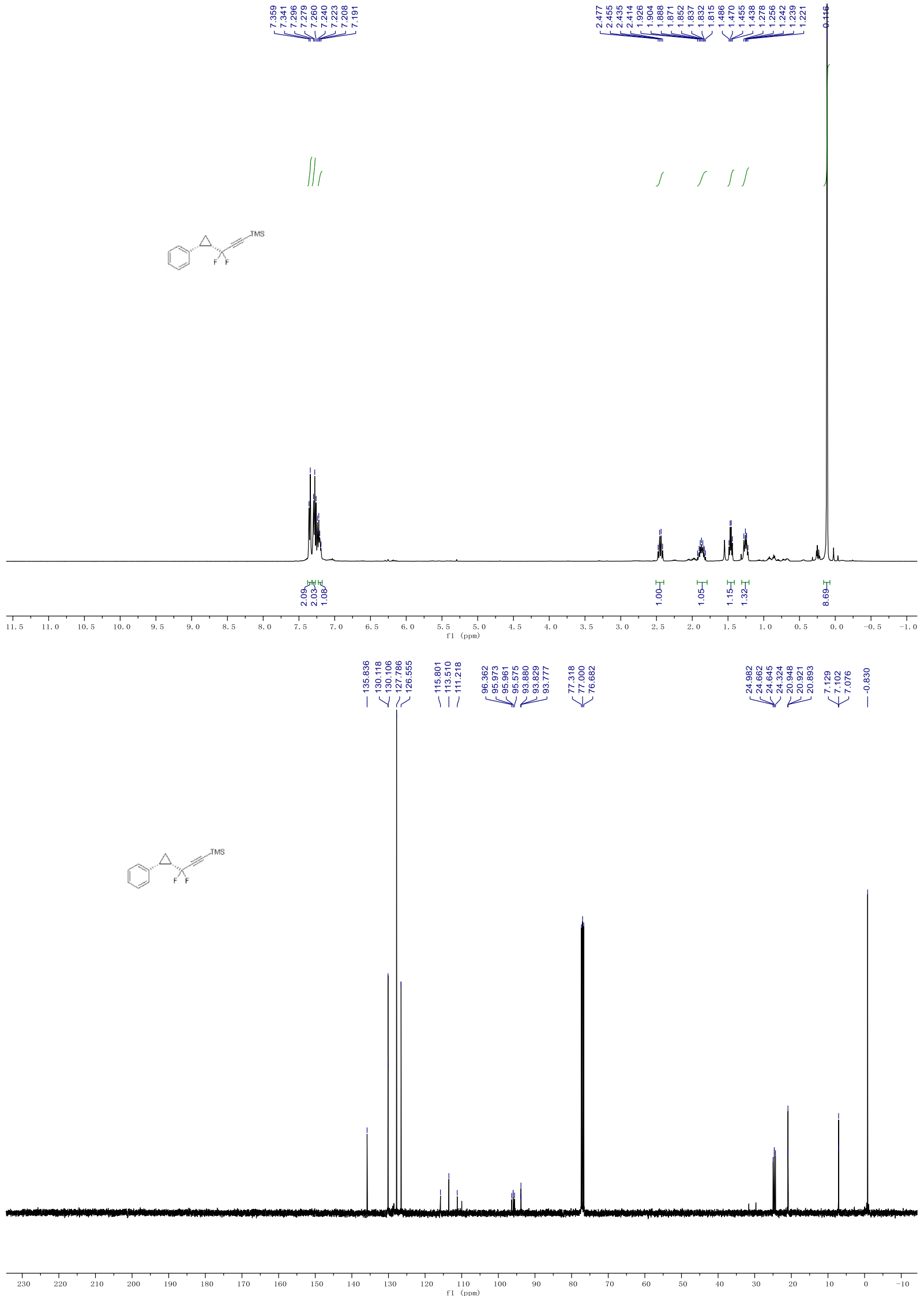

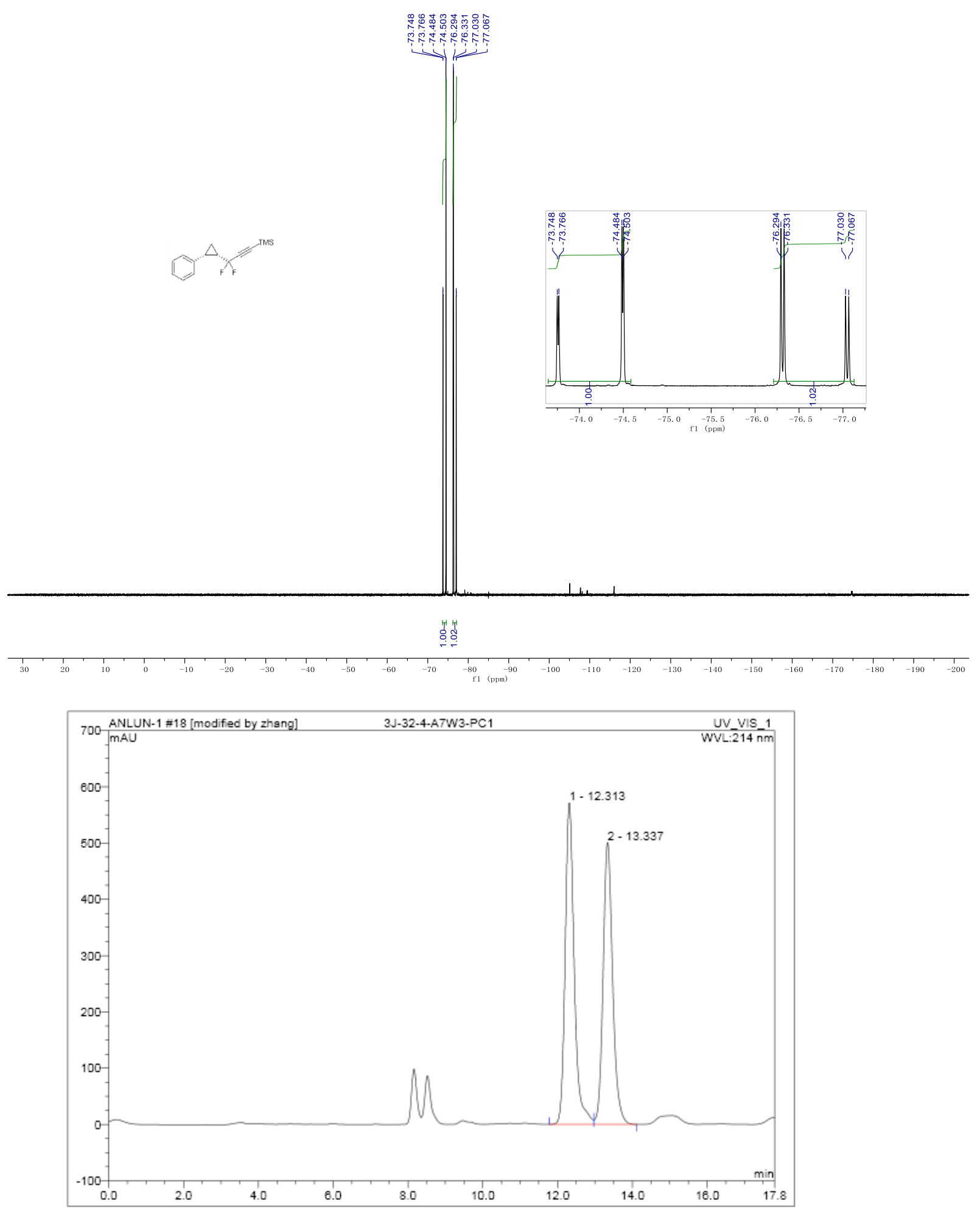

\begin{tabular}{|r|ccccccc|}
\hline No. & $\begin{array}{c}\text { Ret.Time } \\
\text { min }\end{array}$ & Peak Name & $\begin{array}{c}\text { Height } \\
\text { mAU }\end{array}$ & $\begin{array}{c}\text { Area } \\
\text { mAU*min }\end{array}$ & $\begin{array}{c}\text { Rel.Area } \\
\%\end{array}$ & Amount & Type \\
\hline 1 & 12.31 & n.a. & 571.252 & 159.895 & 51.88 & n.a. & BM \\
2 & 13.34 & n.a. & 500.634 & 148.318 & 48.12 & n.a. & MB \\
\hline Total: & & & 1071.886 & 308.213 & 100.00 & 0.000 & \\
\hline
\end{tabular}




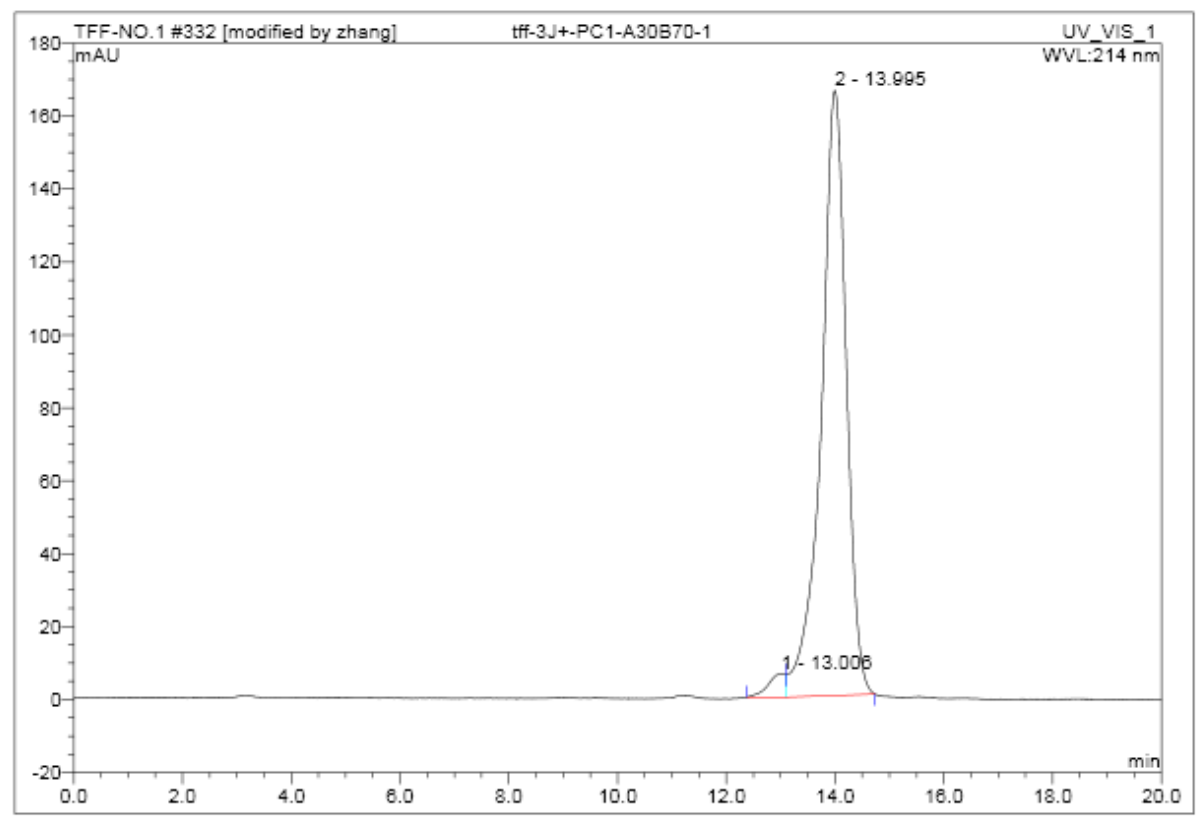

\begin{tabular}{|c|c|c|c|c|c|c|c|}
\hline No. & $\begin{array}{c}\text { Ret.Time } \\
\text { min }\end{array}$ & Peak Name & $\begin{array}{c}\text { Height } \\
\text { mAU }\end{array}$ & $\begin{array}{c}\text { Area } \\
\mathrm{mAU}^{*} \min \end{array}$ & $\begin{array}{c}\text { Rel.Area } \\
\% \\
\end{array}$ & $\begin{array}{c}\text { Amount } \\
\%\end{array}$ & Type \\
\hline 1 & 13.01 & n.a. & 6.307 & 2.285 & 2.51 & n.a. & $\mathrm{BM}^{*}$ \\
\hline 2 & 14.00 & n.a. & 165.917 & 88.728 & 97.49 & n.a. & $\mathrm{MB}^{\star}$ \\
\hline Total: & & & 172.225 & 91.012 & 100.00 & 0.000 & \\
\hline
\end{tabular}

(3,3-Difluoro-3-((1S,2R)-2-phenylcyclopropyl)prop-1-yn-1-yl)triisopropylsilane (3k).

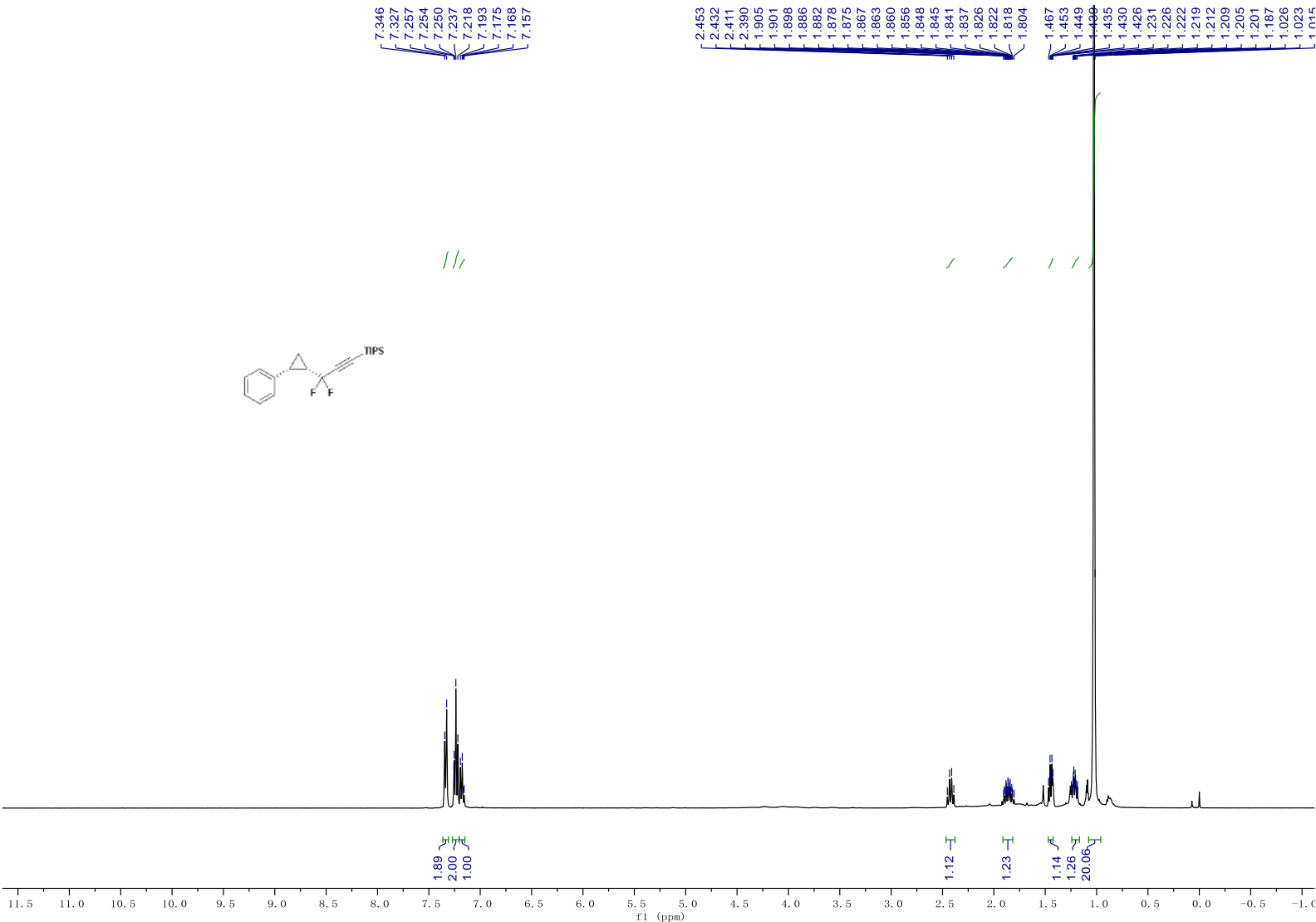

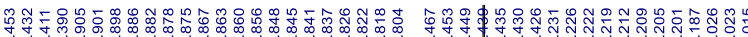


$\Delta F_{F}{ }^{n P S}$
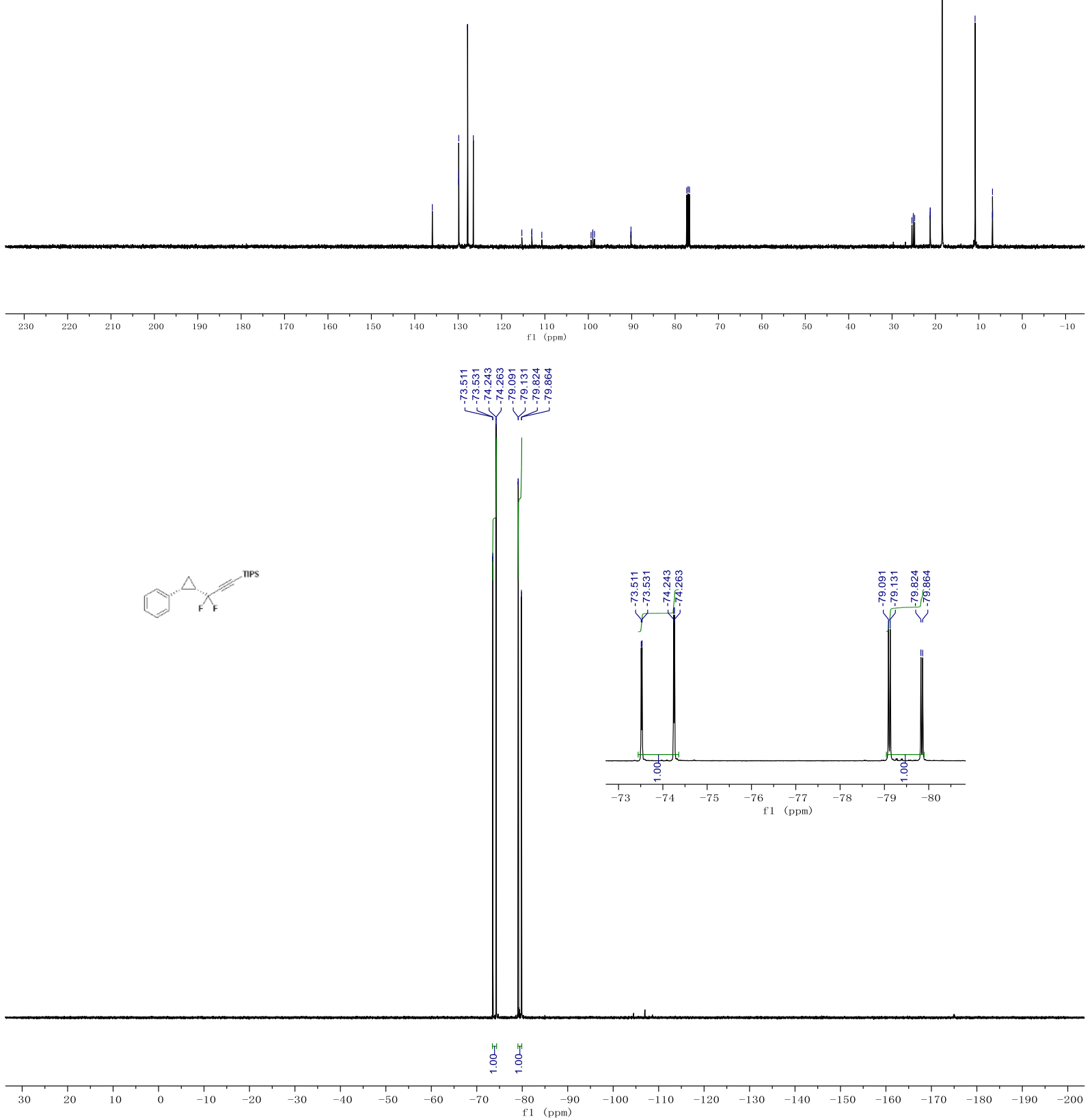


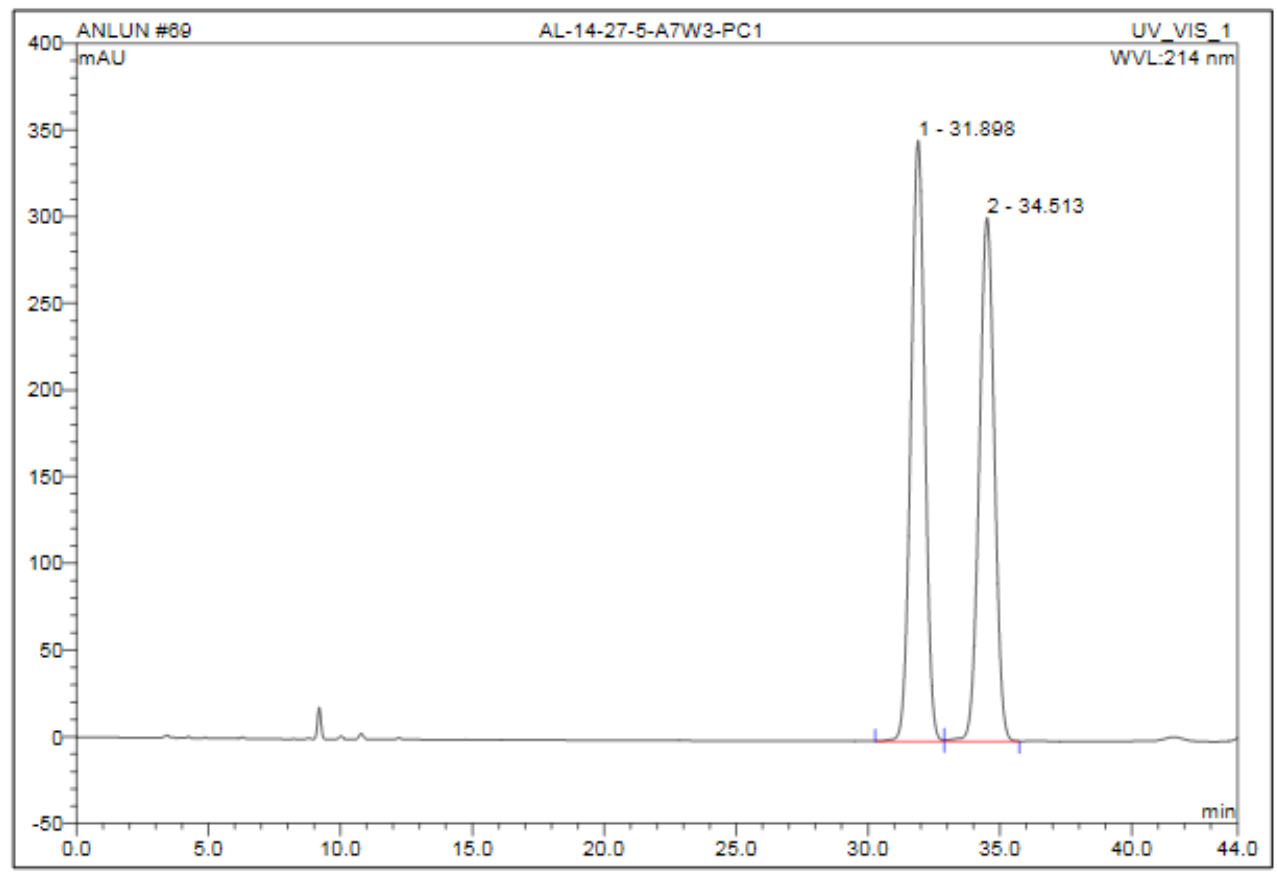

\begin{tabular}{|r|ccccccc|}
\hline No. & $\begin{array}{c}\text { Ret.Time } \\
\text { min }\end{array}$ & Peak Name & $\begin{array}{c}\text { Height } \\
\text { mAU }\end{array}$ & $\begin{array}{c}\text { Area } \\
\text { mAU*min }\end{array}$ & $\begin{array}{c}\text { Rel.Area } \\
\%\end{array}$ & Amount & Type \\
\hline 1 & 31.90 & n.a. & 346.559 & 211.895 & 51.27 & n.a. & BM \\
2 & 34.51 & n.a. & 301.979 & 201.393 & 48.73 & n.a. & MB \\
\hline Total: & & & 648.538 & 413.288 & 100.00 & 0.000 & \\
\hline
\end{tabular}

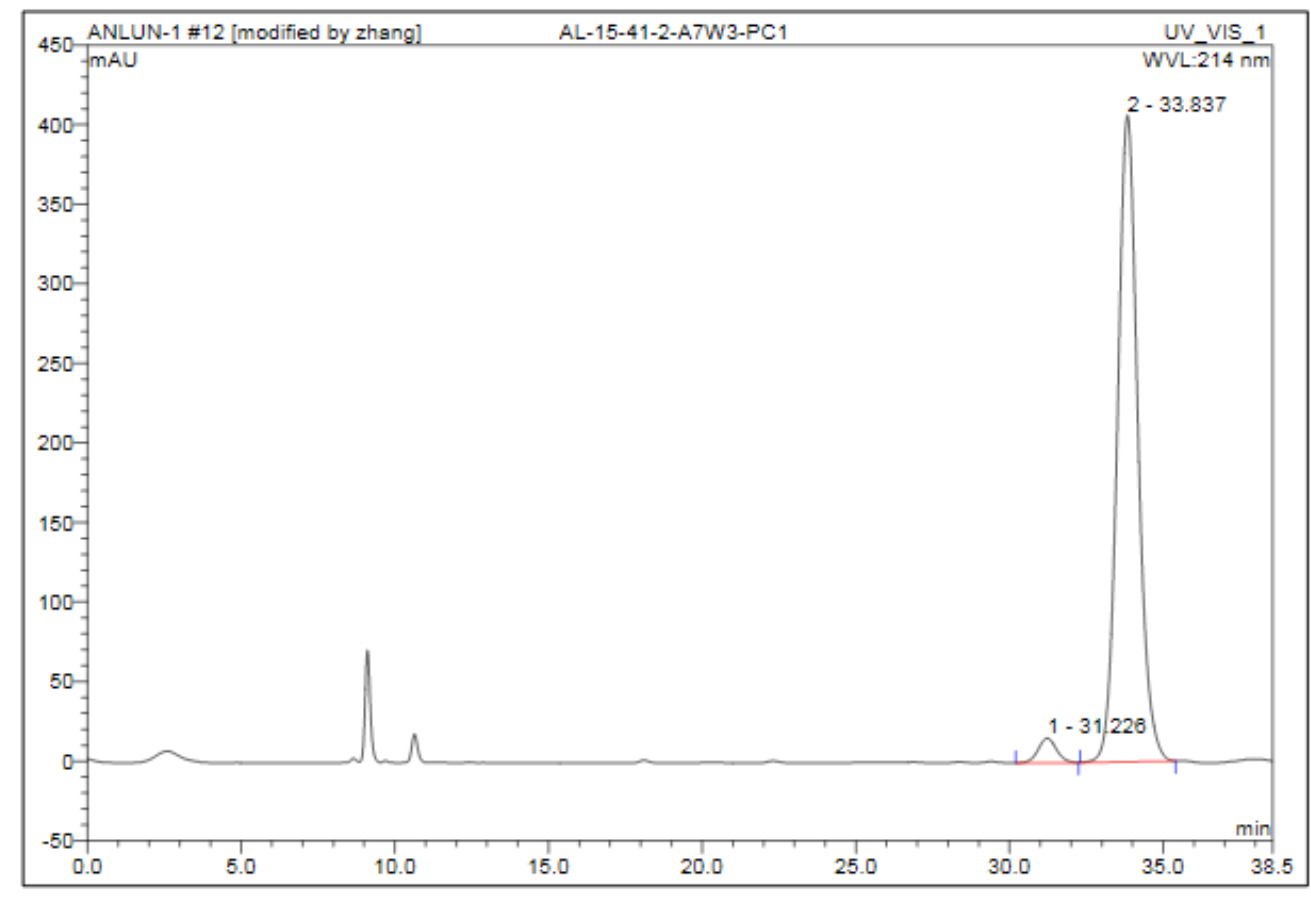

\begin{tabular}{|r|ccrrrrr|}
\hline No. & $\begin{array}{c}\text { Ret.Time } \\
\text { min }\end{array}$ & Peak Name & $\begin{array}{c}\text { Height } \\
\text { mAU }\end{array}$ & $\begin{array}{c}\text { Area } \\
\text { mAU*min }\end{array}$ & $\begin{array}{r}\text { Rel.Area } \\
\%\end{array}$ & Amount & Type \\
\hline 1 & 31.23 & n.a. & 15.423 & 10.687 & 3.31 & n.a. & BMB \\
2 & 33.84 & n.a. & 406.127 & 312.410 & 96.69 & n.a. & BMB \\
\hline Total: & & & 421.550 & 323.096 & 100.00 & 0.000 & \\
\hline
\end{tabular}


tert-Butyl-((6,6-difluoro-6-((1S,2R)-2-phenylcyclopropyl)hex-4-yn-1-yl)oxy)dimethylsilane (31).

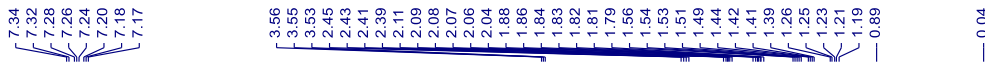
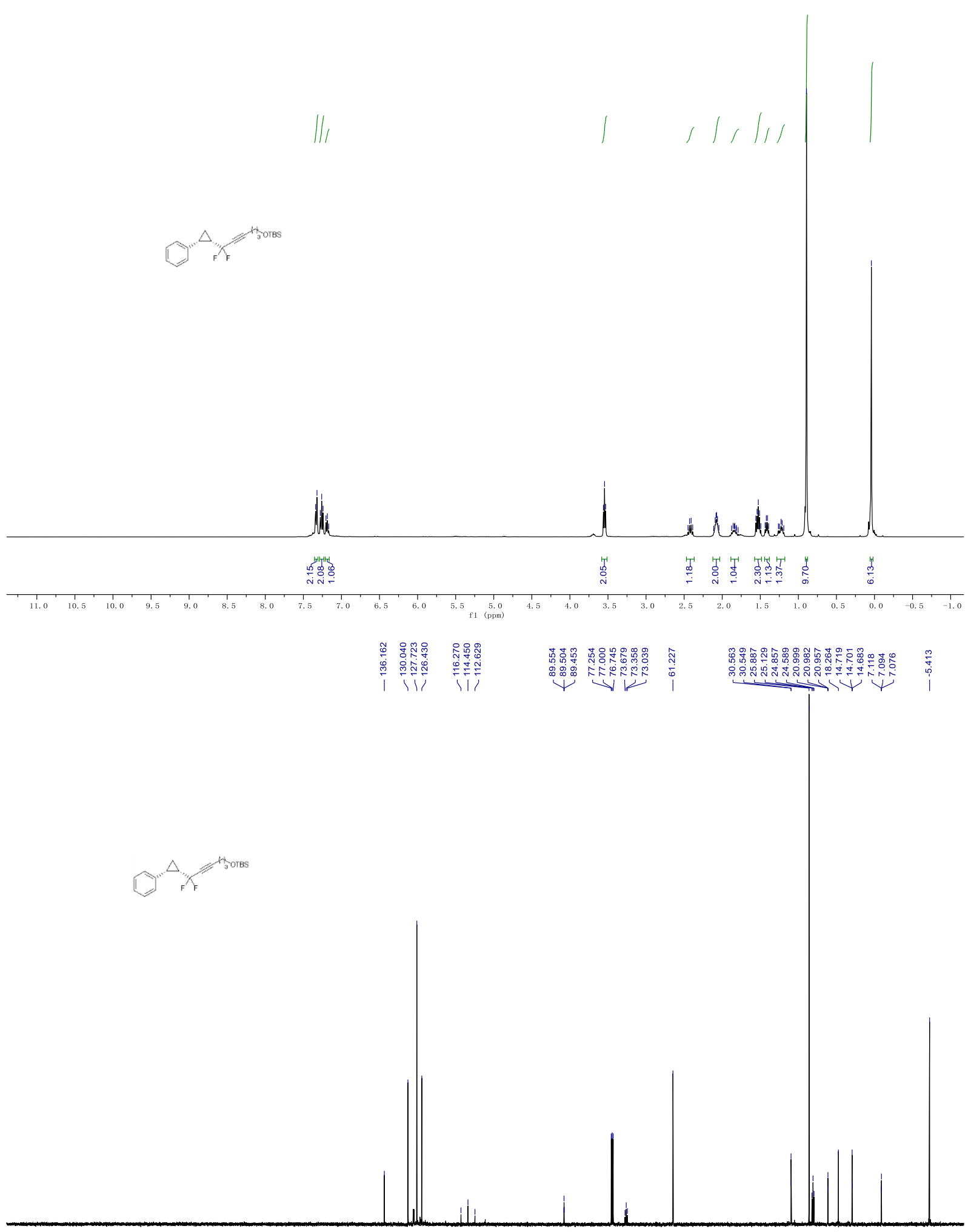

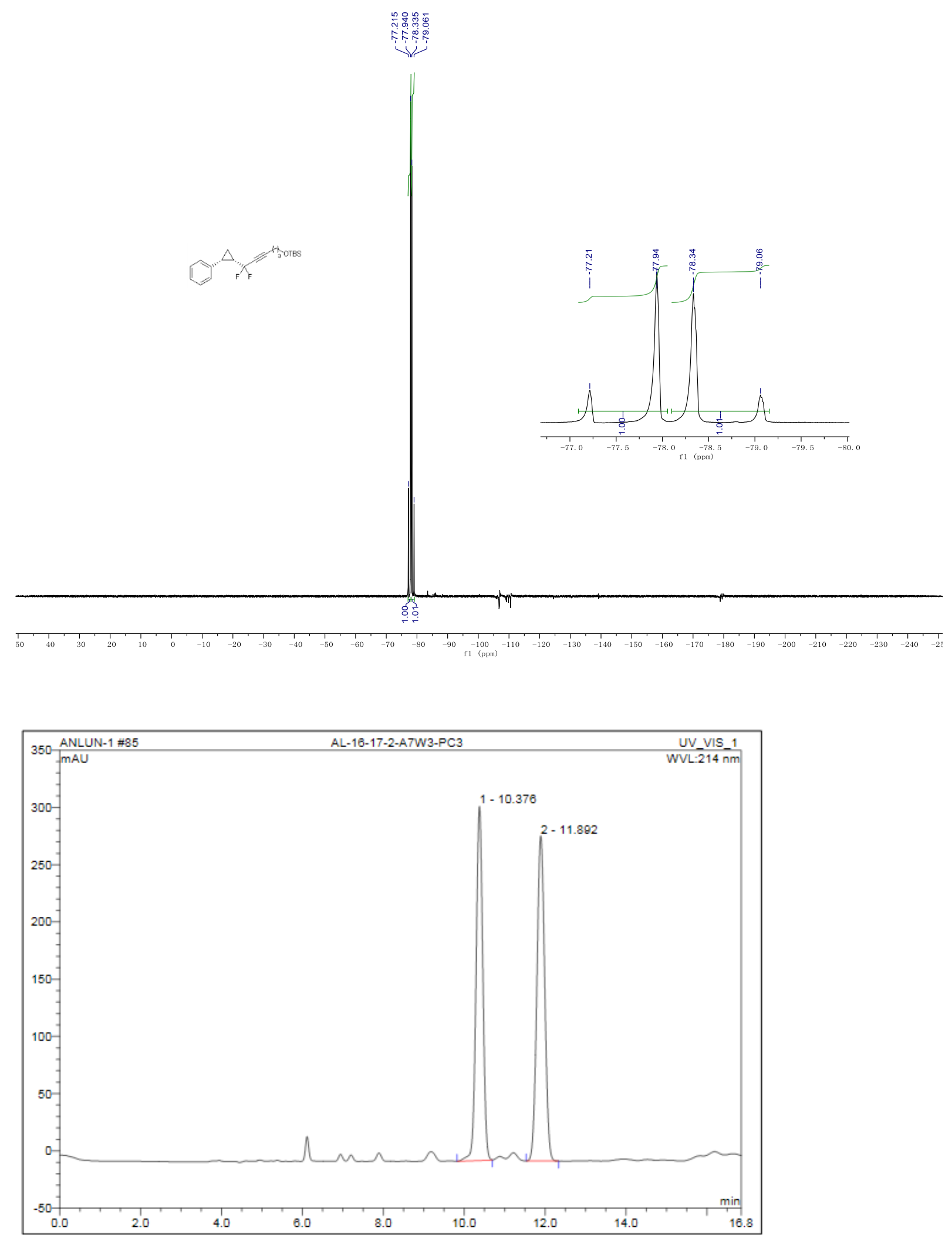

\begin{tabular}{|r|cccrrrr|}
\hline No. & $\begin{array}{c}\text { Ret. Time } \\
\text { min }\end{array}$ & Peak Name & $\begin{array}{c}\text { Height } \\
\text { mAU }\end{array}$ & $\begin{array}{c}\text { Area } \\
\text { mAU*min }\end{array}$ & $\begin{array}{r}\text { Rel.Area } \\
\%\end{array}$ & Amount & Type \\
\hline 1 & 10.38 & n.a. & 309.281 & 59.478 & 48.42 & n.a. & BMB \\
2 & 11.89 & n.a. & 283.986 & 63.359 & 51.58 & n.a. & BMB \\
\hline Total: & & & 593.267 & 122.837 & 100.00 & 0.000 & \\
\hline
\end{tabular}




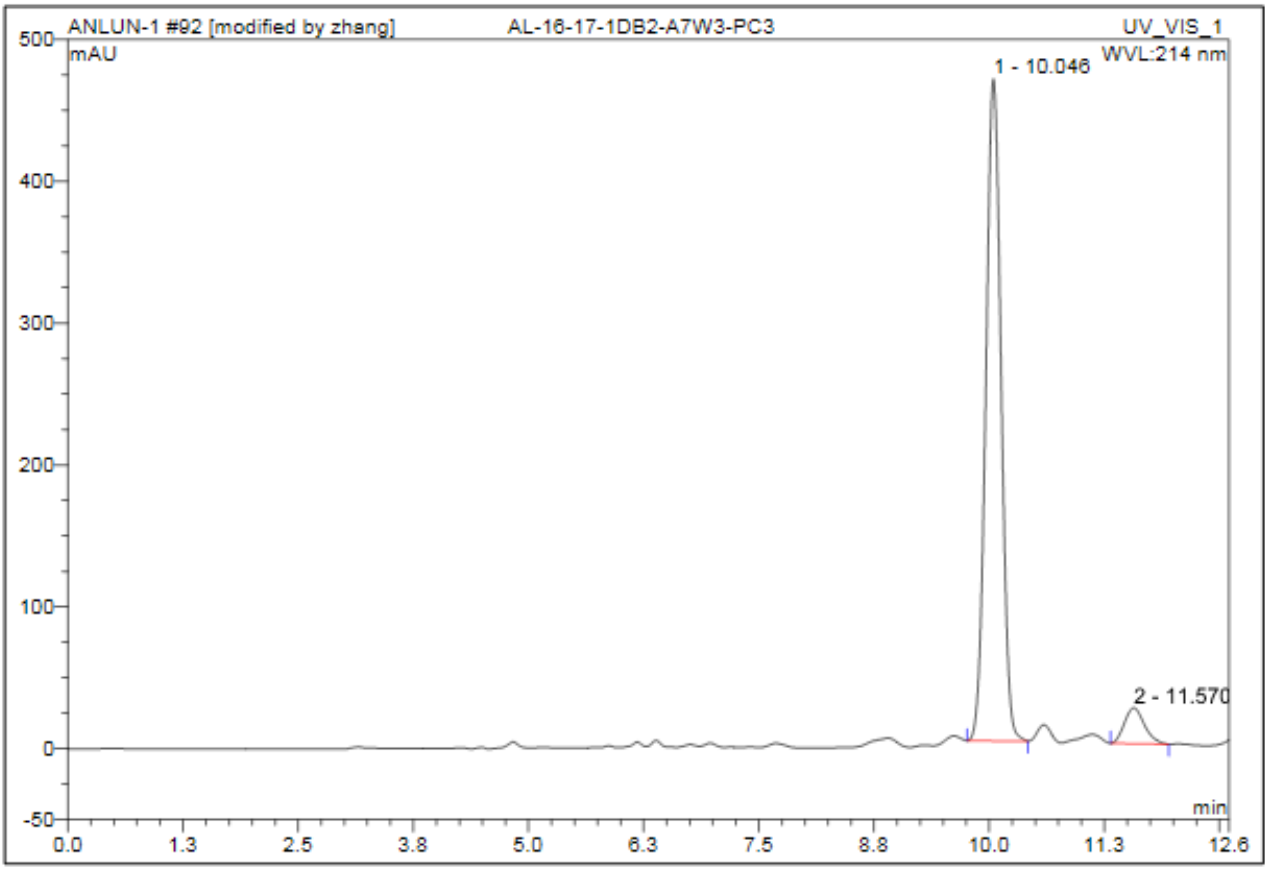

\begin{tabular}{|c|c|c|c|c|c|c|c|}
\hline No. & $\begin{array}{c}\text { Ret.Time } \\
\text { min }\end{array}$ & Peak Name & $\begin{array}{c}\text { Height } \\
\text { mAU }\end{array}$ & $\begin{array}{c}\text { Area } \\
\mathrm{mAU}^{\star} \min \end{array}$ & $\begin{array}{c}\text { Rel.Area } \\
\%\end{array}$ & Amount & Type \\
\hline 1 & 10.05 & n.a. & 466.628 & 89.120 & 93.37 & n.a. & BMB \\
\hline 2 & 11.57 & n.a. & 25.129 & 6.327 & 6.63 & n.a. & $\mathrm{BMB}$ \\
\hline Total: & & & 491.756 & 95.448 & 100.00 & 0.000 & \\
\hline
\end{tabular}

((1R,2S)-2-(3-(1-(Benzyloxy)cyclopentyl)-1,1-difluoroprop-2-yn-1-yl)cyclopropyl)benzene (3m).

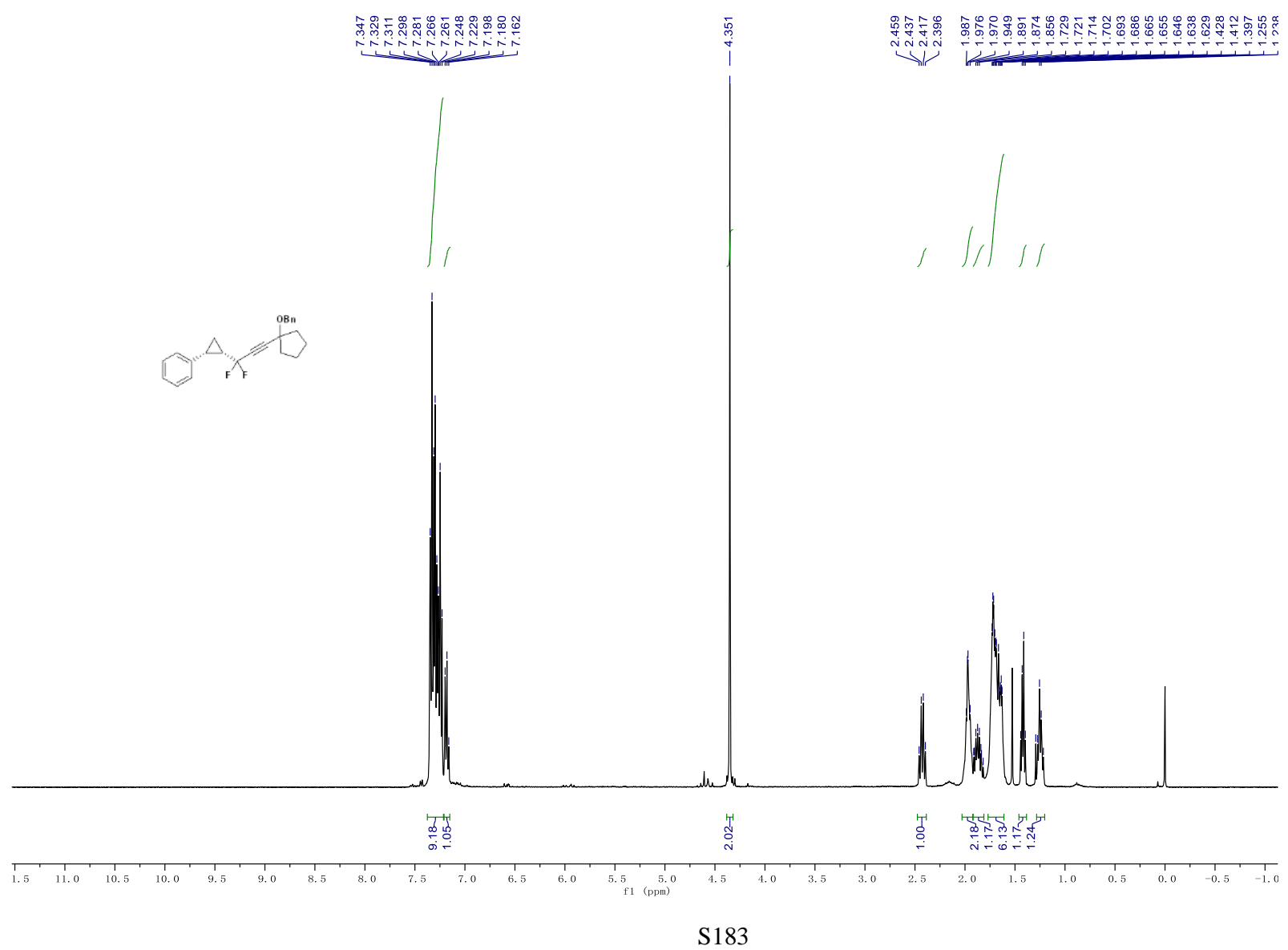



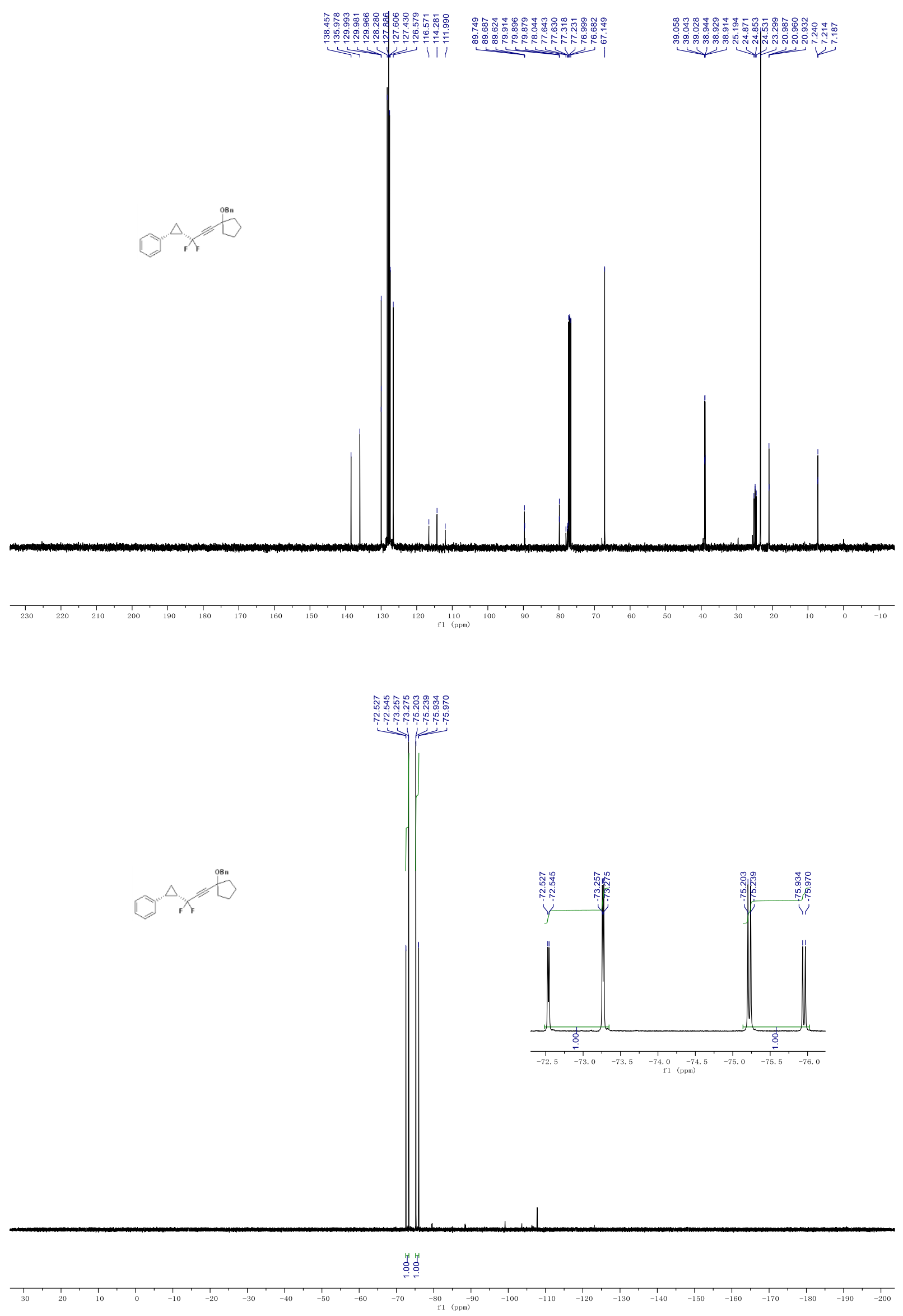


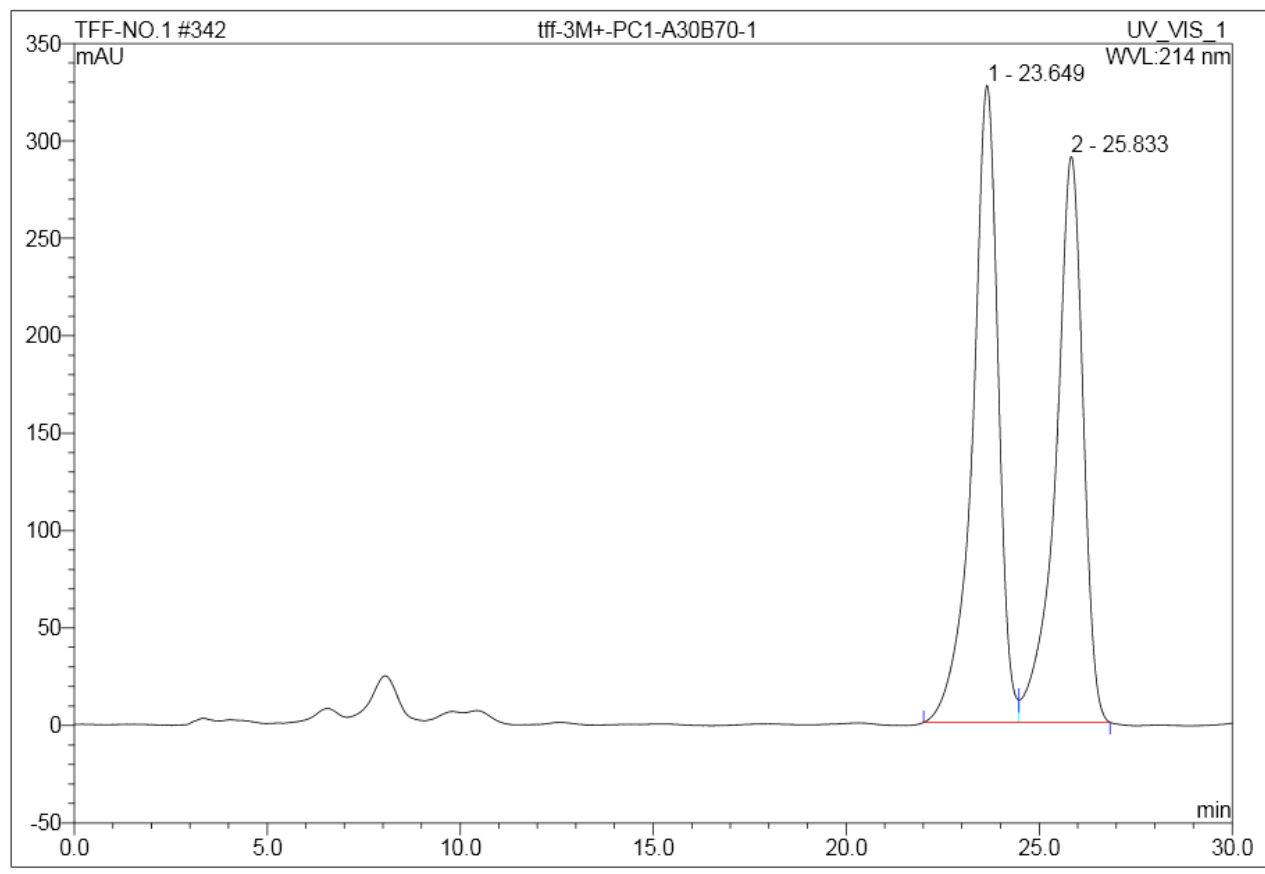

\begin{tabular}{|r|cccrrrr|}
\hline No. & $\begin{array}{c}\text { Ret.Time } \\
\text { min }\end{array}$ & Peak Name & $\begin{array}{c}\text { Height } \\
\text { mAU }\end{array}$ & $\begin{array}{c}\text { Area } \\
\text { mAU*min }\end{array}$ & $\begin{array}{c}\text { Rel.Area } \\
\%\end{array}$ & $\begin{array}{c}\text { Amount } \\
\%\end{array}$ & Type \\
\hline 1 & 23.65 & n.a. & 326.896 & 251.987 & 51.44 & n.a. & BM \\
2 & 25.83 & n.a. & 290.359 & 237.883 & 48.56 & n.a. & MB \\
\hline Total: & & & 617.255 & 489.870 & 100.00 & 0.000 & \\
\hline
\end{tabular}

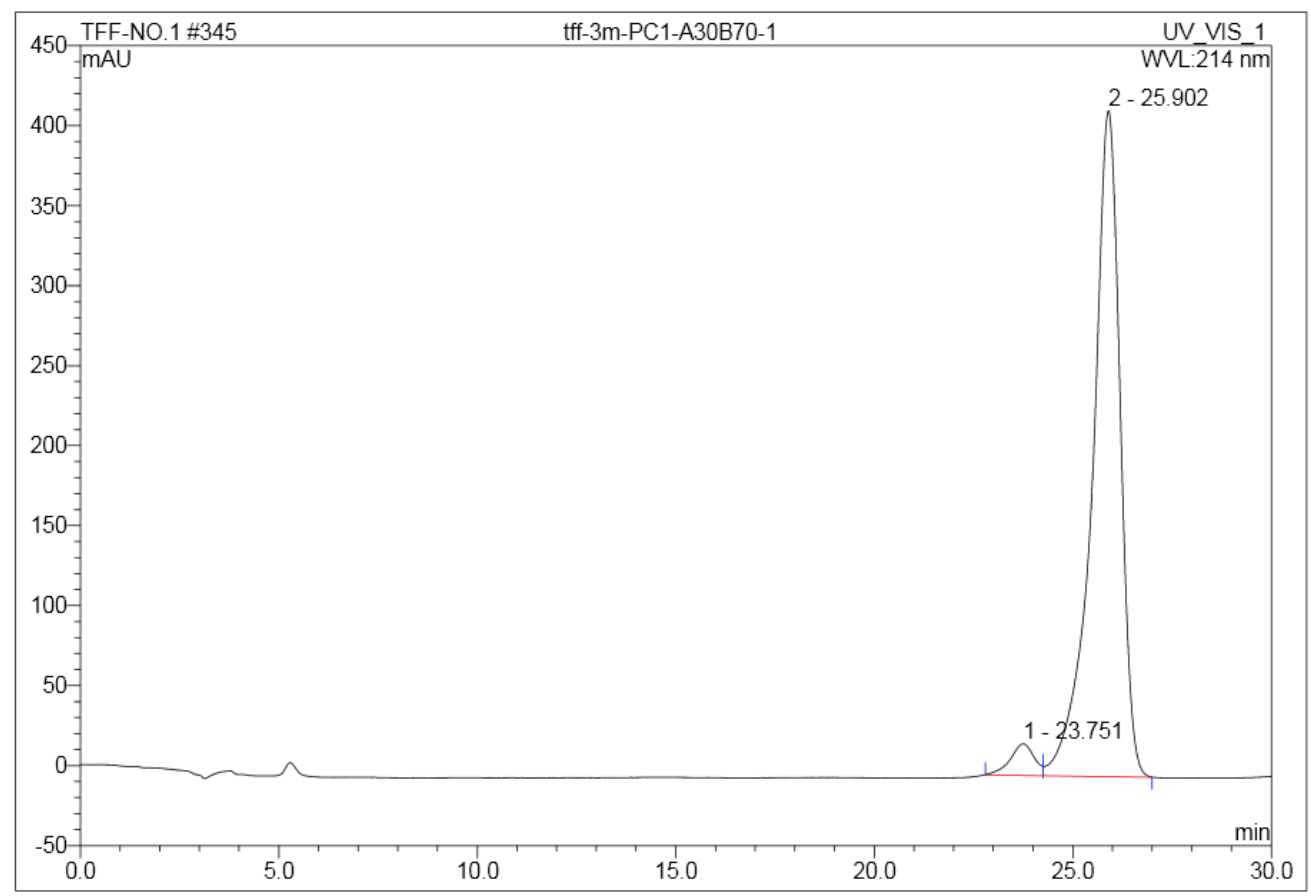

\begin{tabular}{|r|ccrrrrr|}
\hline No. & $\begin{array}{c}\text { Ret. Time } \\
\text { min }\end{array}$ & Peak Name & $\begin{array}{c}\text { Height } \\
\text { mAU }\end{array}$ & $\begin{array}{c}\text { Area } \\
\text { mAU*min }\end{array}$ & $\begin{array}{c}\text { Rel.Area } \\
\%\end{array}$ & $\begin{array}{c}\text { Amount } \\
\%\end{array}$ & Type \\
\hline 1 & 23.75 & n.a. & 19.658 & 13.740 & 3.91 & n.a. & BM \\
2 & 25.90 & n.a. & 416.245 & 337.373 & 96.09 & n.a. & MB \\
\hline Total: & & & 435.903 & 351.113 & 100.00 & 0.000 & \\
\hline
\end{tabular}


tert-Butyl-4-(benzyloxy)-4-(3,3-difluoro-3-((1S,2R)-2-phenylcyclopropyl)prop-1-yn-1-yl)piperid ine-1-carboxylate (3n).
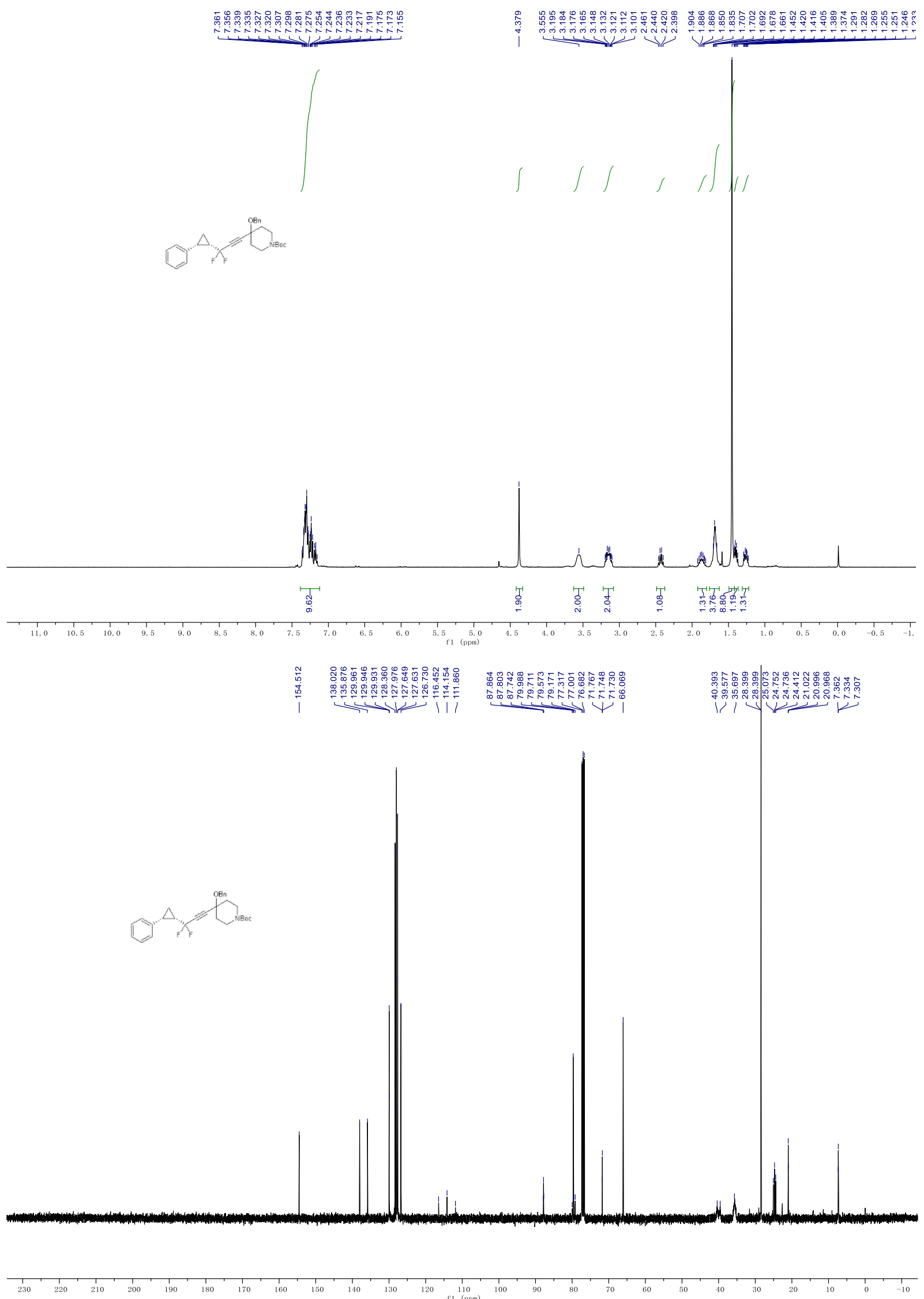

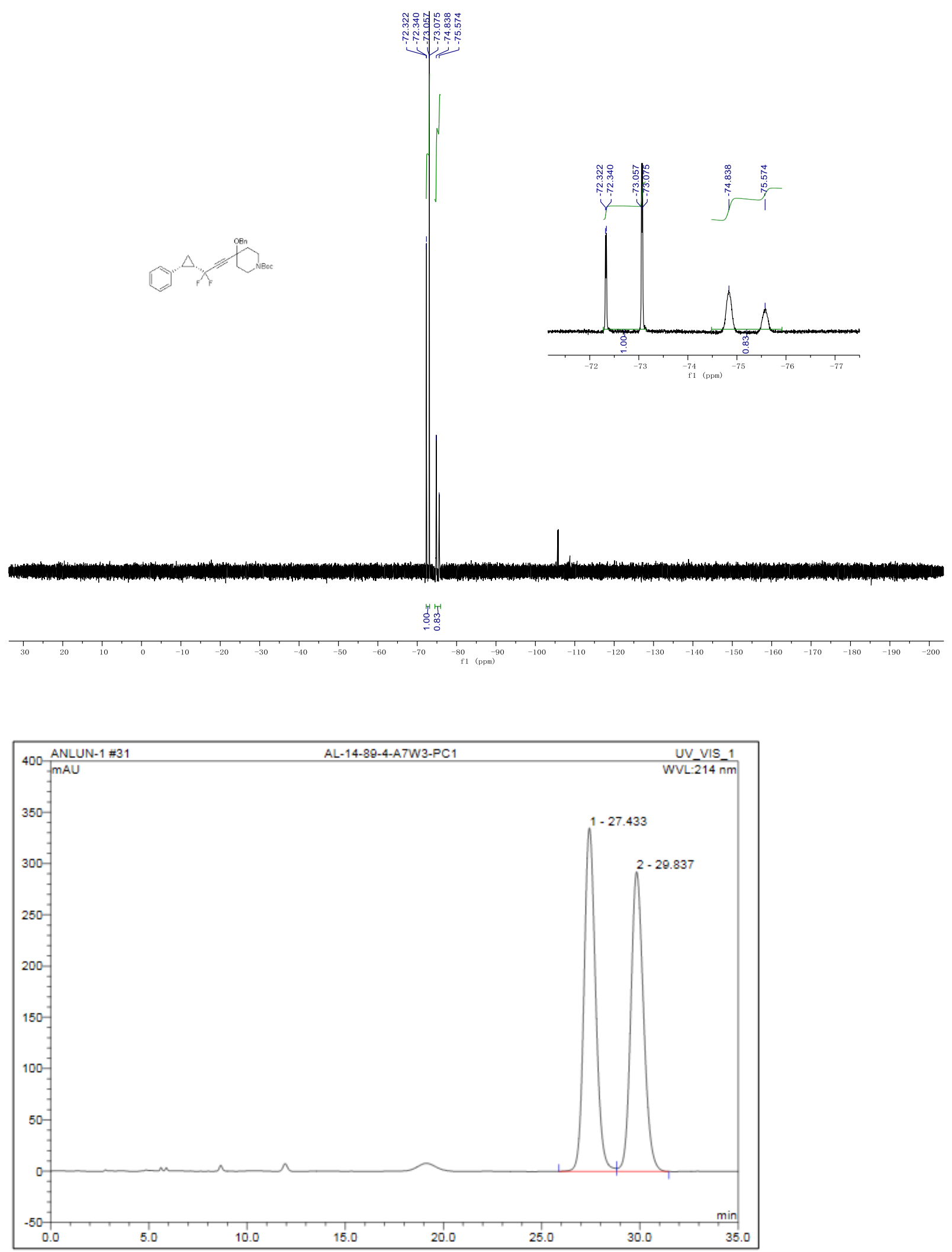

\begin{tabular}{|r|cccrccc|}
\hline No. & $\begin{array}{c}\text { Ret.Time } \\
\text { min }\end{array}$ & Peak Name & $\begin{array}{c}\text { Height } \\
\text { mAU }\end{array}$ & $\begin{array}{c}\text { Area } \\
\text { mAU*min }\end{array}$ & $\begin{array}{r}\text { Rel.Area } \\
\%\end{array}$ & Amount & Type \\
\hline 1 & 27.43 & n.a. & 334.922 & 228.630 & 51.05 & n.a. & BM \\
2 & 29.84 & n.a. & 292.348 & 219.213 & 48.95 & n.a. & MB \\
\hline Total: & & & 627.270 & 447.843 & 100.00 & 0.000 & \\
\hline
\end{tabular}




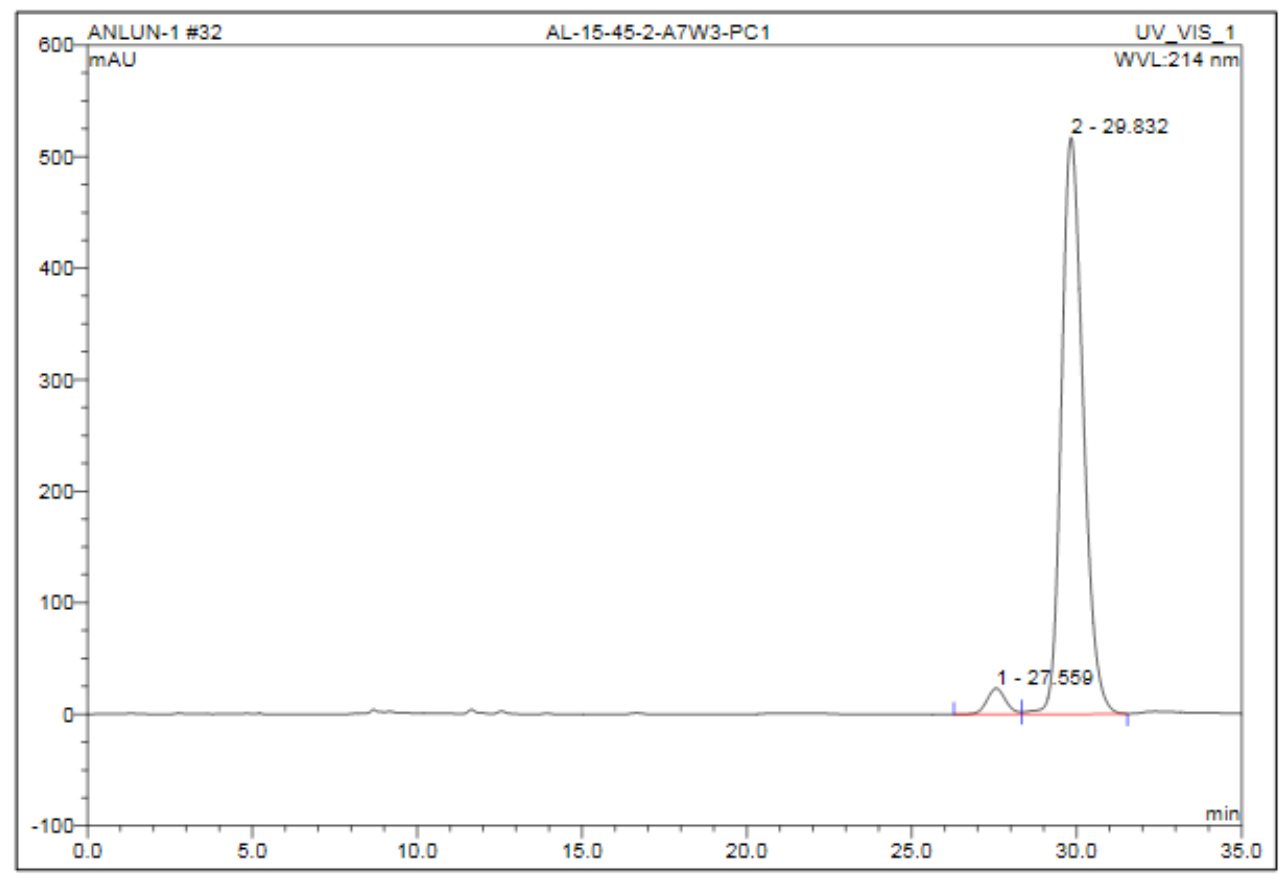

\begin{tabular}{|c|c|c|c|c|c|c|c|}
\hline No. & $\begin{array}{c}\text { Ret.Time } \\
\text { min }\end{array}$ & Peak Name & $\begin{array}{c}\text { Height } \\
\text { mAU }\end{array}$ & $\begin{array}{c}\text { Area } \\
\text { mAU*min } \\
\end{array}$ & $\begin{array}{c}\text { Rel.Area } \\
\% \\
\end{array}$ & Amount & Type \\
\hline 1 & 27.56 & n.a. & 23.253 & 15.528 & 3.77 & n.a. & BM \\
\hline 2 & 29.83 & n.a. & 517.259 & 396.082 & 96.23 & n.a. & $\mathrm{MB}$ \\
\hline Total: & & & 540.513 & 411.609 & 100.00 & 0.000 & \\
\hline
\end{tabular}

(3aR,5R,6aR)-6-(benzyloxy)-5-(3,3-difluoro-3-((1S,2R)-2-phenylcyclopropyl)prop-1-yn-1-yl)-2,2 -dimethyltetrahydrofuro[2,3-d][1,3]dioxole (3o).

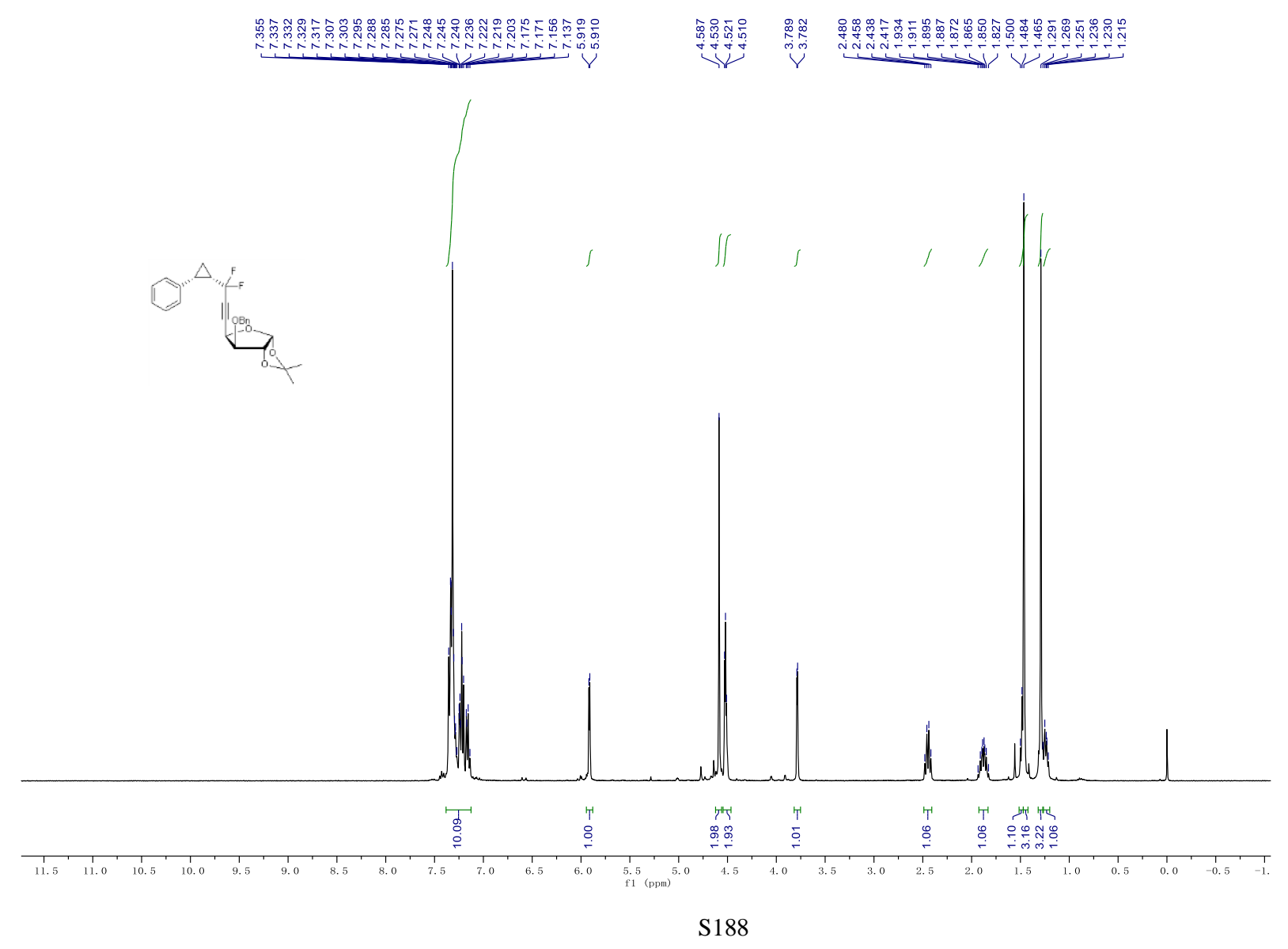



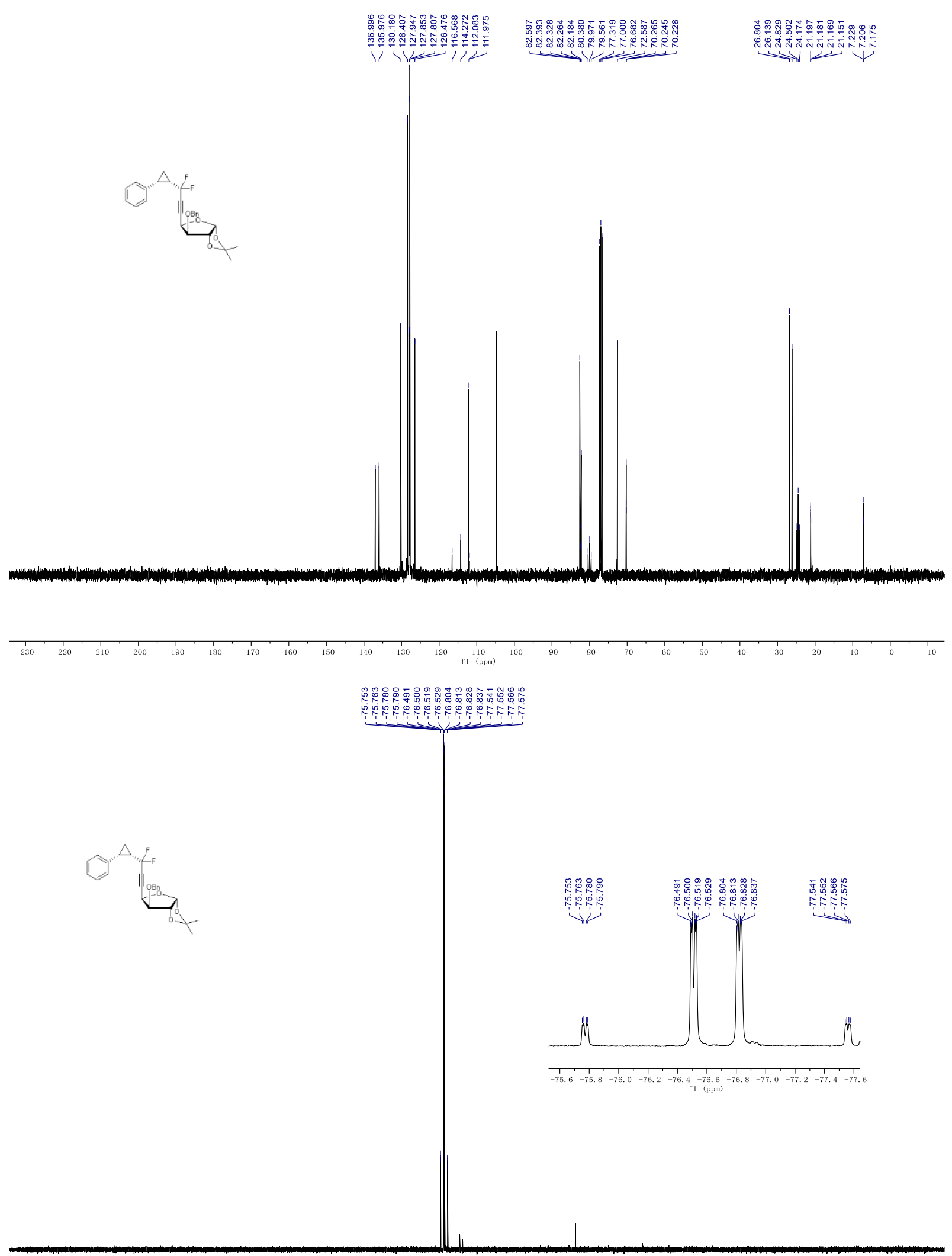


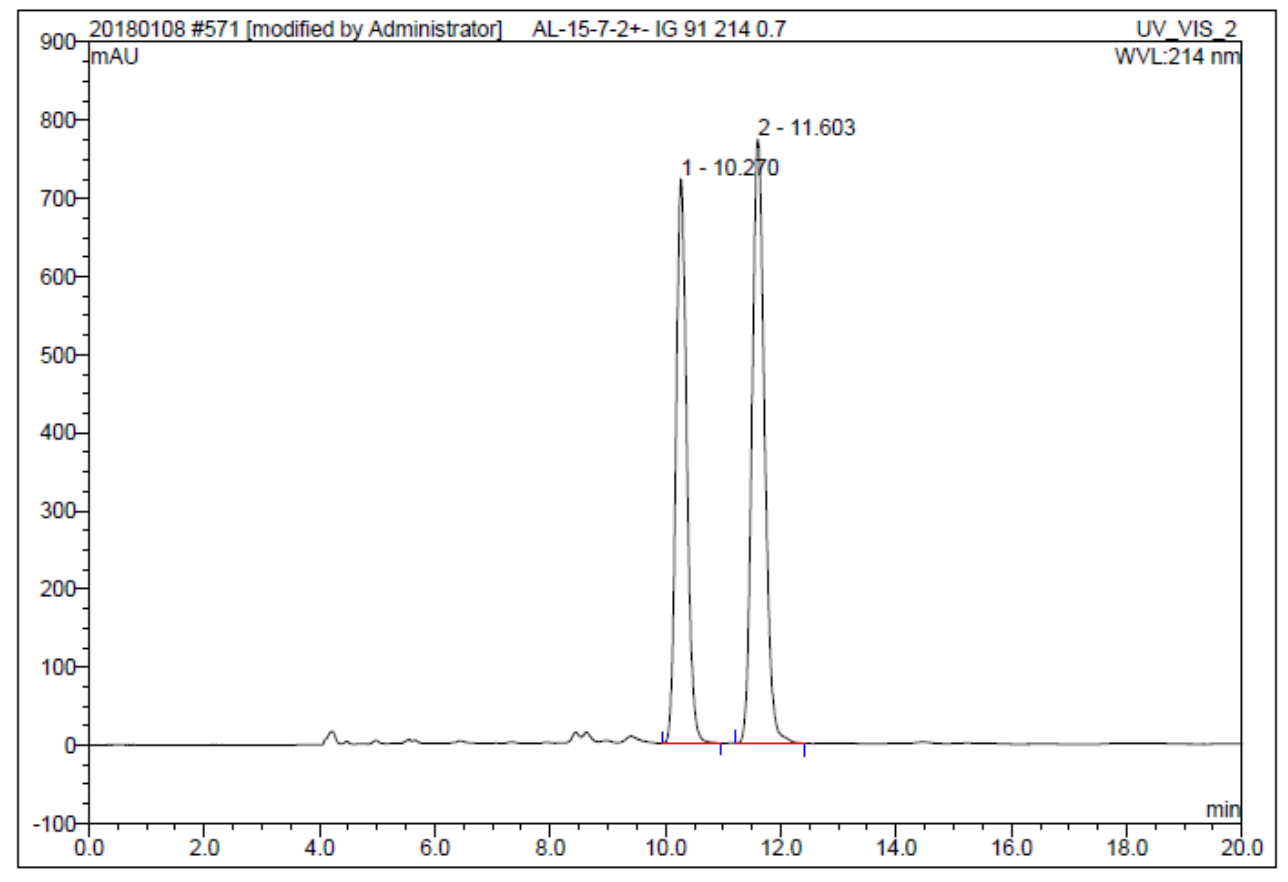

\begin{tabular}{|r|cccrrrr|}
\hline No. & $\begin{array}{c}\text { Ret.Time } \\
\text { min }\end{array}$ & Peak Name & $\begin{array}{c}\text { Height } \\
\text { mAU }\end{array}$ & $\begin{array}{c}\text { Area } \\
\text { mAU*min }\end{array}$ & $\begin{array}{c}\text { Rel.Area } \\
\%\end{array}$ & Amount & Type \\
\hline 1 & 10.27 & n.a. & 722.834 & 157.033 & 44.50 & n.a. & BMB \\
2 & 11.60 & n.a. & 773.947 & 195.811 & 55.50 & n.a. & BMB \\
\hline Total: & & & 1496.781 & 352.843 & 100.00 & 0.000 & \\
\hline
\end{tabular}

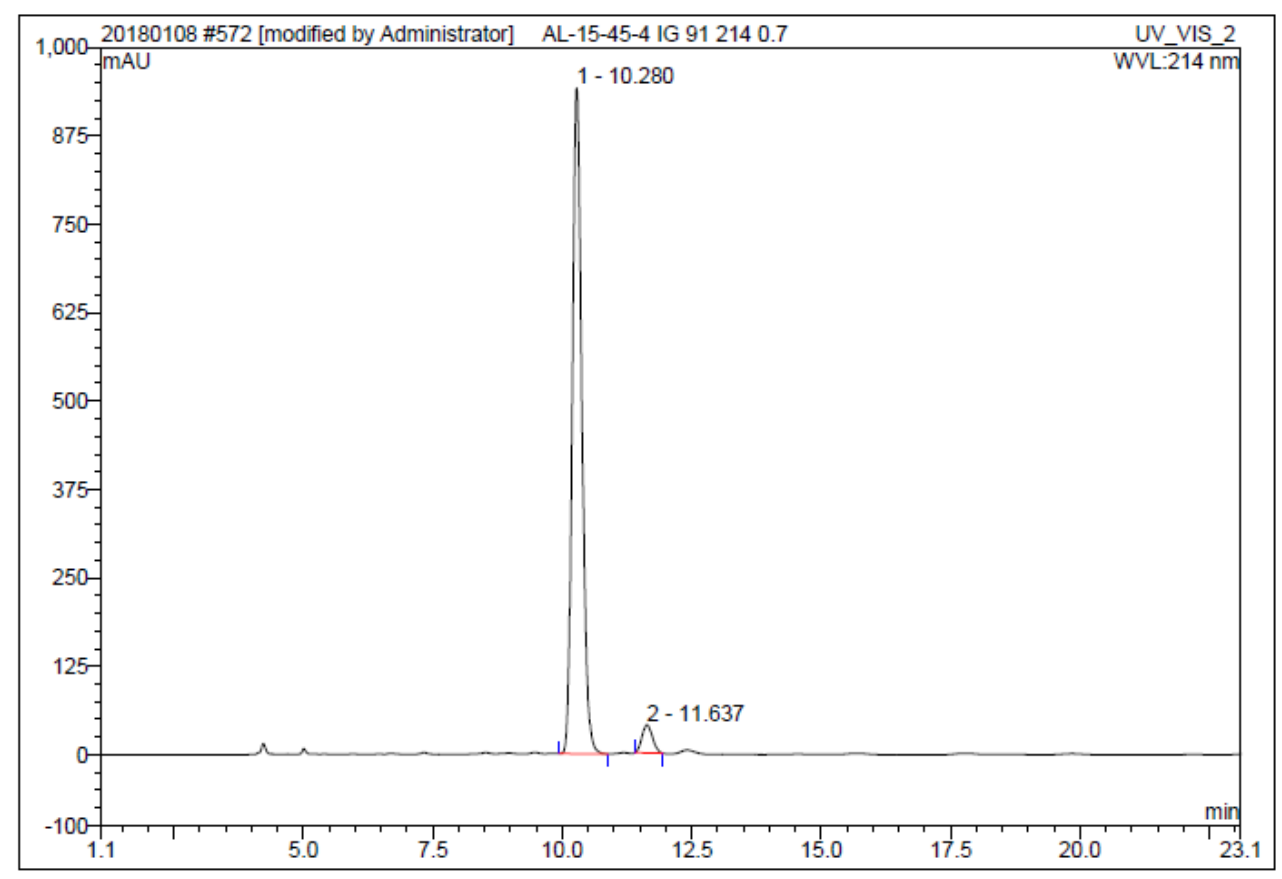

\begin{tabular}{|r|ccrrrrr|}
\hline No. & $\begin{array}{c}\text { Ret.Time } \\
\text { min }\end{array}$ & Peak Name & $\begin{array}{c}\text { Height } \\
\text { mAU }\end{array}$ & $\begin{array}{c}\text { Area } \\
\text { mAU*min }\end{array}$ & $\begin{array}{r}\text { Rel.Area } \\
\%\end{array}$ & Amount & Type \\
\hline 1 & 10.28 & n.a. & 941.042 & 202.314 & 95.61 & n.a. & BMB \\
2 & 11.64 & n.a. & 39.766 & 9.281 & 4.39 & n.a. & BMB $^{*}$ \\
\hline Total: & & & 980.808 & 211.595 & 100.00 & 0.000 & \\
\hline
\end{tabular}




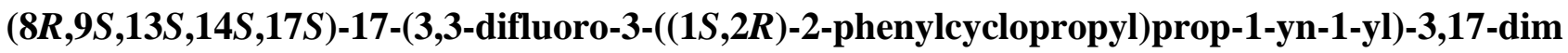
ethoxy-13-methyl-7,8,9,11,12,13,14,15,16,17-decahydro-6H-cyclopenta[a]phenanthrene (3p).

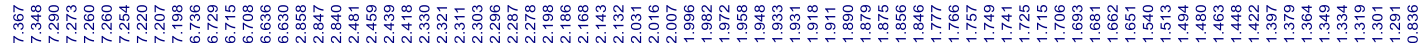
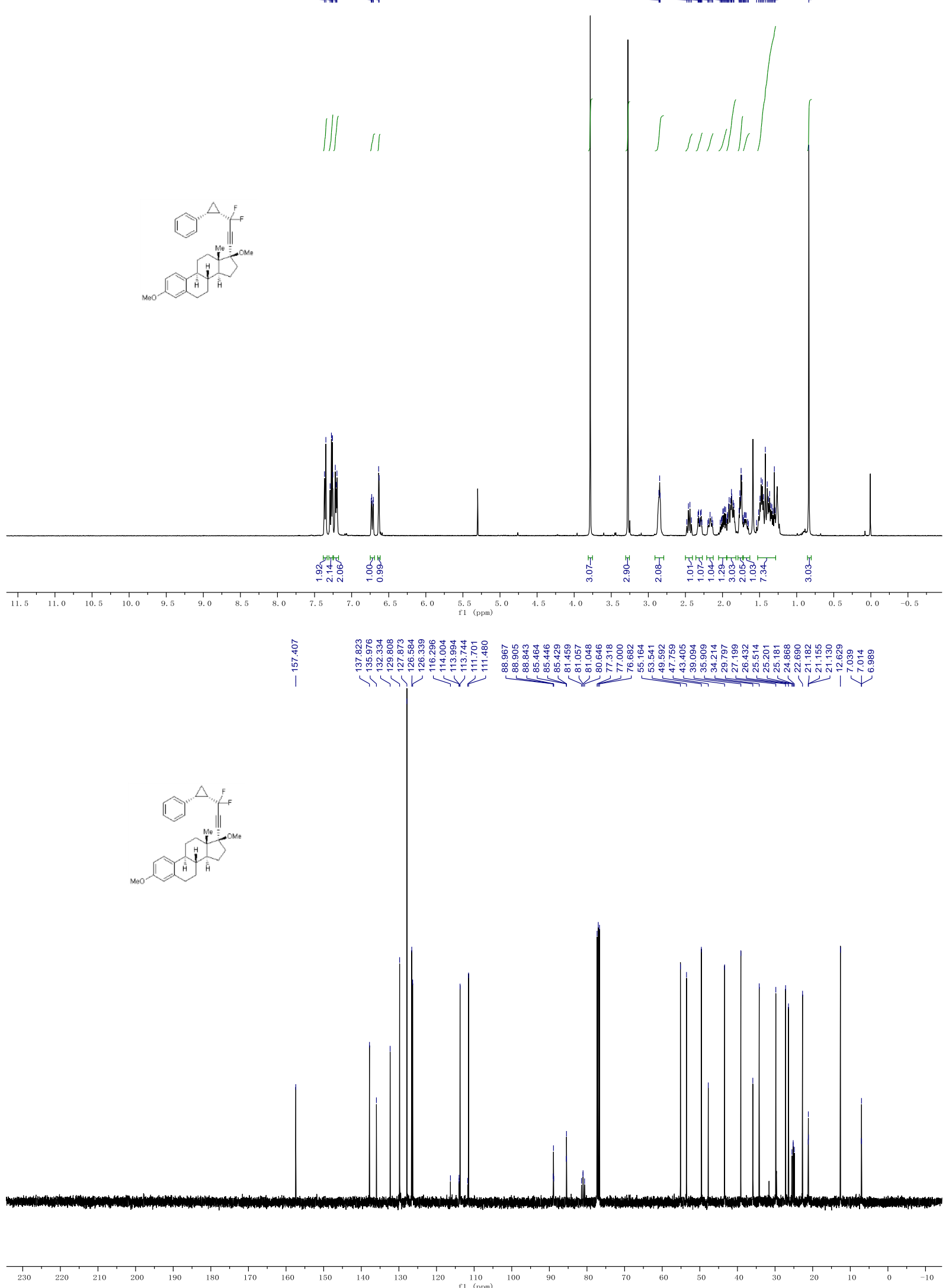

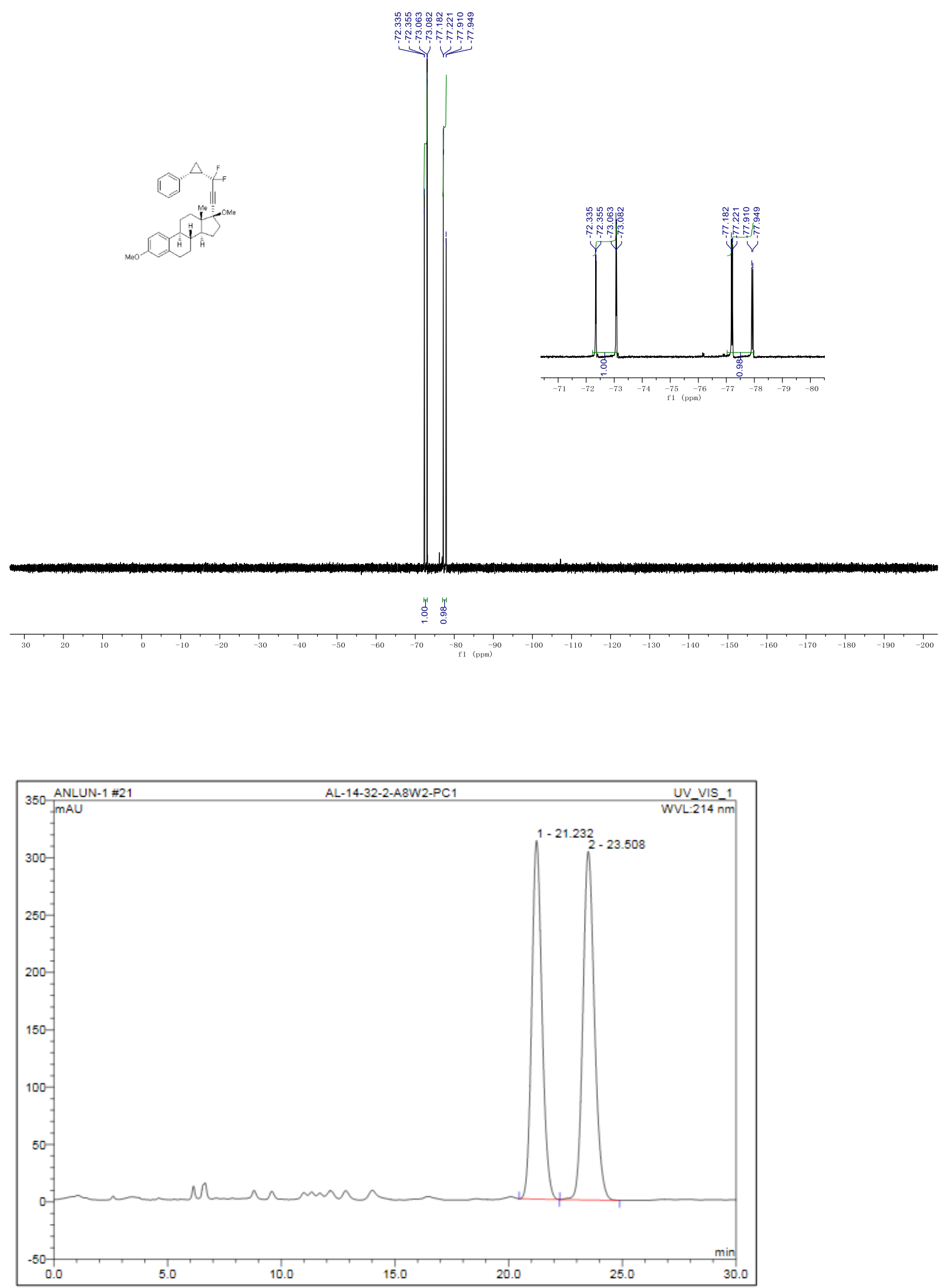

\begin{tabular}{|r|cccrrrr|}
\hline No. & $\begin{array}{c}\text { Ret.Time } \\
\text { min }\end{array}$ & Peak Name & $\begin{array}{c}\text { Height } \\
\text { mAU }\end{array}$ & $\begin{array}{c}\text { Area } \\
\text { mAU*min }\end{array}$ & $\begin{array}{c}\text { Rel.Area } \\
\%\end{array}$ & Amount & Type \\
\hline 1 & 21.23 & n.a. & 312.511 & 162.685 & 47.65 & n.a. & BMB \\
2 & 23.51 & n.a. & 303.823 & 178.713 & 52.35 & n.a. & BMB \\
\hline Total: & & & 616.334 & 341.398 & 100.00 & 0.000 & \\
\hline
\end{tabular}




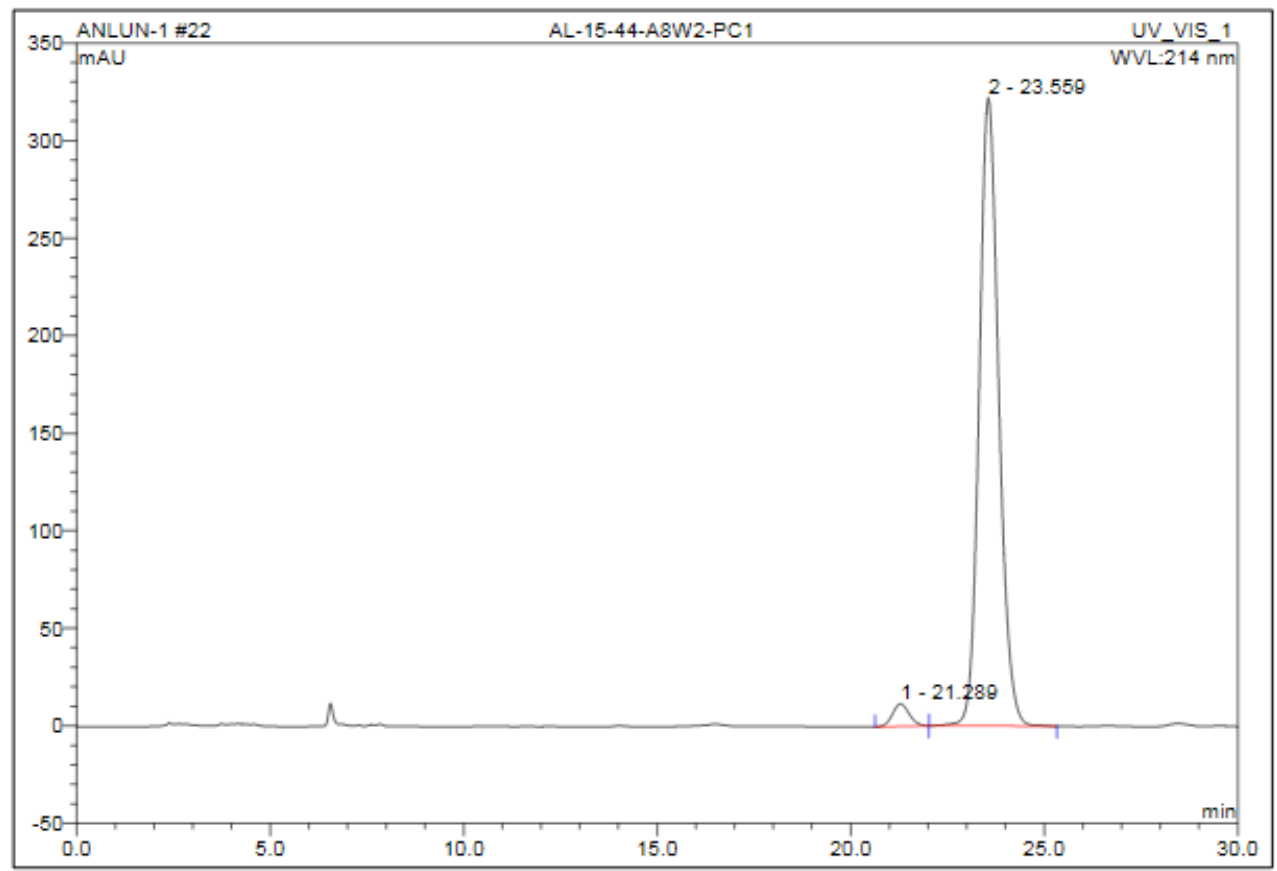

\begin{tabular}{|r|ccrrrrr|}
\hline No. & $\begin{array}{c}\text { Ret.Time } \\
\text { min }\end{array}$ & Peak Name & $\begin{array}{c}\text { Height } \\
\text { mAU }\end{array}$ & $\begin{array}{r}\text { Area } \\
\text { mAU*min }\end{array}$ & $\begin{array}{r}\text { Rel.Area } \\
\%\end{array}$ & Amount & Type \\
\hline 1 & 21.29 & n.a. & 11.583 & 5.824 & 2.99 & n.a. & BMB \\
2 & 23.56 & n.a. & 322.058 & 189.222 & 97.01 & n.a. & BMB \\
\hline Total: & & & 333.641 & 195.046 & 100.00 & 0.000 & \\
\hline
\end{tabular}

(3,3-Difluoro-3-((1S,2R,3R)-2-methyl-3-phenylcyclopropyl)prop-1-yn-1-yl)triethylsilane (3q).

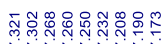

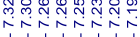

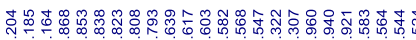

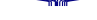

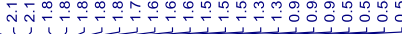

Whe

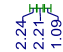

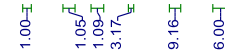



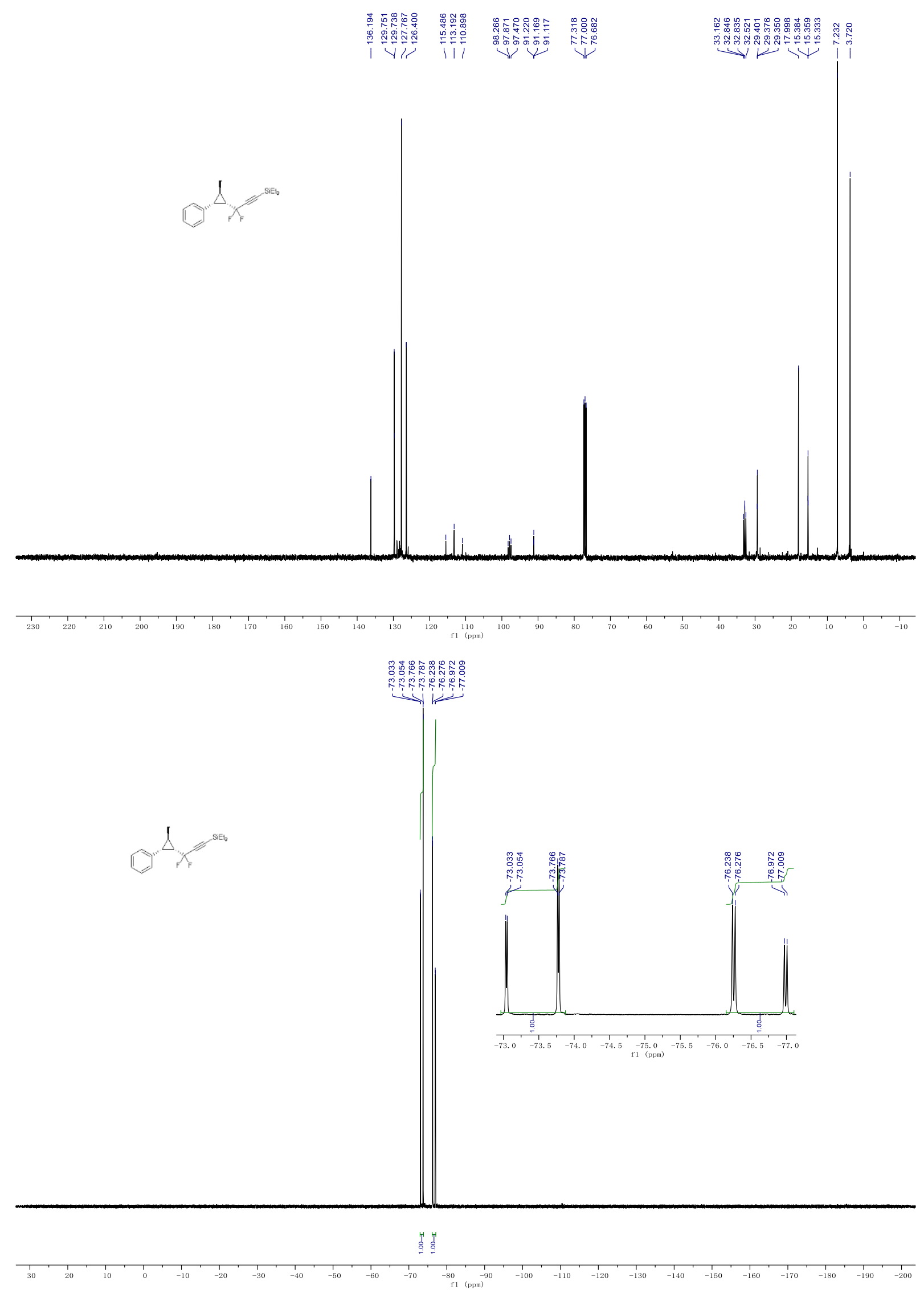


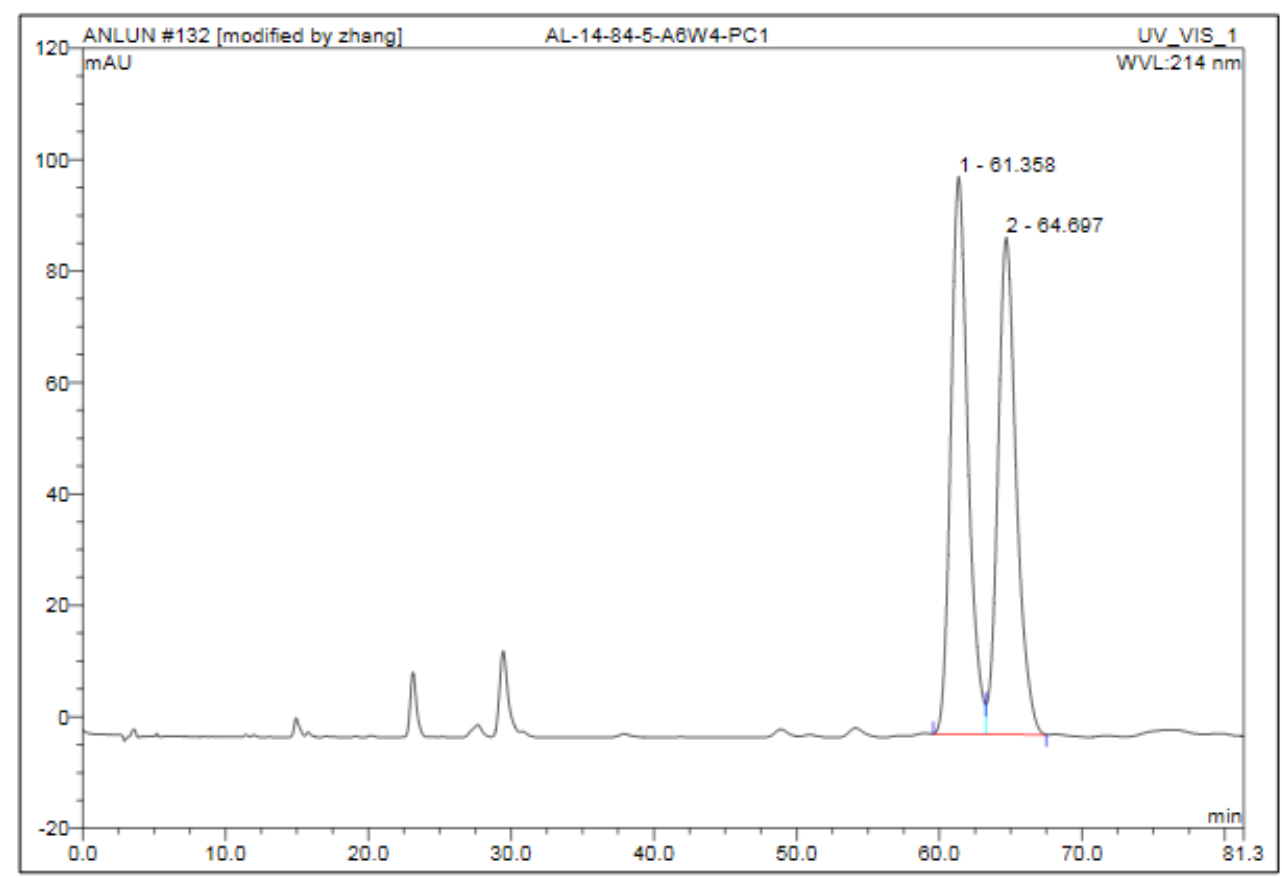

\begin{tabular}{|r|ccrrrrr|}
\hline No. & $\begin{array}{c}\text { Ret.Time } \\
\text { min }\end{array}$ & Peak Name & $\begin{array}{c}\text { Height } \\
\text { mAU }\end{array}$ & $\begin{array}{c}\text { Area } \\
\text { mAU*min }\end{array}$ & $\begin{array}{r}\text { Rel.Area } \\
\%\end{array}$ & Amount & Type \\
\hline 1 & 61.36 & n.a. & 100.028 & 140.081 & 51.13 & n.a. & BM \\
2 & 64.70 & n.a. & 89.169 & 133.892 & 48.87 & n.a. & MB \\
\hline Total: & & & 189.197 & 273.973 & 100.00 & 0.000 & \\
\hline
\end{tabular}

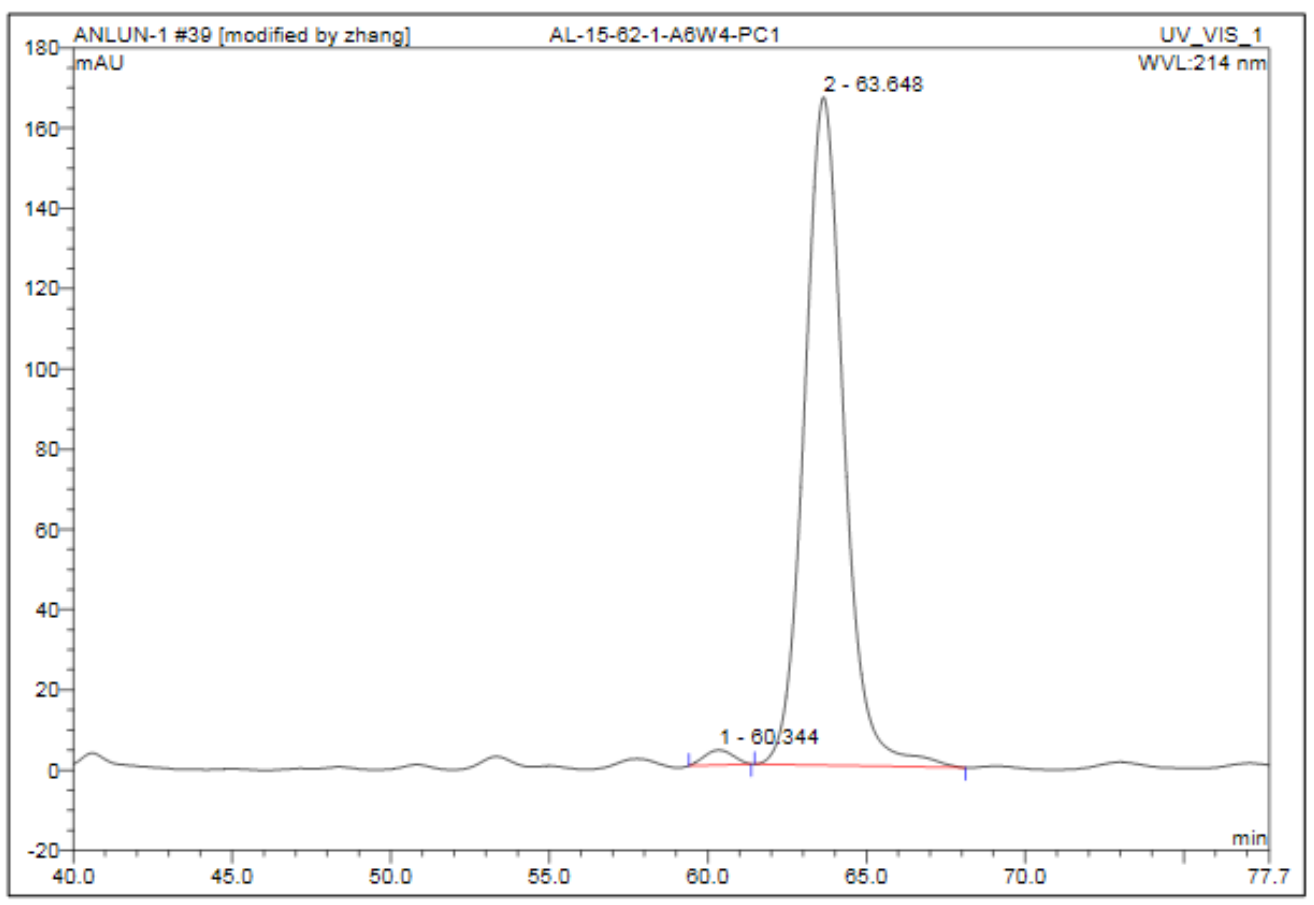

\begin{tabular}{|r|rrrrrrr|}
\hline No. & $\begin{array}{c}\text { Ret.Time } \\
\text { min }\end{array}$ & Peak Name & $\begin{array}{c}\text { Height } \\
\text { mAU }\end{array}$ & $\begin{array}{r}\text { Area } \\
\text { mAU*min }\end{array}$ & $\begin{array}{r}\text { Rel.Area } \\
\%\end{array}$ & Amount & Type \\
\hline 1 & 60.34 & n.a. & 3.710 & 3.891 & 1.59 & n.a. & BMB $^{\star}$ \\
2 & 63.65 & n.a. & 166.547 & 240.654 & 98.41 & n.a. & BMB \\
\hline Total: & & & 170.258 & 244.545 & 100.00 & 0.000 & \\
\hline
\end{tabular}


(3-((1S,2R,3R)-2-Butyl-3-phenylcyclopropyl)-3,3-difluoroprop-1-yn-1-yl)triethylsilane (3r).

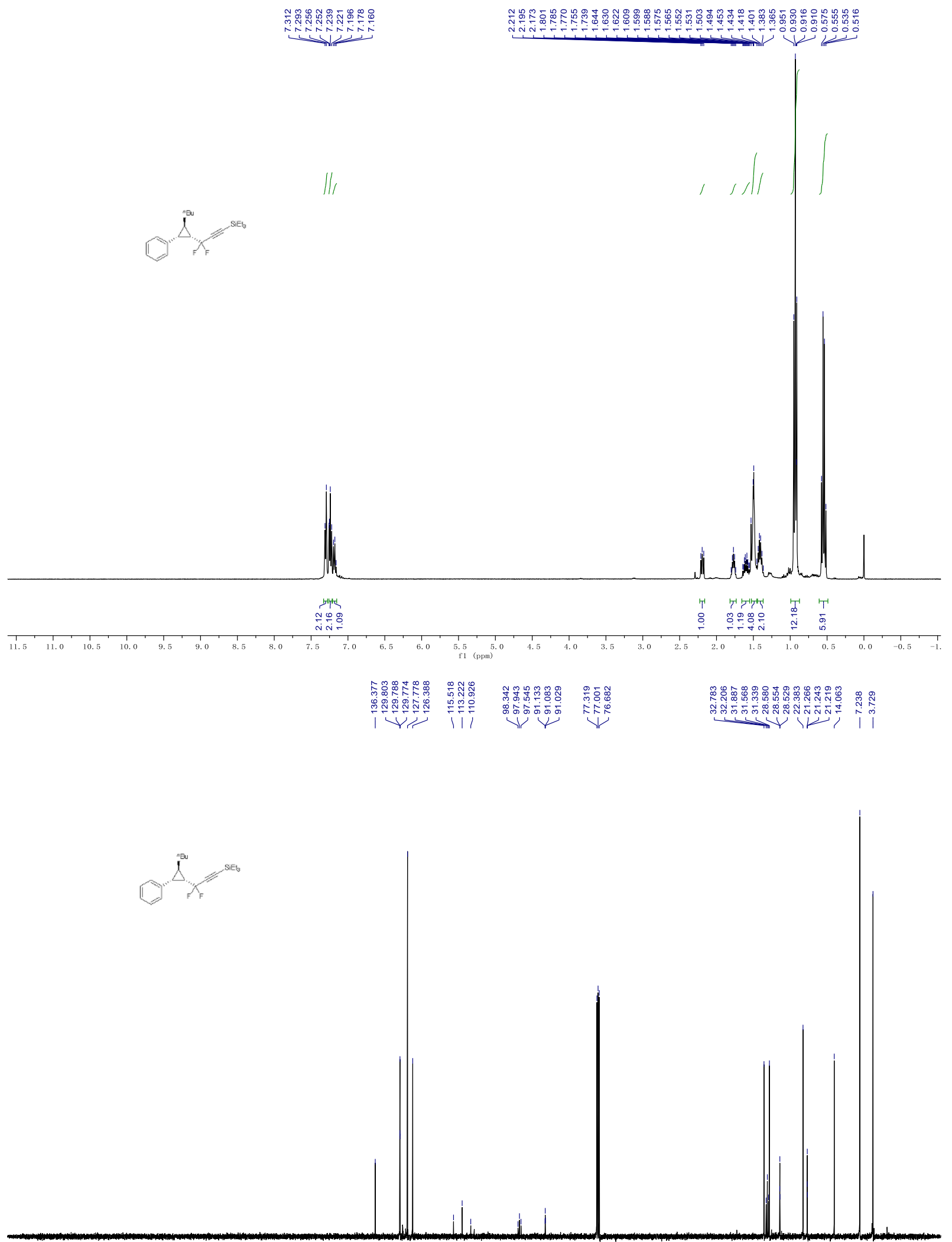




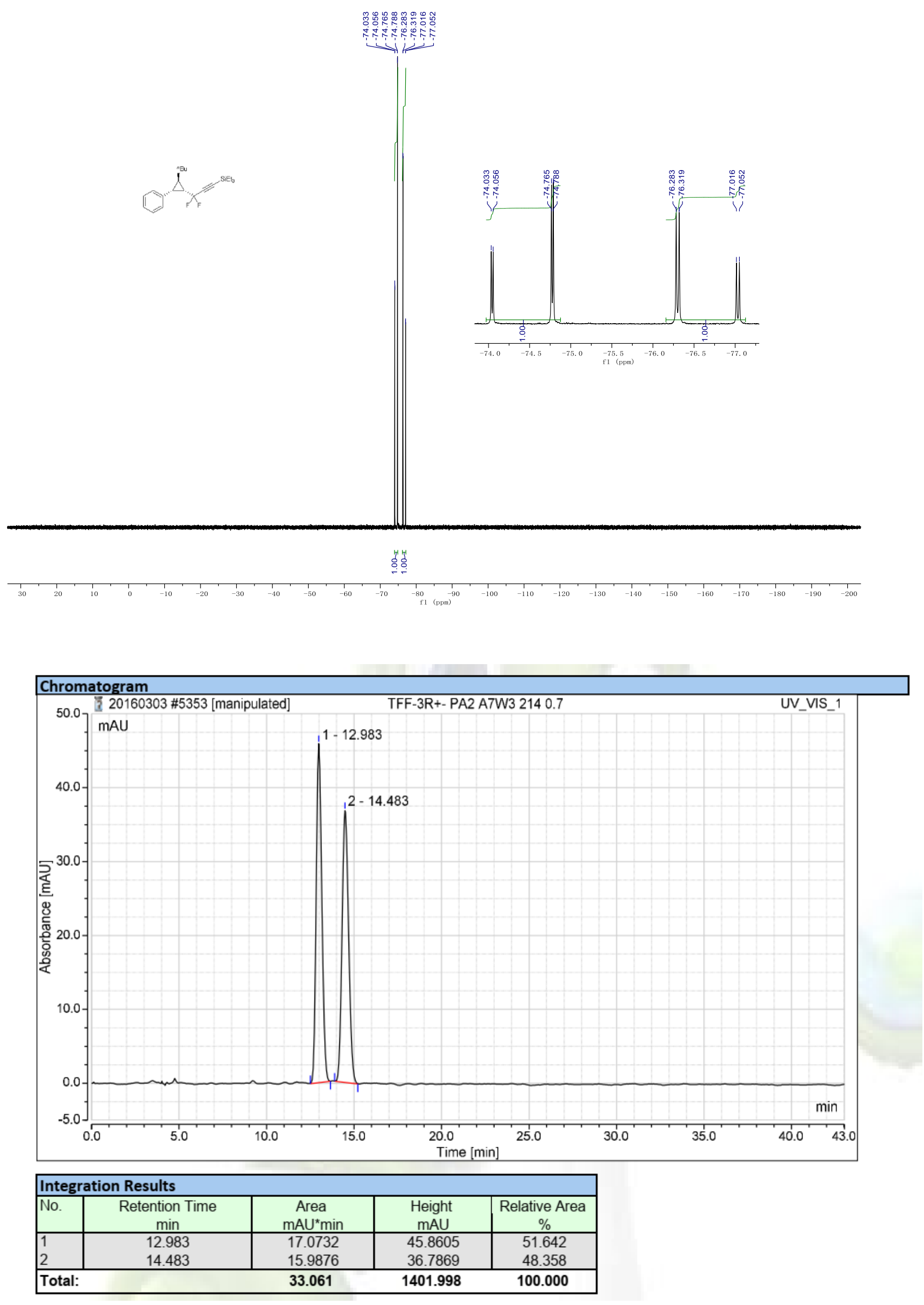




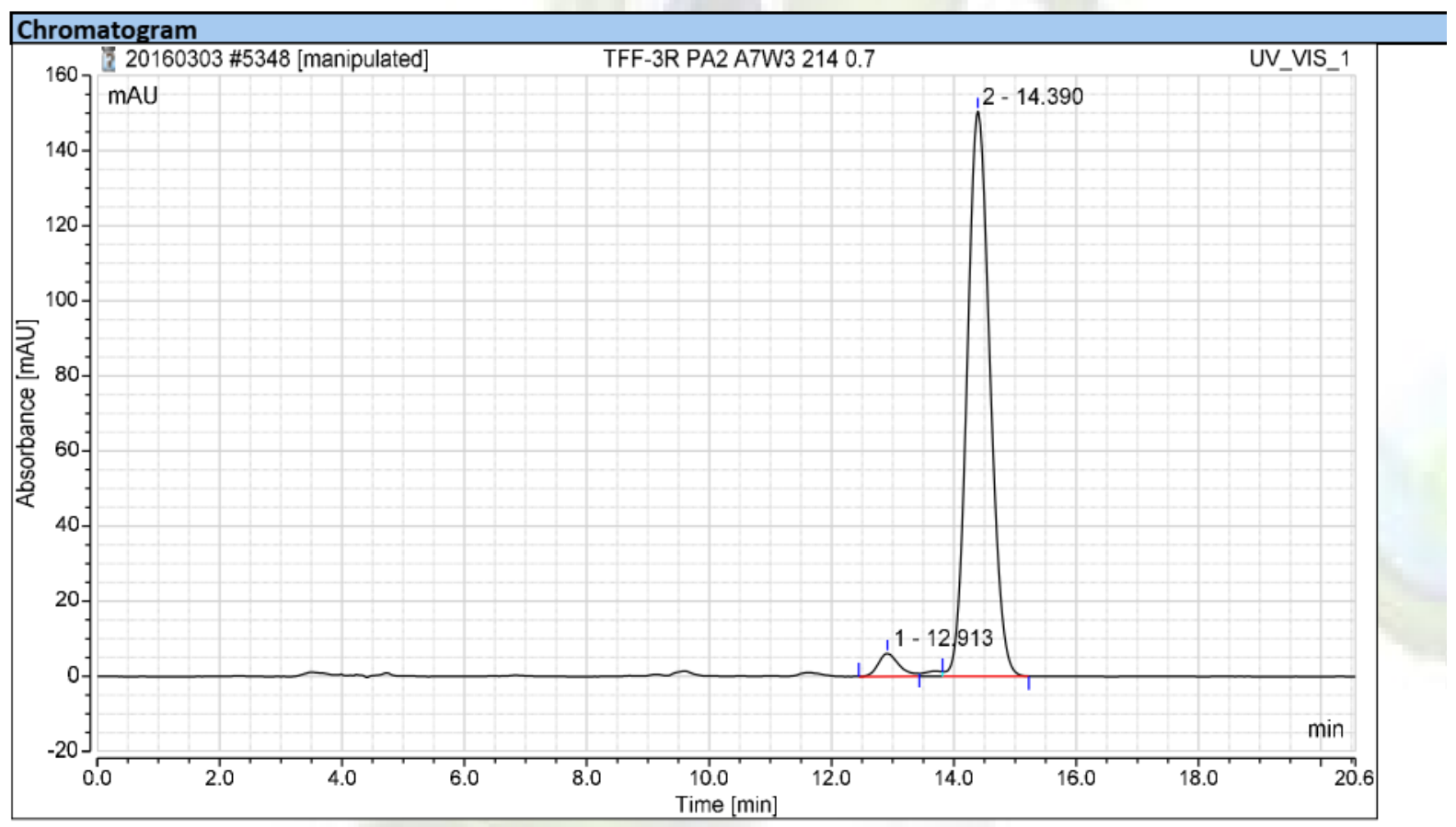

\begin{tabular}{|l|c|c|c|c|}
\hline \multicolumn{5}{|l|}{ Integration Results } \\
\hline No. & $\begin{array}{c}\text { Retention Time } \\
\text { min }\end{array}$ & $\begin{array}{c}\text { Area } \\
\mathrm{mAU}^{\star} \text { min }\end{array}$ & $\begin{array}{c}\text { Height } \\
\mathrm{mAU}\end{array}$ & $\begin{array}{c}\text { Relative Area } \\
\%\end{array}$ \\
\hline 1 & 12.913 & 2.4420 & 6.0794 & 3.633 \\
2 & 14.390 & 64.7777 & 150.4238 & 96.367 \\
\hline Total: & $\mathbf{6 7 . 2 2 0}$ & $\mathbf{1 4 0 1 . 9 9 8}$ & $\mathbf{1 0 0 . 0 0 0}$ \\
\hline
\end{tabular}

(3-((1R,2R,3R)-2-((benzyloxy)methyl)-3-phenylcyclopropyl)-3,3-difluoroprop-1-yn-1-yl)triethylsilane (3s).

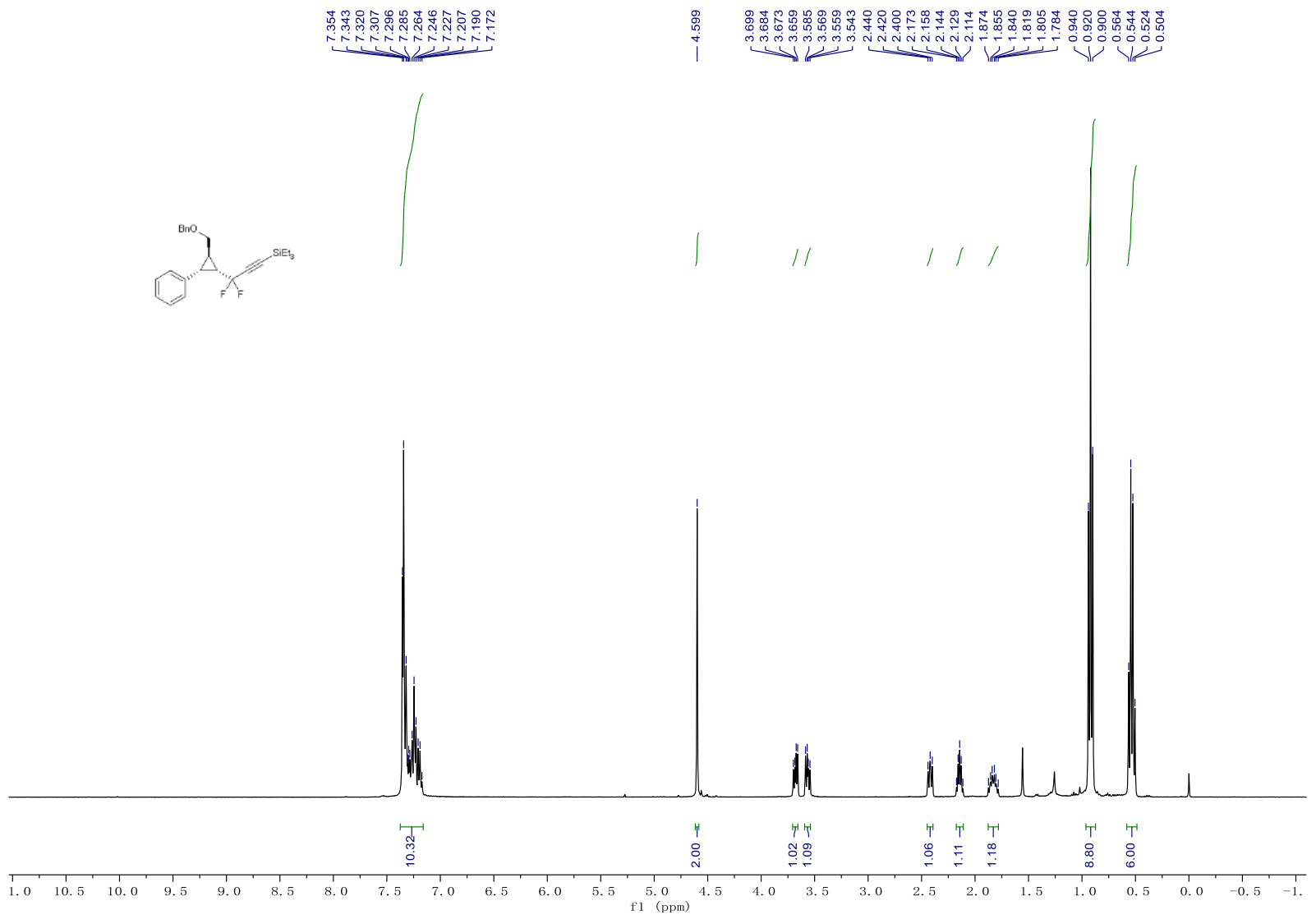



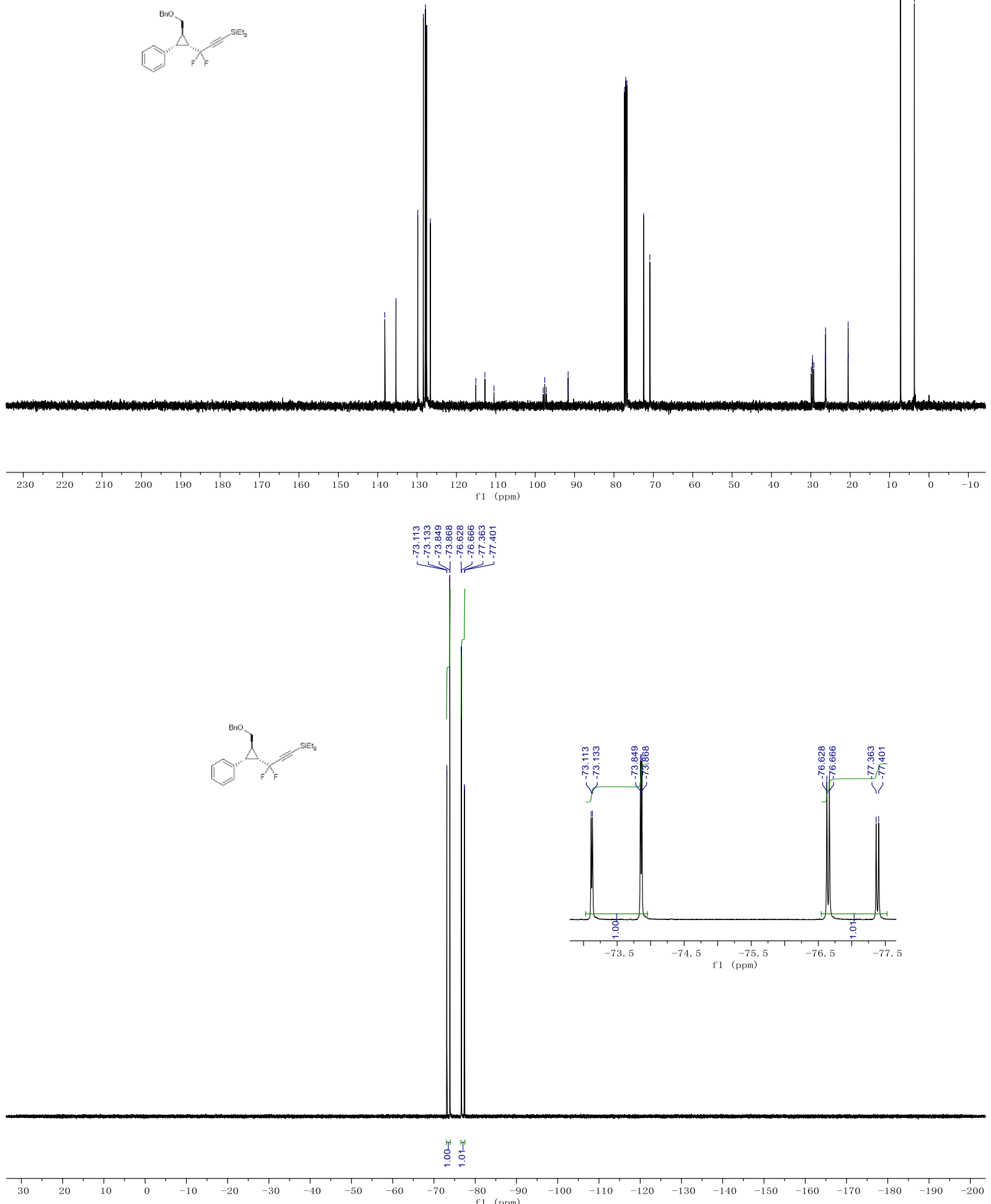


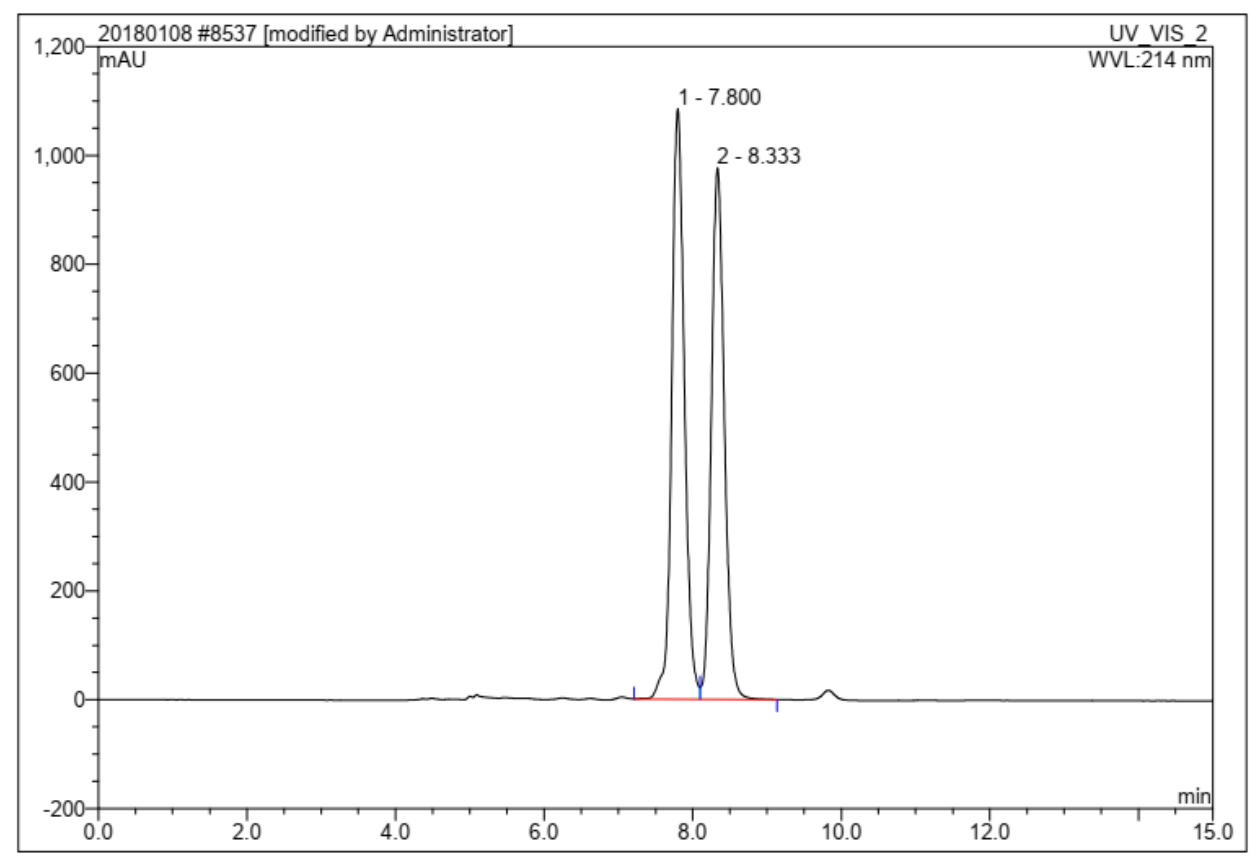

\begin{tabular}{|r|ccccccc|}
\hline No. & $\begin{array}{c}\text { Ret.Time } \\
\text { min }\end{array}$ & Peak Name & $\begin{array}{c}\text { Height } \\
\text { mAU }\end{array}$ & $\begin{array}{c}\text { Area } \\
\text { mAU*min }\end{array}$ & $\begin{array}{c}\text { Rel.Area } \\
\%\end{array}$ & Amount & Type \\
\hline 1 & 7.80 & n.a. & 1084.383 & 215.726 & 52.13 & n.a. & BM $^{*}$ \\
2 & 8.33 & n.a. & 976.624 & 198.066 & 47.87 & n.a. & MB $^{\star}$ \\
\hline Total: & & & 2061.008 & 413.792 & 100.00 & 0.000 & \\
\hline
\end{tabular}

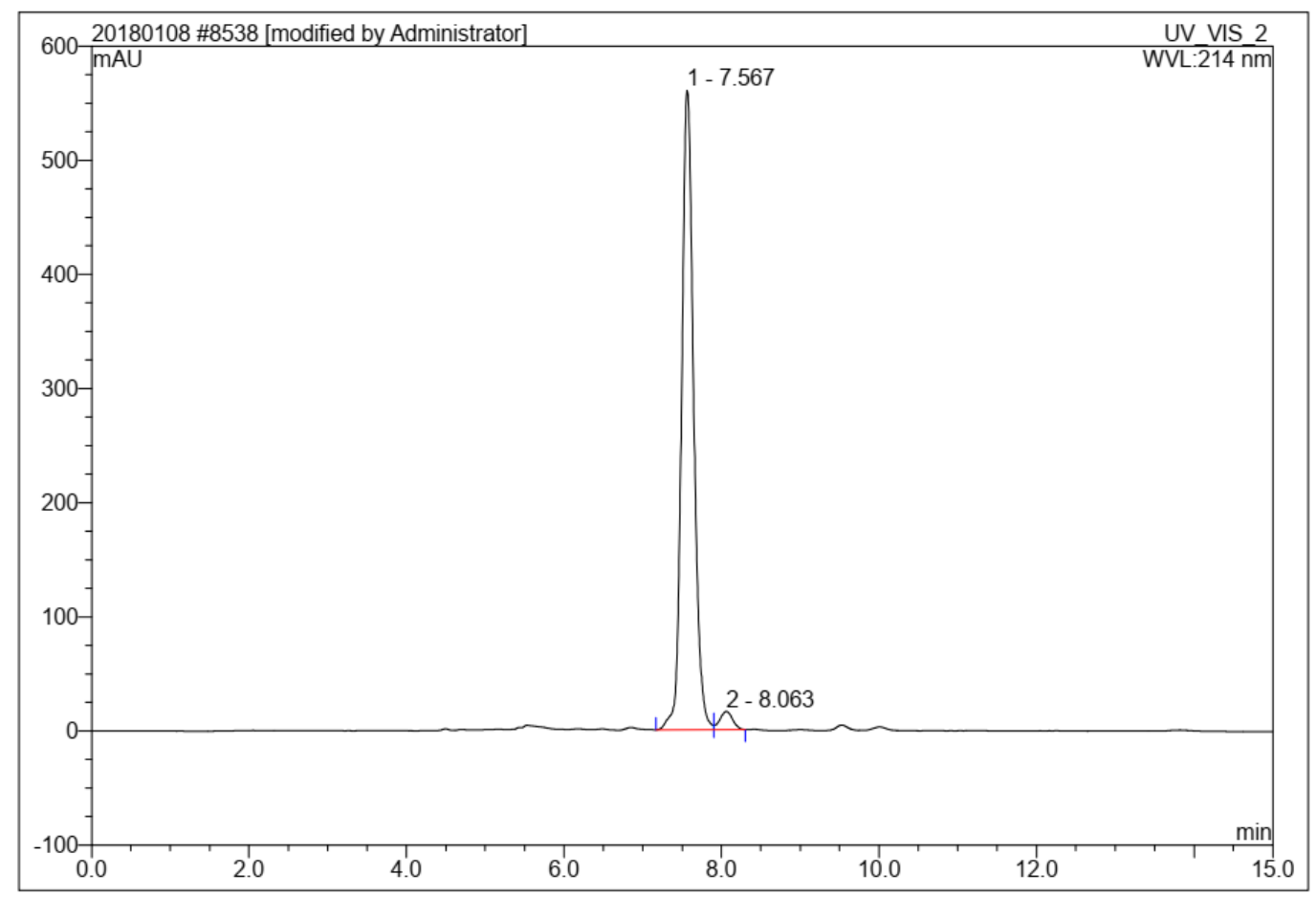

\begin{tabular}{|c|c|c|c|c|c|c|c|}
\hline No. & $\begin{array}{l}\text { Ret.Time } \\
\text { min }\end{array}$ & Peak Name & $\begin{array}{c}\text { Height } \\
\text { mAU }\end{array}$ & $\begin{array}{c}\text { Area } \\
\text { mAU*min }\end{array}$ & $\begin{array}{c}\text { Rel.Area } \\
\%\end{array}$ & Amount & Type \\
\hline 1 & 7.57 & n.a. & 560.112 & 99.038 & 97.08 & n.a. & BM \\
\hline 2 & 8.06 & n.a. & 15.922 & 2.982 & 2.92 & n.a. & MB \\
\hline Total: & & & 576.034 & 102.020 & 100.00 & 0.000 & \\
\hline
\end{tabular}


Triisopropyl-(3-((1R,2R)-2-phenylcyclopropyl)prop-1-yn-1-yl)silane (8a).
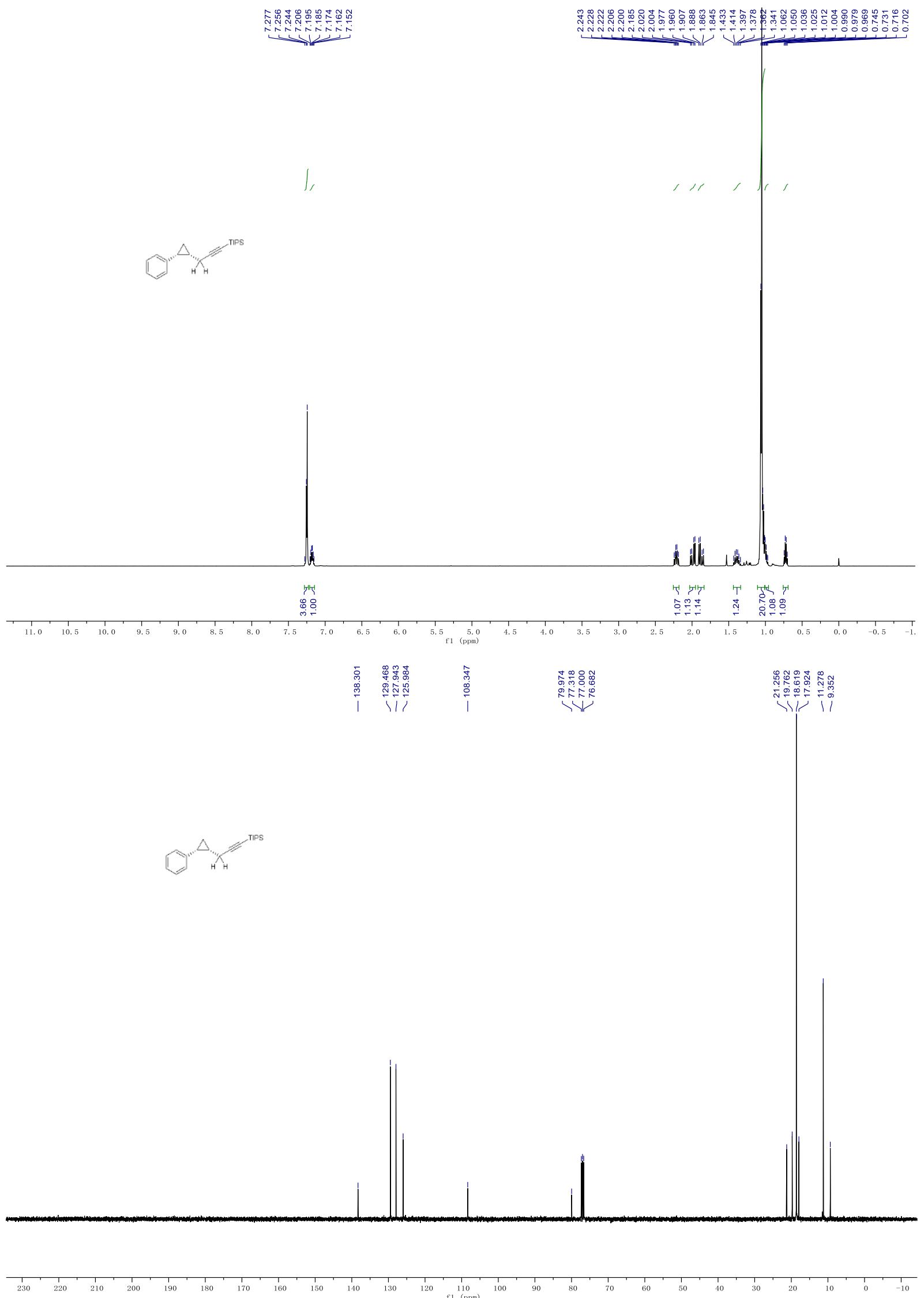


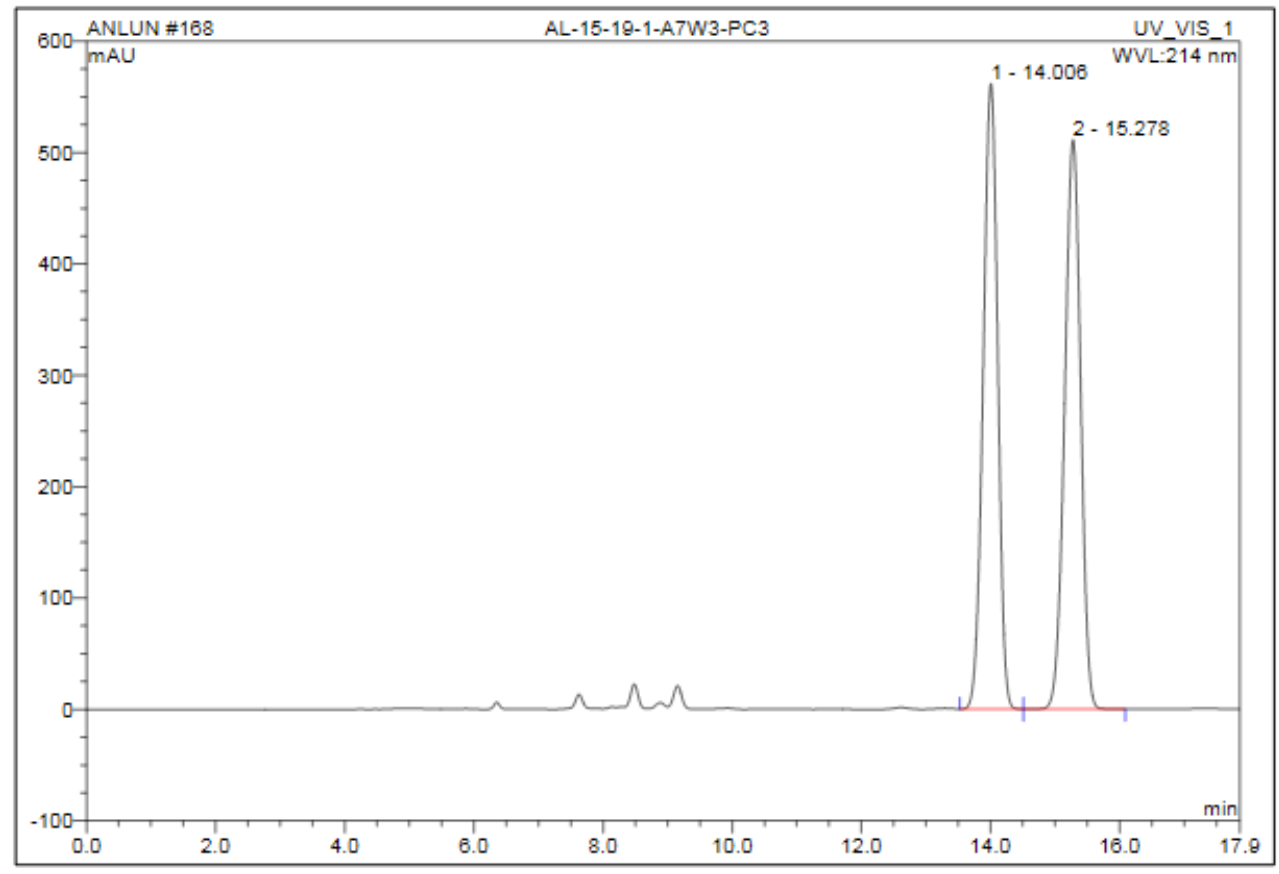

\begin{tabular}{|r|ccrrrrr|}
\hline No. & $\begin{array}{c}\text { Ret.Time } \\
\text { min }\end{array}$ & Peak Name & $\begin{array}{c}\text { Height } \\
\text { mAU }\end{array}$ & $\begin{array}{c}\text { Area } \\
\text { mAU*min }\end{array}$ & $\begin{array}{r}\text { Rel.Area } \\
\%\end{array}$ & Amount & Type \\
\hline 1 & 14.01 & n.a. & 561.848 & 146.640 & 49.99 & n.a. & BM \\
2 & 15.28 & n.a. & 511.499 & 146.713 & 50.01 & n.a. & MB \\
\hline Total: & & & 1073.347 & 293.353 & 100.00 & 0.000 & \\
\hline
\end{tabular}

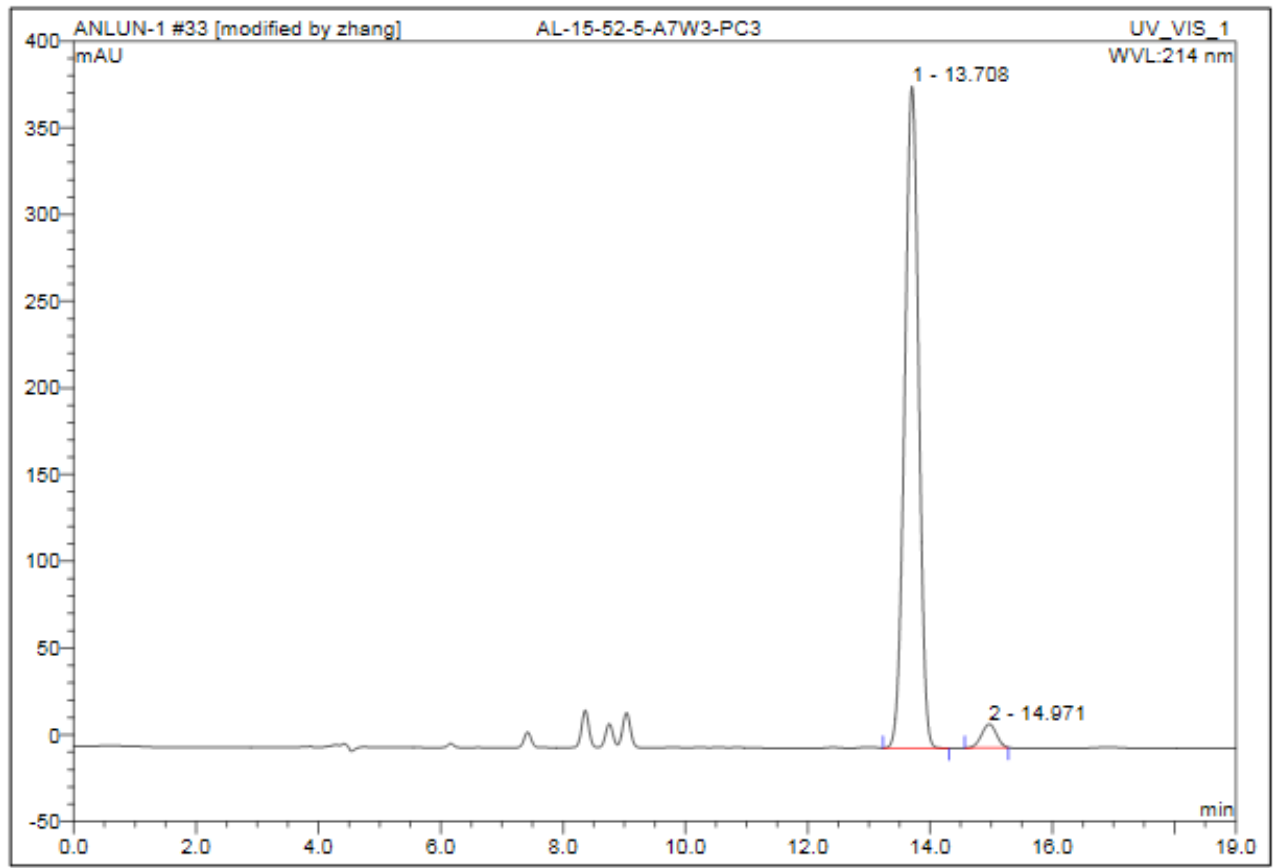

\begin{tabular}{|r|ccrrrrr|}
\hline No. & $\begin{array}{c}\text { Ret.Time } \\
\text { min }\end{array}$ & Peak Name & $\begin{array}{c}\text { Height } \\
\text { mAU }\end{array}$ & $\begin{array}{r}\text { Area } \\
\text { mAU*min }\end{array}$ & $\begin{array}{r}\text { Rel.Area } \\
\%\end{array}$ & Amount & Type \\
\hline 1 & 13.71 & n.a. & 381.548 & 101.385 & 96.34 & n.a. & BMB \\
2 & 14.97 & n.a. & 13.611 & 3.849 & 3.66 & n.a. & BMB $^{\star}$ \\
\hline Total: & & & 395.159 & 105.234 & 100.00 & 0.000 & \\
\hline
\end{tabular}


Triisopropyl-(3-((1R,2R)-2-(4-(trifluoromethoxy)phenyl)cyclopropyl)prop-1-yn-1-yl)silane (8b).

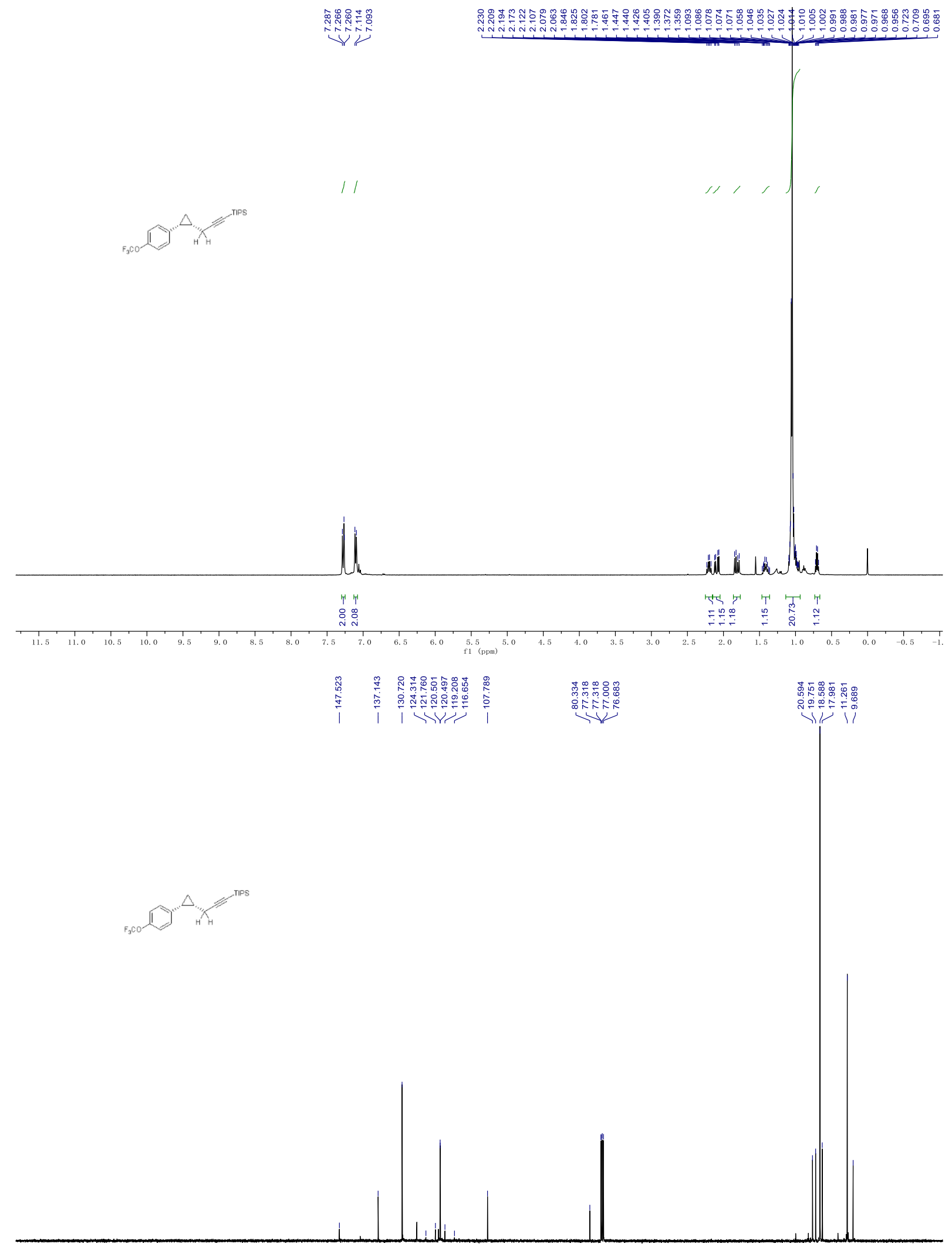

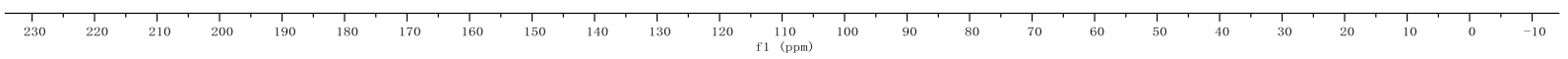



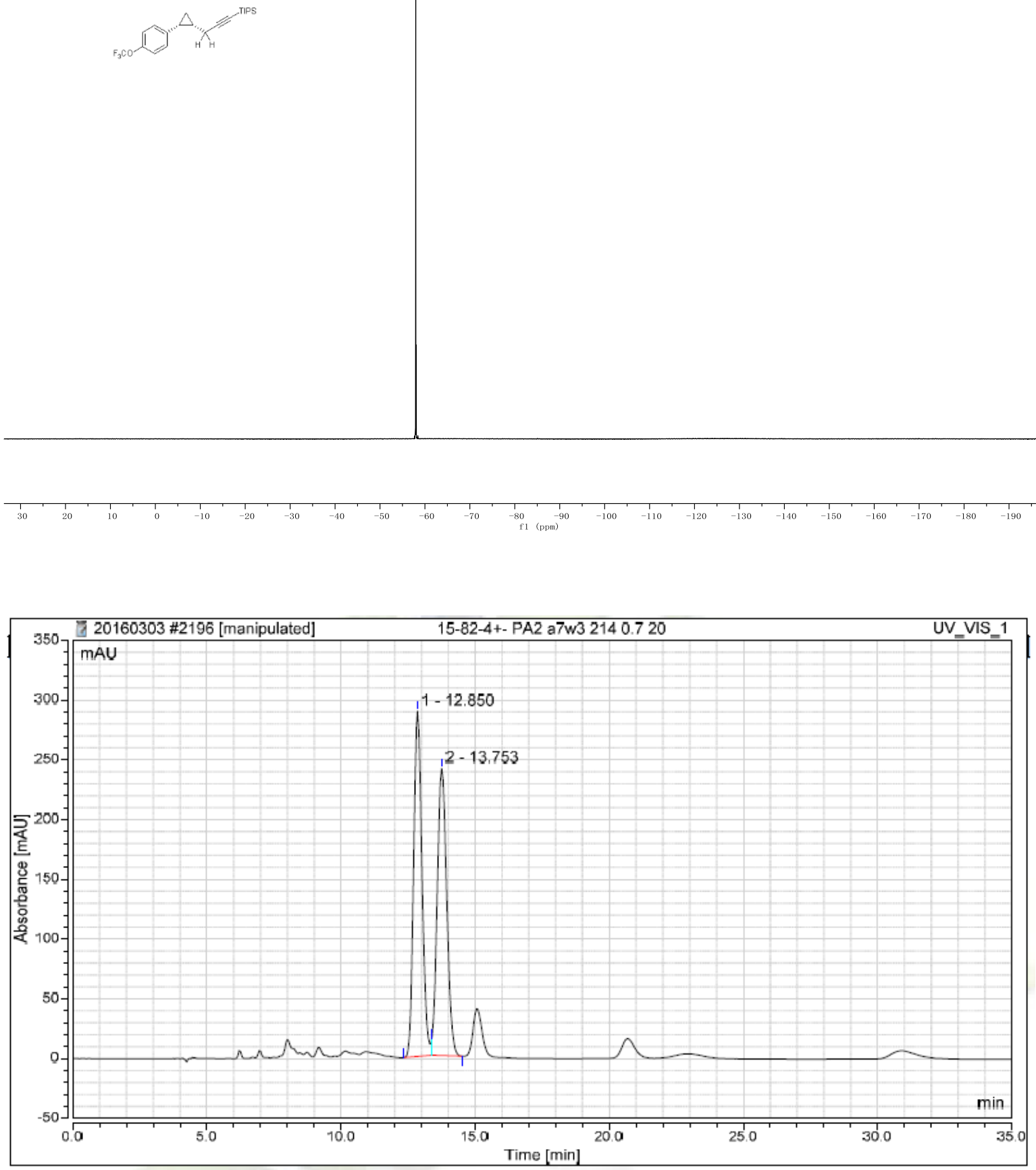

\begin{tabular}{|c|c|c|c|c|c|c|}
\hline \multicolumn{7}{|c|}{ Integration Results } \\
\hline No. & $\begin{array}{l}\text { Retention Time } \\
\text { min }\end{array}$ & $\begin{array}{c}\text { Retention Time } \\
\text { min }\end{array}$ & $\begin{array}{c}\text { Area } \\
\mathrm{mAU}^{*} \text { min }\end{array}$ & $\begin{array}{c}\text { Height } \\
\text { mAU }\end{array}$ & $\begin{array}{c}\text { Relative Area } \\
\%\end{array}$ & \multirow[t]{3}{*}{ Relativ } \\
\hline 1 & 13 & 12.850 & 104.197 & 288.898 & 51.41 & \\
\hline \multicolumn{2}{|c|}{ Total: } & 202.683 & 529.321 & 100.00 & & \\
\hline
\end{tabular}




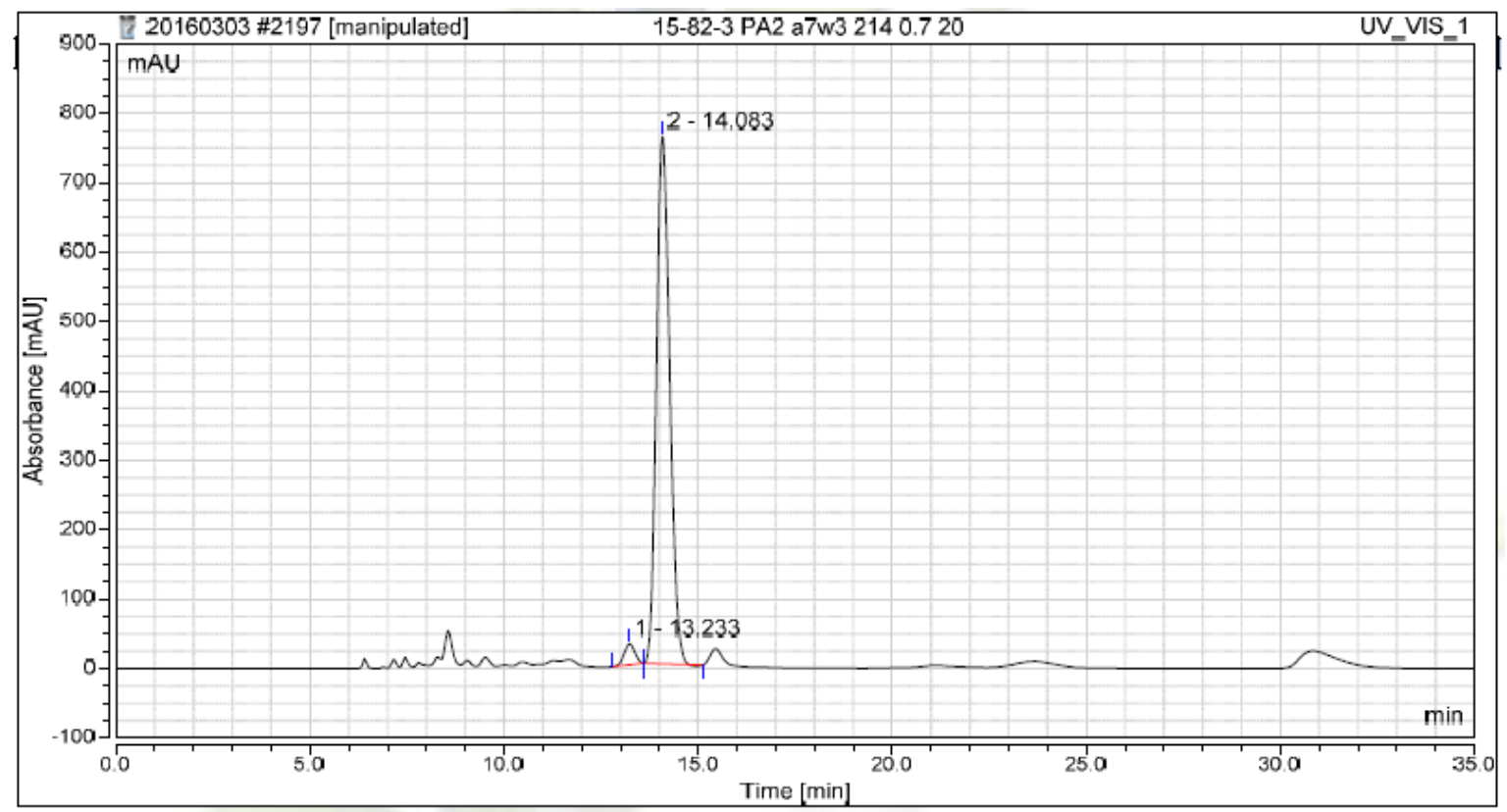

\begin{tabular}{|c|c|c|c|c|c|c|}
\hline \multicolumn{7}{|c|}{ Integration Results } \\
\hline No. & \begin{tabular}{|l|} 
Retention Time \\
min
\end{tabular} & $\begin{array}{c}\text { Retention Time } \\
\text { min }\end{array}$ & $\begin{array}{c}\text { Area } \\
\mathrm{mAU}^{*} \min \end{array}$ & $\begin{array}{c}\text { Height } \\
\text { mAU }\end{array}$ & $\begin{array}{c}\text { Relative Area } \\
\%\end{array}$ & \multirow[t]{3}{*}{ Relativ } \\
\hline 1 & 13 & 13.233 & 9.617 & 30.304 & 3.07 & \\
\hline \multicolumn{2}{|c|}{ Total: } & 313.423 & 790.312 & 100.00 & & \\
\hline
\end{tabular}

Triisopropyl(3-((1R,2R)-2-(4-(trimethylsilyl)phenyl)cyclopropyl)prop-1-yn-1-yl)silane (8c).

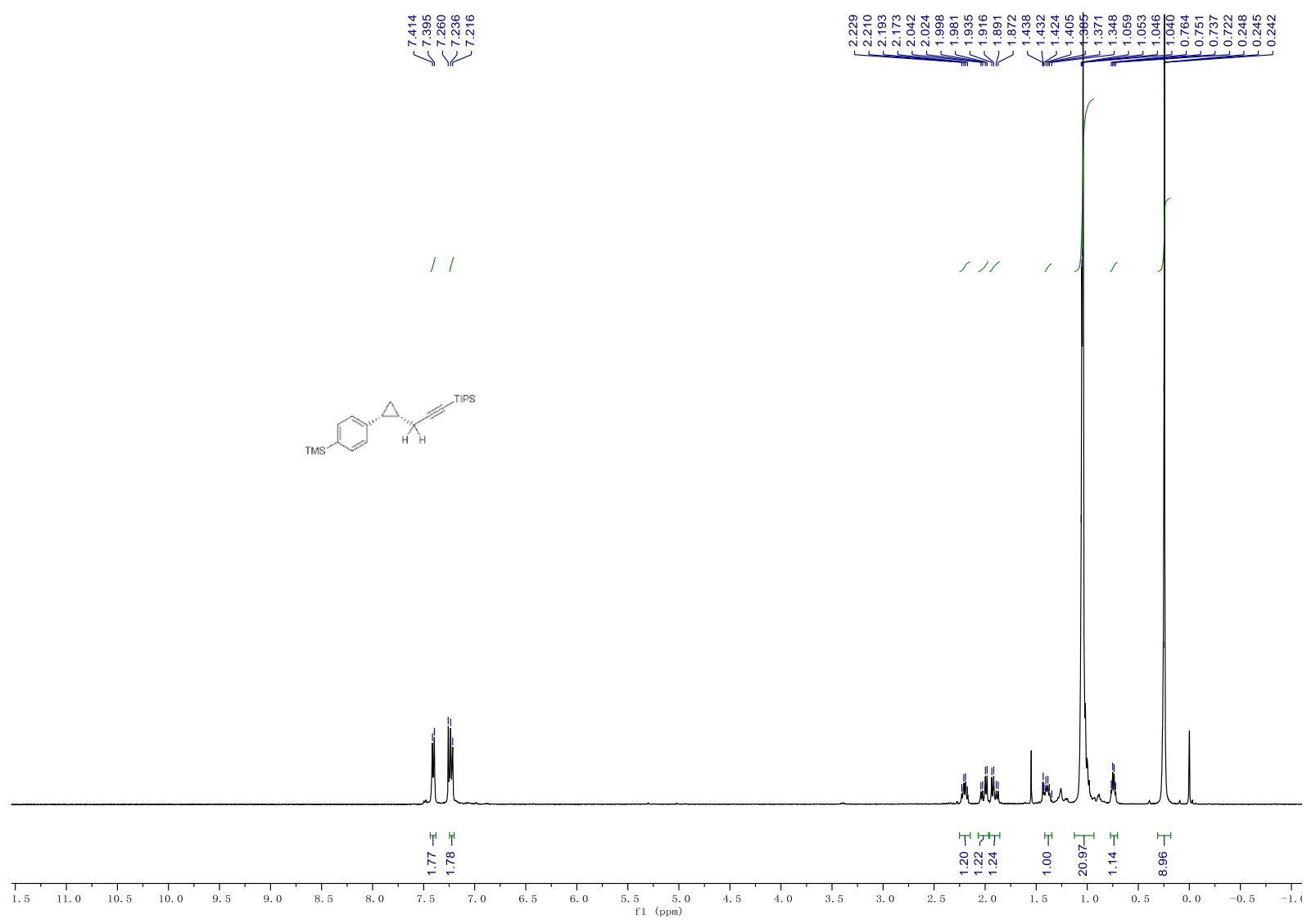



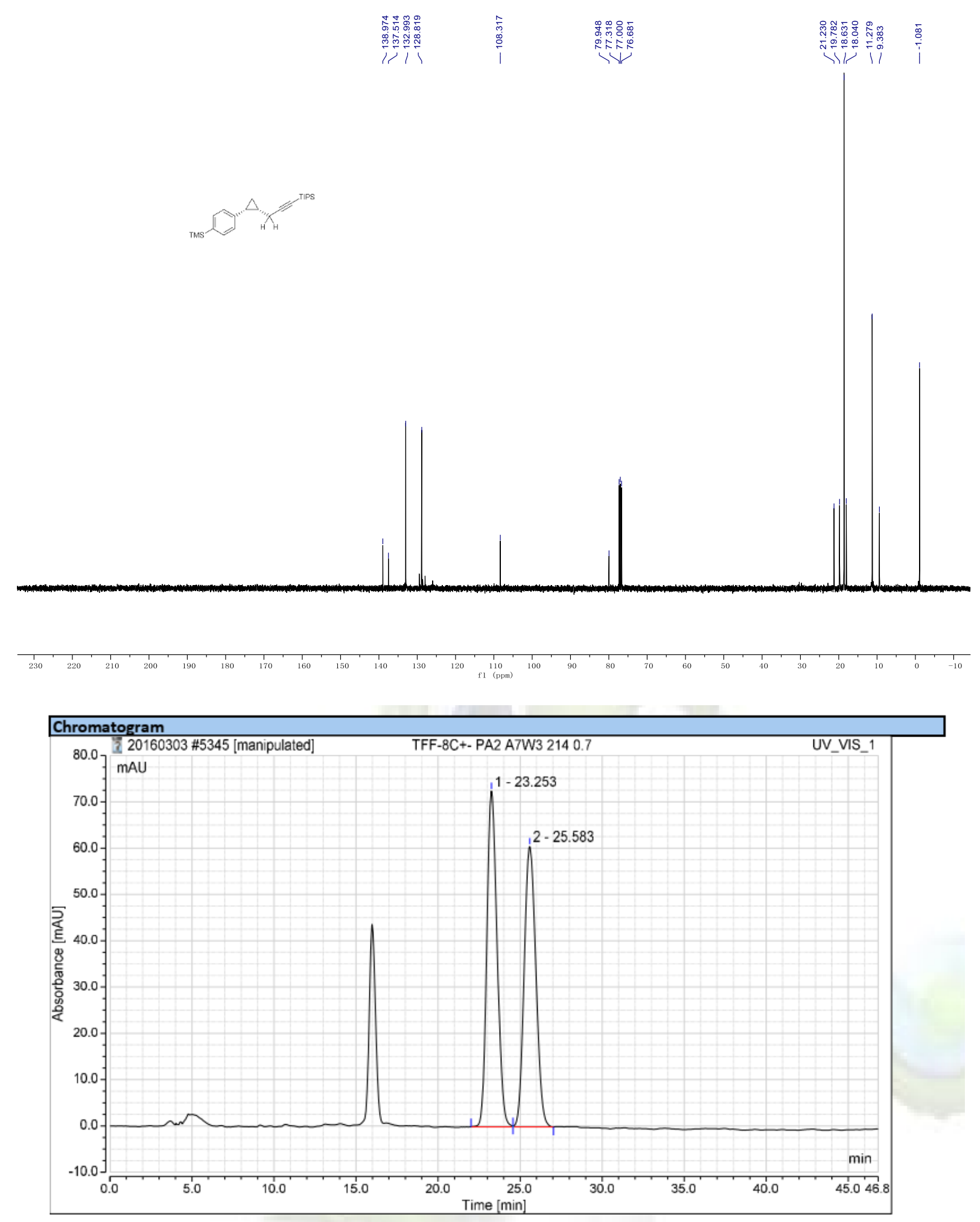

\begin{tabular}{|c|c|c|c|c|}
\hline \multicolumn{5}{|c|}{ Integration Results } \\
\hline No. & $\begin{array}{l}\text { Retention Time } \\
\text { min }\end{array}$ & $\begin{array}{c}\text { Area } \\
m A U^{*} \text { min }\end{array}$ & $\begin{array}{c}\text { Height } \\
\text { mAU }\end{array}$ & $\begin{array}{c}\text { Relative Area } \\
\%\end{array}$ \\
\hline 1 & 23.253 & 52.5110 & 72.5472 & 51.315 \\
\hline 2 & 25.583 & 49.8197 & 60.5998 & 48.685 \\
\hline \multicolumn{2}{|c|}{ Total: } & 102.331 & 1401.998 & 100.000 \\
\hline
\end{tabular}




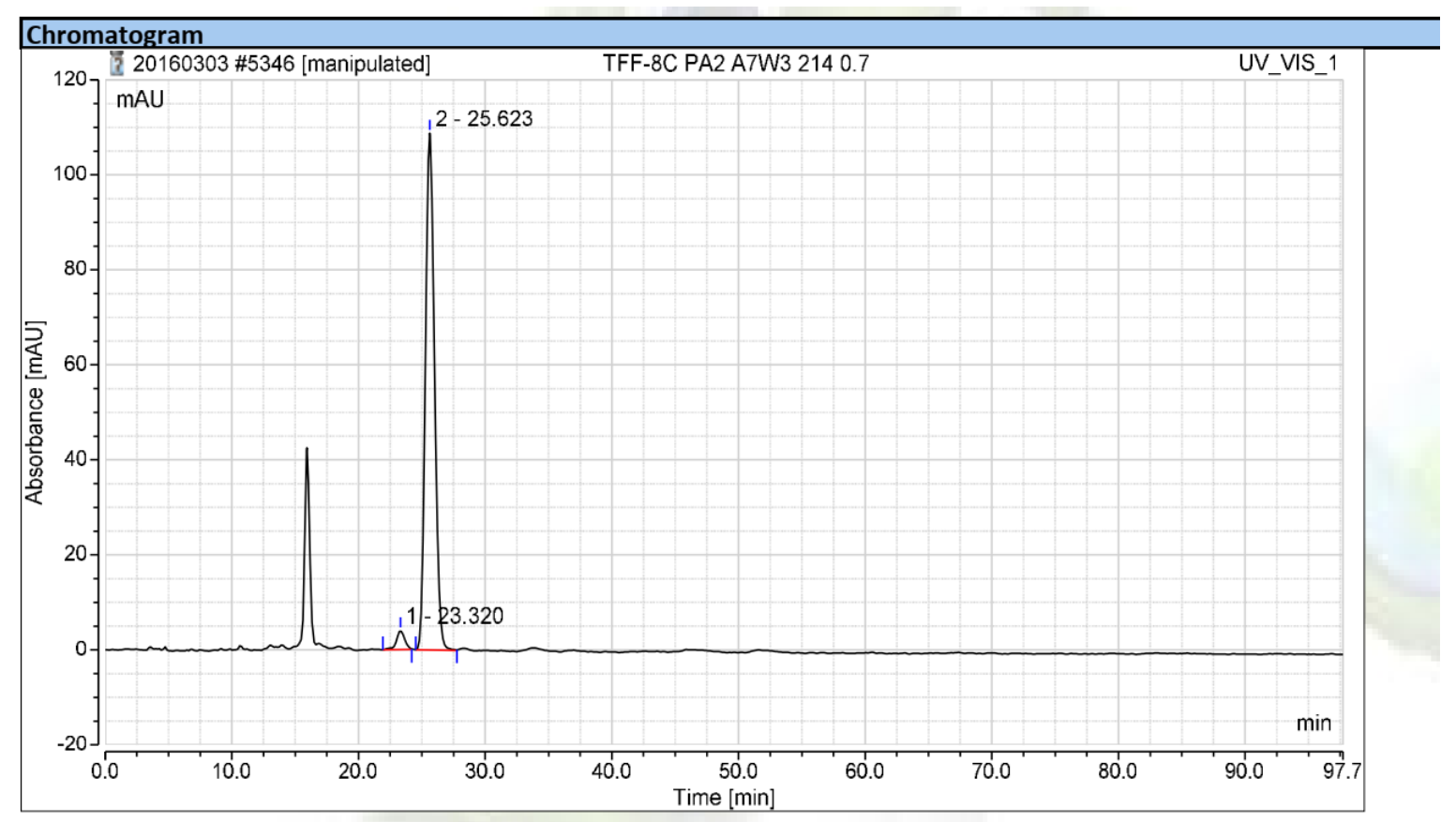

\begin{tabular}{|c|c|c|c|c|}
\hline \multicolumn{5}{|c|}{ Integration Results } \\
\hline No. & $\begin{array}{c}\text { Retention Time } \\
\text { min }\end{array}$ & $\begin{array}{c}\text { Area } \\
m A U^{*} \min \end{array}$ & $\begin{array}{l}\text { Height } \\
\text { mAU }\end{array}$ & $\begin{array}{c}\text { Relative Area } \\
\%\end{array}$ \\
\hline $\begin{array}{l}1 \\
2 \\
\end{array}$ & $\begin{array}{l}23.320 \\
25.623 \\
\end{array}$ & $\begin{array}{c}2.9894 \\
91.0194 \\
\end{array}$ & $\begin{array}{c}3.9510 \\
108.7552 \\
\end{array}$ & $\begin{array}{c}3.180 \\
96.820 \\
\end{array}$ \\
\hline \multicolumn{2}{|c|}{ Total: } & 94.009 & 1401.998 & 100.000 \\
\hline
\end{tabular}

\section{(3-((1R,2R)-2-(4-Chlorophenyl)cyclopropyl)prop-1-yn-1-yl)triisopropylsilane (8d).}

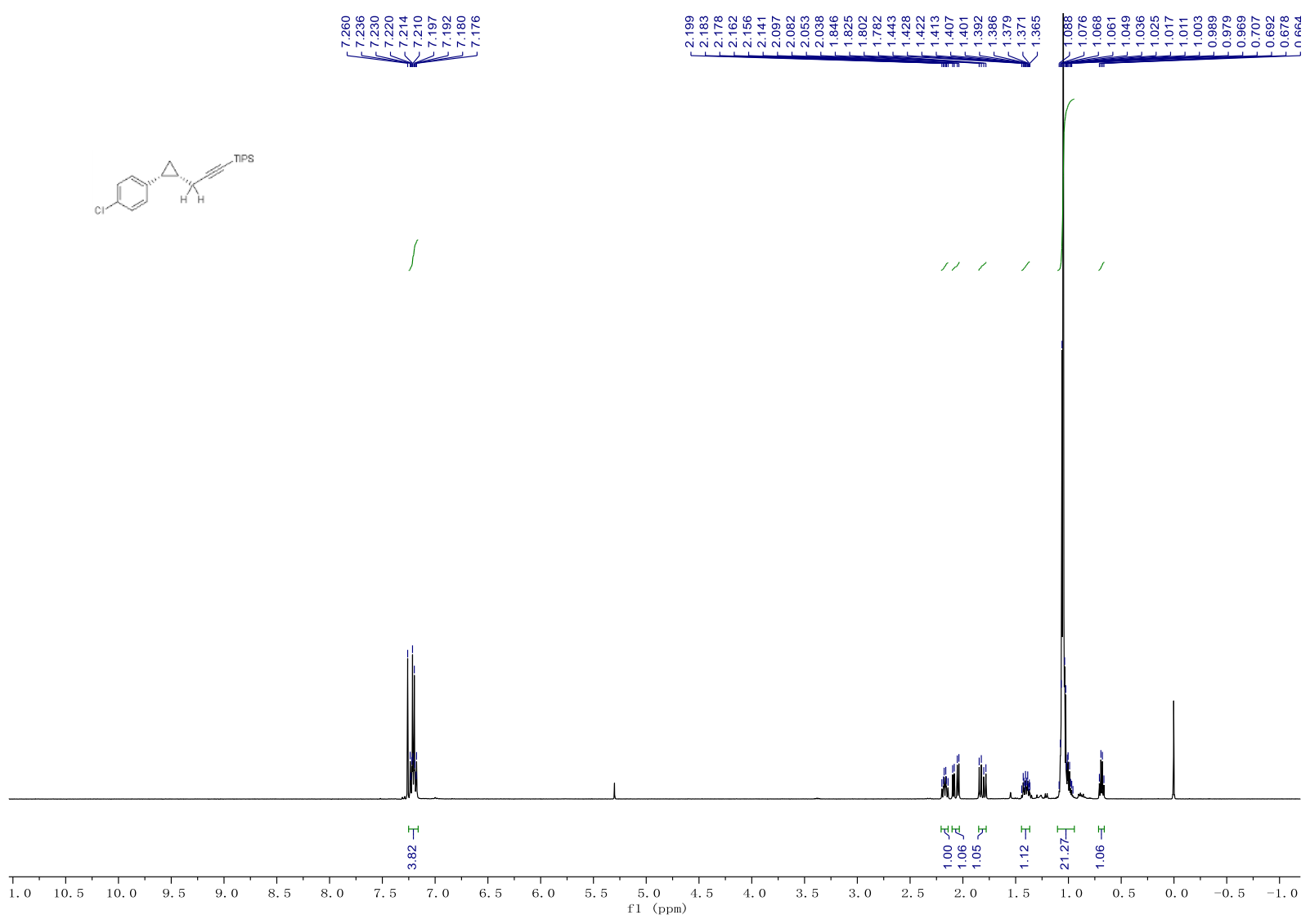



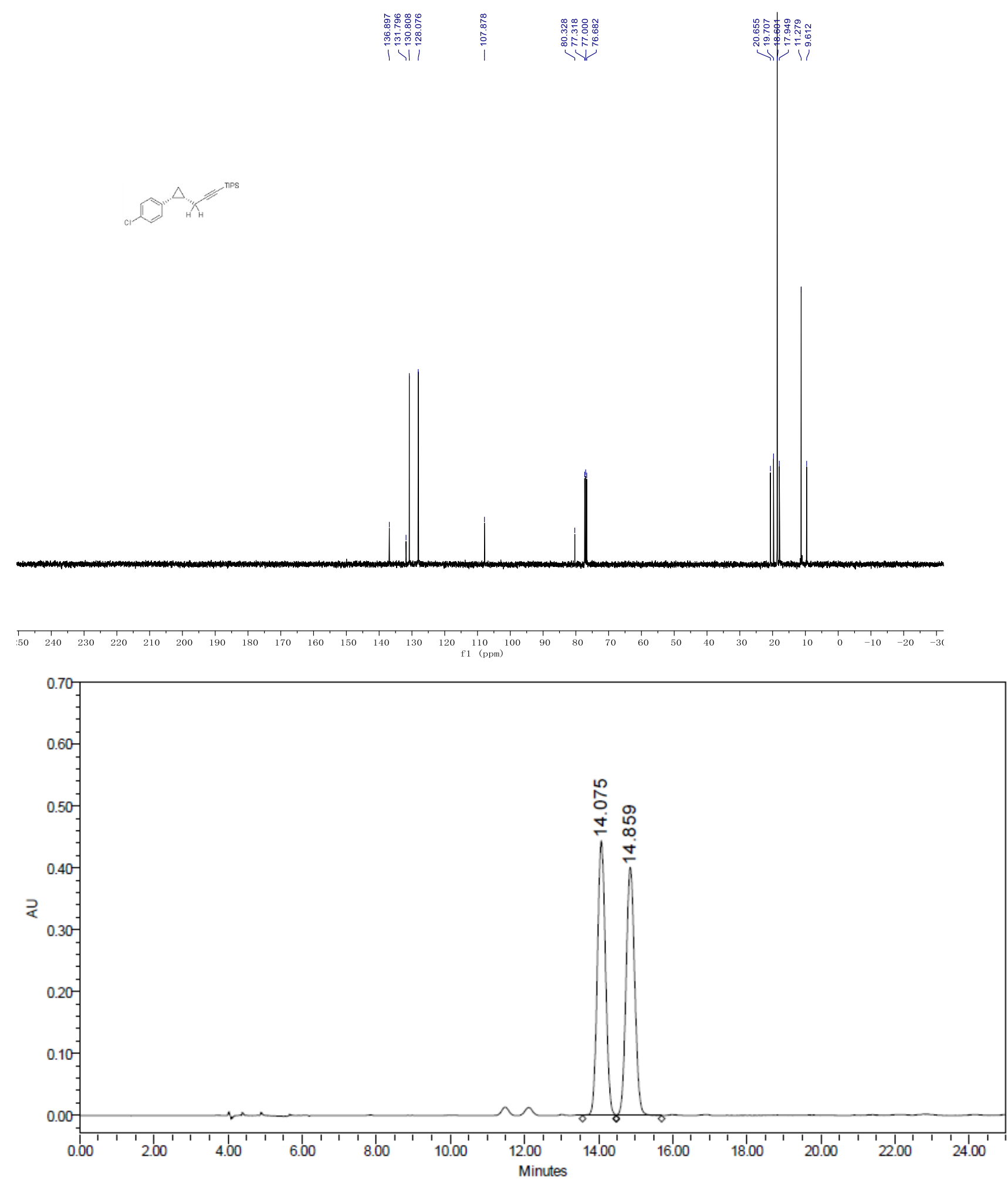

Peak Results
\begin{tabular}{|c|c|c|c|r|}
\hline & RT & Area & Height & $\%$ Area \\
\hline 1 & 14.075 & 6756042 & 444433 & 51.01 \\
\hline 2 & 14.859 & 6487852 & 401438 & 48.99 \\
\hline
\end{tabular}




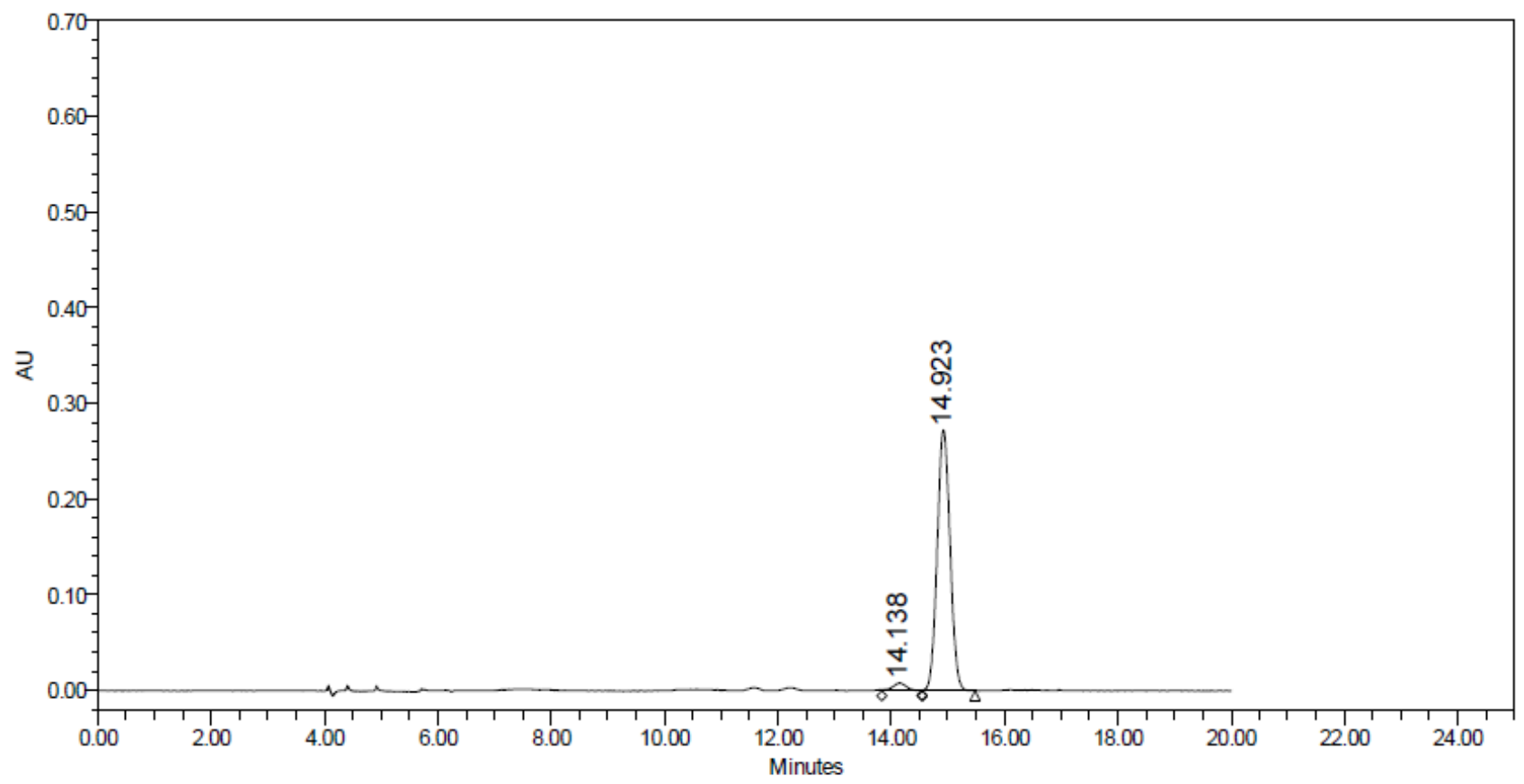

Peak Results
\begin{tabular}{|c|c|c|r|r|}
\hline & RT & Area & Height & $\%$ Area \\
\hline 1 & 14.138 & 145684 & 8270 & 3.27 \\
\hline 2 & 14.923 & 4309182 & 272672 & 96.73 \\
\hline
\end{tabular}

Triisopropyl-(3-((1S,2S,3R)-2-methyl-3-phenylcyclopropyl)prop-1-yn-1-yl)silane (8e).

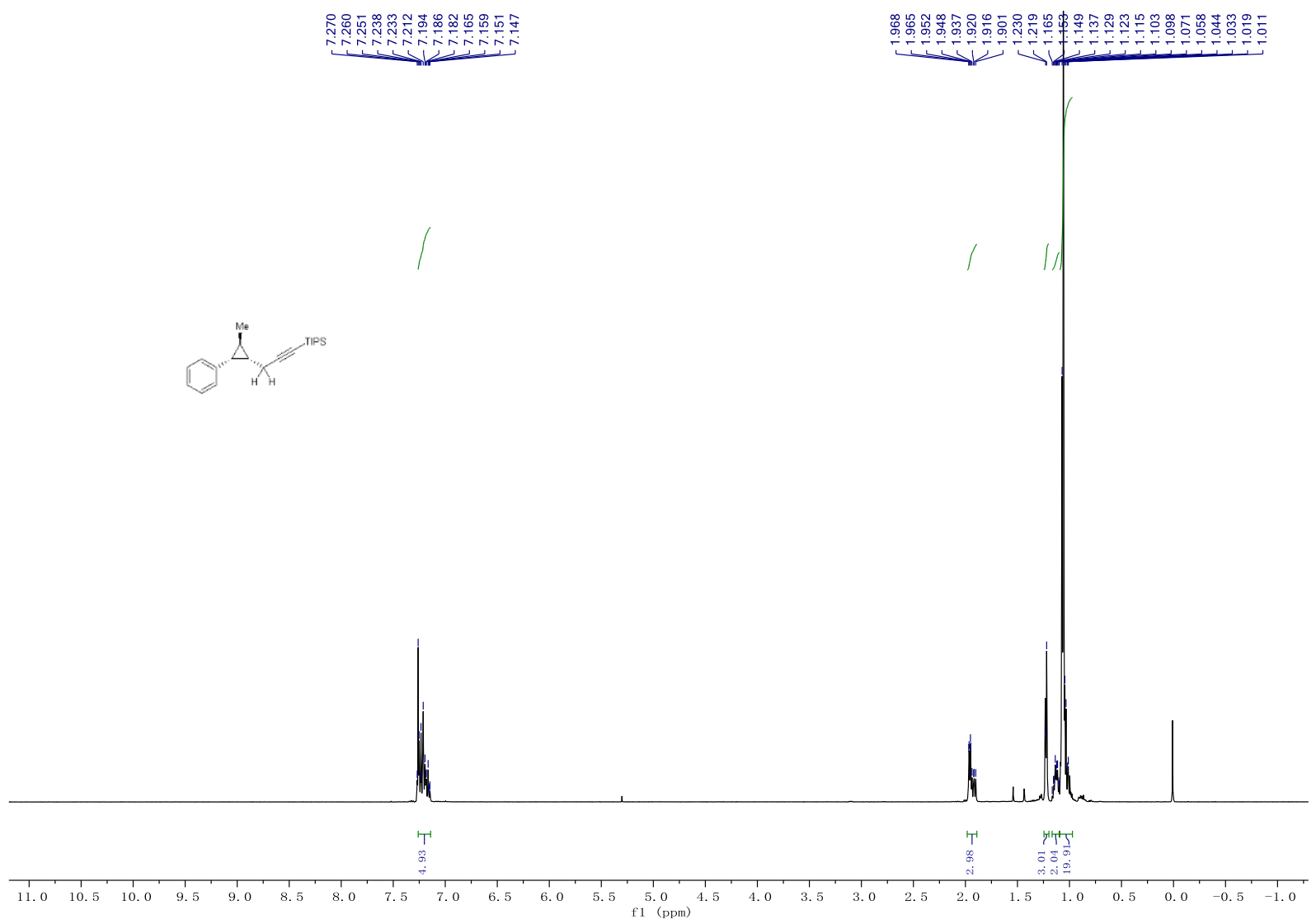



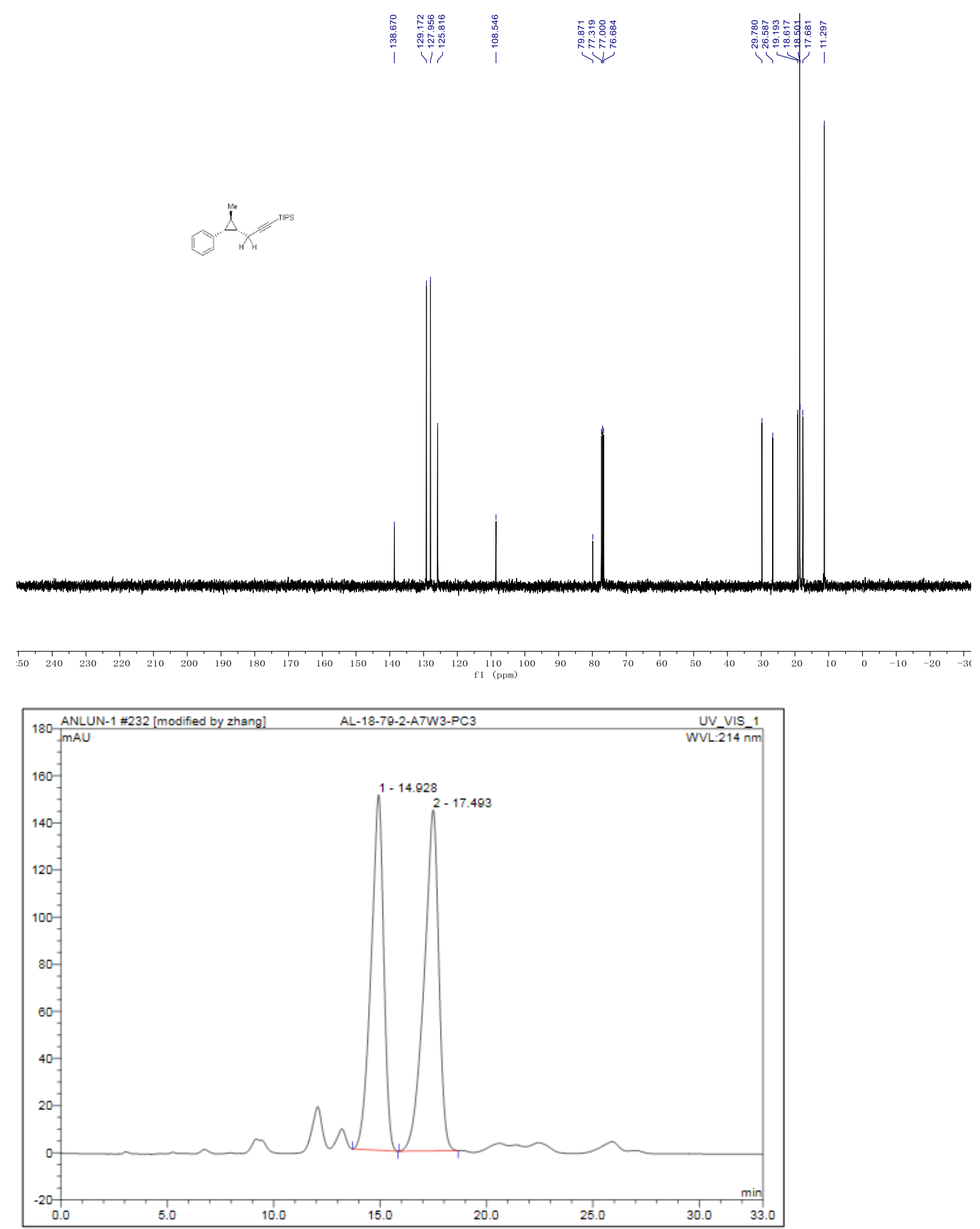

\begin{tabular}{|r|cccrccc|}
\hline No. & $\begin{array}{c}\text { Ret.Time } \\
\text { min }\end{array}$ & Peak Name & $\begin{array}{c}\text { Height } \\
\text { mAU }\end{array}$ & $\begin{array}{c}\text { Area } \\
\text { mAU*min }\end{array}$ & $\begin{array}{c}\text { Rel.Area } \\
\%\end{array}$ & Amount & Type \\
\hline 1 & 14.93 & n.a. & 150.912 & 104.104 & 47.32 & n.a. & BMB \\
2 & 17.49 & n.a. & 144.742 & 115.907 & 52.68 & n.a. & BMB \\
\hline Total: & & & 295.654 & 220.012 & 100.00 & 0.000 & \\
\hline
\end{tabular}




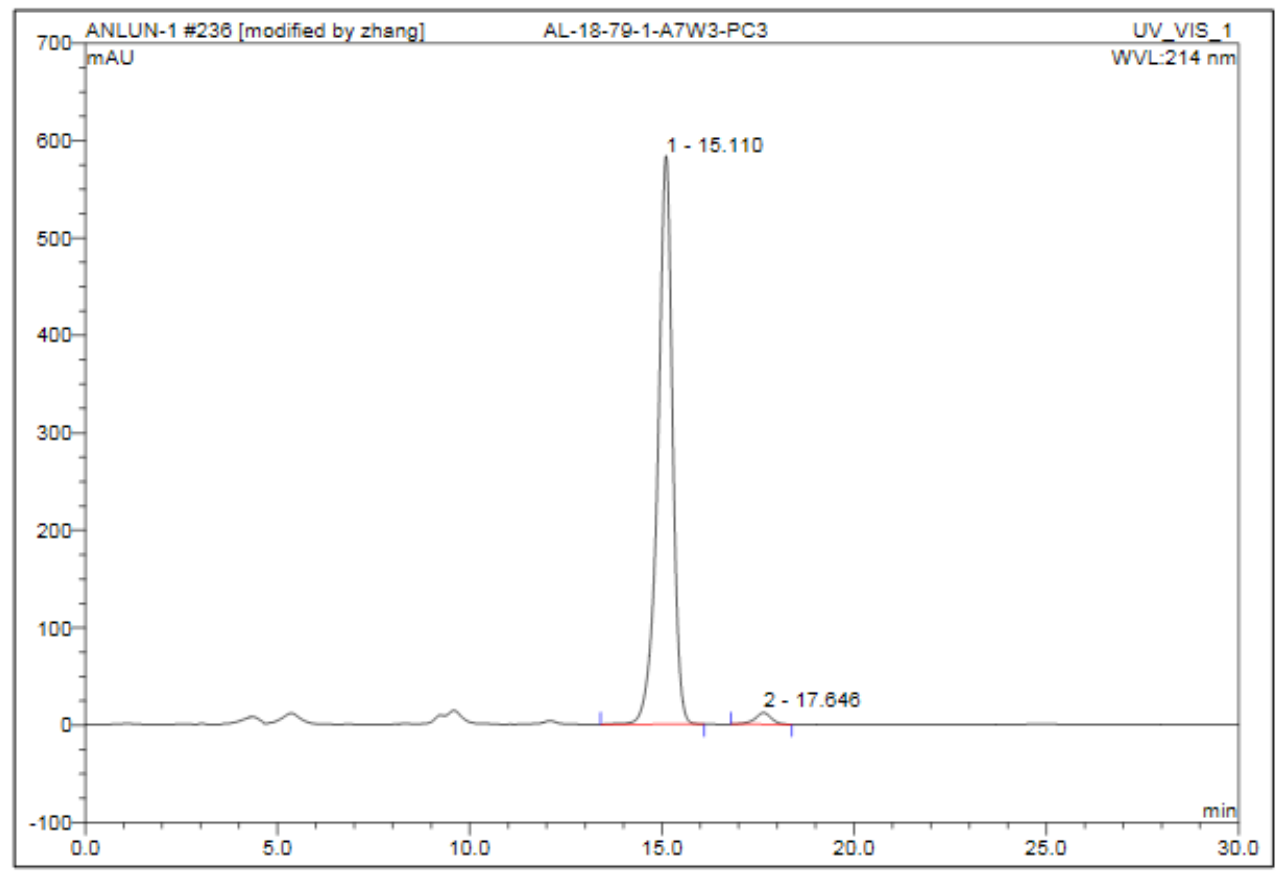

\begin{tabular}{|r|ccrrrrr|}
\hline No. & $\begin{array}{c}\text { Ret.Time } \\
\text { min }\end{array}$ & Peak Name & $\begin{array}{c}\text { Height } \\
\text { mAU }\end{array}$ & $\begin{array}{c}\text { Area } \\
\text { mAU*min }\end{array}$ & $\begin{array}{r}\text { Rel.Area } \\
\%\end{array}$ & Amount & Type \\
\hline 1 & 15.11 & n.a. & 583.102 & 257.519 & 97.75 & n.a. & BMB \\
2 & 17.65 & n.a. & 11.946 & 5.936 & 2.25 & n.a. & BMB \\
\hline Total: & & & 595.049 & 263.455 & 100.00 & 0.000 & \\
\hline
\end{tabular}

((1R,2S)-2-Allylcyclopropyl)benzene (9a).

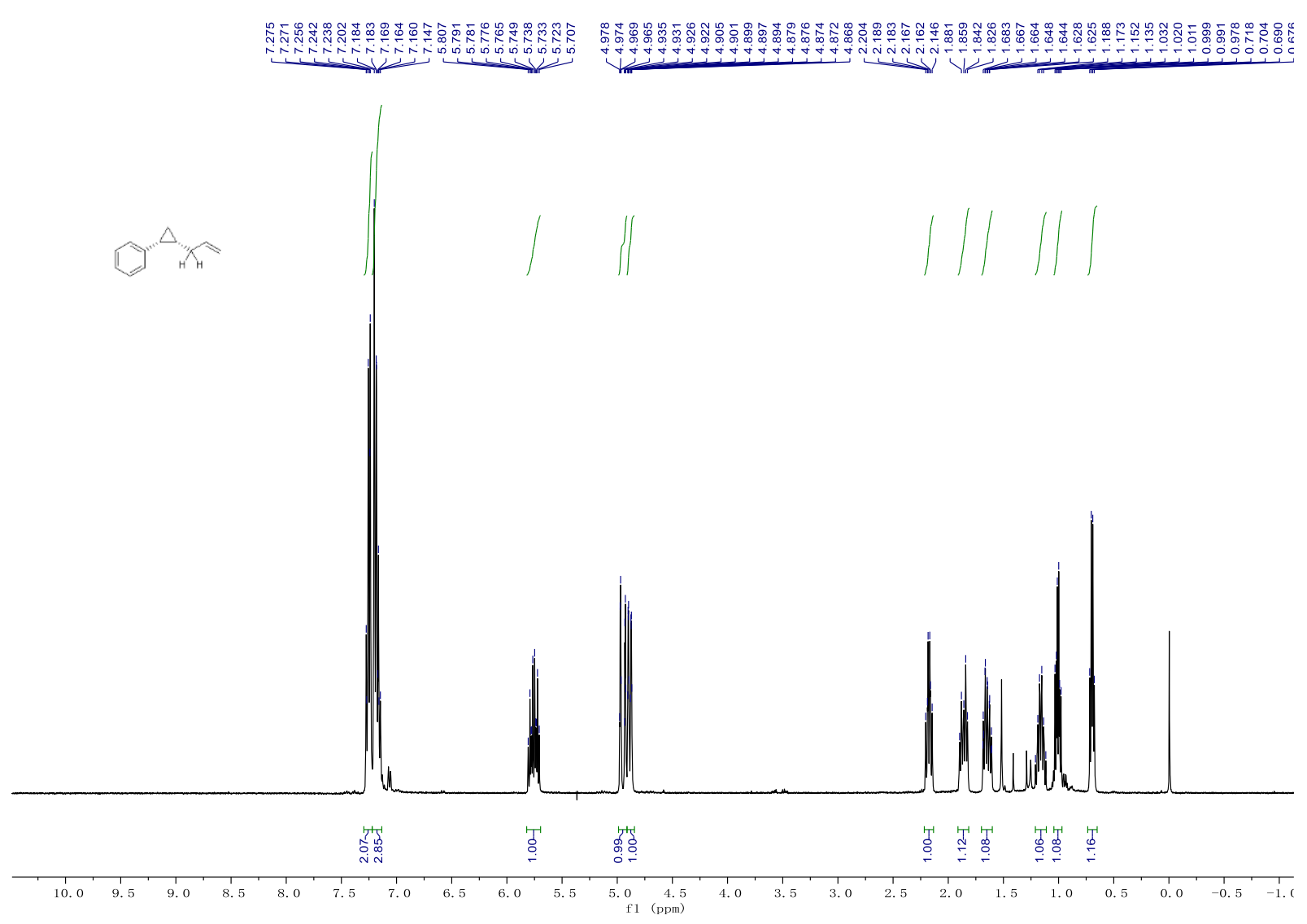



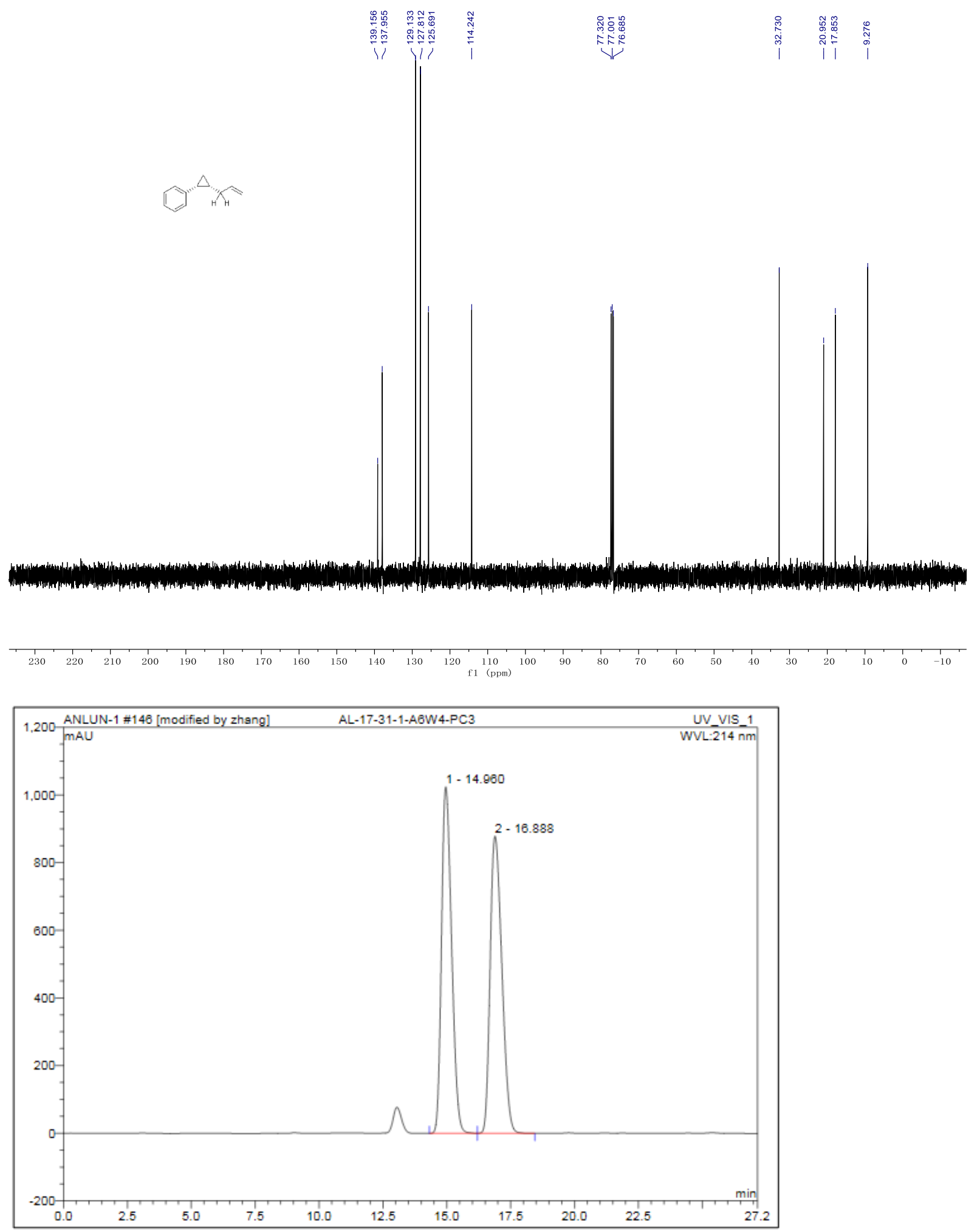

\begin{tabular}{|r|ccrrrrr|r|}
\hline No. & $\begin{array}{c}\text { Ret.Time } \\
\text { min }\end{array}$ & Peak Name & $\begin{array}{c}\text { Height } \\
\text { mAU }\end{array}$ & $\begin{array}{c}\text { Area } \\
\text { mAU*min }\end{array}$ & $\begin{array}{c}\text { Rel.Area } \\
\%\end{array}$ & Amount & Type \\
\hline 1 & 14.96 & n.a. & 1024.563 & 468.212 & 50.00 & n.a. & BM \\
2 & 16.89 & n.a. & 879.023 & 468.210 & 50.00 & n.a. & MB \\
\hline Total: & & & 1903.586 & 936.422 & 100.00 & 0.000 & \\
\hline
\end{tabular}




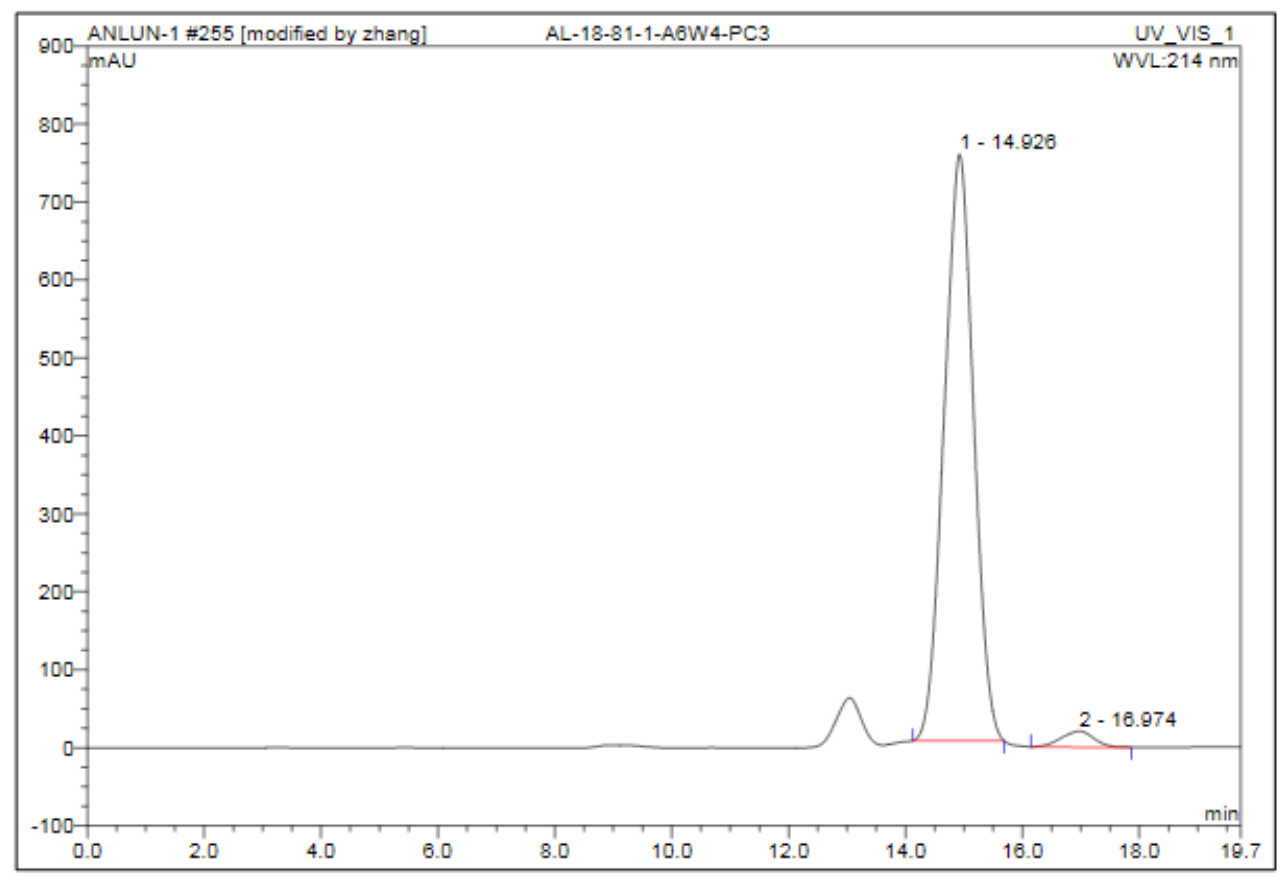

\begin{tabular}{|r|ccrrrrr|}
\hline No. & $\begin{array}{c}\text { Ret.Time } \\
\text { min }\end{array}$ & Peak Name & $\begin{array}{c}\text { Height } \\
\text { mAU }\end{array}$ & $\begin{array}{c}\text { Area } \\
\text { mAU*min }\end{array}$ & $\begin{array}{r}\text { Rel.Area } \\
\%\end{array}$ & Amount & Type \\
\hline 1 & 14.93 & n.a. & 752.301 & 438.867 & 97.13 & n.a. & BMB $^{\star}$ \\
2 & 16.97 & n.a. & 20.378 & 12.989 & 2.87 & n.a. & BMB \\
\hline Total: & & & 772.679 & 451.856 & 100.00 & 0.000 & \\
\hline
\end{tabular}

((1R,2R)-2-(2-Methylallyl)cyclopropyl)benzene (9b).

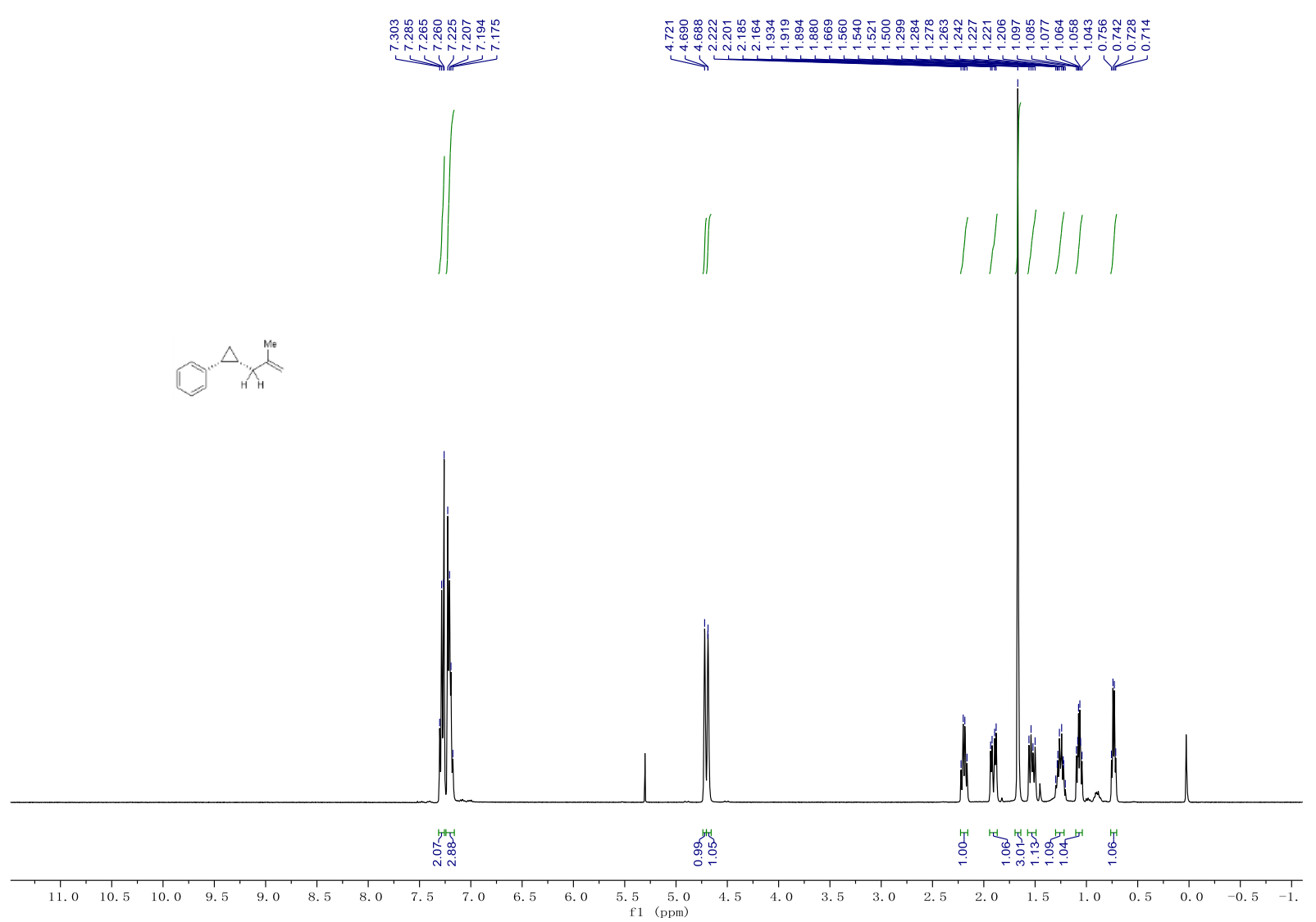



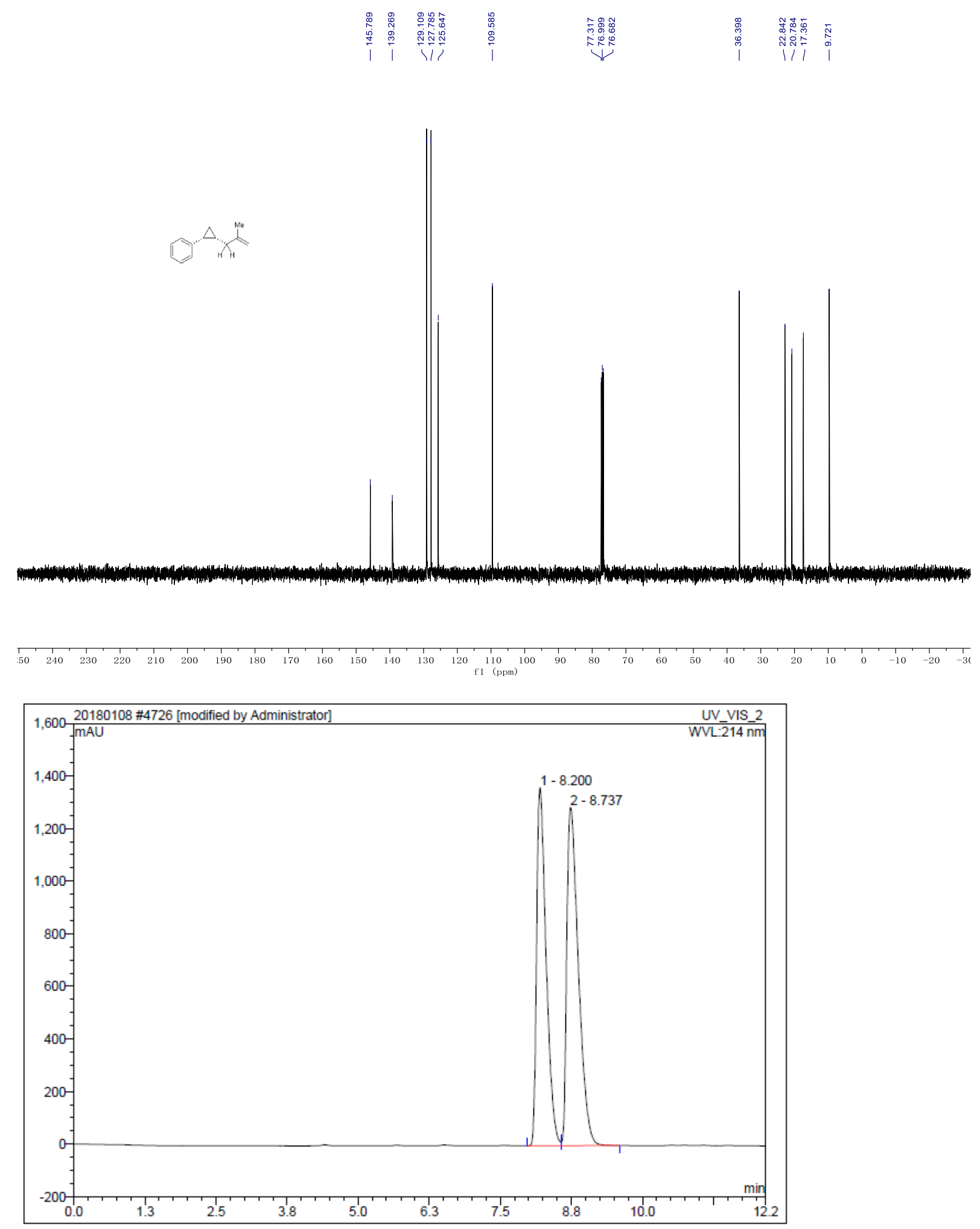

\begin{tabular}{|c|cccrrrr|}
\hline No. & $\begin{array}{c}\text { Ret.Time } \\
\text { min }\end{array}$ & Peak Name & $\begin{array}{c}\text { Height } \\
\text { mAU }\end{array}$ & $\begin{array}{c}\text { Area } \\
\text { mAU*min }\end{array}$ & $\begin{array}{c}\text { Rel.Area } \\
\%\end{array}$ & Amount & Type \\
\hline 1 & 8.20 & n.a. & 1362.158 & 265.217 & 46.50 & n.a. & BM \\
2 & 8.74 & n.a. & 1286.714 & 305.163 & 53.50 & n.a. & MB \\
\hline Total: & & & 2648.872 & 570.379 & 100.00 & 0.000 & \\
\hline
\end{tabular}




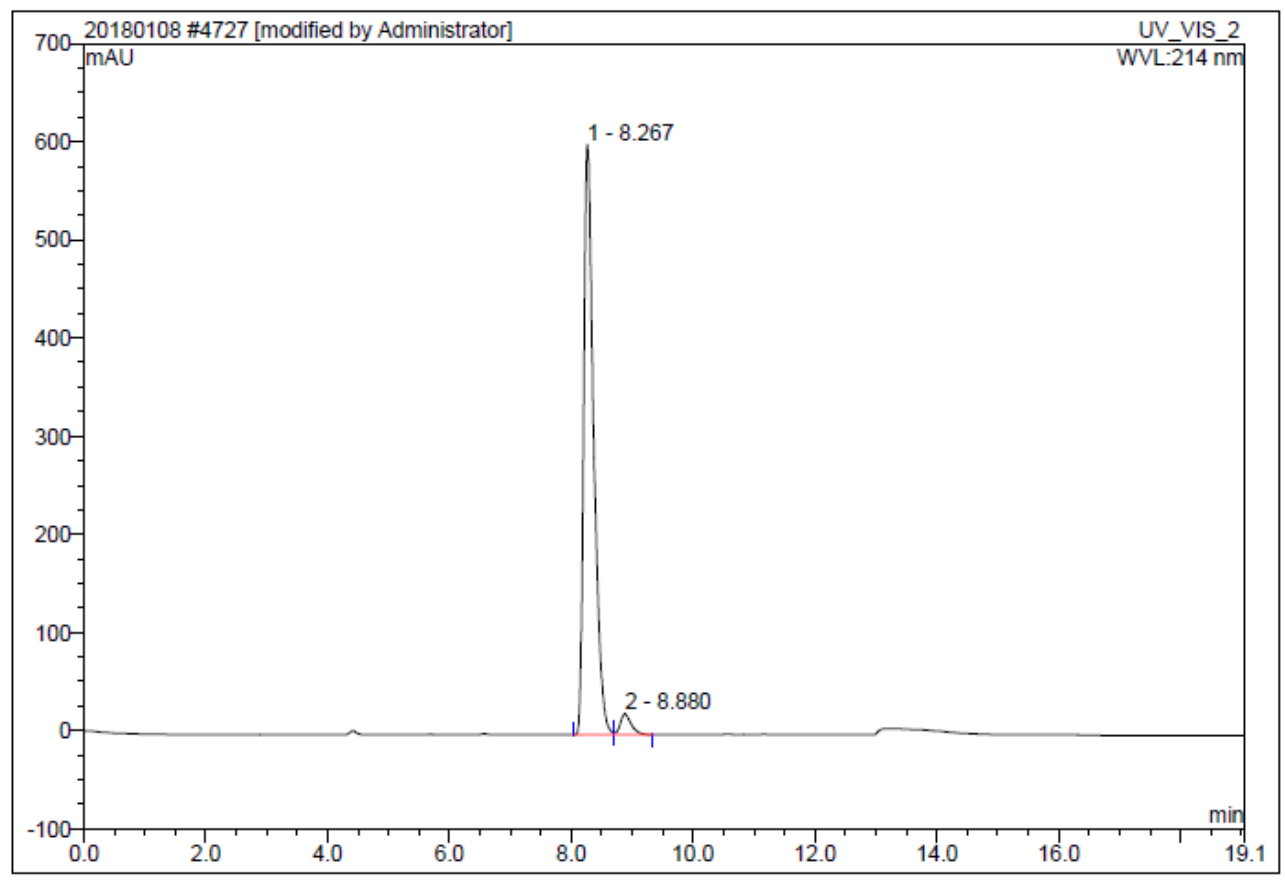

\begin{tabular}{|r|ccrrrrr|}
\hline No. & $\begin{array}{c}\text { Ret.Time } \\
\text { min }\end{array}$ & Peak Name & $\begin{array}{c}\text { Height } \\
\text { mAU }\end{array}$ & $\begin{array}{c}\text { Area } \\
\text { mAU*min }\end{array}$ & $\begin{array}{c}\text { Rel.Area } \\
\%\end{array}$ & Amount & Type \\
\hline 1 & 8.27 & n.a. & 601.513 & 114.976 & 96.36 & n.a. & BM \\
2 & 8.88 & n.a. & 21.224 & 4.342 & 3.64 & n.a. & MB \\
\hline Total: & & & 622.737 & 119.318 & 100.00 & 0.000 & \\
\hline
\end{tabular}

Ethyl-2-(((1R,2R)-2-phenylcyclopropyl)methyl)acrylate (9c).

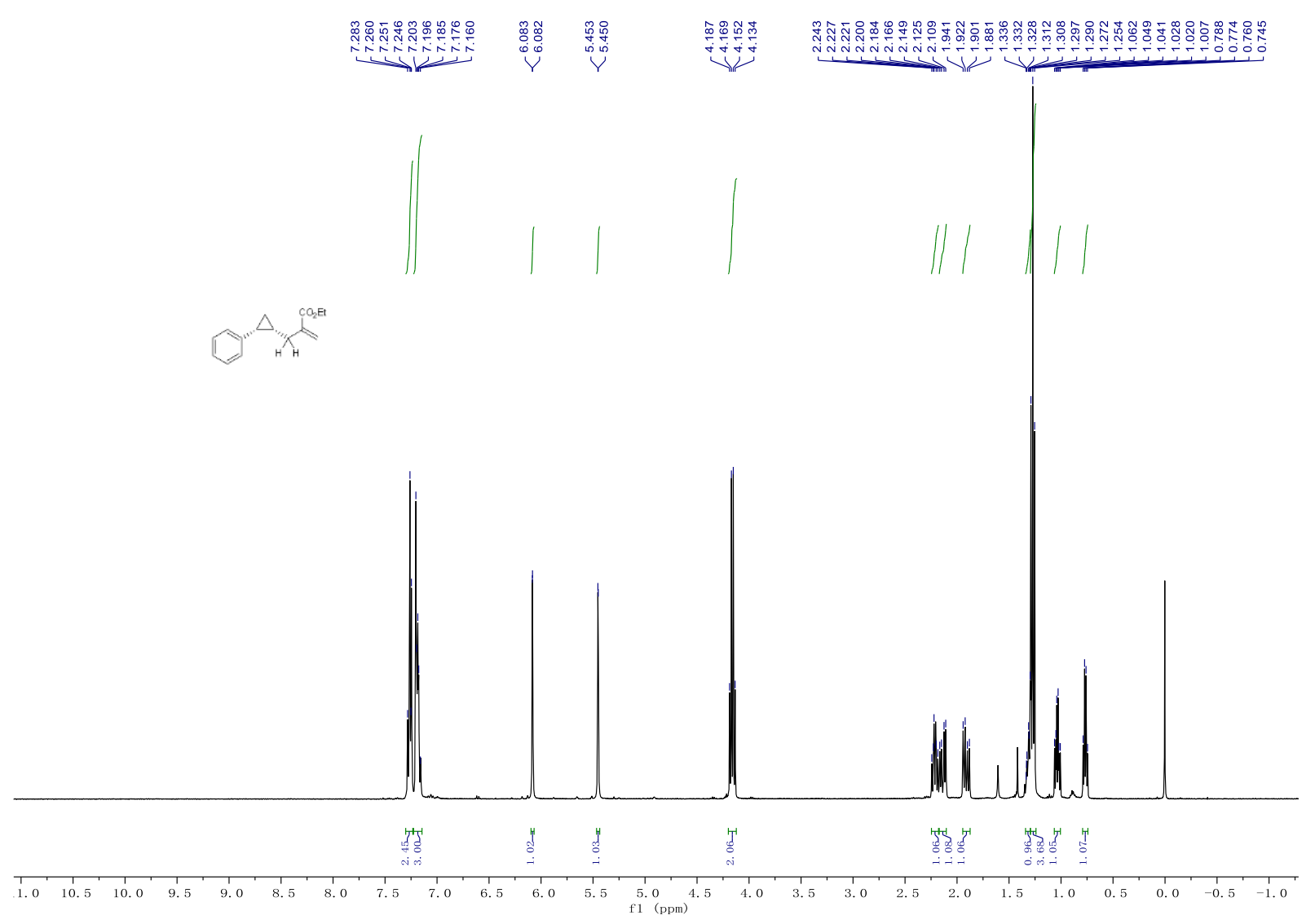



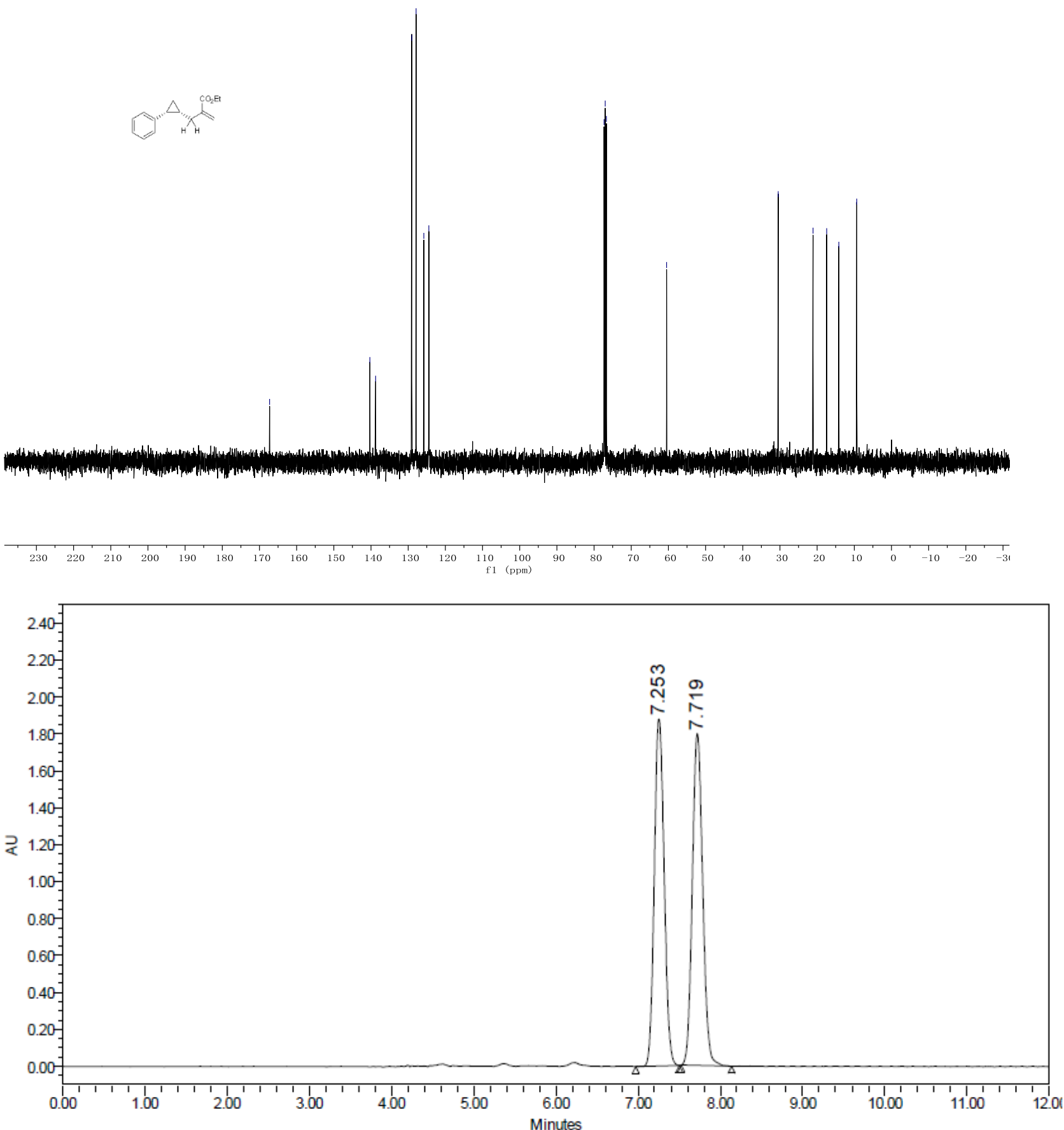

Peak Results
\begin{tabular}{|c|c|c|c|r|}
\hline & RT & Area & Height & $\%$ Area \\
\hline 1 & 7.253 & 15575726 & 1878093 & 49.73 \\
\hline 2 & 7.719 & 15746294 & 1796008 & 50.27 \\
\hline
\end{tabular}




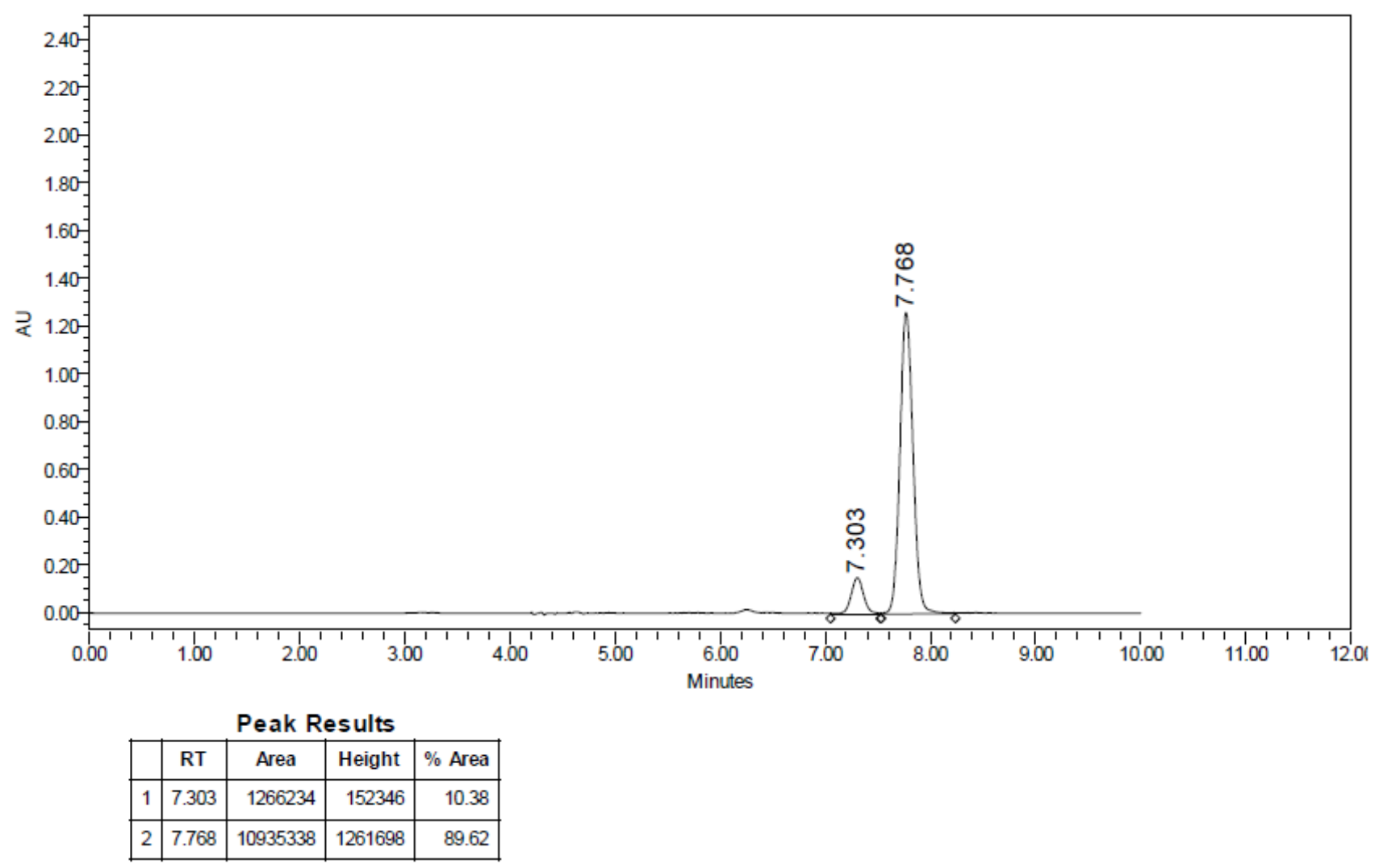

\section{3-((1S,2R)-2-Phenylcyclopropyl)propyl benzoate (9d).}

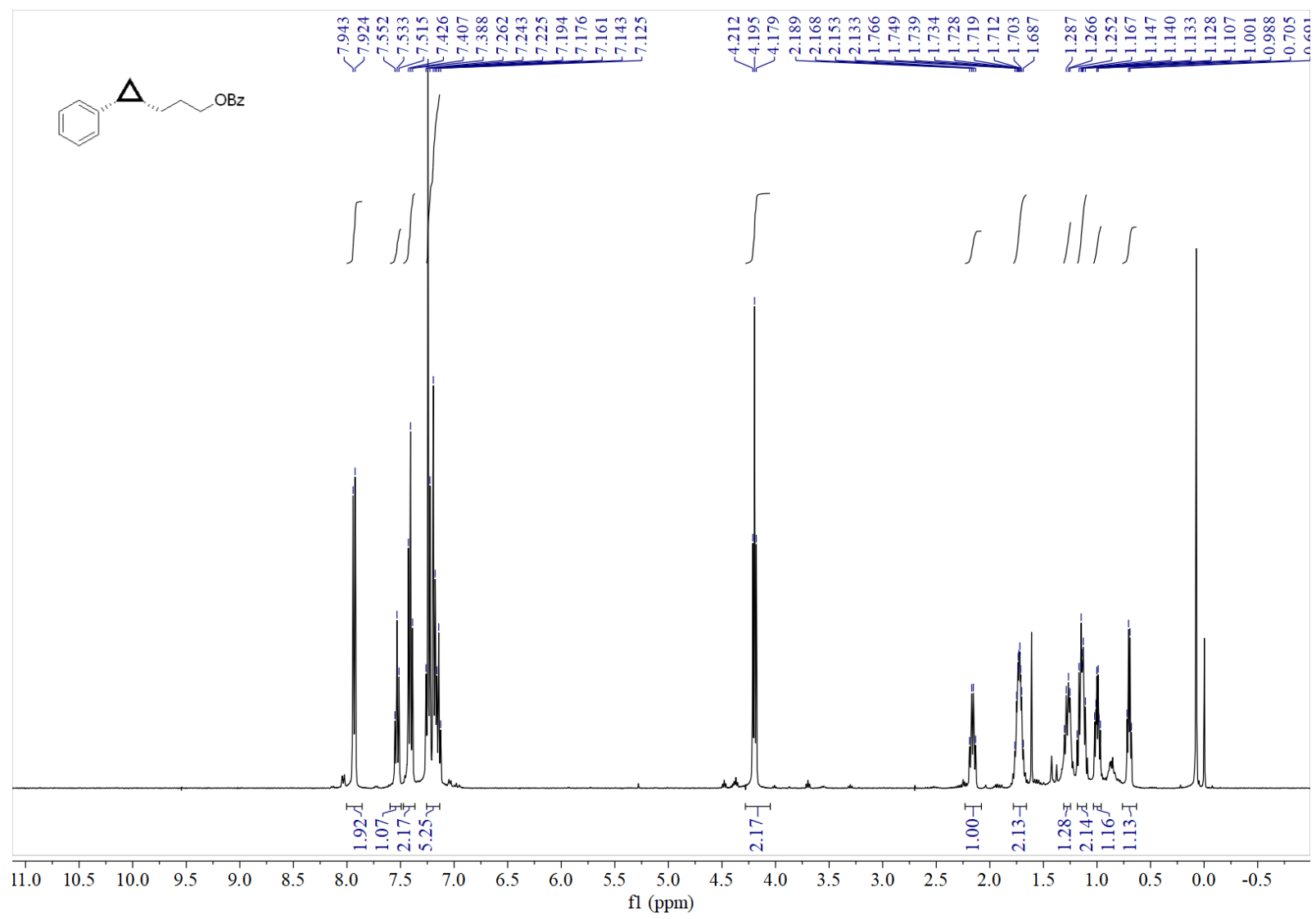



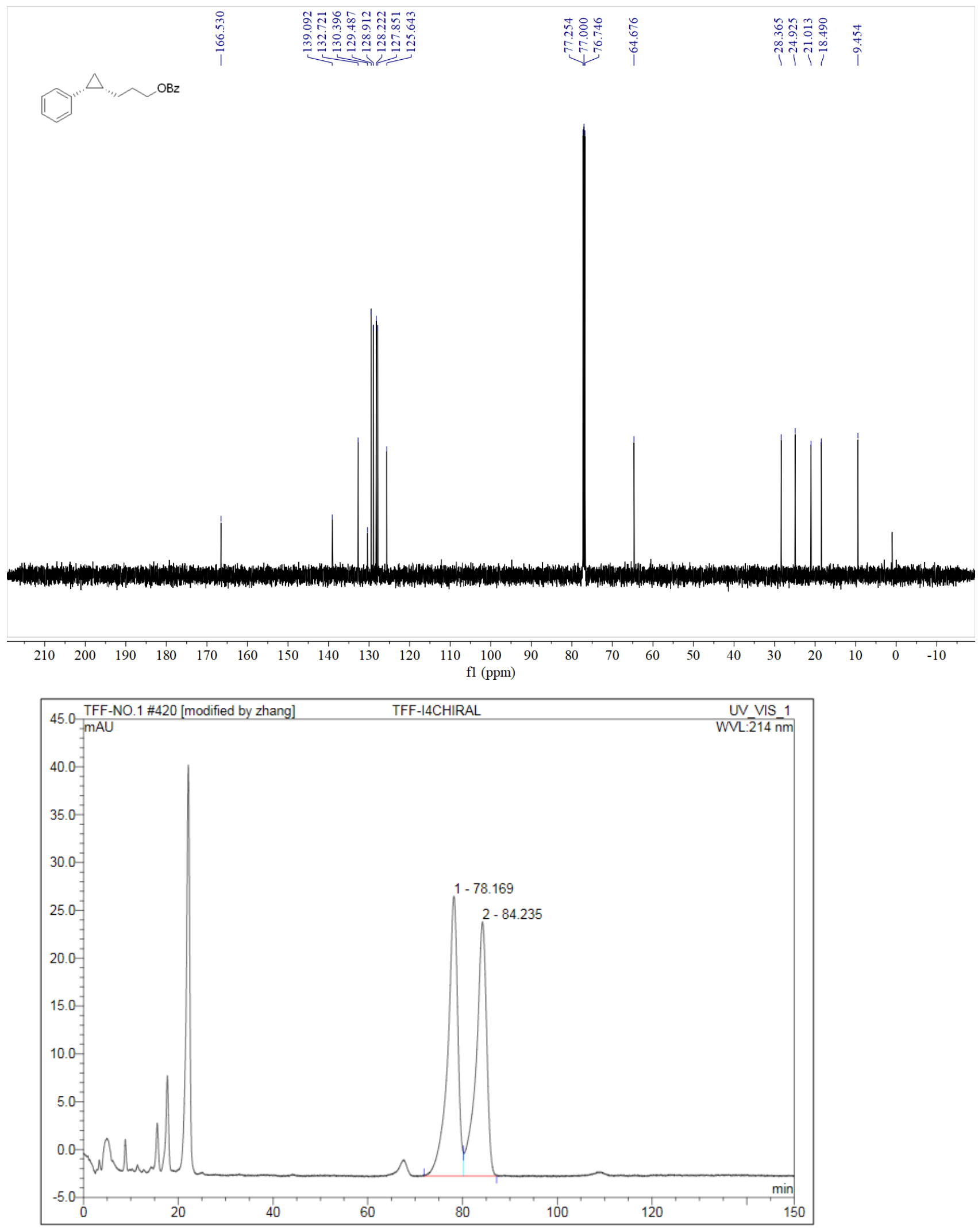

\begin{tabular}{|r|ccrrrrr|}
\hline No. & $\begin{array}{c}\text { Ret.Time } \\
\text { min }\end{array}$ & Peak Name & $\begin{array}{c}\text { Height } \\
\text { mAU }\end{array}$ & $\begin{array}{c}\text { Area } \\
\text { mAU*min }\end{array}$ & $\begin{array}{c}\text { Rel.Area } \\
\%\end{array}$ & $\begin{array}{c}\text { Amount } \\
\%\end{array}$ & Type \\
\hline 1 & 78.17 & n.a. & 29.259 & 71.815 & 51.26 & n.a. & BM $^{*}$ \\
2 & 84.24 & n.a. & 26.595 & 68.271 & 48.74 & n.a. & MB $^{*}$ \\
\hline Total: & & & 55.855 & 140.086 & 100.00 & 0.000 & \\
\hline
\end{tabular}




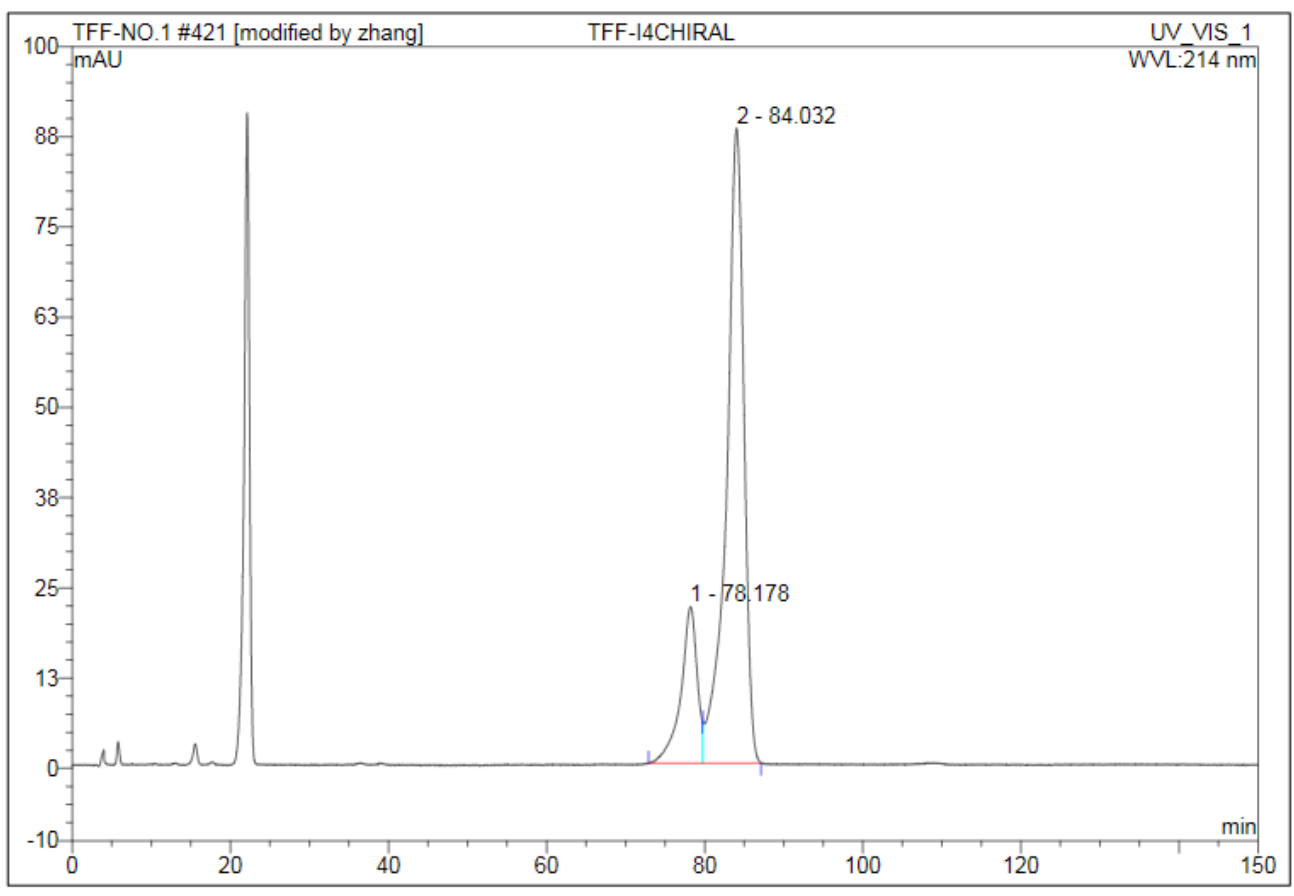

\begin{tabular}{|r|ccrrrrr|}
\hline No. & $\begin{array}{c}\text { Ret.Time } \\
\text { min }\end{array}$ & Peak Name & $\begin{array}{c}\text { Height } \\
\text { mAU }\end{array}$ & $\begin{array}{r}\text { Area } \\
\text { mAU*min }\end{array}$ & $\begin{array}{c}\text { Rel.Area } \\
\%\end{array}$ & $\begin{array}{c}\text { Amount } \\
\%\end{array}$ & Type \\
\hline 1 & 78.18 & n.a. & 21.717 & 51.605 & 18.73 & n.a. & BM $^{*}$ \\
2 & 84.03 & n.a. & 87.981 & 223.918 & 81.27 & n.a. & MB $^{*}$ \\
\hline Total: & & & 109.699 & 275.523 & 100.00 & 0.000 & \\
\hline
\end{tabular}

(3,3-Difluoro-3-(2-phenylcyclopropyl)prop-1-yn-1-yl)triethylsilane (3aa)

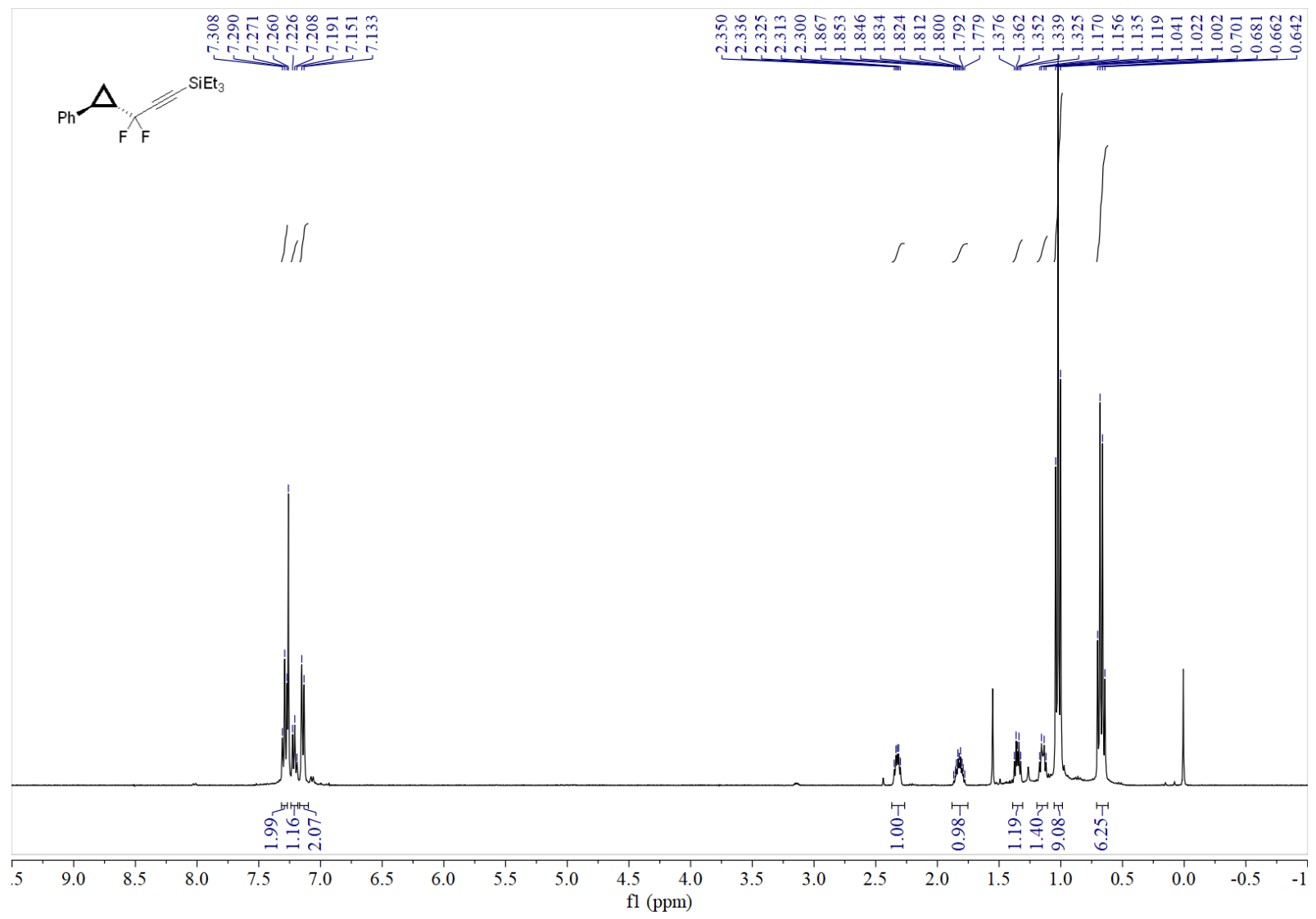




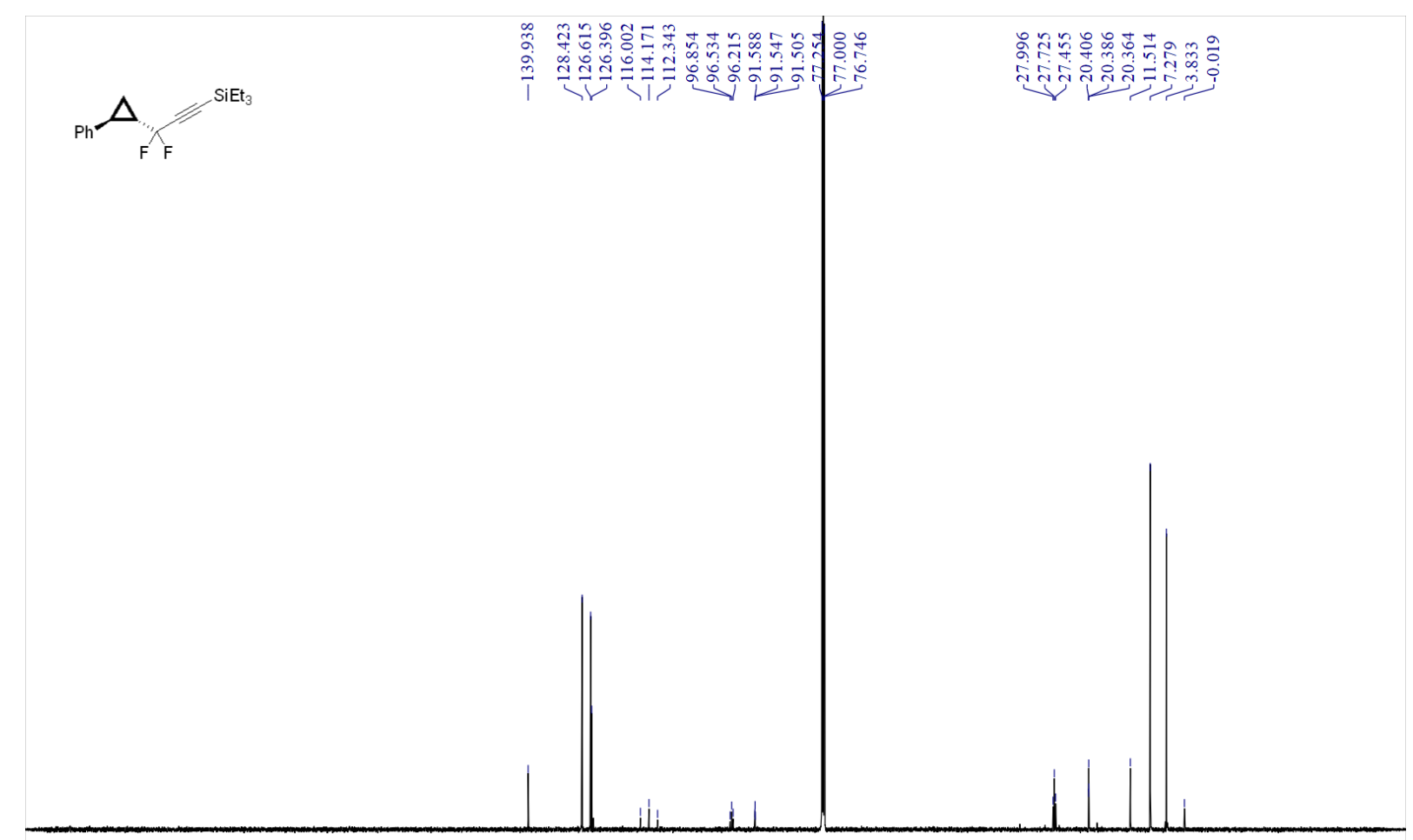

$\begin{array}{lllllllllllllllllllllllllllllllllllllll}240 & 230 & 220 & 210 & 200 & 190 & 180 & 170 & 160 & 150 & 140 & 130 & 120 & 110 & 100 & 90 & 80 & 70 & 60 & 50 & 40 & 30 & 20 & 10 & 0 & -10 & -20 & -30 & -40\end{array}$ fl (ppm)

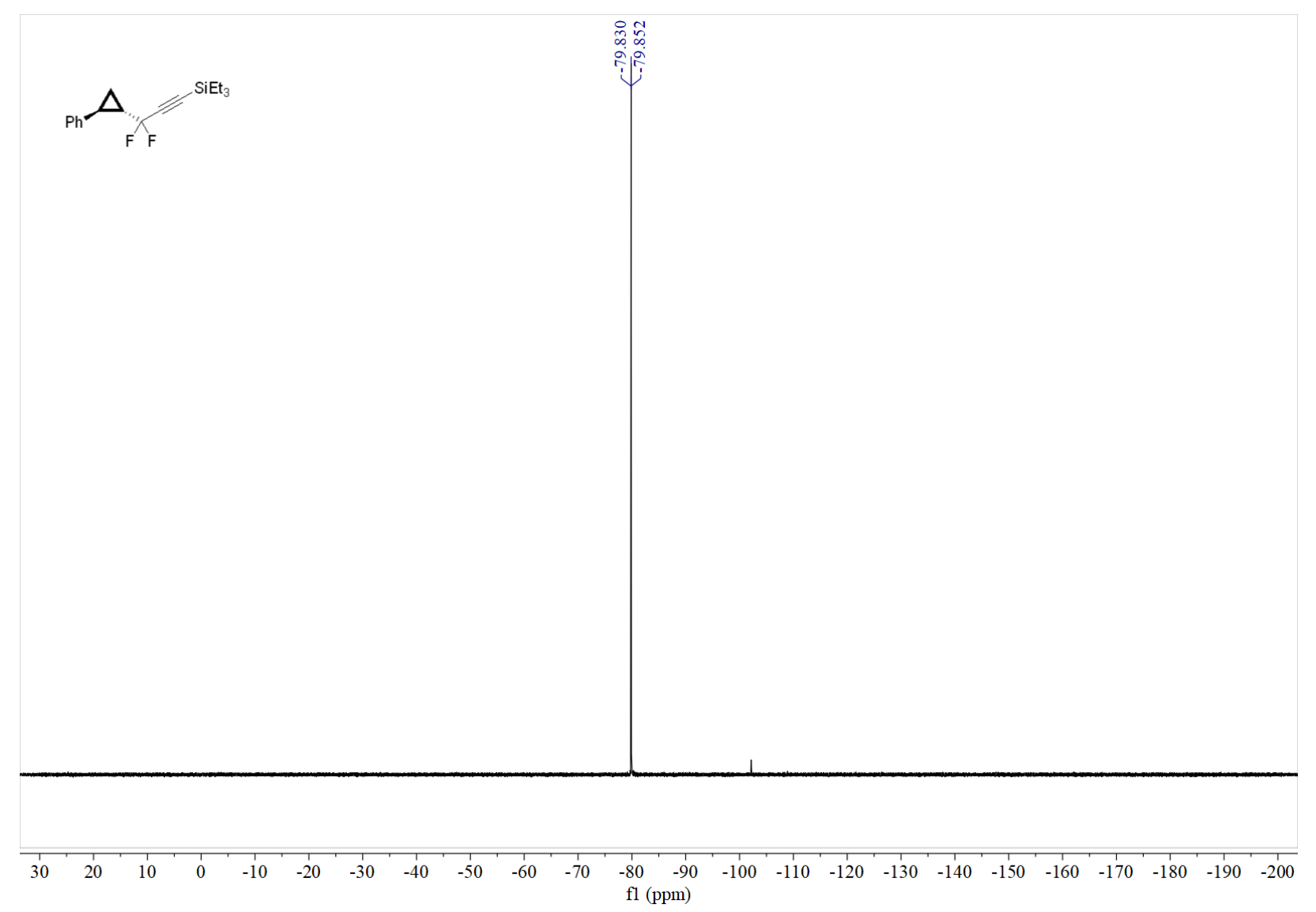




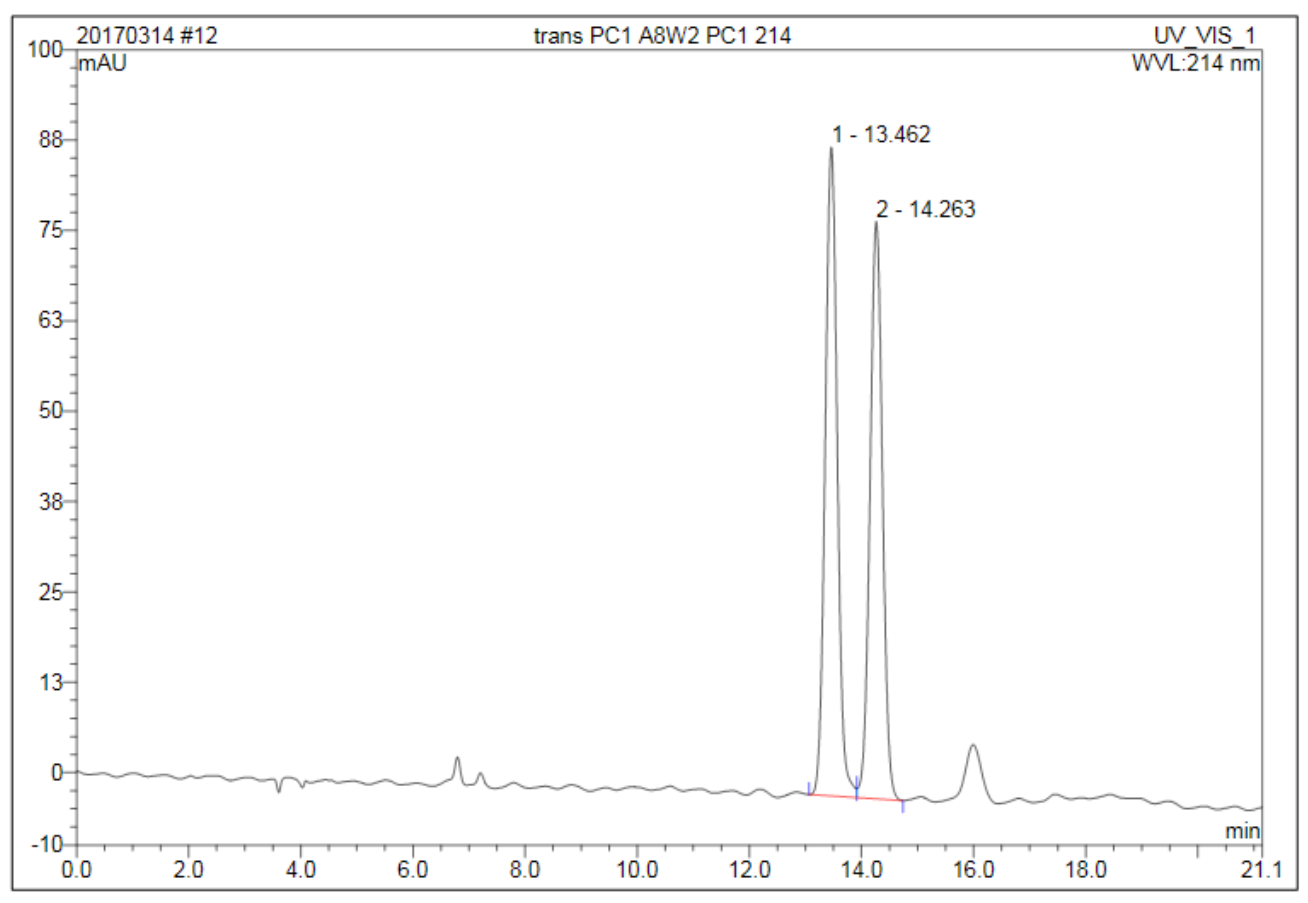

\begin{tabular}{|r|ccrrrrr|}
\hline No. & $\begin{array}{c}\text { Ret.Time } \\
\text { min }\end{array}$ & Peak Name & $\begin{array}{c}\text { Height } \\
\text { mAU }\end{array}$ & $\begin{array}{c}\text { Area } \\
\text { mAU*min }\end{array}$ & $\begin{array}{c}\text { Rel.Area } \\
\%\end{array}$ & Amount & Type \\
\hline 1 & 13.46 & n.a. & 89.727 & 22.438 & 52.13 & n.a. & BM \\
2 & 14.26 & n.a. & 79.840 & 20.604 & 47.87 & n.a. & MB \\
\hline Total: & & & 169.567 & 43.041 & 100.00 & 0.000 & \\
\hline
\end{tabular}

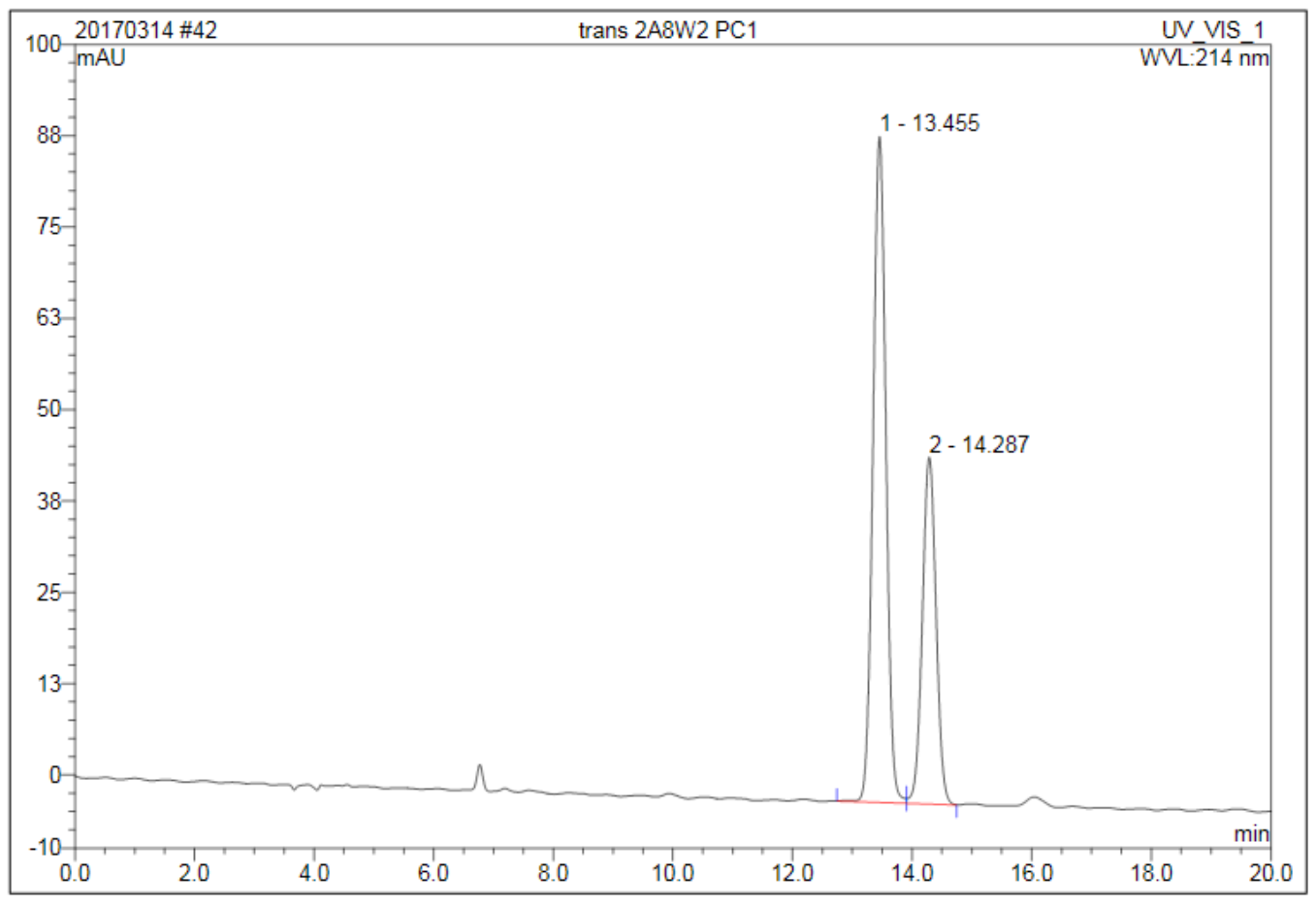

\begin{tabular}{|r|ccrrrrr|}
\hline No. & $\begin{array}{c}\text { Ret.Time } \\
\text { min }\end{array}$ & Peak Name & $\begin{array}{c}\text { Height } \\
\text { mAU }\end{array}$ & $\begin{array}{c}\text { Area } \\
\text { mAU*min }\end{array}$ & $\begin{array}{c}\text { Rel.Area } \\
\%\end{array}$ & Amount & Type \\
\hline 1 & 13.46 & n.a. & 91.139 & 22.838 & 64.44 & n.a. & BM \\
2 & 14.29 & n.a. & 47.463 & 12.603 & 35.56 & n.a. & MB \\
\hline Total: & & & 138.602 & 35.441 & 100.00 & 0.000 & \\
\hline
\end{tabular}


((S)-4,4-difluoro-3-((1R,2S)-2-phenylcyclopropyl)but-1-yn-1-yl)triisopropylsilane (10a).

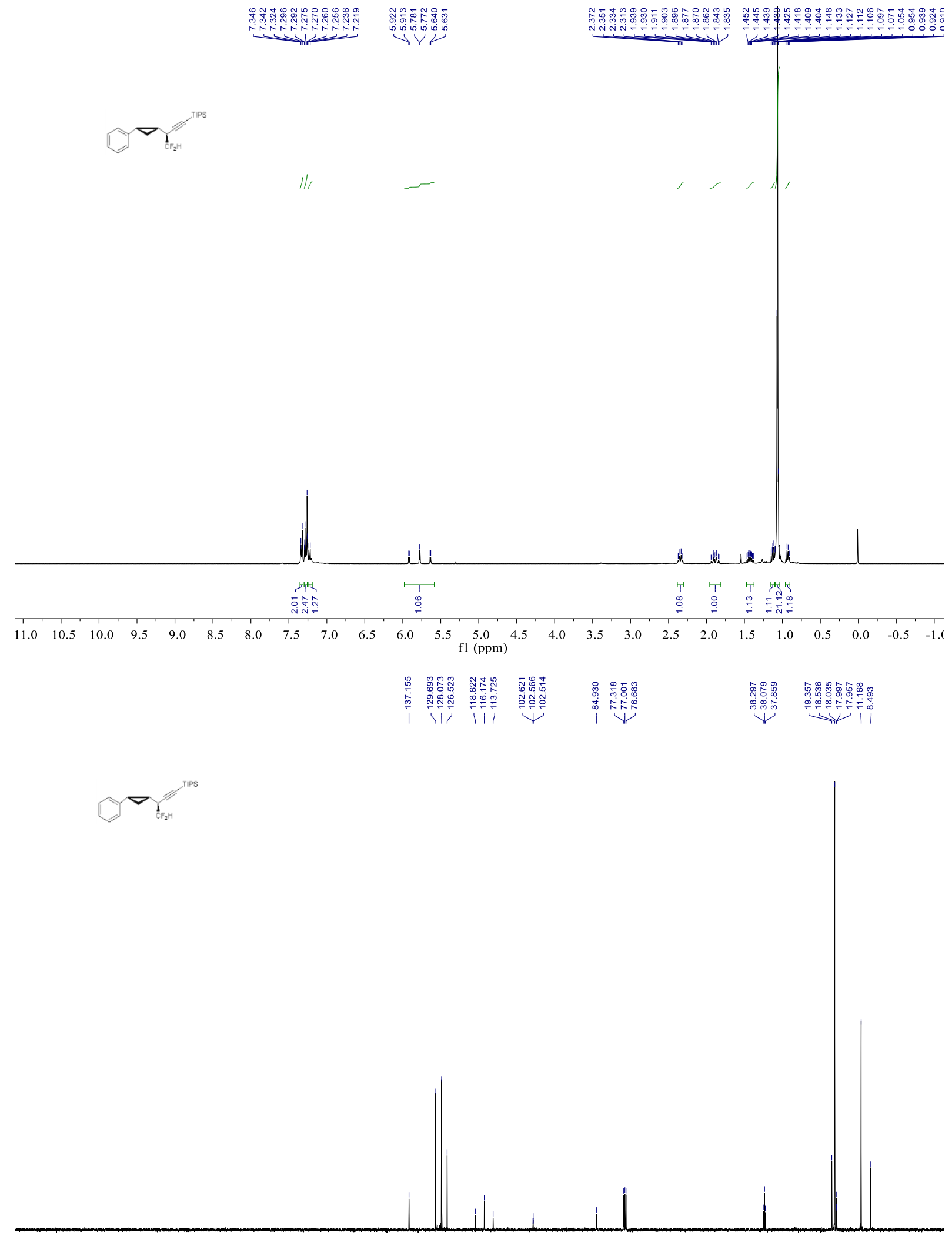

$\begin{array}{llllllllllllllllllllllllll}240 & 230 & 220 & 210 & 200 & 190 & 180 & 170 & 160 & 150 & 140 & 130 & 120 & 110 & 100 & 90 & 80 & 70 & 60 & 50 & 40 & 30 & 20 & 10 & 0 & -11\end{array}$ 


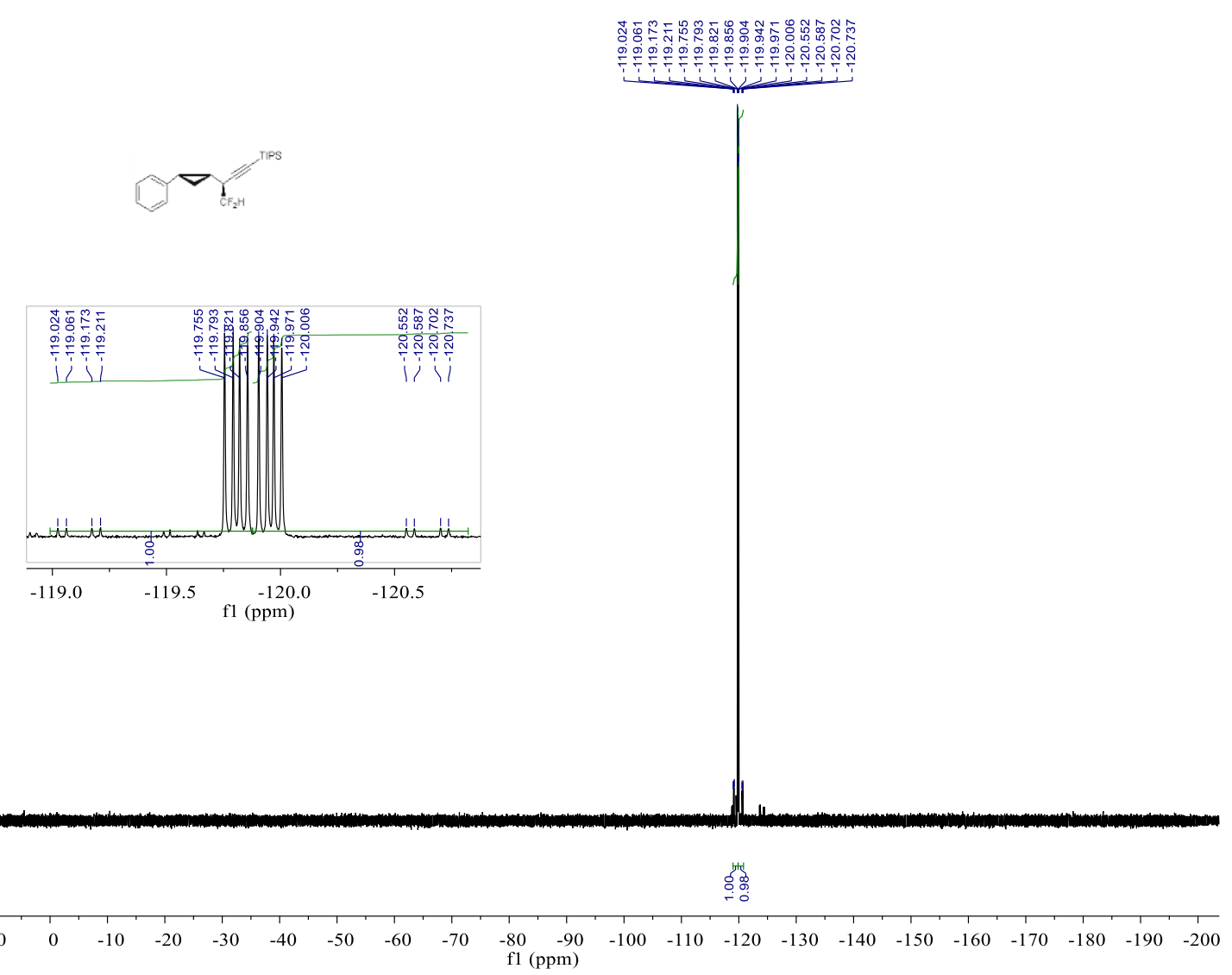

1-benzyl-4-((S)-2,2-difluoro-1-((1R,2S)-2-phenylcyclopropyl)ethyl)-1H-1,2,3-triazole (10a').

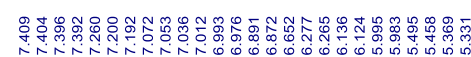

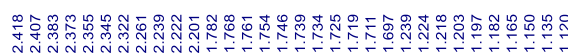

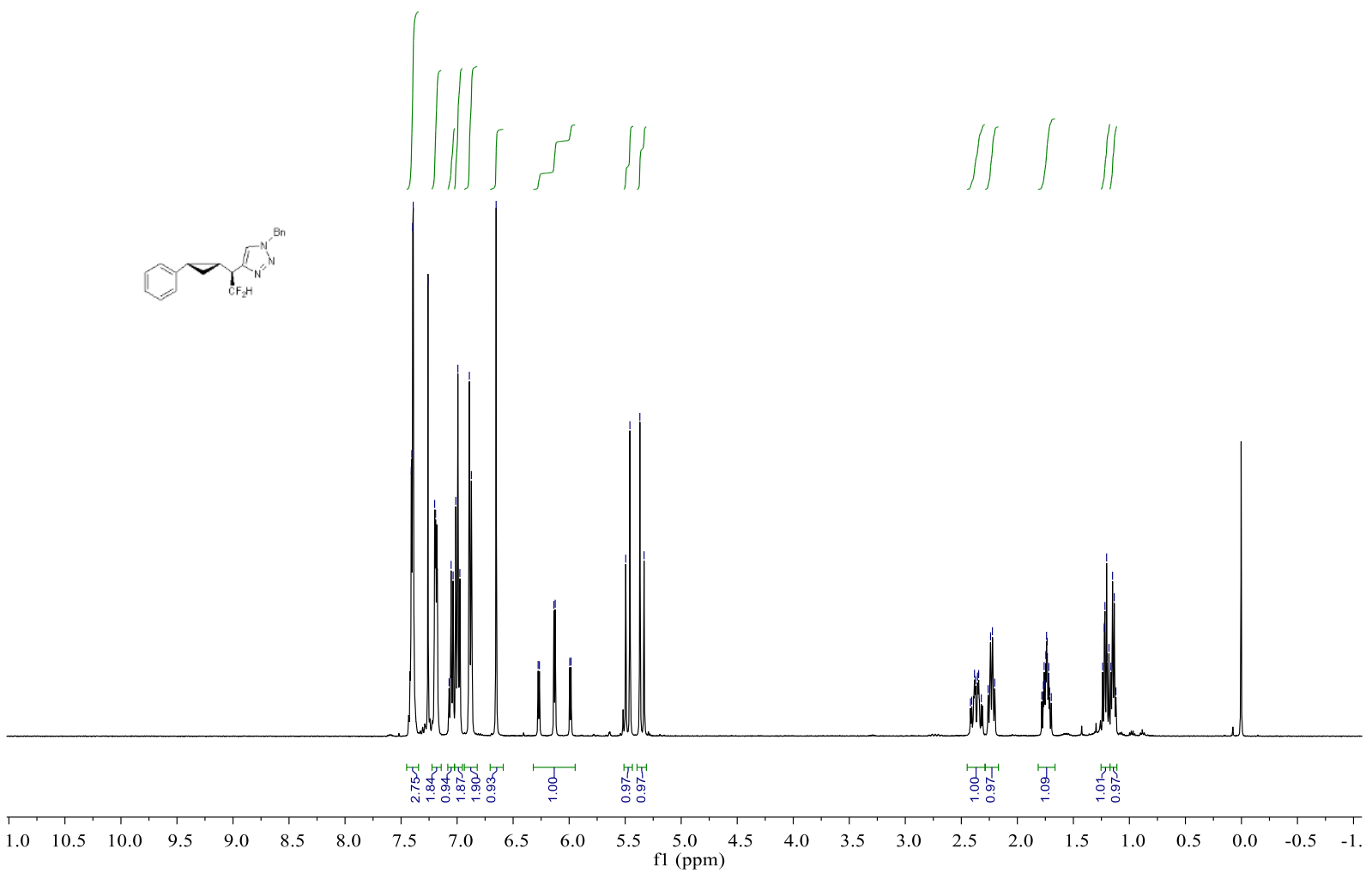




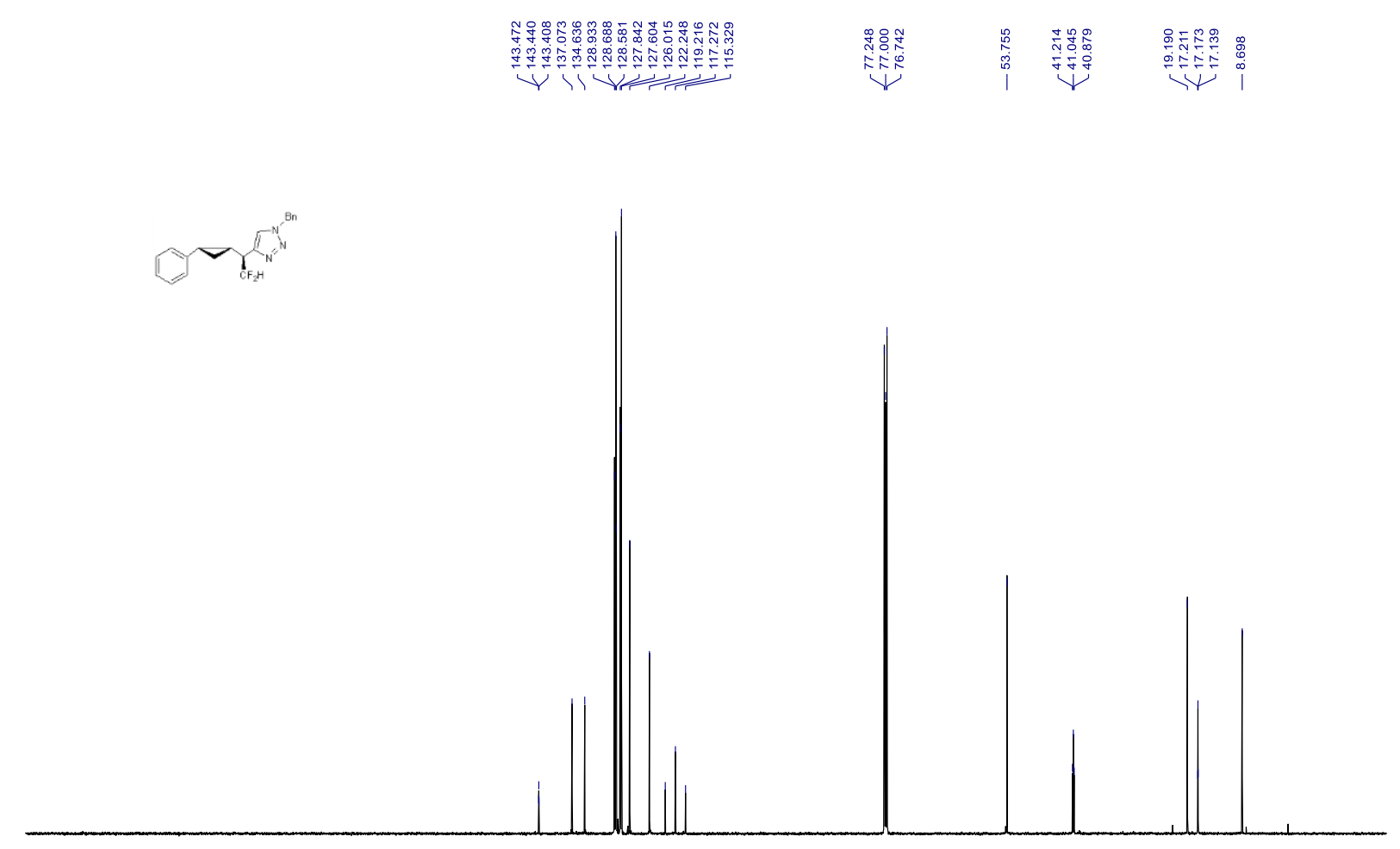

$\begin{array}{lllllllllllllllllllllllllllllll}240 & 230 & 220 & 210 & 200 & 190 & 180 & 170 & 160 & 150 & 140 & 130 & 120 & 110 & 100 & 90 & 80 & 70 & 60 & 50 & 40 & 30 & 20 & 10 & 0 & -10\end{array}$

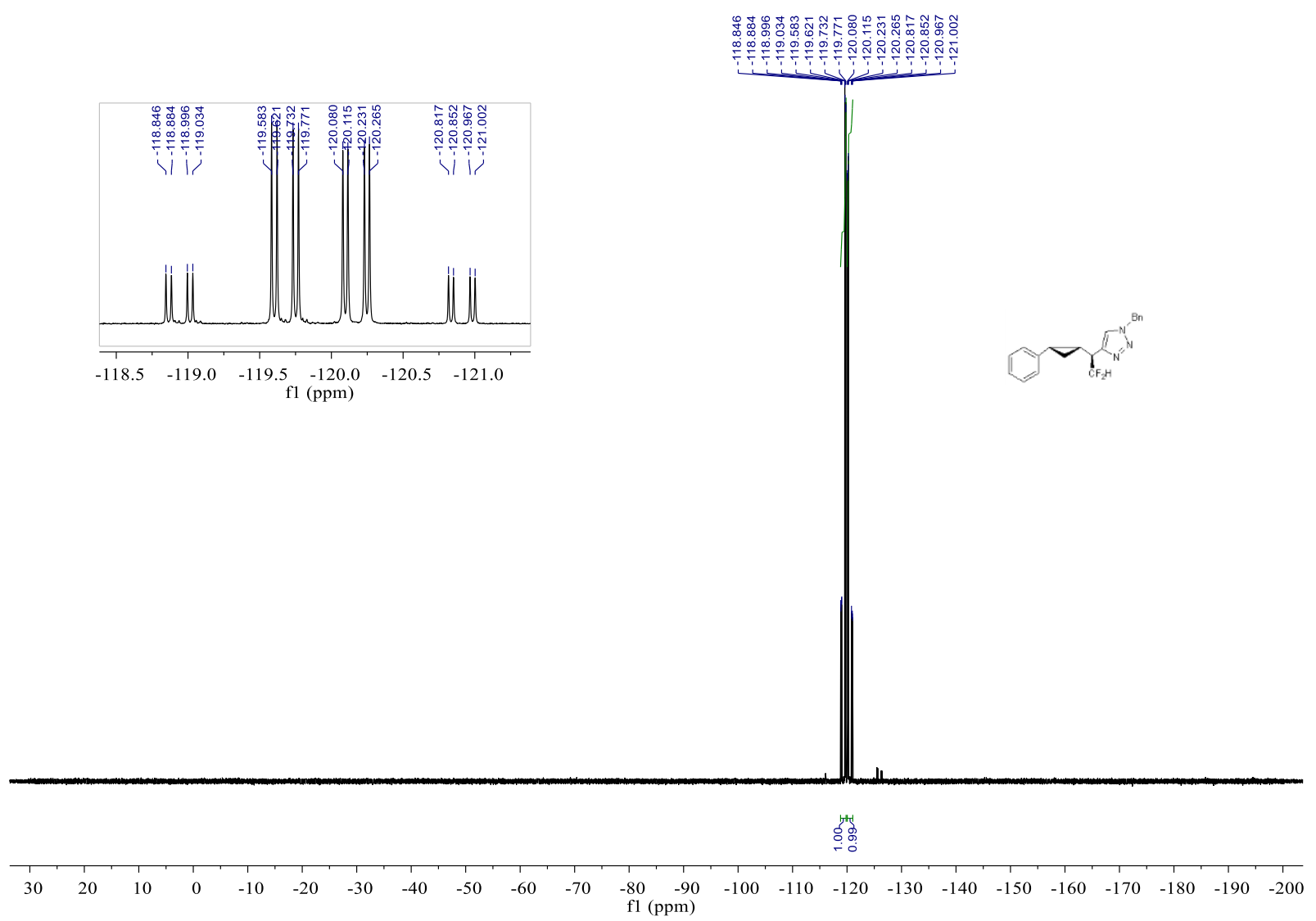




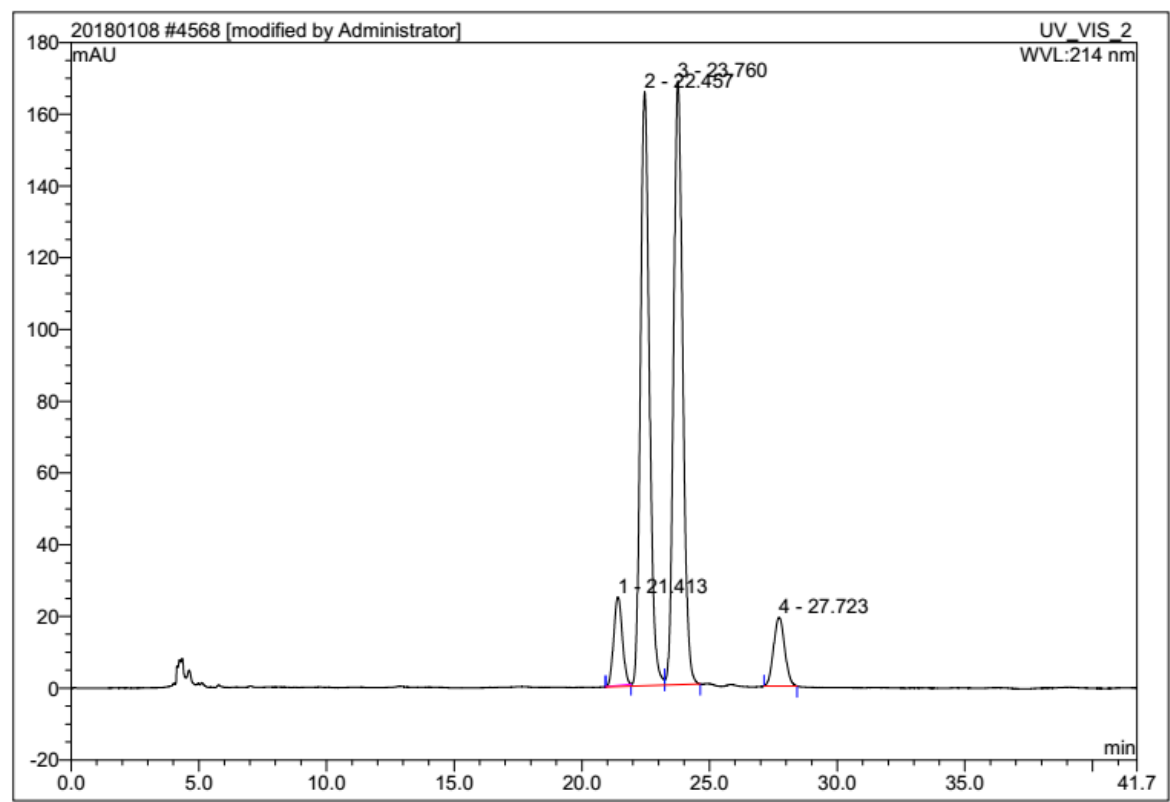

\begin{tabular}{|r|ccrrrrr|}
\hline No. & $\begin{array}{c}\text { Ret.Time } \\
\text { min }\end{array}$ & Peak Name & $\begin{array}{c}\text { Height } \\
\text { mAU }\end{array}$ & $\begin{array}{c}\text { Area } \\
\text { mAU*min }\end{array}$ & $\begin{array}{c}\text { Rel.Area } \\
\%\end{array}$ & Amount & Type \\
\hline 1 & 21.41 & n.a. & 24.737 & 9.597 & 6.12 & n.a. & Ru \\
2 & 22.46 & n.a. & 165.558 & 67.520 & 43.07 & n.a. & BM \\
3 & 23.76 & n.a. & 168.223 & 69.934 & 44.61 & n.a. & MB \\
4 & 27.72 & n.a. & 19.204 & 9.703 & 6.19 & n.a. & BMB \\
\hline Total: & & & 377.722 & 156.754 & 100.00 & 0.000 & \\
\hline
\end{tabular}

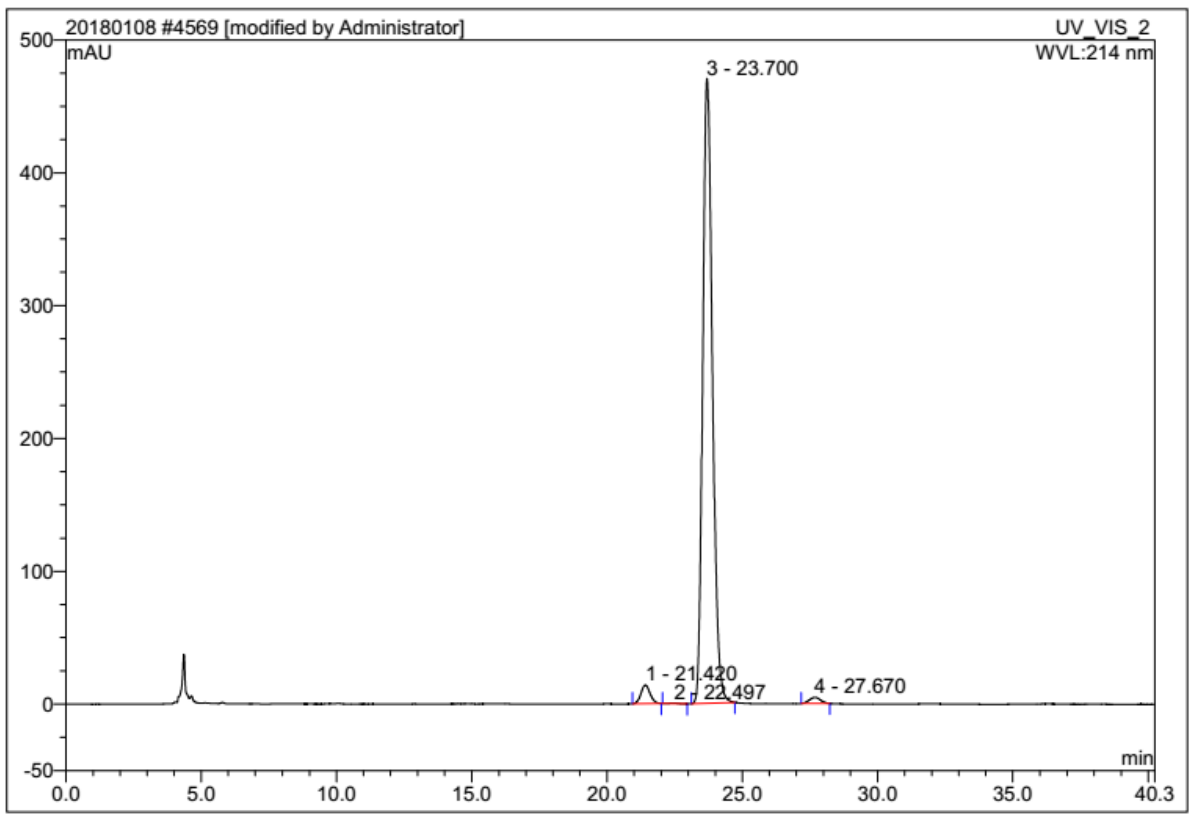

\begin{tabular}{|r|ccrrrrr|}
\hline No. & $\begin{array}{c}\text { Ret.Time } \\
\text { min }\end{array}$ & Peak Name & $\begin{array}{c}\text { Height } \\
\text { mAU }\end{array}$ & $\begin{array}{c}\text { Area } \\
\text { mAU*min }\end{array}$ & $\begin{array}{r}\text { Rel.Area } \\
\%\end{array}$ & Amount & Type \\
\hline 1 & 21.42 & n.a. & 13.884 & 5.425 & 2.66 & n.a. & BMB \\
2 & 22.50 & n.a. & 0.457 & 0.129 & 0.06 & n.a. & BMB $^{*}$ \\
3 & 23.70 & n.a. & 470.262 & 195.830 & 96.19 & n.a. & BMB \\
4 & 27.67 & n.a. & 4.622 & 2.194 & 1.08 & n.a. & BMB $^{*}$ \\
\hline Total: & & & 489.225 & 203.578 & 100.00 & 0.000 & \\
\hline
\end{tabular}

((S)-4,4-Difluoro-3-((1R,2S)-2-(4-methoxyphenyl)cyclopropyl)but-1-yn-1-yl)triisopropylsilane (10b). 


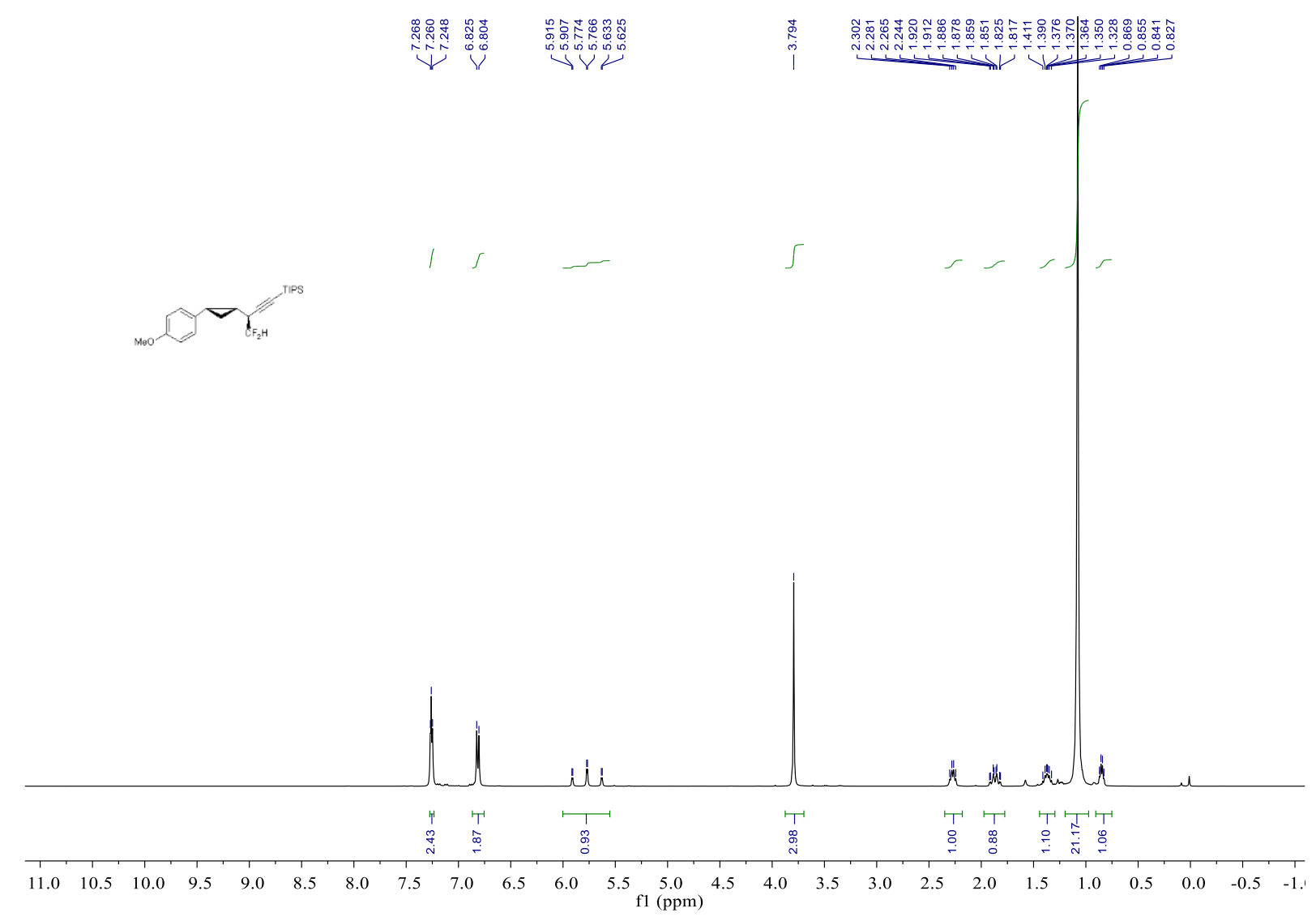

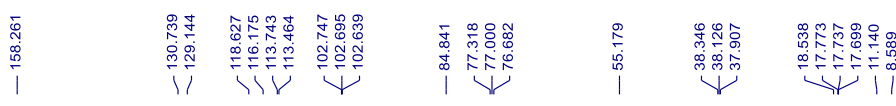

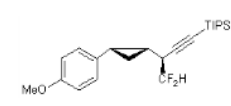

$\begin{array}{rlllllllllllllllllllllllllllll}230 & 220 & 210 & 200 & 190 & 180 & 170 & 160 & 150 & 140 & 130 & 120 & 110 & 100 & 90 & 80 & 70 & 60 & 50 & 40 & 30 & 20 & 10 & 0 & -10 & -20 & -3\end{array}$ 


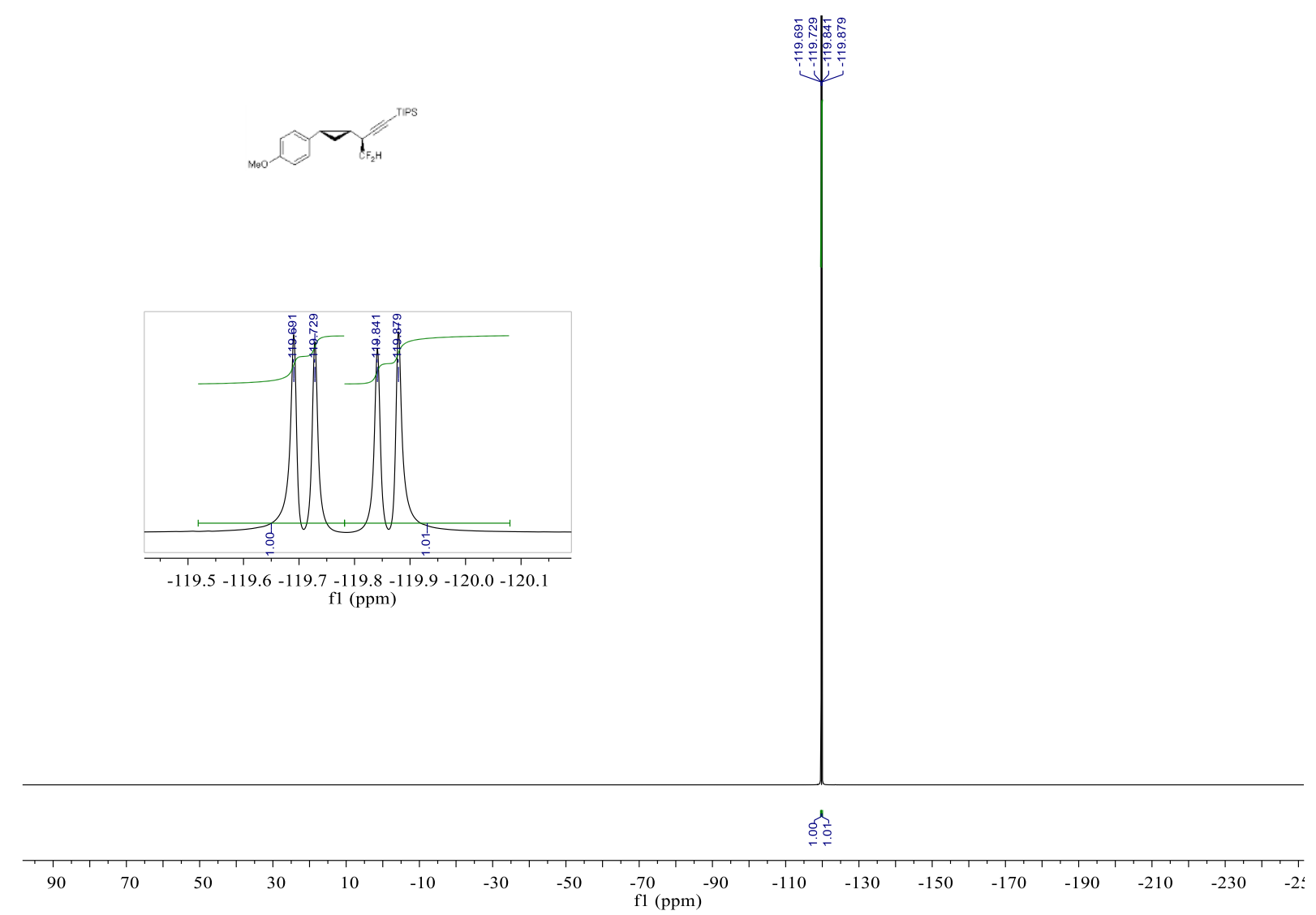




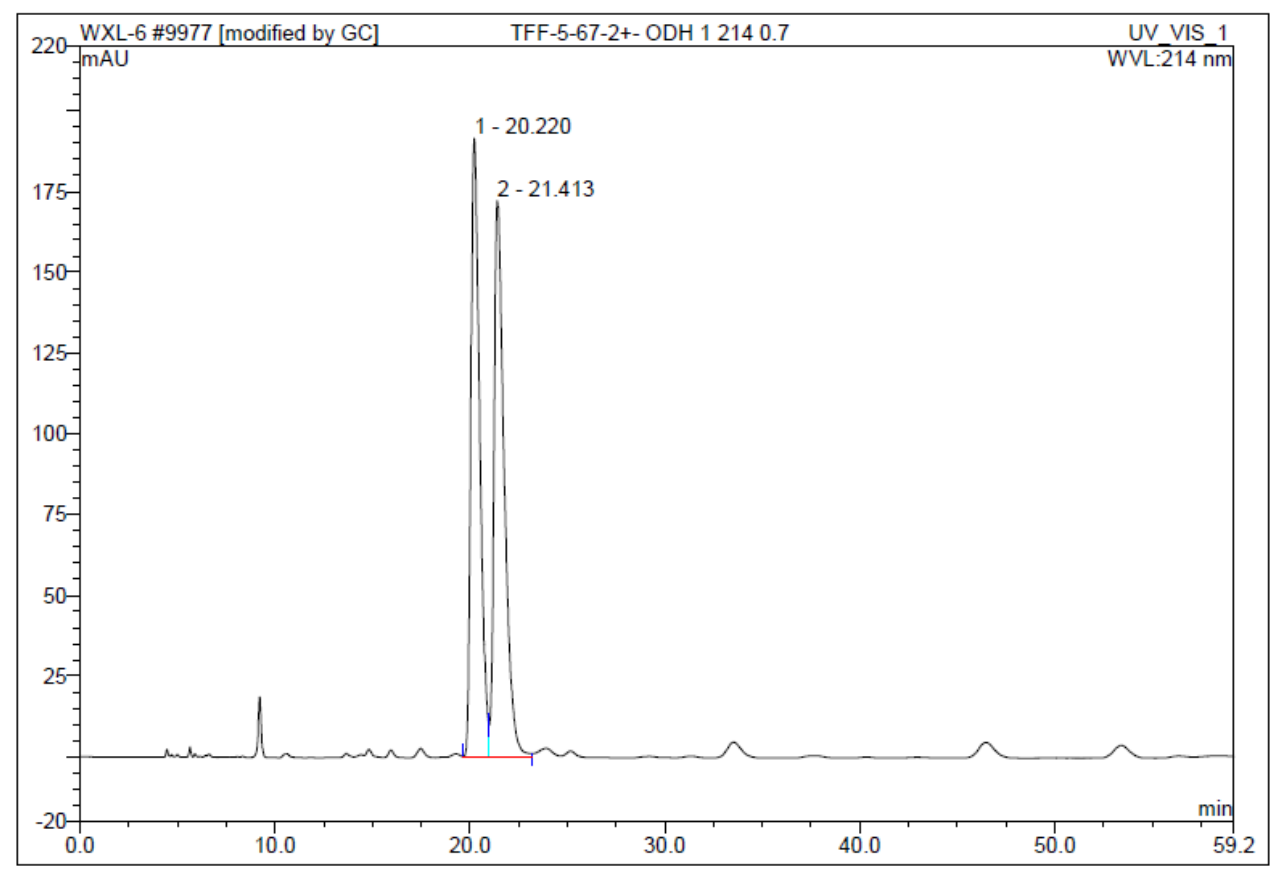

\begin{tabular}{|r|ccrrrrr|}
\hline No. & $\begin{array}{c}\text { Ret.Time } \\
\text { min }\end{array}$ & Peak Name & $\begin{array}{c}\text { Height } \\
\text { mAU }\end{array}$ & $\begin{array}{c}\text { Area } \\
\text { mAU*min }\end{array}$ & $\begin{array}{r}\text { Rel.Area } \\
\%\end{array}$ & Amount & Type \\
\hline 1 & 20.22 & n.a. & 191.534 & 101.026 & 49.01 & n.a. & BM $^{*}$ \\
2 & 21.41 & n.a. & 172.095 & 105.126 & 50.99 & n.a. & M * $^{*}$ \\
\hline Total: & & & 363.629 & 206.152 & 100.00 & 0.000 & \\
\hline
\end{tabular}

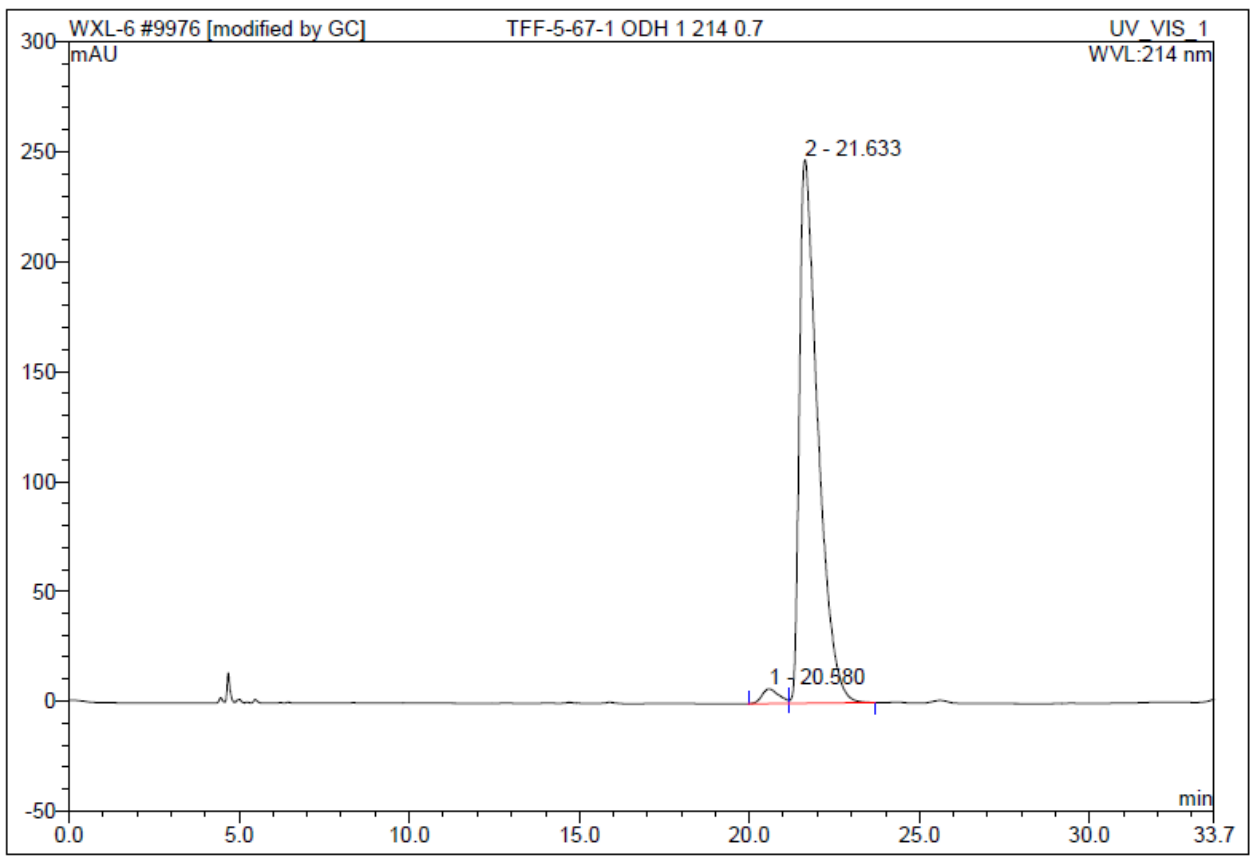

\begin{tabular}{|r|ccrrrrr|}
\hline No. & $\begin{array}{c}\text { Ret.Time } \\
\text { min }\end{array}$ & Peak Name & $\begin{array}{c}\text { Height } \\
\text { mAU }\end{array}$ & $\begin{array}{r}\text { Area } \\
\text { mAU*min }\end{array}$ & $\begin{array}{r}\text { Rel.Area } \\
\%\end{array}$ & Amount & Type \\
\hline 1 & 20.58 & n.a. & 6.634 & 3.845 & 2.41 & n.a. & BM \\
2 & 21.63 & n.a. & 247.320 & 155.950 & 97.59 & n.a. & MB \\
\hline Total: & & & 253.955 & 159.795 & 100.00 & 0.000 & \\
\hline
\end{tabular}


((S)-3-((1R,2S)-2-(4-Chlorophenyl)cyclopropyl)-4,4-difluorobut-1-yn-1-yl)triisopropylsilane (10c).

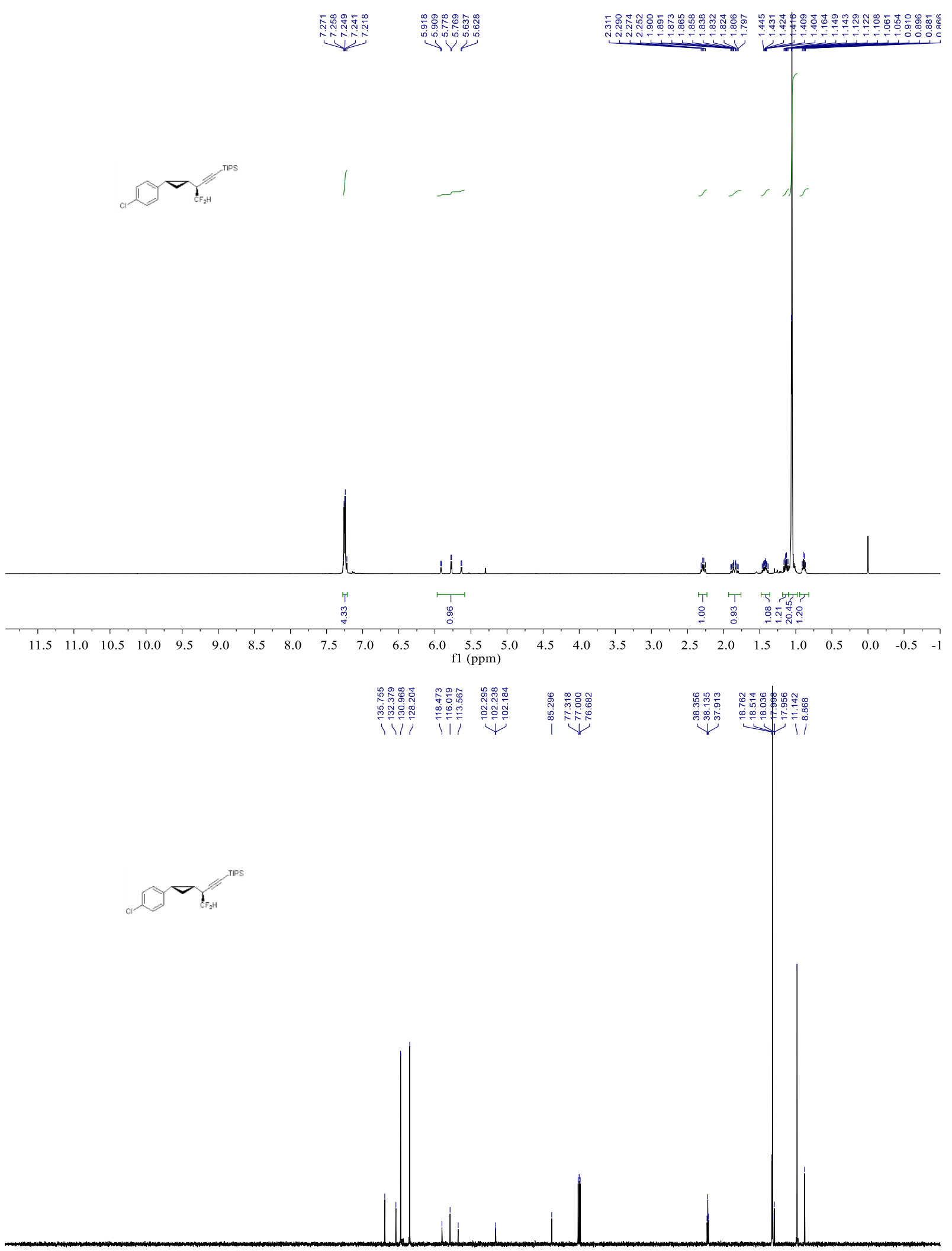

$\begin{array}{lllllllllllllllllllllllllllllllllllllllllllll}50 & 240 & 230 & 220 & 210 & 200 & 190 & 180 & 170 & 160 & 150 & 140 & 130 & 120 & 110 & 100 & 90 & 80 & 70 & 60 & 50 & 40 & 30 & 20 & 10 & 0 & -10 & -20 & -31\end{array}$ 


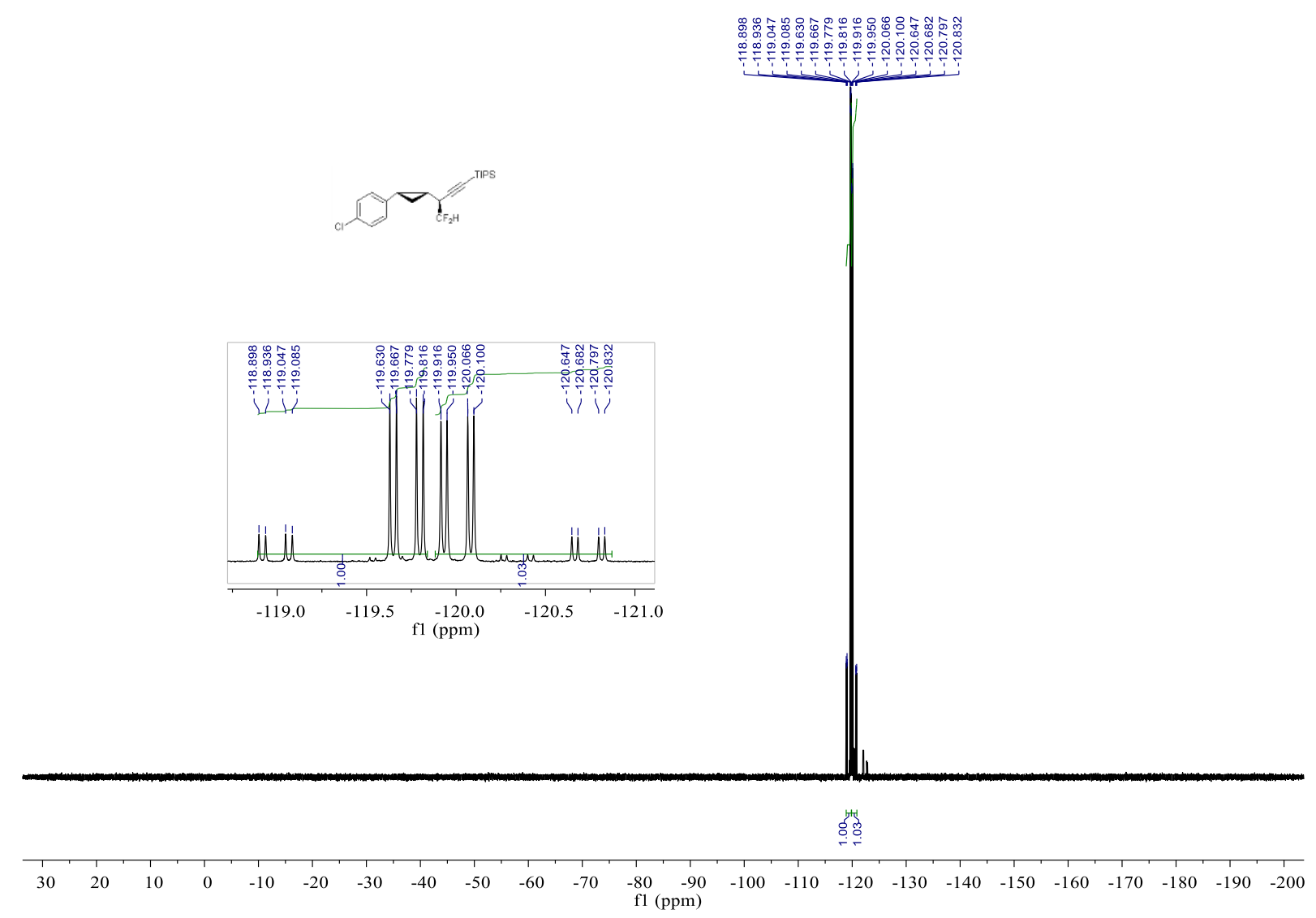

1-Benzyl-4-((S)-1-((1R,2S)-2-(4-chlorophenyl)cyclopropyl)-2,2-difluoroethyl)-1H-1,2,3-triazole (10c').

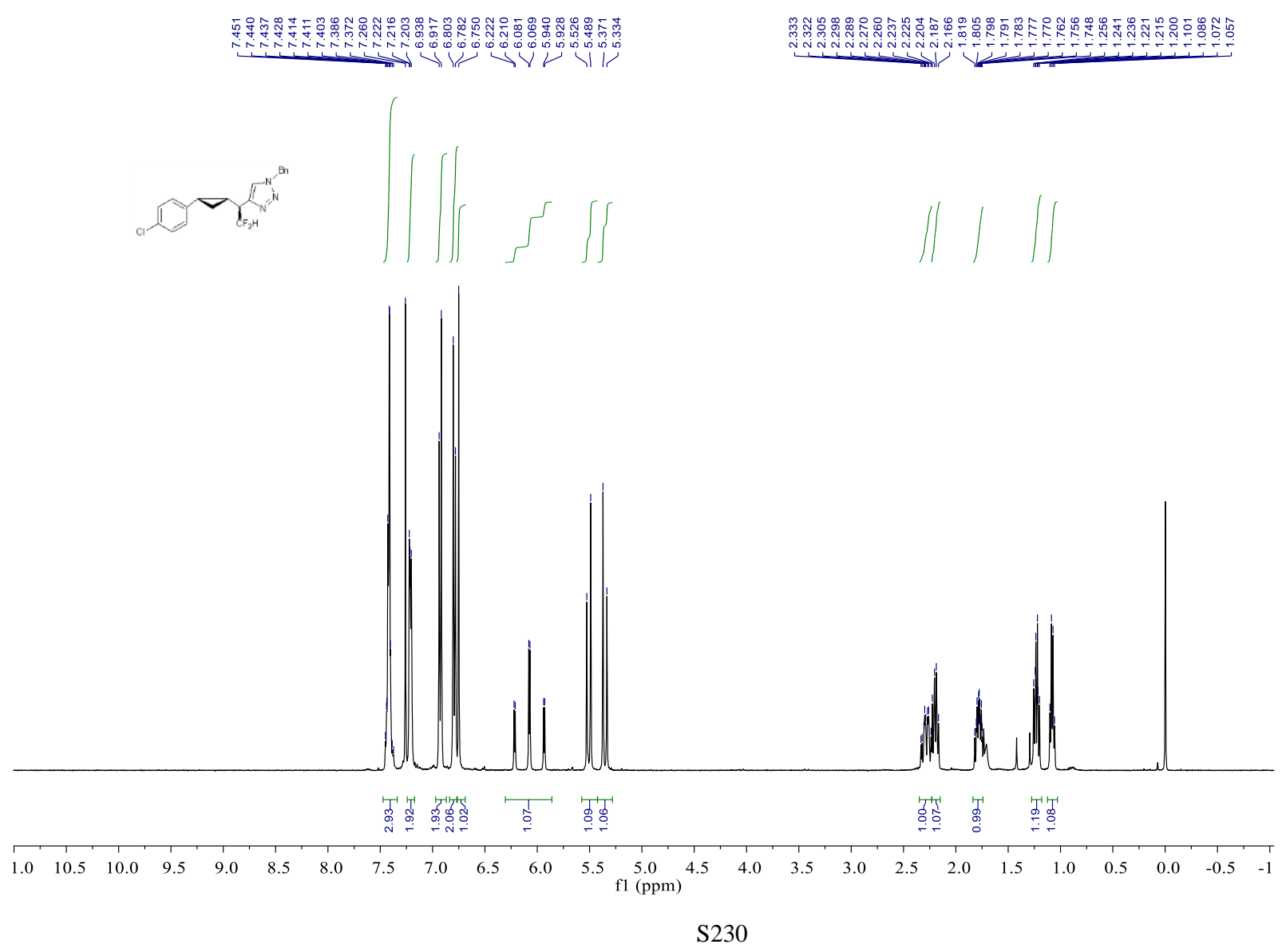




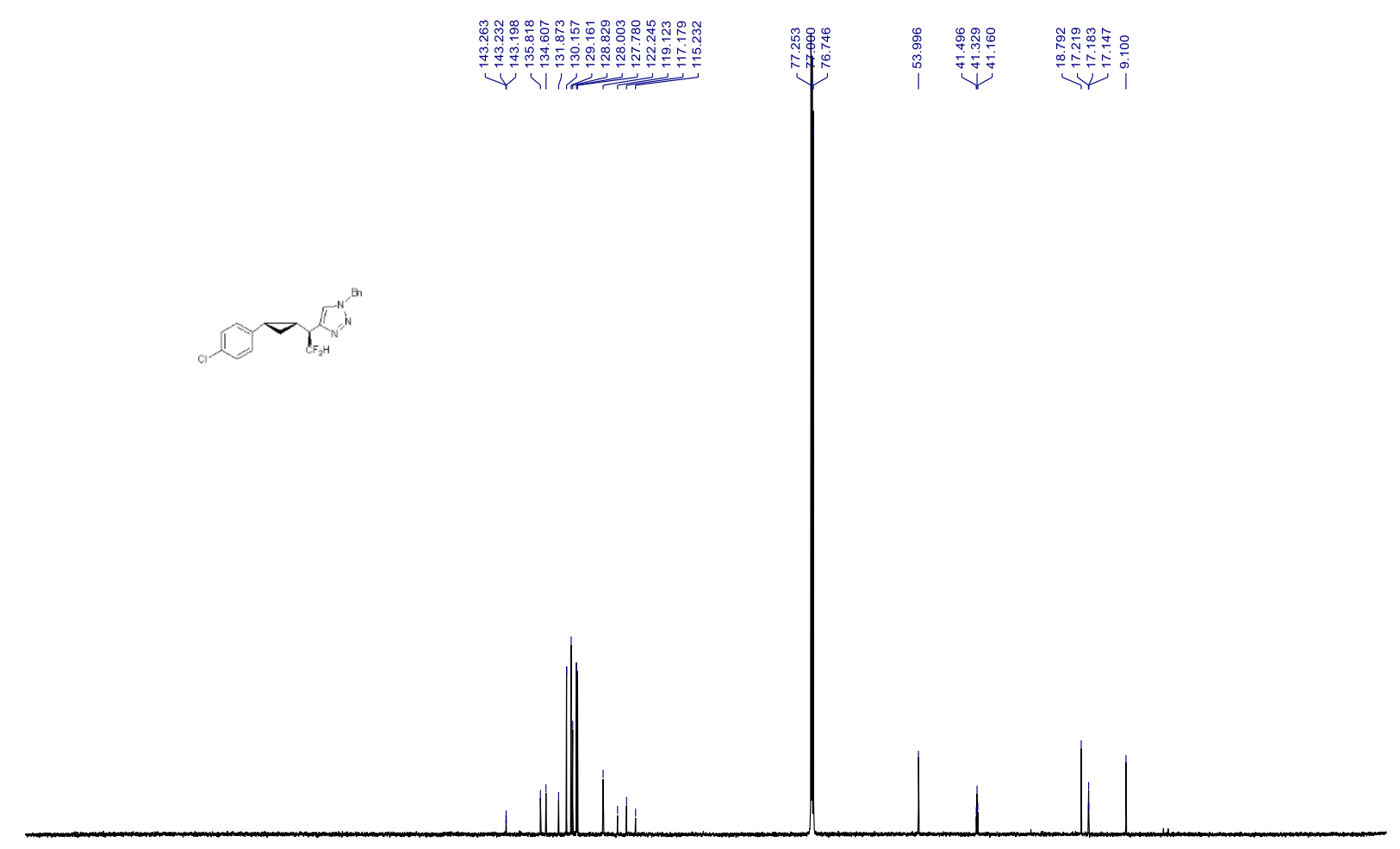

$\begin{array}{llllllllllllllllllllllllllllllllllllllllll}240 & 230 & 220 & 210 & 200 & 190 & 180 & 170 & 160 & 150 & 140 & 130 & 120 & 110 & 100 & 90 & 80 & 70 & 60 & 50 & 40 & 30 & 20 & 10 & 0 & -10 & -20 & -30 & -40\end{array}$

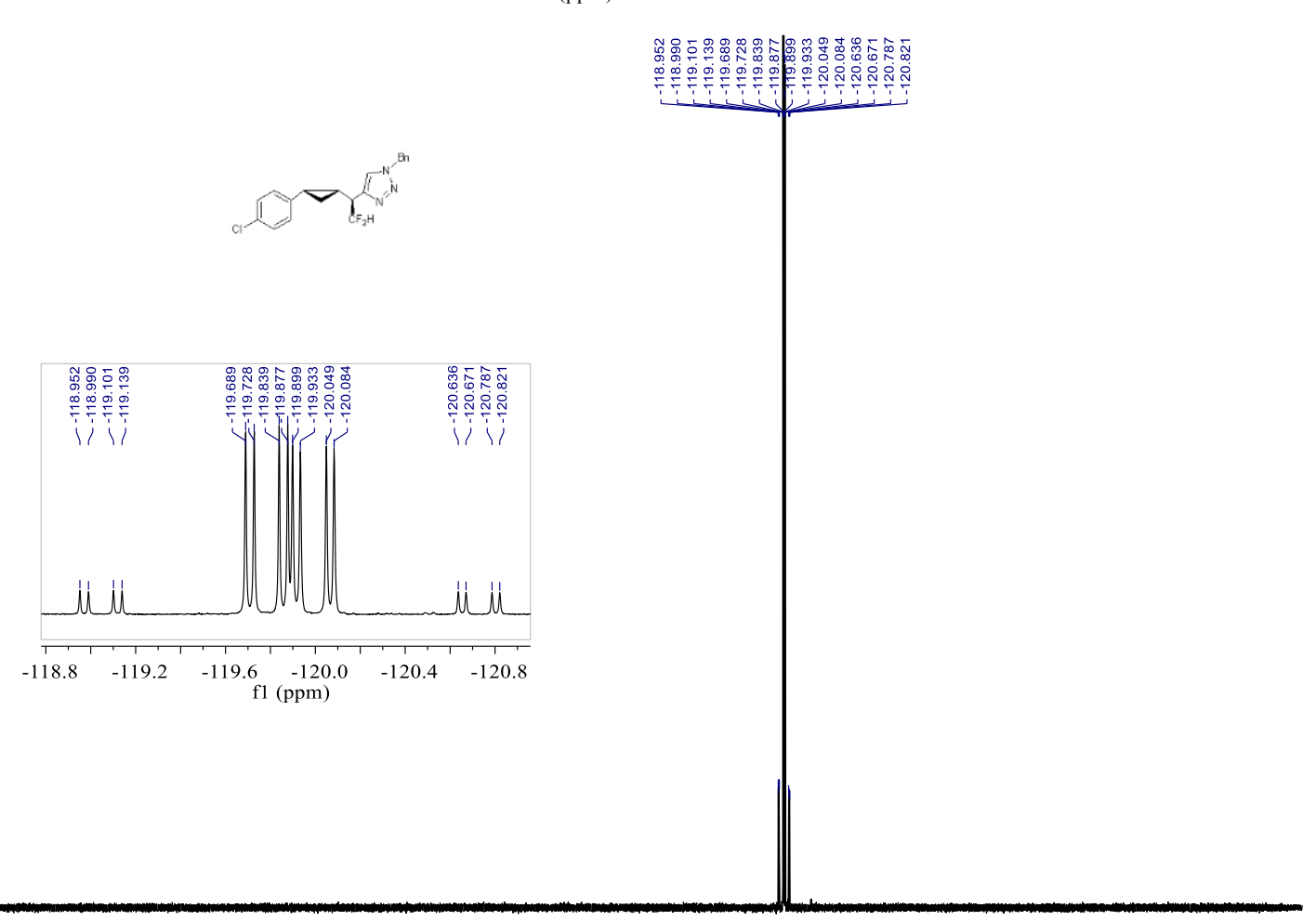

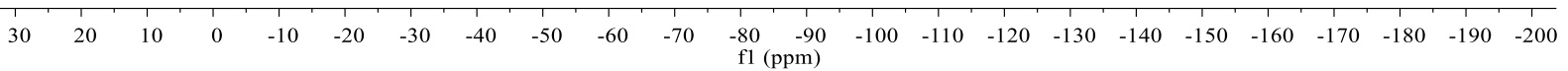




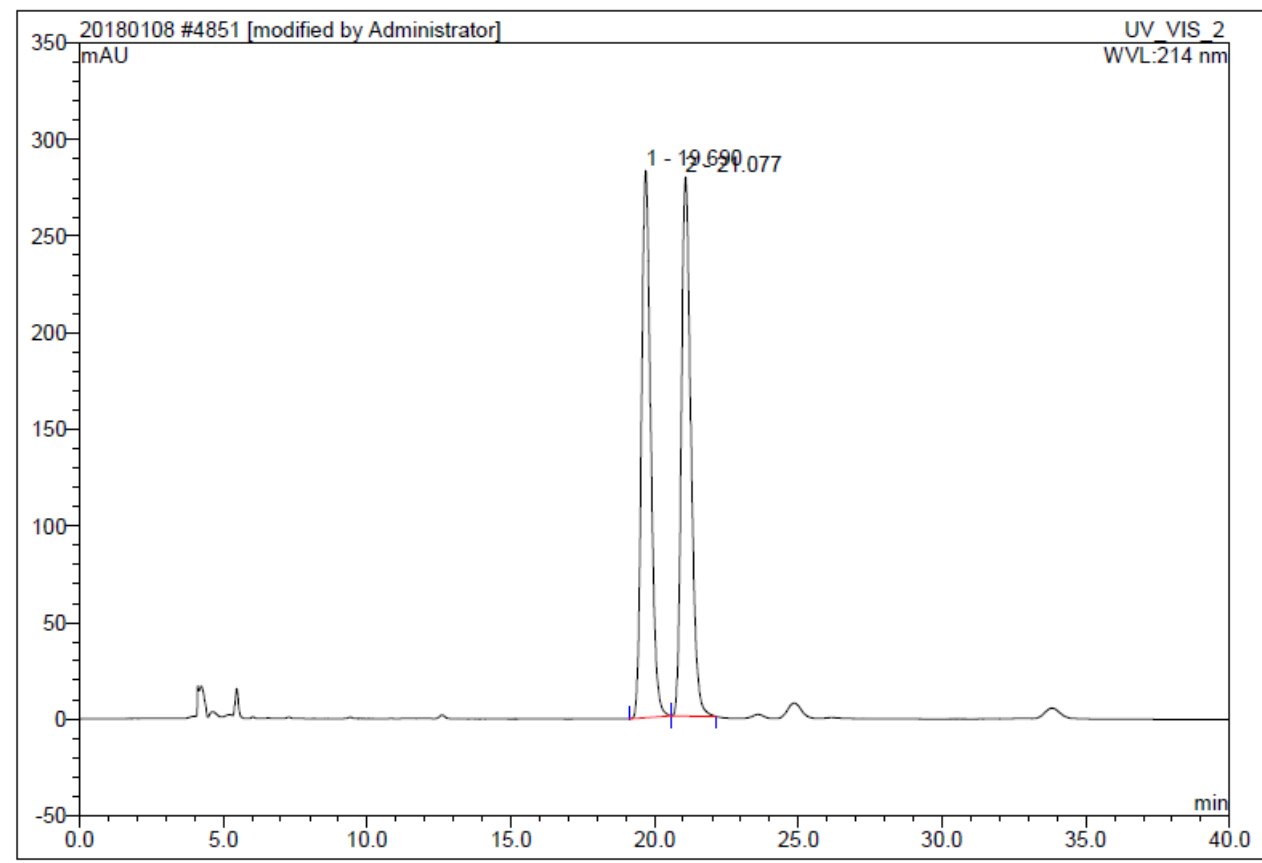

\begin{tabular}{|r|ccccccc|}
\hline No. & $\begin{array}{c}\text { Ret.Time } \\
\text { min }\end{array}$ & Peak Name & $\begin{array}{c}\text { Height } \\
\text { mAU }\end{array}$ & $\begin{array}{c}\text { Area } \\
\text { mAU*min }\end{array}$ & $\begin{array}{r}\text { Rel.Area } \\
\%\end{array}$ & Amount & Type \\
\hline 1 & 19.69 & n.a. & 283.381 & 105.562 & 49.48 & n.a. & BMB $^{*}$ \\
2 & 21.08 & n.a. & 279.149 & 107.798 & 50.52 & n.a. & BMB $^{*}$ \\
\hline Total: & & & 562.530 & 213.360 & 100.00 & 0.000 & \\
\hline
\end{tabular}

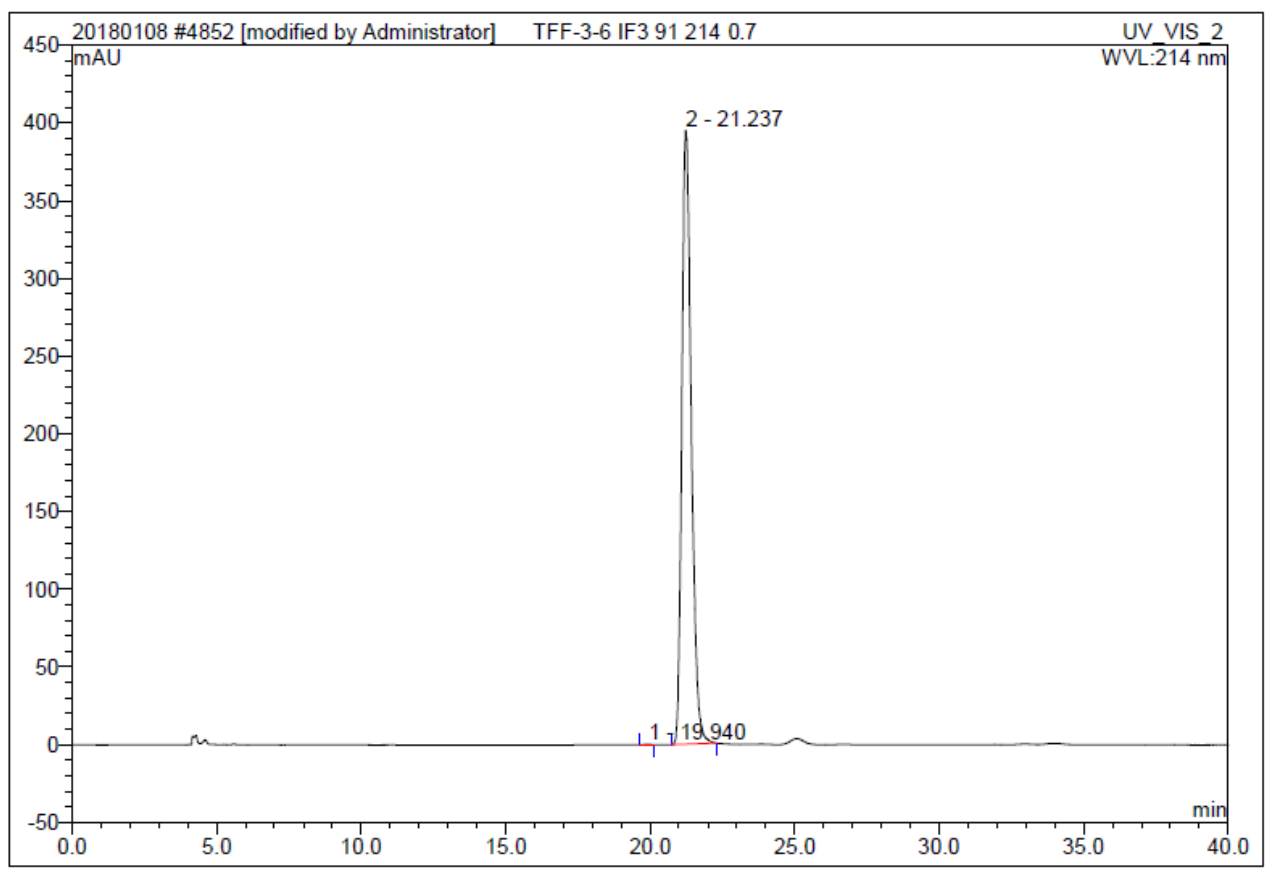

\begin{tabular}{|r|ccrrrrr|}
\hline No. & $\begin{array}{c}\text { Ret.Time } \\
\text { min }\end{array}$ & Peak Name & $\begin{array}{c}\text { Height } \\
\text { mAU }\end{array}$ & $\begin{array}{r}\text { Area } \\
\text { mAU*min }\end{array}$ & $\begin{array}{r}\text { Rel.Area } \\
\%\end{array}$ & Amount & Type \\
\hline 1 & 19.94 & n.a. & 0.196 & 0.044 & 0.03 & n.a. & BMB $^{*}$ \\
2 & 21.24 & n.a. & 394.965 & 150.241 & 99.97 & n.a. & BMB $^{*}$ \\
\hline Total: & & & 395.161 & 150.285 & 100.00 & 0.000 & \\
\hline
\end{tabular}



(10d).

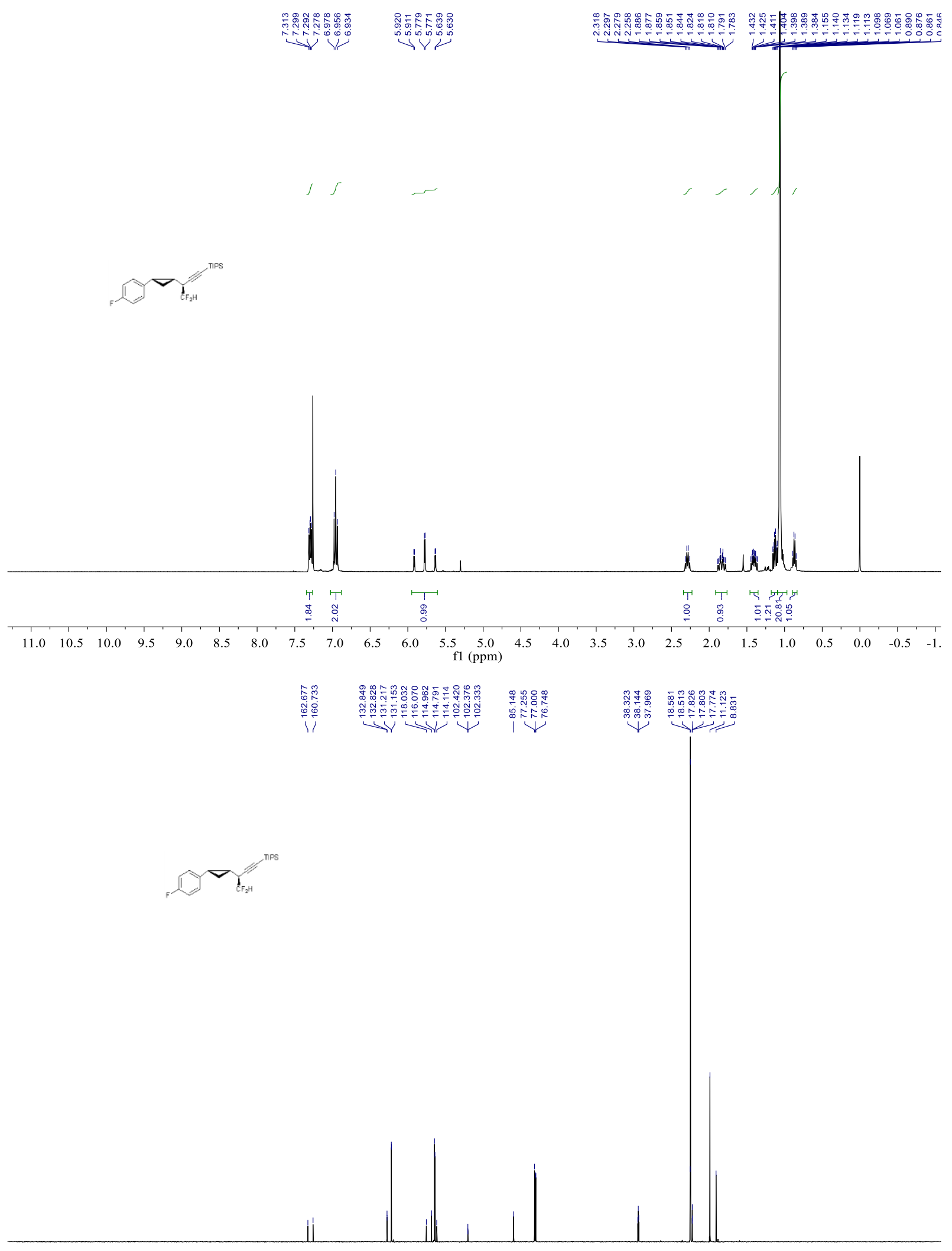




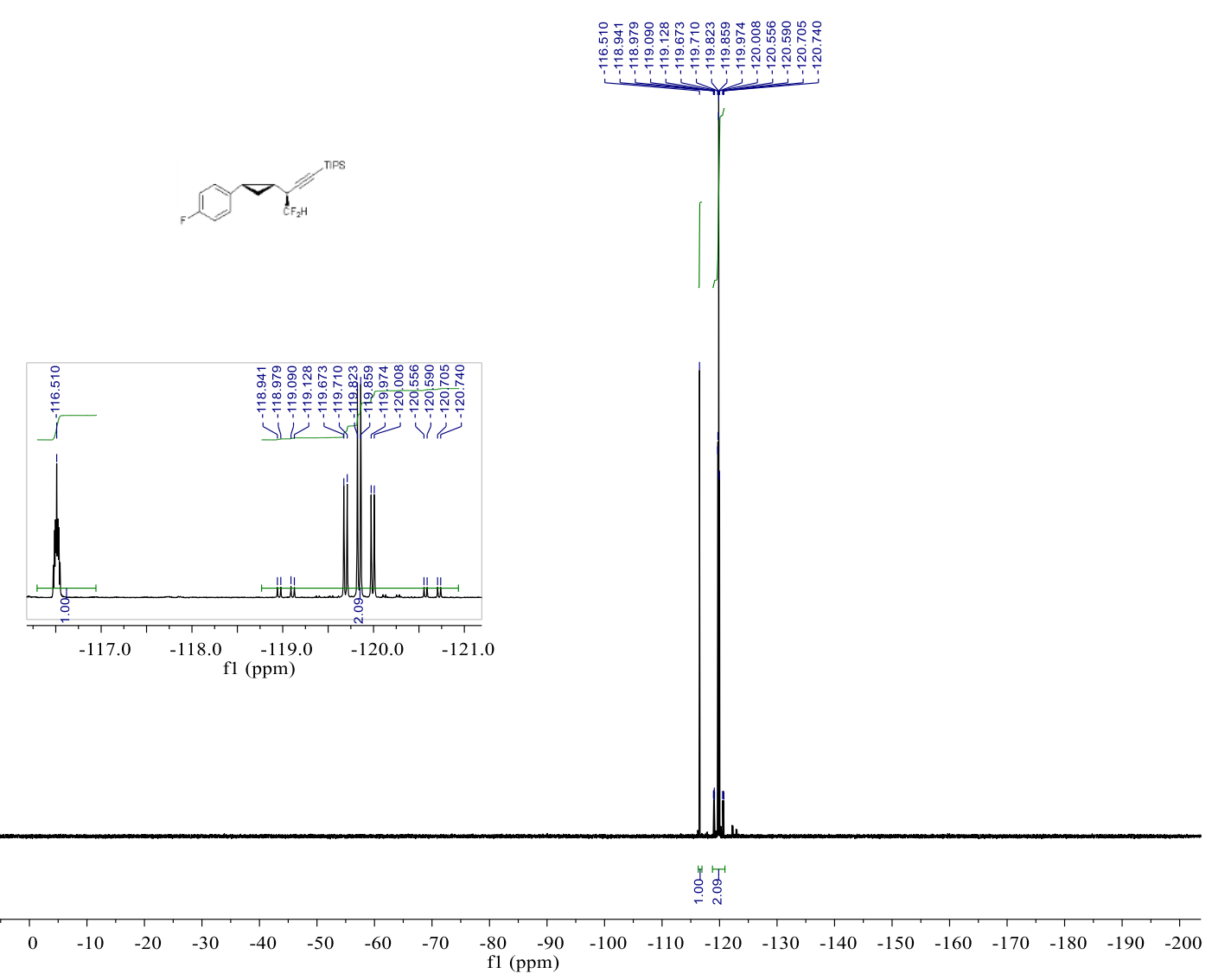

1-Benzyl-4-((S)-2,2-difluoro-1-((1R,2S)-2-(4-fluorophenyl)cyclopropyl)ethyl)-1H-1,2,3-triazole (11).

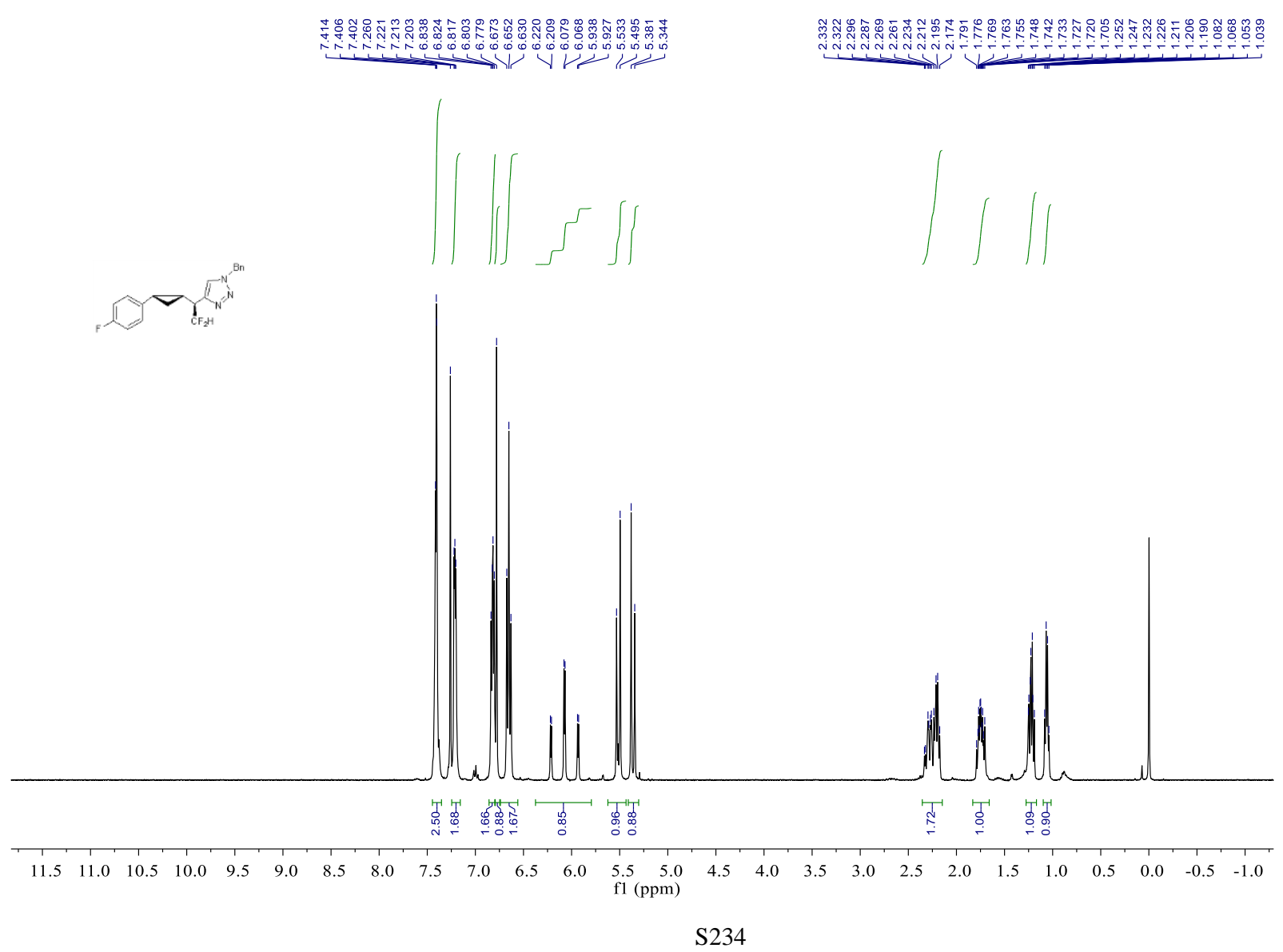




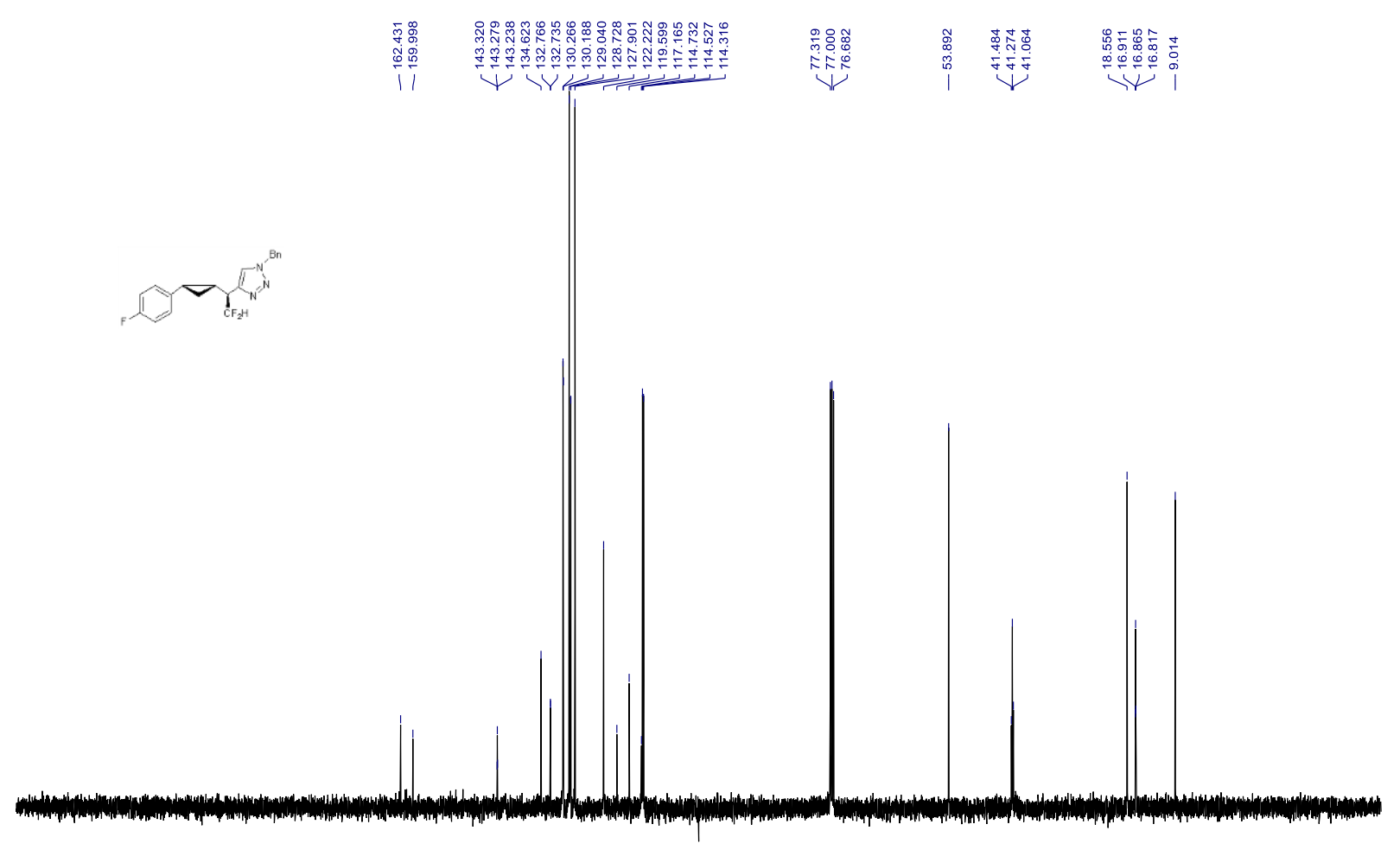

$\begin{array}{llllllllllllllllllllllllllllllll}230 & 220 & 210 & 200 & 190 & 180 & 170 & 160 & 150 & 140 & 130 & 120 & 110 & 100 & 90 & 80 & 70 & 60 & 50 & 40 & 30 & 20 & 10 & 0 & -10 & -20 & -31\end{array}$
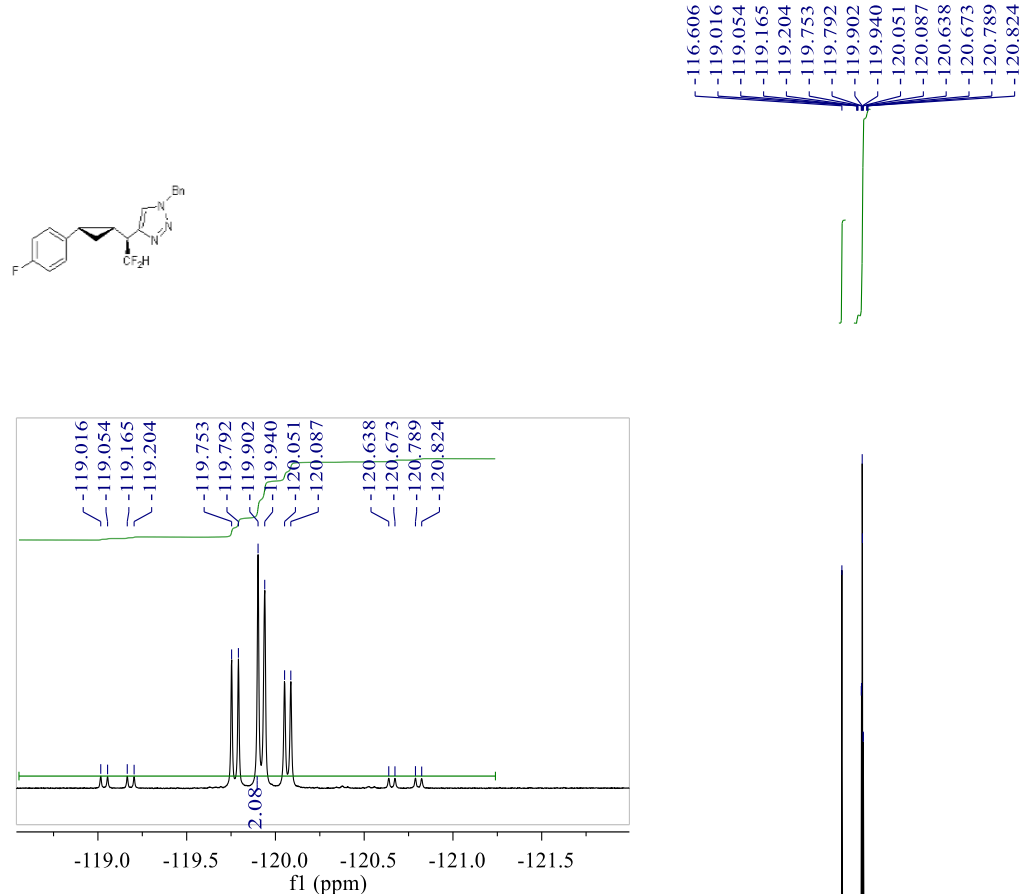

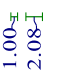

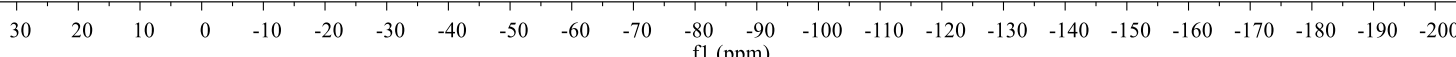




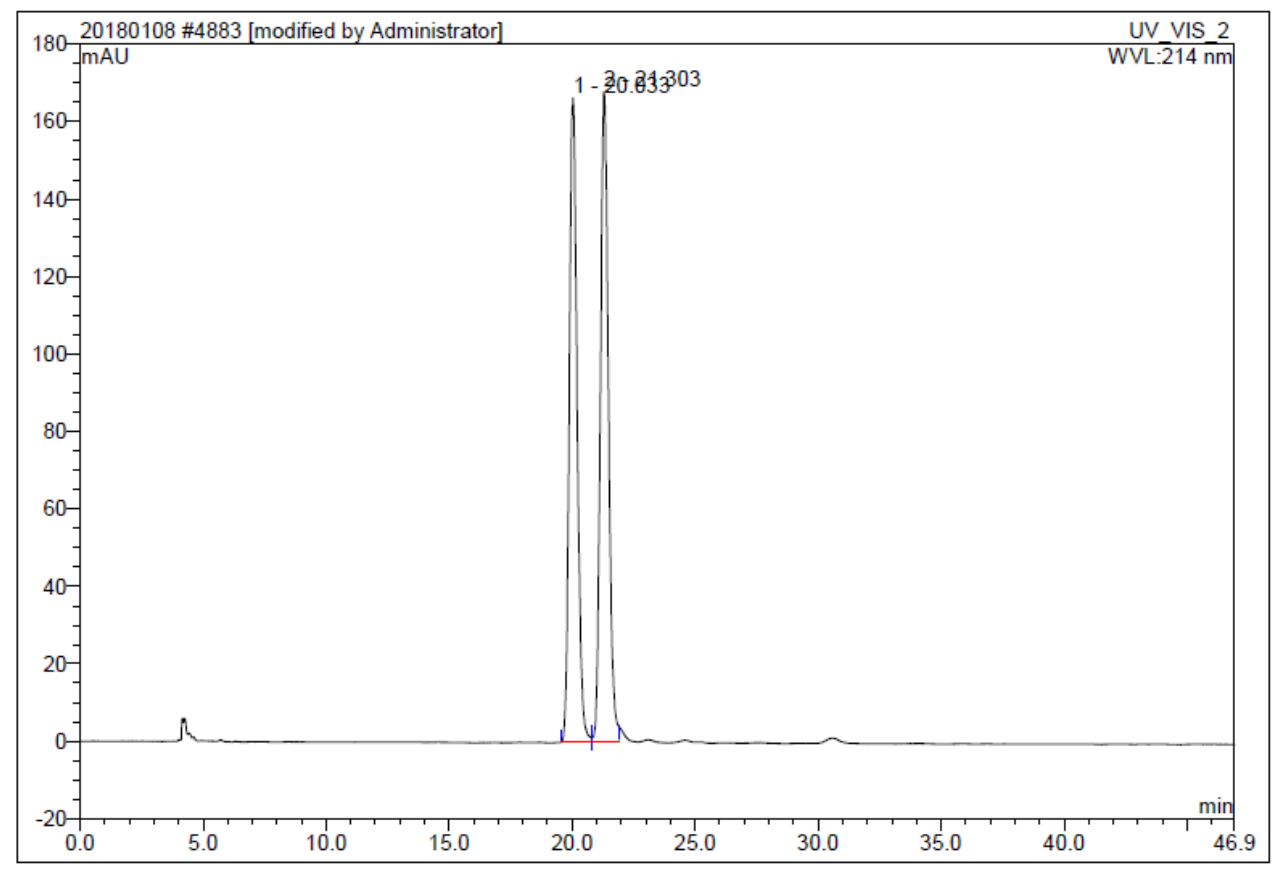

\begin{tabular}{|r|cccrrrr|}
\hline No. & $\begin{array}{c}\text { Ret.Time } \\
\text { min }\end{array}$ & Peak Name & $\begin{array}{c}\text { Height } \\
\text { mAU }\end{array}$ & $\begin{array}{c}\text { Area } \\
\text { mAU*min }\end{array}$ & $\begin{array}{c}\text { Rel.Area } \\
\%\end{array}$ & Amount & Type \\
\hline 1 & 20.03 & n.a. & 166.370 & 61.609 & 49.17 & n.a. & BM $^{*}$ \\
2 & 21.30 & n.a. & 168.062 & 63.694 & 50.83 & n.a. & M $^{*}$ \\
\hline Total: & & & 334.432 & 125.304 & 100.00 & 0.000 & \\
\hline
\end{tabular}

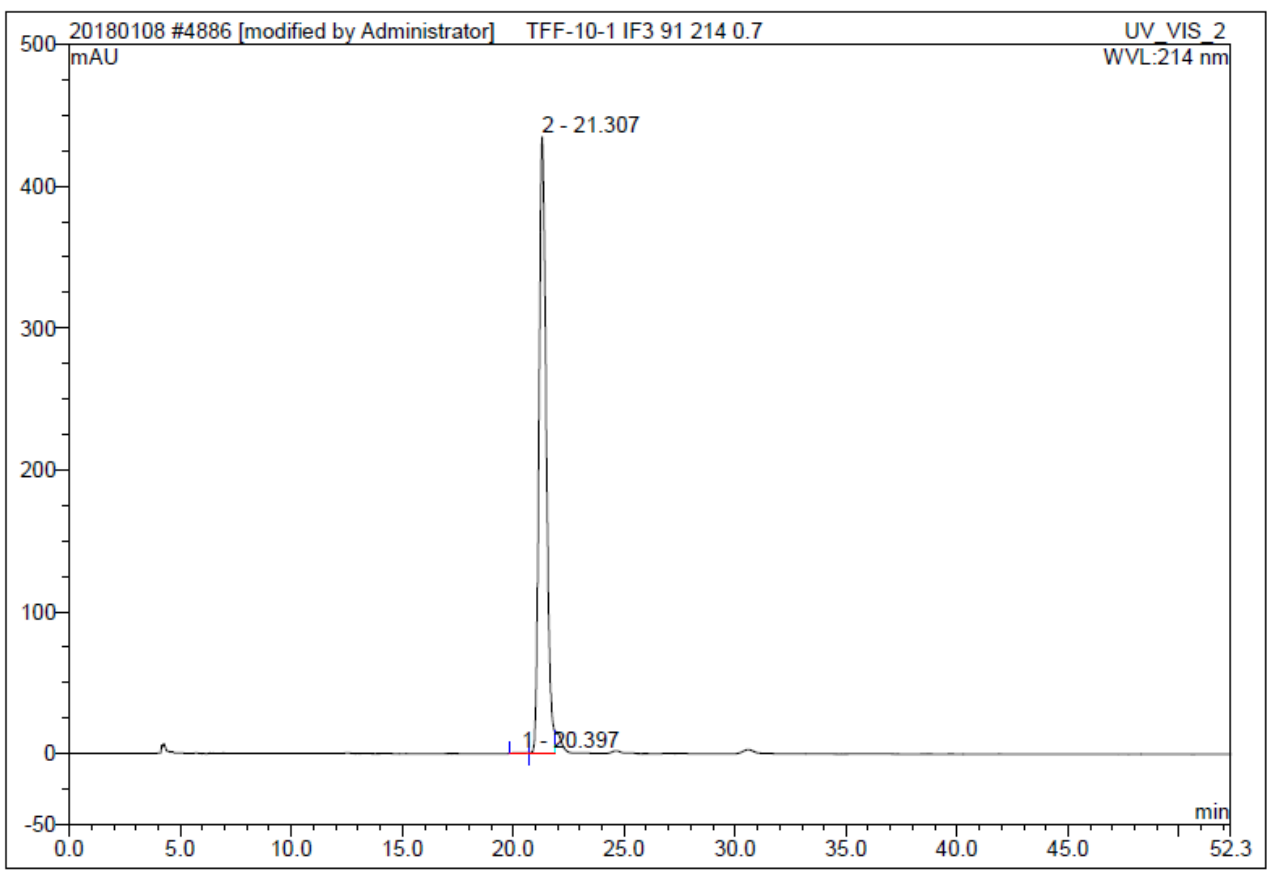

\begin{tabular}{|r|ccrrrrr|}
\hline No. & $\begin{array}{c}\text { Ret.Time } \\
\text { min }\end{array}$ & Peak Name & $\begin{array}{c}\text { Height } \\
\text { mAU }\end{array}$ & $\begin{array}{r}\text { Area } \\
\text { mAU*min }\end{array}$ & $\begin{array}{r}\text { Rel.Area } \\
\%\end{array}$ & Amount & Type \\
\hline 1 & 20.40 & n.a. & 0.444 & 0.193 & 0.12 & n.a. & BMB $^{*}$ \\
2 & 21.31 & n.a. & 434.675 & 164.942 & 99.88 & n.a. & BM $^{*}$ \\
\hline Total: & & & 435.119 & 165.135 & 100.00 & 0.000 & \\
\hline
\end{tabular}




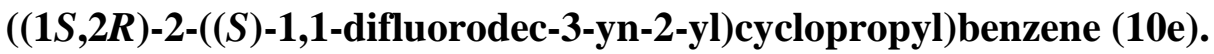
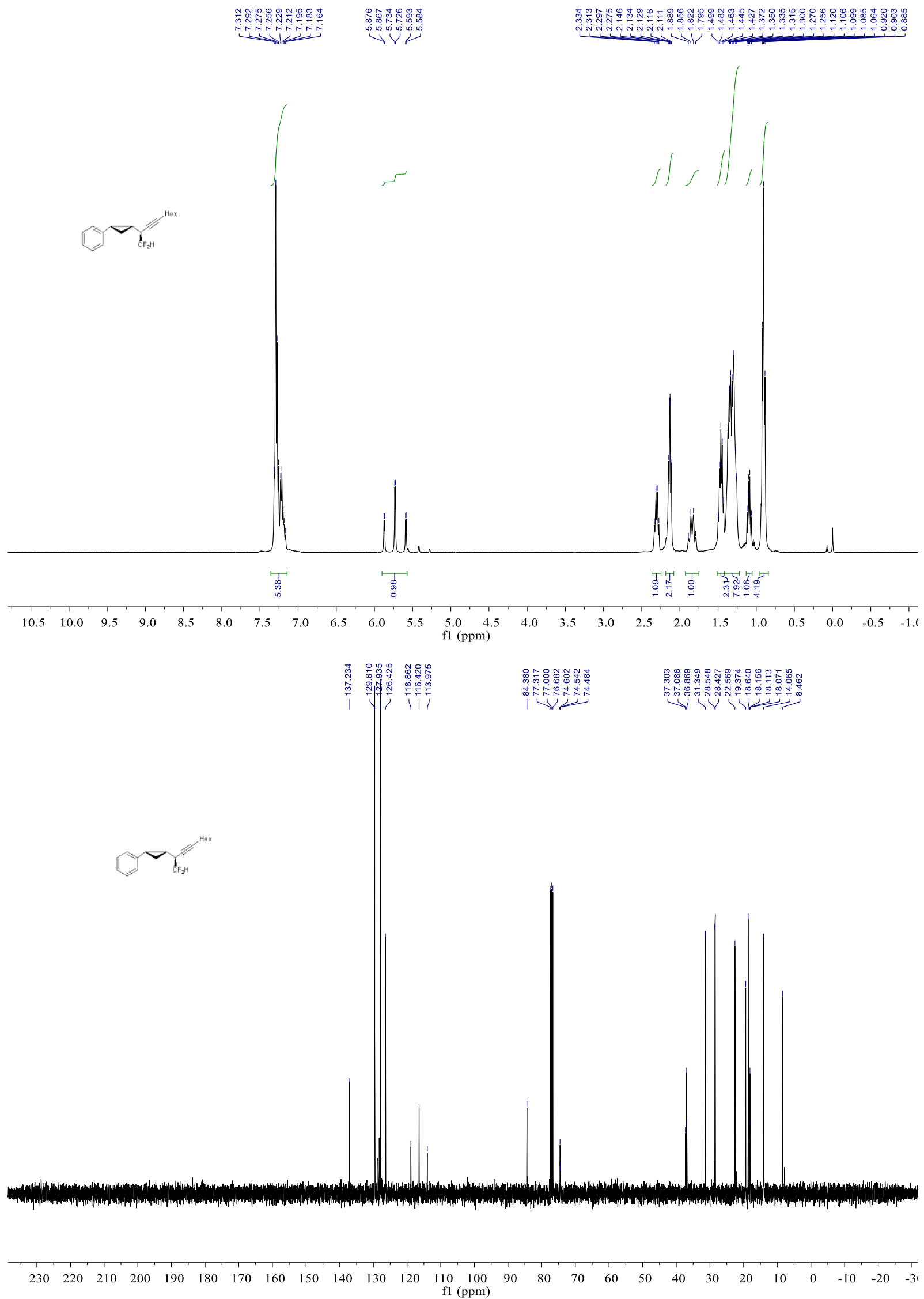

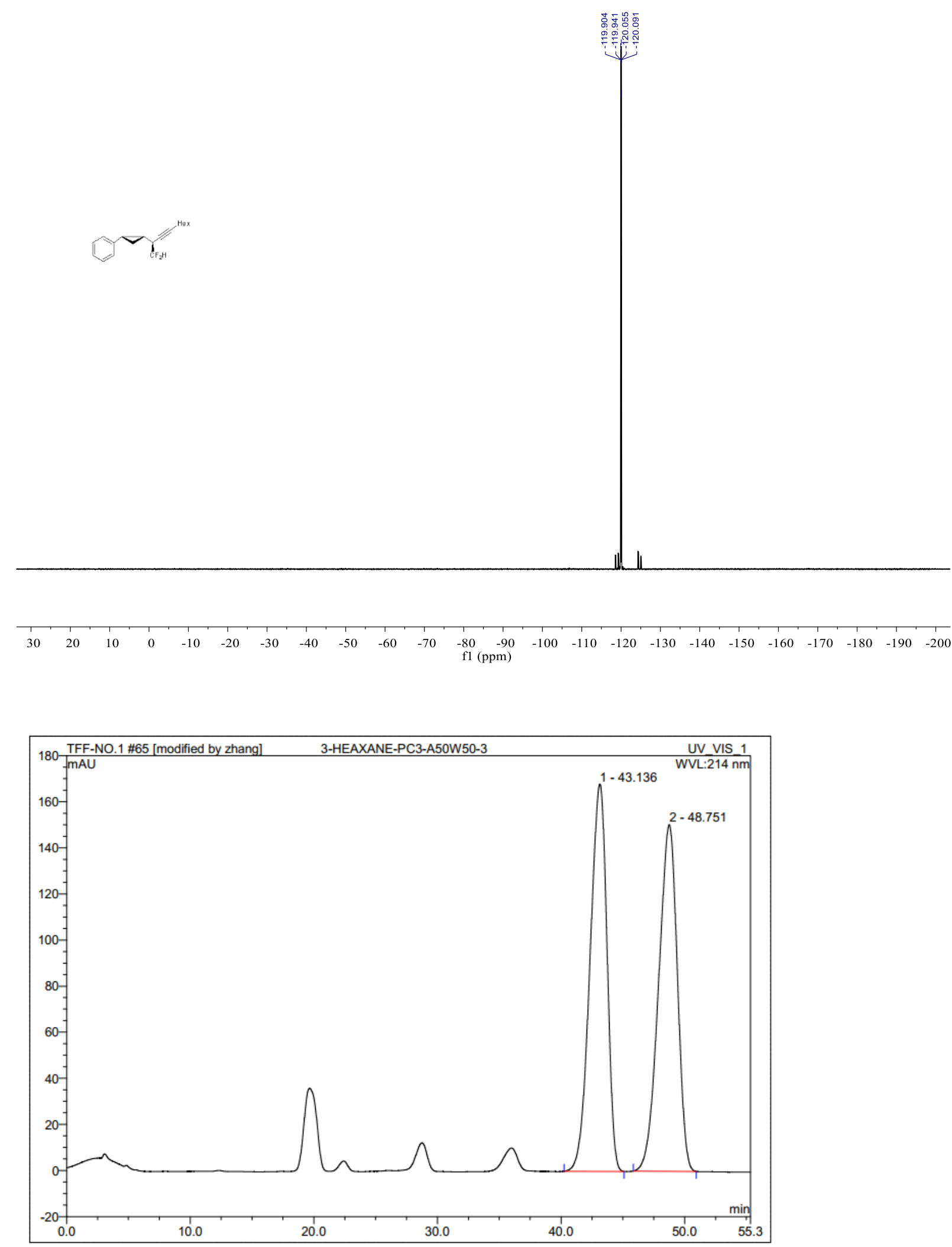

\begin{tabular}{|r|ccccccc|}
\hline No. & $\begin{array}{c}\text { Ret.Time } \\
\text { min }\end{array}$ & Peak Name & $\begin{array}{c}\text { Height } \\
\text { mAU }\end{array}$ & $\begin{array}{c}\text { Area } \\
\text { mAU*min }\end{array}$ & $\begin{array}{c}\text { Rel.Area } \\
\%\end{array}$ & $\begin{array}{c}\text { Amount } \\
\%\end{array}$ & Type \\
\hline 1 & 43.14 & n.a. & 168.080 & 267.415 & 50.35 & n.a. & BMB \\
2 & 48.75 & n.a. & 150.453 & 263.669 & 49.65 & n.a. & BMB \\
\hline Total: & & & 318.533 & 531.084 & 100.00 & 0.000 & \\
\hline
\end{tabular}




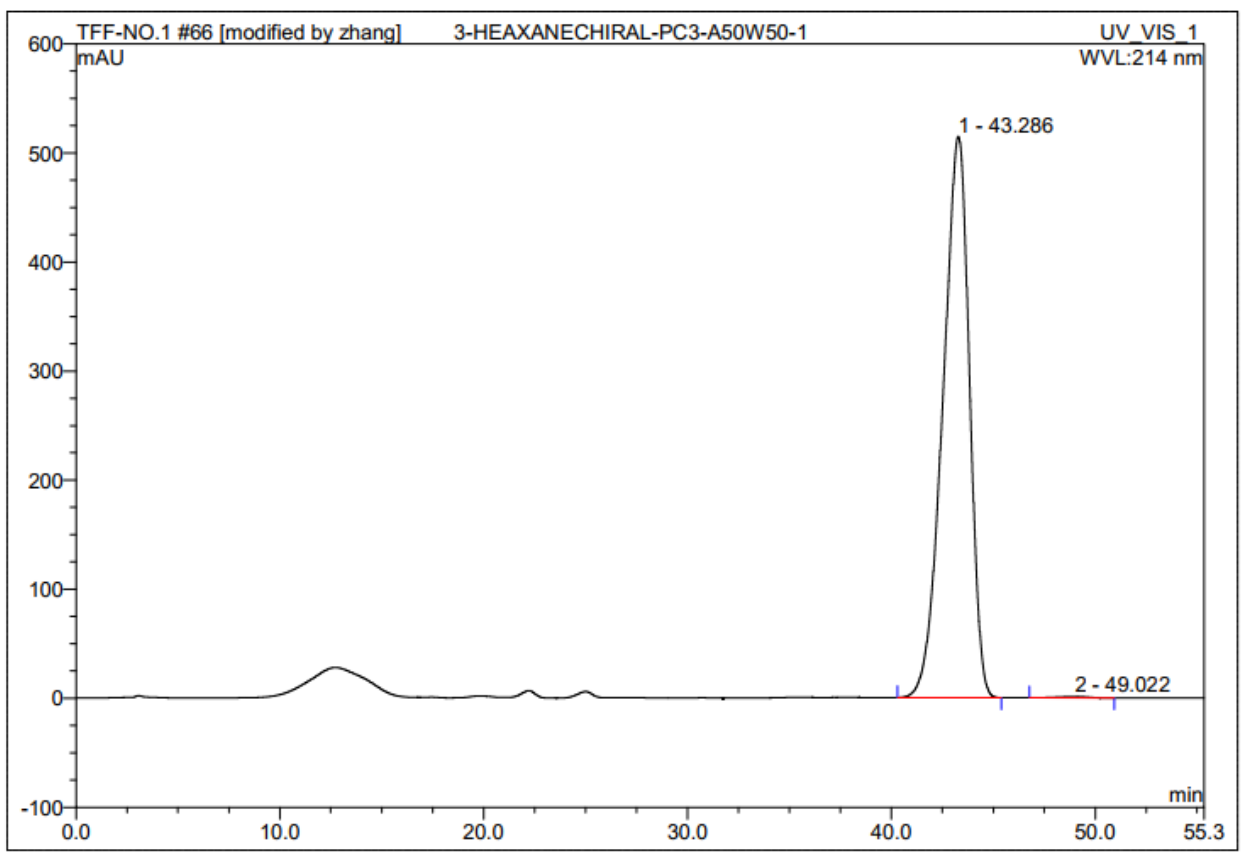

\begin{tabular}{|r|rrrrrrr|}
\hline No. & $\begin{array}{c}\text { Ret.Time } \\
\text { min }\end{array}$ & Peak Name & $\begin{array}{c}\text { Height } \\
\text { mAU }\end{array}$ & $\begin{array}{c}\text { Area } \\
\text { mAU*min }\end{array}$ & $\begin{array}{c}\text { Rel.Area } \\
\%\end{array}$ & $\begin{array}{c}\text { Amount } \\
\%\end{array}$ & Type \\
\hline 1 & 43.29 & n.a. & 514.801 & 775.858 & 99.74 & n.a. & BMB \\
2 & 49.02 & n.a. & 1.200 & 2.006 & 0.26 & n.a. & BMB $^{*}$ \\
\hline Total: & & & 516.001 & 777.865 & 100.00 & 0.000 & \\
\hline
\end{tabular}

((1S,2R)-2-((S)-7-(Benzyloxy)-1,1-difluorohept-3-yn-2-yl)cyclopropyl)benzene (10f).

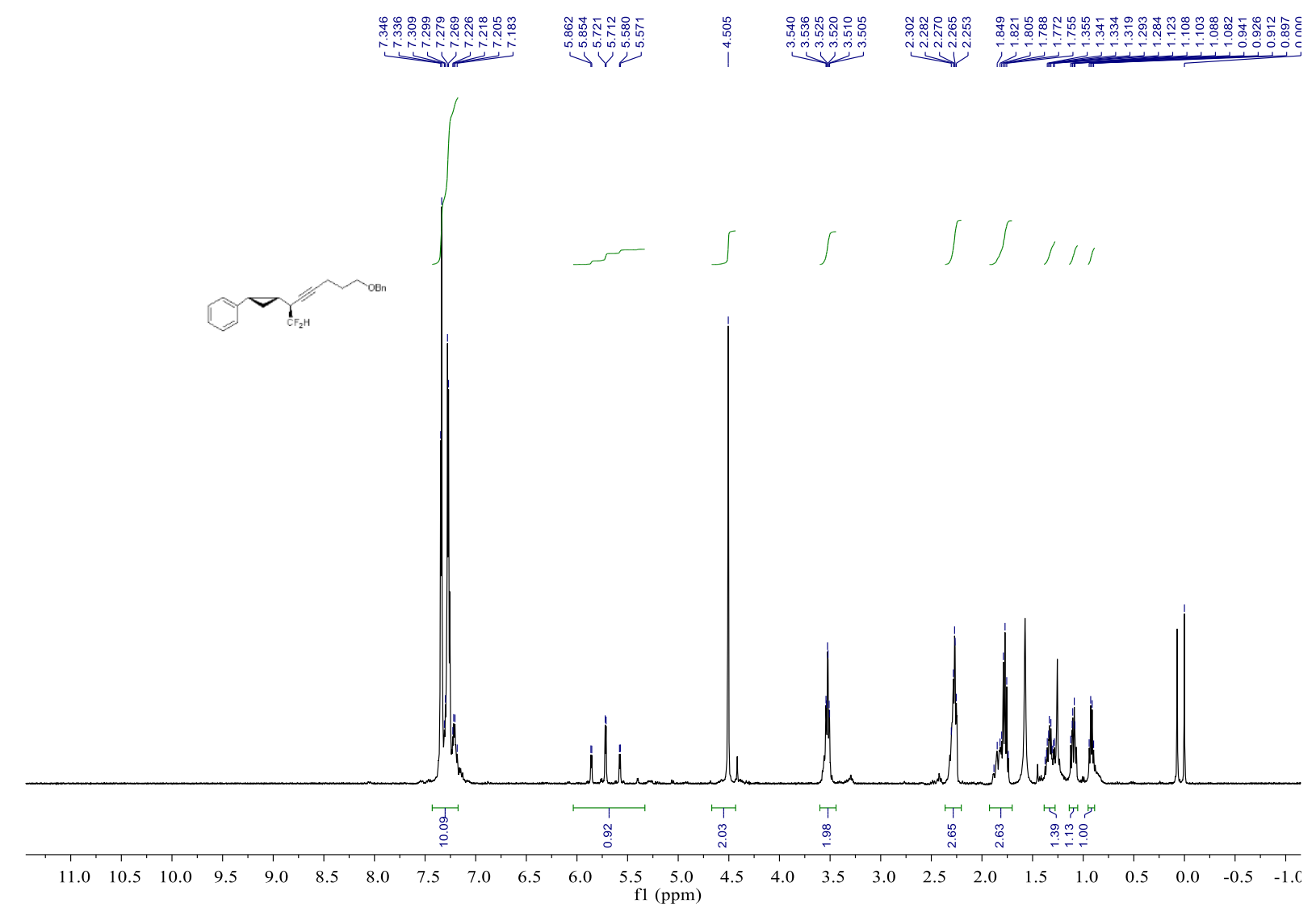



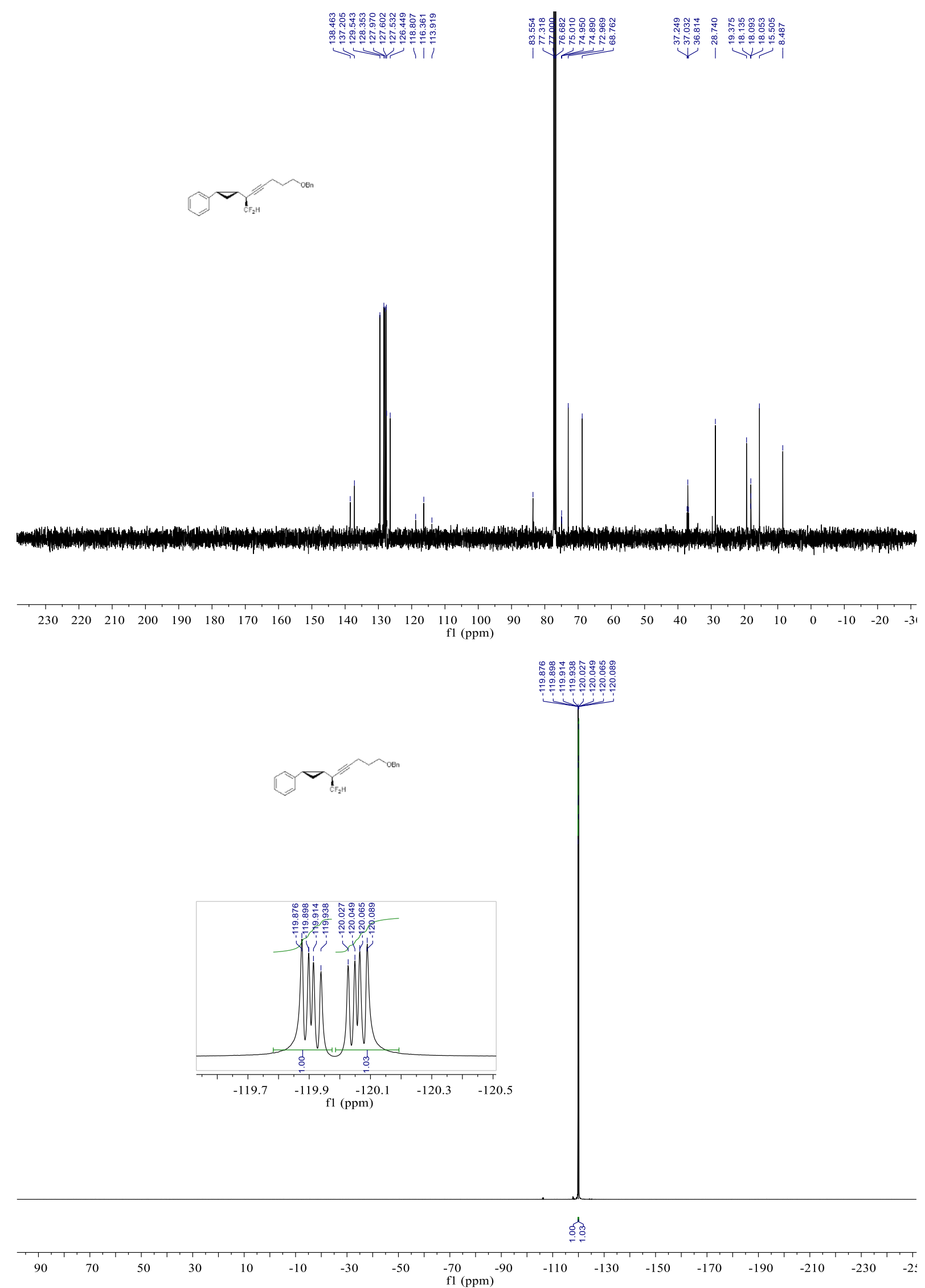


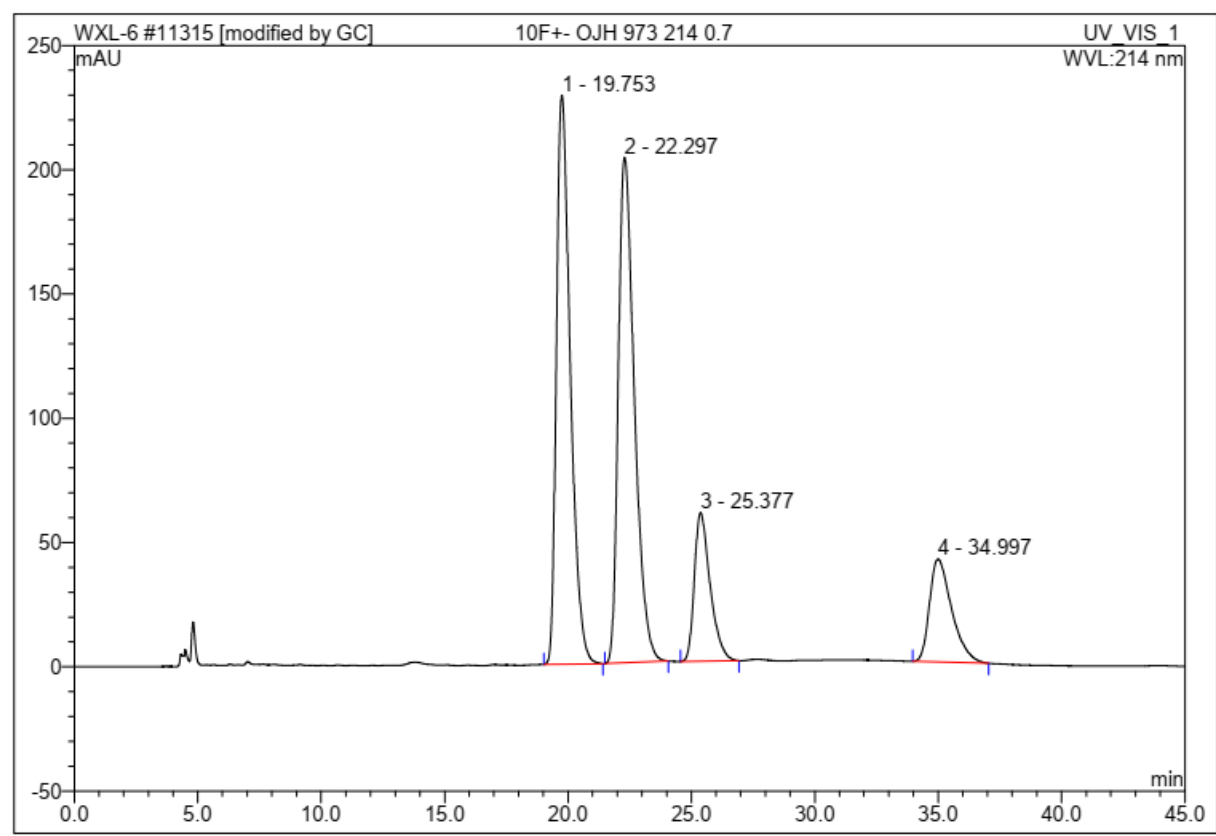

\begin{tabular}{|r|ccrrrrr|}
\hline No. & $\begin{array}{c}\text { Ret.Time } \\
\text { min }\end{array}$ & Peak Name & $\begin{array}{c}\text { Height } \\
\text { mAU }\end{array}$ & $\begin{array}{c}\text { Area } \\
\text { mAU } \text { min }\end{array}$ & $\begin{array}{c}\text { Rel.Area } \\
\%\end{array}$ & Amount & Type \\
\hline 1 & 19.75 & n.a. & 229.035 & 149.652 & 38.25 & n.a. & BMB \\
2 & 22.30 & n.a. & 203.482 & 153.795 & 39.31 & n.a. & BMB \\
3 & 25.38 & n.a. & 59.857 & 44.484 & 11.37 & n.a. & BMB \\
4 & 35.00 & n.a. & 41.549 & 43.309 & 11.07 & n.a. & BMB \\
\hline Total: & & & 533.923 & 391.240 & 100.00 & 0.000 & \\
\hline
\end{tabular}

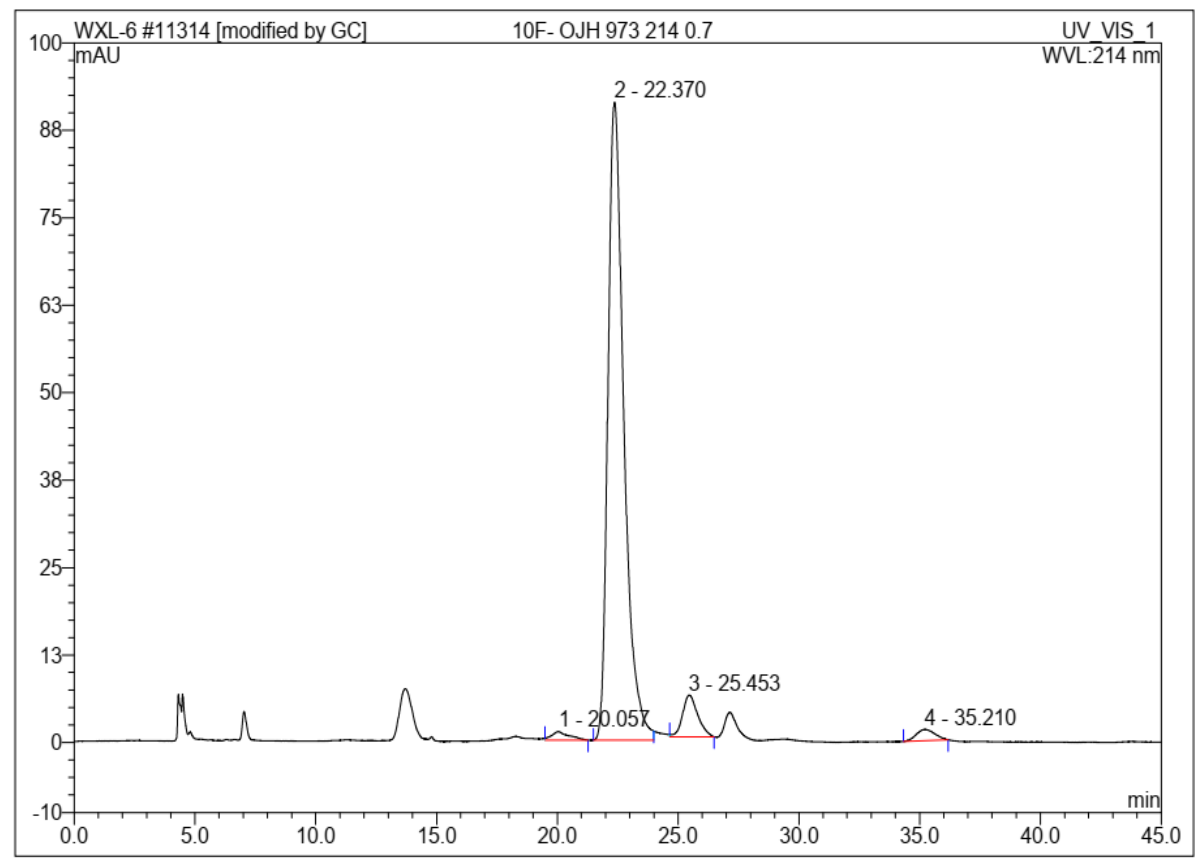

\begin{tabular}{|r|ccrrrrr|}
\hline No. & $\begin{array}{c}\text { Ret.Time } \\
\text { min }\end{array}$ & Peak Name & $\begin{array}{c}\text { Height } \\
\text { mAU }\end{array}$ & $\begin{array}{c}\text { Area } \\
\text { mAU*min }\end{array}$ & $\begin{array}{r}\text { Rel.Area } \\
\%\end{array}$ & Amount & Type \\
\hline 1 & 20.06 & n.a. & 1.221 & 1.018 & 1.33 & n.a. & MB $^{*}$ \\
2 & 22.37 & n.a. & 91.185 & 69.735 & 91.13 & n.a. & BM $^{*}$ \\
3 & 25.45 & n.a. & 5.950 & 4.353 & 5.69 & n.a. & MB $^{*}$ \\
4 & 35.21 & n.a. & 1.606 & 1.419 & 1.85 & n.a. & BMB $^{*}$ \\
\hline Total: & & & 99.962 & 76.525 & 100.00 & 0.000 & \\
\hline
\end{tabular}



(10g).

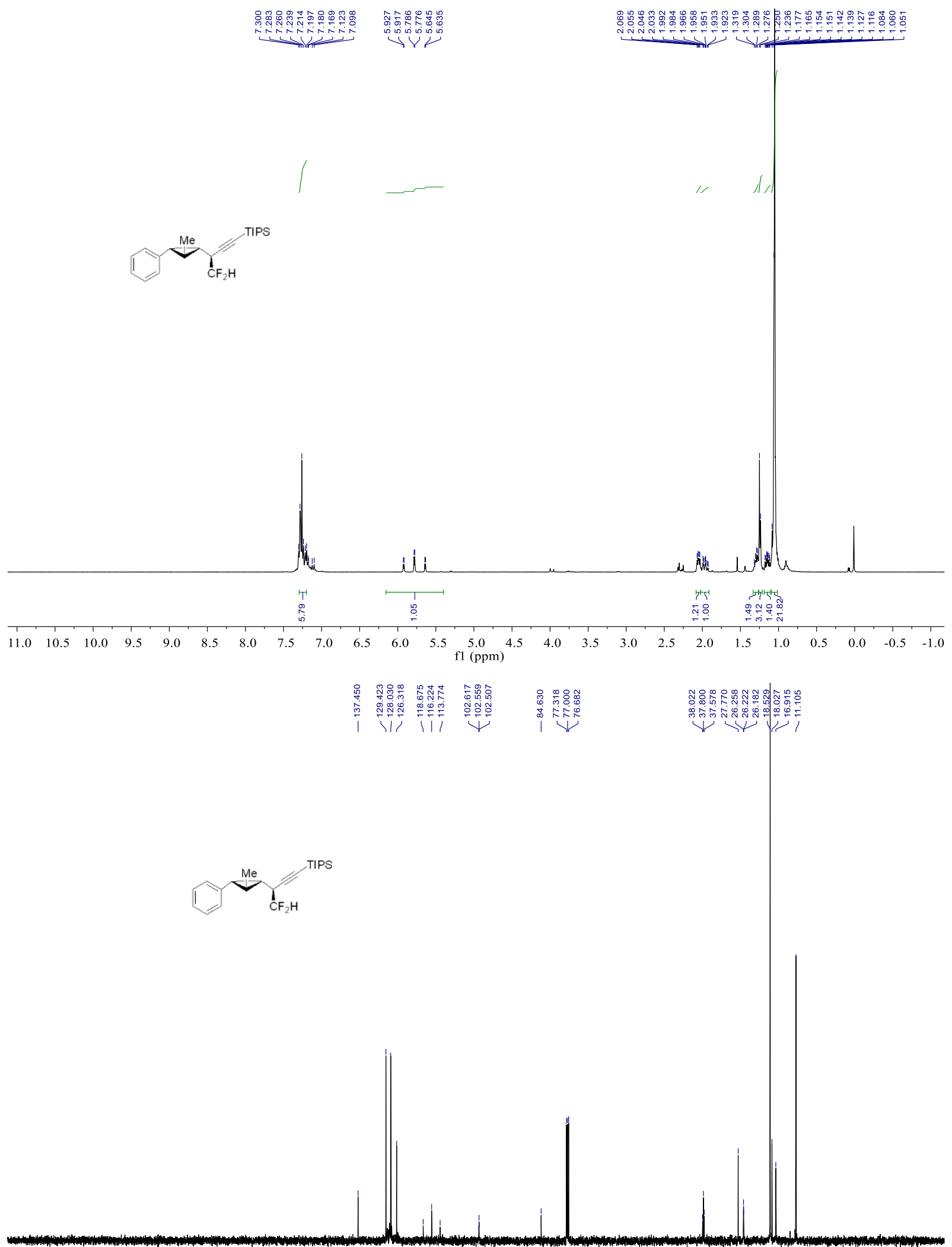




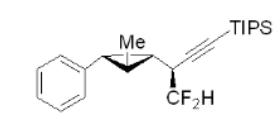
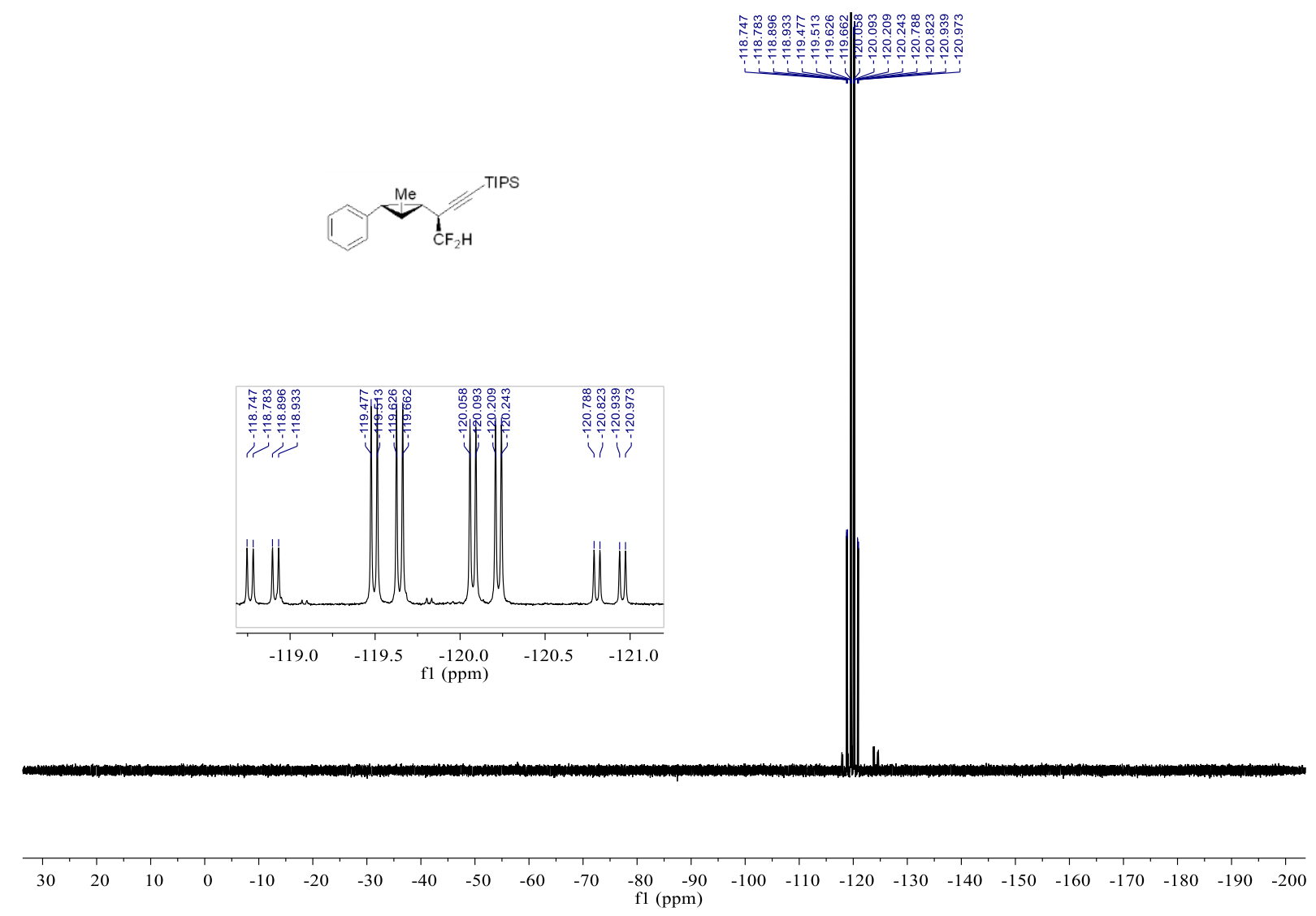

1-Benzyl-4-((S)-2,2-difluoro-1-((1R,2R,3S)-2-methyl-3-phenylcyclopropyl)ethyl)-1H-1,2,3-triazo le $\left(10 g^{\prime}\right)$.

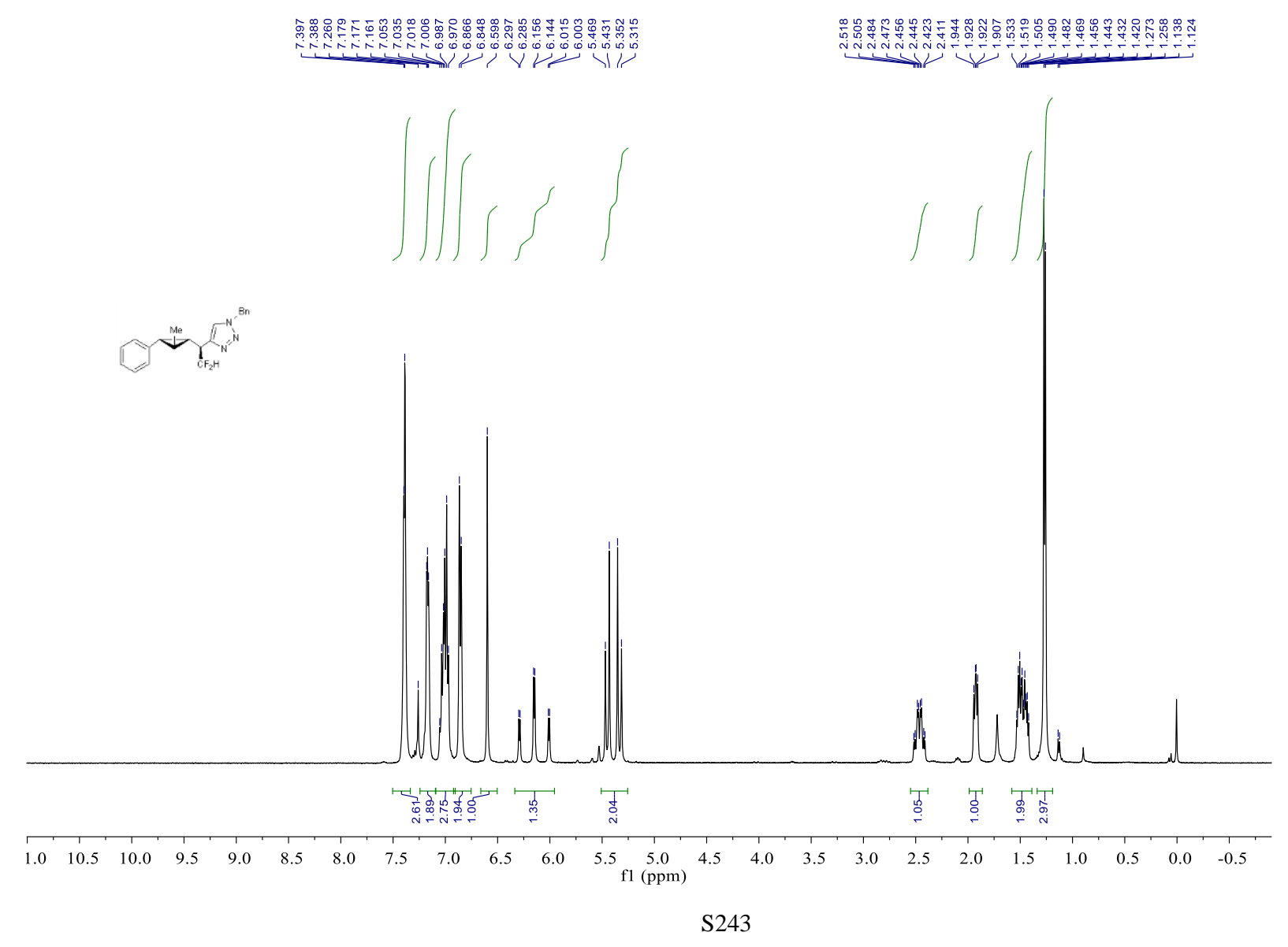




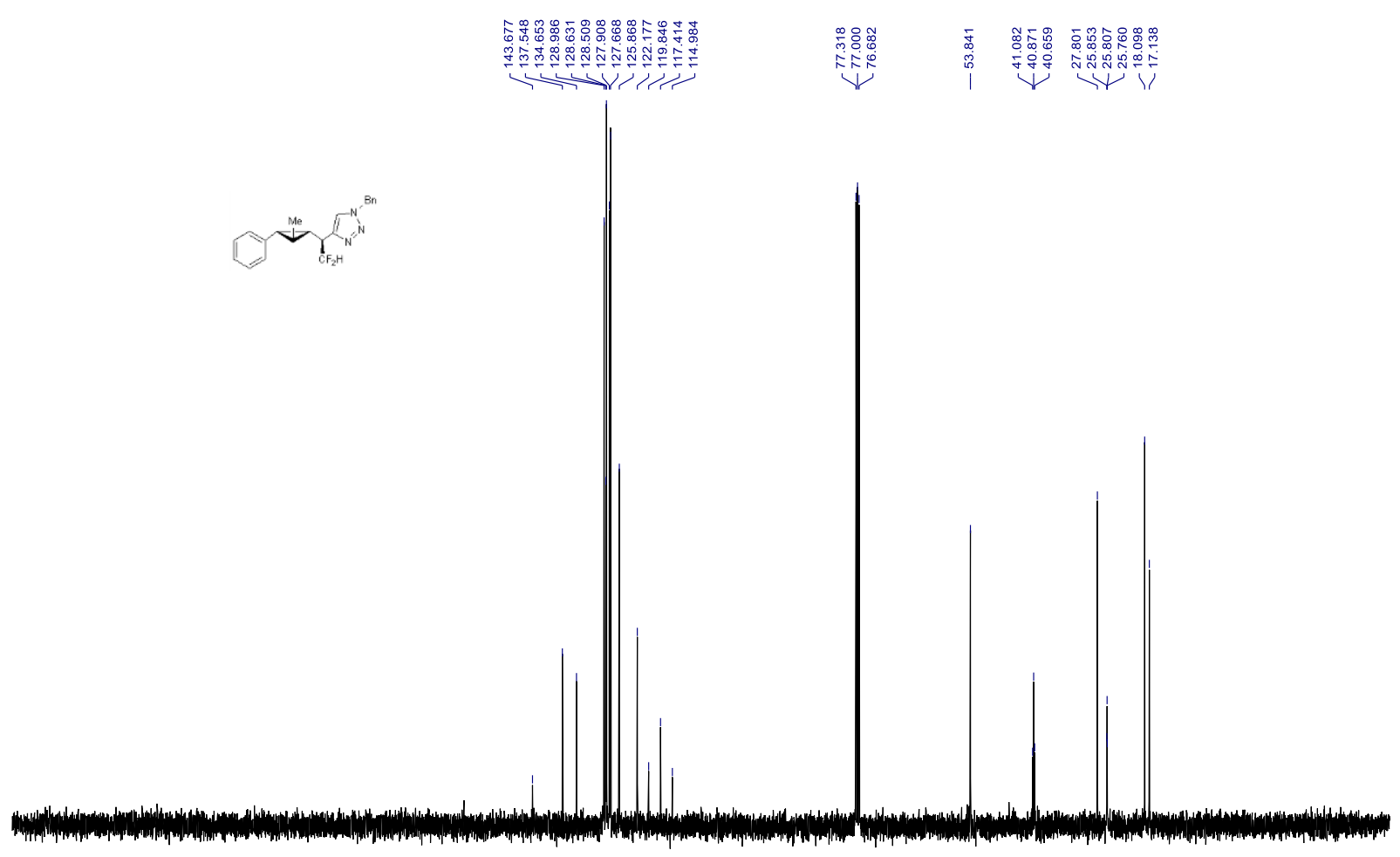

\begin{tabular}{lllllllllllllllllllllllllllllllllllllllllll}
\hline 50 & 240 & 230 & 220 & 210 & 200 & 190 & 180 & 170 & 160 & 150 & 140 & 130 & 120 & 110 & 100 & 90 & 80 & 70 & 60 & 50 & 40 & 30 & 20 & 10 & 0 & -10 & -20 & -31
\end{tabular}

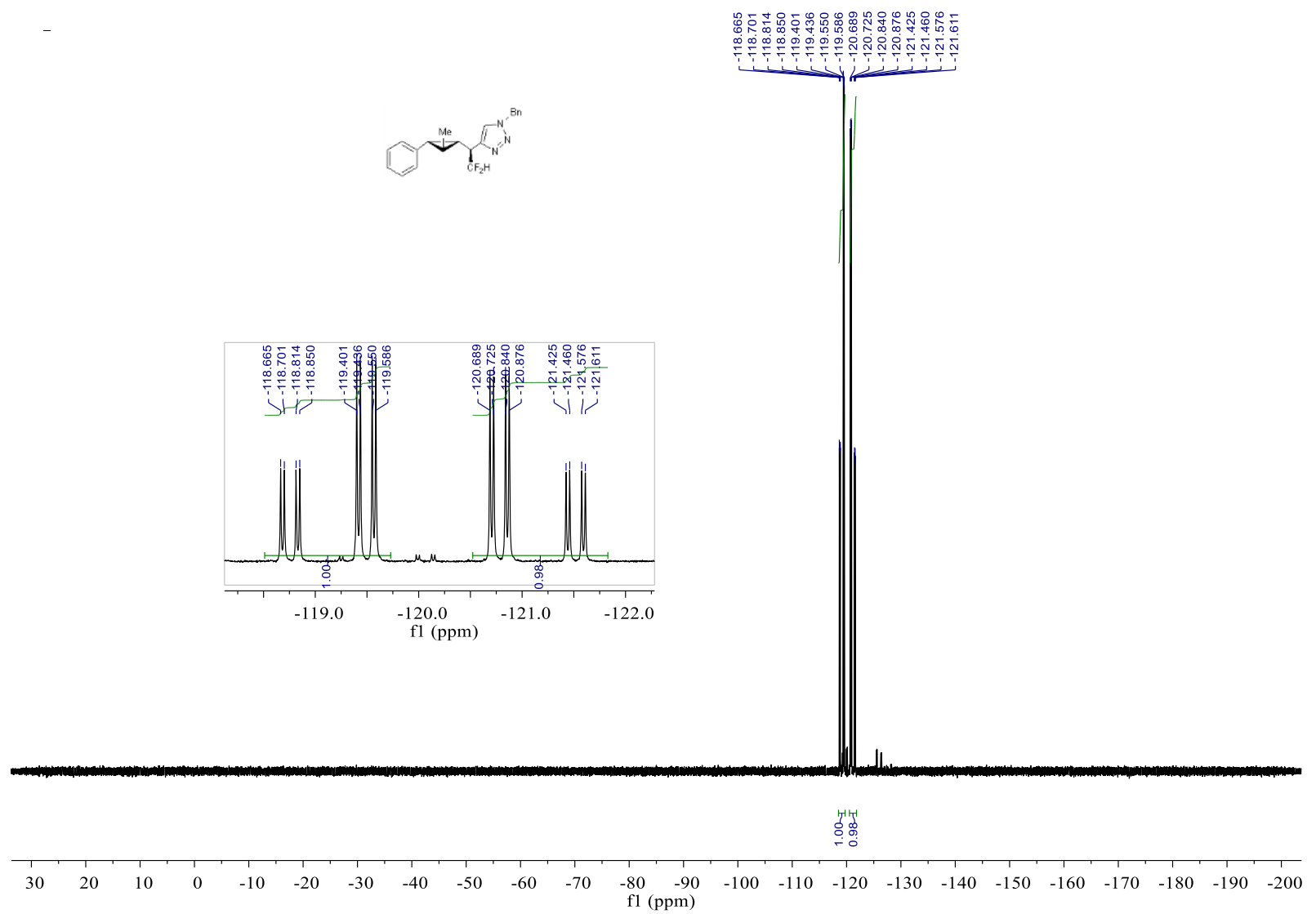




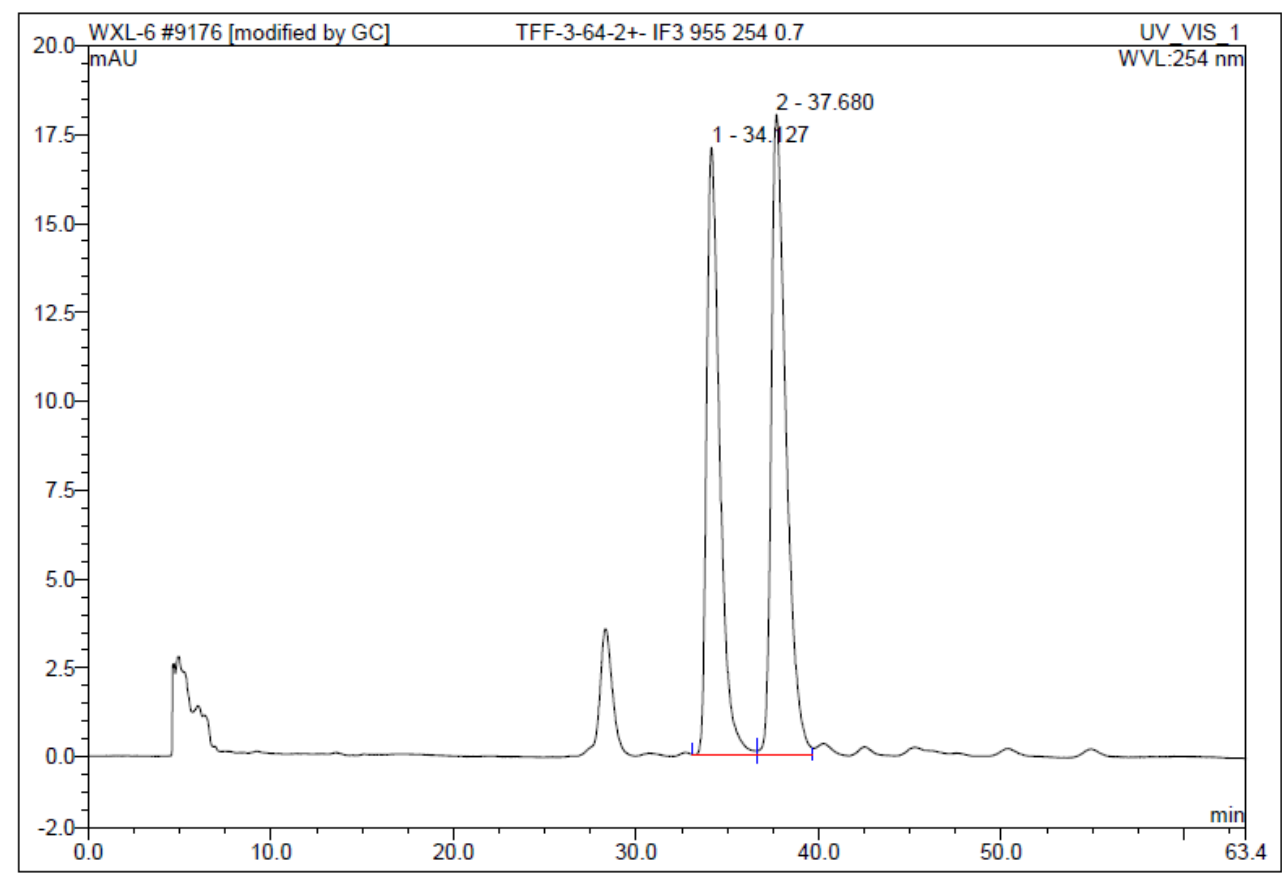

\begin{tabular}{|r|ccrrrrr|}
\hline No. & $\begin{array}{c}\text { Ret.Time } \\
\text { min }\end{array}$ & Peak Name & $\begin{array}{c}\text { Height } \\
\text { mAU }\end{array}$ & $\begin{array}{c}\text { Area } \\
\text { mAU }^{*} \text { min }\end{array}$ & $\begin{array}{r}\text { Rel.Area } \\
\%\end{array}$ & Amount & Type \\
\hline 1 & 34.13 & n.a. & 17.095 & 14.325 & 45.85 & n.a. & BM $^{*}$ \\
2 & 37.68 & n.a. & 18.015 & 16.916 & 54.15 & n.a. & M * $^{*}$ \\
\hline Total: & & & 35.110 & 31.241 & 100.00 & 0.000 & \\
\hline
\end{tabular}

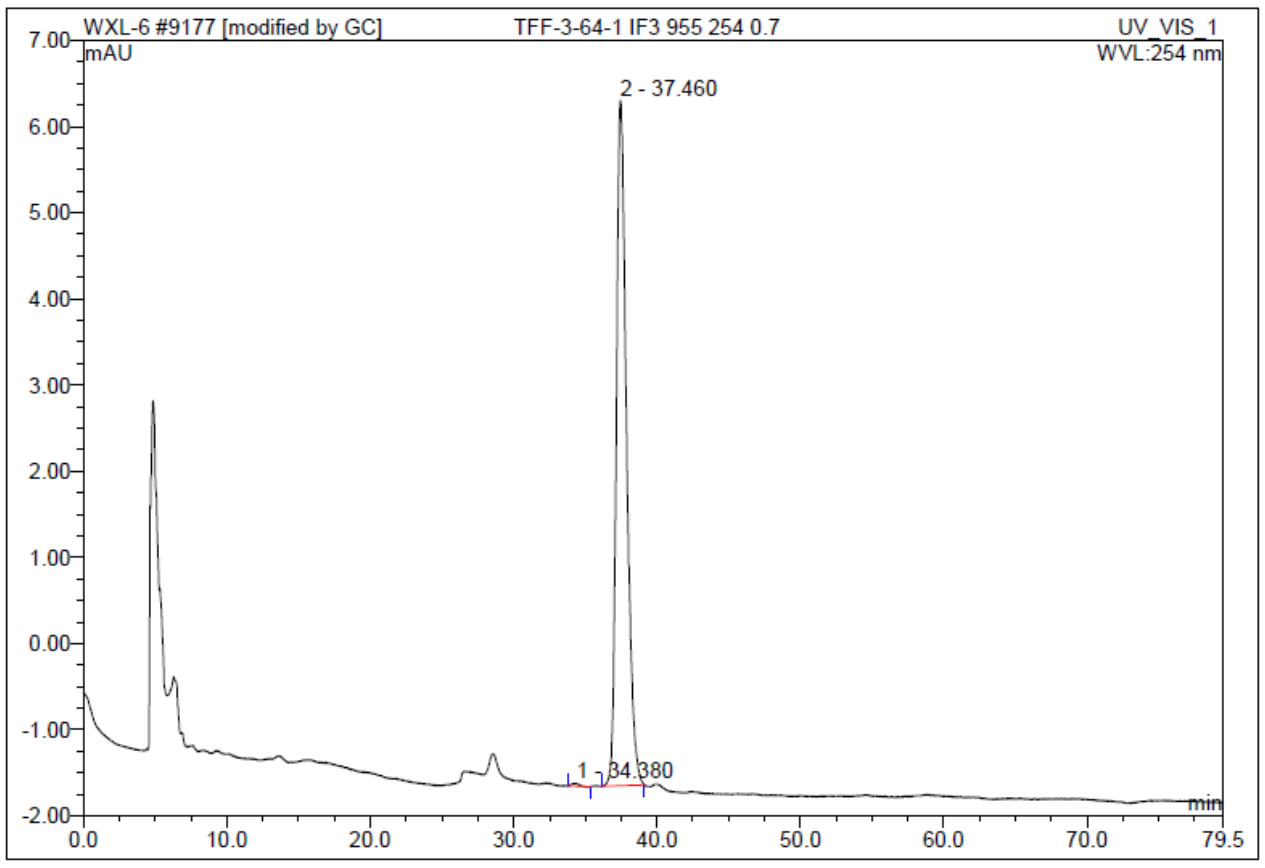

\begin{tabular}{|r|ccrrrrr|}
\hline No. & $\begin{array}{c}\text { Ret.Time } \\
\text { min }\end{array}$ & Peak Name & $\begin{array}{c}\text { Height } \\
\text { mAU }\end{array}$ & $\begin{array}{r}\text { Area } \\
\text { mAU*min }\end{array}$ & $\begin{array}{r}\text { Rel.Area } \\
\%\end{array}$ & Amount & Type \\
\hline 1 & 34.38 & n.a. & 0.037 & 0.019 & 0.29 & n.a. & BMB $^{*}$ \\
2 & 37.46 & n.a. & 7.953 & 6.546 & 99.71 & n.a. & BMB $^{*}$ \\
\hline Total: & & & 7.990 & 6.565 & 100.00 & 0.000 & \\
\hline
\end{tabular}


Triisopropyl((S)-3-((1S,2S)-2-phenylcyclopropyl)but-1-yn-1-yl)silane (10h).

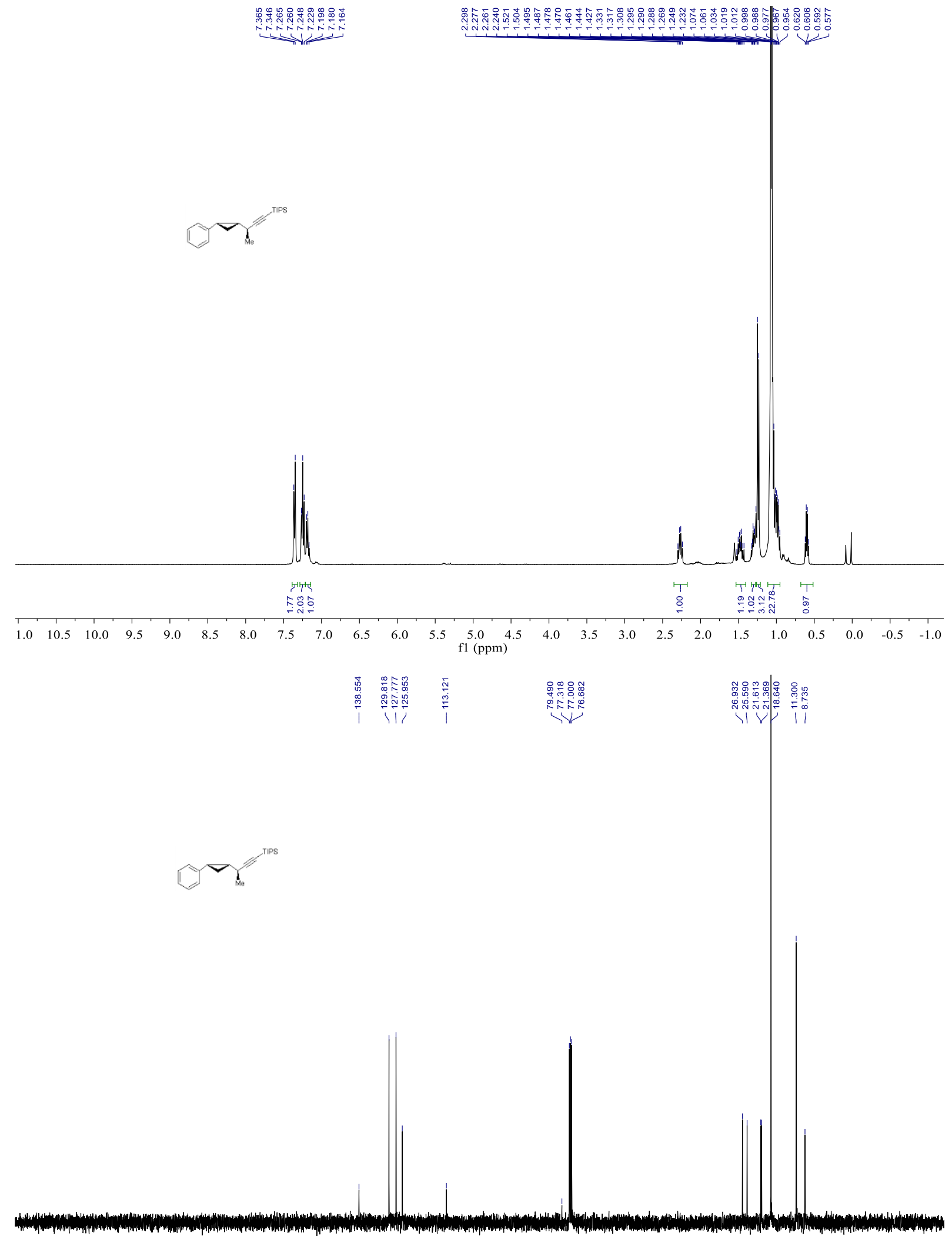

$\begin{array}{lllllllllllllllllllllllllllllllll}230 & 220 & 210 & 200 & 190 & 180 & 170 & 160 & 150 & 140 & 130 & 120 & 110 & 100 & 90 & 80 & 70 & 60 & 50 & 40 & 30 & 20 & 10 & 0 & -10 & -20 & -3\end{array}$ 


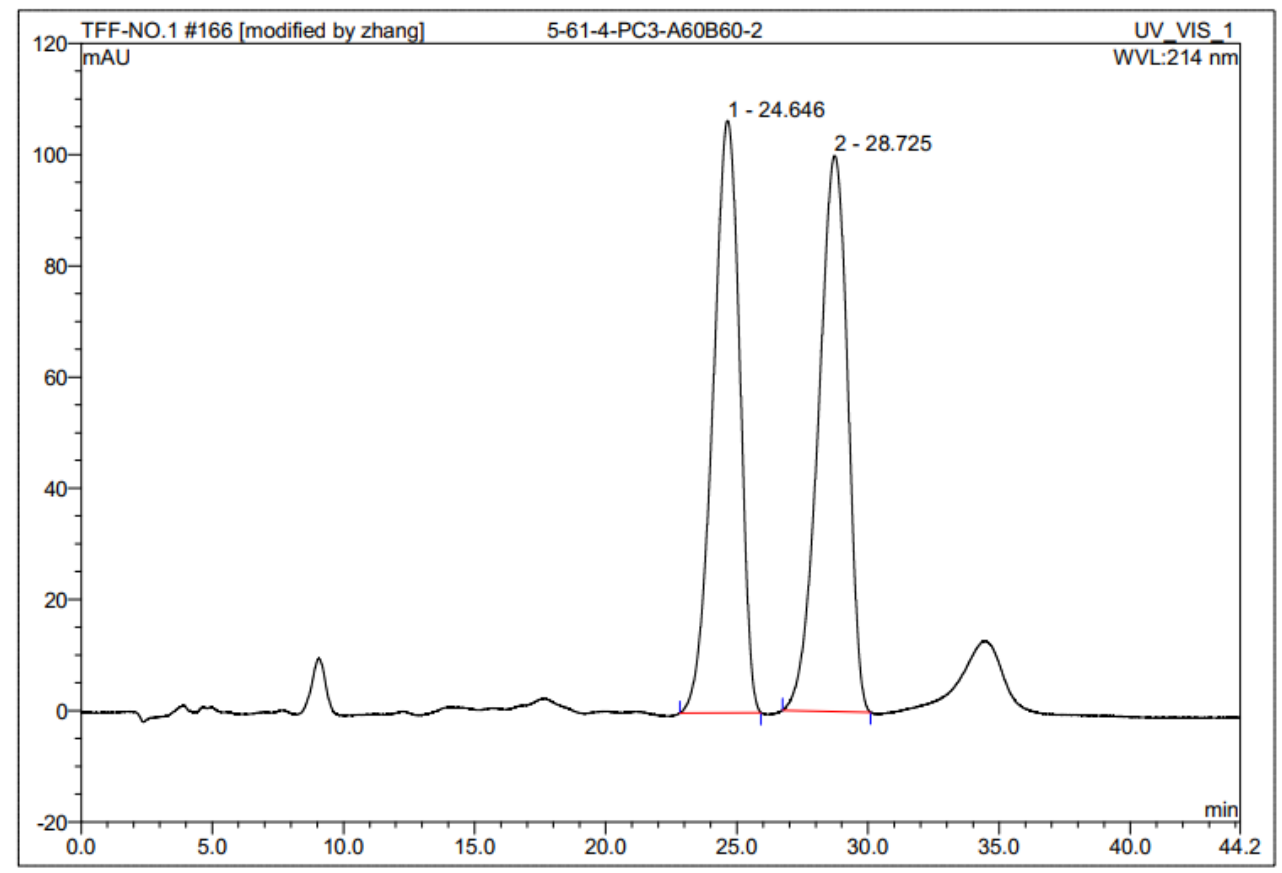

\begin{tabular}{|r|ccrrrrr|}
\hline No. & $\begin{array}{c}\text { Ret.Time } \\
\text { min }\end{array}$ & Peak Name & $\begin{array}{c}\text { Height } \\
\text { mAU }\end{array}$ & $\begin{array}{c}\text { Area } \\
\text { mAU*min }\end{array}$ & $\begin{array}{c}\text { Rel.Area } \\
\%\end{array}$ & $\begin{array}{c}\text { Amount } \\
\%\end{array}$ & Type \\
\hline 1 & 24.65 & n.a. & 106.505 & 125.675 & 49.36 & n.a. & BMB \\
2 & 28.73 & n.a. & 99.971 & 128.935 & 50.64 & n.a. & BMB $^{*}$ \\
\hline Total: & & & 206.476 & 254.610 & 100.00 & 0.000 & \\
\hline
\end{tabular}

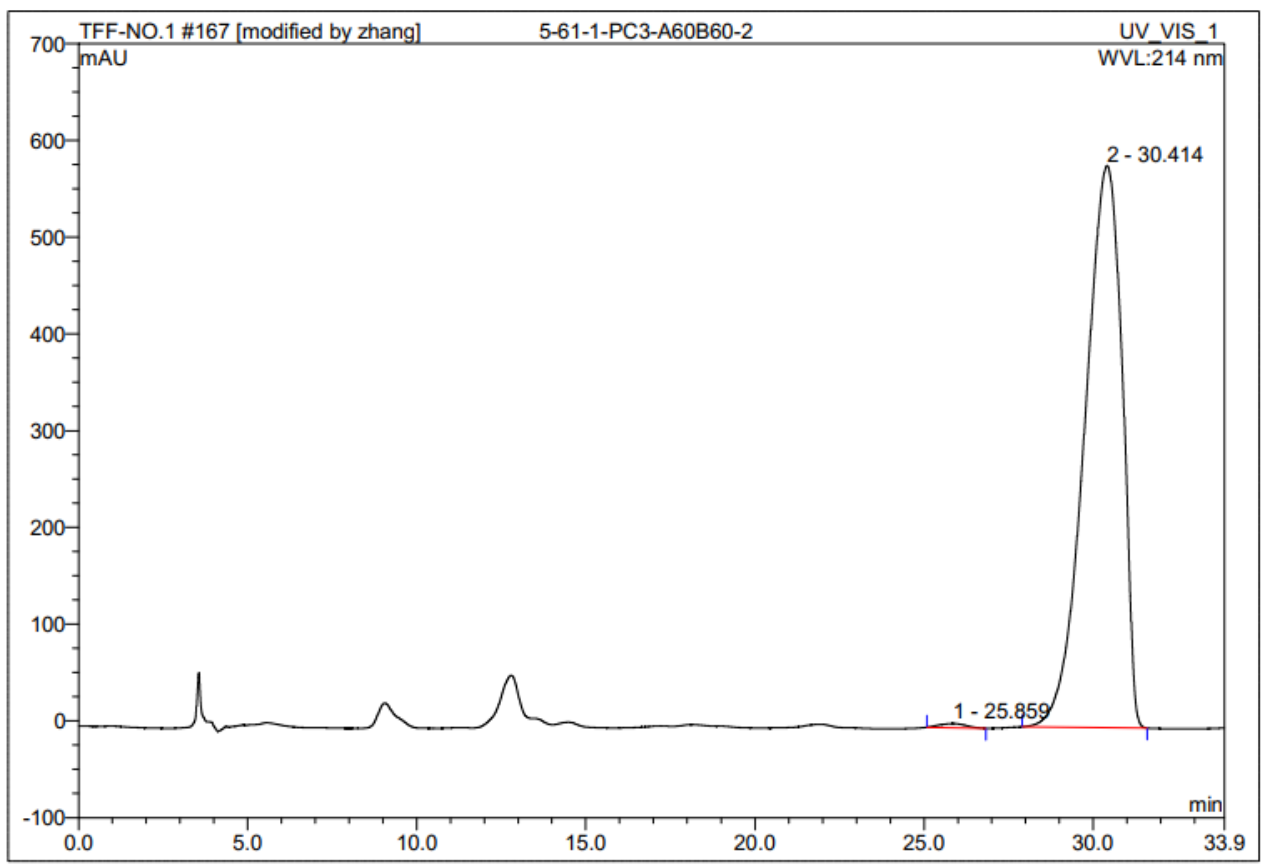

\begin{tabular}{|c|c|c|c|c|c|c|c|}
\hline No. & $\begin{array}{l}\text { Ret.Time } \\
\min \end{array}$ & Peak Name & $\begin{array}{c}\text { Height } \\
\text { mAU }\end{array}$ & $\begin{array}{c}\text { Area } \\
\text { mAU*min }\end{array}$ & $\begin{array}{c}\text { Rel.Area } \\
\%\end{array}$ & $\begin{array}{c}\text { Amount } \\
\%\end{array}$ & Type \\
\hline 1 & 25.86 & n.a. & 4.303 & 3.787 & 0.51 & n.a. & BMB* $^{*}$ \\
\hline 2 & 30.41 & n.a. & 580.944 & 742.159 & 99.49 & n.a. & BMB \\
\hline Total: & & & 585.247 & 745.946 & 100.00 & 0.000 & \\
\hline
\end{tabular}


Triisopropyl((R)-4,4,4-trifluoro-3-((1S,2R)-2-phenylcyclopropyl)but-1-yn-1-yl)silane (10i).

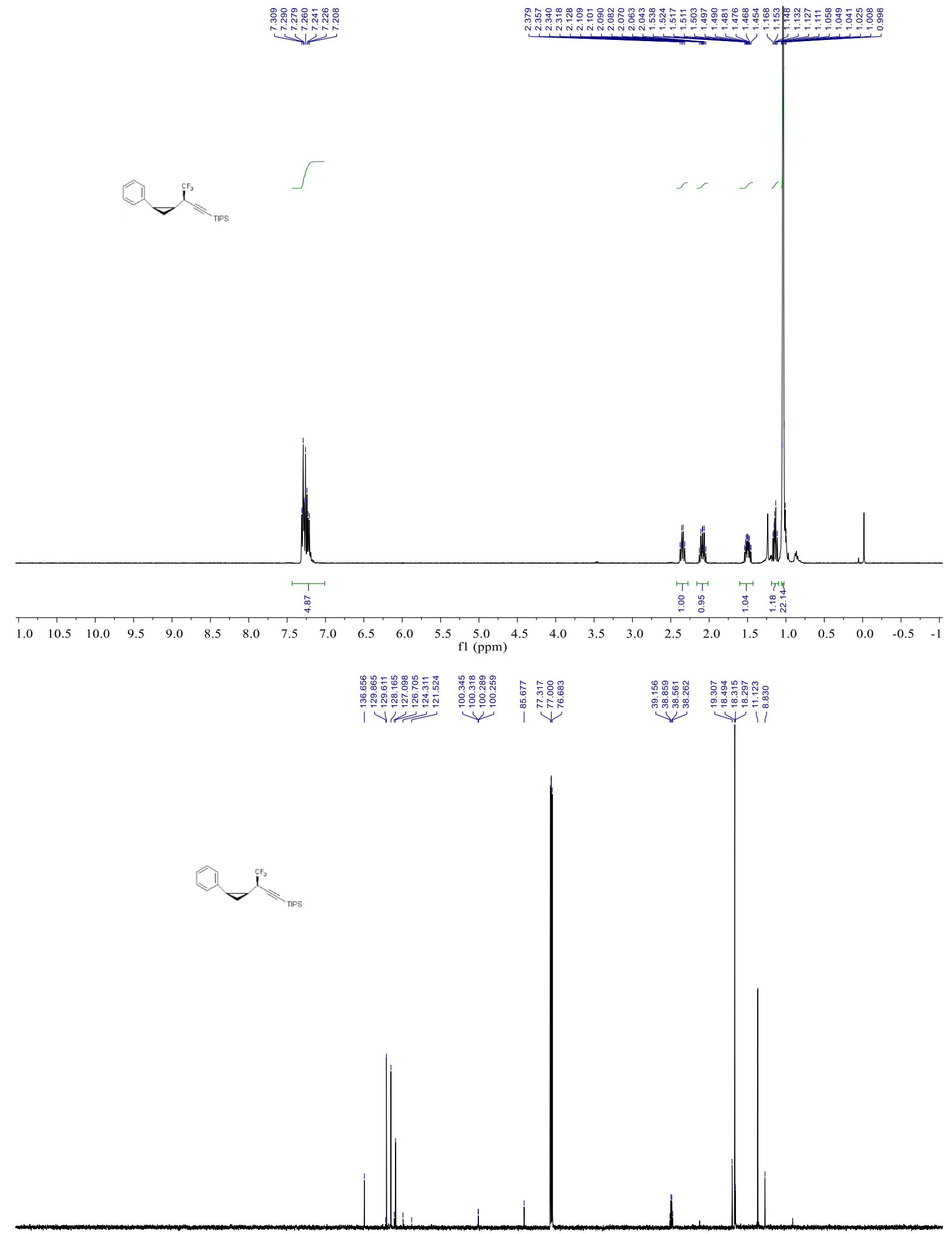

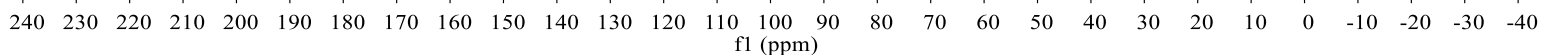




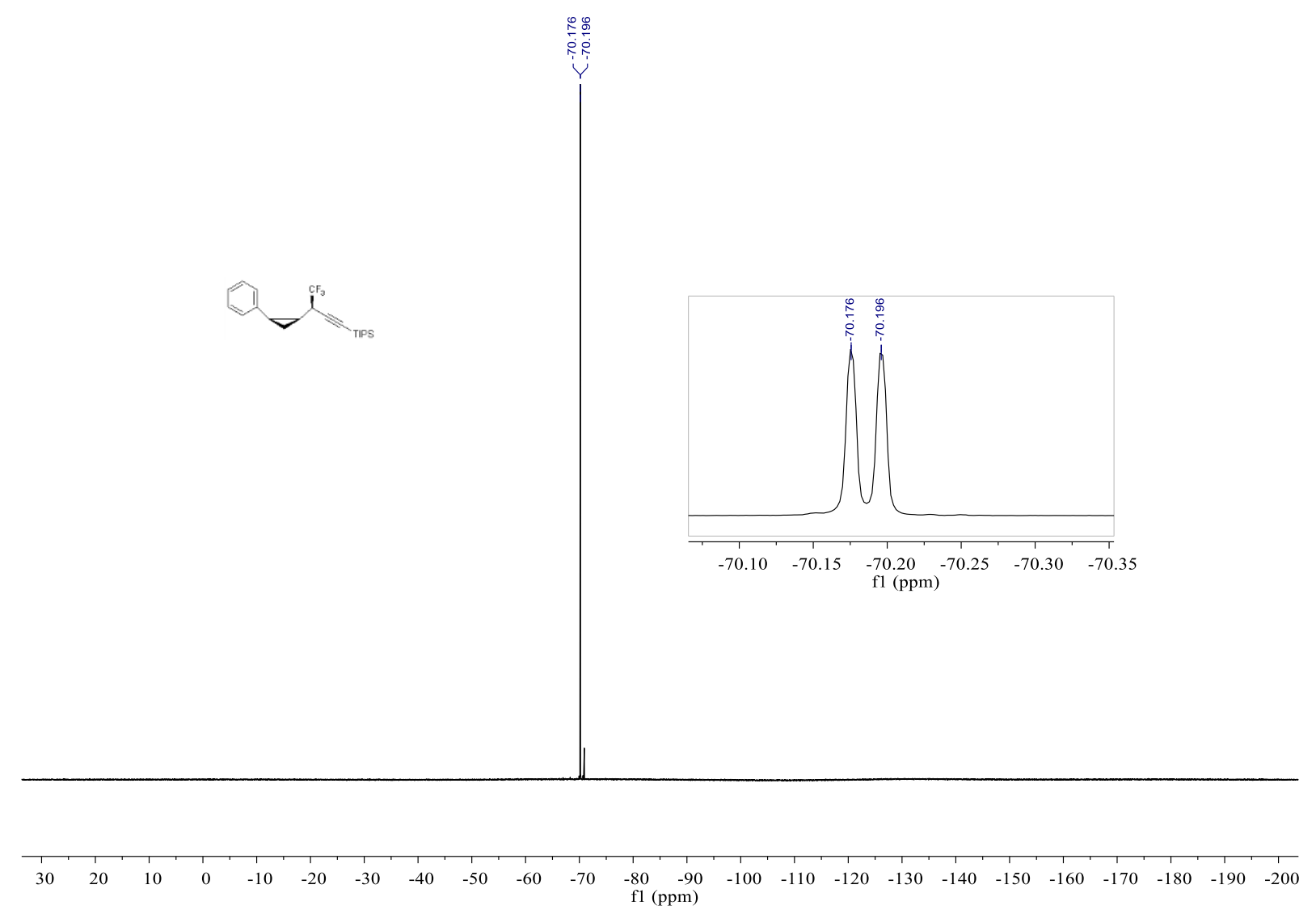

1-benzyl-4-((R)-2,2,2-trifluoro-1-((1S,2R)-2-phenylcyclopropyl)ethyl)-1H-1,2,3-triazole (10i').

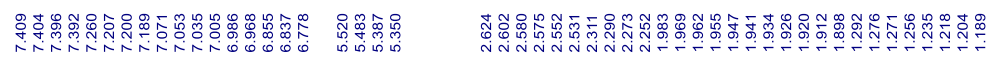

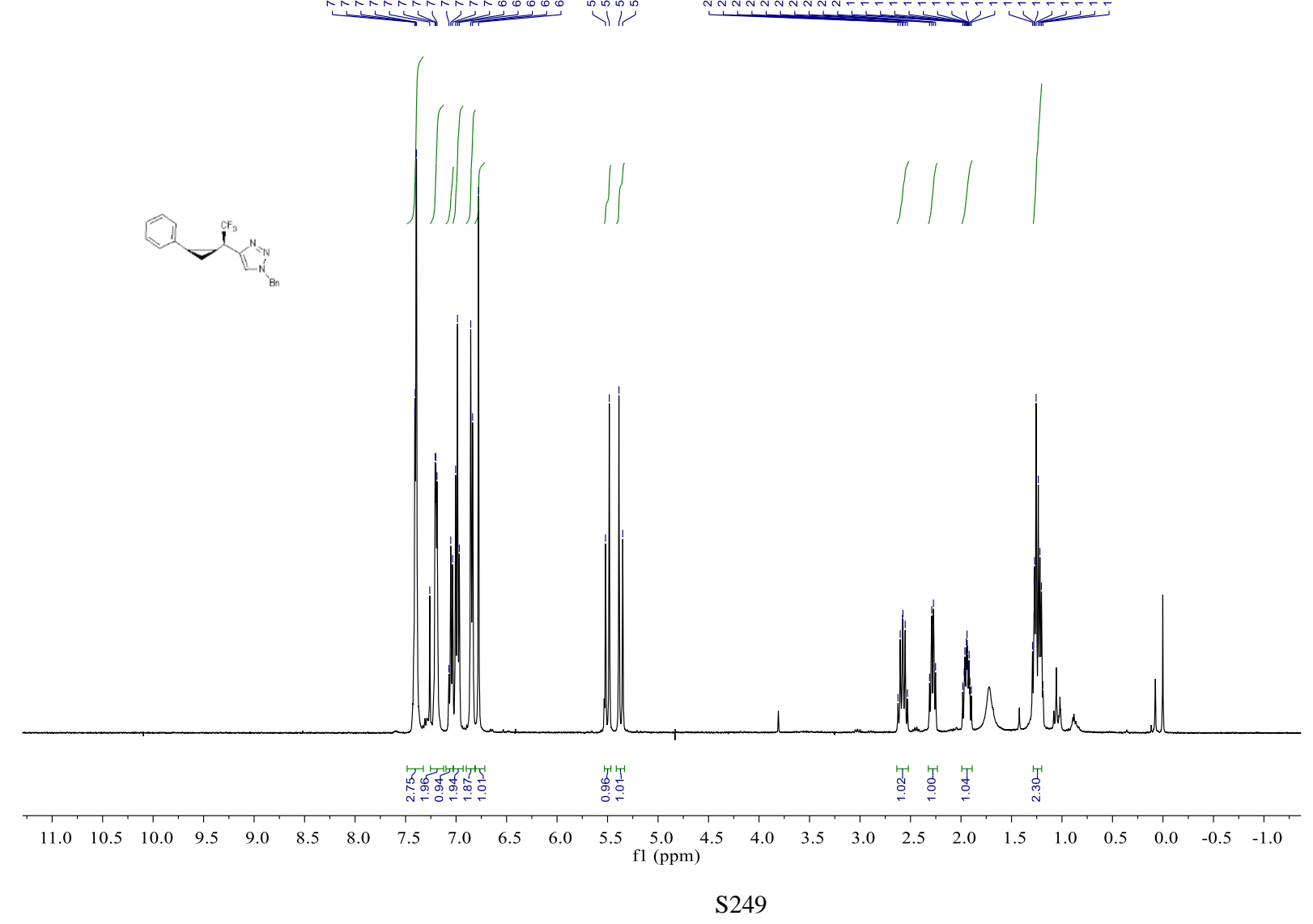




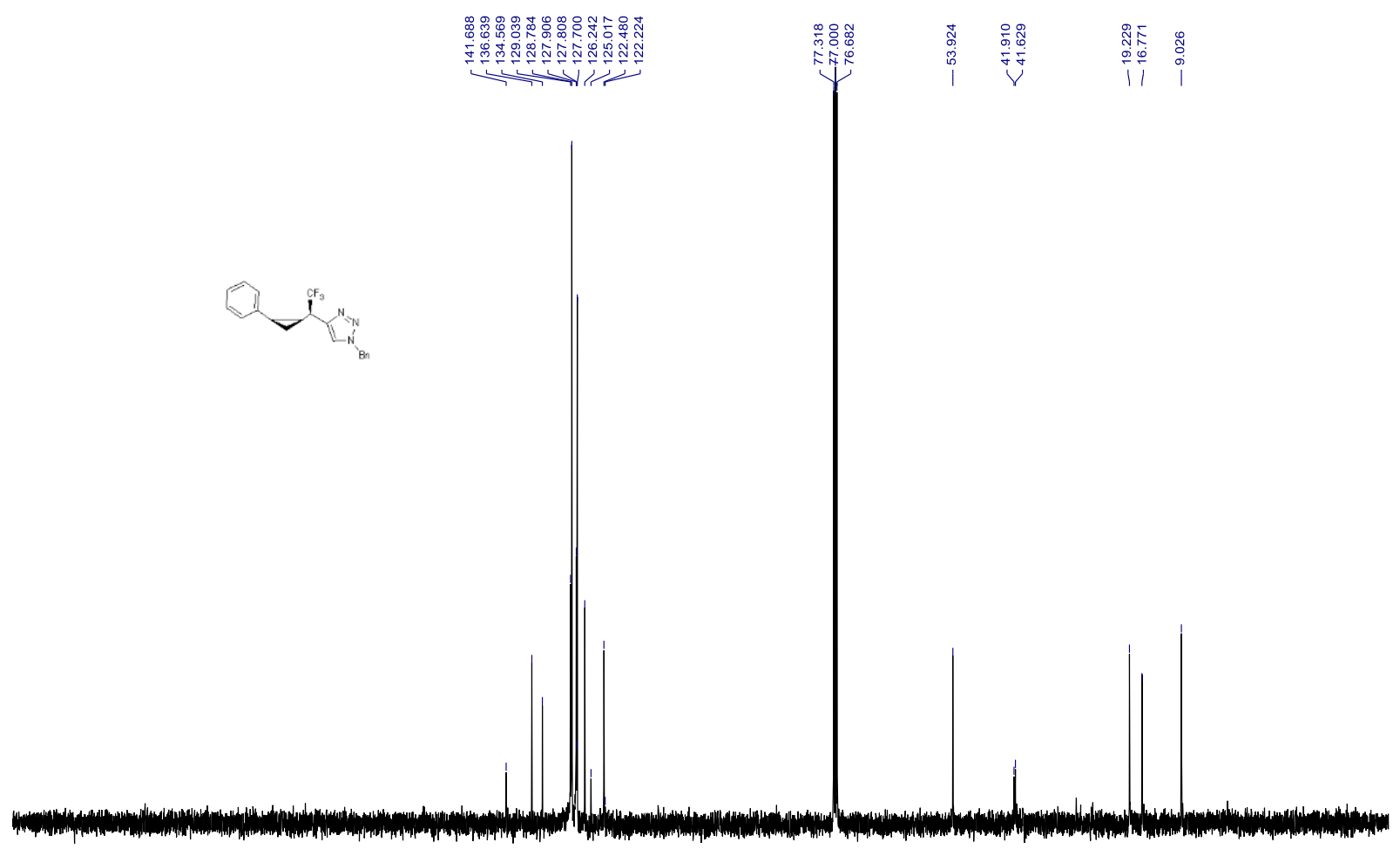

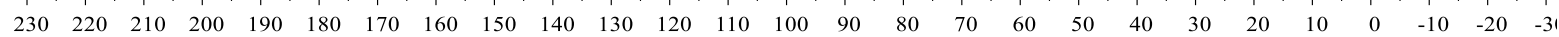
f1 (ppm)

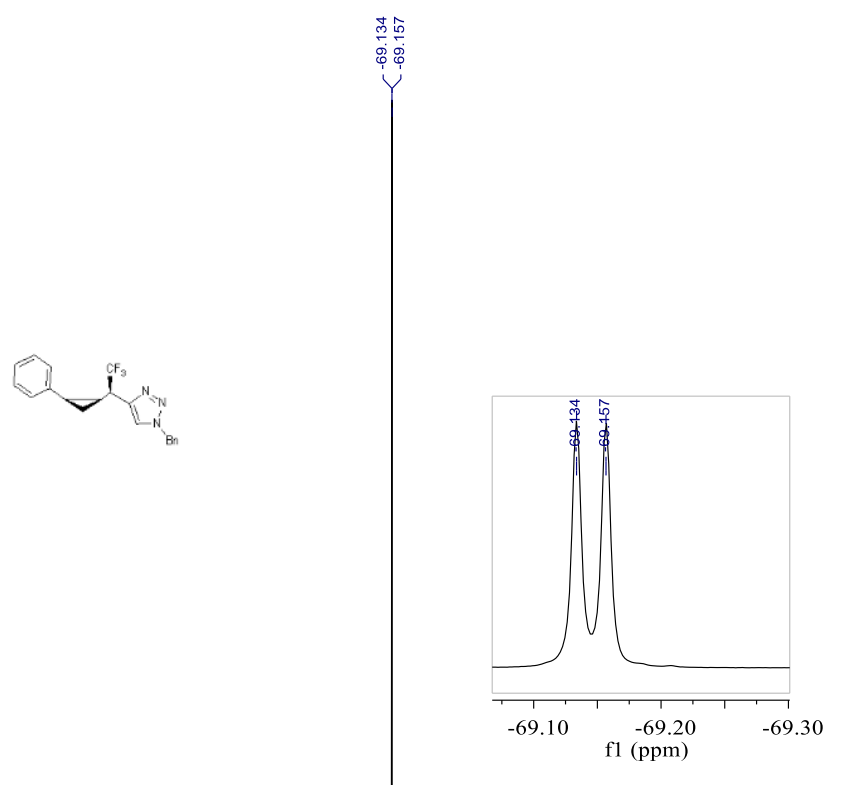

$\begin{array}{llllllllllllllllllllllllll}30 & 20 & 10 & 0 & -10 & -20 & -30 & -40 & -50 & -60 & -70 & -80 & -90 & -100 & -110 & -120 & -130 & -140 & -150 & -160 & -170 & -180 & -190 & -200\end{array}$ 
$<$ Chromatogram $>$

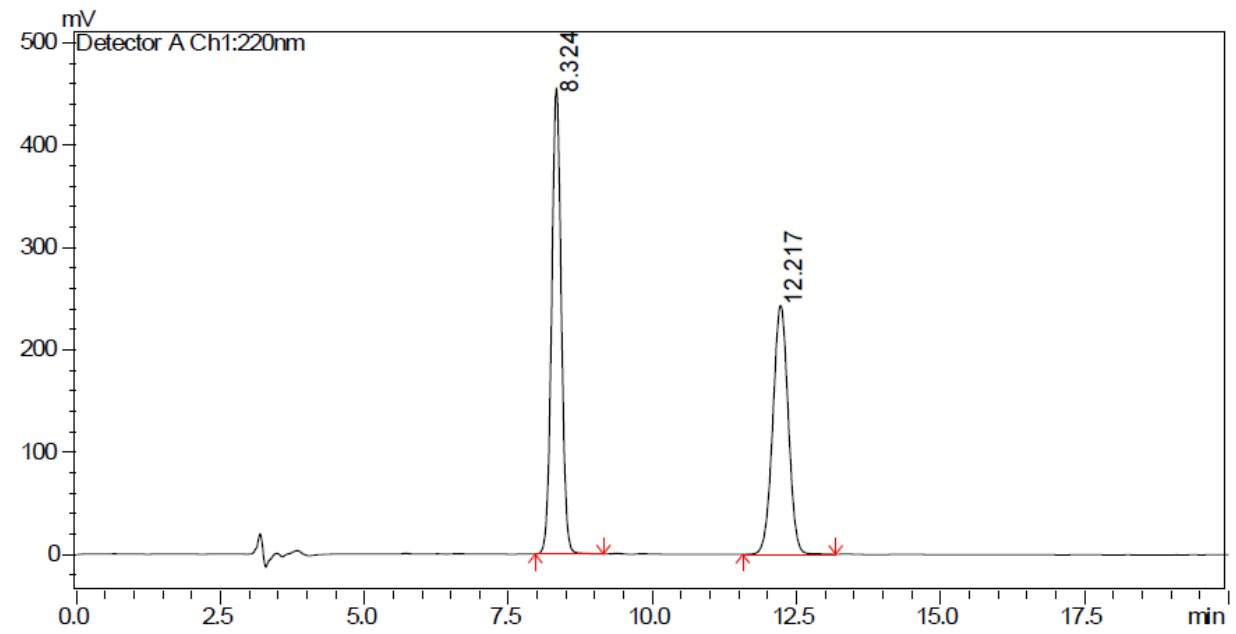

$<$ Column Performance Report $>$

$\begin{array}{ccccccc}\text { Peak No. } & \text { Time } & \text { Area } & \text { Area \% } & \text { Plate number } & \text { Tailing } & \text { Resolution } \\ 1 & 8.324 & 5325618 & 54.0717 & 9988.397 & 1.069 & -- \\ 2 & 12.217 & 4523565 & 45.9283 & 9333.130 & 1.022 & 9.279\end{array}$

$<$ Chromatogram $>$

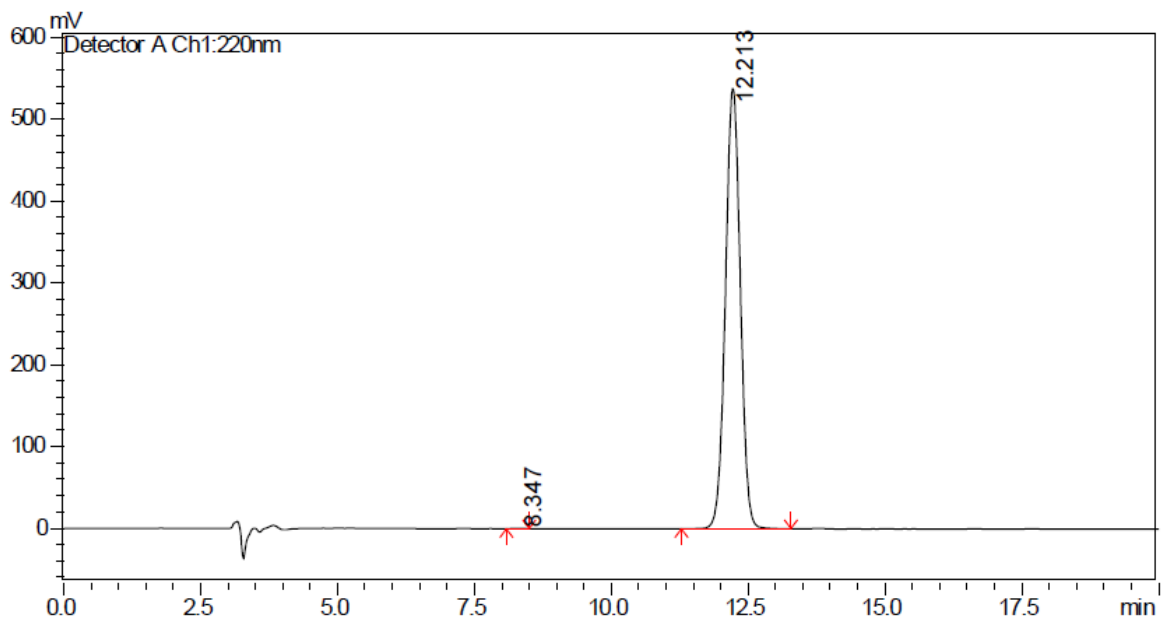

$<$ Column Performance Report $>$

$\begin{array}{ccccccc}\text { Peak No. } & \text { Time } & \text { Area } & \text { Area \% } & \text { Plate number } & \text { Tailing } & \text { Resolution } \\ 1 & 8.347 & 5320 & 0.0523 & 7628.502 & 0.759 & -- \\ 2 & 12.213 & 10160052 & 99.9477 & 9046.938 & 1.035 & 8.631\end{array}$


Triisopropyl((R)-4,4,4-trifluoro-3-((1S,2R)-2-(4-(trimethylsilyl)phenyl)cyclopropyl)but-1-yn-1yl)silane (10j).
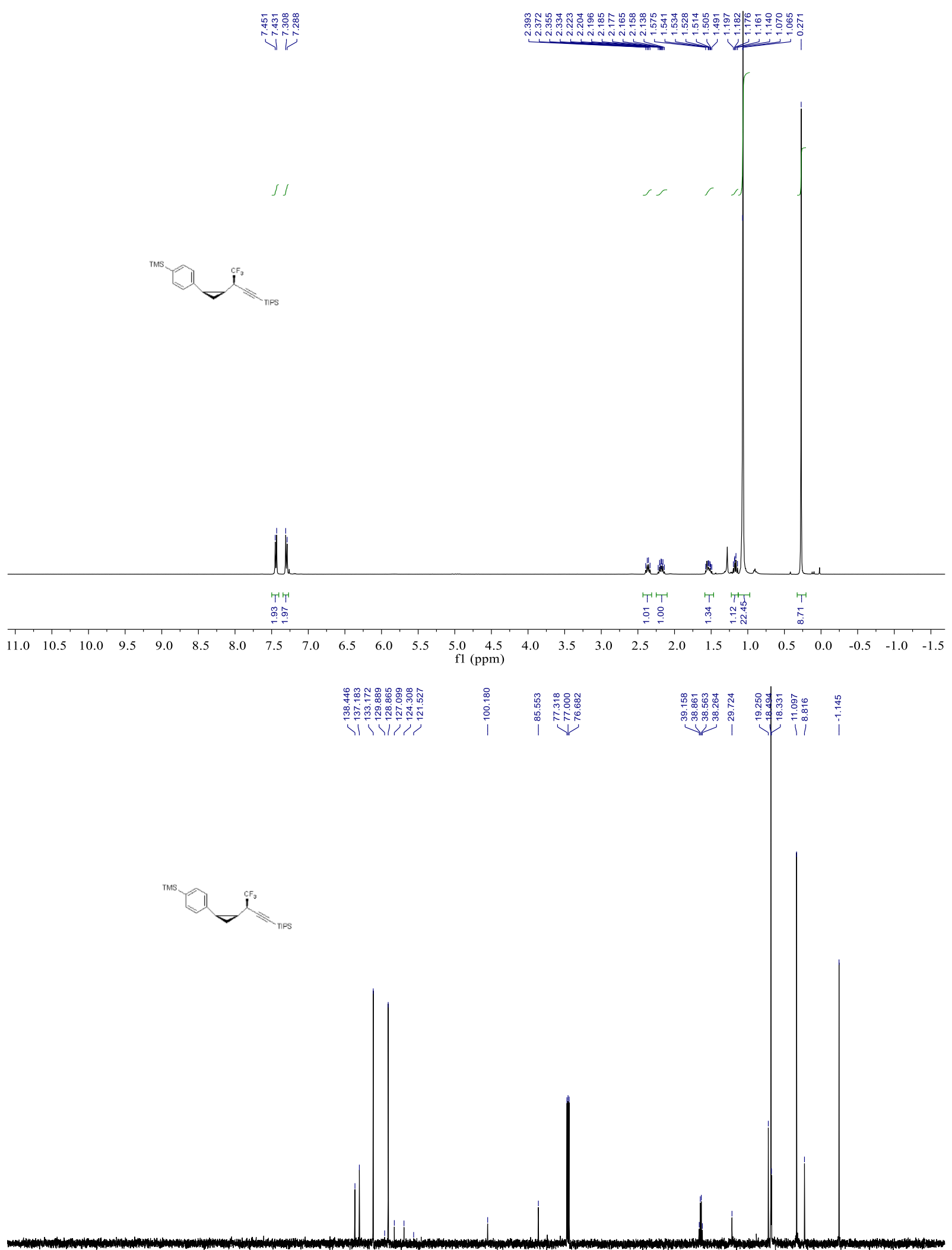

$\begin{array}{lllllllllllllllllllllllllllllllllll}230 & 220 & 210 & 200 & 190 & 180 & 170 & 160 & 150 & 140 & 130 & 120 & 110 & 100 & 90 & 80 & 70 & 60 & 50 & 40 & 30 & 20 & 10 & 0 & -10 & -20 & -3\end{array}$ 


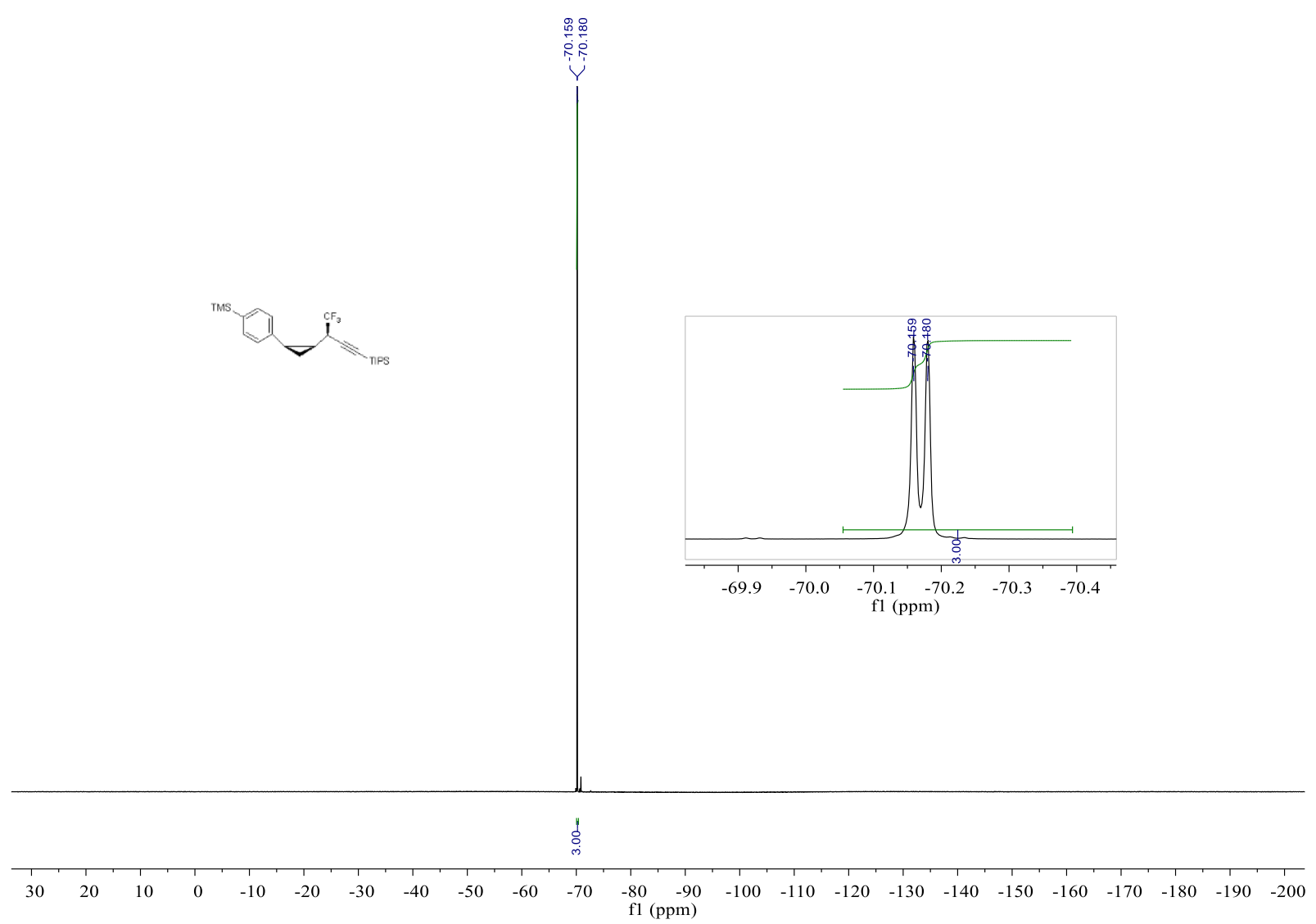

1-Benzyl-4-((R)-2,2,2-trifluoro-1-((1S,2R)-2-(4-(trimethylsilyl)phenyl)cyclopropyl)ethyl)-1H-1,2 ,3-triazole (10j').

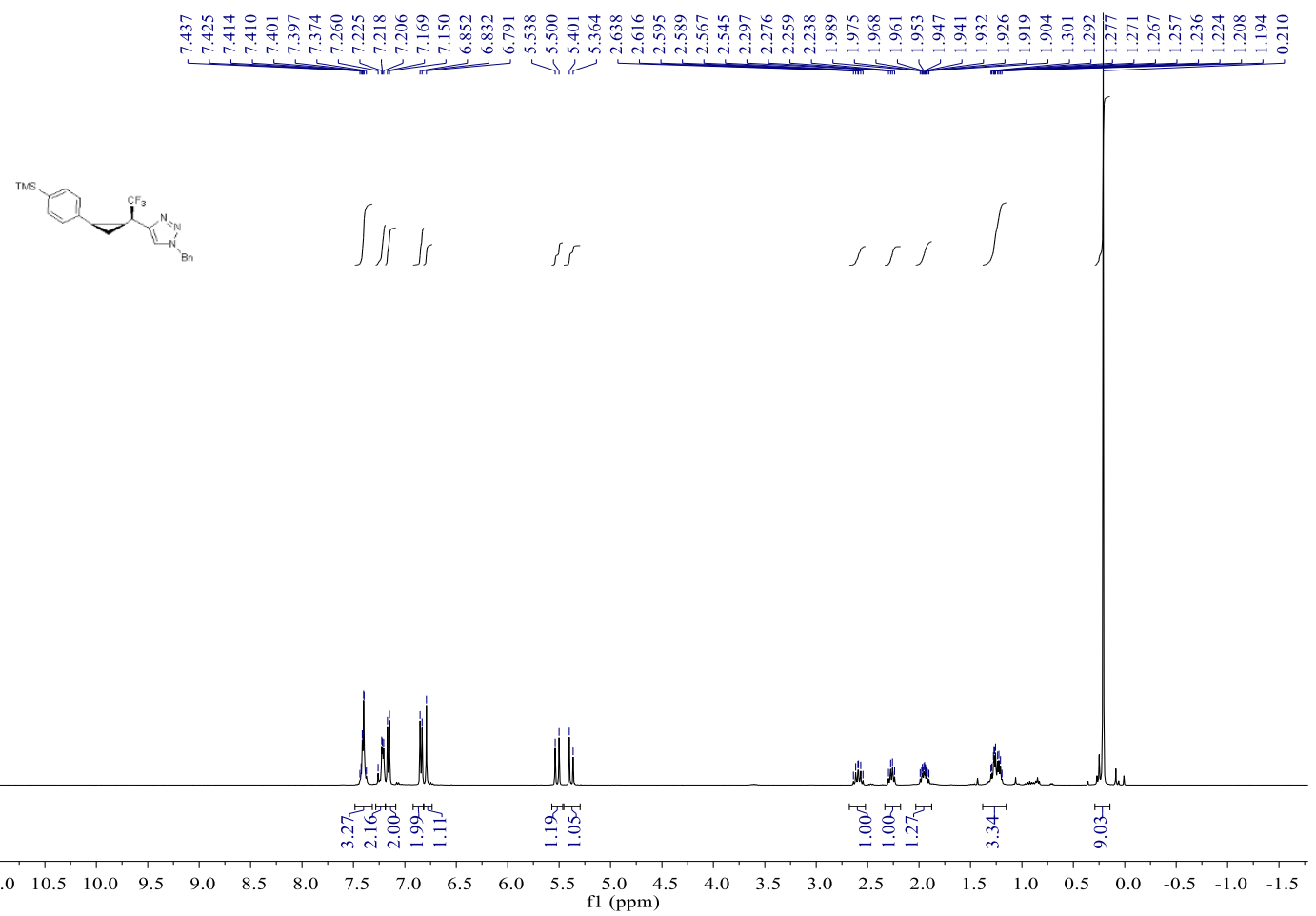



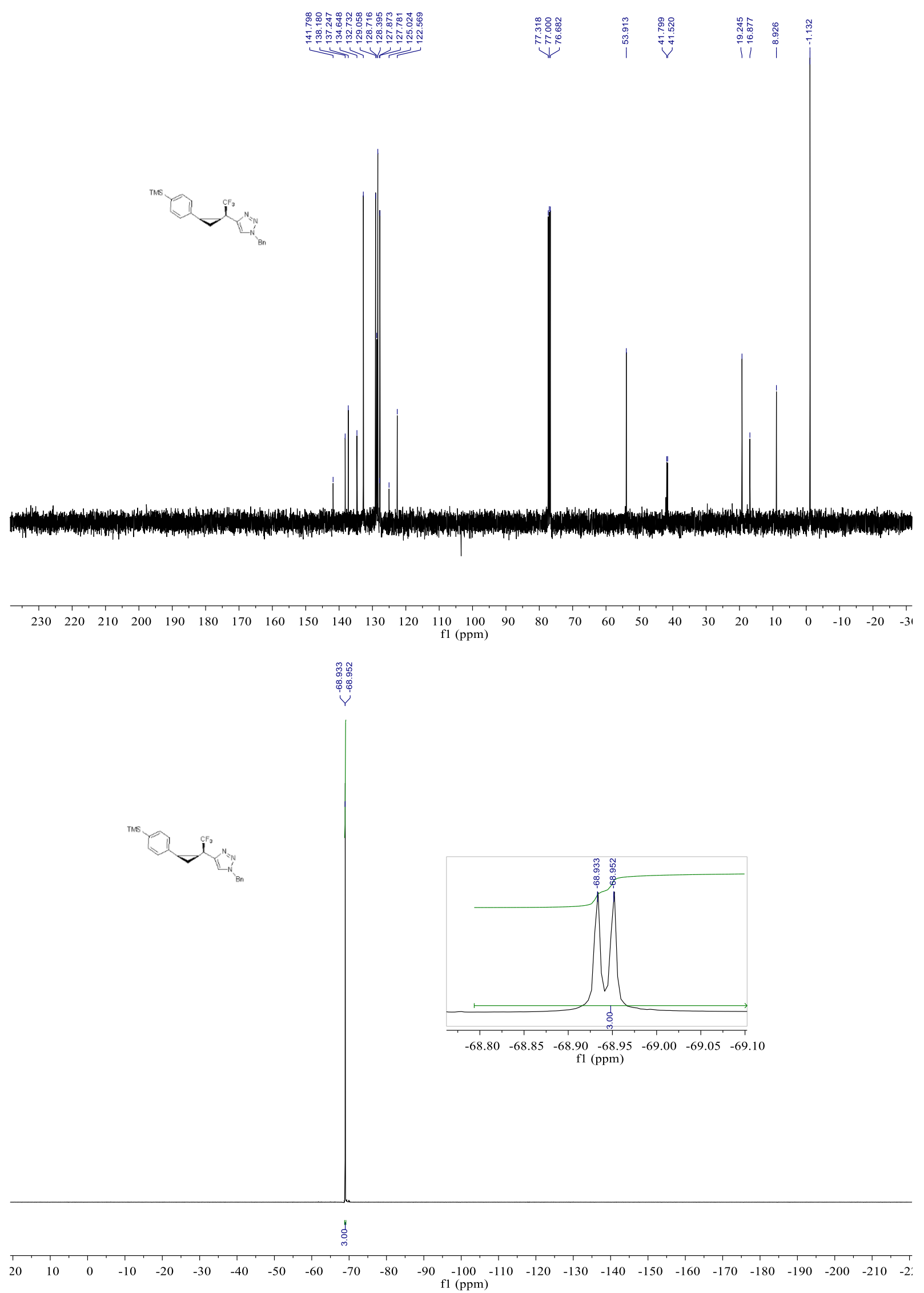


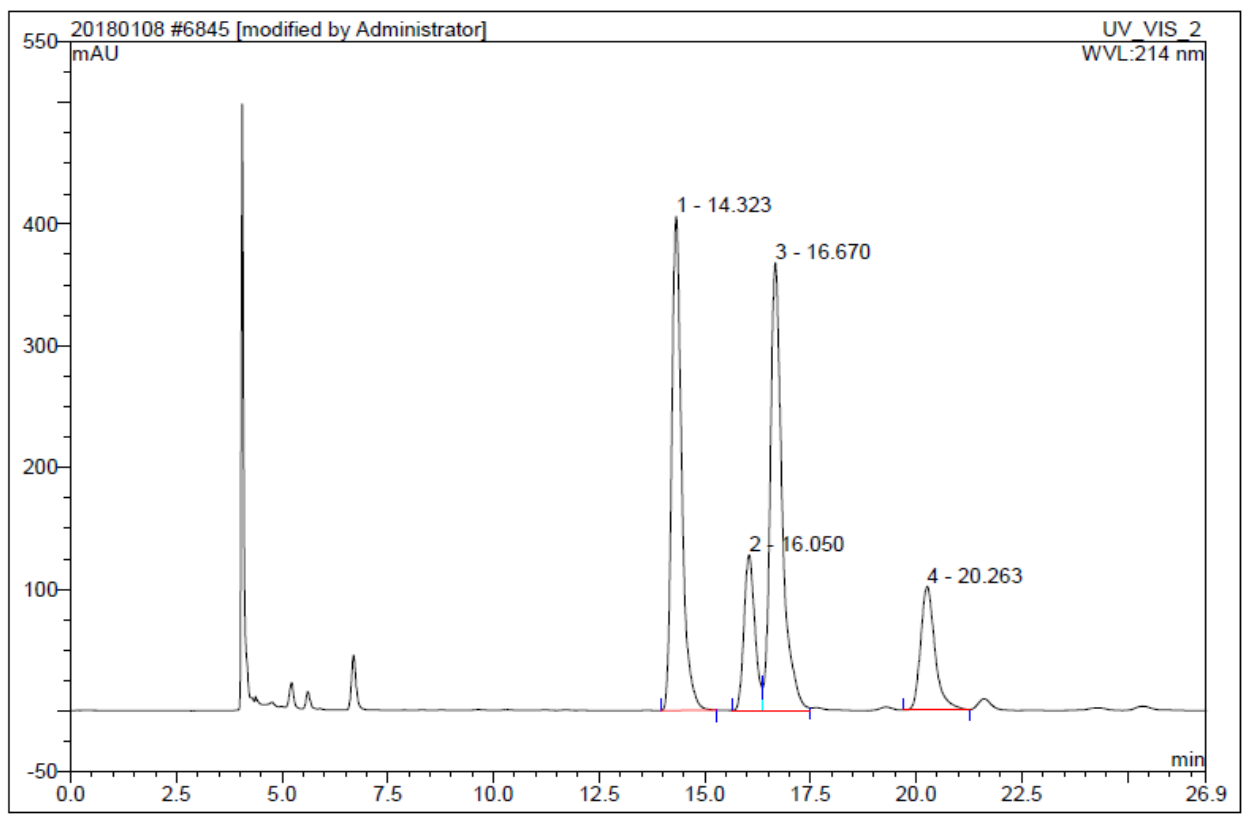

\begin{tabular}{|r|ccrrrrr|}
\hline No. & $\begin{array}{c}\text { Ret.Time } \\
\text { min }\end{array}$ & Peak Name & $\begin{array}{c}\text { Height } \\
\text { mAU }\end{array}$ & $\begin{array}{c}\text { Area } \\
\text { mAU*min }\end{array}$ & $\begin{array}{c}\text { Rel.Area } \\
\%\end{array}$ & Amount & Type \\
\hline 1 & 14.32 & n.a. & 406.365 & 111.618 & 35.93 & n.a. & BMB \\
2 & 16.05 & n.a. & 128.094 & 38.303 & 12.33 & n.a. & BM $^{*}$ \\
3 & 16.67 & n.a. & 368.082 & 120.104 & 38.67 & n.a. & M $^{*}$ \\
4 & 20.26 & n.a. & 101.945 & 40.593 & 13.07 & n.a. & BM $^{*}$ \\
\hline Total: & & & 1004.485 & 310.619 & 100.00 & 0.000 & \\
\hline
\end{tabular}

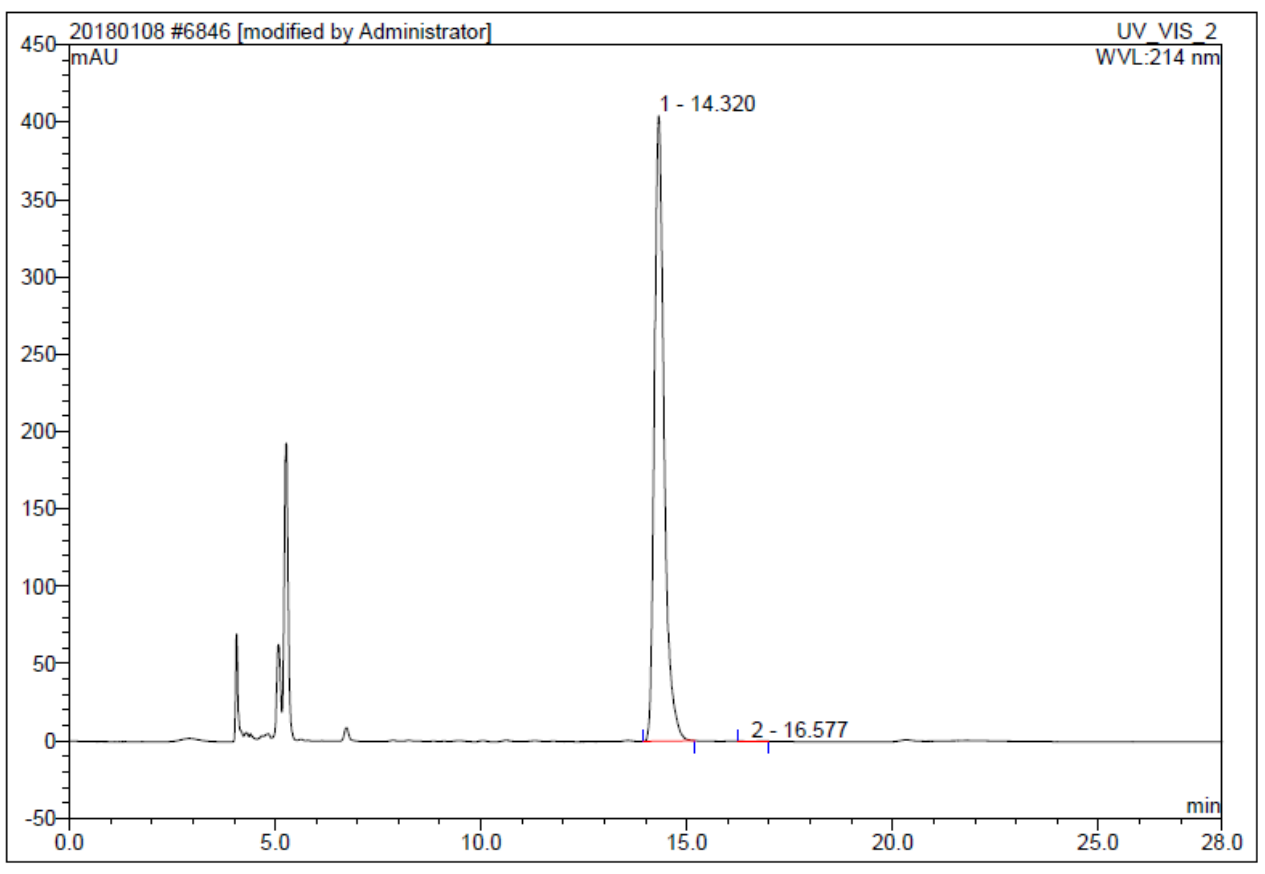

\begin{tabular}{|c|c|c|c|c|c|c|c|}
\hline No. & $\begin{array}{c}\text { Ret.Time } \\
\text { min }\end{array}$ & Peak Name & $\begin{array}{c}\text { Height } \\
\text { mAU }\end{array}$ & $\begin{array}{c}\text { Area } \\
\mathrm{mAU}^{\star} \mathrm{min}\end{array}$ & $\begin{array}{c}\text { Rel.Area } \\
\% \\
\end{array}$ & Amount & Type \\
\hline 1 & 14.32 & n.a. & 404.213 & 110.839 & 99.96 & n.a. & BMB \\
\hline 2 & 16.58 & n.a. & 0.255 & 0.047 & 0.04 & n.a. & $\mathrm{BMB}^{\star}$ \\
\hline Total: & & & 404.467 & 110.886 & 100.00 & 0.000 & \\
\hline
\end{tabular}



(10k).

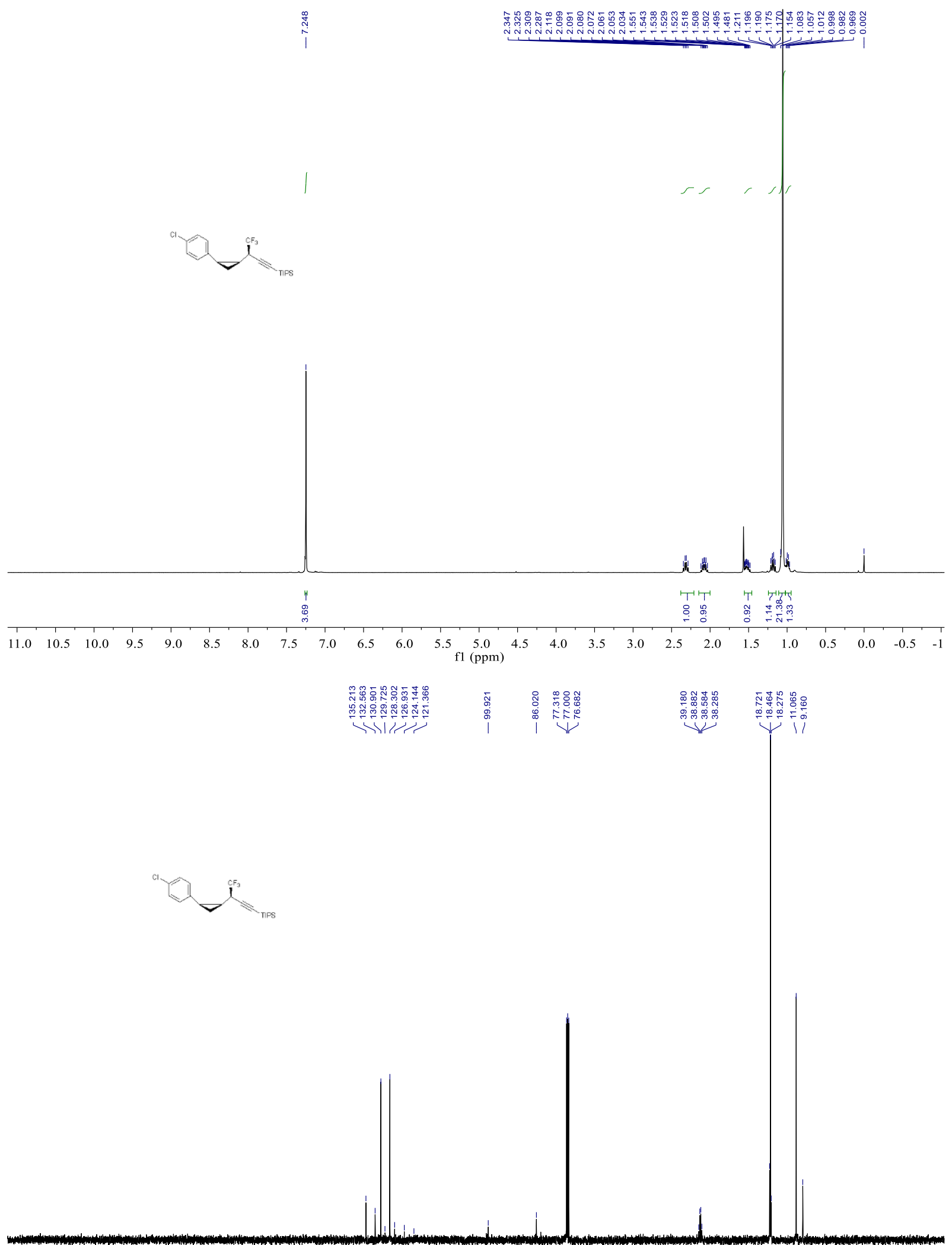

$\begin{array}{llllllllllllllllllllllllllll}230 & 220 & 210 & 200 & 190 & 180 & 170 & 160 & 150 & 140 & 130 & 120 & 110 & 100 & 90 & 80 & 70 & 60 & 50 & 40 & 30 & 20 & 10 & 0 & -10 & -20 & -3\end{array}$ 


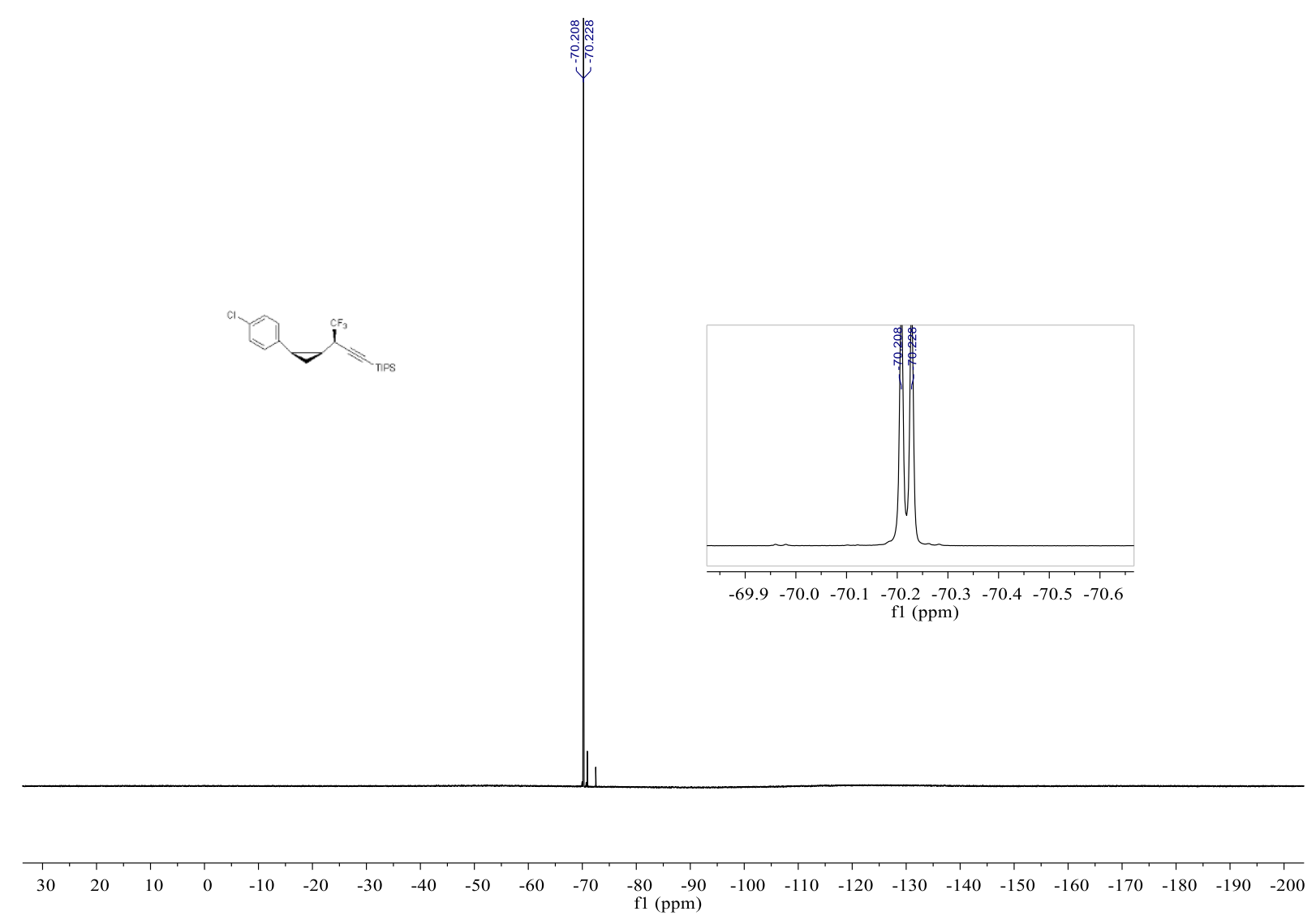

1-Benzyl-4-((R)-1-((1S,2R)-2-(4-chlorophenyl)cyclopropyl)-2,2,2-trifluoroethyl)-1H-1,2,3-triazo le (12).

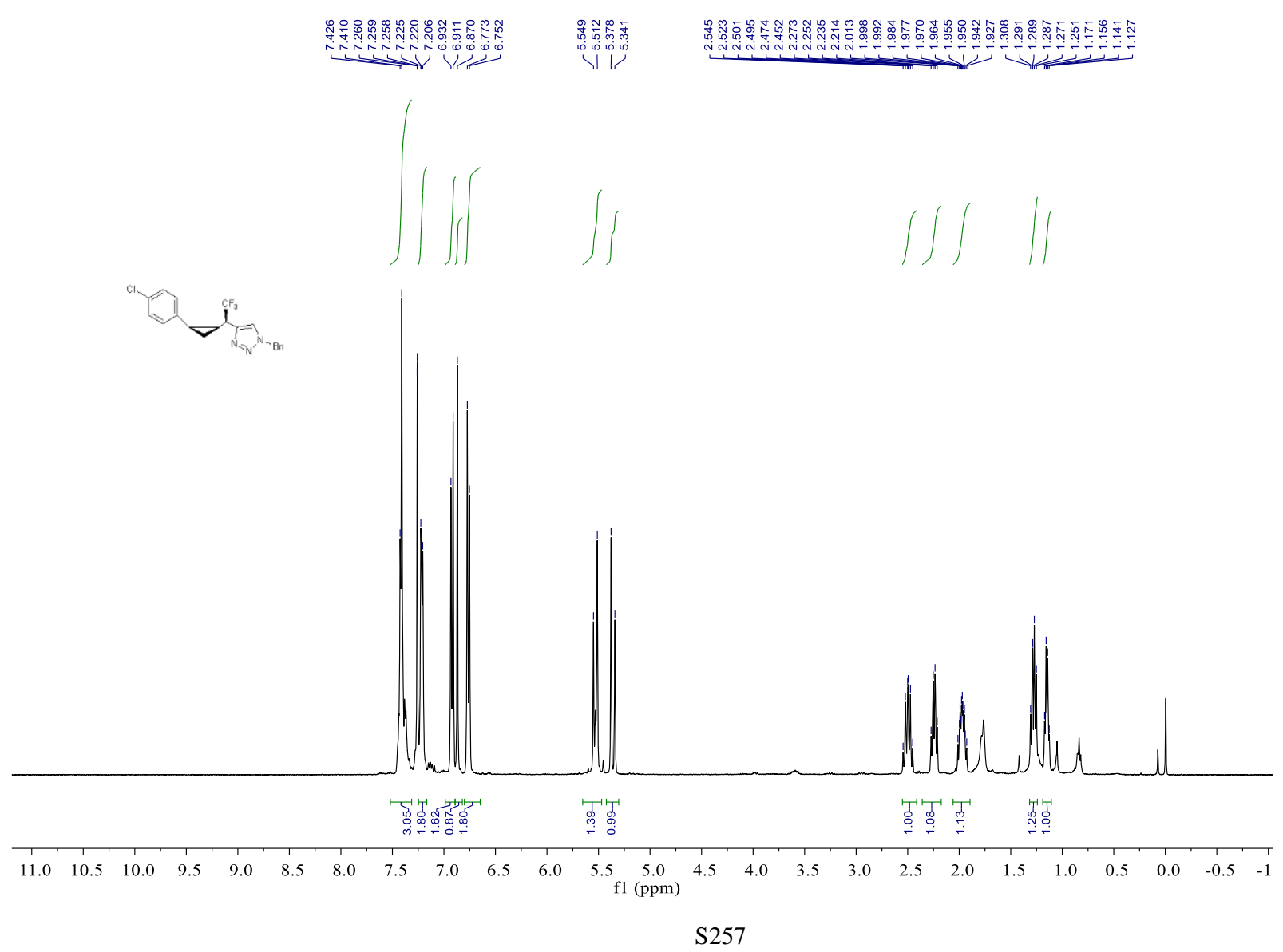




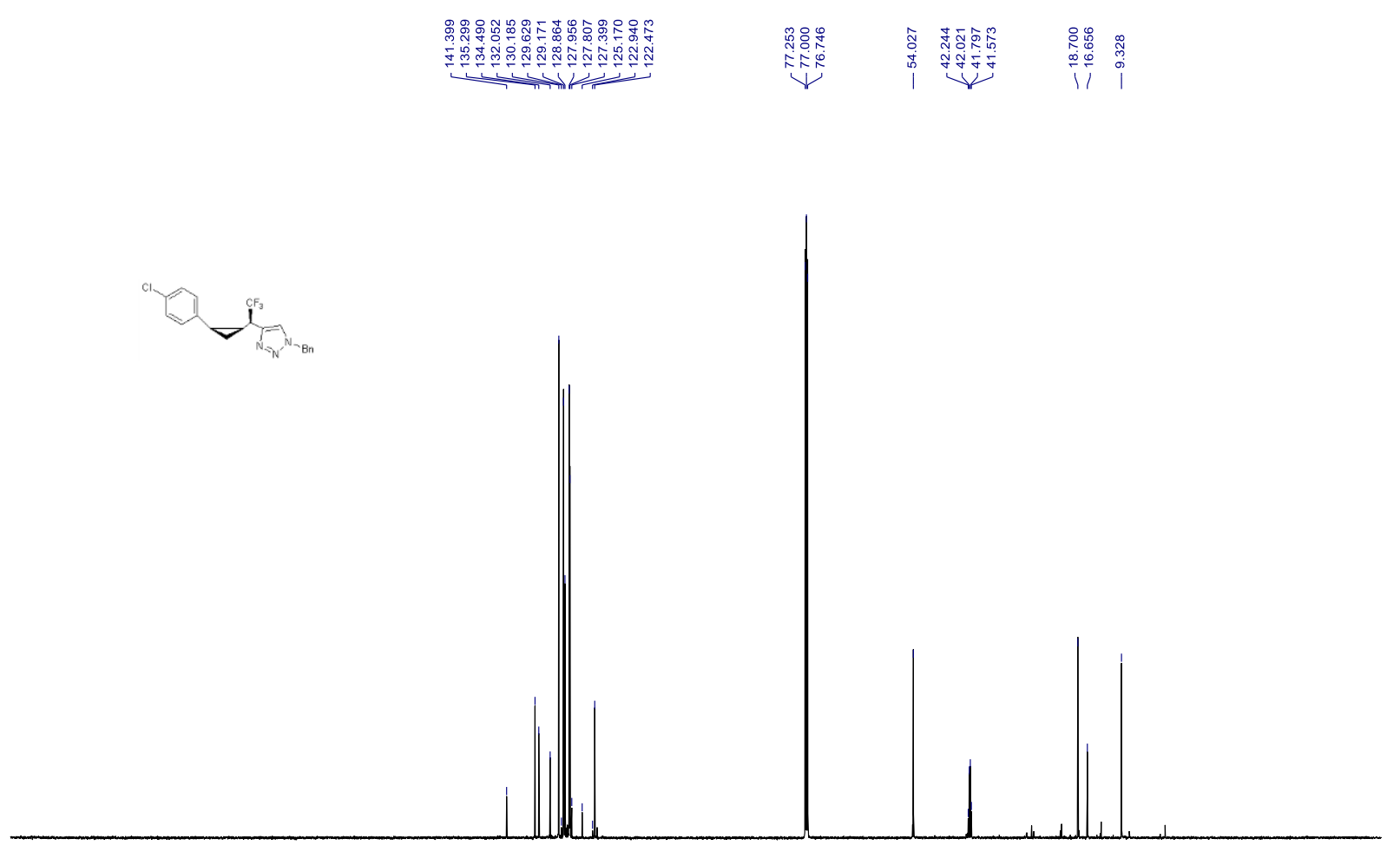

$\begin{array}{lllllllllllllllllllllllllllllllllllllllllllll}240 & 230 & 220 & 210 & 200 & 190 & 180 & 170 & 160 & 150 & 140 & 130 & 120 & 110 & 100 & 90 & 80 & 70 & 60 & 50 & 40 & 30 & 20 & 10 & 0 & -10 & -20 & -30 & -40\end{array}$

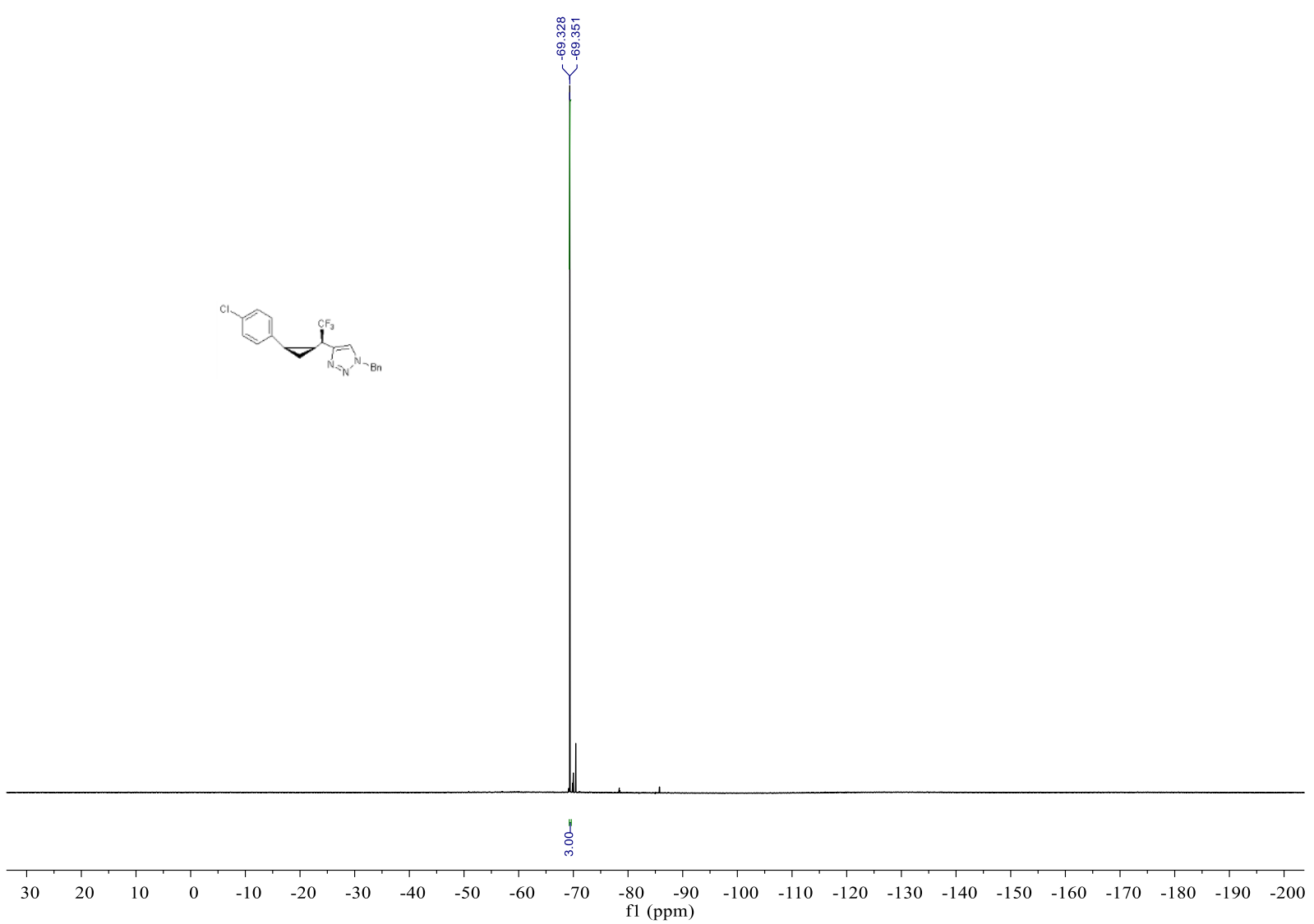




\begin{tabular}{|c|c|c|c|}
\hline & SAMPLE & INFORMATION & \\
\hline Sample Name: & TFF-5-80-2+- IF3 912140.7 & Acquired By: & System \\
\hline Sample Type: & Unknown & Sample Set Name: & 20190821 \\
\hline Vial: & $1: \mathrm{C}, 1$ & Acq. Method Set: & 1008 \\
\hline Injection\#. & 1 & Processing Method & 1 \\
\hline Injection Volume: & $200 \mathrm{ul}$ & Channel Name: & PDA Ch1 214nm@4.8nm \\
\hline Run Time: & 25.0 Minutes & Proc. Onnl. Descr:: & PDA Ch1 214nm@4.8nm \\
\hline Date Acquired: & 9/10/2019 11:57:30 AMCST & & \\
\hline Date Processed: & 12/19/2019 1:36:50 PM CST & & \\
\hline
\end{tabular}

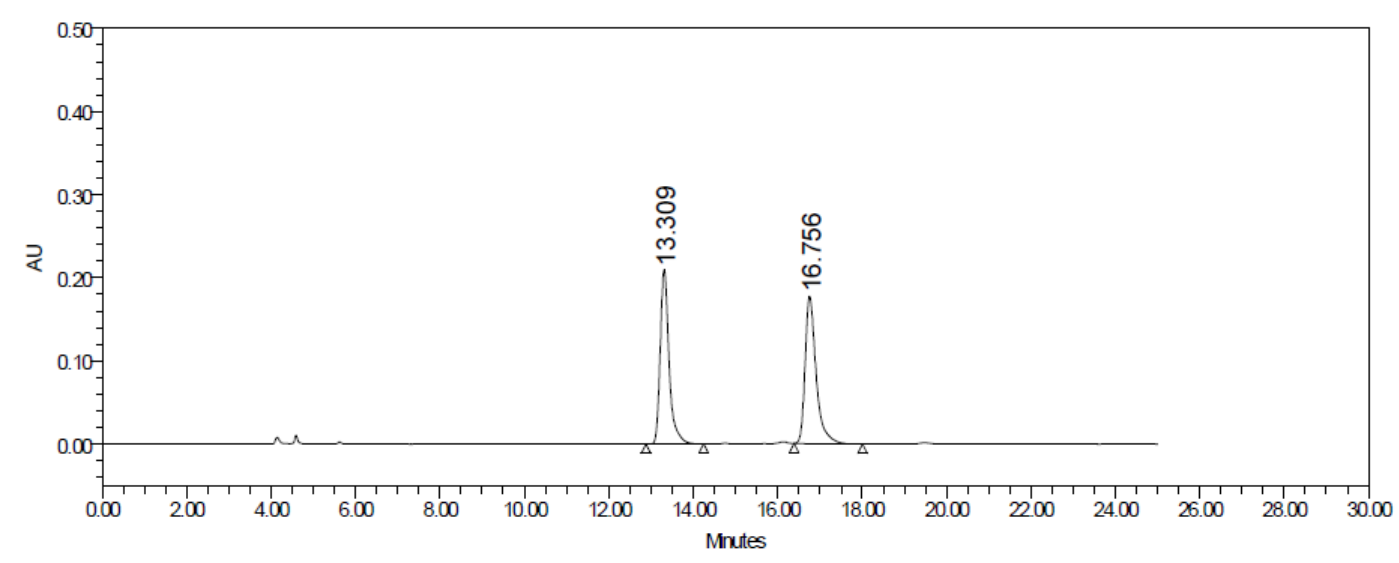

\begin{tabular}{|l|c|c|r|c|}
\hline & RT & Area & $\%$ Area & Height \\
\hline 1 & 13.309 & 3052246 & 48.82 & 209639 \\
\hline 2 & 16.756 & 3200320 & 51.18 & 177336 \\
\hline
\end{tabular}

\begin{tabular}{|c|c|c|c|}
\hline & SAMPLE & INFORMATION & \\
\hline Sample Name: & TFF-5-80-1 IF3 912140.7 & Acquired By: & System \\
\hline Sample Type: & Unknown & Sample Set Name: & 20190821 \\
\hline Vial: & $1: C, 2$ & Acq. Method Set: & 1008 \\
\hline Injection\#. & 1 & Processing Method & 1 \\
\hline Injection Volume: & $200 \mathrm{ul}$ & Channel Name: & PDA Ch1 214nm@4.8nm \\
\hline Run Time: & 25.0 Minutes & Proc. Arnl. Descr.: & PDA Ch1 214nm@4.8nm \\
\hline Date Acquired: & 9/10/2019 1:01:36 PM CST & & \\
\hline Date Processed: & 12/19/2019 1:37:24 PMCST & & \\
\hline
\end{tabular}

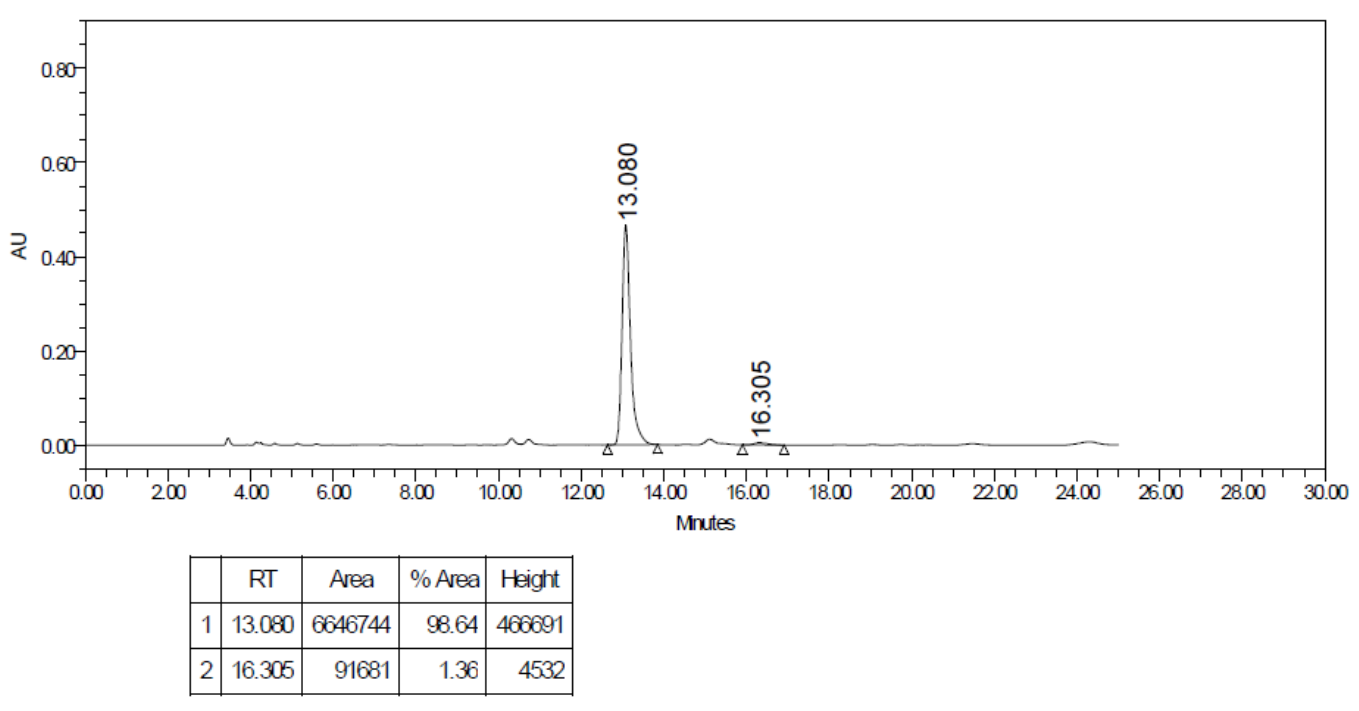


Ethyl(R)-2,2-difluoro-3-((1S,2R)-2-phenylcyclopropyl)-5-(triisopropylsilyl)pent-4-ynoate (101).
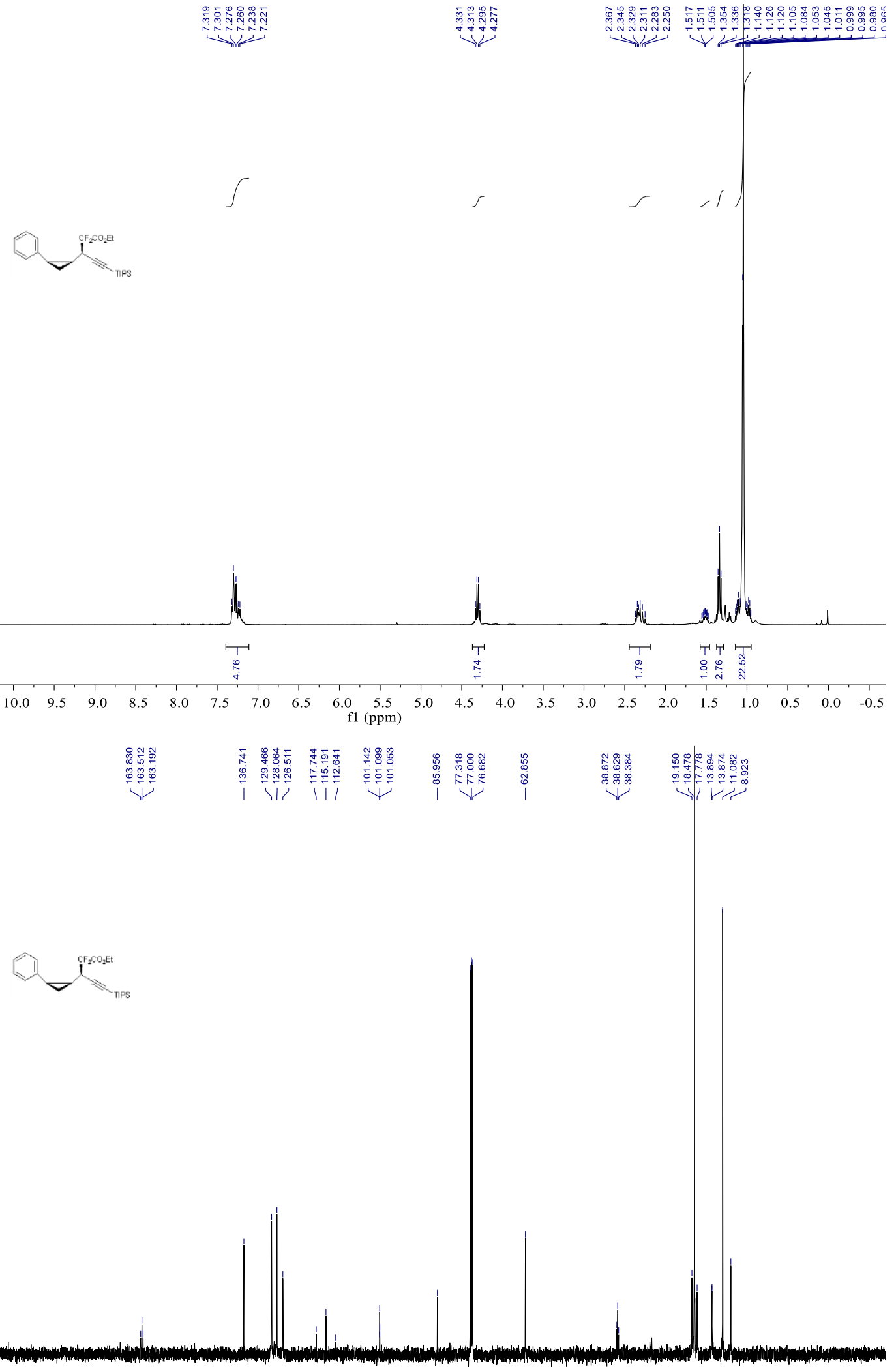

$\begin{array}{lllllllllllllllllllllllllllllll}230 & 220 & 210 & 200 & 190 & 180 & 170 & 160 & 150 & 140 & 130 & 120 & 110 & 100 & 90 & 80 & 70 & 60 & 50 & 40 & 30 & 20 & 10 & 0 & -10 & -20 & -3\end{array}$ 


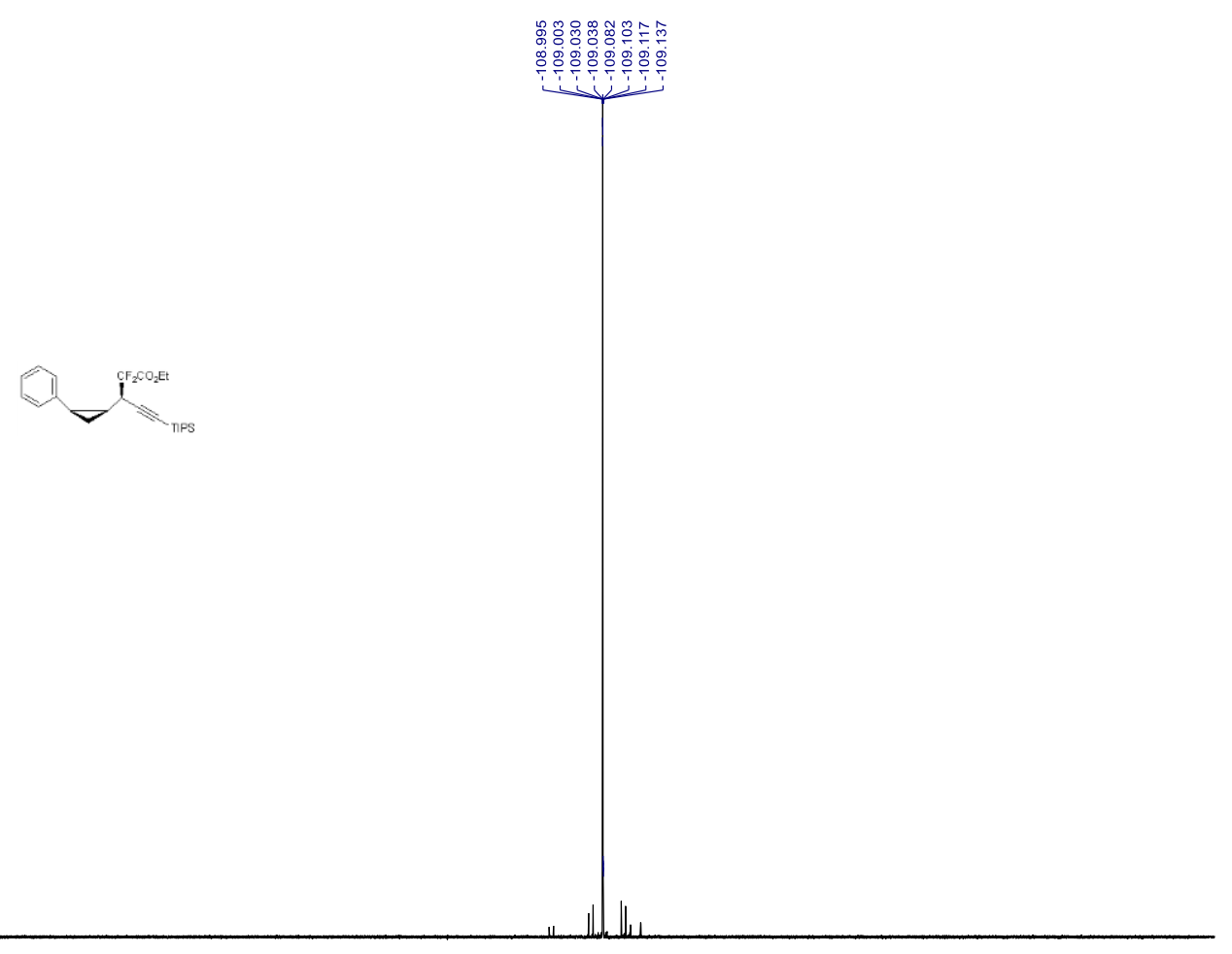

\begin{tabular}{llllllllllllllllllllllll}
\hline 30 & 20 & 10 & 0 & -10 & -20 & -30 & -40 & -50 & -60 & -70 & -80 & -90 & -100 & -110 & -120 & -130 & -140 & -150 & -160 & -170 & -180 & -190 & -200
\end{tabular}

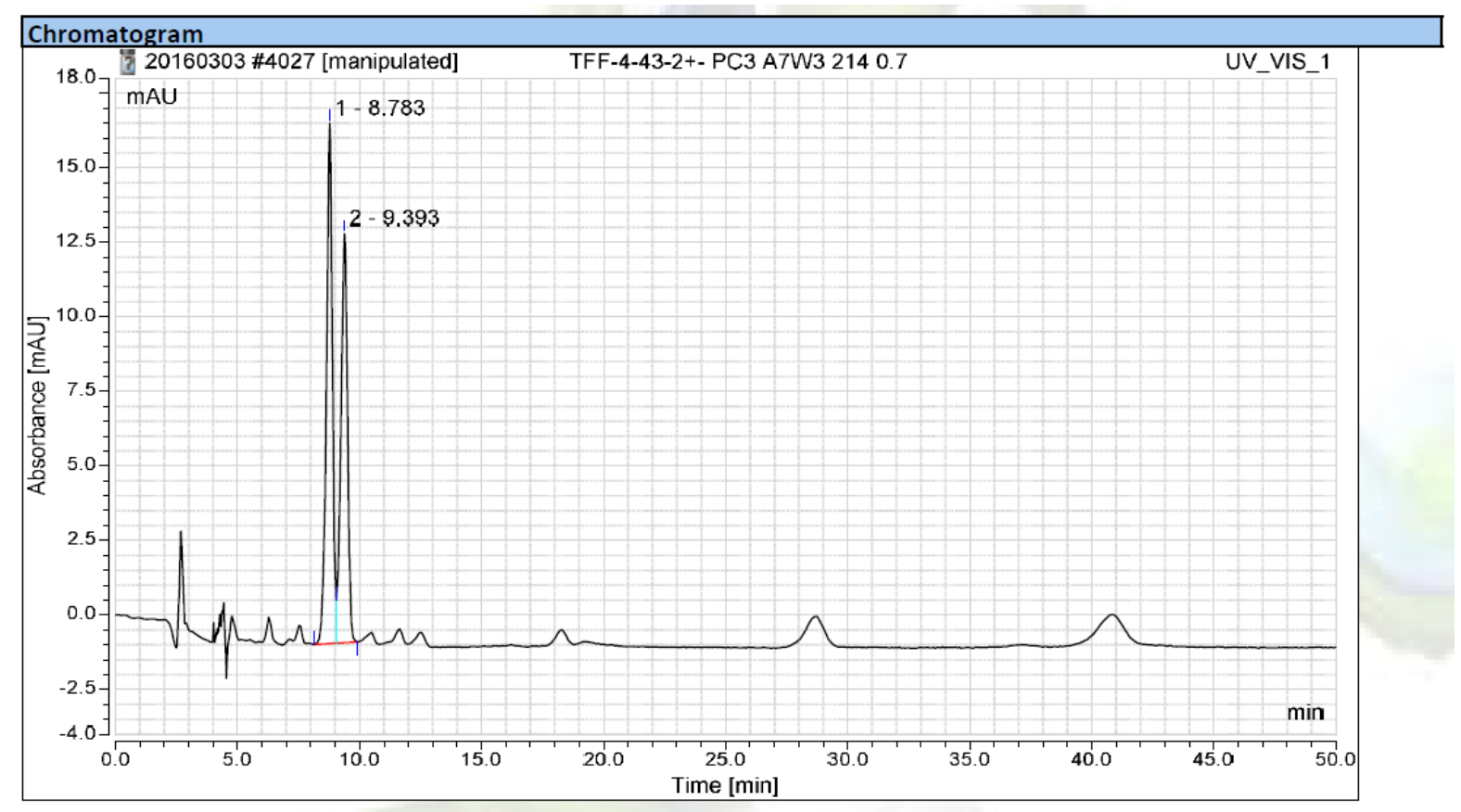

\begin{tabular}{|l|c|c|c|c|}
\hline \multicolumn{5}{|l|}{ Integration Results } \\
\hline No. & $\begin{array}{c}\text { Retention Time } \\
\text { min }\end{array}$ & $\begin{array}{c}\text { Area } \\
\text { mAU*min }^{*}\end{array}$ & $\begin{array}{c}\text { Height } \\
\text { mAU }\end{array}$ & $\begin{array}{c}\text { Relative Area } \\
\%\end{array}$ \\
\hline 1 & 8.783 & 4.9597 & 17.4467 & 54.149 \\
2 & 9.393 & 4.1996 & 13.7175 & 45.851 \\
\hline Total: & 9.159 & 1401.998 & 100.000 \\
\hline
\end{tabular}




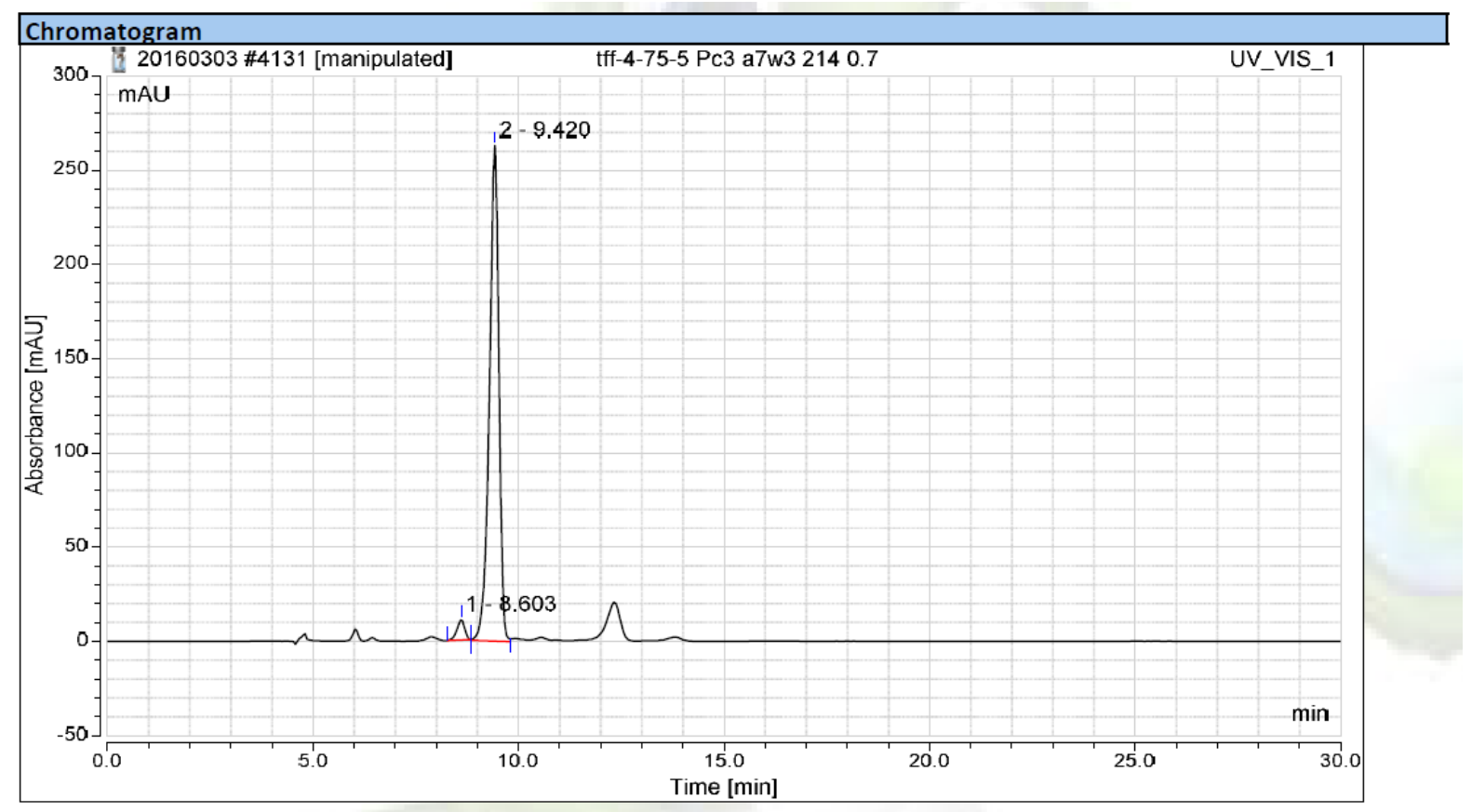

\begin{tabular}{|c|c|c|c|c|}
\hline \multicolumn{5}{|c|}{ Integration Results } \\
\hline No. & $\begin{array}{c}\text { Retention Time } \\
\text { min }\end{array}$ & $\begin{array}{c}\text { Area } \\
\text { mAUmin }\end{array}$ & $\begin{array}{c}\text { Height } \\
\text { mAU }\end{array}$ & $\begin{array}{c}\text { Relative Area } \\
\%\end{array}$ \\
\hline 1 & 8.603 & 2.3468 & 10.8459 & 3.245 \\
\hline 2 & 9.420 & 69.9770 & 263.1462 & 96.755 \\
\hline \multicolumn{2}{|c|}{ Total: } & 72.324 & 1401.998 & 100.000 \\
\hline
\end{tabular}

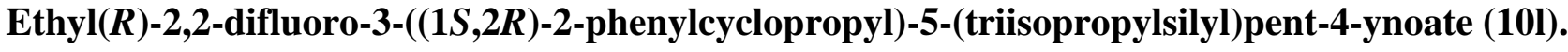

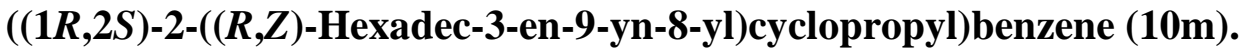

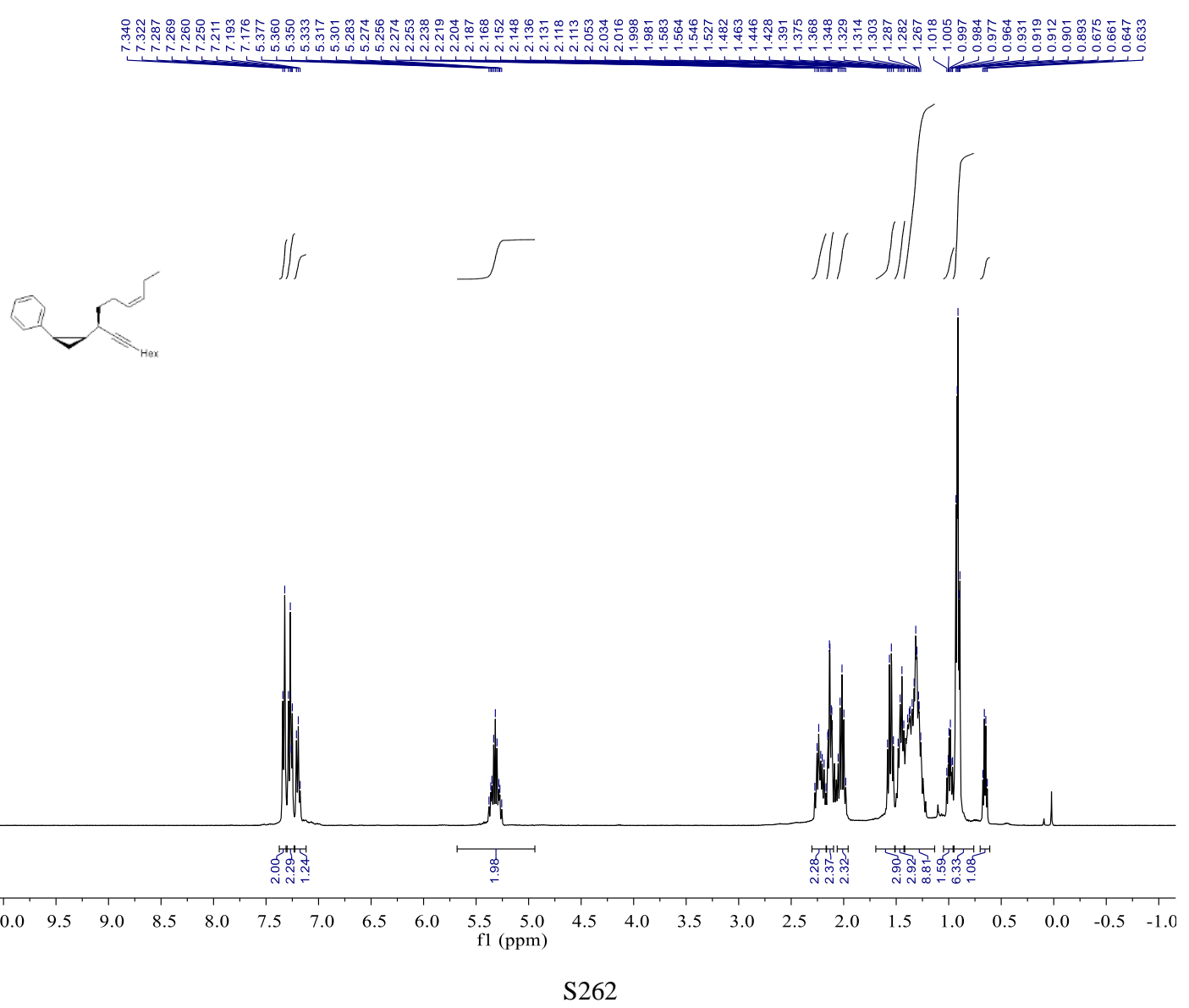




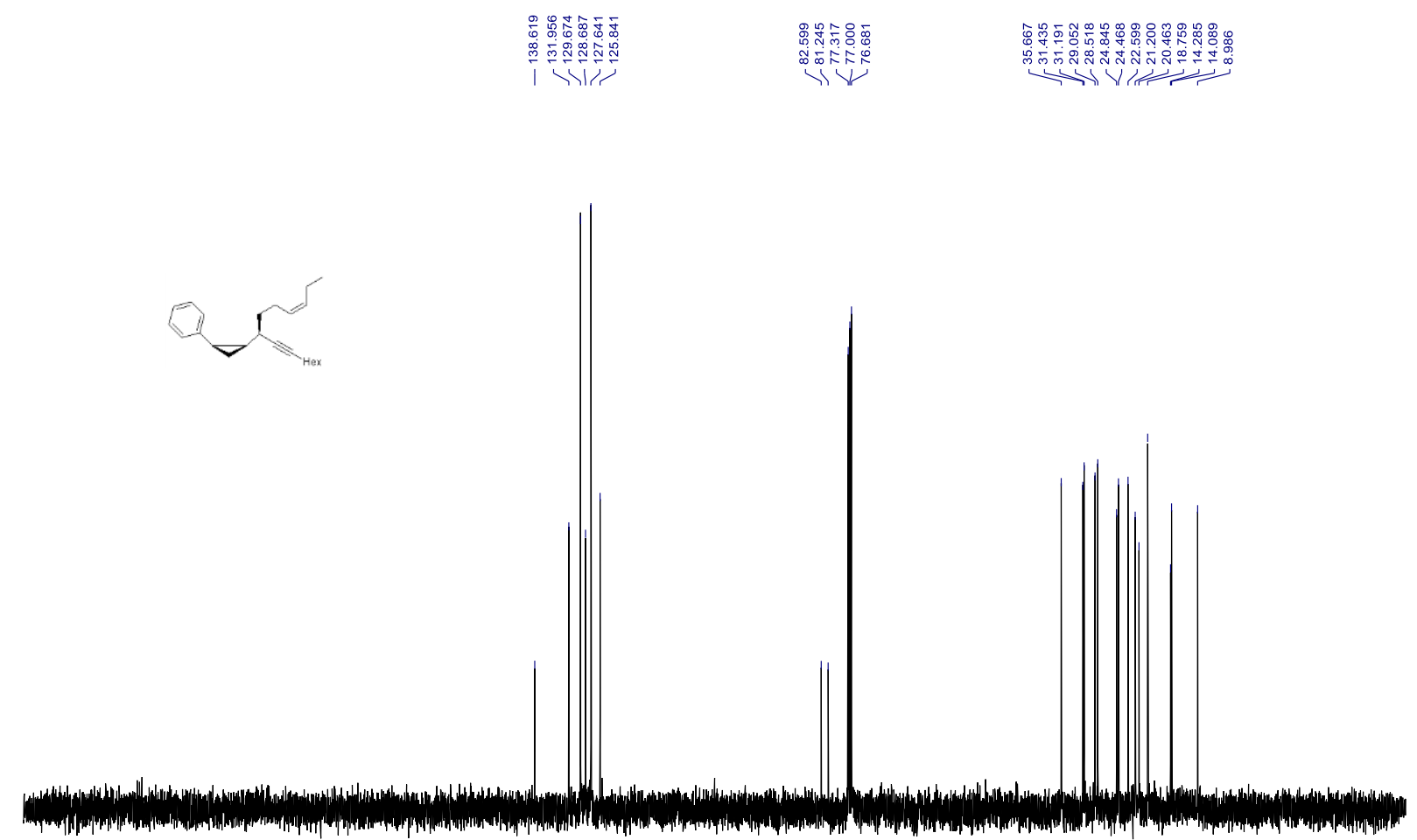

$\begin{array}{lllllllllllllllllllllllllllllll}230 & 220 & 210 & 200 & 190 & 180 & 170 & 160 & 150 & 140 & 130 & 120 & 110 & 100 & 90 & 80 & 70 & 60 & 50 & 40 & 30 & 20 & 10 & 0 & -10 & -20 & -3\end{array}$

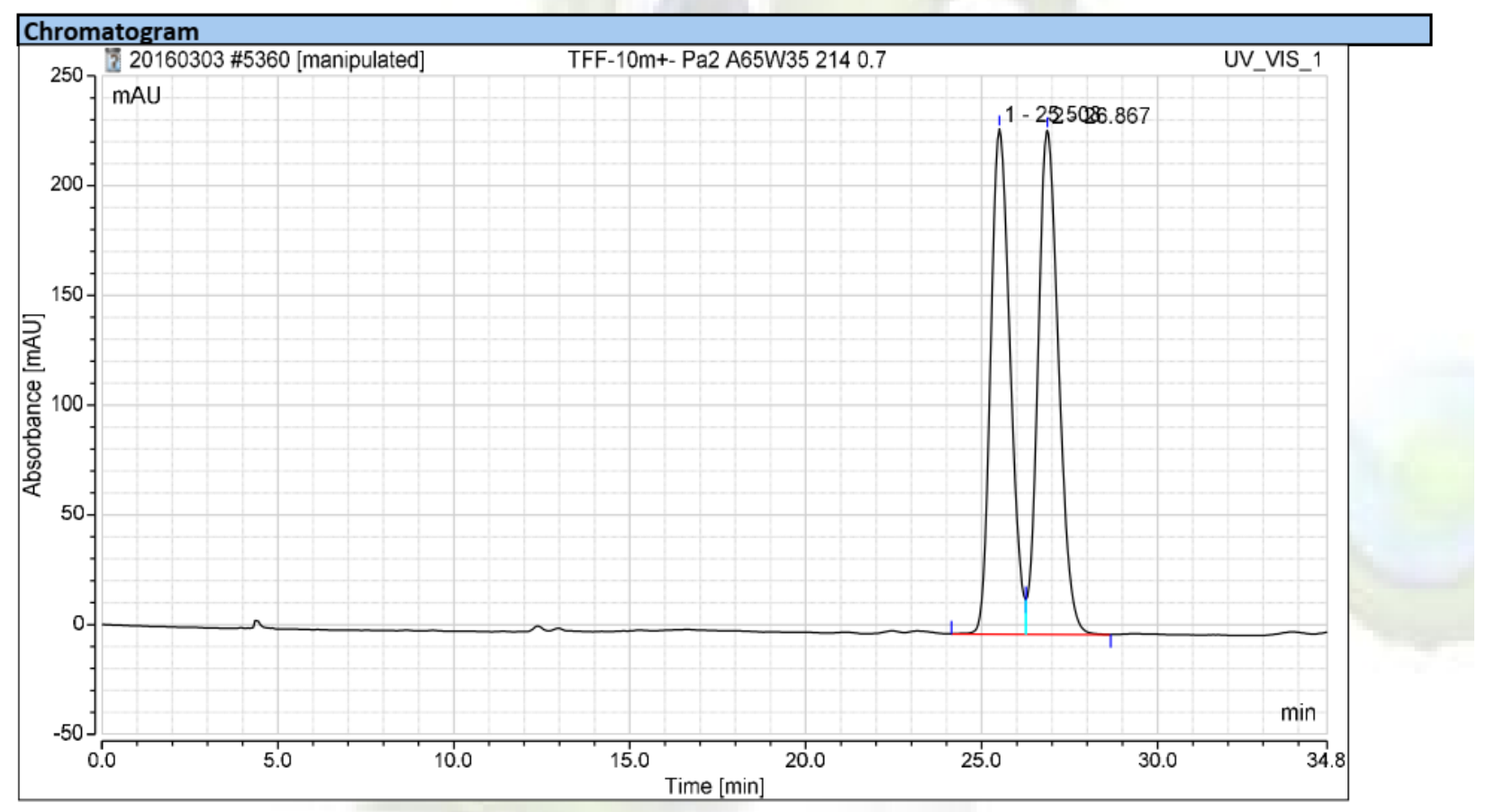

\begin{tabular}{|c|c|c|c|c|}
\hline \multicolumn{5}{|c|}{ Integration Results } \\
\hline No. & $\begin{array}{l}\text { Retention Time } \\
\text { min }\end{array}$ & $\begin{array}{c}\text { Area } \\
m A U^{\star} \min \end{array}$ & $\begin{array}{c}\text { Height } \\
\text { mAU }\end{array}$ & $\begin{array}{c}\text { Relative Area } \\
\%\end{array}$ \\
\hline 1 & 25.503 & 147.5561 & 230.3014 & 48.624 \\
\hline 2 & 26.867 & 155.9087 & 229.6383 & 51.376 \\
\hline \multicolumn{2}{|c|}{ Total: } & 303.465 & 1401.998 & 100.000 \\
\hline
\end{tabular}




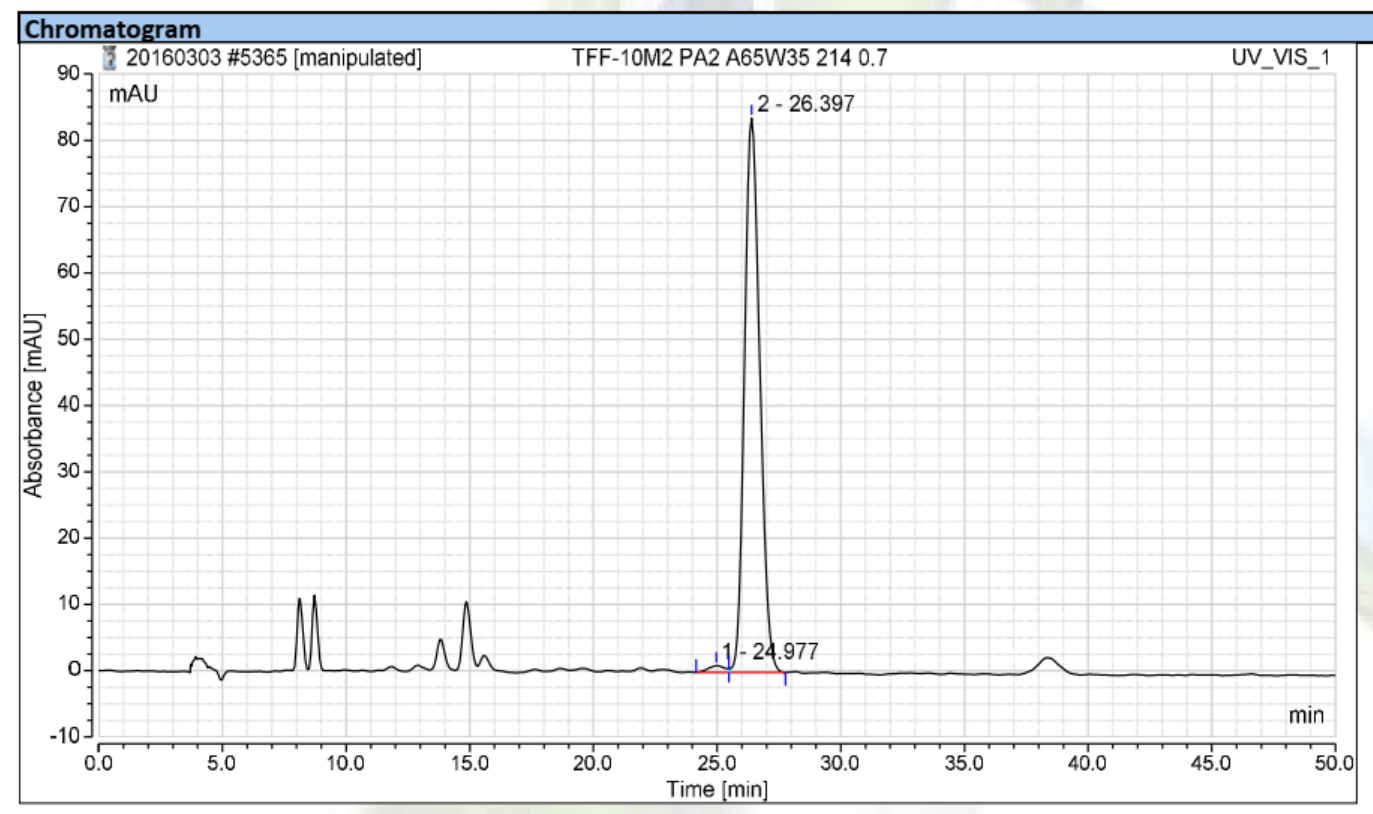

\begin{tabular}{|l|c|c|c|c|}
\hline Integration Results \\
\hline No. & $\begin{array}{c}\text { Retention Time } \\
\text { min }\end{array}$ & $\begin{array}{c}\text { Area } \\
\mathrm{mAU} \text { min }\end{array}$ & $\begin{array}{c}\text { Height } \\
\mathrm{mAU}\end{array}$ & $\begin{array}{c}\text { Relative Area } \\
\%\end{array}$ \\
\hline 1 & 24.977 & 0.7597 & 1.0077 & 1.253 \\
2 & 26.397 & 59.8829 & 83.5905 & 98.747 \\
\hline \multicolumn{5}{|l}{$\mathbf{6 0 . 6 4 3}$} \\
\hline
\end{tabular}

EDRC Reaction with Primary Propargyl Bromides

$((1 S, 2 R)$-2-Allylcyclopropyl)benzene (5b).

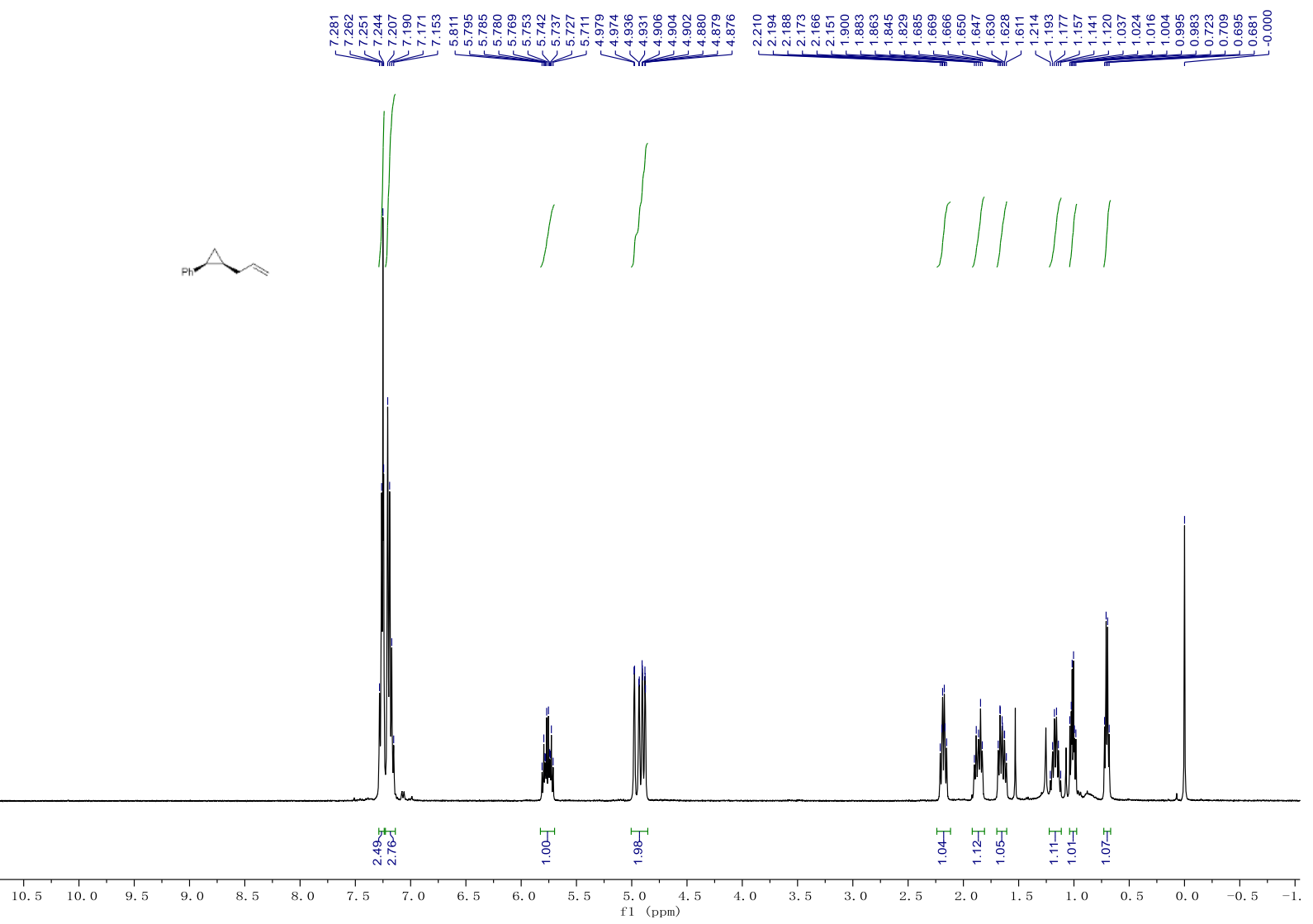



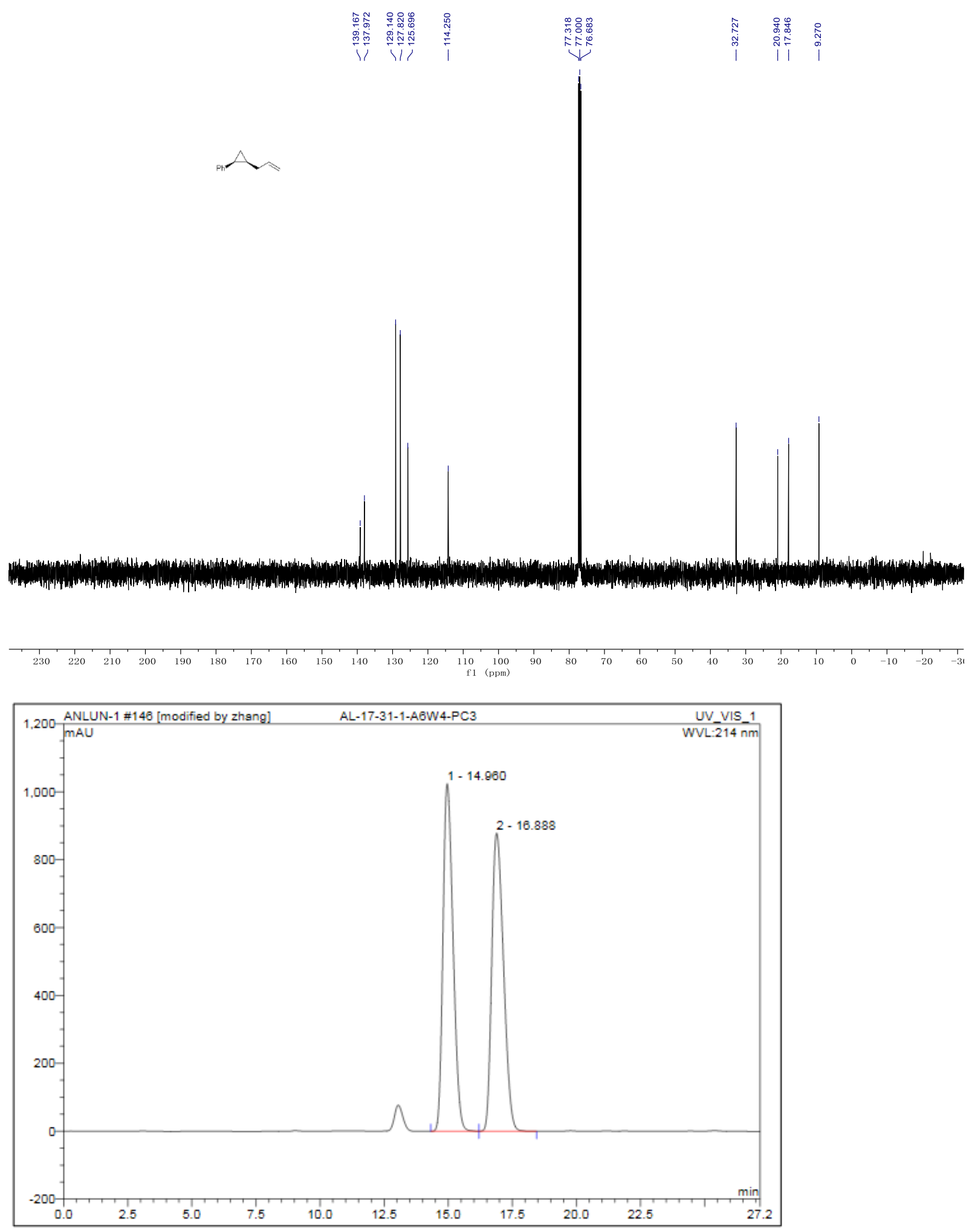

\begin{tabular}{|r|ccrrrrr|}
\hline No. & $\begin{array}{c}\text { Ret.Time } \\
\text { min }\end{array}$ & Peak Name & $\begin{array}{c}\text { Height } \\
\text { mAU }\end{array}$ & $\begin{array}{c}\text { Area } \\
\text { mAU*min }\end{array}$ & $\begin{array}{c}\text { Rel.Area } \\
\%\end{array}$ & Amount & Type \\
\hline 1 & 14.96 & n.a. & 1024.563 & 468.212 & 50.00 & n.a. & BM \\
2 & 16.89 & n.a. & 879.023 & 468.210 & 50.00 & n.a. & MB \\
\hline Total: & & & 1903.586 & 936.422 & 100.00 & 0.000 & \\
\hline
\end{tabular}




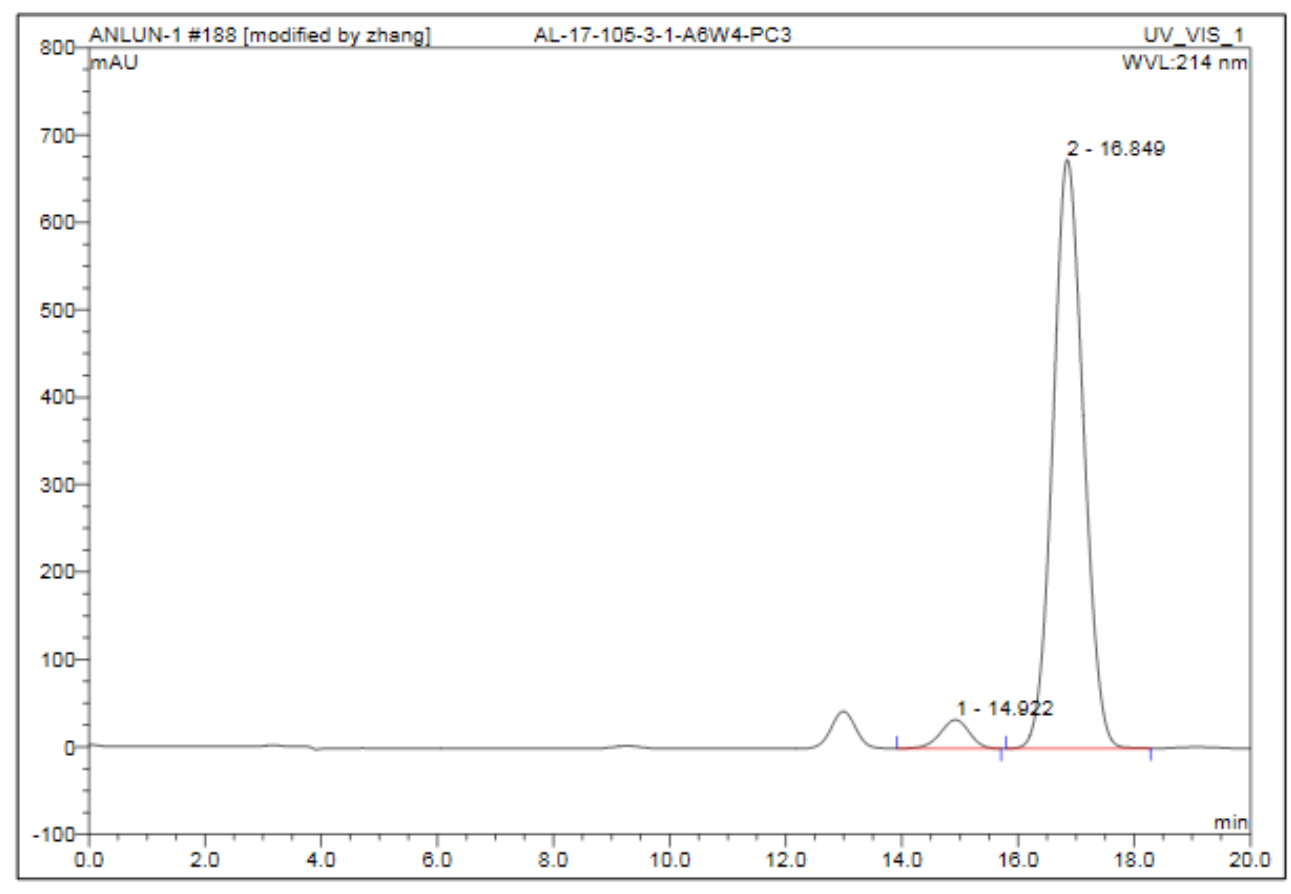

\begin{tabular}{|r|ccrrrrr|}
\hline No. & $\begin{array}{c}\text { Ret.Time } \\
\text { min }\end{array}$ & Peak Name & $\begin{array}{c}\text { Height } \\
\text { mAU }\end{array}$ & $\begin{array}{c}\text { Area } \\
\text { mAU*min }\end{array}$ & $\begin{array}{r}\text { Rel.Area } \\
\%\end{array}$ & Amount & Type \\
\hline 1 & 14.92 & n.a. & 32.716 & 18.937 & 4.41 & n.a. & BMB $^{\star}$ \\
2 & 16.85 & n.a. & 673.683 & 410.856 & 95.59 & n.a. & BMB \\
\hline Total: & & & 706.399 & 429.793 & 100.00 & 0.000 & \\
\hline
\end{tabular}

Ethyl-2-(((1S,2S)-2-phenylcyclopropyl)methyl)acrylate (5c).

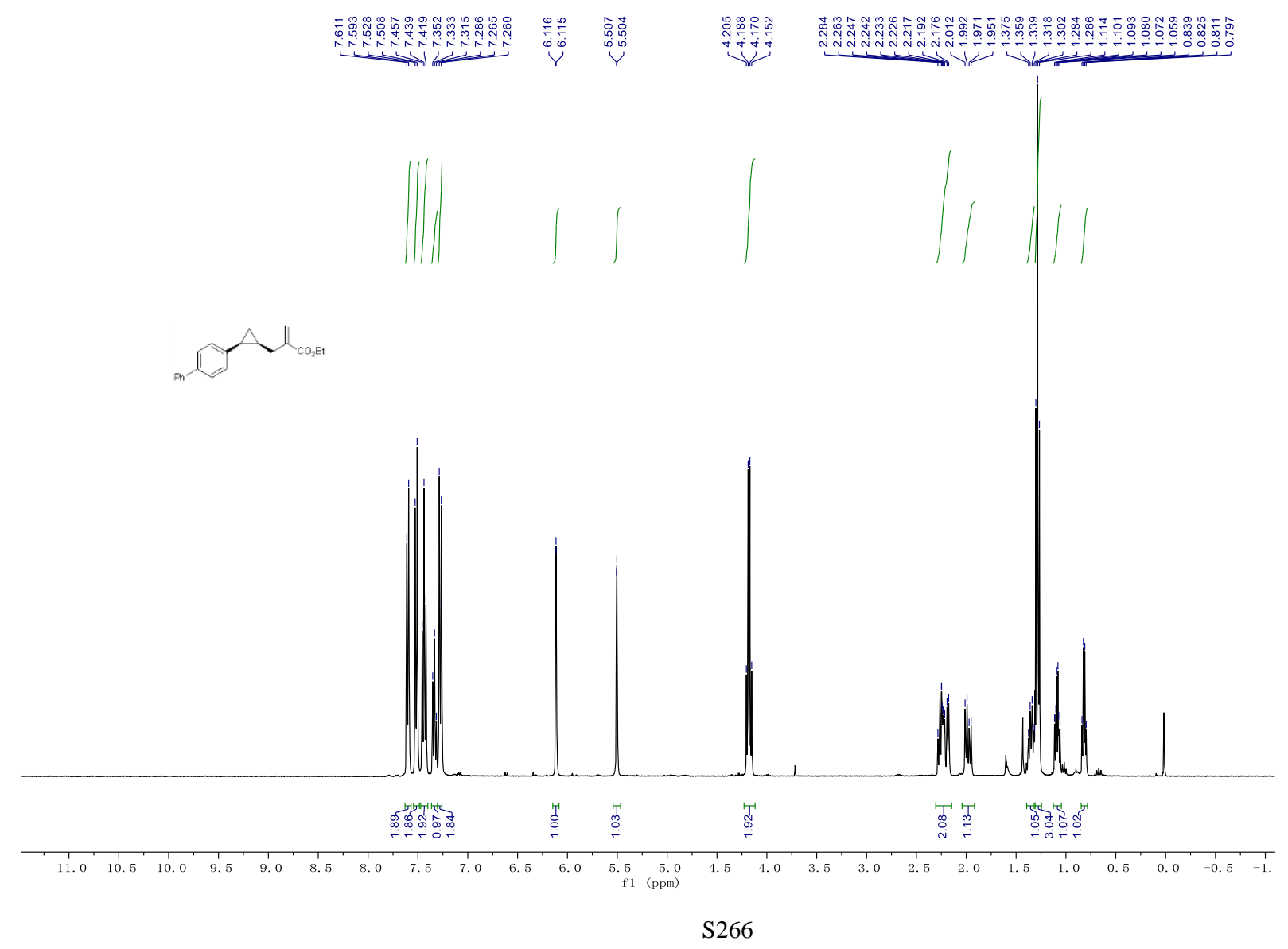



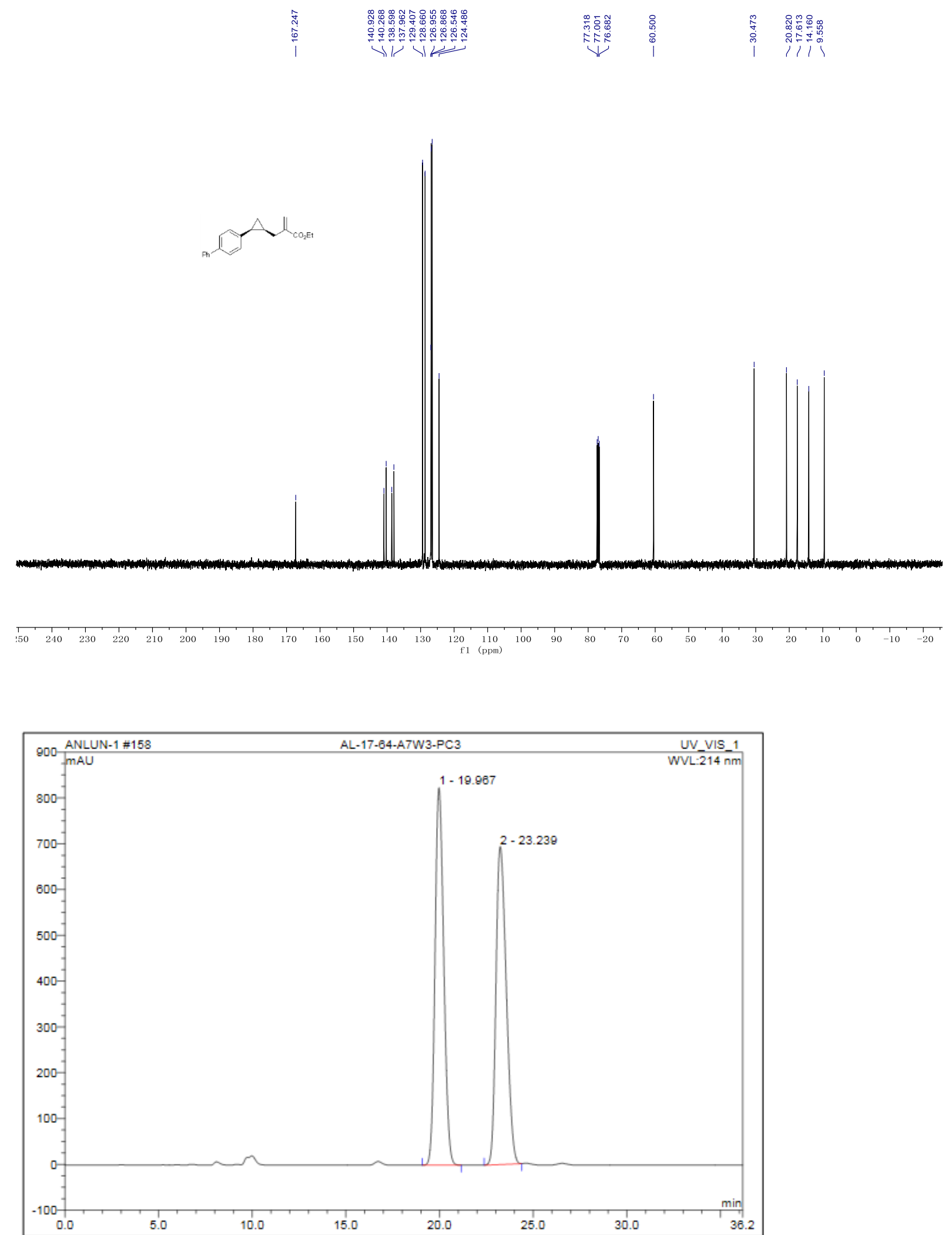

\begin{tabular}{|r|ccrrrrr|}
\hline No. & $\begin{array}{c}\text { Ret.Time } \\
\text { min }\end{array}$ & Peak Name & $\begin{array}{c}\text { Height } \\
\text { mAU }\end{array}$ & $\begin{array}{r}\text { Area } \\
\text { mAU*min }\end{array}$ & $\begin{array}{c}\text { Rel.Area } \\
\%\end{array}$ & Amount & Type \\
\hline 1 & 19.97 & n.a. & 824.206 & 444.225 & 50.10 & n.a. & BMB \\
2 & 23.24 & n.a. & 694.341 & 442.432 & 49.90 & n.a. & BMB \\
\hline Total: & & & 1518.547 & 886.657 & 100.00 & 0.000 & \\
\hline
\end{tabular}




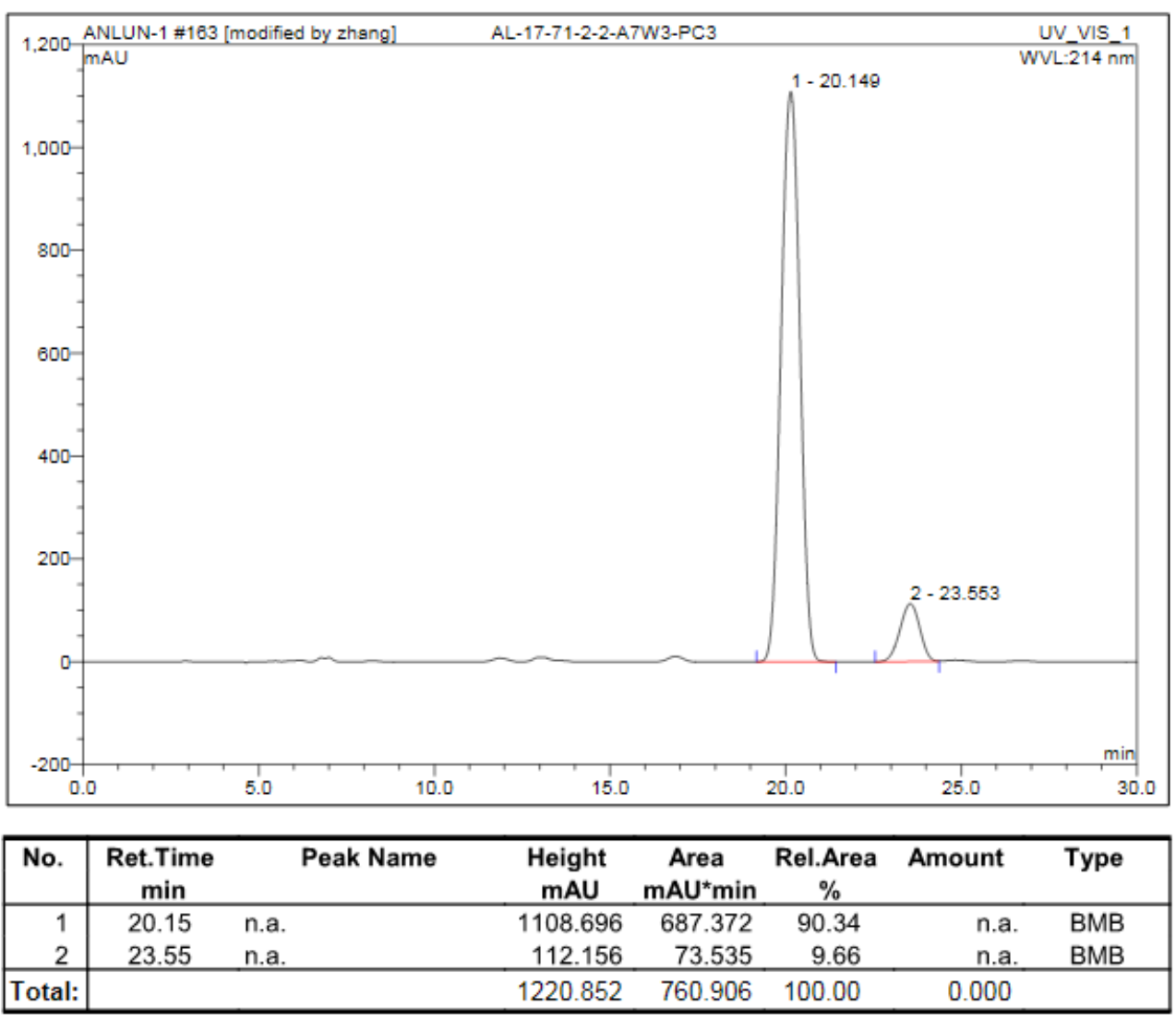

Phenyl((1R,2S)-2-phenylcyclopropyl)methanone (5d).

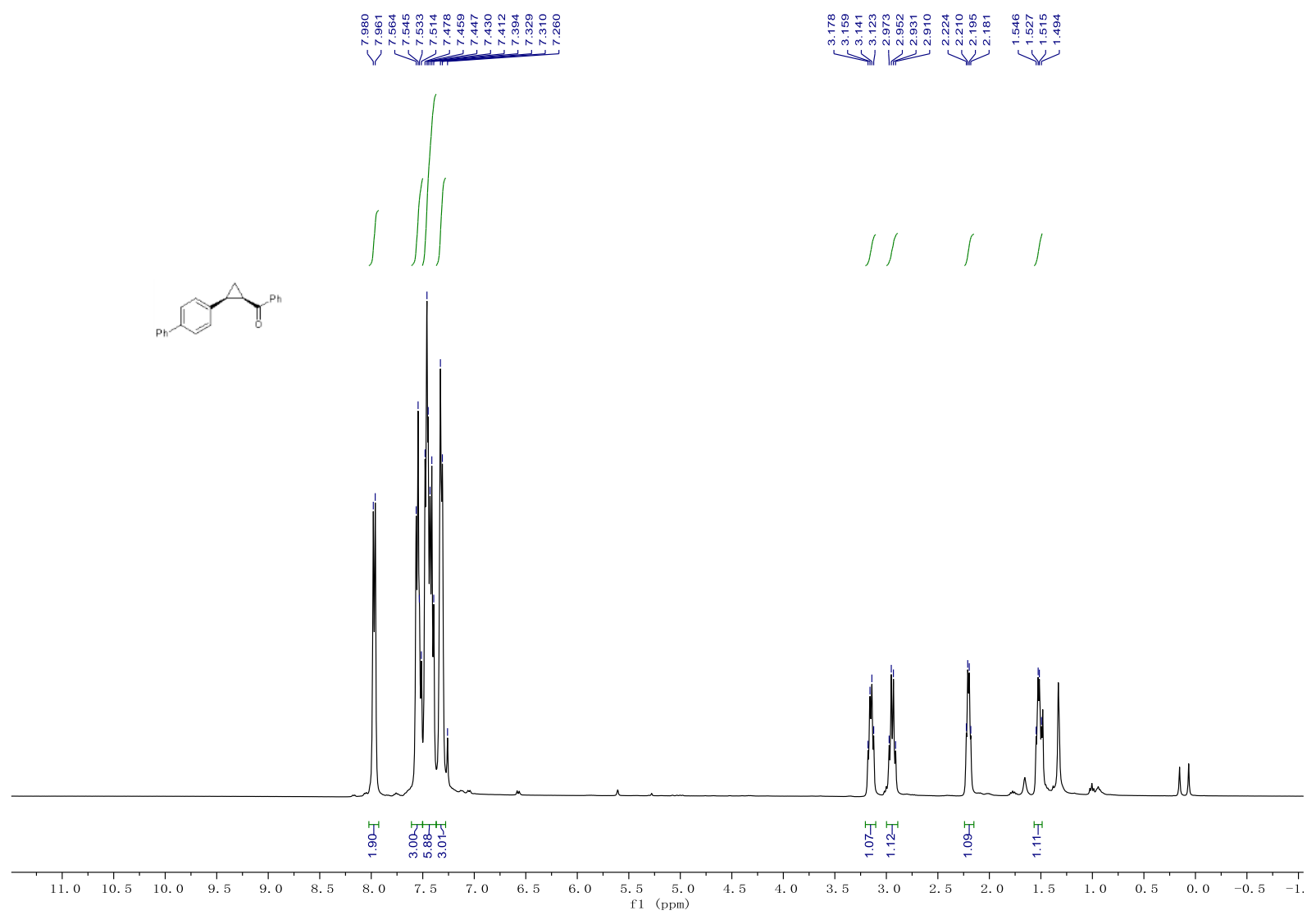



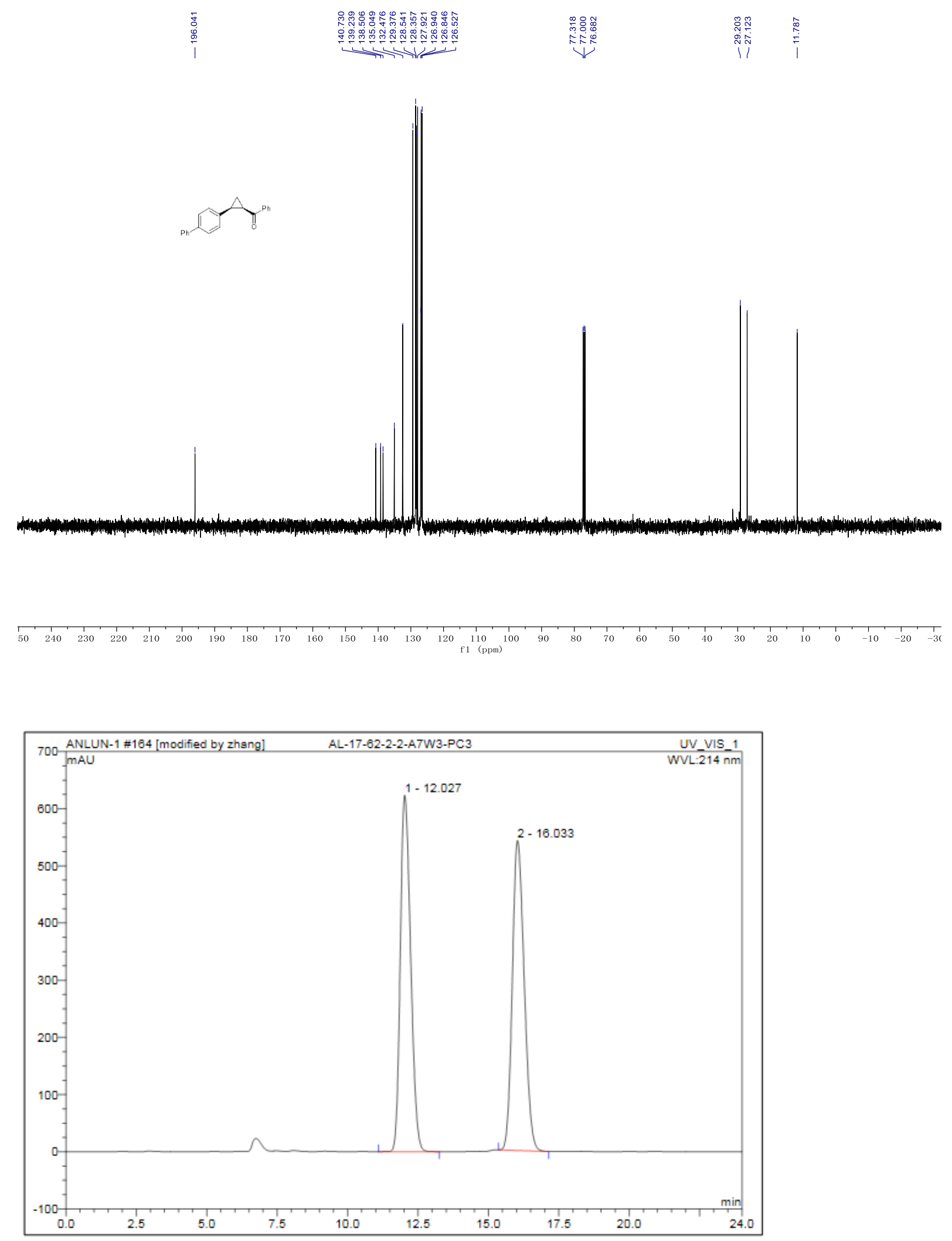

\begin{tabular}{|r|ccrrrrr|}
\hline No. & $\begin{array}{c}\text { Ret.Time } \\
\text { min }\end{array}$ & Peak Name & $\begin{array}{c}\text { Height } \\
\text { mAU }\end{array}$ & $\begin{array}{c}\text { Area } \\
\text { mAU*min }\end{array}$ & $\begin{array}{r}\text { Rel.Area } \\
\%\end{array}$ & Amount & Type \\
\hline 1 & 12.03 & n.a. & 623.427 & 264.856 & 50.01 & n.a. & BMB \\
2 & 16.03 & n.a. & 542.111 & 264.715 & 49.99 & n.a. & BMB \\
\hline Total: & & & 1165.538 & 529.571 & 100.00 & 0.000 & \\
\hline
\end{tabular}




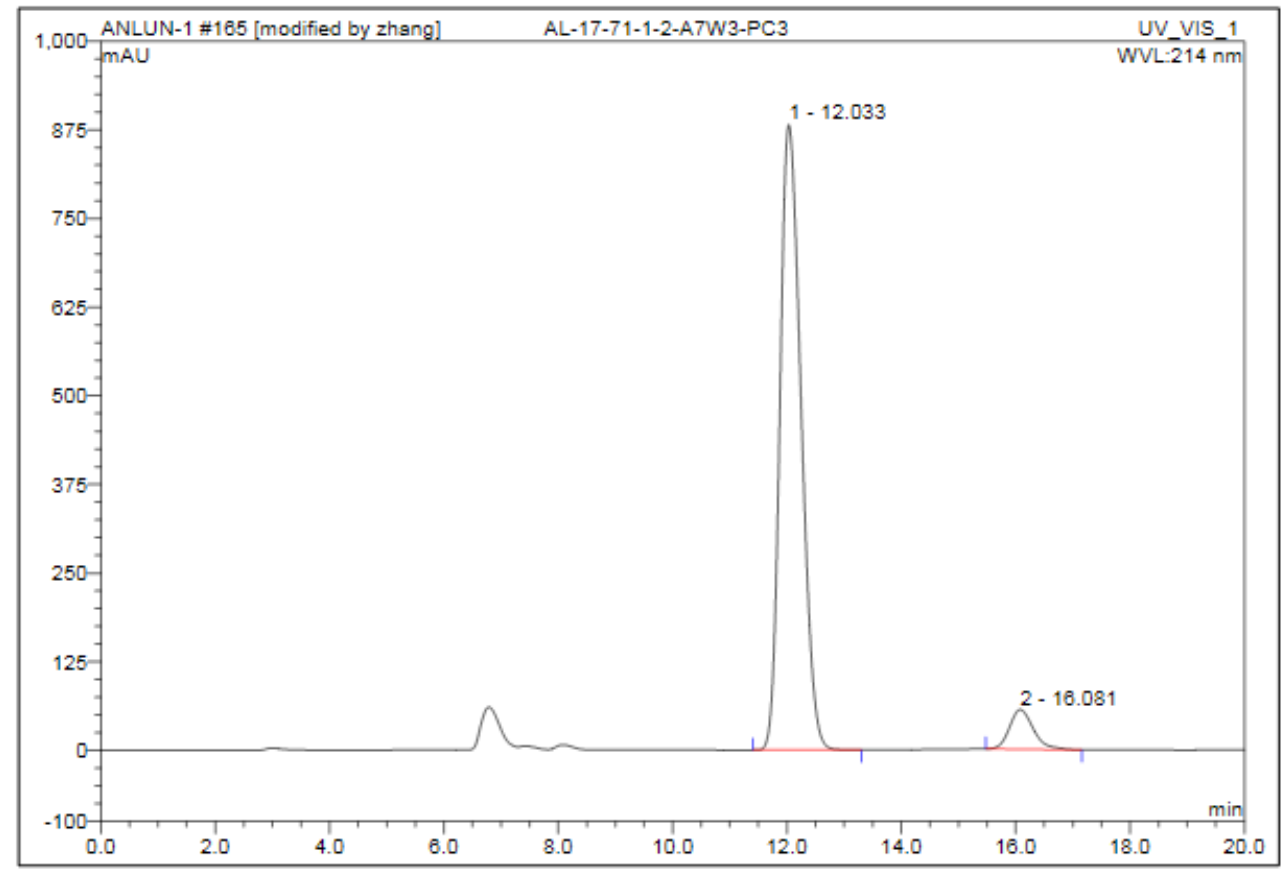

\begin{tabular}{|r|ccrrrrr|}
\hline No. & $\begin{array}{c}\text { Ret.Time } \\
\text { min }\end{array}$ & Peak Name & $\begin{array}{c}\text { Height } \\
\text { mAU }\end{array}$ & $\begin{array}{r}\text { Area } \\
\text { mAU*min }\end{array}$ & $\begin{array}{r}\text { Rel.Area } \\
\%\end{array}$ & Amount & Type \\
\hline 1 & 12.03 & n.a. & 882.079 & 375.958 & 93.29 & n.a. & BMB \\
2 & 16.08 & n.a. & 55.701 & 27.039 & 6.71 & n.a. & BMB \\
\hline Total: & & & 937.780 & 402.996 & 100.00 & 0.000 & \\
\hline
\end{tabular}

4-((1S,2R)-2-(Phenylethynyl)cyclopropyl)-1,1'-biphenyl (5e).

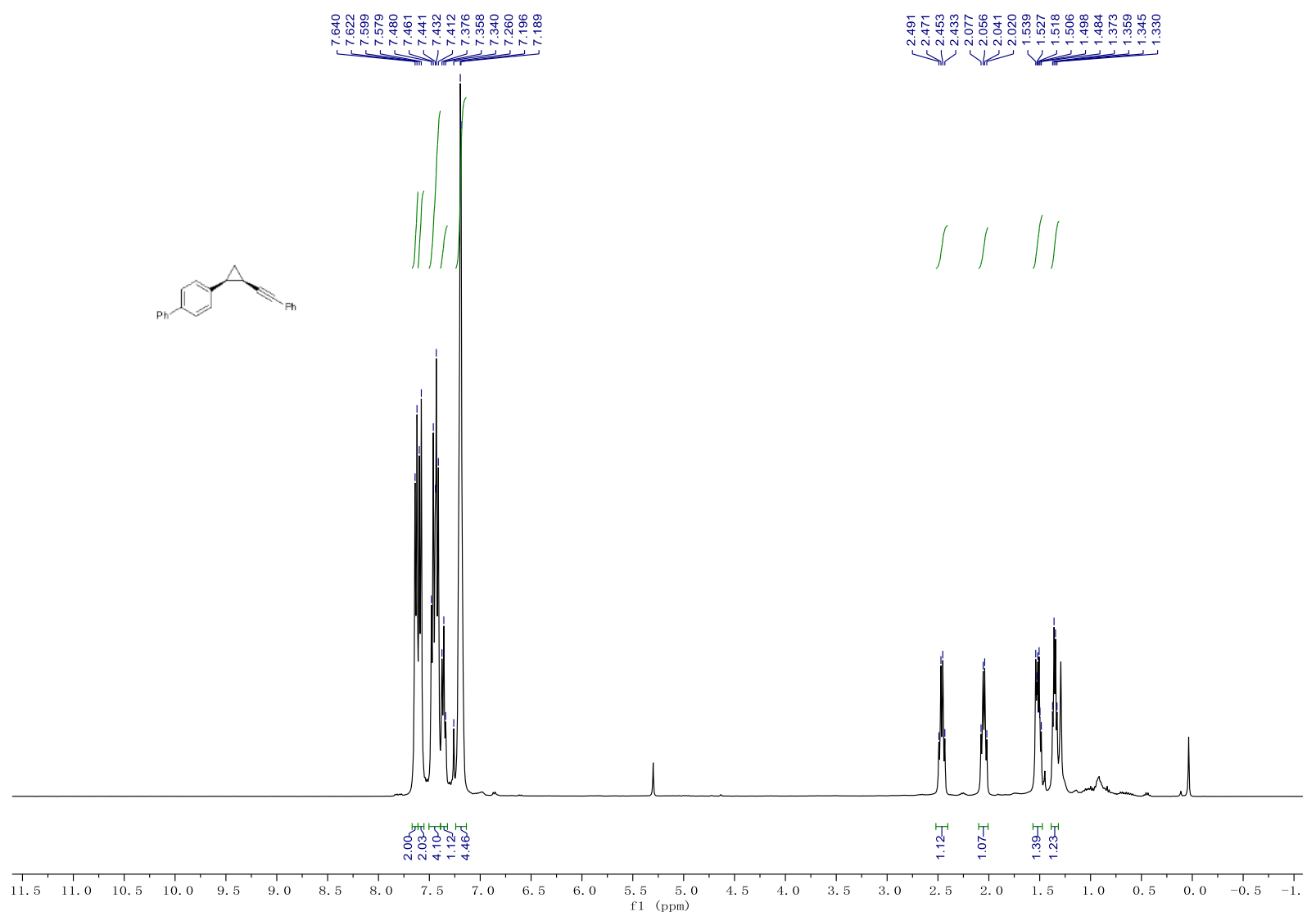



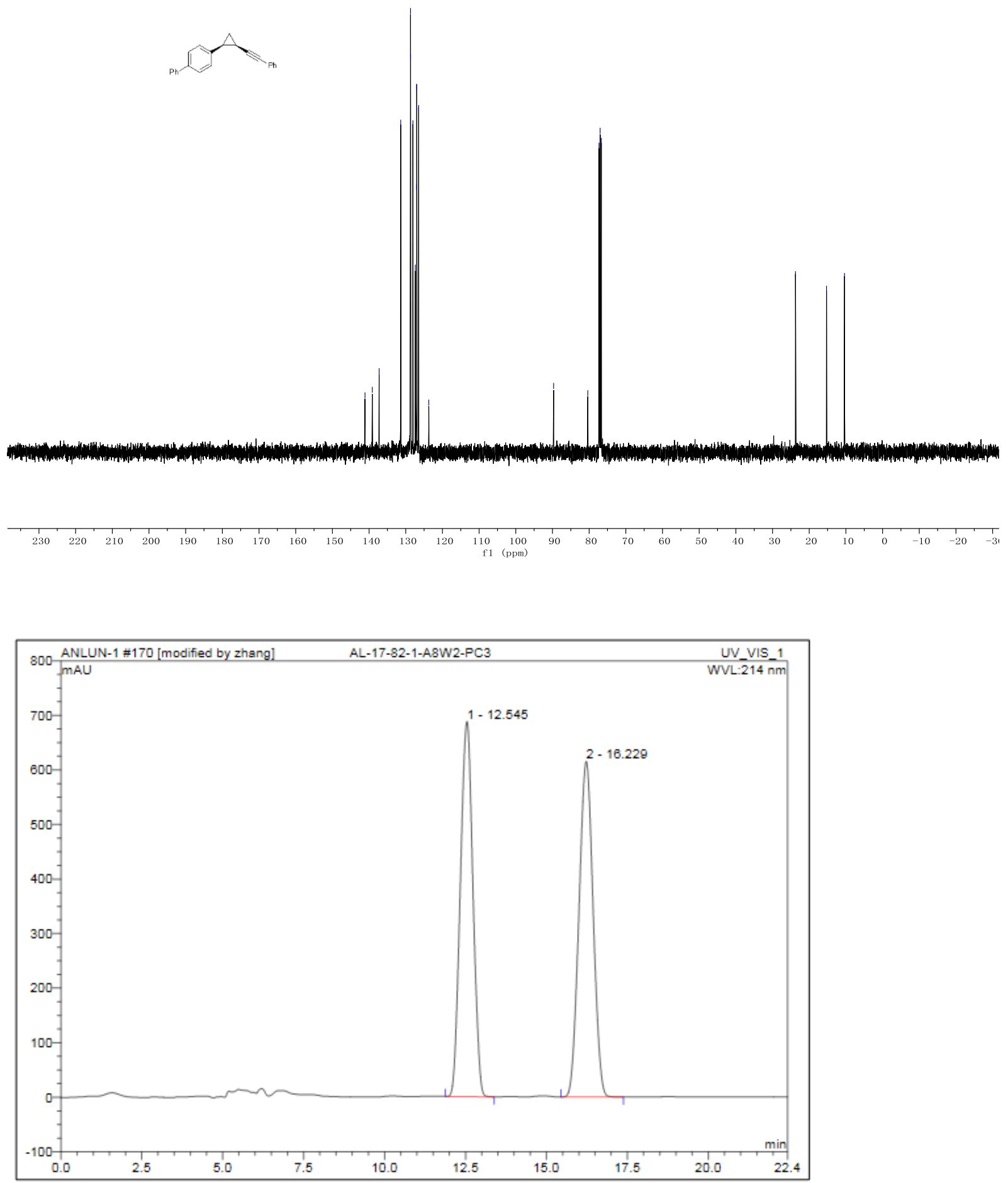

\begin{tabular}{|r|ccrrrrr|}
\hline No. & $\begin{array}{c}\text { Ret.Time } \\
\text { min }\end{array}$ & Peak Name & $\begin{array}{c}\text { Height } \\
\text { mAU }\end{array}$ & $\begin{array}{c}\text { Area } \\
\text { mAU*min }\end{array}$ & $\begin{array}{c}\text { Rel.Area } \\
\%\end{array}$ & Amount & Type \\
\hline 1 & 12.55 & n.a. & 687.992 & 308.505 & 49.80 & n.a. & BMB \\
2 & 16.23 & n.a. & 615.387 & 310.921 & 50.20 & n.a. & BMB \\
\hline Total: & & & 1303.379 & 619.426 & 100.00 & 0.000 & \\
\hline
\end{tabular}




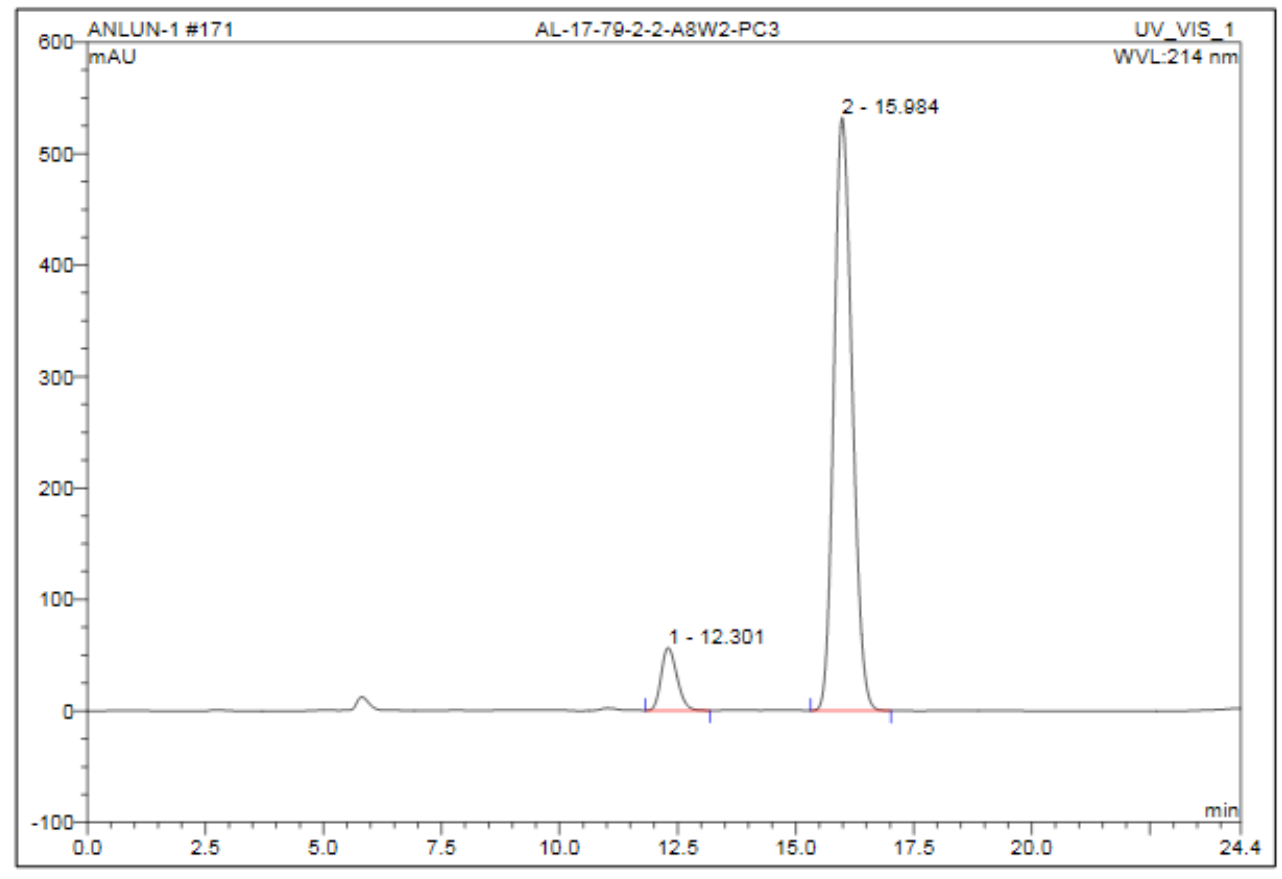

\begin{tabular}{|r|ccrrrrr|}
\hline No. & $\begin{array}{c}\text { Ret.Time } \\
\text { min }\end{array}$ & Peak Name & $\begin{array}{c}\text { Height } \\
\text { mAU }\end{array}$ & $\begin{array}{c}\text { Area } \\
\text { mAU*min }\end{array}$ & $\begin{array}{r}\text { Rel.Area } \\
\%\end{array}$ & Amount & Type \\
\hline 1 & 12.30 & n.a. & 56.286 & 21.932 & 8.40 & n.a. & BMB \\
2 & 15.98 & n.a. & 532.291 & 239.266 & 91.60 & n.a. & BMB \\
\hline Total: & & & 588.577 & 261.198 & 100.00 & 0.000 & \\
\hline
\end{tabular}

((1S,2R)-2-(Penta-1,2-dien-3-yl)cyclopropyl)benzene (5f).

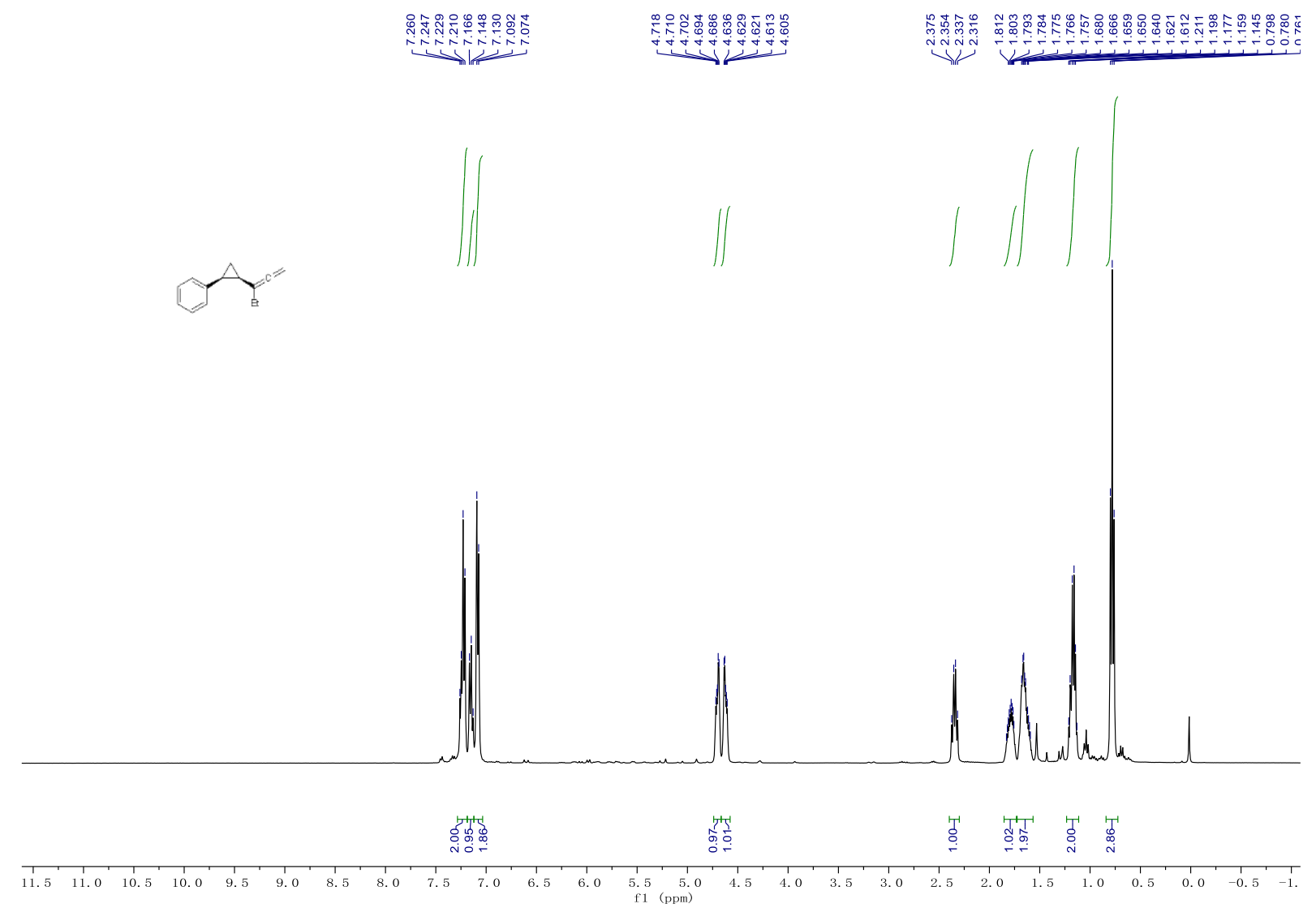



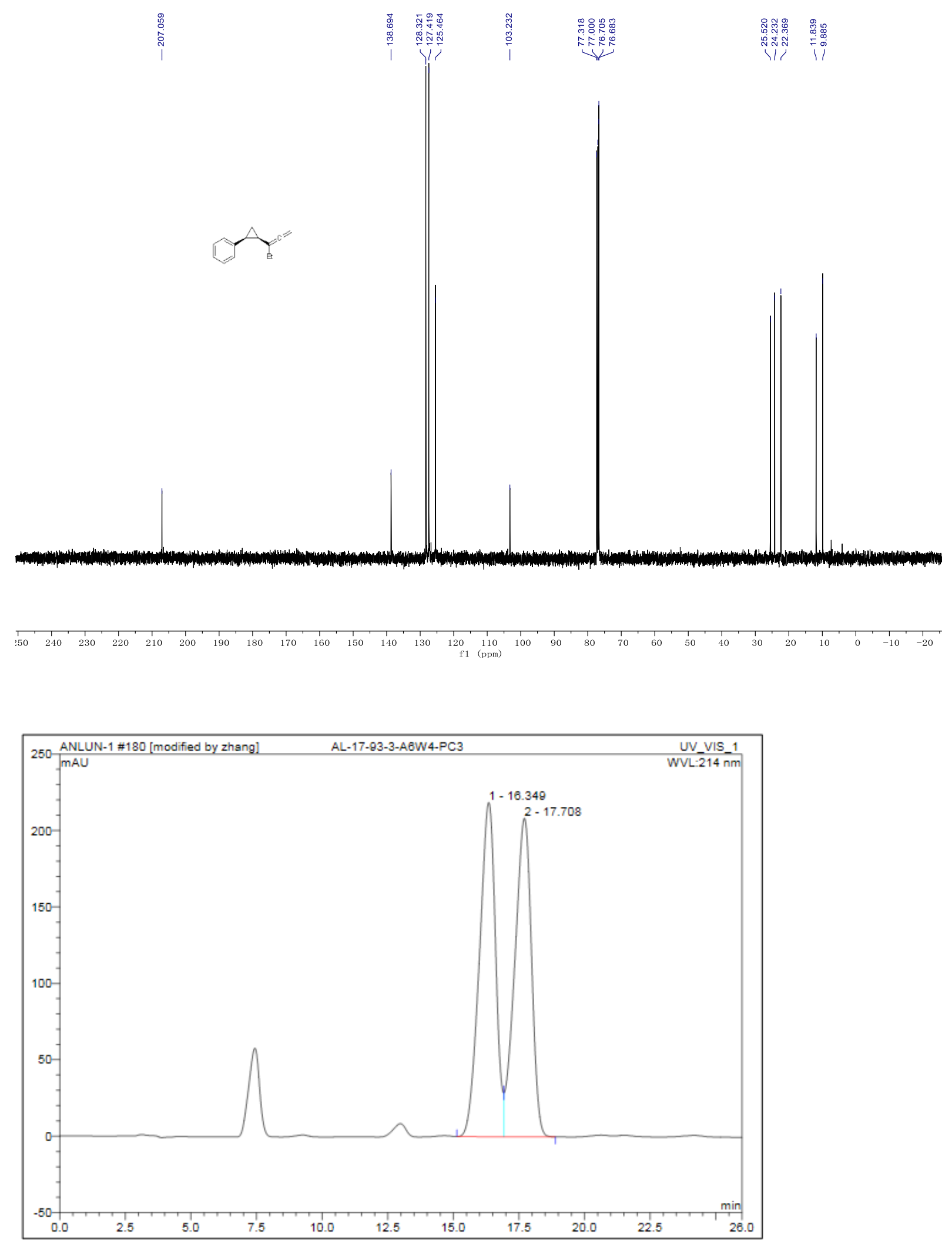

\begin{tabular}{|r|ccccccc|}
\hline No. & $\begin{array}{c}\text { Ret.Time } \\
\text { min }\end{array}$ & Peak Name & $\begin{array}{c}\text { Height } \\
\text { mAU }\end{array}$ & $\begin{array}{c}\text { Area } \\
\text { mAU*min }\end{array}$ & $\begin{array}{c}\text { Rel.Area } \\
\%\end{array}$ & Amount & Type \\
\hline 1 & 16.35 & n.a. & 218.697 & 156.285 & 50.28 & n.a. & BM \\
2 & 17.71 & n.a. & 208.475 & 154.530 & 49.72 & n.a. & MB \\
\hline Total: & & & 427.173 & 310.815 & 100.00 & 0.000 & \\
\hline
\end{tabular}




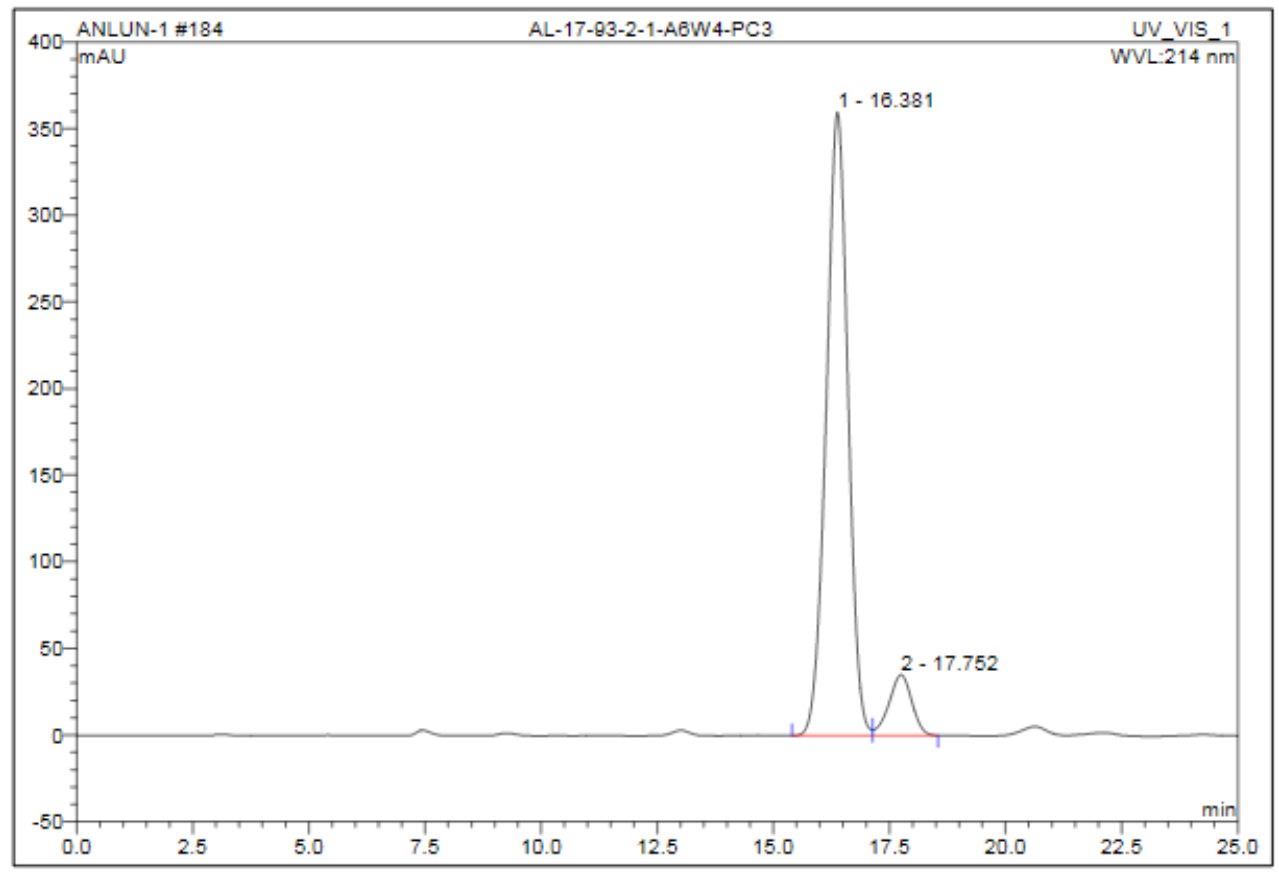

\begin{tabular}{|r|ccrrrrr|}
\hline No. & $\begin{array}{c}\text { Ret.Time } \\
\text { min }\end{array}$ & Peak Name & $\begin{array}{c}\text { Height } \\
\text { mAU }\end{array}$ & $\begin{array}{r}\text { Area } \\
\text { mAU*min }\end{array}$ & $\begin{array}{r}\text { Rel.Area } \\
\%\end{array}$ & Amount & Type \\
\hline 1 & 16.38 & n.a. & 359.946 & 194.835 & 90.70 & n.a. & BM \\
2 & 17.75 & n.a. & 35.044 & 19.983 & 9.30 & n.a. & MB \\
\hline Total: & & & 394.990 & 214.817 & 100.00 & 0.000 & \\
\hline
\end{tabular}

4-((1R,2R)-2-Bromocyclopropyl)-1,1'-biphenyl (5g).

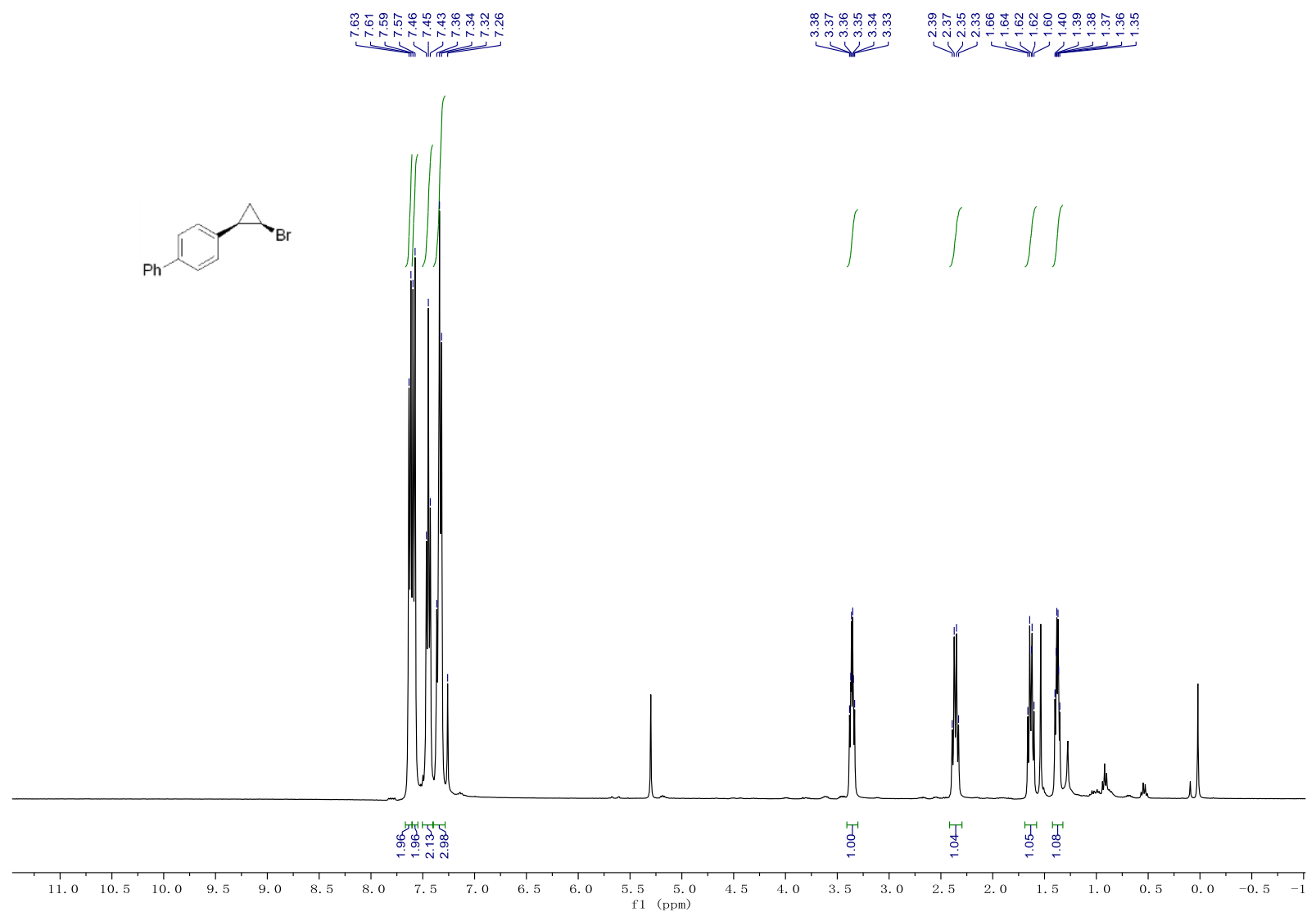



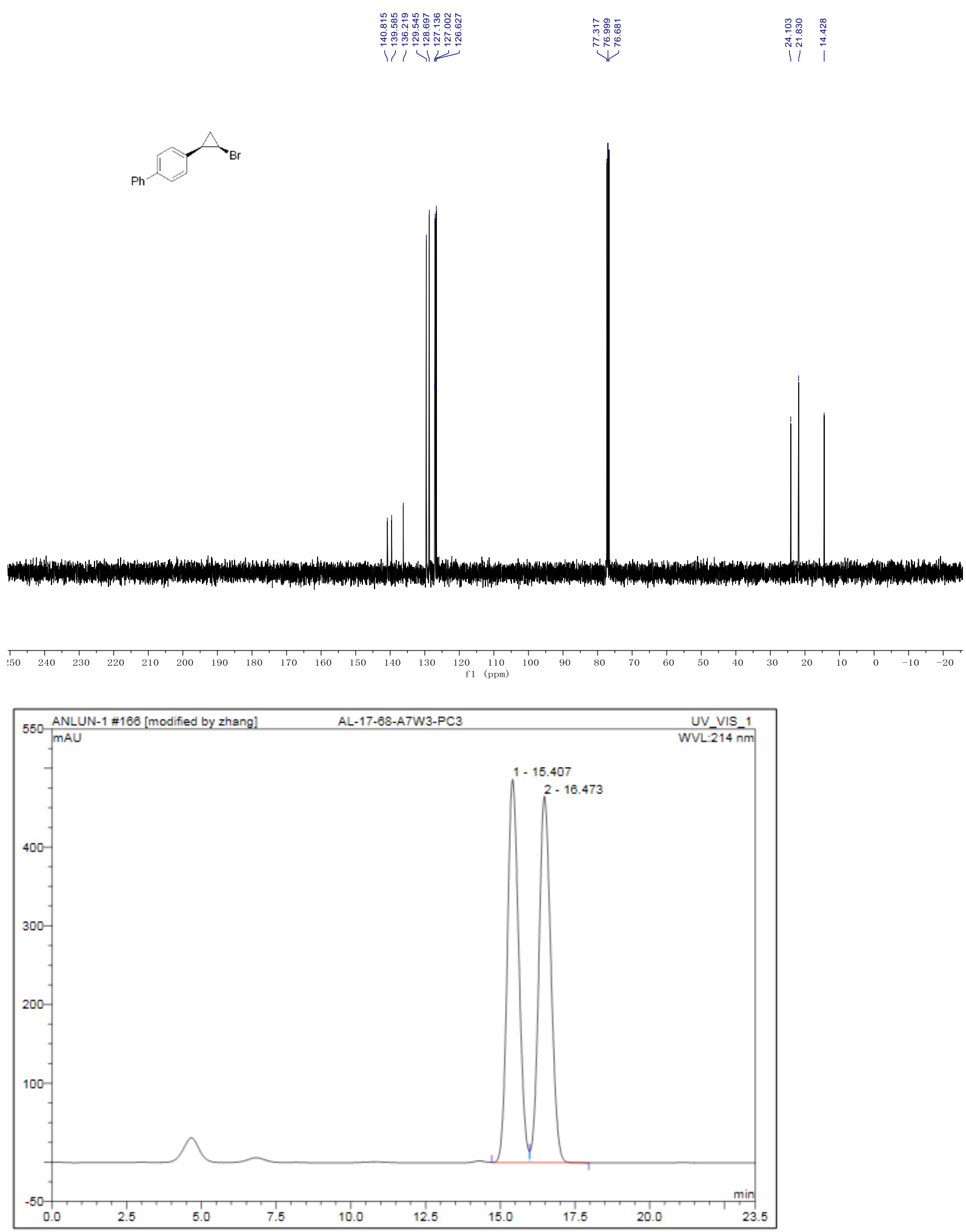

\begin{tabular}{|r|cccrrrr|}
\hline No. & $\begin{array}{c}\text { Ret.Time } \\
\text { min }\end{array}$ & Peak Name & $\begin{array}{c}\text { Height } \\
\text { mAU }\end{array}$ & $\begin{array}{c}\text { Area } \\
\text { mAU*min }\end{array}$ & $\begin{array}{r}\text { Rel.Area } \\
\%\end{array}$ & Amount & Type \\
\hline 1 & 15.41 & n.a. & 486.388 & 215.270 & 49.91 & n.a. & BM \\
2 & 16.47 & n.a. & 465.309 & 216.010 & 50.09 & n.a. & MB \\
\hline Total: & & & 951.698 & 431.279 & 100.00 & 0.000 & \\
\hline
\end{tabular}




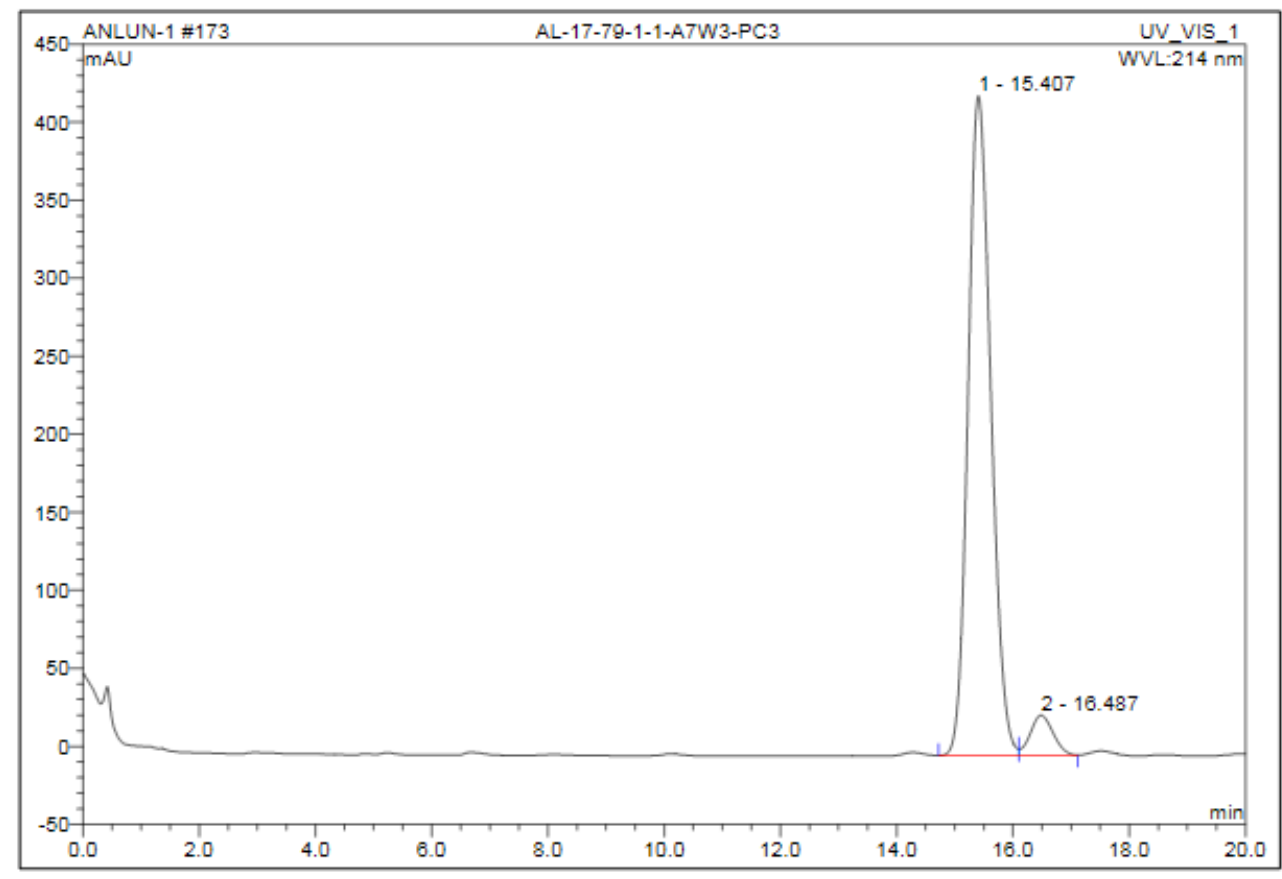

\begin{tabular}{|r|ccrrrrr|}
\hline No. & $\begin{array}{c}\text { Ret.Time } \\
\text { min }\end{array}$ & Peak Name & $\begin{array}{c}\text { Height } \\
\text { mAU }\end{array}$ & $\begin{array}{r}\text { Area } \\
\text { mAU*min }\end{array}$ & $\begin{array}{r}\text { Rel.Area } \\
\%\end{array}$ & Amount & Type \\
\hline 1 & 15.41 & n.a. & 422.698 & 192.295 & 94.32 & n.a. & BM \\
2 & 16.49 & n.a. & 25.637 & 11.571 & 5.68 & n.a. & MB \\
\hline Total: & & & 448.335 & 203.866 & 100.00 & 0.000 & \\
\hline
\end{tabular}

$((1 R, 2 R)-2$-Bromocyclopropyl)benzene (5h).

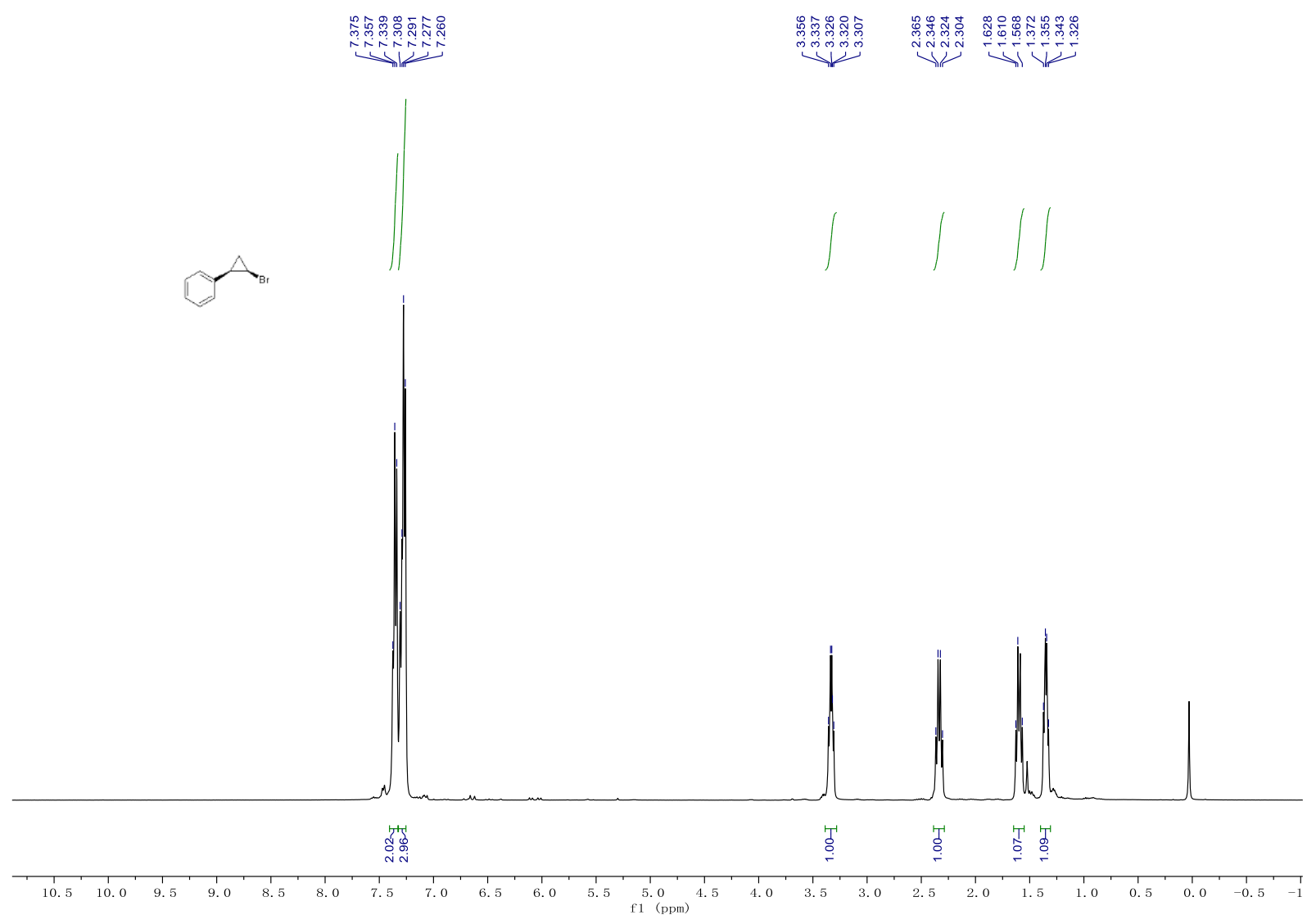




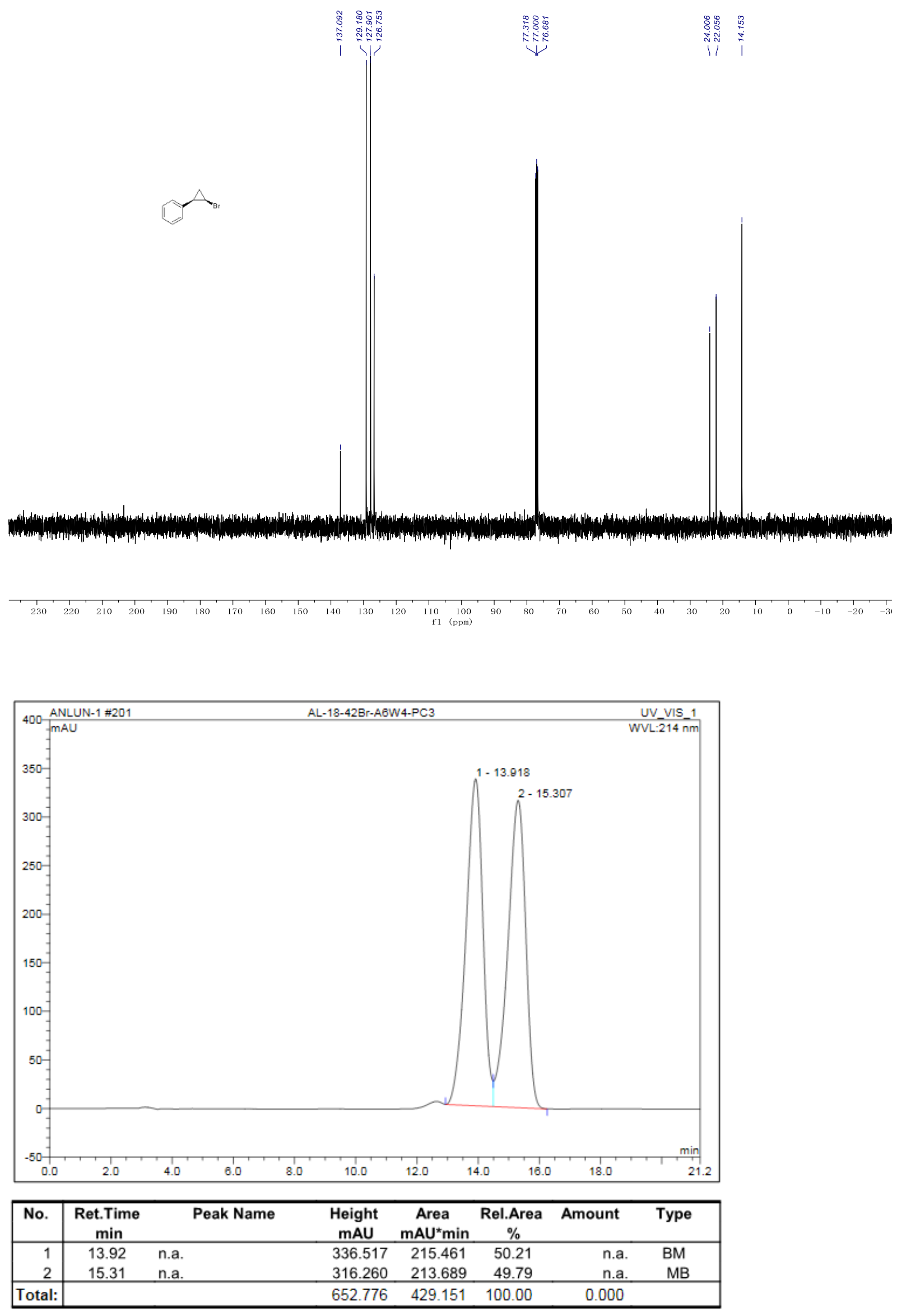




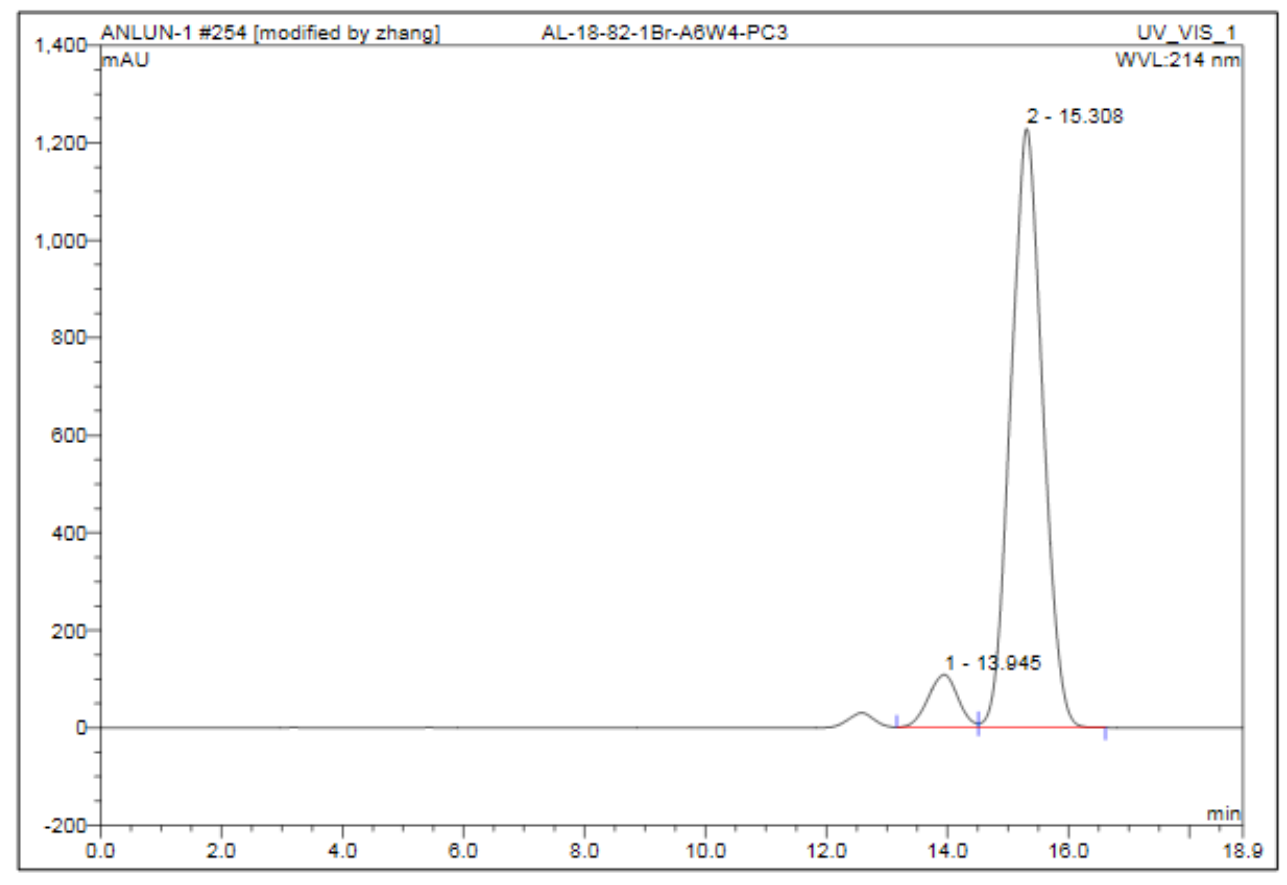

\begin{tabular}{|r|ccrrrrr|}
\hline No. & $\begin{array}{c}\text { Ret.Time } \\
\text { min }\end{array}$ & Peak Name & $\begin{array}{c}\text { Height } \\
\text { mAU }\end{array}$ & $\begin{array}{c}\text { Area } \\
\text { mAU*min }\end{array}$ & $\begin{array}{r}\text { Rel.Area } \\
\%\end{array}$ & Amount & Type \\
\hline 1 & 13.95 & n.a. & 107.935 & 61.997 & 7.64 & n.a. & BM * $^{*}$ \\
2 & 15.31 & n.a. & 1228.074 & 749.745 & 92.36 & n.a. & MB $^{*}$ \\
\hline Total: & & & 1336.009 & 811.742 & 100.00 & 0.000 & \\
\hline
\end{tabular}

\section{4-((1R,2R)-2-Chlorocyclopropyl)-1,1'-biphenyl (5i).}

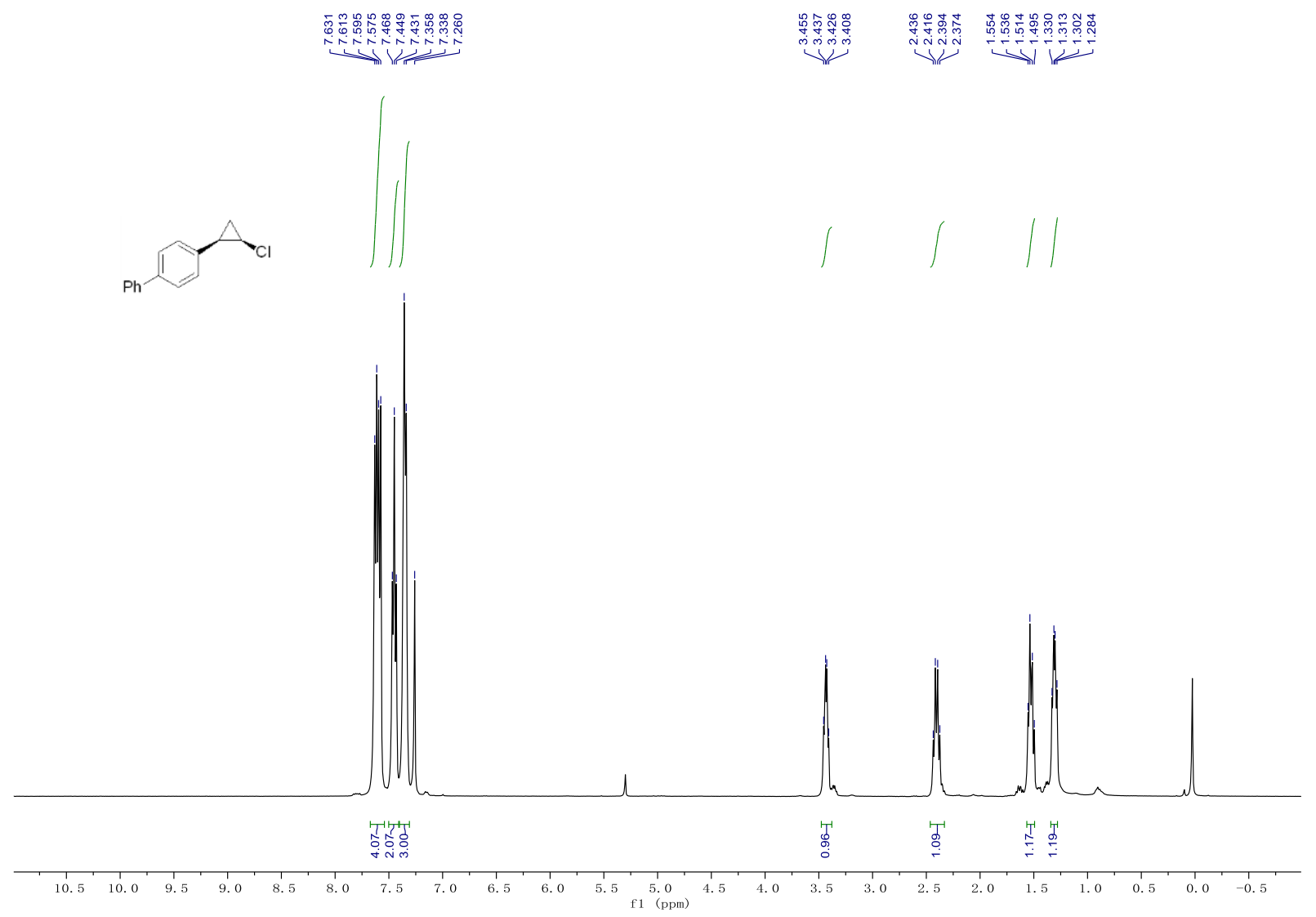



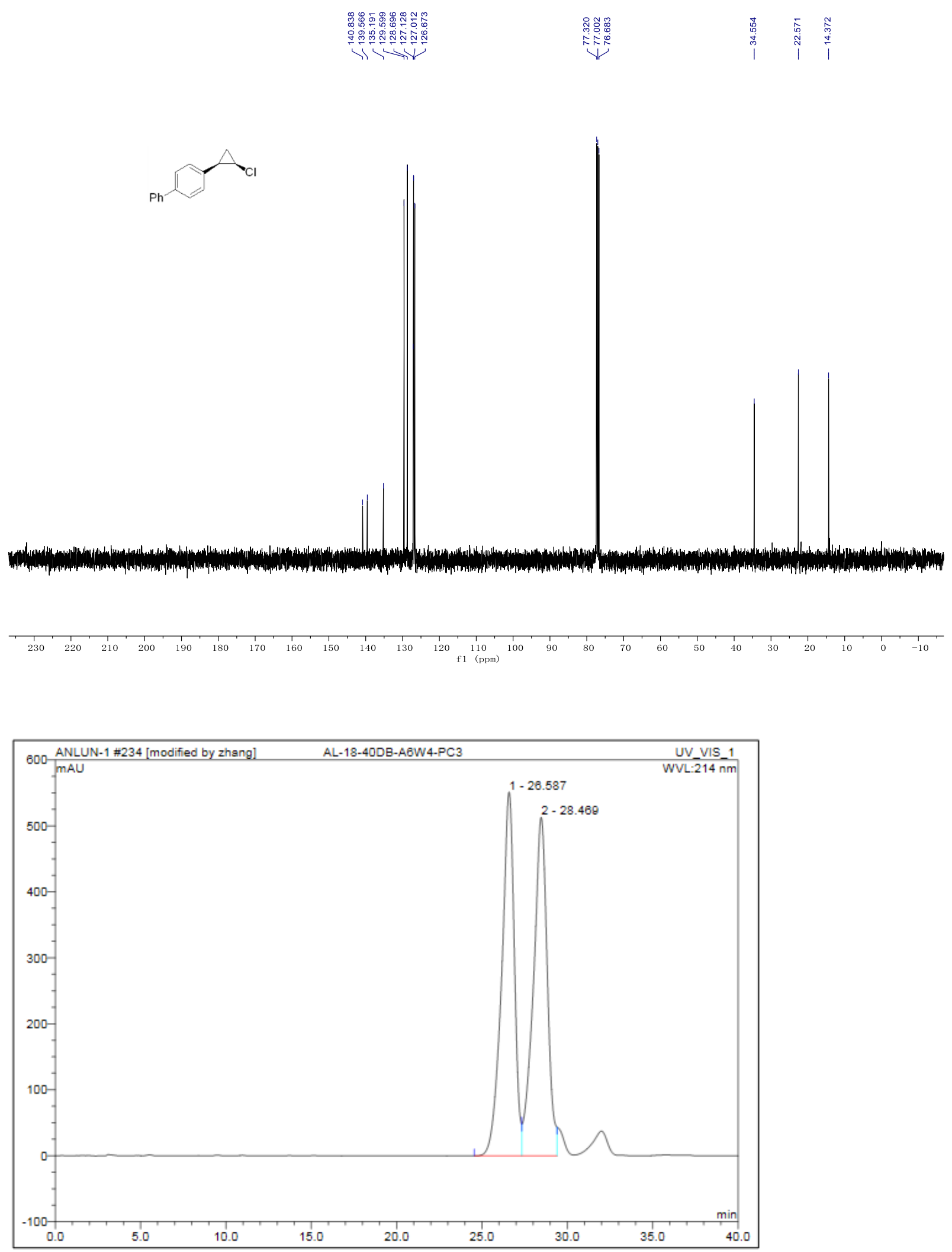

\begin{tabular}{|r|ccccccc|}
\hline No. & $\begin{array}{c}\text { Ret.Time } \\
\text { min }\end{array}$ & Peak Name & $\begin{array}{c}\text { Height } \\
\text { mAU }\end{array}$ & $\begin{array}{c}\text { Area } \\
\text { mAU*min }\end{array}$ & $\begin{array}{c}\text { Rel.Area } \\
\%\end{array}$ & Amount & Type \\
\hline 1 & 26.59 & n.a. & 551.727 & 476.006 & 49.50 & n.a. & BM * $^{*}$ \\
2 & 28.47 & n.a. & 513.245 & 485.677 & 50.50 & n.a. & $M^{*}$ \\
\hline Total: & & & 1064.972 & 961.683 & 100.00 & 0.000 & \\
\hline
\end{tabular}




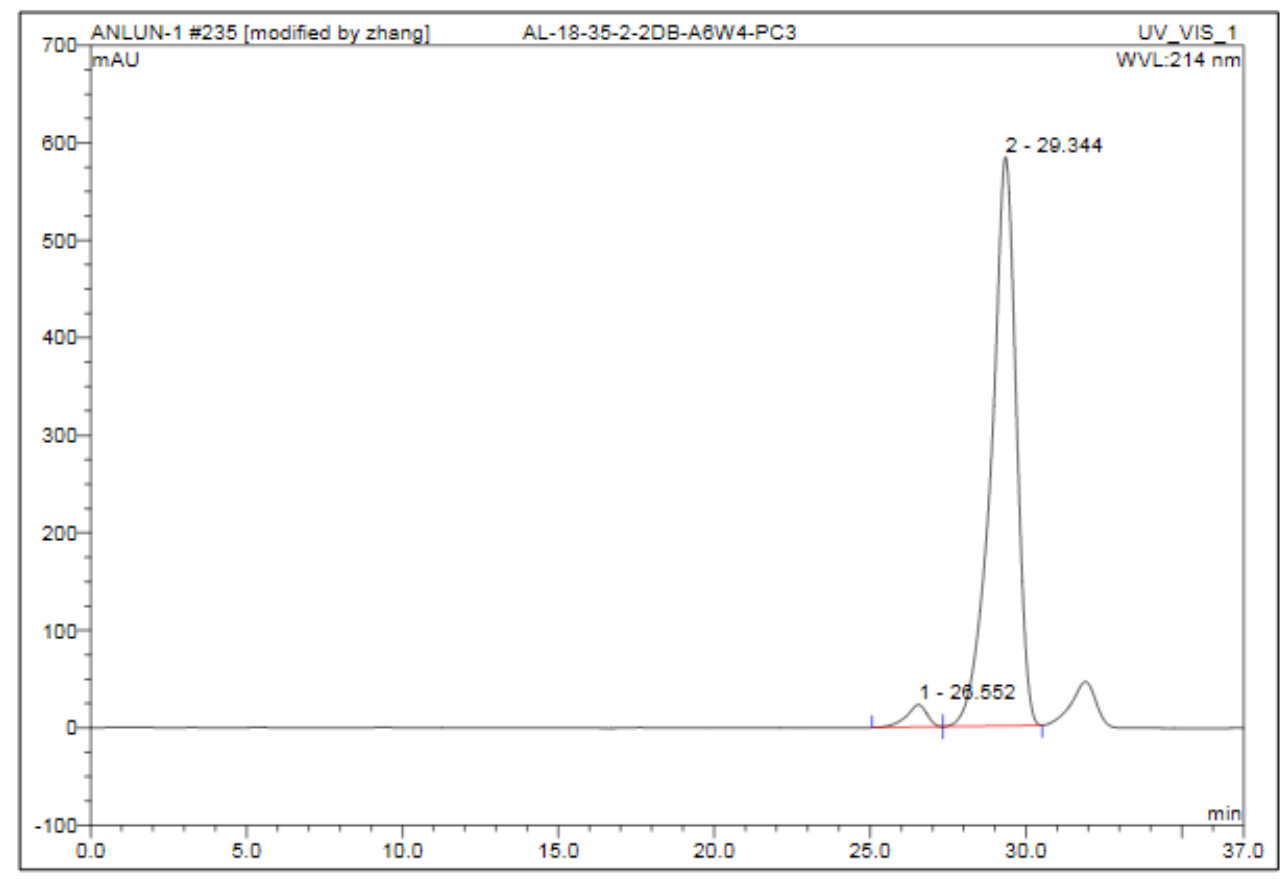

\begin{tabular}{|r|ccrrrrr|}
\hline No. & $\begin{array}{c}\text { Ret.Time } \\
\text { min }\end{array}$ & Peak Name & $\begin{array}{c}\text { Height } \\
\text { mAU }\end{array}$ & $\begin{array}{c}\text { Area } \\
\text { mAU*min }\end{array}$ & $\begin{array}{r}\text { Rel.Area } \\
\%\end{array}$ & Amount & Type \\
\hline 1 & 26.55 & n.a. & 22.850 & 17.764 & 3.19 & n.a. & BM $^{*}$ \\
2 & 29.34 & n.a. & 583.156 & 539.731 & 96.81 & n.a. & MB $^{*}$ \\
\hline Total: & & & 606.006 & 557.495 & 100.00 & 0.000 & \\
\hline
\end{tabular}

((1R,2R)-2-Iodocyclopropyl)benzene (5j).

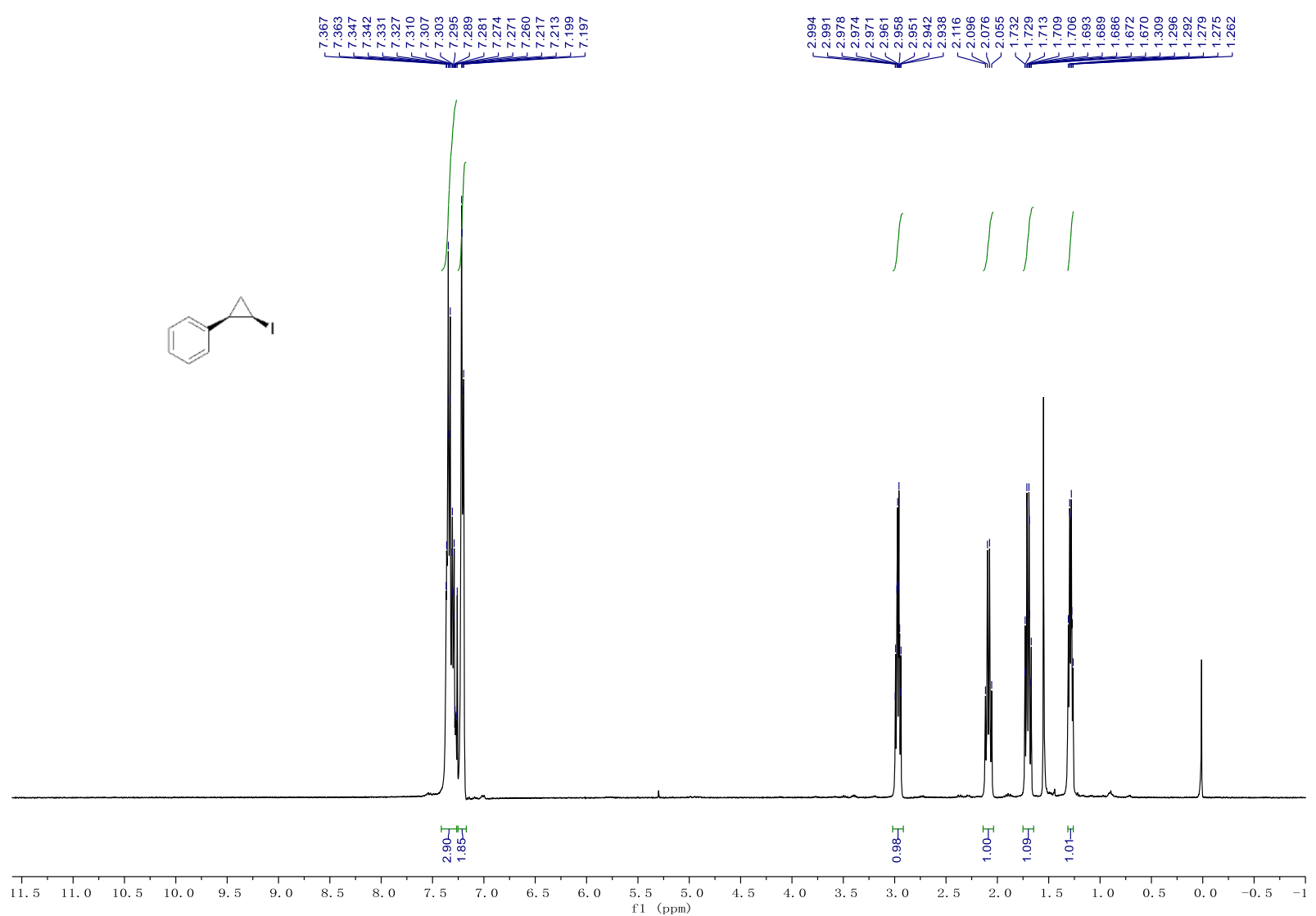



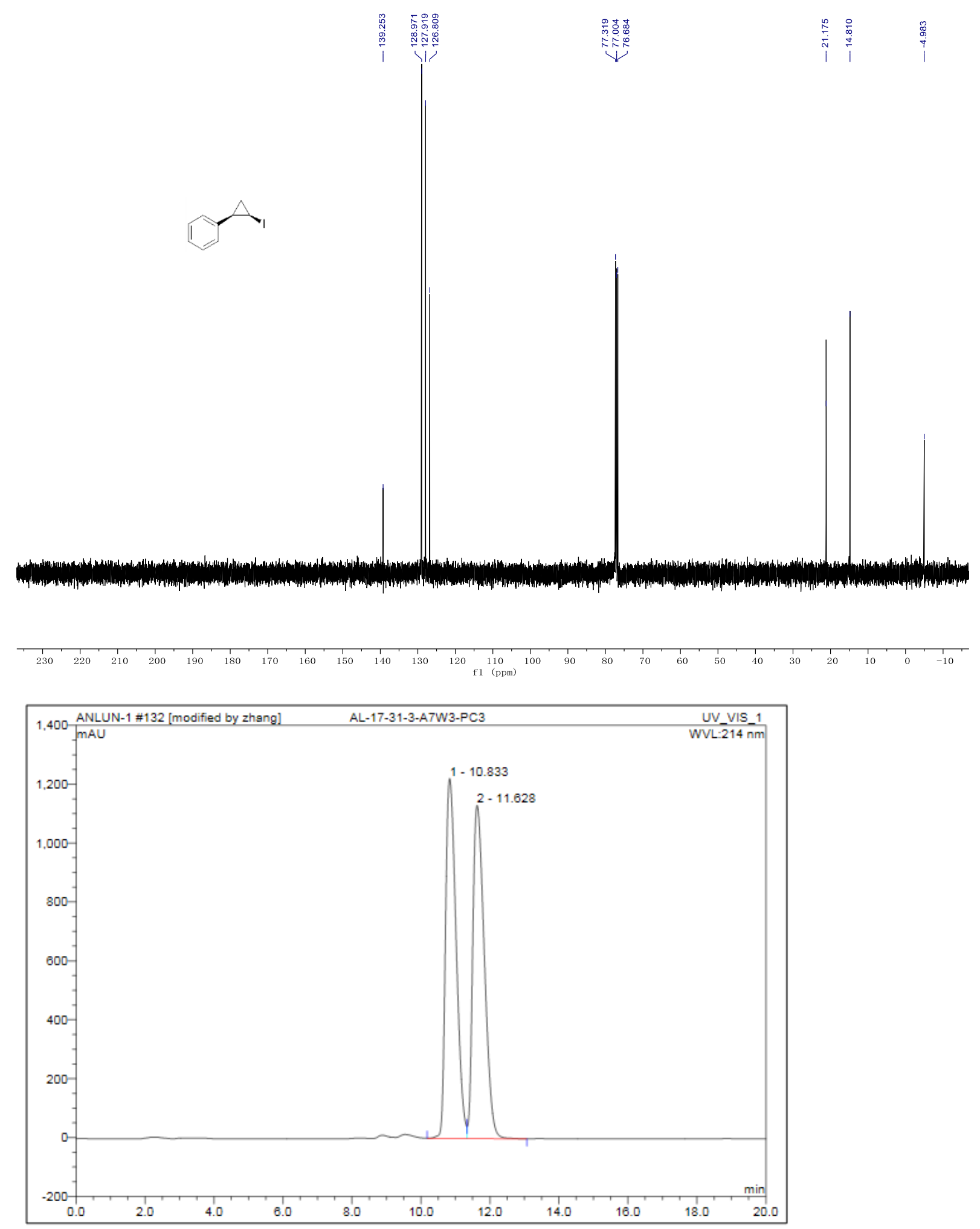

\begin{tabular}{|r|cccrrrr|}
\hline No. & $\begin{array}{c}\text { Ret.Time } \\
\text { min }\end{array}$ & Peak Name & $\begin{array}{c}\text { Height } \\
\text { mAU }\end{array}$ & $\begin{array}{c}\text { Area } \\
\text { mAU*min }\end{array}$ & $\begin{array}{c}\text { Rel.Area } \\
\%\end{array}$ & Amount & Type \\
\hline 1 & 10.83 & n.a. & 1221.941 & 435.979 & 49.81 & n.a. & BM \\
2 & 11.63 & n.a. & 1131.269 & 439.286 & 50.19 & n.a. & MB \\
\hline Total: & & & 2353.210 & 875.265 & 100.00 & 0.000 & \\
\hline
\end{tabular}




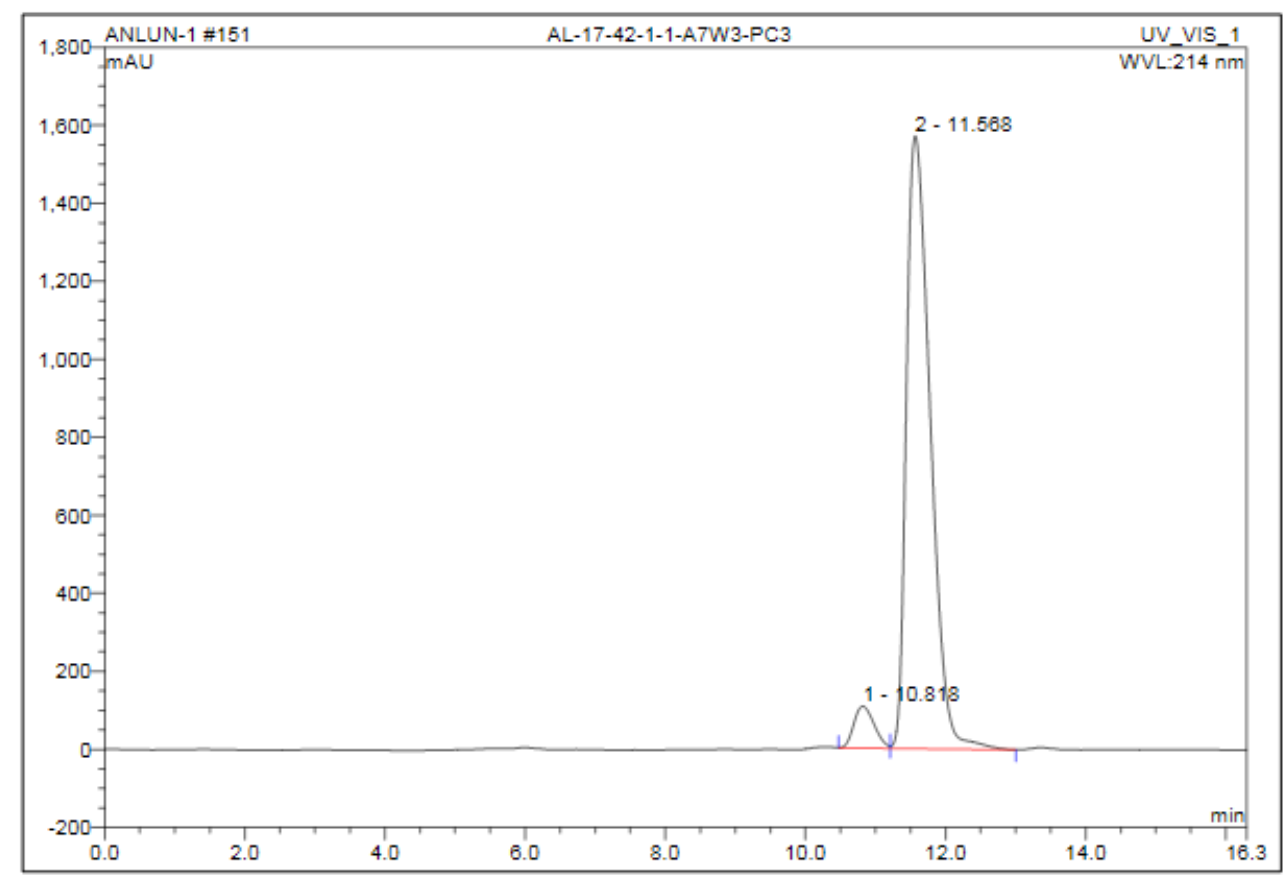

\begin{tabular}{|r|ccrrrrr|}
\hline No. & $\begin{array}{c}\text { Ret.Time } \\
\text { min }\end{array}$ & Peak Name & $\begin{array}{c}\text { Height } \\
\text { mAU }\end{array}$ & $\begin{array}{c}\text { Area } \\
\text { mAU*min }\end{array}$ & $\begin{array}{r}\text { Rel.Area } \\
\%\end{array}$ & Amount & Type \\
\hline 1 & 10.82 & n.a. & 107.205 & 35.893 & 5.48 & n.a. & BM \\
2 & 11.57 & n.a. & 1571.131 & 619.649 & 94.52 & n.a. & MB \\
\hline Total: & & & 1678.336 & 655.542 & 100.00 & 0.000 & \\
\hline
\end{tabular}

Phenyl((1R,2R)-2-phenylcyclopropyl)selane (5k).

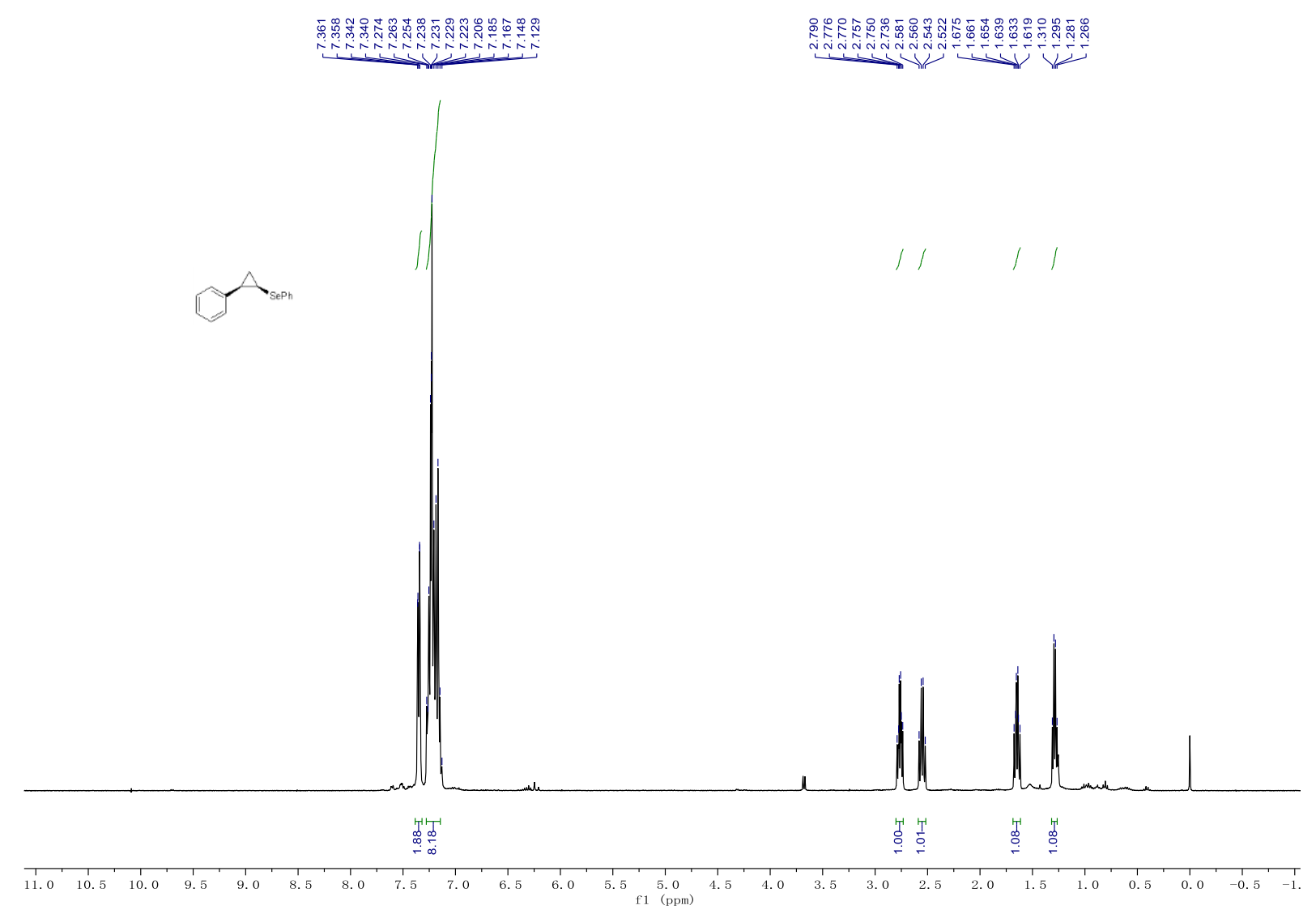




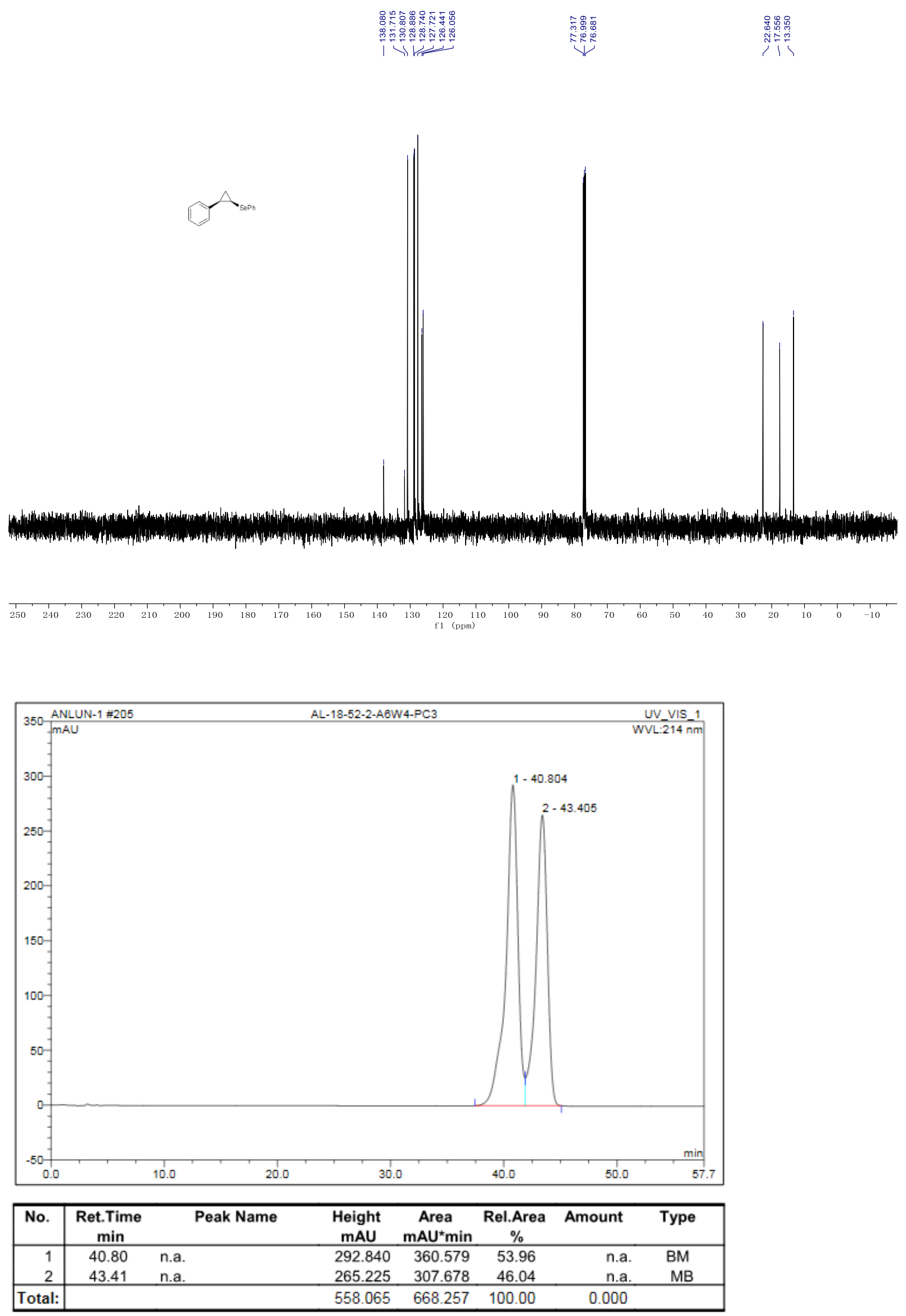




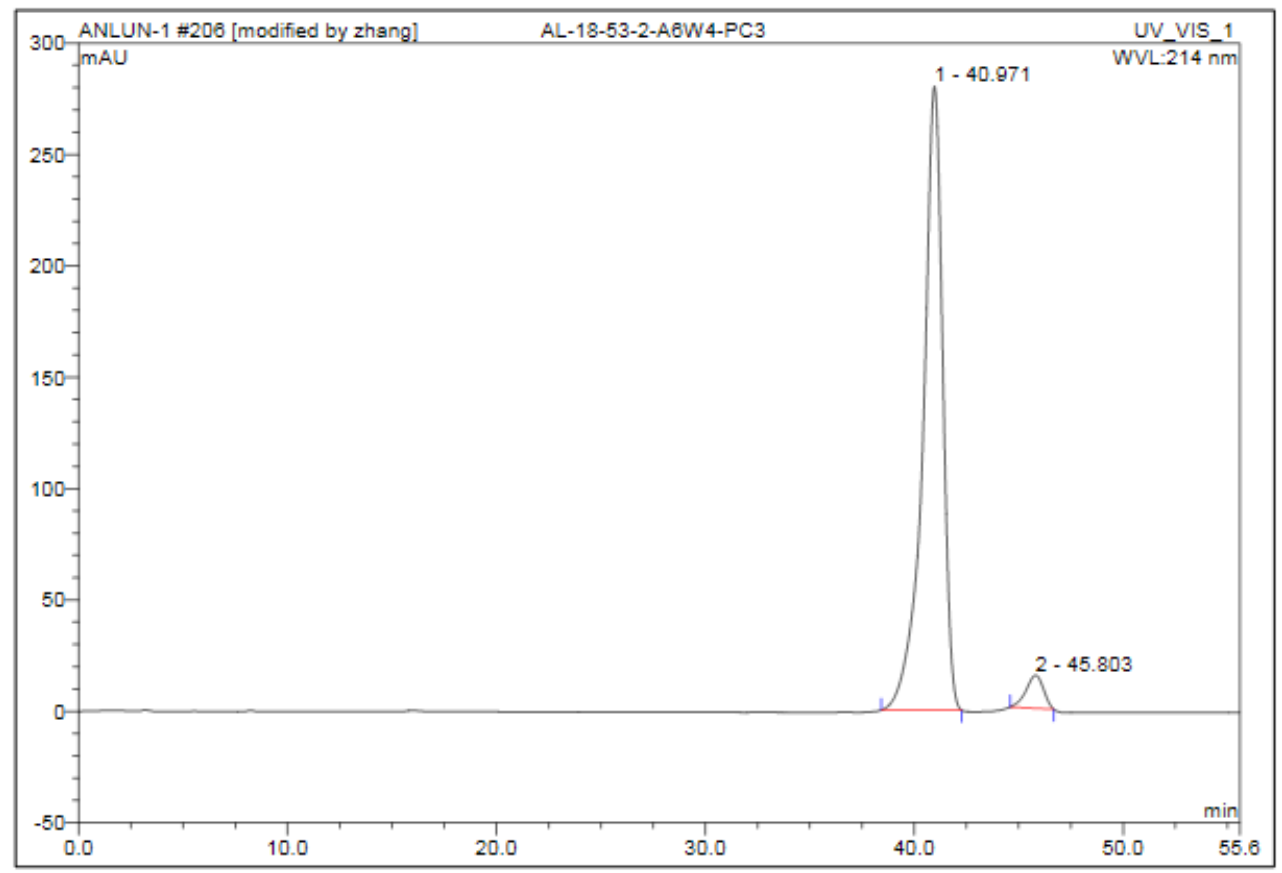

\begin{tabular}{|r|ccrrrrr|}
\hline No. & $\begin{array}{c}\text { Ret.Time } \\
\text { min }\end{array}$ & Peak Name & $\begin{array}{c}\text { Height } \\
\text { mAU }\end{array}$ & $\begin{array}{c}\text { Area } \\
\text { mAU*min }\end{array}$ & $\begin{array}{r}\text { Rel.Area } \\
\%\end{array}$ & Amount & Type \\
\hline 1 & 40.97 & n.a. & 280.103 & 303.578 & 95.49 & n.a. & BMB $^{\star}$ \\
2 & 45.80 & n.a. & 14.828 & 14.337 & 4.51 & n.a. & BMB $^{\star}$ \\
\hline Total: & & & 294.931 & 317.915 & 100.00 & 0.000 & \\
\hline
\end{tabular}

((1R,2R)-2-([1,1'-Biphenyl]-4-yl)cyclopropyl)tributylstannane (5l).

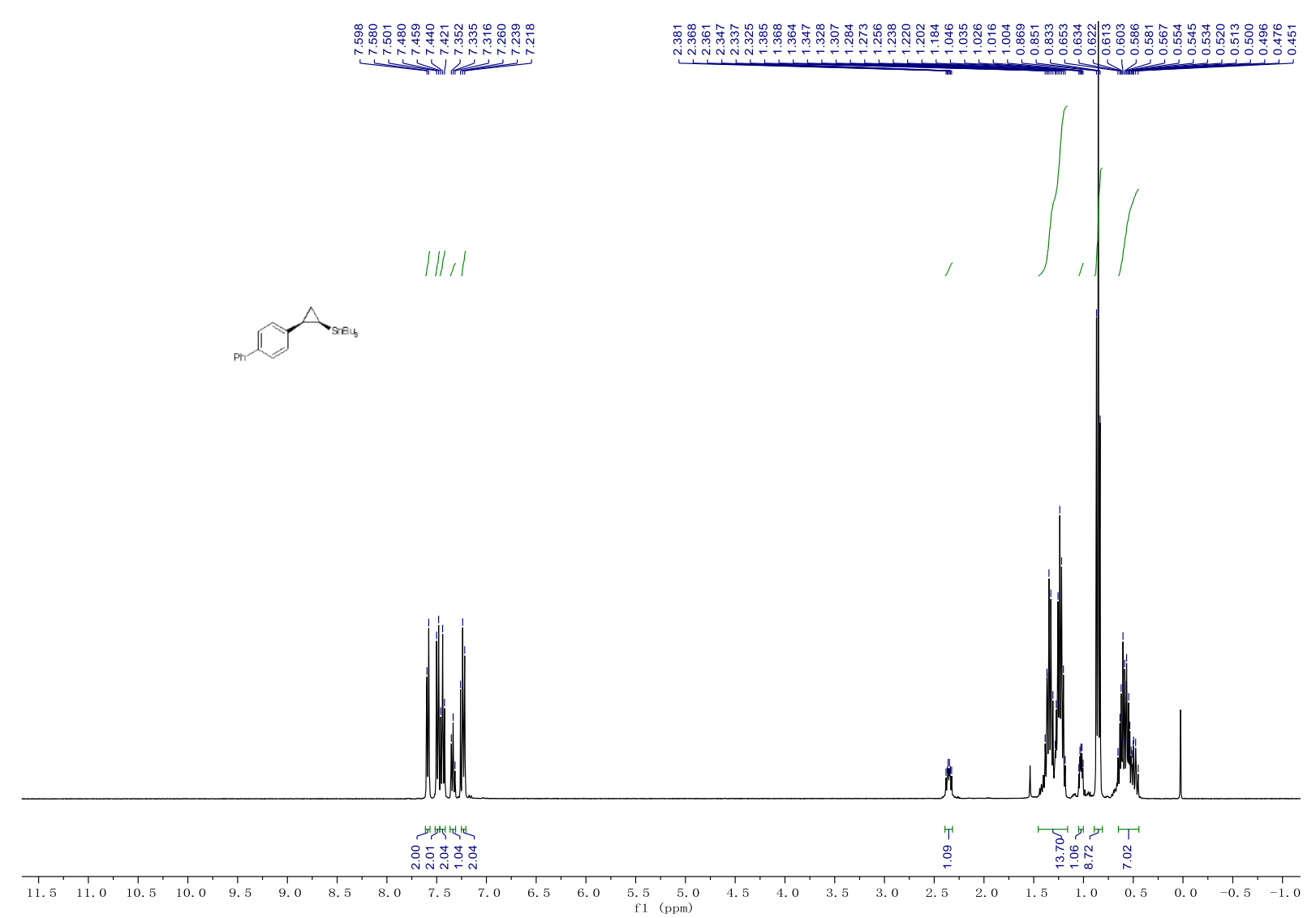




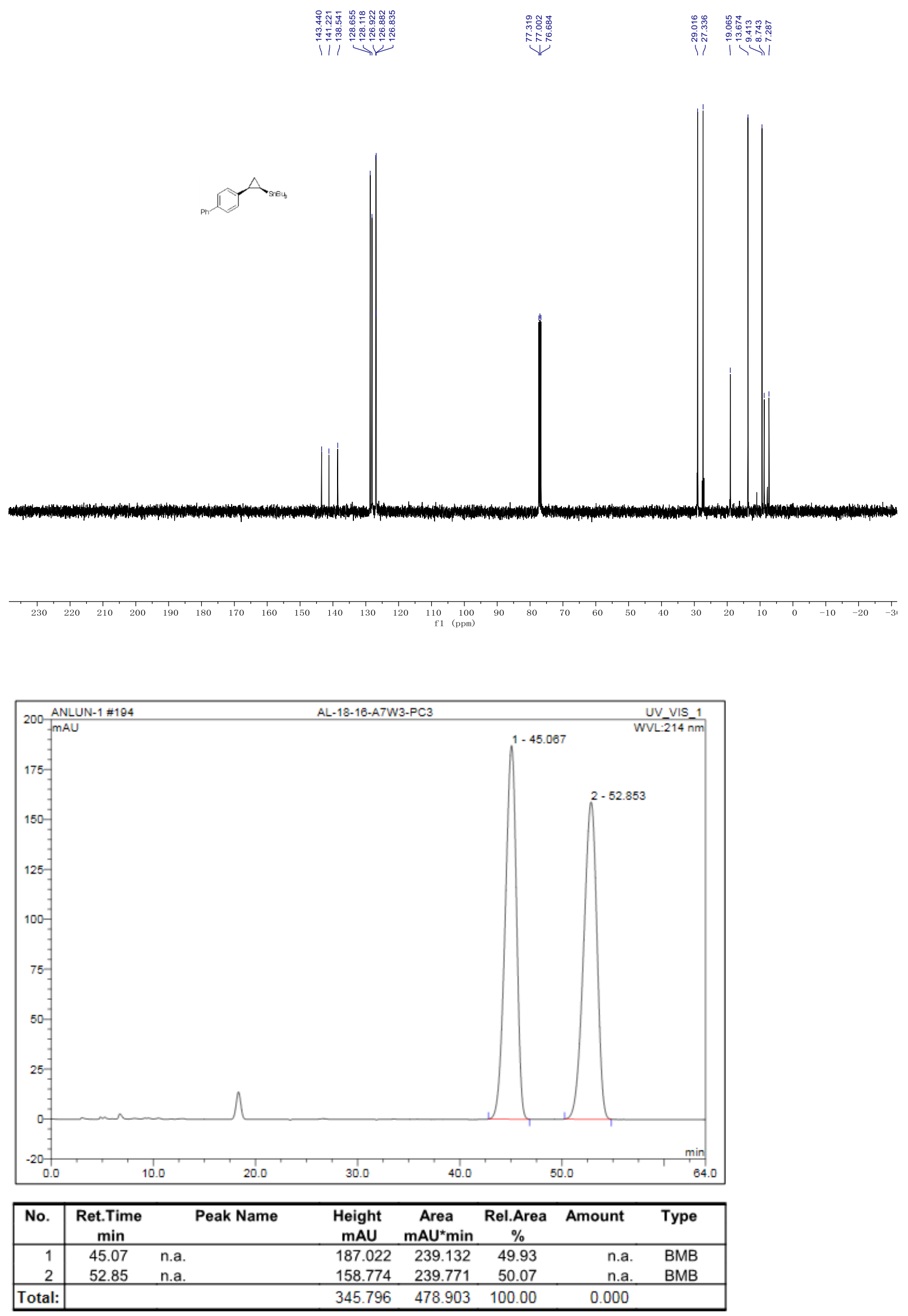




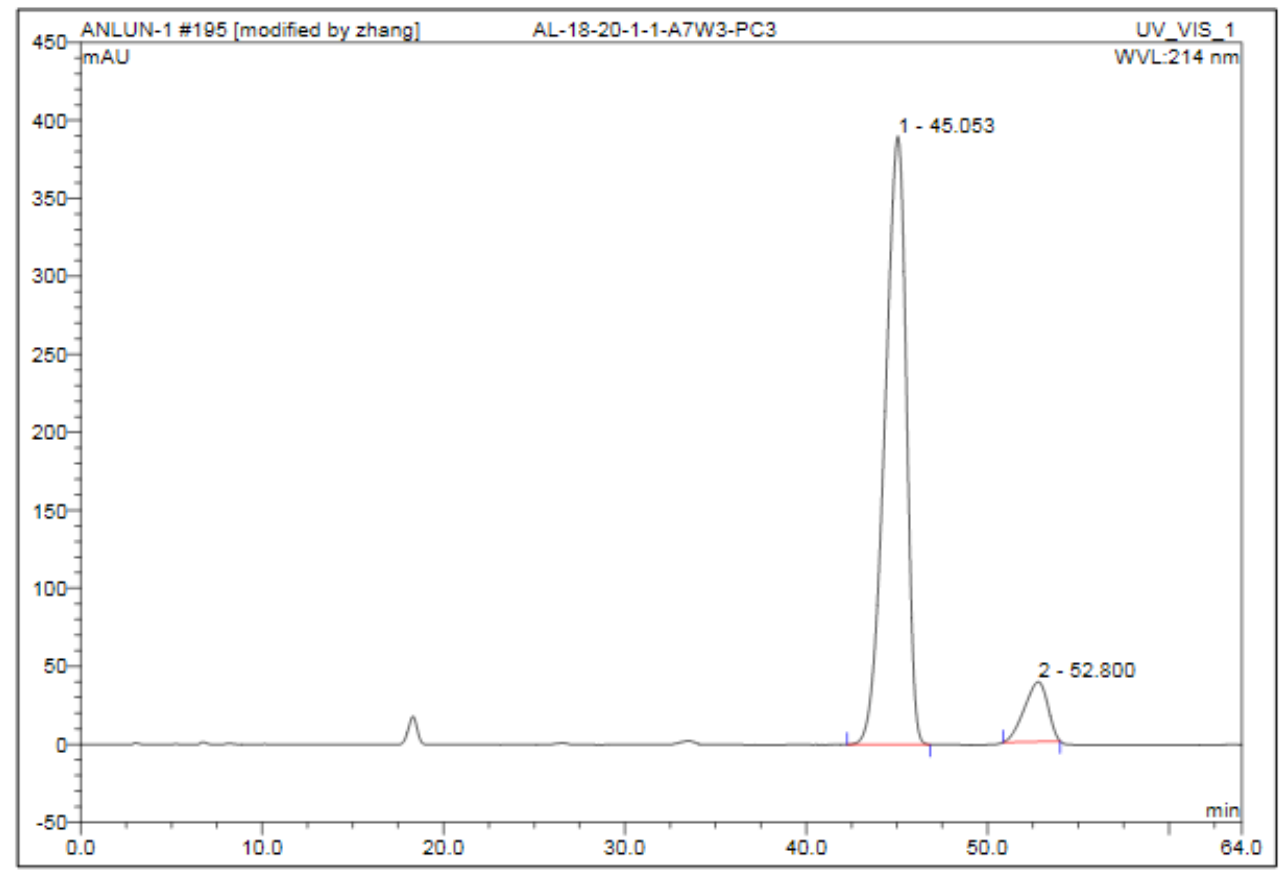

\begin{tabular}{|r|ccrrrrr|}
\hline No. & $\begin{array}{c}\text { Ret.Time } \\
\text { min }\end{array}$ & Peak Name & $\begin{array}{c}\text { Height } \\
\text { mAU }\end{array}$ & $\begin{array}{c}\text { Area } \\
\text { mAU*min }\end{array}$ & $\begin{array}{r}\text { Rel.Area } \\
\%\end{array}$ & Amount & Type \\
\hline 1 & 45.05 & n.a. & 389.910 & 533.187 & 90.30 & n.a. & BMB \\
2 & 52.80 & n.a. & 38.260 & 57.262 & 9.70 & n.a. & BMB $^{\star}$ \\
\hline Total: & & & 428.170 & 590.449 & 100.00 & 0.000 & \\
\hline
\end{tabular}

EDRC Reaction with Secondary Propargyl Bromides

\section{$((1 R, 2 S)$-2-Allylcyclopropyl)benzene (9a).}

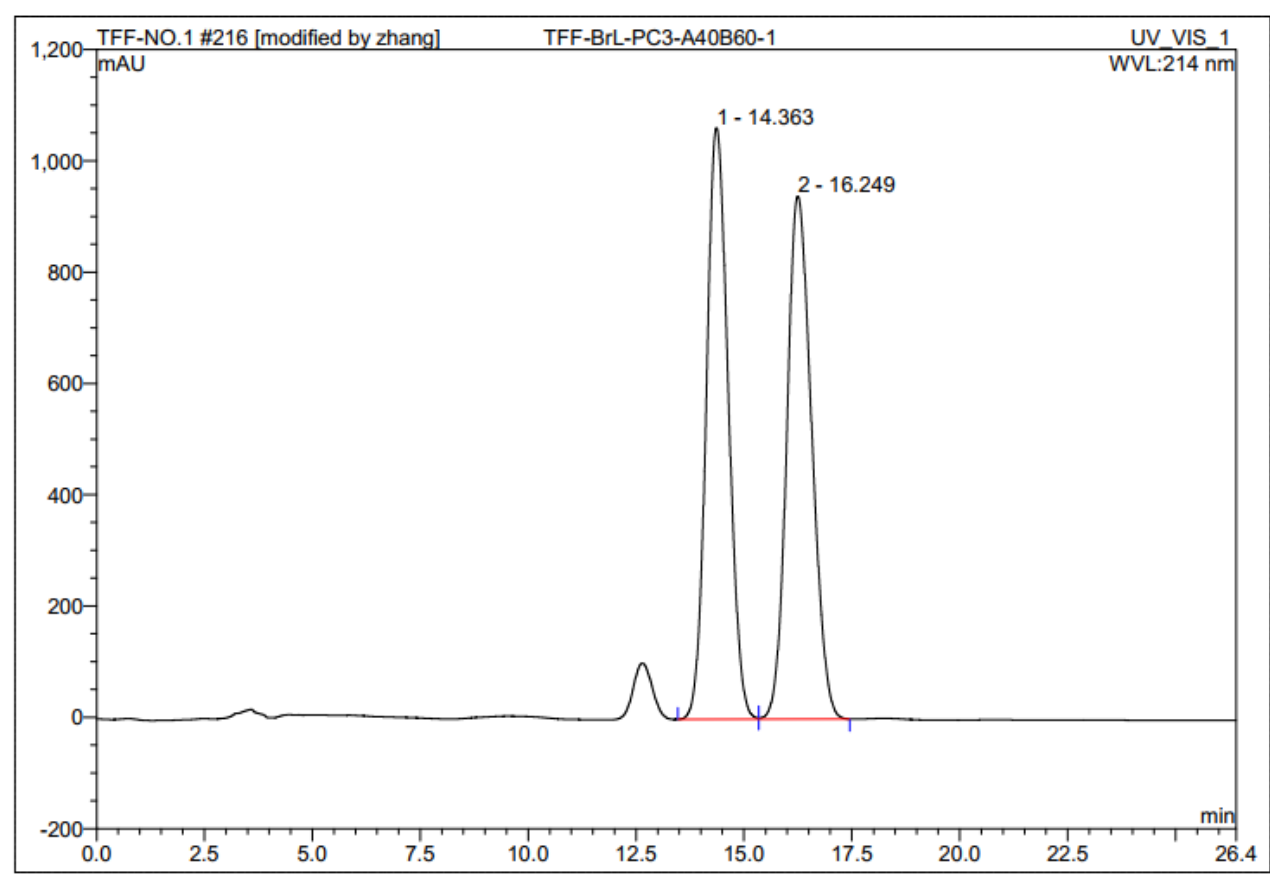

\begin{tabular}{|r|ccrrrrrr|}
\hline No. & $\begin{array}{c}\text { Ret.Time } \\
\text { min }\end{array}$ & Peak Name & $\begin{array}{c}\text { Height } \\
\text { mAU }\end{array}$ & $\begin{array}{c}\text { Area } \\
\text { mAU*min }\end{array}$ & $\begin{array}{c}\text { Rel.Area } \\
\%\end{array}$ & $\begin{array}{c}\text { Amount } \\
\%\end{array}$ & Type \\
\hline 1 & 14.36 & n.a. & 1062.840 & 625.614 & 49.93 & n.a. & BM \\
2 & 16.25 & n.a. & 939.713 & 627.408 & 50.07 & n.a. & MB \\
\hline Total: & & & 2002.552 & 1253.022 & 100.00 & 0.000 \\
\hline
\end{tabular}




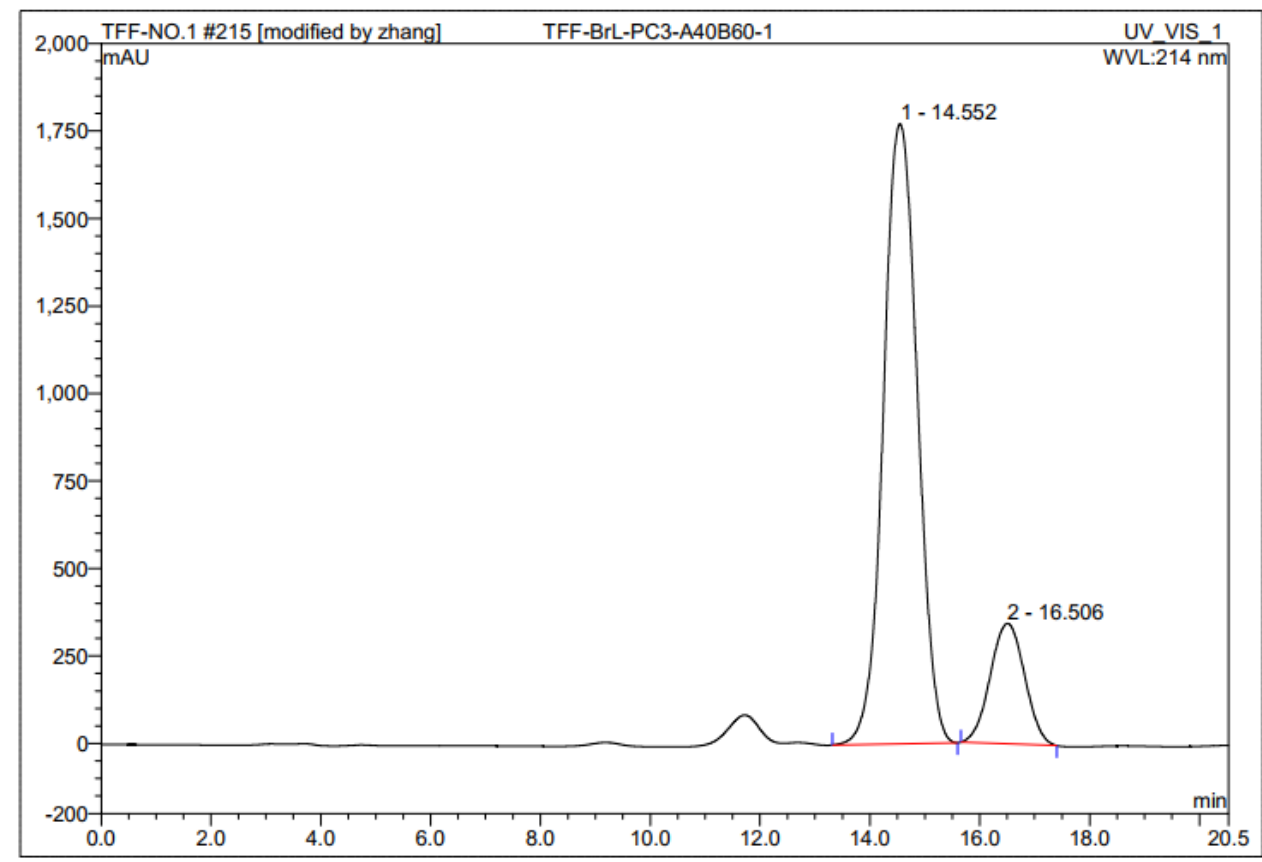

\begin{tabular}{|r|ccrrrrr|}
\hline No. & $\begin{array}{c}\text { Ret.Time } \\
\text { min }\end{array}$ & Peak Name & $\begin{array}{c}\text { Height } \\
\text { mAU }\end{array}$ & $\begin{array}{r}\text { Area } \\
\text { mAU*min }\end{array}$ & $\begin{array}{r}\text { Rel.Area } \\
\%\end{array}$ & $\begin{array}{c}\text { Amount } \\
\%\end{array}$ & Type \\
\hline 1 & 14.55 & n.a. & 1771.687 & 1267.782 & 83.58 & n.a. & BMB $^{*}$ \\
2 & 16.51 & n.a. & 343.365 & 249.144 & 16.42 & n.a. & BMB $^{*}$ \\
\hline Total: & & & 2115.052 & 1516.926 & 100.00 & 0.000 & \\
\hline
\end{tabular}

((1S,2S)-2-Bromocyclopropyl)benzene (5m).

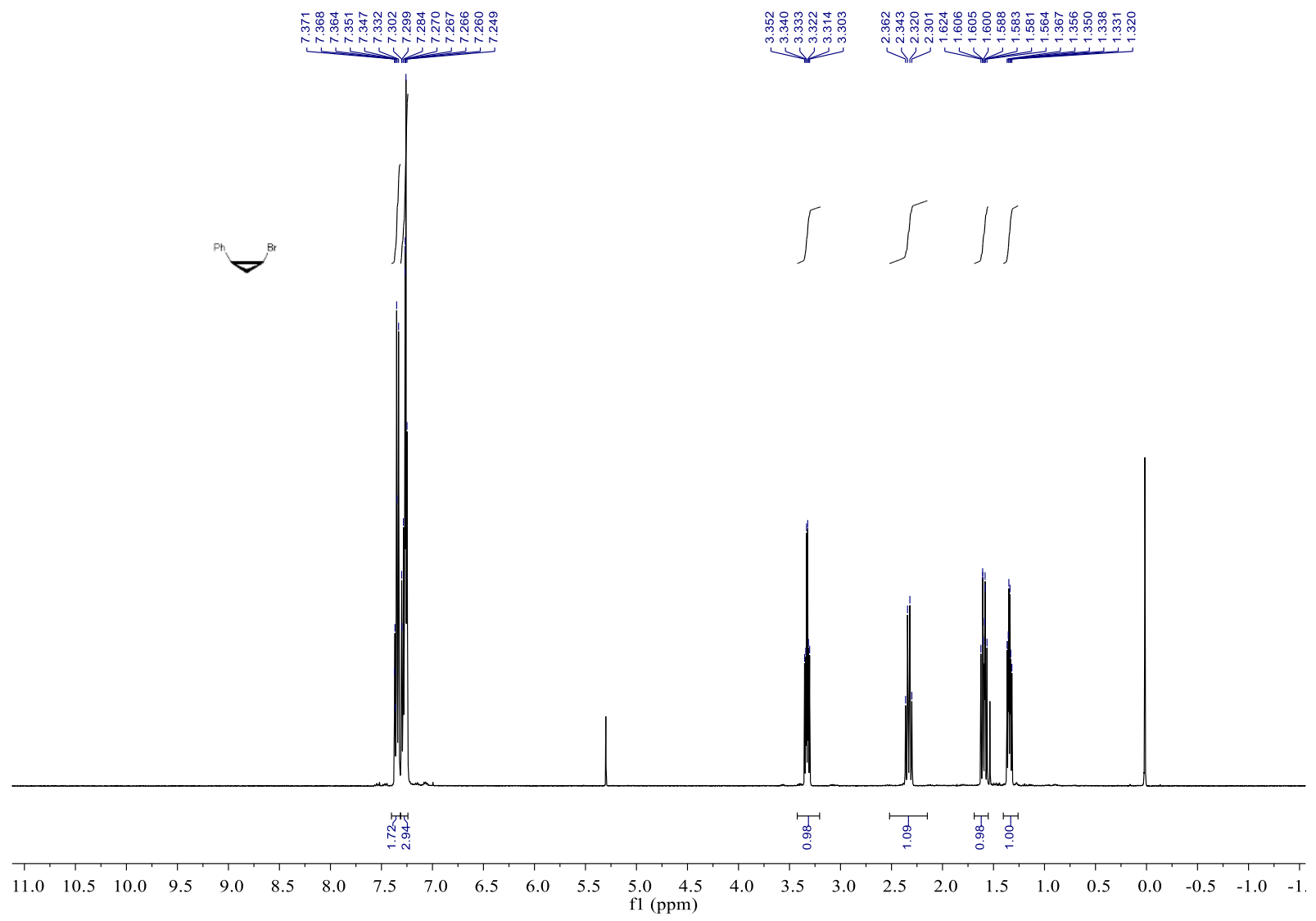




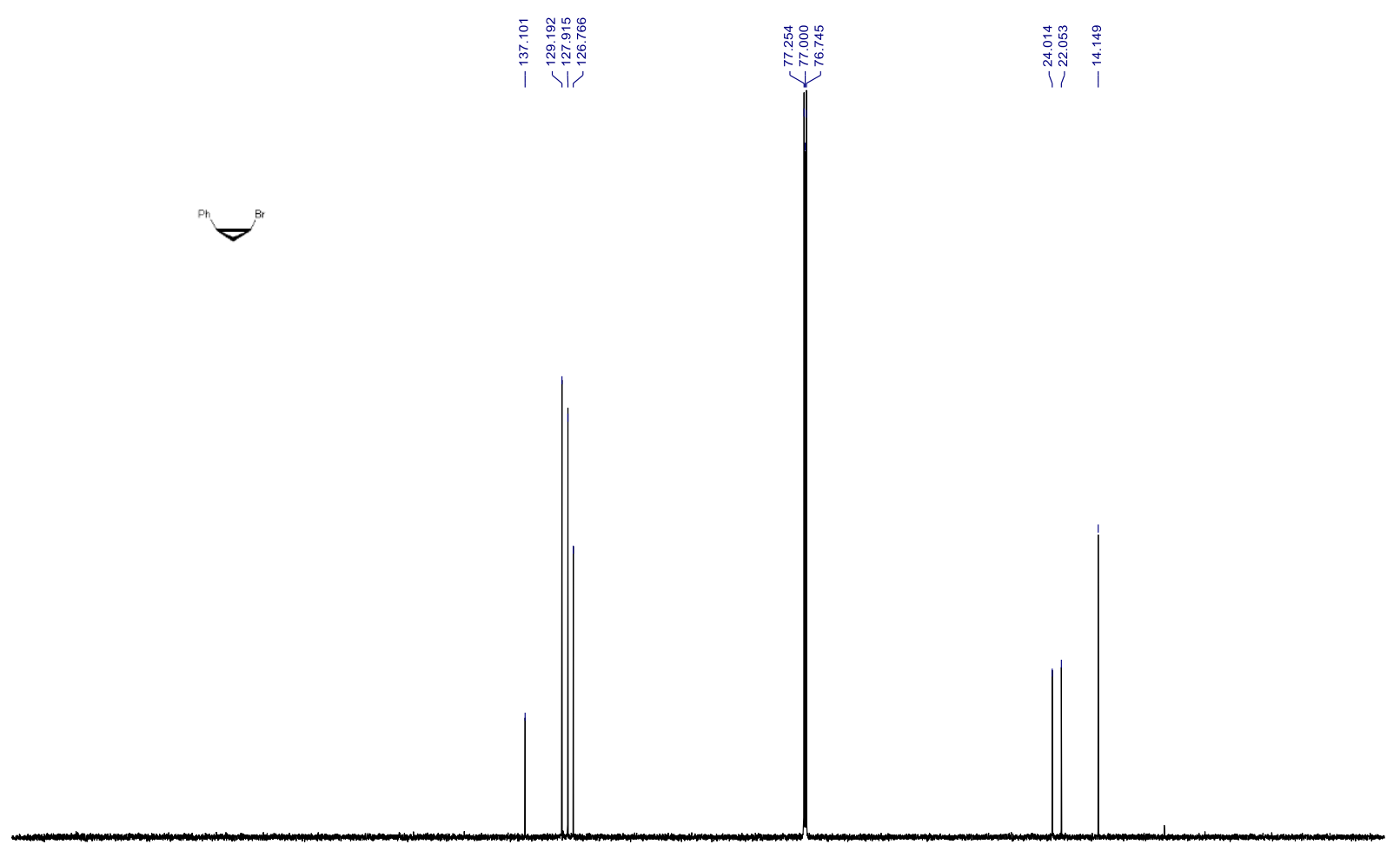

$\begin{array}{llllllllllllllllllllllllllllllll}240 & 230 & 220 & 210 & 200 & 190 & 180 & 170 & 160 & 150 & 140 & 130 & 120 & 110 & 100 & 90 & 80 & 70 & 60 & 50 & 40 & 30 & 20 & 10 & 0 & -10 & -20 & -30 & -40\end{array}$

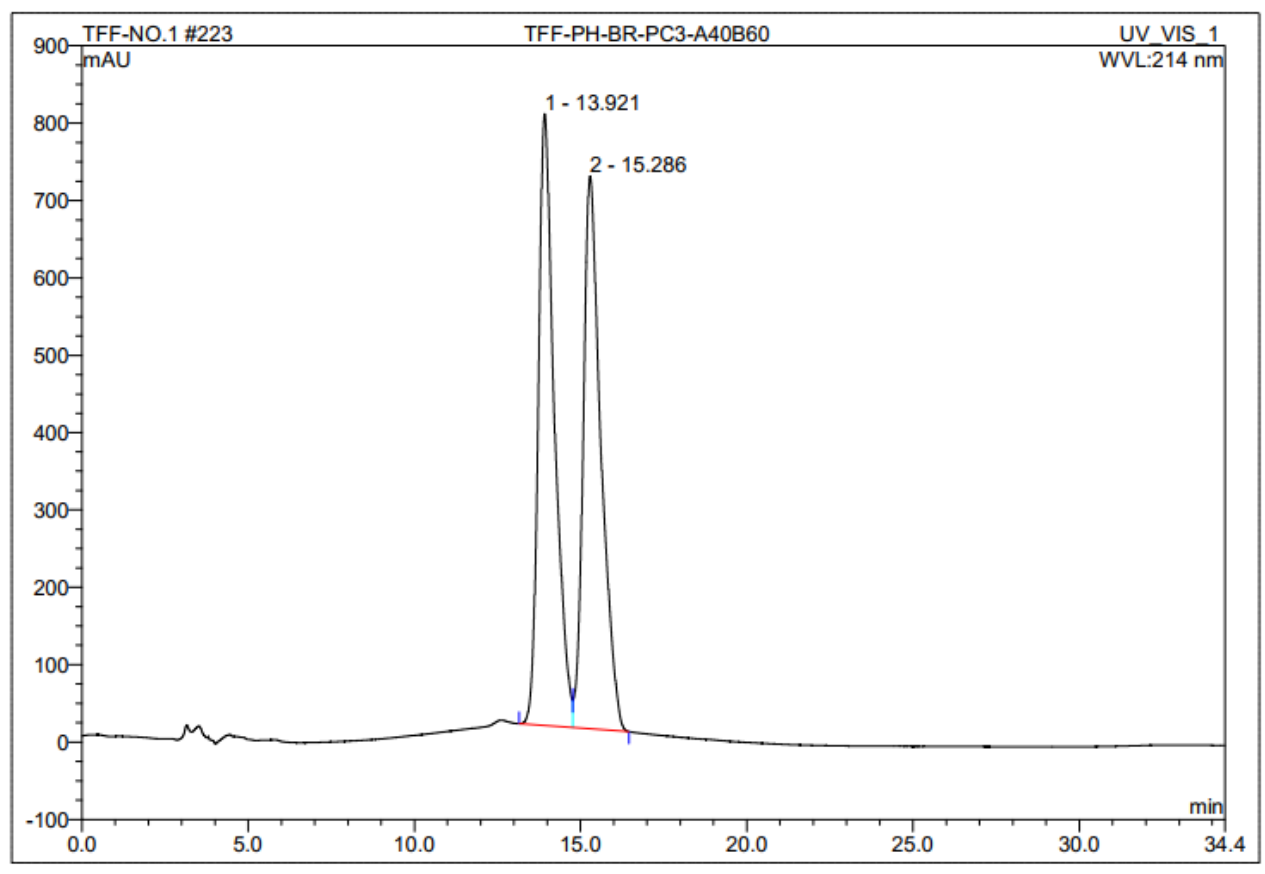

\begin{tabular}{|r|ccccccc|}
\hline No. & $\begin{array}{c}\text { Ret.Time } \\
\text { min }\end{array}$ & Peak Name & $\begin{array}{c}\text { Height } \\
\text { mAU }\end{array}$ & $\begin{array}{c}\text { Area } \\
\text { mAU*min }\end{array}$ & $\begin{array}{c}\text { Rel.Area } \\
\%\end{array}$ & $\begin{array}{c}\text { Amount } \\
\%\end{array}$ & Type \\
\hline 1 & 13.92 & n.a. & 790.847 & 445.265 & 50.21 & n.a. & BM \\
2 & 15.29 & n.a. & 714.514 & 441.592 & 49.79 & n.a. & MB \\
\hline Total: & & & 1505.361 & 886.857 & 100.00 & 0.000 & \\
\hline
\end{tabular}




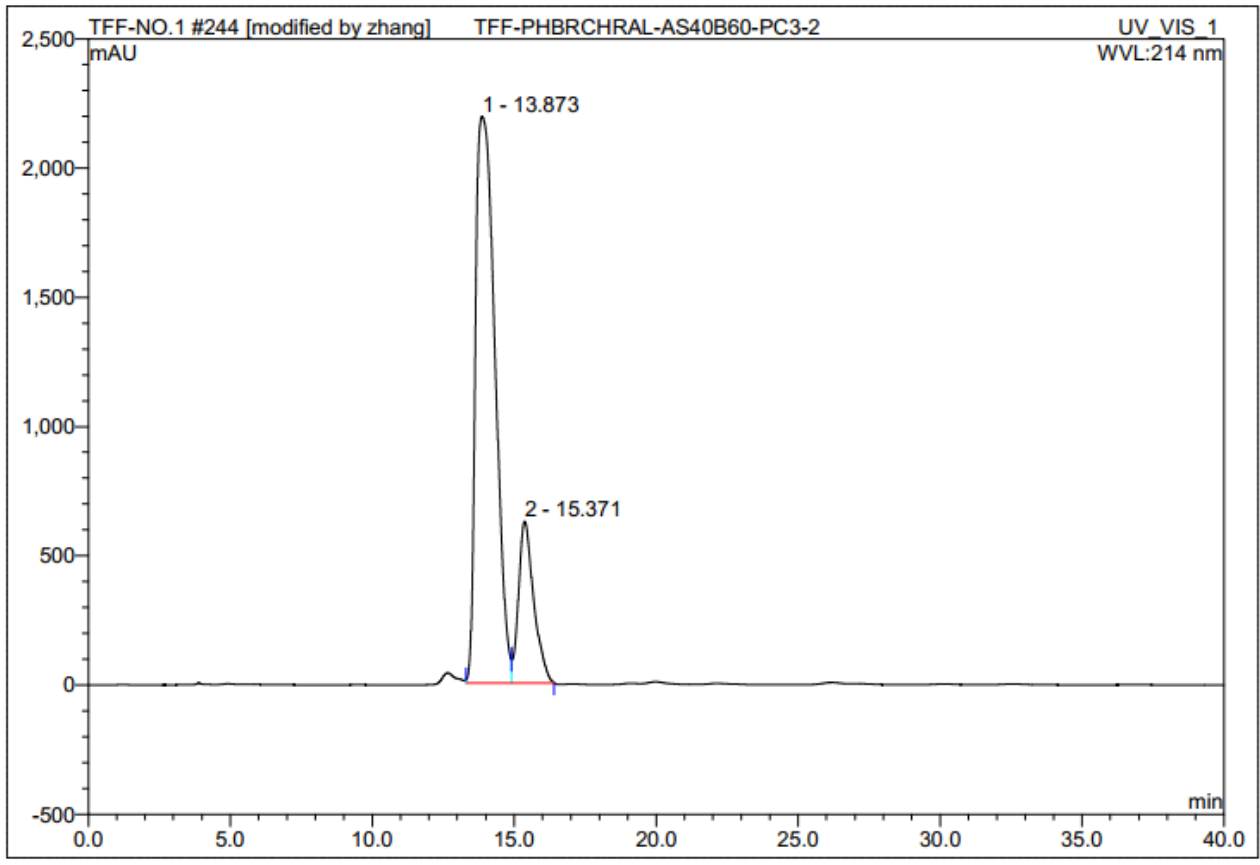

\begin{tabular}{|r|ccrrrrr|}
\hline No. & $\begin{array}{c}\text { Ret.Time } \\
\text { min }\end{array}$ & Peak Name & $\begin{array}{c}\text { Height } \\
\text { mAU }\end{array}$ & $\begin{array}{c}\text { Area } \\
\mathrm{mAU}^{*} \min \end{array}$ & $\begin{array}{c}\text { Rel.Area } \\
\%\end{array}$ & $\begin{array}{c}\text { Amount } \\
\%\end{array}$ & Type \\
\hline 1 & 13.87 & n.a. & 2191.531 & 1737.300 & 81.62 & n.a. & $\mathrm{M}^{*}$ \\
2 & 15.37 & n.a. & 625.089 & 391.336 & 18.38 & n.a. & MB $^{*}$ \\
\hline Total: & & & 2816.620 & 2128.636 & 100.00 & 0.000 & \\
\hline
\end{tabular}

(((1S,2R)-2-Phenylcyclopropyl)ethynyl)benzene (5n).

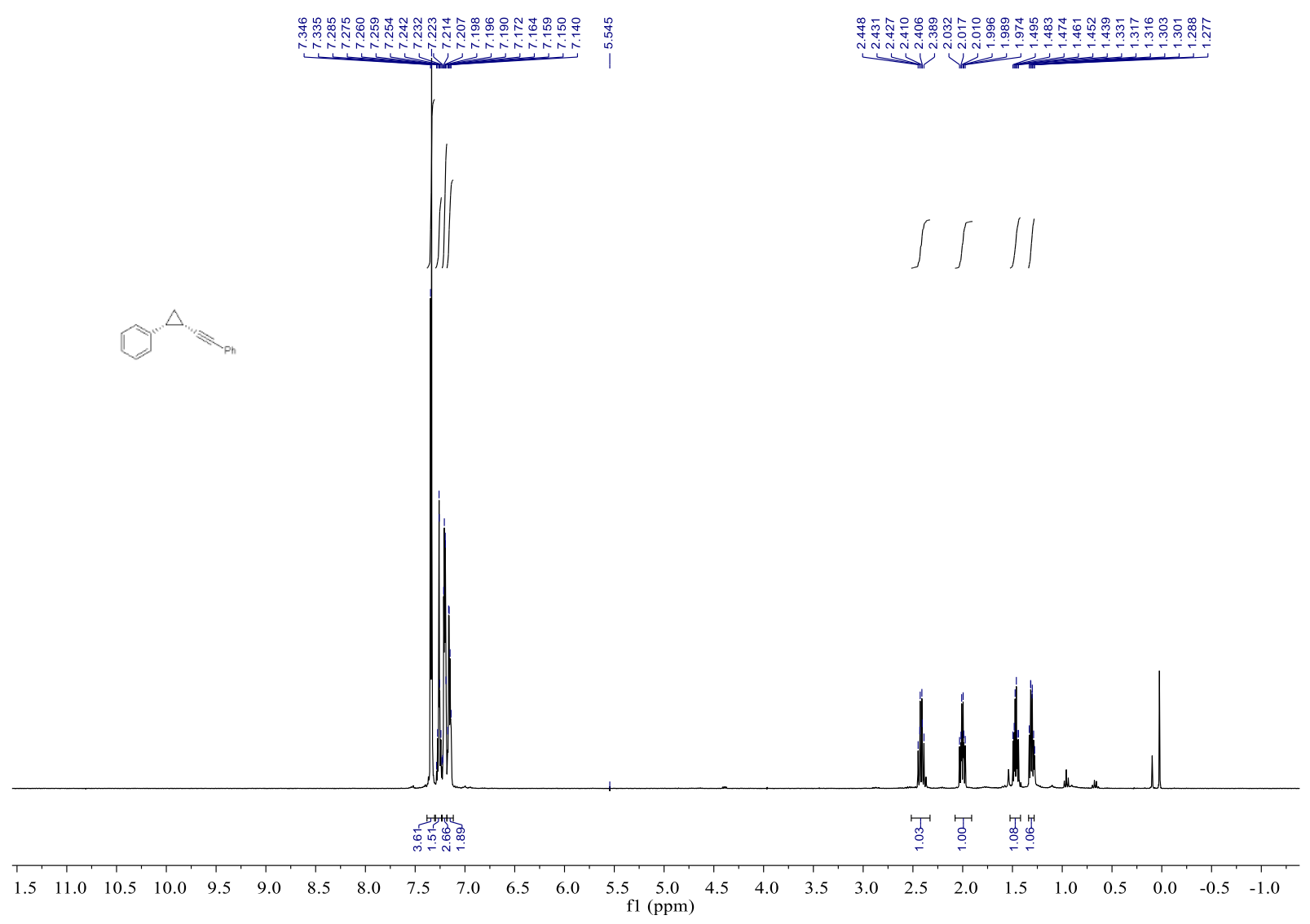




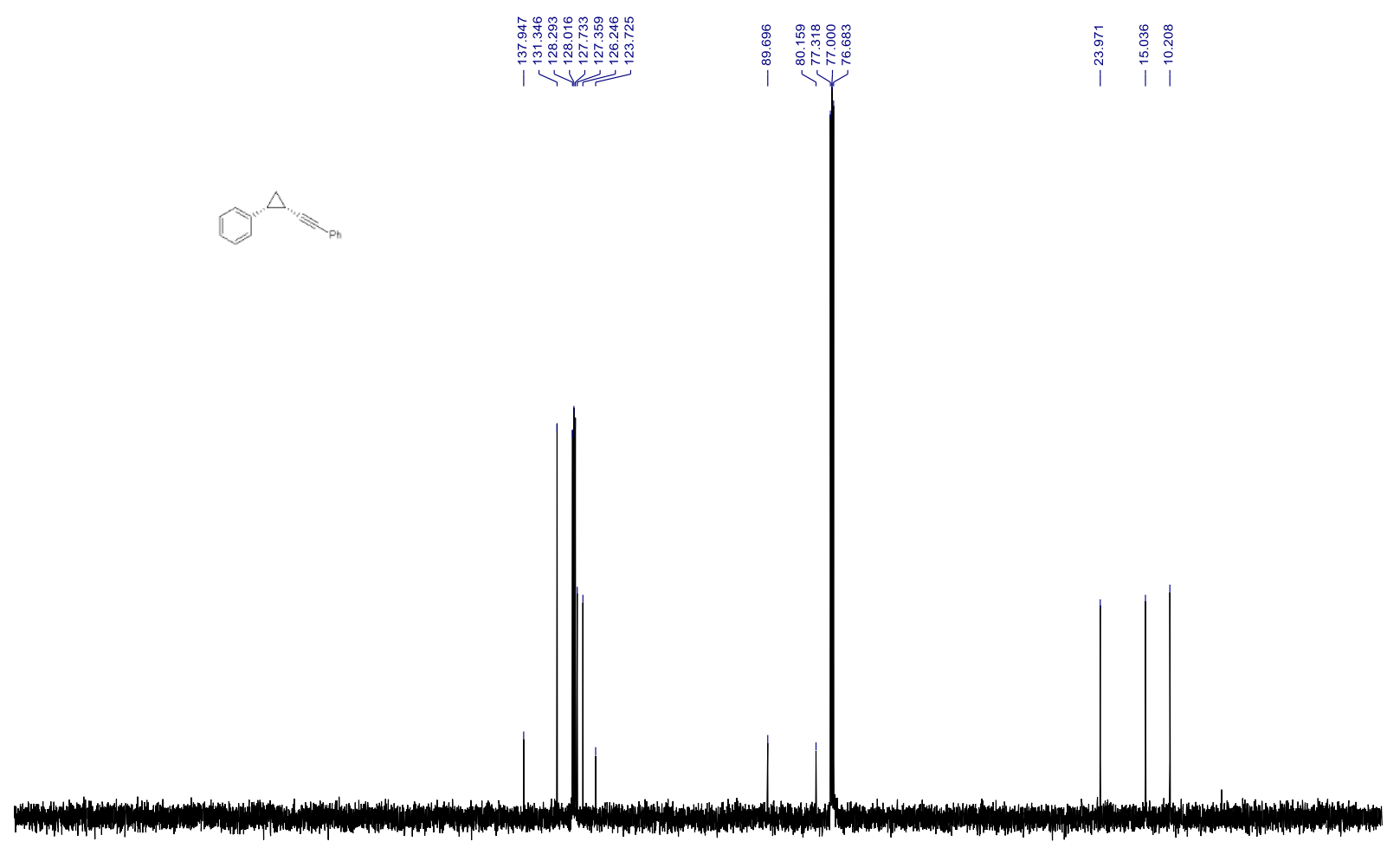

$\begin{array}{llllllllllllllllllllllllllllll}230 & 220 & 210 & 200 & 190 & 180 & 170 & 160 & 150 & 140 & 130 & 120 & 110 & 100 & 90 & 80 & 70 & 60 & 50 & 40 & 30 & 20 & 10 & 0 & -10 & -20 & -3\end{array}$

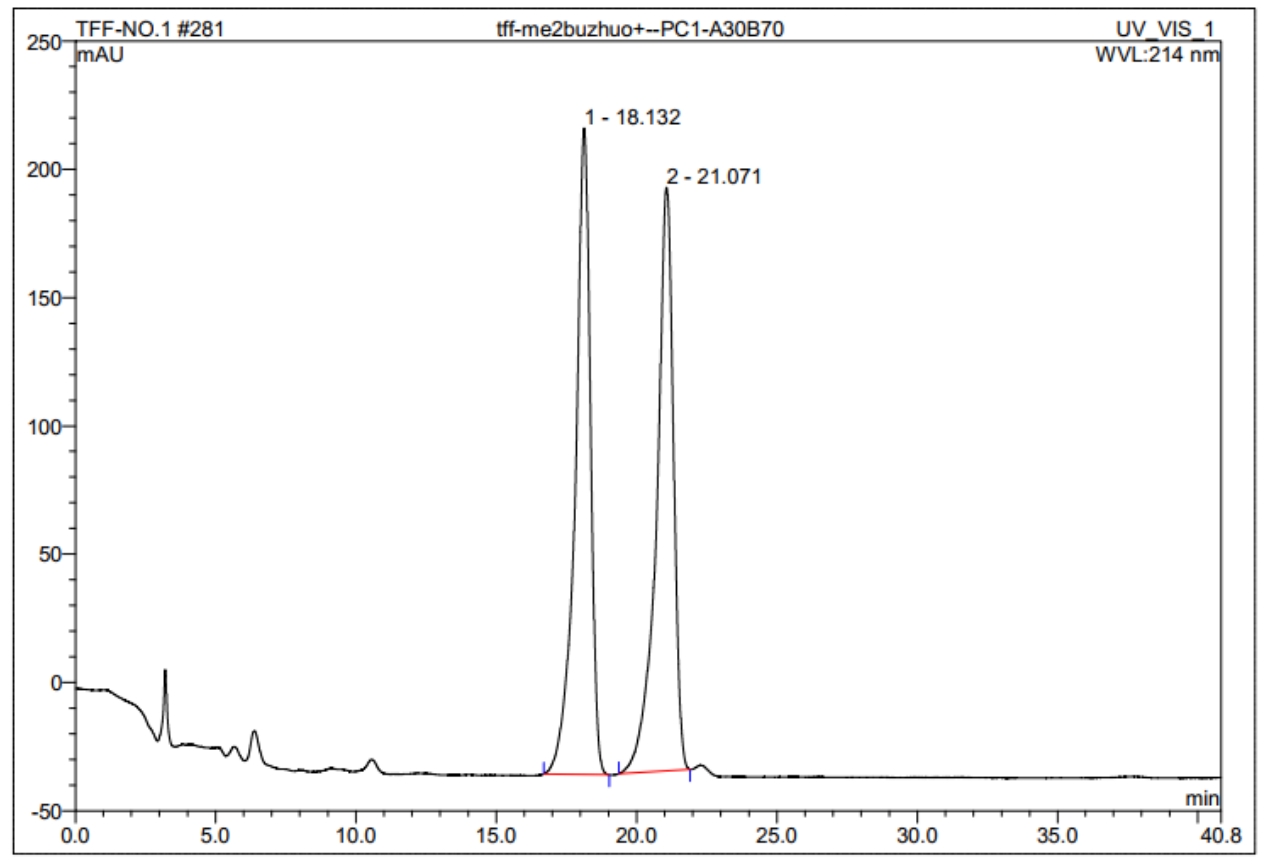

\begin{tabular}{|r|ccccccc|}
\hline No. & $\begin{array}{c}\text { Ret.Time } \\
\text { min }\end{array}$ & Peak Name & Height & Area & Rel.Area & Amount & Type \\
& & mAU & mAU*min & $\%$ & $\%$ & n.a. & BMB \\
\hline 1 & 18.13 & n.a. & 251.991 & 157.679 & 49.94 & n.a. & BMB \\
\hline 2 & 21.07 & n.a. & 227.470 & 158.053 & 50.06 & 0.000 & \\
\hline Total: & & & 479.461 & 315.732 & 100.00 & 0 \\
\hline
\end{tabular}




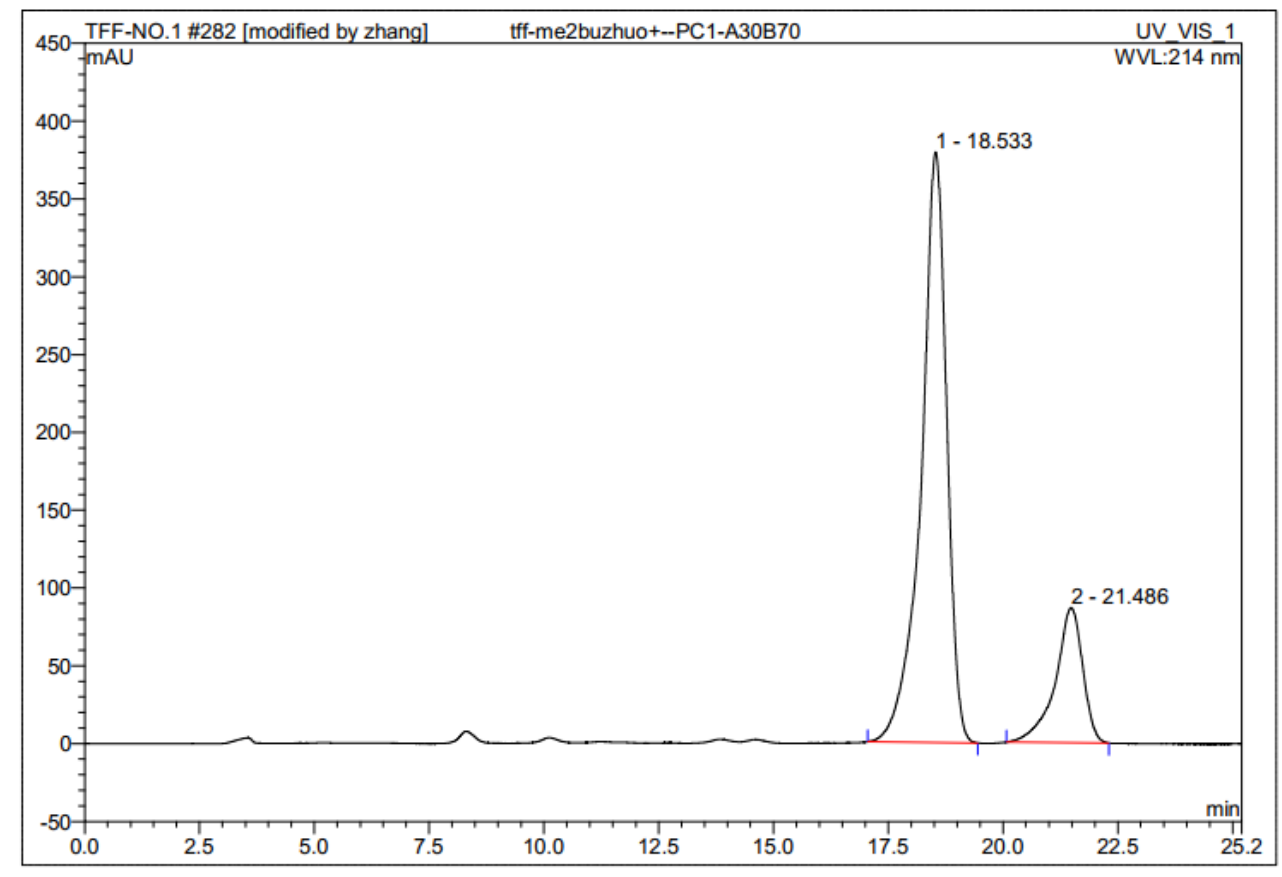

\begin{tabular}{|r|ccrrrrr|}
\hline No. & $\begin{array}{c}\text { Ret.Time } \\
\text { min }\end{array}$ & Peak Name & $\begin{array}{c}\text { Height } \\
\text { mAU }\end{array}$ & $\begin{array}{c}\text { Area } \\
\text { mAU*min }\end{array}$ & $\begin{array}{c}\text { Rel.Area } \\
\%\end{array}$ & $\begin{array}{c}\text { Amount } \\
\%\end{array}$ & Type \\
\hline 1 & 18.53 & n.a. & 379.617 & 243.257 & 80.20 & n.a. & BMB \\
2 & 21.49 & n.a. & 86.771 & 60.054 & 19.80 & n.a. & BMB $^{*}$ \\
\hline Total: & & & 466.387 & 303.311 & 100.00 & 0.000 & \\
\hline
\end{tabular}

2-(3,3-Difluoro-3-((1S,2R)-2-phenylcyclopropyl)prop-1-yn-1-yl)pyridine (14a).

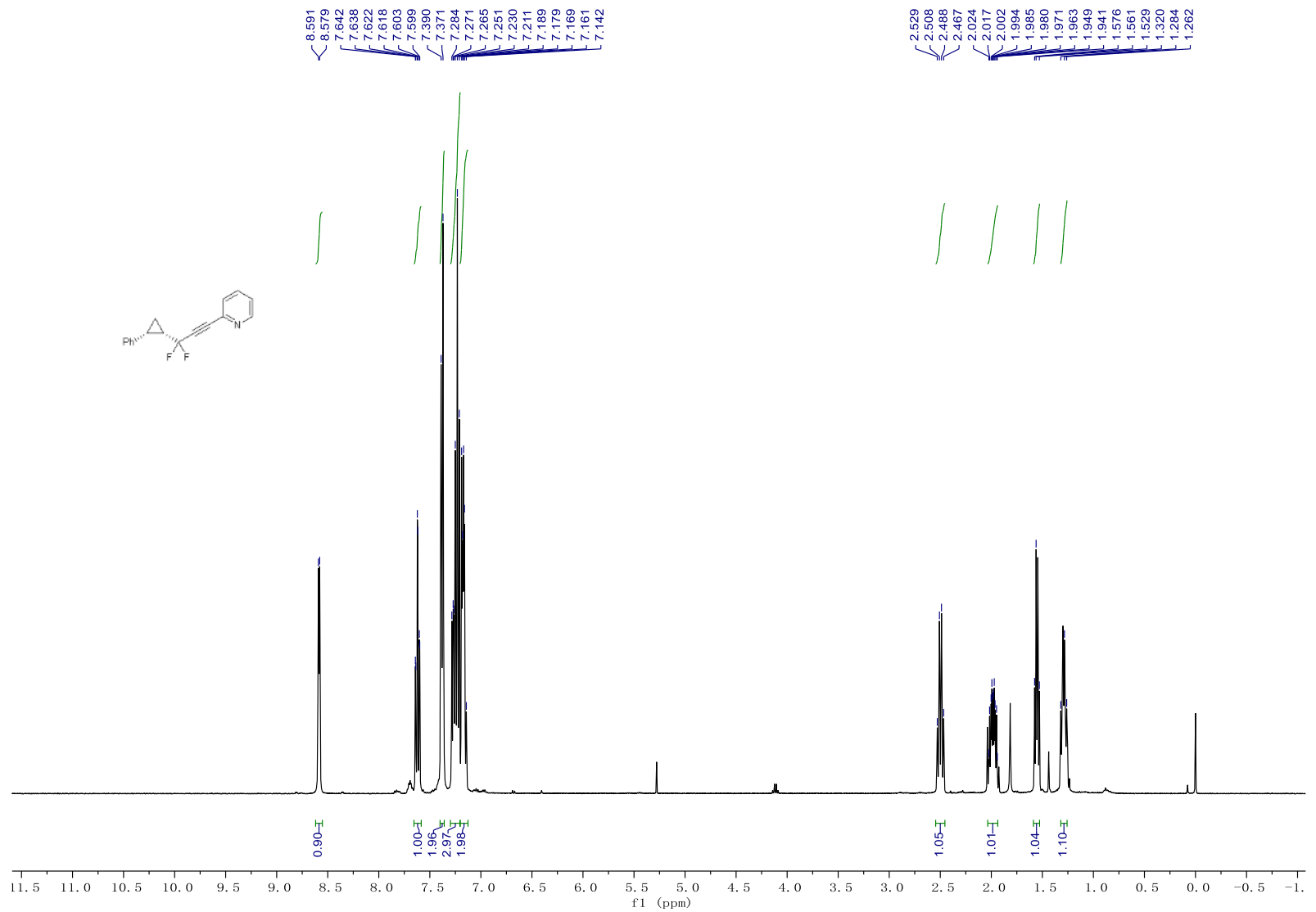



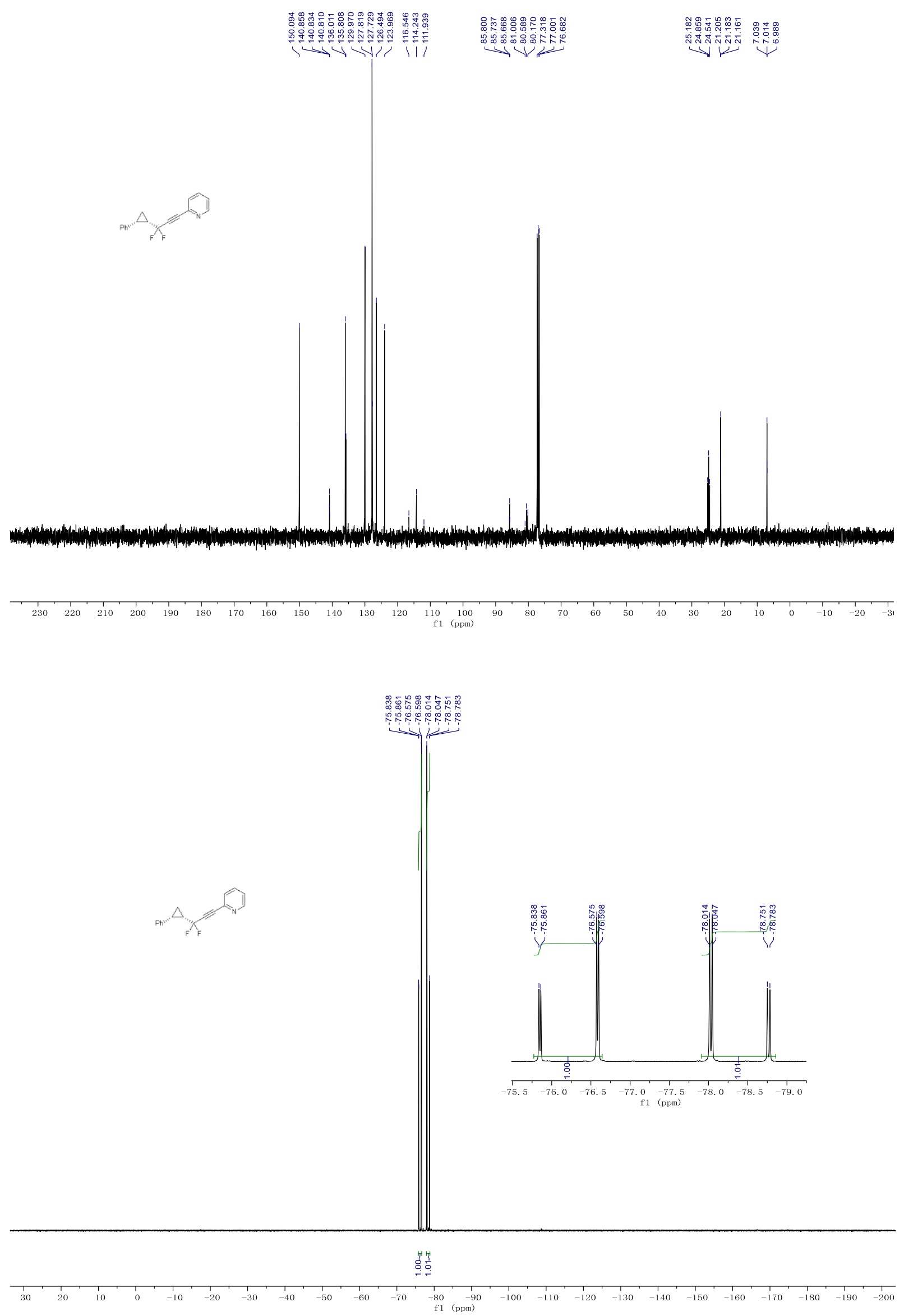


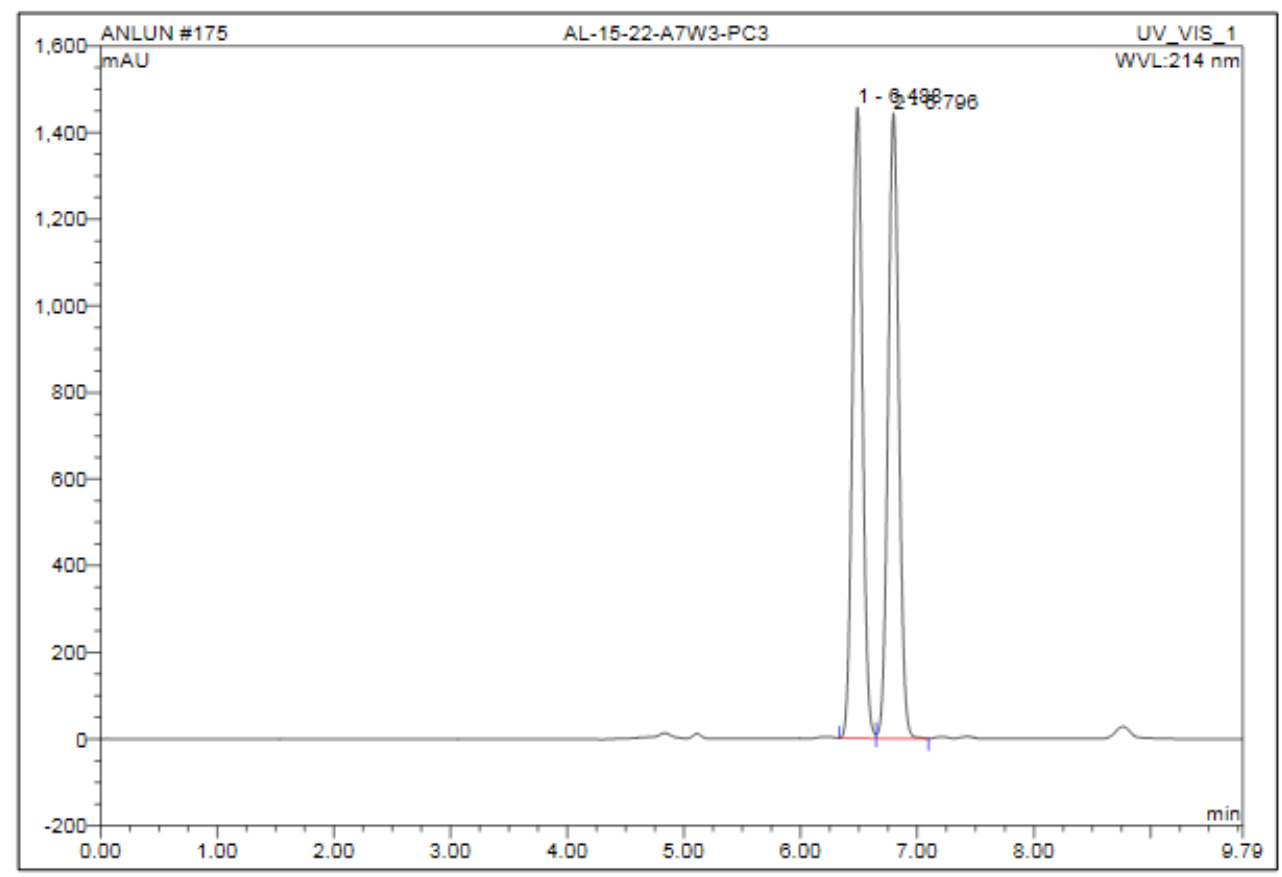

\begin{tabular}{|r|ccccccc|}
\hline No. & $\begin{array}{c}\text { Ret.Time } \\
\text { min }\end{array}$ & Peak Name & $\begin{array}{c}\text { Height } \\
\text { mAU }\end{array}$ & $\begin{array}{c}\text { Area } \\
\text { mAU*min }\end{array}$ & $\begin{array}{r}\text { Rel.Area } \\
\%\end{array}$ & Amount & Type \\
\hline 1 & 6.49 & n.a. & 1457.173 & 146.222 & 48.59 & n.a. & BM \\
2 & 6.80 & n.a. & 1443.287 & 154.737 & 51.41 & n.a. & MB \\
\hline Total: & & & 2900.460 & 300.959 & 100.00 & 0.000 & \\
\hline
\end{tabular}

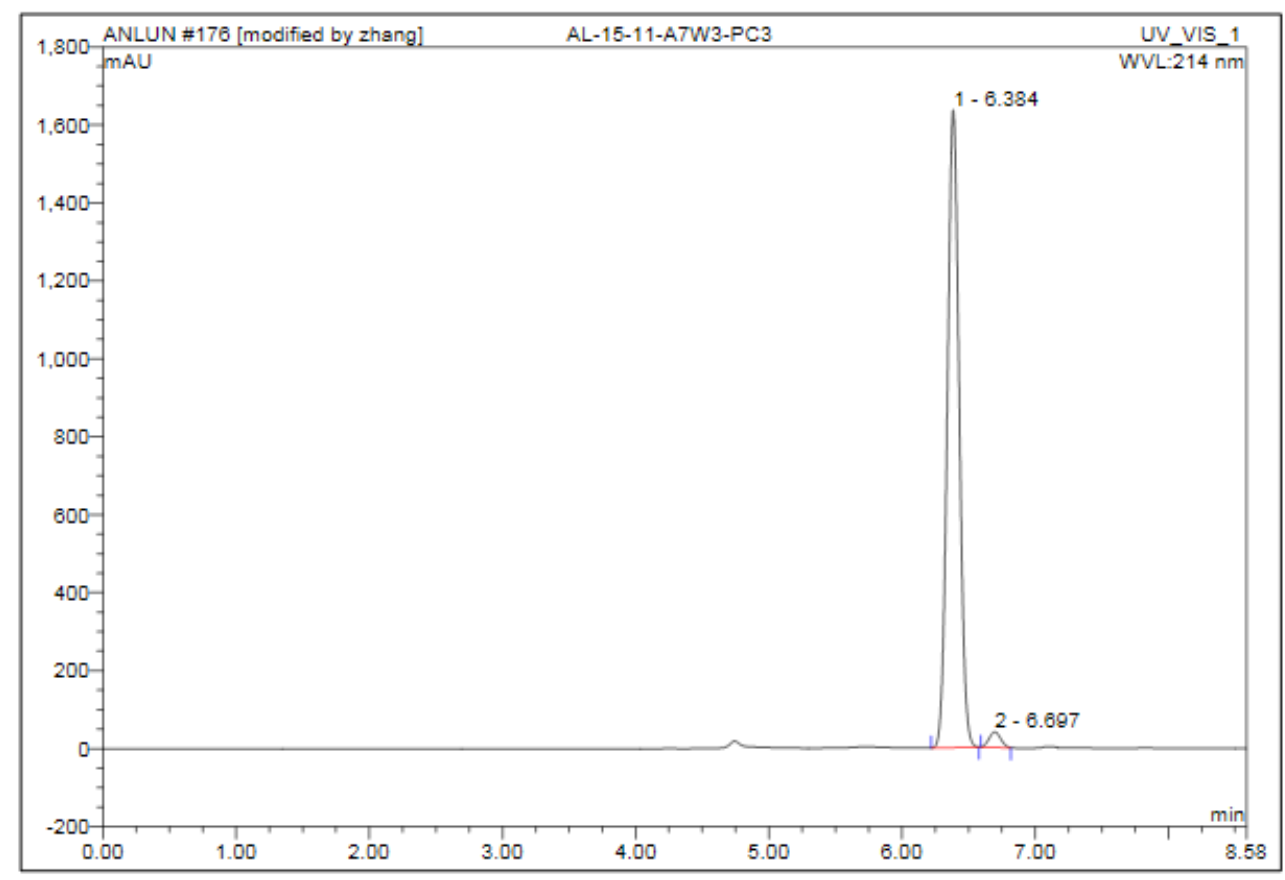

\begin{tabular}{|r|rrrrrrr|}
\hline No. & $\begin{array}{c}\text { Ret.Time } \\
\text { min }\end{array}$ & Peak Name & $\begin{array}{c}\text { Height } \\
\text { mAU }\end{array}$ & $\begin{array}{c}\text { Area } \\
\text { mAU*min }\end{array}$ & $\begin{array}{c}\text { Rel.Area } \\
\%\end{array}$ & Amount & Type \\
\hline 1 & 6.38 & n.a. & 1636.343 & 167.593 & 97.67 & n.a. & BMB \\
2 & 6.70 & n.a. & 39.538 & 3.998 & 2.33 & n.a. & BMB $^{\star}$ \\
\hline Total: & & & 1675.882 & 171.591 & 100.00 & 0.000 & \\
\hline
\end{tabular}


4,4-Difluoro-N,N-dimethyl-4-((1S,2R)-2-phenylcyclopropyl)but-2-ynamide (14b).
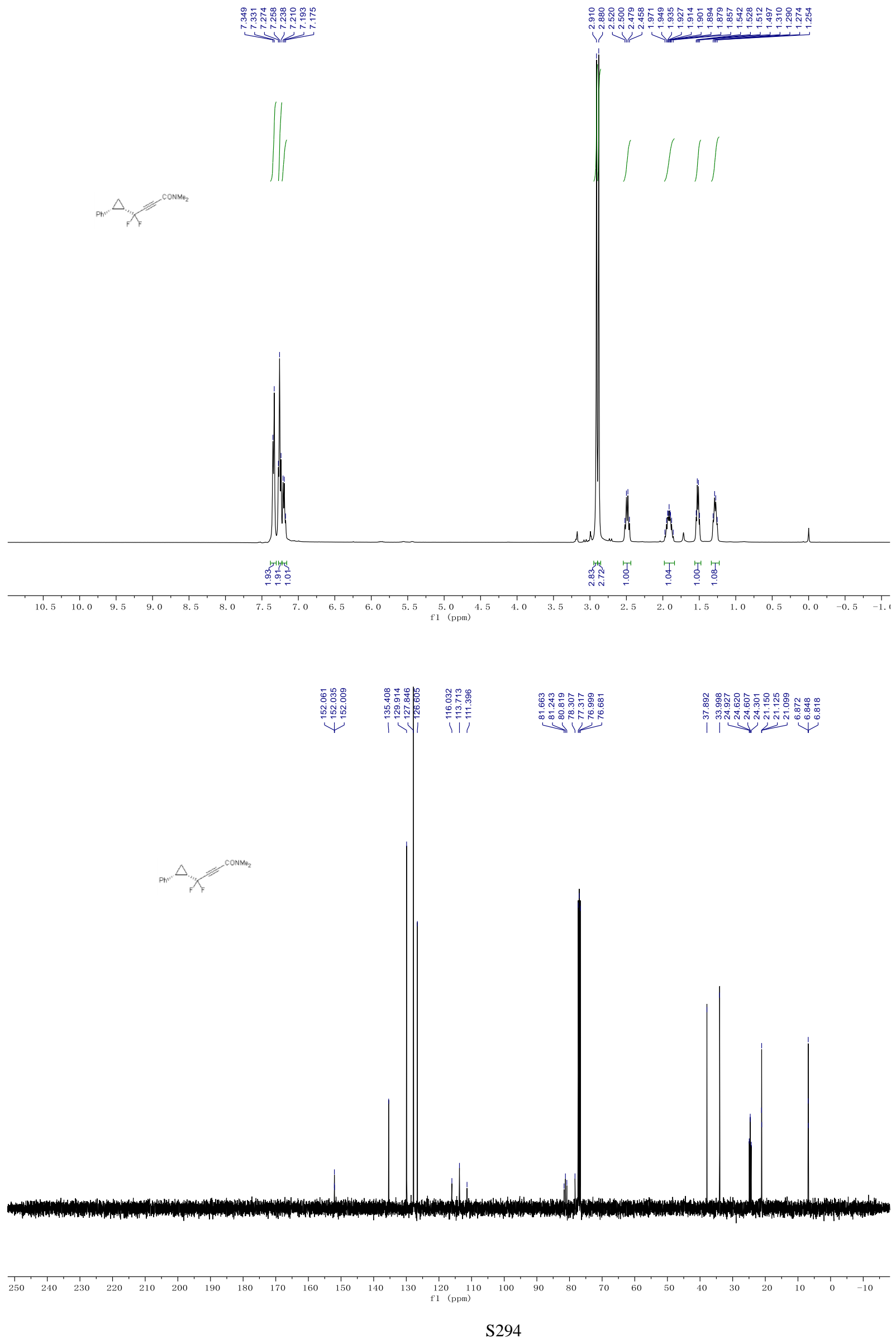

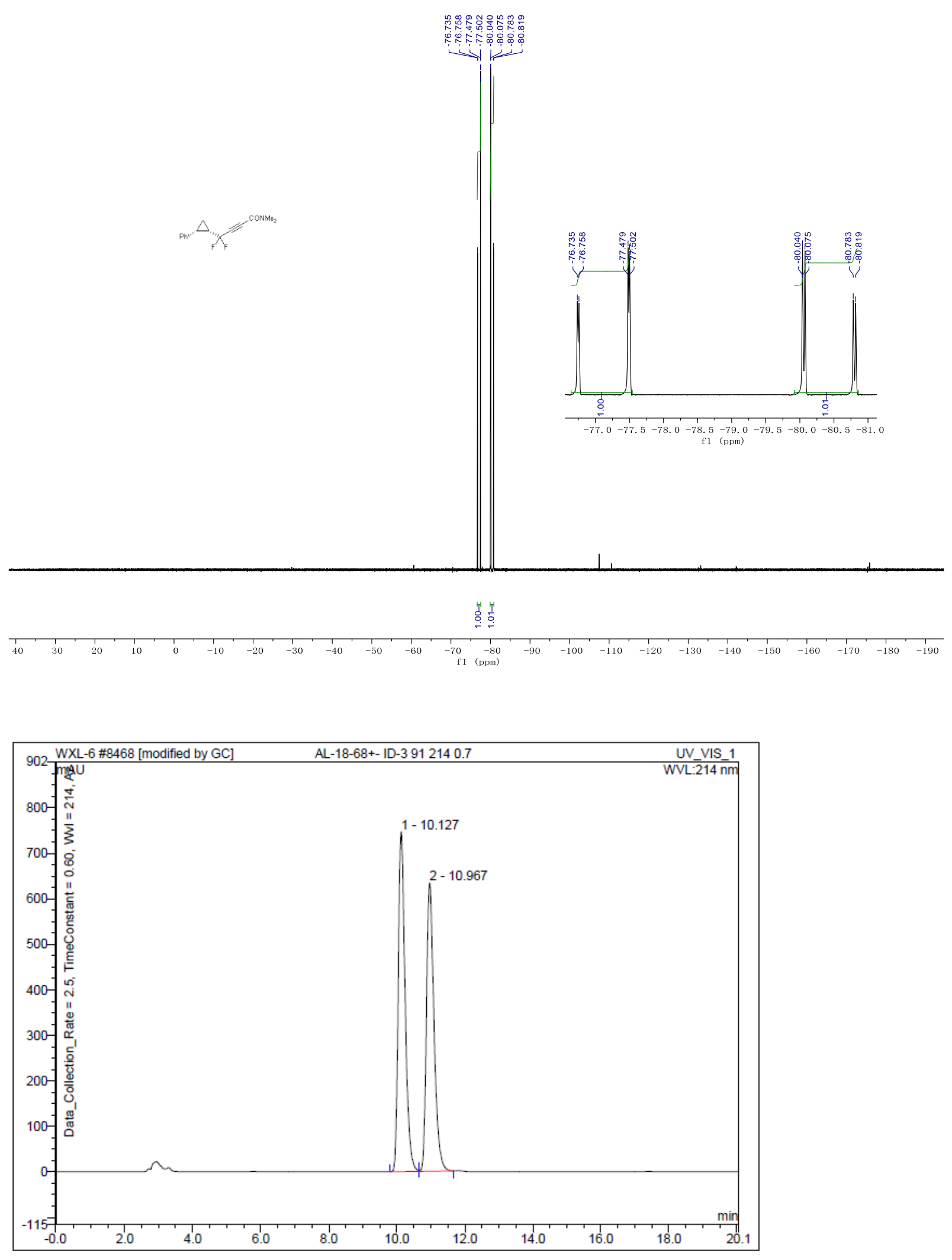

\begin{tabular}{|r|ccrrrrr|}
\hline No. & $\begin{array}{c}\text { Ret.Time } \\
\text { min }\end{array}$ & Peak Name & $\begin{array}{c}\text { Height } \\
\text { mAU }\end{array}$ & $\begin{array}{c}\text { Area } \\
\text { mAU*min }\end{array}$ & $\begin{array}{r}\text { Rel.Area } \\
\%\end{array}$ & Amount & Type \\
\hline 1 & 10.13 & n.a. & 747.103 & 174.131 & 51.85 & n.a. & BM \\
2 & 10.97 & n.a. & 633.353 & 161.706 & 48.15 & n.a. & MB \\
\hline Total: & & & 1380.455 & 335.837 & 100.00 & 0.000 & \\
\hline
\end{tabular}




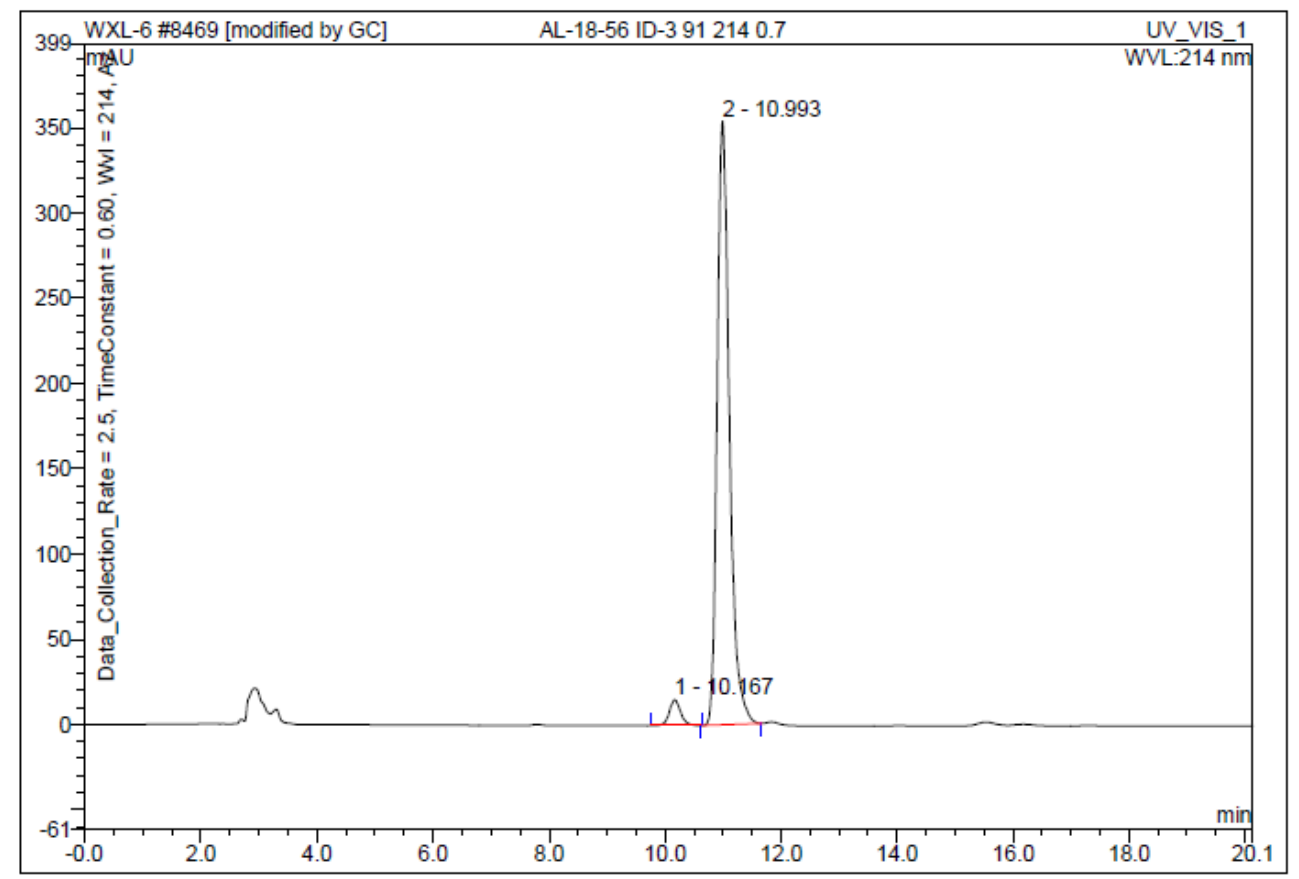

\begin{tabular}{|c|c|c|c|c|c|c|c|}
\hline No. & $\begin{array}{c}\text { Ret.Time } \\
\text { min }\end{array}$ & Peak Name & $\begin{array}{c}\text { Height } \\
\text { mAU }\end{array}$ & $\begin{array}{c}\text { Area } \\
\text { mAU*min }\end{array}$ & $\begin{array}{c}\text { Rel.Area } \\
\%\end{array}$ & Amount & Type \\
\hline 1 & 10.17 & n.a. & 15.066 & 3.424 & 3.79 & n.a. & BMB \\
\hline 2 & 10.99 & n.a. & 353.902 & 86.902 & 96.21 & n.a. & $\mathrm{BMB}$ \\
\hline Total: & & & 368.969 & 90.326 & 100.00 & 0.000 & \\
\hline
\end{tabular}

(E)-4,4-Difluoro-1-phenyl-4-((1S,2R)-2-phenylcyclopropyl)but-2-en-1-one (14c).

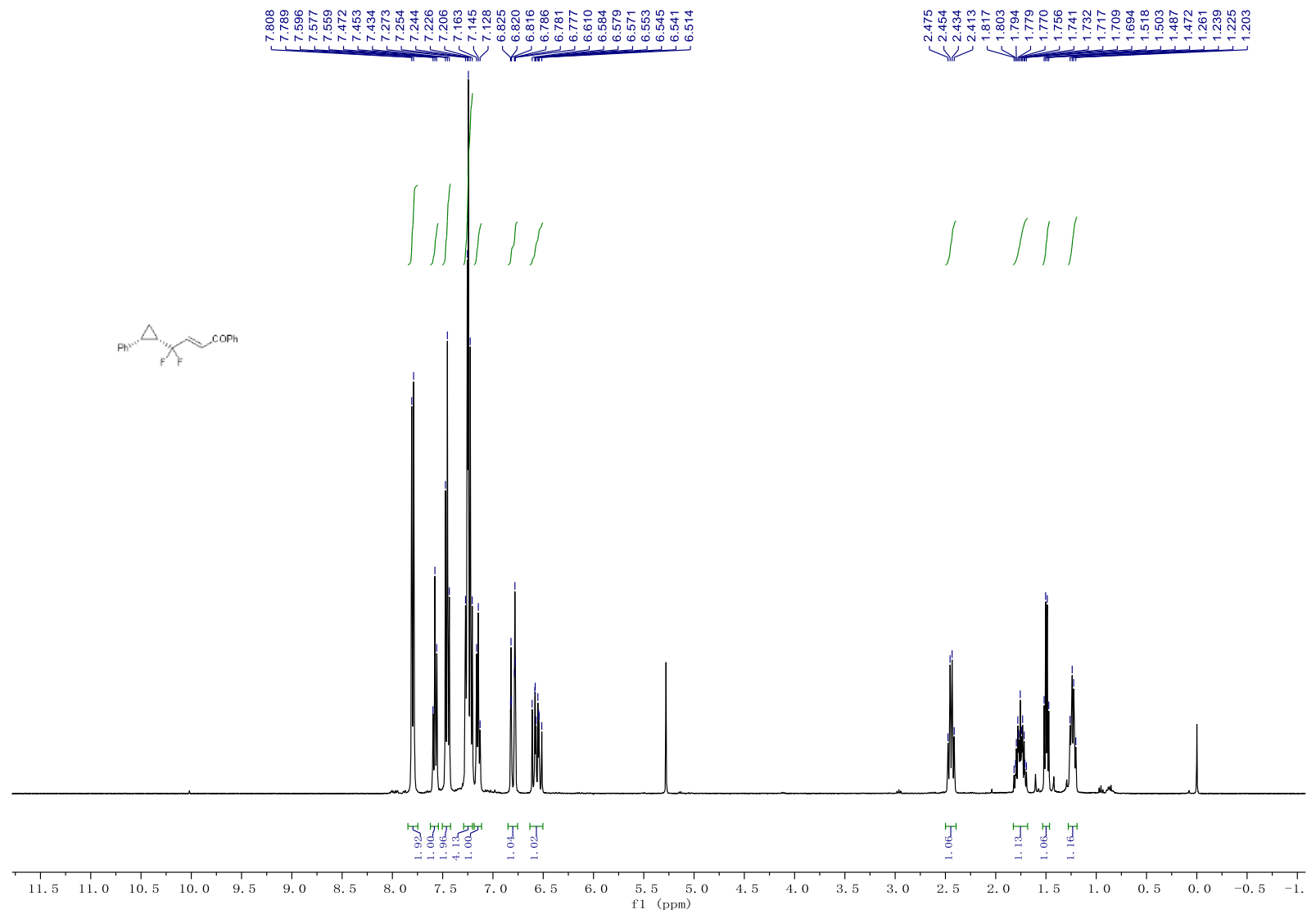



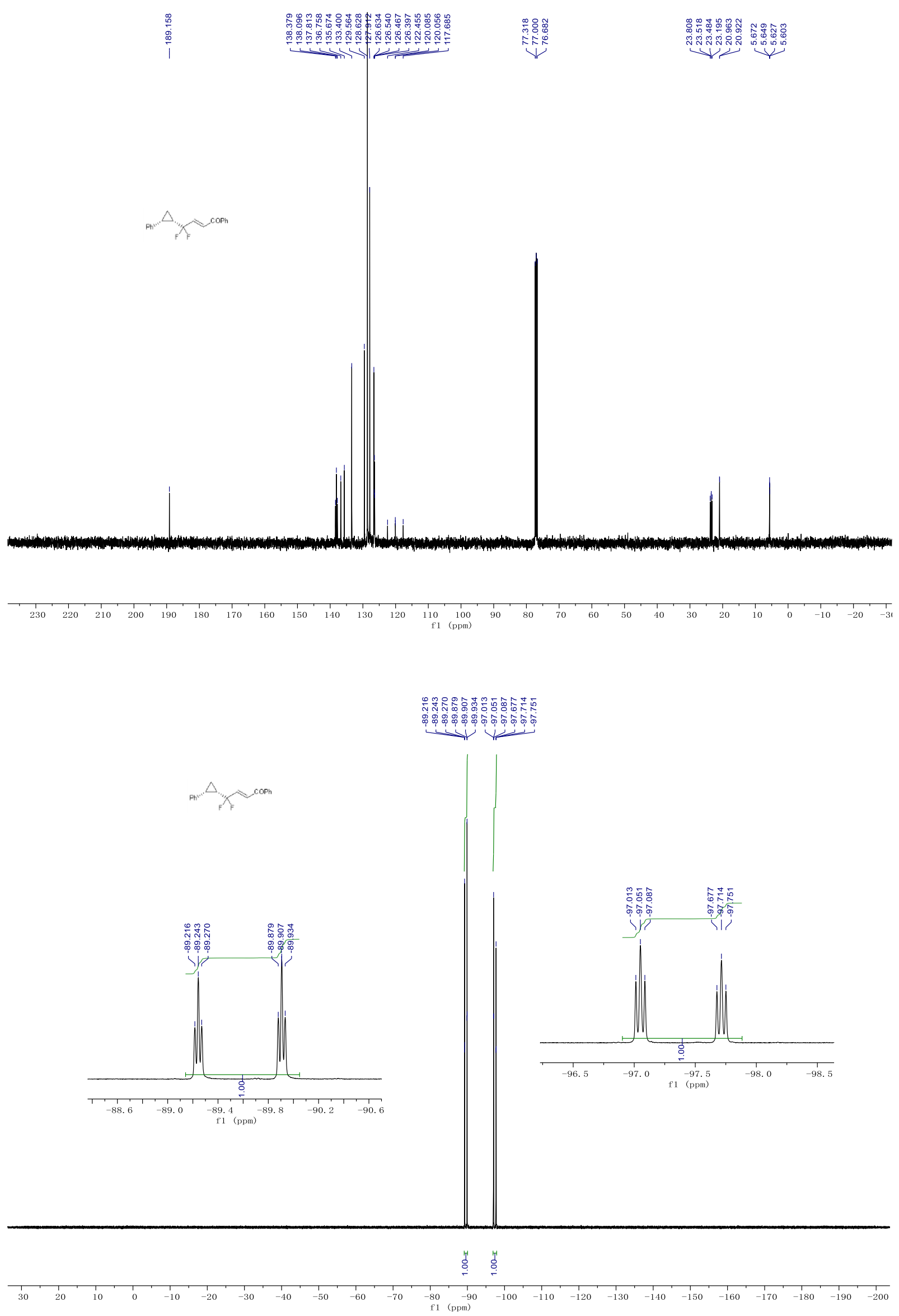


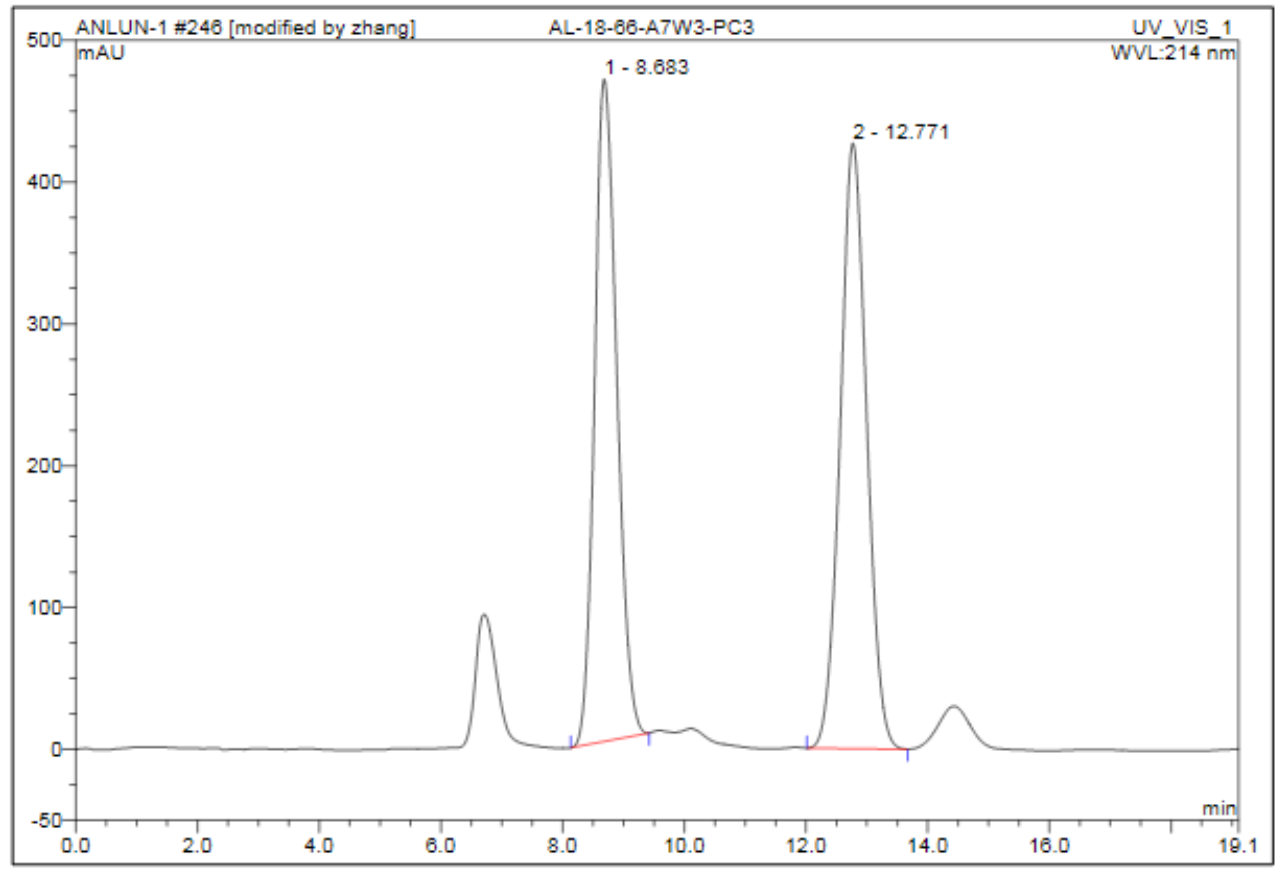

\begin{tabular}{|r|rrrrrrr|}
\hline No. & $\begin{array}{c}\text { Ret.Time } \\
\text { min }\end{array}$ & Peak Name & $\begin{array}{c}\text { Height } \\
\text { mAU }\end{array}$ & $\begin{array}{c}\text { Area } \\
\text { mAU*min }\end{array}$ & $\begin{array}{r}\text { Rel.Area } \\
\%\end{array}$ & Amount & Type \\
\hline 1 & 8.68 & n.a. & 466.919 & 198.046 & 48.01 & n.a. & BMB \\
2 & 12.77 & n.a. & 426.837 & 214.450 & 51.99 & n.a. & BMB \\
\hline Total: & & & 893.756 & 412.496 & 100.00 & 0.000 & \\
\hline
\end{tabular}

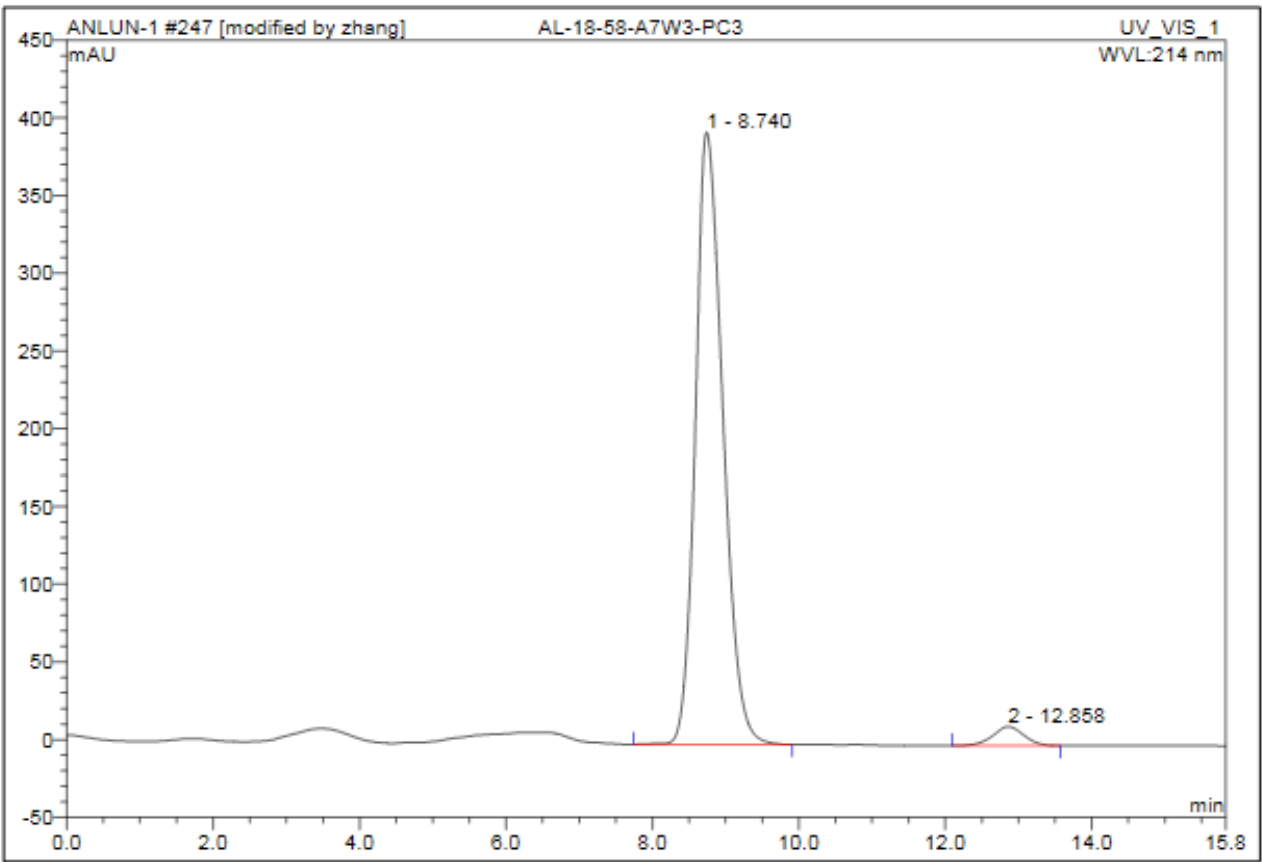

\begin{tabular}{|r|rrrrrrr|}
\hline No. & $\begin{array}{c}\text { Ret.Time } \\
\text { min }\end{array}$ & Peak Name & $\begin{array}{c}\text { Height } \\
\text { mAU }\end{array}$ & $\begin{array}{r}\text { Area } \\
\text { mAU*min }\end{array}$ & $\begin{array}{r}\text { Rel.Area } \\
\%\end{array}$ & Amount & Type \\
\hline 1 & 8.74 & n.a. & 393.931 & 171.403 & 96.61 & n.a. & BMB \\
2 & 12.86 & n.a. & 12.245 & 6.011 & 3.39 & n.a. & BMB \\
\hline Total: & & & 406.176 & 177.414 & 100.00 & 0.000 & \\
\hline
\end{tabular}


5-(Difluoro((1S,2R)-2-phenylcyclopropyl)methyl)-3-phenylisoxazole (14d).

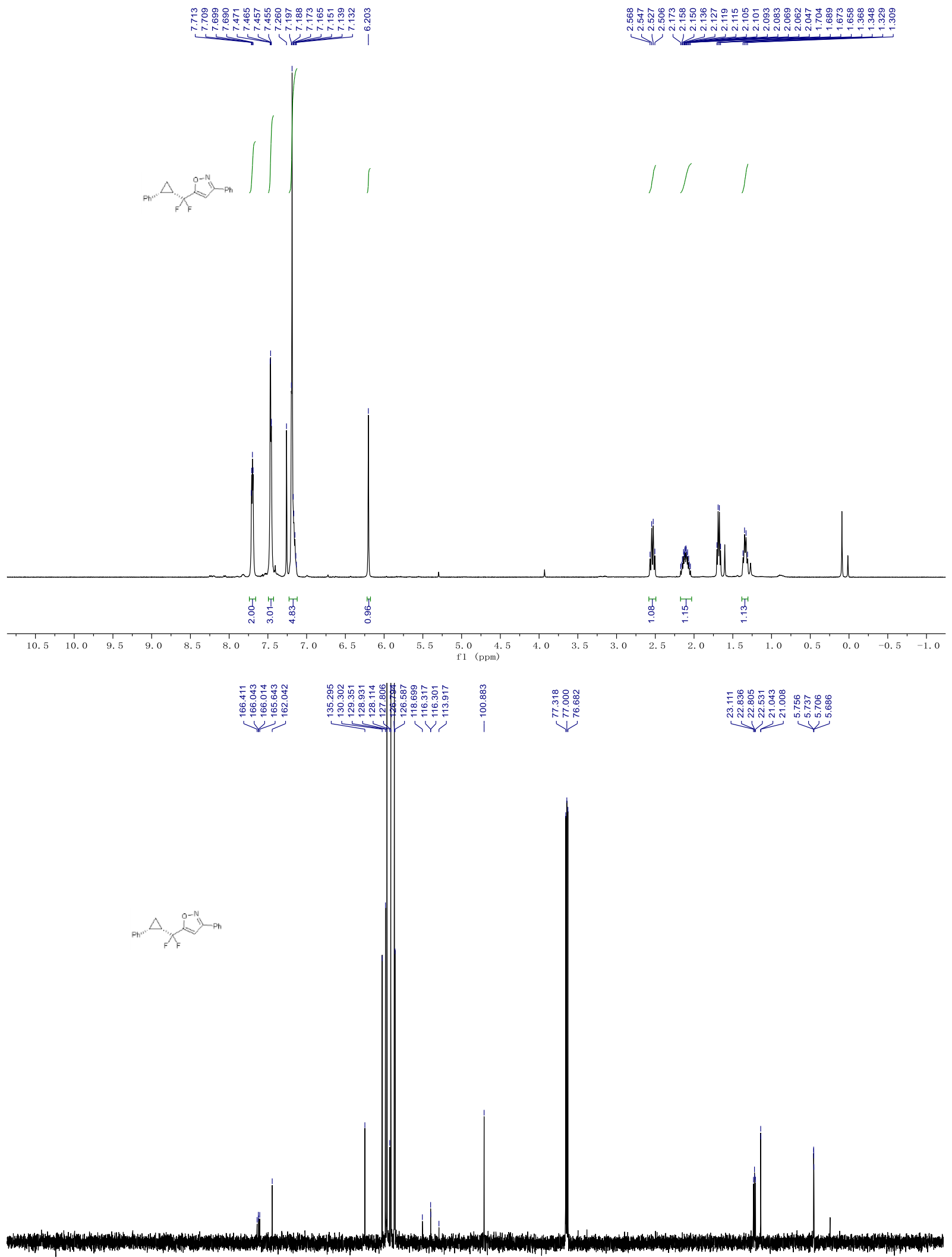



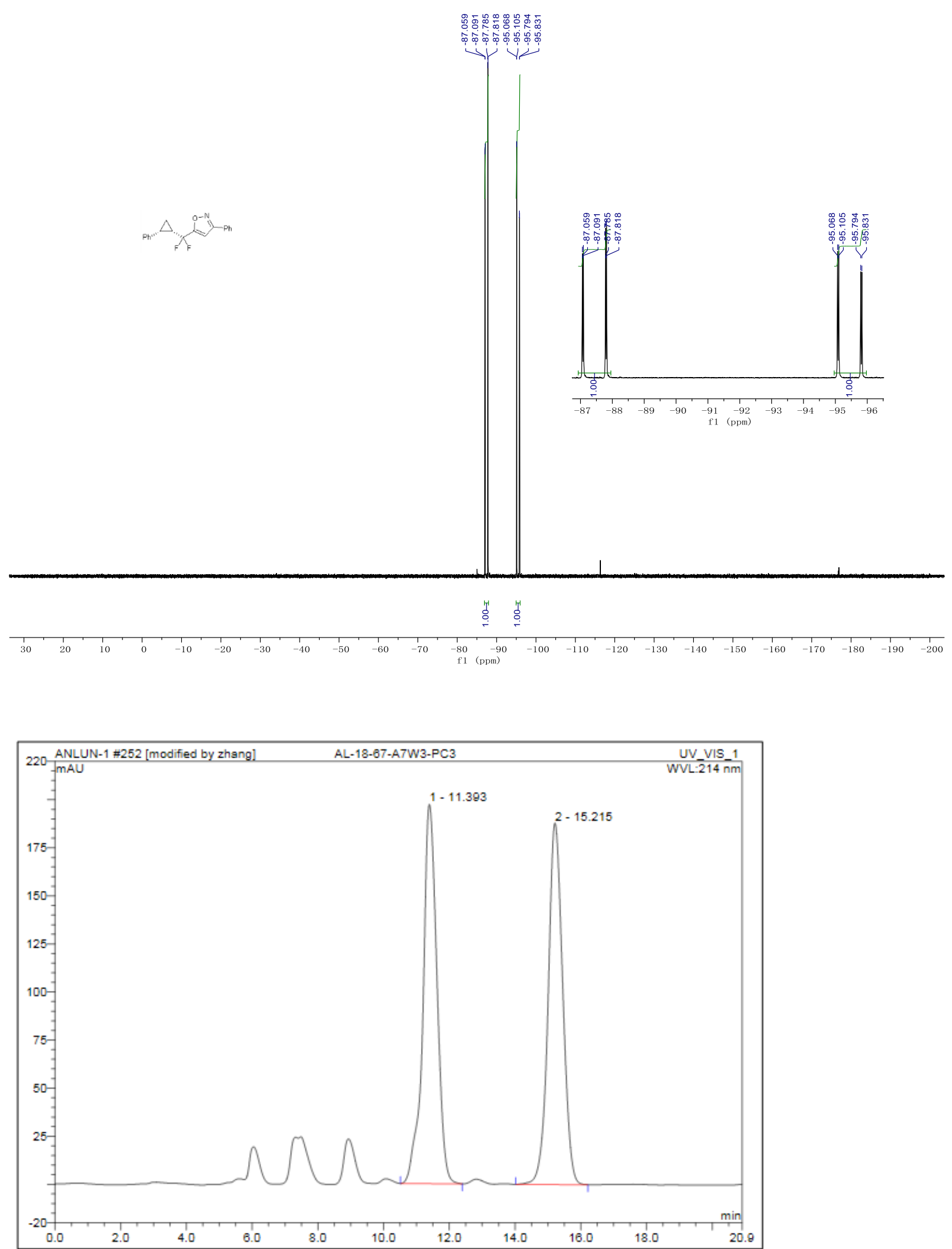

\begin{tabular}{|r|ccrrrrr|}
\hline No. & $\begin{array}{c}\text { Ret.Time } \\
\text { min }\end{array}$ & Peak Name & $\begin{array}{c}\text { Height } \\
\text { mAU }\end{array}$ & $\begin{array}{c}\text { Area } \\
\text { mAU*min }\end{array}$ & $\begin{array}{r}\text { Rel.Area } \\
\%\end{array}$ & Amount & Type \\
\hline 1 & 11.39 & n.a. & 197.283 & 97.338 & 50.20 & n.a. & BMB \\
2 & 15.22 & n.a. & 187.994 & 96.563 & 49.80 & n.a. & BMB \\
\hline Total: & & & 385.276 & 193.901 & 100.00 & 0.000 & \\
\hline
\end{tabular}




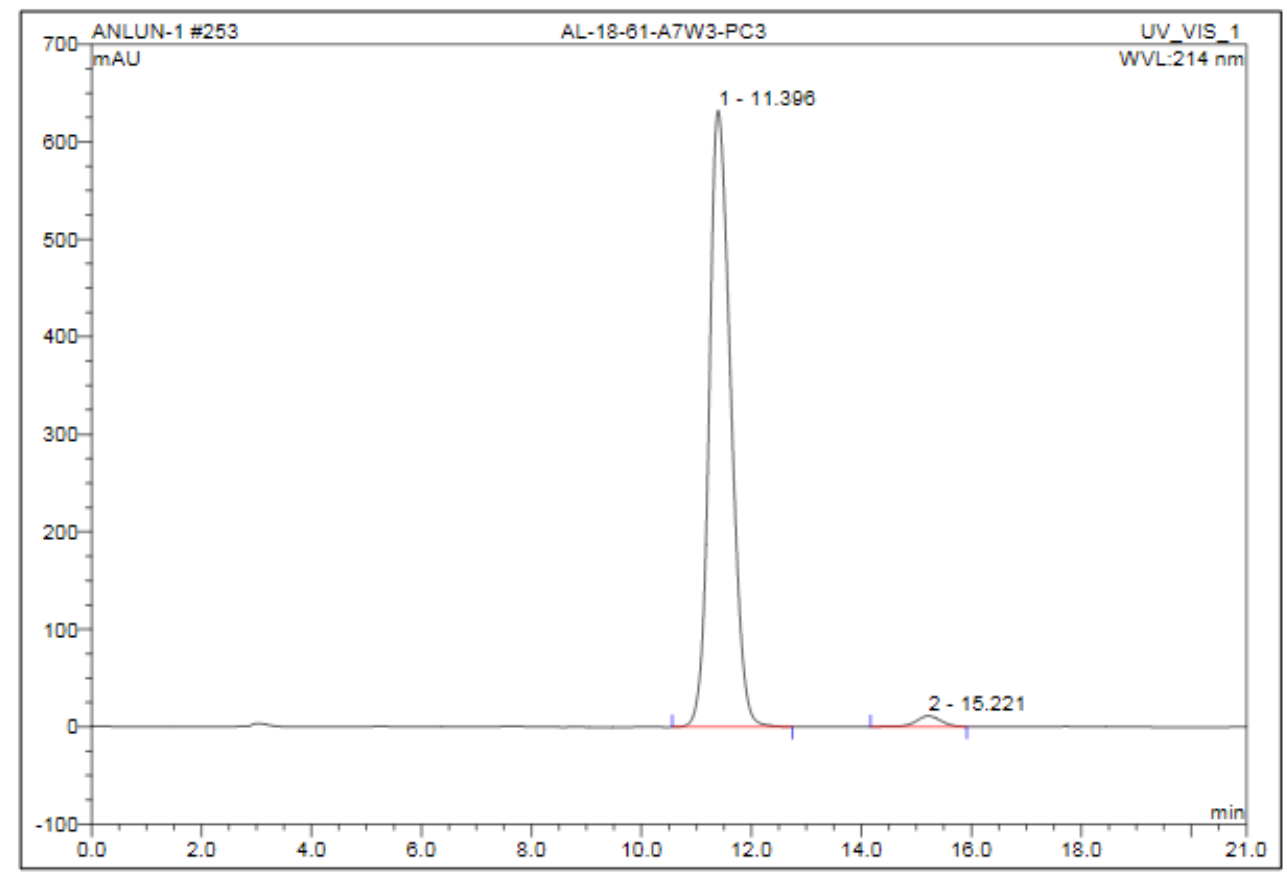

\begin{tabular}{|c|c|c|c|c|c|c|c|}
\hline No. & $\begin{array}{c}\text { Ret.Time } \\
\text { min }\end{array}$ & Peak Name & $\begin{array}{c}\text { Height } \\
\text { mAU }\end{array}$ & $\begin{array}{c}\text { Area } \\
\text { mAU*min }\end{array}$ & $\begin{array}{c}\text { Rel.Area } \\
\%\end{array}$ & Amount & Type \\
\hline 1 & 11.40 & n.a. & 632.200 & 286.132 & 98.01 & n.a. & BMB \\
\hline 2 & 15.22 & n.a. & 11.012 & 5.819 & 1.99 & n.a. & BMB \\
\hline Total: & & & 643.212 & 291.951 & 100.00 & 0.000 & \\
\hline
\end{tabular}

$N$-(3-(4/5-(Difluoro((1S,2R)-(2-phenylcyclopropyl)methyl)-1H-1,2,3-triazol-1-yl)propyl)-5-(dim ethylamino)naphthalene-1-sulfonamide (14e).

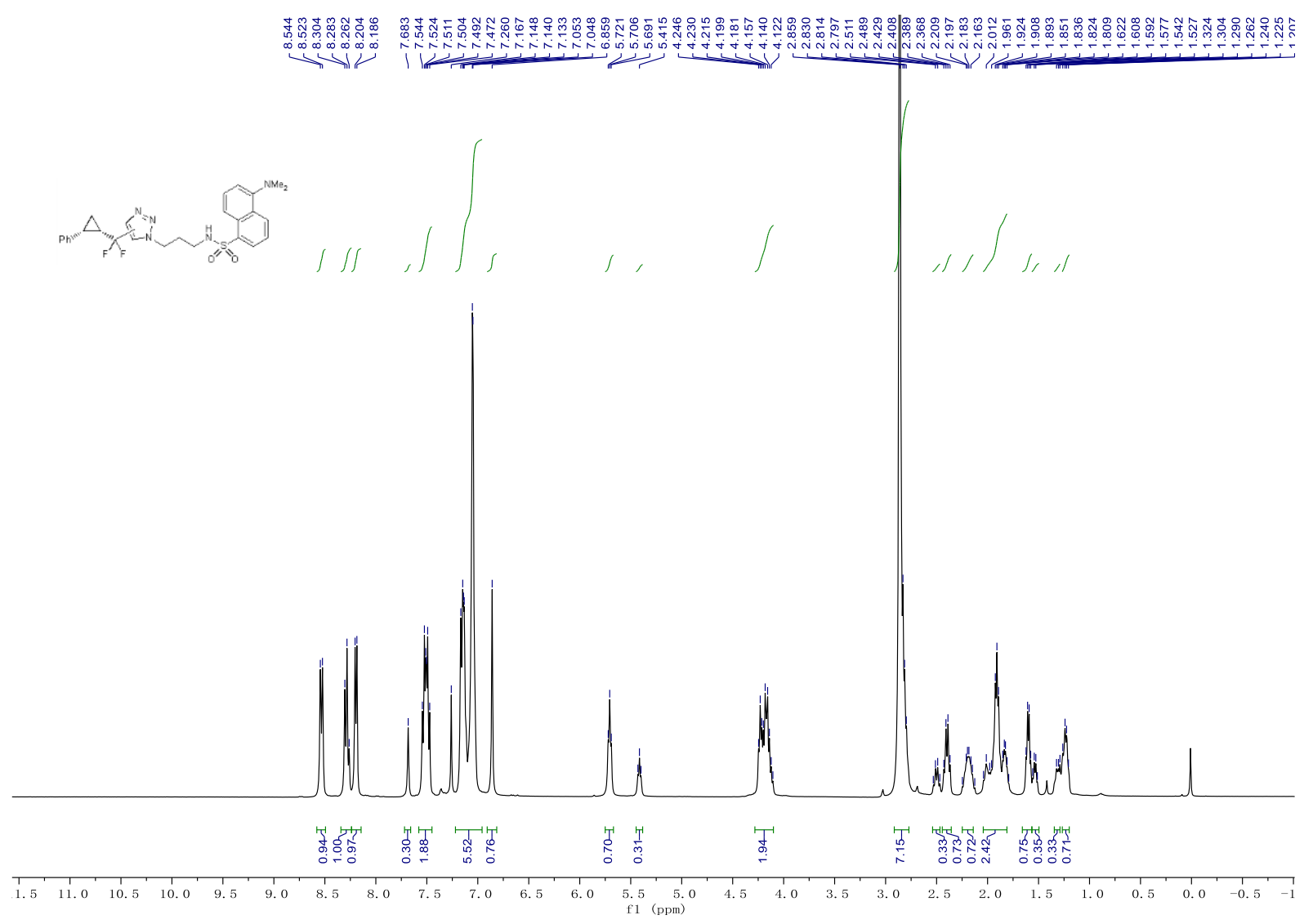



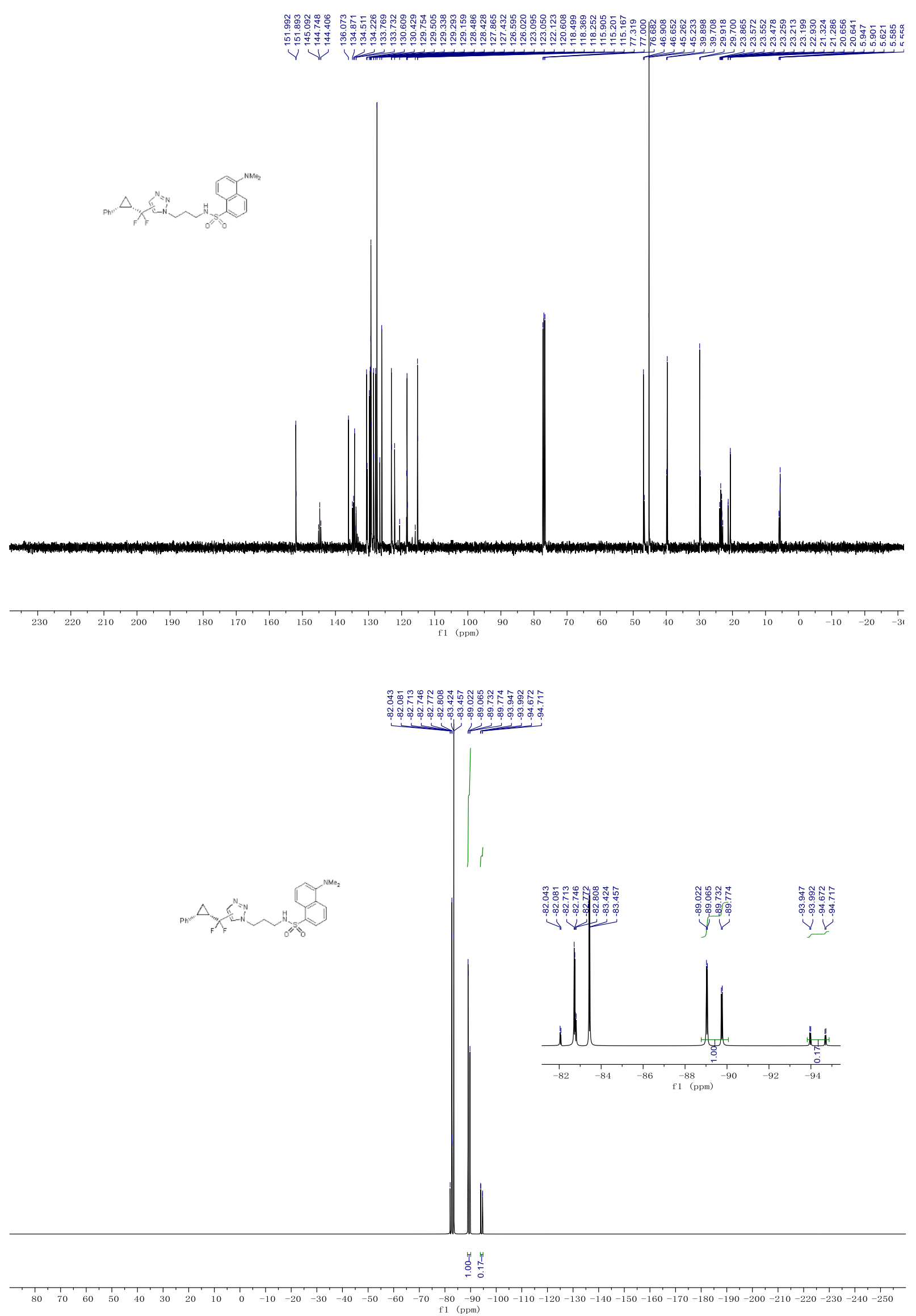
Diphenyl((1R,2R)-2-phenylcyclopropyl)phosphine (15a).

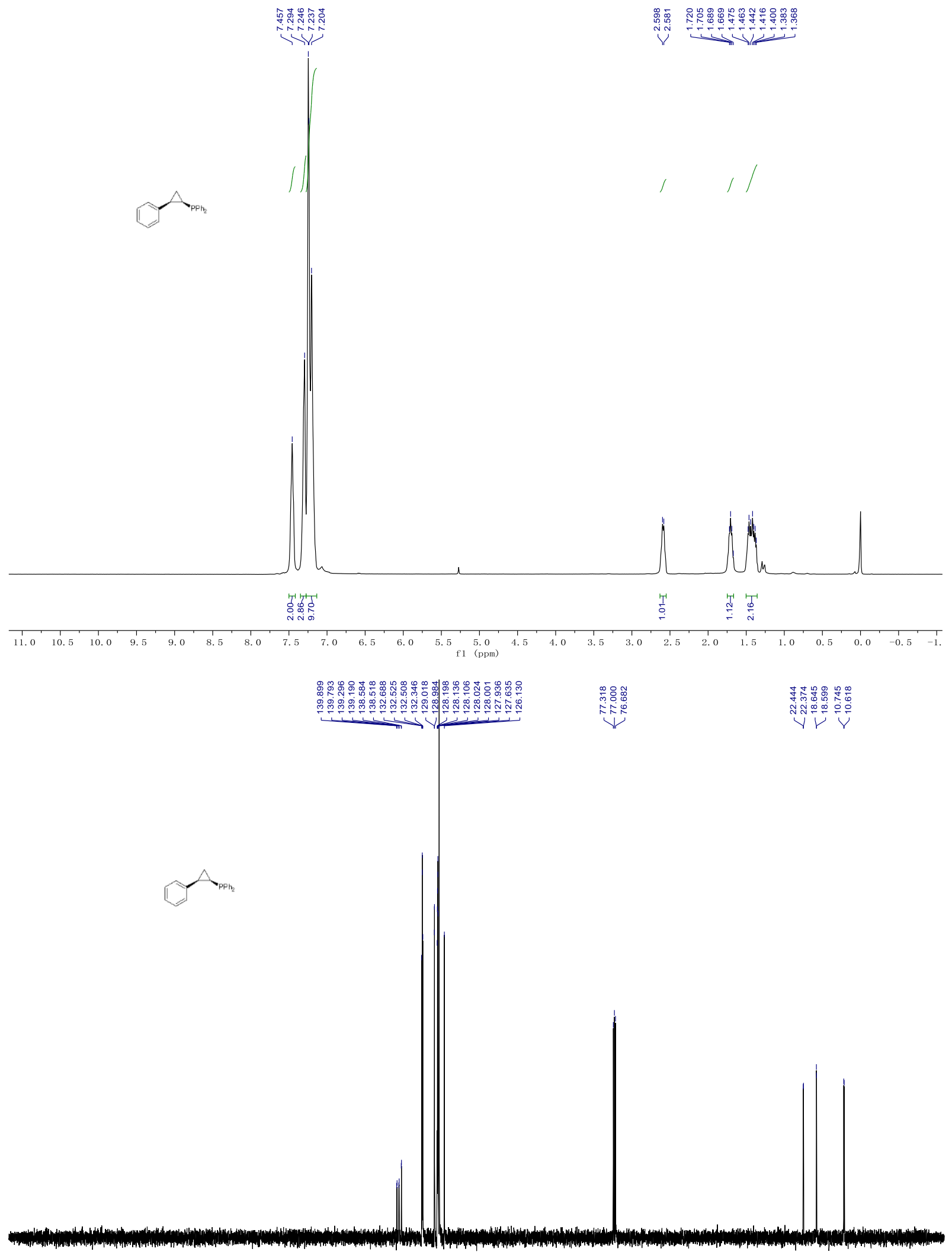

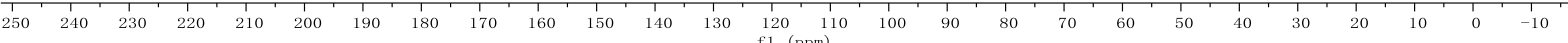




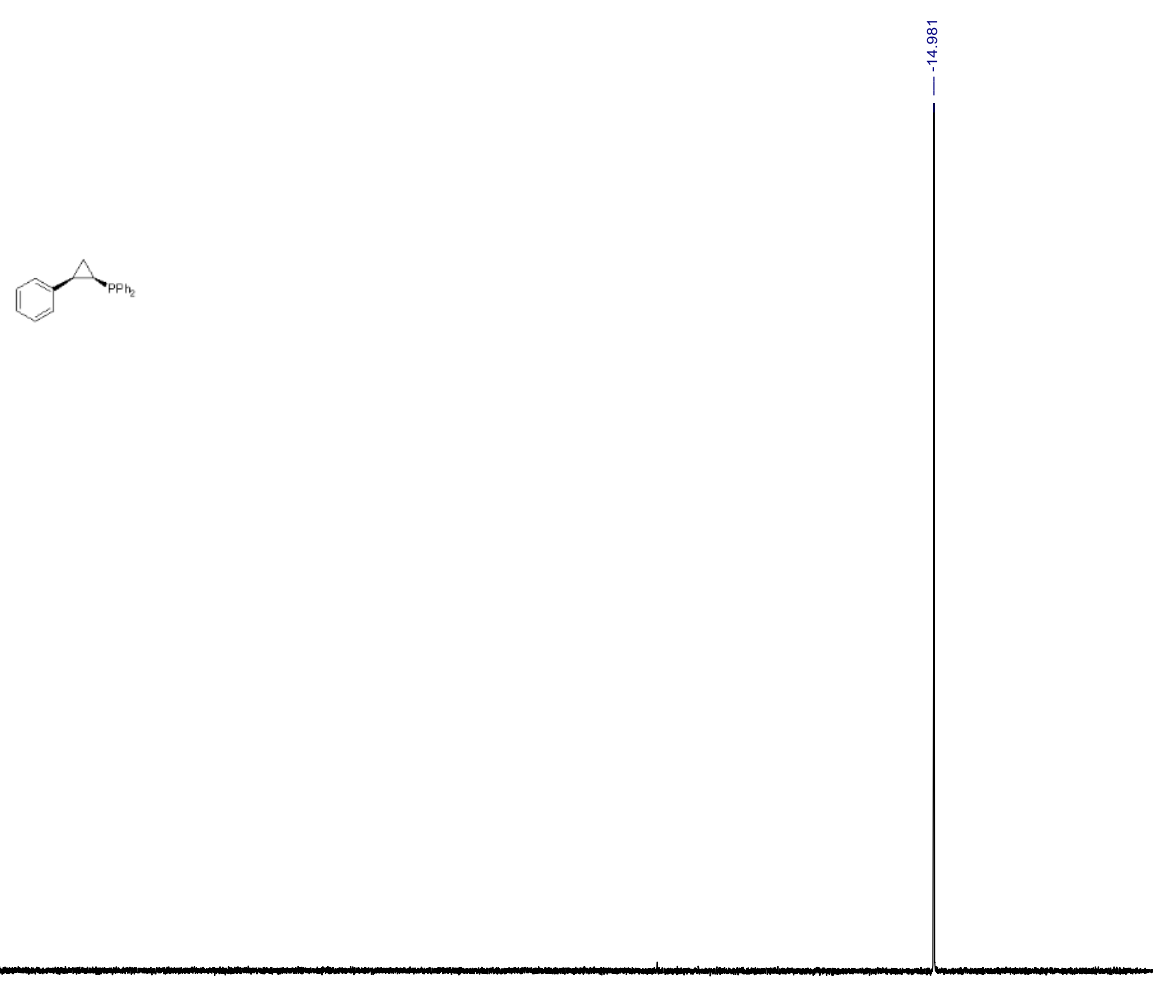

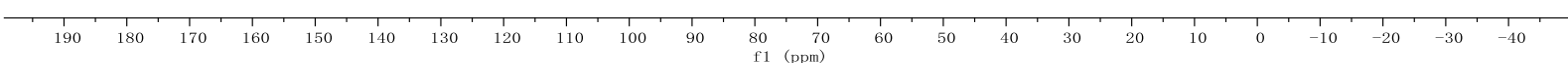

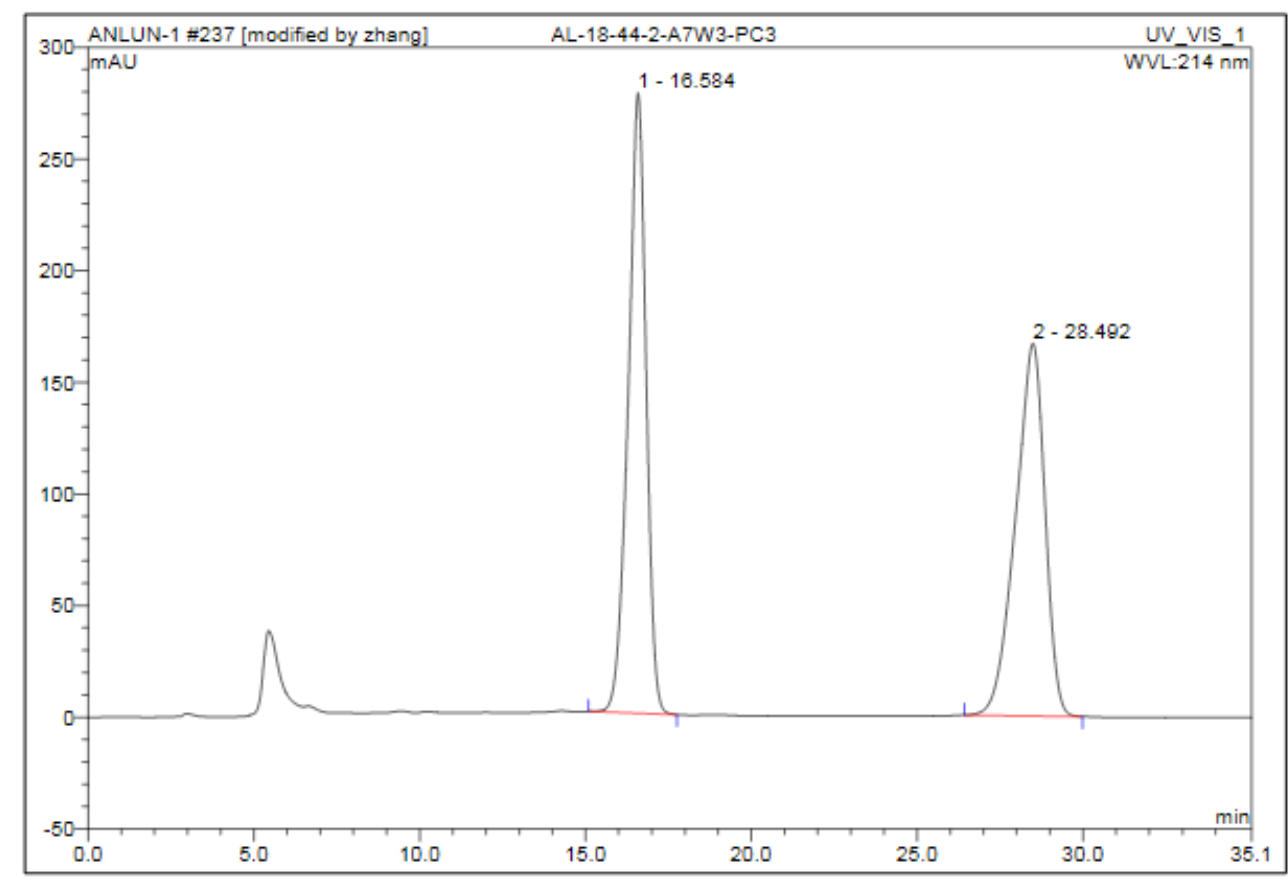

\begin{tabular}{|r|ccccccc|}
\hline No. & $\begin{array}{c}\text { Ret.Time } \\
\text { min }\end{array}$ & Peak Name & $\begin{array}{c}\text { Height } \\
\text { mAU }\end{array}$ & $\begin{array}{c}\text { Area } \\
\text { mAU*min }\end{array}$ & $\begin{array}{c}\text { Rel.Area } \\
\%\end{array}$ & Amount & Type \\
\hline 1 & 16.58 & n.a. & 277.790 & 173.805 & 50.31 & n.a. & BMB \\
2 & 28.49 & n.a. & 166.812 & 171.692 & 49.69 & n.a. & BMB \\
\hline Total: & & & 444.603 & 345.497 & 100.00 & 0.000 & \\
\hline
\end{tabular}




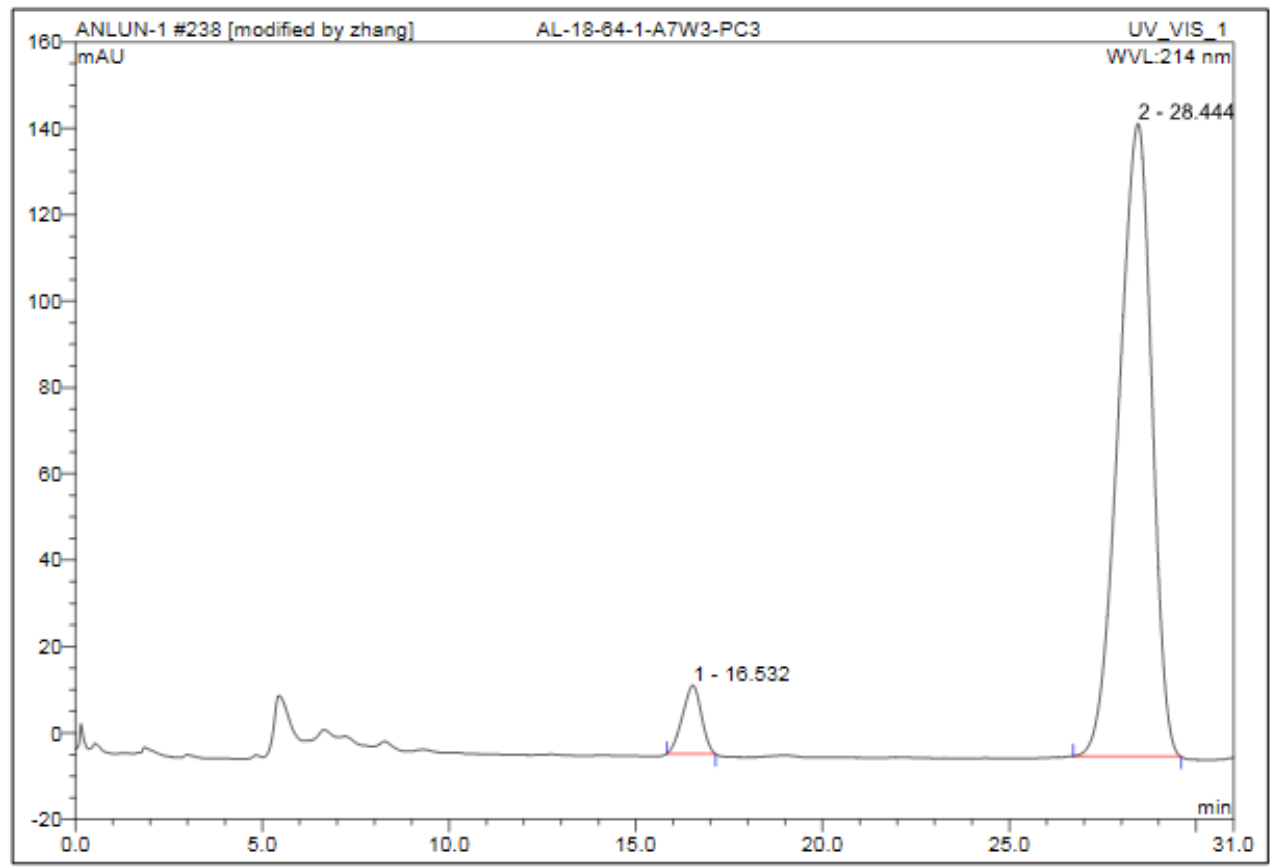

\begin{tabular}{|r|ccrrrrr|}
\hline No. & $\begin{array}{c}\text { Ret.Time } \\
\text { min }\end{array}$ & Peak Name & $\begin{array}{c}\text { Height } \\
\text { mAU }\end{array}$ & $\begin{array}{c}\text { Area } \\
\text { mAU*min }\end{array}$ & $\begin{array}{r}\text { Rel.Area } \\
\%\end{array}$ & Amount & Type \\
\hline 1 & 16.53 & n.a. & 15.885 & 9.301 & 5.83 & n.a. & BMB $^{\star}$ \\
2 & 28.44 & n.a. & 146.567 & 150.315 & 94.17 & n.a. & BMB $^{\star}$ \\
\hline Total: & & & 162.452 & 159.616 & 100.00 & 0.000 & \\
\hline
\end{tabular}

4,4,5,5-Tetramethyl-2-((1R,2S)-2-phenylcyclopropyl)-1,3,2-dioxaborolane (15b).

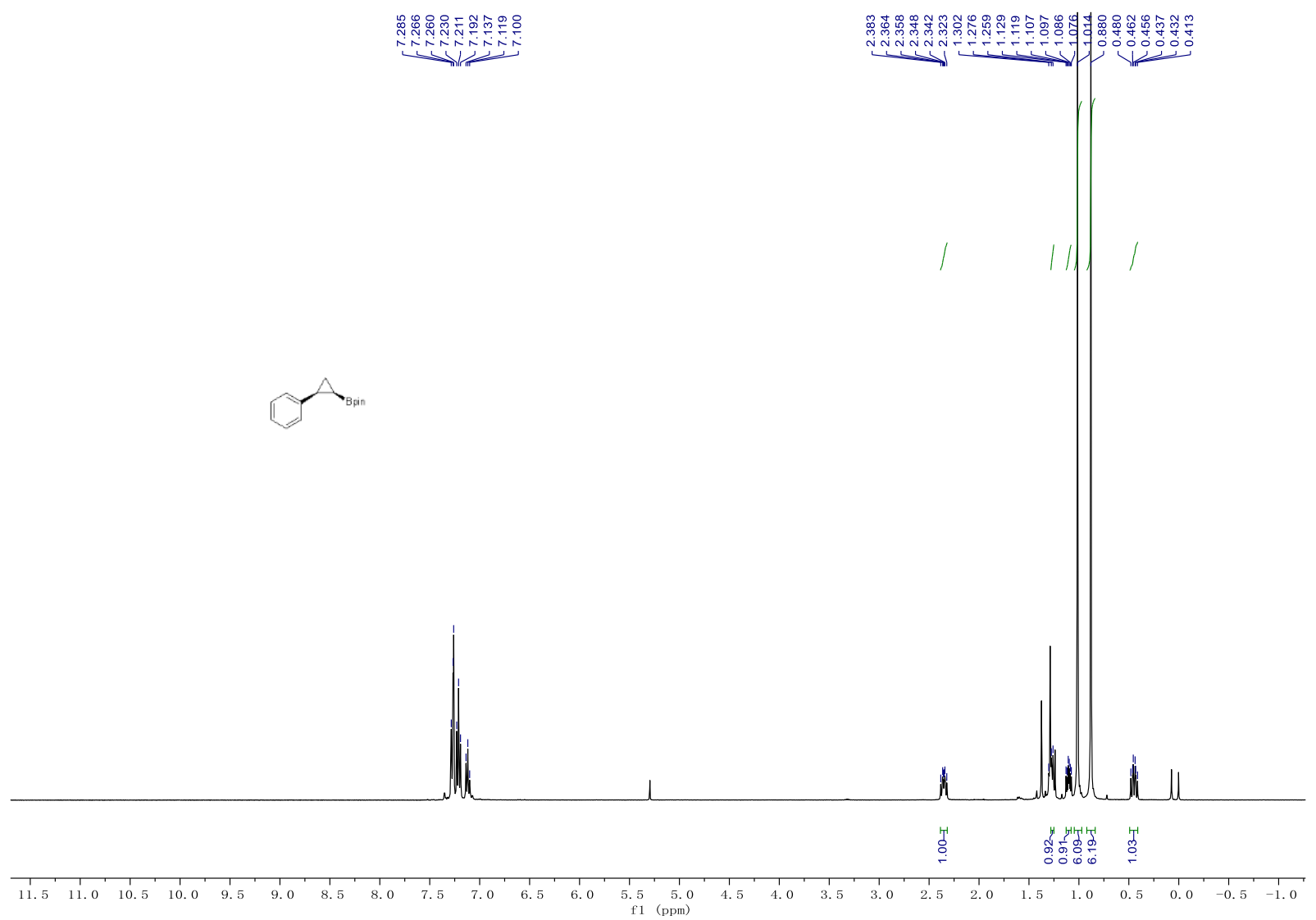




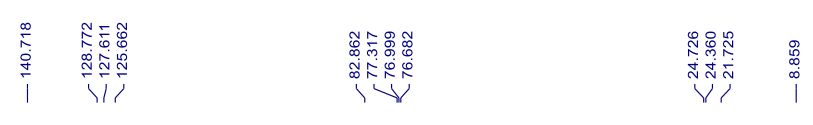
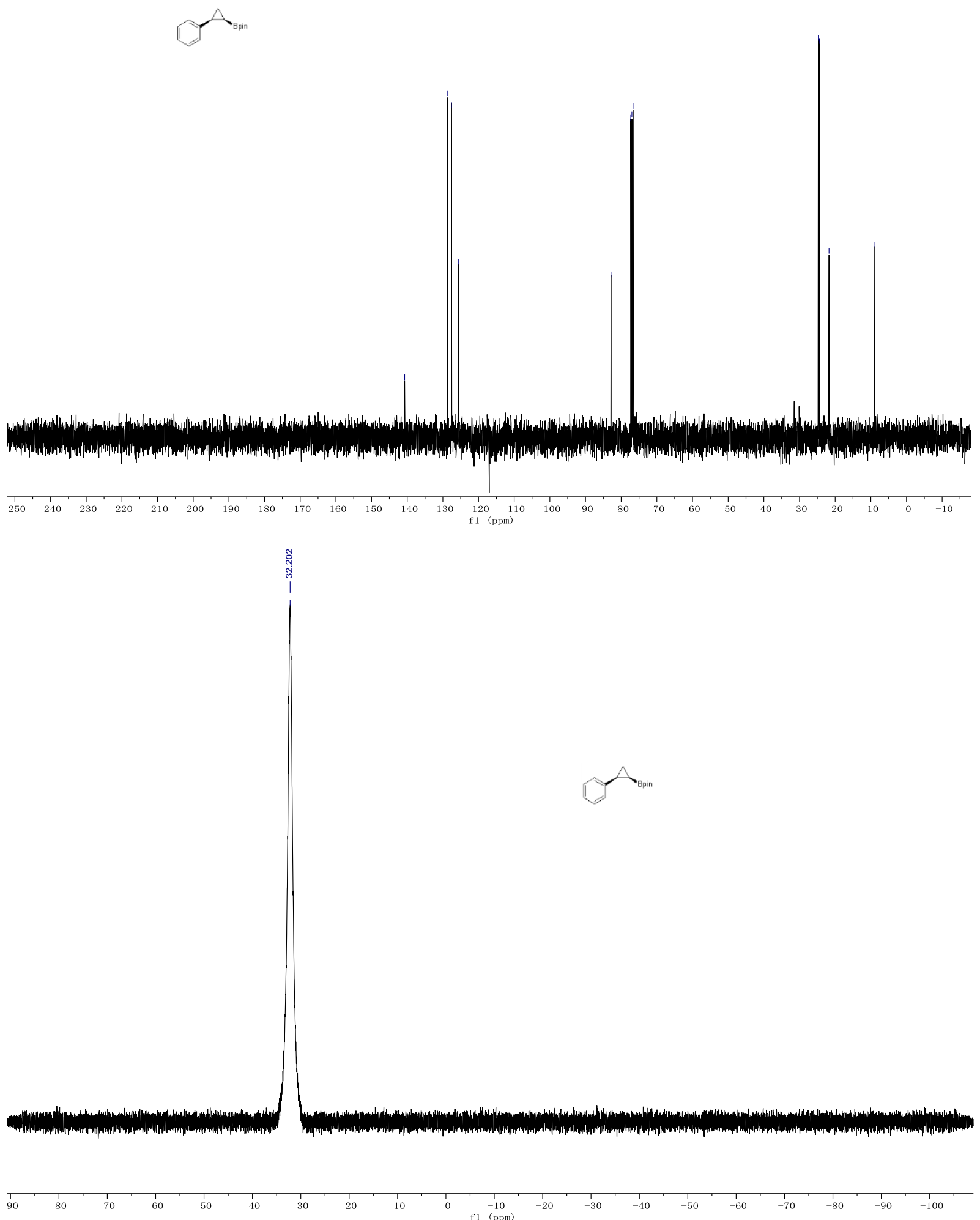


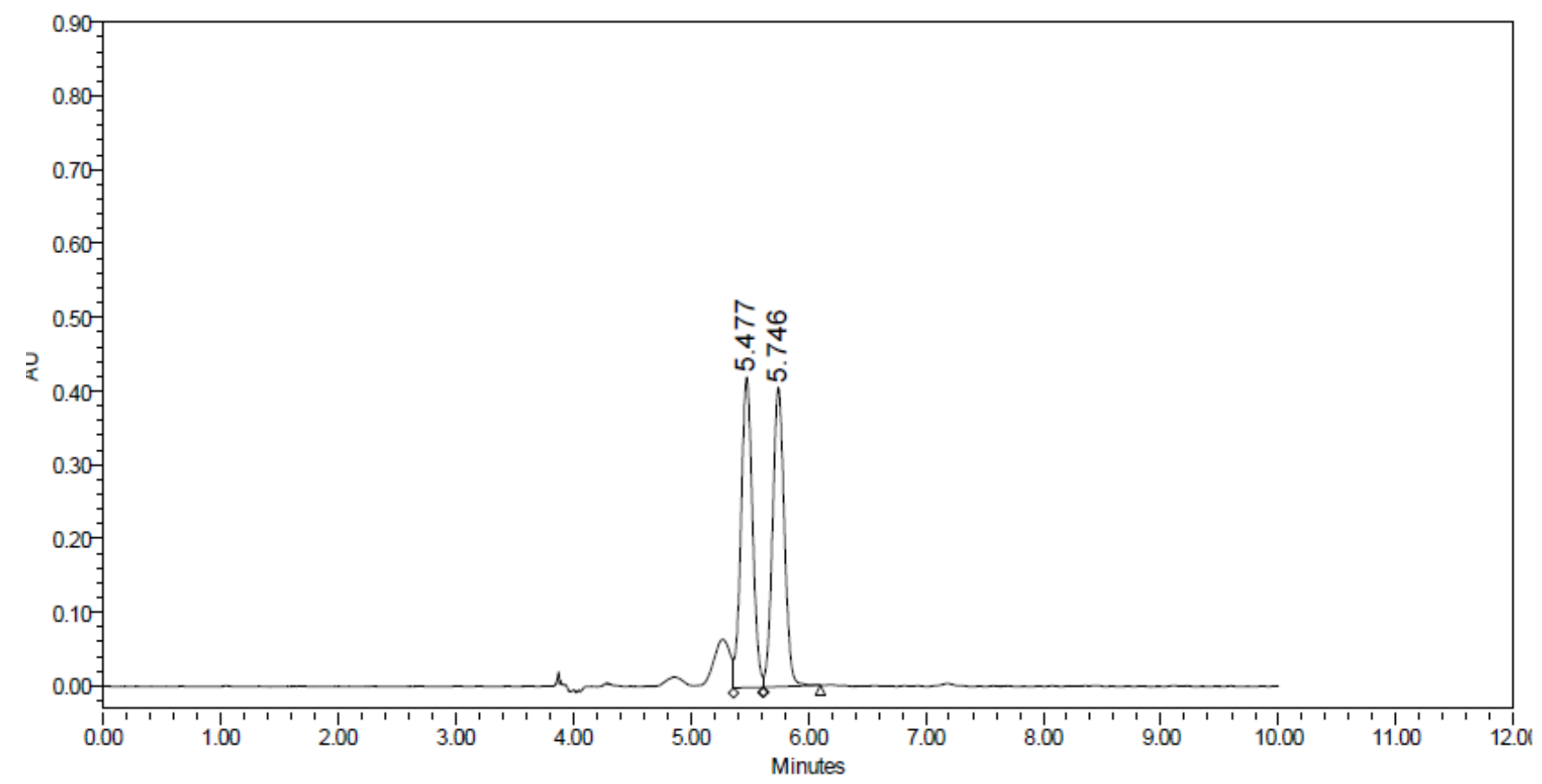

Peak Results

\begin{tabular}{|c|c|c|c|r|}
\hline & RT & Area & Height & $\%$ Area \\
\hline 1 & 5.477 & 2842922 & 420464 & 50.24 \\
\hline 2 & 5.746 & 2816236 & 405543 & 49.76 \\
\hline
\end{tabular}

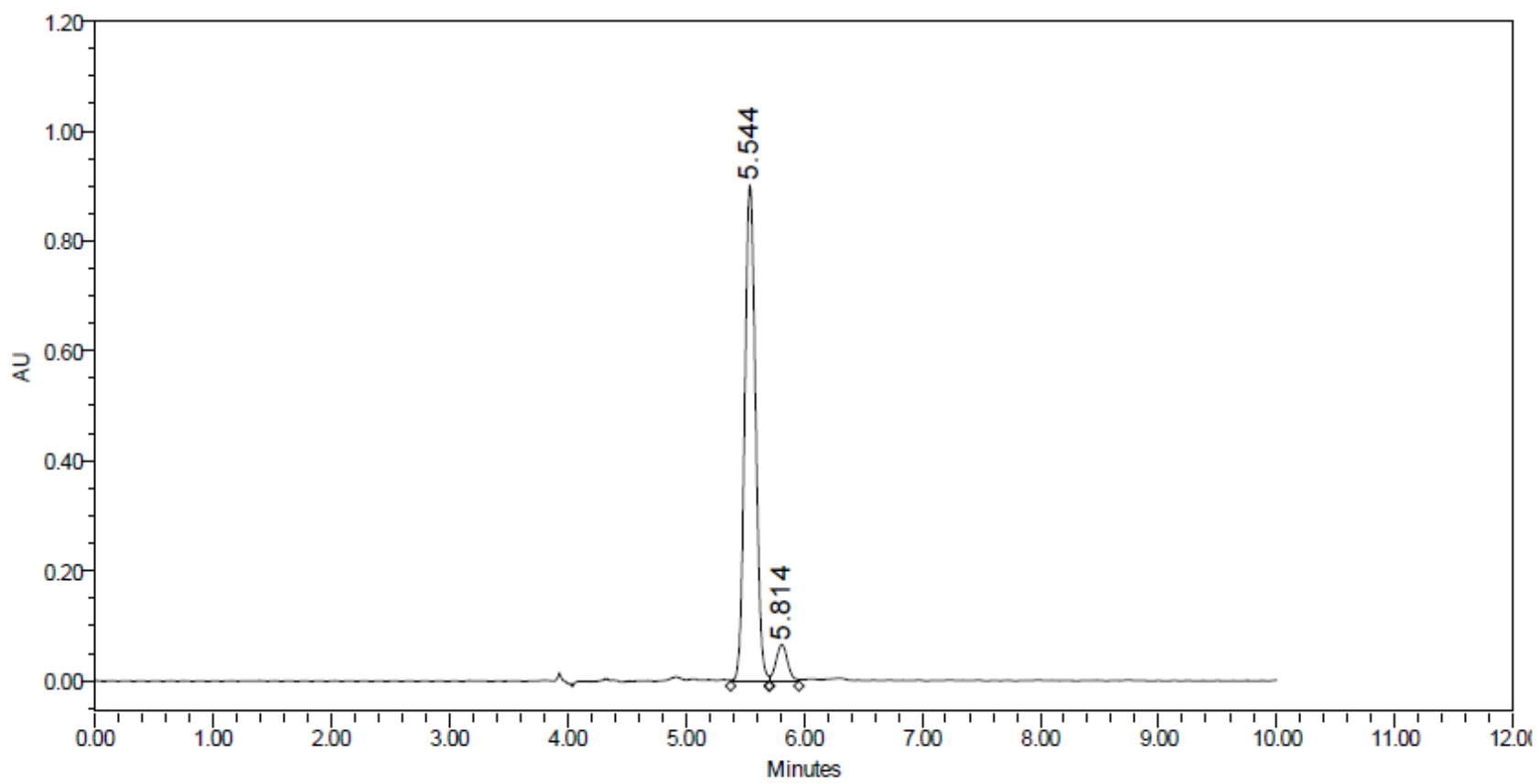

Peak Results
\begin{tabular}{|l|c|c|r|r|}
\hline & RT & Area & Height & $\%$ Area \\
\hline 1 & 5.544 & 5739901 & 902956 & 92.71 \\
\hline 2 & 5.814 & 451105 & 66845 & 7.29 \\
\hline
\end{tabular}


Methyldiphenyl((1R,2R)-2-phenylcyclopropyl)silane (15c).
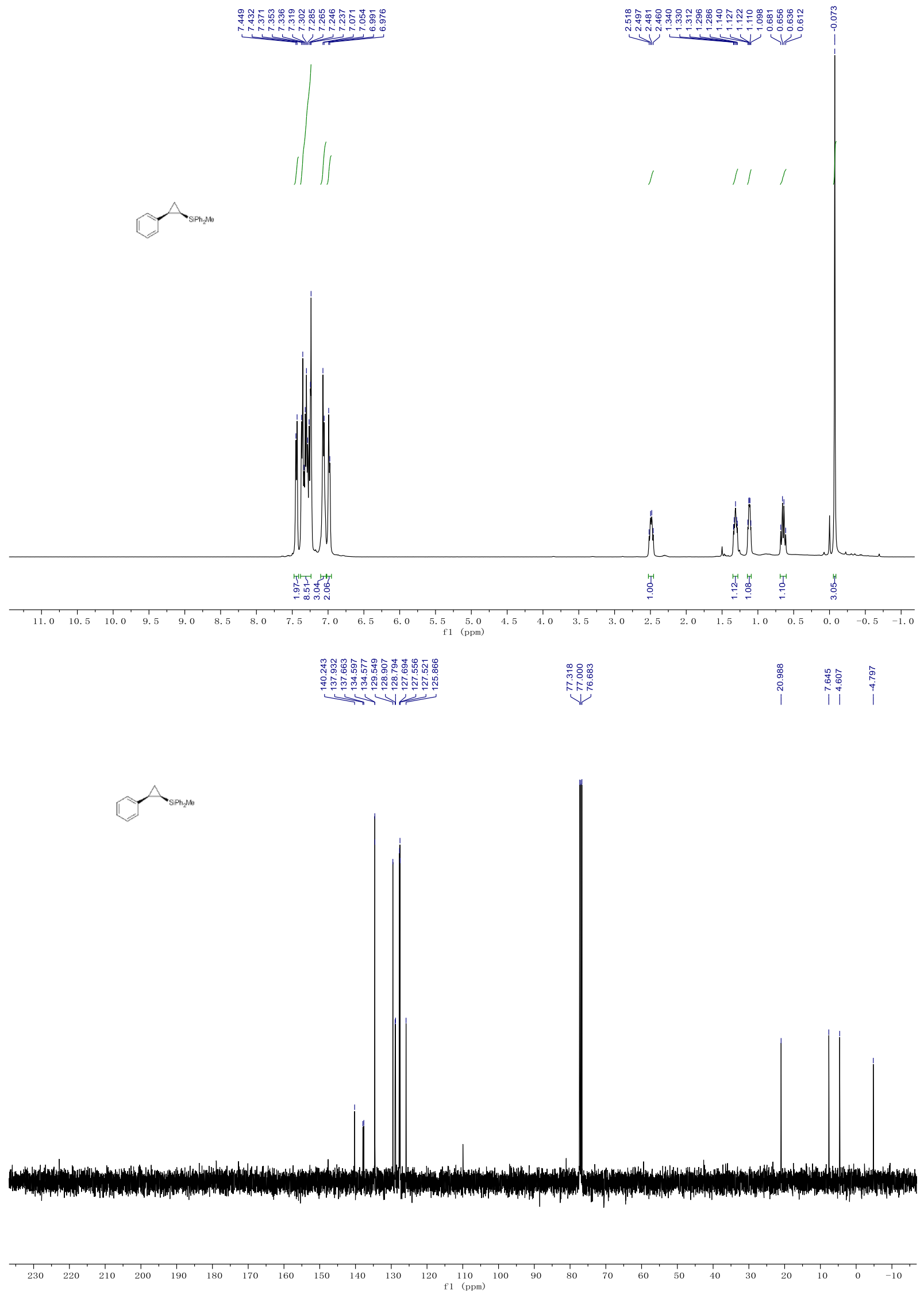


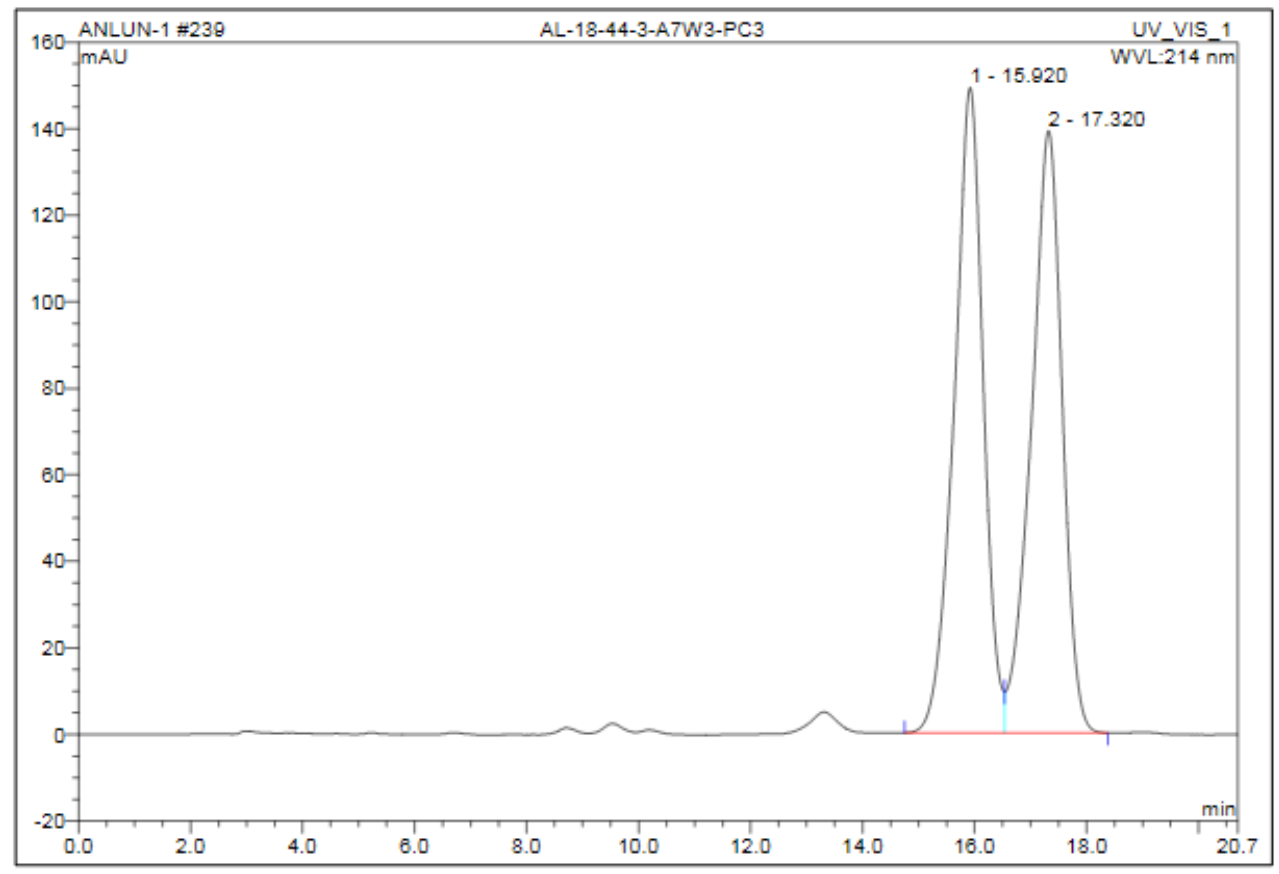

\begin{tabular}{|r|cccrrrr|}
\hline No. & $\begin{array}{c}\text { Ret.Time } \\
\text { min }\end{array}$ & Peak Name & $\begin{array}{c}\text { Height } \\
\text { mAU }\end{array}$ & $\begin{array}{c}\text { Area } \\
\text { mAU*min }\end{array}$ & $\begin{array}{r}\text { Rel.Area } \\
\%\end{array}$ & Amount & Type \\
\hline 1 & 15.92 & n.a. & 149.210 & 89.838 & 50.20 & n.a. & BM \\
2 & 17.32 & n.a. & 139.243 & 89.111 & 49.80 & n.a. & MB \\
\hline Total: & & & 288.453 & 178.948 & 100.00 & 0.000 & \\
\hline
\end{tabular}

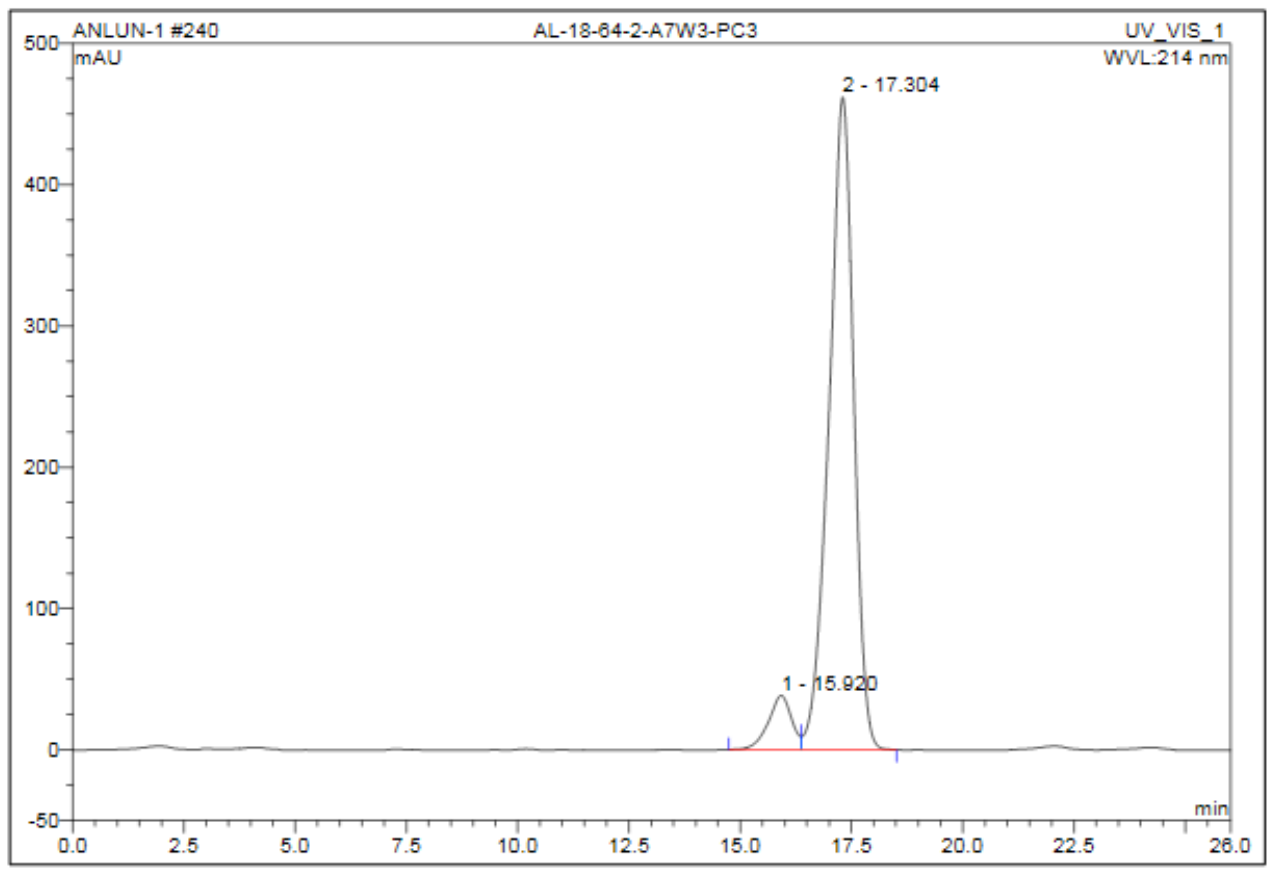

\begin{tabular}{|r|ccrrrrr|}
\hline No. & $\begin{array}{c}\text { Ret.Time } \\
\text { min }\end{array}$ & Peak Name & $\begin{array}{c}\text { Height } \\
\text { mAU }\end{array}$ & $\begin{array}{c}\text { Area } \\
\text { mAU*min }\end{array}$ & $\begin{array}{r}\text { Rel.Area } \\
\%\end{array}$ & Amount & Type \\
\hline 1 & 15.92 & n.a. & 38.441 & 23.453 & 7.29 & n.a. & BM \\
2 & 17.30 & n.a. & 461.866 & 298.233 & 92.71 & n.a. & MB \\
\hline Total: & & & 500.307 & 321.686 & 100.00 & 0.000 & \\
\hline
\end{tabular}


Phenyl((1R,2R)-2-phenylcyclopropyl)sulfane (15d).

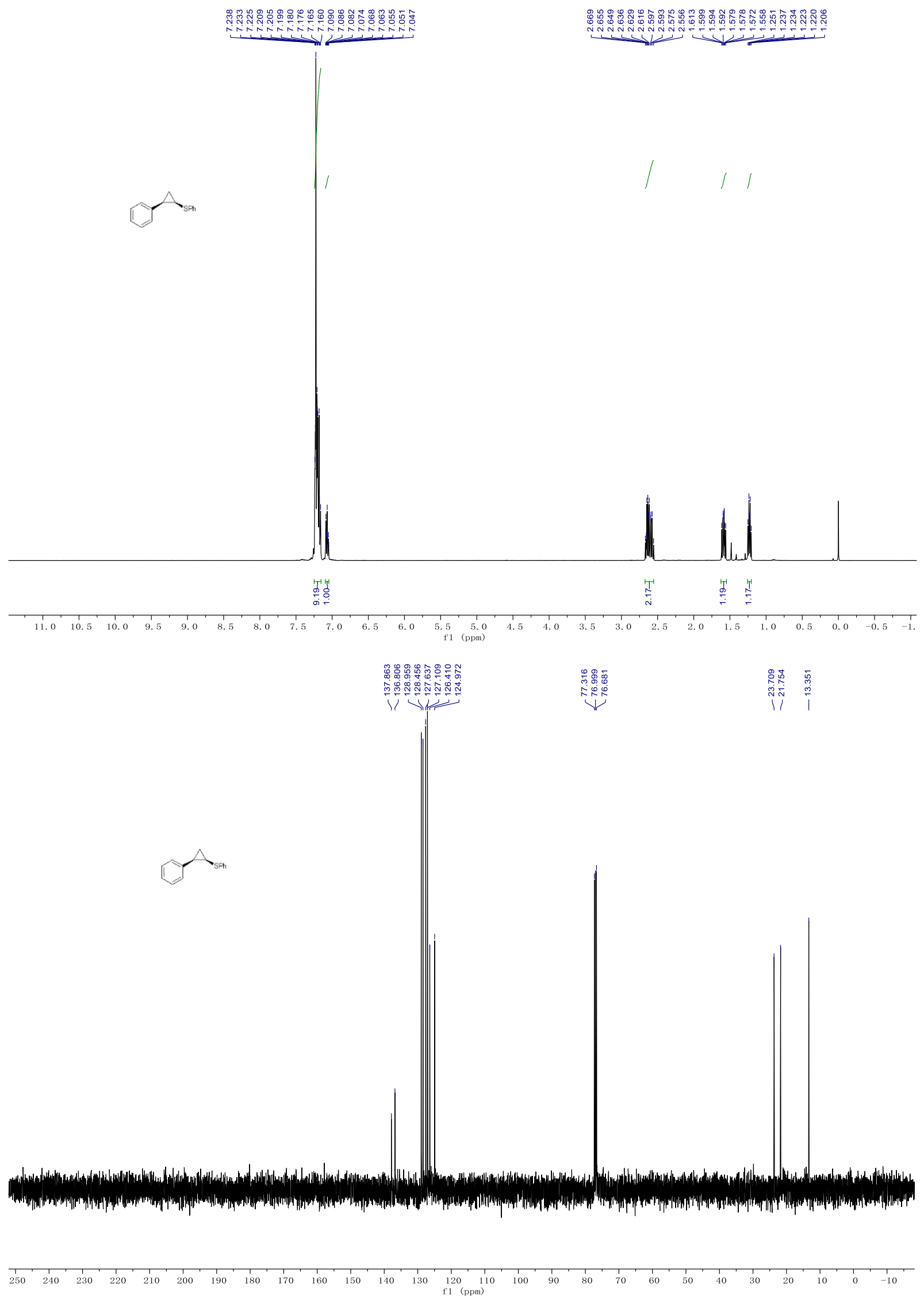




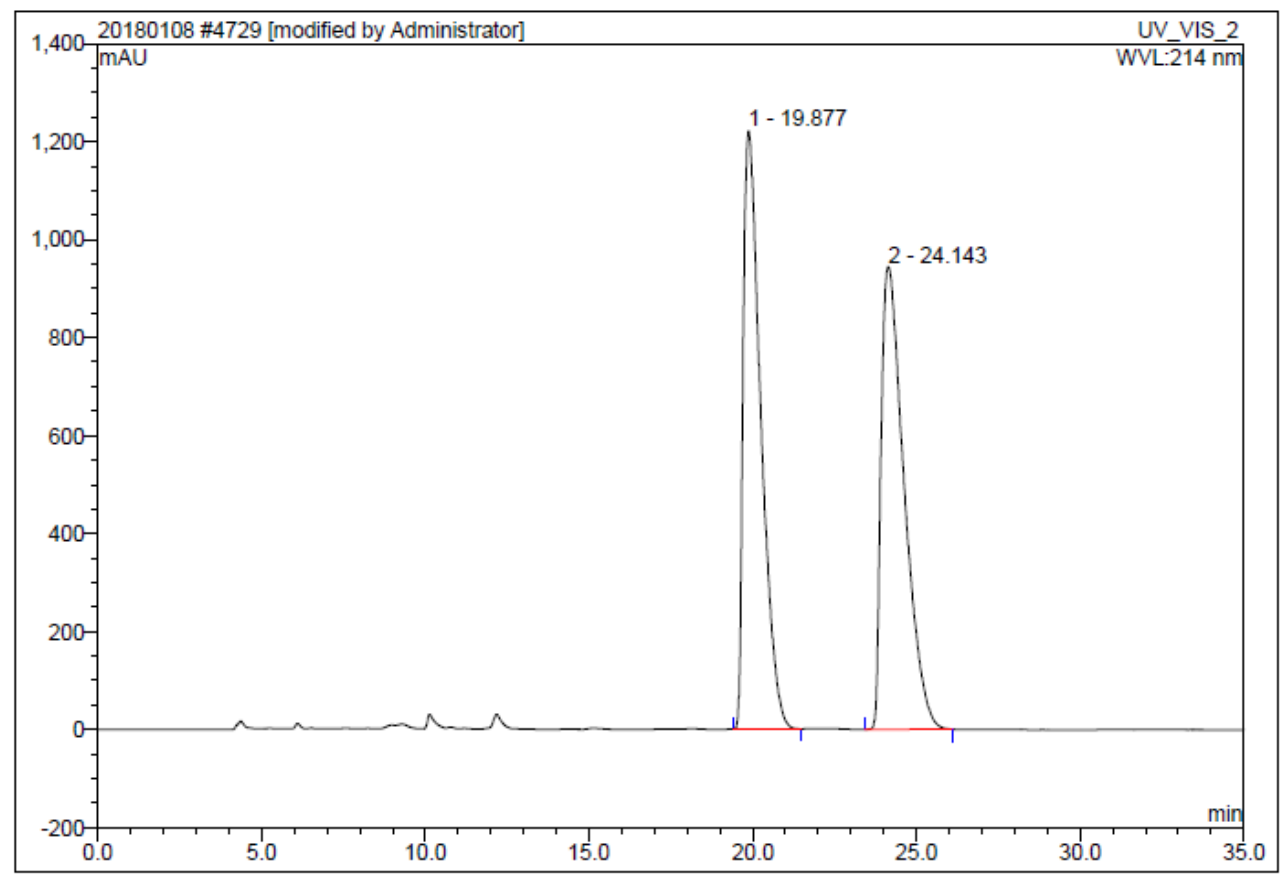

\begin{tabular}{|r|ccrrrrr|}
\hline No. & $\begin{array}{c}\text { Ret.Time } \\
\text { min }\end{array}$ & Peak Name & $\begin{array}{c}\text { Height } \\
\text { mAU }\end{array}$ & $\begin{array}{c}\text { Area } \\
\text { mAU*min }\end{array}$ & $\begin{array}{c}\text { Rel.Area } \\
\%\end{array}$ & Amount & Type \\
& 19.88 & n.a. & 1220.761 & 764.629 & 49.87 & n.a. & BMB \\
2 & 24.14 & n.a. & 943.723 & 768.516 & 50.13 & n.a. & BMB \\
\hline Total: & & & 2164.485 & 1533.146 & 100.00 & 0.000 & \\
\hline
\end{tabular}

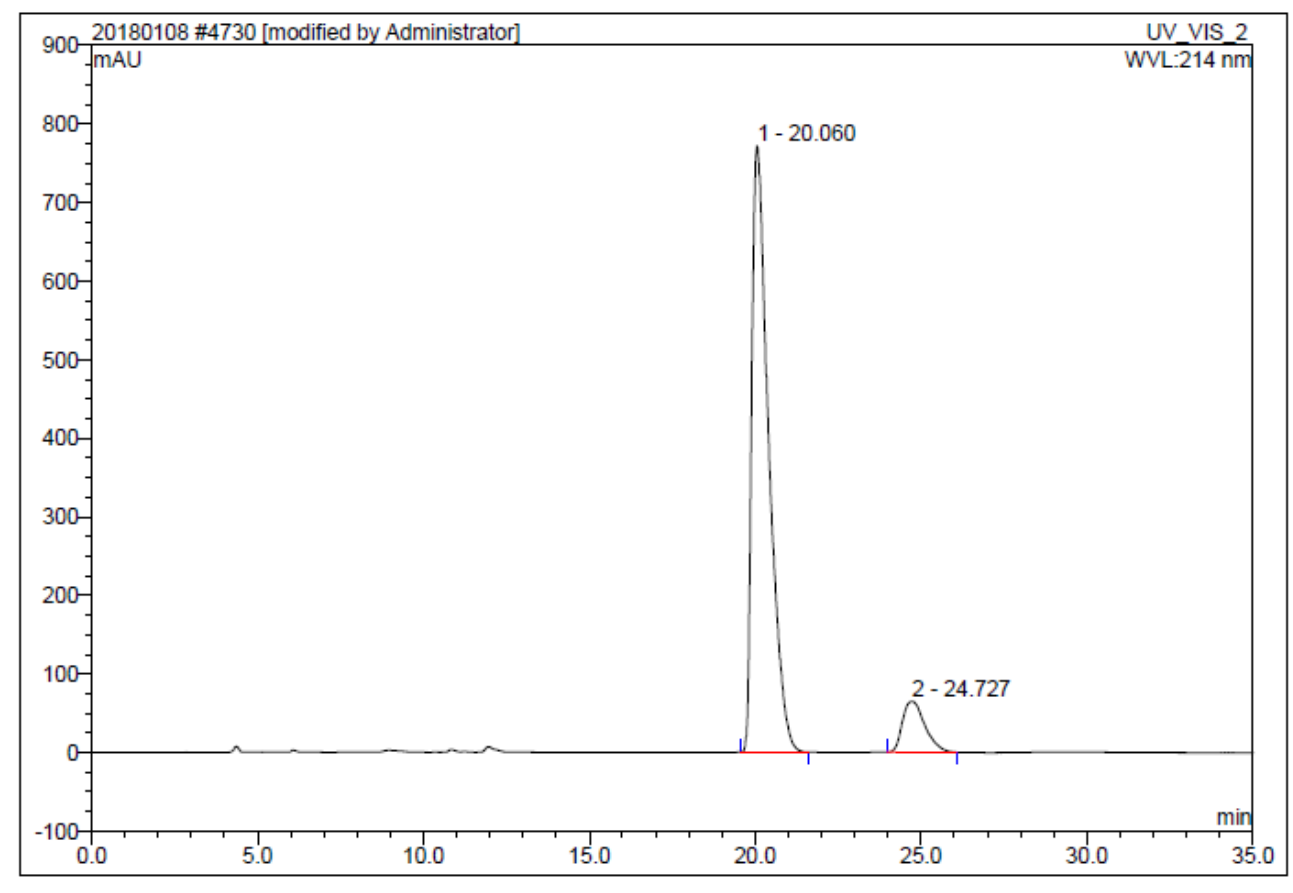

\begin{tabular}{|r|ccrrrrr|}
\hline No. & $\begin{array}{c}\text { Ret.Time } \\
\text { min }\end{array}$ & Peak Name & $\begin{array}{c}\text { Height } \\
\text { mAU }\end{array}$ & $\begin{array}{r}\text { Area } \\
\text { mAU*min }\end{array}$ & $\begin{array}{r}\text { Rel.Area } \\
\%\end{array}$ & Amount & Type \\
\hline 1 & 20.06 & n.a. & 771.949 & 454.985 & 89.95 & n.a. & BMB \\
2 & 24.73 & n.a. & 64.757 & 50.817 & 10.05 & n.a. & BMB \\
\hline Total: & & & 836.706 & 505.802 & 100.00 & 0.000 & \\
\hline
\end{tabular}


$(1 R, 2 S)$-2-Phenylcyclopropanecarboxylic acid (15e).
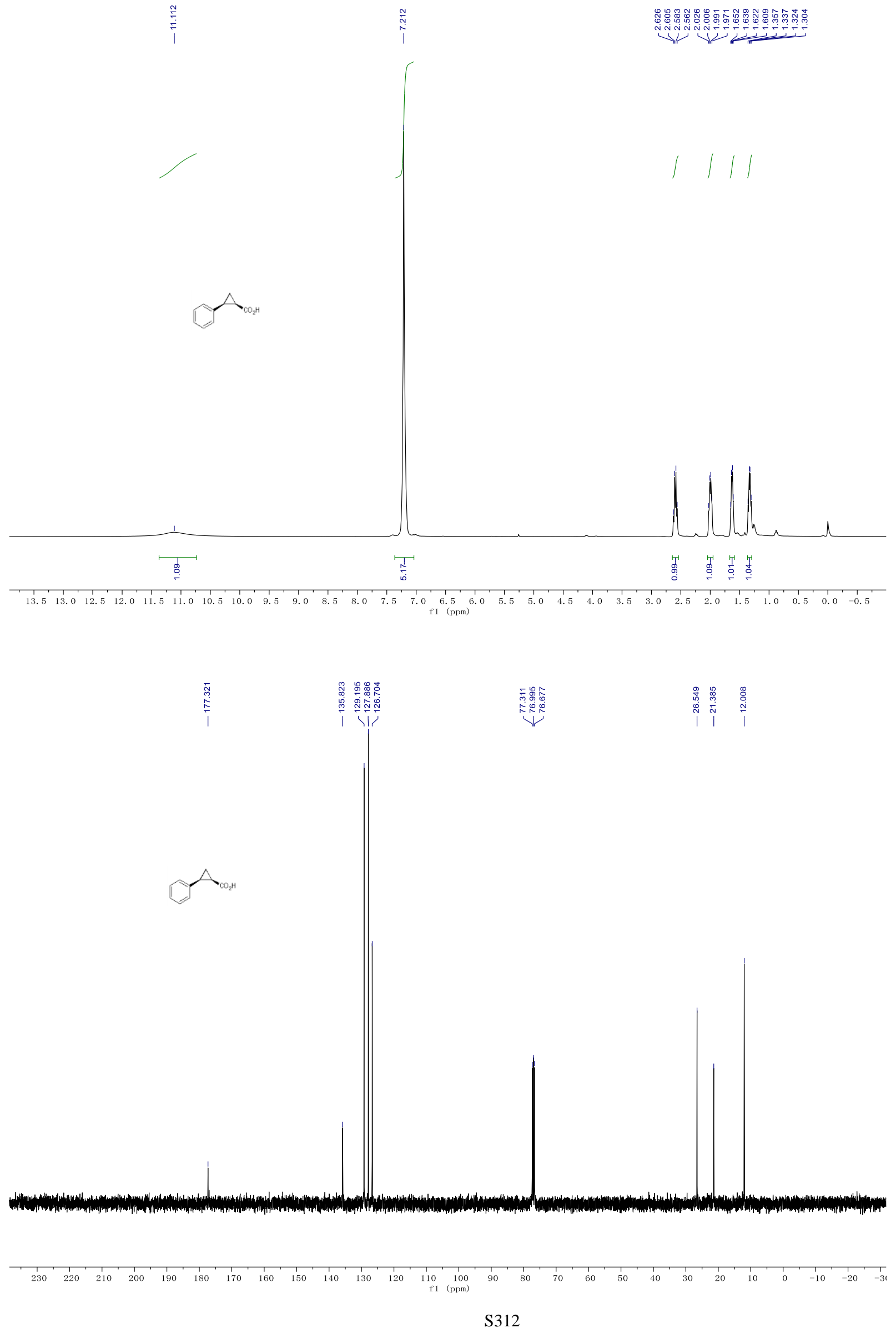


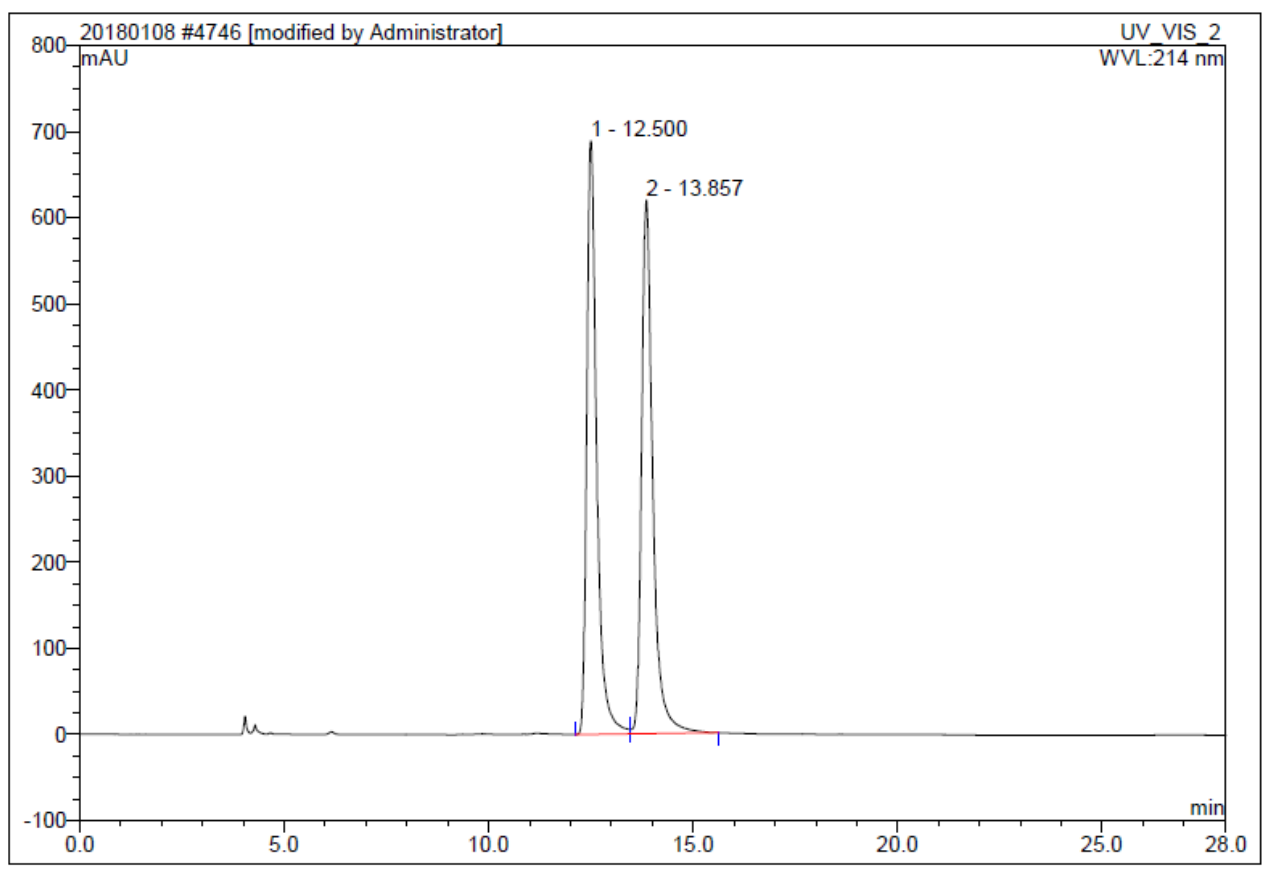

\begin{tabular}{|r|ccrrrrr|}
\hline No. & $\begin{array}{c}\text { Ret.Time } \\
\text { min }\end{array}$ & Peak Name & $\begin{array}{c}\text { Height } \\
\text { mAU }\end{array}$ & $\begin{array}{c}\text { Area } \\
\text { mAU*min }^{*}\end{array}$ & $\begin{array}{c}\text { Rel.Area } \\
\%\end{array}$ & Amount & Type \\
\hline 1 & 12.50 & n.a. & 689.611 & 194.735 & 49.57 & n.a. & BM \\
2 & 13.86 & n.a. & 620.174 & 198.108 & 50.43 & n.a. & MB \\
\hline Total: & & & 1309.785 & 392.843 & 100.00 & 0.000 & \\
\hline
\end{tabular}

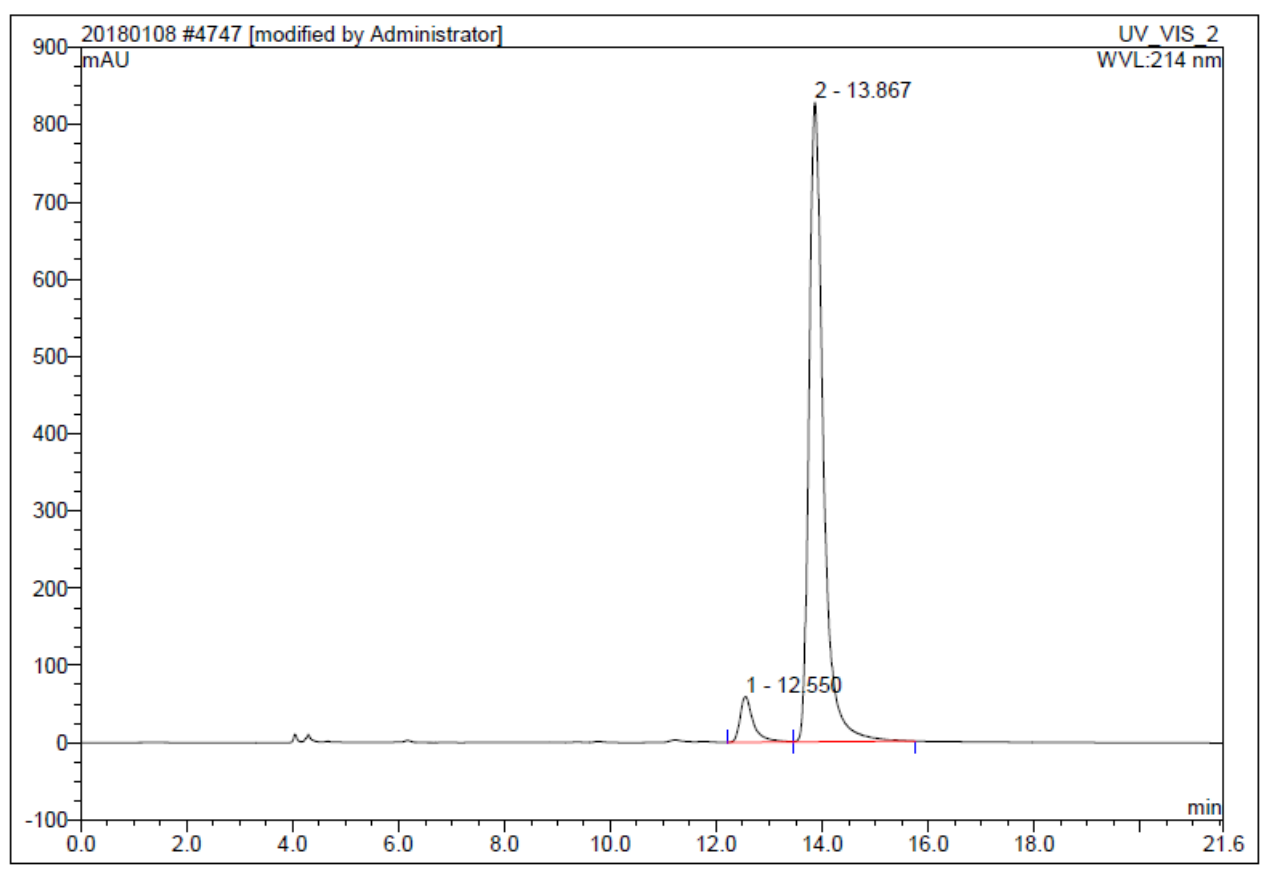

\begin{tabular}{|r|ccrrrrr|}
\hline No. & $\begin{array}{c}\text { Ret.Time } \\
\text { min }\end{array}$ & Peak Name & $\begin{array}{c}\text { Height } \\
\text { mAU }\end{array}$ & $\begin{array}{r}\text { Area } \\
\text { mAU*min }\end{array}$ & $\begin{array}{r}\text { Rel.Area } \\
\%\end{array}$ & Amount & Type \\
\hline 1 & 12.55 & n.a. & 58.853 & 17.296 & 6.27 & n.a. & BM \\
2 & 13.87 & n.a. & 827.766 & 258.513 & 93.73 & n.a. & MB \\
\hline Total: & & & 886.619 & 275.809 & 100.00 & 0.000 & \\
\hline
\end{tabular}


$(1 R, 2 S)-N, N$-Dimethyl-2-phenylcyclopropanecarboxamide (15f).
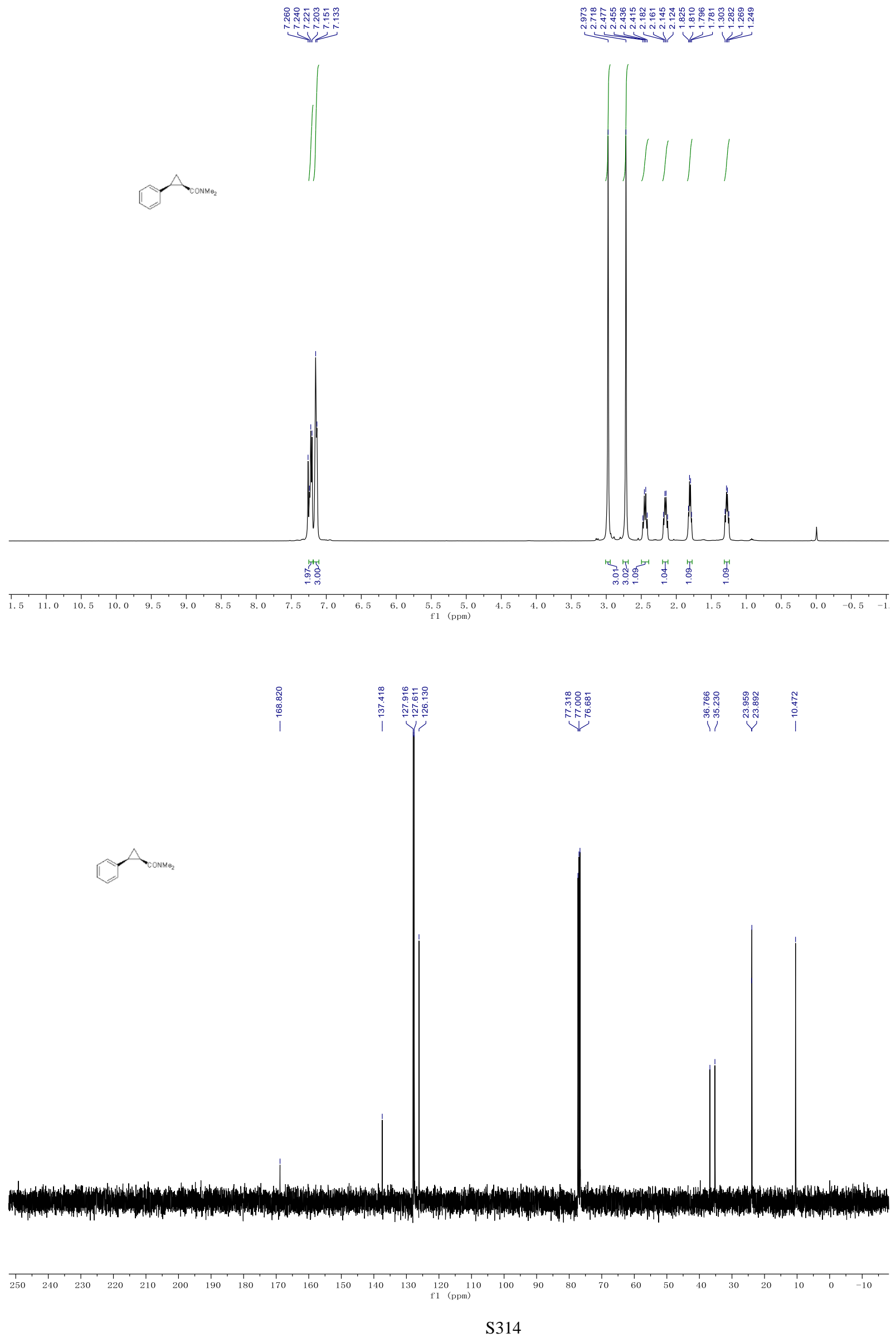


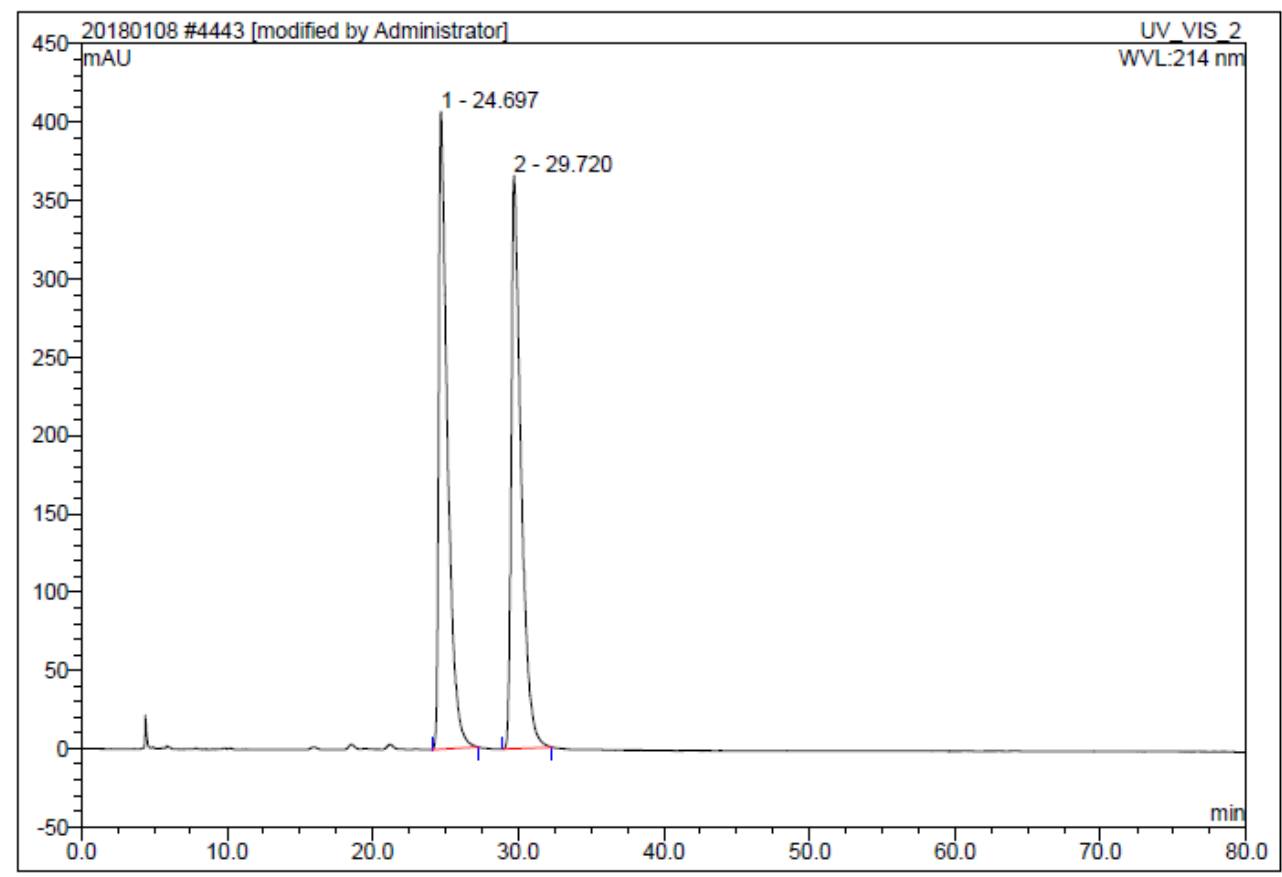

\begin{tabular}{|r|cccrrrr|}
\hline No. & $\begin{array}{c}\text { Ret.Time } \\
\text { min }\end{array}$ & Peak Name & $\begin{array}{c}\text { Height } \\
\text { mAU }\end{array}$ & $\begin{array}{c}\text { Area } \\
\text { mAU*min }\end{array}$ & $\begin{array}{c}\text { Rel.Area } \\
\%\end{array}$ & Amount & Type \\
\hline 1 & 24.70 & n.a. & 407.201 & 286.142 & 50.04 & n.a. & BMB \\
2 & 29.72 & n.a. & 366.022 & 285.639 & 49.96 & n.a. & BMB \\
\hline Total: & & & 773.223 & 571.782 & 100.00 & 0.000 & \\
\hline
\end{tabular}

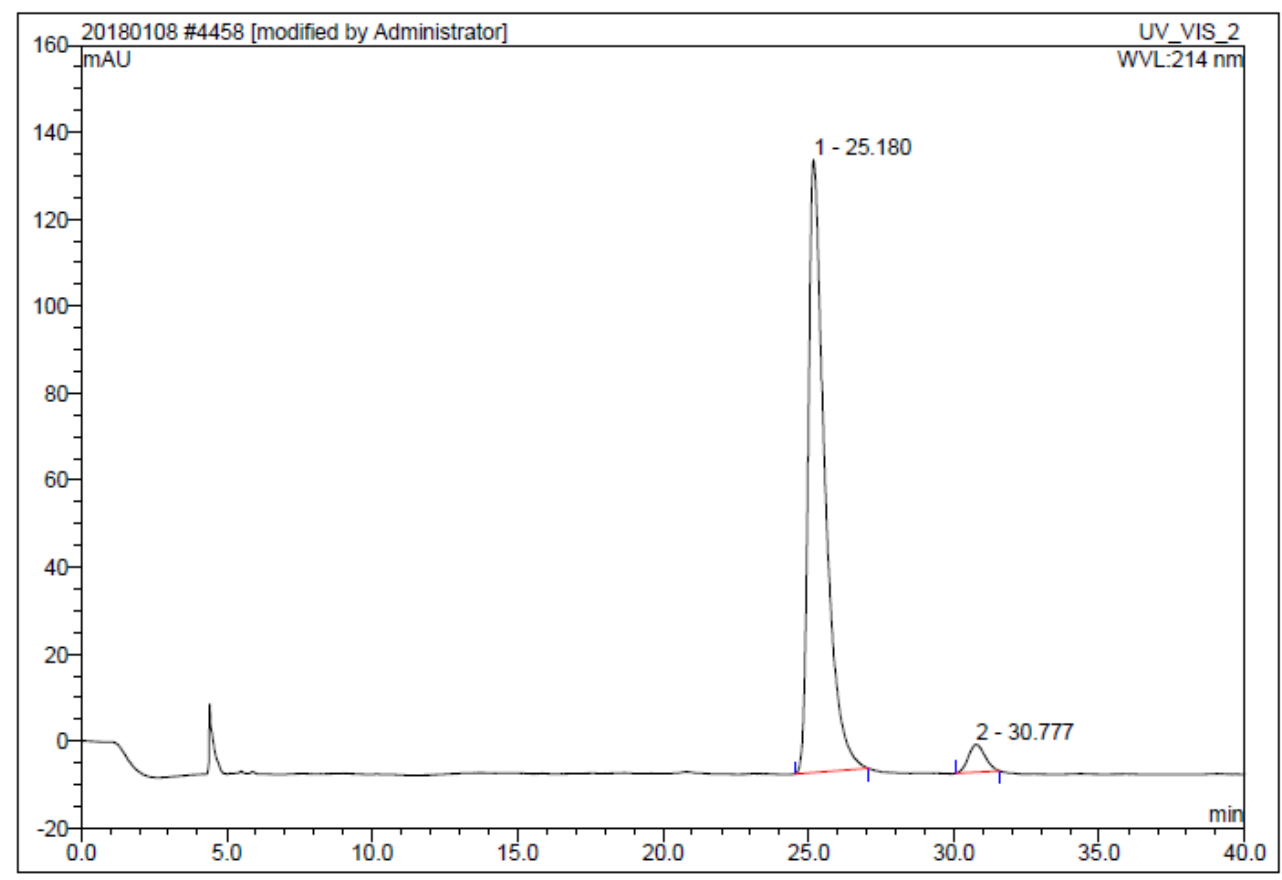

\begin{tabular}{|r|ccrrrrr|}
\hline No. & $\begin{array}{c}\text { Ret.Time } \\
\text { min }\end{array}$ & Peak Name & $\begin{array}{c}\text { Height } \\
\text { mAU }\end{array}$ & $\begin{array}{c}\text { Area } \\
\text { mAU*min }\end{array}$ & $\begin{array}{c}\text { Rel.Area } \\
\%\end{array}$ & Amount & Type \\
\hline 1 & 25.18 & n.a. & 140.931 & 93.156 & 95.69 & n.a. & BMB \\
2 & 30.78 & n.a. & 6.325 & 4.199 & 4.31 & n.a. & BMB \\
\hline Total: & & & 147.256 & 97.355 & 100.00 & 0.000 & \\
\hline
\end{tabular}


$(1 R, 2 S)$-2-Phenylcyclopropanecarbonitrile (15g).
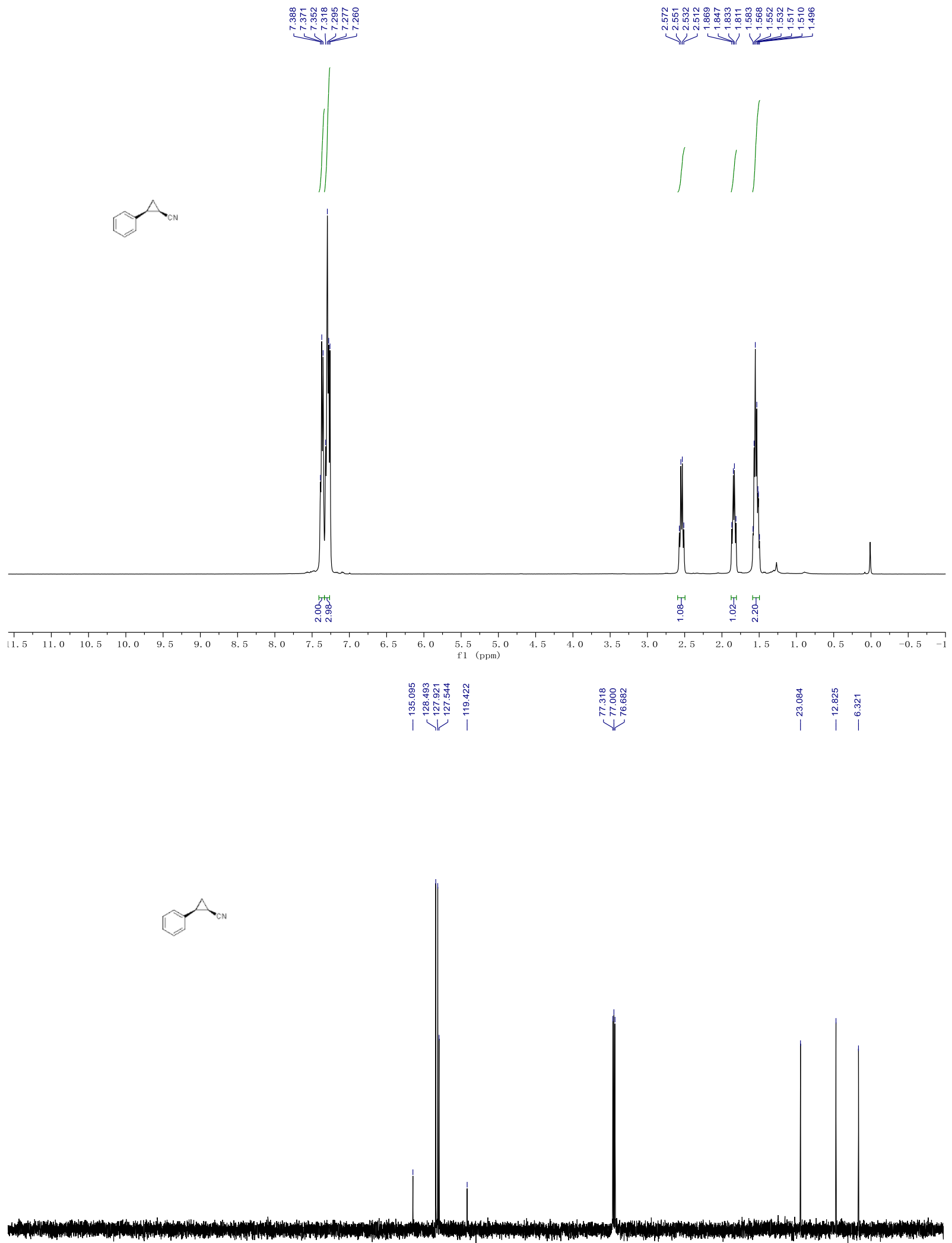

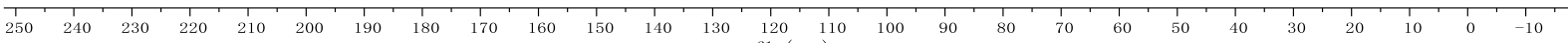




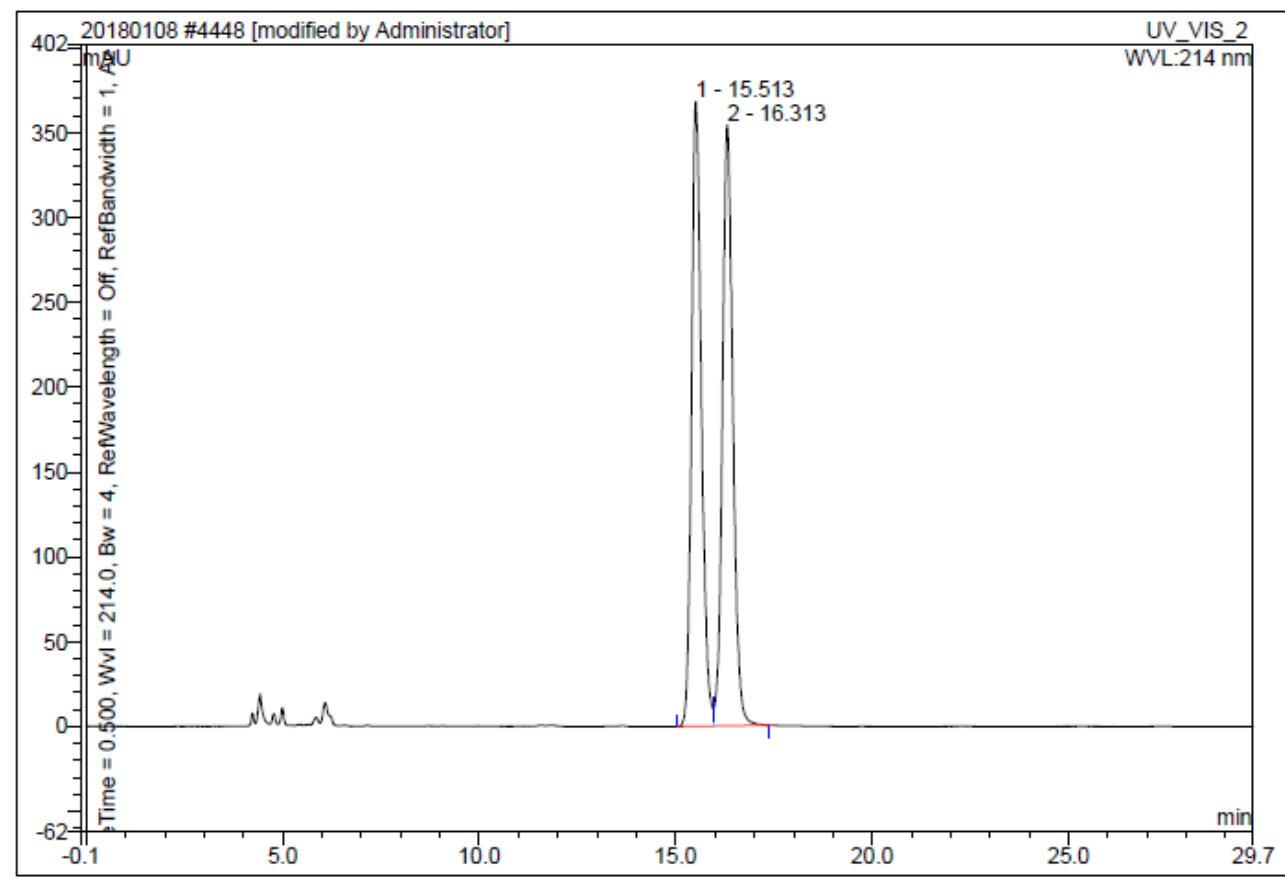

\begin{tabular}{|r|cccrrrr|}
\hline No. & $\begin{array}{c}\text { Ret.Time } \\
\text { min }\end{array}$ & Peak Name & $\begin{array}{c}\text { Height } \\
\text { mAU }\end{array}$ & $\begin{array}{c}\text { Area } \\
\text { mAU*min }\end{array}$ & $\begin{array}{r}\text { Rel.Area } \\
\%\end{array}$ & Amount & Type \\
\hline 1 & 15.51 & n.a. & 368.373 & 107.437 & 49.52 & n.a. & BM \\
2 & 16.31 & n.a. & 354.365 & 109.503 & 50.48 & n.a. & MB \\
\hline Total: & & & 722.738 & 216.940 & 100.00 & 0.000 & \\
\hline
\end{tabular}

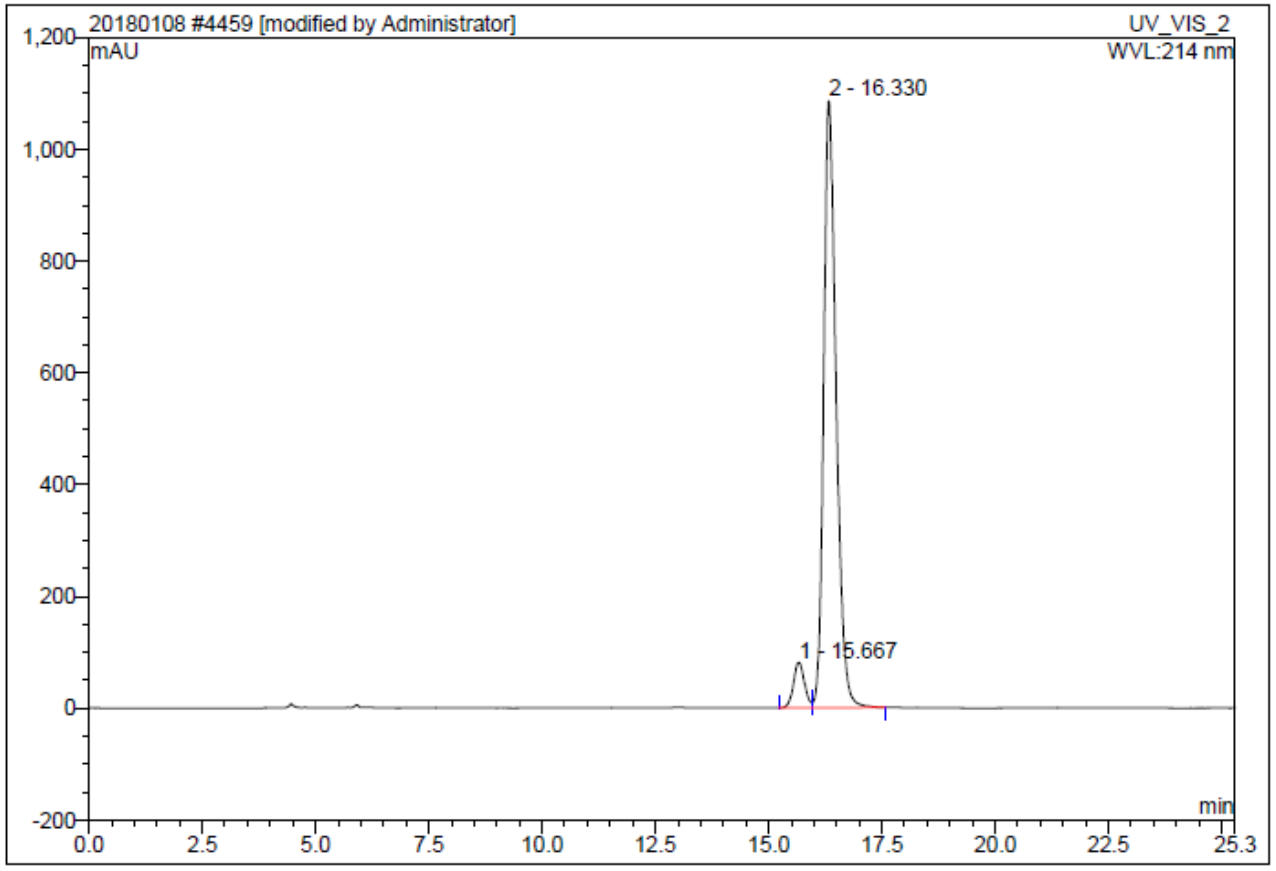

\begin{tabular}{|r|ccrrrrr|}
\hline No. & $\begin{array}{c}\text { Ret.Time } \\
\text { min }\end{array}$ & Peak Name & $\begin{array}{c}\text { Height } \\
\text { mAU }\end{array}$ & $\begin{array}{c}\text { Area } \\
\text { mAU*min }\end{array}$ & $\begin{array}{r}\text { Rel.Area } \\
\%\end{array}$ & Amount & Type \\
\hline 1 & 15.67 & n.a. & 81.597 & 22.915 & 6.20 & n.a. & BM \\
2 & 16.33 & n.a. & 1085.307 & 346.427 & 93.80 & n.a. & MB \\
\hline Total: & & & 1166.904 & 369.342 & 100.00 & 0.000 & \\
\hline
\end{tabular}

